Nadine Bernhard

\title{
Durch Europäisierung zu mehr Durchlässigkeit?
}

Veränderungsdynamiken des Verhältnisses von beruflicher Bildung zur Hochschulbildung in Deutschland und Frankreich 
Nadine Bernhard

Durch Europäisierung zu mehr Durchlässigkeit? 
Nadine Bernhard

\section{Durch Europäisierung zu mehr Durchlässigkeit?}

Veränderungsdynamiken des Verhältnisses von beruflicher Bildung zur Hochschulbildung in Deutschland und Frankreich

Budrich UniPress Ltd.

Opladen • Berlin • Toronto 2017 
Bibliografische Information der Deutschen Nationalbibliothek

Die Deutsche Nationalbibliothek verzeichnet diese Publikation in der Deutschen

Nationalbibliografie; detaillierte bibliografische Daten sind im Internet über

http://dnb.d-nb.de abrufbar.

(C) 2017 Dieses Werk ist bei Budrich UniPress erschienen und steht unter folgender Creative Commons Lizenz: http://creativecommons.org/licenses/by-nc-nd/3.0/de/ Verbreitung, Speicherung und Vervielfältigung erlaubt, kommerzielle Nutzung und Veränderung nur mit Genehmigung des Verlags Budrich UniPress.

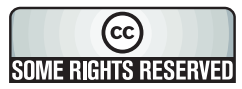

Dieses Buch steht im OpenAccess Bereich der Verlagsseite zum kostenlosen Download bereit (http://dx.doi.org/10.3224/86388261)

Eine kostenpflichtige Druckversion (Printing on Demand) kann über den Verlag bezogen werden. Die Seitenzahlen in der Druck- und Onlineversion sind identisch.

D188

$$
\begin{array}{lll}
\text { ISBN } & 978-3-86388-706-3 & \text { (Paperback) } \\
\text { eISBN } & 978-3-86388-261-7 & \text { (eBook) }
\end{array}
$$

Das Werk einschließlich aller seiner Teile ist urheberrechtlich geschützt. Jede Verwertung außerhalb der engen Grenzen des Urheberrechtsgesetzes ist ohne Zustimmung des Verlages unzulässig und strafbar. Das gilt insbesondere für Vervielfältigungen, Übersetzungen, Mikroverfilmungen und die Einspeicherung und Verarbeitung in elektronischen Systemen.

Umschlaggestaltung: Bettina Lehfeldt, Kleinmachnow - www.lehfeldtgraphic.de Typographisches Lektorat: Anja Borkam, Jena 


\section{Inhaltsverzeichnis}

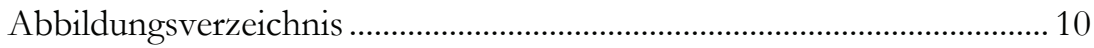

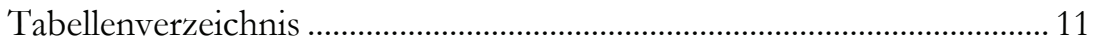

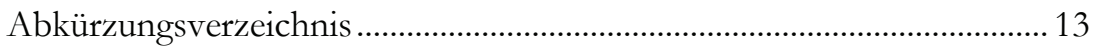

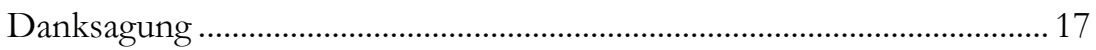

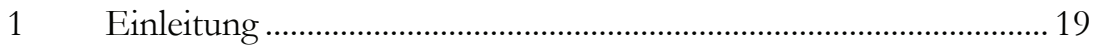

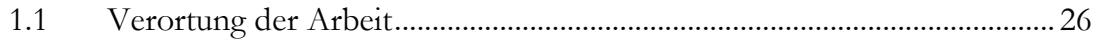

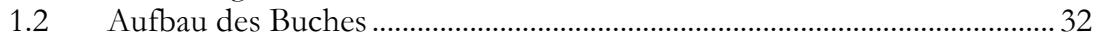

2 Durchlässigkeit - Eine konzeptionelle und theoretische Annäherung..................................................................................... 39

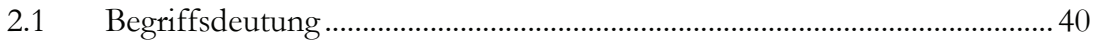

2.1.1 Soziale und institutionelle Durchlässigkeit ....................................................... 40

2.2 Identifikation eines Durchlässigkeitskonzepts.............................................. 42

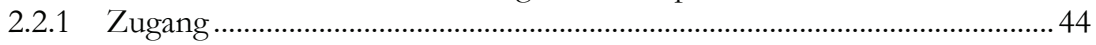

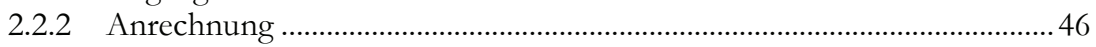

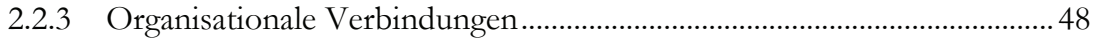

2.2.4 Heterogenität der Lernenden......................................................................... 51

2.2.5 Anerkennung in anderen gesellschaftlichen Teilsystemen .........................56

2.2.6 Zusammenschau der Aspekte von Durchlässigkeit.................................... 57

2.3 Bildungsstrukturen als funktionales Erfordernis oder Legitimation bestehender Herrschaftsverhältnisse? ............................................................. 62

2.3.1 Selektions- und Sozialisationsfunktion von Bildung - Eine strukturfunktionalistische Perspektive .......................................................... 63

2.3.2 Allokations- und Legitimationsfunktion von Bildung - Eine
institutionelle und konflikttheoretische Perspektive...................................65

3 Theoretischer Rahmen zur Analyse institutionellen Wandels ...... 69

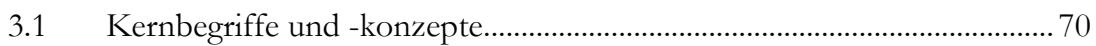

3.1.1 Diskursbegriff und Diskursanalyse ................................................................ 70

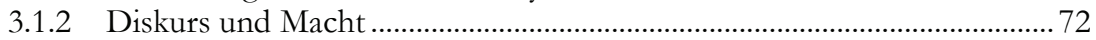

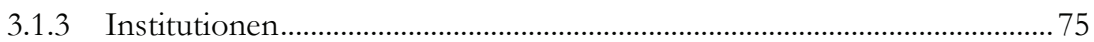

3.1.4 Diskurs und Institutionen ........................................................................... 79 
3.1.5 Organisation und Organisationsfeld............................................................ 80

3.1.6 Legitimität und lose Kopplung.................................................................... 82

3.2 Institutionellen Wandel konzeptualisieren ...................................................... 84

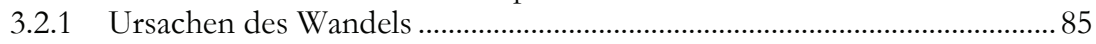

3.2.2 Ergebnisse des Wandels.................................................................................... 91

3.3 Gründe für die Verbindung einer diskursanalytischen
Forschungsperspektive mit neoinstitutionellen Theorien ........................ 95

3.3.1 Soziologischer Neoinstitutionalismus und seine Kritik ............................. 95

3.3.2 Stärken der diskurstheoretischen Perspektive .............................................. 96

3.3.3 Stärken der neoinstitutionalistischen Perspektive ......................................... 99

$4 \quad$ Design und Methoden ....................................................................... 103

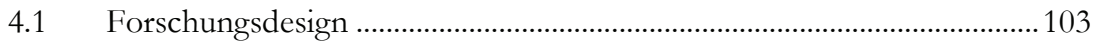

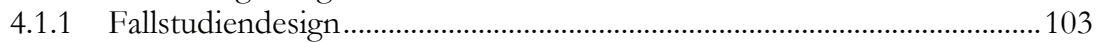

4.1.2 Fallauswahl und Vergleich.............................................................................. 110

4.2 Erhebungs- und Analyseverfahren ............................................................116

4.2.1 Wissenssoziologische Diskursanalyse.........................................................116

4.2.2 Theoriegeleitete qualitative Inhaltsanalyse ................................................130

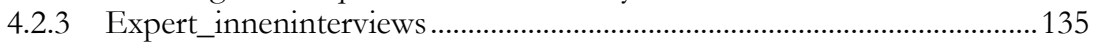

4.2.4 Dokumentenanalyse .................................................................................... 140

$4.3 \quad$ Güte der Arbeit ....................................................................................... 142

5 Durchlässigkeit - ein Fokus europäischer Bildungspolitik? ........ 147

5.1 Berufs- und Hochschulbildung in der europäischen Bildungspolitik -

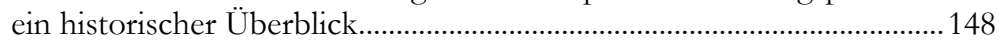

5.1.1 Europäische Bildungspolitik 1951-2000 ……………………..................... 148

5.1.2 Überblick über die Bologna- und Kopenhagen-Prozesse ........................155

5.2 Durchlässigkeit in den Bologna- und Kopenhagen-Prozessen ...............163

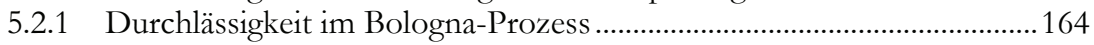

5.2.2 Durchlässigkeit im Kopenhagen-Prozess ....................................................... 176

5.2.3 Durchlässigkeit in den Bologna- und Kopenhagen-Prozessen im Vergleich.

6 Der deutsche Fall - Bildungssystem, Geschichte und das Problem der Durchlässigkeit............................................................. 195

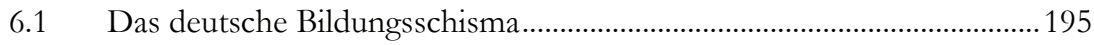

6.2 Beschreibung der Organisationsfelder .......................................................199

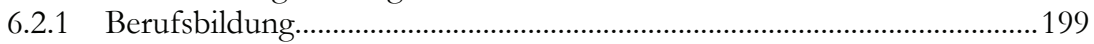

6.2.2 Hochschulbildung .....................................................................................204 
6.2.3 Bildungswege zwischen Berufs- und Hochschulbildung ..........................2207

6.3 Verhältnis von Berufs- und Hochschulbildung aus historischer

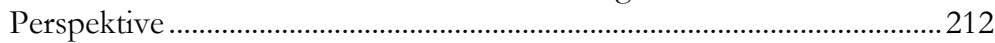

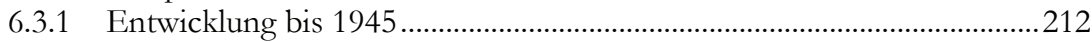

6.3.2 Entwicklung von 1945 bis 1989 ..................................................................215

7 Entwicklung institutioneller Durchlässigkeit in Deutschland.....219

7.1 Gesellschaftliche Wissensbestände zu Durchlässigkeit vor Bologna und Kopenhagen (1990-1998) ................................................................... 220

7.1.1 Schlaglichter der deutschen Debatten zu Durchlässigkeit (1990-1998) 220

7.1.2 Deutsche Diskurse 1990-1998: Durchlässigkeit als Diskurs über Zugang und Gleichwertigkeit .225

7.2 Gesellschaftliche Wissensbestände zu Durchlässigkeit in Zeiten von Bologna und Kopenhagen (1999-2012) ......................................................248

7.2.1 Schlaglichter der deutschen Debatten zu Durchlässigkeit (1999-2012) 249

7.2.2 Deutsche Diskurse 1999-2012: Durchlässigkeit als Norm zwischen Bildungsschisma und neuer Gleichwertigkeit ..........................................259

7.3 Analyse des institutionellen Wandels ...........................................................290

7.3.1 Veränderungen der institutionellen Strukturen in Richtung Durchlässigkeit

7.3.2 Fortführung institutioneller Entwicklungspfade - Barrieren für

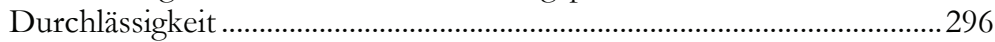

7.3.3 Zusammenfassung und Schlussfolgerungen ..................................................301

8 Der französische Fall: Bildungssystem, Geschichte und das Problem der Durchlässigkeit.......................................................... 303

8.1 Das Problem der ,segregativen Demokratisierung“ in Frankreich ........303

8.2 Beschreibung der Organisationsfelder ..........................................................307

8.2.1 Bildungswege nach dem, collège' ...................................................................307

8.2.2 Berufsbildung der Sekundarstufe II.............................................................309

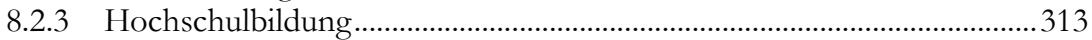

8.2.4 Bildungswege zwischen Berufs- und Hochschulbildung ............................319

8.3 Verhältnis von Berufs- und Hochschulbildung aus historischer Perspektive

8.3.1 Französisches Bildungswesen bis 1958: Klare Trennung zwischen höherer Allgemein- und beruflicher Bildung... 324

8.3.2 Entstehung des modernen französischen Bildungssystems: Prozesse der Entwertung beruflicher Bildung im Sekundarbereich. .327

9 Entwicklung institutioneller Durchlässigkeit in Frankreich .........331 
9.1 Gesellschaftliche Wissensbestände zu Durchlässigkeit vor Bologna und Kopenhagen (1990-1998)

9.1.1 Schlaglichter der französischen Debatten zu Durchlässigkeit (1985-1998)

9.1.2 Französischer Diskurs 1985-1998: Durchlässigkeit via Weiterbildung

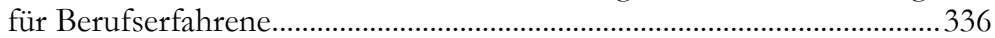

9.2 Gesellschaftliche Wissensbestände zur Durchlässigkeit in Zeiten von Bologna und Kopenhagen (1999-2012)

9.2.1 Schlaglichter der französischen Debatten zu Durchlässigkeit (1999-2012)

9.2.2 Französischer Diskurs 1999-2012: Durchlässigkeit im Spannungsverhältnis universalistischer und funktionalistischer Vorstellungen. 353

9.3 Analyse des institutionellen Wandels 389

9.3.1 Veränderungen institutioneller Durchlässigkeitsstrukturen und Fortführung institutioneller Entwicklungspfade

9.3.2 Zusammenfassung und Schlussfolgerungen

10 Durchlässigkeit in Deutschland und Frankreich und der Einfluss europäischer Bildungsprozesse: Die Frage nach der Konvergenz.....

10.1 Konvergenz zwischen den nationalen Bildungssystemen? 402

10.1.1 Nationale Durchlässigkeitsstrukturen im Vergleich (1985/90-1998) .... 403

10.1.2 Nationale Durchlässigkeitsstrukturen im Vergleich (1999-2012).......... 410

10.2 Durchlässigkeit durch Europäisierung? Nationale Entwicklungen und europäische Vorgaben

10.2.1 Passung zwischen nationalen Strukturen und europäischen Vorgaben vor 1999

10.2.2 Annäherung an europäische Durchlässigkeitsvorgaben seit 1999?........ 427

10.2.3 Einfluss der Bologna- und Kopenhagen-Prozesse auf die nationalen Durchlässigkeitsstrukturen.

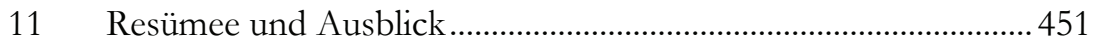

11.1 Anlage der Untersuchung und Ergebnisse ................................................. 451

11.1.1 Entwicklung der nationalen Durchlässigkeitsstrukturen in Deutschland und Frankreich 456

11.1.2 Vergleich institutioneller Durchlässigkeit in Deutschland und Frankreich 462

11.1.3 Einfluss europäischer Bildungsprozesse 465

11.2 Limitationen, weiterführende Forschung und Implikationen 470 
11.2.1 Grenzen der Studie und mögliche Erweiterungen......................................470

11.2.2 Gesellschaftspolitische Implikationen..........................................................473

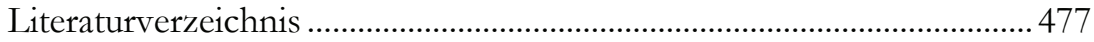

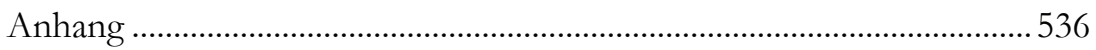

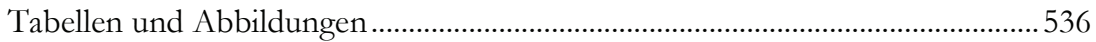

Übersicht über die geführten Expert_inneninterviews ..........................................546

Online Anhang: ................................................... DOI 10.3224/86388706A

Anhang I: Tabellen

Anhang II: Interviewleitfäden

Anhang III: Datengrundlage der Diskursanalyse

Anhang IV: Datengrundlage der regulativen Analyse

Den Online-Anhang finden Sie auf der Webseite zum Buch unter https://shop.budrich-academic.de/produkt/durch-europaeisierung-zu-mehrdurchlaessigkeit $/ ?_{v}=3 a 52 \mathrm{f} 3 \mathrm{c} 22 \mathrm{ed} 6$ 


\section{Abbildungsverzeichnis}

Abbildung 1 Durchlässigkeitskonzept........................................................................ 58

Abbildung 2 Methodische Anlage der Untersuchung ..............................................111

Abbildung 3 Verteilung der Neuzugänge auf die drei Sektoren des

Berufsbildungssystems ......................................................................201

Abbildung 4 Chronologie der Debatten und Politiken in Deutschland

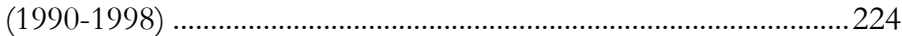

Abbildung 5 Positional map der deutschen Akteure (1990-1998) ...........................245

Abbildung 6 Chronologie der Debatten und Politiken in Deutschland

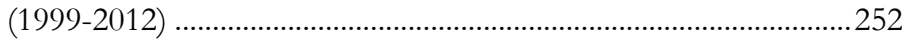

Abbildung 7 Positional map der deutschen Akteure (1999-2012) ...........................286

Abbildung 8 Entwicklung des Abiturient_innenanteils nach Typ des Abiturs (\%) ........................................................................................ 310

Abbildung 9 Chronologie der Debatten und Politiken in Frankreich (1985-1998)

Abbildung 10 Chronologie der Debatten und Politiken in Frankreich (1999-2012)

Abbildung 11 Positional map der französischen Akteure (1999-2012) ..................386

Abbildung 12 Entwicklung der Zahl der Validierungen mit dem VAE Verfahren seit 2002 in Frankreich .543 


\section{Tabellenverzeichnis}

Tabelle 1 Überblick über Durchlässigkeitsaspekte ..............................................59

Tabelle 2 Analysefokus der Untersuchung .............................................................. 109

Tabelle 3 Anzahl der analysierten Dokumente nach Organisationen in Deutschland und Frankreich................................................................125

Tabelle 4 Kategorien für die Dokumentenanalyse ................................................141

Tabelle 5 Durchlässigkeits-Standards im Bologna-Prozess ...............................175

Tabelle 6 Durchlässigkeitsrelevante Standards im Kopenhagen-Prozess .......188

Tabelle 7 Das deutsche Bildungsschisma nach Baethge (2006) ........................197

Tabelle 8 Bevölkerung in Deutschland nach Altersgruppen und

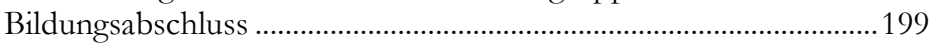

Tabelle 9 Hochschultypen und Studierendenverteilung....................................205

Tabelle 10 Argumentationslogiken der deutschen Diskurse (1990-1998) ........231

Tabelle 11 Vergleich der Diskursstränge zur Frage des Zugangs in Deutschland (1990-1998)

Tabelle 12 Vergleich der Diskursstränge zur Bedeutung organisationaler Verbindungen in Deutschland (1990-1998)

Tabelle 13 Vergleich der Diskursstränge zur Frage des Umgangs mit Heterogenität in Deutschland (1990-1998)

Tabelle 14 Argumentationslogiken der deutschen Diskurse (1999-2012) ........263

Tabelle 15 Vergleich der Diskursstränge zur Frage des Zugangs in Deutschland (1999-2012)

Tabelle 16 Vergleich der Diskursstränge zur Bedeutung von Anrechnung in Deutschland (1999-2012)

Tabelle 17 Vergleich der Diskursstränge zur Bedeutung organisationaler Verbindungen in Deutschland (1999-2012) 279

Tabelle 18 Vergleich der Diskursstränge zur Frage des Umgangs mit Heterogenität in Deutschland (1999-2012)

Tabelle 19 Bildungskarrieren aller Abiturient_innen nach Typ des Abiturs

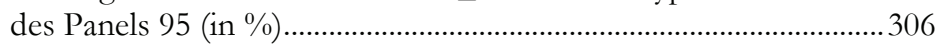

Tabelle 20 Entwicklung der Lehrlingszahlen in den centres de formation d'apprentis (CFA)

Tabelle 21 Direkte Einschreibungsrate der Abiturient_innen 2012 in die unterschiedlichen Studiengänge (in \%).

Tabelle 22 Studienerfolg in der licence innerhalb von vier Jahren je nach Abiturschwerpunkt im Abiturjahrgang 2007

Tabelle 23 Argumentationslogiken des französischen Diskurses (1985-1998) 338

Tabelle 24 Struktur des Durchlässigkeitsdiskurses (1985-1998):

Durchlässigkeit über Weiterbildung

Tabelle 25 Argumentationslogiken des französischen Diskurses (1999-2012) 361 
Tabelle 26 Struktur des französischen Diskurses 1999-2012: Zugang und

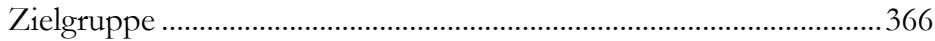

Tabelle 27 Struktur des französischen Diskurses 1999-2012: Anrechnung .....368

Tabelle 28 Vergleich der Diskursstränge zur Bedeutung organisationaler Verbindungen in Frankreich (1999-2012)

Tabelle 29 Vergleich der Diskursstränge zur Frage des Umgangs mit Heterogenität in Frankreich (1999-2012)

Tabelle 30 Vergleich der durchlässigkeitsrelevanten Zielgruppe für die Hochschulbildung vor 1999 .406

Tabelle 31 Unterschiede und Ähnlichkeiten in den nationalen Durchlässigkeitsstrukturen vor 1999

Tabelle 32 Diskursiv behandelte Aspekte von Durchlässigkeit im Vergleich vor und nach 1999

Tabelle 33 Vergleich der durchlässigkeitsrelevanten Zielgruppe für die Hochschulbildung nach 1999

Tabelle 34 Unterschiede und Ähnlichkeiten in den Durchlässigkeitsstrukturen nach 1999.

Tabelle 35 Kompatibilität rechtlicher Durchlässigkeitsstrukturen mit den europäischen Vorgaben

Tabelle 36 Thematisierte Aspekte von Durchlässigkeit in Deutschland, Frankreich und den europäischen Bildungsprozessen

Tabelle 37 Institutionelle Charakteristika der Berufs- und Hochschulbildung in Deutschland und Frankreich

Tabelle 38 Darstellung des europäischen Kernkonzeptes von

Durchlässigkeit

Tabelle 39 Überblick regulativer Veränderungen 1990-2012................................538

Tabelle 40 Glossar zum französischen Bildungssystem ........................................540

Tabelle 41 Studienerfolg in der licence innerhalb von vier Jahren je nach Abiturschwerpunkt im Abiturjahrgang 2007

Tabelle 42 Studienerfolg am IUT nach Typ des Abiturs innerhalb von drei Jahren in \% (Kohorte 2008)....

Tabelle 43 Studienerfolg in den STS nach Typ des Abiturs im Jahr 2012 ........543

Tabelle 44 Überblick regulativer Veränderungen in Frankreich 1985-2012 ....544

Tabelle 45 Expert_inneninterviews in Deutschland.............................................546

Tabelle 46 Expert_inneninterviews mit bildungspolitischen Akteuren in Frankreich .547

Tabelle 47 Expert_inneninterviews mit (Bildungs-)Forscher_innen in Frankreich .548 


\section{Abkürzungsverzeichnis}

$\begin{array}{ll}\text { Abkürzung } & \text { Langform } \\ \text { ADIUT } & \text { Association des Directeurs d'IUT } \\ \text { aHR } & \text { Allgemeine Hochschulreife } \\ \text { ANKOM } & \begin{array}{l}\text { Anrechnung beruflicher Kompetenzen auf Hochschulstu- } \\ \text { diengänge }\end{array} \\ \text { APB } & \text { Admission Post Bac } \\ \text { B.A. } & \text { Bachelor Abschluss/Bachleor of Arts } \\ \text { Bac } & \text { Baccalauréat } \\ \text { BBiG } & \text { Berufsbildungsgesetz } \\ \text { BEP } & \text { Brevet d'études professionnelles } \\ \text { BEPC } & \text { Brevet d'études de Premier Cycle } \\ \text { BIBB } & \text { Bundesinstitut für Berufsbildung } \\ \text { BMBF } & \text { Ministerium für Bildung und Forschung } \\ \text { BMBW } & \text { Bundesministerium für Bildung und Wissenschaft } \\ \text { BOS } & \text { Berufsoberschulen } \\ \text { BRD } & \text { Bundesrepublik Deutschland } \\ \text { BTS } & \text { Brevet de technicien supérieur/Zertifikat für den höheren } \\ \text { CA } & \text { Techniker } \\ \text { CAP } & \text { Centre d' apprentissage } \\ \text { CEREQ } & \text { Certificat d'aptitude professionnelle } \\ \text { CFA } & \text { Centre d'études et de recherches sur les qualifications } \\ \text { CGPME } & \text { Centre de formation d'apprentis/Ausbildungszentrum für } \\ \text { duale Ausbildung } \\ \text { CGT } & \text { Confédération Générale des Petites et Moyennes Entre- } \\ \text { CMH } & \text { prises } \\ \text { CPGE } & \text { Confédération Générale du Travail } \\ \text { CPO } & \text { Centre Maurice-Halbwachs } \\ \text { CPU } & \text { Classes préparatoires aux grandes écoles/Vorbereitungs- } \\ \text { CQR } & \text { klassen für die ,grandes écoles“ } \\ \text { DAEU } & \text { Causal-process observation } \\ \text { DDR } & \text { Conférence des présidents d'université } \\ \text { Certificats de qualification professionnelle } \\ \text { den Zugang zum Hochschulstudium gewährleistet } \\ & \text { Deutsche Demokratische Republik } \\ & \end{array}$



Abkürzung
Langform
$\mathrm{DE}$
Deutschland
DECVET
Leistungspunktesystem für die berufliche Bildung in Deutschland
DESS
Diplôme d'Etudes Supérieures Specialisées
DGB
Deutscher Gewerkschaftsbund
DIHK
Deutsche Industrie- und Handelskammertag
DIHT
Deutsche Industrie- und Handelskammertag
DQR
Deutscher Qualifikationsrahmen
DUT
EAES
Diplôme universitaire de technologie/Technologisches Hochschuldiplom
Examen d'accès à l.enseignement supérieur / Hochschulein- gangsprüfung
ECTS European Credit Transfer System
ECVET European Credit System for Vocational Education and Training (Europäisches Leistungspunktesystem für die Berufsbildung)
EFTA European Free Trade Association (Europäische Freihan- delsassoziation)
EG
Europäische Gemeinschaft
EGKS
Europäische Gemeinschaft für Kohle und Stahl
EGV
EHEA
Vertrag zur Gründung der Europäischen Gemeinschaft
EHR
European Higher Education Area
ENP
Europäischer Hochschulraum
EPCI
Ecoles Nationales Professionnelles
Ecoles Pratiques de Commerce et d'Industrie/Staatlich be- zahlte Hochschule für Handel und Industrie
EQAR
European Quality Assurance Register for Higher Education
EQARF
European Quality Assurance Reference Framework for VET
EQF
European Qualifications Framework
EQR
Europäischer Qualifikationsrahmen
ESEU
Examen spécial d'entrée à l'université
EU
Europäische Union
EUGH
Europäischer Gerichtshof
EURATOM
Europäische Atomgemeinschaft
EWG
Europäische Wirtschaftsgemeinschaft
$\mathrm{FH}$
Fachhochschule (heute Hochschule genannt) 


\begin{tabular}{|c|c|}
\hline Abkürzung & Langform \\
\hline FHR & Fachhochschulreife \\
\hline FOS & Fachoberschulen \\
\hline FR & Frankreich \\
\hline FSU & Fédération Syndicale Unitaire \\
\hline GTM & Grounded Theory Methodology \\
\hline HR & Hochschulreife \\
\hline HRG & Hochschulrahmengesetz \\
\hline HRK & Hochschulrektorenkonferenz \\
\hline $\mathrm{HE}$ & Higher education \\
\hline $\mathrm{HwO}$ & Handwerksordnung \\
\hline INVEST & $\begin{array}{l}\text { DFG Projekt „Internationalization of Vocational and } \\
\text { Higher Education Systems in Transition“ }\end{array}$ \\
\hline ISCED & International Standard Classification of Education \\
\hline IUP & $\begin{array}{l}\text { Institut universitaire professionnels/Universitätsinstitut für } \\
\text { berufliche Bildung }\end{array}$ \\
\hline IUT & $\begin{array}{l}\text { Institut universitaire technologique/Technisches Hoch- } \\
\text { schulinstitut }\end{array}$ \\
\hline KMK & Kultusministerkonferenz \\
\hline LHG & Landeshochschulgesetz \\
\hline LLL & Lebenslanges Lernen \\
\hline LMD & Licence-Master-Doctorat (neue Zyklenstruktur) \\
\hline LRU & Loi relative aux libertés et responsabilités des universités \\
\hline M.A. & Master Abschluss/Master of Arts \\
\hline MDEP & Ministère Délégué à l'Enseignement Professionnel \\
\hline $\mathrm{MEDEF} / \mathrm{CNPF}$ & $\begin{array}{l}\text { Mouvement des entreprises de France/Conseil national du } \\
\text { patronat français }\end{array}$ \\
\hline MEN & Ministère de l'Education Nationale/Bildungsministerium \\
\hline MENJS & $\begin{array}{l}\text { Ministère de l'Education Nationale de la Jeunesse et des } \\
\text { Sports }\end{array}$ \\
\hline MENRT & $\begin{array}{l}\text { Ministere de l'Education Nationale de la Recherche et de } \\
\text { Technologie }\end{array}$ \\
\hline MENSR & $\begin{array}{l}\text { Ministère de l'Education Nationale, de l'Enseignment supé- } \\
\text { rieur et de la Recherche }\end{array}$ \\
\hline MESR & Ministère de l'Enseignement Supérieur et de la Recherche \\
\hline MINT & Mathematik, Informatik, Naturwissenschaft und Technik \\
\hline MJENR & $\begin{array}{l}\text { Ministère de la jeunesse, de l'éducation nationale de la re- } \\
\text { cherche }\end{array}$ \\
\hline
\end{tabular}




\begin{tabular}{|c|c|}
\hline Abkürzung & Langform \\
\hline MSA & Mittlerer Schulabschluss (Mittlere Reife) \\
\hline NQR & Nationaler Qualifikationsrahmen \\
\hline OECD & $\begin{array}{l}\text { Organisation für wirtschaftliche Zusammenarbeit und Ent- } \\
\text { wicklung / Organisation for Economic Co-operation and } \\
\text { Development }\end{array}$ \\
\hline RNCP & $\begin{array}{l}\text { Répertoire national des certifications professionnelles } \\
\text { (Register der Berufszertifikate) }\end{array}$ \\
\hline SekII & Sekundarstufe II \\
\hline SNESUP & Syndicat National de l'Enseignement Supérieur \\
\hline SPD & Sozialdemokratische Partei Deutschland \\
\hline STS & $\begin{array}{l}\text { Section de technicien supérieur (Sektionen für höhere tech- } \\
\text { nische Bildung in Oberschulen) }\end{array}$ \\
\hline $\mathrm{U} 3 \mathrm{M}$ & $\begin{array}{l}\text { Université du troisième millénaire (Plan für die (franz.) } \\
\text { Universität im 3. Jahrtausend) }\end{array}$ \\
\hline VAE & $\begin{array}{l}\text { Validation des acquis de l'expérience/Zertifizierung beruf- } \\
\text { lich und außerberuflich erworbener Kompetenzen }\end{array}$ \\
\hline VAP & $\begin{array}{l}\text { Validation des acquis professionnels/Validierung allein be- } \\
\text { ruflicher Kompetenzen }\end{array}$ \\
\hline VET & Vocational education and training \\
\hline
\end{tabular}




\section{Danksagung}

Für Paps (1958-2009) und meine Familie

Dieses Buch ist eine geringfügig überarbeitete Fassung meiner Dissertation im Fach Soziologie, die ich im Juni 2014 an der Freien Universität Berlin verteidigt habe.

Die Entstehung des Buches wäre ohne die Hilfe und Unterstützung vieler Personen und Organisationen nicht möglich gewesen. Gefördert wurde diese Arbeit durch die DFG im Rahmen des Projekts "Berufliche Bildung im Wandel: Institutioneller Wandel von Berufsbildungs- und Hochschulsystemen in Europa"1, kurz INVEST, und durch das Wissenschaftszentrum Berlin für Sozialforschung (WZB).

Mein besonderer Dank gilt dem Forschungsteam des INVEST ${ }^{2}$ Projekts, insbesondere Heike Solga, Justin J. W. Powell und Lukas Graf. Ohne die vielen inspirierenden und motivierenden Arbeitstreffen, die kritischen Kommentare, die vielfältigsten Anregungen, aber auch die kontinuierliche Unterstützung, die mir im Projektteam zuteil wurde, hätte ich diese Arbeit nicht verfassen können. Heike Solga und Justin J. W. Powell danke ich zudem, dass sie sich bereit erklärt haben, diese Arbeit zu betreuen und zu begutachten. Für die konstruktiven Kommentare während der Disputation danke ich Katharina Blum und Reinhard Pollack. Ebenfalls möchte ich mich für die wertvolle Unterstützung bei den studentischen Mitarbeiterinnen des INVEST-Projektes Claudia Finger, Anne Piezunka, Katja Pomianowicz und Kerstin Albrecht bedanken.

Ich danke auch dem Doktorand_innenkolloquium sowie meinen Kolleginnen und Kollegen der Abteilung „Ausbildung und Arbeitsmarkt“ am WZB, die mich im Laufe der Promotion unterstützt haben. Für ihre fortwährende Ermutigung und ihren Rat zu einzelnen Kapiteln danke ich im Besonderen Alessandra Rusconi, Lisa Pfahl, Paula Protsch und Franziska Scheier sowie den Teilnehmer_innen der qualitativen Forschungswerkstatt. Für ihre wertvolle Hilfe beim Lektorat danke ich Friederike Theilen-Kosch, Mario Schimanski, Sarah Rögl vom Budrich UniPress Verlag und insbesondere Lena Förster. Der Abteilung Vergleichende und Internationale Erziehungswissenschaften an der Humboldt Universität zu Berlin bin ich dankbar für die Ermutigungen bei der Manuskriptüberarbeitung.

Bedanken möchte ich mich zudem bei Annie Bouder, Laurence Coutrot und Annick Kieffer, die mir im Rahmen meiner Forschung in Frankreich mit Rat und

1 DFG-Geschäftszeichen: SO 430/5-1

2 INVEST steht als Akronym für den englischen Titel „Internationalization of Vocational Training and Higher Education Systems in Transition" des oben genannten DFGForschungsprojektes. 
Tat zur Seite standen und geholfen haben, ein besseres Verständnis des französischen Bildungssystems zu entwickeln. Für die Gastfreundschaft am Centre Maurice Halbwachs und im Maison Suger der Fondation Maison des Sciences de l'Homme in Paris sowie am Centre d'études et de recherche sur les qualifications (CEREQ) in Marseille bin ich ebenfalls dankbar. Gleichfalls danke ich Christine Musselin, David Baker und Reiner Keller, da mir ihr Feedback und ihre Kommentare halfen, meine Argumente weiter zu schärfen. Zu Dank verpflichtet bin ich auch meinen vielen Interviewpartner_innen in Deutschland und Frankreich. Vor allem durch ihre Expertise ist es mir möglich gewesen, die notwendigen Puzzleteile zusammenzufügen.

Dem Wissenschaftszentrum Berlin für Sozialforschung danke ich für das anregende Umfeld und die ausgezeichneten Forschungsbedingungen während meiner Promotionszeit.

Nicht zuletzt möchte ich meinen Freundinnen und Freunden und vor allem meiner Familie danken, ohne die es nicht möglich gewesen wäre, diese Arbeit zu schreiben. Meinen Eltern danke ich für ihre stete Ermutigung, auch große Ziele voller Zuversicht und Selbstbewusstsein anzugehen. Meinen Kindern Jonathan und Julius bin ich dankbar, dass sie mich immer wieder in die ,reale' Welt zurückholen, wodurch ich neue Kraft schöpfe und nicht vergesse, was mir wichtig ist. Und Gregor danke ich schließlich von Herzen für seine unendliche Geduld, sein Verständnis und die tatkräftige Unterstützung während all dieser Jahre.

Berlin, Oktober 2016

Nadine Bernhard 


\section{Einleitung}

Das Bürgerrecht auf Bildung ist zunächst ein soziales Grundrecht aller Bürger, das gleichsam den Fußboden absteckt, auf dem jeder Staatsbürger stehen darf und stehen muß, um als solcher tätig zu werden. Dieses Recht ergänzt die anderen großen Bürgerrechte vor dem Gesetz und im Staate. Es hat in der allgemeinen Schulpflicht seine erste konkrete Gestalt gefunden und ist seither ständig durch neue Inhalte ergänzt worden. Dieser Prozeß der Ergänzung wird nie abgeschlossen sein. (Ralf Dahrendorf 1965: 27)

In seinem einflussreichen Werk „Bildung ist Bürgerrecht“ konstatiert Ralf Dahrendorf, dass Bildung die fundamentale Voraussetzung für Menschen ist, an der Gesellschaft als freie und gleiche Individuen zu partizipieren. Um dieses Bürgerrecht jedoch zu verwirklichen, ist eine ,aktive Bildungspolitik“" (Dahrendorf 1965) notwendig. Das Ziel einer derartigen Politik lautet dabei „mehr Bildung für mehr Menschen" (Dahrendorf 1965: 28), d.h. eine Expansion der Bildungsbeteiligung im gesamten Bildungssystem. Der Bildungsbegriff, welcher verwendet wird, zielt in dieser Hinsicht nicht nur auf Bildung zur Erhöhung von Humanressourcen, sondern primär auch auf die individuelle Regulierungsfähigkeit sowie gesellschaftliche Teilhabe und Chancengleichheit. Baethge et al. (2007) betonen, dass ein derartiges Verständnis von Bildung dazu führt, den Begriff Bildungsmobilität als relevante Kategorie in das Zentrum der Analyse von Bildungssystemen zu stellen.

Institutionell bedeutet Bildungsmobilität, dass die Grenzen zwischen den unterschiedlichen Bildungsbereichen durchlässig sind, individuell äußert sie sich darin, dass die Jugendlichen [bzw. Individuen, NB] die Kompetenzen erwerben, die für eine selbständige Organisation ihrer Bildungsbiografie in der Perspektive lebenslangen Lernens erforderlich sind. (Baethge et al. 2007: 7)

Die vorliegende Studie fokussiert die institutionelle Perspektive - die der Durchlässigkeit im Bildungswesen, spezifisch zwischen beruflicher Bildung und Hochschulbildung. Durchlässige Bildungssysteme sollen dabei verstanden werden als Systeme, in denen in jeder Lebensphase an vorhandene Abschlüsse und Kompetenzen angeknüpft und begonnene Bildungskarrieren in verschiedene Richtungen fortgesetzt werden können. Durchlässigkeit heißt demnach, dass es keine Bildungssackgassen geben darf. Die Möglichkeit zur Höherqualifikation wird bereits in Dahrendorfs Werk in den 1960er Jahren als signifikant erachtet. Seitdem hat die Bedeutung der Ermöglichung des kontinuierlichen Weiterlernens in modernen Gesellschaften allerdings noch zugenommen, denn heutzutage ist ein geringes Qualifikationsniveau mit größeren Nachteilen für das Individuum und die Gesellschaft verbunden als früher (vgl. Pfahl/Powell 2010; Protsch 2014; Solga 2002; Solga 2005b; Solga/Dombrowski 2009).

Analysen hinsichtlich Strukturen des Bildungssystems und damit auch die Untersuchung der institutionellen Durchlässigkeit erbringen dabei Hinweise zum 
Verständnis der Ursachen und Bedingungen sozialer Ungleichheit in modernen Gesellschaften. Bildung stellt sowohl eine bedeutende Determinante als auch eine Dimension sozialer Ungleichheit dar (vgl. Solga et al. 2009). Mayer (2004) unterstreicht, dass die größte Varianz der Unterschiede in individuellen Lebensläufen durch externe Strukturen, welche besonders eng mit der Arbeitsteilung, Arbeitsstruktur und Berufsstruktur eines Landes assoziiert sind, verursacht wird. Diese Strukturen, wie bspw. das Bildungssystem, bilden die Grundlage für die Verteilung der anfänglichen Ressourcen sowie die daraus resultierenden späteren Einkommen. „Already the institutional contexts [...] narrow down to a large extent which life avenues are open and which are closed“ (Mayer 2004: 165). Veränderungen der Bildungssysteme sind somit verbunden mit Veränderungen der Lebenschancen der Individuen im Lebenslauf und beeinflussen dann den Verlauf von kumulativen Prozessen von Bildungsungleichheiten über den Lebensverlauf.

Die Institutionen der Hochschul- und Berufsbildung bestimmen individuelle Möglichkeiten im Lebenslauf mindestens in Bezug auf vier Dimensionen: erstens die Chance auf einen Arbeitsplatz bei Eintritt in den Arbeitsmarkt, zweitens die mit der Ausbildung verbundenen Aufstiegsmöglichkeiten, drittens die Pfade der Weiterbildung und viertens das mit der Ausbildung und Arbeitsposition verbundene Einkommen sowie die Statusposition. Zusammengefasst ist die Frage nach Durchlässigkeit zwischen beruflicher und Hochschulbildung insofern wichtig, als stratifizierte (Aus-)Bildungssysteme ohne Brücken zwischen einzelnen Bildungssektoren den möglichst gleichen Zugang zu Bildungs- und Beschäftigungsopportunitäten behindern. Das Recht auf Bildung wäre demnach eingeschränkt.

Aber nicht nur aus Perspektive individueller Bildungsmobilität, sondern auch aus gesellschaftlicher Perspektive sind die Durchlässigkeit von Bildungssystemen und die Verringerung von sozialen Ungleichheiten relevant, denn Bildung ermöglicht gesellschaftliche Teilhabe. Soziale Ausschlüsse, hervorgerufen durch fehlende Teilhabemöglichkeiten, ziehen dagegen hohe gesellschaftliche Kosten nicht nur für die Sozialversicherungssysteme, sondern auch für die soziale Kohäsion, den sozialen Zusammenhalt einer Gesellschaft, nach sich (Autorengruppe Bildungsberichterstattung 2014).

Die Ermöglichung der Erhöhung des Bildungsstandes der Bevölkerung, für die ein durchlässiges Bildungssystem eine Voraussetzung darstellt, wird jedoch nicht nur aus der Perspektive der individuellen Grundrechte und des Abbaus sozialer Ungleichheiten als gesellschaftlich relevant erachtet. Vielmehr wird auch ein gestiegener Bedarf an gut ausgebildeten Individuen in modernen Gesellschaften festgestellt.

Seit mehr als vierzig Jahren (Dostal 2001) vollzieht sich ein sektoraler Wandel von der Ökonomie der industriellen Produktion zu einer Ökonomie der Dienstleistungen und des Wissens (Baethge et al. 2007: 11). Damit einhergehend hat sich der Druck für bessere und mehr Bildung intensiviert, denn der sektorale Strukturwandel (Dostal 2001) läuft parallel zu einer Transformation der Arbeit und 
führt auch zu tiefgreifenden Implikationen für die Prozesse des Kompetenzerwerbs. Es geht bei dieser Transformation nicht allein um die Entwicklung von arbeitsspezifischen Fähigkeiten hin zu mehr analytischen allgemeinen Fähigkeiten, sondern auch um den Wandel von einer eher hierarchisch klar strukturierten Aufgabenbeschreibung hin zu einer autonomen Arbeitsweise in den Betrieben (vgl. Helmrich 2008; Mayer/Solga 2008). Auf dem Arbeitsmarkt werden durch diese Entwicklungen verstärkt allgemeines und theoretisches Wissen und Fähigkeiten im Gegensatz zu eingegrenzten berufsbezogenen Kompetenzen verlangt, was eine Herausforderung für die berufliche Bildung darstellt (Mayer/Solga 2008: 1 ff.; Streeck 2012). Insgesamt kommt es damit zu einem Anstieg der wissensintensiven Beschäftigtenkategorien und der durchschnittlichen Qualifikationsanforderungen (Baethge et al. 2007; Cordes/Gehrke 2012). Mit diesem Trend ist zudem ein viel schnellerer Verfall des bereits erworbenen Wissens verbunden, wodurch sich die Notwendigkeit eines kontinuierlichen Weiterlernens erhöht.

Außerdem verändert sich die Zusammensetzung der Population durch die demografischen Entwicklungen, d.h. durch eine niedrige Geburtenzahl in vielen Ländern Europas und die Zunahme der Lebenserwartung. Es wird argumentiert, dass beide Prozesse zu einer Verlängerung der Lebensarbeitszeit führen, was die Bedeutung von lebenslangem Lernen unterstreicht (vgl. Jakobi/Rusconi 2008; Spermann 2013; Wolter 2011). Menschen tendieren allerdings immer noch zu der Annahme, wenn sie die Ausbildung abgeschlossen haben, reichten diese Investitionen für ihr gesamtes Leben aus (Mayer/Solga 2008: 1). Und auch institutionell ist trotz der Veränderungen der letzten Jahrzehnte die Erstausbildung weiterhin von äußerster Relevanz für die individuellen Karriereverläufe. Um mit dem Wandel Schritt zu halten, ist es wichtig, dass sich Bildungsinstitutionen den neuen Gegebenheiten und Bedürfnissen anpassen. Es geht daher um einen Perspektivenwechsel weg von einem Lebensarbeitsplatz beruhend auf der Erstausbildung hin zu einem kontinuierlichen Weiterentwickeln der individuellen Fähigkeiten im Lebenslauf, weg von starren, hin zu flexiblen Bildungssystemen, in denen die künstliche Segregation der verschiedenen Lernorte und -pfade überwunden wird.

Schließlich wird auch auf globaler und vor allem europäischer Ebene immer stärker auf die Notwendigkeit der Höherqualifizierung der Bevölkerungen hingewiesen. Internationalisierungsprozesse sind daher als bedeutende Faktoren anzusehen, welche die Transformation von Bildungssystemen beeinflussen (Powell/Solga 2010: 707; 2008). So versuchen Organisationen wie die OECD, bestimmte Bildungsmodelle, wie das des lebenslangen Lernens (vgl. Jakobi 2009) in den Nationalstaaten zu verbreiten. Auf europäischer Ebene wird Bildungspolitik primär als Marktschaffungspolitik gesehen (Trampusch 2008). Dabei haben in den letzten Jahren die Initiativen und Prozesse zugenommen, welche auf eine höhere Bildung der europäischen Bevölkerungen drängen. In der Lissabon-Strategie aus dem Jahr 2000 wird Bildung eine Schlüsselrolle zugeschrieben, um Europa zum ,wettbewerbsfähigsten und dynamischsten wissensbasierten Wirtschaftsraum der Welt werden [zu lassen, NB] - einem Wirtschaftsraum, der fähig 
ist, ein dauerhaftes Wirtschaftswachstum mit mehr und besseren Arbeitsplätzen und einem größeren sozialen Zusammenhalt zu erzielen" (Europäischer Rat 2000).

Als relevanter für Fragen der Durchlässigkeit zwischen Berufs- und Hochschulbildung können allerdings die intergouvernementalen Bildungsprozesse Bologna und Kopenhagen angesehen werden. Die Bologna-Erklärung wurde 1999 von 29 europäischen Ländern unterzeichnet. Mit ihr wollen die europäischen Staaten die Entstehung eines europaweiten Hochschulraums fördern, um individuelle Mobilität, Transparenz und Anerkennung von Qualifikationen sowie eine Erhöhung von deren Qualität zu erleichtern. Die Kopenhagen-Erklärung wurde 2002 von 31 Ländern unterschrieben, um die europäische Zusammenarbeit in der beruflichen Bildung zu verstärken (vgl. Balzer/Rusconi 2007). Wichtige Ziele des Prozesses sind die Schaffung eines einheitlichen Qualifikations- und Kompetenzrahmens, ein System von Leistungspunkten in der beruflichen Bildung, die Validierung informell erworbener Qualifikationen, die Schaffung gemeinsamer Instrumente zur Qualitätssicherung und insgesamt die Verbesserung des Zugangs der Bürger zum lebenslangen Lernen. Generell versuchen beide Prozesse einen europäischen Bildungsraum zu erschaffen, der die Wettbewerbsfähigkeit der Mitgliedsländer im internationalen Vergleich stärkt und somit zu dem Erreichen der Lissabon-Ziele beiträgt. Ein gemeinsames Anliegen in beiden Prozessen ist dabei auch die Förderung durchlässiger Bildungsstrukturen (vgl. Bernhard et al. 2010; Powell et al. 2012b).

Eine Höherqualifizierung der Bevölkerung wird demnach aus differenten Perspektiven - dem Blick auf individuelle Bildungsrechte, dem Abbau von sozialen Ungleichheiten, dem Fokus auf Veränderungen der Wirtschaftsstruktur und der Demografie sowie auch durch europäische Einflüsse - als wichtig angesehen. Durchlässigkeit von Bildungsstrukturen, insbesondere auch zwischen Berufs- und Hochschulbildung, ist dafür eine Voraussetzung, denn dies sind die Bildungsbereiche, in denen nach Beendigung der Schulzeit die weitere formale Bildung erfolgt. Insofern stellt sich die Frage, wie europäische Gesellschaften auf die hier skizzierten Herausforderungen reagieren. Ändern sich ihre Bildungsstrukturen hin zu mehr Durchlässigkeit? Dieser Frage soll in der vorliegenden Arbeit in einem Vergleich zwischen Deutschland und Frankreich nachgegangen werden.

Insbesondere Länder, deren Bildungssysteme sich durch eine Trennung zwischen Berufs- und Hochschulbildung auszeichnen, wie die sogenannten DACHLänder Deutschland, Österreich und die Schweiz, stehen somit vor besonders großen Herausforderungen (vgl. auch Graf 2013). Speziell in Deutschland ist eine starke Segmentierung der Berufs- und Hochschulbildung festzustellen. Baethge (2006) nennt es das deutsche „Bildungsschisma“, welches die Etablierung von durchlässigen Bildungsstrukturen zwischen Berufs- und Hochschulbildung erschwert. So sind kaum Übergänge aus dem einen in das andere System möglich. Des Weiteren werden durch diese strikte Segmentierung die Qualifikationen, wel- 
che in einem Bereich erlernt worden sind, wenig bis gar nicht im anderen anerkannt (Haugg 2008). So entstehen neben Bildungsbarrieren auch solche für Karrierewege, wenn z.B. Absolvent_innen einer beruflichen Ausbildung keinen $\mathrm{Zu}-$ gang zu Führungspositionen erhalten, da für diese implizit oder explizit ein akademischer Abschluss vorausgesetzt wird (Thielen 2008). Solga und Dombrowski (2009: 20) fassen die deutsche Situation folgendermaßen zusammen:

Insgesamt ist für Deutschland eine mangelhafte Durchlässigkeit zwischen beruflicher Bildung und Hochschule zu konstatieren, so dass frühe Bildungsungleichheiten nur selten und dann auch nur partiell nachträglich korrigiert werden können.

Insofern würde eine zunehmende Durchlässigkeit zwischen den Bildungsbereichen zu einer Erhöhung der Bildungs- und sozialen Mobilität in Deutschland führen (vgl. Powell/Solga 2010; Powell/Solga 2008).

Aber stellt sich die Frage nach Durchlässigkeit zwischen beruflicher und Hochschulbildung wirklich nur in Ländern, in denen diese Bereiche getrennt institutionalisiert sind? Welche Entwicklungen sind in Ländern wie Frankreich zu erkennen, die sich formal durch eine große Durchlässigkeit zwischen Berufs- und Hochschulbildung auszeichnen?

Das französische Bildungssystem wird im Vergleich zum deutschen oft als nur moderat stratifiziert (Kerckhoff 2001) und als ein System beschrieben, welches den Zugang zur Hochschulbildung weniger stark einschränkt als das deutsche Bildungssystem, welches durch seine starke Berufsausbildung charakterisiert ist (vgl. Shavit/Müller 1998, 2000a, b). In Frankreich ist eine strikte Trennung zwischen Berufs- und Hochschulbildung nicht vorhanden, zumal beruflich orientierte Bildung zu weiten Teilen ebenfalls im Hochschulsystem selbst stattfindet. Im Gegensatz zu Deutschland ist in Frankreich auch kein stark ausgebautes postsekundäres berufliches Weiterbildungssystem außerhalb der Hochschulen existent. Durchlässigkeit zwischen Berufs- und Hochschulbildung ist formal viel stärker gewährleistet als in Deutschland, da Zugänge über die Berufs- zur Hochschulbildung möglich sind. Auch bestehen Anrechnungsverfahren in der Hochschulbildung, in denen berufliche Kompetenzen anerkannt werden. Trotzdem kann für Frankreich gezeigt werden, dass die formale Durchlässigkeit nicht zu einer tatsächlichen Durchlässigkeit für beruflich Qualifizierte führt, denn diese sind systematisch stärker von Studienabbrüchen betroffen als andere Bildungsgruppen (vgl. Beaud/Pialoux 2001; Duru-Bellat/Kieffer 2008). Ein Vergleich zwischen Deutschland und Frankreich bietet sich aber nicht nur aus der Logik der Unterschiedlichkeit an. Beide Länder sind wichtige und einflussreiche Mitgliedsstaaten der Europäischen Union sowie Mitbegründer und Initiatoren der Bologna- und Kopenhagen-Prozesse. Zudem zeichnen sich beide Länder durch traditionell einflussreiche Bildungsmodelle aus (vgl. z.B. Goldschmidt 1991; Greinert 2005), welche die transnationalen europäischen Bildungsprozesse mit beeinflussen können (vgl. Powell et al. 2012b).

Vor dem Hintergrund der zuvor dargestellten Herausforderungen, denen sich europäische Länder stellen müssen, soll in der vorliegenden Arbeit daher die 
Entwicklung der institutionellen Durchlässigkeitsstrukturen in Frankreich und Deutschland vergleichend untersucht werden. Durchlässigkeit kann als ein Merkmal der Ausgestaltung von Bildungssystemen erachtet werden. Sie wird aber durch die institutionelle Ausgestaltung des Bildungssystems, spezifisch der Strukturen, welche die Beziehung zwischen den Organisationsfeldern Berufs- und Hochschulbildung charakterisieren, mitbestimmt. Diese institutionellen Strukturen, welche beeinflussen, inwieweit Bildungsmobilität zwischen Berufs- und Hochschulbildung möglich ist, werden in dieser Arbeit institutionelle Durchlässigkeitsstrukturen genannt.

Folgende drei Forschungsfragen stehen im Zentrum der Analyse:

1. Inwiefern haben sich die institutionellen Durchlässigkeitsstrukturen in Deutschland und in Frankreich in dem Zeitraum von 1985/1990 bis 2012 geändert und wie können diese Entwicklungen beschrieben werden?

2. In welchem Ausmaß kann mit den nationalen Veränderungen auch eine Annäherung der institutionellen Durchlässigkeitsstrukturen zwischen den beiden Ländern festgestellt werden?

3. Welchen Einfluss hatten die europäischen Bildungsprozesse Bologna und Kopenhagen auf die festgestellten Entwicklungen bzw. inwiefern waren diese stärker endogen bedingt?

Die vorliegende Studie zielt damit darauf ab, zum einen den institutionellen Wandel des Verhältnisses von Berufsbildung zu Hochschulbildung in Bezug auf Durchlässigkeit in Deutschland und Frankreich zu rekonstruieren und zum anderen zu eruieren, welche Faktoren jewels die Richtung des Wandels sowie das Ausmaß an Stabilität mitbestimmen.

Der Untersuchungszeitraum in Deutschland beginnt mit der deutschen Vereinigung 1990, da diese als Chance für grundlegende Veränderungen im Bildungssystem hätte genutzt werden können. In Frankreich wird der Zeitraum ab 1985 betrachtet, da in diesem Jahr das berufliche Abitur und damit die Berechtigung zum Hochschulstudium über berufliche Bildungswege eingeführt wurde. Für beide Fallanalysen endet der Untersuchungszeitraum für das empirische Material $2012 .^{3}$

Um die Forschungsfragen zu beantworten, wird in dieser Arbeit ein analytisches Konzept entwickelt, in welchem eine diskursanalytische Perspektive (vgl. Keller 2006, 2007b, 2008, 2009) mit neoinstitutionellen Ansätzen, dem soziologischen Neoinstitutionalismus (vgl. z.B. DiMaggio/Powell 1983; Hasse/ Krücken 2005; Meyer 1977; Meyer/Rowan 1977; 1992; Scott 2008) und dem historischen Institutionalismus (vgl. Mahoney 2000; North 1994; Streeck/ Thelen 2005; Thelen 2004) verbunden wird. Auf diese Weise kann zudem eine 
stärker institutionen- und konflikttheoretische Perspektive auf Bildung (vgl. Meyer 1977) und gesellschaftliche Entwicklungsprozesse eingenommen werden, aus der heraus betont wird, dass institutioneller Wandel von Durchlässigkeitsstrukturen keine bloße funktionale Anpassung an gesellschaftliche Veränderungen wie etwa die demografische Entwicklung darstellt, sondern gleichfalls Ausdruck von veränderten Machtverhältnissen in der Gesellschaft einerseits und veränderter Legitimität bestehender Institutionen andererseits sein kann (vgl. Graf 2013; Mahoney 2000: 517). Insbesondere wenn Institutionen wie die untersuchten institutionellen Durchlässigkeitsstrukturen im Bildungssystem maßgeblich zukünftige Lebenschancen der Individuen und deren soziale Platzierung in der Gesellschaft mitbestimmen, ist davon auszugehen, dass deutliche Verteilungskonflikte in der Gesellschaft bestehen.

Mithilfe der diskursanalytischen Forschungsperspektive, welche sich primär an den Ansatz der wissenssoziologischen Diskursanalyse (vgl. Keller 2006, 2008, 2009) und das Diskursverständnis von Foucault (u.a. Foucault 1981, 2003) anlehnt, werden die Veränderungen der Wissensordnungen in Bezug auf Durchlässigkeit sowie die in den Diskursen kristallisierten Machtkonstellationen und Deutungskämpfe analysiert. In Diskursen können Akteure Ereignisse, wie z.B. die europäischen Bildungsprozesse, als Bestätigung bestehender symbolischer Ordnungen interpretieren oder aber sie zum Anlass für Forderungen nach Umdeutungen oder institutionellen Änderungen nehmen und dadurch sozialen Wandel ermöglichen sowie fördern. Insofern ist anzunehmen, dass insbesondere in Zeiten, in denen Institutionen sich ändernden Bedingungen (wie Europäisierungsprozessen) ausgesetzt sind, in den Diskursen verschiedenste Vorstellungen über den Wandel von Institutionen sichtbar werden. So werden durch die diskursanalytische Perspektive der prozesshafte Charakter sowie die Konflikthaftigkeit, welche mit der Transformation oder auch dem Erhalt institutioneller Ordnungen verbunden sind, in den Vordergrund gerückt.

Gleichzeitig werden durch den differenzierten Institutionenbegriff des soziologischen Neoinstitutionalismus, der die kulturell-kognitive, normative und regulative institutionelle Dimension analytisch unterscheidet (Scott 2008), institutionelle Veränderungen fokussiert. So sollen durch das breite Verständnis von Institutionen institutionelle Durchlässigkeitsstrukturen in allen Dimensionen - auch in der kulturell-kognitiven und normativen - untersucht werden. Es sind somit nicht nur die formalen Regelungen, welche die Durchlässigkeit von Bildungssystemen bestimmen, sondern auch die vorherrschenden Vorstellungen zu Durchlässigkeit, die Normen und Standards. Weiterhin bietet der soziologische und historische Neoinstitutionalismus eine Varianz an theoretischen Erklärungen an, wie Wandel beschrieben werden kann, wie er zustande kommt - z.B. über Diffusionsprozesse von Regeln, Normen und Vorstellungen (vgl. Boli/Thomas 1997; Dobbin et al. 2007), - aber auch Hinweise darauf, was die Richtung des Wandels mitbestimmt. Durch Einbeziehung des historischen Institutionalismus fokussiert 
die Analyse zudem nicht nur Diskontinuitäten, wie meist üblich in Diskursanalysen, sondern berücksichtigt auch historisch gewachsene Strukturen und Abhängigkeiten.

\section{$1.1 \quad$ Verortung der Arbeit}

Diese Studie ist an der Schnittstelle verschiedenster Forschungsausrichtungen, z.B. der Bildungs- und Wissenssoziologie, der vergleichenden Erziehungswissenschaft, der politischen und soziologischen Europäisierungsforschung, der Diskursforschung sowie sozialen Ungleichheitsforschung und damit primär in der Soziologie, aber auch in Politik- und Erziehungswissenschaft verortet und soll auch einen Beitrag für diese leisten.

Durch den Fokus dieser Arbeit auf Durchlässigkeit zwischen Berufs- und Hochschulbildung steht die Analyse der strukturellen Veränderungen des Verhältnisses dieser beiden Organisationsfelder im Blickpunkt. Wie Graf (2013) sowie Powell und Solga (2010) kritisieren, besteht demgegenüber in der Forschung oft die Tendenz dazu, Veränderungen nur in einem der beiden Bildungsbereiche zu betrachten. Eine solche Begrenzung der Forschung ignoriert, dass Systeme der Berufs- und Hochschulbildung miteinander verbunden sind und Veränderungen in einem Bereich daher Veränderungen im anderen Bereich nach sich ziehen können (Powell/Solga 2010).

Es gibt nur wenige Arbeiten, die sich mit den Dynamiken im Verhältnis beruflicher und hochschulischer Bildung befassen. So untersuchen etwa Forscher wie Young und Raffe (1998) die verschiedensten Szenarien, um Gleichwertigkeit zwischen Berufs- und Hochschulbildung zu erreichen, sowie unterschiedliche konzeptuelle Möglichkeiten, wie das Verhältnis beruflicher Bildung zur Hochschulbildung institutionalisiert sein kann (ebd., aber auch Moodie 2008: 170ff.; Raffe 2003; Raffe et al. 1998; Young et al. 1997). Weitere Studien beschäftigen sich mit der zunehmenden Diversifizierung und Differenzierung zwischen Berufs- und Hochschulbildung (Dunkel et al. 2009) oder mit der Konzeptualisierung verschiedener Arten der Verbindung beruflicher Bildung und Hochschulbildung (vgl. Ebner et al. 2013). Graf (2013) sowie Powell und Solga (2010: 706) weisen insbesondere auf die Bedeutung hybrider Organisationsformen für die Verbindung von Berufs- und Hochschulbildung hin.

Weitere Forschungsarbeiten, welche die Verbindung zwischen Berufs- und Hochschulbildung betrachten, sind Studien zur Durchlässigkeit zwischen diesen, primär im Hinblick auf Deutschland, aber auch auf andere Länder mit bestehender Trennung zwischen Berufs- und Hochschulbildung. Auch wenn die Frage von Durchlässigkeit bereits im 20. Jahrhundert untersucht wurde (vgl. z.B. Hegelheimer 1986; Wolter 1994; Wolter/Reibstein 1991; Wolter/Scholz 1986), 
hat die diesbezügliche Forschung insbesondere in den letzten Jahren zugenommen (vgl. z.B. Buhr et al. 2008; Freitag et al. 2011, 2015; Hanft/Brinkmann 2013). Auffällig ist dabei Folgendes: Erstens werden selten institutionelle Entwicklungen der Durchlässigkeitsstrukturen über einen längeren Zeitraum fokussiert (siehe aber Freitag 2012; Nickel/Duong 2012; Schwabe-Ruck 2010; Ulbricht 2012a; Wolter 2012), zweitens wird Durchlässigkeit mit jeweils unterschiedlichem Fokus untersucht, wobei die meisten Studien sich mit den Fragen des Zugangs von beruflicher Bildung zur Hochschulbildung befassen (vgl. Hillmert/Jacob 2003; Nickel/Duong 2012; Nickel/Leusing 2009; Teichler 1990b; Teichler/Wolter 2004; Ulbricht 2012a, 2014; Wolter 1994, 2012). Drittens wird die Frage von Durchlässigkeit selten ländervergleichend analysiert (siehe aber z.B. Bernhard et al. 2013; Ebner et al. 2013; Graf 2013) und viertens stehen im Zentrum der Analyse fast immer die rechtlichen Regelungen und ganz selten normative sowie kulturell-kognitive Strukturen (siehe aber Alheit 2009).

Dagegen sollen in der vorliegenden Arbeit die Veränderungen der Durchlässigkeitsstrukturen sowohl in den rechtlichen Änderungen als auch im Verständnis von Durchlässigkeit sowie die Argumentation zu Durchlässigkeit, d.h. die kulturell-kognitive und z.T. die normative Dimension, über Diskurse analysiert werden. ${ }^{4}$ Auf diese Weise ist es möglich, Veränderungen im Selbstverständnis von Gesellschaften wahrzunehmen, auch wenn diese noch nicht rechtlich festgeschrieben sind. Gleichzeitig soll darüber jedoch ebenfalls deutlich werden, wenn Diskrepanzen zwischen Rechten und den Vorstellungen zu Durchlässigkeit bestehen.

Um die institutionellen Durchlässigkeitsstrukturen adäquat analysieren zu können, wird in der vorliegenden Arbeit zuerst ein Konzept zu Durchlässigkeit aus der bestehenden Literatur erarbeitet, denn Durchlässigkeit stellt keinen klar definierten soziologischen Begriff dar (Freitag 2008), sondern kann äußerst different verstanden werden. Ein solches Konzept soll auch dazu dienen, die Frage von Durchlässigkeit in Ländern wie Frankreich, in denen Durchlässigkeit vor allem als Folge eines begrenzten Verständnisses von Zugangs- und Anrechnungsmöglichkeiten gemeinhin nicht als Forschungsproblem thematisiert wird, umfassender zu untersuchen und zu begreifen. In Frankreich befassen sich Studien zum Verhältnis von Berufs- und Hochschulbildung bisher vor allem mit der Verberuflichung der Hochschulbildung, es wird darin also eine immer stärkere Integration beruflich orientierter Bildungsgänge im tertiären Bildungssystem in den Blick genommen (vgl. z.B. Agulhon 2007, 2011; Maillard/Veneau 2008; Maillard et al. 2004; Pinto 2008; Tanguy 2005).

Die Analyse der Entwicklung von Bildungssystemen aus diskursanalytischer Perspektive hat in den letzten Jahren deutlich zugenommen (vgl. Pfahl 2011; Rothe 2011, 2013; Spilker 2012; Waldow 2007; Wrana 2006). Insbesondere auch der Bologna-Prozess wurde dabei in den Blick genommen (Angermüller/Scholz 2013; Maeße 2009, 2010). Aber wenn die Bedeutung der diskursiven Ebene für Bildungssysteme auch zunehmend in den Blick gerät, so fehlen dennoch Studien im Hinblick auf Durchlässigkeit oder das Verhältnis von Berufs- und Hochschulbildung. 
Das hier erarbeitete Konzept von Durchlässigkeit sowie die Analyse nicht nur rechtlicher, sondern vor allem auch kulturell-kognitiver institutioneller Entwicklungen ermöglichen es, Durchlässigkeit auch als relevantes Forschungsproblem für Frankreich zu untersuchen. Bei der Analyse von Durchlässigkeit interessieren dann nicht nur der Zugang, sondern auch Fragen der Anrechnung, der organisationalen Verbindung und Strukturen, welche durch die Anerkennung der Heterogenität der Lernenden ein erfolgreiches Abschließen des Bildungsgangs in den Fokus rücken. So stellt sich für Frankreich heraus, dass die Durchlässigkeit zwischen Berufs- und Hochschulbildung zwar formal vielfach geregelt ist, in der Praxis jedoch beruflich Qualifizierte auf Sekundarbildungsniveau kaum eine Chance haben, ein Studium abzuschließen. Anhand dieser Durchlässigkeitsproblematik in Frankreich wird daher deutlich, dass Durchlässigkeit nicht bedeutet, lediglich die theoretische Möglichkeit zu erhalten, im anderen Bildungsbereich weiterzulernen, sondern auch eine realistische Chance, dies erfolgreich zu tun.

Dies ist nur ein weiterer Hinweis, warum sich auch ein deutsch-französischer Vergleich als wertvoll für die Frage von Durchlässigkeitsstrukturen erweisen kann. Zudem ermöglicht eine solche Studie, das Verhältnis von Berufs- und Hochschulbildung in ländervergleichender Perspektive zu untersuchen. Der vergleichende Ansatz dient dazu, nationale Selbstverständlichkeiten und Regelungen als kontingente Konstrukte aufzudecken, kulturelle Unterschiede zu verdeutlichen und nationale Entwicklungen in eine neue Perspektive zu rücken. Gleichzeitig kann durch den Vergleich untersucht werden, inwiefern exogene Einflüsse, wie die europäischen Bildungsprozesse, auf die Durchlässigkeitsstrukturen der beiden Länder wirken und ob sich im Laufe der Zeit auch die jeweiligen Bildungsstrukturen angleichen.

Deutsch-französische Vergleiche der Bildungssysteme wurden bereits einige angestellt, allerdings konzentrieren sie sich meist, wie auch die Forschung allgemein, auf nur einen Bildungsbereich und nicht auf deren Verbindung (siehe aber Bernhard et al. 2013; Powell et al. 2012c). So existieren mehrere Studien zu den institutionellen Unterschieden und Gemeinsamkeiten der Berufsbildungssysteme (vgl. Brauns et al. 1999b; Deißinger 2001b; Géhin/Méhaut 1993; Greinert 2005; Hörner 2006; Koch 1998; Lutz 1976; Moebus/Verdier 1998) und der Hochschulsysteme (vgl. Böhlke et al. 2009; Friedberg/Musselin 1993; Goldschmidt 1991; Liebeskind 2011; Musselin 2005). Die vorliegende Studie jedoch leistet einen Beitrag zur vergleichenden Forschung dieser Bildungssysteme, indem das Verhältnis der Berufs- und Hochschulbildung in den Blick genommen wird.

Mit der zweiten und dritten Forschungsfrage, der Frage nach der Annäherung zwischen den deutschen und französischen Bildungssystemen sowie dem Einfluss Europas auf institutionelle Veränderungen, knüpft diese Arbeit an eine breite Literatur zur Internationalisierung, Europäisierung sowie dem Transfer von Politiken, Normen und Vorstellungen an. Bereits seit Jahren sind Globalisierung und Europäisierung Schlagworte für den Veränderungsdruck, welcher auf nationale Gesellschaften wirkt. Dabei gibt es in der Literatur keinen Konsens darüber, 
ob diese Prozesse auch zu einer übernationalen Konvergenz führen. Vertreter der vergleichenden Institutionenanalyse hinterfragen zumindest die Selbstverständlichkeit und angenommene Geschwindigkeit solcher Prozesse. Stattdessen betonen sie die den Veränderungsprozessen unterliegenden Mechanismen, die kausalen Konzepte von evolutionärem und inkrementellem Wandel sowie die bleibenden Differenzen zwischen den einzelnen Nationalstaaten bedingt durch deren Einbettung in die jeweiligen komplexen institutionellen Gefüge (vgl. Campbell 2004; Hall/Soskice 2001; Meyer/Ramirez 2007; Streeck/Thelen 2005). Andererseits erkennen insbesondere world-polity-Forscher_innen die Durchsetzung westlicher Standards, d.h. die weltweite Diffusion von Werten und Normen, die strukturierend auf Staaten, Organisationen und Individuen wirken. Bereits seit dem Beginn der vergleichenden internationalen Bildungsforschung haben Wissenschaftler sich mit Fragen des grenzüberschreitenden Imports und Exports von Bildungskonzepten beschäftigt. Dabei wurde die Diffusion einer quasi ideologischen bildungspolitischen Charta festgestellt (Meyer et al. 1977; Ramirez/Boli-Bennett 1982). So kam es nicht allein zu einer Bildungsexpansion, sondern auch zur Angleichung nationaler Curricula (Benavot et al. 1991; Kamens et al. 1996; Meyer et al. 1992, Meyer/Ramirez 2007) und der zunehmenden Betonung von Bildung als Menschenrecht (Chabbott 2003) ${ }^{5}$. Andere dagegen argumentieren, dass die weltweite Diffusion von Erwartungen, Werten und Strukturen eher zu heterogenen Ergebnissen in Bildung und Wissenschaft geführt hat (u.a. Baker/LeTendre 2005; Krücken 2003; Schriewer 1987, 2007; Blanck et al. 2013).

In der vorliegenden Arbeit wird die Frage nach dem Einfluss der Bolognaund Kopenhagen-Prozesse auf die Durchlässigkeitsstrukturen zwischen Berufsund Hochschulbildung in Deutschland und Frankreich im Zentrum stehen. Der Einfluss dieser europäischen Prozesse wurde in den letzten Jahren zunehmend untersucht, wobei ein stärkeres Interesse an den Entwicklungen im Hochschulsystem zu erkennen ist (u.a. Alesi et al. 2005; Dobbins 2009; Dobbins/Knill 2009; Finger 2011; Krücken 2007; Lörz et al. 2015; Musselin 2008; Powell/Finger 2013; Ravinet 2008; Reinalda/Kulesza 2006; Serrano-Velarde 2009; Voegtle et al. 2011; Witte 2006; Witte et al. 2008). Dobbins (2009) weist darauf hin, dass seit der Initiierung des Bologna-Prozesses im Jahr 1999 die Konvergenz europäischer Hochschulsysteme ein häufig diskutiertes Phänomen in der einschlägigen Konvergenzliteratur ist (vgl. Hackl 2001; Rakic 2001). Was die Auswirkungen des BolognaProzesses anbelangt, beschränkten sich viele Arbeiten auf die Untersuchung der Angleichung von Studienprogrammstrukturen. Zudem wurden teilweise die konkreten nationalen Umsetzungsprozesse untersucht, um auf diese Weise Bedingungen für Konvergenz zu entziffern (Dobbins 2009). Die Annahme, dass durch

5 Diese Betonung von Bildung als Menschenrecht wird dagegen auch durch Globalisierungsprozesse wie die GATS-Verhandlungen eingeschränkt, indem Bildung immer stärker kommodifiziert und als Ware behandelt wird (vgl. Scherrer 2007). 
Bologna nationale Bildungssysteme auf ein gemeinsames Hochschulmodell zusteuern, wird dabei eher infrage gestellt (vgl. Musselin 2008; Witte 2006). Graf (2009) betont in einem Vergleich der Internationalisierungsprozesse von deutschen und britischen Hochschulen, dass die Einbettung der Hochschulsysteme in das jeweilige nationale Kapitalismusmodell zu differenten Internationalisierungsstrategien der Hochschulen beiträgt. Witte et al. (2008) weisen unter anderem darauf hin, dass Bologna partiell als Anlass für tiefgreifende Strukturreformen genutzt wurde, dies aber jeweils nur selektiv und entsprechend der vorherrschenden Präferenzen der beteiligten Akteure. Auch die unterschiedliche Konfiguration von Politiknetzwerken kann zusätzlich zu den verschiedenen nationalen politischen Systemen Wirkungen transnationaler Trends beeinflussen (vgl. Theisens/Enders 2007). Die Mehrzahl der Studien zum Einfluss von Bologna untersucht jeweils nur ein Land, aber mittlerweile gibt es auch eine wachsende Anzahl von Ländervergleichen (u.a. Curaj et al. 2012; Dobbins/Knill 2009; Knill et al. 2013; Kozma 2014; Teichler 2005; Voegtle et al. 2011; Witte 2006; Witte et al. 2008).

Konsequenzen globaler oder transnationaler Entwicklungstrends für die Berufsbildungssysteme werden gemeinhin viel weniger untersucht als im Bereich der Hochschulbildung (siehe aber Deißinger 2001a; Hillmert 2008; Phillips/Ertl 2003). Dies gilt ebenfalls für Studien, welche sich mit dem Einfluss des Kopenhagen-Prozesses beschäftigen, zumal dieser europäische Prozess erst später begonnen hat als der von Bologna. Insofern sind die ersten Studien solche, die Annahmen über mögliche Auswirkungen der europäischen Berufsbildungspolitik anstellen (Baethge et al. 2007; Bouder 2006; Clement 2006; Grollmann et al. 2006; Rauner et al. 2006; Severing 2005). Mit der weiteren Entwicklung des Kopenhagen-Prozesses häuften sich auch die Studien zum konkreten Einfluss des Prozesses (vgl. Baron 2007; Eckert/Zöller 2006; Hanf 2011; Trampusch 2008). Auch hier sind ländervergleichende Analysen deutlich unterrepräsentiert (siehe aber Bieber 2010a; Powell/Trampusch 2012; Trampusch 2009). In Bezug auf die Forschung zum Einfluss der europäischen Bildungsprozesse ist zudem zu konstatieren, dass erstens nicht nur selten die europäischen Prozesse Bologna und Kopenhagen zusammen betrachtet werden (siehe aber Balzer/Rusconi 2007; Bernhard et al. 2013; Graf 2013; Powell et al. 2012a; Powell/Solga 2010; Powell/Trampusch 2012), sondern zweitens auch kaum deren Einfluss auf das Verhältnis von Berufsund Hochschulbildung fokussiert wird (vgl. aber Bernhard et al. 2013; Dobischat et al. 2008; Graf 2013). Mittlerweile gibt es auch Studien zum Einfluss der Europäisierungsprozesse auf die Durchlässigkeit zwischen Berufs- und Hochschulbildung (vgl. z.B. Blings 2012; Blings/Ruth 2012; Freitag 2009a; Freitag 2012; Hartmann et al. 2009; Wanken et al. 2010), wobei Durchlässigkeit in diesem Kontext meist nur im Hinblick auf einen einzelnen Aspekt, z.B. Anrechnung, untersucht wird. 
In dieser Arbeit sollen die bisher meist getrennten Forschungsstränge zur Hochschulbildung und Berufsbildung sowie zu den einzelnen Europäisierungsprozessen zusammengeführt werden. Dabei wird insbesondere auch eine gemeinsame Betrachtung der Bologna- und Kopenhagen-Prozesse als wichtig erachtet, da diese auch auf europäischer Ebene, z.B. durch das europäische Rahmenprogramm „Allgemeine und berufliche Bildung 2020“ (Europäischer Rat 2009), immer stärker miteinander verbunden werden. Vor allem aber ist zu erwarten, dass induzierte Veränderungen im Hochschulsystem gleichzeitig auch Einfluss auf die institutionelle Verfasstheit des beruflichen Bildungssystems haben werden (Powell/Solga 2008, 2010). Um zu verstehen, welche Herausforderungen die Bologna- und Kopenhagen-Prozesse im Hinblick auf die Durchlässigkeit von Berufs- und Hochschulbildung bedeuten, werden diesbezügliche Vorstellungen, Normen und Standards, welche in den Prozessen festgehalten worden sind, analysiert und verglichen und können schließlich zu einem europäischen Durchlässigkeitsmodell zusammengefasst werden. Denn auch in den Prozessen selbst wird die Verbindung zwischen Berufs- und Hochschulbildung fokussiert. Auf diese Weise werden nicht nur einzelne Elemente herausgepickt, die durchlässigkeitsrelevant sein können, wie es in vielen Studien der Fall ist, sondern es wird ein ganzheitliches Bild der europäischen Vorgaben entwickelt. Auch werden zugeschriebene Charakteristika der Prozesse nicht einfach ungeprüft übernommen, sondern die tatsächlich von den Mitgliedsstaaten beschlossenen Forderungen zu Durchlässigkeit aufgegriffen. Auf Basis dieses Modells kann im Anschluss viel umfassender und differenzierter der Einfluss der Prozesse auf die Durchlässigkeitsstrukturen in Deutschland und Frankreich untersucht werden. Zudem verschafft auch die Analyse der diskursiven Strukturen in den beiden Ländern einen Erkenntnisgewinn gegenüber den meisten Studien zum Einfluss europäischer Bildungsprozesse, die allein die regulativen Veränderungen betrachten (vgl. z.B. Bieber 2010b; Trampusch 2008, 2009).

Mit dem Fokus auf Durchlässigkeit steht das Verhältnis zwischen den beiden Organisationsfeldern im Zentrum der Analyse. Fragen zur Bedeutung der europäischen Prozesse und der Konvergenz zwischen dem europäischen Modell und den Entwicklungen in Deutschland und Frankreich werden somit im Hinblick auf das bislang zu wenig beachtete Verhältnis zwischen Berufs- und Hochschulbildung, konkret in Bezug auf Durchlässigkeit, beantwortet.

Die Bearbeitung der Forschungsfragen, wie sich die nationalen Durchlässigkeitsstrukturen in Deutschland und Frankreich verändern, welchen Einfluss europäische Bildungsprozesse dabei haben und inwiefern sich die institutionellen Strukturen der beiden traditionell als deutlich unterschiedlich typisierten Bildungssysteme angleichen, leistet einen wichtigen Beitrag zur Bearbeitung der von Solga und Becker (2012: 29f.) identifizierten Forschungslücken der aktuellen bildungssoziologischen Forschung: So werden ländervergleichend die institutionellen Bildungsstrukturen und deren Entwicklung sowie die Interdependenz von in- 
ternationalen und nationalen institutionellen Entwicklungen untersucht. Gleichzeitig kann in dieser Arbeit gezeigt werden, wie sich Entwicklungsprozesse in unterschiedlichen institutionellen Dimensionen vollziehen und was die Prozesse mitbestimmt. Auch soll die Arbeit ein besseres Verständnis von Durchlässigkeit und deren differenzierten und mehrdimensionalen Institutionalisierungsmöglichkeiten ermöglichen, welches auch Hinweise für mögliche Reformen geben kann.

In welcher Weise in diesem Buch die zuvor skizzierten Forschungsfragen beantwortet werden, soll nachfolgend erläutert werden.

\subsection{Aufbau des Buches}

Das Kapitel 2 dient der Klärung des Konzepts der Durchlässigkeit und seiner theoretischen Einbettung in die Theorieansätze sozialer Ungleichheit. Dabei wird erstens zwischen sozialer und institutioneller Durchlässigkeit unterschieden und herausgearbeitet, inwiefern institutionelle Durchlässigkeit eine Voraussetzung für ein sozial durchlässiges Bildungssystem sein kann. Anschließend wird auf Basis der bestehenden Literatur, welche primär die Durchlässigkeitsproblematik in Deutschland behandelt, ein Konzept institutioneller Durchlässigkeit erarbeitet, das aus mehreren Aspekten besteht. Durchlässigkeit kann danach als Frage des Zugangs zu Bildungsbereichen, der Anrechnung von bereits Gelerntem im jeweils anderen Bildungsbereich, der organisationalen Verbindung zwischen Bildungsbereichen und schließlich auch als Frage des Umgangs mit heterogenen Bedürfnissen der Lernenden gefasst werden. An diese Konzeption von Durchlässigkeit anknüpfend, wird anschließend die Frage nach Bildungsdurchlässigkeit aus einer strukturfunktionalistischen und einer stärker institutionen- und konflikttheoretischen Perspektive betrachtet. Dabei werden die Fragen dieser Arbeit stärker aus letzterer Perspektive analysiert, da angenommen wird, dass institutioneller Wandel von Durchlässigkeitsstrukturen keine bloße funktionale Anpassung an Umweltveränderungen darstellt, sondern auch Ausdruck veränderter Machtverhältnisse in der Gesellschaft einerseits und veränderter Legitimität bestehender Institutionen andererseits ist.

In Kapitel 3 wird der theoretische Rahmen dargestellt, welcher die Analyse des institutionellen Wandels der nationalen Durchlässigkeitsstrukturen anleitet. Entsprechend der stärker institutionen- und konflikttheoretischen Perspektive wird die Frage des institutionellen Wandels anhand der Verbindung des soziologischen und historischen Neoinstitutionalismus mit einer wissenssoziologischen Diskursperspektive untersucht. So kann mithilfe des soziologischen Neoinstitutionalismus analysiert werden, inwiefern die Institutionen im Hinblick auf Durchlässigkeit an Legitimität gewinnen oder verlieren und welche institutionellen Änderungen sich vollziehen. Andererseits werden aus der diskursanalytischen For- 
schungsperspektive die Konflikthaftigkeit und der prozesshafte Charakter, welche mit institutionellem Wandel verbunden sind, betrachtet. Da in Diskursen die Prozesse der Institutionalisierung, der Reproduktion und der Veränderung von gesellschaftlichen Wissensvorräten, also den Ideologien, Leitbildern und Vorstellungen, sichtbar werden, ist anzunehmen, dass auch gesellschaftliche Konfliktlinien nachvollziehbar werden. Das Kapitel ist in drei Abschnitte eingeteilt. Der erste dient der Klärung zentraler Begriffe und Konzepte, welche die Grundlage der empirischen Untersuchung sind, sowie deren Verhältnis zueinander. Im zweiten Abschnitt wird dargestellt, was unter institutionellem Wandel verstanden wird und wie dieser erklärt und beschrieben werden kann. Und schließlich wird gezeigt, warum sich die institutionelle und die diskursanalytische Forschungsperspektive fruchtbar ergänzen und nur durch ihre Kombination für diese Arbeit den passenden Rahmen liefern.

Darauf aufbauend werden in Kapitel 4 das qualitativ-explorative Forschungsdesign und der verwendete Methodenmix erläutert. So werden in einem ersten Schritt das vergleichende Fallstudiendesign, welches einen diskursanalytischen Forschungsansatz mit dem des process tracing verbindet, und die Anlage der Untersuchung, d.h. die Logik der einzelnen Analyseschritte, die Auswahl des Untersuchungszeitraums, die Fallauswahl sowie die Bedeutung des Vergleichs für diese Arbeit erklärt. Dabei beruht die Analyse der institutionellen Entwicklung primär auf der diskursanalytischen Auswertung von mehr als 250 bildungspolitischen Dokumenten der wichtigsten Akteure der Berufs- und Hochschulbildung in Frankreich und Deutschland. Um auch die regulative institutionelle Dimension zu erfassen, wird die Diskursanalyse durch eine Dokumentenanalyse rechtlicher Texte sowie durch 31 Expert_inneninterviews und eine Analyse von Sekundärliteratur ergänzt. Der Ansatz des process tracing leitet die Untersuchung im Hinblick auf die schrittweise Rekonstruktion der nationalen Entwicklungen und deren Erklärung durch exogene, d.h. europäische, aber auch endogene Einflüsse an. Die Analyse der europäischen Bildungsprozesse Bologna und Kopenhagen erfolgt primär auf Basis einer theoriegeleiteten qualitativen Inhaltsanalyse der Deklarationen und Kommuniqués dieser Prozesse. Die Untersuchung der Frage nach deren Einfluss auf die nationalen Bildungssysteme erfolgt wiederum auf Basis des Vergleichs der nationalen Durchlässigkeitsstrukturen mit dem aus den europäischen Dokumenten rekonstruierten Durchlässigkeitsmodell sowie durch Expert_inneninterviews. Da die europäischen Prozesse noch nicht sehr lange bestehen, sind es insbesondere Expert_innen, welche einen Eindruck der Wirkung europäischer Prozesse vermitteln können. Die Frage der Konvergenz zwischen den Ländern kann anhand eines Vergleichs der analysierten Entwicklungen der nationalen Durchlässigkeitsstrukturen beantwortet werden.

Im zweiten Abschnitt von Kapitel 4 werden die methodischen Verfahren und das konkrete Vorgehen in dieser Arbeit für die erfolgte wissenssoziologische Dis- 
kursanalyse, die theoriegeleitete qualitative Inhaltsanalyse, die Expert_inneninterviews sowie die Dokumentenanalyse erklärt. Der dritte Abschnitt dient der Reflexion der Güte der durchgeführten Analysen.

Mit Kapitel 5, in welchem die europäischen Entwicklungen aufgezeigt werden, beginnt der empirische Teil der vorliegenden Studie. Ziel dieses Kapitels ist es, ein Bild davon zu erhalten, wie die Bologna- und Kopenhagen-Prozesse in die supranationale europäische Bildungspolitik eingebettet sind, welchen Stellenwert Durchlässigkeit zwischen Berufs- und Hochschulbildung einnimmt und welches Modell von Durchlässigkeit durch die Prozesse in die Nationalstaaten diffundieren könnte, d.h., welche Art von Durchlässigkeitsstrukturen auf europäischer Ebene legitimiert werden. Dafür wird in einem ersten Schritt ein kurzer historischer Überblick über die zunehmende Relevanz von Bildungsfragen in der europäischen Bildungspolitik sowie das Verhältnis von Allgemein-, Hochschul- und Berufsbildung innerhalb dieser Politik vermittelt. Diese historische Einbettung dient vor allem der Abgrenzung und der Betonung der Spezifika der intergouvernementalen Bildungsprozesse Bologna und Kopenhagen und liefert die Begründung dafür, warum speziell diese Prozesse als besonders untersuchenswert für das Verhältnis von Berufs- und Hochschulbildung in den Nationalstaaten gelten können.

Im zweiten Schritt werden die Bologna- und Kopenhagen-Prozesse mithilfe der Inhaltsanalyse der verabschiedeten Deklarationen und Kommuniqués beider Prozesse genauer daraufhin untersucht, inwiefern und auf welche Weise Durchlässigkeit zwischen Berufs- und Hochschulbildung in ihnen thematisiert wird. So interessiert es, wie Durchlässigkeit jeweils verstanden und begründet wird, mithilfe welcher Standards sie umgesetzt werden soll und wie die Forderungen regulativ umzusetzen sind. Schließlich wird anhand des Vergleichs der beiden Prozesse ein gemeinsames europäisches Durchlässigkeitsmodell rekonstruiert.

Die Kapitel 6 und 7 dienen der Untersuchung der institutionellen Entwicklung in Deutschland. In Kapitel 6 wird die deutsche Durchlässigkeitsproblematik dargestellt. Dafür werden in einem ersten Schritt das von Baethge (2006) identifizierte Bildungsschisma und daran anschließend die Organisation sowie Steuerung der Hochschul- und Berufsbildung in Deutschland beschrieben. Auch bestehende Übergänge zwischen den Bildungsbereichen werden identifiziert. Um die Veränderungen in Bezug auf Durchlässigkeit zwischen beruflicher und Hochschulbildung in den letzten Jahrzehnten verstehen und einordnen zu können, wird in einem dritten Schritt die historische Entwicklung der deutschen Durchlässigkeitsproblematik vor dem Untersuchungszeitraum dargestellt. Der Fokus liegt dabei auf der Frage des Hochschulzugangs für beruflich Qualifizierte, da dieser Aspekt in den Quellen zur historischen Entwicklung der dominante war, an dem sich das Verhältnis der beiden Organisationsfelder herauskristallisierte. Zudem wird ein kurzer Einblick in die durchlässigkeitsrelevanten Strukturen des DDRSystems gegeben, denn mit der Wiedervereinigung hätten auch die Strukturen aus den neuen Bundesländern Einfluss auf die weitere Entwicklung haben können. 
Ziel dieses Kapitels ist es, ein Verständnis des deutschen Bildungssystems zu erlangen, welches notwendig ist, um die Entwicklungen der institutionellen Durchlässigkeitsstrukturen in den letzten 25 Jahren zu verstehen.

Die Analyse der institutionellen Veränderungen in Kapitel 7 erfolgt in drei Schritten. Entsprechend der analytischen Unterscheidung in einen Untersuchungszeitraum vor dem Beginn der Bologna- und Kopenhagen-Prozesse (19901998) und dem Zeitraum seitdem (1999-2012), welche wichtig ist, um einen Wandel besser darstellen und vor allem auch den Einfluss der europäischen Prozesse feststellen zu können, werden die institutionellen Veränderungen für die Untersuchungszeiträume unabhängig voneinander beschrieben. In einem dritten Schritt werden die Entwicklungen verglichen und eingeordnet. Diese Untersuchungen der institutionellen Entwicklung der Durchlässigkeitsstrukturen basieren primär auf der Diskursanalyse der Dokumente bildungspolitischer Akteure der Berufsund Hochschulbildung, so dass der Fokus auf die Analyse der kulturell-kognitiven und teilweise auch der normativen institutionellen Dimension gelegt wird. Gezeigt wird, wie in den jeweiligen Zeiträumen Durchlässigkeit von den bildungspolitischen Akteuren konstruiert wird, aber auch, welche Deutungskämpfe und damit Infragestellungen der Legitimität bestehender Institutionen sichtbar werden. Auf diese Weise kann die Dynamik institutioneller Entwicklungen dargestellt und bereits zum Teil erklärt werden. Aber auch regulative Entwicklungen, welche auf Basis der Dokumentenanalyse erfasst wurden, werden in die Analyse eingearbeitet.

Das Ziel des Kapitels besteht darin, die Entwicklungen der deutschen Durchlässigkeitsstrukturen darzustellen und zu analysieren, inwiefern der Wandel seit 1990 zu einer Fortführung eingeschlagener Entwicklungspfade, ihrer Veränderung oder sogar einer gänzlichen Abkehr von historisch gewachsenen Institutionen geführt haben. Während eine Weiterführung bedeuten würde, dass die historisch gewachsene Hierarchie der Bildungsbereiche und die institutionelle Trennung zwischen Berufs- und höherer Allgemeinbildung ohne Übergänge weiterbestünde, hieße eine Abkehr von diesen institutionellen Prinzipien faktisch entweder eine Gleichwertigkeit oder sogar Höherbewertung beruflicher Bildung sowie eine aufgehobenen Trennung der Bereiche. Insgesamt kann in Deutschland eine institutionelle Veränderung in Richtung einer größeren Durchlässigkeit zwischen Berufs- und Hochschulbildung, die sich sowohl in der kulturell-kognitiven als auch in der normativen und regulativen Dimension niederschlägt, festgestellt werden. Doch wie ist diese Entwicklung genau einzuordnen? Im Rahmen der Analyse soll schließlich auch deutlich werden, inwiefern bestehende Barrieren zu Durchlässigkeit verringert wurden und worin weiterhin Barrieren bestehen.

In Kapitel 8 stehen das französische Bildungssystem und die identifizierte Durchlässigkeitsproblematik für den französischen Fall im Vordergrund. Es dient als Grundlage für die Analyse des institutionellen Wandels der Durchlässigkeitsstrukturen in Frankreich in Kapitel 9. Es kann gezeigt werden, dass Durchlässigkeit zwischen Berufs- und Hochschulbildung nicht nur ein Problem in Ländern 
mit stark segmentierten Bildungsbereichen im Sekundar- und Tertiärbereich, wie in Deutschland, ist, sondern durchaus auch in einem weniger segmentierten und stratifizierten System wie Frankreich. Um das Problem des systematisch erhöhten Studienmisserfolgs beruflich Qualifizierter besser verstehen zu können, werden in einem zweiten Schritt die Organisationsfelder Berufs- sowie Hochschulbildung und deren Steuerung sowie die bestehenden Bildungswege zwischen den Organisationsfeldern beschrieben. Schließlich erfolgt eine historische Einbettung der Durchlässigkeitsproblematik, die ebenfalls dem besseren Verständnis der gewachsenen Bildungsstrukturen und Hierarchien zwischen Berufs- und Hochschulbildung dient. Zudem ist die Kenntnis der historischen Evolution für die Analyse des institutionellen Wandels notwendig, denn nur so können endogene Strukturen als pfadabhängige Faktoren identifiziert werden, was die Einschätzung darüber ermöglicht, ob sich das Bildungssystem entlang bestehender Pfade oder durch die Schaffung neuer Strukturen weiterentwickelt.

In Kapitel 9 sollen aufbauend auf den Darstellungen in Kapitel 8 die Veränderungen der institutionellen Durchlässigkeitsstrukturen von 1985 bis 2012 in Frankreich analysiert werden. Im Fokus der Analyse steht dabei die Durchlässigkeit aus dem beruflichen Zweig der Sekundarbildung in das Hochschulsystem. Auch für den französischen Fall werden zwei Zeiträume unterschieden, die Phase vor 1999 und den Zeitraum danach, somit erfolgt die Analyse analog der Vorgehensweise zum deutschen Fall in Kapitel 7. Zuerst werden die institutionellen Entwicklungen in den beiden Zeiträumen dargestellt, wobei sowohl die durchlässigkeitsrelevanten Debatten als auch die Struktur der Diskurse analysiert werden. Anschließend soll die Frage nach dem Wandel der Durchlässigkeitsstrukturen in Frankreich zusammenfassend beantwortet werden. Auch in Frankreich können signifikante Veränderungen der Durchlässigkeitsstrukturen festgestellt werden, wobei sich diese jedoch verstärkt in der kulturell-kognitiven institutionellen Dimension gezeigt haben. Die Einordnung dieser Veränderungen im Hinblick auf bestehende Entwicklungspfade erfolgt ebenfalls in Kapitel 9.

Während in den Länderkapiteln gezeigt wird, dass und wie sich jeweils in Deutschland und Frankreich die institutionellen Dimensionen zur Durchlässigkeit zwischen beruflicher und Hochschulbildung gewandelt haben, stehen in Kapitel 10 der Vergleich zwischen den Ländern und die Frage nach dem Einfluss Europas im Vordergrund. So wird in einem ersten Schritt untersucht, inwiefern sich das Verständnis von einem durchlässigen Bildungssystem und dessen diskursive Behandlung sowie die gesetzlichen Regelungen zu Durchlässigkeit zwischen Deutschland und Frankreich is heute angeglichen haben. Durch den Vergleich können nationale Selbstverständlichkeiten und Regelungen als kontingente Konstrukte aufgedeckt werden. Dabei wird festgestellt, dass zwar eine Annäherung in einem gewissen Ausmaß stattgefunden hat, diese aber nicht zu einer Konvergenz der Durchlässigkeitsstrukturen führt. 
Im zweiten Teil dieses Kapitels wird eruiert, wie die obige und die nationalen Entwicklungen zu erklären sind. Welchen Einfluss haben die europäischen Bildungsprozesse Bologna und Kopenhagen? Um diese Frage zu beantworten, wird untersucht, inwiefern es zu einer Annäherung der nationalen Strukturen an das in Kapitel 5 rekonstruierte europäische Modell von Durchlässigkeit in Deutschland und Frankreich gekommen ist. Dafür wird zuerst untersucht, inwiefern die institutionellen Strukturen der beiden Länder bereits vor 1999 den europäischen Vorgaben entsprachen, so dass die vermeintliche Stärke des normativen Anpassungsdrucks auf die Länder sichtbar wird. Insbesondere da Frankreich und Deutschland zu den Vorreiterstaaten der beiden untersuchten europäischen Bildungsprozesse gehören und die Prozesse mit der Methode der offenen Koordinierung (OMK) arbeiten und sich stark an bestehende Praktiken und Standards in den Mitgliedsstaaten anlehnen, ist es wichtig zu ergründen, inwiefern in den gemeinsam verfassten europäischen Dokumenten nicht bereits die in den Ländern bestehenden Standards eingeschrieben wurden. In diesem Fall wäre der Handlungsdruck wesentlich geringer. Anschließend wird analysiert, inwiefern die Veränderung der nationalen Durchlässigkeitsstrukturen zu einer Annäherung an die europäischen Standards geführt hat. Für beide Länder kann dabei eine gewisse Entwicklung in Richtung europäischer Vorgaben festgestellt werden. In einem dritten Schritt werden die Annahmen über den Einfluss der europäischen Prozesse, welche aus der vorhergehenden Analyse entstanden sind, anhand der Bedeutungszuschreibung der europäischen Prozesse in den Diskursen und in den geführten Expert_inneninterviews ${ }^{6}$ geprüft. Die Kombination von Diskursen und der Befragung von Expert_innen erscheint hier sinnvoll, da politische Akteure nicht immer zugeben, dass Reformen durch exogene Kräfte beeinflusst wurden (vgl. Waldow 2009: 478). Wurde in den Diskursen oder Interviews direkt auf europäische Prozesse oder Standards verwiesen? Welche Rolle messen die Expert_innen den europäischen Prozessen zu?

Der letzte Abschnitt des Vergleichskapitels verbindet die Erkenntnisse zu den Entwicklungen der nationalen Durchlässigkeitsstrukturen mit dem analtisch erfassten Einfluss der Europäisierungsprozesse, um die nationalen Entwicklungen unter Einbezug sowohl des Europäisierungseinflusses als auch endogener Faktoren zu erklären. Dabei können für Deutschland und Frankreich äußerst differente Einflüsse auf die nationalen institutionellen Entwicklungen identifiziert werden, wobei in Deutschland europäische Prozesse einen stärkeren Einfluss auf die Entwicklungen genommen haben als in Frankreich. Kapitel 10 soll daher auch Aufschluss über die potenzielle Wirkmächtigkeit europäischer Prozesse und deren Begrenzung durch nationale Entwicklungspfade geben.

Abschließend werden in Kapitel 11 die theoretischen Überlegungen und zentralen Ergebnisse dieser Arbeit zusammengefasst, wobei die Beantwortung der drei leitenden Untersuchungsfragen im Zentrum steht. Anschließend werden 
Grenzen der empirischen Untersuchung, wie die Beschränkung auf spezifische Analyseebenen und die damit einhergehende Vernachlässigung anderer, aufgezeigt und mögliche ergänzende Forschungsansätze erwogen. Schließlich werden in einem letzten Schritt gesellschaftliche Implikationen der Untersuchungsergebnisse diskutiert. Was kann aus dieser Studie für Reformen von Bildungssystemen gelernt werden? So wird z.B. auf die Vielseitigkeit des Durchlässigkeitskonzepts und die Bedeutung der nicht zu vernachlässigenden unterschiedlichen institutionellen Dimensionen, auf denen dieses institutionalisiert wird, verwiesen. 


\section{Durchlässigkeit - Eine konzeptionelle und theoretische Annäherung}

In der vorliegenden Arbeit sollen die Veränderungen nationaler Durchlässigkeitsstrukturen untersucht werden. Doch was ist Durchlässigkeit? Was steht hinter diesem häufig benutzten, dennoch wenig definierten und konzeptualisierten Begriff?

Die Frage nach der Durchlässigkeit von Bildungssystemen ist seit mehreren Jahren in aller Munde, jedoch wird selten explizit dargelegt, was genau mit Durchlässigkeit gemeint sein soll. Dies gilt sowohl für politische als auch für sozialwissenschaftliche Publikationen (siehe aber BIBB 2011; Freitag 2008, 2009c; Wolter et al. 2014) ${ }^{7}$. Das liegt partiell ebenfalls daran, dass Durchlässigkeit keinen klassischen soziologischen Begriff mit fester Definition darstellt. Dessen ungeachtet wird mit dem Begriff Durchlässigkeit in den Sozialwissenschaften gearbeitet. Anhand dieser Arbeiten und dem darin verwendeten Verständnis von Durchlässigkeit soll demgemäß ein Konzept von Durchlässigkeit herausgearbeitet werden, um dieses für die weitere Analyse fruchtbar zu machen. Dabei wurden insbesondere Arbeiten verwendet, welche sich mit der Durchlässigkeit zwischen Berufsund Hochschulbildung beschäftigen.

Bevor das analytische Konzept vorgestellt wird, steht eine allgemeine Begriffsklärung im Vordergrund, wobei eine alltagspraktische Ebene von einer stärker soziologischen Definitionsebene unterschieden wird. Aus letztgenannter Perspektive wird das allgemeine Verständnis von sozialer und institutioneller Durchlässigkeit sowie deren Verhältnis zueinander dargestellt. Dabei wird deutlich, dass die Analyse von institutionellen Durchlässigkeitsstrukturen gleichfalls aus einer sozialen Ungleichheitsperspektive relevant ist. Demgemäß wird in einem letzten Schritt, nachdem das erarbeitete Konzept institutioneller Durchlässigkeit dargelegt wurde, das Problem von Durchlässigkeit aus zwei differenten theoretischen Perspektiven auf soziale Ungleichheit, einer stärker funktionalistischen und einer institutionellen konflikttheoretischen, betrachtet.

7 Dieses Buch basiert auf der im April 2014 eingereichten Version der Dissertation, so dass neuere Literatur wie Wolter et al. 2014, die ebenfalls ein ausführlicheres Konzept von Durchlässigkeit entwickelt haben, bei der Erarbeitung dieses Konzepts noch nicht berücksichtigt werden konnte. Auf Ähnlichkeiten wird im Verlauf des Kapitels verwiesen. 


\subsection{Begriffsdeutung}

Wie von Freitag (2008: 111) bereits festgestellt, ist Durchlässigkeit ein wissenschaftlich unscharfer Begriff und bedarf einer genaueren Einbettung in die sozialwissenschaftliche Literatur. Dies soll sowohl über eine stärker alltagspraktische als auch über eine soziologische Sichtweise erfolgen.

Für die alltägliche Praxis, ist im Duden folgende Bedeutung von durchlässig zu finden:

(von einem abgeschlossenen System o. Ä.) einen Austausch, Wechsel, Wandel o. Ä. ermöglichend (Duden 1976: 590)

Aus dieser Begriffserklärung geht hervor, dass es zwar um die Möglichkeit des Austausches bzw. des Passierens aus einem Bereich in den anderen geht, aber nicht darum, die Bereiche oder Systeme an sich aufzulösen. Die Grenze zwischen den Systemen oder Bereichen wird passierbar, sie bleibt aber bestehen - ebenso wie die Bereiche selbst. Dies ist insofern wichtig, als die Frage der Durchlässigkeit einen eher strukturkonservativen Blick auf Systeme bietet. Eine Auflösung ist per se nicht vorgesehen. Diese Bedeutung von Durchlässigkeit erklärt damit aber wiederum auch, warum vor allem in Bildungssystemen mit starken Grenzziehungen zwischen einzelnen Bildungsbereichen und Organisationsfeldern Durchlässigkeit ein wichtiges Thema der Bildungspolitik und -forschung ist, während in anderen Ländern, welche weniger stratifizierte und segmentierte Bildungssysteme aufweisen, Durchlässigkeit im Bildungssystem weniger problematisiert wird. Auf die Organisationsfelder Hochschul- und Berufsbildung bezogen erklärt sich daher auch die starke Fokussierung der deutschen sozialwissenschaftlichen Literatur auf Durchlässigkeit vor allem im Vergleich zu Großbritannien, den USA oder auch Frankreich - also Ländern, in welchen die Trennung zwischen der höheren Allgemeinbildung und der Berufsbildung viel weniger ausgeprägt ist (vgl. Powell/Solga 2008). Gleichwohl weisen aber auch diese Bildungssysteme eine starke Differenzierung im Bildungssystem mit dazugehörigen Vor- und Nachteilen auf.

\subsubsection{Soziale und institutionelle Durchlässigkeit}

Der Begriff Durchlässigkeit wird in der Bildungssoziologie im Großen und Ganzen auf zweierlei Weise genutzt: einmal im Sinne sozialer Durchlässigkeit, d.h. als soziale Bildungsmobilität, und zum anderen als strukturelle Durchlässigkeit im Sinne der Möglichkeit des Wechsels von Bildungswegen und zwischen Bildungssystemen (vgl. Freitag 2008).

Für erstere Bedeutung ist eher die Frage des sozialen Aufstieges über Bildung von Belang. Untersuchungen mit diesem Fokus analysieren die intra- oder intergenerationale Mobilität im Bildungs- und Lebensverlauf - zumeist basierend auf 
den Bildungsabschlüssen oder dem beruflichen Status sowie weiteren Merkmalen. (siehe z.B. Erikson/Goldthorpe 1992; Mayer/Solga 1994; Pollak 2010; Reimer/Pollak 2010). Es kann zwischen horizontaler und vertikaler Mobilität unterschieden werden. Der Wechsel von Individuen in statusähnliche Positionen fokussiert horizontale Mobilität während der Wechsel in Positionen mit differentem Status vertikale Mobilität untersucht. Bei letzterem geht es daher um die Frage, ob Bildung in der Gesellschaft eher zur Auflösung von Klassen- oder Schichtzugehörigkeiten führt oder deren Reproduktion unterstützt.

Die zweite Perspektive, die strukturelle Durchlässigkeit, richtet ihren Blick auf die institutionelle Ausgestaltung des Bildungssystems selbst. Der Fokus der Analyse ruht hier auf der Organisations- und Systemebene. Es wird eine mesound makrosoziologische Perspektive eingenommen und analysiert, inwiefern das Bildungssystem mit seiner institutionellen Verfasstheit Übergänge von Lernenden aus Bildungsbereichen oder -gängen ermöglicht. Durch diesen Fokus auf Institutionen wird in der vorliegenden Arbeit daher von institutioneller Durchlässigkeit gesprochen. Unterscheiden kann man auch hier horizontale institutionelle Durchlässigkeit, d.h. die Ermöglichung des Wechsels von Bildungsgängen auf formal gleichem Bildungsniveau, von der vertikalen institutionellen Durchlässigkeit, d.h. der institutionellen Ermöglichung des Wechsels in Bildungsgänge mit einem formal höheren Bildungsniveau.

Räumliche Durchlässigkeit kann beide Formen der institutionellen Durchlässigkeit beinhalten, denn hier handelt es sich um die Möglichkeit der geografischen Bildungsmobilität meist zwischen Nationalstaaten, aber auch zwischen Bundesländern, wobei die Mobilität dann sowohl horizontal als auch vertikal ausgerichtet sein kann. Um diese zu ermöglichen, müssen durchlässige Strukturen nicht nur innerhalb eines Bildungssystems, sondern auch zwischen Bildungssystemen aufgebaut werden. Insbesondere die in der vorliegenden Arbeit betrachteten Europäisierungsprozesse - der Bologna- und der Kopenhagen-Prozess - zielen im Kern auf die Mobilität über nationalstaatliche Grenzen hinweg. Freitag (2008: 111, Hervorhebung im Original) stellt einen Diskurswandel fest:

Wird Durchlässigkeit in den 1960er - 1980er Jahren als „soziale Durchlässigkeit“ stark im Zusammenhang mit sozialer (Bildungs-)Ungleichheit und Schicht thematisiert, verliert der Erklärungszusammenhang gegen Ende des 20. Jahrhunderts an Bedeutung.

Seit den 1990er Jahren vollzieht sich ein Paradigmenwechsel und Durchlässigkeit wird im Diskurs vielmehr als strukturelle Durchlässigkeit mit einem Hauptfokus auf räumliche Mobilität gefasst (Freitag 2008). Letztere ist zwar eng mit den Europäisierungsprozessen verknüpft, soll aber in dieser Arbeit nicht im Vordergrund stehen.

Auch wenn soziale und institutionelle Durchlässigkeit partiell und zwar vor allem auch in der politischen Sphäre getrennt behandelt werden (vgl. Finger 2011; Powell et al. 2012a), sind diese jedoch keine unabhängigen Phänomene. So schreibt Archer (1986: 83) „educational systems are differentially penetrable to 
different social groups; they are never equally permeable to all, and at any given time they are usually impenetrable to some". Aber warum gibt es eine systematisch unterschiedliche soziale Durchlässigkeit der Bildungssysteme und damit unterschiedliche soziale Mobilitätschancen für bestimmte Gruppen? Eine der Erklärungen in diesem Kontext ist auch in der institutionellen Ausgestaltung der Bildungssysteme zu suchen. Das bedeutet, dass die Grenzziehung zwischen Bildungsbereichen als Mechanismus zu der sozialen Reproduktion gesellschaftlicher Ungleichheitsstrukturen beiträgt. Umgekehrt können dann institutionell durchlässige Systeme eine Voraussetzung für soziale Durchlässigkeit, d.h. für soziale Mobilität, sein. Dabei können institutionell durchlässige Bildungsstrukturen aber nur als eine von mehreren Bedingungen für soziale Durchlässigkeit oder Mobilitätschancen gelten. So zeigen auch Studien, dass Opportunitätsstrukturen allein, die z.B. das Nachholen von Abschlüssen ermöglichen, zumindest in Deutschland bisher nicht $\mathrm{zu}$ einer Verringerung sozialer Ungleichheit beitragen (vgl. Hillmert/Jacob 2005). Gäbe es allerdings institutionalisierte Übergangsmöglichkeiten nicht und wären die einzelnen Bildungsbereiche abgeschottet und würden weiterhin systematisch unterschiedliche Lebenschancen für die Lernenden bereitstellen, wäre jede (Fremd- und Selbst-)Selektion im Bildungssystem eine finale. Auf diese Weise würden Sackgassen im Bildungssystem kreiert, welche eine barrierefreie Bildungsbiografie sowie soziale Aufstiege verhindern (vgl. StammRiemer/Hartmann 2011).

Es wird dementsprechend durch die dargestellte Verbindung zwischen sozialer und institutioneller Durchlässigkeit deutlich, dass die Untersuchung der Entwicklung der Durchlässigkeitsstrukturen aus Perspektive der sozialen Ungleichheitsforschung hochgradig relevant ist. Aus diesem Grund soll zum einen in Abschnitt 2.3 eine Einbettung des Durchlässigkeitskonzepts in die Theorien der sozialen Ungleichheitsforschung erfolgen. Zuvor wird das Konzept der institutionellen Durchlässigkeit genauer vorgestellt.

\subsection{Identifikation eines Durchlässigkeitskonzepts}

Institutionelle Durchlässigkeit zielt aus Systemsicht darauf, bestehende institutionelle Trennungen von Bildungsbereichen, Bildungsgängen und Bildungsstufen so aufzuheben, dass flexible und funktionsfähige Übergänge im nationalen Bildungssystem innerhalb eines Bildungsbereichs sowie an unterschiedlichen Schnittstellen von Bildungsbereichen möglich werden. Für Individuen eröffnet Durchlässigkeit im Bildungssystem (und darüber hinaus) die Möglichkeit, in jeder Lebensphase an vorhandene Abschlüsse und Kompetenzen anzuknüpfen und Bildungskarrieren in verschiedene Richtungen fortzusetzen und vorherige Qualifikationen sowie Kompetenzen, unabhängig davon, von wem oder wo sie erworben wurden, anerkennen zu lassen. 
Der Fokus der vorliegenden Arbeit ruht auf der Analyse von institutioneller Durchlässigkeit, spezifisch zwischen beruflicher Bildung und Hochschulbildung. Jedoch ist Durchlässigkeit auch zwischen anderen Bildungsbereichen ein wichtiges Thema. So kann z.B. die Frage nach Durchlässigkeit gestellt werden, wenn man sich die tatsächlichen Bildungsmöglichkeiten in Deutschland nach einem Hauptschulabschluss oder Sonder- bzw. Förderschulabschluss betrachtet, welche mittlerweile sehr stark begrenzt sind (vgl. Pfahl 2011; Pfahl/Powell 2011; Protsch 2014; Solga/Wagner 2001). Ähnliches gilt auch für die Strukturen des deutschen Übergangssystems ${ }^{8}$, welches für diejenigen Lernenden eingerichtet wurde, welche Probleme haben, eine Berufsausbildung zu finden (vgl. Baethge et al. 2007; Solga 2009). Obwohl gerade das Übergangssystem als Brücke zwischen Schul- und Berufsbildungssystem eingerichtet wurde, um Durchlässigkeit zu erhöhen, kann konstatiert werden, dass dies nicht immer wie gewünscht gelingt und sogar negative Auswirkungen für die weitere Bildungskarriere haben kann (Gei et al. 2011: 9).

Institutionelle Durchlässigkeit zwischen getrennten Bildungsbereichen kann unterschiedlich erreicht und konzeptualisiert werden. Im Folgenden soll daher das analytische Konzept Durchlässigkeit, welches aus vier Aspekten besteht, entwickelt werden. Die Aspekte beruhen dabei vor allem auf der Rezeption und Analyse der sozialwissenschaftlichen Literatur zu Durchlässigkeit zwischen Berufsund Hochschulbildung (vgl. z.B. BIBB 2011; Buhr et al. 2008; Freitag et al. 2011; Frommberger 2009; Graf 2013; Hegelheimer 1986; Raffe et al. 1998; Wolter 1994), sie können aber in den meisten Fällen auch davon abstrahiert und auf Durchlässigkeit zwischen Bildungsbereichen im Bildungssystem generell angewendet werden, weshalb die Erklärung der einzelnen Durchlässigkeitsaspekte allgemeiner gehalten wird. Analytisch unterscheide ich diese vier Aspekt:

1. Zugang in Bildungsbereiche,

2. Anrechnung von Erlerntem,

3. organisationale Verbindungen von Bildungsbereichen und

4. institutioneller Umgang mit den heterogenen Bedürfnissen der Lernenden.

Die betrachteten Aspekte sind dabei nicht als notwendige Komponenten von Durchlässigkeit zu verstehen, so dass nur, wenn alle Komponenten vorhanden sind, Durchlässigkeit erreicht ist. Es handelt sich vielmehr um unterschiedliche analytische Betrachtungsweisen, aber auch Möglichkeiten, Durchlässigkeit zu er-

8 Das Konsortium Bildungsberichterstattung (2006: 79) definiert als Übergangssystem alle Bildungsgänge, „die unterhalb einer qualifizierten Berufsausbildung liegen bzw. zu keinem anerkannten Ausbildungsabschluss führen, sondern auf eine Verbesserung der individuellen Kompetenzen von Jugendlichen zur Aufnahme einer Ausbildung oder Beschäftigung zielen und zum Teil das Nachholen eines allgemein bildenden Schulabschlusses ermöglichen“. 
reichen. Trotzdem bauen die analytisch getrennten Aspekte partiell auch aufeinander auf bzw. integrieren Eigenschaften der anderen Aspekte. Nachfolgend wird dies auch verdeutlicht, u.a. darin wie sie institutionalisiert werden können und auch darin, für wen sie konzipiert sind. So zielen sie entweder auf die Erleichterung von Durchlässigkeit für Gruppen, für das einzelne Individuum oder eine Kombination von beiden. Im Folgenden soll dieser Fokus ebenfalls systematisch mitbetrachtet werden.

Da jedoch Durchlässigkeit im Bildungssystem und damit auch die Anerkennung der unterschiedlichen Bildungswege nicht nur für das Bildungssystem an sich und für individuelle Bildungskarrieren von Bedeutung ist, geht es in einem fünften Punkt schließlich um die Anerkennung der Gleichwertigkeit von Hochschul- und Berufsbildung in anderen gesellschaftlichen Teilsystemen, insbesondere auf dem Arbeitsmarkt. Die bestehende Trennung zwischen den Bereichen kann somit sowohl im Bildungssystem als auch außerhalb wirksam sein, wenn z.B. im Arbeitsmarkt bestimmte Positionen systematisch nur mit Qualifizierten aus dem einen Bereich besetzt werden. In diesem Sinn kann sich die Segmentierung von Bildungsbereichen in unterschiedliche Lernwege, Organisationen, Curricula auch in anderen gesellschaftlichen Teilbereichen systematisch fortsetzen. Deren Aufhebung wird daher zusammen mit den Aspekten von Durchlässigkeit mitdiskutiert, zumal in der Literatur ebenfalls auf diese Dimension des Verhältnisses von Berufs- und Hochschulbildung hingewiesen wird (vgl. Hegelheimer 1986).

Während also die vier Aspekte institutionelle Durchlässigkeit innerhalb des Bildungssystems betrachten, wird bei der Frage der Anerkennung in anderen gesellschaftlichen Teilbereichen die Bedeutung der differenten Bildungswege außerhalb des Bildungssystems und insbesondere auf dem Arbeitsmarkt näher beleuchtet. Allen vier Aspekten sowie dem Blick auf Anerkennung in anderen gesellschaftlichen Teilbereichen ist der Fokus auf institutionelle Bedingungen gemeinsam, welche die Grenzen zwischen Bildungsbereichen aufweichen.

\subsubsection{Zugang}

Wenn über Durchlässigkeit nachgedacht wird, dann ist ein Aspekt, der stets als Strukturvoraussetzung für durchlässige Bildungssysteme verstanden wird, die Ermöglichung des Zugangs von einem Bildungsbereich/-gang in den anderen. So haben sich viele Arbeiten, insbesondere im Hinblick auf Durchlässigkeit zwischen Berufs- und Hochschulbildung mit den Fragen des Zugangs beschäftigt (vgl. Banscherus 2007; Husemann et al. 1995; Nickel/Duong 2012; Teichler 1990b; Teichler/Wolter 2004; Ulbricht 2014, 2012b; Wolter/Scholz 1986).

Es existieren mehrere Möglichkeiten, den Zugang zu Bildungsgängen zu realisieren. Eine Variante wäre der unbeschränkte Zugang. Alle Lernenden könnten den Bildungsgang aufnehmen, ohne vorher festgesetzte Bedingungen erfüllen zu 
müssen. Formal wäre der Zugang zur dualen Ausbildung in Deutschland ein Beispiel für diese Art Zugangsregelung, da keine spezifischen Schulabschlüsse als Voraussetzung für die Aufnahme einer Ausbildung rechtlich festgelegt sind. ${ }^{9}$ Der Zugang zur Hochschule ist allerdings gemeinhin beschränkt.

Eine zweite Variante ist die Gewährung des Zugangs in Bildungsgänge über erworbene generelle Berechtigungen im Bildungssystem, wobei die Art der Berechtigungen zuvor (meist politisch) für den Bildungsgang festgelegt wird. Durchlässigkeit zwischen Bildungsbereichen wäre dann gegeben, wenn auch im jeweils anderen Bereich Berechtigungen erworben werden können. Konkret heißt das, dass es möglich sein müsste, nicht nur über die höhere Allgemeinbildung (Abitur) sondern auch über die berufl iche Bildung Berechtigung für das Hochschulsystem und über die höhere Allgemeinbildung Berechtigungen für Bildungsgänge im Berufsbildungssystem zu erwerben.

Eine dritte Variante rückt den Fokus auf die aufnehmende Institution, welche anhand von Abschlüssen und Zertifikaten einerseits oder verschiedenen Formen von Eingangsprüfungen andererseits über den Zugang entscheidet (vgl. BIBB 2011), wobei Mischformen ebenfalls denkbar sind. Hier wäre Durchlässigkeit lediglich möglich, wenn auch die Abschlüsse und Zertifikate aus dem anderen Bildungsbereich anerkannt werden würden. Die Eingangsprüfungen dagegen müssten so aufgebaut sein, dass eine erfolgreiche Teilnahme gleichermaßen für Individuen aus beiden Bildungsbereichen möglich ist. Wäre letzteres nicht der Fall, wäre vielleicht formell Durchlässigkeit gegeben, denn jeder könnte an den Zulassungstests teilnehmen - faktisch wären aber bestimmte Gruppen von einem erfolgreichen Bestehen des Tests ausgeschlossen, da diese aufgrund der abgefragten Kompetenzen systematisch benachteiligt wären. Ein Beispiel für eine wenig Durchlässigkeit fördernde Hochschulzugangsprüfung wäre, wenn diese für ein erfolgreiches Absolvieren die in der Abiturstufe erworbenen Kenntnisse voraussetzt. In Frankreich besteht bereits seit längerem eine Tradition der Selektion durch die aufnehmenden Institutionen und auch in Deutschland wurde 2004 mit der Novellierung des Hochschulrahmengesetzes den Hochschulen mehr Autonomie bei der Auswahl ihrer Studierenden zugesprochen.

Der unbeschränkte Zugang, der Zugang über Berechtigungen ebenso wie die zertifikatsbasierte Zulassung der aufnehmenden Institutionen bestimmen die Zugangschancen für Gruppen, denen entweder universell oder auf Basis von Abschlüssen ohne weitere Prüfung der Zugang gewährt wird. Der Zugang über Eignungstests oder Auswahlgespräche dagegen ist stärker individuumszentriert, denn jedes Individuum wird einzeln nach seinen/ihren Fähigkeiten bewertet.

9 Faktisch ist die Aufnahme einer Ausbildung nicht unabhängig von den Schulabschlüssen. Dieser Zusammenhang hat sich zudem im Zeitverlauf vergrößert (vgl. Protsch 2011, 2014). 


\subsubsection{Anrechnung}

Eine weitere Möglichkeit, Bildungssysteme durchlässig zu gestalten, ist die Institutionalisierung von Anrechnungsverfahren von vorgängigem Lernen aus einem Bereich in den anderen (vgl. z.B. BIBB 2011; Freitag 2009b; Freitag et al. 2011). Loroff et al. (2011) unterscheiden drei Varianten:

- die individuelle Anrechnung,

- die pauschale Anrechnung und

- $\quad$ eine Kombination von individueller und pauschaler Anrechnung.

Bei der individuellen Anrechnung wird für jeden Einzelfall geprüft, ob und in welchen Maße zuvor erworbene Kenntnisse und Fähigkeiten mit den im Zielbildungsgang vermittelten Kenntnissen und Fähigkeiten übereinstimmen und damit dann bestimmte Bestandteile des Zielbildungsgangs ersetzen können. Die pauschale Anrechnung dagegen erfolgt personenunabhängig, denn die Äquivalenzbestimmung besteht nicht für die individuellen Kenntnisse, sondern für formale Abschlüsse und Zertifikate allgemein, und wird dann auf die Absolvent_innen dieser Abschlüsse sowie Besitzer_innen der Zertifikate übertragen. Insofern sind bei diesem Verfahren vor allem formales Lernen, nur sehr bedingt non-formales Lernen (Ausnahmen könnten stark verbreitete Weiterbildungskurse sein) und nicht informelles Lernen für die Anrechnung relevant. Das kombinierte Verfahren umfasst beides, die pauschale Anrechnung von formalen Abschlüssen und Zertifikaten sowie die Beurteilung der weiteren nicht formellen und non-formalen erworbenen Kenntnisse sowie Fertigkeiten. Auf diese Weise könne „das vorliegende Anrechnungspotenzial umfangreicher erschlossen werden" als allein durch pauschale Anrechnung (Loroff et al. 2011: 79). Auch hier ist der Fokus der Verfahren entweder auf Gruppen bei der pauschalen Anrechnung oder auf das Individuum bei der individuellen und der kombinierten Anrechnung gerichtet.

Zentral bei der Frage der Anrechnung von vorgängigem Lernen, egal bei welchem Verfahren, ist die Frage der Äquivalenz der erworbenen Kompetenzen aus dem einen Bereich X für den Bildungsgang im anderen Bereich Y. Die Anerkennung von Gleichwertigkeit ist damit dem Anrechnungsverfahren inhärent, denn ohne diese wäre Anrechnung nicht notwendig und nicht möglich. Dabei stellt sich die Frage der Äquivalenz noch viel deutlicher, wenn es um Erlerntes aus unterschiedlichen und stark voneinander separierten Organisationsfeldern oder Bildungssystemen geht, die unterschiedliche Logiken, Lernorte, Lernpraxen, Curricula, Governance aufweisen, da hier die Übertragbarkeit eine Abstraktion von diesen Logiken verlangt. Als Reaktion auf diese Notwendigkeit erfolgte in den letzten Jahren vor allem auch auf europäischer Ebene ein Paradigmenwechsel hin zur Lernergebnisorientierung, welche eine Vergleichbarkeit des Gelernten ermöglichen soll. Lernergebnisse nach europäischer Definition sind: 
die Gesamtheit der Kenntnisse, Fähigkeiten und/oder Kompetenzen, die eine Person nach Durchlaufen eines Lernprozesses erworben hat und/oder nachzuweisen in der Lage ist. Lernergebnisse (learning outcomes) sind Aussagen über das, was ein Lernender am Ende einer Lernperiode wissen, verstehen, können soll. (Europäische Kommission 2005: 13)

Es stellt sich mit dieser Perspektive nicht mehr die Frage danach, welchen Inhalt man in einem Kurs gelernt hat (Frage des Inputs), um das Zertifikat zu erhalten, sondern vielmehr welche Fähigkeiten mit dem Abschluss des Bildungsgangs erworben worden sind (die Frage des Output/der Ergebnisse) ${ }^{10}$.

Insbesondere in Ländern wie Deutschland, in welchen sich historisch eine stark institutionalisierte Trennung zwischen den Organisationsfeldern der Hochschul- und Berufsbildung, die sich jeweils genau durch ihre unterschiedlichen Logiken auszeichnen, entwickelt hat, sind daher Diskussionen um Gleichwertigkeit seit Jahrzehnten sowohl in der Fachliteratur als auch in der politischen Diskussion präsent (vgl. z.B. Blankertz 1963; Frommberger 2009; Hegelheimer 1986). Sie spielt daher auch in Fragen der Anrechnung eine besondere Rolle.

Anrechnung von Gelerntem kann, um Durchlässigkeit zu fördern, einerseits dazu beitragen, dass Zugang zu Bildungsgängen gewährt wird. Dies trifft dann zu, wenn z.B. vorhandene Abschlüsse per se keine Berechtigungen für den Zugang liefern, aber vorhandene Kompetenzen als äquivalent zu den Kompetenzvoraussetzungen des Bildungsgangs eingestuft werden ${ }^{11}$. Ein Beispiel vermittelt die fachgebundene Studienzulassung für beruflich Qualifizierte, welche seit 2009 in Deutschland gilt (KMK 2009d). Hier können Absolvent_innen einer beruflichen Erstausbildung nach dreijähriger Berufserfahrung und einem möglichen Einstufungstest Zugang zur Hochschule erhalten. Bestehende Berufserfahrungen werden in diesem Beispiel angerechnet. Anrechnungsverfahren ermöglichen jedoch nicht nur den Zugang zu Bildungsgängen, sondern primär auch das Überspringen von Lerneinheiten innerhalb eines Bildungsgangs, um auf diese Weise doppelte Lernzeiten zu verhindern. An dieser Stelle wird deutlich, dass Anrechnung und Zugang eng miteinander verbunden sind: Erstens kann Anrechnung ein Verfahren sein, um Zugang zu gewährleisten und zweitens ist Zugang eine Voraussetzung für die Ermöglichung von Anrechnung innerhalb des Bildungsgangs. Trotz dieser engen Verknüpfung soll Anrechnung als eigener Aspekt des Konzepts gelten, da eben durch die Feststellung von Äquivalenz viel mehr als nur Zugang gewährt werden kann, denn ohne vorher festgelegte Begrenzung wäre die Anerkennung von ganzen Abschlüssen möglich.

10 Für eine kritische Auseinandersetzung mit dem Konzept der Lernergebnisorientierung siehe u.a.: Bohlinger (2006).

11 Auch für den Fall von Berechtigungen, die in unterschiedlichen bereichen erworben werden können, kann argumentiert werden, dass eine Form von Anrechnung vorliegt, da ja das Erlernte als gleichwertig und ausreichend für eine Zulassung anerkannt wird. 
Resümierend führt Anrechnung also dazu, dass differente Lernwege und daraus resultierende Lernergebnisse in den unterschiedlichen Settings als gleichwertig betrachtet werden, sofern sie auch zu gleichwertigen Kompetenzen und Kenntnissen sowie Fähigkeiten bei den Lernenden führen. Gesellschaftlich gewachsene Hierarchisierungen von Bildungswegen bzw. -bereichen können auf diese Weise aufgehoben oder zumindest verringert werden. Anrechnung von beruflicher Vorbildung bspw. und damit auch der Erlass von bestimmten Prüfungsleistungen im Hochschulstudium stellen dann eine Gleichwertigkeit der erlernten Kompetenzen dar.

\subsubsection{Organisationale Verbindungen}

Der dritte Aspekt von Durchlässigkeit beschäftigt sich mit der Möglichkeit der organisationalen Verbindung von Bildungsbereichen bis hin zu ihrer Integration in einer Organisationsform oder einem Bildungsgang. Angelehnt an die idealtypische Kategorisierung von Young et al. (1997) kann zwischen Bildungssystemen unterschieden werden, welche ,tracked", „linked" oder ,unified" sind (vgl. auch Raffe et al. 1998). „,Tracked" sind jene Systeme, in denen Hochschul- und Berufsbildung in unterschiedlichen Organisationsfeldern mit klar abgrenzbaren Lernwegen stattfinden. Abschottung der Bereiche und nicht Durchlässigkeit ist die Charakteristik. Durchlässigkeit wäre dann eine Möglichkeit, die starren Grenzen aufzubrechen. „Linked", also verbunden, sind Systeme, wenn es zwischen diesen Bildungsbereichen fest institutionalisierte Verbindungen gibt. „Unified" oder integrierte bzw. vereinigte Systeme sind dann solche, die keine separaten Lernwege für berufliche oder hochschulische Bildung anbieten (Young et al. 1997). Im letzteren Fall wäre Durchlässigkeit zwischen den Organisationsfeldern der Berufsund Hochschulbildung kein Thema mehr. Young et al. (1997) betonen jedoch, dass es mehrere Ebenen gibt, welche ein Bildungssystem ausmachen: Dies sind Abschlüsse, die Curricula, die Organisationsformen, die Methodik etc. Aus diesem Grund gilt: ,national systems can be unified on some dimensions and tracked or linked on others" (Young et al. 1997: 532). Für die Konzeption des dritten Aspekts von Durchlässigkeit kann Integration der Bildungsbereiche daher nicht auf Systemebene in allen Ebenen stattfinden, denn ohne Grenzen zwischen Bereichen wäre Durchlässigkeit nicht mehr notwendig. Stattdessen ist jedoch eine Integration oder Verbindung von Bildungsbereichen auf der Mesoebene als organisationale Verbindung vorstellbar. In diesem Fall gäbe es die mehr oder minder getrennten Bildungsbereiche noch, aber sie wären miteinander verbunden.

Vor diesem Hintergrund unterscheide ich für den Aspekt der organisationalen Verbindungen zwei Varianten: die Verbindung von separaten Bildungsgängen aus zwei Bildungsbereichen oder Organisationsfeldern (Übergänge) und die Verbindung der Bildungsbereiche in einem Bildungsgang oder einer Organisationsform (Integration). 


\section{Organisationale Verbindung als institutionalisierte Übergänge ${ }^{12}$}

Bei getrennten Bildungsbereichen, wie Berufs- und Hochschulbildung, kann trotz Grenzziehung Durchlässigkeit gefördert werden, wenn Verbindungen zwischen den getrennten Bildungsbereichen eingerichtet werden. Derartige institutionalisierte Übergänge können dann den Zugang zu Bildungsgängen in einem Bereich $\mathrm{X}$ nach Abschluss des Bildungsgangs im anderen Bereich Y ermöglichen (Möglichkeit A). Dies entspricht der Zugangsvariante über generelle Berechtigungen und soll nicht weiter als institutionalisierter Übergang behandelt werden.

Verbindungen können aber auch dergestalt institutionalisiert werden, dass es möglich ist, mit dem Abschluss aus einem Bildungsbereich X in einen Bildungsgang im anderen Bereich $\mathrm{Y}$ in ein fortgeschrittenes Stadium einzusteigen (Möglichkeit B). So ist es in Frankreich z.B. möglich, nach Abschluss der zwei Jahre dauernden Vorbereitung auf die französischen Elitehochschulen grandes écoles in den sogenannten Vorbereitungsklassen (CPGE) ein drittes Jahr an der Universität anzuschließen und den licence-Abschluss, das Äquivalent zum B.A., zu erhalten. Eine Wiederholung der ersten zwei licence-Jahre wird nicht als notwendig erachtet. Auch der geregelte Einstieg nach einer IT-Weiterbildung in ein bestimmtes Semester der Informatik in Deutschland wäre als Beispiel denkbar.

Eine dritte Möglichkeit ist der Übergang von einem Bildungsgang im Bereich $\mathrm{X}$ in einen Bildungsgang im Bereich Y (Möglichkeit C). Bei diesem Szenario ist kein vorheriger Abschluss für den Übergang notwendig, der Wechsel erfolgt im Verlauf des Bildungsgangs. In Frankreich besteht z.B. die Möglichkeit insbesondere für Studierende, welche einen anderen Schwerpunkt suchen, aus dem allgemeinbildenden licence-Studiengang in einen berufsorientierten Studiengang zu wechseln, ohne die Semester wiederholen zu müssen (Interview MESR2) ${ }^{13}$. Ein anderes Beispiel bestünde, wenn es die institutionalisierte Möglichkeit gäbe, während des Studiums nach z.B. drei Semestern in einen fachnahen beruflichen Weiterbildungsgang zu wechseln. Zu einem institutionalisierten Übergang würde dieses Beispiel dann werden, wenn durch vorher festgelegte Regelungen, für z.B. bestimmte Fachrichtungen, die Studierenden immer in denselben Lernabschnitt der beruflichen Weiterbildung einsteigen, ohne von vorn beginnen zu müssen.

Generell beinhalten die Möglichkeiten B und C der institutionalisierten Übergänge die beiden Durchlässigkeitsaspekte Zugang und Anrechnung. Institutionalisierte Übergänge wurden dessen ungeachtet als Teil des Aspekts organisationaler Verbindungen aufgenommen, da sie jeweils mehr als nur Zugang oder Anrechnung allein umfassen - diese stattdessen fest institutionalisiert verbinden. Im Gegensatz zum ersten Aspekt des Zugangs geht es bei Übergängen um den Zugang

12 Dieser Teilaspekt, im Unterschied zu den anderen, beruht vor allem auf der Kenntnis des französischen Falls und der Bildungsstrukturen in diesem System.

13 Die komplette Liste durchgeführter Expert_inneninterviews befindet sich im im Anhang (Tabellen 45-47). 
nicht am Beginn, sondern im Verlauf des Bildungsgangs. Im Gegensatz zum Aspekt Anrechnung ist beim institutionalisierten Übergang Zugang schon inbegriffen. Insofern stehen die institutionalisierten Übergänge von Bildungsbereichen in dieser Arbeit analytisch getrennt von den Durchlässigkeitsaspekten Zugang und Anrechnung, um den stärkeren Grad der Institutionalisierung deutlich zu machen. Ein stärkerer Grad an Institutionalisierung bedeutet auch, dass diese Übergänge im Bildungssystem auf institutioneller Ebene bestehen, unabhängig von der Nachfrage der Individuen nach diesen Möglichkeiten. Es handelt sich demnach um institutionalisierte Lernwege, die bestimmte Organisationsformen oder Bildungsgänge miteinander verbinden, wobei aber das Lernen konsekutiv organisiert ist, also erst in einem Bereich und dann im anderen stattfindet.

\section{Organisationale Verbindung als Integration}

Die zweite Variante der Integration beinhaltet viel stärker die institutionelle Verbindung von Elementen aus separaten Bildungsbereichen in einem Bildungsgang oder auch einer Organisationsform. Dies ist auf mehreren Ebenen möglich, auf der Qualifikationsebene mit Doppelqualifikationen z.B., in den Lehrplänen und der Didaktik und Methodik, den Lernorten, etc. (vgl. Graf 2013; Young et al. 1997). Insofern geht es bei der organisationalen Verbindung in Form der Integration um Durchlässigkeit im Sinne eines Aufbrechens der bestehenden Grenzen durch die Institutionalisierung der für beide Bildungsbereiche typischen Logiken, Normen und/oder Regularien. Wenn die Integration der typischen Logiken, Normen und/oder Regularien systematisch auf verschiedenen Ebenen erfolgt, kann man auch von hybriden Organisationsformen sprechen (vgl. Graf 2013).

Die strukturelle Ermöglichung von individueller Bildungsmobilität kann, aber muss in diesem Fall nicht gefördert werden (ebd.). Eine Unterstützung von Bildungsmobilität findet aber vor allem dann statt, wenn die Bildungsgänge einerseits zu Doppelqualifikationen führen, d.h. zu einer Qualifikation aus dem Bereich $\mathrm{X}$ sowie aus dem Bereich $\mathrm{Y}$, und damit gleichzeitig den Zugang in weitere Bildungsgänge in den beiden Bildungsbereichen eröffnen. Andererseits ist eine Förderung von Bildungsmobilität auch dann möglich, wenn durch die Integration der Bildungsbereiche auch der Zugang von Lernenden aus beiden Bereichen ermöglicht wird.

Ein Beispiel für eine Form der Integration wäre das duale Studium ${ }^{14}$ in Deutschland, in welchem u.a. sowohl der für die berufliche Ausbildung typische Lernort Betrieb als auch der Lernort Hochschule systematisch miteinander verbunden werden (vgl. Graf 2013; Graf et al. 2014; Mucke/Kupfer 2011). Studierende lernen auf diese Weise beide Welten mit ihren je spezifischen Logiken kennen und verstehen. Auch die beteiligten Akteure sind gezwungen, sich mit den Mucke/Kupfer 2011). 
jeweiligen Spezifika des anderen Bildungsbereichs auseinanderzusetzen. Auf diese Weise ist eine Annäherung vorher getrennter Welten möglich, die letztlich auch zu einer größeren gegenseitigen Akzeptanz der Bildungsbereiche führen können.

Eine andere Möglichkeit verschiedene Bildungsbereiche miteinander zu verbinden wäre die Entkopplung von Zertifikaten von Organisationsformen. Es wäre also nicht mehr wichtig, in welcher Organisation gelernt wird, wenn Abschlüsse bereichsübergreifend vergeben werden. Als Beispiel kann im deutschen Schulsystem auch in der Hauptschule ein Realschulabschluss erworben werden. Für Durchlässigkeit zwischen Berufs- und Hochschulbildung angewendet würde das dann bedeuten, das der Bachelor oder Master über berufliche Bildungswege erworben wird und der Meister z.B. an der Fachhochschule. Zu differenzieren ist hierbei ob eine Entkopplung innerhalb eines segmentierten Organisationsfeldes wie dem Schulbereich stattfindet, um Durchlässigkeit zu erhöhen. Dies könnte einfacher zu institutionalisieren sein als zwischen getrennten Organisationsfeldern wie der Berufs- und Hochschulbildung, da diese sich meist durch ganz unterschiedliche Governancestrukturen, Zuständigkeiten für die Zertifikatsvergabe und Logiken auszeichnen. Nichts desto trotz können in spezifischen dualen Studiengängen (z.B. ausbildungsintegrierende Studiengänge) Doppelabschlüsse erworben werden. Allerdings bleibt der Lernort Firma hier erhalten, so dass keine reine Entkopplung stattfindet. Es stellt sich die Frage, ob eine Entkopplung von Abschlüssen zwischen getrennten Organisationsfeldern, die gleichsam einhergehen müsste mit einer Anpassung und Erweiterung der curricula in den Organisationen noch Durchlässigkeit bedeutet oder nicht bereits Integration der Organisationsfelder zu einem stärker "unified system“, wenn es egal wäre an welcher Organisationsform man einen beruflichen oder akademischen Abschluss macht.

Betrachtet man beide Formen der organisationalen Verbindung, wird deutlich, dass sowohl die institutionalisierten Übergänge als auch integrierte Bildungsgänge eng verbunden sind mit der Frage der Anerkennung der Gleichwertigkeit unterschiedlicher Bildungsbereiche, denn lediglich dergestalt ist ihre Verbindung überhaupt möglich.

\subsubsection{Heterogenität der Lernenden}

Der vierte Aspekt des Durchlässigkeitskonzepts bezieht sich auf die institutionellen Rahmenbedingungen, welche auf die Anpassung an heterogene Bedürfnisse der Lernenden abzielen. Die Schaffung von Strukturen, welche es ermöglichen, mit der Heterogenität von Lernenden umzugehen, ist allgemein eine Voraussetzung zur Realisierung eines inklusiven Bildungssystems, das jedem Individuum die Möglichkeit der freien Entfaltung seiner Persönlichkeit und Fähigkeiten bietet (vgl. z.B. Pfahl/Powell 2010; Powell 2011). Für die Frage von Durchlässigkeit ist dieser Aspekt insbesondere relevant. Angenommen, dass einzelne Bildungsbereiche und vor allem Organisationsfelder unterschiedlichen Logiken unterworfen 
sind, kann man auch davon ausgehen, dass diese unterschiedlichen Umgebungen zu einer unterschiedlichen Sozialisation der Lehrenden und Lernenden sowie einer unterschiedlichen Entwicklung im Lebensverlauf führen. Umso wichtiger wird die Frage des Umgangs mit Heterogenität, wenn sich in dem Bildungssystem die Lernenden in den Bildungsbereichen auch noch anderweitig systematisch unterscheiden, wie z.B. nach sozialer Herkunft, Migrationshintergrund, Geschlecht oder einer Kombination mehrerer askriptiver Merkmale. Will man nun jedoch sämtliche Bildungswege und damit auch Bildungsbereiche für alle Individuen gleichermaßen offen halten, bedeutet dies ferner, dass man den Lernenden ihren Bedürfnissen entsprechende institutionelle Lernbedingungen ermöglicht. Es geht insofern nicht nur darum, den Zugang formal zu erhalten, sondern auch um das Ermöglichen eines erfolgreichen Absolvierens des Bildungsgangs. Hierfür bedarf es Strukturen, die auf die Heterogenität der Lernenden ausgerichtet sind. Das Erschaffen von förderlichen Bedingungen kann zu unterschiedlichen Zeitpunkten stattfinden. Zum einen geht es darum, den Individuen Bedingungen zu bieten, die dabei unterstützen und motivieren, auch den individuell passenden Bildungsgang zu wählen und Zugang zu ,bereichsfremden' Bildungswegen zu suchen. Hier müssen die Strukturen vor dem Beginn des Bildungsgangs wirken. Zum anderen bedarf es Strukturen, welche das erfolgreiche Lernen im ,fremden' Bildungsbereich selbst erleichtern.

In der Literatur zur Durchlässigkeit zwischen Berufs- und Hochschulbildung können verschiedene strukturelle Möglichkeiten, welche eine Anpassung an heterogene Bedürfnisse fördern, identifiziert werden (vgl. z.B. Banscherus et al. 2015; Freitag et al. 2011; Hegelheimer 1986; Mucke/Kupfer 2011; Teichler/Wolter 2004; Wolter 1994; Wolter et al. 2014). Diese sind auch auf andere Bildungsbereiche übertragbar, würden aber dann andere Schwerpunkte oder Spezifizierungen aufweisen. Strukturen, welche vor allem erst den Weg in einen ,fremden' Bildungsbereich ebnen, sind zum einen a) Informations- und Beratungssysteme über die verschiedenen Lernwege und zum anderen Unterstützungsstrukturen, z.B. b) Finanzierungsmöglichkeiten, welche eine Aufnahme des Lernweges ermöglichen. In den Bildungsorganisationen selbst sind dann für das Lernen förderliche Bedingungen zu finden: $\mathrm{c}$ ) in der Art und Weise wie das Lernen organisiert ist und unterstützt wird und d) welche Lern- und Lehrkultur vorherrscht und inwiefern diese offen bzw. flexibel auf Veränderungen reagiert. Gleichsam sind weiterhin Beratungs- und Informationsangebote auch während des Bildungsgangs wichtig für die Lernendenorientierung und -motivation. Es bleibt darauf hinzuweisen, dass die Zuordnung zur zeitlichen Wirksamkeit der Strukturen lediglich eine analytische ist und letztlich auch Strukturen, die das Lernen selbst erleichtern, wie die Lernorganisation auch dazu beiträgt, ob der Bildungsgang überhaupt in Betracht bezogen wird oder nicht. Im Folgenden sollen die einzelnen Strukturen, die den Umgang mit heterogenen Bedürfnissen erleichtern vorgestellt werden. 


\section{a) Informationen und Beratung über Lernwege}

$\mathrm{Ob}$ die Individuen sich für bestimmte Bildungswege entscheiden, also den $\mathrm{Zu}-$ gang suchen, ist nicht nur signifikant von den individuellen Präferenzen abhängig, sondern auch davon, ob sie über Möglichkeiten im Bildungssystem ausreichend informiert sind. Speziell durch die Sozialisation in getrennten Bildungsbereichen entstehen Informationsasymmetrien, welche ausgeglichen werden müssen. Für die Studienbeteiligung beruflich Qualifizierter in Deutschland haben z.B. Nickel und Leusing (2009) herausgefunden, dass ein Grund für die geringe Beteiligung in der mangelnden Infrastruktur von Informations- und Beratungsdienstleistungen zu finden ist. Es reicht daher nicht, formal durch Zugangs- oder Anrechnungsverfahren die Systeme durchlässiger zu machen. Die Lernenden müssen auch über diese Möglichkeiten und über das, was sie erwartet, informiert sein, damit diese ihre Wirkung entfalten. Auf diese Weise wird der Informationsnachteil, der besteht, da sie nicht zu den ,typischen' Studierenden gehören, ausgeglichen. Fördernde Strukturen wären dann z.B. spezifische Beratungsangebote explizit für beruflich Qualifizierte über deren Möglichkeiten im Hochschulsystem.

\section{b) Finanzierung}

Für das Beispiel der beruflich Qualifizierten, welche den Zugang in das Hochschulsystem suchen, stellt Sicherstellung der Finanzierung eine weitere wichtige Opportunitätsstruktur dar (vgl. Mucke/Kupfer 2011). Die Frage der Finanzierung stellt sich dabei auf mehreren Ebenen. Das Standardstudium in Deutschland ist derzeit noch oft als Vollzeitstudium konzipiert. Beruflich Qualifizierte müssten dann ihre Berufstätigkeit stark einschränken und sind mit der Frage konfrontiert, wie sie ihren Lebenserhalt und ihren Lebensstandard finanzieren und aufrechterhalten. Kompliziert wird die Situation zudem, wenn die meist etwas älteren beruflich Qualifizierten einen erschwerten Zugang zu Stipendien oder anderen Förderungsmöglichkeiten erhalten, da diese oft noch altersgebunden und ausgerichtet auf den jüngeren ,Durchschnittsstudierenden“ sind. Im Hinblick auf fördernde Bedingungen zwischen Berufs- und Hochschulbildung existieren mehrere Möglichkeiten, das Finanzierungsproblem zu lösen. Zum einen könnten Stipendien explizit für bestimmte Zielgruppen eingerichtet werden oder bestehende Unterstützungsmaßnahmen angepasst werden.

\section{c) Lernorganisation und Unterstützungsstrukturen}

Auch die Bedürfnisse, wie Lernen organisiert ist, können sich zwischen den einzelnen Bildungsteilnehmer_innen und auch systematisch zwischen den Lernenden, welche aus unterschiedlichen Bildungsbereichen kommen, unterscheiden. Differenzen kann es so z.B. erstens in den Wünschen zur zeitlichen und örtlichen 
Ausgestaltung geben. So weisen Mucke und Kupfer (2011) darauf hin, dass beruflich Qualifizierte in Deutschland, welche sich für ein Hochschulstudium entscheiden, oft bereits andere familiäre und berufliche Verpflichtungen haben können als traditionelle Studierende ${ }^{15}$ und damit flexible Studienmodelle wie Teilzeit, berufsbegleitende Studiengänge oder das Fernstudium bevorzugen, da sie so besser Beruf, Familie und Studium vereinbaren können. Insofern dienen flexible Studienstrukturen, die unterschiedliche Lernrhythmen und -orte ermöglichen, dazu, jene Lernenden zu gewinnen, die ein traditionelles Vollzeitstudium nicht realisieren könnten.

Ähnlich wichtig sind zweitens entsprechende Anpassungen der Infrastruktur. Wenn nämlich die Lernzeiten und -orte flexibler gestaltbar sind, müssen auch Serviceeinrichtungen für die Lernenden angepasst werden. Beispiele wären flexible Zeiten der Beratungsdienste, Sprechstunden, Bibliotheken, Kindereinrichtungen. Dementsprechend würden alle und nicht nur die traditionellen Lernenden von der für ein erfolgreiches Lernen notwendigen Infrastruktur profitieren.

Drittens, sollte auch in der Didaktik und Methodik der Lernangebote sowie in den Curricula auf die Bedürfnisse und auch Vorerfahrungen der Lernenden eingegangen werden. Am Beispiel der beruflich Qualifizierten, welche ein Hochschulstudium aufnehmen, ginge es darum, auf berufliche Vorerfahrungen Bezug zu nehmen, aber auch auf die neue Lernumgebung z.B. durch Einführungskurse in das wissenschaftliche Arbeiten oder Brückenkurse vorzubereiten. Auch die Integration von berufspraktischen Elementen in die Lehrpläne wäre eine Möglichkeit, das Angebot an die Bedürfnisse anzupassen (Mucke/Kupfer 2011). Andere Beispiele wären die Einführung von Tutorien, die helfen, Gelerntes in Kleingruppen zu vertiefen. Auf diese Weise könnte es besser möglich sein, auf die individuellen Fähigkeiten zu reagieren und diese gezielt zu fördern. Umgekehrt könnten Absolvent_innen eines Studiums Praktika helfen, sich in die Spezifik des Berufsfeldes, in denen sie sich weiterbilden wollen, vertraut zu machen.

Viertens sind Beratung und Information nicht nur vor dem Beginn des Bildungsgangs von Bedeutung, sondern auch im Verlauf desselbigen wichtig (Hanft et al. 2013; Hartmann-Bischoff/Brunner 2013). Insbesondere Beratung verschafft die Möglichkeit, bestehende Probleme zu erkennen und anzugehen oder auch Bildungsentscheidungen im Bildungsgang zu treffen. Im Gegensatz zu traditionellen Bildungsteilnehmer_innen kann vor allem für solche aus dem anderen Bildungsbereich eine Beratung dabei helfen, die neuen Regeln, Logiken und die

15 Im Gegensatz zu traditionellen Studierenden definieren Teichler und Wolter nicht-traditionelle Studierende folgendermaßen: „Studierende, die nicht auf dem geraden Weg bzw. in der vorherrschenden zeitlichen Sequenz und Dauer zur Hochschule gekommen sind; nicht die regulären schulischen Voraussetzungen für den Zugang erfüllen; und solche, die nicht in der üblichen Form des Vollzeit- und Präsenzstudiums studieren (sondern als Teilzeit-, Abend- und Fernstudierende). Eine genaue Abgrenzung ist auch deshalb nicht zu treffen, weil manche Studierende in einigen Aspekten als ,traditionell', in anderen dagegen als ,nicht-traditionell ${ }^{\text {* }}$ eingestuft werden können.“ (Teichler/Wolter 2004: 72) 
Kultur der Bildungsorganisation und des -bereichs zu verstehen und darin zu reüssieren.

\section{d) Kultur der Offenheit in den Organisationen}

Ein weiterer bedeutender Punkt, welcher in einigen Studien betrachtet wird (vgl. Alheit 2009; Völk 2011), besteht in der Frage der Kultur der aufnehmenden Organisationen. Besteht eine Kultur der Offenheit gegenüber der Heterogenität der Lernenden und ihren Bedürfnissen, ist eine Integration von Lernenden aus anderen Bildungsbereichen wahrscheinlicher und erfolgversprechender als bei einer Orientierung auf eine vermeintlich bedürfnishomogene Gruppe traditioneller Lernender. Völk (2011) macht dies am Beispiel der Hochschullehrenden deutlich, welche mit ihren Annahmen hinsichtlich der Studierfähigkeit von beruflich Qualifizierten bzw. auch mit ihren Positionen zur Frage der Anrechenbarkeit von beruflichen Abschlüssen den beruflich Qualifizierten das Lernen in Hochschulen erleichtern oder erschweren können. Die vorherrschende Kultur kann in einem engen Zusammenhang mit der Lernorganisation und der Ausgestaltung der Informations- und Beratungsstrukturen stehen. Es ist aber auch denkbar, dass sowohl eine adäquate Lernorganisation als auch passende Informations- und Beratungsstrukturen gegeben sind, die Kultur in der aufnehmenden Organisation dies (eventuell noch) nicht widerspiegelt. Dies entspräche einem klassischen Fall der losen Kopplung (vgl. DiMaggio/Powell 1983; Meyer/Rowan 1977) zwischen Regelungen und tatsächlicher Praxis bzw. Kultur.

Auch Alheit (2009) konnte darlegen, dass in deutschen Universitäten vorherrschende Kulturen bzw. der „universitäre Habitus“ (Alheit 2009: 224) einen negativen Einfluss auf den Erfolg von nicht-traditionellen Studierenden haben können. Er identifiziert „Symptome für eine latente symbolische Schließung des deutschen Hochschulsystems" (Alheit 2009: 224). Dies arbeitet er heraus anhand von Interviews mit Studienberatern der unterschiedlichen Fächergruppen, welche gerade für nicht-traditionelle Studierende als Gatekeeper wirken können. Auch Teichler und Wolter (2004: 69f.) stellen fest, dass der Studienerfolg nicht nur für die sogenannten nicht-traditionellen Studierenden stärker vom Studienfach und dem damit verbundenen Klima als von der Art der Studienberechtigung bestimmt werden. Es wird somit auch an dieser Stelle die besondere Rolle der Organisationskultur angesprochen. Deutlich wird jedoch ebenfalls, dass es nicht nur eine Kultur in Organisationen geben muss, sondern, dass diese auch fachspezifisch variieren kann.

In den Ausführungen zum Umgang mit heterogenen Bedürfnissen wird deutlich, dass dieser Aspekt stark auf Chancengleichheit abzielt. Dabei geht es darum, dass diejenigen im Bildungsgang, welche durch z.B. unterschiedliche Sozialisation in anderen Bildungsbereichen andere Voraussetzungen mitbringen, in dem Bildungsgang die gleichen Chancen auf erfolgreiches Lernen haben. Durch Infor- 
mations- und Beratungsangebote werden auf diese Weise mögliche Informationsasymmetrien aufgehoben, welche ebenfalls durch das Lernen im anderen Organisationsfeld zustande kommen. Die Anpassung der Finanzierungsangebote oder auch der Lernorganisation und Kultur an die Bedürfnisse, d.h. diesen ein Studium z.B. finanziell und auch zeitlich zu ermöglichen, zielen ebenfalls auf Chancengleichheit.

An dieser Stelle soll darauf hingewiesen werden, dass diese Strukturen, die den Umgang mit den heterogenen Bedürfnissen von Lernenden erleichtern, nicht nur relevant sind für Fragen der Durchlässigkeit, d.h. des Bildungserfolges von Lernenden aus einem Bereich in einem anderen, sondern vielmehr allgemein für ein erfolgreiches Absolvieren von Bildungsgängen wichtig sind. Zudem besteht die Gefahr, dass durch spezifische Förderinstrumente für eine fest definierte Gruppe deren Andersartigkeit unterstrichen und reproduziert wird und somit auch die Trennung in der Organisation auf diese Weise bestehen bleibt. Wichtig für Strukturen, welche den Umgang mit der Heterogenität der Lernenden unterstützen, ist somit, dass sie allgemein die Homogenitätsannahmen von Lernenden hinterfragen und nicht zur Stigmatisierung von Gruppen beitragen. Es gibt nicht ,die ${ }^{6}$ traditionellen Studierenden und auch nicht ,die beruflich Qualifizierten. Beide Gruppen sind auch in sich heterogen. Die Lösung dieses Problems ist keine einfache, aber eine Möglichkeit besteht darin, dass unterstützende Angebote nicht nur für eine Zielgruppe eröffnet werden, sondern allgemein allen Lernenden der Organisation offenstehen, aber mögliche spezifische Bedürfnisse integrieren. Auf diese Weise wird eine größere Durchlässigkeit zwischen Bildungsbereichen gefördert, ohne die Trennung der Bildungsbereiche zu reproduzieren.

\subsubsection{Anerkennung in anderen gesellschaftlichen Teilsystemen}

Da die Trennung von Bildungsbereichen nicht nur im Bildungssystem selbst ihre Wirkung entfaltet, sondern auch in anderen gesellschaftlichen Teilbereichen wirksam sein kann, soll in diesem Abschnitt die Frage der Anerkennung des Gelernten in anderen gesellschaftlichen Teilsystemen und vor allem aber dem Arbeitsmarkt mitbedacht werden. Es ist bekannt, dass Bildung, insbesondere formale Bildungsabschlüsse, in unseren Gesellschaften signifikante und dauerhafte Konsequenzen im Lebensverlauf nach sich ziehen (Mayer 2004; Solga 2005b). Diese Konsequenzen beziehen sich einerseits im engeren Sinn auf Beschäftigungschancen, den beruflichen Status, Einkommen, andererseits im weiteren Sinn auf soziokulturelle Teilhabe in den gesellschaftlichen Teilbereichen außerhalb des Arbeitsmarkts (vgl. z.B. Hillmert 2011). Im Folgenden fokussiere ich den Arbeitsmarkt, da dieser in unseren Gesellschaften von besonderer Bedeutung für die Lebenschancen von Individuen ist. Sicherlich ließen sich die folgenden Überlegungen aber auch auf andere wichtige gesellschaftliche Teilbereiche übertragen, in denen Status und Positionen durch Bildungsabschlüsse vermittelt werden. Es handelt sich dabei nicht 
um einen neuen Aspekt von Durchlässigkeit im Bildungssystem. Vielmehr soll darauf aufmerksam gemacht werden, dass das Bildungssystem in der Gesellschaft eingebettet ist und dass Durchlässigkeit im Bildungssystem nicht per se soziale Durchlässigkeit in der Gesellschaft bedeutet.

Im Umkehrschluss könnte aber auch ohne Durchlässigkeit im Bildungssystem größere soziale Durchlässigkeit in der Gesellschaft erreicht werden. Dies ist dann der Fall, wenn die unterschiedlichen getrennten Bildungswege zu gleichen Positionen und Ressourcen außerhalb des Bildungssystems führen, also als gleichwertig anerkannt werden. Am Beispiel des Arbeitsmarkts soll dies verdeutlicht werden: Ist das Erwerbssystem segmentiert und zwar gekoppelt an getrennte Bildungsbereiche im Bildungssystem, wäre eine Auflösung dieser Kopplung einerseits oder die gesellschaftliche Gleichstellung von den so getrennten Berufswegen andererseits eine Möglichkeit, soziale Durchlässigkeit zu erreichen. Wenn es keine Unterschiede im Status der segregierten Erwerbsbereiche gibt, geht es bei Auflösung der Kopplung um horizontale soziale Durchlässigkeit. Wenn mit dieser Kopplung aber auch eine Hierarchisierung von Positionen einhergeht, wäre durch eine Auflösung dieser Kopplung auch eine vertikale soziale Durchlässigkeit erreichbar. Die Hierarchisierung der Qualifikationen aus den unterschiedlichen Bildungsbereichen würde dann aufgehoben werden. Wie bei dem Aspekt Anrechnung wäre es nicht mehr wichtig, wo oder wie etwas erlernt wurde. Ausschließlich relevant wäre das sogenannte Lernergebnis, d.h. die Kenntnisse, Fähigkeiten und Kompetenzen der Lernenden, eine bestimmte Arbeit durchzuführen. Im Grunde handelt es sich daher um Anerkennung des Gelernten außerhalb des Bildungssystems. Folglich ist auch hier insbesondere für vertikale soziale Durchlässigkeit die Frage der Gleichwertigkeit virulent. So schreibt auch Hegelheimer (1986: 5) zum Thema Gleichwertigkeit von Berufs- und Hochschulbildung, die

Anerkennung der Gleichwertigkeit entscheidet sich daher nicht allein im Bildungswesen. Auch die privaten und öffentlichen Arbeitgeber müssen ihren Beitrag leisten, dass der Aufstieg im Beruf aufgrund beruflicher Qualifikationen und Leistungen in Zukunft einen gleichen Stellenwert erhält wie der Aufstieg aufgrund von Berechtigung, die im Schul- und Hochschulwesen erworben werden.

Eine Gleichwertigkeit zwischen Berufs- und Hochschulbildung auf dem Arbeitsmarkt ist demnach gegeben, wenn unabhängig davon, welcher Bildungsweg durchlaufen wurde, Absolvent_innen beider Bereiche die gleichen Chancen erhalten, bestimmte Arbeitsmarkt- und Statuspositionen zu erreichen.

\subsubsection{Zusammenschau der Aspekte von Durchlässigkeit}

Wie dargelegt, bestehen verschiedene Möglichkeiten, wie über institutionelle Durchlässigkeit nachgedacht und wie Durchlässigkeit ermöglich werden kann (vgl. Tabelle 1 und Abbildung 1). Wichtig hierbei ist zu betonen, dass die einzelnen Aspekte sich nicht gegenseitig ausschließen. Im Gegenteil, partiell hängen die 
analytisch getrennten Aspekte auch zusammen, bauen aufeinander auf oder unterstützen die jeweilige Durchlässigkeitswirkung. So ist z.B. die Erteilung des Zugangs zu einem Bildungsbereich oder -gang aufgrund der vorangegangen Abschlüsse und Zertifikate auch verbunden mit der Anerkennung und impliziten Anrechnung des zuvor Gelernten. Anrechnung dagegen ist nicht ohne die Ermöglichung des Zugangs realisierbar. Institutionalisierte Übergänge verbinden Anrechnung und Zugang. Zudem ist zu erwarten, dass insbesondere integrierte Bildungsgänge oder Organisationen wahrscheinlicher Strukturen aufweisen, welche an heterogene Bedürfnisse der Lernenden angepasst sind, als Bildungsgänge oder Organisationen, die nicht Bildungsbereiche verbinden. Dies gilt vor allem, wenn der Zugang zu diesen Organisationen Lernenden aus den getrennten Bildungsbereichen offen steht. Anpassung an die heterogenen Bedürfnisse verstärkt zudem die Durchlässigkeitswirkung der anderen drei Aspekte, da die Lernenden nach Zugang, Übergang oder Anrechnung nicht allein gelassen, sondern gemäß ihren Bedürfnissen unterstützt werden.

Abbildung 1 Durchlässigkeitskonzept

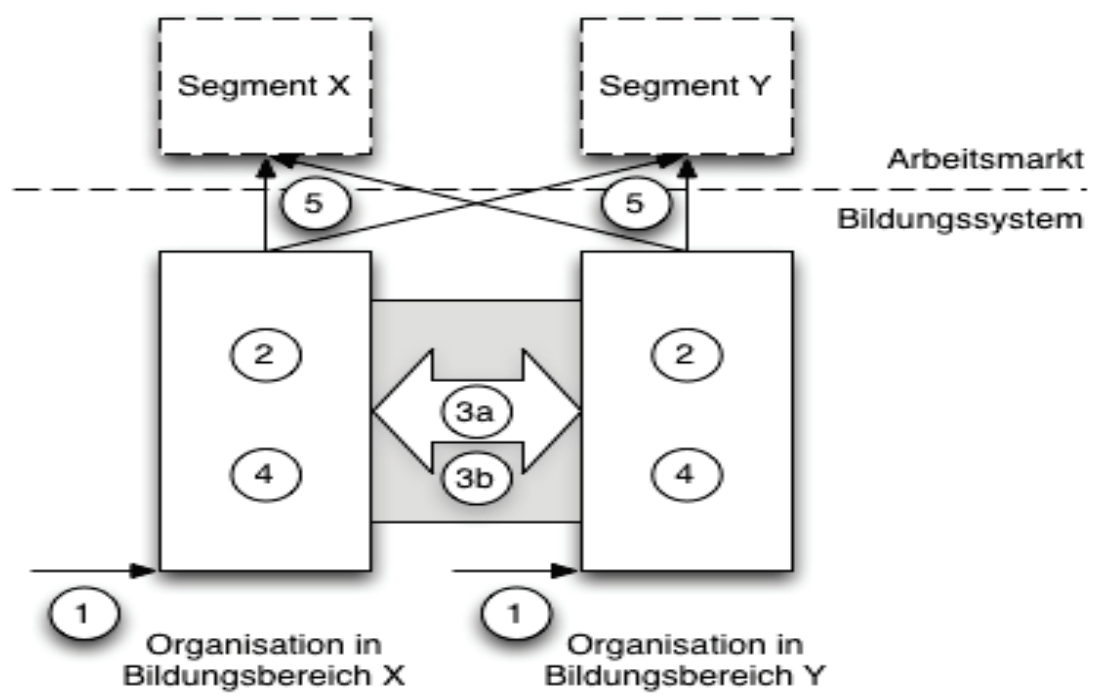

1 Zugang 2 Anrechnung; 3a institutionalisierte Übergänge; 3b Integration 4 Anpassung an heterogene Bedürfnisse 5 Anerkennung im Erwerbssystem

Quelle: Darstellung der Verfasserin

In der Darstellung der einzelnen Aspekte in Abbildung 1 wird ebenfalls deutlich, dass Durchlässigkeit ein vielfältiges Konzept ist und es viele Institutionalisierungsmöglichkeiten von durchlässigkeitsfördernden Bildungsstrukturen gibt. So 
kann unterschieden werden zwischen Strukturen, welche stärker innerhalb der Bildungsorganisationen institutionalisiert werden müssten (Aspekt 2 und 4), während andere mehr die Verbindungen der unterschiedlichen Bildungsbereiche darstellen (Aspekt 3a und 1). Die Organisationen selbst werden in diesen Fällen nicht weiter angepasst werden.

Tabelle 1 Überblick über Durchlässigkeitsaspekte

\begin{tabular}{|c|c|c|c|}
\hline Aspekte & $\begin{array}{l}\text { Ausrich- } \\
\text { tung }\end{array}$ & Förderliche Bedingung & Durchlässigkeitsrisiken \\
\hline \multicolumn{4}{|l|}{ Zugang } \\
\hline $\begin{array}{l}\text { universeller Zu- } \\
\text { gang }\end{array}$ & $\begin{array}{l}\text { gruppen- } \\
\text { zentriert }\end{array}$ & rechtsbasiert, einklagbar & $\begin{array}{l}\text { Ungenügende Vorbereitung } \\
\text { bzw. fehlende Unterstützungs- } \\
\text { strukturen }\end{array}$ \\
\hline $\begin{array}{l}\text { über Berechtigun- } \\
\text { gen }\end{array}$ & $\begin{array}{l}\text { gruppen- } \\
\text { zentriert }\end{array}$ & rechtsbasiert, einklagbar & $\begin{array}{l}\text { nicht in beiden Bildungsberei- } \\
\text { chen erwerbbar, statisches } \\
\text { Konzept von Studierfähigkeit }\end{array}$ \\
\hline $\begin{array}{l}\text { Entscheidung auf- } \\
\text { nehmender Institu- } \\
\text { tion über } \\
\text { Zertifikate }\end{array}$ & $\begin{array}{l}\text { gruppen- } \\
\text { zentriert }\end{array}$ & $\begin{array}{l}\text { Entscheidungsspielräume } \\
\text { der Institutionen }\end{array}$ & $\begin{array}{l}\text { Fehlende Anerkennung von } \\
\text { Gleichwertigkeit der Zertifikate } \\
\text { Abhängigkeit von einzelnen } \\
\text { Organisationen } \\
\text { statisches Konzept von Stu- } \\
\text { dierfähigkeit }\end{array}$ \\
\hline $\begin{array}{l}\text { Entscheidung auf- } \\
\text { nehmender Institu- } \\
\text { tion über Eig- } \\
\text { nungsprüfungen }\end{array}$ & $\begin{array}{l}\text { individu- } \\
\text { umszen- } \\
\text { triert }\end{array}$ & $\begin{array}{l}\text { Entscheidungsspielräume } \\
\text { der Institution } \\
\text { tatsächliche Kompeten- } \\
\text { zen geprüft }\end{array}$ & $\begin{array}{l}\text { Bevorzugung von Kompeten- } \\
\text { zen aus einem Bereich } \\
\text { statisches Konzept von Stu- } \\
\text { dierfähigkeit (Ausnahme Pro- } \\
\text { bestudium) }\end{array}$ \\
\hline \multicolumn{4}{|l|}{ Anrechnung } \\
\hline $\begin{array}{l}\text { Pauschale } \\
\text { Anrechnung }\end{array}$ & $\begin{array}{l}\text { gruppen- } \\
\text { zentriert }\end{array}$ & $\begin{array}{l}\text { Schnell mit weniger Auf- } \\
\text { wand für Individuen und } \\
\text { Organisationen }\end{array}$ & $\begin{array}{l}\text { Zuschreibung von (zu viel/zu } \\
\text { wenig) Kompetenzen, } \\
\text { nur formale Kompetenzen } \\
\text { Anrechnungsbegrenzung }\end{array}$ \\
\hline $\begin{array}{l}\text { Individuelle } \\
\text { Anrechnung }\end{array}$ & $\begin{array}{l}\text { individu- } \\
\text { umszen- } \\
\text { triert }\end{array}$ & $\begin{array}{l}\text { Anerkennung der konkre- } \\
\text { ten Kompetenzen (inkl. } \\
\text { non- und informell) }\end{array}$ & $\begin{array}{l}\text { Abhängig von Unterstützungs- } \\
\text { strukturen für Beantragende } \\
\text { (aufwendig), } \\
\text { Anrechnungsbegrenzung }\end{array}$ \\
\hline $\begin{array}{l}\text { Kombination } \\
\text { beider Verfahren }\end{array}$ & $\begin{array}{l}\text { individu- } \\
\text { umszen- } \\
\text { triert }\end{array}$ & $\begin{array}{l}\text { Einbezug nicht nur forma- } \\
\text { ler Qualifikationen und } \\
\text { weniger aufwendig als nur } \\
\text { individuell }\end{array}$ & $\begin{array}{l}\text { Abhängig von Unterstützungs- } \\
\text { strukturen für Beantragende } \\
\text { Anrechnungsbegrenzung }\end{array}$ \\
\hline
\end{tabular}


Aspekte Ausrich- Förderliche Bedingung Durchlässigkeitsrisiken

\begin{tabular}{|c|c|c|c|}
\hline \multicolumn{4}{|c|}{ Organisationale Verbindung } \\
\hline $\begin{array}{l}\text { Institutionalisierte } \\
\text { Übergänge }\end{array}$ & $\begin{array}{l}\text { gruppen- } \\
\text { zentriert }\end{array}$ & $\begin{array}{l}\text { Feste Institutionalisierung } \\
\text { als Unterstützung bei } \\
\text { Übergängen }\end{array}$ & $\begin{array}{l}\text { Förderung von Einseitigkeit } \\
\text { der Übergänge }\end{array}$ \\
\hline Integration & $\begin{array}{l}\text { gruppen- } \\
\text { zentriert }\end{array}$ & $\begin{array}{l}\text { Größere Kenntnis und } \\
\text { Akzeptanz für unter- } \\
\text { schiedliche Bereiche bei } \\
\text { Lernenden und Organisa- } \\
\text { tionen } \\
\text { Weiterbildungsmöglich- } \\
\text { keiten (z.B. Doppelquali- } \\
\text { fikation) }\end{array}$ & $\begin{array}{l}\text { Begrenzter Zugang, fehlende } \\
\text { Integration auf Augenhöhe }\end{array}$ \\
\hline \multicolumn{4}{|c|}{ Heterogenität der Lernenden } \\
\hline $\begin{array}{l}\text { Information und } \\
\text { Beratung }\end{array}$ & $\begin{array}{l}\text { individu- } \\
\text { umszen- } \\
\text { triert }\end{array}$ & $\begin{array}{l}\text { Ausgleich von Informati- } \\
\text { onsasymmetrien }\end{array}$ & $\begin{array}{l}\text { einseitige Information (Orien- } \\
\text { tierung nur zu best. Bildungs- } \\
\text { gängen) }\end{array}$ \\
\hline Finanzierung & $\begin{array}{l}\text { individu- } \\
\text { umszen- } \\
\text { triert }\end{array}$ & $\begin{array}{l}\text { Verringerung finanzieller } \\
\text { Abhängigkeiten }\end{array}$ & $\begin{array}{l}\text { Orientierung an ,traditionellen } \\
\text { Studierenden (Bsp. Altersbe- } \\
\text { grenzung) }\end{array}$ \\
\hline $\begin{array}{l}\text { Lernorganisation - } \\
\text { und Unterstüt- } \\
\text { zungsstrukturen }\end{array}$ & $\begin{array}{l}\text { individu- } \\
\text { umszen- } \\
\text { triert }\end{array}$ & $\begin{array}{l}\text { Individuelle Unterstüt- } \\
\text { zung/Begleitung zum } \\
\text { Lernerfolg (Infrastruktur, } \\
\text { Beratung etc.) }\end{array}$ & $\begin{array}{l}\text { Stigmatisierungsgefahr, wenn } \\
\text { Strukturen exklusiv für be- } \\
\text { stimmte Gruppen } \\
\text { u.U. Gefahr der Defizitorientie- } \\
\text { rung (bei Anpassungsforde- } \\
\text { rung an die Lernenden an den } \\
\text {,Standard') }\end{array}$ \\
\hline $\begin{array}{l}\text { Kultur der } \\
\text { Offenheit }\end{array}$ & $\begin{array}{l}\text { individu- } \\
\text { umszen- } \\
\text { triert }\end{array}$ & $\begin{array}{l}\text { Anerkennung aller Studie- } \\
\text { rendengruppen }\end{array}$ & \\
\hline
\end{tabular}

Quelle: Darstellung der Verfasserin

Zudem unterscheiden sich auch die Aspekte mit ihren verschiedenen Varianten in Bezug darauf, ob sie eher individuumszentrierte oder gruppenzentrierte Strukturen eröffnen (vgl. Tabelle 1). Anhand dieses unterschiedlichen Fokus kann aber auch die Widersprüchlichkeit des Durchlässigkeitskonzepts deutlich gemacht werden. Denn jeweilige Ausrichtungen bedingen unterschiedliche Vorund Nachteile. Gruppenspezifische Strukturen können dabei einerseits als stärker zugänglich bzw. barriereärmer gelten. So ist die Umsetzung von einer Regelung für viele mit weniger Aufwand sowohl für die umsetzende Organisation als auch für das Individuum verbunden, zumal solche Regelungen oft mit Berechtigungen für das Individuum einhergehen, welches somit aufgrund angenommener gruppenspezifischer Eigenschaften das Recht z.B. auf Zugang oder eine bestimmten 
Anrechnungshöhe hat. Implizit sind aber solchen gruppenspezifischen Strukturen Homogenitätsannahmen über die Gruppen eingebaut, welche andererseits auch nachteilig sein können, wenn es keine Strukturen gibt, die das ausgleichen und somit die Heterogenität der Individuen auch innerhalb der Gruppen anerkennen. So gehen z.B. pauschale Anrechnungsverfahren mit der Gefahr einher, dass einerseits bestimmte Kompetenzen, welche zusätzlich bestehen, unberücksichtigt bleiben. Zudem ist es möglich, dass durch die Zuschreibung eines gewissen Kenntnisstandes eventuell notwendige Unterstützung nicht angeboten wird, da das Individuum mit diesem spezifischen Zertifikat dies alles können müsste.

Individuumszentrierte Verfahren dagegen sind ressourcenaufwendiger, meist insbesondere für die aufnehmende Organisation aber teilweise auch für das Individuum, da hier der Fokus der Strukturen auf die individuellen Biografien und Lebensverläufe gerichtet ist. Dies ist insofern ein Vorteil, dass man der Person mit ihren ganz spezifischen Erfahrungen und Bedürfnissen gerecht werden kann. Andererseits kann es aber auch nachteilig sein, wenn es keine vorgefertigten Regelungen gibt, da das Individuum nicht auf diese bestehen kann, sondern die eigenen Bedürfnisse und Rechte individuell einfordern muss bzw. sich dieser erst selbst bewusst werden muss.

Eine Schlussfolgerung wäre demnach, dass für Durchlässigkeit in der Umsetzung beides benötigt wird, der Fokus auf Gruppen, damit eine stärkere Institutionalisierung stattfindet, und der Fokus auf das Individuum, für eine stärkerer Flexibilität und Anpassung. Ebenfalls ist festzustellen, dass die strukturellen Möglichkeiten Durchlässigkeit zu schaffen nicht nur förderlich wirken können (vgl. Tabelle 1).

Einerseits sind Barrieren dabei teilweise konzeptionell inhärent. So ist der Zugang über Berechtigung anhand von Zertifikaten oder einmaligen Prüfungen mit einem statischen Konzept von Studierfähigkeit verbunden, d.h., es wird angenommen, dass eine Studierfähigkeit a priori vor dem Studium besteht und nicht erst mit dem Vorgang des Studierens erworben wird. Dies kann dazu führen, dass fähige Individuen nicht zugelassen werden, während andere im Studium feststellen, dass dieses Ihnen nicht liegt. Andererseits gibt es bei der Einführung der unterschiedlichen durchlässigkeitsfördernden Strukturen immer auch die Gefahr, dass diese bei unzureichender Institutionalisierung ihre Wirkung nicht entfalten können. So helfen individualisierte Anrechnungsverfahren z.B. nur, wenn auch Unterstützung bei der intensiven Vorbereitung gewährt wird. Auch fördern institutionalisierte Übergänge Durchlässigkeit, wenn diese zum Beispiel in alle Richtungen genutzt werden können, von der Berufs- in die Hochschulbildung und umgekehrt.

Eine weitere Schlussfolgerung, die sich aus dem Durchlässigkeitskonzept mit den unterschiedlichen Aspekten, deren möglichen Ausrichtungen und Perspektiven ergibt, ist, dass es nicht zu erwarten sein wird, dass die europäischen Bildungsprozesse Bologna und Kopenhagen ein ganz spezifisches Modell von Durchlässigkeit vorgeben. Zum einen haben die unterschiedlichen Mitgliedsstaaten, welche 
den Prozess gestalten, auch unterschiedliche Traditionen in Bezug auf Durchlässigkeit, so dass zu spezifische Vorgaben möglicherweise nicht mit bestehenden Systemen kompatibel wären. Dies ist jedoch unwahrscheinlich, da die Deklarationen einstimmig von allen Ländern beschlossen wurden. So dürften die Vorgaben nicht mit dem bestehenden Subsidiaritätsprinzip in Konflikt stehen. Zum anderen scheint es auch nicht einfach, ein passendes Modell, in dem alle Eigenschaften aufeinander abgestimmt sind, vorzugeben, da wie zuvor dargelegt, zum einen unterschiedlich Vor- und Nachteile bestehen und zum anderen national jeweils differente Organisationen in den Reformprozess eingebunden sein können. Insofern wird es auch in den Nationalstaaten interessant zu untersuchen sein, wie Durchlässigkeit institutionalisiert ist. Welche Aspekte besonders wichtig erscheinen und welche Inkongruenzen dort bestehen.

Nachdem das Konzept von Durchlässigkeit mit den verschiedenen Ausprägungsmöglichkeiten vorgestellt wurde, soll nachfolgend die Frage der Bedeutung durchlässiger Bildungsstrukturen aus der Perspektive von zwei theoretischen Sichtweisen der sozialen Ungleichheitsforschung dargestellt werden.

\subsection{Bildungsstrukturen als funktionales Erfordernis oder Legitimation bestehender Herrschaftsverhältnisse?}

Wie bereits deutlich wurde, sind Analysen über die Strukturen des Bildungssystems, d.h. die Untersuchung der strukturellen Durchlässigkeit, für die Frage von sozialer Ungleichheit, relevant. Soziale Ungleichheit kann dabei aus unterschiedlichsten Perspektiven analysiert werden (Solga et al. 2009). Auch die Bedeutung von Bildung und deren Strukturen kann verschieden bewertet werden. Meyer (1977) unterscheidet einmal die Perspektive, in welcher das Bildungssystem eine Sozialisationsfunktion der Individuen übernimmt und eine weitere Sichtweise, in der das Bildungssystem stärker eine Allokations- und Legitimationsfunktion einnimmt ${ }^{16}$. Die erste Perspektive entspricht einer eher strukturfunktionalistischen Sicht auf Bildung während die zweite eine konflikttheoretische und institutionelle Perspektive verbindet. Im Folgenden sollen diese beiden Perspektiven mit Bezug auf Durchlässigkeitsstrukturen von Bildungssystemen und deren Wandel dargestellt werden.

16 Meyer (1977) kritisiert die Sicht der klassischen Vertreter der Allokationstheorie, da sie die Bedeutung von Bildung als Institution in der Gesellschaft meist auf individuelle Konsequenzen verkürzen und den Einfluss auf die gesellschaftlichen Strukturen als Ganzes aus dem Auge verlieren. In dieser Arbeit sollen beide Ansätze verbunden werden. So soll die stärker konflikttheoretische Perspektive im Fokus stehen, ohne die institutionellen Wirkungen von Bildung aus dem Blick zu verlieren. 


\subsubsection{Selektions- und Sozialisationsfunktion von Bildung - Eine strukturfunktionalistische Perspektive}

Eine dominante Sichtweise schreibt dem Bildungssystem die Aufgabe der Sozialisation der Individuen der Gesellschaft zu (siehe Meyer 1977: 57), d.h., es wird angenommen, dass durch den Besuch von Bildungsorganisationen die Individuen ihr Wissen, ihre Kompetenzen ausbauen sowie verstärkt moderne Werte und Orientierungen sozialisiert werden. Die im Bildungssystem erworbenen Kompetenzen sind daraufhin die Grundlage, auf deren Basis die Positionen in der Gesellschaft vergeben werden. Aufbauend auf dem im Bildungssystem erworbenen $\mathrm{Hu}-$ mankapital werden die Personen somit ihren Funktionen in der Gesellschaft zugeführt.

Es handelt sich hierbei vor allem um eine funktionalistische Perspektive, in welcher soziale Ungleichheiten als notwendige Voraussetzung des Funktionierens moderner Gesellschaften betrachtet werden und dass, nur wenn auch Ungleichheit besteht, sich Gesellschaften fortschrittlich entwickeln (Davis/Moore 1945). Jede Gesellschaft hat die Aufgabe, ihre fähigsten Mitglieder auf die gesellschaftlich wichtigen Positionen zu verteilen. Um dies zu gewährleisten, bedarf es einer Anreizstruktur in Form von unterschiedlichen Belohnungen für die differenten notwendigen gesellschaftlichen Aufgaben und Verantwortlichkeiten, damit die Individuen bereit sind, in zusätzliche Qualifikationen für den Erwerb von sozialen Positionen zu investieren. Dem Bildungssystem kommt dann die Aufgabe zu, die Individuen entsprechend ihrer Begabung und Fähigkeiten erst zu sozialisieren und dann zu selektieren (vgl. Fend 1980, 2006; Parsons 1959). Die Sozialisationsund damit zusammenhängende Selektionsfunktion des Bildungssystems spielt somit aus funktionalistischer Perspektive eine signifikante Rolle. Dabei sollen in modernen Gesellschaften askriptive Merkmale wie soziale Herkunft nicht mehr die Selektion bestimmen. Denn der gesellschaftliche Wandel erfordert, dass nicht mehr nur die höheren Schichten einer Gesellschaft Bildung erhalten können, sondern alle gesellschaftlichen Gruppen entsprechend ihrem Talent, der Begabung oder Fähigkeiten (Bell 1994). Der Allokationsmodus in modernen Gesellschaften ist stattdessen die meritokratische Selektion (Solga 2005a; Waldow 2014a, b).

Meritokratie ist eine Herrschaftsordnung, in der die Begabung und Leistungsfähigkeiten der Individuen über die gesellschaftlichen Positionen, die sie erreichen, bestimmen. Leistung setzt sich in dieser Perspektive zusammen aus der individuellen Begabung ${ }^{17}$, ihrem Talent und der Anstrengung, die aufgewendet

17 Die Annahme, dass es natürliche Begabungsunterschiede gibt, ist zentral für die, meritokratische Leitfigur' nach (Solga 2005a). Solga weist aber daraufhin, dass Begabung selbst ein soziales Konstrukt ist, die im Rahmen der meritokratische Leitfigur ausgeblendet wird. Schon allein durch die unterschiedlichen Auffassungen, die es zum Konzept Begabung in unterschiedlichen Gesellschaften gibt, wird die soziale Konstruktion deutlich. So unterscheidet Lenhardt (2002) zwischen einem demokratischen Glauben an universelle Bildungsfähigkeit, der in den USA vorherrsche und einem naturalisierten Begabungsglauben, der typisch für das deutsche 
wird, um etwas zu erreichen (vgl. Ruß 2012). Becker und Hadjar (2009: 40f.) fassen die von Goldthorpe (1996) entwickelten Prämissen der bildungsbasierten Meritokratie folgendermaßen zusammen. Die erste Prämisse ist, dass „Verantwortungspositionen einzig nach demonstrierter Kompetenz im Sinne von Fähigkeiten zu besetzen sind“. Die zweite Prämisse ist, dass die „Chancen auf Bildungserwerb einzig von natürlichen Begabungen abhängen“. Insofern werden allein begabte Lernende für die höheren Bildungsgänge selektiert. Die dritte Prämisse unterstreicht die Bedeutung des „Leistungskriterium[s] als Basis jeglicher Selektionen in modernen Gesellschaften“. Leistung dient dann der Rechtfertigung für soziale Ungleichheiten.

Doch wie kann man nun Durchlässigkeit aus funktionalistischer Perspektive betrachten, und inwiefern ist Durchlässigkeit ebenfalls ein Problem, welches behandelt werden sollte? Fehlende Durchlässigkeit aus funktionalistischer Sicht ist in modernen Gesellschaften erst dann problematisch, wenn erstens die Selektion im Bildungssystem nicht meritokratisch erfolgt und Korrekturen im Bildungsverlauf notwendig erscheinen. Zweitens wird Durchlässigkeit wichtig, wenn die Trennung von Bildungsbereichen nicht mehr den Funktionserfordernissen der Gesellschaft entspricht. Spezifisch auf die Trennung von beruflicher Bildung und Hochschulbildung bezogen stellt sich die Frage, ob sich die Trennung mit dem Wandel der industriellen Gesellschaft, in welcher sich die berufliche Bildung entwickelt hat (vgl. Thelen 2004) hin zu einer postindustriellen oder Wissensgesellschaft (vgl. Bell 1975), weiterhin als funktional erweist. Bilden berufliche und Hochschulbildung weiterhin die in der Gesellschaft benötigten Fähigkeiten aus, trotz des Wandels, der mit einem Anstieg an wissensintensiven Berufen einhergeht, die klassischerweise durch die Hochschulbildung beliefert werden? Wird diese Frage negativ beantwortet, wäre auch hier anhand funktioneller Erfordernisse eine größere Durchlässigkeit eine Möglichkeit auf diese Erfordernisse zu reagieren. So unterstreicht auch Parsons (1959) die Bedeutung von Bildung für moderne Gesellschaften und die Bildungsexpansion als Voraussetzung für die gesellschaftliche Modernisierung. Durchlässigkeitsstrukturen sind vor diesem Hintergrund aber nicht für alle Lernenden eines Bildungssystems notwendig, sondern nur für diejenigen, die aufgrund ihrer Leistung im Bildungssystem auch für weitere höhere Bildungsgänge selektiert werden können.

Bildungssystem sei. Während letzterer davon ausgeht, dass es verschiedene natürliche Begabungen, die sich im Verlauf nicht verändern, gibt, die dann auch unterschiedlich behandelt werden sollten, weshalb unterschiedliche Bildungsgänge entwickelt werden, geht man in der ersten Variante davon aus, dass jedes Individuum per se bildungsfähig ist und dass Leistungsversagen durch erneute Anstrengung beantwortet werden solle. 


\subsubsection{Allokations- und Legitimationsfunktion von Bildung - Eine institutionelle und konflikttheoretische Perspektive}

Der funktionalistische Ansatz, in welchem angenommen wird, dass das Besuchen von Bildungsorganisationen tatsächlich zu einem Kompetenz- und Produktivitätszuwachs führt - auf Basis derer die gesellschaftlichen Positionen meritokratisch verteilt werden -, wird aus konflikt- und institutionentheoretischer Sicht infrage gestellt. Während die Erfordernisse der Gesellschaft aus funktionalistischer Perspektive im Vordergrund stehen, werden hier die bestehenden Herrschaftsverhältnisse und deren Legitimation in den Fokus der Erklärung gerückt. Der Aufbau von Bildungssystemen, deren Stratifikation sowie die darin bestehenden unterschiedlichen individuellen Möglichkeiten zur Höherqualifizierung werden dann als Ausdruck institutionalisierter Schließungsprozesse gefasst.

So wird im Rahmen der Allokationstheorie angenommen, dass die Aufgabe von Bildungsinstitutionen weniger die Sozialisation als vielmehr die legitimierte Verteilung von Individuen auf Positionen qua Bildungsabschluss ist. Auf Grundlage der Dauer und Art der Bildung, die abgeschlossen wurde (Meyer 1977: 59), werden Individuen klassifiziert und zu gesellschaftlichen Positionen zugeordnet, unabhängig, ob und wie sich deren Humankapital tatsächlich vergrößert hat. Das Vertrauen auf die Bildungszertifikate reicht aus, um den Zugang zu wichtigen gesellschaftlichen Positionen zu kontrollieren. Dies ist die Kernannahme über das Funktionieren kredentialistischer Gesellschaften. Aus dieser Perspektive können nach Collins (1979) Bildungszertifikate als zentrales Kriterium für soziale SchlieBungsprozesse angesehen werden. So schrieb bereits Weber (1922 [1980]: 577):

Wenn wir auf allen Gebieten das Verlangen nach der Einführung von geregelten Bildungsgängen und Fachprüfungen laut werden hören, so ist selbstverständlich nicht ein plötzlich erwachender ,Bildungsdrang`, sondern das Streben nach Beschränkung des Angebots für die Stellungen und deren Monopolisierung zugunsten der Besitzer von Bildungspatenten der Grund.

Kredentialismus ist somit ein Weg der Reproduktion sozialer Ungleichheiten, in dem Ressourcen und Lebenschancen über das Erreichen von Bildungszertifikaten kontrolliert wird. Das Erreichen der Bildungszertifikate wird jedoch durch die ungleiche Ressourcenausstattung der Lernenden der unterschiedlichen sozialen Schichten über im Lernkontext institutionell verankerte Selektionsmechanismen beeinflusst (vgl. Bourdieu/Passeron 1971), so dass Kinder aus höheren sozialen Schichten wesentlich wahrscheinlicher einen höheren Abschluss erreichen. Die soziale Ungleichheit wird so in Form von Bildungszertifikaten manifestiert und verschleiert (vgl. Bourdieu 2009), denn wenn angenommen wird, dass Bildungszertifikate leistungsbasiert vergeben werden, sind die erzielten Zertifikate auch die Ergebnisse individueller Begabung und Anstrengungen. Herkunftsbedingte Unterschiede werden auf diese Weise in ,erworbene' Merkmale umgewandelt (siehe Solga 2005b). 
Aus konflikttheoretischer Perspektive sind Bildungsinstitutionen demnach nicht nur an der Reproduktion von sozialer Ungleichheit beteiligt, sie legitimieren auch Ungleichheit und tragen so zu ihrer Persistenz bei. So sieht Solga (2005a) in der meritokratischen Leitfigur moderner Gesellschaften eine Ideologie, die der Rechtfertigung von Ungleichheiten dient, da sie Bildung als Chance präsentiert und damit Zugang zu privilegierten Positionen über Bildungszertifikate legitimiert. Auch Baker (2011: 27) betont, "The belief that formal education is the proper context for the playing out of merit is widely held in postindustrial society." Und gerade dieser Glaube „could be education's deepest sociological impact as an institution over the course of the education revolution."

Es geht demnach nicht nur um die Konsequenzen von Bildung für die einzelnen Individuen, sondern um den Einfluss von Bildung als Institution. Denn Bildung kann das Leben der Menschen, auch unabhängig ihrer individuellen Bildungswege beeinflussen, da durch Bildung die Perzeption der Realität der Menschen mitbestimmt und legitimiert wird. Durch Bildung werden Wissenskategorien aber auch Personenkategorien geschaffen. Auch wird im Bildungssystem ein bestimmtes Wissen als ,richtig' und geltend vermittelt. Bildung besteht demnach aus einem Regelnetzwerk „creating public classifications of persons and knowledge. It defines which individuals belong to these categories and possess the appropriate knowledge. And it defines which persons have access to valued positions in society" (Meyer, 1977: 55). Diese institutionalisierten Regeln stellen einen mächtigen Mythos moderner Gesellschaften dar, die dabei helfen, die Welt $\mathrm{zu}$ interpretieren und bestehende Organisationsstrukturen zu legitimieren (Meyer/Rowan 1992). Wenn Bildung nun als Mythos moderner Gesellschaften angesehen werden kann, dann beruht nach Meyer (1977) deren Geltungskraft auf dem Folgenden:

The effects of myths inhere, not in the fact that individuals believe them, but in the fact that they „know" everyone else does, and thus that „for all practical purposes“ the myths are true. (Meyer 1977: 75)

Was bedeutet dieser eher generelle Blick auf die institutionellen und herrschaftsrelevanten Konsequenzen von Bildung für das Problem von Durchlässigkeit? Zum einen soll deutlich gemacht werden, dass Durchlässigkeit eng verbunden mit der Problematik sozialer Schließung ist. Wenn also Positionen in der Gesellschaft vermehrt über das Bildungssystem vermittelt und legitimiert werden, ist der $\mathrm{Zu}$ gang zu Bildung eine Ressource, die es zu monopolisieren gilt. Die Stratifizierung von Bildungssystemen, in denen die Absolvent_innen aus bestimmten Bildungsbereichen eher in statusniedrige Positionen gelangen und aus anderen Bereichen den Erwerb von statushöheren Positionen ermöglichen, ist ein Weg, Bildung als Ressource zu monopolisieren. In der Literatur zu tracking zwischen beruflichen und akademischen Programmen wird dieser Prozess der sozialen Schließung deutlich. So dienen getrennte Bildungswege zu einer Ablenkung von Kindern aus einem Haushalt mit niedrigem sozialem Status weg vom Hochschulsystem in ei- 
nen vermeintlich sicheren Berufsbildungsweg, der aber perspektivisch nur begrenzte Aufstiegsmöglichkeiten bietet (Shavit/Müller 2000a). Tracking ist dann ein Mechanismus zur intergenerationalen Reproduktion sozialer Ungleichheit. Insbesondere in Ländern wie Deutschland, in denen die Schüler_innen früh durch ein mehrgliedriges Schulsystem in bestimmte Bildungswege sortiert werden, ist die Frage des tracking zwischen Berufs- und Hochschulbildung besonders wichtig (vgl. Allmendinger 1989; Shavit/Müller 2000a). Aber auch in Ländern, welche weniger stark getrennte Bildungsbereiche aufweisen sind Mechanismen der Ablenkung festzustellen. So zeigen Brint und Karabel $(1989,1991)$ dieses eindrücklich für die Rolle der Community Colleges in den USA.

Durchlässige Bildungsstrukturen können dann aber ein Weg sein, dass, auch wenn ein bestimmter Bildungsweg eingeschlagen wurde, dieser wieder verlassen werden kann, da es von jedem Punkt im Bildungssystem Anschlussmöglichkeiten zur Weiterbildung auch in andere Bildungsbereiche gibt. Aus der stärker konflikttheoretischen Perspektive gilt es demnach zu untersuchen, inwiefern durchlässige Strukturen zu einer geringeren Schließung im Bildungssystem führen? Wie müssen Strukturen gebaut werden, um dieses Ziel zu erreichen. Und inwiefern ist es zu beobachten, dass einerseits Strukturen durchlässiger gemacht werden, um an anderer Stelle neue Schließungsmechanismen einzubauen?

So kann z.B. in Sachen Durchlässigkeit des Bildungssystems wichtig sein, inwiefern es rein kredentialistisch funktioniert und ob, wenn das der Fall ist, lediglich bestimmte Zertifikate aus einem Bildungsbereich Zugang ermöglichen oder auch Zertifikate aus allen anderen? Wenn also Zertifikate weiterhin über Zugang entscheiden sollen, ist aus Durchlässigkeitsperspektive wichtig, dass die Gleichwertigkeit von Zertifikaten aus unterschiedlichen Bildungsbereichen anerkannt wird. Eine Möglichkeit, Zugang nicht kredentialistisch zu organisieren, wäre dann der universale Zugang oder der Zugang über Eignungstests. Während der universale Zugang keine/n ausschließt, kann ein Zugang über Eignungstests auch ein Mittel sein, um Individuen aus bestimmten Bildungsbereichen auszuschließen. Wichtig wäre dann, dass der Zugang nicht spezifische Formen des inkorporierten Kapitals (vgl. Bourdieu 1987), welches nur durch bestimmte Bildungswege erlangt wird, erfordert, sondern von allen Interessierten gleichermaßen erfolgreich bestanden werden kann.

Wenn man an Anrechnung denkt, sind es theoretisch gerade nicht allein die Zertifikate, sondern die in den einzelnen Bildungsgängen vermittelten Kompetenzen, die verglichen werden. Dies gilt vor allem, wenn auch non-formale und informelle Kompetenzen wie Berufserfahrung bei der Bewertung miteinfließen und es sich somit nicht nur um pauschale Verfahren handelt. Über Anrechnung kann demnach mitunter ein rein kredentialistischer Blick eingegrenzt werden.

Vor allem aber die Einführung von Strukturen, welche auf die heterogenen Bedürfnissen der Lernenden eingehen und das Ziel verfolgen, alle Lernenden zum Bildungserfolg zu führen, können Schließungsmechanismen, die Bildungsteilneh- 
mer_innen aus einem Bereich benachteiligen, außer Kraft gesetzt werden. So können z.B. Informationsangebote herrschaftsbedingte Chancenungleichheiten, wie Informationsasymmetrien, ausgleichen. Auch die angepasste Lernorganisation und die Kultur in den Bildungsorganisationen spielen somit eine wichtige Rolle. Anhand des differenzierten Durchlässigkeitskonzepts wird demnach deutlich, dass es mehrere Stellschrauben gibt, um institutionelle Durchlässigkeit aus einer stärker konflikttheoretischen Perspektive zu gewährleisten oder zu erschweren.

Aber nicht nur die Bildungssysteme an sich, sondern auch deren Wandel werden aus den unterschiedlichen Perspektiven unterschiedlich erklärt (vgl. auch Graf 2013). So kann einerseits der Wandel von Bildungsstrukturen als funktionale Anpassung gesehen werden und somit die Notwendigkeit von Reformen im Vordergrund stehen. In der stärker konflikttheoretischen institutionellen Perspektive wird andererseits dieser Funktionalismus dahingehend hinterfragt, inwiefern Veränderungen der Bildungsstrukturen dazu beitragen, dass sowohl bestimmte Gruppen systematisch von Bildungschancen sowie gesellschaftlichen Positionen ferngehalten als auch bestehende Herrschaftsverhältnisse manifestiert werden. Zugleich ist aus der institutionen- und konflikttheoretischen Sicht bedeutend, inwiefern bestehende Bildungsstrukturen weiterhin als legitim gelten bzw. deren Legitimität infrage gestellt wird. Vor dem Hintergrund konflikttheoretischer Theorien sozialer Ungleichheit ist fehlende Durchlässigkeit dann problematisch, wenn durch die Trennung von Bildungsbereichen bestimmte Gruppen systematisch von dem Erreichen von gesellschaftlich wertvollen Positionen ausgeschlossen werden. $\mathrm{Ob}$ und wie Durchlässigkeit umgesetzt wird, hängt damit eng damit zusammen, inwiefern bestehende Herrschaftsverhältnisse verändert werden.

Die vorliegende Arbeit versucht, diese konflikttheoretische und institutionentheoretische Perspektive auf Bildung und Durchlässigkeit aufzunehmen, indem die Perspektive des soziologischen Neoinstitutionalismus mit einer diskursanalytischen Forschungsperspektive verbunden wird. So werden in dieser Arbeit folgende Fragen untersucht: Wie wird argumentiert? Welche Schließungsprozesse und damit Barrieren zu Durchlässigkeit werden in den Diskursen sichtbar? Werden Schließungsstrategien deutlich? Aber auch die Frage des Funktionalismus wird in dieser Arbeit aufgegriffen. Inwiefern spielen funktionalistische Argumentationen eine Rolle? Wird die Funktion der Trennung von Berufs- und Hochschulbildung als notwendig angesehen oder infrage gestellt? Diese Fragen sollen in den Länderkapiteln nachgegangen werden. Nachfolgend soll allerdings beginnend der weitere theoretische Rahmen zur Analyse institutionellen Wandels dargelegt werden. 


\section{Theoretischer Rahmen zur Analyse institutionellen Wandels}

Das nun folgende Kapitel soll den theoretischen Rahmen, welcher die Analyse des institutionellen Wandels der nationalen Durchlässigkeitsstrukturen anleitet, darstellen. Im Zentrum des Buches stehen drei Fragenkomplexe, die alle institutionelle Wandelprozesse sowie deren Ergebnisse untersuchen. Erstens wird erfragt, inwiefern seit 1990 in Deutschland und 1985 in Frankreich ein institutioneller Wandel von Durchlässigkeitsstrukturen festzustellen ist. Zweitens wird untersucht, inwieweit diese nationalen Entwicklungen durch die europäischen Bildungsprozesse Bologna und Kopenhagen beeinflusst worden sind. Und drittens wird erörtert, ob die nationalen Entwicklungen in Deutschland und Frankreich eine Annäherung ihrer institutionellen Durchlässigkeitsstrukturen und damit auch der Bildungssysteme zur Folge hatte.

Durchlässigkeitsstrukturen von Bildungssystemen bestimmen die Bildungsund Lebenschancen von Individuen eines Landes mit. Ihre Untersuchung ist insofern ebenfalls aus Perspektive sozialer Ungleichheitsforschung wichtig. Entsprechend einer stärker institutionen- und konflikttheoretischen Perspektive (vgl. Kapitel 2) wird in der vorliegenden Arbeit angenommen, dass institutioneller Wandel von Durchlässigkeitsstrukturen keine bloße funktionale Anpassung an Veränderungen darstellt, sondern auch Ausdruck ist von veränderten Machtverhältnissen in der Gesellschaft einerseits und veränderter Legitimität bestehender Institutionen andererseits. Die vorliegende Studie versucht, diese konflikt- und institutionentheoretische Perspektive auf Bildung und Durchlässigkeit aufzunehmen, indem die theoretischen Ansätze des Neoinstitutionalismus, vor allem des soziologischen aber auch historischen, mit dem der wissenssoziologischen Diskursperspektive verbunden werden. Einerseits gilt es, mit dem soziologischen Neoinstitutionalismus zu untersuchen, inwiefern Institutionen im Hinblick auf Durchlässigkeit an Legitimität gewinnen oder verlieren und welche institutionellen Änderungen sich vollziehen. Andererseits werden mit der diskursanalytischen Forschungsperspektive die Konflikthaftigkeit, die mit institutionellem Wandel verbunden ist und damit auch die Frage der Veränderung von Herrschaftsverhältnissen in den Fokus gerückt. So schreibt Archer (1979), dass die Stabilität von Systemen, auch Bildungssystemen, durch die Interessen von herrschenden Gruppen der Gesellschaft erklärt werden können.

Neither in principle nor in practice is there any factor or force which ultimately prevents the appropriate decision-makers [...] from deciding to alter the structure of those systems [...]. In reality, however, structures distribute vested interests in their maintenance and it is the fact that groups do defend these which make patterns of interaction and change durable in the long run. (Archer 1979: 788-789)

Das Kapitel ist in drei größere Abschnitte eingeteilt. Der erste dient der Klärung von zentralen Begriffen und Konzepten, welche die Grundlage der empirischen 
Untersuchung bilden. Zweitens wird dargestellt, was unter institutionellem Wandel verstanden wird und wie es dazu kommt. Drittens soll gezeigt werden, warum sich die institutionelle Forschungsperspektive und die diskursanalytische ergänzen und nur durch ihre Kombination für diese Arbeit den passenden Rahmen aufbieten.

\subsection{Kernbegriffe und -konzepte}

Die Begriffe Institutionen und Diskurse werden in den Sozialwissenschaften häufig und differierend verwendet. Aus diesem Grund werden die verwendeten Definitionen dieser zwei und weiterer Schlüsselbegriffe, welche diese Arbeit anleiten, nachfolgend dargelegt. Zudem soll die Konzeption der Begrifflichkeiten bereits erste Hinweise darauf geben, wie und warum die Verbindung neoinstitutioneller Theoriestränge mit einer wissenssoziologischen Diskursperspektive fruchtbar sein kann. In einem ersten Schritt wird daher das in dieser Arbeit verwendete Diskursverständnis dargestellt, wobei insbesondere auch die dem Diskursverständnis innewohnende Verknüpfung von Macht und Diskurs erläutert wird. In einem zweiten Schritt wird der Institutionenbegriff erörtert, um anschließend darzustellen, in welchem Verhältnis Diskurse und Institutionen zueinander stehen. Schließlich werden zentrale Begrifflichkeiten des soziologischen Neoinstitutionalismus, welche für die Frage des Wandels der Durchlässigkeitsstrukturen relevant sind und das Verständnis von Institutionen, Organisationen und ihr Verhältnis zueinander erklären, erläutert.

\subsubsection{Diskursbegriff und Diskursanalyse}

Diskurs ist ein viel und unterschiedlich verwendeter Begriff. ${ }^{18}$ In der vorliegenden Arbeit verwende ich eine diskursanalytische Perspektive, welche stark auf den Überlegungen Foucaults aufbaut, jedoch an das Programm einer wissenssoziologischen Diskursanalyse anknüpft. Diese Verbindung ist möglich, da nach Keller (2008) und Bublitz (2001) diskurstheoretische und -analytische Perspektiven schon aufgrund ihrer Forschungsinteressen und Methoden in der Tradition der Wissenssoziologie verortet werden können. Besonders der von Foucault präzisierte Diskursbegriff eignet sich dabei, um die konfliktbehaftete und machtdurch-

Keller et al. (2001) unterscheiden dabei die discourse analysis im Sinne der Gesprächs- und Konversationsanalyse, den Diskursbegriff der Diskursethik nach Habermas, den Diskursbegriff aus der Diskurstheorie in Anschluss an Saussure sowie den Diskursbegriff der kulturalistischen Diskursanalyse mit Fokus auf die die handlungstheoretische Grundlage bei der Konstruktion gesellschaftlicher Wissensordnungen. 
setzte Hervorbringung von Wissen und dessen sozialen Dimensionen miteinander zu verknüpfen (Bechmann 2007). Sein Ansatz trägt deutlich wissenssoziologische Züge und nimmt insbesondere die gesellschaftlichen Machtprozesse in die Analyse auf, verortet die Konstruktion von Wissen nicht mehr auf der Mikroanalyse - wie die klassischen wissenssoziologischen Arbeiten z.B. von Berger und Luckmann (2007) -, sondern betrachtet vor allem die machtvollen institutionellen Mechanismen der Wissenszirkulation. Ebenjene machtvollen Prozesse werden in dieser Arbeit im Fokus stehen.

Foucault definiert Diskurse als „Praktiken, die systematisch die Gegenstände bilden, von denen sie sprechen“ (Foucault 1981: 74). Diese kollektiven Praktiken der Wissensproduktion sind dabei in den jeweiligen historischen und gesellschaftlichen Kontext situiert und erzeugen, reproduzieren und transformieren Weltund Wirklichkeitsordnungen, stellen zum einen Wissen auf Dauer und tragen aber auch zur Transformation und Auflösung institutionalisierter Deutungen bei (Keller 2006: 131). Wandel ist diesem Verständnis von Diskurs somit inhärent. Dabei unterscheiden sich Diskurse durch die Regeln voneinander, welche sie befolgen und nicht durch den unterschiedlichen Gegenstand, der thematisiert und konstruiert wird. In dem Sinne bilden Diskurse verschiedene „perspektivische Sichtweisen des Gegenstands" (Bublitz 2006: 258). Der Diskursbegriff von Foucault entwickelte sich während seines Werkes weiter, kann aber nicht als einheitliche Theorie gefasst werden. Der Diskursbegriff steht jedoch immer verbunden mit seinen Ausführungen zur Archäologie (vgl. Klemm/Glasze 2004). Eine Diskursanalyse im Sinne der Archäologie meint entsprechend der obigen Diskursdefinitionen „die Rekonstruktion der Erzeugungsmuster gesellschaftlicher Wissensformationen, die als Abfolge von ,Regimen des Sagbaren““ definiert werden können (vgl. Keller 2008: 128, Hervorhebung im Original).

Diskurse treten in Erscheinung in Form von Aussageereignissen. Dabei wird unterschieden zwischen Äußerung und der Aussage. Während erstere ein einmaliges Ereignis darstellt und Fragmente von Diskursen beinhaltet, enthalten Aussagen bereits das typische für den Diskurs. Die gleiche Aussage kann dabei in mehreren Äußerungen produziert werden (Keller 2008: 236). Das Verhältnis von Aussageereignissen und Diskurs beschreibt Keller (2008) in Anlehnung an Giddens folgendermaßen:

Das Verhältnis von Diskurs und diskursivem Ereignis entspricht dem Verhältnis von Struktur bzw. Strukturierung und einzelner Handlung (Praktik). Aus der Handlung entsteht die Struktur, aus der Struktur im Prozess der Strukturierung die Handlung. Ohne Aussageereignisse gibt es keine Diskurse; ohne Diskurse können Aussageereignisse nicht verstanden, typisiert und interpretiert werden. Dieses Verhältnis von Diskurs und diskursivem Ereignis bildet die Dualität von Struktur. (Keller 2008: 205)

Somit kann der „Zusammenhang zwischen einzelnem diskursiven Ereignis und Gesamtdiskurs [...] als aktualisierende Reproduktion oder Transformation einer Diskursstruktur verstanden werden, die nur in dieser Aktualisierung realisiert 
wird“ (Keller 2008: 206). Dabei stehen bei Foucault Macht und Diskurs in einem unzertrennlichen Verhältnis zueinander.

Weil Diskurse konsequent in ihrer wirklichkeitsschaffenden Macht in den Blick genommen werden, geht es der diskursanalytischen Perspektive konkret immer auch um Wissens-(-an-)ordnungen (um vorherrschende Bedeutungen, Sinngehalte, Deutungsmuster, ihre Wirkungsbedingungen und Folgen), die durch institutionalisierte diskursive Praktiken im Sinne von Wissenspolitiken hergestellt, durchgesetzt, stabilisiert, verändert oder umgestürzt werden. Weil es in diesem Sinne um Wissenspolitiken als Wahrheitspolitiken geht, sind Diskursanalysen immer auch Machtanalysen. (Bührmann/Schneider 2007: Abs. 12)

Zusammenfassend sind Diskurse „geregelte Praktiken der Deutungsproduktion und Wirklichkeitskonstruktion" (Keller 2006: 125). Sie produzieren Wahrheiten - nicht basierend auf dem Modus des Verstehens oder besseren Arguments -, sondern auf Ordnungs- und Distributionsstrukturen fußend, die Sinn als ihren Effekt hervorbringen. Die diskursive Praxis kann so als ein „Wahr-Sprechen“ (dire-vrai) bezeichnet werden (Bührmann/Schneider 2007: Abs. 1).

Anschließend an dieses Verständnis von Diskurs soll die Diskursanalyse demzufolge die Prozesse der sozialen Konstruktion, Kommunikation sowie Legitimation von Deutungs- und Handlungsweisen auf der Ebene von institutionellen Feldern, Organisationen, sozialen Kollektiven und Akteuren analysieren, rekonstruieren und die gesellschaftlichen Wirkungen aufzeigen (vgl. Keller 2006, 2007b). Es geht also um „die Erschließung von übersituativen Sinnfiguren und deren situativen und strukturellen Bestandsbedingungen, die insbesondere in Machtverhältnissen gesehen werden“" (Traue et al. 2014: 495, Hervorhebung im Original).

In der vorliegenden Arbeit wird eine entsprechende diskusanalytische Perspektive eingenommen. Untersucht werden die Veränderungen in den Diskursen und damit auch der Wissensordnungen zur Durchlässigkeit zwischen Berufs- und Hochschulbildung in Deutschland und Frankreich.

\subsubsection{Diskurs und Macht}

Im Werk Foucaults kamen neben der Frage der Archäologie immer stärker Überlegungen zu den spezifischen Machtwirkungen der Diskurse hinzu. Diese lassen sich im Konzept der Genealogie wiederfinden (vgl. Klemm/Glasze 2004). Eine Diskursanalyse im Sinne der Genealogie untersucht die Entstehung von Diskursen unter Betrachtung sich ändernder Machtkonstellationen sowie Machtspiele und analysiert „Diskurse als Wirkungen von Machtpraktiken“ (Bublitz 2006: 259). „Denn der Diskurs [...] ist die Macht, deren man sich zu bemächtigen sucht“ (Foucault 2007: 11). In diesem Abschnitt steht das bereits angesprochene Verhältnis von Diskurs und Macht im Blickpunkt, da dieses speziell eine Stärke des Diskursansatzes nach Foucault ausmacht und anknüpft an die Perspektive der 
Konflikttheorien (vgl. Kapitel 2), welche die Bedeutung von Herrschaftsverhältnissen für die Ausgestaltung von gesellschaftlichen Ungleichheitsstrukturen betonen.

Wie definiert Foucault Macht? Was soll unter Macht verstanden werden?

Unter Macht, scheint mir, ist zunächst zu verstehen: die Vielfältigkeit von Kräfteverhältnissen, welche ein Gebiet bevölkern und organisieren; das Spiel, das in unaufhörlichen Kämpfen und Auseinandersetzungen diese Kräfteverhältnisse verwandelt, verstärkt, verkehrt; die Stützen, die diese Kraftverhältnisse aneinander finden, indem sie sich zu Systemen verketten - oder die Verschiebungen und Widersprüche, die sie gegeneinander isolieren; und schließlich die Strategien, in denen sie zur Wirkung gelangen und deren große Linien und institutionelle Kristallisierungen sich in den Staatsapparaten, in der Gesetzgebung und in den gesellschaftlichen Hegemonien verkörpern. (Foucault 2003: 93)

Macht wird also definiert als Kräfteverhältnisse. Dabei ist Macht nicht singulär und geht nicht von einer bestimmten Gruppe aus, sondern ist plural zu verstehen - es gibt viele Kräfteverhältnisse in der Gesellschaft, in der Familie, in der Schule in den Betrieben etc.

Die Macht ist nicht eine Institution, ist nicht eine Struktur, ist nicht eine Mächtigkeit einiger Mächtiger. Die Macht ist der Name, den man einer komplexen strategischen Situation in einer Gesellschaft gibt (Foucault 2003: 94).

Macht ist dabei auch nicht etwas Definitives - sie ist vielmehr eine Möglichkeit, auf das Handeln anderer einzuwirken (Lemke 2008: 41). Eine Machtbeziehung besteht also ab dem Moment, wenn A das Handeln von B beeinflusst, unabhängig ob das im Einverständnis mit B war oder gegen seinen Willen geschieht (vgl. auch Definition von Weber (1972: 28). Insofern ist Macht bei Foucault allgegenwärtig (Lemke 2008). Diese omnipräsenten strategischen Beziehungen sind prinzipiell veränderbar. Jedoch räumt Foucault ein, dass es Herrschaftszustände gibt, in welchen Macht institutionalisiert wird durch ökonomische, politische oder militärische Mittel - und die in starre Machtbeziehungen münden: In dem Fall sind Herrschaftszustände ein Extrempunkt von Machtbeziehungen, in dem es einer gesellschaftlichen Gruppe möglich war „,das Feld der Machtbeziehungen zu blockieren und eine dauerhafte Asymmetrie zu etablieren“ (Lemke 2008: 42). In Demokratien, wie in den Untersuchungsländern Deutschland und Frankreich, sollte hingegen eher die Möglichkeit existent sein, bestehende Machtverhältnisse zu ändern.

Macht und Wissen sind zwei Dimensionen des Diskurses, welche in enger Beziehung zueinander stehen. Wissen kann sich nicht unter neutralen Gegebenheiten entfalten. Bublitz (2001) beschreibt sie als gegenseitige Zwangswirkungen, denn wenn etwas als Wissenselement in Diskursen auftritt - ist es Teil eines Machtsystems spezifischer Zwänge. Machtmechanismen allerdings sind wiederum begründet in Wissenssystemen.

Tatsächlich ist jeder Punkt der Machtausübung zur gleichen Zeit ein Ort der Wissensbildung. Und umgekehrt erlaubt und sichert jedes etablierte Wissen die Ausübung einer Macht. (Foucault 1976: 118) 
Wissen bedeutet Macht und Macht bringt Wissen hervor (Lederle 2008: 127). Es gibt demnach keine Machtbeziehungen ohne dazugehöriges Wissensfeld. Die Machtwirkung von Diskursen besteht dann darin, dass sie die Ordnung der Dinge, die Wissensordnung, herstellen. Die Kräfteverhältnisse, welche diese Ordnung hervorbringen, lassen sich insofern analysieren, als dass sich die eigentlich instabilen Macht-Wissens-Systeme verfestigen können und sich durch Wiederholung kristallisieren und zwar in akzeptierte Praktiken, Routinen, Selbstverständlichkeiten, Normen oder Regeln, kurz in Institutionen.

Wichtig ist in diesem Zusammenhang, dass Foucault Macht und Widerstand als zusammengehörig begreift. Widerstände entstehen aus der inneren Inkonsistenz der Macht. So konstituieren beide ein zirkuläres Verhältnis. Damit gibt es quasi keinen Ort außerhalb von Macht. Eine Umkehrung von Machtverhältnissen ist aber als generell möglich gedacht (vgl. Bublitz 2001). Insofern zielt die Macht stets darauf, gefährliche Kräfte des Diskurses zu kontrollieren. Diese Prozeduren der Gesellschaft zur Kontrolle des Diskurses benennt Foucault in seiner Vorlesung zur Ordnung des Diskurses; es sind Verbote, der Ausschluss, die Tabuisierung, Ritualisierung von Redesituationen, die Grenzziehung zwischen Vernunft und Wahnsinn, Wahrem und Falschen (Foucault 2007).

Zusammenfassend lässt sich sagen, dass Diskurse Wissen als Effekt hervorbringen und konstruieren damit die gesellschaftliche Wirklichkeit. Diese Konstruktion ist immer mit einer historisch situierten Machtkonstellation verbunden. Die gesellschaftlichen Wissensvorräte müssen in dieser Hinsicht konzipiert werden als sich in ständiger Bewegung befindend und als untereinander in Konkurrenzbeziehungen stehend. Es gibt nicht nur eine Deutung der Wirklichkeit. Macht und Interessen konkreter Personengruppen entscheiden, welche konkurrierenden Deutungen Geltung erlangen und sich in Institutionen kristallisieren. In Diskursen produzieren somit institutionell-organisatorisch bestimmbare Akteure wesentliche Elemente symbolischer Ordnungen einer Gesellschaft und damit ihr Verständnis der Wirklichkeit. Diese Produktion und Vermittlung von Deutungsangeboten ist immer konfliktbehaftet (vgl. Schwap-Trapp 2001). Konflikthaftigkeit besteht, weil die Deutungen Vorgaben für politische und soziale Handlungszusammenhänge produzieren und darüber entscheiden, wie sie wahrgenommen und bewertet werden. Sie legitimieren soziales und politisches Handeln, aber auch Institutionen, indem sie sagen, was ,gut ${ }^{`}$ und ,böse‘ ist.

Aus dieser Perspektive der Konflikthaftigkeit erscheint Geschichte demgemäß als eine permanente Konfrontation von Kräften, als Aneinanderreihung von Machtkämpfen verbunden mit Niederlagen, Siegen und Überwältigungen (vgl. Fink-Eitel 1990). Dabei müssen die Ereignisse, welche zu Wandel führen, nicht notwendigerweise spektakulär sein.

Die großen Veränderungen kommen auf Taubenfüßen. Es sind Wendepunkte, Verschiebungen und Unterbrechungen, in denen unvermerkt Bedeutungen wechseln, Machtbeziehungen kippen, Kräfteverhältnisse umschlagen. (Konersmann 2007: 84) 
Für die Frage des Wandels nationaler Durchlässigkeitsstrukturen wird dann anhand der diskursanalytischen Perspektive zu untersuchen sein, inwiefern sich die Diskurse und ihre Strukturierungen im Lauf der Zeit ändern? Wie wird Durchlässigkeit diskursiv konstruiert? Welche konkurrierenden Deutungsangebote gibt es? Inwiefern setzen sich bestimmte Deutungsangebote über Durchlässigkeit im Lauf der Zeit durch? Durch diese Analyse können insofern auch Aussagen über die Kräfteverhältnisse zwischen Diskursen und zugehörigen Akteursgruppen gemacht werden.

Wenn Diskurse nun als geregelte Praktiken der Deutungsproduktion und Wirklichkeitskonstruktion verstanden werden, welche Rolle spielen dann Institutionen bzw. in welchem Verhältnis stehen Diskurse und Institutionen? Bevor diese Fragen beantwortet werden, soll zuerst der verwendete Institutionenbegriff vorgestellt werden.

\subsubsection{Institutionen}

Es gibt eine große Anzahl von Definitionen, was Institutionen sind (vgl. Csigó 2006: 63; Powell 2011: 39) - auch innerhalb des Theoriestrangs des soziologischen Neoinstitutionalismus ${ }^{19}$. Diese Arbeit übernimmt die umfassende Institutionendefinition von Scott (2008: 48), der seinerseits seine Konzeption an die Arbeiten von Powell und DiMaggio (z.B. 1991) anlehnt.

Institutions are social structures that have attained a high degree of resilience. (Scott 2001: 48)

Institutions are compromised of regulative, normative and cultural-cognitive elements that, together with associated activities and resources, provide stability and meaning to social life. (Scott 2008: 48)

Institutionen verringern demgemäß Unsicherheit in menschlichen Beziehungen und spezifizieren die Grenzen legitimen Handelns. Insofern steht im Zentrum des neoinstitutionalistischen Paradigmas das Konzept von eingeschränkter Wahlfreiheit, von „choice within constraints“ (Nee 2001: 9). Die drei Dimensionen von Institutionen (kulturell-kognitive, normative und regulative) formen dabei ein Kontinuum zwischen Unbewusstem und Bewusstem - den alltäglichen Selbstverständlichkeiten und dem Verordneten (Walgenbach/Meyer 2008: 57).

Regulative Elemente von Institutionen generieren nach Scott (2008) Handlungen bzw. Handlungsbeschränkungen durch festgelegte Regeln in der Gesellschaft und werden über Zwang durchgesetzt (vgl. auch DiMaggio/Powell 1983). Diese Ebene ist diejenige, die in den meisten Institutionstheorien mit dem Phänomen Institution assoziiert wird. Die Verhaltensregulierung erfolgt über for-

19 Der soziologische Neoinstitutionalismus wird auch als organisationssoziologischer Neoinstitutionalismus bezeichnet. 
melle Regelungen wie Gesetze und informelle Regelungen wie Traditionen. Indikatoren für diese Ebene sind demnach Regeln, Gesetze und Sanktionen, wobei über letztere die Einhaltung der Regeln sichergestellt werden (vgl. Scott 2008: 52ff.). In Bezug auf die Entwicklung von Durchlässigkeitsstrukturen wäre demnach zu untersuchen, welche Veränderungen durch Zwangsmechanismen wie nationale Gesetze zustande kommen. Wird Durchlässigkeit zwischen Berufs- und Hochschulbildung gesetzlich geregelt? In welchen Aspekten von Durchlässigkeit? Gibt es Regelungen zum Zugang oder zur Anrechnung vorgängigen Wissens? Welche gesetzlichen Festlegungen gibt es hinsichtlich der Zuständigkeiten der Akteure im Bildungssystem und der Finanzierung. All dies sind Elemente der regulativen Dimension.

Normative Elemente definieren Rollen sowie Erwartungen und sind Spezifizierungen, wie Dinge gehandhabt werden sollten. Sie umfassen somit auch die Bewertung der Angemessenheit (vgl. March/Olsen 1989), von gut und schlecht. Die normative Dimension basiert auf Normen, Werten und Standards und setzt sich über den Mechanismus von (moralischer) Verpflichtung durch. Das bedeutet, Akteure entscheiden sich aufgrund ihrer Wahrnehmung, ihrer sozialen Rolle und des Wissens, welches Verhalten mit diesen Rollen in spezifischen Situationen verbunden ist (Scott 2008: 55). Auf der normativen Dimension ist zudem festgelegt, wie das als richtig und gut befundene, erreicht werden soll (Scott 2008: 56). Wenn die normative Ebene analysiert wird, stehen die Praxen der Akteure und die vorherrschenden Standards im Vordergrund. Die Analyse erfolgt hier am ehesten auf Organisationsebene. Um die normative Dimension von Durchlässigkeit dementsprechend zu untersuchen, könnten bestehende professionelle Standards in Organisationen auf ihre durchlässigkeitsfördernden Wirkungen geprüft werden. Zu untersuchen wären auch die Curricula (vgl. Powell/Solga 2008) oder Best-Practice-Anleitungen, z.B. wie mit der Heterogenität von Studierenden umgegangen werden soll. Auch die Definition von Zielgruppen für Bildungsbereiche sind Elemente der normativen Dimension, da durch solche Definition entschieden wird, wer ein_e angemessene_r Bildungsteilnehmer_in ist und wer nicht.

Die kulturell-kognitiven Elemente beziehen sich darauf, wie die empirische Wirklichkeit erfahren wird. Insofern sollen kulturell-kognitive Elemente von Institutionen als die sozial vermittelte Konstruktion einer gemeinsamen Konzeption von der Beschaffenheit der Wirklichkeit ,the shared conceptions that constitute the nature of social reality and the frames through which meaning is made" (Scott 2008: 57) verstanden werden. Kognitive Elemente können dabei als Basis für die anderen beiden Dimensionen gesehen werden, denn auch diese besitzen kognitive Aspekte - Wissen ist gemäß Berger und Luckmann (2007: 117) der Wertkomponente inhärent.

Im soziologischen (Neo-)Institutionalismus ist die Einführung dieser kognitiven Dimension die zentrale Kategorie, welche diese Ansätze insbesondere von denen des älteren soziologischen Institutionalismus (z.B. Werke von Parsons), welcher den Fokus auf die normative Dimension legt, trennt (Scott 2008). Diese 
Ebene umfasst symbolische und kulturelle Ansätze, welche die institutionelle Prägung von Akteuren, ihre Wahrnehmungen, Interpretationen und Situationsdefinitionen betonen, aus der die Selbstverständlichkeit organisationaler Praktiken und Formen resultiert.

So beschreibt Zucker (1983: 25) den Prozess der Institutionalisierung als einen „fundamentally cognitive process“. Kognitionen werden im soziologischen Institutionalismus als Regeln, Rahmen, Schemata oder Skripte verstanden und bezeichnen die differenten Formen von Wissen. Dabei ist dieses Wissen erstens durch seine Selbstverständlichkeit als ,taken for granted' gekennzeichnet. Zweitens wird Wissen aus einer konstruktivistischen Perspektive heraus als Realität erzeugend verstanden (Klatetzki 2006). Wenn soziale Realität in sozialen Interaktionen entsteht, müssen somit auch die kognitiven Dimensionen untersucht werden, um zu verstehen ,which actions are produced, repeated, and come to evoke stable, similar meanings in self and other" (Scott 2008: 16). Schließlich hat Wissen einen externen Charakter, d.h., es ist überindividuell ,in den sozialen Beziehungen zwischen Individuen und kulturellen Artefakten außerhalb des Individuums ver-

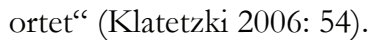

Es handelt sich also bei der kulturell-kognitiven Dimension von Institutionen um geteilte Vorstellungen der sozialen Wirklichkeit, Glaubens- bzw. Bedeutungssysteme - die über den Mechanismus der Selbstverständlichkeit ihre Wirkung entfalten.

Was ein Akteur wahrnimmt, wie er/sie diese Wahrnehmungen interpretiert und wie er/sie damit umgeht, wird wesentlich von seinen/ihren kognitiven Repräsentationen der Umwelt beeinflusst. (Walgenbach/Meyer 2008: 60)

Um die kulturelle-kognitive Dimension der Durchlässigkeitsstrukturen zu untersuchen, muss primär herausgearbeitet werden, was unter Durchlässigkeit verstanden wird und inwiefern sich dieses Verständnis von Durchlässigkeit verändert hat und warum? Wie wird z.B. die Notwendigkeit von Durchlässigkeit begründet? Warum wird Durchlässigkeit prinzipiell thematisiert? Dazu gehört auch die Analyse der gesellschaftlichen Interpretation, was Berufs- und Hochschulbildung in der Gesellschaft sind und welche Aufgaben sie zu erfüllen haben.

Es bleibt anzumerken, dass die Unterteilung zwischen den Dimensionen eine analytische ist, und dass insbesondere die Übergänge zwischen normativer und kulturell-kognitiver Dimension fließend sind (Berger/Luckmann 2007: 117).

Am Beispiel einer roten Ampel im Straßenverkehr sollen die unterschiedlichen Dimensionen und ihr Einfluss auf das Handeln von Institutionen dargestellt werden. Im Straßenverkehrsgesetz ist geregelt, dass man nicht bei Rot über die Ampel gehen darf. Wenn man erwischt wird, wird das Nichtbefolgen dieser Regel sanktioniert, z.B. mit einem Bußgeld. Die Handlungsregel, bei Rot stehen zu bleiben, hat demnach eine klar regulative Komponente. Will man nun doch, auch auf die Gefahr einer Sanktion hin, über eine rote Ampel gehen, weil man es eilig hat und gerade kein Auto kommt, so existiert die Norm zu schauen, ob man erstens niemanden gefährdet und ob zweitens nicht ein Kind mit an der Ampel steht. Ist 
letzteres der Fall, besagt die gesellschaftliche Norm, dass man kein schlechtes Vorbild für ein Kind sein sollte und wartet, bis die Ampel grün wird. Die Handlungsregel wird somit durch normative institutionelle Dimension unterstützt. Auf der kulturell-kognitiven Ebene ist das Konzept Ampel selbst angesiedelt, d.h., man muss erst ein Verständnis bzw. Wissen haben, was eine Ampel ist und wie sie funktioniert. Dabei ist dieses Wissen stark kulturell bedingt. Individuen aus Gesellschaften, welche keine Ampeln kennen, müssen sich erst mit dem Konzept, der Idee Ampel, vertraut machen und dieses inkorporieren, bevor sie entsprechend angemessen handeln können.

Die analytische Unterscheidung in die drei Säulen begründet Scott (2008: 51) damit, dass die Annahme, sämtliche Institutionen würden von allen drei Säulen gleichermaßen getragen werden, wenig sinnvoll erscheint. Dies würde nur zu einer Überdeterminierung von Handlungsweisen und -strukturen führen. Um somit Unterschiede zwischen Institutionen analysieren zu können, erscheint die Differenzierung in drei Ebenen vorteilig. Zudem weist Scott daraufhin, dass die drei Säulen miteinander verwoben sind, dies aber nicht sein müssen, sondern sogar Widersprüche zwischen ihnen bestehen können. Anhand eines Beispiels soll dies verdeutlicht werden.

Regulativ kann so z.B. Durchlässigkeit durch ein Gesetz, das Anrechnung in Bildungsorganisationen ermöglicht, gefördert werden. Die Konsequenz wäre, dass Universitäten z.B. Standards einrichten müssen, die festhalten, wie in beruflicher Ausbildung erlernte Kompetenzen im Studium anzuerkennen sind. Wenn nun aber in den Selbstverständlichkeiten der Gesellschaft dieser Wandel, die Anerkennung von vorgängig Gelerntem in anderen Bildungsbereichen, nicht ebenso vollzogen wird, so wird es sich schwierig gestalten, faire Standards der Anerkennung zu entwickeln. Das deutsche ANKOM-Projekt (Anrechnung beruflicher Kompetenzen auf Hochschulstudiengänge) ist ein Pilotprojekt, welches mit einzelnen Universitäten und Ausbildungsgängen die praktische Möglichkeit der Anrechnung von Kompetenzen untersucht. Es wurde festgestellt, dass Anrechnung möglich ist, aber schwierig, da vor allem von der akademischen Seite das Vertrauen in die Qualität von Berufsbildung vermittelter Kompetenzen fehlt (vgl. Buhr et al. 2008). Das heißt, selbst wenn es in einer institutionellen Dimension entsprechende Änderungen gibt, bedeutet es nicht, dass dies auch auf den anderen der Fall sein muss. Natürlich kann festgehalten werden, dass offensichtlich im Verständnis der Expert_innen, welche die neue Regelung erlassen haben, Anrechnung bereits verankert ist. Dies muss aber nicht für andere gesellschaftliche Gruppen gelten, die letztlich mitentscheidend für die Umsetzung von Anrechnung sind.

Zusammenfassend bleibt zu sagen, dass die drei Säulen somit analytisch unterscheidbare Elemente sind, aber keine Typen von Institutionen definieren sollen. Empirisch vorfindbare Institutionen werden sich vielmehr aus allen drei Elementen unterschiedlich stark gewichtet konstituieren. Institutionen sind im Sinne Scotts (2008) keine externe Handlungsbeschränkung. Sie konstituieren vielmehr 
soziale Identitäten von Akteuren, Interessen und Rationalitätskriterien. Individuen übernehmen dann mit ihrer sozialen Identität eine bestimmte Perspektive auf die Welt. An dieser Stelle machen Walgenbach und Meyer (2008: 61) deutlich, dass insbesondere die kognitive Ebene dazu verleitet, Akteure passiv, übersozialisiert und mit einem zu geringen Handlungsspielraum zu konzeptionalisieren, was zahlreich kritisiert wurde (vgl. z.B Powell 1991; Scott 2008) ${ }^{20}$.

\subsubsection{Diskurs und Institutionen}

Das Verhältnis von Diskursen und Institutionen wird von Foucault als gegenseitiger Ermöglichungszusammenhang dargestellt. Diaz-Bone (2002: 88) fasst es wie folgt zusammen: „Diskurse werden von Institutionen angereizt und Institutionen ermöglichen Diskursen die Entfaltung und Machtwirkung.“

Auch wenn Foucault in seinem Werk einen eher begrenzten Institutionenbegriff verwendet (Lederle 2008), so ist doch allein die Betonung von Diskursen als Wissen erzeugend, eng mit dem neoinstitutionalistischen Institutionsbegriffs verbunden. Wissen erhält zum einen nur in Form des Diskurses soziale Relevanz, zum anderen müssen Institutionen kognitiv repräsentiert sein, um wirksam zu werden. Auch Keller (2006) betont, dass die diskurstheoretische und diskursanalytische Perspektiven schon aufgrund ihrer Forschungsinteressen und Methoden in der Tradition der Wissenssoziologie verortet werden können. Die Ansätze verbindet folgende Grundannahme:

Alles, was wir wahrnehmen, erfahren, spüren, auch die Art, wie wir handeln, ist über sozial konstruiertes, typisiertes, in unterschiedlichen Graden als legitim anerkanntes und objektiviertes Wissen vermittelt. Dieses Wissen ist nicht auf ein ,angeborenes kognitives Kategoriensystem rückführbar, sondern auf gesellschaftlich hergestellte symbolische Systeme. (Keller 2006: 115)

Die Anschlussfähigkeit der Diskursanalyse an den soziologischen Neoinstitutionalismus ist gegeben, denn auch dieser beruht in seinen Wurzeln auf der Wissenssoziologie (vgl. Hasse/Krücken 2005; Scott 2008). So verortet sich gemäß Keller (2008: 67) überdies einer der prominentesten Vertreter des soziologischen Institutionalismus, John Meyer (1992), in dieser Tradition. ${ }^{21}$ Wissen stellt damit in beiden Ansätzen einen Brückenbegriff dar.

In der wissenssoziologischen Diskursanalyse werden Institutionen und Diskurse nahezu gleichgesetzt. Die Diskursanalyse begreift dann Institutionen „im

20 Diese Kritik wird zu einem späteren Zeitpunkt nochmal aufgenommen.

21 Diskussionen der Grundlegung des Neo-Institutionalismus in der Wissenssoziologie von Berger und Luckmann finden sich gemäß Keller (2008: 67) u.a. auch bei Dobbin (1994), Thomas et al. (1987) sowie DiMaggio und Powell (1991). Zusätzlich betonen Meyer und Jepperson (2000: 102) die Nähe zu Foucault's Arbeiten, der ebenfalls davon ausgeht, dass das Handeln von institutionellen Strukturen bedint wird. 
Sinne des interpretativen Paradigmas der Soziologie als umstrittene, vorübergehend kristallisierte symbolische Strukturen der Ordnung von Welt, die das individuelle Handeln zugleich ermöglichen und beschränken“ (Keller 2008: 11). Diskurse sind abgrenzbare übersituative Zusammenhänge und Äußerungsformen sowie Inhalte, welche unterschiedlich stark institutionalisiert sind. Sie werden in spezifischen Praktiken produziert, reproduziert und transformiert. So beschreibt Lederle (2008: 134) die Beziehung folgendermaßen: „Institutionen sind geronnene Diskurse“. Jedoch führen nicht alle Diskurse zu Institutionen, auch wenn Institutionen als Ergebnisse von Diskursen verstanden werden können (vgl. Philips et al. 2004). Diskurse bringen verschiedenste Ergebnisse vor, die aber nicht notwendig die Steuerungsfunktion von Institutionen besitzen. So kann in dieser Arbeit herausgearbeitet werden, wie die durch Europäisierungsprozesse induzierten Diskurse unterschiedlich in Deutschland und Frankreich auf die institutionellen Durchlässigkeitsstrukturen wirken.

Zusammenfassend lässt sich festhalten, dass Institutionen durch Diskurse erzeugt und legitimiert werden, sie setzen Diskurse voraus und sie werden durch sie aufrechterhalten oder gewandelt. Zum anderen sind es aber auch Institutionen, die gleichsam Diskurse beschränken können.

Setzt man nun diesen Institutionenbegriff in Relation zu dem Begriff der Wissensordnung, die durch Diskurse konstruiert werden, wird deutlich, dass Wissensordnung weit mehr umfasst als Institutionen, so z.B. auch die Praktiken. Diaz-Bone (2003) betont, dass mit der Theorie von Foucault die Wissensordnung nicht mehr direkt an den Individuen haftet:

[...]anstatt sich in den ,Köpfen der Subjekte' zu befinden, stellt das Wissen eine Denkordnung in einem sozialen Feld dar. Es ist nicht die Denktätigkeit von Sprechern, die die Bedeutung und die Ordnung des Wissens hervorbringt. Es ist die diskursive Praxis innerhalb eines Feldes, die für FOUCAULT die sinnhervorbringende überindividuelle Praxis darstellt. (Diaz-Bone 2003, Abs. 6, Hervorhebung im Original)

Die Nutzung des Institutionenbegriffs bietet somit eine praktikable Eingrenzung und Systematisierung des Untersuchungsgegenstandes an. So kann anhand von Veränderungen der drei Dimensionen nach Scott Institutionenwandel beschrieben werden. Wie sich diese Institutionen allerdings ändern können, wird mithilfe der diskurstheoretischen Perspektive offenbar und als Prozess rekonstruierbar. Dies gilt vor allem für die Ideen-Ebene, die kulturell-kognitive Ebene, da diese insbesondere im Diskurs als Wissen konstruierender Prozess erschließbar wird.

\subsubsection{Organisation und Otganisationsfeld}

$\mathrm{Da}$ in der vorliegenden Studie die institutionellen Veränderungen der Durchlässigkeitsstrukturen zwischen Berufs- und Hochschulbildung untersucht werden, sind Organisationen und Organisationsfelder zwei zentrale Begriffe, welche es zu 
definieren gilt. Denn es sind die verschiedenen Organisationen und ihre Umwelten, die als Organisationsfeld beschrieben werden können, zusammen mit den darin vorherrschenden Institutionen, die ein Bildungssystem ausmachen.

Die Unterscheidung zwischen Organisation und Institution ist insbesondere im soziologischen Neoinstitutionalismus nicht trivial, da beide Strukturierungsaspekte aufweisen (Bluhm 2005: 267). So wird kritisiert, dass zum Teil überhaupt keine Unterscheidung mehr zwischen Organisationen in Institutionen gemacht wird (vgl. Bluhm 2005). In dieser Arbeit sollen Organisationen als in institutionelle Umwelten eingebettet verstanden werden - d.h., dass das Handeln der Organisationen, welche als Akteure wahrgenommen werden bzw. das ihrer Mitglieder von Institutionen angeleitet wird.

Organisation ist ein Begriff, der von vielen Soziolog_innen genutzt wurde. Nach Jansen (2006: 2) ähneln sich die Definitionen von Organisation in drei Aspekten: Organisationen sind soziale Gebilde, sie haben ein gemeinsames meist kodifiziertes Ziel und dieses verfolgen sie mithilfe ihrer Mitglieder. Mitgliedschaften in Organisationen sind oft reguliert, so dass zwischen Mitgliedern und Nichtmitgliedern unterschieden wird. Schließlich weisen Organisationen eine formale meist hierarchische Struktur auf und funktionieren über sozial konstruierte Routinen, welche das Handeln der Mitglieder anleiten (Hannan/Carroll 1995). Organisationen in dieser Arbeit sind dann z.B. sowohl die Bildungseinrichtungen, d.h. die Hochschulen, die Berufsschulen, die Betriebe als auch die Akteure, welche die Berufs- und Bildungspolitik bestimmen, die Gewerkschaften, Kammern, Verbände.

Diese unterschiedlichen Organisationen, die miteinander in Interaktion stehen, bilden dann das Organisationsfeld. Das Konzept des Organisationsfeldes bezieht sich damit auf die Gesamtheit der Akteure in einer institutionellen Arena. Es betont die Beziehungen zwischen den Organisationen, die Vielzahl von Netzwerken, in welchen sich die Organisationen befinden und operieren sowie die strukturellen Äquivalente bestimmter Organisationsformen, die eine ähnliche Position im Netzwerk besitzen (vgl. Powell/Solga 2008). Präzise ausgedrückt: „Those organizations that together constitute a recognized area of institutional life“" (DiMaggio/Powell 1983: 64f.). Zudem sind die Organisationsfelder die Hauptarenen, in welchen neue Praktiken, Verfahren und Regeln diffundieren. In dieser Analyse stehen die Bildungsbereiche Berufs- und Hochschulbildung als Organisationsfelder mit den darin handelnden Organisationen im Blickpunkt. Untersucht werden soll dabei vor allem, wie sich die Institutionen, welche das Handeln der Organisationen und der Mitglieder innerhalb dieser anleiten, verändern. Dabei bleibt die Analyse auf der Ebene der Organisationsfelder und untersucht weniger die veränderten Praktiken innerhalb der Organisationen. Es interessiert, inwiefern sich z.B. die Regeln für die Hochschulen im Hinblick auf Durchlässigkeit geändert haben, ebenso wie das Verständnis, die Ideen von Durchlässigkeit, welche in den Debatten zwischen den Akteuren innerhalb und zwischen den Organisationsfeldern Berufs- und Hochschulbildung herausbilden. 
Nicht untersucht wird, ob die institutionellen Anforderungen z.B. innerhalb der Hochschulen umgesetzt werden.

\subsubsection{Legitimität und lose Kopplung}

Ein zentraler Aspekt im soziologischen Institutionalismus, welcher insbesondere auch für den Wandel bzw. die Stabilität von Institutionen und Organisationen von Bedeutung ist, ist das Konzept der Legitimität.

Bereits Meyer und Rowan (1977) zeigten, dass das Überleben bzw. der Erfolg von Organisationen nicht allein von ihrer Formalisierung oder Effizienz abhängt, wie Rational-Choice-Institutionalisten argumentieren würden, sondern vielmehr von ihrer Fähigkeit, bestimmten Erwartungen der Umwelt gerecht zu werden. Einerseits können u.U. auf diese Weise auch Organisationen weiterbestehen, welche wegen Effizienz oder aus Rationalitätsgesichtspunkten längst nicht mehr bestehen dürften (Hasse/Krücken 2005: 36-41). Andererseits wandeln sich Organisationen demnach, um als legitim anerkannt zu werden.

Suchman (1995: 574) definiert Legitimität folgendermaßen:

Legitimacy is a generalized perception or assumtion that the actions of an entity are desirable, proper, or appropriate within some socially constructed system of norms, values, beliefs and definitions.

Nach Scott (2008: 59-62) können drei Arten von Legitimität unterschieden werden. Die pragmatische Legitimität betont die Konformität mit Regeln und Gesetzen sowie dem Erfolg einer Organisation, welcher sich in wirtschaftlichem Erfolg oder auch dem Überleben der Organisation zeigen kann. Legitimität in Bezug auf die zweite Säule resultiert aus einem Normen und Werte konformen Verhalten. Es handelt sich nach Suchman (1995) um eine moralische Legitimität. Im Hinblick auf die dritte Säule ist es die Übereinstimmung mit kulturellen Deutungsmustern, aus der die Legitimität resultiert. Es werden zwei Varianten der kulturellkognitiven Legitimität unterschieden - zum einen basiert sie auf Verständlichkeit bzw. Nachvollziehbarkeit, welche durch geteilte Interpretationsrahmen, ein geteiltes Wissen, entsteht. Die zweite ist die Legitimität der unhinterfragten Selbstverständlichkeiten. Durch diese analytische Unterscheidung ist es möglich, kulturell kognitive Aspekte von Institutionen auch jenseits unreflektierter Selbstverständlichkeiten zu thematisieren (Walgenbach/Meyer 2008: 65).

Zentral in diesem Konzept ist, dass Legitimität nicht besessen werden kann, sondern nur durch einen Prozess der Zuschreibung entsteht (vgl. Suchman 1995). Inhärent ist somit in diesem Konzept auch eine Referenzgruppe von Akteuren, die Legitimität anerkennen, der sogenannte „legitimatorische Resonanzraum von Institutionen“ (Rehberg 1995: 184 nach Lederle 2008: 68). Damit ist aber auch die Legitimität an eine bestimmte Referenzgruppe, ein soziales Feld gebunden - 
nicht überall muss die Institution legitim sein. Das heißt, unterschiedliche Gruppen können die Legitimität von Institutionen divergent bewerten (Scott 2008: 60). Legitimität ist nicht stabil, sondern kann entzogen werden.

Es geht damit nicht nur darum, dass Organisationen als legitim anerkannt werden müssen, um weiterzubestehen, sondern auch Institutionen. So können z.B. bestehende Gesetze als illegitim anerkannt werden und daraufhin verändert werden, wenn sich Normen und Vorstellungen einer Gesellschaft ändern und die Gesetze aus dem Blickwinkel dieser gesellschaftlichen Vorstellungen nicht mehr legitim sind.

Das heißt, während des Prozesses der Institutionalisierung, aber auch danach müssen Institutionen, um wirksam zu bleiben, Legitimität erhalten. Diese erhalten sie demnach auf Basis bestehender gesellschaftlicher Institutionen, auf deren Grundlage die Bewertungen der Legitimität erfolgen. Organisationen können dann versuchen, den Prozess der Legitimation aktiv zu gestalten, indem sie versuchen, die Interpretationen, Vorstellungen und Erwartungen der Referenzgruppe zu beeinflussen (Walgenbach/Meyer 2008). Es geht hierbei also um die Macht der Wirklichkeitskonstruktion. „Legitimierung verstanden als sozialer Prozess des Erklärens und Rechtfertigens (Berger/Luckmann 1986: 100) verweist auf die herausragende Bedeutung der Sprache in diesen Prozessen" (Walgenbach/Meyer 2008: 67). An dieser Stelle ist die diskursive Grundlage von Legitimation offenbar.

Dabei ist zu beachten, dass nicht alle Akteure einer Referenzgruppe notwendigerweise die gleichen Legitimitätsvorstellungen haben und Organisationen so mit widersprüchlichen Erwartungen konfrontiert sein können, wobei aber die Relevanz der unterschiedlichen Anspruchsgruppen für die Organisation differieren kann (vgl. Hasse/Krücken 2005). Ein Beispiel stellen in diesem Kontext Universitäten dar. Diese sollen gleichzeitig gute Forschung und Lehre leisten, die Studierenden allgemein und auf einen hin Beruf ausbilden, dabei effizient mit ihren Mitteln umgehen, sich auf Kerngebiete spezialisieren, Transfer der Forschung in die Wirtschaft leisten und nicht in Bezug auf Geschlecht, Behinderung, Migrationshintergrund etc. diskriminieren. Ähnlich diversen Anforderungen müssen sich auch die Organisationen der Berufsausbildung stellen.

Mit dem Blick auf die Analyse der institutionellen Durchlässigkeitsstrukturen ist weiterhin zu untersuchen, wie bestehende Institutionen legitimiert oder delegitimiert werden. Inwiefern kommt es z.B. zum Wandel von Regelungen, weil es ein neues Verständnis von Durchlässigkeit in der Gesellschaft gibt? Setzen sich Organisationen für eine stärkere oder schwächere Institutionalisierung von Durchlässigkeit ein?

Eine Strategie, um von Organisationen Legitimität zu erhalten, ist die Entkopplung der formalen Strukturen von den tatsächlichen Organisationsabläufen. Dieses Phänomen wurde von Meyer und Rowan (1977) als loose coupling benannt. Dabei signalisieren die Organisationen durch formale Anpassung ihrer Strukturen mit den Umweltanforderungen übereinzustimmen. Auch für die Untersuchung 
von Durchlässigkeitsstrukturen wäre es dann wichtig zu analysieren, inwiefern z.B. nationale und europäische Vorgaben letztlich auch von den Berufsbildungsund Hochschulakteuren umgesetzt werden. Da aber, wie bereits hingewiesen, nicht die Praxis innerhalb von Bildungsorganisationen untersucht wird, werden derartige Praxen des Entkoppelns in der vorliegenden Arbeit weniger im Blickpunkt stehen. Dessen ungeachtet können Inkongruenzen zwischen der diskursiven Praxis und bestehenden Regelungen aufgezeigt werden.

Generell gilt aber, selbst wenn loose coupling in Organisationen stattfindet, also unterschieden wird von einer offiziellen Rhetorik oder formalen Regelungen und der tatsächlichen Praxis, kann auch die Institutionalisierung auf formaler Ebene als ein erster Schritt in Richtung Wandel angesehen werden. Nur wenn beispielsweise regulativ Anrechnung von beruflichen Qualifikationen im Hochschulbereich geordnet ist, kann auch die nicht existierende Praxis als illegitim angeprangert werden. Kritiker des Konzepts halten aber eine langfristige Entkopplung nicht für möglich (Walgenbach/Meyer 2008: 82) und es kommt schließlich entweder zu einer Anpassung und Wandel innerhalb der Organisation oder deren Delegitimierung.

\subsection{Institutionellen Wandel konzeptualisieren}

Was soll in der Arbeit unter institutionellem Wandel verstanden werden? Woran kann entschieden werden, dass sich Dinge verändern oder doch so bleiben, wie sie waren? Ab wann handelt es sich um Wandel?

Es existiert nicht die eine Definition institutionellen Wandels, vielmehr ist die jeweilige Definition abhängig vom gewählten theoretischen Rahmen. Gleichwohl lassen sich Kernmerkmale institutionellen Wandels festmachen: Institutioneller Wandel ist per se ein Phänomen, welches relativ und relational beschrieben werden kann. Wandel kann nur in Beziehung zu etwas anderem festgestellt werden. Zudem ist dem Konzept Wandel auch immer eine temporale Beziehung inhärent. "Change is the movement away from a present state toward a future state" (Tippets 2011: 2). Wandel kann selbst als Prozess aber auch als Ergebnis eines Prozesses gesehen werden.

Die Untersuchung institutionellen Wandels in der vorliegenden Arbeit baut auf dem dreidimensionalen Verständnis von Institutionen nach Scott (2008) auf. Eine Analyse institutionellen Wandels macht es demnach notwendig, festzustellen, inwiefern die unterschiedlichen Dimensionen allgemein und in welchem Aus$\mathrm{ma}$ von Veränderungen betroffen sind. Denn es ist möglich, dass sich Wandel nicht notwendigerweise in allen Dimensionen ebenso stark und ebenso schnell vollzieht. So kann es durchaus dazu kommen, dass Durchlässigkeit zwischen Berufs- und Hochschulbildung wichtiger wird in den Diskursen, sich dies aber noch 
nicht in gesetzlichen Regelungen oder den Standards der Bildungseinrichtungen bemerkbar macht.

Um institutionellen Wandel zu analysieren, schlägt North (1994) vier verschiedene Analysedimensionen vor. Wer sind die Agenten des Wandels? Wo liegen die Ursachen? In welche Richtung entwickeln sich die Institutionen und wie ist das Ausmaß der Veränderung? Im Folgenden sollen vor allem theoretische Konzepte, die die Ursachen von Wandel und Stabilität sowie das Ergebnis des Wandels, die Richtung und das Ausmaß beschreiben, näher vorgestellt und auf die Fragestellungen bezogen werden.

\subsubsection{Ursachen des Wandels}

Die Auslöser bzw. Quellen institutionellen Wandels können unterschiedliche Faktoren sein. Gemeinhin werden analytisch exogene und endogene Faktoren unterschieden. Endogene Faktoren sind dabei innerhalb der Analyseeinheit wurzelnde Faktoren während exogene Faktoren außerhalb der Untersuchungseinheit liegen (Scott 2008). In der vorliegenden Arbeit sind die Analyseeinheit die nationalen Bildungssysteme, d.h. die organisationalen Felder Hochschule und Berufsbildung.

\section{Exogene Quellen des Wandels}

Exogene Faktoren können Veränderungen der Wettbewerbsbedingungen, technologische Innovationen, Kriege, Abschlüsse internationaler Verträge bzw. ihre Veränderung oder auch nur internationale Kooperationen sein. Exogene Einflussfaktoren eröffnen dann ein window of opportunity für Akteure, welche den bisherigen Strukturelementen kritisch gegenüberstehen, ihre Kritik zu äußern und aktiv die Institutionalisierung neuer Praktiken zu forcieren. Es kann zu einem neuen Gleichgewicht durch z.B. die Diffusion neuer Ideen, alternativer Sichtweisen und Logiken kommen (Walgenbach/Meyer 2008). In dieser Arbeit werden die europäischen Bildungsprozesse analytisch als exogen eingestuft und deren Einfluss auf die nationalen Durchlässigkeitsstrukturen in Deutschland und Frankreich untersucht.

In der Definition von Scott (2008: 47f.) existieren Institutionen auf den unterschiedlichsten Ebenen vom Weltsystem bis hin zu lokalen interpersonalen Beziehungen. Sie implizieren per Definition Stabilität, sind aber gleichzeitig Wandlungsprozessen ausgesetzt. Ein mögliches Ergebnis von Wandel wird von DiMaggio und Powell (1983) Isomorphie benannt, d.h., es kommt zu einer strukturellen Vereinheitlichung von Organisationen in einem Organisationsfeld. Faktoren, welche zu Isomorphie führen, sind analog der drei Dimensionen von Institutionen zum einen Zwang, normativer Druck oder Imitation, welche ausgelöst 
wird durch Unsicherheit. Als Modelle werden dann meist solche gewählt, die besonders erfolgreich scheinen. Die europäische offene Methode der Koordinierung $(\mathrm{OMK})^{22}$ setzt unter anderem auf diese Prozesse des Best-Practice-ModellLernens. Diffusionskonzepte stellen folglich eine zentrale Erklärung von institutionellem Wandel hin zu Homogenität eines Feldes dar (DiMaggio/Powell 1983).

Das Konzept der Diffusion wurde durch Rogers (1995: 5f.) definiert als ,the process by which an innovation is communicated through certain channels over time among the members of a social system“. Diffusion kann sich auf alle institutionellen Dimensionen beziehen, die Diffusion von Ideen, von Werten, aber auch Regeln. Verschiedene Mechanismen, die zur Diffusion führen, können unterschieden werden:

Dobbin et al. (2007), aber auch Maggetti und Gilardi (2014) unterscheiden ganz ähnliche Mechanismen. Erstens kann es zur Diffusion über Zwang durch mächtige Akteure kommen. Auch Wettbewerb wird als Mechanismus anerkannt. Hier werden erfolgreiche Politiken übernommen, die Organisationen oder Ländern einen Wettbewerbsvorteil in bestimmten Bereichen ermöglichen. Steuerpolitik wird oft als Beispiel angeführt. Weiterhin wird das Lernen als wichtiger Mechanismus der Diffusion anerkannt (vgl. auch Knill/Dobbins 2013) Dabei werden aus den Erfahrungen von anderen Organisationen und Ländern mögliche Ansatzpunkte zur Veränderung der eigenen Strukturen geschlossen. Beim Mechanismus des Lernens ist in diesem Zusammenhang wichtig, dass ein Lernprozess vorherige Annahmen über Ursache und Wirkung verändert hat.

Learning does not occur when policy makers simply adapt to the policy shifts of others, but only when their beliefs about cause and effect change. (Dobbin et al. 2007: 12)

Schließlich gibt es noch den Mechanismus, den Dobbin et al. (2007) soziale Konstruktion nennen und welcher insbesondere in Arbeiten, die dem world-polityAnsatz (vgl. Meyer 2004) folgen, zu finden ist. Hier wird angenommen, dass sich in den letzten paar hundert Jahren eine Weltgesellschaft entwickelt hat, in welcher sich geteilte kulturelle Modelle herausgebildet haben, welche zu einer weltweit ähnlichen Struktur in Bereichen der Wirtschaft, Politik, Kultur, Wissenschaft und Bildung geführt haben (vgl. Meyer/Ramirez 2007 für die Bedeutung von Bildung). Schriewer (2007: 7) spricht von „Leitideen von individueller Persönlichkeitsentfaltung und Staatsbürgerschaft, von gesellschaftlichem Fortschritt und wirtschaftlicher Entwicklung sowie [dem] Modell des Nationalstaats, der als Garant und legitimer Ordnungsrahmen für die Aktivitäten gesellschaftlicher Gruppen und Akteure gesehen wird". Expert_innengruppen und internationale Organisationen fördern diese kulturellen Leitideen bzw. Mythen im Zusammenhang durch daran anknüpfende Politiken (Dobbin et al. 2007). Zudem sind es auch die

22 Die OMK funktioniert über Selbstverpflichtung der Mitglieder und basiert auf Prozessen gegenseitigen Lernens sowie der regelmäßigen Planung und Prüfung von gemeinsam gesetzten Zielen, sogenannten Benchmarks (vgl. Ravinet 2008; Sabel/Zeitlin 2007). 
Staaten selbst, welche sich als Mitglied der Weltgesellschaft sehen und dann die als legitim anerkannte Politiken oder Ideen aus Staaten, welche ebenfalls dieser Weltgesellschaft zugehörig zählen, imitieren. Im Unterschied zum Lernen ist bei diesem Mechanismus die symbolische Ebene die signifikante. Institutionen, die übernommen werden sollen, haben dann mehr Legitimität, wenn sie auch den kulturellen Leitbildern der Weltgesellschaft und damit den Umwelterwartungen entsprechen. Dies gilt unabhängig davon, ob sie im eigenen Kontext funktionieren oder nicht. Analog gibt es dann nicht nur legitime Modelle, die diffundieren, sondern auch solche, die entsprechend tabu sind (Maggetti/Gilardi 2014). Dies gilt z.B. für alle Institutionen, die Menschenrechte verletzen.

Jakobi und Martens (2007) zeigen in ihrer Studie, wie internationale Organisationen als Agenten von Diffusion tätig werden, indem sie das betreffende Organisationsfeld, welches insbesondere in Bezug auf Bildungspolitik eher national angesiedelt ist, auf die internationale Ebene erweitern. Sie schaffen dabei Strukturen, „um ein Feld zu konstituieren und damit langfristig Angleichungsprozesse zwischen den Staaten in Gang zu setzen" (Jakobi/Martens 2007: 250). Voraussetzung für die Konstitution des erweiterten Organisationsfeldes sind die Entwicklungen von geteilten Zielen, eine „Zunahme der Informationsmenge zu einem bestimmten Thema“ und die „Verstärkung von Interaktion“ (Jakobi/Martens 2007: 250). Überträgt man diese in Bezug auf die OECD gemachten Annahmen auf die europäischen Prozesse Bologna und Kopenhagen, können für diese ebenfalls derartige Strukturmerkmale festgestellt werden: Erstens werden innerhalb der europäischen Prozesse in den Deklarationen und Kommuniqués gemeinsame Ziele und auch die Wege dorthin festgelegt. Zweitens kommt es durch diese Konferenzen sowie die Arbeitsgruppen und deren regelmäßige Treffen zu einer verstärkten Interaktion. Dies gilt nicht nur für die Regierungen der Mitgliedsstaaten, sondern auch für die relevanten Stakeholder in der Berufs- und Hochschulbildung, die in den Prozess integriert sind. Schließlich sind im Rahmen von Bologna und Kopenhagen verschiedenste Formen des Informationsaustauschs institutionalisiert. ${ }^{23}$ Zum einen erfolgt dies über die nationalen Berichte, in denen die Länder ihre Fortschritte im Hinblick auf die gemeinsam verabschiedeten Benchmarks darstellen. Auch werden Best-practice-Beispiele aus den Ländern vorgestellt und schließlich sind die institutionalisierten Arbeitstreffen selbst Orte, an denen Informationen über z.B. Möglichkeiten der Anrechnung ausgetauscht werden. Die Bildungsprozesse Bologna und Kopenhagen erfüllen damit die Voraussetzungen, dass eine Diffusion von Ideen, Normen und Politiken innerhalb des „erweiterten“ Organisationsfeldes stattfinden kann.

Inwiefern exogene Einflüsse, wie europäische Bildungsprozesse oder singuläre Ereignisse dann auch wirklich zu Wandel führen, ist dabei abhängig, wie sie diskutiert oder ob sie überhaupt diskutiert werden. An dieser Stelle wird die Rolle 
der diskursiven Herstellung der Realität deutlich: Denn nur wenn exogene Faktoren auch so diskursiv genutzt bzw. interpretiert werden und neue Ideen und Leitbilder hervorbringen, entfalten sie eine Wirkung (vgl. Schmidt/Radaelli 2004).

Druck von außen, sei es über internationale oder europäische Prozesse, muss daher nicht immer auch zu Wandel bzw. Handlungsnotwendigkeiten auf nationaler Ebene führen. Diese Notwendigkeit liegt vor allem dann vor, wenn durch die europäischen Prozesse deutliche Politikveränderungen auf nationaler Ebene nötig sind. So wird in der Politikwissenschaft darauf hingewiesen, dass dieses von dem goodness of fit zwischen vorgeschlagener EU-Politik und den nationalen historisch gewachsenen Strukturen abhängt (vgl. Börzel/Risse 2003; Green Cowles et al. 2001; Héritier 2001), ob ein Strukturwandel entsprechend europäischer Vorgaben vollzogen wird oder nicht. Der goodness of fit-Ansatz wird dabei eher in Bereichen der positiven Integration, d.h. in Politikbereichen erwartet, in welchen die EU eindeutige Regelungskompetenzen vorweist und ein Politikmodell vorgibt, angewendet (vgl. Auel 2006). Aber auch wenn die europäischen Bildungsprozesse Bologna und Kopenhagen nicht Teil der supranationalen Politiksetzung der EU sind, kann von ihnen doch normativer Druck ausgehen, zumal das Erreichen der freiwillig gemeinsam vereinbarten Ziele kontinuierlich überprüft wird. Kritisch ist allerdings, dass insbesondere Europäisierungsprozesse, die keine eindeutig operationalisierten Modelle mit klaren Handlungsempfehlungen oder Politiken vorgeben (Powell et al. 2012b), auch schwierig zu nutzen sind, um eine Inkompatibilität der Strukturen auszumachen. Hier wird somit ebenso zu untersuchen sein, was in den Bologna- und Kopenhagen-Prozessen in Bezug auf Durchlässigkeit gefordert wird, um diese Forderungen dann mit den nationalen Strukturen zu vergleichen.

Radaelli (2003) weist allerdings darauf hin, dass dieses Konzept von goodness of fit seine Grenzen hat. Denn inwiefern und vor allem wie groß die Passung europäischer Forderungen mit nationalen Strukturen ist, ist zuerst einmal politisch zu definieren. Es liegt also an den politischen und gesellschaftlichen Akteuren, welcher Handlungsbedarf gesehen wird. „This often means that interpretation, inter-subjective understandings and discourse shape the notion of ,goodness of fit““ (Schmidt/Radaelli 2004: 187).

Inwiefern in dem vorliegenden Fall die europäischen Prozesse von Bologna und Kopenhagen, die klassisch als exogen einzustufen sind, auch als exogen angesehen werden können, ist ebenfalls zu hinterfragen. Gerade diese Prozesse, die in ihremFunktionieren der Methode der offenen Koordinierung entsprechen, sind permanent an die nationalen Systeme rückgekoppelt - so dass deren Akteure auch Einfluss auf die europäischen Prozesse nehmen können. Die Bologna- und Kopenhagen-Prozesse werden nicht nur hierarchisch von oben über supranationale Institutionen entschieden, zumal Bildungspolitik noch vorwiegend in den nationalstaatlichen Kompetenzbereich fällt. So waren es vor allem die Regierungen aber auch nationale Universitäten, die Sozialpartner, Studierende und europäische Organisationen, welche die Prozesse beeinflusst haben (Walter 2006). Auch wenn 
Europäisierung als vorwiegend exogen betrachtet wird, so kann sie nicht als Einbahnstraße gesehen werden. Insbesondere mächtige Akteure wie Frankreich und Deutschland beeinflussen europäische Politiken bereits im Prozess ihrer Entstehung. EU-Politiken sind somit nicht in einem Vakuum zu sehen, sondern in einem Feld, in dem EU-Institutionen und die Mitgliedsstaaten aufeinander einwirken, einem Feld, in welchem sich ebenfalls Diskurse verschränken (vgl. Schmidt/Radaelli 2004).

An dieser Stelle wird deutlich, dass in einer komplexen Welt nicht einfach zwischen endogen und exogen unterschieden werden kann. Jedoch werden im Folgenden aus analytischen Gründen europäische Prozesse als quasi-exogene Faktoren betrachtet, ohne die strukturelle Rückkopplung an die Mitgliedsstaaten zu vergessen.

\section{Endogene Quellen des Wandels und der Stabilität}

Wie kann dann aber endogener Wandel im soziologischen Neoinstitutionalismus erklärt werden? Was löst endogenen Wandel aus? Walgenbach und Meyer (2008) weisen auf die Herausforderung in der neoinstitutionellen Theorie hin, endogenen institutionellen Wandel zu beschreiben. So konstatiert auch Scott (1981: 187), dass ,in highly institutionalized systems, endogenous change seems almost to contradict the meaning of institutions“. Walgenbach und Meyer (2008: 105-108) finden aber innerhalb institutioneller Literatur folgende Erklärungen für endogen ausgelösten Wandel:

Erstens können Widersprüche zwischen institutionellen Elementen zu Wandel führen: Es wird argumentiert, dass Institutionen multidimensionale Phänomene sind. Divergente Veränderungsgeschwindigkeiten in den Dimensionen oder auch Inkonsistenzen und Widersprüchlichkeiten zwischen den Ebenen können Wandel initiieren (Walgenbach/Meyer 2008: 105f.). So kann Durchlässigkeit zwischen Berufs- und Hochschulbildung vielleicht bereits durch Expert_innengruppen und auf Basis deren Deutungen der Wirklichkeit in Gesetzen gefördert werden, ohne dass sich dieses Verständnis zu Durchlässigkeit bereits in anderen gesellschaftlichen Gruppen durchgesetzt hat. Die Veränderung der regulativen Ebene kann aber nun dazu führen, dass Durchlässigkeit zwischen Berufs- und Hochschulbildung mehr und mehr an Selbstverständlichkeit in der Gesellschaft gewinnt. Andererseits ist es auch möglich, dass sich erst in weiten Gruppen der Gesellschaft Deutungen zu Durchlässigkeit geändert haben und diese Veränderungen dann schließlich auch in der regulativen und normativen Dimension umgesetzt werden.

Als zweites Beispiel für endogene Faktoren, die zu Wandel führen, wird in der Literatur darauf hingewiesen, dass Akteure durch multiple Institutionen und institutionelle Logiken beeinflusst werden (vgl. Walgenbach/Meyer 2008: 106). Akteure partizipieren in einer Mehrzahl von institutionellen Umwelten und haben 
somit Kenntnis verschiedenster institutioneller Regeln, die durchaus konkurrierenden Logiken entsprechen können. Ein Aufeinandertreffen dieser unterschiedlichen Logiken in einem organisationalen Feld erhöht die Wahrscheinlichkeit von institutionellem Wandel. Gerade durch die europäischen Prozesse und die Einbindung der Akteure in die europäischen Netzwerke kann es somit aber auch auf nationaler Ebene neue Arbeitsgruppen zu durchlässigkeitsrelevanten Themen wie dem Qualifikationsrahmen geben, die dann ebenfalls zu Veränderungen von Vorstellungen über Durchlässigkeit und dessen, was als normativ richtig angesehen wird, führen.

Die Anwendung abstrakter Regeln auf konkrete Handlungssituationen wird ebenfalls als endogene Quelle von Wandel identifiziert (Walgenbach/Meyer 2008: 107): Da die Anwendung von Regeln als interpretativer Prozess gesehen wird, kann es allein dabei schon zu Veränderungen kommen, denn eine neue Erarbeitung der Interpretation der Regeln für eine konkrete Situation kann von der alten abweichen.

Wie bereits im Abschnitt zum Konzept der Legitimität deutlich wurde, können auch widersprüchliche Umwelterwartungen Transformationen auslösen, insbesondere dann wenn sich die Relevanzvorstellungen der Referenzgruppen und somit auch ihre Erwartungen und Interessen verändern. So wurde in den letzten Jahren den Hochschulen immer stärker auch eine wichtige Rolle zur Ermöglichung lebenslangen Lernens zugeschrieben. Eine Reaktion darauf könnte eine stärkere Ausrichtung der Hochschulen auf beruflich Qualifizierte als neue Zielgruppe sein. Es ist insofern zu fragen, ob sich neue Umwelterwartungen im Hinblick auf Durchlässigkeit feststellen lassen.

Endogene Faktoren können aber nicht nur Wandel induzieren, sondern auch Stabilität bzw. inkrementellen Wandel erklären. Meyer und Rowan (2006) betonen, dass vor allem auch Bildungsorganisationen durch äußerst stabile Institutionen geprägt sind, die substanzielle Reformen im Bildungssystem signifikant erschweren. Vor diesem Hintergrund ist es notwendig, das u.a. im historischen Institutionalismus geprägte Konzept der Pfadabhängigkeiten bei der Analyse von institutionellen Wandel zu betrachten (Powell/Solga 2008). Pfadabhängigkeit ist ein zentrales Konzept des historischen Institutionalismus, welches über die einfache Aussage hinausgeht, dass „history matters“ (Thelen 2004). Ebbinghaus (2005) unterscheidet zwei Ansätze im Hinblick auf das Konzept der Pfadabhängigkeit, ein eher starres deterministisches Konzept, in dem Wandel kaum möglich ist und eine zweite Interpretation, welche Wandel zulässt. Auch Thelen (2004) sowie Streeck und Thelen (2005) kritisieren, dass das Konzept der Pfadabhängigkeit viel zu starr genutzt wurde und dass im Gegensatz zur Möglichkeit von inkrementellen Wandel vielfach in der Literatur nur critical junctures als Auslöser von Wandel gesehen werden. In der vorliegenden Arbeit wird das dynamischere Konzept von Pfadabhängigkeit als Analyserahmen verwendet. 
Methodologisch kann Pfadabhängigkeit als ein Mittelweg zwischen totalem Zufall und historischer Vorherbestimmung verstanden werden (Leipold 1996: 95). So unterstreicht North (1990: 98f.):

Path dependence is a way to narrow conceptually the choice set and link decisions making through time. It is not a story of inevitability in which path predicts the future.

Pfadabhängigkeit hebt damit hervor, dass die Entscheidungen von Akteuren immer auch durch alte und entstehende Institutionen mitbestimmt werden. Dabei sieht North jedoch ebenfalls, dass die Erklärung von Pfadabhängigkeit nun also nicht nur daher kommen darf, wie Institutionen Alternativen binden, sondern auch daher, auf welche Art und Weise Wahrnehmungen die Wahlmöglichkeiten begrenzen (North 1995: 24). An dieser Stelle wird die Anschlussfähigkeit zum soziologischen Neoinstitutionalismus besonders deutlich. Es ist somit wichtig zu analysieren, inwiefern Wandel innerhalb bestehender Systeme vorangetrieben wird, indem bestehende Institutionen so verändert werden, dass sie neuen Zielen entsprechen (Streeck/Thelen 2005: 19).

\subsubsection{Ergebnisse des Wandels}

In diesem Abschnitt sollen primär im Neoinstitutionalismus entwickelten Konzepte, welche die Ergebnisse von Wandel beschreiben, dargestellt werden. Dabei liegt einerseits der Fokus auf dem Ausmaß des Wandels. Hier geht es primär um die Beschreibung der Entwicklungen innerhalb von Analyseeinheiten - in dieser Arbeit um das nationale Bildungssystem. Andererseits werden die Ergebnisse des Wandels einer Analyseeinheit in Relation zur Entwicklung in anderen Analyseeinheiten oder zu einem globalen Modell beschrieben.

\section{Ausmaß des Wandels}

Verschiedene Autoren (vgl. Campbell 2004; Greenwood/Hinings 1996; Scott 2008) unterscheiden dabei das Ausmaß von Wandel in zwei größere Kategorien: So kann er eher inkrementell und evolutionär sein und eine geringfügige Anpassung oder Veränderung der Institutionen bedürfen, aber er kann auch revolutionär sein. So wird auch von first order und second order changes gesprochen (Meyer et al. 1990). Veränderungen erster Ordnung sind inkrementelle Anpassungen, welche reversibel sind und keine Transformation der Kernprinzipen, Regeln, Werte und Normen beinhalten. Es akkumulieren sich hier lediglich kleinere Veränderungen im Laufe der Zeit, so dass ",todays institutional arrangements differ but still resemble those of yesterday" (Campbell 2004: 65).

Veränderungen zweiter Ordnung oder diskontinuierlicher Wandel dagegen sind radikale Veränderungen der Kernprinzipien, Werte und Regeln. Sie sind ir- 
reversibel und umfassen somit einen Wechsel der zugrunde liegenden institutionellen Logik, den verwendeten „Mustern des Organisierens“ (vgl. Walgenbach/Meyer 2008, 88), sowie in der Strukturierung des Feldes (Greenwood/Hinings 1996). ${ }^{24}$ Dabei betont u.a. North (1994), dass der meiste Wandel inkrementell auftritt.

The reason is that the economies of scope, the complementarities, and the network externalities that arise from a given institutional matrix of formal rules, informal constraints, and enforcement characteristics will typically bias costs and benefits in favor of choices consistent with the existing framework. The larger the number of rule changes, ceterus paribus the greater the number of losers and hence opposition. (North 1994: 6)

Insofern ist nach North (1994) auch die Richtung, in die sich der meiste Wandel vollzieht, meist pfadabhängig. Diese Einteilung von evolutionärem und revolutionärem Wandel soll dabei mehr als Analysemuster dienen, denn als nominale Einteilung, da das Ausmaß institutionellen Wandels eher auf dem Kontinuum zwischen geringer Anpassung und revolutionären Veränderungen liegen wird (vgl. Campbell 2004). Zudem konnte Thelen (2004) zeigen, dass auch inkrementeller Wandel zu großen Veränderungen von Institutionen führen kann.

Ebbinghaus (2005: 17) unterscheidet drei analytische Szenarien institutionellen Wandels im Rahmen des dynamischen Pfadabhängigkeits-Konzepts: Die Stabilisierung des Pfads (path stabilization): Es kommt nur zu einer geringen Adaption zu den Umweltbedingungen, ohne dass sich die Kernprinzipien wandeln. Das Abweichen vom Pfad (path departure): Hier kommt es zu einer stärkeren Anpassung der Institution mit partieller Erneuerung von institutionellen Arrangements und einer begrenztem Neuausrichtung der Kernprinzipien. Die Beendigung eines Pfads und/oder der Wechsel zu einem neuem Pfad (path cessation or switching): Hier kommt es zu einem Eingriff, welcher das Ende einer sich selbstverstärkenden Institution herbeiführt und damit Platz für die Entstehung einer neuen Institution mit neuen Kernprinzipien machen kann.

Diese analytische Unterscheidung ermöglicht es, die verschiedenen Formen von Wandel und Stabilität zu untersuchen. In der vorliegenden Arbeit wäre demnach zu untersuchen, inwiefern die Reformprozesse, ausgelöst einerseits durch die verstärkte europäische Zusammenarbeit im Rahmen von Bologna und Kopenhagen, aber auch durch nationale Faktoren, zu einer Stabilisierung vorhandener Durchlässigkeitsinstitutionen führen, zu einer Veränderung oder zur Entstehung neuer.

In der Policy Analyse wird in Anlehnung an Hall (1993) unterschieden in Wandel erster Ordnung (first order change): Dieser ist verbunden mit dem veränderten Einsatz der verfügbaren Instrumente bzw. deren Weiterentwicklung. Dem Wandel zweiter Ordnung (second order change) der Veränderung des Sets der politischen Instrumente und dem Wandel dritter Ordnung (third order change): Veränderung der politischen Ziele. Eine solche Veränderung der Politikziele begründet nach Hall einen Paradigmenwechsel (vgl. Hall 1993: 273-279). 


\section{Frage der Konvergenz}

Wie bereits im Abschnitt zu den exogenen Faktoren von Wandel dargestellt, wird eine mögliche Konsequenz von internationalen Diffusionsprozessen in der Entstehung von Isomorphie innerhalb eines Organisationsfeldes gesehen. Theoretisch ist Isomorphie vor allem auf die sich immer ähnlicher werdenden Organisationsstrukturen von Organisationen in einem Feld bezogen (DiMaggio/Powell 1983). Ein ähnliches Konzept, welches allerdings weniger explizit Organisationsstrukturen fokussiert, sondern generell Ähnlichkeit von Strukturen zwischen Analyseeinheiten, ist das der Konvergenz (vgl. Holzinger/Knill 2005). Oft wird es im Zusammenhang mit dem Transfer oder der Diffusion von Politiken oder generell Institutionen verwendet (vgl. z.B Bennett 1991; do Amaral 2011; Holzinger/Knill 2005; Jakobi/Teltemann 2011; Steiner-Khamsi 2012). Dabei wird Konvergenz entweder als Angleichung von Strukturen zwischen einzelnen Analyseeinheiten, meist OECD Ländern, oder im Hinblick auf externe Modelle untersucht. Konvergenzanalysen sind besonders häufig in der Politikwissenschaft, wobei in den Analysen vor allem die Politiken, d.h. die regulative Ebene, im Blickpunkt stehen, weniger die Konvergenz von Ideen, Vorstellungen, der kulturell-kognitiven Ebene.

Insbesondere in quantitativen Analysen werden verschiedene Formen von Konvergenz unterschieden (Holzinger et al. 2007). Für vorliegende Arbeit sind lediglich die Konzepte der Sigma- und Delta-Konvergenz von Belang, auch wenn die Analyse qualitativer Natur sein wird und nicht auf Berechnungen beruht. Sie stehen aber als Konzepte Pate für zwei zentrale Forschungsfragen der vorliegenden Arbeit.

Erstens geht es um die Frage, welchen Einfluss die europäischen Bildungsprozesse auf die Entwicklung der nationalen Durchlässigkeitsstrukturen in Deutschland und Frankreich haben. Hier steht im Zentrum der Analyse, ob es zu einer Annäherung der nationalen Strukturen an europäische Vorgaben gekommen ist. Analytisch handelt es sich bei dieser Frage um die Analyse der DeltaKonvergenz - d.h. die Frage nach der Annäherung an ein Referenzmodell (ebd.), wobei ein Hinweis der Entwicklung in Richtung Delta-Konvergenz nicht gleichgesetzt werden kann mit dem Beweis des Einflusses europäischer Prozesse. Es könnten auch endogene Faktoren zu einer Entwicklung in Richtung des Referenzmodells geführt haben. Aus diesem Grund kann die Delta-Konvergenz nur ein wichtiger Anhaltspunkt zur Beantwortung der Frage nach dem Einfluss sein.

Zweitens soll in dieser Arbeit untersucht werden, inwiefern sich die institutionellen Durchlässigkeitsstrukturen zwischen Frankreich und Deutschland angenähert haben. Bei dieser Frage dreht es sich um die Sigma-Konvergenz, d.h. um die Angleichung von zwei unterschiedlichen Analyseeinheiten innerhalb eines bestimmten Zeitraums (ebd.). Entsprechend dem Konzept von Isomorphie und der Annahme, dass sich die Organisationsfelder durch die europäischen Bildungsprozesse vergrößert haben, wäre anzunehmen, dass eine mögliche Konvergenz auf 
europäische Diffusionsprozesse zurückzuführen ist, da ein Anpassen an das europäische Modell als legitim angesehen wird. Herauszufinden wäre demnach, inwiefern sich die Anforderungen der Legitimität an das deutsche und französische Hochschul- und Berufsbildungssystem durch Europäisierungsprozesse analog ändern und welchen Einfluss dies auf Durchlässigkeit zwischen Berufs- und Hochschulbildung hat. Tatsächlich ist es aber auch möglich, dass es zu einer gröBeren Delta-Konvergenz, also einer Annäherung an ein europäisches Referenzmodell in beiden Ländern gekommen ist, ohne dass sich die Strukturen zwischen den Ländern deutlich ähneln ${ }^{25}$.

Gründe für eine fehlende Konvergenz werden meist in nationalen Strukturen gesehen. Das Konzept der Translation akzentuiert z.B., dass Organisationsformen und Praktiken, wenn sie aus einem Kontext in einen anderen übertragen werden bzw. diffundieren (z.B. über Europäisierungsprozesse), erst übersetzt werden müssen, was stets eine Veränderung des Übertragenen mitbringt. Diese Übersetzungsleistung wird deshalb notwendig, da der Zielkontext nicht mit dem Empfangskontext identisch ist. Veränderungen finden dabei in zwei Richtungen statt - zum einen werden die Praktiken bzw. Organisationsformen geändert zum anderen aber auch das aufnehmende Bedeutungs- und Handlungssystem. BeckerRitterspach (2006) nennt diesen Prozess dialektische Transformation. Das Konzept der Translation begrenzt das Konzept der Isomorphie dahingehend, dass gemäß diesem Konzept keine eins zu eins Übertragungen stattfinden können und es daher kaum zu einer perfekten Isomorphie kommen kann (vgl. Senge/Hellmann 2006).

Schließlich wird mit der Einbeziehung von Pfadabhängigkeit theoretisch dem Konzept von Isomorphie ein Konzept entgegengesetzt, welches viel stärker die jeweilige historische Einbettung betont und eher national differente Entwicklungen annehmen würde. Die Einbettung der Bildungssysteme in institutionell gewachsene Strukturen verhindert eine gleichförmige Veränderung der institutionellen Settings in den Ländern. Europäisierung führt dann zu national spezifischen Antworten auf Reformimpulse, die nicht zu einer starken Angleichung zwischen den Ländern führen muss.

Beide Perspektiven, die der Pfadabhängigkeit und der Isomorphie bzw. Konvergenz sind für die Frage nach der Veränderung der nationalen Durchlässigkeitsstrukturen von Bedeutung, da der soziologische Institutionalismus vor allem Makrolevel-Analysen mit dem Fokus auf kulturell-kognitive Aspekte und Normen im Zusammenhang mit dem Einfluss internationalen Organisationen, Staaten und Verbänden in den Vordergrund rückt. Der Fokus vom historischen Institutionalismus ruht dagegen viel stärker auf der regulativen, der GovernanceEbene - auf Regime-, Staats- oder Industrieebene (Scott 2008: 88-90). Die vorliegende Arbeit verbindet diese Ebenen, da analysiert werden soll, wie von supranationaler Ebene kommende Transformationsimpulse sich auf die Entwicklung

Auch umgekehrt kann eine Zunahme an Delta-Konvergenz möglich sein ohne eine nationale Zunahme an Sigma-Konvergenz. 
der Organisationsfelder Berufsbildung und Hochschule auswirken, ohne endogene nationalstaatliche Transformationskräfte zu vernachlässigen. (Powell/Solga 2008).

\subsection{Gründe für die Verbindung einer diskursanalytischen Forschungsperspektive mit neoinstitutionellen Theorien}

Während in den letzten beiden Abschnitten mögliche Erklärungsansätze für institutionellen Wandel sowie die für diese Arbeit zentralen Konzepte und Begrifflichkeiten der wissenssoziologischen Diskursperspektive als auch neoinstitutioneller Theoriestränge erläutert worden sind, soll im nächsten Abschnitt dargestellt werden, warum eine Kombination von beiden ein ergiebiger Ansatz für die Analyse des Wandels von institutionellen Durchlässigkeitsstrukturen ist.

\subsubsection{Soziologischer Neoinstitutionalismus und seine Kritik}

Verallgemeinernd kann die hauptsächliche Kritik dergestalt zusammengefasst werden, dass der soziologische Neoinstitutionalismus vor allem die Rolle von Akteuren, ihren Interessen und Macht sowie den stark prozesshaften Charakter von Institutionen, ihrem Erhalten aber auch ihrer Veränderung vernachlässigen. Während Wandel noch als Prozess betrachtet wird, so muss insbesondere auch Stabilität von Institutionen mit Blick auf die diese Stabilität konstruierenden Prozesse beleuchtet werden. Hierbei spielen auch Akteure und die herrschenden Machtverhältnisse eine starke Rolle.

Jedoch, und das zeigen Walgenbach und Meyer (2008), wurde vielfach kritisiert, dass die Rolle von Akteuren bei Entstehung, Aufrechterhaltung und Veränderung institutioneller Praktiken oft nicht oder nur wenig berücksichtigt werden. Dieses Fehlen intentionalen und strategischen Handelns ließ den Eindruck von übersozialisiertem Verhalten von Individuen entstehen (Powell/DiMaggio 1991). Dabei zeigen Walgenbach und Meyer (2008: 115), dass die Kritik sowohl von außen (vgl. Stinchcombe 1997) als auch aus den eigenen Reihen des Theoriestrangs (vgl. DiMaggio 1991; Dobbin 1994; Powell 1991) aufkam. Die fehlende Aufmerksamkeit kam insbesondere insofern zustande, als der soziologische Neoinstitutionalismus als ,important break with rational-actor models and a promising strategy for modeling and explaining instances of organizational change that are not driven by processes of interest mobilization " verstanden wurde (DiMaggio 1988: 3 nach Walgenbach/Meyer 2008: 119).

Es wird allerdings von Walgenbach und Meyer (2008: 125ff.) darauf hingewiesen, dass zunehmend auch innerhalb der Theorie versucht wird, Akteure als 
institutionelle Unternehmer in die Untersuchung von institutionellen Wandel hereinzunehmen. Wenn nun also zwar Akteure in die Analyse gebracht werden, so bleibt aber die Rolle von Macht weiterhin wenig thematisiert. Wird Macht thematisiert, dann ist sie quasi oft exogen, und wird in den Umwelten, die Einfluss ausüben, verortet (siehe Lederle 2008: 102). Dadurch bleibt jedoch unbeantwortet, durch wen Leitideen oder Bedeutungsverschiebungen institutionalisiert werden und wie sich diese Leitideen durchsetzen lassen.

In neueren Ansätzen wird versucht, diesem Problem zu begegnen. Dabei erfolgt die Thematisierung von Macht in neoinstitutionellen Analysen oft auch im Zusammenhang mit einer diskursanalytischen Perspektive. So zeigen zum einen Lawrence und Suddaby (2006) in ihrem Überblick über die Arbeiten zu institutional work ${ }^{26}$, dass institutional work, d.h. die Arbeit von Akteuren zur Institutionalisierung, Stabilisierung und Deinstitutionalisierung von Institutionen, oft auf sprachlicher Ebene stattfindet und damit diskursanalytische Zugänge vielversprechende Ansätze zur Analyse davon seien. Arbeiten im Rahmen von institutional work beziehen sich aber meist auf die Veränderungen von Organisationstrukturen einzelner Organisationen und weniger wie in dieser Arbeit auf institutionelle Strukturen von (Bildungs-)Systemen.

Zum anderen wird die Verbindung von institutionellen Ansätzen und Diskursanalyse besonders deutlich im diskursiven Institutionalismus (vgl. Schmidt 2002, 2008, 2010). Dieser wird von Schmidt als Dachkonzept für die Ansätze gesehen, die die Ebene von Vorstellungen und Ideen nutzen, um politischen Wandel und Stabilität in institutionellen Kontexten zu erklären (Schmidt 2010: 2). Wichtig im diskursiven Institutionalismus ist, dass Institutionen nicht nur als Ideen oder Leitbilder konzeptualisiert werden, sondern dass Institutionen umgeben sind von kommunikativen Prozessen, den Diskursen mit ihrer jeweils eigenen Strukturlogik, welche beeinflussen, wie die Institutionen handlungsorientierend auf politische Akteure wirken (Schmidt 2008: 309). Entsprechend dieser Definition kann auch die vorliegende Arbeit als eine Arbeit im Strang des diskursiven Institutionalismus angesehen werden. Im Unterschied zu der stärker auf den politischen Prozess und die Beeinflussung politischer Akteure ausgerichteten Forschung bei Schmidt (vgl. auch Crespy/Schmidt 2014; Schmidt 2014) ruht in dieser Arbeit aufgrund des wissenssoziologischen Unterbaus vor allem der Fokus auf dem Diskurs als Ort der Institutionalisierung, der Schaffung der Wirklichkeit von Wissensordnungen, und damit insbesondere der kulturell-kognitiven auch normativen institutionellen Dimension.

\subsubsection{Stärken der diskurstheoretischen Perspektive}

Insgesamt können diskursanalytische Ansätze als komplementär zum historischen und soziologischen Neoinstitutionalismus gesehen werden (Schmidt 2008).

Neuere Arbeiten zu institutional work sind zu finden in Lawrence et al. (2009, 2013). 
Letztere geben vor allem auch Hintergrundinformationen, welche für die Analyse institutionellen Wandels wichtig sind (vgl. Schmidt 2008). Dies gilt im besonderen $\mathrm{Ma}$ für den historischen Institutionalismus, denn vor allem mit dem klassischen Ansatz kann eher selten Wandel erklärt werden, da die Betonung vielmehr auf locked-in-Effekten und positiven Verstärkungsprozessen liegt (vgl. auch Rixen/Viola 2014). Wenn critical junctures aber als Auslöser für Wandel identifiziert werden, wird im Allgemeinen jedoch nicht erklärt, wie es zu diesem kritischen Zeitpunkt kam. Diskursanalytische Ansätze können dagegen vielmehr Einblicke gewähren, inwiefern die pfadabhängigen Strukturen immer wieder neu rekonstruiert werden und wie und warum critical junctures entstehen können (Schmidt 2008). Aber auch in den dynamischeren Ansätzen des historischen Institutionalismus (Thelen 2004, Streeck/Thelen 2005) ruht der Analysefokus stärker auf Veränderungen der normativen und regulativen institutionellen Dimension, die kulturell-kognitive dagegen wird oft vernachlässigt (siehe Graf 2013: 44).

In Bezug auf den soziologischen Institutionalismus betont Schmidt (2008: 320) zuerst einmal die Gemeinsamkeiten. Dieser wie auch diskursanalytische Ansätze akzentuieren die kulturell-kognitive Ebene und es stehen vor allem Ideen und Vorstellungen im Vordergrund. Beide Ansätze sind konstruktivistisch und teilen eine Ablehnung gegenüber Rational-Choice-Ansätzen in Bezug auf Individuen. Zudem lehnen sie beide die Annahme, dass es eine objektive Rationalität gäbe, ab. Interessen bestehen nicht unabhängig von Ideen. Alle Interessen sind Ideen und Ideen machen Interessen aus (Hay 2006). Wenn auch in beiden Ansätzen Ideen bzw. Wissen im Vordergrund stehen, sind Diskurse jedoch im Gegensatz zu Ideen nicht nur der Text oder Inhalt (was gesagt wird) sondern auch der Kontext, die Formationsregeln dessen, was gesagt wird. Diskurse umfassen mehr als die bloße Struktur, sie erfassen ebenfalls die dazugehörige Agency. So betont Wendt (1987) in Anlehnung an Giddens Konzept der Dualität der Struktur, dass Strukturen einen inhärenten diskursiven Charakter besitzen, d.h., sie sind untrennbar mit den Überlegungen und dem Selbstverständnis, die Akteure zu ihrem Handeln bringen, verbunden, während aber die Agenten gleichzeitig diese Strukturen konstituieren.

Die Hauptdifferenz zwischen dem soziologischen und diskursiven Institutionalismus besteht nach Schmidt (2008: 320) darin, inwiefern Ideen als dynamische Konstrukte gesehen werden und inwiefern Machtaspekte als relevant betrachtet werden. Das Spektrum der Fragen einer Diskursanalyse sei größer, denn es interessiert viel stärker ebenfalls, wer als Träger von Ideen identifiziert wird und in welche Richtung sich diese entwickeln - ob eher hierarchisch von oben oder auch in einem Prozess von unten nach oben. Es ergibt sich auf diese Weise die Möglichkeit, anstatt nur von einer hierarchischen Vermittlung auszugehen, auch zu beobachten, wie Ideen und Glaubensgrundsätze sich in einem Prozess der kontinuierlichen Infragestellung ändern. Welche gesellschaftlichen Kräfte in Deutschland und Frankreich sind aktiv bei der Gestaltung institutionellen Wandels von Durchlässigkeit? Für diese Arbeit wäre folglich gleichermaßen spannend 
zu untersuchen, woher mögliche Ideen stammen, die institutionellen Wandel zwischen den Organisationsfeldern der Hochschulbildung und Berufsbildung induzieren. Handelt es sich vor allem um exogene neu über z.B. europäische Koordinierungsprozesse eingebrachte Ideen oder um andere endogene, in diesem Fall nationalstaatliche bzw. dem Organisationsfeld entstammende Vorstellungen, welche die institutionelle Entwicklung um Durchlässigkeit prägen?

Zudem erlaubt die Diskursanalyse eine stärkere Prozessorientierung, da Sinnund Deutungsmuster nicht nur festlegen, wie Akteure ihre Welt begrifflich fassen, sondern auch durch mögliche Rekonzeptualisierung als Ressource genutzt werden können, um Wandel zu ermöglichen (Schmidt 2008, 2010: 4). Wie bereits im Abschnitt zum Verhältnis vom Diskurs zu Institutionen herausgearbeitet wurde, dass Institutionen durch Diskurse erzeugt und legitimiert werden, begrenzen aber bestehende Institutionen gleichsam wiederum Diskurse. Diskursanalysen können demnach bei der Rekonstruktion von institutionellem Wandel helfen, indem sie Untersuchungen der diskursiven Auseinandersetzungen um die Transformation gesellschaftlicher Definitionsverhältnisse anleiten. Auch wenn in vielen Analysen gezeigt werden konnte, dass institutionelle Arrangements sich eher langsam ändern und sich durch eine gewisse Trägheit auszeichnen, so konnte jedoch auch gezeigt werden, wie durch neue Diskurse alte ,Wahrheiten` bzw. altes Wissen seiner Selbstverständlichkeit enthoben wird, unter Rechtfertigungsdruck gelangt und einem Prozess der Delegitimation ausgesetzt wird, aus dem die Institutionen schließlich verändert hervorgehen (Keller 2008). Insbesondere Michel Foucaults Arbeiten fordern auf, die Herkunft von Sinn-Setzungen durch Raum und Zeit zurückzuverfolgen. Zudem rückt er die Ausbildung und Verbreitung neuer Diskurse in Auseinandersetzung mit den bestehenden symbolischen Ordnungen und deren Transformationen in den Mittelpunkt der Diskursanalyse, wobei durch den inhärenten Konnex zwischen Wissen und Macht Diskursanalysen auch immer die Veränderungen der Kräfteverhältnisse in den Blick nehmen und somit Macht und Konfliktanalysen sind ${ }^{27}$. Gerade wenn Institutionen wie Durchlässigkeitsstrukturen im Bildungssystem - maßgeblich zukünftige - die Lebenschancen der Individuen und deren sozialen Platzierung in der Gesellschaft mitbestimmen, ist davon auszugehen, dass deutliche Verteilungskonflikte in der Gesellschaft vorherrschen, wobei bestehende Strukturen von den herrschenden Gruppen einer Gesellschaft verteidigt werden, während sie von den Benachteiligten infrage gestellt wird. Da Diskurse als Ort der Institutionalisierung, der Reproduktion und der Veränderung von gesellschaftlichen Wissensvorräten, den Ideologien, Leitbildern, Vorstellungen, gelten, ist anzunehmen, dass auch hier die gesellschaftlichen Konfliktlinien

27 Keller (2008) kritisiert die hermeneutische Wissenssoziologie für ihren zu starken Fokus auf die Mikroanalyse von Wissen, da so der Analyse systematisch die Karrieren öffentlicher Diskurse als Prozesse der Wissenskonstitution und -zirkulation entgehen. Aber auch stärker expert_innengestütztes Wissen bzw. institutionelles Wissen sickert in die Wirklichkeitskonstruktion der Individuen ein, so dass die Untersuchung solcher Phänomene notwendigerweise zu den Untersuchungsgegenständen einer wissenssoziologischen Perspektive gehört. 
sichtbar sind. So schlägt auch Scherrer (2001) vor, zu analysieren, von welchen Institutionen Verharrungsmomente ausgehen oder auch Konkurrenzsituationen entstehen, welche ebenfalls diskursiv wahrgenommen werden. Es öffnet sich der Blick für die politischen Auseinandersetzungen um den Institutionenwandel. $\mathrm{Zu}$ fragen wäre insofern z.B., welche Akteure eine erhöhte Durchlässigkeit zwischen Hochschulbildung und Berufsbildung befördern oder behindern? Welche Strategien werden verfolgt?

Die diskursanalytische Erweiterung der neoinstitutionalistischen Theorien betont neben der Ideenebene somit auch die Rolle von Macht und handelnden Akteuren. Die diskursanalytische Annahme einer gegenseitigen Subversion von Struktur und Subjekt bietet dabei einen Ansatz zur Analyse von institutionellem Wandel (Scherrer 2001). Auch wenn auf diese Dualität von Handlung und Struktur ebenfalls im soziologischem Institutionalismus hingewiesen wird (u.a. Scott 2008, Hasse/Krücken 2005), so bleiben diese Aspekte in den Analysen doch weitgehend ausgeblendet (Scherrer 2001, Schmidt 2008, siehe aber Meyer/Jepperson 2000). Searle (1995) betont, dass Institutionen sich nicht nur unbewusst ändern, indem die Institutionen anders genutzt werden, sondern auch bewusst geändert werden, indem man sich entscheidet, sie anders zu nutzen. Diskurs ist ein interaktiver Prozess, der es erlaubt, Institutionen zu verändern, weil der deliberative Charakter von Diskursen es erlaubt, über Institutionen zu reden.

Insbesondere in Zeiten, in denen Institutionen sich ändernden Bedingungen ausgesetzt sind, wie z.B. durch die Bologna- und Kopenhagen-Prozesse, ist anzunehmen, dass über den Diskurs über die Institutionen verschiedene Vorstellungen über den Wandel von Institutionen sichtbar werden.

Inwiefern können sich neue Vorstellungen zu Durchlässigkeit durchsetzen? Inwiefern können europäische Prozesse genutzt werden, da diese durch ihre Vorgaben Durchlässigkeitsforderungen eine größere Legitimität geben könnten? Kommt es quasi zu diskursiven Machtverschiebungen in Frankreich und Deutschland?

\subsubsection{Stärken der neoinstitutionalistischen Perspektive}

Nachdem die Stärken der Diskursanalyse und der erweiterte Analysefokus für die Analysen institutionellen Wandels dargestellt wurden, soll jedoch auch gezeigt werden, warum eine Diskursanalyse allein die Dynamiken von institutionellem Wandel nicht so gut fassen kann.

Der große Vorteil, den der soziologische neoinstitutionalistische Ansatz für diese Arbeit bietet, ist der ausdifferenzierte Institutionenbegriff, welcher drei Dimensionen von Institutionen unterscheidet und darauf hinweist, dass institutioneller Wandel in allen drei Dimensionen stattfinden kann und zwar auch in einem unterschiedlichen Maß. Das heißt, auch wenn der Fokus in dieser Arbeit durch die Verbindung von neoinstitutionalistischen Analysen mit der Diskursanalyse 
vor allem auf den Veränderungen des Wissens, der kulturell-kognitiven und normative Dimension liegt, sollen regulative Änderungen durchaus mitberücksichtigt werden und mögliche Inkonsistenzen aufgezeigt werden. Insbesondere derartige Inkonsistenzen können dann auch wiederum eine Erklärung für Wandel sein (vgl. Abschnitt 3.2.1). In dieser Arbeit werden somit nicht nur Entwicklungen des Verständnisses, der gesellschaftlichen Vorstellungen von Durchlässigkeit und deren Legitimationen (kulturell-kognitive Dimension), sondern z.B. auch Rollenzuschreibungen für Absolvent_innen beruflicher und allgemeinbildender Bildungswege (normative Dimension) sowie gesetzliche Veränderungen zur Durchlässigkeit zwischen Berufs- und Hochschulbildung (regulative Dimension) in den Blick genommen werden. Auf diese Weise entsteht ein differenziertes Bild über die institutionellen Entwicklungen, welches auch eine ganzheitlichere Beurteilung von Wandelprozessen ermöglicht.

Die Diskursanalyse bietet einen derartig differenzierten Begriff nicht, entweder fokussiert sie auf die Veränderungen oder Rekonstruktion der Wissensebene oder sie befasst sich im Rahmen von z.B. Dispositivanalysen ${ }^{28}$ mit deutlich mehr als Institutionen (vgl. Bührmann/Schneider 2008; Keller 2007a).

Ein weiterer Vorteil der Verbindung beider Ansätze besteht in Folgendem: In Diskursanalysen werden zwar Wissensveränderungen und damit zusammenhängend Veränderungen von Machtverhältnissen in den Blick gerückt und ein erster Teil einer Erklärung geliefert, wie es zu Wandel kommt. Trotzdem bleibt es relativ unbeleuchtet, wie z.B. neue Diskurse in die nationalen Diskurse eindringen. Hier bietet der neoinstitutionalistische Ansatz mit dem Diffusionskonzept einen wichtigen Ansatzpunkt. Auf diese Weise könnte institutioneller Wandel auch bei gleichen Machtverhältnissen möglich sein. Diffusion von Ideen ist somit ein wichtiger Mechanismus, welcher auch zur Innovation von Diskursen beiträgt. Inwiefern aber die diffundierenden Ideen überhaupt aufgenommen werden, wie sie und ob sie überhaupt interpretiert werden und welche Interpretationen sich verfestigen und inwiefern sie die Legitimität bestehender Institutionen untergraben, wird dann erst in den Diskursen sichtbar.

28 Bührmann und Schneider (2007: Abs. 19) beschreiben, dass Foucault (1978: 119f.) „mit Dispositiv ein ,heterogenes Ensemble‘ von solchen unterschiedlichen Elementen wie Diskursen, Institutionen, architekturalen Einrichtungen, reglementierenden Entscheidungen, Gesetzen usw. bezeichnet." Das Dispositiv bildet dabei aber nicht die Summe dieser Elemente, sondern vielmehr die Verbindung, das Netz, das zwischen diesen Elementen besteht (Bührmann/Schneider 2007). Während in Diskursanalysen zwar auch Praktiken und zwar diskursive untersucht werden, stehen bei Dispositivanalysen auch die nicht-diskursiven Praktiken mit im Fokus. Angermüller (2010) fasst den Dispositivbegriff von Bührmann und Schneider (2008) in seiner Rezension auf der Homepage wmw.litkult.de folgendermaßen zusammen „Ein Dispositiv bezeichnet demnach ein soziohistorisches Arrangement symbolisch objektivierbarer Wissensordnungen wie material vergegenständlichbarer Handlungsmuster, das sich als Reaktion auf einen gesellschaftlichen ,Notstand` bzw. bestimmte soziale Problemfeldern formiert und Subjektivitätsangebote bereit hält.“ 
Zudem bietet der Neoinstitutionalismus wertvolle Konzepte zur Beschreibung institutionellen Wandels an, die sich ferner auf Diskursebene untersuchen lassen können, und zwar sowohl für die Entwicklungsprozesse innerhalb eines Landes als auch im Vergleich zu internationalen Modellen und zu anderen Ländern. Auch hier wird die konzeptuelle Nähe der Ansätze offenbar. In der vorliegenden Arbeit soll somit erstens die Frage beantwortet werden, inwiefern institutioneller Wandel in Deutschland und Frankreich eher zu einer Stabilisierung vorhandener Durchlässigkeitsinstitutionen führt, zu einer Veränderung dieser oder zur Entstehung ganz neuer Muster, die nicht mehr den traditionellen nationalen Entwicklungspfaden entsprechen. Und zweitens interessiert auch, inwiefern der Wandel zu einer Konvergenz in Richtung europäischer Vorgaben einerseits und einer Konvergenz zwischen den nationalen Strukturen in Deutschland und Frankreich andererseits geführt hat.

Schließlich sind Diskursanalysen zwar immer historisch in einem bestimmten Kontext verortet (Keller 2008), aber der Fokus ihrer Analysen liegt eher in der Suche nach Bruchstellen in der Wissensformation, nach Diskontinuitäten, welche zu Bedeutungsverschiebungen führen (Traue et al. 2014: 494) Der historische Neoinstitutionalismus weist dagegen auch auf die Bedeutung vorhandener institutioneller Arrangements und ihrer Wirkmächtigkeit hin. In diesem Rahmen wird somit von Relevanz sein zu untersuchen, wie die traditionellen Durchlässigkeitsstrukturen in Deutschland und Frankreich die weitere Entwicklung bestimmen, aber wie auch auf diskursiver Ebene mit diesen Kontinuitäten gebrochen wird. ${ }^{29}$

In diesem Abschnitt sollte gezeigt werden, dass beide Ansätze Stärken aber auch blinde Flecken mit sich bringen, aber dass Neoinstitutionalismus und die diskursanalytische Forschungsperspektive zusammen für die Erklärung komplexer Prozesse wie von institutionellem Wandel eine sinnvolle und fruchtbare gegenseitige Ergänzung bieten. Während einerseits die konfliktbehafteten Prozesse der Deutungsproduktion und damit auch Prozesse der Institutionalisierung in den Vordergrund geraten, werden andererseits institutionelle Kontexte und Analyseebenen nicht vernachlässigt.

Aufbauend auf dem in diesem Kapitel dargelegten theoretischen Konzept soll im nachfolgenden Kapitel anhand des Forschungsdesigns gezeigt werden, wie in dieser Arbeit die Forschungsfragen beantwortet werden sollen.

29 Die Bedeutung historischer Gewachsenheit von Diskursen und die Beschränkung diskursiver Praktikten durch bestehende Diskurse und Diskurshistorie werden auch in Foucaults Arbeiten thematisiert. Allerdings arbeitet er weniger die sich selbstverstärkenden Mechanismen, die im Rahmen des historischen Neoinstitutionalismus betont werden, heraus. 



\section{Design und Methoden}

Diese Arbeit hat drei Ziele: erstens zu rekonstruieren, ob und wie ein Wandel der institutionellen Durchlässigkeitsstrukturen in Deutschland und Frankreich stattgefunden hat, zweitens zu vergleichen, inwiefern sich die institutionellen Durchlässigkeitsstrukturen in den beiden Ländern angeglichen haben, und drittens zu untersuchen, welchen Einfluss europäische Bildungsprozesse auf die Durchlässigkeitsstrukturen hatten und welche anderen Faktoren mit bestimmend waren. Vor dem Hintergrund dieser Forschungsfragen und des dargestellten theoretischen Zugangs soll in diesem Kapitel das Forschungsdesign beschrieben werden. Es handelt sich um eine Mehrebenenanalyse unter Verwendung verschiedener methodischer sowie theoretischer Ansätze. Nachfolgend sollen daher das Design und die Analyseverfahren in zwei Unterkapiteln vorgestellt werden. Der letzte Abschnitt dient der Reflexion der Güte der durchgeführten Analysen.

\subsection{Forschungsdesign}

Der Abschnitt zum Forschungsdesign zielt darauf ab, diese Arbeit allgemein als Fallstudie zu verorten und die konkrete Anlage der Untersuchung zu begründen. Dafür wird zuerst das spezifische Fallstudiendesign, in dem eine diskursanalytische Forschungsperspektive mit der des process tracing verbunden wird, erklärt. Dabei werden der Analysefokus der Untersuchung, die Logik der einzelnen Analyseschritte und die Auswahl des Untersuchungszeitraums erläutert. In einem zweiten Teil wird die Fallauswahl begründet und die Bedeutung des Vergleichs für diese Arbeit dargestellt.

\subsubsection{Fallstudiendesign}

\section{Fallstudien}

Die vorliegende Arbeit kann als vergleichendes Fallstudiendesign beschrieben werden. Doch was sind Fallstudien? Gerring (2004: 341) definiert sie folgendermaßen: ,a case study is best defined as an in-depth study of a single unit (a relatively bounded phenomenon) where the scholar's aim is to elucidate features of a larger class of similar phenomena". Das zu untersuchende Phänomen sind die Entwicklungen der nationalen institutionellen Durchlässigkeitsstrukturen zwischen Berufs- und Hochschulbildung in Deutschland und Frankreich. Ziel ist zu zeigen, dass Durchlässigkeit - abhängig von dem jeweiligen Bildungssystem - 
ganz unterschiedlich institutionalisiert sein kann und mit unterschiedlichen Problematiken einhergeht. Zudem sollen auch die Entwicklungen der jeweiligen Durchlässigkeitsstrukturen nachvollzogen werden. Aber nicht nur die Unterschiede, sondern auch Ähnlichkeiten sollen untersucht werden. Ausgehend von der Analyse der jeweiligen Entwicklung im deutschen oder französischen Fall (unit of analysis), die sich aus der diachronen Betrachtung der Fälle ergibt, soll auch gezeigt werden, inwiefern sich die Strukturen angleichen, und schließlich, welchen Einfluss die Europäisierung auf die Entwicklungen hatte. Nach der Einteilung von Gerring würde dieses Design am ehesten dem historisch vergleichenden Design entsprechen, in dem die Analyse ,across and within units synchronically and diachronically" (Gerring 2004: 343) stattfindet.

Fallstudien sollen gemäß Snow und Trom (2002: 153) offen und flexibel, multiperspektivisch, longitudinal und mit verschiedenen Methoden analysiert werden. Offenheit und Flexibilität unterstreichen, dass diese qualitative Forschung nicht als rein linearer Prozess verstanden werden kann. Vielmehr werden Annahmen im Verlauf des Forschungsprozesses reformuliert, Erhebung und Interpretation können im Verlauf Erklärungslücken aufzeigen, was die Erhebung weiterer Daten nötig macht.

Das Vorgehen der Triangulation bietet sich für ein solches Fallstudiendesign an, da es verschiedene Perspektiven und Methoden berücksichtigt, um unterschiedliche Aspekte eines Problems zu erfassen. Triangulation bedeutet, dass verschiedene Datenquellen, theoretische Ansätze, und/oder Auswertungsmethoden miteinander kombiniert werden (Flick 2004). Auch in dieser Arbeit fand eine Triangulation von Daten, Methoden und Theorien statt. Es wurden verschiedene Datenquellen verwendet: Interviews sowie unterschiedlichste Dokumente in Form von Gesetzen, Berichten, Beschlüssen und Kommuniqués sowie Protokolle teilnehmender Beobachtungen. Die Verbindung unterschiedlicher theoretischer Stränge - die der Diskursforschung und des Neoinstitutionalismus - ermöglicht die Berücksichtigung verschiedener Einflussfaktoren, um den Fall möglichst umfassend zu erklären. Mit der Triangulation der Methoden kann die Begrenztheit von Einzelmethoden durch ihre Kombination überwunden werden. Diese Arbeit basiert primär auf der diskursanalytischen Auswertung bildungspolitischer Dokumente. Die Diskursanalyse wird aber ergänzt um eine Dokumentenanalyse rechtlicher Texte, um Expert_inneninterviews sowie um eine qualitative Inhaltsanalyse europäischer Deklarationen und Kommuniqués. Um den Einfluss der europäischen Prozesse bestimmen zu können und Erklärungsvorschläge für die vorgefundene institutionelle Entwicklung zu liefern, wird sich zudem an das Verfahren des process tracing angelehnt, wobei der Fokus der Arbeit primär auf der Darstellung der nationalen Änderungsdynamiken liegt. 


\section{Process tracing und Diskursperspektive}

Process tracing dient als Verfahren zur Rekonstruktion einzelner Sequenzen eines Handlungsablaufs bzw. einer Entwicklung. George und Bennett (2005: 206) definieren dieses Verfahren folgendermaßen:

The process-tracing method attempts to identify the intervening causal process, the causal chain and causal mechanism between an independent variable (or variables) and the outcome of the dependent variable.

Im Gegensatz zu statistischen Auswertungen, in denen zwar Kausalzusammenhänge, nicht aber deren Zustandekommen gezeigt werden können, ist es anhand einer Fallstudie möglich zu rekonstruieren, wie sich ein bestimmtes Ergebnis im Zeitverlauf ergibt:

The causal analysis takes the form of a tree diagram, showing how a case progresses from step to step in the story, each step understood as preceding in time the one that follows it. (Becker 1992: 226)

Hierbei ist aber anzumerken, dass in dieser Arbeit der traditionelle Fokus auf Mechanismen (vgl. George/Bennett 2005; Mahoney 2010), die ein Outcome erklären, weniger ausgeprägt ist. Eine genaue Rekonstruktion der politischen Prozesse, die klassischerweise im Zentrum von Process-tracing-Analysen stehen, findet nicht statt. Im Fokus steht vor allem die Darstellung des institutionellen Wandels der Durchlässigkeitsstrukturen. Die Frage nach den genauen Mechanismen, die erklären, wie genau z.B. Europäisierungsprozesse gewirkt haben, d.h., ob es z.B. eher zu einer Diffusion kam primär durch transnationale Kommunikation oder durch europäische Wettbewerbsanreize (Bieber 2011: 6), oder ob eine Sozialisierung durch Überzeugung (Checkel 2005) stattgefunden hat, kann in dieser Arbeit nicht abschließend beantwortet werden. Vielmehr wird von dem Ansatz des process tracing übernommen, Wandel Schritt für Schritt zu rekonstruieren und zu beschreiben (Collier 2011; Mahoney 2010), um Einflüsse aufzudecken. Daher werden in den Fallanalysen, auch wenn es zwei Untersuchungszeiträume gibt, die Entwicklungen innerhalb der Zeiträume genauer betrachtet, so dass dieses Wissen auch die Fragen der Einflussfaktoren des Wandels bereichert. Folgt man Mahoneys (2010) Unterscheidung in unterschiedliche Arten von process tracing oder auch „causal-process observation" (CPO), dann entspricht die in dieser Arbeit durchgeführte Version am ehesten der ,independent variable CPO“ (Mahoney 2010: 125128). Es geht darum zu zeigen, dass z.B. Europäisierung im Rahmen der Bolognaund Kopenhagen-Prozesse einen Einfluss auf die nationalen Durchlässigkeitsstrukturen hat. Die Annahme dahinter ist, dass erstens in den Europäisierungsprozessen Durchlässigkeit als Thema relevant ist. Zweitens wird - wie im Theoriekapitel gezeigt - angenommen, dass es durch Europäisierung zu einer Diffusion von Vorstellungen, Normen und Regeln kommen kann, die durch die sozialen Akteure als Bestätigung der bestehenden Strukturen interpretiert oder aber 
infrage gestellt werden können, wodurch ein Wandel in Gang gesetzt wird. Beides wird in dieser Arbeit untersucht.

Eine Schwierigkeit, die mit dem Verbinden der Methoden des process tracing mit der Diskursanalyse einhergeht, ist die Kopplung des eher positivistischen Ansatzes des process tracing (Checkel 2005), der nach kausalen Zusammenhängen fragt, mit einem postpositivistischen konstruktivistischen Ansatz. Die Untersuchung von gesellschaftlichen Problemen aus diskurstheoretischer Perspektive muss mit der Problematik umgehen, dass nicht einfach von einer Ursache-Wirkungsrelation ausgegangen werden kann. Es wird vielmehr ein reziprokes oder zirkuläres Verhältnis von Ursache und Wirkung angenommen (Quenzel 2005). Auch im process tracing wird ein zirkuläres Verhältnis angenommen, da eine erkannte Auswirkung gleichzeitig mit Ursache für neuere Entwicklungen sein kann. Aus diesem Grund ist eine klare Unterscheidung von abhängiger und unabhängiger Variable nicht möglich, woraus letztlich eine stärker rekonstruktive Herangehensweise an den Untersuchungsgegenstand folgt. Es geht primär darum, welchen Platz bestimmte Phänomene im Gegensatz zu anderen einnehmen. Trotzdem soll die diskursanalytische Forschungsperspektive in diese Arbeit nicht allein auf die Rekonstruktion verkürzt werden, denn die theoretischen Annahmen bieten sehr wohl auch Erklärungsmöglichkeiten, wenn man die machttheoretische Einbettung des Ansatzes ernst nimmt (vgl. Kapitel 3). Was aber zu Veränderungen diskursiver Machtstrukturen führt, kann stärker aus einer Perspektive des process tracing erklärt werden. Letztlich würde ein reines Berücksichtigen positivistischer oder diskursanalytischer Methoden nicht notwendigerweise einen adäquaten Rahmen für die Studie komplexer Sachverhalte wie die des institutionellen Wandels sein.

Versucht man, trotz der genannten Prämissen für ein besseres Verständnis des Forschungsdesigns zwischen abhängiger und unabhängiger Variable zu unterscheiden, könnte Wandel im Sinne eines Ergebnisses und nicht als Prozess als abhängige Variable verstanden werden. Dabei ist zu beachten, dass die veränderten Bedingungen zu einem Zeitpunkt aber immer auch Ausgangsbedingungen für die weitere Entwicklung sind. Als mögliche Einflussfaktoren, die vermeintlich unabhängigen Variablen, können die folgenden gelten: Erstens wird der Einfluss des ,quasi-exogenen' europäischen Veränderungsdrucks, d.h. die mit den Bolognaund Kopenhagen-Prozessen verbundene Europäisierung, betrachtet. Dabei gilt, dass insbesondere die Bologna- und Kopenhagen-Prozesse nicht nur von oben instruiert werden, zumal gerade die Bildungspolitik noch in die nationalen Verantwortungsbereiche fällt. So waren die nationalen Regierungen wie auch die nationalen Hochschulen, die Studierende, die Sozialpartner sowie europäische Institutionen an den Prozessen beteiligt (Walter 2006). In diesem Sinn können die Prozesse nicht als rein exogen bezeichnet werden (vgl. auch Schmidt 2014). Europäisierung wirkt zudem nicht nur in eine Richtung. So können auch die nationalen Entwicklungen aufgrund von Europäisierung multidirektional sein. 
Europeanization can be defined as internal differentiation and external adaptation. It turns national societies more dynamic and accounts for accelerated social change. As such, it is linked to harmonizing processes through which national societies become increasingly similar, but it is also manifested in new heterogeneous practices, which increase internal diversity. (Trenz 2008: 6)

Zweitens müssen als den Wandel beeinflussende Faktoren die nationalen pfadabhängigen Strukturen in die Analyse einbezogen werden. Inwiefern verhindern diese institutionellen Strukturen Veränderungen? Inwiefern sind sie aber auch Teil einer Weiterentwicklung bzw. beeinflussen die Aufnahme von diffundierten Konzepten? Es werden auch die im jeweiligen Organisationsfeld beteiligten Akteure als potenzielle Einflussfaktoren in die Analyse aufgenommen. Durch die Diskursanalyse von Dokumenten der bildungspolitischen Akteure können dann entsprechende Machtkonstellationen und damit verbundenen Strategien aufgedeckt werden.

Um insbesondere den Einfluss der europäischen Prozesse nachzuvollziehen, werden für die Analyse zwei Zeiträume unterschieden. Der erste Zeitraum umfasst die Zeit vor den Bologna- und Kopenhagen-Prozessen bis einschließlich 1998. ${ }^{30}$ Der zweite Zeitraum reicht von 1999 bis 2012. Wie lange vor dem ,offiziellen' Beginn der beiden Europäisierungsprozesse die Diskurse untersucht werden, ist abhängig von dem spezifischen Fall. In Deutschland wurde 1990 als Startpunkt bestimmt, da erstens mit dem Fall der Mauer eine neue Dynamik der Europäisierung begann. Zweitens war durch die Wiedervereinigung auch eine Art window of opportunity in Deutschland geschaffen worden, die institutionellen Settings des Bildungssystems und somit auch das Bildungsschisma infrage zu stellen. Denn damals musste in Gesamtdeutschland ein einheitliches System institutionalisiert werden, wobei in Ostdeutschland das Bildungsschisma viel weniger stark institutionalisiert war. In Frankreich bietet es sich an, bis zur Einführung des baccalauréat professionnel, des Berufsabiturs, im Jahr 1985 zurückzugehen, da dies einen entscheidenden Einfluss auf die Entwicklung einer größeren Durchlässigkeit des Bildungssystems bedeutete. Jedoch zeigte sich, dass die für die Diskursanalyse notwendigen Dokumente für den Zeitraum vor 1989 schwer zugänglich waren, so dass die Untersuchungszeiträume nun doch fast gleich sind.

\section{Anlage der Untersuchung}

Im Folgenden soll anhand von Abbildung 2 vereinfacht gezeigt werden, was in dieser Arbeit untersucht wird und auf Basis welcher Analyseverfahren.

Erstens wird angenommen, dass von europäischer Ebene durch die Bolognaund Kopenhagen-Prozesse Vorstellungen, Normen, und Regeln auf die nationale

30 Zwar begann der Bologna-Prozess quasi schon 1998 mit der Sorbonne-Deklaration, jedoch wird davon ausgegangen, dass der durch diesen Prozess induzierte Wandel sich nicht bereits 1998 voll entfaltet hat. 
Ebene über unterschiedlichste Mechanismen der Diffusion der sozialen Konstruktion von Problemen, des gegenseitigen Lernens oder des Wettbewerbs (Dobbin et al. 2007) diffundieren. Diese Vorstellungen, Normen und Regeln können dann Einfluss auf die nationalen Bildungssysteme, die Organisationsfelder Hochschulbildung und Berufsbildung und damit auch die darin verankerten institutionellen Durchlässigkeitsstrukturen ausüben. Wie und ob die europäischen Prozesse aber eine Wirkung entfalten können, hängt auch von den bestehenden nationalen Strukturen, den Pfadabhängigkeiten sowie den Akteuren und ihren Machtverhältnissen ab. Gleichzeitig können auch endogen durch das nationale Setting Änderungsprozesse in Gang gesetzt werden.

Die Fallstudien für Deutschland und Frankreich untersuchen jeweils die nationalen institutionellen Veränderungen des Verhältnisses von Hochschul- und Berufsbildungsstrukturen im Hinblick auf Durchlässigkeit. Doch wie soll nun die Veränderung der Institutionen in den unterschiedlichen Organisationsfeldern rekonstruiert werden?

Entsprechend der an Scott (2008) angelehnten analytischen Unterteilung von Institutionen in drei Dimensionen (kulturell-kognitiv, normativ und regulativ) sollten diese auch analysiert werden, wobei mein Fokus vor allem auf der Untersuchung der Veränderungen in der kulturell-kognitiven Dimension liegen wird.

Ein Wandel in der regulativen Dimension kann über die Analyse von Gesetzen, Vorschriften und Beschlüssen untersucht werden. Das heißt ich untersuche, inwiefern sich die gesetzlichen Regelungen in Bezug auf Durchlässigkeit geändert haben. Die kulturell-kognitive Ebene lässt sich vor allem über eine Diskursanalyse erfassen. Auch die normative Dimension ist in den Diskursen nachzuzeichnen. Dabei ist zu bedenken, dass es nicht immer möglich sein wird, bei der Diskursanalyse die idealtypische Unterscheidung der drei Dimensionen von Institutionen aufrechtzuerhalten (vgl. auch Kapitel 3). Hellmann (2006) weist darauf hin, dass entsprechend der asymmetrischen Unterscheidung des Legitimationsbegriffs von Berger und Luckmann (2007: 100) die kognitive Dimension der normativen vorangestellt ist, „bei der Legitimation von Organisationen geht das Wissen den Werten voraus". Insofern sind in Wertungen, was z.B. gute Standards für Universitäten sind, bereits kulturell-kognitive Skripte eingelagert. Zum anderen ist es aber auch denkbar, dass normativ bereits Veränderungen gefordert werden, die aber im Gegensatz zur kulturell-kognitiven Dimension stehen.

Die normative Dimension lässt sich anhand der Standards und Normen untersuchen, die z.B. an die Organisationen in Diskursen herangetragen werden. Auch wäre es möglich zu untersuchen, welche Standards und Normen im Hinblick auf Durchlässigkeit in den Organisationen selbst gelten. Der Fokus dieser Arbeit liegt aber auf der Makroebene sowie auf den Entwicklungen in den Organisationsfeldern auf der Mesoebene, so dass eine Analyse von Entwicklungen in Organisationen nicht geleistet wird. Auch die Individualebene, also die Mikroebene, wird nur indirekt über die Betrachtung von Beteiligungsraten aufgenommen (vgl. Tabelle 2). 
Die stärkere Fokussierung dieser Arbeit auf die Analyse von Diskursen bietet sich an, weil die europäischen Bildungsprozesse noch nicht sehr lange bestehen. Dies gilt umso mehr für den Kopenhagen-Prozess. Folglich macht sich der Einfluss dieser Prozesse mitunter noch nicht über regulative Veränderungen bemerkbar. Zudem sind auch Veränderungen in den anderen institutionellen Dimensionen wichtig, weil auch sie Entwicklungen aufzeigen. Zudem können sie aber auch Inkongruenzen zwischen den institutionellen Dimensionen offenbaren und damit Wandel verstärken oder bremsen. Insofern ist eine ganzheitlichere Betrachtung auch hilfreich zur Erklärung institutioneller Änderungen.

Tabelle 2 Analysefokus der Untersuchung

\begin{tabular}{|c|c|c|}
\hline Erklärungsfaktoren & Analyseebene & Analysefokus \\
\hline Europa & Makroebene & $\begin{array}{l}\text { Entwicklung der europäischen Bildungspolitik und der } \\
\text { Bologna- und Kopenhagen-Prozesse }\end{array}$ \\
\hline $\begin{array}{l}\text { Nationale } \\
\text { Gesellschaften }\end{array}$ & Makroebene & $\begin{array}{l}\text { Entwicklung von Vorstellungen und Ideologien zu Bil- } \\
\text { dung und Durchlässigkeit }\end{array}$ \\
\hline Nationale Politik & Makroebene & $\begin{array}{l}\text { Nationale und föderale Strukturen und Akteure wie In- } \\
\text { teressenvertretungen }\end{array}$ \\
\hline Organisationsfelder & Mesoebene & $\begin{array}{l}\text { Governance der Organisationsfelder sowie institutio- } \\
\text { nelle Strukturen der Berufsbildung und Hochschulbil- } \\
\text { dung im Hinblick auf Durchlässigkeit }\end{array}$ \\
\hline Organisationen & Mesoebene & $\begin{array}{l}\text { Hochschulen, Betriebe, Berufsbildungsschulen (vor- } \\
\text { herrschende Standards, Kulturen, Umsetzung der Re- } \\
\text { gelungen in den Organisationen) }\end{array}$ \\
\hline Individuen & Mikroebene & $\begin{array}{l}\text { Studierende, Auszubildende }{ }_{31} \text { Schüler_innen, Erwach- } \\
\text { sene (z.B. Beteiligungsraten }{ }^{2} \text { ) }\end{array}$ \\
\hline
\end{tabular}

Quelle: In Anlehnung an Powell (2004: 29) angewandt auf diese Arbeit durch die Verfasserin

Aber nicht nur Europäisierungsprozesse, sondern auch die historische Gewachsenheit des Verhältnisses von Berufs- und Hochschulbildung sind für die Erklärung der Entwicklungen notwendig. Primär über die Analyse von historischen Arbeiten zu den nationalen Bildungssystemen, aber auch durch Expert_inneninterviews werden die traditionell gewachsenen Strukturen sowie Kontexte der untersuchten Entwicklungen in dieser Arbeit aufgearbeitet. Die Rolle der nationalen Akteure und der Machtverhältnisse wird wiederum im Rahmen der Diskursanalyse betrachtet, da die Positionen von bedeutenden bildungspolitischen Akteuren der beiden Organisationsfelder untersucht werden.

31 Auf Individualebene sind zudem die demografischen und individuellen Eigenschaften wie Herkunft, Geschlecht, Alter, Ethnizität usw. wichtig. Zudem können auf Individualebene auch die Bildungsentscheidungen, die sich dann in den Beteiligungsraten abbilden, untersucht werden, wie dies im Rahmen des Berliner-Studienberechtigten-Panel geschieht. 
Der Ansatz des process tracing leitet die Untersuchung im Hinblick auf eine schrittweise Rekonstruktion der Entwicklungen und deren Erklärung durch vor allem exogene, d.h. europäische, aber auch endogene Einflüsse an. Die Analyse der Entwicklung und vor allem der Inhalte der europäischen Bildungsprozesse Bologna und Kopenhagen erfolgt auf Basis einer theoriegeleiteten qualitativen Inhaltsanalyse der entsprechenden europäischen Deklarationen und Kommuniqués. Die Untersuchung des Einflusses dieser Prozesse erfolgt wiederum anhand der Analyse der institutionellen Durchlässigkeitsstrukturen in den Fallstudien sowie durch Expert_inneninterviews.

Die Anlage der Untersuchung wird in Abbildung 2 zusammengefasst: Für den Vergleich von Frankreich und Deutschland erfolgt zuvor eine analoge Analyse der Einzelfälle. In den Formen, die die einzelnen Einflussfaktoren und bzw. der zu untersuchenden Strukturen darstellen, stehen jeweils kursiv geschrieben die angewendeten Erhebungs- und Analyseverfahren, die genauer in Abschnitt 4.2. beschrieben werden.

\subsubsection{Fallauswahl und Vergleich}

Bevor die einzelnen durchgeführten Analyseverfahren genauer beschrieben werden, soll in einem nächsten Schritt die Fallauswahl begründet und damit auch die Bedeutung des Vergleichs für diese Arbeit dargestellt werden. Warum vergleichen und nicht eine einzelne Fallstudie?

Der Vergleich in dieser Arbeit hat zwei große Ziele: Erstens soll der Vergleich dazu dienen, die Entwicklungen der nationalen Fälle besser zu verstehen. Es können die Spezifika des deutschen und des französischen Falls besser herausgearbeitet werden. Kulturelle Unterschiede werden vergleichend erst deutlich und nationale Entwicklungen in eine neue Perspektive gerückt. Aber nicht nur Unterschiede, sondern auch Ähnlichkeiten in der institutionellen Evolution und in Konfigurationen sollen analysiert werden, die dann auf verallgemeinerbare Entwicklungen oder Konstellationen oder auch typische Durchlässigkeitsproblematiken hinweisen.

Zweitens geht es auch darum zu erklären, welchen Einfluss die europäischen Prozesse in den beiden Ländern hatten, ob sie gleichgerichtet auf die Durchlässigkeitsstrukturen wirken oder inwiefern sie ganz unterschiedlich einflussreich waren. Anhand des Vergleichs könnten dann auch Kontextfaktoren für Einflüsse von Europäisierung deutlich gemacht werden. Schließlich ist auch nur anhand des Vergleichs die Frage zu beantworten, inwiefern die jeweiligen Entwicklungen zu einer Annäherung der Durchlässigkeitsstrukturen in Frankreich und Deutschland geführt haben. 


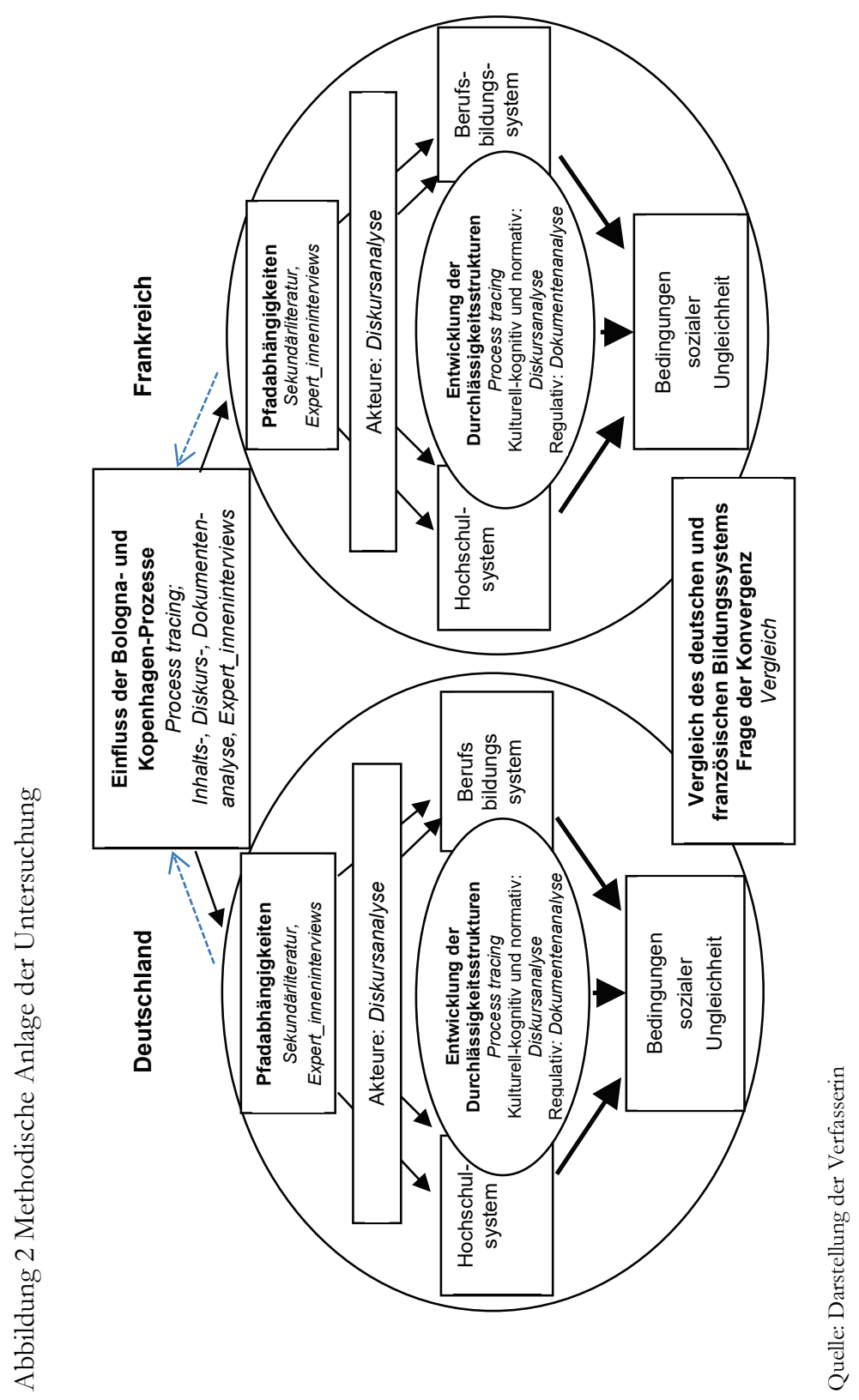




\section{Einordnung in die klassischen Vergleichsdesigns}

Doch wie gestaltet sich die Fallauswahl? Für diese Studie bleibt festzuhalten, dass die klassischen nomothetischen Vergleichsdesigns (vgl. Jahn 2005) nach Mill (1872) mit der Konkordanz- bzw. Differenzmethode nicht angewendet werden können und auch die Ansätze nach Przeworski und Teune (1970) mit dem most similiar system und dem most different system design nicht zu $100 \%$ auf die Fallauswahl in dieser Arbeit zutreffen. Daraus ergibt sich aber auch, dass über die Fälle in dieser Arbeit hinaus nur begrenzt Verallgemeinerungen möglich sein werden.

Warum nicht Mills Ansatz? Mill (1872) geht in seinen Überlegungen davon aus, dass man bereits bei der Fallauswahl die Ausprägung der abhängigen Variable kennt. Entweder kann man diese konstant halten und anhand eines Vergleichs untersuchen, welche gemeinsamen Einflussfaktoren identifiziert werden können (Konkordanz), oder man nimmt Fälle, die sich in der abhängigen Variable unterscheiden und untersucht Einflussfaktoren und kann dann Faktoren, die in beiden Fällen auftauchen, als erklärende Faktoren ausschließen (Differenzmethode) (Jahn 2005). In dieser Arbeit ist jedoch die Ausprägung der quasi-abhängigen Variable, ob Wandel in den Durchlässigkeitsstrukturen stattfindet oder nicht, bei der Fallauswahl nicht bekannt, sondern Ergebnis der Untersuchung.

Mit Blick auf das Verhältnis von Berufs- und Hochschulbildung und den Einfluss europäischer Bildungsprozesse können der deutsche und der französische Fall nicht als most similar systems, wohl aber als dissimilar systems (Przeworski/Teune 1970) angesehen werden. Die Analyse folgt somit einer Logik der Unterschiedlichkeit. Die Fälle Frankreich und Deutschland können aber trotz dieser Unterschiedlichkeit nicht als most different klassifiziert werden, weil z.B. beide Länder als meritokratische Demokratien gelten, traditionell zu den katholisch konservativen Wohlfahrtsstaaten gehören, über ein institutionalisiertes Berufsbildungssystem verfügen, die teilweise auch korporatistisch geregelt werden. Zudem sind beide Länder wichtige und mächtige Mitgliedsstaaten in der Europäischen Union und bedeutende Akteure in den europäischen Bildungsprozessen. Sie gehören zu den Initiatoren der Bologna- und Kopenhagen-Prozesse und haben daher auch beide ein Interesse an deren Fortgang.

Dennoch wurden einige Charakteristika des most-different-system-Ansatzes übernommen (Jahn 2005). So wurden erstens, abgesehen von diesen gerade benannten Ähnlichkeiten, die quasi als Einflussfaktoren in beiden Ländern konstant gehalten werden, relativ unterschiedliche Fälle ausgesucht im Hinblick auf die institutionellen Strukturen und die Organisation der Berufs- und Hochschulbildung, die primär Durchlässigkeitsstrukturen mitbestimmen. ${ }^{32}$ Zweitens sollte in-

32 Es stellt sich die Frage, ab wann ein Fall als most different eingeordnet werden kann. Im europäischen Vergleich sticht z.B. England heraus, weil dort die berufliche Ausbildung nicht stark institutionalisiert ist und Bildung vor allem Allgemeinbildung bedeutet. Der Unterschied 
nerhalb der Fälle nach Zusammenhängen in Bezug auf die Durchlässigkeitsentwicklungen gesucht werden. Können trotz der Unterschiedlichkeit der Fälle gleiche Zusammenhänge oder Entwicklungen durch z.B. Europäisierungseinflüsse festgestellt werden, kann von relativ stabilen Zusammenhängen ausgegangen werden (Jahn 2005: 13). Inwiefern zeigen sich trotz der unterschiedlichen institutionellen Ausgestaltung ähnliche Reaktionen auf die Europäisierungsprozesse? Aber auch im Hinblick auf die Entwicklung der Durchlässigkeitsstrukturen an sich ist es interessant zu untersuchen, ob trotz unterschiedlicher Ausgangssituationen ähnliche Probleme bestehen oder sich ähnliche Entwicklungen vollziehen, die vielleicht einen Hinweis auf die allgemeine Gestaltung von Durchlässigkeit ermöglichen. Weichen drittens aber die gefunden Erklärungen für die Länder stark voneinander ab, dann sollen auch diese Spezifika im Fokus stehen (Jahn 2005).

\section{Fallauswahl auf Basis der Organisation und Struktur der Berufs- und der Hochschulbildung}

Nachdem die Logik der Fallauswahl in dieser Arbeit in Relation zu den klassischen Vergleichsansätzen eingeordnet wurde, soll nachfolgend die Auswahl der beiden Fälle Deutschland und Frankreich erklärt werden. Diese bezieht sich vor allem auf die institutionellen Strukturen und die Organisation der Berufs- und Hochschulbildung, wobei die Charakteristika der drei institutionellen Dimensionen in den Blick genommen werden. Dabei waren bestehende Typologien für die Berufs- und Hochschulbildung sowie für das Verhältnis von Bildungssystem und Arbeitsmarkt hilfreich. Zuerst werden überblicksartig und mithilfe von Typologien die beruflichen Bildungssysteme gegenübergestellt, dann die Hochschulsysteme und danach deren Verhältnis untereinander.

Der Vergleich der Entwicklungen in Deutschland und Frankreich bietet sich aus folgenden Gründen an: Beide Länder sind entscheidende Akteure in den europäischen Bildungsprozessen. Zudem zeichnen sich beide Länder durch unterschiedliche Bildungsstrukturen, -traditionen und -ideale aus. Frankreich und Deutschland werden in Bezug auf ihre Bildungssysteme oft gegenübergestellt und als einflussreiche Modelle gehandelt (Powell et al. 2012c). So zählen nach Greinert (2005) die beruflichen Bildungssysteme in Frankreich und Deutschland zu den drei klassischen Modellen der Berufsbildung: Frankreich verkörpert das staatlich regulierte bürokratische Modell, Deutschland das dual-korporatistische Modell und Großbritannien das liberale marktwirtschaftliche Modell. Dabei kann das französische Berufsbildungssystem als stark zentralisiert und reguliert beschrieben werden. Der wichtigste Akteur ist der Staat, der die Planung, Regulierung und Finanzierung übernimmt. Zwar bezahlen die Betriebe eine Ausbildungssteuer, jedoch ist ihr Einfluss auf die berufliche Ausbildung begrenzt, weil sie überwiegend

zwischen Deutschland und Frankreich dagegen ist nicht so groß, aber doch entscheidend, weil es in Frankreich kein institutionalisiertes Bildungsschisma ohne $\mathrm{Zu}$ - und Übergänge gibt. 
in vollzeitschulischen Einrichtungen stattfindet. In den meisten Fällen sind daher die Lernenden auch Schüler_innen und nicht Angestellte (Powell et al. 2009). Die betriebliche Praxis bleibt unterbelichtet, was sich auch in den Curricula widerspiegelt, wo die Theorievermittlung im Mittelpunkt steht (Greinert 2005). Als zentrale Charakteristik des französischen Modells gilt zudem die staatliche Planung von Qualifizierungsbedarfen für die nationale Volkswirtschaft (Lutz 1976) und damit auch die Regulierung des Angebots von Ausbildungsplätzen und folglich auch von Fachkräften (Koch 1998).

In Greinerts (2005) Typologie wird das französische Modell primär auf die schulische Berufsbildung begrenzt und die alternierenden Ausbildungen, bei denen auch die Betriebe stärkeren Einfluss nehmen können, bleiben unterbelichtet. Auch in der Beschreibung des deutschen Systems findet eine ebensolche Zuspitzung statt. Im deutschen Modell steht das duale System im Mittelpunkt, und die schulische Berufsausbildung sowie das Übergangssystem (vgl. Autorengruppe Bildungsberichterstattung 2014; Baethge et al. 2007) wird vernachlässigt. Das deutsche Berufsbildungssystem wird als ein auf korporatistischen Regelungen basierendes System beschrieben, das sich durch institutionalisierte Absprachen des Staates, der Arbeitgeber- und Arbeitnehmervertreter auszeichnet und auch auf diese Weise gesteuert wird Die Finanzierung erfolgt dabei primär durch die Betriebe. Die Lernenden sind nicht nur Schüler_innen, sondern gleichzeitig auch Angestellte. Entsprechend dem Berufsprinzip sollen die Auszubildenden den Beruf in der Praxis erlernen (Kraus 2007). Im Gegensatz zu Frankreich, wo die berufliche Sekundarbildung als individueller Makel gesehen wird bzw. Lernende in der Berufsbildung als Problemschüler_innen stigmatisiert werden (Brauns 1998), kann in Deutschland die berufliche Bildung auf Sekundarniveau als gesellschaftlich anerkannt gelten. Es ist der Bildungsweg für einen Großteil einer Kohorte.

Darüber hinaus unterscheiden sich Deutschland und Frankreich deutlich in Bezug auf das Verhältnis zwischen Ausbildung, Arbeitsmarkt und Karrieremobilität. Maurice et al. (1986) charakterisieren Frankreich als organisational space, in dem berufliche Bildung als Investition in firmenspezifische Fähigkeiten angesehen wird und auch Karrieren vor allem in Firmen stattfinden. ${ }^{33}$ In Deutschland besteht dagegen ein qualificational space. Durch einen berufsspezifisch segmentierten Arbeitsmarkt ist Mobilität zwischen Berufen in Deutschland weniger üblich. Durch den hohen Grad an Standardisierung der beruflichen Qualifikationssysteme ist es den Arbeitnehmern aber leicht möglich, zwischen Firmen im gleichen Beruf zu wechseln.

Neben den Unterschieden im Berufsbildungssystem ist auch das Hochschulbildungssystem durch einige Unterschiede gekennzeichnet. So beschreibt Goldschmidt (1991) das französische Hochschulsystem als ,administrativen Zentralismus", d.h., die Rolle des Zentralstaates ist auch für das Funktionieren des Hochschulwesens von wesentlicher Bedeutung. Deutschland wird dagegen beschrieben

33 Dass sich dies teilweise in den letzten Jahren auch in Frankreich geändert hat, zeigt CampinosDubernet (1995). 
als ein System des ,political legalism ", in dem Konflikte auf rechtlicher Ebene gelöst werden (Goldschmidt 1991: 5f.). Reguliert wird das Hochschulwesen in Deutschland aber nicht zentralstaatlich, sondern föderal durch die Bundesländer. Die Finanzierung des Hochschulwesens erfolgt in Frankreich und Deutschland aus öffentlichen Mitteln.

Auch wenn die Aufgabe der Hochschulbildung in beiden Ländern klassisch die gleiche ist - nämlich eine gesellschaftliche Elite auszuwählen und auszubilden (Ben-David 1992) - unterscheiden sich die Strukturen der Länder deutlich. So weist Teichler (2002: 349f.) darauf hin, dass das deutsche System sich vor allem dadurch auszeichnet, dass die Universitäten forschungsorientiert sind und im Großen und Ganzen das gleiche Qualitätsniveau haben. ${ }^{34}$ Zudem besteht das deutsche Hochschulsystem vor allem aus Universitäten und Fachhochschulen (jetzt Hochschulen) und einigen wenigen Berufsakademien als Organisationen der höheren Bildung, während das französische System viel stärker differenziert und stratifiziert ist. Es kann unterteilt werden in selektive und nichtselektive Bereiche, die klar hierarchisiert sind. Typisch ist insbesondere die Elitenförderung durch die grandes ecoles. ${ }^{35}$

Anhand der Gegenüberstellung der Strukturen der Organisationsfelder Berufs- und Hochschulbildung auf Grundlage von bestehenden Typologien konnten deutliche Unterschiede zwischen Frankreich und Deutschland herausgearbeitet werden. So ist es nicht verwunderlich, dass sich auch das Verhältnis zwischen Berufs- und Hochschulbildung in Deutschland und Frankreich traditionell unterscheidet. In Deutschland besteht ein System, in dem berufliche Bildung und Allgemeinbildung getrennt sind. Baethge (2006) nennt es das deutsche Bildungsschisma. Es handelt sich um eine institutionelle Segmentierung, d.h. eine gegenseitige Abschottung der Bildungsbereiche mit ihren eigenen institutionellen Ordnungen. Gekennzeichnet ist diese Trennung durch eine unterschiedliche Ausgestaltung der institutionellen Strukturen der Organisationsfelder. So unterscheidet sich die Form der Regulation, der Standards, der Lernorte und der Ziele und Ideale (Baethge 2006). Durch diese strikte Segmentierung sind sowohl $\mathrm{Zu}$ - als auch Übergänge von einem Bereich in den anderen schwierig, und zudem werden die Qualifikationen, die in einem Bereich erlernt worden sind, wenig bis gar nicht im anderen anerkannt oder angerechnet (Haugg 2008), was häufig zu Doppelqualifikationen führt (Hörner 2006).

In Frankreich ist eine solche strikte Trennung zwischen Berufs- und Hochschulbildung nicht vorhanden, zumal beruflich orientierte Bildung zu weiten Teilen auch im Hochschulsystem selbst stattfindet. Im Gegensatz zu Deutschland gibt es in Frankreich kein ausgebautes postsekundäres berufliches Weiterbildungssystem außerhalb der Hochschulen. Zudem sind die Curricula sowohl der

34 Die Auswirkungen von zeitlich begrenzten Programmen wie der Exzellenzinitiative sind momentan noch nicht abzusehen.

35 Ausführlichere Darstellungen der Bildungssysteme sind in Kapitel 6 und 8 nachzulesen. 
Berufsbildung zu weiten Teilen als auch der Hochschulbildung mehr auf allgemeinbildende Inhalte aufgebaut. Für das französische System wird daher auch die Gefahr gesehen, dass die berufliche Bildung ihre Unabhängigkeit als eigenständiger Bildungsbereich in Relation zur Allgemeinbildung verliert (Culpepper/Thelen 2008). Außerdem ist die Durchlässigkeit zwischen Berufsbildung und Hochschulbildung zumindest formal viel eher gewährleistet, weil das Berufsabitur (baccalauréat professioned) 1985 eingeführt wurde und man somit auch mit einer beruflichen Ausbildung eine Hochschulzugangsberechtigung erlangen konnte und kann und auch Anrechnungsverfahren seit den 1980er Jahren bestehen, die kontinuierlich ausgebaut wurden.

Es ist somit deutlich geworden, dass Frankreich und Deutschland durch ihre Unterschiedlichkeit der Organisationsfelder der Berufs- zur Hochschulbildung und dem Verhältnis der Bereiche zueinander interessante Fälle für einen Vergleich der Durchlässigkeitsstrukturen, deren Entwicklung und den Einfluss europäischer Prozesse ausmachen.

\subsection{Erhebungs- und Analyseverfahren}

Wie bereits in der Beschreibung des Forschungsdesigns deutlich wurde, erfolgte für die Bearbeitung der Forschungsfragen eine Kombination von Daten sowie methodischen Herangehensweisen. Daher werden im Folgenden die methodischen Verfahren und das konkrete Vorgehen in dieser Arbeit vorgestellt: die wissenssoziologische Diskursanalyse, die qualitative Inhaltsanalyse, die Expert_inneninterviews sowie die Dokumentenanalyse.

\subsubsection{Wissenssoziologische Diskutsanalyse}

Nachfolgend soll es um das diskursanalytische Vorgehen gehen, da dessen Ergebnisse im Zentrum der Analyse stehen. Wie bereits dargestellt, dient die Diskursanalyse der Untersuchung der normativen, aber vor allem der kulturell-kognitiven institutionellen Entwicklung in Deutschland und Frankreich. Zudem soll sie auch zeigen, welche Rolle den Europäisierungsprozessen in den nationalen Debatten zugestanden wird. Ich lehne mich an das Vorgehen der wissenssoziologischen Diskursanalyse nach Keller (2005, 2006, 2007a, b, 2008) und Truschkat (2008) an und werde nachfolgend die Schritte des Forschungsprozesses genauer darstellen: Als erstes geht es darum, offenzulegen, woher das für eine Diskursanalyse notwendige Kontextwissen stammt. Anschließend wird die Korpusbildung, d.h. die Selektion der zu analysierenden Dokumente, und schließlich das Vorgehen der Feinanalyse dargestellt. 


\section{Kontextwissen}

Keller (2007b: 82) weist darauf hin, dass es bereits vor der Datenerhebung notwendig ist, sich näher mit dem Gegenstandsbereich der Forschung zu befassen. Sowohl für den deutschen als auch für den französischen Fall bedeutet dies, eine gute Kenntnis der jeweiligen Bildungssysteme, der dazugehörigen wichtigen Akteure und damit auch der Diskursträger zu erlangen. Für diese Arbeit erfolgte dies vor allem über Sekundärliteratur, aber auch über explorative Expert_inneninterviews sowie teilnehmende Beobachtungen an Fachtagungen. Die Erarbeitung des notwendigen Kontextwissens führte auch dazu, dass der der Forschungsgegenstand von dem allgemeineren Fokus auf das Verhältnis der Berufszur Hochschulbildung in Deutschland und Frankreich auf den spezifischen Fokus auf Durchlässigkeitsstrukturen eingegrenzt wurde.

Diese Eingrenzung auf Durchlässigkeit erfolgte nach einem ersten Forschungsaufenthalt in Frankreich im März 2010 durch die Vertiefung des Wissens über das französische Bildungssystem und dessen Problematiken, die kaum durch eine einfache Rezeption von Bildungssystembeschreibungen in der Sekundärliteratur zu erfassen gewesen wären. Da die in Deutschland offensichtliche Problematik von Durchlässigkeit in Frankreich aufgrund des differenzierten Bildungssystems, das diese für Deutschland typische Segmentierung in berufliche und Hochschulbildung nicht aufweist, nicht offensichtlich war, wurde mithilfe von explorativen Expert_inneninterviews und Sekundäranalysen untersucht, worin in Frankreich die Problematiken im Verhältnis von beruflicher und Hochschulbildung bestehen könnten. Es stellte sich heraus, dass auch in Frankreich Durchlässigkeitsprobleme existieren, die mit dem Verhältnis von beruflicher Bildung und höherer Allgemeinbildung verbunden sind. ${ }^{36}$ Auf diesen Weg konnte der Fokus für die Diskursanalysen begrenzt werden.

\section{Korpusbildung}

Nachdem das Thema eingegrenzt worden war, musste der zu analysierende Textkorpus für beide Fälle erstellt werden. Die Daten des Korpus wurden gemäß der grounded theory nach dem Verfahren des theoretical samplings ausgewählt (Strauss/Corbin 1996). Das heißt, nicht nur die Analyse, sondern auch die Auswahl der Daten erfolgte nach theoriegeleiteten und reflektierten Kriterien. Bei der Datenauswahl war damit das Konzept der theoretischen Sensitivität (Strauss/Corbin 1996) leitend. „Theoretische Sensibilität setzt sich nach STRAUSS und CORBIN aus Literaturkenntnissen, beruflichen und persönlichen Erfahrungen und aus den Erkenntnissen zusammen, die im Rahmen des laufenden Forschungsprojekts gewonnen werden" (Truschkat et al. 2005: Abs. 12, Hervorhebung im Original). Die Datenauswahl wurde in zweifacher Hinsicht

Für eine ausführliche Darstellung siehe Kapitel 8. 
theoretisch angeleitet. Erstens wurden die zu untersuchenden Akteure bzw. relevante Organisationen identifiziert. Zweitens wurde auch die Begrenzung auf Durchlässigkeit theoretisch beeinflusst.

\section{Auswahl der Akteure}

Bei der Analyse unterscheide ich in Anlehnung an Schmidt (2008) zwischen zwei verschiedenen Diskursräumen: dem koordinativen und dem kommunikativen Diskursraum, die jeweils einer unterschiedlichen Logik unterliegen. Der koordinative Diskursraum wird getragen von Akteuren, die im Zentrum der Politikformulierung stehen und die beteiligt sind an der Weiterentwicklung von Programmen und Politiken. Diese Akteure umfassen politische Akteure, Beamte, Expert_innen sowie die unterschiedlichen organisierten Interessengruppen in dem zu untersuchenden Politikbereich. Die Nähe des Konzepts des koordinierten Diskursraums zu dem des Organisationsfeldes soll an dieser Stelle unterstrichen werden. Die vorherrschende Logik der Diskurse hier ist die des Verhandelns, da es sich um eher geschlossene Debatten handelt außerhalb des Blickfeldes der Öffentlichkeit (Schmidt 2008).

Die prävalente Logik im kommunikativen Feld ist die des Argumentierens, da hier Entscheidungen vor einem größeren Publikum, der Öffentlichkeit, begründet werden müssen (Schmidt 2008) Beispiele von solchen Diskursräumen wären das Parlament oder auch die Medienöffentlichkeit. Hier stehen stärker die Notwendigkeit und Angemessenheit von Politiken oder Veränderungen im Blickpunkt. Akteure sind vor allem Politiker, aber auch die jeweiligen Interessengruppen und Journalisten (Schmidt 2008).

Im Rahmen der Arbeit werde ich die Diskursanalyse mit Fokus auf den koordinativen Diskursraums durchführen. Dieser Fokus erklärt sich anhand von drei Gründen: Erstens gibt es, vor allem für Deutschland, ein generelles Ungleichgewicht in der medialen Berichterstattung zu Hochschulbildung und beruflicher Bildung, wobei Letzterer traditionell weniger Beachtung geschenkt wird. Um über Entwicklungen im deutschen und französischen Bildungssystem informiert zu sein, wurden zudem regelmäßig die deutsche und französische Presse ab 2009 verfolgt und auch rückwirkend über Lexis Nexis $^{37}$ durchgeschaut. ${ }^{38}$ Zweitens zeigte sich neben dem Ungleichgewicht in der Berichterstattung über Hochschulund Berufsbildung, dass in beiden Ländern auch über den Bologna-Prozess viel

37 Lexis Nexis ist ein Portal, das den Zugriff auf verschiedenste Datenbanken, u.a. auch Datenbanken internationaler Presse erlaubt. Es bietet die Möglichkeit, in den Volltexten von z.B. Zeitungen nach Stichworten zu suchen.

38 Im Rahmen der Datenbank Lexis Nexis wurde in Deutschland die taz und in Frankreich die Zeitung Le Monde nach Stichworten, die im Zusammenhang mit den europäischen Prozessen stehen könnten (z.B. Europa, Bologna, ECTS, Kopenhagen, ECVET, Qualifikationsrahmen), sowie nach allgemeineren Stichworten wie Hochschulbildung und Berufsbildung durchsucht. So war es möglich, einen Einblick über die Relevanz der Prozesse in den Medien zu erhalten. Es wurden stärker links-orientierte Zeitungen ausgesucht, da diese traditionell viel über Bildungsproblematiken berichten. 
stärker berichtet wurde als über den Kopenhagen-Prozess. Aus dem deutschen Fall war aber bekannt, dass der Kopenhagen-Prozess relevant war und vor allem Diskussionen um den Qualifikationsrahmen in Expert_innenkreisen stattfanden, so dass deutlich wurde, dass diese Entwicklungen über eine Medienanalyse nicht abgebildet werden würden. Da der Kopenhagen-Prozess zu Beginn des Forschungsprozesses drittens auch nocht nicht sehr lange bestand, ist anzunehmen, dass Beschlüsse oder Prozesse der Umsetzung kaum in Gang gesetzt worden waren, die medial hätten wirksam sein können.

Nach der Entscheidung für die Untersuchung des koordinativen Diskurses musste eine Auswahl der zu untersuchenden Akteure erfolgen. Diese erfolgte anhand der Kenntnis der Governance der Organisationsfelder der Hochschul- und Berufsbildung in Frankreich und Deutschland (vgl. Kapitel 6 und 8). Die ausgesuchten Akteure sind jeweils kursiv geschrieben.

In Deutschland ist Hochschulbildung größtenteils Ländersache, wobei es auch ein Hochschulrahmengesetz gab, welches auf Bundesebene beschlossen und verändert wurde, das bis zum Beschluss 2007 in der Föderalismuskommission eine größere Reichweite hatte. Zudem wird das Hochschulsystem z.B. durch die Exzellenzinitiative von Bundesebene weiterhin mit beeinflusst (Leibfried 2010; Simon 2010), so dass neben den Länderregierungen auch das Bildungs- und Forschungsministerium (BMBF) als Akteur im Hochschulwesen eine Rolle spielt. Für die Länder wird stellvertretend die Kultusministerkonferenz (KMK) als Akteur aufgenommen, da im Rahmen dieser Arbeit eine Analyse der Entwicklungen in den einzelnen Bundesländern mit den bestehenden Forschungsressourcen nicht möglich gewesen wäre. Zudem sind die Kultusminister bedeutend für die Regelungen der allgemeinbildenden Schulen und für Fragen der Zulassung zum Studium. Wichtig sind außerdem die Hochschulen, wobei die Hochschulrektorenkonferenz (HRK) als ihre Vertreterorganisation untersucht wurde. Weitere Akteure im Bereich Hochschulbildung in Deutschland sind Studierende, Professor_innen und Gewerkschaften.

Berufsbildung in Deutschland wird sowohl auf Bundesebene (Berufspraxis) als auch auf Landesebene (Berufsschulen und schulische Berufsausbildungen) geregelt. Insofern wurden sowohl das Bildungsministerium ${ }^{39}$ als auch die Kultusministerkonferenz als Akteure betrachtet. Zudem ist das deutsche Berufsbildungssystem durch seine korporatistische Organisation gekennzeichnet. Wichtige Akteure sind die Arbeitgeber und Kammern sowie die Gewerkschaften. Da es eine größere Menge an Gewerkschaften und Arbeitgeberverbändern wie auch Handwerks-, Industrie- und Handelskammern gibt, musste sich forschungspragmatisch auf eine kleine Auswahl beschränkt werden. Auf Gewerkschaftsseite wird der Deutsche Gewerkschaftsbund (DGB) näher beleuchtet, da er viele Gewerkschaften mit ihren

39 Neben dem Bildungsministerium sind auch noch andere Ministerien an der Regulation beteiligt (z.B. Wirtschaftsministerium, Landwirtschaftsministerium), aber nur Ersteres wurde in die Untersuchung aufgenommen. 
Überzeugungen vertritt. Außerdem wurde der Deutscher Industrie- und Handelskammertag (DIHK) als zu untersuchender Akteur ausgewählt, ${ }^{40}$ da er als Dachorganisation der Industrie- und Handelskammern die Interessen der gewerblichen deutschen Wirtschaft und somit nicht nur einen Industriezweig vertritt. Zudem finden unter der Aufsicht der Kammern die Prüfungen der beruflichen Aus- und Weiterbildung statt.

In Frankreich wird sowohl die Hochschul- als auch die Berufsbildung stark von zentralstaatlicher Ebene gesteuert. Wichtige Ministerien sind das Bildungs- und das Forschungsministerium, wobei das erste eher für die die Bildung bis Ende der Sekundarstufe II verantwortlich ist und das zweite stärker für die Hochschulbildung. Im Laufe des Untersuchungszeitraums gab es unterschiedliche Benennungen und Kompetenzzuordnungen der Ministerien. Es wurde aber versucht, jeweils die wichtigen für die sekundäre Allgemein- und Berufs- und für die Hochschulbildung in die Analyse mitaufzunehmen. Ähnlich wie in Deutschland sind gerade bei der Berufsbildung oft mehrere Ministerien verantwortlich. Hier wurde sich auf das Bildungsministerium beschränkt. In Frankreich wurden zusätzlich zu den Vertretern der Ministerien auch fünf ausgewählte Reden des Präsidenten und des Premierministers (aber allein für den ersten Zeitraum) mit aufgenommen. Dies war notwendig, da es allgemein schwierig war, für den ersten Zeitraum entsprechende Dokumente zu erhalten. Begründet werden kann das damit, dass sowohl der Premierminister als auch der Staatspräsident als Vertreter der nationalen Regierungspolitik im semipräsidentiellen französischen politischen System gelten können. Für den zweiten Zeitraum in Frankreich und generell in Deutschland wurde aber auf eine Ausweitung der Regierungsakteure verzichtet, um das zu untersuchende Material zu begrenzen.

Ähnlich wie in Deutschland sind im Hochschulbereich zunehmend auch die Hochschulen selbst wichtige Akteure, da diesen im Laufe der Zeit mehr Autonomie zuerkannt wurde. Als Hauptakteur wurde das französische Pendant der Hochschulrektorenkonferenzen, die Conférence des présidents d'université $(\mathrm{CPU})^{41}$ in die Analyse einbezogen sowie die Association des Directeurs d'IUT (ADIUT) ${ }^{42}$ als Vertreter der berufsorientierten technischen Hochschulen. Auch wenn Frankreich sowohl in der Hochschulbildung als auch in der sekundären Berufsbildung

40 Auch der Zentralverband des Handwerks $(\mathrm{ZdH})$ und die Handwerkskammern sind sehr wichtige Organisationen in der deutschen Berufsbildung, aber nur eine Auswahl an Akteuren kann untersucht werden.

41 Die CPU vertritt dabei vor allem Universitäten, aber auch die an den Universitäten angegliederten Technischen Hochschulen (IUT) sowie ausgewählte grandes ecoles. Der Fokus der Vertretung liegt aber auf den Universitäten.

42 ADIUT habe ich mit aufgenommen (wenn auch nur für den zweiten Zeitraum), da diese speziell eine Vertretung der technologischen Hochschulen ist, die durch ihre langjährige finanzielle Sonderstellung im Vergleich zu den Universitäten und der Selektivität bei der Studierendenaufnahme andere Positionen im Diskurs einnehmen könnten als die, die in der CPU vertreten werden. 
vor allem als staatszentriert klassifiziert wird, darf der Einfluss der Gewerkschaften und Unternehmen nicht unterschätzt werden. So haben Gewerkschaften durch Generalstreiks schon auf einige Reformen Einfluss genommen. Zudem sind die Sozialpartner besonders in der Berufsbildung bei der Gestaltung der Diplome institutionalisierte Partner. Dies gilt auch für berufliche Abschlüsse im Hochschulbereich (vgl. Kapitel 8). Da die französische Gewerkschaftslandschaft aber stark zersplittert ist, war es nicht einfach, sich auf einen Akteur zu beschränken. Aus diesem Grund wurde einerseits ein Gewerkschaftsbund ausgewählt, die Confédération générale du travail (CGT), die eine Ansammlung verschiedener linksorientierter Gewerkschaften vertritt und den zweitgrößten Gewerkschaftsbund in Frankreich darstellt. Zum anderen wurde explizit die größte Gewerkschaftsunion für den Erziehungsbereich ausgewählt, die Fédération Syndicale Unitaire (FSU), um einen noch stärkeren Fokus auf die Entwicklungen im Bildungssystem von Gewerkschaftsseite aufzunehmen. ${ }^{43}$ Für die Unternehmensseite wurde der Arbeitgeberverband Mouvement des entreprises de France MEDEF (vorher CNPF) als Akteur ausgesucht. MEDEF gilt als die größte Unternehmensunion in Frankreich. MEDEF ist ein Dachverband von rund 840 lokalen und regionalen Branchenvereinigungen und vertritt mehr als 700.000 Unternehmen (Martin 2005). Zudem gehört der MEDEF zu den wenigen staatlich anerkannten Tarifpartnern auf Arbeitgeberseite. Die regionale Ebene wurde für den französischen Fall nicht einbezogen, da die Entscheidungsbefugnis auf dieser Ebene noch wesentlich durch die Bundesebene begrenzt wird, auch wenn die regionalen Regierungen gerade in der Berufsbildung kontinuierlich durch Prozesse der Regionalisierung (Powell et al. 2012c) an Einfluss gewonnen haben.

Anzumerken bleibt, dass die Auswahl der Akteure immer auch aus einer forschungspragmatischen Logik erfolgte. Die Begrenzung auf den koordinativen Diskursraum sowie die Selektion von Akteuren innerhalb dieses Diskursraums bedeutet zudem auch, dass in dieser Arbeit nur Aussagen über ein begrenztes Diskursspektrum gemacht werden können und nicht der gesamtgesellschaftliche Diskurs zu Durchlässigkeit analysiert wird. Dennoch wird mit diesem begrenzten Fokus auf die wichtigsten Organisationen, die die Steuerung der Bildungssysteme in Deutschland und Frankreich mit bestimmen, ein wichtiger Diskursauschnitt untersucht, da es diese Akteure sind, die einen großen Einfluss auf die Gestaltung des Bildungssystems haben.

43 Beide Gewerkschaften können als politisch stärker linksorientiert eingeordnet werden. Die Auswahl wurde aus einer Logik der maximalen Kontrastierung getroffen, um ein breites Meinungsspektrum abzubilden. 


\section{Erstellung des Samples}

\section{Bestimmung der Daten- und Textsorten}

Zur Analyse des koordinativen Diskurses bieten sich verschiedene Formate an. So können das Beschlüsse, Reden, Pressemitteilungen, offizielle Statements, Interviews in Organisationszeitschriften sein. Da nicht alle Organisationen die gleiche Art von Datenformaten verwenden, um ihre Organisationsmeinung zu publizieren, wird sich nicht auf ein Format beschränkt und gleichzeitig die Breite des Diskurses besser erfasst. Dieses Vorgehen muss mit der Schwierigkeit umgehen, dass die verschiedenen Textsorten nicht immer gleich aufgebaut sind. So sind z.B. Pressemitteilungen meist wesentlich verdichteter, während in Stellungnahmen meist die Argumentationslinie besser nachzuvollziehen ist. ${ }^{44}$ Wichtig bei den Textsorten ist, dass sie eine Stellungnahme, eine Position der Organisation widerspiegeln und nicht ausschließlich empirische Daten ohne weitere Bewertung aufzählen. Wissend, dass auch diese Form der Daten zur Untermauerung von Positionen genutzt werden und eine Form der Konstruktion von Wirklichkeit darstellen, stehen in dieser Arbeit trotzdem Positionsäußerungen im Vordergrund, da anzunehmen ist, dass hier die den Argumentationen unterliegenden Strukturen und Vorstellungen eher rekonstruiert werden können. Auch ist in Stellungnahmen die Abgrenzung zu anderen Positionen eher zu erwarten.

\section{Strategie der Dokumentengewinnung}

Aus dem Forschungsdesign ergibt sich, dass Akteursdokumente für beide Untersuchungszeiträume benötigt werden: für Frankreich 1985-1998 und für Deutschland 1990-1998 für den ersten Zeitraum und jeweils 1998 bis $2012^{45}$ für den zweiten Zeitraum. Doch wie genau erfolgte die weitere Beschränkung des Korpus?

Auf der Suche nach den Positionspapieren wurden in einem ersten Schritt die Homepages der Organisationen nach relevanten Stellungnahmen untersucht. An dieser Stelle ergab sich das Problem, dass auf den jeweiligen Organisationshomepages nur Positionen der letzten Jahre, aber selten der letzten zwei Jahrzehnte zu finden sind. Aus diesem Grund wurde eine mehrdimensionale Strategie der Materialgewinnung verfolgt. Neben den Homepages der Organisationen, die gezielt nach Reden, Pressemitteilungen und anderen Stellungnahmen durchsucht wurden, wurden nationale Archive aufgesucht (das HRK-Archiv ${ }^{46}$, das Archiv der $\mathrm{CPU}^{47}$, das Archiv des französischen Bildungs- und Forschungsministerium in

Für die Feinanalyse aber werde ich mich nicht auf Interviews stützen, weil sie erstens teilweise auch dem kommunikativen Diskurs angehören und zweitens anders aufgebaut sind und natürlich auch von den Fragen des Journalisten abhängen.

45 Während auch wichtige Entwicklungen, die 2013 stattfanden, noch in die regulative Analyse einflossen, erfolgte die Diskursanalyse nur bis Ende 2012.

46 Archiv/Bibliothek der Hochschulrektorenkonferenz, Ahrstraße 39, Bonn.

47 Archiv der CPU im „La Maison des Universités“, 103 Bvd St Michel, Paris. 
Paris $^{48}$ sowie die Bibliothek der Documentation Francaise $\left.{ }^{49}\right)$. Des Weiteren wurden einige Organisationen direkt angeschrieben, um entweder eine Materialsammlung zu erhalten (BMBF, CGT, MEDEF) oder bestimmte Dokumente, auf die im Verlauf der Untersuchung hingewiesen wurde (KMK, DGB, CPU). Auch wurden Konferenz- bzw. Tagungsdokumentationen, in denen z.B. Reden oder auch wichtige Positionspapiere gesammelt waren, sowie archivierte Organisationszeitschriften (DGB, FSU) verwendet. Für den französischen Fall war zudem eine Onlinedatenbank ${ }^{50}$ besonders hilfreich, in der Reden und Stellungnahmen, vor allem der französischen Akteure wie Politiker_innen, von Gewerkschaften und auch Unternehmen, digital archiviert aufzufinden sind.

Die Datensuche kann als ein kontinuierlicher Prozess beschrieben werden, da die Datenerhebung nicht vollständig abgeschlossen war, bevor die Analyse begann. Zum Teil gaben auch bereits bestehende Dokumente Hinweise auf andere wichtige zu untersuchende Positionspapiere, die dann gezielt gesucht wurden.

\section{Auswabl der Daten für die Feinanalyse}

$\mathrm{Da}$ in der Datenauswertung eine qualitative Strategie verfolgt werden soll, muss der verfügbare Datenbestand nach systematisch reflektierten Kriterien reduziert werden, so dass eine Analyse unter der Bedingung der vorhandenen Ressourcen machbar ist. Ausgehend von der Vielzahl von Stellungnahmen, die man recherchieren kann, erfolgte die Datenauswahl für die Feinanalyse in mehreren Schritten. Erstens wurden nur die Dokumente ausgewählt, die das Thema Durchlässigkeit zwischen Berufs- und Hochschulbildung thematisieren. Im Prozess der Auswahl bekam man auch einen generellen Überblick über die bildungspolitischen Diskurse, die die Berufs- und die Hochschulbildung in den beiden Ländern betreffen. Dieses Kontextwissen ist insofern wichtig, als es einen Eindruck vermittelt, welche Rolle Durchlässigkeit im Vergleich zu anderen Themen spielt.

Die Schwierigkeit der Selektion von durchlässigkeitsrelevanten Dokumenten bestand darin, dass mit der Diskursanalyse auch darauf abgezielt wird, die Vorstellungen von Durchlässigkeit erst zu rekonstruieren. Erschwerend kam hinzu, dass insbesondere in Frankreich Durchlässigkeit (perméabilité) als Begriff nicht verwendet wird, so dass eine Suche, die allein den Begriff Durchlässigkeit als Anhaltspunkt nimmt, nicht erfolgversprechend sein konnte. Keller (2007b: 68) beschreibt das Problem der Datensammlung folgendermaßen:

Wenn Gegenstände durch Diskurse erst in ihrer spezifischen, erkennbaren Gestalt geschaffen werden, kann nicht einfach vom Gegenstand ausgehend ein Diskurs erschlossen werden.

48 Archives du ministère de l'Education nationale et de l'enseignement supérieur, 97 rue de Grenelle, Paris.

49 Librairie der Documentation Française, 29 quai Voltaire, Paris.

50 Vgl. http://www.vie-publique.fr/discours/. 
Insofern war Durchlässigkeit allein als Gegenstand nicht ausreichend. Um aber trotzdem eine Auswahl treffen zu können, half das heuristische Konzept Durchlässigkeit, welches in Kapitel 2 erarbeitet wurde. Relevant waren Thematisierungen von Zugangsmöglichkeiten zwischen den Bildungsbereichen, von Anrechnungsmöglichkeiten, von bestimmten Formen organisationaler Verbindungen, z.B. duales oder alternierendes Lernen bzw. institutionalisierte Übergänge, sowie von der Frage nach Strukturen, die ein erfolgreiches Lernen im jeweils anderen Bildungsbereich ermöglichen bzw. erst darüber informieren und dafür interessieren. Kurzum, alle möglichen thematisierten Verbindungen bzw. verbindungsfördernden Strukturen zwischen Berufs- ${ }^{51}$ und Hochschulbildung wurden als relevant erachtet und in einem ersten Schritt ausgewählt. Dabei musste nicht das gesamte Dokument diese Themen behandeln. Es reichte auch, wenn dies nur in Abschnitten der Fall war. Um der Gefahr der zu starken Einengung auf vorgefertigte Kategorien zu verringern, wurden die Dokumente explizit nicht nur nach diesen Kategorien ,abgesucht', sondern reflexiv und offen nach weiteren in der Empirie wichtigen Merkmalen gelesen. Zudem ist anzumerken, dass die verwendete Arbeitsdefinition quasi eine bereits interpretierte Konzeption von Durchlässigkeit ist, die dem wissenschaftlichen Diskurs entspringt (Kapitel 2). Insofern stammt die Arbeitsdefinition quasi aus der Empirie. Zudem kann der wissenschaftliche Diskurs dabei nicht als abgeschnitten von dem nicht-wissenschaftlichen Diskurs der politischen Akteure aufgefasst werden, sondern steht in einem sich gegenseitig beeinflussenden Wechselverhältnis.

Die Auswahl der weiteren Dokumente wurde gemäß den in der Grounded Theorry Methodology (GTM) begründeten Prinzipien, der minimalen und maximalen Kontrastierung, vorgenommen (Strauss/Corbin 1996). Innerhalb des größeren Datenkorpus durchlässigkeitsrelevanter Dokumente wurde nach stark unterschiedlichen (maximale Kontrastierung) bzw. sehr ähnlichen (minimale Kontrastierung) Aussageereignissen bzw. Dokumenten gesucht. Auf diese Weise konnten einerseits die heterogenen Bestandteile des Diskurses, was schon allein durch die unterschiedlichen Akteure gegeben war, erfasst, aber auch die einzelnen Diskursteile möglichst genau rekonstruiert werden, bis die Analyse gesättigt war. Dabei war wichtig, dass bei der Auswahl auch die zeitliche Dimension Berücksichtigung fand. Die minimale und maximale Kontrastierung durfte dabei nicht nur innerhalb eines Zeitraums stattfinden. So wurden Dokumente mit ganz ähnlichem Fokus von einem Akteur trotzdem in das Sample aufgenommen, wenn eine gewisse Zeitspanne (ca. ein Jahr) zwischen den Veröffentlichungsdaten der Dokumente lag, so dass auch Kontinuität oder wiederkehrende Muster feststellbar waren. Die Referenzen aller untersuchten Dokumente können im Online-Anhang konsultiert werden ${ }^{52}$.

51 Hierbei wird Berufsbildung jedoch nicht allein auf das institutionalisierte Bildungssystem begrenzt, sondern auf das berufliche Lernen allgemein erweitert.

52 Im Literaturverzeichnis finden sich daher nur die als Quellen exemplarisch zitierten Dokumente, nicht der ganze untersuchte Dokumentenkorpus. 
Tabelle 3 Anzahl der analysierten Dokumente nach Organisationen in Deutschland und Frankreich

\begin{tabular}{|c|c|c|c|c|c|c|}
\hline & Deutschland & $1 . \mathrm{UZ}$ & $2 . U Z$ & Frankreich & $1 . \mathrm{UZ}$ & 2.UZ \\
\hline $\begin{array}{l}\text { Nationale } \\
\text { Regierung } \\
\text { (Bildungs- } \\
\text { ministerien) }\end{array}$ & $\begin{array}{l}\text { Ministerien für For- } \\
\text { schung und Hoch- } \\
\text { schulbildung, Berufs- } \\
\text { bildung und Schulbil- } \\
\text { dung }\end{array}$ & 11 & 22 & $\begin{array}{l}\text { Ministerien für } \\
\text { Forschung und } \\
\text { Hochschulbildung, } \\
\text { Berufsbildung und } \\
\text { Schulbildung }\end{array}$ & 21 & 59 \\
\hline Länderebene & $\begin{array}{l}\text { Kultusministerkonfe- } \\
\text { renz }\end{array}$ & 5 & 24 & $\begin{array}{l}\text { Regionen nicht unter- } \\
\text { sucht }\end{array}$ & - & - \\
\hline Hochschulen & $\begin{array}{l}\text { Hochschulrektorenkon- } \\
\text { ferenz }\end{array}$ & 8 & 14 & $\begin{array}{l}\text { Conférence des prési- } \\
\text { dents d'université; } \\
\text { Association des Direc- } \\
\text { teurs d'IUT }\end{array}$ & 0 & 21 \\
\hline Unternehmen & $\begin{array}{l}\text { Deutscher Industrie- } \\
\text { und Handelskammer- } \\
\text { tag }\end{array}$ & 4 & 18 & $\begin{array}{l}\text { Mouvement des entre- } \\
\text { prises de France }\end{array}$ & 0 & 10 \\
\hline Gewerkschaften & $\begin{array}{l}\text { Deutscher Gewerk- } \\
\text { schaftsbund }\end{array}$ & 8 & 22 & $\begin{array}{l}\text { Confédération générale } \\
\text { du travai; Fédération } \\
\text { Syndicale Unitaire }\end{array}$ & 0 & 14 \\
\hline $\begin{array}{l}\text { Gemeinsame } \\
\text { Autorenschaft }\end{array}$ & & & 8 & & & \\
\hline $\mathrm{N}$ gesamt & & 36 & 108 & & 21 & 104 \\
\hline
\end{tabular}

Quelle: Darstellung durch die Verfasserin

In Tabelle 3 wird für einen Überblick das Dokumentensample dargestellt. Dabei wird deutlich, dass wesentlich weniger Dokumente für den ersten Zeitraum analysiert wurden und in Frankreich eine deutliche Überrepräsentanz von Dokumenten staatlicher Akteure in beiden Zeiträumen vorzufinden ist. Letzteres kann zum einen durch die noch immer dominierende Rolle der zentralstaatlichen Ebene in der französischen Bildungspolitik erklärt werden. Es kann zum anderen aber auch damit begründet werden, dass nicht nur ein Ministerium, sondern mindestens zwei und bis zu drei Organisationen verantwortlich waren und untersucht wurden.

Die geringe Zahl der Dokumente für den ersten Zeitraum hatte zweierlei Gründe. Primär waren Aspekte von Durchlässigkeit nicht so präsent in den Bildungsdiskursen des ersten Zeitraums. Zweitens, und das gilt insbesondere für

53 Nur Dokumente der ADIUT aus dem zweiten Untersuchungszeitraum gingen in die Analyse mit ein. 
Frankreich, war es sehr schwierig, die Dokumente anderer Akteure als der Regierung zu bekommen - trotz direkter Kontaktaufnahme mit den Organisationen. ${ }^{54}$ Im Gegensatz zu Deutschland ist der erste Zeitraum daher nur durch Dokumente der Regierung abgebildet. Dies ist problematisch und musste auch bei der Auswertung beachtet werden. Aber gerade in Frankreich, wo noch ein Großteil der bildungspolitischen Entscheidungen zentralstaatlich getroffen wird, ist zumindest der Hauptakteur des koordinativen Diskurses repräsentiert. Die meisten Dokumente stammten aus der digitalen Datenbank Discours public ${ }^{55}$, in der aber auch Dokumente anderer Akteure (allerdings deutlich weniger) vorhanden sind. Bei der Selektion der Dokumente wurde in den Positionspapieren dieser Nichtregierungsakteure jedoch keine thematische Relevanz zu Durchlässigkeit festgestellt. Auch in den Dokumentationen der CPU, die auch für den ersten Zeitraum zugänglich waren, wurden keine Dokumente für die Feinanalyse ausgewählt. Insofern kann das Fehlen auch als empirisches Resultat gewertet werden. In den Medien, die durch ein gezieltes Suchen in der Datenbank Lexis Nexis auch für den ersten Zeitraum untersucht wurden, waren zudem auch keine konkurrierenden Deutungen sichtbar. ${ }^{56} \mathrm{Zu}$ beachten ist zudem, dass Durchlässigkeit generell im ersten Zeitraum in Frankreich nur ein sehr kleines Randthema in den Debatten war, was in der Medienanalyse Bestätigung fand. Weiterhin ist anzumerken, dass der Diskurs zu Durchlässigkeit im zweiten Zeitraum wiederum von den Bildungsministerien dominiert wurde und nur ein Diskurs - und nicht konkurrierende Diskurse - bestimmt werden konnte, an dem die anderen Akteure auch beteiligt waren. Es wird davon ausgegangen, dass dies im ersten Zeitraum nicht anders war. Vor dem Hintergrund all dieser Argumente ist das Fehlen der Dokumente zwar problematisch und muss reflektiert werden. Nichtsdestoweniger würde ich die vorliegende Analyse als repräsentativ für einen wichtigen Ausschnitt des koordinativen Diskursraums in Frankreich im ersten Zeitraum ansehen, zumal die geringe Relevanz der Durchlässigkeit von der Berufs- zur Hochschulbildung für den ersten Zeitraum sich auch in den geführten Expert_inneninterviews widerspiegelte.

54 Die Organisationen besitzen zum Teil selbst kein (aufgearbeitetes) Archiv für diesen Zeitraum.

55 Einige der Dokumente waren leider nicht in Volltextversionen, sondern nur in Zusammenfassungen der wichtigsten Argumentationen in den Reden erhältlich. Dies gilt besonders für die ersten Untersuchungsjahre (1985-1992). Diese Dokumente wurden trotzdem in die Analyse aufgenommen, aber nur, wenn auch in den Zusammenfassungen Hinweise und Argumentationen nachvollziehbar waren.

56 Natürlich bedeuten fehlende konkurrierende Aussagen im kommunikativen Diskursraum zu dem herausgearbeiteten Diskurs nicht, dass es nicht doch solche gegeben hat. Der kommunikative Diskurs kann aber als ein weiterer Anhaltspunkt gesehen werden. 


\section{Vorgehen bei der Feinanalyse}

Aus dem Vorgehen der Dokumentauswahl für die Feinanalyse wurde deutlich, dass durch das Verfahren der minimalen und maximalen Kontrastierung der Prozess der Feinanalyse und der Prozess der Korpusbildung parallel verliefen. Im Folgenden soll der Prozess der Feinanalyse beschrieben werden. Die wissenssoziologische Diskursanalyse ist hierbei ,immer und notwendig ein Prozess hermeneutischer Textauslegung“ (Keller 2007b: 72).

\section{Situiertheit der Aussageereignisse}

Bevor man sich die genauere Struktur der Diskurse anschaut, ist es wichtig, in welchem Kontext der Text entstanden ist bzw. in welchem Zusammenhang der relevante Abschnitt eines Textes steht. Das heißt, der erste Schritt der Analyse ist, folgende Frage zu beantworten: Wer wann wie und für wen die jeweilige Aussage produziert hat. Diese Analyse ist die Grundlage der chronologischen Beschreibung der Debatten (vgl. Kapitel 7 und 9), in denen Durchlässigkeit zwischen Hochschulbildung und Berufsbildung in Deutschland und Frankreich thematisiert wurde. Zudem stellt sie einen Teil der Diskursstruktur dar.

\section{Analysefokus}

Zur Analyse von Diskursen stehen dem Forscher gemäß Keller (2008) verschieden Möglichkeiten zur Verfügung. In dieser Arbeit sollen die sogenannte Phänomenstruktur sowie die story line herausgearbeitet werden.

Nach Keller (2008) gehe ich davon aus, dass Diskurse in der Konstitution ihres Themas unterschiedliche Dimensionen und Elemente ihres Themas benennen, die sich zu einer spezifischen Gestalt oder Konstellation zusammenfassen lassen. Diese spezifische Gestalt nennt Keller Phänomenstruktur. Bereits Karl Mannheim untersuchte die Aspektstruktur von Themen, d.h. die „benutzten Begriffe einschließlich ihrer Bedeutungsdifferenz zu anderen möglichen Begriffen, der Zusammenhang dieser Begriffe, Kausalschemata“" (Mannheim 1969: 234 bei Keller 2008: 248). Damit die Konstruktion eines Themas als Problem auf die öffentliche Agenda kommt, behandeln die Protagonisten immer verschiedene Dimensionen eines Handlungsproblems (Gerhards 1992). Keller (2007b: 99; 2008) zählt beispielsweise folgende Dimensionen auf: „die Bestimmung der Art des Problems oder des Themas, die Benennung von Merkmalen, kausale Zusammenhängen (Ursache-Wirkung) und ihre Verknüpfung mit Zuständigkeiten (Verantwortung)“. Da die tatsächlichen Bausteine sowie die Ausprägungen der Phänomenstruktur nicht vor der Analyse bekannt sind, müssen diese erst am Material erarbeitet werden.

Die in der Problemstruktur herausgearbeiteten Deutungsbausteine eines Diskurs(-strangs) sowie andere analysierbare Bausteine des Interpretationsrepertoires 
wie typische Deutungsmuster werden gemäß Keller durch narrative Muster miteinander verknüpft. Diese Muster nennt Keller $(2008,2009)$ story line ${ }^{57}$. Sie entspricht dem roten Faden, der die anscheinend willkürlichen Äußerungen in einen spezifischen Zusammenhang stellt und verbindet. In dieser Arbeit spreche ich in Anlehnung an das Konzept der story line von Argumentationslogiken, die den Diskurs(-strang) bestimmen und zusammenhalten. Es geht daher dabei weniger um narrative Muster als um argumentative, die dennoch ebenso bestimmend für den Diskurs sein können.

\section{Modellierung der Diskursstruktur}

In einem nächsten Schritt soll gezeigt werden, wie die Struktur der Diskurse erhoben wurde. Hierbei ist wichtig zu betonen, dass es sich bei der wissenssoziologischen Diskursanalyse um ein Verfahren der interpretativen Analytik handelt (Keller 2005). Im Unterschied zu anderen Ansätzen in der qualitativen Forschungsrichtung geht es nicht so sehr um die erschöpfende Analyse eines Dokuments, in dem eine geschlossene konsistente Sinnstruktur rekonstruiert wird, vielmehr werden mehrere Daten zueinander in Beziehung gesetzt (Keller 2005). Denn in Dokumenten können beispielswiese nur Teilstücke oder Diskursfragmente (Jäger 1999) identifiziert werden, die in ihrer Vielzahl und durch ihren spezifischen Zusammenhang die Diskurse bilden.

Bei der Analyse kommt es zu einer ,ko-konstruktiven Leistung des Forschenden, die ihren Ursprung zwar in den Ausgangstexten hat, dennoch aber, Zusammenhänge in den Daten ,neu ordnet ${ }^{6}$ bzw. ,neue 'Gesichtspunkte hervorhebt ${ }^{6}$ (Dausien 2002: 174, Hervorhebung im Original) “ (Truschkat 2008: 79). In diesem Sinn soll in Anlehnung an Truschkat (2008: 78) die Analyse der Diskursstruktur als ein Prozess der Modellierung und damit als „ein Prozess der aktiven, idealtypischen Beschreibung von Zusammenhängen", verstanden werden. Die Benennung von Diskursen und Diskurssträngen zu Durchlässigkeit und die Beschreibung ihrer spezifischen Struktur sind somit Ergebnisse von Modellierungsprozessen.

Dieser Prozess der Modellierung erfolgte in Anlehnung an die Methodologie der Grounded Theory (GTM). Truschkat (2008) unterscheidet eine objektivistische Variante der GTM (z.B. Glaser 1992) und die stärker konstruktivistische (Charmaz 2006) sowie die pragmatische (Strauss/Corbin 1996) Variante, wobei der Unterschied zwischen den letzten beiden Ansätzen als weniger eindeutig beschrieben wird. In der objektivistischen Variante geht Glaser von einer Realität einer externen Welt aus, wobei die Daten Beispiele dieser externen Welt sind und die Kategorien von einem neutralen Beobachter aus den Daten generiert werden

„Während der Begriff des Interpretationsrepertoires das Gesamt der typisierten Grundannahmen eines Diskurses bezeichnet, bezieht sich ,story line' auf die interne Strukturierung des Zusammenhangs dieser Grundannabmen." (Keller 2009: 46f., Hervorhebung im Original) 
(Truschkat 2008). Der neutrale Beobachter soll dabei Vorwissen ausblenden und bezieht seine Informationen nur aus den Daten.

In der konstruktivistischen Variante und ebenso in der pragmatischen wird der Einfluss der Forschenden auf den Forschungsprozess und das Forschungsergebnis viel stärker reflektiert. Es sind die Forschenden, der die Daten konstruieren und mit einem bestimmten Fokus interpretieren. Ohne einen solchen Fokus wäre die zu analysierende Realität nicht zu begrenzen (Truschkat 2008: 71f.). Es ist somit die Forschungsperspektive, die u.a. durch bestimmte theoretische Konzepte, Erfahrungen des Wissenschaftlers, Kenntnisse des Forschungsfeldes bestimmt wird, die die Interpretation anleitet. Truschkat (2008) betont, dass ein solches pragmatistisches bzw. konstruktivistisches Verständnis der GTM besonders für einen diskursanalytischen Ansatz geeignet ist. Wie bereits bei der Korpusbildung beschrieben, kann dann insbesondere das theoretisch-heuristische Konzept von Durchlässigkeit als Beispiel der ,sensibilisierenden Vorannahmen“ (Truschkat 2008: 72) bei der Auswahl der Daten und Modellierung der Diskurstruktur verstanden werden. ${ }^{58}$ Trotzdem bietet die GTM ein systematisches Vorgehen an, das neben diesen Vorannahmen aber eine Offenheit für die Daten und neue Konzepte beinhaltet und somit als ergebnisoffen charakterisiert werden kann. Diese Ergebnisoffenheit kommt durch einen ,iterativ-zyklischen“ Forschungsprozess zustande, in dessen Verlauf die gefunden Kategorien verdichtet werden (Strübing 2004: 47). Dies geschieht vor allem über die Methode des permanenten Vergleichens (Strauss/Corbin 1996). So werden Materialsegmente zum gleichen Gegenstand immer wieder systematisch miteinander verglichen. Ähnlichkeiten und Unterschiede bei der Gegenstandskonstruktion helfen dann, die unterschiedlichen Dimensionen von Kategorien zu erarbeiten.

Die Modellierung in Anlehnung an die GTM erfolgte dabei in mehreren Schritten, die gleichzeitig auch Schritte der Samplingstrategie waren. Strauss und Corbin (1996) unterscheiden einen dreistufigen Kodierprozess, der das offene, das axiale und das selektive Kodieren beinhaltet. In einem ersten Schritt dem offenen Kodieren, werden die Texte gelesen und alle relevanten Passagen zu Durchlässigkeit eingegrenzt. Zudem werden die gefundenen Textabschnitte aufgeschlüsselt und verkodet. Die Kodes beschreiben nah am Material, worum es in dem zu untersuchenden Materialsegment geht. Diese Kodes werden in einem nächsten Schritt in Beziehung zueinander gesetzt, so dass allgemeinere Kategorien gebildet werden können. Handelt es sich z.B. um eine Begründung, warum über Durchlässigkeit geredet wird? Oder wird eine Lösung vorgeschlagen? Auch die Frage des Zusammenhangs, in dem Durchlässigkeit thematisiert wird, wird im Prozess der offenen Kodierung festgehalten. Ziel ist, die Kategorien und ihre Ausprägungen zu ermitteln, die den Diskurs zu Durchlässigkeit bestimmen.

58 An dieser Stelle soll darauf hingewiesen werden, dass die Forschungslogik nicht in allen Schritten auch der Darstellungslogik in dieser Arbeit entspricht. So wurde erst im Verlauf des Forschungsprozesses der Fokus auf Durchlässigkeit eingegrenzt und auch parallel das theoretisch-heuristische Konzept erarbeitet. 
Im Schritt des axialen Kodierens geht es darum, diese Kategorien in Beziehung zueinander zu setzen. Auf diese Art werden die Daten auf eine neue Art zusammengesetzt. In welchem Verhältnis stehen z.B. bestimmte Problemwahrnehmungen zu Lösungsansätzen oder einem bestimmten Verständnis von Durchlässigkeit? Im Rahmen des offenen und des axialen Kodierens konnten somit zuerst inhaltliche Kategorien entwickelt werden, die im axialen Kodieren zu der jeweiligen Phänomenstruktur zusammengefasst wurden. Zudem wurden während dieser Kodierschritte die Ausprägungen der Kategorien verfeinert.

In einem letzten Schritt, dem selektiven Kodieren, wird auf Basis des offenen und axialen Kodierens versucht, Kernkategorien oder Muster zu identifizieren, die das Phänomen erklären. In dieser Arbeit sind das die Identifikation von unterschiedlichen Diskursen bzw. Diskurssträngen, ihrer spezifischen Argumentationslogik, die den gesamten Diskursstrang bestimmt, aber auch der Muster, die die Spezifik des Diskursstrangs ausmachen. Dabei können diese Typisierungen nur durch die bereits herausgearbeitete Phänomenstruktur und den Vergleich der einzelnen Deutungsbausteine der Diskurse vorgenommen werden.

So unterscheide ich im deutschen Fall einen strukturkritischen Diskurs, der aus zwei Diskurssträngen besteht, die jeweils durch eigene Argumentationslogiken, eine funktionalistische und eine universalistische Logik, bestimmt werden, von einem strukturkonservativen Diskurs, der vor allem auf einer funktionalistischen Argumentationslogik beruht. In Frankreich konnte ein hegemonialer reformorientierter Diskurs identifiziert werden, der ebenfalls einen universalistischen und einen funktionalistischen Diskursstrang beinhaltet.

Das Kodieren selbst fand in dieser Arbeit mithilfe der Software Atlas ti statt. Diese ermöglichte es, die Kategorien zu verfeinern und den Analyseprozess zu dokumentieren.

\subsubsection{Theoriegeleitete qualitative Inhaltsanalyse}

Das inhaltsanalytische Vorgehen wurde ausgewählt, um zu zeigen, ob und wie Durchlässigkeit in den europäischen Bildungsprozessen Bologna und Kopenhagen gefördert werden soll und welche konkreten Vorgaben und Vorstellungen überhaupt von europäischer Ebene in die Nationalstaaten diffundieren konnten. Nachfolgend wird daher das Vorgehen inklusive Datenauswahl und Analyseschritte dargestellt.

\section{Datenauswahl}

Gerade in Bezug auf die beiden europäischen Bildungsprozesse Bologna und Kopenhagen gibt es eine große Vielzahl und Vielfalt an Dokumenten. Beispiele sind 
Stocktaking Reports (vgl. Rauhvargers et al. 2009; Zgaga 2003) oder Berichte einzelner nationaler oder europäischer Stakeholder sowie Dokumentationen und Berichte der Arbeitsgruppen der intergouvernementalen Prozesse.

Für die Analyse in dieser Arbeit untersuche ich die europäischen Deklarationen, die zu Beginn der Bologna- und Kopenhagen-Prozesse von nationalstaatlichen Vertretern unterschrieben worden sind. Dabei wurde die Sorbonne-Erklärung, auch wenn sie im Jahr vor dem offiziellen Beginn von Bologna von den vier Ländern Deutschland, Frankreich, Italien und Großbritannien verabschiedet wurde, als Teil des Bologna-Prozesses behandelt und in die Analyse mit einbezogen. Weiterhin besteht das Sample aus den Abschlusskommuniqués der alle zwei Jahre stattfindenden Nachfolgekonferenzen, die wiederum von allen an den Prozessen beteiligten Staaten verabschiedet werden. Insgesamt waren es neun Dokumente für den Bereich der Hochschulbildung und fünf im Bereich der beruflichen Bildung aus dem Zeitraum 1998 bis 2012. ${ }^{59}$ Diese Dokumente definieren gemeinsame Ziele, begründen diese, geben Standards vor, die von den Mitgliedsstaaten auf freiwilliger Basis implementiert werden sollten, und beurteilen, wie die Prozesse sich entwickelt haben.

Gerade, wenn neben der Beschreibung der Prozesse auch ihr Einfluss auf nationale Systeme beurteilt werden soll, ist es wichtig, die Dokumente zu untersuchen, die den Konsens aller Mitgliedsstaaten widerspiegeln und somit auch eine stärkere normative Wirkmächtigkeit haben als Interpretationen nationaler Akteure.

Während die Akteure, geprägt durch ihren jeweiligen nationalen Hintergrund, spezielle Modellvorstellungen und damit verbunden unterschiedliche Perspektiven auf Bildungssysteme haben, stellen die europäischen Erklärungen und Kommuniqués das Resultat von Aushandlungsprozessen über konfligierende Vorstellungen und Perspektiven dar. (Powell et al. 2012a: 443) ${ }^{60}$

59 Folgende Dokumente wurden untersucht: Sorbonne Declaration (1998); Bologna Declaration (1999); Prague Communiqué (2001); Berlin Communiqué (2003); Bergen Communiqué (2005); London Communiqué (2007); Leuven/Louvain-la-Neuve Communiqué (2009); Budapest/Vienna Declaration (2010) und Bucharest Communiqué (2012) sowie Copenhagen Declaration (2002); Maastricht Communiqué (2004); Helsinki Communiqué (2006); Bordeaux Communiqué (2008) und das Bruges Communiqué (2010). Da es nicht alle Dokumente in deutscher Übersetzung gibt und Übersetzungen bereits auch eine stärkere Interpretation bedeuten könnten, wurden ausschließlich die englischen Versionen für die Analyse verwendet, die auch offiziell verabschiedet wurden.

60 Dieser Minimalkonsens ist auch ein Grund, warum ein inhaltsanalytisches und kein diskursanalytisches Verfahren zur Untersuchung der Dokumente gewählt wurde. Die Dokumente sind das Produkt von Aushandlungsprozessen verschiedenster Akteure (Walter 2006). Es ist nicht möglich, die Entstehung der Politiken und der darin wichtigen Akteure nachzuvollziehen. Damit ist unklar, inwiefern es mehrere Diskurse gab und sich einer durchgesetzt hat und welche Konsequenzen dies hat. Diese Fragen müssen für die Fragestellung dieser Arbeit aber auch gar nicht beantwortet werden. Vielmehr soll gezeigt werden, was von europäischer Ebene an Ideen und Vorgaben über die Kommuniqués und Deklarationen in die 


\section{Analyseverfahren und Vorgehen}

Im folgenden Abschnitt soll das inhaltsanalytische Vorgehen beschrieben und begründet werden.

Die qualitative Inhaltsanalyse ist ein Verfahren zur systematischen Beschreibung und Klassifikation von (Text-)Bedeutung. Mayring (2003) unterscheidet vier Formen der qualitativen Inhaltsanalyse: die zusammenfassende Inhaltsanalyse, die explizierende Inhaltsanalyse, die induktive Kategorienbildung und die strukturelle Inhaltsanalyse. In dieser Arbeit wurde die strukturierende Inhaltsanalyse mit induktiver Kategorienbildung verbunden, wobei auch zusammenfassende Elemente vorhanden waren. Letztere dienten der Zusammenfassung durchlässigkeitsrelevanter Textpassagen aus den einzelnen Dokumenten, die für die chronologische Darstellung der Europäisierungsprozesse benutzt wurden (vgl. Kapitel 5). Dabei flossen aber bereits Erkenntnisse aus der strukturierten induktiven Analyse mit ein. Bei dieser Analyse wurde das Material nach bestimmten Kriterien, den institutionellen Dimensionen nach Scott (2008) und den Aspekten von Durchlässigkeit, im Sinne einer strukturierten Inhaltsanalyse untersucht, wobei die Entwicklung von Kategorien sowie die Erarbeitung der Ausprägungen anhand des Textmaterials erfolgte.

Die methodische Vereinigung von beiden Strängen, d.h. die Synthese von Offenheit und theoretischer Vorgehensweise, von Induktion und Deduktion, wird von Gläser und Laudel $(1999,2006)$ auch als theoriegeleitete qualitative Inhaltsanalyse bezeichnet. ${ }^{61}$ Diese methodische Herangehensweise wird als besonders sinnvoll erachtet bei der Analyse von komplexen Untersuchungsgegenständen.

Das Prinzip der Offenheit fordert, daß eine qualitative Untersuchung durch den Untersuchungsgegenstand, das heißt durch die im empirischen Material enthaltenen Informationen, strukturiert werden soll [...]. Die Forderung nach einem theoriegeleiteten Herangehen betont die Notwendigkeit, an vorhandenes theoretisches Wissen über den Untersuchungsgegenstand anzuschließen, da nur so auch zu diesem Wissen beigetragen werden kann. (Gläser/Laudel 1999: 3)

\section{Vier Phasen der Analyse}

Gläser und Laudel (2006) unterscheiden vier Phasen bei einer theoriegeleiteten qualitativen Inhaltsanalyse: Vorbereitung der Extraktion, Prozess der Extraktion, Aufbereitung und Auswertung. Der Kern der Analyse besteht in der Extraktion,

Nationalstaaten diffundieren könnte. Insofern ist ein Verfahren, das nah am Text bleibt und aus diesem gezielt Informationen extrahiert, besser geeignet.

61 Gläser und Laudel (1999, 2006) lehnen sich dabei explizit an Mayrings Verfahren der qualitativen Inhaltsanalyse an. Im Gegensatz aber zu Mayring wird das Kategoriensystem nicht bereits vorher durch einen Probedurchlauf festgelegt, sondern im gesamten Analyseprozess entwickelt. 
d.h. dem Lesen des Textes, der Entscheidung, ob relevante Informationen bestehen, und einer Zuordnung zu Kategorien. ${ }^{62}$ Nachfolgend soll der in dieser Arbeit stattgefundene Analyseprozess anhand der vier Schritte beschrieben werden.

Im Rahmen der Vorbereitung der Datenanalyse wird auf Basis theoretischer Vorüberlegungen und natürlich in engem Bezug zur Fragestellung ein Kategorienschema angelegt, welches aber im Verlauf der Extraktion erweitert werden kann. ${ }^{63}$ Für diese Arbeit waren die institutionellen Dimensionen von Scott (2008) sowie die Durchlässigkeitsaspekte leitend bei der Kategorienentwicklung.

Im Hinblick auf die kulturell-kognitive Dimension von Scott (2008) interessiert, wie und warum Durchlässigkeit thematisiert wird. Welche Vorstellungen von Durchlässigkeit werden sichtbar? Unterschieden habe ich dann anhand des theoretisch-heuristischen Durchlässigkeitskonzepts, ob Fragen des Zugangs, der Anrechnung, der organisationalen Verbindung von Berufs- und Hochschulbildung oder Fragen des Umgangs mit der Heterogenität der Lernenden aus dem jeweils anderen Bildungsbereich thematisiert wurden. Insbesondere in den Argumentationen, warum Durchlässigkeit erreicht werden sollte bzw. warum es ein wichtiges Thema ist, werden dann die zugrunde liegenden Annahmen über das Verständnis und die Funktionen von Bildung sowie das Verhältnis von Berufszu Hochschulbildung für den europäischen Bildungsraum sichtbar.

In der normativen Dimension wird untersucht, ob im Text diskutiert wird, wie Durchlässigkeit zwischen Berufs- und Hochschulbildung erreicht werden kann. Werden Vorgaben gemacht bezüglich Standards, die für Durchlässigkeit einzuführen sind? An dieser Stelle ist auch interessant, in welchem Kontext, d.h. für welche Aspekte letztlich Standards definiert werden. Für die normative Dimension interessiert auch, für wen standardmäßig Durchlässigkeit zwischen Berufs- und Hochschulbildung eingerichtet werden soll.

Antworten auf folgende Fragen sollen dagegen Hinweise auf die regulative Dimension geben. Wer soll die institutionelle Durchlässigkeit national umsetzen, d.h., welche Akteure sind verantwortlich? Wie sollen Strukturen finanziert werden? Es ist insgesamt nicht zu erwarten, dass in den Deklarationen und Kommuniqués aufgrund ihrer fehlenden rechtlichen Verbindlichkeit konkrete Vorgaben für die regulative Umsetzung identifiziert werden.

Neben der Vorstrukturierung des Kategoriensystems gehört in die Phase der Vorbereitung auch die Auswahl der zu analysierenden Daten. Diese Auswahl der europäischen Deklarationen und Kommuniqués habe ich bereits begründet.

62 Aus diesem Grund wird diese Form der Inhaltsanalyse von Schreier (2014: Absatz 43) als „qualitative Inhaltsanalyse mittels Extraktion" bezeichnet.

63 Diese Inhaltsanalyse stellt eine auf Durchlässigkeit fokussierte Weiterentwicklung der im DFGProjekt „Internationalization of Vocational and Higher Education Systems in Transition (INVEST) " durchgeführten Inhaltsanalyse der Deklarationen und Kommuniqués dar (vgl. Powell et al. 2012a). 
Auch die Analyseeinheiten sollen festgelegt werden. Für die Analyse der Deklarationen stellte es sich als am sinnvollsten heraus, Absätze als Analyseeinheiten zu definieren, da diese auch meist inhaltlich kohärent sind.

In der zweiten Phase, der Extraktion, findet die tatsächliche Arbeit mit dem Textmaterial statt. Es wurde nach Abschnitten gesucht, die für Fragen der Durchlässigkeit relevant sind. Wurden solche Abschnitte identifiziert, wurde entschieden, ob die Informationen bereits bestehenden Kategorien und ihren Ausprägungen zugeordnet werden können oder ob neue Kategorien bzw. Ausprägungen festgelegt werden müssen. Die Analyse erfolgte mithilfe der Software Atlas ti, die es ermöglichte, den Kodierprozess mithilfe von Memos und Kommentaren festzuhalten. Auf diese Weise entsprechen zwar die Kodierungen der subjektiven $\mathrm{Zu}-$ ordnung der Forscherin, aber sie sind rekonstruierbar. Die Kategorien, die in dieser Arbeit genutzt wurden, waren dabei sehr breit gewählt ${ }^{64}$. Eine genauere Spezifizierung und auch analytische Bearbeitung der gefundenen Ausprägungen erfolgte erst im nächsten Analyseschritt.

Im Verlauf der Aufbereitung der Daten, der dritten Phase, wurde das kodierte Material nach gleichen Inhalten sortiert. Dies geschah am einfachsten, indem alle Textbausteine, die zu einem Kode gehörten oder auch einer institutionellen Dimension zugerechnet wurden, in einem Extradokument miteinander verglichen wurden. Auf diese Weise wurden Ausprägungen spezifiziert. Dadurch, dass die Kodes durch die Software immer auch in Referenz zum Originaldokument blieben, wurde die Gefahr der Dekontextualisierung minimiert.

Im letzten Schritt der Auswertung wurden die gefundenen Kategorien und ihre Ausprägungen miteinander in Beziehung gesetzt, um das sich in den europäischen Dokumenten darstellende Konzept von Durchlässigkeit entlang der institutionellen Dimensionen zu beschreiben. Ziel war es, aus den Texten herauszukristallisieren, wie Durchlässigkeit verstanden wird und welche Vorstellungen, Normen, Standards und Regeln von europäischer Ebene auf die nationale Ebene diffundieren könnten. Dabei wird nicht unterschieden, wie häufig bestimmte Kategorien Erwähnung fanden. Stattdessen sollte das vermittelte Durchlässigkeitskonzept in der Breite dargestellt werden.

Ecker-Ehrhardt (2006) weist darauf hin, dass bei qualitativen Analysen wie der Inhaltsanalyse zwei Gütekriterien der empirischen Sozialforschung miteinander in Konflikt stehen. Zum einen ist das die Validität als Anspruch, ,das Richtige‘ gültig zu messen, zum anderen die Reliabilität als Anspruch, ,das Richtige، zuverlässig zu messen. Das Konkurrenzverhältnis, das zwischen den beiden besteht, wird besonders deutlich, wenn man an den Prozess der Extraktion, also der Zuordnung von Kategorien zu Textstellen, denkt. Der polyseme Charakter von Sprache ist substanzieller Bestandteil des Untersuchungsgegenstands, so dass schon allein aus diesem Grund eine ganz eindeutige Zuordnung fraglich bleibt. Zugangs“, „Zielgruppe“. 
Es besteht das Problem, dass dadurch, dass der Extraktionsprozess auf der Interpretation der Forschenden beruht, die extrahierten Daten dem Referenzrahmen der Forschenden widerspiegeln. Auch Galonska (2004) betont, dass einer der Hauptkritikpunkte von Inhaltsanalysen die Reliabilität sei, und zwar sowohl in Bezug auf den Interpretationsspielraum der Kodierenden („Inter-Coder-Reliabilität") als auch auf den Lerneffekt während des Kodierens („Intra-Coder-Reliabilität"). Das bedeutet, es könnte zu möglichen Verzerrungen und Verfälschungen im Verlauf der Analyse kommen. Diesem Problem wurde auf mehreren Wegen zu begegnen versucht: zum einen durch die Kombination eines strukturierten und induktiven Vorgehens. So wurde einerseits durch das offene Kodieren von Variablen ein zu leichtfertiges Aussortieren von interessanten Textstellen, die nicht in ein vorgefertigtes Kategoriensystem passen, verhindert. Zum anderen hilft ein möglichst eindeutiges Kategoriensystem mit einer klaren Kodieranleitung. ${ }^{65}$ Das Kodieren bzw. Extrahieren für diese Arbeit wurde aber nur von der Autorin vorgenommen, so dass auf diesem Weg Fehler durch Fremdkodierende nicht auftreten konnten.

\subsubsection{Expert_inneninterviews}

In der Beschreibung des Forschungsdesigns wurde deutlich, dass die Expert_inneninterviews zu zwei Zwecken durchgeführt wurden: zum einen, um ein besseres Verständnis des jeweiligen Falles zu bekommen und das notwendige Kontextwissen für die Interpretation der Akteursdokumente zu erhöhen, und zum anderen, um spezifisch nach den Einflüssen europäischer Prozesse auf die nationalen Durchlässigkeitsstrukturen zu fragen.

Um diese Aufgaben zu erfüllen, können die geführten Expert_inneninterviews als explorative, aber auch als systematisierende Interviews beschrieben werden. Nach Bogner und Menz (2009: 64) helfen explorative Interviews Orientierung in einem neuen Forschungsfeld zu bekommen und das zu untersuchende Phänomen einzugrenzen bzw. zu strukturieren. Systematisierende Interviews zielen dagegen zuerst auf eine systematische und möglichste lückenlose Informationsgewinnung. Die Hauptfunktion des Interviews ist die Aufklärung im Hinblick auf eine spezifische Fragestellung. Mithilfe des Interviews soll so die Rekonstruktion von Prozessen oder sozialen Situationen ermöglicht werden (Bogner/Menz 2009).

65 Da die Inhaltsanalyse in dieser Arbeit auf eine bereits durchgeführte Analyse im Rahmen des INVEST-Projekts aufbaute (vgl. Powell et al. 2012a), konnten zudem auch bereits getestete Kodierregeln und Kategorien verwendet werden. Im Rahmen des Projekts wurden diese Regeln von bis zu vier Personen erarbeitet und geprüft. Durch diese Familiarität bereits intersubjektiv geprüfter Kategorien kann auch für diese Arbeit ein höheres Maß an Reliabilität angenommen werden. 
Was aber ist ein Experte bzw. eine Expertin? Gläser und Laudel (2006: 10) definieren sie und diese Interviewform folgendermaßen: „Experten sind Menschen, die ein besonderes Wissen über soziale Sachverhalte besitzen, und Experteninterviews sind eine Methode, dieses Wissen zu erschließen." Der Expert_innenstatus ist somit immer auch von der Forschungsfrage abhängig. Meuser und Nagel (2009: 37) betonen daher, dass der Expert_innenstatus von den Forschenden vergeben wird, da angenommen wird, dass die Person über ein exklusives Wissen über z.B. die Organisation, Entscheidungsprozesse, Entwicklungen in einem bestimmten Feld verfügt, das für Außenstehende nicht zugänglich ist. Aber auch wenn die Zuschreibung eines Expert_innenstatus diesen erst konstruiert, so weisen Bogner und Menz (2009: 69) darauf hin, „dass der Forscher in seiner Auswahl des Expert_innen immer schon praktisch davon geleitet ist, in welcher Form er die soziale Welt bedeutungsstrukturiert vorfindet." In dem Sinn hat nicht nur das Forschungsthema, sondern auch die soziale Repräsentativität der Interviewten einen Einfluss (Bogner/Menz 2009; Bogner et al. 2014: 11).

\section{Sampling}

Als Expert_innen für diese Arbeit galten Personen, die durch ihre Arbeit in bestimmten Organisationen einen spezifischen Blick auf und Wissen über die berufliche und hochschulische Bildung sowie ihr Verhältnis zueinander, die institutionellen Entwicklungen und die Einflüsse europäischer Bildungsprozesse hatten. Die konkrete Expert_innenauswahl gestaltete sich in Deutschland und Frankreich dabei unterschiedlich. In Frankreich dienten die Interviews vor allem in dem ersten Forschungsaufenthalt 2010 viel stärker der Exploration des Forschungsthemas, so dass zwar auch Repräsentant_innen für die Bildungsbereiche relevanter Organisationen systematisch über Organigramme angeschrieben wurden. Als relevante Organisationen wurden dieselben erachtet, die auch im Rahmen der Diskursanalyse untersucht wurden: Ministerien, Hochschulvertretung, Unternehmen und Gewerkschaften. Neben dieser Taktik wurden aber noch einige weitere Expert_innen über das Verfahren des Schneeballprinzips gewonnen. Außerdem wurden auch Interviews mit im Bildungsbereich Forschenden geführt. ${ }^{66}$ Insofern wurde die Samplingstrategie des Schneeballprinzips mit dem des theoretischen Samplings verbunden. Es wurden 13 Interviews geführt, wobei drei der Interviews mit zwei Interviewpartnern durchgeführt wurden. Im zweiten Forschungsaufenthalt im Mai 2011, als die Forschungsfrage bereits auf Durchlässigkeit begrenzt und auch das für Frankreich bestehende Problem herausgearbeitet war, wurden viel gezielter zusätzliche Expert_innen im Hinblick auf diese Frage und die Europäisierungsprozesse ausgesucht. Zehn Interviews fanden statt, auch hier gab es zwei Interviews mit zwei Expert_innen zusammen. Interviewt wurden vor

66 Eine komplette Liste der interviewten Organisationen in Frankreich und Deutschland findet sich im Anhang (vgl. Tabellen 45-47). 
allem Vertreter_innen aus Ministerien, Hochschulen und dem staatlichen Forschungsinstitut CEREQ. ${ }^{67}$ Im CEREQ wurde mit Forscher_innen gesprochen, die sich mit der Entwicklung des bac pro, der licence pro und der Verberuflichung der Hochschulbildung, den Europäisierungsprozessen und der Frage des Studienerfolgs beschäftigten.

In Deutschland wurden keine explorativen Interviews durchgeführt, da das Bildungssystem und die bestehende Durchlässigkeitsproblematik der Autorin viel besser bekannt waren. Zudem gab es die Möglichkeit, sich bei Fachtagungen genauer über das Forschungsphänomen zu informieren. Ausgesucht wurden daher die Expert_innen aufgrund ihres Wissens über die Entwicklung der Durchlässigkeitsstrukturen in Deutschland sowie der europäischen Bildungsprozesse. Mitarbeiter_innen folgender Organisationen wurden interviewt: das Bundesinstitut für Berufsbildung (BIBB), das Bundesministerium für Bildung und Forschung (BMBF) sowie die Hochschulrektorenkonferenz (HRK). Insgesamt wurden sieben Interviews mit acht Expert_innen geführt. Es fällt auf, dass nur zwei der vier Organisationen untersucht worden sind, die auch als Akteure für die Diskursanalyse selektiert wurden. Zum einen wurde in den Diskursen aller untersuchten Akteure ein Einfluss der europäischen Prozesse ersichtlich. Die Interviews sollten quasi die Erkenntnisse der Diskursanalyse absichern. Dies ist am besten möglich mit Organisationen, die direkt auch an der Gestaltung der europäischen Prozesse und der nationalen Umsetzung beteiligt sind. Das gilt für die ausgesuchten Organisationen im besonderen Maße. Auch ging es bei den Interviews weniger darum, die Diskurse zu rekonstruieren und die unterschiedlichen Positionen der Befragten in den Vordergrund zu stellen. Dies wurde in der Diskursanalyse vorgenommen. Vielmehr ging es darum, Einschätzungen zu den Entwicklungen des Bildungssystems und dem europäischen Einfluss zu erhalten. Das BIBB wurde als Organisation ausgewählt, da es in dieser Organisation viele Expert_innen gibt, die entweder direkt bei der Ausarbeitung der europäischen Prozesse, der Umsetzung der nationalen Prozesse beteiligt waren und zudem bereits langjährig mit dem Thema Durchlässigkeit zwischen Hochschul- und Berufsbildung, sei es über Duale Studiengänge oder Anrechnungsfragen, arbeiten und forschen. Insofern war insbesondere im BIBB eine Expertise über den Einfluss von Europäisierung auf Durchlässigkeit zu erwarten.

67 Das „Centre d'études et de recherches sur les qualifications“ (CEREQ) ist ein staatliches Forschungszentrum mit den Schwerpunkten Arbeitsmarkt, Qualifikationen und berufliche Bildung. Zu den Aufgaben des Instituts gehört es, die Arbeitsmarktentwicklung und die Verbindung der Qualifikationsentwicklung im Bildungssystem mit dieser zu beforschen, um so Maßnahmen und Vorschläge für eine bessere Kohärenz zwischen Qualifikationsentwicklung und Arbeitsmarktbedürfnissen zu entwickeln. 


\section{Interview und Leitfaden}

Die Interviews wurden in Form teilstandardisierter Leitfadeninterviews ${ }^{68}$ durchgeführt. Ein Leitfadeninterview ist dadurch charakterisiert, dass dem Interviewenden eine Liste vorbereiteter, meist offener Fragen als Grundlage für das Gespräch dient, wobei die Reihenfolge der Fragen sich aus Gesprächsverlauf und Wichtigkeit ergeben. Der Leitfaden dient daher als Gesprächsorientierung und soll sicherstellen, dass wesentliche Punkte nicht vergessen werden. Die Konstruktion des Leitfadens ist theoriegeleitet aus der Forschungsfrage, den Vorüberlegungen und in Abhängigkeit von konkreten Expert_innen (Gläser/Laudel 2006). Der Leitfaden für diese Studie bestand aus vier Themenblöcken. Das galt auch für die Phase der Exploration in Frankreich. Nachfolgend sollen diese Themenblöcke kurz beschrieben werden.

In dem ersten Block ging es zum einen um das Verständnis von Durchlässigkeit und die wichtigsten Entwicklungen im deutschen und französischen Bildungssystem in Bezug auf Durchlässigkeit für die letzten 20 bis 25 Jahre. Wie haben sich die Diskussionen um Durchlässigkeit geändert, und was muss von Seiten der Berufs- und Hochschulbildung für eine verbesserte Durchlässigkeit getan werden? Im französischen Leitfaden wurde dabei auch das Wort Durchlässigkeit, welches in Frankreich nicht gängig ist, definiert, so dass die Expert_innen wussten, was gemeint ist, wenn ich nach Durchlässigkeit fragte. Dabei wurde zuerst allgemein über die Möglichkeiten der Bildungsmobilität zwischen Berufs- und Hochschulbildung gesprochen. Auch wenn in den Frage eine erste grobe Definition im Sinne von Strukturen zur Ermöglichung von Bildungsmobilität vorgegeben wurde, konnte trotzdem das Verständnis von Durchlässigkeit untersucht werden, je nachdem, welche Aspekte von den Expert_innen als wichtig erachtet wurden.

Im Leitfaden für die explorativen Interviews für den französischen Fall wurde das Thema noch wesentlich allgemeiner gehalten. Es wurde im ersten Abschnitt nach den wichtigsten Entwicklungen im französischen Hochschul- und Berufsbildungssystem gefragt sowie nach Reformen, die möglicherweise das Verhältnis zwischen Hochschul- und Berufsbildung verändern.

Der zweite Interviewabschnitt zielte auf eine Vertiefung der Durchlässigkeitsfragen und umfasste je nach Expertise unterschiedliche Schwerpunkte. So wurden z.B. die Entwicklung dualer Studiengänge, die Validierung von informellem und non-formalem Lernen, Pilotprogramme und Fragen der Anrechnung thematisiert. Im französischen Teil fand diese Spezialisierung ebenfalls statt. AuBerdem wurde explizit, wenn es nicht schon vorher thematisiert wurde, das Problem der beruflich qualifizierten Studierenden angesprochen, es wurden Einschätzungen zu bestehenden Reformen erfragt und relevante Themen wie die

68 Beispiele für die entwickelten Leitfäden für die Befragungen in Deutschland 2011 und Frankreich 2010 und 2011 finden sich im Online-Anhang II, wobei diese Versionen jeweils auf die interviewten Expert_innen weiter abgestimmt wurden. 
Orientierung in Richtung Hochschule sowie die Selektion im Hochschulsystem angesprochen.

Im Leitfaden für das explorative Interview ging es im zweiten Teil viel stärker darum, ein Gefühl für das französische Verständnis ihres Bildungssystems zu bekommen. So wurde gefragt, was die unterschiedlichen Bildungszweige unterscheidet. Was sind die Ideale der französischen Berufs- und Hochschulbildung bzw. was sind die bestehenden Leitlinien? Wie sind die verschiedenen Bildungswege hierarchisiert? Welche Organisationsformen haben in den Jahren an Bedeutung gewonnen, und inwiefern gibt es Verbindungen und Übergangsmöglichkeiten zwischen gewählten Bildungswegen?

Der dritte Interviewabschnitt zielte darauf, den Einfluss der Europäisierungsprozesse zu erkunden. Hier wurde zuerst allgemein nach dem Einfluss der europäischen Ebene, dann spezieller zu den Bologna- und Kopenhagen-Prozessen und deren Wirkung auf die nationalen Bildungssysteme gefragt. Weiterhin wurden spezifische Standards wie der Qualifikationsrahmen thematisiert. Auch wenn die Interviews vor allem in Bezug auf die Einschätzung der europäischen Prozesse ausgewertet wurden, war es sinnvoll, diesen Interviewabschnitt erst später zu thematisieren. Auf diese Weise wurde auch aus den Beschreibungen der wichtigsten Entwicklungen in den Bildungssystem und der dortigen Thematisierung europäischer Prozesse der Einfluss derselben sichtbar, ohne dass konkret danach gefragt wurde.

Der letzte Interviewabschnitt zielte auf die gewünschten und erwarteten Änderungen im Bildungssystem in Bezug auf Durchlässigkeit, d.h., es wurde die normativ erwünschte und die vom Expert_innenwissen ausgehende wahrscheinliche Entwicklung erfragt.

\section{Auswertung}

Bei der Auswertung orientierte ich mich an den Bearbeitungsphasen von Meuser und Nagel (2009) und integrierte das inhaltsanalytische Vorgehen nach Gläser und Laudel (2006: 56f.). Zuerst erfolgte die Transkription fast aller Interviews. Ausnahmen bildeten zwei wenig ergiebige französische Interviews sowie solche, bei denen keine Aufzeichnung möglich war. Hier erfolgte die Auswertung anhand der Interviewnotizen. Im Folgenden wird die Auswertung im Hinblick auf den Einfluss der Europäisierungsprozesse dargestellt. ${ }^{69}$

69 Die Informationen aus den Interviews, die für ein besseres Verständnis, also zur Exploration genutzt wurden, wurden nicht nach einem bestimmten Auswertungsschema erworben, sondern einfach durch ein wiederholtes Querlesen der Interviewtexte mit anschließenden Notizen zu interessanten Punkten und Hinweise darauf, was im Forschungsverlauf genauer betrachtet werden sollte. Diese Hinweise verdichteten sich, wenn in mehreren Interviews das gleiche Thema angesprochen wurde. Insofern erfolgte auch hier die Analyse nicht sequenziell und Interview für Interview, sondern über den Querschnitt aller Texte. 
Im zweiten Schritt erfolgte die Kodierung des Materials. Der Arbeitsschritt, der bei Meuser und Nagel (2009) Kodierung genannt wird, umfasst die Schritte der Vorbereitung der Extraktion und die Extraktion nach Gläser und Laudel (2006). Auch wenn die einzelnen Interviews nacheinander durchgearbeitet worden sind, ging es nicht um eine Einzelfallrekonstruktion oder genaue sequenzielle Bearbeitung eines Interviews (vgl. Mäuser/Nagel 2009). Vielmehr ging es um die thematisch relevanten Abschnitte, die an verschiedensten Stellen im Interview auftauchen konnten. Kodiert wurden dann die Abschnitte, die sich auf den Einfluss Europas auf die Entwicklungen im Bildungssystem allgemein und spezifisch auf die Durchlässigkeitsstrukturen bezogen. In einem zweiten Schritt wurde analysiert, ob deutlich wurde, welche Prozesse für welche Entwicklungen verantwortlich gemacht wurden, welche Standards mit Durchlässigkeit in Verbindung gebracht und welche Aspekte von Durchlässigkeit nach Meinung der Expert_innen durch Europa beeinflusst wurden. Diese Fragen waren leitend bei der Analyse, aber wie bei der theoriegeleiteten qualitativen Inhaltsanalyse nach Gläser und Laudel (2006) wurde auch offen untersucht, was noch in Bezug auf die europäischen Prozesse und Durchlässigkeit thematisiert worden war.

Der nächste Schritt, die „soziologische Konzeptualisierung“ (Mäuser/Nagel 2009: 57), zielte auf eine Generalisierung der empirischen Daten. Dies geschieht über eine interviewübergreifende Kategorienbildung, wobei die Kategorien zu großen Teilen bereits den im Kodierprozess leitenden Fragen entsprechen. Dieser Prozess beinhaltet damit auch die Schritte der Aufbereitung der Daten und der beginnenden Auswertung nach Gläser und Laudel (2006).

Die „theoretische Generalisierung“ (Meuser/Nagel 2009: 57) ist der letzte Schritt im Auswertungsprozess. Nun erfolgt eine Darstellung der Ergebnisse nicht mehr aus der Logik der Interviews, sondern im Zusammenhang der verwendeten theoretischen Perspektive. Werden von den Expert_innen Diffusionsprozesse von der europäischen Ebene auf die nationale festgestellt? Wo und wie waren sie besonders einflussreich? Welche Verallgemeinerungen lassen sich für den Einfluss der europäischen Bildungsprozesse ablesen? Ist der Einfluss auf bestimmte Durchlässigkeitsaspekte besonders groß? Diese theoretisch unterfütterten Fragen sollten dann anhand der Interviews beantwortet werden (vgl. Kapitel 10).

\subsubsection{Dokumentenanalyse}

Das Analyseverfahren der Dokumentenanalyse ist nach Mayring (2002) breit zu definieren. Es geht bei der Dokumentenanalyse darum, Material zu untersuchen, das nicht erst vom Forschenden erschaffen wird. Die Art der zu untersuchenden Dokumente kann dabei sehr vielfältig sein.

Die Dokumentenanalyse in dieser Arbeit diente zur Analyse der regulativen Entwicklungen. Auch wenn aus forschungspragmatischen Gründen diese primär 
aus der Sekundärliteratur synthetisiert werden sollte, stellte sich heraus, dass dies nicht in Bezug auf alle relevanten Analysefoki möglich war. Insofern wurden Dokumente mit Rechtscharakter untersucht. Die Auswahl der Dokumente erfolgte somit eng orientiert an der Fragestellung und den theoretischen Vorüberlegungen zu den institutionellen Dimensionen.

Tabelle 4 Kategorien für die Dokumentenanalyse

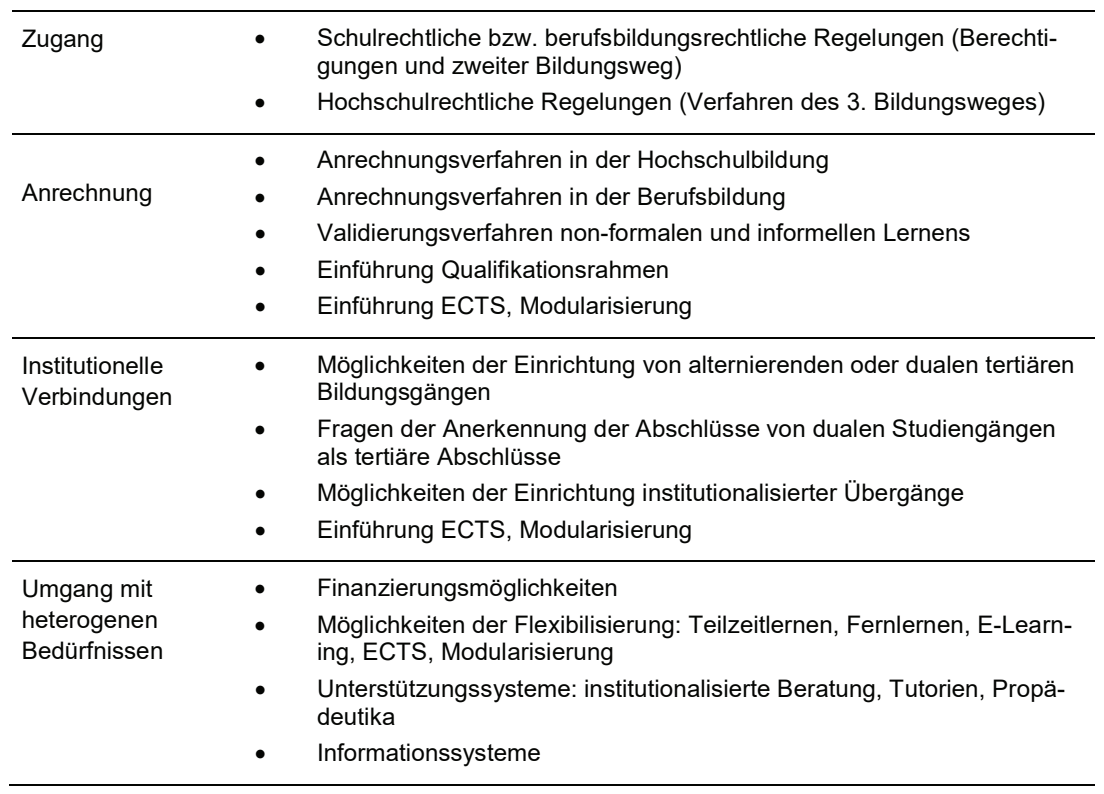

Quelle: Darstellung der Verfasserin

Für den deutschen Fall wurden bundesweite Regelungen untersucht wie das Hochschulrahmengesetz (HRG) oder das Berufsbildungsgesetz (BiGG) genauso wie die Beschlüsse der KMK, die als Richtlinien für die Schul- und Hochschulgesetze der Länder gelten. Auch wenn diese nicht rechtlich bindend sind (Ulbricht 2012a), setzen sie doch starke regulative Anreize. Aus diesem Grund werden KMK-Beschlüsse im Folgenden auch bei der regulativen Analyse einbezogen. Auch Regelungen der Hochschulgesetze der Bundesländer wurden berücksichtigt $^{70}$. Für Frankreich, wo die richtungsgebenden Entscheidungen über die Bildungsprogramme vor allem auf Zentralstaatsebene entschieden werden, fokussierte ich mich auf die nationalen Gesetzestexte (loi) und Verordnungen (décret und

70 Ein Überblick über die untersuchten Dokumente für die Regulative findet sich im OnlineAnhang, wobei die Dokumente der KMK auch als Dokumente der Diskursanalyse miteingegangen sind (vgl. auch untersuchte Dokumente für die Diskursanalyse im OnlineAnhang III). 
arrèté) der Bildungsministerien. Auch hier war die Sekundärliteratur aber die erste Quelle zur Rekonstruktion der rechtlichen Entwicklung ${ }^{71}$.

Der Fokus der Analyse lag auf den vier Aspekten von Durchlässigkeit, d.h., die Sekundärliteratur und rechtlichen Dokumente wurden nach Regelungen zum Zugang und zur Anrechnung, nach der organisationalen Verbindung sowie in Bezug auf den Umgang mit Heterogenität untersucht. Aufbauend auf diesen vier Aspekten und dem in Kapitel 2 dargestellten Verständnis der Aspekte sowie den in Kapitel 5 herausgearbeiteten europäischen Forderungen wurden grobe Kategorien gebildet, nach denen dann die Dokumente durchsucht wurden.

Bei der Untersuchung der rechtlichen Dokumente wurde immer darauf geachtet, dass die Regelungen für die untersuchte Zielgruppe gelten. So wurde z.B. explizit nach Finanzierungsmöglichkeiten für beruflich Qualifizierte, die ein Hochschulstudium aufnehmen wollen, gesucht.

\subsection{Güte der Arbeit}

Nachdem das Forschungsdesign und die genauen Erhebungs- und Analyseverfahren dargestellt wurden, sollen im Folgenden Überlegungen zur Güte dieser Forschungsarbeit, die nur eine Darstellung der Wirklichkeit sein kann und keine Abbildung, vorgenommen werden.

Gerade für die qualitative Forschung wird die Frage der Gütekriterien eingehend diskutiert (vgl. Flick 1998, 2014; Freikamp 2008; Mayring 2002; Steinke 1999), da diese nicht so deutlich an Maßzahlen festgehalten werden kann wie in quantitativer Forschung. Auch gibt es verschiedene Herangehensweisen, wie die Qualität qualitativer Forschung gesichert werden kann (vgl. Flick 1998, 2014; Freikamp 2008). Folgende Kriterien sollen mit Bezug zu meiner Arbeit betrachtet werden: intersubjektive Nachvollziehbarkeit (Steinke 1999), Indikation (Steinke 1999), Triangulation (Flick 2014; Mayring 2002) sowie „reflektierte Subjektivität“ (Steinke 1999: 231).

„Intersubjektive Nachvollziehbarkeit“, d.h., dass der Forschungsprozess vom Publikum bzw. Lesenden nachvollzogen werden kann, definiert Steinke (1999: 207) als wichtigstes Kriterium zur Sicherung der Qualität qualitativer Arbeiten. Dies kann erreicht werden über eine genaue Dokumentation des Vorgehens, die Anwendung regelgeleiteter Verfahren und die Interpretation in Gruppen. Die ersten beiden Punkte entsprechen auch Kriterien von Mayring (2002). In dieser Arbeit soll das Kapitel zum Design und Methoden diese größere intersubjektive Nachvollziehbarkeit ermöglichen, da sowohl eine Beschreibung des

71 Eine Übersicht über die wenigen konsultierten französischen Gesetzestexte findet sich im Online-Anhang IV. 
Forschungsdesigns und Forschungsprozesses als auch der Regeln, die im Forschungsprozess befolgt worden sind (z.B. systematisches Vorgehen bei der Analyse sowie bei der Sampleerstellung und der Kodierung in der Diskursanalyse), dokumentiert wurden. Festzuhalten ist aber auch, dass z.B. das Vorgehen bei der Kodierung für die Diskursanalyse nur teilweise dem Ideal nach GTM entsprach, da durch die begrenzte Zeit der Forschungsaufenthalte in Frankreich kein permanenter Wechsel zwischen Analyse und Dokumentenauswahl möglich war.

Die Interpretation des Materials in Gruppen konnte in dieser Arbeit nur vereinzelt stattfinden. Durchgeführt wurden zwei solcher Gruppeninterpretation im Rahmen einer qualitativen Forschungswerkstatt einerseits und als Interpretationstandems mit jeweils einer weiteren Person andererseits. Diese Gruppeninterpretation erfolgte dabei ausschließlich im Rahmen der Diskursanalyse, und auch nur für ausgewählte deutsche und zwei französische Schlüsseldokumente. Die gemeinsame Interpretation half dabei, Deutungen und Verständnisse zu hinterfragen, aber auch zu verfestigen. Wie bereits bei der qualitativen Inhaltsanalyse beschrieben, wurde die Analyse zwar allein durchgeführt, baute aber auf vorherigen Gruppeninterpretationen im Rahmen des Projektkontextes auf, so dass auch dadurch eine größerer intersubjektive Nachvollziehbarkeit erreicht worden ist. Die Möglichkeit des Nachvollziehens der Interpretation und Argumentation wird in dieser Arbeit zudem durch empirische Beispiele in Form von Zitaten ermöglicht.

Ein weiterer Punkt, den Steinke (1999) betont, ist die Indikation, d.h. die Frage, inwiefern der gesamte Forschungsprozess auch gegenstandsangemessen erfolgte. Begründet wurde die Angemessenheit für diese Arbeit in diesem Kapitel vor allem in der Beschreibung des Forschungsdesigns und der Anlage der Untersuchung sowie den jeweiligen Samplingstrategien.

Mit Triangulation weist Mayring (2002) in Anlehnung an Denzin (1978) darauf hin, dass Triangulation die Qualität der Forschung vergrößert, da versucht wird, über verschiedene Datenquellen, Methoden, Interpreten und theoretische Ansätze für die Forschungsfrage unterschiedliche Lösungsansätze zur vollständigeren Beantwortung der Fragen zu finden. Aus diesem Grund wurden für die Analyse der institutionellen Entwicklungen in Deutschland und Frankreich z.B. sowohl die Diskurse als auch die regulativen Strukturen anhand der Dokumentenanalyse untersucht. Um den Einfluss der europäischen Prozesse besser einschätzen zu können, wurde neben der untersuchten Entwicklung der Durchlässigkeitsstrukturen und deren Abgleich mit den geforderten europäischen Standards, die mittels Inhaltsanalyse herausgearbeitet wurden, Hinweise auf Europäisierungseinflüsse in den Diskursen gesucht und auch Expert_inneninterviews durchgeführt.Letztere können auch als kommunikative Validierung von Ergebnisen angesehen werden (Flick 2014: 415). Triangulation, wie bereits auch im Abschnitt zum Forschungsdesign beschrieben, ist demnach ein zentrales Merkmal dieser Studie. 
Schließlich betont Steinke (1999: 231) das Prinzip der „reflektierten Subjektivität", dass im gesamten Forschungsprozess die Rolle des Forschenden selbst als integrativer Bestandteil dieses Prozesses und des Ergebnisses der Forschung reflektiert werden soll. Im Fokus stehen Selbstbeobachtungen und -erkenntnisse im Forschungsverlauf sowie persönliche Voraussetzungen des Forschenden. Auch Beziehungen zu den Interviewpartner_innen könnten hier mit bedacht werden. Neben den Reflexionen zur möglichen Auswahl von Methoden und der Samplingstrategie, die in diesem Kapitel auch deutlich gemacht worden sind, sind drei weitere Punkte wichtig, da sie Interpretationsmöglichkeiten der Forscherin dieser Arbeit beeinflussen.

Erstens muss die Biografie der Forscherin betrachtet werden. Sie ist in Deutschland aufgewachsen und hat einen Großteil ihrer hochschulischen Ausbildung in Deutschland verbracht. Aus dieser biografischen Situiertheit ergibt sich eine viel bessere Kenntnis des deutschen Bildungssystems und der deutschen Sprache und Kultur als des französischen Pendants. Problematisch wird dies vor allem bei der Diskursanalyse, da dort auch kulturelle Feinheiten eine Rolle spielen. Es ist anzunehmen, dass in der französischen Analyse nicht alle diese Feinheiten identifiziert wurden. Diesem Problem wurde auf zweierlei Weise begegnet: einmal durch das explorative Vorgehen im ersten Forschungsaufenthalt in Frankreich, in dem versucht wurde, ein größeres Verständnis für diese kulturellen Feinheiten und Selbstverständnisse zu erhalten Zudem war das französische Hochschulsystem durch einen halbjährigen Studienaufenthalt während des Studiums der Forscherin bekannt. Auch war es der Forscherin möglich, während ihrer Forschungsaufenthalte bei Unklarheiten Französ_innen um die Interpretation des untersuchten Problems zu bitten, so dass dieser „deutsche Bias“ abgeschwächt wurde.

Zweitens ergibt sich aus der biografischen Situiertheit nicht nur ein Bias im Hinblick auf das Verständnis der Landesspezifika, auch der rein akademischer Hintergrund könnte bei der Interpretation der Dokumente und Entwicklungen dazu führen, dass bestimmte Elemente wichtiger bewertet werden oder Selbstverständlichkeiten der Berufsbildung unentdeckt bleiben. Diesem Problem wurde vor allem durch eine sehr gute Kenntnis der Berufsbildungssysteme, die Gruppeninterpretationen und den Besuch auch von Fachtagungen zu begegnen versucht.

Schließlich ist die Frage nach Durchlässigkeit zwischen Berufs- und Hochschulbildung stark inspiriert durch die Kenntnis des deutschen Falls. Es könnte sein, dass durch eine, zu deutsche Brille vielleicht andere wichtige Probleme für das Verhältnis zwischen Berufs- und Hochschulbildung in Frankreich nicht ausreichend Beachtung fanden. Andererseits sollte genau der zweimalige Forschungsaufenthalt mit einer ersten Exploration und Zuschneidung des Themas und einer Vertiefung anschließend einer verkürzten Sicht auf die französische Problematik vorbeugen. Auch wenn die Durchlässigkeitsproblematik in Frankreich nicht so offensichtlich ist wie in Deutschland, konnte im Rahmen dieser Arbeit eine solche für Frankreich herausgearbeitet werden. Dies kann auch als 
Stärke dieser Arbeit gelten, da zwar das Problem der Studienabbrüche bekannt ist, dieses aber nicht unbedingt als ein Problem von Durchlässigkeit bzw. eines in Bezug auf das bestehenden Verhältnis der Berufs- zur höheren Allgemeinbildung untersucht wird. 



\section{Durchlässigkeit - ein Fokus europäischer Bildungspolitik?}

In diesem Kapitel sollen die europäischen Entwicklungen im Mittelpunkt stehen. Dabei wird zum einen die Bildungspolitik bis zu den Bologna- und KopenhagenProzessen dargestellt, da die historische Gewachsenheit der europäischen Politiken ebenfalls die Evolution der Bologna- und Kopenhagen-Prozesse mitbestimmt. Auf diese Weise soll gezeigt werden, wie berufliche Bildung, Allgemeinund Hochschulbildung auf EU-Ebene auf die Agenda kamen und in welchem Verhältnis die Bildungsbereiche zueinander institutionalisiert wurden. Es werden die Bologna- und Kopenhagen-Prozesse sowie deren generelle Ausrichtung und Einbettung in der europäischen Bildungspolitik beschrieben. Gleichfalls sollen auch wichtige Instrumente oder Schlüsselbegriffe näher erläutert werden. Dieser Abschnitt soll zeigen, warum die Bologna- und Kopenhagen-Prozesse als besonders einflussreich im Hinblick auf Durchlässigkeitsstrukturen, welche das Verhältnis von Berufs- und Hochschulbildung mitbestimmen, gelten können.

In einem zweiten Schritt werden die Bologna- und Kopenhagen-Prozesse genauer daraufhin untersucht, inwiefern Durchlässigkeit zwischen Berufs- und Hochschulbildung in ihnen thematisiert wird. Diese Analyse beruht auf einer qualitativen Inhaltsanalyse der verabschiedeten Deklarationen und Kommuniqués beider Prozesse. So wird zunächst chronologisch dargestellt, wie in den einzelnen Dokumenten das Thema insgesamt thematisiert wurde. Darauf aufbauend wird analysiert, wie jeweils in dem Bologna- und Kopenhagen-Prozess Durchlässigkeit entlang der institutionellen Analysekategorien eingefordert wird. So interessiert, wie Durchlässigkeit verstanden und begründet wird oder mithilfe welcher Standards sie umgesetzt werden. Schließlich werden die beiden Prozesse verglichen und untersucht, inwiefern die jeweils analysierten institutionellen Dimensionen zu einem europäischen Durchlässigkeitsmodell integriert werden können. Das Ziel des Kapitels besteht darin, ein Bild davon zu erhalten, wie die Bologna- und Kopenhagen-Prozesse in die supranationale europäische Bildungspolitik eingebettet sind, welchen Stellenwert Durchlässigkeit zwischen Berufs- und Hochschulbildung einnimmt und welches Modell von Durchlässigkeit durch die Prozesse in die Nationalstaaten diffundieren könnte, d.h., welche Art von Durchlässigkeitsstrukturen durch die Prozesse legitimiert werden. 


\subsection{Berufs- und Hochschulbildung in der europäischen Bildungspolitik - ein historischer Überblick}

Die Entwicklung der europäischen Bildungspolitik wird häufig in drei Phasen eingeteilt: die erste bis zum Vertrag von Maastricht 1993, die zweite bis 1999 oder 2000 dem Beginn des Bologna-Prozesses bzw. der Lissabon-Strategie und die dritte startend mit den Bologna- und Kopenhagen-Prozessen (vgl. Becker/Primova 2009; Cort 2009; Powell/Trampusch 2012; Walkenhorst 2008). Jener Unterteilung wird an dieser Stelle weitgehend gefolgt - zuerst werden die Jahre bis zum Beginn des Bologna-Prozesses und in einem zweiten Abschnitt die Bologna- und Kopenhagen-Prozesse näher sowie deren Einbettung in der weiteren europäischen Bildungspolitik dargestellt.

\subsubsection{Eutopäische Bildungspolitik 1951-2000}

\section{Entwicklungen bis 1992}

Im Folgenden wird in der entsprechenden Kürze die Entwicklung der europäischen Bildungspolitik bis zu den Bologna- und Kopenhagen-Prozessen dargestellt - mit zwei Schwerpunkten: Der erste ist aufzuzeigen, inwiefern berufliche Bildung sowie Hochschul- und Allgemeinbildung auf die europäische Agenda kamen. Des Weiteren wird das Verhältnis von beruflicher Bildung und Hochschulbildung in der europäischen Bildungspolitik untersucht.

Bereits vor den Römischen Verträgen ${ }^{72} 1957$ war Anerkennung von beruflichen Qualifikationen und berufliche Mobilität ein Thema in der Europäischen Gemeinschaft für Kohle und Stahl (EGKS), wenn auch nur in der Kohle- und Stahlindustrie. Diese sektorspezifische Herangehensweise wurde auch nach 1957 beibehalten und zwar für die Bereiche, welche nach und nach als Gemeinschaftspolitikfelder aufgenommen wurden (z.B. Landwirtschaft in Artikel 41 des EWGVertrages). Ziel war jeweils, die Mobilität der Arbeitnehmer_innen in diesen Sektoren durch Anerkennung ihrer Qualifikationen zu ermöglichen (vgl. Cort 2009).

Mit der Gründung der Europäischen Wirtschaftsgemeinschaft (EWG) 1957 wurde Berufsbildung im Artikel 128 demgemäß als Gemeinschaftspolitikfeld aufgenommen, ganz im Gegensatz zur Hochschulbildung und Allgemeinbildung, welche keine Erwähnung fanden (Ertl 2003). Der Europäische Rat in Zusammenarbeit mit der Europäischen Kommission sollte allgemeine Prinzipien für eine gemeinsame europäische berufliche Bildungspolitik festlegen.

72 Gegründet wurde sowohl die europäische Wirtschaftsgemeinschaft (EWG) als auch die europäische Atomgemeinschaft (EURATOM). 
The Council shall, acting on a proposal from the Commission and after consulting the Economic and Social Committee, lay down general principles for implementing a common vocational training policy capable of contributing to the harmonious development both of the national economies and of the common market. (EWG-Vertrag 1957: 128)

Zudem wurde bereits in Artikel 118 des EWG Vertrags auf die Notwendigkeit der Förderung von Berufs- und Weiterbildung in den Mitgliedsstaaten als sozialpolitische Maßnahme hingewiesen, welche die Europäische Kommission in enger Zusammenarbeit mit den Mitgliedsstaaten fördern soll (Petrini 2004). Auch die Frage der Anerkennung von Abschlüssen wurde im EWG-Vertrag im Artikel 57 bereits verhandelt (Ertl 2003).

In den folgenden Jahren versuchte nun vor allem die Kommission - auf Basis des EWG-Vertrags - ihren Einfluss in der beruflichen Bildung auszuweiten, mit dem Ziel der Harmonisierung der beruflichen Bildung in Europa. Die Mitgliedsstaaten wehrten sich allerdings, da sie ein derartiges Vorgehen als Eingriff in ihre Souveränität betrachteten (Cort 2009). Insbesondere Deutschland und Frankreich verhinderten die von der Kommission initiierten weitreichenden Integrationsvorschläge im Bereich der Berufsbildung (Petrini 2004). Ungeachtet der Verabschiedung eines umfassenden von der Kommission initiierten 10 PunkteGrundsatzprogramms für die Durchführung einer gemeinsamen Politik der Berufsausbildung (siehe Petrini 2004: 53f.) durch den Europäischen Rat 1963 wurde daher nicht viel im Bereich der beruflichen Bildung bewegt (vgl. Becker/Primova 2009; Cort 2009; Ertl 2003). Diese Entwicklung wurde zudem verstärkt, da die Generaldirektion, welche für Berufsbildung zuständig war, der traditionellen Dichotomie von Allgemeinbildung und Berufsbildung folgte und letztere insbesondere aus wirtschaftlichen Überlegungen heraus betrachtete (Ertl 2003: 17).

Bereits Ende der 1960er und insbesondere auch in den 1970ern waren es jedoch die Mitgliedsstaaten und nicht mehr die Kommission, welche eine verstärkte gemeinsame europäische Politik im Bereich der beruflichen Bildung vorantrieben, da wirtschaftliche Probleme und Arbeitslosigkeit, insbesondere auch Jugendarbeitslosigkeit, gemeinsame Herausforderungen darstellten (vgl. Cort 2009; Ertl 2003; Petrini 2004). So fand 1974 das erste Bildungsministertreffen im Europäischen Rat statt, wodurch Bildungspolitik auf europäischer Ebene weiter institutionalisiert wurde (Balzer/Rusconi 2007). In ihrer gemeinsamen Resolution zur Kooperation im Bereich Bildung wurde u.a. festgehalten, dass die bildungspolitische Zusammenarbeit nicht nur als Bestandteil der wirtschaftlichen Entwicklung der Gemeinschaft gesehen werden könne. Bildungszusammenarbeit findet jedoch vornehmlich im Rahmen der Harmonisierungsbestrebungen in der gemeinsamen Wirtschafts- und Sozialpolitik statt, wobei die nationalen Traditionen im Bildungsbereich gewahrt werden sollten. Harmonisierung im Bildungswesen wurde dagegen explizit nicht als Ziel der gemeinsamen Kooperation angesehen. „Harmonization of these systems or policies cannot, therefore, be considered an end in itself“" (European Ministers Responsible for Education 1974). 
Ziele einer europäischen Bildungszusammenarbeit waren die Verbesserung der Korrespondenz der Bildungssysteme in Europa, verbesserte Statistiken und Dokumentationen über die Bildungssysteme, die Verstärkung der Zusammenarbeit der Hochschulen in Europa, verbesserte Möglichkeiten einer Anerkennung von Abschlüssen und Studienzeiten sowie eine verstärkte Bildungsmobilität und schließlich der chancengleiche Zugang zu Bildungseinrichtungen für alle Bürger (European Ministers Responsible for Education 1974). An ebendieser Stelle ist auffällig, dass obwohl Hochschulbildung nicht zum vertraglich festgelegten Aufgabengebiet der europäischen Gemeinschaft gehörte, sie trotzdem verstärkt auf die politische Agenda gelangte.

Während zu Beginn allein von beruflicher Bildung die Rede war, wurde am Anfang der 1970er Jahre in den europäischen Dokumenten darauf hingewiesen, dass sowohl Allgemeinbildung als auch berufliche Bildung wichtige Bereiche sind, um den gemeinsamen Markt zu realisieren. Aber auch mit der Frage von Übergängen von der Schule in den Arbeitsmarkt wurde Allgemeinbildung in die Politikdokumente als Thema aufgenommen, ebenso wie „permanent education“, was sich später zur Thematik lebenslanges Lernen entwickelte (Cort 2009). Nach und nach wurden somit die Bildungsbereiche, wenn auch nicht vertraglich festgehalten, von der Berufsbildung auf Allgemein- und Hochschulbildung ausgeweitet.

Bereits 1973 wurde von einer Sachverständigengruppe unter Führung des Belgiers Henri Janne der sogenannte ,Janne Report" verfasst. In ihm wurde ebenfalls die „Notwendigkeit der Überwindung der Teilung zwischen beruflicher und allgemeiner Bildung im Gemeinschaftsrecht betont" (Becker/Primova 2009: 6). Ertl (2003) weist darauf hin, dass der Janne Report die Trennung von Berufs- und Allgemeinbildung als größtes Hindernis einer gemeinsamen europäischen Politik in beiden Bildungsbereichen darstellt. Dieser Report bildete den Grundstein für die nachfolgenden europäischen Bildungsprogramme (Becker/Primova 2009). Im gleichen Jahr wurde ebenfalls die erste Generaldirektion für Forschung, Wissenschaft und Bildungspolitik unter der Führung des deutschen Kommissars Lord Dahrendorf gegründet. Die Institutionalisierung der europäischen Bildungspolitik fand somit nicht nur auf intergouvernementaler Ebene mit dem ersten Europäischen Rat der Bildungsminister 1974 sondern auch auf supranationaler Ebene statt.

Das erste Aktionsprogramm im Bereich Bildung wurde 1976 ins Leben gerufen und war prägend für die Entwicklung der Bildungspolitik auf europäischer Ebene in den folgenden Jahren (Becker/Primova 2009). Auch wurde Bildungspolitik durch diesen Beschluss der Minister zu einem legitimen Politikfeld der EWG. Dieses Aktionsprogramm beinhaltete bereits Reformvorschläge für den Bereich der Vorschule bis hin zum Hochschulstudium, wobei letzteres einen Schwerpunkt bildete. So wurde die Notwendigkeit der Zusammenarbeit ebenfalls auf dem Gebiet des Hochschulwesens betont. Zu fördern sei Freizügigkeit und Mobilität von Studierenden sowie die Anerkennung von Studienabschlüssen und Leistungen. Schließlich sei auch die 
Verwirklichung der Chancengleichheit für den uneingeschränkten Zugang zu allen Bildungsformen [...] ein wesentliches Ziel der Bildungspolitik aller Mitgliedsstaaten, und ihre Bedeutung für die Erreichung der Chancengleichheit in der Gesellschaft muß in Verbindung mit der übrigen Politik im wirtschaftlichen und sozialen Rahmen betont werden. (Aktionsprogramm 1976)

Mit diesem Programm wird Bildungspolitik erstmals nicht mehr nur auf die wirtschaftliche Integration begrenzt, sondern zielt auch auf eine Entwicklung der sozialen Kohäsion und europäischen Kultur ab (Becker/Primova 2009: 6). So ist es gleichfalls nicht verwunderlich, dass mit der Zunahme der Bedeutung von Hochschulbildung zudem 1976 das Europäische Hochschulinstitut in Florenz seine Türen öffnete.

Aber auch wenn sich in den 1970er Jahren der Politikbereich der beruflichen Bildung durch die Einführung der Programmmethode in Europa vergrößerte, Allgemeinbildung inklusive Hochschul- und Weiterbildung versucht wurde mit einzubeziehen, wurde andererseits von den Mitgliedsländern eine weitergehende Kooperation im Bereich der Allgemeinbildung kontinuierlich zurückgewiesen (Cort 2009). Die tatsächliche Ausweitung der Kompetenzen auf den Bereich der Allgemeinbildung, inklusive der Hochschulbildung ist vornehmlich eine Entwicklung, welche durch wegweisende Urteile des Europäischen Gerichtshofs (EUGH) vorangetrieben wurde (Becker/Primova 2009; Cort 2009; Ertl 2003). Seit dem Urteil des EUGH im Fall „Gravier gegen die Stadt Lüttich“ 1985 wurde der Politikbereich der beruflichen Bildung ausgeweitet, und zwar auf die Hochschulbildung, da auch Hochschulkurse als berufliche Bildung definiert wurden ${ }^{73}$. Der Gerichtshof entschied, dass der Freizügigkeitsgrundsatz für Arbeitnehmer_innen ebenfalls auf Studierende als künftige Arbeitnehmer_innen angewendet werden sollte.

Das Gravier-Urteil war der tatsächliche Beginn der europäischen Hochschulpolitik, die trotz des Urteils nicht in den Bereich der beruflichen Bildung aufgenommen, sondern parallel dazu weiterentwickelt wurde, mit eigenen Gemeinschaftsprogrammen, Prozessen, wie z.B. der Einführung des Erasmusprogramms 1987 (Cort 2009). Am Ende der 1980er Jahre wurde eine Reihe von Programmen sowohl in beruflicher Bildung als auch in Hochschul- und Weiterbildung von der Europäischen Gemeinschaft (EG) ins Leben gerufen. Es kam somit zu einer Konsolidierung aller drei Bildungsbereiche unter Artikel 128 der Römischen Verträge (Cort 2009). Trotz dieser ,schleichenden Vergemeinschaftung bildungspolitischer Zuständigkeiten“ (Martens/Wolf 2006: 153) blieben die Auswirkungen auf die nationalen Bildungssysteme bis dahin gering (Ertl 2003).

Die gegenseitige Anerkennung von Berufsabschlüssen war seit Beginn der europäischen Zusammenarbeit ein gemeinsames Ziel - schon allein weil dies eine Voraussetzung für Freizügigkeit ist (vgl. Berggreen-Merkel 2006). Mit der Unterzeichnung der Einheitlichen Europäischen Akte 1986, in welcher die Mitgliedsstaaten sich auf die Finalisierung des Gemeinsamen Markts bis 1992 einigten, kam

Weitere Urteile sind in Becker/Primova 2009 sowie Ertl 2003 nachzulesen. 
diese Frage jedoch wieder verstärkt auf die Agenda. So wurden Anerkennungsrichtlinien sowohl im Hochschulbereich als auch im Bereich der beruflichen Bildung eingeführt (vgl. Graf 2013), die zuerst arbeitsmarktsektorspezifisch eingeführt und später zumindest im Hochschulbereich verallgemeinert wurden (Teichler 1990a).

\section{Entwicklungen 1992 bis 1998}

Obwohl oder gerade weil die Europäische Kommission ihren Einfluss im Bildungsbereich zumindest indirekt mit der Programmmethode und den Anerkennungsrichtlinien kontinuierlich weiter ausgebaut hatte, wurde im Vertrag vom Maastricht, dem Gründungsvertrag der Europäischen Union (EU), die Aufgabenbereiche der EU und der Mitgliedsstaaten klar geregelt (vgl. Busemeyer 2009; Cort 2009; Martens/Wolf 2006). Insgesamt kann konstatiert werden, dass der Vertrag von Maastricht den bestehenden Pluralismus der Bildungssysteme in Europa schützen soll. Harmonisierung der Bildungssysteme wird ausdrücklich ausgeschlossen. Ziel ist stattdessen seitdem eher die Schaffung von Transparenz und Vergleichbarkeit der Bildungssysteme. Walkenhorst (2008) beschreibt die zweite Periode als Interimsperiode, in welcher Bildung zwar relevant war, jedoch andere Probleme, wie die Wirtschafts- und Währungsunion, noch eindeutig dominierten.

Seit dem Vertrag von Maastricht wird mit dem Artikel 126 (heute Artikel 149) zum ersten Mal auch Allgemeinbildung in den Kompetenzbereich der EU aufgenommen, wobei der Kommission hier nur eine Unterstützungsfunktion zukommt. Ziel ist die „Entwicklung einer qualitativ hochstehenden Bildung“ wobei die alleinige Verantwortung ,für die Lehrinhalte und die Gestaltung des Bildungssystems sowie [die] Vielfalt ihrer Kulturen und Sprachen“" bei den Mitgliedsländern liegt (Artikel 126 EGV). Artikel 127 des EGV wurde als Ersatz für den bisherigen stark umstrittenen Artikel 128 der Römischen Verträge eingeführt. Gemäß Ertl (2003) stellt er eine Ausweitung der Kompetenzen der EU dar. Denn während zuvor nur allgemeingültige Prinzipien aufgestellt werden durften, stehen der EU jetzt zu, ebenso Maßnahmen zur Erreichung einer gemeinsamen Berufsbildungspolitik zu erlassen. Ungeachtet der Ausweitung der Kompetenzen einerseits wird der Handlungsfähigkeit der EU durch das eingeführte Prinzip der Subsidiarität ${ }^{74}$ enge Grenzen gesetzt. So unterstreicht auch Busemeyer (2009: 3) dass die Aufnahme von Bildungspolitik in den Vertrag von Maastricht bedeutete, , dass nun zum ersten Mal der Europäischen Union formale Kompetenzen im Bereich der Bildungspolitik zuerkannt wurden, auch wenn diese im Vergleich zu den zuvor faktisch angeeigneten zunächst eingeschränkt waren." Bildungspolitik kam

„Der Maastrichter Vertrag von 1992 nimmt das Subsidiaritätsprinzip als allgemeine Maxime in die Verträge auf: Art. 5 EG-Vertrag bestimmt, dass die Gemeinschaft in Bereichen, die nicht in ihre ausschließliche Zuständigkeit fallen, nur tätig wird, sofern und soweit die Ziele der in Betracht gezogenen Maßnahmen auf der Ebene der Mitgliedsstaaten nicht ausreichend und daher auf europäischer Ebene besser erreicht werden können. “ (bpb Lexikon 2014) 
demnach in die supranationale Säule der EU, wobei bei Fragen der Allgemeinbildung die bildungspolitischen Instrumente auf transgouvernementale Kooperationen begrenzt wurden (Walkenhorst 2008).

Während bis zu den 1990er Jahren die Bildungsbereiche Berufs- und höhere Allgemeinbildung noch weitgehend getrennt behandelt wurden, änderte sich dies nach 1990 trotz der getrennten Artikel im Vertrag von Maastricht, als die Bereiche zu einer Politik des Lebenslangen Lernens integriert wurden (Cort 2009). ${ }^{75}$ Das „europäische Jahr des lebensbegleitenden Lernens“ 1996 zeigt die Bedeutung, die dieses Konzept in der europäischen Politik einnahm. Dem Jahr ging ein Weißbuch „Lehren und Lernen. Auf dem Weg zur kognitiven Gesellschaft“ 1995 voraus. Im Rahmen dieses Weißbuchs wird gefordert, dass die Aneignung neuer Kenntnisse das ganze Leben notwendig sei und ermöglicht werden solle. Voraussetzung für diese lebenslange Aneignung sei ein hohes $\mathrm{Maß}$ an Allgemeinbildung, auf welche dann spezifischere Berufsbildung aufbauen kann. Notwendig sei die Ausrichtung auf lebensbegleitendes Lernen durch einen festgestellten Strukturwandel (Kraus 2001).

Eine weitere Entwicklung, welche die zunehmende Bedeutung der Bildungspolitik in Europa verdeutlicht war die Anerkennung der Rolle von Bildung für die Entwicklung der Individuen und europäischen Gesellschaften in der Präambel des Amsterdamer Vertrags (Jakobi/Rusconi 2009).

\section{Lissabon-Strategie}

Bevor nachfolgend der Fokus auf den Bologna- und Kopenhagen-Prozessen ruht, soll zuvor noch die Lissabon-Strategie, welche 2000 entworfen wurde, dargestellt werden, da sie für die europäische Bildungspolitik sowie für die transnationalen Bildungsprozesse Bologna und Kopenhagen von äußerster Relevanz war. Ziel der sogenannten Lissabon-Strategie war es, die Europäische Union

[...] zum wettbewerbsfähigsten und dynamischsten wissensbasierten Wirtschaftsraum der Welt zu machen - einem Wirtschaftsraum, der fähig ist, ein dauerhaftes Wirtschaftswachstum mit mehr und besseren Arbeitsplätzen und einem größeren sozialen Zusammenhalt zu erzielen. (Europäischer Rat 2000)

Bildung und insbesondere auch berufliche Bildung kam in der Lissabon-Strategie eine zentrale Rolle zu, denn die veränderten wirtschaftlichen und gesellschaftlichen Bedingungen führen zu einem erhöhten Bedarf an gut ausgebildeten Indivi-

75 Trotz dieser Entwicklung blieben die europäischen Bildungsprogramme Leonardo da Vinci für die Berufsbildung und Sokrates für die Hochschulbildung weiterhin getrennt. Eine Zusammenführung der Programme findet erst seit 2014 statt, seitdem es nur noch das Programm Erasmus+ gibt. In diesem sind aber die anderen europäischen Bildungsprogramme wie Leonardo da Vinci und Erasmus weiterhin integriert. 
duen. Lebenslanges Lernen soll eine große Rolle im europäischen Gesellschaftsmodell spielen, denn nur über Bildung sei es möglich, die gesellschaftlich notwendigen Kompetenzen anzueignen (Europäischer Rat 2000).

Der Europäische Rat in Lissabon legte ebenfalls den Grundstein dafür, dass die offene Methode der Koordinierung (OMK) im Bereich der Bildungspolitik eingeführt wurde (Gornitzka 2005). Diese zielt darauf ab, ein hohes Maß an Verpflichtung gegenüber gemeinsam beschlossenen Zielen und Standards zu erreichen, ohne die engen Grenzen der EU-Kompetenzen in sensiblen Politikfeldern, wie der Bildungspolitik zu überschreiten (Scharpf 2000). Im Rahmen des Europäischen Rates in Lissabon wurden daraufhin die europäischen Bildungsminister aufgefordert, zukünftige Ziele der Bildungspolitik zu formulieren, welche diese in einem ersten Schritt dem europäischen Rat von Stockholm 2001 vorlegten und die schließlich im Arbeitsprogramm über die „Allgemeine und berufliche Bildung 2010“, welches 2002 verabschiedet wurde, einmündeten. Übergeordnete Ziele lauteten: Erstens die Erhöhung der Qualität der Systeme der allgemeinen und beruflichen Bildung, zweitens eine Erleichterung des Zugangs zur allgemeinen und beruflichen Bildung für alle sowie drittens die Öffnung der allgemeinen und beruflichen Bildung gegenüber der Welt (Europäischer Rat 2002; European Council 2001). Insbesondere der zweite Punkt fokussierte dabei nicht nur den Zugang zu Bildung an sich, sondern ebenfalls eine Erleichterung der Übergänge zwischen Bildungsbereichen, wie Berufs- und Hochschulbildung. Es wird somit deutlich, dass mit Lissabon nicht nur Bildung an sich eine zentrale Rolle zur Erreichung der wirtschaftlichen und gesellschaftlichen Ziele zugesprochen wurde, sondern, dass insbesondere Berufs- und höhere Allgemeinbildung dies zusammen zu leisten haben.

Resümierend bleibt festzuhalten, dass erste europäische Initiativen auf den Bereich der Berufsbildung begrenzt waren und nur wenig Einfluss entwickeln konnten. Auch wenn Allgemeinbildung erst mit dem Vertrag von Maastricht offiziell zum europäischen Kompetenzbereich gehörte, wurde bereits seit den 1970er Jahren der Arbeitsbereich auf Allgemein- und Hochschulbildung insbesondere durch die Urteile des Europäischen Gerichthofs ausgeweitet. Programme zur Weiterentwicklung der Hochschulbildung wurden dabei jedoch nicht in die Programme für Berufsbildung integriert, sondern wurden parallel zu ihnen entwickelt, so dass auch auf europäischer Ebene in der Behandlung der Berufs- und Hochschulbildung eine Trennung sichtbar wurde. Mit dem Vertrag von Maastricht blieb die Trennung zwischen Allgemein- und Berufsbildung formal durch die beiden separaten Artikel bestehen. Zudem wurden auch wiederum getrennt die Programme SOKRATES und LEONARDO weitergeführt. Andererseits begann mit der Einführung der Politik des lebenslangen Lernens eine integrierte Betrachtung der beiden Bildungsbereiche, welche mit der Lissabon-Strategie und den daraufhin folgenden Bildungs- und Arbeitsprogrammen ihren Höhepunkt erreichte. 


\subsection{2 Überblick über die Bologna- und Kopenhagen-Prozesse}

Nachfolgend werden die Kopenhagen- und Bologna-Prozesse und ihre Entwicklung, ihre allgemeinen Ziele sowie deren Einbettung in die EU-Bildungspolitik vorgestellt. ${ }^{76}$ Dabei geht es nicht darum zu zeigen, wie die einzelnen Dokumente zustande kamen. Vielmehr sollen die Prozesse im Überblick kurz dargestellt werden, bevor sie in einem weiteren Schritt näher im Hinblick auf ihre Durchlässigkeitsrelevanz untersucht werden.

\section{Bologna-Prozess}

Auch wenn der Bologna-Prozess offiziell mit der Konferenz 1999 in Bologna seinen Anfang nahm, muss die Sorbonne Deklaration, welche von den vier groBen EU-Mitgliedsstaaten, Deutschland, Frankreich, Großbritannien und Italien, 1998 unterzeichnet wurde, als eigentlicher Beginn des Prozesses gelten (Witte 2006), da viele der Ziele und Forderungen sich bereits in der gemeinsamen Deklaration zur "harmonisation of the architecture of the European Higher Education system" wiederfinden (Sorbonne Declaration 1998). So wird die Sorbonne Deklaration ferner als „hochschulpolitischer Urknall“ (Toens 2007: 38) bezeichnet. Denn es waren die Nationalstaaten und nicht wie zuvor meist die Kommission, welche eine intensive Zusammenarbeit in der sensiblen Bildungspolitik voranbringen wollten.

Zum 800-jährigen Geburtstag der Sorbonne Universität trafen sich die für die Hochschulbildung zuständigen Minister_innen dieser vier Staaten und konstatierten ,that Europe is not only that of the Euro, of the banks and the economy: it must be a Europe of knowledge as well." Um dies zu erreichen und den Anforderungen, welche sich durch den Strukturwandel der Wirtschaft verändert hatten, gerecht zu werden, verabschiedeten sie folgende Ziele (vgl. Brändle 2010):

1. Etablierung eines europäischen Raums für Hochschulbildung

2. Förderung von Mobilität (im Sinne geografischer Bildungsmobilität)

3. Einführung einer gestuften Studienstruktur (bestehend aus 2 Zyklen)

4. Einführung eines Systems zur Anrechnung von Studienleistungen (Leistungspunktesysteme wie ECTS) und Semestern für eine größere Flexibilität der Studierenden

5. Internationale gegenseitige Anerkennung von Studienabschlüssen

Viele der Ideen, insbesondere die Mobilitätsförderung und die Anerkennung von Hochschulabschlüssen, hatte die europäische Kommission bereits zuvor mit ihren Aktionsprogrammen, wie ERASMUS, gegen die Souveränitätsbedenken der

76 Für genauere Analysen insbesondere zum Ablauf des Bologna-Prozesses siehe u.a. Brändle 2010; Ravinet 2008, 2009; Voegtle et al. 2011; Witte 2006. 
Mitgliedsstaaten versucht voranzutreiben (Toens 2007). Die vier Länder entschieden sich aber gerade explizit für eine intergouvernementale Zusammenarbeit auBerhalb der EU und erhofften sich auf diese Weise ebenfalls, ihre Souveränitätsrechte besser kontrollieren zu können (Ravinet 2008). Auch wenn die Sorbonne Erklärung nur von den vier Staaten unterzeichnet wurde, wurden die anderen europäischen - und explizit nicht nur EU-Mitgliedsländer - zu einer Kooperation in dieser Sache aufgefordert. Toens (2007) weist daraufhin, dass die Zugkraft der Sorbonne Erklärung auch damit zu erklären ist, dass die Unterzeichnerstaaten die drei wichtigsten Universitätsmodelle Europas darstellten: das liberale Modell Großbritanniens, das Humboldt'sche Modell Deutschlands und das professionsorientierte Modell Frankreichs.

Während der Alleingang der vier großen europäischen Nationen von 1998 von den anderen europäischen Staaten kritisch beäugt wurde (Ravinet 2008), war es dennoch der Startschuss für einen stetig wachsenden gesamteuropäischen Prozess für die Hochschulbildung. Die Dynamik des Bologna-Prozesses wurde daher auch bereits mit einem Schneeballeffekt (Zgaga 2004: 105) verglichen, denn die Beteiligungsrate von ursprünglich vier Staaten stieg rasant an und betrug 201548 Nationen. Zudem sind nicht mehr nur die Regierungen, sondern auch eine Vielzahl von Stakeholdern sowie die Europäische Kommission am Prozess beteiligt. Aber auch die Ziele wurden kontinuierlich erweitert (Brändle 2010).

Die Bologna-Deklaration wurde im Juni 1999 von 29 Bildungsminister_innen unterzeichnet. Diese einigten sich auf sechs grundlegende Ziele, welche zu den sogenannten Bologna-Aktionslinien wurden (Bologna Declaration 1999). Sie galten als die Voraussetzung zur Schaffung eines europäischen Hochschulraums, der bis zum Ende der erstens Dekade des neuen Jahrtausends bestehen soll:

1. Einführung eines Systems leicht verständlicher und vergleichbarer Abschlüsse: Dies soll gewährleistet werden durch das Diploma Supplement, d.h. eine standardisierte Beschreibung der einzelnen Studiengänge.

2. Einführung einer gestuften Studienstruktur: Diese Struktur besteht aus einem ersten Zyklus, welcher mindestens drei Jahre dauern und eine arbeitsmarktrelevante Ausbildung sein soll. Der zweite Zyklus sollte in der Bologna-Deklaration entweder zum Master oder zu einem Doktorabschluss führen. Seit der Nachfolgekonferenz in Berlin umfasst der zweite Zyklus nur den Master und das Promotionsstudium (als dritter Zyklus) wurde als neue Aktionslinie aufgenommen.

3. Einführung eines Leistungspunktesystems: Dieses System, das dem bereits bestehenden System der ECTS 77 ähneln sollte, soll studentische

77 Ursprünglich war das mit dem ERASMUS-Austauschprogramm entstandene ECTS (European Credit Transfer System) nur ein System zum Transfer von Leistungspunkten. Eine Akkumulationsfunktion war nicht vorgesehen. Dies änderte sich mit dem Bologna-Prozess, so dass ECTS erweitert wurde auf das „European Credit Transfer and Accumulation System“ (vgl. Brändle 2010). 
Mobilität und Flexibilität fördern. Die Leistungspunkte sollen der Anerkennung von Leistungen der Studierenden an unterschiedlichen Universitäten oder auch außerhalb der Hochschulen dienen. ECTS sollen das Arbeitspensum, den sogenannten workload darstellen, den Studierende für die Bewältigung der einzelnen Kurse und Vorlesungen inklusive der Vorbereitung und Nachbereitungszeit benötigen, wobei als ein Kredit 25-30 Arbeitsstunden gerechnet werden.

4. Förderung der Mobilität: Neben der Einführung von Leistungspunktesystemen sollen auch weitere Barrieren, welche die Mobilität sowohl der Studierenden als auch der im Bildungssystem beteiligten Professionsgruppen behindern, überwunden werden.

5. Förderung der Zusammenarbeit bei der Qualitätssicherung: Qualitätssicherung wird im Verlauf des Bologna-Prozesses als wichtige Voraussetzung für das Vertrauen in die nationalen Abschüsse und ihre gegenseitige Anerkennung identifiziert. Daher sollen gemeinsame vergleichbare Kriterien und Methoden der Qualitätssicherung entwickelt werden, nach welchen im Anschluss die Akkreditierung der Studiengänge erfolgen soll.

6. Förderung der europäischen Dimension im Hochschulwesen: Gefördert werden soll die „europäische Dimension“ sowohl in der Gestaltung der Lehrpläne als auch durch institutionalisierte Austauschprogramme sowie durch grenzüberschreitende Kooperationen von Hochschulorganisationen.

Seit der Konferenz in Bologna fanden meist in einem zweijährigen Turnus Nachfolgekonferenzen statt, in welchen die Ziele erweitert, die Instrumente spezifiziert und die bisherigen Entwicklungen auf dem Weg zu einem europäischen Hochschulraum begutachtet wurden. Nach Voegtle et al. (2011) findet somit gleichzeitig eine Erweiterung und Konsoldierung statt, wobei in den Nachfolgekonferenzen die unterschiedlichen Aspekte nicht immer gleichermaßen diskutiert worden sind, sondern unterschiedliche Schwerpunkte gesetzt wurden (Powell/Finger 2013).

In den Konferenzen von Prag 2001 und Berlin 2003 wurden die sechs Aktionslinien, welche in der Bologna-Deklaration festgehalten worden sind, auf insgesamt zehn erweitert (Berlin Communiqué 2003; Prague Communiqué 2001).

7. Förderung von Lebenslangem Lernen: Diese Aktionslinie wird das erste Mal im Prager Kommuniqué 2001 aufgenommen. Es wird darauf hingewiesen, dass Europa als wissensbasierte Gesellschaft lebenslanges Lernen ermöglichen muss, damit einerseits die Individuen durch kontinuierliche (Weiter-)Bildung im Lebensverlauf für den Arbeitsmarkt konkurrenzfähig bleiben und andererseits, um gesellschaftlichen Herausforderungen gerecht zu werden. 
8. Einbezug von Hochschulorganisationen und Studierenden: Um einen europäischen Hochschulraum zu errichten, wird seit Prag die Mitarbeit sowohl der Hochschuleinrichtungen als auch der Studierenden als notwendig erachtet.

9. Förderung der Attraktivität des europäischen Hochschulraums: Diese Aktionslinie zielt auf eine größere Wettbewerbsfähigkeit der europäischen Hochschullandschaft: Wichtig sei die Erhaltung der Qualität der Hochschulausbildung und Forschung, um so international an Attraktivität zu gewinnen.

10. Einbezug der Promotion als dritter Zyklus und die Förderung eines europäischen Hochschul- und Forschungsraums: Die Einbeziehung des Promotionsstudium in den Bologna-Prozess seit Berlin soll zu einer stärkeren Verbindung des europäischen Hochschul- mit dem europäischen Forschungsraum führen. Dies sei notwendig, auch um die Wettbewerbsfähigkeit der europäischen Hochschulen zu steigern.

Zudem wurde in Berlin die sogenannte soziale Dimension als Querschnittsdimension hervorgehoben. Im Rahmen des Bologna-Prozesses soll sozialer Zusammenhalt gestiftet und soziale Ungleichheiten sollen abgebaut werden.

Nach der Konferenz in Berlin wurden keine weiteren Aktionslinien hinzugefügt. Ritualsiert wurden die Ziele der jeweiligen Vorgängerkonferenzen bekräftigt (Brändle 2010). Während zu Beginn des Prozesses noch vornehmlich eher allgemeine Ziele und Intentionen in den Deklarationen festgehalten wurden, wurden diese in den weiteren Kommuniqués konkretisiert, wenn auch in einem unterschiedlichen Maße, und wichtige Instrumente wurden eingeführt ${ }^{78}$. Zwei sollen an dieser Stelle genannt werden, ein System zur Qualitätssicherung und der Qualifikationsrahmen für den (europäischen oder nationalen) Hochschulbereich.

In Sachen der Qualitätssicherung wurden so z.B. gemeinsame Standards und Leitlinien (Bergen Communiqué 2005) sowie die Einführung eines komplexen Qualitätssicherungssystems (London Communiqué 2007), dem European Quality Assurance Register for Higher Education (EQAR) beschlossen.

Auf der Grundlage der Erklärungen von Bologna und Berlin wurde in Bergen die Einführung eines Qualifikationsrahmens für die europäische Hochschulbildung eingeführt. Dieser, das wurde in den nachfolgenden Konferenzen bestätigt, soll sich in den europäischen Qualifikationsrahmen für lebenslanges Lernen, welcher im Kopenhagen-Prozess angestoßen und 2007 vom Europäischen Rat beschlossen wurde, integrieren. Die KMK beschreibt einen Qualifikationsrahmen als ,eine systematische Beschreibung der Qualifikationen, die das Bildungssystem eines Landes hervorbringt" (DHQR 2005). Der Qualifikationsrahmen beschreibt demnach die „Lernergebnisse“, das was in einem Bildungsgang gelernt wird, d.h.

78 Weitere relevante Standards werden in der nachfolgenden auf Durchlässigkeit fokussierten Beschreibung dargestellt. 
was ein Lernender weiß, versteht und in der Lage ist zu tun. Zudem kann er aufzeigen, wie die differenten Qualifikationen im Bildungssystem zueinander stehen. Insbesondere für Länder wie Deutschland bedeutet die Orientierung auf Lernergebnisse eine große Umstellung, da die deutschen Studienprogramme bis dato vor allem durch ihre Studieninhalte, Zulassungskriterien sowie Studiendauer beschrieben wurden (DHQR 2005). Es handelt sich demnach um eine geforderte Umstellung von Input- auf Outputorientierung, wodurch die Transparenz im Bildungssystem gefördert werden soll (DHQR 2005) ${ }^{79}$.

Auch in den zuletzt stattgefundenen Konferenzen in Leuven 2009, Budapest 2010 und Bucharest 2012 wurde die Umsetzung der bestehenden Aktionslinien wiederholt beschlossen, wobei es immer stärker um die Implementierung und Verbesserung der zusammen entwickelten Standards, wie den ECTS, den Qualifikationsrahmen, der Diploma Supplement und der Qualitätssicherungssysteme ging. Zudem wurde auch darauf hingewiesen, dass die Förderung der internationalen Mobilität im Hochschulsystem noch nicht ausreichend sei und auch hier weiterer Entwicklungsbedarf bestünde (vgl. Bucharest Communiqué 2012; Budapest-Vienna Declaration 2010; Leuven Communiqué 2009).

\section{Der Kopenhagen-Prozess}

Der Kopenhagen-Prozess startete zwar nur vier Jahre später als der Bologna-Prozess, war aber direkt von Beginn an mit der Lissabon-Strategie und der EU-Bildungspolitik verbunden, während sich der Bologna-Prozess viel unabhängiger von der supranationalen Politiksphäre entwickelte. Im Gegensatz zu Bologna wurde der Kopenhagen-Prozess explizit in Gang gesetzt, um Antworten im Rahmen der Berufsbildungspolitik auf die Herausforderungen zu finden, welche in der Lissabon-Strategie festgestellt wurden (Copenhagen Declaration 2002). Am 30. November 2002 einigten sich daher die Bildungsminister_innen der Mitgliedsstaaten der EU sowie assoziierter Staaten (EFTA), die Europäische Kommission und die Sozialpartner in Kopenhagen auf gemeinsame Prioritäten und Strategien für die Berufsbildungspolitik in den kommenden Jahren. Vier zentrale Handlungsfelder wurden beschlossen:

79 Qualifikationsrahmen zielen somit darauf ab, die Qualifikationen, die im Bildungssystem eines Landes erworben werden können, systematisch in ein Raster einzuordnen und damit vergleichbar zu machen. In der Diskussion muss unterschieden werden zwischen dem europäischen Qualifikationsrahmen, der die Qualifikationen transnational vergleichbar machen soll und den nationalen Qualifikationsrahmen. Beiden ist gemein, dass sie alle Bildungsbereiche (Allgemein, Hochschul- und Berufsbildung) in ein gemeinsame Raster integrieren. Qualifikationsrahmen bilden zudem die unterschiedlichsten Bildungsniveaus ab mit dem Ziel das gesamte Spektrum von Bildungsergebnissen darstellen. Der europäische Qualifikationsrahmen unterscheidet acht Bildungsniveaus, wobei mit den Stufen das Kontinuum von grundlegenden allgemeinen Kenntnissen und Fertigkeiten (Stufe 1) bis zur Beherrschung eines hoch spezialisierten Wissensgebiets (Stufe 8) abgedeckt wird. 
1. Zur Förderung der europäischen Dimension sollen die Ermöglichung von Mobilität und das Erlernen von interkulturellen Kompetenzen, eine verstärkte europäische Zusammenarbeit und die Einbettung europäischer Themen in Lehrplänen beitragen.

2. Die Transparenz der europäischen Berufsbildungsabschlüsse soll insbesondere über die Erarbeitung eines einheitlichen Rahmens zur Klassifizierung von Kompetenzen und Qualifikationen erleichtert werden. Dieser Rahmen soll die bisher nicht aufeinander abgestimmten Instrumente wie den europäischen Lebenslauf, das Diploma Supplement und den Europass weiterentwickeln (Fahle/Thiele 2003). Solch ein Rahmen kann dann auch dem Individuum eine Transparenz über individuelle Kompetenzen ermöglichen. Auf nationaler Ebene sollen zudem verstärkt auch Informations- und Beratungssysteme die Transparenz steigern.

3. Im Hinblick auf eine Förderung der Anerkennung und Übertragung von Kompetenzen und Qualifikationen werden in der Kopenhagen-Erklärung drei Ziele formuliert. Erstens soll ein Anrechnungs- und Übertragungssystem basierend auf Leistungspunkten für die Berufsbildung entwickelt werden. Zweitens sollen auf sektorieller Ebene Qualifikationsprofile herausgearbeitet werden. Drittens sollen gemeinsame Prinzipien zur Validierung von non-formalen und informellen Lernen erarbeitet werden.

4. Um das Vertrauen in die beruflichen Abschlüsse zu gewährleisten, sollen schließlich auch gemeinsame Kriterien und Grundsätze für die Qualität in der beruflichen Bildung entstehen.

Im Gegensatz zum Bologna-Prozess werden in der Kopenhagen-Erklärung keine eindeutigen Aktionslinien formuliert. Stattdessen handelte es sich um grobe Arbeitsschwerpunkte, welche bis 2010 bearbeitet werden sollten. Auch fand in den nachfolgenden Konferenzen keine lineare Weiterentwicklung der Arbeitspakete statt. Vielmehr wurden Fortschritte begutachtet und Schwerpunkte für das weitere Vorgehen gesetzt.

In Maastricht 2004 fand die zweite Konferenz statt, auf der das bis dato Erreichte evaluiert wurde. Zum einen wurde der Kopenhagen-Prozess noch enger mit den bestehenden EU-Bildungsprozessen verknüpft, insbesondere mit dem 2001 beschlossenen Arbeitsprogramm „Allgemeine und berufliche Bildung 2010“. Im Maastricht Kommuniqué wurden zudem das erste Mal explizit Aufgaben zur nationalen Umsetzung formuliert - diese waren u.a. eine bessere Bekanntmachung des Prozesses mit den dazugehörigen Instrumenten, ein Fokus der Berufsbildungspolitik auf die benachteiligten Gruppen der Gesellschaft, verstärkte Einrichtung individualisierbarer Bildungswege und offener Lernansätze sowie eine verbesserte Berufsbildungsplanung. Auf europäischer Ebene sollte weiter an 
den Instrumenten wie dem europäischen Leistungspunktesystem für die berufliche Bildung (ECVET) und dem europäischen Qualifikationsrahmen (EQR) gearbeitet werden (vgl. Maastricht Communiqué 2004).

Während in der Kopenhagen-Erklärung noch allgemein von einem einheitlichen Rahmen zur Schaffung von Transparenz gesprochen wurde, wurde der europäische Qualifikationsrahmen im Maastricht Kommuniqué erstmals als solcher erwähnt. Um diesen zu entwickeln, fand 2005 ein Konsultationsverfahren auf europäischer Ebene zur Ausgestaltung des EQR statt. Konsens war dabei, dass der Rahmen, um die Bildungssysteme vergleichbar zu machen, auf Lernergebnissen beruhen sollte (Follow up Copenhagen 2006). Im Rahmen der Überarbeitung und nochmaligen Zustimmung der Mitgliedsstaaten einigte man sich darauf, dass der EQR ein Referenzrahmen für lebenslanges Lernen sein und die Leistungen der nationalen Bildungssysteme auf acht Niveaustufen abbilden soll. Diese Niveaustufen sollen das gesamte Spektrum von Bildungsergebnissen abdecken. Dabei werden die Niveaus über Deskriptoren in Bezug auf erworbene Kenntnisse (Theorie- und Faktenwissen), Fertigkeiten sowie Kompetenzen beschrieben. Fertigkeiten können dabei kognitive oder auch praktische sein - wie z.B. Problemlösungsfähigkeit und der Umgang mit bestimmten Instrumenten. Kompetenzen umfassen „Selbständigkeit und Verantwortlichkeit“, „Lernkompetenz“, „kommunikative und soziale Kompetenzen“ sowie die „professionelle und berufliche Kompetenz" (Hanf/Fahle 2005). Mit ansteigender Niveaustufe steigt ebenfalls der Anspruch an die Lernergebnisse, so dass die Niveaustufen von einfachen allgemeinen Grundfähigkeiten bis zu hochspezialisierten Expert_innenwissen reichen $^{80}$.

In den nachfolgenden Konferenzen in Helsinki 2006 und Bordeaux 2008 wurden die bisher beschlossenen Prioritäten und Ziele bestätigt. Schwerpunkte der weiteren Arbeit soll daher auf der Entwicklung und Erprobung gemeinsamer europäischer Instrumente, insbesondere auf dem Qualifikationsrahmen, dem Leistungspunkte- sowie dem Qualitätssicherungssystem, liegen. Zudem sollte das gegenseitige Lernern der Mitgliedsstaaten durch eine verbesserte Berufsbildungsstatistik erleichtert werden. Schließlich sei es wichtig, insgesamt das Image, die Attraktivität und Qualität von Berufsbildung zu erhöhen (Bordeaux Communiqué 2008; Helsinki Communiqué 2006).

Auch im bisher letzten Kommuniqué des Kopenhagen-Prozesses in Brügge wurden diese Arbeitsschwerpunkte weitgehend wiederholt und ergänzt. Folgende Kernbereiche wurden identifiziert:

- Verbesserung der Qualität und Effizienz der beruflichen Bildung und Erhöhung ihrer Attraktivität und Relevanz

- Lebenslanges Lernen und Mobilität als Realität

- Förderung von Kreativität, Innovation und Unternehmergeist

80 Für die genaue Beschreibung des EQR siehe European Commission (2008). 
- Förderung von Gerechtigkeit, sozialem Zusammenhalt und aktivem Bürgersinn.

Deutlich wurde zudem die wiederum starke Einbindung des Kopenhagen-Prozesses in den „Strategischen Rahmen Bildung und Ausbildung 2020" der EUBildungsminister vom Mai 2009 (Council of the European Union 2009), da sich die formulierten Ziele stark entsprechen. Zudem wurde ein Aktionsplan 2011 bis 2014 festgelegt, welcher konkrete Maßnahmen für die einzelnen Arbeitsbereiche beinhaltet.

\section{Ziele und Argumentationen der Bologna- und Kopenhagen- Prozesse}

Nachdem beide Prozesse in ihrem Verlauf und ihren Schwerpunkten dargestellt wurden, sollen nachfolgend die Ziele und Argumentationen der Prozesse verglichen werden, um so in einem nächsten Schritt einen besseren Eindruck zu erhalten, in welchen allgemeinen Rahmen Durchlässigkeitsfragen in den europäischen Bildungsprozessen diskutiert worden sind. Zudem wird durch den Vergleich deutlich, worin sich die Prozesse in ihrer allgemeinen Ausrichtung ähneln und zum Teil auch unterscheiden.

Im Querschnitt über die Jahre 1998 bis 2010 konnte eine weitgehende Übereinstimmung der Ausrichtung der Prozesse festgestellt werden (Powell et al. 2012a). Primär dienen die Bologna- und Kopenhagen-Prozesse der Ermöglichung Lebenslangen Lernens, der Stärkung der Wettbewerbsfähigkeit Europas, einer Erhöhung der Beschäftigungsfähigkeit europäischer Bürger, der Ermöglichung von Mobilität innerhalb Europas, einer besseren Vergleichbarkeit von nationalen Qualifikationen sowie allgemein einer Stärkung der Attraktivität und Qualität der europäischen Bildung (Powell et al. 2012a: 445f.). Dafür sollen in beiden Prozessen Standards entwickelt werden, die die Vergleichbarkeit und Übertragbarkeit der nationalen Qualifikationen ermöglichen. Im KopenhagenProzess sind deutlich stärker als im Bologna-Prozess ökonomische Ziele ausgeprägt. Zudem wird im ersteren auch stärker die Frage der sozialen Kohäsion gestellt. Im Bologna-Prozess wird zusätzlich eine Einbettung in den europäischen Forschungsraum gefordert (Powell et al. 2012a: 446).

Während die Ziele der beiden Prozesse weitgehend ähnlich waren, existieren doch Differenzen bei der Begründung der Reformnotwendigkeiten. Der wichtigste Grund in beiden Prozesse ist der festgestellte Strukturwandel, welcher die Reformen im Bildungssystem bedingt. In den Bologna-Dokumenten wird dieser Wandel aber nicht näher bestimmt, während im Kopenhagen-Prozess die spezifischen gewandelten Bedarfe des Arbeitsmarkts aufgrund des Wachstums des Dienstleistungssektors und einer gestiegenen Qualifikationsanforderung festgestellt wird. Des Weiteren werden ein gestiegener Fachkräftebedarf und ein gewachsener globaler Wettbewerb in beiden Prozessen als Grund genannt. Im Bo- 
logna-Prozess werden die angestrebten Reformen damit begründet, dass sie einerseits zu einer stärkeren Demokratisierung der Mitgliedsstaaten sowie der Förderung von nachhaltiger Entwicklung beitragen. Im Kopenhagen-Prozess werden hingegen die Reformen mit den Herausforderungen der Osterweiterung und dem demografischen Wandel mitbegründet (Powell et al. 2012a). Powell et al. (2012a: 448) fassen zusammen:

Die Berufsbildung reagiert auf konkrete quantifizierbare Beschäftigungsbedarfe, während die Hochschulbildung eher prospektiv, viel weniger auf bestimmte Probleme hin ausgerichtet ist, Innovationen fördern und die europäischen Wissensgesellschaften voranbringen soll.

Insgesamt sind sich jedoch die beiden europäischen Prozesse in ihren Zielen und ihren Argumentationen relativ ähnlich - auch wenn sie unterschiedlich gestartet sind: Der Bologna-Prozess begann als rein transnationaler intergouvernementaler Prozess, der erst nach und nach auch die EU-Kommission und andere nationale Nichtregierungsakteure beteiligte. Der Kopenhagen-Prozess andererseits war von Beginn an stark mit der vorherrschenden EU-Bildungspolitik und der LissabonStrategie verwebt. Trotz dieser Unterschiede war das Vorgehen in den beiden Prozessen ein ähnliches, es handelte sich um eine wiederholte Selbstverpflichtung der Regierungen zu Reformen über gemeinsam gesetzte Benchmarks, einen Prozess des gegenseitigen Lernens über Best Practices und des ständigen Vergleichens von Entwicklungen über Evaluationsberichte.

Gemeinsam ist den beiden Prozessen ebenfalls, dass aus den beschrieben Zielen, Arbeitsschwerpunkten und Aktionslinien abgeleitet werden kann, dass Durchlässigkeit im und vor allem zwischen Bildungssystemen ein relevantes Thema ist. Dies wird deutlich an dem Ziel der Förderung des lebenslangen Lernens, die Ermöglichung von Anrechnung und die Entwicklung von Leistungspunktesystemen und Validierungsverfahren von vorgängigem Lernen. Inwiefern institutionelle Durchlässigkeit zwischen Berufs- und Hochschulbildung ganz konkret im Blickpunkt steht, soll nachfolgend gezeigt werden.

\subsection{Durchlässigkeit in den Bologna- und Kopenhagen- Prozessen}

Dieser Abschnitt soll zeigen, wie die Frage von Durchlässigkeit zwischen beruflicher und Hochschulbildung in den Bologna- und Kopenhagen-Prozessen thematisiert wird und wie sich das Konzept Durchlässigkeit im Zeitverlauf entwickelt hat. Die Analyse erfolgt in drei Schritten und basiert auf einer theoriegeleiteten qualitativen Inhaltsanalyse der Deklarationen und Kommuniqués (vgl. Kapitel 4): Erstens wird chronologisch für die beiden einzelnen Prozesse beschrieben, ob 
und wie in den Dokumenten Durchlässigkeit thematisiert wird. Diese chronologische Darstellung ist notwendig, da sie zum einen die mögliche gegenseitige Einflussnahme der beiden Europäisierungsprozesse nachvollziehen lässt. Zum anderen ist jedoch für die Analyse der nationalen Fälle relevant, wann bestimmte Konzepte in Europa beschlossen wurden und ab wann sie ihren vollen Einfluss ausüben konnten. Gewiss gab es Konzepte bereits, bevor sie auf den Konferenzen beschlossen wurden und sie könnten daher auch schon vorher nationale Systeme beeinflusst haben. Aber ich argumentiere, dass sie einen größeren normativen Druck ausüben, wenn sie in Kommuniqués und Deklarationen verabschiedet worden sind. ${ }^{81}$

In einem zweiten Schritt verdichte ich die gewonnenen Informationen anhand der institutionellen Analysekategorien. In diesem Punkt unterscheide ich zwischen der kulturell-kognitiven, der normativen und der regulativen Dimension, wobei die ersten beiden im Vordergrund stehen. Regulative Aussagen sind kaum zu erwarten, da Bildung noch immer primär in das Aufgabengebiet der Mitgliedsstaaten fällt. Schließlich werden die Ergebnisse beider Prozesse miteinander verglichen und in ein europäisches Modell zur Durchsetzung von Durchlässigkeit zusammengeführt.

\subsubsection{Durchlässigkeit im Bologna-Prozess}

\section{Eine chronologische Darstellung der Thematisierung von Durchlässigkeit}

Durchlässigkeit im Sinne von Bildungsmobilität allgemein ist von Anfang ein Thema in den Bologna-Dokumenten. Primär geht es dabei um eine erhöhte geografische Mobilität von Individuen zwischen nationalen Bildungssystemen - aber auch um die Steigerung von Durchlässigkeit innerhalb des Hochschulsystems eines Landes, also zwischen den Studienzyklen und den einzelnen Hochschulorganisationen. Diese Thematik wird seit der Sorbonne-Erklärung 1999 in allen Kommuniqués wiederholt. Durchlässigkeit zwischen beruflicher Bildung und Hochschulbildung wird dagegen in den Bologna-Dokumenten kaum explizit angesprochen. Stattdessen werden Fragen der Durchlässigkeit allgemeiner zur Ermöglichung von lebenslangem Lernen sowie zur Erreichung von Chancengleichheit, im Sinne einer größeren Diversität der Studierendenschaft, d.h. einer Inklusion nicht-traditioneller Studierender, sogenannte ,underrepresented groups“, angesprochen (siehe Sorbonne Declaration 1998). Diese allgemeinen Formulierungen schließen damit aber implizit ebenfalls Fragen der Durchlässigkeit zwischen den beiden Organisationsfeldern der Hochschul- und Berufsbildung ein, so dass eine weitere Analyse der Dokumente durchaus sinnvoll ist.

81 Es interessiert in dieser Arbeit aber nicht, wie die Konzepte auf europäischer Ebene zustande gekommen sind oder welche Akteure dabei besonders einflussreich waren. 
Bereits in der Sorbonne-Erklärung von 1998 wird auf die Notwendigkeit hingewiesen, dass Individuen zu jedem Zeitpunkt ihres Berufslebens die Möglichkeit haben sollten, Zugang zum Hochschulsystem zu erhalten und dass die Zielgruppe der Hochschulen eine heterogene Student_innenschaft mit verschiedensten Hintergründen ist.

Indeed, students should be able to enter the academic world at any time in their professional life and from diverse backgrounds. (Sorbonne Declaration 1998) ${ }^{82}$

In der Bologna-Erklärung von 1999 (sowie bereits auch in der von Sorbonne) ist die Einführung von Leistungspunktesystemen zentral, um Mobilität und damit auch Flexibilität innerhalb der Hochschulsysteme durch Anerkennung von Studienleistungen zu ermöglichen. Zum ersten Mal wird jedoch in der Bologna-Erklärung auch die Möglichkeit in Betracht gezogen, Leistungen, die außerhalb des Hochschulbereichs erworben worden sind, für ein Studium anzuerkennen. Diese Möglichkeit bestünde aber nur insoweit, wie die aufnehmenden Institutionen, die Universitäten bzw. Hochschulen, dazu bereit sind.

Establishment of a system of credits - such as in the ECTS system - as a proper means of promoting the most widespread student mobility. Credits could also be acquired in non-higher education contexts, including lifelong learning, provided they are recognised by receiving Universities concerned. (Bologna Declaration 1999)

Wichtig sei, im gesamten Prozess die Autonomie der Hochschulen zu bewahren (Bologna Declaration 1999), mit der Folge, dass Anerkennungsfragen im Entscheidungsbereich der Hochschulen verbleiben.

Die erste Nachfolgekonferenz 2001 in $\operatorname{Prag}^{83}$ wurde durch die im Jahr 2000 verabschiedete Lissabon-Strategie beeinflusst, in welcher lebenslanges Lernen als ein Schlüssel angesehen wurde, die Wettbewerbsfähigkeit Europas zu erhöhen. So wird auch 2001 im Prager Kommuniqué erstmals die Rolle von lebenslangem Lernen als elementarer Bestandteil eines zu erschaffenden europäischen Hochschulraums unterstrichen. Diese Entwicklung ist wichtig, da in den darauffolgenden Konferenzen die Frage von Durchlässigkeit mit der Notwendigkeit in Verbindung gebracht wird, lebenslanges Lernen und zwar auch an den Hochschulen zu ermöglichen.

Im Prager Kommuniqué wird zudem die Transfer- und Akkumulationsfunktion des einzuführenden Leistungspunktesystems hervorgehoben, wenn auch nur im Zusammenhang von Mobilität innerhalb des Hochschulbereichs und des Arbeitsmarkts. Über Leistungspunkte kann demnach Erlerntes in andere Bereiche

82 Nicht alle Dokumente weisen Seitenangaben aus.

83 „Im Hinblick auf die Mobilität hoben die Ministerinnen und Minister aufgrund wiederholt vorgetragener Forderungen der Studierenden (ESIB 2001b: 2f.) in Prag erstmals hervor, dass auch die soziale Dimension von Mobilität Gegenstand des Bologna-Prozesses sein müsse. In diesem Zusammenhang verweist das Prager Kommuniqué auf entsprechende EU-Programme - etwa im Rahmen des ,Mobility Action Plan‘, den der Europäische Rat 2000 in Nizza verabschiedet hat.“ (Keller 2003) 
übertragen werden. Des Weiteren wird unterstrichen, dass funktionierende nationale Qualitätssicherungssysteme für Fragen der Anrechnung sowie Anerkennung von Studienzeiten im Hochschulsystem von elementarer Bedeutung seien. Schließlich wird in Anbetracht der Diversität der Qualifikationen in Europa auch eine faire Anerkennung von sowohl akademischen als auch beruflichen Zertifikaten und Abschlüssen im Hochschulsystem gefordert, so dass die Bürger_innen ihre Qualifikationen, Fähigkeiten und Kompetenzen im gesamten europäischen Hochschulraum nutzen können (Prague Communiqué 2001).

Die nächste Bologna-Folgekonferenz fand 2003 in Berlin statt, und zwar ein Jahr nach dem offiziellen Beginn des Kopenhagen-Prozesses. Ab diesem Zeitpunkt wären eine gegenseitige Beeinflussung der beiden transnationalen Bildungsprozesse und somit auch eine direktere Bezugnahme zur beruflichen Bildung erwartbar. Das Berliner Kommuniqué nimmt zwar keinen direkten Bezug auf berufliche Bildung. Aber seit dem Berliner Kommuniqué wird die Anerkennung von vorgängigem Lernen als zentrale Aufgabe von Hochschulorganisationen gesehen, um lebenslanges Lernen zu ermöglichen. Was unter vorgängigem Lernen zu verstehen ist, wird allerdings erst in den nachfolgenden Konferenzen ausbuchstabiert.

Ministers underline the important contribution of higher education in making lifelong
learning a reality. They are taking steps to align their national policies to realise this
goal and urge Higher Education Institutions and all concerned to enhance the possi-
bilities for lifelong learning at higher education level including the recognition of prior
learning. (Berlin Communiqué 2003: 6)

Die Frage des Zugangs zum Hochschulsystem wird unter dem Gesichtspunkt der Chancengleichheit thematisiert. Alle sollen Zugang zur Universität erhalten dürfen, aber nur, wenn sie die notwendigen Fähigkeiten besitzen, ein Studium auch abzuschließen.

Ministers stress their commitment to making higher education equally accessible to all, on the basis of capacity, by every appropriate means. (Berlin Communiqué 2003:

4)

sowie

[...] the need to improve opportunities for all citizens, in accordance with their aspirations and abilities, to follow the lifelong learning paths into and within higher education. (Berlin Communiqué 2003: 6)

Hier werden zwei Punkte deutlich, zum einen der meritokratische Gedanke bei der Selektion der Student_innenschaft. Zum zweiten jedoch ebenfalls eine Abgrenzung von der Gruppe der ,Nicht-Fähigen'. Wer über den Zugang entscheidet, wird nicht festgelegt, jedoch mit dem Hinweis auf die zu erhaltende Autonomie der Hochschulen im Allgemeinen (nicht speziell in diesem Kontext) ein möglicher Hinweis gegeben.

Im Bergen-Kommuniqué von 2005 wird ein Qualifikationsrahmen erstmals innerhalb des Bologna-Prozesses thematisiert. Da 2004 in Maastricht, der ersten 
Kopenhagen-Folgekonferenz, die Einführung von nationalen Qualifikationsrahmen (NQR) beschlossen wurde, wird im Bergen Kommuniqué auf diese Weise zum ersten Mal ersichtlich der Brückenschlag zum Kopenhagen-Prozess gemacht. Es wird gefordert, dass der in Bergen beschlossene übergreifende Qualifikationsrahmen des europäischen Hochschulraums (EHR) komplementär zu den $\mathrm{zu}$ entwickelnden nationalen Qualifikationsrahmen, die Allgemeinbildung und Berufsbildung vereinigen, sein sollte. An dieser Stelle wird die Frage, warum im Hochschulbereich ein eigener Qualifikationsrahmen entwickelt werden soll und warum es der Komplementarität zum NQR bedarf, nicht beantwortet. An anderer Stelle im Beschluss wird jedoch die Einführung eines nationalen Qualifikationsrahmens als Möglichkeit gesehen, um lebenslanges Lernen im Hochschulbereich zu stärken.

We see the development of national and European frameworks for qualifications as an opportunity to further embed lifelong learning in higher education. (Bergen Communiqué 2005)

Um flexiblere Lernwege zu ermöglichen, sollen zudem die Hochschulorganisationen verstärkt Möglichkeiten zur Anerkennung von vorgängigem Lernen schaffen. Dies soll dazu beitragen, den Weg in die Hochschule zu erleichtern, aber auch die Möglichkeit bieten, Studienabschnitte anzuerkennen. In Bergen wird das vorgängige Lernen, im Sinne von Lernen außerhalb der Hochschule, diesmal näher definiert, denn neben formalen sollen auch informell und non-formal erworbene Kompetenzen Anerkennung finden.

We will work with higher education institutions and others to improve recognition of prior learning including, where possible, non-formal and informal learning for access to, and as elements in, higher education programmes. (Bergen Communiqué 2005: 3)

Schließlich wird im Bergen-Kommuniqué auch der Zugang zum Hochschulbereich ausführlich thematisiert: So soll dieser gleichberechtigt allen offenstehen, unabhängig von sozialen und ökonomischen Hintergründen der Individuen. Eine Öffnung der Hochschule insbesondere für sozial benachteiligte Gruppen, was seit Berlin im Bologna-Prozess als soziale Dimension des Prozesses definiert wird, soll über staatliche finanzielle Unterstützung sowie durch Beratungsdienste erreicht werden.

The social dimension includes measures taken by governments to help students, especially from socially disadvantaged groups, in financial and economic aspects and to provide them with guidance and counselling services with a view to widening access. (Bergen Communiqué 2005: 4)

An dieser Stelle wird soziale Durchlässigkeit im Bildungssystem gefordert, wobei nicht speziell auf eine größere Durchlässigkeit aus anderen Bildungsbereichen gedrungen wird. Stellen aber Absolvent_innen aus anderen Bildungsbereichen systematisch benachteiligte Gruppen dar, so wären diese inkludiert. Expliziert wird dies im Kommuniqué nicht. 
Anerkennung bildet ein zentrales Thema im Londoner Kommuniqué von 2007. Als Ziel wird die faire Anerkennung insbesondere von Hochschulqualifikationen über Grenzen hinweg, aber auch von außerhochschulischen Vorkenntnissen inklusive non-formalen und informellen Lernens, formuliert.

Fair recognition of higher education qualifications, periods of study and prior learning, including the recognition of non-formal and informal learning, are essential components of the EHEA, both internally and in a global context. (London Communiqué 2007: 3)

Um dies zu erreichen, sind vergleichbare Abschlüsse, die Studienstruktur aus drei Zyklen, öffentlich zugängliche Informationen über Bildungssysteme und Qualifikationsrahmen notwendig. Letztere sollen im Hochschulbereich die Umstellung auf Studienmodule und Lernergebnisorientierung, ${ }^{84}$ aber auch die Anerkennung von Vorkenntnissen, erleichtern und zu einer größeren Transparenz sowie Vergleichbarkeit im europäischen Hochschulraum, jedoch auch innerhalb der nationalen Systeme und damit auch zu einer größeren Mobilität führen (London Communiqué 2007). Wenn im Bologna-Prozess Qualifikationsrahmen thematisiert werden, ist in der Regel der Rahmen im Hochschulbereich gemeint, es sei denn es wird direkt Bezug auf den EQR oder NQR genommen. Komplementarität zwischen den Qualifikationsrahmen wird allerdings gefordert. Auf diese Weise wird der Notwendigkeit der Anschlussfähigkeit von akademischen Abschlüssen und Fertigkeiten mit nicht-akademischem Hintergrund Ausdruck verliehen.

Ein großes Problem der Hochschulsysteme in Europa seien die wenig institutionalisierten Verfahren für die Anerkennung von vorgängigem Lernen. Derartige Systeme sind jedoch notwendig, um flexible Lernwege zu ermöglichen und lebenslanges Lernen an den Hochschulorganisationen zu gewährleisten, denn sie können sowohl bei der Anrechnung von Studienleistungen als auch bei der Zulassung zum Hochschulsystem, hilfreich sein. Insgesamt sollen die Zugangsbarrieren zur Hochschule sowie die innerhalb des Hochschulsystems verringert und die Hochschulpartizipation ausgeweitet werden. Die Studierenden, die Zielgruppe des Hochschulbereichs, soll die Diversität der nationalen Gesellschaften widerspiegeln d.h., es darf keine systematische Diskriminierung beim Zugang und beim Fortkommen im Hochschulsystem stattfinden. Chancengleichheit ist das Ziel. Es soll dafür gesorgt werden, dass soziale und ökonomische Hintergründe keine Behinderung beim Studium darstellen. U.a. sollen Beratungsleistungen Individuen bei der Zulassung und im Studium unterstützen. Ziel ist die Leistungspotenziale der Individuen zu maximieren (London Communiqué 2007).

84 Im EQR sind Lernergebnisse definiert als Aussagen darüber, was Lernende wissen, verstehen und in der Lage sind zu tun, nachdem sie einen Lernprozess abgeschlossen haben. Lernergebnisse werden als Kenntnisse, Fertigkeiten und Kompetenzen definiert (vgl. European Commission 2008). 
Zehn Jahre nach dem Beginn des Bologna-Prozesses fand in Leuven 2009 eine weitere Folgekonferenz statt, in welcher die Fortführung des Prozesses beschlossen wurde. Wie bereits in den Kommuniqués zuvor wird die Ermöglichung von lebenslangem Lernen als elementarer Bestandteil des Hochschulsystems und als öffentliche Aufgabe deklariert. Die nationale Umsetzung der Politik des lebenslangen Lernens wird als Gemeinschaftsaufgabe der beteiligten Stakeholder definiert. Dies sind die öffentlichen Verwaltungen und Ministerien, die Hochschulorganisationen, Studierende sowie die Sozialpartner (Leuven Communiqué 2009).

Lediglich über lebenslanges Lernen, welches darauf abzielt, die Talente und Fähigkeiten der europäischen Bürger zu maximieren, können die Herausforderungen der europäischen Gesellschaften, welche sich durch den demografischen Wandel ergeben, bewältigt werden. Lebenslanges Lernen bedeutet die Ermöglichung flexibler Lernwege. Ausdrücklich wird an dieser Stelle die Möglichkeit von Teilzeitstudium und beruflich orientierter Lernwege genannt.

Es werden differente Aufgaben für die Nationalstaaten identifiziert, welche $\mathrm{zu}$ einer besseren Realisierung von lebenslangem Lernen in den nationalen Hochschulsystemen führen können. Die Einführung von Qualifikationsrahmen im Hochschulbereich, welche mit dem Europäischen Qualifikationsrahmen für lebenslanges Lernen koordiniert werden sollen, ist eine davon. Zudem sollen Systeme entwickelt werden, die vorgängiges Lernen systematisch - egal ob formal, non-formal oder informell - auf Basis von Lernergebnissen erfassen und anerkennen.

Successful policies for lifelong learning will include basic principles and procedures for recognition of prior learning on the basis of learning outcomes regardless of whether the knowledge, skills and competences were acquired through formal, nonformal, or informal learning paths. (Leuven Communiqué 2009: 3)

Auf diese Weise soll ebenfalls die Beteiligung im Hochschulsystem ausgeweitet werden, insbesondere gilt dieses für den Zugang für bisher unterrepräsentierte Gruppen auf allen Studienebenen. Aber nicht nur Zugangsanforderungen, sondern auch die Studienbedingungen sollen an die Bedürfnisse dieser Gruppen angepasst werden. Hier scheint immer wieder durch, dass die Studierenden, welche bisher nicht im Hochschulsystem waren, besondere Bedürfnisse haben, auf welche eingegangen werden muss. Als Beispiel werden die Lernumwelt, ökonomische Barrieren aber auch andere nicht weiter identifizierte Barrieren angeführt.

Access into higher education should be widened by fostering the potential of students from underrepresented groups and by providing adequate conditions for the completion of their studies. This involves improving the learning environment, removing all barriers to study, and creating the appropriate economic conditions for students to be able to benefit from the study opportunities at all levels. (Leuven Communiqué 2009: 2) 
Aber auch die Existenz intermediärer Studiengänge innerhalb des ersten Studienzyklus kann ein Mittel darstellen, die Beteiligung im Hochschulsektor zu erhöhen. Eine Weiterführung oder Erklärung des Vorschlags findet im Kommuniqué jedoch nicht statt, so dass hier ein großer Interpretationsspielraum bleibt. So könnten intermediäre Studiengänge beispielsweise ein- oder zweijährige Studiengänge sein, wie es sie auch in Frankreich gibt.

Im Gegensatz zu den Kommuniqués zuvor setzt die Deklaration der Konferenz in Budapest und Wien von 2010 keinen starken Fokus auf Fragen des lebenslangen Lernens. Aber es werden zwei durchlässigkeitsrelevante Punkte kurz angesprochen: Der Zugang zur Hochschullandschaft soll gleichberechtigt allen offen stehen, insbesondere für die bisher unterrepräsentierten Gruppen und flexible Lernwege sollen ermöglicht werden (Budapest-Vienna Declaration 2010).

Im Kommuniqué von Bukarest 2012 wurden keine neuen Wege zu größerer Durchlässigkeit identifiziert. Auch wurde weiterhin nicht direkt von Durchlässigkeit zwischen Berufs- und Hochschulbildung gesprochen, jedoch die bereits zuvor in den Kommuniqués erwähnten Ziele und Standards in ihrer Bedeutung unterstrichen. So wurde auch in Bukarest Kommuniqué die größere Öffnung der Hochschulen für bisher unterrepräsentierte Gruppen, welche durch Beratungsund Informationsstrukturen besser erreicht werden sollen, die Ermöglichung des lebenslangen Lernens, die Anrechnung vorgängigen Lernens auch non-formaler und informeller Lernergebnisse, die Verbindung des hochschulischen Qualifikationsrahmen mit dem EQR sowie die Implementierung von einer stärkeren Studierendenzentrierung, um den Lernenden gerecht zu werden, gefordert (Bucharest Communiqué 2012).

\section{Institutionelle Dimensionen von Durchlässigkeit im Bologna- Prozess}

Während zuvor chronologisch für die spezifischen Bologna-Dokumente dargestellt wurde, was an durchlässigkeitsrelevanten Aspekten vorkam, findet die Darstellung der institutionellen Dimensionen für den gesamten Prozess zusammen statt. Dabei wird nicht unterschieden, wie häufig bestimmte Aspekte Erwähnung gefunden haben. Ziel ist stattdessen, das Spektrum des vermittelten Durchlässigkeitskonzepts darzustellen. Es handelt sich somit um eine Synthese und weitergehenden Analyse des zuvor Dargestellten.

Was sagen uns die europäischen Dokumente des Bologna-Prozesses zum Thema Durchlässigkeit, insbesondere zwischen beruflicher und Hochschulbildung? Warum ist Durchlässigkeit wichtig? Welche Aspekte von Durchlässigkeit werden thematisiert, d.h., wie wird Durchlässigkeit verstanden? Dies sind die Fragen, die helfen sollen, die kulturell-kognitive Dimension zu erfassen. Wie genau soll Durchlässigkeit bzw. Bildungsmobilität zwischen den Bildungsbereichen erreicht werden? Welche Standards sollen eingeführt werden. Für welche Zielgruppe wird Durchlässigkeit thematisiert? Diese Fragen dienen der Analyse der 
normativen institutionellen Dimension. Wer soll die institutionelle Durchlässigkeit national umsetzen? Wie sollen Durchlässigkeitsstrukturen finanziert werden? Antworten auf diese Fragen geben Hinweise auf die regulative institutionelle Dimension (vgl. Abschnitt 4.2.2).

Bevor die institutionellen Dimensionen ausführlich dargestellt werden, folgen zuerst ein paar allgemeine Feststellungen:

1. Auch wenn das Wort Durchlässigkeit nicht in den englischen Dokumenten des Bologna-Prozess vorkommt, werden doch durchlässigkeitsrelevante Themen, im Sinne der Entwicklung von flexiblen Lernwegen zur Ermöglichung von Bildungsmobilität, behandelt, aus denen schließlich das in den europäischen Dokumenten vermittelte Bild von Durchlässigkeit ersichtlich wird.

2. Durchlässigkeitsrelevante Aussagen treten vor allem im Kontext von Passagen zu lebenslangem Lernen und der sogenannten sozialen Dimension auf, wobei es bis auf sehr wenige Ausnahmen nicht explizit um Durchlässigkeit zwischen den Organisationsfeldern der Berufs- und Hochschulbildung geht, sondern die Aussagen allgemein für das gesamte Bildungssystem oder nur hochschulspezifisch gehalten werden.

3. Im Zeitverlauf mit Ausnahme der Konferenz in Wien/Budapest 2010, sieht man eine Bedeutungszunahme von Themen der Durchlässigkeit, und zwar qualitativ und quantitativ. Dies hängt eng mit der Konjunktur des Themas lebenslanges Lernen zusammen (vgl. Jakobi 2009; Jakobi/Rusconi 2009). Quantitativ heißt, dass in einer größer werdenden Zahl von Absätzen durchlässigkeitsrelevante Themen diskutiert werden, und qualitativ bedeutet, dass die Themen eingehender besprochen werden. So wurde z.B. in Prag und Berlin noch allgemein die Anerkennung von erworbenen Zertifikaten bzw. vorgängigem Lernen gefordert. Seit Bergen ist dieses vorgängige Lernen spezifiziert in formales, non-formales und informelles Lernen. Auch die Rolle von Qualifikationsrahmen und lebenslangem Lernen werden im Zeitverlauf ausführlicher diskutiert, wobei ebenfalls Wege und Mittel aufgezeigt werden, wie lebenslanges Lernen oder eine Ausweitung der Studienbeteiligung auf alle sozialen Gruppen zu erreichen sei.

\section{Kulturell-kognitive Dimension:}

In den Bologna-Dokumenten konnten drei Aspekte institutioneller Durchlässigkeit identifiziert werden: die Frage des Zugangs, Anerkennung und Anrechnung, sowie der Umgang mit Heterogenität.

Der erste Aspekt, die Frage des Zugangs zum Hochschulsystem und zu den einzelnen Zyklen wird in dreifacher Weise thematisiert: Erstens soll der Zugang diskriminierungsfrei und chancengleich für alle Bürger_innen sein. In diesem $\mathrm{Zu}$ sammenhang wird zweitens auf die Integration von bisher benachteiligten Gesellschaftsgruppen gedrungen und damit für eine Erhöhung der Partizipation im 
Hochschulbereich plädiert. Hier sind indirekt beruflich Qualifizierte angesprochen, wenn diese bisher nicht zur traditionellen Student_innenschaft zählten. Jedoch sollen sich drittens die Hochschulen nur für diejenigen öffnen, welche sich als leistungsfähig erweisen. Auf diese Weise wird auf meritokratische Ideale, welche unsere Bildungsgesellschaften prägen (Solga 2005a), verwiesen. Wie genau die Hochschulreife definiert wird und welche Kompetenzen die Individuen mitbringen müssen, wird nicht weiter erörtert, d.h. eine genaue Charakterisierung dessen, was den Hochschulbereich spezifisch macht, findet in diesem Zusammenhang nicht statt.

Mit dem Thema der Anerkennung und Anrechnung im Hochschulbereich primär von bisherigen Studienleistungen, aber auch von außerhochschulisch erworbenen Vorkenntnissen, dem informellen oder non-formalen Lernen wird auch der zweite Aspekt von Durchlässigkeit eingefordert. Ein direkter Bezug zur Berufsbildung wird ebenfalls nicht hergestellt, jedoch kann diese als in den außerhochschulisch erworbenen Kenntnissen integriert betrachtet werden.

Der dritte Aspekt, welcher in den Bologna-Dokumenten extrahiert werden konnte, ist die Institutionalisierung von Strukturen, welche den Umgang mit heterogenen Bedürfnissen der Studierenden erleichtern und darauf abzielen, Barrieren zum und im Hochschulsystem abzubauen. Im Rahmen des Bologna-Prozesses wird wiederholt auf die Existenz von Hindernissen hingewiesen, welche einem erfolgreichen Studium im Wege stehen. So sind es sowohl Barrieren finanzieller Art als auch die Lernumwelt, d.h. eine fehlende Unterstützung beim Studium, sowie fehlendes Wissen bzw. Informationen über das Hochschulsystem, die als hinderlich erkannt werden. Interessanterweise wird vor allem den bisher nicht traditionellen Studierenden ein besonderer Bedarf der Unterstützung zugeschrieben.

Doch wie wird die Notwendigkeit von durchlässigeren Strukturen begründet? Wenn es um die Frage des auf unterrepräsentierte Gruppen zu erweiternden Zugangs geht, wird mit der Förderung von Chancengleichheit und sozialer Kohäsion argumentiert, der sogenannten sozialen Dimension des Bologna-Prozesses. Durchlässigkeitsrelevante Forderungen im Kontext von Lebenslangem Lernen werden vor allem mit dem Zugewinn an Mobilität, primär innerhalb der Hochschullandschaft Europas sowohl national als auch transnational, legitimiert. Auch der demografische Wandel als Herausforderung für die europäischen Gesellschaften wird als eine weitere Begründung für die Integration der Politik des lebenslangen Lernens in den Bologna-Prozess aufgenommen.

\section{Normative Dimension}

Wie genau sollen durchlässigere Strukturen geschaffen werden? Welche Wege und Instrumente werden aufgezeigt? Was sind die zu implementierenden Standards und in welchem Zusammenhang mit welchen Aspekten werden diese thematisiert? 
Dadurch, dass Mobilität insbesondere geografische, aber auch Bildungsmobilität, wenn auch vor allem innerhalb der Hochschulsysteme, eine zentrale Stellung im Bologna-Prozess einnimmt, können eine ganze Reihe von Standards identifiziert werden, welche durchlässige Bildungsstrukturen befördern. Fast alle Standards haben daher zum Ziel, innerhalb der Hochschullandschaft flexible Lernwege zu fördern. Aber auch eine erhöhte Mobilität zwischen Organisationsfeldern kann mit vielen dieser Standards befördert werden. Analytisch unterscheide ich die Standards daraufhin, auf welcher Ebene, d.h. auf der der Bildungsorganisationen oder der des -systems, sie angesiedelt sind und ob sie stärker auf die Organisation der Bildungsgänge oder der Unterstützung von Bildungsteilnehmer_innen abzielen.

So werden erstens Standards identifiziert, welche auf Hochschulebene und einem Fokus auf die Organisation der Bildungsgänge angesiedelt sind. Das wären die Einführung der Lernergebnisorientierung, der Leistungspunktesysteme, der Modularisierung, der Studienzyklenstruktur sowie von Verfahren der Anerkennung und Anrechnung und Qualitätssicherung. Allein Verfahren zur Anerkennung von formalem, non-formalem und informellem Lernen zielen in den Dokumenten stärker auf eine erhöhte Mobilität zwischen Organisationsfeldern. Diese könnten die berufliche Bildung, die Erwerbsarbeitssphäre oder auch die Nichterwerbsarbeitssphäre sein. Anerkennungsverfahren dienen in diesem Zusammenhang dem Transfer von Kenntnissen von einem Bereich in den nächsten und können daher sowohl für den Zugang zur Hochschule als auch für die Anrechnung von (nicht-akademisch) erworbenen Leistungen innerhalb des Hochschulsystems eine Rolle spielen.

Die Implementierung der dreigliedrigen Studienstruktur und Studienmodule sowie die Einführung von Qualitätssicherungssystemen werden in den Dokumenten allein im Zusammenhang von Mobilität im (europäischen) Hochschulbereich angesprochen. Die Einführung der Studienzyklen, dem Bachelor, Master und Doktor, und ihre Modularisierung soll dabei die Vergleichbarkeit der Abschlüsse erhöhen, so dass diese leichter an anderen Hochschulorganisationen anerkannt werden und die Zulassung zum nächsten Zyklus erleichtert wird. Qualitätssicherungssysteme wie die Akkreditierung von Studiengängen haben dagegen die Aufgabe, Vertrauen in die Abschlüsse zu erhöhen, so dass ihre Anerkennung und Zulassung einfacher vonstatten gehen kann.

Die Einführung von Qualifikationsrahmen, von Leistungspunktesystemen und der Lernergebnisorientierung sind zentral bei der Förderung von durchlässigeren Strukturen im Hochschulbereich sowie zwischen Organisationsfeldern und zwar in den Aspekten des Zugangs und der Anrechnung. Lernergebnisorientierung soll nicht nur die Entwicklung von Studiengängen an sich anleiten, sondern ist auch die Grundlage für das Funktionieren von Leistungspunktesystemen und Qualifikationsrahmen. 
The development, understanding and practical use of learning outcomes is crucial to the success of ECTS, the Diploma Supplement, recognition, qualifications frameworks and quality assurance - all of which are interdependent. (Bucharest Communiqué 2012: 3)

Die Orientierung von Bildungsgängen an Lernergebnissen soll daher die Transparenz bzw. Vergleichbarkeit und damit auch die Transferierbarkeit von Gelerntem erleichtern. Leistungspunkte sind dann die Währung, sozusagen die Möglichkeit, Wissen, Fertigkeiten und Kompetenzen zählbar zu akkumulieren und zu transferieren. Auch der Erwerb von Leistungspunkten außerhalb der Hochschule und dessen Übertragung auf die Hochschulebene wird generell als möglich erachtet.

Zweitens werden stärkere Standards eingefordert, welche auch innerhalb von Bildungsorganisationen eingeführt werden müssen, aber stärker auf die Unterstützung der Bildungsteilnehmer_innen zielen und einen Umgang mit der Heterogenität dieser Lernenden ermöglichen. Dies wären institutionalisierte Beratungsangebote, Unterstützungssysteme und Flexibilisierungsmöglichkeiten. Durch ihre Einführung wird zum einen Durchlässigkeit im Hochschulbereich gefördert, aber zum anderen ist ebenfalls erhöhte Durchlässigkeit zwischen verschiedenen Organisationsfeldern denkbar. Letzteres hängt davon ab, woher die Gruppe kommt, auf welche die zu implementierenden Standards zugeschnitten werden. So werden erstens bessere und institutionalisierte Beratungsangebote gefordert. Diese sollen sowohl bei der Orientierung im Studium als auch auf dem Weg zum Studium helfen und den Bürger_innen die notwendigen Informationen geben, welche sie benötigen, um sich selbstständig fortzubilden. Fehlende Informations- und Beratungsangebote werden auch als ein Grund angesehen, warum bestimmte Bevölkerungsgruppen nicht den Weg in die Hochschule finden, insofern stellt ihre Institutionalisierung die Anpassung an die Bedarfe nicht-traditioneller Studierender dar. Eine ebensolche Anpassungshilfe sollen zweitens auch Unterstützungssysteme, wie Finanzhilfen, aber auch drittens Flexibilisierungsmöglichkeiten des Studiums - z.B. durch die Einführung von Teilzeitstudiengängen oder verstärktem E-Learning - sein. Ziel der Standards ist somit die Ermöglichung eines erfolgreichen Studiums entsprechend der differenten Bedürfnisse der Lernenden.

Drittens wird schließlich mit dem Qualifikationsrahmen auch ein Standard eingefordert, welcher stärker auf Ebene des gesamten Hochschulbereichs bzw. in Verbindung mit den nationalen Qualifikationsrahmen auf Ebene des Bildungssystems angesiedelt ist. Die Entwicklung und Einführung von Qualifikationsrahmen im Hochschulbereich, welche auf gemeinsamen Referenzniveaus fußen und lernergebnisorientiert beschrieben werden, sollen primär zu einer größeren Transparenz der angebotenen Studiengänge Europas führen und damit eine bessere Orientierungsmöglichkeit bieten. Zudem sollen die Qualifikationsrahmen im Hochschulbereich aber auch mit dem europäischen Qualifikationsrahmen für lebenslanges Lernen und den nationalen komplementären Rahmen koordiniert werden, 
so dass sie anschlussfähig sind und Transparenz dann für das gesamte Bildungssystem also auch zwischen Organisationsfeldern gewährleistet ist.

Tabelle 5 fasst die identifizierten Standards zusammen und zeigt ihre Zuordnung zu den in den Bologna-Dokumenten thematisierten Durchlässigkeitsaspekten sowie dem Ort, an welchem die Durchlässigkeitsstrukturen wirken sollen.

Tabelle 5 Durchlässigkeits-Standards im Bologna-Prozess

\begin{tabular}{|c|c|c|}
\hline Standards & $\begin{array}{l}\text { Durchlässigkeitsaspekt(e), in } \\
\text { deren Kontext die Standards } \\
\text { thematisiert wurden }\end{array}$ & $\begin{array}{c}\text { Wo wird Durchlässigkeit } \\
\text { gefördert? }\end{array}$ \\
\hline \multicolumn{3}{|c|}{ Ebene der Bildungsorganisationen mit Fokus auf Bildungsgang } \\
\hline Lernergebnisorientierung & Zugang und Anrechnung & $\begin{array}{l}\text { Im Hochschulbereich und } \\
\text { zwischen Organisationsfeldern }\end{array}$ \\
\hline Leistungspunktesystem & Zugang und Anrechnung & $\begin{array}{l}\text { Im Hochschulbereich und } \\
\text { zwischen Organisationsfeldern }\end{array}$ \\
\hline Modularisierung & Zugang und Anrechnung & im Hochschulbereich \\
\hline Studienzyklenstruktur & Zugang und Anrechnung & Im Hochschulbereich \\
\hline $\begin{array}{l}\text { Verfahren zur Anerkennung } \\
\text { von vorgängigem Lernen }\end{array}$ & Zugang und Anrechnung & $\begin{array}{l}\text { Im Hochschulbereich und } \\
\text { zwischen Organisationsfeldern }\end{array}$ \\
\hline Qualitätssicherungssysteme & Zugang und Anrechnung & Im Hochschulbereich \\
\hline \multicolumn{3}{|c|}{ Ebene der Bildungsorganisationen mit Fokus auf das Bildungssubjekt } \\
\hline $\begin{array}{l}\text { Institutionalisierte } \\
\text { Beratungsangebote }\end{array}$ & Umgang mit Heterogenität & $\begin{array}{l}\text { Im Hochschulbereich und } \\
\text { zwischen Organisationsfeldern }\end{array}$ \\
\hline $\begin{array}{l}\text { Unterstützungssysteme ins } \\
\text { und im Studium }\end{array}$ & Umgang mit Heterogenität & $\begin{array}{l}\text { Im Hochschulbereich und } \\
\text { zwischen Organisationsfeldern }\end{array}$ \\
\hline $\begin{array}{l}\text { Flexibilisierungsmöglichkei- } \\
\text { ten des Studiums }\end{array}$ & Umgang mit Heterogenität & $\begin{array}{l}\text { Im Hochschulbereich und } \\
\text { zwischen Organisationsfeldern }\end{array}$ \\
\hline \multicolumn{3}{|c|}{ Ebene des Bildungssystems } \\
\hline $\begin{array}{l}\text { Qualifikationsrahmen für die } \\
\text { Hochschulbildung }\end{array}$ & Zugang und Anrechnung & $\begin{array}{l}\text { Im Hochschulbereich und } \\
\text { zwischen Organisationsfeldern }\end{array}$ \\
\hline
\end{tabular}

Quelle: Darstellung der Verfasserin

Neben den zu implementierenden Standards ist auch die Zielgruppe, für die durchlässigere Strukturen entwickelt werden sollen, für die normative Dimension zu untersuchen. Konkrete Gruppen, auf die das zutrifft, werden in den Dokumenten kaum hervorgehoben. Stattdessen werden sie als bisher unterrepräsentiert bezeichnet. Dazu gehören aber explizit genannt ebenfalls beruflich Qualifizierte. Die Zielgruppe für das Studium allgemein wird wie folgt dargestellt: Die Gruppe der Studierenden soll einerseits die Diversität der Gesellschaft widerspiegeln, so dass auch diejenigen einbezogen werden, welche bisher nicht traditionell an die Hochschulen gekommen sind. Voraussetzung für den Zugang zur Hochschule ist 
aber andererseits eine nicht näher definierte Leistungsfähigkeit, welche die Lernenden befähigt, ein Studium auch abzuschließen. In den Dokumenten wird damit einerseits deutlich zwischen Fähigen und Nicht-Fähigen unterschieden. Andererseits wird auch insbesondere den bisher unterrepräsentierten Gruppen der Bedarf von spezifischen Zuwendungen zugeschrieben, der ihnen ein erfolgreiches Studieren eher ermöglicht. Insofern wird auch innerhalb der Gruppe der Studierenden unterschieden zwischen den traditionellen Studierenden und den nicht traditionellen mit erhöhtem Zuwendungsbedarf.

\section{Regulative Dimension}

Die regulative Dimension wird in den Dokumenten kaum angesprochen. Allgemein werden jedoch die Frage der Finanzierung der Hochschulen und der Durchsetzung von lebenslangem Lernen als öffentliche Aufgabe dargestellt. Zudem soll in die Autonomie der Hochschulen nicht eingegriffen werden, so dass die Umsetzung der Anerkennungsverfahren direkt in ihr Aufgabengebiet fallen. Insgesamt wird die Implementierung von lebenslangem Lernen als gemeinsame Aufgabe von Ministerien, den Hochschulorganisationen, Studierenden, Arbeitgeber_innen und -nehmer_innen gesehen.

\subsubsection{Durchlässigkeit im Kopenhagen-Prozess}

\section{Eine chronologische Darstellung der Thematisierung von Durchlässigkeit}

Bereits seit Beginn des Kopenhagen-Prozesses 2002 wird anerkannt, dass die berufliche Bildung lediglich im Kontext mit anderen Bildungsbereichen und europäischen Bildungskooperationen weiterentwickelt werden kann. So ist der Kopenhagen-Prozess integriert in die Lissabon-Strategie von 2000, in der die Rolle der beruflichen Bildung für das Erreichen der gesetzten Ziele herausgehoben wird. Zudem wird bereits in der Kopenhagen-Erklärung auf den bestehenden Bologna-Prozess für die Hochschulbildung verwiesen, welcher den Beginn einer neuen Qualität der europäischen Zusammenarbeit in Bildungsfragen markiert (Copenhagen Declaration 2002). Auch durchlässigkeitsrelevante Aspekte nehmen seit dem Start des Prozesses eine zentrale Rolle in den Zielsetzungen ein. Ebenso wie im Bologna-Prozess liegt das Hauptaugenmerk auf Ermöglichung von geografischer Bildungsmobilität, für die Anerkennungsmechanismen, Transparenz der Qualifikationen und Qualitätssicherung Voraussetzung sind. Aber auch Bildungsmobilität innerhalb der Systeme zwischen Bildungsbereichen wird von Anfang an mitgedacht. So ist die Ermöglichung von lebenslangem Lernen seit der Kopenhagener-Erklärung ein zentrales Ziel, in dessen Kontext wiederum die Implementation von Standards gefordert wird, welche die institutionelle Durchlässigkeit von Bildungssystemen verbessern: Die diesbezüglichen Prioritäten in der 
Kopenhagener Erklärung sind die Schaffung von Transparenz und die Möglichkeit der Validierung sowie Anerkennung von Kompetenzen und Qualifikationen. Transparenz soll gewährleistet werden durch Beratungsangebote und öffentlich zugängliche Informationen über das berufliche Bildungssystem insbesondere Informationen über Zugangsmöglichkeiten zu Bildungsgängen aber auch über Transfer- und Anerkennungsmöglichkeiten von Erlerntem. Transparenz soll daher auch nicht allein der geografischen grenzüberschreitenden Mobilität, sondern auch der beruflichen und der Bildungsmobilität innerhalb der nationalen Bildungssysteme dienen.

\begin{abstract}
Strengthening policies, systems and practices that support information, guidance and counselling in the Member States, at all levels of education, training and employment, particularly on issues concerning access to learning, vocational education and training, and the transferability and recognition of competences and qualifications, in order to support occupational and geographical mobility of citizens in Europe. (Copenhagen Declaration 2002)
\end{abstract}

Wie genau Vergleichbarkeit, Transferierbarkeit und Anerkennung von Qualifikationen und Kompetenzen erreicht werden soll, wird zu Beginn des KopenhagenProzesses noch nicht deutlich. Folglich wird daher anstatt der Implementation von klar definierten Standards, welche dies leisten könnten, vielmehr deren gemeinsame Entwicklung gefordert, so dass Kompatibilität zwischen den Ländern gewährleistet ist. Beratungsdienste und Leistungspunktesysteme für die berufliche Bildung (ECVET) werden bereits als nützliches Mittel anerkannt.

Im Gegensatz zum Bologna-Prozess wird im Kopenhagen-Prozess Anerkennung von vorgängigem Lernen nicht nur von Beginn an thematisiert, es wird zugleich als formales, sowie non-formales und informelles Lernen definiert. Zudem werden im Unterschied zum Bologna-Prozess auch von Beginn an die verantwortlichen Akteure benannt. So sollen insbesondere bei Fragen der Anerkennung und der Entwicklung von Anerkennungsverfahren die Sozialpartner mitwirken. Insgesamt wird in der Kopenhagen-Erklärung die Notwendigkeit, lebenslanges Lernen zu fördern, damit begründet, dass es die Beschäftigungsfähigkeit der Individuen erhöht, zu einer aktiveren Bürgerschaft, sozialen Inklusion aber auch der Persönlichkeitsentwicklung der Lernenden beiträgt.

Die erste Folgekonferenz des Kopenhagen-Prozesses fand 2004 in Maastricht statt. Die Ermöglichung von lebenslangem Lernen wird wiederum als ein wichtiger Schritt gesehen, diese Ziele zu erreichen (Maastricht Communiqué 2004). Wichtig ist zudem, dass im Kommuniqué von Maastricht erstmals explizit das Verhältnis beruflicher und Hochschulbildung thematisiert wird und zugleich ein wichtiges Thema im Kommuniqué darstellt.

Da berufliche Bildung zunehmend auf allen Bildungsebenen stattfindet, wird erstens Gleichwertigkeit zwischen beruflicher und Allgemeinbildung, insbesondere Hochschulbildung, und zweitens die Einrichtung von Übergängen von einem Bereich in den anderen gefordert. Es müsse möglich sein, auch innerhalb der 
Berufsbildung höhere Qualifikationsniveaus zu erwerben, denn nur auf diese Weise könne man mehr Auszubildende für die berufliche Bildung gewinnen.

VET is increasingly taking place at all educational levels and, therefore, the parity of esteem and links between VET and general education, in particular with higher education, need to be fostered by innovative strategies and instruments at the national and European levels. This should include designing VET systems which attract more students to higher qualifications. (Maastricht Communiqué 2004: 2)

An dieser Stelle wird auf europäischer Ebene auf eine zweifache Konkurrenzsituation hingewiesen. Zum einen geht es um die gesellschaftliche Wertschätzung zwischen den Bildungsbereichen, insbesondere dem Status der Hochschul- und Berufsbildung, wobei eine zu geringe Wertschätzung der beruflichen Bildung konstatiert wird. Dies zu ändern ist ein zentrales Ziel des Kopenhagen-Prozesses. Zum anderen geht es ebenfalls um den Wettbewerb um die klügsten Köpfe.

Es wird jedoch ebenfalls eine fehlende institutionelle Durchlässigkeit zwischen den Bildungsbereichen kritisiert. Bestehende Barrieren zwischen Berufsbildung und Allgemeinbildung sollen verringert werden. Dabei geht es demnach nur um eine Verringerung und nicht um eine gänzliche Abschaffung der Barrieren. Dies soll erstens geschehen durch die Entwicklung offener Lernansätze, welche zusammen mit Berufsorientierungs- und Beratungsleistungen Individuen befähigen, ihren Bildungsweg selbstständig zu planen, und zweitens sollen flexible und offene Rahmenbedingungen in der Berufsbildung geschaffen werden, welche die Durchlässigkeit zwischen Aus-, Weiterbildung und Hochschulbildung befördern. Notwendig ist:

$[\ldots]$ the development and implementation of open learning approaches, enabling people to define individual pathways, supported by appropriate guidance and counselling. This should be complemented by the establishment of flexible and open frameworks for VET in order to reduce barriers between VET and general education, and increase progression between initial and continuing training and higher education. (Maastricht Communiqué 2004: 3).

Die bestehenden Barrieren zwischen Berufs- und Allgemeinbildung werden auf unterschiedlichen Ebenen identifiziert. Einmal auf der individuellen Ebene: Die Bürger_innen müssen immer mehr in die Lage versetzt werden, durch Beratungsund Orientierungsangebote selbst ihre Bildungswege zu planen. Zum anderen werden strukturelle Probleme erkannt - zu geschlossene und unflexible Strukturen im Berufsbildungssystem, welche an dieser Stelle nicht näher identifiziert werden.

Weitere strukturelle Probleme, welche Durchlässigkeit zwischen den Bildungssektoren (aber auch nationalen Bildungssystemen) behindern, werden bei Fragen der Anerkennung und Übertragbarkeit von Qualifikationen sowie der Validierung und Anerkennung non-formal und informell erworbener Kompetenzen gesehen. Zwei Instrumente sollen insbesondere helfen, diese Probleme zu überwinden. Zum einen wird die Entwicklung eines offenen und flexiblen Europäischen Qualifikationsrahmens, der auf Transparenz und gegenseitigem Vertrauen 
beruht, gefordert. Zum anderen die Entwicklung und Einführung von Leistungspunktesystem für die berufliche Bildung (ECVET) (vgl. Maastricht Communiqué 2004).

Im Kommuniqué von Maastricht wird damit zum ersten Mal die Entwicklung von Qualifikationsrahmen thematisiert. Dies ist bedeutend, denn nationale und der europäische Qualifikationsrahmen werden zu zentralen Standards im Kopenhagen- aber auch im Bologna-Prozess. Sie werden explizit als Facilitatoren von Durchlässigkeit in Bildungssystemen anerkannt, da sie als gemeinsame Bezugsrahmen für die Anerkennung und den Transfer von Qualifikationen sowie nonformal und informell erworbener Kompetenzen zwischen den beruflichen und allgemeinbildenden Sektoren (Sekundar- und Hochschulbildung) dienen sollen. Um dies zu leisten, müssen Qualifikationsrahmen auf gemeinsamen Referenzniveaus fußen, welche wiederum über Beschreibungen von zu erreichenden Lernergebnissen und Kompetenzen definiert werden.

The framework will provide a common reference to facilitate the recognition and transferability of qualifications covering both VET and general (secondary and higher) education, based mainly on competences and learning outcomes. It will improve permeability within education and training systems [...].(Maastricht Communiqué 2004: 4)

Um das Funktionieren von Qualifikationsrahmen zu gewährleisten, wird im Kommuniqué von Maastricht die Entwicklung von Qualitätssicherungsmechanismen als sehr wichtig erachtet, da nur so das notwendige Vertrauen in die - in den einzelnen Bildungsbereichen erworbenen - Qualifikationen und Kompetenzen entstehen kann.

Die Entwicklung und Umsetzung von Leistungspunktesystemen für die Berufsbildung (European Credit Transfer System for VET; ECVET) soll ebenfalls Mittel sein, Lernende beim Wechsel zwischen Bildungsbereichen zu unterstützen, indem sie auf ihre Fähigkeiten, welche sie im Rahmen ihrer Lernlaufbahn erworben haben, aufbauen können. Ebenso wie Qualifikationsrahmen sollen ECTS auf erworbenen Kompetenzen und Lernergebnissen basieren. Ziel ist ihre Umsetzung in einem ersten Schritt für das System formaler Qualifikationen, aber es soll ebenfalls in einem zweiten Schritt auf nicht formale Qualifikationen anwendbar gemacht werden (Maastricht Communiqué 2004).

Um Fragen der Durchlässigkeit geht es auch, wenn über den Zugang zur beruflichen Bildung gesprochen wird. Dieser soll allen europäischen Bürgern offenstehen, aber insbesondere soll berufliche Bildung der Förderung von benachteiligten Gruppen, wie Schulabbrecher_innen, Arbeitslosen, Migrant_innen, Geringqualifizierten, Personen mit Behinderung dienen, welche ansonsten oft gefährdet sind, sozial exkludiert zu werden. Ihre Integration soll vor allem auch über Anerkennung von Lernerfahrungen und auf sie abgestimmte Schulungs-, Beratungs- und Lernangebote erfolgen (Maastricht Communiqué 2004). Hier wird Durchlässigkeit gefordert - zum einen zwischen den Bildungsbereichen der Schule und Berufsbildung aber auch zwischen der Sphäre des Arbeitsmarkts, bzw. 
der Arbeitslosigkeit und der beruflichen Bildung. Es wird im Gegensatz zur Hochschulbildung nicht zwischen einer Gruppe der Fähigen und Nicht-Fähigen unterschieden. Leistung als wichtiges Zugangskriterium findet keine Erwähnung. Stattdessen soll berufliche Bildung für High Potentials, aber auch für Geringqualifizierte offen stehen.

Das Kommuniqué von Helsinki von 2006 nimmt die wichtigsten durchlässigkeitsrelevanten Aspekte des Kommuniqués von Maastricht wieder auf. Sie sind wiederum eingebettet in die Bemühungen, lebenslanges Lernen in Europa zu ermöglichen. Im Folgenden sollen vor allem die Aspekte angesprochen werden, welche die Durchlässigkeit bzw. Bildungsmobilität zwischen Berufs- und Hochschulbildung betreffen. Angemerkt sei aber, dass Fragen der institutionellen Durchlässigkeit zwischen anderen Bildungsbereichen sowie auch zwischen dem Arbeitsmarkt, der Nichterwerbsarbeitssphäre und der beruflichen (Weiter-)Bildung im Kommuniqué von Helsinki thematisiert werden. Es wird gefordert, dass die beruflichen Bildungssysteme verstärkt Übergänge zur Weiterbildung insbesondere von der beruflichen Bildung zur Hochschulbildung ermöglichen, und so zur Flexibilisierung der Lernverläufe beitragen.

VET systems should, as part of flexible educational pathways, increasingly enable progression to further education and training, especially from VET to higher education. (Helsinki Communiqué 2006: 2)

So wird z.B. eine hochqualitative berufliche Erstausbildung als förderlich für die Verbindung der beiden Organisationsfelder angesehen. Auch soll berufliche Bildung auf allen Qualifikationsebenen als wichtiger Bestandteil des Regimes des lebenslangen Lernens weiterentwickelt werden und zwar mit engen Verbindungen zur Allgemeinbildung (Helsinki Communiqué 2006).

Wiederum wird explizit auf die Statusprobleme der beruflichen Bildung aufmerksam gemacht. Ihre Attraktivität und ihr Image sollen daher verbessert werden und zwar durch eine offene Ausgestaltung der Berufsbildungssysteme, welche flexibel und individuell genutzt werden können und bessere Voraussetzungen für Übergange ins Arbeitsleben, in die Weiterbildung, einschließlich Hochschulbildung schaffen. Ebenfalls zentral ist die Entwicklung von Systemen zur Anerkennung non-formalen und informellen Lernens, um so berufliche Weiterentwicklung und lebensbegleitendes Lernen zu unterstützen (Helsinki Communiqué 2006).

Auch die Arbeit an den gemeinsamen europäischen Instrumenten sollte fortgeführt werden: Dies beinhaltet erstens die Entwicklung von Leistungspunktesystemen für die Berufsbildung, um die Akkumulation und den Transfer von erworbenen Qualifikationen zu gewährleisten; zweitens in Zusammenarbeit mit den Hochschulen den Ausbau der Systeme der Qualitätssicherung, um gegenseitiges Vertrauen in Qualifikationen zu schaffen und drittens die Weiterentwicklung des EQR, um zum einen Gleichwertigkeit zwischen Berufs- und Hochschulbildung und zum anderen eine bessere Verknüpfung zwischen den beiden Bildungsbereichen zu erzielen. 
Further development of common European tools in which VET plays a major role, by: developing and testing a European Qualifications Framework (EQF) based on learning outcomes, providing greater parity and better links between the VET and HE sectors. (Helsinki Communiqué 2006)

Es zeigt sich, dass insbesondere das Instrument des Qualifikationsrahmens mit der Schaffung einer größeren Durchlässigkeit zwischen Berufs- und Hochschulbildung in Verbindung gebracht wird.

2008 trafen sich die Bildungsminister in Bordeaux, um die Entwicklung des Kopenhagen-Prozesses zu evaluieren und die Arbeitsschwerpunkte für die nächsten Jahre festzulegen. Als Hauptergebnis des Kopenhagen-Prozesses wird hier die Entwicklung von Instrumenten genannt, welche die Transparenz von Qualifikationen erhöhen, die Anerkennung von Wissen, Fertigkeiten und Kompetenzen befördern sowie die Qualität der Bildungssysteme sichern (Bordeaux Communiqué 2008).

Ebendiese Instrumente, der Qualifikationsrahmen (EQR und NQR), die Leistungspunktesysteme für die berufliche Bildung (ECVET), Prinzipien der Anerkennung von vorgängigem Lernen sowie Qualitätssicherungsinstrumente (EQARF) sind Instrumente, welche nicht nur Mobilität zwischen Ländern, sondern auch innerhalb von Bildungssystemen befördern können.

Wie in den Kommuniqués zuvor wird berufliche Bildung als wesentlicher Bestandteil der Realisierung von lebenslangem Lernen angesehen. Um dieses zu ermöglichen, bedarf es Brücken zwischen Allgemein-, Berufs- und Hochschulbildung. Während traditionell der Zugang von der Berufs- zur Hochbildung gefordert wird, wird in diesem Kommuniqué auch von der umgekehrten Möglichkeit ausgegangen - berufliche Bildung soll auch jene auffangen, die ihr Studium abgebrochen haben.

VET should offer individualised career paths in order to integrate those who have left their studies or training premature. (Bordeaux Communiqué 2008: 5)

Ziel ist es, individualisierte flexible Karrierewege zu eröffnen, die Erstausbildung, Weiterbildung und alle Formen des Lernens sowie alle Lernumfelder enger miteinander zu verknüpfen. Den Bürger_innen sollte ermöglicht werden, ihre Lernergebnisse zu akkumulieren und in andere Systeme, sei es transnational oder national zu übertragen. Um dies zu realisieren, ist es notwendig, alle Stakeholder bei der Weiterentwicklung von Berufsbildungspolitik einzubinden und diese entsprechend zu finanzieren und zwar durch einen Mix von öffentlichen, privaten und europäischen Geldern (Bordeaux Communiqué 2008).

Insbesondere Durchlässigkeit zur Hochschule steht im Kommuniqué von Bordeaux im Fokus und deren Bedarf wird erstmals ausführlicher begründet: Hauptargument für die Verbindung zwischen Berufs- und Hochschulbildung ist der Bedarf an hochqualifizierten Bürgern, welche für eine gesteigerte Wettbewerbs- und Innovationsfähigkeit Europas unabdingbar sind (Bordeaux Communiqué 2008). An dieser Stelle wird wiederum die enge Einbindung in die Lissabon- 
Strategie offenbar. Da die Struktur des Arbeitsmarkts sich ändert, müsse die Verbindung zwischen Hochschullandschaft und Berufsbildung ausgebaut werden: Es wird prognostiziert, dass $2020 \mathrm{ca} .80 \%$ aller Arbeiten von Individuen mit Abschlüssen auf mittlerer und hoher Qualifikationsebene ausgeführt werden, dass auch einfache Tätigkeiten, die bisher eher von Geringqualifizierten ausgeführt worden sind, anspruchsvoller werden und sich der Bedarf an gut ausgebildeten Bürgern in dem Zeitraum von 2006 bis 2020 verdoppelt. Um dem Bedarf an hochwertigen Qualifikationen und der wachsenden Entwertung von alten Qualifikationen Rechnung zu tragen, sind daher Brücken zwischen Schulbildung, Arbeitsmarkt, Ausbildung und Hochschulbildung wichtig (vgl. Bordeaux Communiqué 2008).

Es werden vier Prioritäten für die kommenden Jahre festgesetzt. Allen vier Prioritäten ist gemein, dass sie Durchlässigkeit zwischen Hochschule und Berufsbildung befördern und fordern (Bordeaux Communiqué 2008):

1. Einfübrung der oben genannten Durchlässigkeit fördernden Instrumente (EQR/NQR, ECVET, EQARF sowie Verfahren der Anerkennung und Validierung von vorgängigem Lernen)

2. Die Erböbung der Qualität und Attraktivität von Berufsbildung:

Hier wird wiederum explizit Durchlässigkeit zwischen Berufsbildung und Allgemein- und Hochschulbildung gefordert. Denn eine derartige Verbindung ermöglicht erst, von einem Qualifikationsniveau zu dem nächsten aufzusteigen.

Facilitate paths enabling people to progress from one level of qualification to another by strengthening links between general education, VET, higher education and adult learning (Bordeaux Communiqué 2008: 9)

Wichtig ist, dass der Zugang allen, insbesondere auch den benachteiligten Gruppen, offensteht. Beratungsangebote sollen hierbei helfen, die möglichen individuellen Bildungswege zu identifizieren.

3. Verbesserte Verbindung zwischen Berufsbildung und dem Arbeitsmarkt

Für dieses Ziel müssen insbesondere Verfahren zur Anerkennung von allen Lernformen weiterentwickelt und eingeführt werden. Zudem wird berufliche (Weiter-)Bildung in Hochschulen als ein wichtiger Weg gesehen auch die Integration in den Arbeitsmarkt zu gewährleisten. In diesem Kontext wird explizit auf die Charta der europäischen Universitäten für lebenslanges Lernen Bezug genommen.

4. Die Stärkung europäischer Kooperation

Hier wird u.a. eine verstärkte Zusammenarbeit mit dem Bologna-Prozess gefordert. Zudem wird als notwendig erachtet, dass die Rolle der beruflichen 
Bildung im zu entwickelnden Rahmenprogramm für die europäische $\mathrm{Zu}$ sammenarbeit auf dem Gebiet der allgemeinen und beruflichen Bildung (ET 2020) Anerkennung findet und es verstärkt Verbindungen gibt zwischen den Politikprogrammen für die Allgemein-, Hochschul- und Erwachsenenbildung.

Das Kommuniqué von Brügge wurde 2010 unter dem Einfluss von mehreren Ereignissen erarbeitet: Insbesondere sind dies die Wirtschaftskrise und damit steigenden Arbeitslosigkeitszahlen in Europa, aber auch der 2009 von den EU-Mitgliedsstaaten verabschiedete strategische Rahmen für die europäische Zusammenarbeit auf dem Gebiet der allgemeinen und beruflichen Bildung „Allgemeine und berufliche Bildung 2020 (ET 2020)“, indem der Kopenhagen-Prozess ein fester Bestandteil ist. Das Kommuniqué zeigt wiederum die Herausforderungen, denen sich die Mitgliedsstaaten stellen müssen. Dies sind Arbeitsmarktwandel und die Tatsache, dass die Halbwertzeit von Qualifikationen immer stärker abnehmen, der demografische Wandel, soziale Kohäsion und eben die herrschende Wirtschaftskrise. Vor diesem Hintergrund werden im Kommuniqué von Brügge die Kernziele für die Entwicklung der beruflichen Bildung in Europa bis zum Jahr 2020 festgelegt. Zu den übergeordneten Zielen gehört dabei, den Anteil der 30bis 34-Jährigen, welche über einen tertiären oder vergleichbaren Abschluss verfügen, auf mindestens $40 \%$ zu erhöhen und lebenslanges Lernen in Europa zu verwirklichen. Aus diesem Grund sind auch viele der formulierten Kernziele aus Durchlässigkeitsperspektive relevant (vgl. Bruges Communiqué 2010).

Durchlässigkeit zu anderen Bildungssegmenten und auch explizit zur Hochschulbildung wird als notwendig erachtet, um die berufliche Bildung attraktiver zu machen, aber auch, um lebenslanges Lernen und damit Bildungsmobilität zu ermöglichen.

\title{
Die Erhöhung der Attraktivität von Berufsbildung erfolgt durch
}

\begin{abstract}
Flexible systems of VET, based on a learning outcomes approach, which support flexible learning pathways, which allow permeability between the different education and training subsystems (school education, VET, higher education, adult education) and which cater for the validation of non-formal and informal learning, including competences acquired in the work place. (Bruges Communiqué 2010: 6)
\end{abstract}

Lebenslanges Lernen und Mobilität wird ermöglicht, indem die Mitgliedsstaaten

Promote flexible pathways between VET, general education and higher education, and enhance permeability by strengthening the links between them. (Bruges Communiqué 2010: 11)

Die Einführung von Lernergebnisorientierung und das Verfahren der Anerkennung vorgängigen Lernens, sowohl von formalen als auch von informellen und non-formalen Lernformen, ermöglichen flexible Lernwege im Berufsbildungssystem selbst und auch außerhalb. Zur Verwirklichung dieses Ziels und zur Steigerung der Teilnahme am lebenslangen Lernen wird der Erstellung und Umsetzung 
von nationalen Qualifikationsrahmen, die ebenfalls auf Lernergebnissen basieren, eine Katalysatorfunktion zuerkannt. Qualifikationsrahmen, welche Allgemeinbildung, Berufs- und Hochschulbildung in einem Instrument vereinigen, schaffen Transparenz und erhöhen die Durchlässigkeit und Flexibilität der nationalen Bildungssysteme, insbesondere zwischen Hochschul- und Berufsbildung. Zudem können sie die Entwicklung von Verfahren zur Validierung des nicht formalen und informellen Lernens unterstützen und zur Verbesserung von integrierten Beratungsdiensten, welche alle Bildungssegmente betrachten, beitragen (vgl. Bruges Communiqué 2010).

An mehreren Stellen im Kommuniqué von Brügge wird der Wunsch deutlich, dass berufliche Bildung als der Hochschulbildung gleichwertig anerkannt wird. Auch auf europäischer Ebene ist somit die Frage der Gleichwertigkeit und den damit im Zusammenhang stehenden Statusfragen kontinuierlich präsent. So wird einerseits unterstrichen, dass in der Wissensgesellschaft nicht allein akademische Fertigkeiten gefragt sind, sondern auch die der beruflichen Bildung.

If Europe is to maintain its position as the strongest exporter of industrial products in the world, it must have world class VET. In the knowledge society vocational skills and competences are just as important as academic skills and competences. (Bruges Communiqué 2010: 3)

Zudem muss es ein Ziel sein, auch der Bevölkerung berufliche Bildung als genauso attraktive Lernmöglichkeit wie die allgemeinbildenden Lernwege näherzubringen.

High quality initial VET (I-VET) which learners, parents and society at large may regard as an appealing option, of the same value as general education. (Bruges Communiqué 2010: 6)

Außerdem wird für die Berufsbildung neben beruflichen Kompetenzen zunehmend stärker die Vermittlung von sogenannten Schlüsselkompetenzen gefordert, welche dann auch Übergänge in eher allgemeinbildende Bildungsgänge erleichtern. Andererseits wird die Integrationskraft von praxisorientiertem Lernen am Arbeitsplatz hervorgehoben. Denn genau diese Praxisorientierung kann Personen, welche in anderen Lernumgebungen eher scheitern, eine Möglichkeit bieten, ihr Potenzial zu entwickeln. Berufliche Bildung muss demnach den Balanceakt schaffen: auf der einen Seite muss sie integrativ auch für eher Niedrigqualifizierte wirken, andererseits soll sie auch dazu beitragen, die Anzahl der hochqualifizierten Bürgern in Europa zu erhöhen (Bruges Communiqué 2010).

Es lässt sich zusammenfassen, dass, während in der Kopenhagen-Deklaration 2002 noch allgemein lebenslanges Lernen und damit auch Bildungsmobilität innerhalb der nationalen Bildungssysteme befördert werden soll, seit Maastricht 2004 explizit auch institutionelle Durchlässigkeit zwischen beruflicher Bildung und Hochschulbildung ein Ziel ist, welches in allen weiteren Kommuniqués thematisiert wird. Im Gegensatz zum Bologna-Prozess ist keine deutliche Zunahme an durchlässigkeitsrelevanten Aspekten in den Kommuniqués zu erkennen, da das 
Thema seit dem Beginn des Prozesses eine sehr zentrale Stellung einnimmt. Allein die Frage, wie Durchlässigkeit umgesetzt werden soll, wurde im Verlauf konkretisiert.

\section{Institutionelle Dimensionen von Durchlässigkeit im Kopenhagen- Prozess}

Der Kopenhagen-Prozess wird von Anfang stark von anderen europäischen Initiativen und Prozessen mit beeinflusst. Zu nennen sind die Lissabon-Strategie, der strategische Rahmen für Rahmen für die europäische Zusammenarbeit auf dem Gebiet der allgemeinen und beruflichen Bildung 2010 und 2020 sowie der Bologna-Prozess. Insbesondere die Lissabon-Strategie ist für den KopenhagenProzess von Bedeutung, da sie Bildung, und vor allem auch lebenslanges Lernen, als einen zentralen Hebel identifiziert, um Europas Wissensökonomien wettbewerbsfähig zu machen (vgl. Jakobi 2009: 94).

Die Ermöglichung von lebenslangem Lernen bzw. flexibler Lernwege im Lebensverlauf ist somit auch ein zentrales Ziel des Kopenhagen-Prozesses, welches von Anfang an in den Dokumenten festgehalten wurde und in dessen Kontext Durchlässigkeit im Kopenhagen-Prozess primär thematisiert wird. Durchlässigkeit wird im Kopenhagen-Prozess für das gesamte Bildungssystem, zwischen Berufs- und Schul- sowie Hochschul- und Weiterbildung, aber ebenso zwischen beruflicher Bildung und der Erwerbsarbeitssphäre sowie Nichterwerbsarbeitssphäre inkl. Arbeitslosigkeit gefordert. Ähnlich wie im Bologna-Prozess sind es auch Fragen der sozialen Kohäsion, die durchlässigkeitsrelevante Aspekte ansprechen. Hier geht es jedoch stärker um die Integration benachteiligter Gruppen wie Geringqualifizierter, Arbeitsloser, Schulabbrecher_innen, Älterer und Migrant_innen in die berufliche Bildung. Diese Foki auf Durchlässigkeit sollen aber hier nicht näher betrachtet werden. Vielmehr steht Durchlässigkeit zwischen beruflicher und Hochschulbildung im Zentrum.

Im Folgenden sollen die institutionellen Dimensionen des spezifisch für die Berufs- und Hochschulbildung relevanten Durchlässigkeitskonzepts analysiert werden.

\section{Kulturell-kognitive Dimension}

Welches Verständnis von Durchlässigkeit wird sichtbar, d.h., welche Aspekte werden thematisiert? Wie wird die Notwendigkeit von Durchlässigkeit im Kopenhagen-Prozess begründet? Mit der Beantwortung dieser Fragen soll die kulturell kognitive Dimension von Durchlässigkeit zwischen Hochschul- und Berufsbildung als Institution dargestellt werden.

Vier Aspekte von Durchlässigkeit können in den Dokumenten identifiziert werden und zeigen, wie Durchlässigkeit im Kopenhagen-Prozess verstanden wird. 
Erstens geht es um Fragen der Zugangsmöglichkeiten zwischen dem Berufsbildungs- und Hochschulbereich, wobei Durchlässigkeit in beide Richtungen gewährleistet werden soll. Es wird explizit gefordert, dass nicht nur beruflich Qualifizierte in die Hochschule gelangen, sondern auch Studierende bzw. Studienabbrecher_innen in der beruflichen Bildung weiterlernen sollen. Die Ausgestaltung der Zugangsregelungen wird in den Dokumenten nicht weiter konkretisiert. Inkludiert wären dann individuumszentrierte oder gruppenspezifische Möglichkeiten.

Zweitens geht es im Kopenhagen-Prozess bereits seit Beginn auch um die Ermöglichung der Fragen der Übertragbarkeit von Kompetenzen und somit um die Anrechnung sowobl von formalem als auch von informellem und non-formalem Lernen. Durch Verfahren der Anerkennung wird die Verbindung der Organisationsfelder Berufs- und Hochschulbildung gestärkt. Teil dieses zweiten Aspektes ist dann auch die Anerkennung von Gleichwertigkeit beruflicher und akademischer Bildung, welche in den Kopenhagen-Dokumenten immer wieder gefordert wird. Denn nur wenn Gleichwertigkeit angenommen wird, ist faire Anerkennung und Anrechnung möglich. Berufliche Bildung findet auf allen Qualifikationsebenen statt und sollte auch dementsprechend angesehen und anerkannt werden. In den Dokumenten wird aber auf eine fehlende Wertschätzung der beruflichen Bildung in der Gesellschaft hingewiesen. Dies gilt es zu ändern.

Der dritte Aspekt, welcher in den Dokumenten thematisiert wird, ist die organisationale Verbindung von beruflicher Bildung und Hochschulbildung. Dabei geht es in den Dokumenten weniger um institutionelle Übergänge als um integrierte Bildungsgänge, im Sinne einer verstärkten beruflichen Ausrichtung von Studiengängen, die berufliche Praxisphasen mit dem Lernen in der Hochschule verbinden.

Schließlich spielt auch der vierte Aspekt, die Institutionalisierung von Strukturen, die den Umgang mit heterogenen Bedürfnissen der Lernenden erleichtern, eine Rolle. Vor allem werden Beratungsangebote, welche die Individuen in die Lage versetzen sollen, ihre weiteren Lernmöglichkeiten wahrzunehmen, gefordert. Dies beinhaltet dann z.B. auch Informationen von Weiterbildungsmöglichkeiten an Hochschulen für beruflich Qualifizierte. Ziel dieser Beratung ist die Stärkung der individuellen Handlungsfähigkeit, indem Bildungsoptionen aufgezeigt werden. Auch die Flexibilisierung der beruflichen Bildung wird in den Dokumenten gefordert, da so für die individuellen Lebensverläufe passgenaue Bildungsangebote entstehen.

Warum ist Durchlässigkeit aber wichtig? Wie wird der Bedarf begründet? Hauptargument speziell für die Verbindung zwischen Berufs- und Hochschulbildung ist der Bedarf an hochqualifizierten Bürgern, die für eine gesteigerte Wettbewerbs- und Innovationsfähigkeit Europas unabdingbar sind. Da die Struktur des Arbeitsmarkts sich ändert und sich durch eine stetig wachsende Zahl wissensbasierter Tätigkeiten auszeichnet, müsse die Verbindung zwischen Hochschulund Berufsbildung ausgebaut werden. Die Notwendigkeit lebenslanges Lernen allgemein zu ermöglichen, wird außerdem begründet mit dem demografischen 
Wandel, welcher dazu führt, dass aufgrund einer sinkenden Zahl von gut qualifizierten Beschäftigten im Verhältnis zu dem Bedarf des Arbeitsmarkts, die individuelle Beschäftigungsfähigkeit aller Bürger erhöht werden müsse. Lebenslanges Lernen soll zudem zu einer aktiveren Bürgerschaft und größerer sozialer Inklusion führen.

Insgesamt stehen demnach funktionale Gründe für den Durchlässigkeitsbedarf eindeutig im Vordergrund. Aber auch der soziale Zusammenhalt der Gesellschaft soll gestärkt werden.

\section{Normative Dimension}

Analysiert man die zu implementierenden Standards, die zu einer größeren Durchlässigkeit zwischen beruflicher und Hochschulbildung führen sollen, fällt auf, dass sie vor allem die ersten beiden Durchlässigkeitsaspekte, die des Zugangs und der Anrechnung, betreffen. Ein Großteil findet sich ebenfalls analytisch auf Ebene der Bildungsorganisationen mit Fokus auf der Organisation der Bildungsgänge wieder (vgl. Tabelle 6). Genannt werden können die Institutionalisierung der Lernergebnisorientierung, von Leistungspunktesystemen (ECVET), einer Modularisierung der Berufsbildungsgänge, Verfahren der Anrechnung und Validierung von vorgängigem Lernen sowie der Qualitätssicherung.

Wie auch im Bologna-Prozess ist die Einführung einer Lernergebnisorientierung Voraussetzung für das Funktionieren weiterer Standards, wie dem Qualifikationsrahmen, Verfahren der Anrechnung, den Leistungspunktesystemen. Denn nur wenn das Lernergebnis und weniger der Lerninhalt und -ort wichtig sind, können abstrahiert von letzteren auch Lernergebnisse unterschiedlicher Lernwege verglichen werden. Die Orientierung von Bildungsgängen an Lernergebnissen soll daher die Transparenz bzw. Vergleichbarkeit und damit auch die Transferierbarkeit von Fähigkeiten erleichtern.

Leistungspunktesysteme sollen gemäß den Kopenhagen-Dokumenten Lernende vor allem beim Wechsel innerhalb und zwischen Bildungsbereichen unterstützen, indem sie auf die Fähigkeiten und Kompetenzen, die sie zuvor erworbenen haben, aufbauen können. Sie dienen daher dem Transfer und der Akkumulation von Lernergebnissen.

Verfahren der Anerkennung und Anrechnung sowie Validierung von formalen, non-formalen und informellen Lernformen sind ebenfalls relevant in Bezug auf die Aspekte Zugang und Anrechnung. Diese Verfahren sollen dabei insbesondere dem Transfer von Erlerntem von einem Bildungsgang sowie -bereich in den anderen ermöglichen. Zum anderen sollen die Verfahren auch die Gleichwertigkeit von beruflicher und Hochschulbildung befördern, insbesondere wenn Praxiselemente stärkere Anerkennung finden.

Qualitätssicherungssysteme haben die Aufgabe, Vertrauen in die Abschlüsse zu erhöhen, so dass ihre Anerkennung beim Zugang und der Anrechnung einfacher von statten gehen kann. Hier wird in den Dokumenten insbesondere die enge Zusammenarbeit mit dem Bologna-Prozess gefordert, so dass Vertrauen 
über Bildungsgrenzen hinweg aufgebaut wird (Helsinki Communiqué 2006, Bruges Communiqué 2010).

Ähnlich wie im Bologna-Prozess wird auch ein Ausbau von institutionalisierten Beratungsangeboten gefordert, welche den Individuen die unterschiedlichsten Bildungswege aufzeigt, so dass Übergänge in andere Bildungsbereiche erleichtert werden. Deutlich wird in den Dokumenten jedoch auch, dass die Orientierungsangebote letztlich die Individuen befähigen sollen, selbstständig dafür zu sorgen, sich adäquat weiterzubilden. Beratungsangebote sowie die verstärkte Vermittlung von Schlüsselkompetenzen (wie der Umgang mit der Informations- und Kommunikationstechnologie, Spracherwerb), welche auch den Übergang zwischen Berufs- und Hochschulbildung erleichtern sollen, sind geforderte Standards, die stärker auf die Bildungsteilnehmenden fokussieren (vgl. Tabelle 6).

Tabelle 6 Durchlässigkeitsrelevante Standards im Kopenhagen-Prozess

\begin{tabular}{|c|c|c|}
\hline Standards & $\begin{array}{l}\text { Durchlässigkeitsaspekt(e), in } \\
\text { deren Kontext die Standards } \\
\text { thematisiert wurden }\end{array}$ & $\begin{array}{c}\text { Wo wird Durchlässigkeit } \\
\text { gefördert? }\end{array}$ \\
\hline \multicolumn{3}{|c|}{ Ebene der Bildungsorganisationen mit Fokus auf Bildungsgang } \\
\hline Lernergebnisorientierung & Zugang und Anrechnung & $\begin{array}{l}\text { in der Berufsbildung, zwischen } \\
\text { Organisationsfeldern }\end{array}$ \\
\hline $\begin{array}{l}\text { Leistungspunktesystem } \\
\text { (ECVET) }\end{array}$ & Zugang und Anrechnung & $\begin{array}{l}\text { in der Berufsbildung, zwischen } \\
\text { Organisationsfeldern }\end{array}$ \\
\hline Modularisierung & $\begin{array}{l}\text { Zugang und Anrechnung, } \\
\text { Umgang mit Heterogenität }\end{array}$ & $\begin{array}{l}\text { in der Berufsbildung und } \\
\text { zwischen Organisationsfeldern }\end{array}$ \\
\hline $\begin{array}{l}\text { Verfahren der Anerkennung } \\
\text { und Validierung von } \\
\text { vorgängigem Lernen }\end{array}$ & Zugang und Anrechnung & $\begin{array}{l}\text { in der Berufsbildung und } \\
\text { zwischen Organisationsfeldern }\end{array}$ \\
\hline Qualitätssicherungssysteme & Zugang und Anrechnung & $\begin{array}{l}\text { in der Berufsbildung und } \\
\text { zwischen Organisationsfeldern }\end{array}$ \\
\hline \multicolumn{3}{|c|}{ Ebene der Bildungsorganisationen mit Fokus auf das Bildungssubjekt } \\
\hline $\begin{array}{l}\text { Institutionalisierte } \\
\text { Beratungsangebote }\end{array}$ & Umgang mit Heterogenität & im gesamten Bildungssystem \\
\hline $\begin{array}{l}\text { Vermittlung von } \\
\text { Schlüsselkompetenzen }\end{array}$ & Zugang & $\begin{array}{l}\text { in der Berufsbildung und } \\
\text { zwischen Organisationsfeldern }\end{array}$ \\
\hline
\end{tabular}

Institutionelle Strukturen zur Verbindung der Organisationsfelder

\begin{tabular}{ll}
\hline $\begin{array}{l}\text { beruflicher Bildung an den } \\
\text { Hochschulen }\end{array}$ & $\begin{array}{l}\text { Organisationale Verbindung zwischen Organisationsfeldern } \\
\text { (Integration) }\end{array}$ \\
\hline
\end{tabular}

Ebene des Bildungssystems

\begin{tabular}{l}
$\begin{array}{l}\text { Qualifikationsrahmen für } \quad \text { Zugang und Anrechnung im gesamten Bildungssystem } \\
\text { lebenslanges Lernen }\end{array}$ \\
\hline
\end{tabular}

Quelle: Zusammenfassung der Verfasserin 
Die Forderung der Einführung beruflicher Bildung an Hochschulen (Bordeaux Communiqué 2008) zielt als Struktur stärker auf die Verbindung von Organisationsfeldern, da berufliche Bildung und Hochschulbildung in einen Bildungsgang zusammengebracht wird. Diese Form der Integration soll insbesondere die Beschäftigungsfähigkeit der Individuen erhöhen. Hier bleibt aber festzuhalten, dass in den Dokumenten nicht spezifisch geregelt ist, wie berufliche Bildung in die Hochschulbildung integriert sein soll. Ob dies z.B. durch verstärkte Praxisorientierung oder durch die Einführung von zweijährigen beruflich orientierten Studiengängen passiert, wird nicht näher ausgeführt. Dass diese Art der organisationalen Verbindung Durchlässigkeit fördern kann, wird ebenfalls nicht expliziert.

Schließlich ist der letzte Standard, die Einführung nationaler Qualifikationsrahmen für lebenslanges Lernen, auf Ebene des gesamten Bildungssystems anzusiedeln. Er wird als Katalysator für die Entwicklung von durchlässigen Strukturen zwischen beruflicher Bildung und Hochschulbildung angesehen. Er soll dadurch, dass er Allgemein-, Berufs- und Hochschulbildung mit gemeinsamen Referenzniveaus in einem Bezugsrahmen vereint, Anerkennung und Transfer von Qualifikationen sowie non-formal und informell erworbener Kompetenzen im Bildungssystem befördern. Indem die Referenzniveaus über Lernergebnisse beschrieben werden, kann der Qualifikationsrahmen für stärkere Transparenz und eine Verbesserung der Beratungsdienste sorgen sowie bei der Entwicklung von Anerkennungsverfahren helfen. Gleichzeitig soll er auch der Darstellung der Gleichwertigkeit von beruflicher und Hochschulbildung dienen.

Neben den Standards ist auch die Frage nach der typischen Zielgruppe für Durchlässigkeit zwischen der Berufs- und Hochschulbildung für die normative Dimension zu analysieren. Eine spezifische Gruppe wird allerdings nicht dargestellt, obwohl Durchlässigkeit zwischen den beiden Organisationsfeldern ein zentrales Thema im Prozess ist. Zu erklären wäre dies vielleicht damit, dass die Organisationen der Berufsbildung in diesem Fall nicht aufnehmende Institution sind und eine Einmischung in den Hochschulbereich nicht stattfinden soll.

Dagegen gelten alle Bürger_innen als potenzielle Zielgruppe für die berufliche Bildung und die Beteiligung am lebenslangen Lernen. Explizit wird im Kopenhagen-Prozess das gesamte Spektrum von sozialer Exklusion gefährdeten Gruppen wie Geringqualifizierten, Arbeitslosen, Migrant_innen, älteren Beschäftigten, Schulabbrecher_innen sowie auch Menschen mit Behinderungen bis hin zu Hochqualifizierten aufgemacht. Der Schwerpunkt in den Dokumenten liegt aber bei den potenziell Benachteiligten (vgl. Powell et al. 2012a). Insbesondere die Praxisorientierung der Berufsbildung sei für diejenigen, die im allgemeinbildenden Bildungssystem scheitern, eine Chance, sich selbst zu verwirklichen und doch erfolgreich zu sein. Andererseits wird unterstrichen, dass berufliche Bildung auf allen Qualifikationsebenen stattfindet und auch die höhere Berufsbildung ausgebaut werden sollte, um dieselbe im Vergleich mit anderen Bildungsangeboten attraktiver zu machen. Ein auf Leistung basierendes Zugangskriterium wird aber für die Berufsbildung nicht gefordert. 


\section{Regulative Dimension}

Die Frage der nationalen Implementierung nimmt zwar keinen großen Raum in den Dokumenten ein, aber es werden wiederkehrend alle Stakeholder, die Ministerien, die Kammern und insbesondere die Sozialpartner zur Zusammenarbeit aufgefordert. Die Hochschulen werden allerdings nicht direkt angesprochen, aber eine engere Kooperation mit dem Bologna-Prozess insbesondere bei Fragen der Kohärenz der Leistungspunktesysteme und bei der Entwicklung der Qualitätssicherung wird gefordert. Auch die Frage der Finanzierung wird thematisiert. Durchlässigkeit befördernde Reformen sollen aus einem Mix von öffentlichen, privaten und europäischen Geldern bezahlt werden.

\subsubsection{Durchlässigkeit in den Bologna- und Kopenhagen- Prozessen im Vergleich}

Nachdem nun die Spezifika der beiden europäischen Bildungsprozesse im Hinblick auf ihre Konzeption von Durchlässigkeit entlang der institutionellen Dimensionen dargestellt worden sind, sollen im Folgenden die größten Gemeinsamkeiten und Unterschiede aufgezeigt werden.

Insgesamt nehmen Fragen der Durchlässigkeit einen wesentlich größeren Raum im Kopenhagen- als im Bologna-Prozess ein. Von Beginn an ist Bildungsmobilität und zwar nicht nur über geografische Grenzen hinweg, sondern auch innerhalb der nationalen Bildungssysteme ein zentrales Ziel. Seit der ersten Folgekonferenz in Maastricht 2004 wird auch Durchlässigkeit zwischen beruflicher und Hochschulbildung gefordert. Das Kommuniqué von Maastricht kann zudem als Schlüsseldokument für das Thema Durchlässigkeit bezeichnet werden, da in ihm fast alle wichtigen Aspekte, welche das Konzept Durchlässigkeit im Kopenhagen-Prozess ausmachen, vorkommen. Sie werden in den weiteren Kommuniqués weiter präzisiert. Im Bologna-Prozess dagegen wird Durchlässigkeit zwischen Berufs- und Hochschulbildung kaum direkt thematisiert. Zudem ist das Thema Durchlässigkeit zu Beginn des Prozesses nicht sehr zentral, auch wenn in den ersten beiden Deklarationen (Sorbonne und Bologna) bereits durchlässigkeitsrelevante Aspekte angesprochen werden. Insgesamt nehmen diese Aspekte aber im Zeitverlauf signifikant zu.

Lebenslanges Lernen ist in beiden Prozessen der Hauptkontext, in dem durchlässige Bildungsstrukturen gefordert werden. Jedoch nimmt lebenslanges Lernen im Kopenhagen-Prozess einen viel größeren Stellenwert ein und wird von Beginn an gefordert. Im Bologna-Prozess ist lebenslanges Lernen seit 2001 relevant, wobei das Thema aber stärker allgemein gehalten wird. Die Ziele sind flexible Lernwege im Hochschulsystem und ins Hochschulsystem. Zwischen welchen Bereichen außerhalb der Hochschule konkret durchlässigere Strukturen erreicht werden sollen, wird nicht expliziert. Im Kopenhagen-Prozess ist man dies- 
bezüglich viel präziser: Es wird Durchlässigkeit sowohl innerhalb der Berufsbildung sowie zwischen den allgemeinbildenden Bildungsbereichen der Schule, Hochschule und Weiterbildung als auch zwischen Berufsbildung und dem Arbeitsmarkt und der Nicht-Erwerbsarbeitssphäre gefordert. In der Analyse wurde sich aber für den Kopenhagen-Prozess auf Durchlässigkeit zwischen Berufs- und Hochschulbildung konzentriert.

Der zweite Kontext im Bologna-Prozess, in dem durchlässigere Strukturen gefordert werden ist die sogenannte soziale Dimension. Der Zugang zur Hochschule für bisher unterrepräsentierte Bevölkerungsgruppen soll Chancengleichheit und soziale Kohäsion fördern. Die Integration von sozial benachteiligten Gruppen ist zwar auch ein Thema im Kopenhagen-Prozess, jedoch geht es dann hier viel stärker um deren Inklusion in die berufliche (Weiter-)Bildung und weniger um deren Bildungsmobilität in die Hochschulbildung. Im Kopenhagen-Prozess ist stattdessen das Ziel, die Attraktivität von beruflicher Bildung zu erhöhen, der weitere Kontext, in welchem Durchlässigkeit zwischen den beiden Organisationsfeldern gefordert wird. Immer wieder wird im Kopenhagen-Prozess auf die Notwendigkeit hingedeutet, die Wertschätzung der beruflichen Bildung in der Bevölkerung zu erhöhen. Es geht somit nicht nur um Verbindungen zwischen den Bildungsbereichen, sondern auch um Statuskämpfe, die in den Europäisierungsprozessen sichtbar werden. Dass berufliche Bildung aber in den Bologna-Dokumenten kein Thema ist, kann in diesem Zusammenhang vielleicht auch als ein Indiz für die Wertigkeit dieses Bildungsbereichs für die europäische Hochschulbildung gesehen werden. Im Gegensatz dazu fordert der Kopenhagen-Prozess von Beginn an eine verstärkte Zusammenarbeit mit dem Bologna-Prozess, während dies andersherum nicht der Fall ist.

Betrachtet man die Aspekte der Durchlässigkeitskonzepte, die aus den Dokumenten herausgearbeitet wurden, so gibt es eine weitgehende Übereinstimmung.

Durchlässigkeit wird diskutiert vor allem anhand von Fragen des Zugangs, der Anrechnung und auch in beiden Prozessen aber verstärkt im Bologna-Prozess wird die Frage des Umgangs mit den heterogenen Bedürfnissen von Lernenden thematisiert, auf die für ein Lernerfolg besser eingegangen werden muss. Im Kopenhagen-Prozess kam zusätzlich auch der Aspekt der organisationalen Verbindung, d.h. einer Integration beruflicher Praxis im Hochschulbereich, in den Dokumenten vor. Dies ist insofern erstaunlich, als dass insbesondere im BolognaProzess die stärkere berufliche Orientierung des Studiums kontinuierlich gefordert wird.

Betrachtet man die beiden europäischen Prozesse im Zeitverlauf ist auch eine wachsende Übereinstimmung in den zu implementierenden Standards festzustellen. In beiden Prozessen wird die Einführung folgender Standards als durchlässigkeitsfördernd beschrieben: Qualifikationsrahmen, Leistungspunktesysteme (ECVET/ECTS), Verfahren zur Anerkennung von vorgängigem Lernen, Lern- 
ergebnisorientierung, Qualitätssicherungssysteme sowie institutionalisierte Beratungsangebote. Die große Kongruenz der Standards kann zum einen erklärt werden durch den Einfluss der Politik des lebenslangen Lernens sowie der LissabonStrategie auf beide Prozesse, aber auch durch die gegenseitige Beeinflussung beider Prozesse. Letzteres wird in den Kopenhagen-Dokumenten auch explizit gemacht.

Die Bologna-Dokumente geben Hinweise auf die Zielgruppen, für die durchlässigere Strukturen zwischen Hochschul- und Berufsbildung geschaffen werden sollen. Dies sind die in den Hochschulen bisher unterrepräsentierten Gruppen der Bevölkerung, welche allerdings in ihrer Gänze nicht näher definiert werden. Menschen mit beruflichen Hintergründen werden aber z.B. explizit genannt. Umgekehrt werden Studienabbrecher als potenzielle Zielgruppe für die berufliche Bildung im Kopenhagen-Prozess dargestellt. Dass Durchlässigkeit keine Einbahnstraße ist und sozusagen in beide Richtungen gehen sollte, wird verdeutlicht. Für den Zugang zur beruflichen Bildung wird keine weitere Vorbedingung festgelegt. Leistung dagegen ist das Kriterium, welches über den Zugang zur Hochschule entscheidet.

Insgesamt werden in beiden Prozessen strukturelle Gründe für eine fehlende Durchlässigkeit identifiziert bzw. über strukturelle Änderungen versucht, größere Bildungsmobilität zu ermöglichen. Aber beiden Prozessen ist auch gemeinsam, dass die Individuen in die Pflicht genommen werden, sich selbstständig mithilfe der zu entstehenden Strukturen flexibel durch die Bildungssysteme zu bewegen. Die Befähigung der Individuen zur Selbsthilfe und damit auch zum Teil die Abgabe der Verantwortung für Bildungsmobilität an das Individuum ist dabei ein zentrales Charakteristikum des entstehenden europäischen Bildungsmodells und nicht nur bei Fragen von Durchlässigkeit relevant (Powell et al. 2012a).

Auch die Frage, wer für die Umsetzung von Bildungsmobilität fördernden Strukturen zuständig ist, wird in den beiden Prozessen ähnlich beantwortet. Alle Stakeholder, die Ministerien, Kammern und vor allem Sozialpartner sollten beteiligt werden. Im Bologna-Prozess wird zudem eine Beteiligung der Studierenden und vor allem der Hochschulorganisationen gefordert. Der Erhalt der Autonomie letzterer ist eine zentrale Forderung. Explizit wird daher auch den Hochschulen das Recht zugesprochen, zu bestimmen, welche Fähigkeiten und Kompetenzen in der spezifischen Hochschule Anerkennung finden. Interessant ist an dieser Stelle, dass im Kopenhagen-Prozess keine direkten Forderungen an die Hochschulen gestellt werden, die Zugangsmöglichkeiten für beruflich Qualifizierte zu verbessern. Entweder finden sich im Kommuniqué Forderungen an die Berufsbildungssysteme für eine Veränderung ihrer Strukturen oder es werden allgemein mehr Übergänge bzw. Durchlässigkeit gefordert. Welche Rolle die Hochschulen dabei übernehmen sollten, findet keine Erwähnung.

Im Bologna-Prozess wird eine Finanzierung der Durchlässigkeitsreformen mit öffentlichen Geldern gefordert, während im Kopenhagen-Prozess von einem 
Finanzierungsmix aus öffentlichen, privaten und europäischen Geldern ausgegangen wird.

Abschließend lässt sich festhalten, dass es zwar Unterschiede in den beiden europäischen Bildungsprozessen gibt, in Bezug darauf wie genau und wie viel Durchlässigkeit thematisiert wird. Es lässt sich jedoch auch eine große Übereinstimmung feststellen. Das gilt sowohl für das allgemeine Verständnis von Durchlässigkeit anhand der Aspekte sowie deren Begründungen in der kulturell kognitiven Dimension und den geforderten Standards in der normativen Dimension als auch für die regulative Dimension im Hinblick auf die bei der Implementierung zu beteiligenden Akteure. Aus diesem Grund soll von einem europäischen Kernkonzept von Durchlässigkeit gesprochen werden (vgl. Tabelle 38 im Anhang).

Wie in Kapitel 2 angenommen, ist dieses Konzept aber kein klar definiertes, sondern lässt bei der Interpretation und Umsetzung einigen Spielraum. Die thematisierten Aspekte von Durchlässigkeit sind breit und ihre Umsetzungsvorschläge wenig spezifisch gefasst. So wird nicht beschrieben, wie die Zugangsregelungen zur Hochschule ausgestaltet sein sollten, ob über Berechtigungen oder hochschulische Regelungen oder ob individuumszentriert oder gruppenspezifisch. Allein dass es keinen universellen Zugang in die Hochschule geben soll, wird vermittelt, da die Auswahl der Studierenden als leistungsbasiert beschrieben wird.

Auch wie Anrechnung stattfinden soll, d.h., inwiefern sie stärker individuell ausgerichtet oder pauschalisiert institutionalisiert wird, wird in den untersuchten Dokumenten offen gelassen. Allerdings wird darauf hingewiesen, dass Richtlinien erarbeitet worden sind, wie Validierungsverfahren aussehen können (Bruges Communiqué 2010). Zudem werden klare Standards, wie die Leistungspunktesysteme, die Lernergebnisorientierung, Qualifikationsrahmen, die Modularisierung, eingefordert, die bei Anrechnung und Validierungsverfahren helfen sollen.

Nicht näher spezifiziert werden desgleichen die zu institutionalisierenden Formen der organisationalen Verbindung zwischen Berufs- und Hochschulbildung: Es soll Übergänge aus einem Bereich in den anderen geben und berufliche Bildung im Hochschulstudium integriert werden. Verschiedenste Modelle sind hier denkbar, z.B. das deutsche Modell der dualen Studien, die französischen beruflichen Kurzstudiengänge oder integrierte Praktika während des Studiums. Etwas spezifischer dagegen sind die Vorgaben, die den Umgang mit Heterogenität der Lernenden erleichtern und zu einem Bildungserfolg führen sollen, da hier Maßnahmen zur Flexibilisierung (e-Learning, Teilzeit, Fernstudium), der Beratung und Unterstützung z.B. durch Lernendenzentrierung und Finanzierungsmöglichkeiten vorgeschlagen werden.

Durch diese allgemeinere Offenheit des europäischen Durchlässigkeitsmodells kann demgemäß ebenfalls nicht geschlussfolgert werden, ob dieses in der Umsetzung eher zu neuen Barrieren in Bildungssystemen und damit Schließungsprozessen führen oder stärker bestehende Barrieren abbauen wird. Die nationale 
Interpretation und Umsetzung des Modells im Rahmen der nationalen Gegebenheiten wird dafür entscheidend sein.

Trotz dieser stärkeren Offenheit der Durchlässigkeitsvorgaben auf europäischer Seite, wird jedoch deutlich, dass Durchlässigkeit ein Thema ist, mit dem sich die Mitgliedsstaaten beschäftigen müssen. Bildungssysteme, die keine Bildungsmobilität zwischen Bildungsbereichen ermöglichen, büßen durch die europäischen Prozesse an Legitimität ein und gelten als Bremser einer wirtschaftlichen und sozialen Entwicklung in Europa. Insofern ist anzunehmen, dass ein Land, wie Deutschland, in welchem die institutionelle Trennung zwischen Berufs- und Hochschulbildung stark ausgeprägt ist und damit eine geringe Bildungsmobilität zwischen den Bereichen gewährleistet, auch unter Druck steht, Strukturen zu schaffen, welche eine stärkere Bildungsmobilität ermöglichen. In Ländern wie Frankreich, in denen Strukturen, die eine Mobilität zwischen Bildungsbereichen fördern, bereits bestehen, ist zu untersuchen, wo und ob überhaupt die Europäisierung einen Einfluss auf die Entwicklung von Durchlässigkeitsstrukturen nehmen kann. Wie sich die Durchlässigkeitsstrukturen in Deutschland und Frankreich verändert haben und wie einflussreich die europäischen Bildungsprozesse dabei waren, soll in den nachfolgenden empirischen Analysen gezeigt werden. 


\section{Der deutsche Fall - Bildungssystem, Geschichte und das Problem der Durchlässigkeit}

Auch wenn bereits in der Fallauswahl wichtige und markante Charakteristika des deutschen Bildungssystems dargestellt wurden, soll dies im Folgenden ausführlicher behandelt werden. Neben einer eingehenden Darstellung des deutschen Bildungsschismas und der daraus resultierenden Problematik für Durchlässigkeit sollen auch die in Deutschland institutionalisierten Bildungswege, vor allem in die Hochschulbildung, und deren quantitative Bedeutung, die Steuerungsspezifika und die Hauptakteure der Organisationsfelder im Mittelpunkt stehen. Schließlich soll auch eine kurze Zusammenfassung der historischen Entwicklung der deutschen Durchlässigkeitsproblematik erfolgen. Ziel dieses Kapitels ist es, ein Verständnis des deutschen Bildungssystems zu erlangen, um die Entwicklungen der institutionellen Durchlässigkeitsstrukturen in den letzten 25 Jahren zu verstehen, die im nachfolgenden Kapitel dargestellt werden.

\subsection{Das deutsche Bildungsschisma}

Warum stellt Durchlässigkeit zwischen Berufs- und Hochschulbildung in Deutschland ein solches Problem dar? Die Antwort findet sich im so genannten deutschen Bildungsschisma, welches in den unterschiedlichsten institutionellen Dimensionen wirksam ist (vgl. Graf 2013). In diesem Abschnitt sollen die Spezifik dieses Schismas und die damit einhergehende Durchlässigkeitsproblematik dargestellt werden

Das deutsche Bildungssystem ist gekennzeichnet durch eine institutionelle Segmentierung der höheren Allgemein- und Berufsbildung, welche Baethge (2006) als das deutsche Bildungsschisma bezeichnet.

Institutionelle Segmentierung meint die dauerhafte wechselseitige Abschottung von Bildungsbereichen gegeneinander, die darauf beruht, dass jeder Bildungsbereich einer anderen institutionellen Ordnung folgt. (Baethge 2006: 16)

Entlang der institutionellen Dimensionen nach Scott (2008) sollen die wichtigsten Unterschiede zwischen den Organisationsfeldern Berufs- und Hochschulbildung, die das Schisma ausmachen, skizziert werden. Dabei beruht die Darstellung dieser Unterschiede zwischen den Bildungsbereichen weitestgehend auf der Ausarbeitung von Baethge (2006). In der kulturell-kognitiven Dimension können die Bildungsziele der Berufs- und der höheren Allgemeinbildung und damit einhergehend auch die differenten Bezugspunkte der Bildungsausrichtung unterschieden werden. Baethge (2006) betont dabei, dass im stärker allgemeinbildenden 
(Hoch-)Schulwesen ${ }^{85}$ das Ziel darin besteht, eine „gebildete Persönlichkeit“ auszubilden, die fähig ist, das individuelle Leben in modernen Gesellschaften selbstständig zu meistern. Um dies zu erlangen, müssen den Individuen spezifische allgemeinbildende Kulturtechniken, d.h. Kompetenzen in Sprachen, Grundlagenkenntnisse der Mathematik und Naturwissenschaften sowie der Geschichte vermittelt werden.

Ihre Grundlage bildet ein Kanon repräsentativen Wissens - gleichsam ein, Weltcurriculum' des Wissens -, bei dem in den letzten Jahrzehnten Wissenschaftsorientierung eine zunehmende Bedeutung gewonnen hat. (Baethge 2006: 17)

In der beruflichen Bildung ist das Ziel die Vermittlung der beruflichen Handlungskompetenz. Selbst wenn auch allgemeinbildende Anteile in der Berufsausbildung relevant sind, zielt die Ausbildung doch primär darauf ab, den Absolvent_innen beizubringen, ihre beruflichen Rollen auszufüllen und sich entsprechend auch in Organisationen, die ihre Arbeitsplätze darstellen, richtig und angemessen zu verhalten. Damit sind auch die Bezugspunkte für die Lehrgestaltung stärker auf die Bedarfe des Arbeitsmarkts, die dort benötigten Qualifikationen, ausgerichtet (Baethge 2006).

Für die normative Dimension heißt das, dass die Lernorganisation in den beruflichen Ausbildungen stärker praxisintegriert erfolgt, und zwar an den zwei Lernorten Schule und Betrieb, während die allgemeinbildenden Bildungsgänge stärker als praxisfern charakterisiert werden können und auch in eigenen Organisationen abseits des Arbeitsalltags stattfinden (Baethge 2006).

Schließlich bestehen auch in der regulativen Dimension klare Unterschiede. Analog zu den verschiedenen Lernorten ist auch der rechtliche Status der Lernenden nicht gleich. In der Allgemeinbildung handelt es sich um Schüler_innen, während in der Berufsbildung die Lernenden Auszubildende mit einem Arbeitsvertrag sind 86. Des Weiteren werden die allgemeinbildenden Bildungsgänge des Schulund Hochschulwesens staatlich durch die Bundesländer und deren Bildungsverwaltung gesteuert, die parlamentarisch kontrolliert werden und damit auch stärker durch Politikwechsel der Länderregierungen beeinflusst werden können. In der beruflichen Bildung dagegen erfolgt die Steuerung zwar auf Basis bundesrechtlicher Regulierungen, aber primär durch korporatistische Zusammenarbeit der Vertreter der Arbeitgeber_innen und -nehmer_innen, der Kammern sowie der Bundesregierung. Auch die Finanzierung der Bildung ist unterschiedlich. Während die duale berufliche Bildung primär als privat durch die Ausbildungsbetriebe finanziert gilt, werden die Schulen und Hochschulen aus öffentlichen Mitteln vor allem der Länder und Kommunen bezahlt. Während Erstere daher vor allem abhängig ist von der Ausbildungsbereitschaft der Betriebe, welche sich in den letz-

85 (Hoch-)Schulwesen wird verwendet, wenn die dargestellten Charakteristika sowohl für die höhere Schul- als auch für die Hochschulbildung gelten.

86 Dies gilt zumindest für die Lernenden einer dualen Ausbildung. 
ten Jahren in Deutschland verringert hat (vgl. Autorengruppe Bildungsberichterstattung 2014; Busemeyer/Thelen 2008; Solga 2009), sind Letztere stärker abhängig von politischen Entscheidungen und der parlamentarischen Kontrolle (Baethge 2006).

Tabelle 7 Das deutsche Bildungsschisma nach Baethge (2006)

\begin{tabular}{|c|c|c|}
\hline $\begin{array}{l}\text { Merkmale institutioneller } \\
\text { Ordnungen im } \\
\text { Bildungswesen }\end{array}$ & Höhere Allgemeinbildung & Berufsbildung \\
\hline \multicolumn{3}{|l|}{ Kulturell-kognitive Dimension } \\
\hline Dominante Zielperspektive & $\begin{array}{l}\text { Gebildete Persönlichkeit / } \\
\text { individuelle Regulationsfä- } \\
\text { higkeit (Autonomie) }\end{array}$ & $\begin{array}{l}\text { Berufliche Handlungskom- } \\
\text { petenz, Beruflichkeit }\end{array}$ \\
\hline $\begin{array}{l}\text { Bezugspunkt für Lernziel- } \\
\text { definition und Curricula }\end{array}$ & $\begin{array}{l}\text { Kanon repräsentativen } \\
\text { systematisierten Wissens/ } \\
\text { Wissenschaftsorientierung, } \\
\text { Forschung, Humboldt }\end{array}$ & $\begin{array}{l}\text { Arbeitsmarkt und Beschäfti- } \\
\text { gungsstruktur; wirtschaftli- } \\
\text { cher Bedarf an Qualifikatio- } \\
\text { nen }\end{array}$ \\
\hline \multicolumn{3}{|l|}{ Normative Dimension } \\
\hline $\begin{array}{l}\text { Instruktionsprinzip / Organi- } \\
\text { sation der Lernprozesse }\end{array}$ & $\begin{array}{l}\text { Praxisenthoben(-fern) in } \\
\text { eigenen Organisationen }\end{array}$ & $\begin{array}{l}\text { Praxisintegriert (Verbindung } \\
\text { von Arbeit und Lernen) }\end{array}$ \\
\hline \multicolumn{3}{|l|}{ Regulative Dimension } \\
\hline Status des Lernenden & Schüler_in, Student_in & $\begin{array}{l}\text { Auszubildende im Arbeits- } \\
\text { verhältnis }\end{array}$ \\
\hline $\begin{array}{l}\text { Politische Steuerung, } \\
\text { Aufsicht, (Qualitäts-) } \\
\text { Kontrolle }\end{array}$ & $\begin{array}{l}\text { Staatlich (demokratische } \\
\text { Kontrolle) durch die Bundes- } \\
\text { länder }\end{array}$ & $\begin{array}{l}\text { Korporatistische Selbstverwal- } \\
\text { tung unter Beteiligung der } \\
\text { Wirtschaft (Verbände, Kam- } \\
\text { mern) und der Arbeitnehmer- } \\
\text { vertreter auf Basis bundes- } \\
\text { staatlicher Regulierung }\end{array}$ \\
\hline Finanzierung & $\begin{array}{l}\text { Öffentlich (Länder, Kommu- } \\
\text { nen) }\end{array}$ & $\begin{array}{l}\text { Primär privat (Ausbildungs- } \\
\text { betriebe) }\end{array}$ \\
\hline
\end{tabular}

Quelle: Baethge (2006) sowie Powell et al. (2009) - erweitert auf die Hochschulbildung und organisiert entlang der institutionellen Dimensionen durch die Verfasserin und Team INVEST

Das Bildungsschisma soll in dieser Arbeit vor allem als eine idealtypische Darstellung verstanden werden, nicht aber als exakte empirische Beschreibung des deutschen Bildungssystems, welches wesentlich komplexer ist. So stehen für die Beschreibung des Berufsbildungssystem vor allem das deutsche duale System und für die höhere Allgemeinbildung die Sekundarstufe II an den Gymnasien und die klassische Forschungsuniversität Pate. Organisationsformen, die beide Bereiche verbinden, wie z.B. duale Studiengänge, werden durch die Beschreibung nicht abgedeckt. Auch ältere und neuere Tendenzen der Verberuflichung der Hochschulbildung z.B. durch die Ausrichtung des Bachelors auf eine Berufsbefähigung (vgl. Rauner 2012) sind hier nicht inkludiert. Zudem kann auch eine strikte Berufs- und Praxisferne der Hochschulen durch mehrere Entwicklungen des 20. Jahrhunderts 
infrage gestellt werden. Zum einen ist die Differenzierung der Hochschularten und Fächer über die klassischen Fakultäten hinaus mit zum Teil explizit berufspraktischen Zielen wie in den Fachhochschulen zu nennen. Auch die klassisch an den Universitäten stattfindende Professionsausbildung z.B. von Ärzt_innen, Jurist_innen und Lehrer_innen sind klar beruflich orientiert und mit Praxisphasen verbunden. Zum anderen können die massive Bildungsexpansion und der Ausbau der Hochschulen als Entwicklung weg vom exklusiven, allein auf die Professionen und Forschung ausgerichteten Bildungsweg gesehen werden. Zudem wurde bereits in den Hochschulreformen seit den 1970er Jahren ein vermehrter Praxisund Berufsbezug als eine Leitlinie im Studium integriert (Wolter 2010b). Zum Teil wird auch eine verstärkte Integration des dualen Prinzips in der Hochschullandschaft erwartet (Grollmann 2012). Andererseits wird in den letzten Jahren auch verstärkt von einer Bewegung der Berufsbildung in Richtung Akademisierung gesprochen. Dies beinhaltet eine Modernisierung der Berufsausbildung, in der vermehrt auch theoretisches disziplinäres Wissen vermittelt wird, da die Anforderungen der Berufe im Verlauf der Zeit gestiegen ${ }^{87}$ bzw. neue Berufe wie im ITBereich hinzugekommen sind. Damit ist aber auch die Entwicklung gemeint, dass Ausbildungen z.B. der Gesundheitsberufe verstärkt als Fachhochschulstudium angeboten werden sollen (vgl. Bischoff-Wanner 2002).

Trotz dieser Trends der Akademisierung einerseits und der Verberuflichung andererseits zeichnen sich die Organisationsfelder der Berufs- und Hochschulbildung weiterhin durch ihre unterschiedlichen institutionellen Logiken aus, die zu einer gegenseitigen Isolierung der Bildungsbereiche führen, die von Baethge (2006) als Segmentierung im Bildungssystem bezeichnet wird.

Aber was bedeutet dann das Bildungsschisma für Durchlässigkeit? Durch die Segmentierung sind Übergänge aus einem Bereich in den anderen schwer möglich. Dass kann auch anhand der institutionalisierten Bildungswege und typischen Weiterbildungsmöglichkeiten gezeigt werden (vgl. Kapitel 6.2). Betrachtet man die Zahlen der beruflich Qualifizierten, die ohne schulische Zugangsberechtigung ein Studium aufnehmen, zeigt sich zwar ein Anstieg, aber das Niveau liegt weiterhin sehr niedrig. So haben 1997 0,6 \% der Studienanfänger_innen ein Studium ohne schulische Hochschulzugangsberechtigung angefangen, und 2010 waren es etwa 2,1 \% (Nickel/Duong 2012: 31). Die Abschottung der Bildungsbereiche und die unterschiedlichen Logiken und Bildungsziele führen auch dazu, dass das bisher Gelernte aus einem Bereich im jeweiligen anderen nicht als gleichwertig anerkannt wird, so dass die Anrechnung von Kompetenzen sich schwierig gestaltet (vgl. Freitag et al. 2011). Eine Folge sind für Deutschland typische Doppelqualifikationen (Hörner 2006).

87 Es kann gezeigt werden, dass ein Upskilling, die Erhöhung der Lernanforderungen, kein genereller Trend in der beruflichen Bildung ist (Protsch 2014). 


\subsection{Beschreibung der Organisationsfelder}

Nachfolgend wird ein Überblick über die beiden Organisationsfelder ${ }^{88}$ Berufsund Hochschulbildung mit ihren Governancestrukturen gegeben und anschlieBend sollen die bestehenden Bildungswege zwischen den Bereichen dargestellt werden.

Tabelle 8 Bevölkerung in Deutschland nach Altersgruppen und Bildungsabschluss

\begin{tabular}{c|c|c|c|c|c}
\multirow{2}{*}{$\begin{array}{c}\text { Alter von } \\
\text { bis unter .. } \\
\text { Jahren }\end{array}$} & $\begin{array}{c}\text { Mit beruflichem Bildungsabschluss } \\
\text { Lehr-/Anlern- } \\
\text { ausbildung }\end{array}$ & $\begin{array}{c}\text { Fachschul- } \\
\text { abschluss }\end{array}$ & $\begin{array}{c}\text { Hochschul- } \\
\text { Ohne beruf- } \\
\text { abschluss }\end{array}$ & $\begin{array}{c}\text { Ohne Angaben } \\
\text { zur } \\
\text { ichen } \\
\text { Aildungs- } \\
\text { abschluss }\end{array}$ \\
\cline { 2 - 4 } & \multicolumn{4}{|c}{ In \% } \\
\hline Insgesamt & 50,2 & 7,6 & 13,6 & 0,2 & 27,8 \\
$15-20$ & 2,5 & - & - & - & 97,3 \\
$20-25$ & 38,1 & $2,-$ & 2,4 & 0,1 & 56,7 \\
$25-30$ & 52,1 & 5,9 & 16,6 & 0,2 & 25,1 \\
$30-35$ & 52,2 & 7,2 & 22,5 & 0,2 & 17,7 \\
$35-40$ & 54,7 & 8,2 & 19,9 & 0,2 & 16,6 \\
$40-45$ & 57,1 & 9,8 & 18,0 & 0,3 & 14,5 \\
$45-50$ & 57,9 & 10,3 & 16,2 & 0,3 & 14,9 \\
$50-55$ & 57,4 & 9,9 & 16,5 & 0,3 & 15,5 \\
$55-60$ & 57,6 & 9,5 & 16,9 & 0,3 & 15,4 \\
$60-65$ & 57,1 & 8,7 & 15,0 & 0,3 & 18,4 \\
65 und älter & 50,5 & 7,9 & 9,6 & 0,2 & 30,3 \\
\hline
\end{tabular}

Quelle: Autorengruppe Bildungsberichterstattung (2012: 236)

\subsubsection{Berufsbildung}

Betrachtet man das deutsche Bildungssystem und die Beteiligungsraten in den einzelnen Bildungszweigen, wird schnell deutlich, welche große Rolle die berufliche Ausbildung im Vergleich zu den anderen Bildungszweigen ab Sekundarstufe II spielt. So gelangten 2010 nach dem Ende der Sekundarstufe I, meist nach mindestens neun oder zehn Schuljahren, um die 71 \% der Schüler_innen in den Bereich der Berufsbildung, während $29 \%$ in allgemeinbildenden Schulen die Sekundarstufe II absolvierten (KMK 2011 in Graf 2013: 92). Dabei ist anzumerken,

88 Das deutsche Bildungssystem ist gekennzeichnet durch einen starken Bildungsföderalismus. Die Darstellung des deutschen Bildungssystems wird daher nur die charakteristischen Züge beinhalten, nicht aber auf die zum Teil deutlichen Unterschiede in den einzelnen Bundesländern eingehen können. 
dass in den $71 \%$ nicht nur Bildungsgänge inkludiert sind, die zu einem beruflichen Abschluss führen, sondern auch z.B. Berufs- und Fachoberschulen, in denen man eine Hochschulzugangsberechtigung erwerben kann. Nichtsdestoweniger ist das Berufsausbildungssystem bestimmend für die deutsche Sekundarstufe II. Und auch bei Betrachtung der Verteilung der Abschlüsse in der Bevölkerung (vgl. Tabelle 8), ist ein beruflicher Abschluss in allen Altersgruppen der Bevölkerung der häufigste Abschluss, selbst wenn in den jüngeren Jahrgängen die Bedeutung des Hochschulabschlusses zugenommen hat (Autorengruppe Bildungsberichterstattung 2014).

Im Folgenden soll zuerst ein kurzer Überblick über das Berufsbildungssystem im Sekundar- und anschließend im postsekundären Bereich gegeben werden.

\section{Berufliche Bildung im Sekundarbereich}

Das Berufsbildungssystem im Sekundarbereich kann in drei große Sektoren mit jeweils eigenen institutionellen Ordnungen eingeteilt werden (vgl. Abbildung 3). Der quantitativ bedeutsamste Sektor ist das duale Ausbildungssystem. In diesem kann eine Ausbildung für einen anerkannten Ausbildungsberuf mit bundeseinheitlich geregelten Qualifikationsanforderungen nach Berufsbildungsgesetz $(\mathrm{BBiG})$ oder Handwerksordnung $(\mathrm{HwO})$ absolviert werden. Die Ausbildung findet sowohl im Betrieb als auch in der Berufsschule statt. Die Lernenden haben einen Arbeitsvertrag und bekommen ein Ausbildungsgehalt. Diese Form der Ausbildung wird oft gleichgesetzt mit dem deutschen Berufsbildungssystem allgemein. Insgesamt macht sie aber nur ungefähr $50 \%$ der sekundären Berufsbildung aus (Autorengruppe Bildungsberichterstattung 2012). ${ }^{89}$

Der zweite Sektor ist das Schulberufssystem, in dem gesetzlich anerkannte Berufe vor allem im Dienstleistungssektor schwerpunktmäßig im Gesundheitsbereich erlernt werden. Dabei kann das Verhältnis zwischen dualem und Schulberufssystem als komplementär beschrieben werden, da unterschiedliche Berufe in dem jeweiligen Sektor gelernt werden und sie keine alternativen Bildungswege für einen Beruf darstellen (Solga 2009). Insgesamt ist für das Berufsbildungssystem das Prinzip der Beruflichkeit leitend (vgl. Deißinger 1998; Kraus 2007). Deutlich wird das an der engen Verzahnung mit der Praxis, die auch im Schulberufssystem durch verstärkte Praxisphasen institutionalisiert ist.

Der dritte Sektor ist das sogenannte Übergangssystem ${ }^{90}$, in dem keine vollqualifizierenden beruflichen Abschlüsse, sondern primär berufsvorbereitende

Auch wenn das deutsche duale System weltweit Vorbildcharakter hat, wird in den letzten Jahrzehnten auch immer stärker Kritik am Funktionieren des Systems geäußert. Unter anderem wird die zurückgegangene Ausbildungsbereitschaft der Betriebe kritisiert (vgl. Busemeyer/Thelen 2008; Solga 2009).

90 Auch wenn sich in den letzten Jahren das Übergangssystem zugunsten der regulären Ausbildungssektoren verkleinert hat (vgl. Abbildung 3), was zu großen Teilen mit den geringeren 
Kompetenzen erworben werden sollen. Ziel des Übergangssystems ist, wie der Name sagt, den Übergang in eine berufliche Ausbildung zu ebnen, wobei dies nur bedingt erfolgreich ist (vgl. Baethge et al. 2007; Beicht/Eberhard 2013; BIBB 2010).

Abbildung 3 Verteilung der Neuzugänge auf die drei Sektoren des Berufsbildungssystems

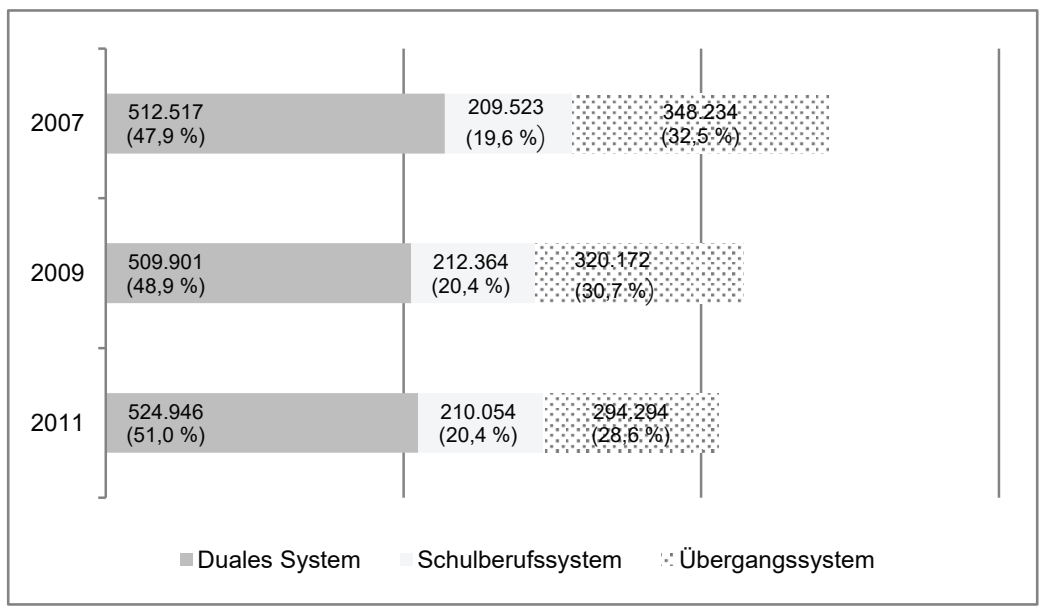

Quelle: Autorengruppe Bildungsberchterstattung (2012: 102)

Das Allgemeinbildungsniveau bei Eintritt in das Berufsbildungssystem bestimmt weitestgehend, in welchen Sektor und welchen Berufszweig die Absolvent_innen einmünden können (Autorengruppe Bildungsberichterstattung 2008, 2010). Im dualen System und im Schulberufssystem sind es vor allem Schüler_innen mit mittlerem Schulabschluss und der allgemeinen Hochschulreife. Schüler_innen mit Hauptschulabschluss machen nur eine kleine Minderheit aus. Dies gilt noch stärker für das Schulberufssystem als für das duale. Der größte Anteil der Hauptschüler_innen und der Schüler_innen ohne Abschluss mündet ohne Ausbildungsvertrag in das Übergangssystem ein (Autorengruppe Bildungsberichterstattung 2008, 2010). Betrachtet man die unterschiedlichen Branchen, sind es vor allem die Handwerksberufe und Berufe in der Landwirtschaft, die von HauptschulAbsolvent_innen erlernt werden. Berufe in der Industrie, im Handel und im öffentlichen Dienst sowie freie Berufe werden meist mit Absolvent_innen mit mittlerem Schulabschluss oder Hochschulreifezeugnis besetzt, wobei einige Berufsausbildungen mittlerweile allein von Abiturient_innen besetzt werden (Autorengruppe Bildungsberichterstattung 2008).

Absolvent_innenzahlen zusammenhängt, stellt das Übergangssystem noch immer den Lernort für über ein Viertel der Neuzugänge im Berufsbildungssystem dar. 
Es wird bereits an dieser Stelle deutlich, dass das stratifizierte Schulsystem Deutschlands, das durch eine kurze gemeinsame Schulzeit (bis Klasse 4 oder 6) und dann segregierten Wegen, die zu unterschiedlichen Bildungsabschlüssen führen, gekennzeichnet ist, auch die Bildungswege im Berufsbildungssystem beeinflusst (vgl. Autorengruppe Bildungsberichterstattung 2010; Protsch 2014). Im Hochschulsystem gilt dies in einem noch viel stärkeren Maße, da hier primär die Hochschulzugangsberechtigung, die am Ende der Sekundarstufe II erworben wird, den Zugang eröffnet (vgl. Powell/Solga 2011). Die anderen Schulwege, die nach der Sekundarstufe I abschließen, sind daher keine Zugangswege in die Hochschule. Die unterschiedlichen allgemeinbildenden Bildungswege der Sekundarstufe I und II finden meist auch in unterschiedlichen Schulformen, den Hauptund Sonderschulen, den Realschulen und den Gymnasien statt, so dass auch eine örtliche Segregation der Schüler_innen vollzogen wird. Begründet wird diese Trennung mit den unterschiedlichen Bedürfnissen der Schüler_innen je nach Leistungsvermögen (Pfahl/Powell 2010). Die Ausnahme bilden Formen der Gesamtschule, in denen alle Abschlüsse erreicht werden können. Zwar ist es möglich, wenn die Leistungen ausreichen, auch von dem einen Schulzweig auf den nächst höheren zu wechseln, allerdings ist dies in Deutschland selten der Fall (Bellenberg 2012). Weiterhin ist bekannt, dass nicht nur Aufstiege schwierig sind, sondern auch der Besuch der unterschiedlichen Schulformen sozial selektiv wirkt (vgl. z.B. Baumert et al. 2010; Ehmke et al. 2004; Solga/Dombrowski 2009). ${ }^{91}$ Insofern überträgt sich auch die soziale Selektivität auf die weiteren Bildungswege nach Verlassen des allgemeinbildenden Schulwesens im Sinne der Kumulation von Ungleichheiten über den Lebenslauf (Mayer 1990, 2004).

\section{Postsekundäre Berufsbildung}

Anders als die Allgemein-, Hochschul- und sekundäre Berufsausbildung ist die berufliche Weiterbildung in Deutschland nur teilweise staatlich geregelt (Kohl et al. 2013). So gibt es für berufliche Weiterbildungen private Anbieter, aber auch Abschlüsse nach Ländergesetzen. Aber es existiert auch analog zu dem eng am Berufsprinzip orientierten Berufsbildungssystem auf Sekundarniveau ein postsekundäres System der beruflichen Aufstiegsfortbildungen. So besteht nach dem Erwerb eines ersten Ausbildungsabschlusses oder nach ausreichender beruflicher Praxis die Möglichkeit, einen bundeseinheitlich geregelten Fortbildungsabschluss zu erwerben. Zu diesen Abschlüssen zählen z.B. die Meister, Techniker, Fachberater, Fachwirte, Fachkaufleute oder Betriebswirte. Fortbildungsordnungen sind wie das duale System nach Berufsbildungsgesetz $(\mathrm{BBiG})$ und Handwerksordnung (HWO) geregelt. Dabei können die Ordnungen vom Bundesministerium für Bildung und Forschung (BMBF), aber auch von den für den Berufsbildungsbereich

91 Zur Frage der sozialen Selektivität des deutschen Schulsystems besteht bereits ein großer einschlägigen Forschungsstand. Die ausgewählten Referenzen sind daher rein exemplarisch ausgewählt. 
zuständigen Stellen (meist Kammern) erlassen werden. Im Unterschied zu den Ausbildungsordnungen beziehen sich die Regelungen für die Fortbildungsabschlüsse jedoch nur auf die Prüfungsanforderungen und definieren nicht, wie auf die Prüfung vorbereitet werden soll (BIBB 2013). Insgesamt gibt es 223 Rechtsverordnungen und Regelungen des Bundes sowie 2.848 Rechtsvorschriften zu 749 Fortbildungsberufen, erlassen von den zuständigen Stellen (BIBB 2013: 369). 2011 fanden in Deutschland rund 118.000 Fortbildungsprüfungen statt (BIBB 2013: 295).

\section{Steuerung der Berufsbildung}

Das Berufsbildungssystem in Deutschland wird in Zusammenarbeit mehrerer Akteure gesteuert (vgl. Tabelle 1 im Online-Anhang). Die wichtigsten sind der Bund, vor allem das BMBF und daran angegliedert das Berufsbildungsinstitut (BIBB), die Länder, die Vertreter der Arbeitgeber_innen und Arbeitnehmer_innen, die Verbände und Gewerkschaften sowie die sogenannten zuständigen Stellen ${ }^{92}$, welche vor allem die Kammern sind. Das duale System wird primär durch das Berufsbildungsgesetz $(\mathrm{BBiG})$, welches vom Bund erlassen wird, reguliert. Zudem werden in Zusammenarbeit von BMBF, BIBB und den Sozialpartnern neue Berufe und Ausbildungsordnungen bzw. Prüfungsordnungen in der beruflichen Fortbildung erarbeitet, die dann vom Bund rechtlich erlassen werden. Die Sozialpartner, d.h. die Gewerkschaften und Verbände der Arbeitgeber_innen, arbeiten nicht nur bei der (Neu-)Ordnung der Ausbildungsrichtlinien mit, sondern dürfen diese auch initiieren. Zudem sind sie beteiligt an der Tarifaushandlung für die Auszubildenden im dualen System und können an den Prüfungen teilnehmen. Diese werden im dualen System überwacht und durchgeführt von den zuständigen Stellen, oft den Handwerks- und Industrie- und Handelskammern. Den Kammern kommt zudem auch die Aufgabe zu, über Zulassungen zur Prüfung, Gleichstellung von Zeugnissen und Anrechnungsmöglichkeiten zu entscheiden (Frommberger/Reinisch 2013: 6f.).

Durch die festgelegte Kulturhoheit der Länder sind diese einerseits für die Ordnung des berufsschulischen Teils des dualen Systems zuständig und andererseits auch für die Einrichtung und Ordnung der vollqualifizierenden Schulberufe nach Landesrecht. Hier wird deutlich, dass sich die Regulierung des dualen Systems größtenteils auf Bundesebene und die des Schulberufssystems auf Länderebene vollzieht.

92 Im Berufsbildungsbildungsgesetz (BBiG) werden die zuständigen Stellen als Organisationen definiert, die auf dem Gebiet der Berufsausbildung die Aufgaben wahrnehmen, für die in der gewerblichen Wirtschaft die „Kammern“ (z.B. die Industrie- und Handelskammern bzw. die Handwerkskammern) zuständig sind. 
Im Übergangssystem arbeiten Bund und Länder zusammen an der Regulierung, die zum Teil nach dem BBiG erfolgt und zum Teil in der schulischen Berufsausbildungsvorbereitung, dem Berufsvorbereitungsjahr, von den Ländern geordnet wird.

\subsubsection{Hochschulbildung}

Das deutsche Hochschulsystem besteht überwiegend aus öffentlichen und einzelnen privaten Hochschulen. Die öffentlichen Hochschulen werden gemeinhin unterschieden in Universitäten, Fachhochschulen sowie Kunst- und Musikhochschulen. Universitäten zeichnen sich durch stärker allgemeinbildende Curricula, eigene Forschungsbereiche und die Möglichkeit, Doktortitel zu verleihen, aus. Im Gegensatz zu anderen Ländern mit stark hierarchisierten Hochschulsystemen und der Tradition in der Elitebildung an einzelnen Organisationen zeichnet sich das deutsche System traditionell dadurch aus, dass die Universitäten im Großen und Ganzen ein gleiches Qualitätsniveau aufweisen (Krüger/Helsper 2014; Teichler 2002). Inwiefern die Exzellenzinitiative oder auch Internationalisierungsprozesse dies ändert, bleibt abzuwarten (vgl. Zymek 2014).

Der Fokus des Studiums an den Fachhochschulen ist stärker anwendungsorientiert, wobei oft ausgedehnte Praxisphasen integriert werden. Der Anwendungsfokus und damit die Ausrichtung auf den Arbeitsmarkt spiegeln sich auch in der Auswahl der Lehrenden der Fachhochschulen wider, die sowohl akademische als auch berufliche Qualifikationen bzw. Erfahrungen nachweisen müssen. Die Kunst- und Musikhochschulen bieten Studiengänge in den bildenden, gestalterischen und darstellenden Künsten sowie im Bereich Film, Fernsehen, Medien und Musik an. Neben diesen drei Hochschularten gibt es zudem in manchen Ländern noch Berufsakademien, die eine Integration von Wissenschafts- und Praxisorientierung durch die Dualität zweier Lernorte, der Hochschulen bzw. Studienakademien und der Betriebe, verbinden. ${ }^{93}$ Wie im dualen System erhalten die Lernenden einen Ausbildungsvertrag und eine Vergütung. Duale Studiengänge dieser Art werden nicht nur an Berufsakademien in Deutschland angeboten, sondern auch an Fachhochschulen, dualen Hochschulen und in sehr geringem Maße an Universitäten (vgl. Graf 2013). Insbesondere in den letzten Jahren hat sich die Zahl der angebotenen dualen Studiengänge massiv erhöht (Graf et al. 2014).

Betrachtet man die Verteilung aller Studierenden auf die unterschiedlichen Hochschulzweige (vgl. Tabelle 9), so lernen fast zwei Drittel an Universitäten, 1,4 \% an den Kunst- und Musikhochschulen und ca. ein Drittel an den Fachhochschulen in Deutschland. Die Berufsakademien nehmen mit $0,4 \%$ aller Studierenden nur einen sehr kleinen Teil auf (Statistisches Bundesamt 2013c).

Für mehr Informationen zur Entwicklung von Berufsakademien und dualen Studienprogrammen generell siehe Graf (2013). 
Tabelle 9 Hochschultypen und Studierendenverteilung

\begin{tabular}{lccccc} 
& Gesamt & $\begin{array}{c}\text { Universi- } \\
\text { täten }\end{array}$ & $\begin{array}{c}\text { Kunst-/Musik- } \\
\text { hochschulen }\end{array}$ & $\begin{array}{c}\text { Fachhoch- } \\
\text { schulen* }\end{array}$ & $\begin{array}{c}\text { Berufs- } \\
\text { akademien }\end{array}$ \\
\hline $\begin{array}{l}\text { Zahl der } \\
\text { Organisationen } \\
\begin{array}{l}\text { Zahl der } \\
\text { Studierenden }\end{array}\end{array}$ & 423 & 121 & 56 & 215 & 31 \\
$\begin{array}{l}\text { Anteil der } \\
\text { Studierenden }\end{array}$ & 2.508 .929 & 1.638 .956 & 34.719 & 825.734 & 9.520 \\
\hline
\end{tabular}

* inkl. Verwaltungshochschulen

Quelle: Statistisches Bundesamt (2013c: 13 und 414) (Anzahl der Studierenden je Organisationsform); HRK (2013) für die Anzahl der Hochschulen ohne Berufsakademien; Anzahl der Berufsakademien über http://www.die-duale-hochschule-kommt.de/berufsakademien-in-deutschland, abgerufen am 27.01.2014; Berechnung der Prozente durch NB

Mit der Umsetzung des Bologna-Prozesses wurden in Deutschland mehr und mehr die traditionellen Studienabschlüsse Diplom und Magister von den Abschlüssen der gestuften Studienstruktur abgelöst. Im ersten Studienzyklus kann ein Bachelorgrad erworben werden. Zugang zum Bachelor erhält man in der Regel über eine Hochschulreife. Diese kann allgemeiner Natur sein und berechtigt zu einer freien Fächerwahl, oder sie kann fachgebunden sein, so dass der Zugang zu einer bestimmten Fächergruppe eröffnet wird. Mit einer Fachhochschulreife wird dagegen der Zugang zu den Fachhochschulen, aber nicht zu den Universitäten eröffnet (KMK 2013). Der Bachelorabschluss gilt als Regelabschluss (KMK 2013), aber bisher scheint in Deutschland eine hohe Neigung zum Weiterstudium zu bestehen (vgl. Heine 2012). Der zweite Studienzyklus endet mit einem Mastergrad, wobei dieser dem alten Diplom und Magister gleichgestellt ist. Zugangsvoraussetzung für einen Masterstudiengang ist in der Regel ein erster berufsqualifizierender Hochschulabschluss (KMK 2013). Der dritte Studienzyklus wird mit der Promotion abgeschlossen. Masterabschlüsse oder äquivalente Abschlüsse wie das Staatsexamen berechtigen zu einer Promotion (KMK 2013).

Wie im Hinblick auf die unterschiedlichen Chancen, einen Ausbildungsplatz in der beruflichen Bildung zu erhalten, gilt in einem noch stärkeren Maße für den Zugang zum Hochschulsystem, dass dieser sozial selektiv ist (vgl. z.B. Dahrendorf 1965; Müller et al. 2009; Wolter 2011). Vorgelagert ist hier vor allem die soziale Selektivität im Hinblick auf die Gruppe, die eine Hochschulreife normalerweise in der gymnasialen Oberstufe erwirbt. Verstärkt wird die soziale Selektivität bei der Entscheidung der Studienberechtigten für oder gegen ein Studium (vgl. Autorengruppe Bildungsberichterstattung 2010: 119; Mayer et al. 2007) bei der Wahl der Studienform Fachhochschule oder Universität (vgl. Mayer et al. 2007; 
Reimer/Pollak 2010) und des Studienfachs (vgl. Becker et al. 2010; Reimer/Pollak 2010). ${ }^{94}$

\section{Steuerung der Hochschulbildung}

Im Gegensatz zum Berufsbildungssystem erfolgt die Steuerung der Hochschulbildung weitestgehend über die Bundesländer, die so ihre Kultur- und Wissenschaftshoheit wahrnehmen. Bis 2008 hatte auch der Bund eine allgemeine Regulierungsmacht durch das Hochschulrahmengesetz. Dieses wurde aber mit den Beschlüssen der Föderalismuskommission 2006 aufgehoben, so dass der Bund nur noch teilweise Kompetenzen in Bezug auf Bereiche der Hochschulzulassung und der Hochschulabschlüsse besitzt und im Rahmen von Gemeinschaftsaufgaben Forschungsvorhaben fördern und Forschungsbauten mitfinanzieren kann.

Die Landeshochschulgesetze sind daher die geltenden Regelungen für die Hochschulen in Deutschland. Somit unterliegen die Rechtsaufsicht, die Gründungsbefugnis, die Organisationsgewalt, die Finanzhoheit sowie die Personalhoheit den Landesministerien. Studiengänge, wenn sie mit Staatsexamen abschlieBen, werden ebenfalls durch die Landesministerien kontrolliert, während die anderen Studiengänge durch die von der Kultusministerkonferenz eingesetzten Akkreditierungsagenturen akkreditiert werden (KMK 2013: 52-53). Gesichert durch das Grundgesetz gilt an den Hochschulen die Wissenschaftsfreiheit, so dass die Hochschulen sich weitestgehend nach dem Prinzip der akademischen Selbstverwaltung selbstständig organisieren und verwalten. Zudem sind den Hochschulen in den letzten Jahren auch mehr Autonomierechte u.a. in Bezug auf die Studierendenzulassung gewährt worden (Frankenberg 2008).

Gewerkschaften und Unternehmen spielen im Hochschulsystem im Gegensatz zum Berufsbildungssystem keine große Rolle, wobei die Unternehmen auch mit dem Anwachsen dualer Studiengänge in den letzten Jahren stärker an Einfluss gewinnen konnten als Gewerkschaften (Graf 2013).

Auch wenn die Beschreibung der institutionellen Ordnungen des Bildungsschismas (vgl. Kapitel 6.1) idealtypisch verstanden werden soll, wird doch anhand der vorgenommenen Darstellung des deutschen Berufs- und Hochschulbildungssystems, dessen Steuerung und des Nebeneinanders beruflicher und stärker allgemeinbildender postsekundärer Bildung die Trennung der Organisationsfelder in Deutschland deutlich. Hervorgehoben werden soll an dieser Stelle vor allem die Rolle des vorgelagerten stratifizierten Schulsystems, das die Trennung der Organisationsfelder vorbereitet und mitbegründet (vgl. Powell/Solga 2011). So ist einerseits die gymnasiale Oberstufe primär als eine Vorbereitung auf die Hochschulreife konzipiert, auch wenn Abiturient_innen teilweise eine Berufsausbildung absolvieren. Der mittlere Schulabschluss und mittlerweile weniger der

94 Die Literaturverweise zur Frage sozialer Ungleichheit beim Hochschulzugang sind nur exemplarisch aus einer breiten Forschungsliteratur zu diesem Thema ausgewählt. 
Hauptschulabschluss sind die Abschlüsse der Schulstränge, die in die Berufsbildung führen, während ein Großteil der Haupt- und Sonderschüler_innen und der Abgänger_innen ohne Schulabschluss in das Übergangssystem gelangen. Wege abseits dieser Standardpfade aus dem deutschen Schulsystem in die Berufs- und Hochschulbildung sind selten.

\subsubsection{Bildungswege zwischen Berufs- und Hochschulbildung}

Trotz gegenseitiger Abschottung haben sich im Laufe der Zeit doch Bildungswege entwickelt, die vor allem einen Übergang aus der Berufs- in die Hochschulbildung ermöglichen. Diese sollen jetzt im Mittelpunkt stehen. Zuerst werden jedoch mögliche Bildungswege aus der höheren Allgemeinbildung in die Berufsbildung zusammengefasst.

\section{Wege in die Berufsbildung}

Ein Zugang in die Berufsbildung zumindest auf Sekundärniveau aus dem allgemeinbildenden Schulwesen ist generell möglich und üblich. Grundvoraussetzung für die Aufnahme einer Berufsausbildung ist das Absolvieren der Vollzeitschulpflicht (mindestens neun Jahre Schulbesuch) (KMK 2013). Prinzipiell gibt es zwar für duale Ausbildungsberufe im Gegensatz zu den schulischen keine weiteren rechtlich festgelegten Zugangsvoraussetzungen, jedoch werden bestimmte Berufsausbildungen stärker mit Absolvent_innen eines bestimmten Niveaus, z.B. dem MSA oder dem Abitur, besetzt (vgl. Autorengruppe Bildungsberichterstattung 2010; Protsch 2014).

Eine weitere Möglichkeit bietet die Externenprüfung. Mit der Externenprüfung kann ein staatlich anerkannter Ausbildungsabschluss nach BBiG oder $\mathrm{HWO}^{95}$ erworben werden, ohne den formalen dualen Ausbildungsgang absolviert zu haben. Die Externenprüfung ist dabei aber nur die Prüfung, ob man an der regulären Abschlussprüfung des dualen Systems teilnehmen kann. Es handelt sich somit um ein Zulassungsverfahren (vgl. Schreiber 2010; Schreiber et al. 2012). Voraussetzung ist nach BBiG (1969: $\$ 40$ (2)), dass der oder die Antragssteller_in nachweist „dass er [oder sie, N.B.] mindestens das Zweifache der Zeit, die als Ausbildungszeit vorgeschrieben ist, in dem Beruf tätig gewesen ist, in dem er [oder sie, NB] die Prüfung ablegen will“. Das heißt, dass die durch den Arbeitsprozess erlernten Kompetenzen und Qualifikationen als Voraussetzung für einen beruflichen Abschluss anerkannt werden. 2012 waren es 7,4 \% der Teilnehmenden an den Abschlussprüfungen, die über die Externenregelungen partizipieren durften (Statistisches Bundesamt 2013b). Während die eigentliche Zielgruppe dieser Regelung un- und angelernte Erwerbstätige sind, zeigt Heckel (1994: 29), dass

95 Die Regelungen in BBiG und der HWO unterscheiden sich nicht stark, so dass im Folgenden nur auf das BBiG Bezug genommen wird. 
deutlich über die Hälfte der Teilnehmer_innen Anfang der 1990er Jahre bereits einen Berufsabschluss erworben hatten und dass auch die schulische Vorbildung der Teilnehmer_innen relativ hoch war, wobei $15 \%$ von ihnen eine Hochschulreife besaßen. Dieser Trend hat sich bis heute fortgesetzt und verstärkt. So zeigte eine Studie des BIBB, dass über $40 \%$ der befragten Externen eine Studienberechtigung und $9 \%$ einen akademischen Abschluss besitzen (Schreiber et al. 2012). Meist wird die Externenprüfung als Um- oder Doppelqualifizierung genutzt (ebd.). Die Anrechnung findet aber gemäß den Regelungen zur Externenprüfung vor allem in Bezug auf die Arbeitserfahrung im Beruf statt, so dass nicht klar ist, inwiefern die Leistungen der Hochschulbildung anerkannt werden.

Für die Zulassung zur Prüfung der Fortbildungsberufe sind gemeinhin eine dreijährige Berufsausbildung und eine mehrjährige Berufspraxis notwendig, zum Teil ist ohne Berufsabschluss auch nur eine langjährige (fünf Jahre) berufsspezifische Berufspraxis Voraussetzung. Zwar gibt es die Regelung der Auffangnorm, aber es hat sich gezeigt, dass diese relativ wenig benutzt wird (1-5\% der Fälle). Neben den geforderten Regelvorgaben ist die Auffangnorm die zweite Möglichkeit, zur Fortbildungsprüfung zugelassen zu werden. Hierfür ist der Nachweis notwendig, dass die berufliche Handlungskompetenz anderweitig erworben wurde (Kohl et al. 2013). Für Absolvent_innen der stärker allgemeinbildenden Bildungsgänge wird in diesem Zusammenhang eine ausschlagkräftige Berufspraxis als besonders wichtig angesehen (ebd.). Wege über die Auffangnorm sind bis dato sehr selten (ebd.).

\section{Wege in die Hochschulbildung - Der zweite und dritte Bildungsweg}

Typischerweise werden die nicht traditionellen Wege in die Hochschulbildung in einen zweiten und dritten Bildungsweg unterschieden, wobei diese in Differenz zum ersten Bildungsweg stehen, der den Erwerb der Hochschulreife innerhalb der ,gesellschaftlichen Normalbiographie“ (Freitag 2012: 9) symbolisiert.

Die Begriffe zweiter und dritter Bildungsweg sind wissenschaftlich geprägte Begriffe, deren Bedeutung sich im Zeitverlauf verändert hat und auch heute nicht eindeutig definiert ist (vgl. Freitag 2012; Lönz 2008; Schwabe-Ruck 2010). Dies gilt insbesondere für den zweiten Bildungsweg, der sowohl der Ordnung im Schul- als auch der im Hochschulsystem zugeordnet werden kann (Freitag 2012). Aus der Logik des Schulsystems handelt es sich allgemein um Möglichkeiten für Erwachsene, formale Schulabschlüsse jeglicher Art nachzuholen. Aus der Logik des Hochschulsystems handelt es sich um die Aufnahme eines Studiums, nachdem eine Hochschulzugangsberechtigung nachträglich nach schulrechtlichen Regelungen erworben wurde (ebd.). Im Folgenden wird der Begriff zweiter Bildungsweg in diesem zweiten Sinn verwendet. 


\section{Zweiter Bildungsweg - Schulrechtliche Regelungen}

Der nachträgliche Erwerb der Hochschulzugangsberechtigung kann an unterschiedlichen Organisationen erfolgen. Auch hier gibt es unterschiedliche Einschätzungen darüber, welche Organisationen dazugezählt werden und welche nicht. Mehrheitlich werden das Abendgymnasium und das Kolleg als Organisationen des zweiten Bildungsweges definiert (vgl. Autorengruppe Bildungsberichterstattung 2012; Lönz 2008). Der Erwerb der Hochschulreife am Kolleg erfolgt meist in Vollzeit und am Abendgymnasium berufsbegleitend. Damit unterscheiden sich auch die Lernenden in den beiden Schulwegen deutlich: Die Kollegiat_innen sind meist jünger, während die Absolvent_innen des Abendgymnasiums älter sind und deutlich mehr Berufserfahrung aufweisen (Freitag 2012: 19). Auch die Nichtschüler_innenprüfung wird als Institution des zweiten Bildungsweges angesehen: Hier können Individuen, ohne zuvor noch einmal an schulischen Bildungsgängen teilgenommen zu haben, die Hochschulreifeprüfung ablegen.

Es gibt aber auch berufsschulische Wege, die nach Abschluss einer beruflichen Erstausbildung oder einer langjährigen Berufserfahrung die Möglichkeit bieten, die verschiedenen Arten der Hochschulreife zu erwerben. Dies wird vor allem an Berufsoberschulen (BOS) und in einigen Ländern auch an Fachoberschulen (FOS) ermöglicht.

In den Fachoberschulen können Absolvent_innen einer Berufsausbildung dann in einem Jahr zu einer allgemeinen Fachhochschulreife (FHR) gelangen, während Schüler_innen mit einem mittleren Schulabschluss zwei Jahre benötigen (KMK 2014b). Die Berufsoberschule führt nach einem zweijährigen Vollzeitunterricht zur fachgebundenen Hochschulreife (fHR) und mit einer zweiten Fremdsprache zur allgemeinen Hochschulreife (aHR). Der Besuch in Teilzeit ist ebenfalls möglich. Die Voraussetzungen für den Besuch einer BOS sind ein mittlerer Schulabschluss und eine mindestens zweijährige Berufsausbildung. Anstatt der abgeschlossenen Ausbildung kann auch eine fünfjährige Berufserfahrung ausreichend für die Aufnahme sein (KMK 2014a).

In der amtlichen Statistik werden diese Wege über berufliche Schulen meist dem ersten Bildungsweg zugeordnet, obwohl auch hier sequenziell nach dem Beruf eine Hochschulzugangsberechtigung erworben werden kann (vgl. Autorengruppe Bildungsberichterstattung 2012). So betrachten dagegen vor allem die Gewerkschaften und die Wirtschaft diese Bildungsgänge als zweiten Bildungsweg (vgl. Freitag 2012: 20), aber auch auf Seiten der Arbeitsagentur werden sie als solcher definiert (Bundesagentur für Arbeit 2014). Ob erster oder zweiter Bildungsweg, Schindler (2012: 3f.) zeigt, dass der Weg über die beruflichen Schulen maßgeblich zur Erhöhung der Hochschulzugangsberechtigtenquoten in den letzten Jahrzehnten beigetragen hat und vor allem die soziale Selektivität der Gruppe der Hochschulzugangsberechtigten verringert hat. 
Betrachtet man die Statistik, so fällt auf, dass im Jahr 2012 noch immer 58 \% der schulrechtlichen Hochschulzugangsberechtigungen über das allgemeinbildende Gymnasium erworben wurden. Dagegen waren es 2012 nur 0,86 \% der Absolvent_innen mit Hochschulzugangsberechtigung, die diese über das Kolleg, und $0,75 \%$, die diese über das Abendgymnasium erworben hatten (Statistisches Bundesamt 2013a). ${ }^{96}$ Da die Fachserien des statistischen Bundesamtes keine Daten der beruflichen Schulen und der allgemeinbildenden Schulen nach Schularten für die gleichen Jahre liefert, werden im Folgenden die Daten für die Fachgymnasien (erster Bildungsweg), die Berufsoberschulen (stärker zweiter Bildungsweg) und die Fachoberschulen (zweiter und zum Teil auch erster Bildungsweg) ${ }^{97}$ für das Jahr 2011 errechnet. Von den Absolvent_innen mit Hochschulzugangsberechtigung in Deutschland im Jahr 2011 haben 2,4\% ihren Abschluss über die Berufsoberschule, 11,4\% ihren Abschluss über die Fachoberschule und 8,3\% über das Fachgymnasium erworben. ${ }^{98}$ Es zeigt sich, dass wiederum die Schulwege, die stärker dem ersten Bildungsweg zuzurechnen sind, am stärksten frequentiert werden, während Absolvent_innen der Berufsoberschulen, der Kollegs und der Abendgymnasien viel geringere Anteile der Gesamtstudienberechtigten ausmachen.

\section{Dritter Bildungsweg - Hochschulrechtliche Regelungen}

Der dritte Bildungsweg ist im Vergleich zum zweiten gekennzeichnet durch das Aufnehmen eines Studiums ohne den vorherigen Erwerb einer schulischen Hochschulzugangsberechtigung. Die Aufnahme des Erststudiums wird hier formal durch die Ausstellung der Studienberechtigung durch die aufnehmende Hochschule ermöglicht. Aber auch hier hat sich das Verständnis des dritten Bildungswegs im Zeitverlauf und in Abhängigkeit von der Definition des zweiten Bildungswegs verändert (vgl. Freitag 2012; Schwabe-Ruck 2010).

Es kann unterschieden werden zwischen der Begabtenprüfung, die bereits 1924 eingeführt wurde, dem Hochschulzugang für beruflich Qualifizierte und der Studienberechtigung ohne formale Hochschulreife, ein über eine Eignungsprüfung angelegter Weg an die Kunst- oder Musikschulen (Freitag 2012). Insbesondere der Hochschulzugang für beruflich Qualifizierte wurde in den letzten beiden Jahrzehnten deutlich ausgebaut.

96 Die Prozente wurden errechnet aus den Angaben des Statistischen Bundesamtes (2013a) anhand der Tabellen 6.7 sowie Tabelle 6.2.

97 Ob Fachoberschulen eher dem zweiten oder dem ersten Bildungsweg zugerechnet werden, hängt auch davon $\mathrm{ab}$, ob die Absolvent_innen vorher eine Berufsausbildung abgeschlossen haben oder nicht. Beides ist an den Schulen möglich. Die Daten geben aber keine getrennte Rechnung an für die mit und die ohne beruflichen Abschluss.

98 Die Prozente wurden errechnet aus den Angaben des Statistischen Bundesamtes (2012) der Fachserie 11, Reihe 2 (Tabellen 5.5, 6.5 und 7.5) sowie den Angaben des Statistischen Bundesamtes (2013a), Fachserie 11, Reihe 1 in Tabelle 6.7. 
Insgesamt können drei unterschiedliche Typen von Regelungen für den Hochschulzugang ohne schulische Berechtigung unterschieden werden (vgl. Ulbricht 2012a): Zulassungsverfahren über eine Prüfung, Zulassung durch die direkte Anerkennung von beruflichen Qualifikationen und das Studium auf Probe, bei dem erst nach einer festgelegten Studienzeit und nach Nachweis eines regulären Studienfortschritts die vorläufige Berechtigung in eine formale umgewandelt wird. Das Zulassungsverfahren für eine Prüfung, bei dem die Studieneignung vor Studienbeginn festgestellt wird, kann dabei unterschiedlich gestaltet sein: in Form einer Eignungsprüfung, eines Eignungsgesprächs oder einer Einstufungsprüfung (vgl. Wolter 1994).

Die 1990er Jahre sowie auch die Jahre nach dem Jahrtausendwechsel waren gekennzeichnet durch eine Welle der Neuregelungen der Hochschulgesetze der Länder in Bezug auf den Zugang von beruflich Qualifizierten ohne schulische Berechtigung (siehe Tabelle 3 im Online-Anhang). Entsprechend dieser Phase der Institutionalisierung wurde die Frage des Hochschulzugangs von beruflich Qualifizierten ohne schulische Berechtigung auch erstmals explizit mit der 4. Novellierung des Hochschulrahmengesetzes 1998 in Artikel 27 zur Zulassung zum Hochschulstudium aufgenommen. ${ }^{99}$ Ihren Höhepunkt erreichte die Erweiterung der Regelungen, als 2009 die KMK festlegte, dass erstens beruflich Qualifizierte mit Fortbildungsabschluss eine allgemeine Hochschulzugangsberechtigung erhalten sollen. Zweitens konnten auch Absolvent_innen der beruflichen Erstausbildung nach mindestens dreijähriger Berufspraxis und einer Eignungsprüfung an den Hochschulen zugelassen werden (KMK 2009d). Trotz all dieser Liberalisierung des Hochschulzugangs sind es aber weiterhin nur 2,1 \% aller Studienanfänger_innen, die in die Hochschulen über den dritten Bildungsweg gelangen, wobei diese 2,1\% eine deutliche Steigerung der noch niedrigeren Quoten in den 1990er Jahren oder zu Beginn des Jahrtausends darstellen (Nickel/Duong 2012: 31). ${ }^{100}$ Dabei nehmen sie häufiger ein Studium an Fachhochschulen auf als an Universitäten, wo sie nur mit $0,6 \%$ der Studienanfängerkohorte vertreten sind (Autorengruppe Bildungsberichterstattung 2010).

Es wird somit deutlich, dass es sowohl Wege in die Berufsbildung als auch Wege in die Hochschulbildung für Absolvent_innen des jeweils anderen Bildungsbereichs gibt. Es wird aber auch deutlich, dass diese Wege zu großen Teilen den bestehenden Logiken des Organisationsfeldes folgen. So sind in den Regelungen zur Externenprüfung und Auffangnorm die berufspraktischen Erfahrungen die entscheidenden, während die meisten Wege in die Hochschulbildung (vor

\27 HRG 1998 ,(1) Alle Deutschen im Sinne des Artikels 116 des Grundgesetzes sind zu dem von ihnen gewählten Hochschulstudium berechtigt, wenn sie die für das Studium erforderliche Qualifikation nachweisen. [...] (2) Der Nachweis nach Absatz 1 Satz 1 wird für den Zugang zu einem Studium, das zu dem ersten berufsqualifizierenden Abschluss führt, grundsätzlich durch den erfolgreichen Abschluss einer auf das Studium vorbereitenden Schulbildung erbracht. In der beruflichen Bildung Qualifizierte können den Nachweis nach näherer Bestimmung des Landesrechts auch auf andere Weise erbringen. [...]“

100 Die Entwicklung der Quoten je Bundesland ist nachlesbar in Nickel/Duong (2012: 33). 
allem der zweite Bildungsweg sowie einige Eignungsprüfungen an den Hochschulen) über die Prüfung des Allgemeinbildungsniveaus führen.

\subsection{Verhältnis von Berufs- und Hochschulbildung aus historischer Perspektive}

In Deutschland haben wir uns bisher schwer getan, den Weg zu einem Hochschulstudium denjenigen zu öffnen, die in die Hochschule etwas anderes einbringen können als schulische Erfahrung und schulische Leistung. (Teichler 1990b: 23)

Um die Entwicklungen zu Durchlässigkeit zwischen beruflicher und Hochschulbildung in den letzten Jahrzehnten verstehen und einordnen zu können, ist es unerlässlich, auch die historische Entwicklung vor dem Untersuchungszeitraum zu kennen. Aus diesem Grund werden im Folgenden wichtige Entwicklungen, die das bestehende Verhältnis von beruflicher Bildung und Hochschulbildung vor allem im Hinblick auf Durchlässigkeit erklären, dargestellt.

Der Fokus liegt dabei vor allem auf der Frage des Hochschulzugangs für beruflich Qualifizierte, da dieser Aspekt in den Quellen zur historischen Entwicklung der dominante Aspekt war, an dem sich das Verhältnis der beiden Organisationsfelder bzw. ihrer Trennung herauskristallisierte. Aber auch die parallele Entwicklung der Berufsbildung und der höheren Allgemeinbildung in Deutschland wird berücksichtigt. Zudem wird ein kurzer Einblick in die durchlässigkeitsrelevanten Strukturen des DDR-Systems gegeben, denn mit der Wiedervereinigung hätten auch die Strukturen aus den neuen Bundesländern Einfluss auf die weitere Entwicklung haben können. Dass dies nicht der Fall war, kann an dieser Stelle bereits erwähnt werden.

\subsubsection{Entwicklung bis 1945}

Wie kam es historisch zu dieser Trennung und der Vorrangstellung des Abiturs für den Hochschulzugang?

1788 wurde in Preußen das Abitur eingeführt, es war aber zu dieser Zeit noch keine Voraussetzung für den Zugang zu den bestehenden Universitäten. Eine solche Verbindung wurde erst später 1812 und 1834 festgelegt. Es wurde beschlossen, dass der Hochschulzugang nur über eine Maturitätsprüfung erfolgen darf (vgl. Wissenschaftsrat 2004: 66; Wolter 1989: 4). Aufgrund dieser Entscheidung wurde auch die erste Regelung, die dem zweiten Bildungsweg zuzuordnen wäre, das Externenabitur eingeführt, das von den Schulen abgenommen wird. Dabei wurde die Einführung des Abiturs als Reifeprüfung als wichtiger Schritt zu einer größeren Chancengleichheit gesehen, weg von ständischen Privilegien (Wolter 
1989). Zum anderen sollte die Einführung aber auch als „Versperrungsmechanismus gegenüber den unteren Sozialgruppen“ (Wolter 1989: 10) genutzt werden. Insgesamt kann aber die Einführung der Reifeprüfung als Voraussetzung für den Hochschulzugang als ein Bruch mit der bestehenden „Tradition der relativen Freizügigkeit des Hochschulzugangs", die keine Zulassungsvoraussetzungen kannte, gesehen werden (Wolter 1989: 13). Eine noch immer nachwirkende Folge dieser Verbindung von Abitur und Hochschulzulassung fasst Wolter (1989: 31) folgendermaßen zusammen:

\begin{abstract}
Sie hat nämlich Bildungstheorie und Lehrplan des Gymnasiums bis in die Gegenwart hinein so eindeutig auf die Funktion einer akademischen Vorbildungseinrichtung festgelegt, daß das Gymnasium quasi zur Einbahnstraße in die Universität wurde. Die Beschränkung der Zulassung wurde zu dieser Zeit begründet vor allem mit dem notwendigen Schutz vor einem Rückgang des Anspruchsniveaus der Universitäten und vor einer Überfüllung dieser durch nicht-fähige. (Wolter 1989: 9)
\end{abstract}

Die Universitäten selbst wurden in dieser Zeit vor allem als Ort angesehen, um den Beamtennachwuchs des königlichen Staates und den Nachwuchs in akademischen Berufen wie Ärzten und Juristen auszubilden, und zwar abseits von Industrie und Gewerbe (Baethge 2006). Insofern wurde das Gymnasium immer stärker als erster Berechtigungsschritt für diese Karrierewege gesehen und wurde zu einer „Verteilerstelle im Bildungswesen für den Zugang zu gehobenen beruflichen Positionen und sozialen Rängen“" (Wolter 1989: 17). Dies war der primäre Zweck der Einführung dieses Bildungsweges. Erst nachträglich wurde dem Gymnasium die Bedeutung als Institution, die den humanistischen Bildungsidealen verhaftet ist, zugeschrieben (vgl. Wolter 1989). Dabei lässt sich zeigen, dass die Institutionalisierung des Gymnasiums in der Konzeption bis 1840 entgegen allgemeinen Annahmen nicht dem Bildungsideal Wilhelm von Humboldts, der individualisierten zweckfreien Bildung zur Persönlichkeitsentfaltung, entsprach, womit die Entstehung des Gymnasiums oft in Verbindung gebracht wird (Wolter 1989). Mit der engen Kopplung von Gymnasium und Universitätsstudium entstand eine weitere für das deutsche Bildungswesen typische Trennung zwischen höherer Gymnasialbildung und niedriger Volksschulbildung. Später entstanden auch Real- und Mittelschulen, die auf eine Berufsausbildung im Gewerbe vorbereiten (Baethge 2006).

Während sich im 19. Jahrhundert die gymnasiale Oberstufe entwickelte, war die berufliche Bildung in Deutschland vor allem durch den Niedergang der Zünfte wenig institutionalisiert. Dies änderte sich erst Ende des 19. Jahrhunderts, als zum Schutz vor der Sozialdemokratie insbesondere das „Handwerkerschutzgesetz" von 1897 erlassen wurde, welches das alte System der Zünfte quasi wiederbelebte. Greinert (2006) bezeichnet diese Gewerberechtsnovelle als Fundament der deutschen Berufsausbildung, des dualen Systems, da sie das Lehrlingswesen regelt und das Handwerk bei der Berufsausbildung bevorrechtigt. Das Modell der Berufsausbildung des Handwerks wurde im 20. Jahrhundert von den deutschen Industriebetrieben übernommen und weiterentwickelt (vgl. Thelen 
2004). Die zweite Entwicklung um die Jahrhundertwende war die Einführung einer beruflichen Fortbildungsschule, wobei sich das Berufsschulwesen in Deutschland langsam entwickelte und erst in den 1930er Jahren die Berufsschule als Pflicht eingeführt wurde (Greinert 2006).

Insgesamt kann man sagen, dass es ein Nebeneinander von Entwicklungen ohne Verbindungen der Institutionalisierungsphasen der Berufs- und der höheren Allgemeinbildung gab. Trotzdem ertönte in der deutschen Bildungsgeschichte wiederholt die Forderung, den Hochschulzugang auch für beruflich Qualifizierte zu öffnen.

In der Weimarer Republik wurde vor allem der zweite Bildungsweg ausgebaut. So wurden Abendgymnasien eingeführt, zudem wurden Erwachsenenschulen gegründet, die den nachträglichen Erwerb der allgemeinen Hochschulreife, meist als Nicht-Schüler-Abitur, ermöglichten (Schwabe-Ruck 2010). Aber auch ein Vorläufer des dritten Bildungsweges wurde eingeführt, die Begabtenprüfung an den Universitäten in Preußen (Schwabe-Ruck 2010; Wolter 1994). Betrachtet man die Debatten um die Öffnung des Hochschulzugangs, konnten einerseits Argumente für die Öffnung als nachholende Chancengleichheit auf Seiten der Gewerkschaften und der Sozialdemokratie festgestellt werden (Schwabe-Ruck 2010: 83). Auf Seiten der Gegner einer stärkeren Öffnung wurde ein Massenansturm auf die Universitäten befürchtet und bestehende Vorbildungsdefizite als Hindernisse angeführt (Wolter 1994: 18).

In der Zeit des Nationalsozialismus ist der Zugang über den zweiten und dritten Bildungsweg vor allem denjenigen geöffnet worden, die ihre Ideologietreue bewiesen. Zwar wurden die Abendgymnasien und die Arbeiter-Abiturientenkurse weitestgehend geschlossen, aber der nachträgliche Erwerb der Hochschulzugangsberechtigung war weiterhin möglich, vor allem in Form des neu eingeführten Langemarck-Studiums. Dies bestand aus zwei Teilen. Erstens konnten regimetreue junge Erwachsene das Abitur nachholen, um dann in einem zweiten Schritt ein Studium anzufangen (Schwabe-Ruck: 2010). Auch der dritte Bildungsweg bestand vor allem über die Begabtenprüfung weiter. Zu dieser wurden primär nicht mehr kriegstaugliche Soldaten bei entsprechender Begabung und Vorbildung zugelassen. Zudem wurde der Hochschulzugang auf Drängen der Rüstungsindustrie und des Militärs weiter geöffnet, und zwar für Offiziersanwärter, Techniker und Landwirte (Schwabe-Ruck 2010). Schwabe-Ruck (2010: 179) fasst das Ziel der Öffnung des Hochschulzugangs in der Zeit des Nationalsozialismus folgendermaßen zusammen:

Dabei stehen aber nicht primär Bildungserwerb, persönliche Entfaltung und Vermehrung der Chancengerechtigkeit im Mittelpunkt der Bemühungen, sondern die optimale Ausbeute der in der Bevölkerung schlummernden Ressourcen für die eigenen inhumanen ideologischen und militaristischen Zwecke. 


\subsubsection{Entwicklung von 1945 bis 1989}

Mit der Teilung Deutschlands nach 1945 entwickelten sich die Bildungssysteme in der ehemaligen DDR und der Bundesrepublik gerade in Bezug auf die $\mathrm{Zu}$ gangswege für beruflich Qualifizierte zum Studium sehr unterschiedlich. Während in der BRD die Länder vor allem an alte Regelungen der Weimarer Republik anknüpften, wurden ganz neue Institutionen in der DDR geschaffen. Im Folgenden soll zuerst die durchlässigkeitsrelevante Entwicklung des Bildungssystems der DDR nach 1945 bis 1989 dargestellt werden. Daran anschließend werden die Entwicklungen in der BRD zusammengefasst.

\section{Entwicklungen in der DDR}

Die Zeit kurz nach Kriegsende kann als Phase der ,antifaschistisch-demokratischen Schulreform“ (Below 2002: 81) bezeichnet werden. Ziel war es, das Bildungsmonopol privilegierter Schichten zu beenden und gezielt bisher benachteiligte Gruppen zu fördern. Im Rahmen dieser Phase wurden die ersten Vorstudienanstalten, die späteren Arbeiter- und Bauernfakultäten, gegründet. Diese hatten als Aufgabe, Mitglieder der Arbeiterklasse und Individuen bäuerlicher Herkunft schulisch so fortzubilden, dass sie ein Studium erfolgreich absolvieren können (vgl. Below 2002; Schwabe-Ruck 2010). Neben diesen Fakultäten wurden auch Abendoberschulkurse zum nachträglichen Erwerb der Hochschulzugangsberechtigung eingeführt (Schwabe-Ruck 2010: 283).

Ab 1949 kann eine zweite Entwicklungsphase des DDR- Bildungssystems unterschieden werden, die „Etappe des Aufbaus der sozialistischen Schule“ (Below 2002: 81), in dieser wurde das Bildungssystem verstärkt nach dem Vorbild der Sowjetunion umgebaut. In dieser Etappe, die bis 1961 anhielt, wurden das Fern- und das Abendstudium eingeführt, so dass bereits Berufstätige Studienabschlüsse erwerben konnten. Ein Großteil, d.h. etwa ein Drittel der Studierenden an Fachhochschulen und ein Zehntel der Studierenden an Hochschulen in der DDR waren Fernstudierende (Fischer 1992 in Below 2002). Aber auch im Schulsystem wurde eine stärkere Verbindung von Berufs- und allgemeiner Bildung institutionalisiert. So wurde 1959 der polytechnische Unterricht eingeführt, in dem die Schüler_innen, die das Produktionssystem der DDR kennenlernten, in sozialistische Betriebswirtschaft eingeführt wurden, technisches Zeichnen lernten und direkte Praxiserfahrungen in Betrieben sammelten. Seit 1959 konnte in der DDR auch eine Berufsausbildung zusammen mit dem Abitur, und somit eine Berechtigung zur Hochschule erworben werden.

1961/62 begann die letzte Phase der Entwicklung des DDR-Bildungssystems, in der die "Gestaltung eines einheitlichen sozialistischen Bildungssystems“ (Below 2002: 81) im Vordergrund stand. In dieser Phase kam es zu einer Begrenzung der Studierendenzahlen, nachdem zuvor durch die geförderte Bildungsexpansion ein massiver Anstieg der Zahl der Studierenden erreicht worden war. So 
verfünffachte sich die Zahl der Studierenden seit 1951 auf ca. $161.000 \mathrm{im}$ Jahr 1971 (Below 2002). 1989 lag die Zahl der Studierenden wieder darunter bei ca. 134.000 (Below 2002: 85). Die Wege in die Hochschulbildung wurden immer stärker quotiert, und der anfänglichen positiven Diskriminierung vor allem von Arbeiter_innen und Bauern sowie Bäuerinnen wich einem System, das seit den 1960er Jahren immer stärker die eigene Elite reproduzierte, so dass vor allem Kinder der staatstreuen Dienstklasse von Studienplätzen profitieren konnten (vgl. Below 2002; Solga 1995). So kann die Gesellschaft in der DDR als eine Klassengesellschaft charakterisiert werden (Solga 1995). Auch das seit dem Beginn der DDR bestehende Postulat des gleichen Rechts auf Bildung wich immer mehr einem Zustand, in dem die Lernenden entweder die Oberschule oder eine Berufsbildung absolvieren konnten (Below 2002: 85). Trotzdem bleibt für das Bildungssystem der DDR festzuhalten, dass eine stärkere Verbindung der höheren Allgemeinbildung und Berufsbildung durch die Berufsausbildung mit Abitur im Sekundarbereich institutionalisiert war. Zudem war durch die Möglichkeiten des Fern- und Abendstudiums ein Schritt in Richtung besserer Vereinbarkeit von Berufstätigkeit und Studium gemacht. Da aber der Zugang zur Hochschulbildung stark begrenzt war, wurde die Verbreiterung der institutionellen Wege, die zu einer Hochschulberechtigung führten, beim Zugang zum Studium wieder zurückgenommen.

\section{Entwicklungen in der BRD}

In der Bundesrepublik Deutschland wurde, anders als in der DDR, nach dem zweiten Weltkrieg sowohl in Bezug auf den zweiten als auch auf den dritten Bildungsweg verstärkt an die Regelungen aus der Weimarer Republik angeknüpft (vgl. Wolter 1994; Schwabe Ruck: 2010). So war der Zugang zum dritten Bildungsweg vor allem via Begabten- und Sonderreifeprüfungen institutionalisiert, die in den Bundesländern sehr divers reguliert waren. 1959 wurden diese dann mit dem Beschluss der damaligen Kultusministerkonferenz reglementiert, so dass als Voraussetzung zur Prüfung eine mindestens dreijährige Berufsausbildung oder fünf Jahre Berufserfahrung festgelegt wurde (Wolter 1994). Auch die Abendgymnasien und später das Kolleg wurden wieder institutionalisiert (Schwabe-Ruck 2010).

In den 1960er und 1970er Jahren wurde das Thema Gleichwertigkeit von Berufs- und Allgemeinbildung als bildungspolitisches Ziel deklariert (vgl. Baethge 2006; Hegelheimer 1986; Schwabe-Ruck 2010; Wolter 1994).

So wird erstmals im Bildungsgesamtplan von 1973 die Herstellung der Gleichwertigkeit von allgemeiner und beruflicher Bildung als eine vordringliche bildungspolitische Aufgabe bezeichnet. (Hegelheimer 1986: 1)

Folgende Maßnahmen werden genannt: Ausbau des beruflichen Bildungswesens; Weiterentwicklung der gymnasialen Oberstufe; Verzahnung der Lehrangebote in 
beiden Feldern (vgl. Bildungsgesamtplan zit. in Hegelheimer 1986: 1). 1974 beschäftigte sich auch der Bildungsrat mit diesem Ziel und empfahl eine Neuordnung der Sekundarstufe II, in der der Gegensatz zwischen Berufs- und Allgemeinbildung aufgehoben werden sollte (Hegelheimer 1986: 2). Hier wäre ein Möglichkeitsfenster gewesen, Bildungsgänge in Deutschland einzuführen, die Berufsausbildung und den Erwerb des Abiturs verbinden. Jedoch fasst Baethge (2006: 21) zusammen:

Die Integration von Allgemein- und Berufsbildung, obwohl deklamatorisch von vielen politischen Stellungnahmen - vor allem von Sozialdemokratie und Gewerkschaften - beschworen, wurde politisch nicht einmal ernsthaft geprüft, obwohl sie von der Bildungskommission des deutschen Bildungsrats als Teil einer differenzierten Sekundarstufe II ins Gespräch gebracht worden war.

Parallel zu diesen Debatten in den 1960er und 1970er Jahren wurde in Deutschland durch die Einführung des BBiG 1969 das duale System mit seiner auf den Korporatismus gestützten Steuerungsform fest institutionalisiert. Die Einführung dieses Gesetzes wird von vielen als der Beginn des dualen Systems Deutschlands identifiziert (Greinert 2006). Es fasste die bis dato zersplitterten Regelungen zusammen und gewährte dem Bund, den Ländern und den Gewerkschaften mehr Mitspracherechte als zuvor, als vornehmlich die Arbeitgeber_innen bestimmend waren (Greinert 2006). Gleichzeitig fand eine Bildungs- und Hochschulexpansion statt. Es kam zu einem starken Anstieg der Nachfrage nach Bildung, gekoppelt mit einer längeren Verweildauer im Bildungssystem. Diese wurde gefördert durch institutionelle Reformen wie den Ausbau des Bildungssystems z.B. durch eine verlängerte Schulpflicht, den Ausbau der weiterführenden Schulen und verbesserte Transportbedingungen (vgl. Hadjar/Becker 2006). Für die Fragen des berufsbildenden Weges in die Hochschulen waren insbesondere die Einführung der Fachhochschulen und des Fachabiturs Anfang der 1970er Jahre bedeutend, da durch diese Gründung die Forderung vor allem von Wirtschaft und Gewerkschaften nach stärker beruflich orientierter höherer Bildung nachgekommen wurde (Freitag 2012). Der Ausbau des dritten Bildungswegs wurde daher in dieser Zeit nicht stark forciert.

In den 1980er Jahren kam die Frage der Gleichwertigkeit von beruflicher und allgemeiner Bildung wieder verstärkt in den bildungspolitischen Diskussionen auf. So wurde 1984 von der Bundesregierung die Realisierung dieses Ziels als Programm ausgerufen (Hegelheimer 1986) und auch der Hauptausschuss des Berufsbildungsinstituts gab 1984 Reformempfehlungen zur Erreichung von Gleichwertigkeit ab: Gleichstellung einer mindestens zweijährigen Ausbildung mit einem mittleren allgemeinbildenden Schulabschluss, Zugang zum tertiären Sektor für Absolvent_innen beruflicher Fortbildungsprüfungen sowie Veränderungen im öffentlichen Dienst in Bezug auf Gleichwertigkeit (Hegelheimer 1986: 7). Trotz dieser Empfehlungen fanden keine weitreichenden Reformen statt, wobei an einzelnen Universitäten und in manchen Bundesländern der Zugang für beruflich Qualifizierte stärker liberalisiert wurde (vgl. Tabelle 3 im Online-Anhang). 
Insgesamt lässt sich festhalten, dass die institutionelle Trennung zwischen Berufs- und höherer Allgemeinbildung insbesondere durch die Einführung der Reifeprüfung als Hochschulzugangsberechtigung (wodurch das Abitur zum primären Weg zur Hochschule wurde) ihren Anfang nahm. Der Ausbau des zweiten und dritten Bildungswegs in der Geschichte Deutschlands hatte dabei meist weniger das Ziel, die Chancengleichheit zu erhöhen, als einem Fachkräftemangel zu verhindern (Schwabe-Ruck 2010). Das Bildungssystem der DDR zeigte zwar Wege auf, wie eine stärkere Verbindung von Berufs- und höherer Allgemeinbildung institutionalisiert werden könnte, jedoch wurde auch dort die anfängliche Öffnung zugunsten der Elitenreproduktion eingeschränkt. Für die BRD ist festzustellen, dass bis 1989 vor allem der zweite Bildungsweg und weniger der dritte als Weg für beruflich Qualifizierte in die Hochschulen gesehen wurde (vgl. Wolter 1994; Schwabe-Ruck 2010). Damit geht der Weg in die Hochschule in Deutschland primär über die Allgemeinbildung. Der eigene bildende Wert der Berufsbildung wurde zwar rhetorisch bei den Forderungen nach Gleichwertigkeit anerkannt, aber nicht institutionalisiert. Inwiefern sich dies nach 1989 geändert hat und wie sich die deutschen Durchlässigkeitsstrukturen entwickelt haben, soll im nachfolgenden Kapitel 7 gezeigt werden. 


\section{Entwicklung institutioneller Durchlässigkeit in Deutschland}

Während im vorangegangenen Kapitel das deutsche Bildungssystem, das Bildungsschisma und damit auch das deutsche Durchlässigkeitsproblem sowie dessen historische Gewachsenheit dargestellt wurden, folgt in diesem Kapitel die Analyse der Veränderungen der institutionellen Durchlässigkeitsstrukturen in Deutschland für den Untersuchungszeitraum. Dies geschieht in drei Schritten. Wie bereits im Methodenkapitel (vgl. Kapitel 4) dargelegt, unterscheide ich zwei Untersuchungszeiträume, um die nationalen Entwicklungen und den Einfluss der europäischen Prozesse auf die nationalen Strukturen besser untersuchen zu können. So differenziere ich den Zeitraum vor den Bologna- und Kopenhagen-Prozessen in Deutschland von 1990 bis 1998 und den Zeitraum der europäischen Prozesse von 1999 bis 2012. Dementsprechend werde ich die Entwicklungen der gesellschaftlichen Wissensbestände zu Durchlässigkeit in einem ersten Schritt von 1990 bis 1998 und in einem zweiten Schritt von 1999 bis 2012 analysieren. Diese Untersuchungen basieren primär auf der Diskursanalyse der ausgewählten Akteursdokumente ${ }^{101}$ (vgl. Kapitel 4). Untersucht wurden dabei Dokumente der Akteursgruppen, ${ }^{102}$ die das Berufs- und Hochschulbildungssystem stark beeinflussen: auf Bundesebene das Ministerium für Bildung und Forschung (BMBW bzw. $\mathrm{BMBF}$ ) als Akteur in der Berufsbildung und, wenn auch weniger, in der Hochschulbildung; auf Länderebene die Kultusministerkonferenz (KMK) als Akteur in der höheren Allgemeinbildung und in Verantwortung für die Berufsschulen; auf Unternehmensseite der Deutsche Industrie- und Handelskammertag (DIHT bzw. DIHK) als Akteur vor allem in der Berufsbildung und auf Gewerkschaftsseite der deutsche Gewerkschaftsbund (DGB) als Akteur primär in der Berufs-, aber auch in der Hochschulbildung.

So soll im Rahmen der Analysen gezeigt werden, wie in den jeweiligen Zeiträumen Durchlässigkeit von den bildungspolitischen Akteuren konstruiert wurde, aber auch, welche Deutungskämpfe und damit Infragestellungen der Legitimität der bestehenden Institutionen sichtbar werden. In einem dritten Schritt soll festgestellt werden, inwiefern sich die institutionellen Durchlässigkeitsstrukturen vom ersten zum zweiten Zeitraum verändert haben.

Insgesamt stehen in diesem Kapitel primär die Ergebnisse der Diskursanalyse und damit die Analyse der kulturell-kognitiven und teilweise normativen institutionellen Dimension im Vordergrund. Wie wird Durchlässigkeit verstanden, wel-

101 Im ersten Zeitraum wurden 36 Dokumente und im zweiten 108 Dokumente analysiert (vgl. Auflistung der untersuchten Dokumente im Online-Anhang III).

102 Eine ausführlichere Begründung der Akteursauswahl findet sich zum einen in Kapitel 4 und eine Beschreibung der Steuerung und damit auch der verantwortlichen Akteure im deutschen Berufsund Hochschulbildungssystem in Kapitel 6. 
che Selbstverständlichkeiten werden sichtbar, welche werden hinterfragt und welche ändern sich? Was ist sag- und denkbar und was nicht? Zudem werden durch die Analyse der Diskurse auch die Praxis der Akteure und ihre Strategien und bestehende Deutungskämpfe aufgezeigt. Aber auch regulative Entwicklungen, welche auf Basis der Dokumentenanalyse erfasst wurden, werden in die Analyse eingearbeitet. Zum einen geschieht dies, wenn ein Überblick über die wichtigsten Debatten zum Thema Durchlässigkeit gegeben wird. Zum anderen werden insbesondere im letzten Abschnitt des Kapitels, in dem die Frage nach dem Wandel beantwortet werden soll, auch die regulativen Veränderungen betrachtet, um ein ganzheitlicheres Bild der Entwicklung zu erhalten. Ziel des Kapitels ist es vor allem, die Entwicklungen der deutschen Durchlässigkeitsstrukturen darzustellen. ${ }^{103}$ Inwiefern werden die institutionellen Strukturen stabilisiert (path stabilisation)? Kommt es zu einer klaren Veränderung historisch gewachsener institutioneller Prinzipien (path departure) oder gar zu einer Abkehr von ihnen (critical juncture) (vgl. Ebbinghaus 2005)?

\subsection{Gesellschaftliche Wissensbestände zu Durchlässigkeit vor Bologna und Kopenhagen (1990-1998)}

Welche institutionellen Entwicklungen im Hinblick auf Durchlässigkeit können für den ersten Untersuchungszeitraum in Deutschland von 1990 bis 1998 festgestellt werden? Dafür wird in einem ersten Schritt ein Überblick über die unterschiedlichen Debatten zum Thema Durchlässigkeit gegeben. Im Rahmen dieses Überblicks werden auch wichtige regulative Änderungen auf Bundesebene benannt. In einem zweiten Schritt werden aus den verschiedenen Aussagen der untersuchten Akteure im ersten Untersuchungszeitraum Diskurse zu Durchlässigkeit rekonstruiert und entlang ihrer typischen Struktur dargestellt.

\subsubsection{Schlaglichter der deutschen Debatten zu Durchlässigkeit (1990-1998)}

Dieser Abschnitt dient der Darstellung der durchlässigkeitsrelevanten Debatten im ersten Zeitraum. Debatten werden dabei als die Kontexte, in denen Durchlässigkeit verhandelt wurde, gefasst. Sie entsprechen somit nicht den einzelnen Themen der analysierten Dokumente und auch nicht den unterschiedlichen Aspekten von Durchlässigkeit. Als Debatten werden vielmehr nur die Themenkomplexe dargestellt, zu denen sich mehrere Akteure in ähnlichen Zusammenhängen und oft auch mit gegenseitigen Bezügen in einem bestimmten Zeitrahmen im Hinblick 
auf Durchlässigkeit geäußert haben. Relevante singuläre Aussageereignisse zu Durchlässigkeit sind zwar in die Diskursanalyse eingegangen, werden aber nicht als Debatten erfasst und dargestellt.

In welchen Kontexten wurde nun primär über Durchlässigkeit diskutiert? Im ersten Zeitraum wurde Durchlässigkeit vor allem im Kontext einer Debatte ${ }^{104}$, und zwar im Zusammenhang mit den Fragen diskutiert, wie die Attraktivität der beruflichen Bildung erhöht und Gleichwertigkeit von Berufs- und Hochschulbildung hergestellt werden kann.

\section{Debatte über Gleichwertigkeit und die Steigerung der Attraktivität von beruflicher Bildung}

Die Debatte zur Steigerung der Attraktivität von beruflicher Bildung begann bereits Ende der 1980er Jahre (Wolter 1994), war aber insbesondere zu Beginn der 1990er Jahre virulent. Vor diesem Hintergrund entfachte sich die im deutschen System immer wieder aufgekommene Diskussion über die Gleichwertigkeit von beruflicher Bildung und Allgemeinbildung (vgl. BIBB 2011; Hegelheimer 1986) erneut. Angestoßen wurde die Debatte zu Beginn der 1990er Jahre durch die Arbeitgeber_innen, die die Gefahr eines großen Fachkräftemangels thematisierten, auf den reagiert werden müsse. Ursache für diesen Fachkräftemangel, der sich an der Zahl der unbesetzten Lehrstellen vor allem in den alten Bundesländern manifestierte, wurde in der mangelnden gesellschaftlich wahrgenommenen Attraktivität der beruflichen Bildung gesehen, die dazu führte, dass Jugendliche sich vermehrt für die weiterführende Allgemeinbildung in der Schule und das Studium anstatt für eine Ausbildung entschieden.

Die Zahl der unbesetzten Lehrstellen, mit 130.000 im letzten Jahr beziffert, belegt deutlich, daß eine Fehlentwicklung bei den Qualifikationen im Gange ist und daß diese eher größer als geringer wird. Wir können aber einen gravierenden Mangel an Fachkräften mittelfristig für den Wirtschaftsstandort Deutschland nicht hinnehmen, sondern wir brauchen hochqualifizierte Fachkräfte, die nach wie vor das Rückgrat unserer Unternehmen sind. Es kann nicht dazu kommen, daß ein Einsatz durch Akademiker das heutige Qualifikationsprofil der Fachkräfte ersetzt. (DIHT 1992a) ${ }^{105}$

Eine Möglichkeit zur Erhöhung der Attraktivität beruflicher Bildung und damit einer Steuerung des Bildungsverhaltens wurde insbesondere mit der Herstellung von Gleichwertigkeit von beruflicher und allgemeiner Bildung gesehen, welche auch durch eine größere Durchlässigkeit im Bildungssystem erfolgen sollte. Die

104 Ich verwende im Folgenden den Debattenbegriff im Singular, um die unterschiedlichsten Kontexte besser voneinander abzugrenzen. Das heißt aber nicht, dass es sich nur um ein Aussageereignis oder einen spezifischen Zeitraum handelt. Vielmehr umfasst die identifizierte Debatte alle Aussagen und Diskussionen zu einem spezifischen Kontext, in dem Durchlässigkeit diskutiert wurde.

105 Die Zitate aus den untersuchten Originaldokumenten werden größtenteils ohne Seitenangaben angegeben, weil die untersuchten Dokumente dann selbst keine Seitenangaben aufweisen. 
Öffnung der Hochschulen für beruflich Qualifizierte stand hierbei im Mittelpunkt (u.a. BMBW 1992; DGB 1990, 1992, 1996). Die Debatte zu Gleichwertigkeit und Attraktivität wurde vor allem von den Akteuren der beruflichen Bildung geführt. Eine Folge war, dass 1992 und 1993, organisiert vom Bundesministerium für Bildung und Wissenschaft, Tagungen zum Thema Gleichwertigkeit von beruflicher und allgemeiner Bildung stattfanden, auf denen Antworten auf die Frage gesucht wurden, wie Gleichwertigkeit hergestellt werden könne. Durchlässigkeit war hierbei ein zentraler Lösungsansatz. Als wichtig wurde auch die verstärkte Anerkennung von beruflicher Bildung im Arbeitsmarkt diskutiert, die sich in verbesserten Karriere- und Entwicklungschancen beruflich Qualifizierter widerspiegeln sollte.

Angestoßen durch die Akteure der Berufsbildung, begann auch die Hochschulrektorenkonferenz (HRK), sich mit dem Thema Durchlässigkeit in Form des Hochschulzugangs beruflich Qualifizierter (ohne Abitur) zu beschäftigen. Wobei die Plenarsitzung 1991 allein dafür genutzt wurde, um zu klären, ob das Thema überhaupt relevant genug sei, um sich weitergehend damit zu befassen (HRK 1991). 1992 beschloss die HRK dann, dass ,besonders qualifizierten Berufstätigen“ ein fachbezogener Hochschulzugang unter der Voraussetzung, dass die Studierfähigkeit der Bewerber_innen geprüft würde, eröffnet werden könne (HRK 1992b).

Der Beschluss der KMK von 1991, dass das Abschlusszeugnis der Berufsschule die Berechtigungen des Mittleren Schulabschlusses (MSA) einschließt, beeinflusste ebenfalls den Verlauf der Debatte. Während u.a. das BMBW sich überwiegend positiv über diese Gleichstellung im Sinne eines Zeichens der Gleichwertigkeit beruflicher und allgemeiner Bildung äußerte, ${ }^{106}$ sahen die Gewerkschaften diesen Beschluss als nicht weitgehend genug und sogar nachträglich für die Herstellung von Gleichwertigkeit von beruflicher Bildung und Allgemeinbildung an, da zu diesem Zeitpunkt bereits viele Bewerber_innen für eine Ausbildung einen MSA besaßen (DGB 1992).

Die KMK befasste sich somit auch mit dem Thema der Gleichwertigkeit von allgemeiner und beruflicher Bildung sowie mit den Fragen nach Studierfähigkeit und der Qualifikationsgrundlage für ein Hochschulstudium (KMK 1994). So wiesen die Kultusminister_innen darauf hin, dass es bereits einige Beschlüsse zu Durchlässigkeitsregelungen von der KMK gebe, aber die Möglichkeiten in diesem Bereich noch nicht ausgeschöpft seien (KMK 1994). Bevor aber Weiteres beschlossen werden könne, bedürfe es einer genaueren Untersuchung des Themas durch die KMK selbst.

Die zweite Hälfte des ersten Untersuchungszeitraums war dadurch geprägt, dass die Forderungen vor allem nach dem Hochschulzugang beruflich Qualifizierter in den Grundsatzprogrammen der Gewerkschaften sowie im Berufsbildungsbericht der Regierung kontinuierlich aufrechterhalten wurden, wobei insbesondere die Länder als die Akteure angesprochen wurden, die sich mit diesem

106 Allein die Voraussetzung des Erreichens eines Notendurchschnitts von 3,0 wurde vom Bildungsministerium als Einschränkung der Gleichwertigkeit kritisiert. 
Problem zu beschäftigen hätten. Die Länder wiederum beschlossen zwar keine Harmonisierung der Möglichkeiten des Hochschulzugangs ohne schulische Berechtigung, jedoch entschieden sie 1997, um die Gleichwertigkeit von beruflicher und Allgemeinbildung voranzubringen, dass unter bestimmten Bedingungen, d.h., wenn ein bestimmtes Maß an allgemeinbildenden Fachstunden nachgewiesen wird, auch in der beruflichen Bildung eine Fachhochschulreife erworben werden kann (KMK 1997b). Inwiefern dieser Beschluss wirklich als ein Schritt zu größerer Gleichwertigkeit angesehen werden könne, wurde jedoch vor allem von den Gewerkschaften hinterfragt, weil mit den angegebenen Kriterien der Hochschulzugang de facto wieder über das Allgemeinbildungsniveau erfolgt (DGB 1998c).

Festzustellen ist, dass die Debatte um Durchlässigkeit an bereits bestehende Diskussionen in der Bundesrepublik anknüpfte. Dass zu Beginn des Untersuchungszeitraums die deutsche Wiedervereinigung stattfand und mit den neuen Bundesländern auch deutlich andere Traditionen, was Durchlässigkeit angeht, dazugekommen sind (vgl. Kapitel 6), wurde in den Aussagen der untersuchten Akteure so gut wie nicht sichtbar. Allein 1991 wurden doppeltqualifizierende Bildungsgänge, wie das in der DDR erwerbbare Abitur mit Berufsausbildung, thematisiert, wobei der DGB (1991) diese Qualifikationsform erhalten und das BMBW (1991) sie, deutlich zurückhaltender, in ihre Überlegungen zu Gleichwertigkeit einbeziehen wollte. Weiteres wurde diesbezüglich aber nicht unternommen.

Die Debatte um Durchlässigkeit, die im ersten Untersuchungszeitraum vor allem als Diskussionen um Gleichwertigkeit von beruflicher und Allgemeinbildung geführt wurde, ging am Ende des Untersuchungszeitraums zwar weiter, aber nicht mit solch einer Dynamik wie zu Beginn. Die Bedeutung des Themas ließ aber nicht nach, was sich auch darin widerspiegelt, dass Gleichwertigkeit sogar im Koalitionsvertrag der neuen Regierung 1998 als zu erreichendes Ziel aufgenommen wurde (Sozialdemokratische Partei Deutschlands/Bündnis 90 Die GRÜNEN 1998). In Abbildung 4 wird die gerade zusammengefasste Debatte im Kontext der nationalen und europäischen Politiken chronologisch dargestellt, um einen Überblick über die wichtigsten Veränderungen zu bekommen. 


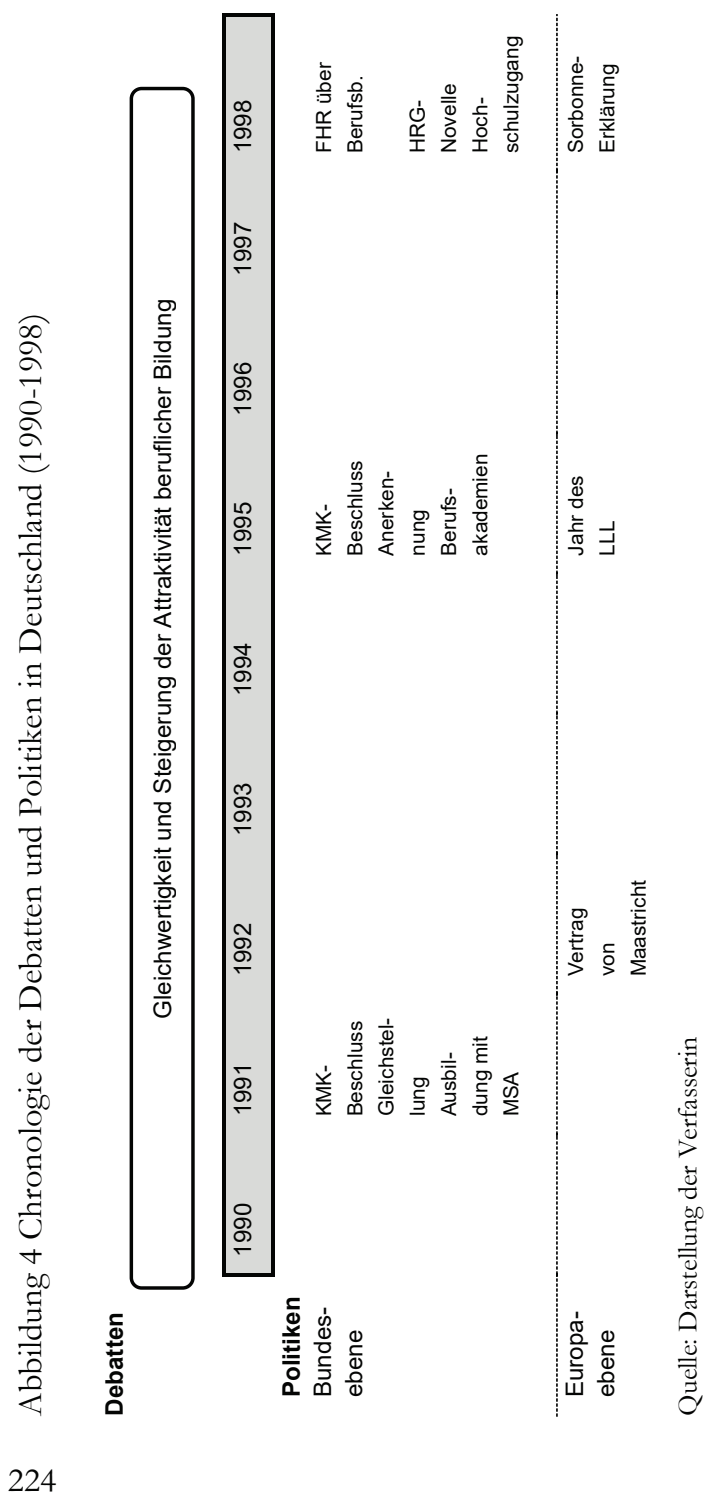




\subsubsection{Deutsche Diskurse 1990-1998: Durchlässigkeit als Diskurs über Zugang und Gleichwertigkeit}

Aus den Aussagen, die im Rahmen der dargestellten Debatte formuliert wurden, sowie aus den wenigen zusätzlichen einzelnen Aussageereignissen (vgl. Kapitel 4) in den 1990er Jahren, die Fragen von Durchlässigkeit thematisierten, wurden zweunterschiedliche Diskurse identifiziert, die sich antagonistisch gegenüberstehen: einen strukturkritischen Durchlässigkeitsdiskurs, der auf eine stärkere Gleichstellung der beruflichen Bildung in der Gesellschaft und damit auch einer größeren Durchlässigkeit zielt sowie einen strukturkonservativen Durchlässigkeitsdiskurs, in dem die Vorrangstellung der höheren Allgemeinbildung reproduziert und Durchlässigkeit von der Berufs- zur Hochschulbildung kritisch betrachtet wird. Es wird also deutlich, dass im ersten Untersuchungszeitraum diskursive Deutungskämpfe um Durchlässigkeit in Deutschland stattfanden.

Im Folgenden soll nun die Struktur der Diskurse (vgl. Kapitel 4) genauer dargestellt werden. Dabei soll noch mal darauf hingewiesen werden, dass die Diskurse als Modellierung der gefundenen Typiken verstanden werden sollen. Es handelt sich demnach um eine Abstraktion vom Ausgangsmaterial und stellt nicht den Forschungsvollzug, sondern das Ergebnis desselben dar, in dem das Material zu Diskursen verdichtet wurde (vgl. Keller 2008). Die Darstellung der Diskursstrukturen erfolgt dabei für den ersten Untersuchungszeitraum im Querschnitt, da keine größeren Veränderungen der Diskurse für diesen Zeitverlauf festzustellen sind. Sind Diskursentwicklungen festzustellen, werden diese hervorgehoben.

Die Darstellung der Diskurse soll in drei Schritten erfolgen. Erstens werden die in den Diskursen dominierenden Argumentationsmuster dargestellt, da diese die Logik der restlichen Strukturmerkmale der Diskurse bestimmen. Zweitens wird die jeweilige spezifische Struktur der Diskursstränge verglichen. Dabei geht es vor allem um das Verständnis von Durchlässigkeit, was auch in den Lösungsvorschlägen sichtbar wird. Das heißt, wie soll Durchlässigkeit erreicht werden? Welche Aspekte von Durchlässigkeit spiegeln sich in den Aussagen zu Durchlässigkeit wider? Für wen soll Durchlässigkeit geschaffen werden? Um die Spezifika der identifizierten Diskurse und Diskursstränge besser herauszuarbeiten, werden diese einander gegenübergestellt. Für die Darstellung nutze ich das in Kapitel 2 entwickelte heuristische Konzept von Durchlässigkeit, um entsprechend den einzelnen Aspekten das jeweilige Verständnis darzustellen. ${ }^{107}$

Drittens sollen auf Basis der dargestellten Diskursstrukturen die sichtbar gewordenen Strategien der Diskursträger dargelegt werden. In diesem Schritt sollen auch noch einmal stärker die Akteurspositionen verortet werden. An dieser Stelle

107 Hierbei ist anzumerken, dass die Logik der Darstellung nicht der reinen Forschungslogik entspricht. Sie dient stattdessen einer besseren Systematisierung. Wie in Kapitel 5 beschrieben, wurden im Forschungsprozess nicht deduktiv die Aspekte anhand von vorgefertigten Kategorien analysiert, sondern er wurde angelehnt an das Vorgehen der GTM (vgl. Kapitel 4.). 
soll darauf hingewiesen werden, dass Diskurse nicht als Meinungen einzelner Akteure gefasst werden können, sondern übergreifend in den Aussagen rekonstruiert werden. Deshalb ist eine eindeutige Zuordnung der untersuchten Akteure zu Diskursen nicht möglich. Die Diskurse sind gekennzeichnet durch eine jeweils spezifische Logik, die die Gründe, die Problematisierung von Durchlässigkeit, die Behandlung des Themas, die Lösungsvorschläge und schließlich auch die Strategien mit strukturiert.

\section{Argumentationslogiken der Diskurse}

Nachfolgend werden die in den Diskursen rekonstruierten Argumentationslogiken dargestellt. Argumentationslogiken sind dabei zentrale Faktoren für die Strukturierung der Diskurse. Sie entsprechen dem roten Faden, der die anscheinend willkürlichen Äußerungen in einen spezifischen Zusammenhang stellt und verbindet. Aus diesem Grund sind es auch die Argumentationslogiken, die die Grundlage für die Unterscheidung einzelner Diskursstränge bilden. In den beiden für Deutschland herausgearbeiteten Diskursen konnten drei vorherrschende Diskursstränge, die jeweils einer bestimmten Argumentationslogik folgen, herausgearbeitet werden. Dabei teilt sich der strukturkritische Diskurs in einen universalistischen und einen funktionalistischen Diskursstrang, und der strukturkonservative Diskurs wird durch einen funktionalistischen Diskursstrang bestimmt. Diese drei unterschiedlichen Argumentationslogiken werden getrennt voneinander behandelt. In ihnen wird deutlich, warum und in welchen Zusammenhängen Durchlässigkeit und damit auch das Verhältnis von Berufs- zu Hochschulbildung thematisiert wird und was eigentlich das wahrgenommene Problem im Hinblick auf Durchlässigkeit ist. Zuerst sollen die Argumentationslogiken des strukturkritischen und anschließend die des strukturkonservativen Diskurses vorgestellt werden. Im Anschluss an die Beschreibung der Logiken werden diese in Tabelle 10 zusammengefasst dargestellt.

\section{Strukturkritisch-funktionalistischer Diskursstrang (1990-1998): Durchläs- sigkeit als Antwort aufden Strukturwandel und das Attraktivitätsproblem}

Im Rahmen des strukturkritischen Diskurses ist die funktionalistische Argumentationslogik die klar dominierende. Der strukturkritisch-funktionalistische Diskursstrang wird daher auch von allen untersuchten Akteuren mehr oder weniger stark getragen.

Auf gesellschaftlicher Ebene werden drei größerer Probleme angesprochen, die durch eine vergrößerte Durchlässigkeit zwischen Berufs- und Hochschulbildung verringert werden können. Erstens soll Durchlässigkeit zu einer Stärkung der Attraktivität der beruflichen Bildung führen. Zweitens ist aufgrund des strukturellen Wandels ein Bedarf der Bevölkerung an Höherqualifizierung festzustellen, auf den auch im Bildungssystem reagiert werden müsse. Und drittens sind gut 
ausgebildete Individuen die Voraussetzung, dass Deutschland weiterhin wettbewerbsfähig bleibt.

Der erste Grund ist der dominierende im Diskurs. So wird festgestellt, dass die fehlende Attraktivität der beruflichen Bildung und damit einhergehend ihre fehlende Wertschätzung in der Gesellschaft zu dem Problem des Facharbeitermangels führe, welcher gesellschaftlich und wirtschaftlich als schädlich eingestuft wurde (BMBW 1992; DIHT 1992a). ${ }^{108}$ Da berufliche Bildung in der Gesellschaft vielfach als ,Sackgasse“ in Bezug auf Karriere- und Entwicklungsmöglichkeiten angesehen werde, seien Reformen der Bildungs- und Beschäftigungsstrukturen notwendig, um zu einer Aufwertung der beruflichen Bildung beizutragen und Eltern und Jugendliche bei der Bildungswahl wieder für den beruflichen Weg zu interessieren. Eine verstärkte Durchlässigkeit von der Berufsbildung zur Hochschulbildung wäre ein Weg, diesen ,immer einseitigeren Zug über das Abitur ins Studium“ (DIHT 1992a) zu stoppen, die Attraktivität der Berufsbildung zu erhöhen und mehr Facharbeiter auszubilden.

Das Problem, das wir mit der Gleichwertigkeit haben und das den Ausgangspunkt auch für Bildungswahlentscheidungen bildet, ist, daß viele Eltern und viele Jugendliche berufliche Bildungsgänge als Sackgasse, Einbahnstraße sehen, die, wenn man sie mal beschritten hat, so leicht nicht wieder verlassen werden kann. Es kommt deshalb darauf an - und darin besteht auch unser Ziel -, die berufliche Bildung und die Berufsperspektiven so attraktiv zu machen, daß der Wettbewerb der verschiedenen Bildungswege untereinander auch tatsächlich stattfinden kann und nicht von vornherein durch die unterschiedlichen Vorausbedingungen nur in verzerrter Form möglich ist. (BMBW 1992: 69)

Eine Ursache für diese (Fehl-)Entwicklung wird teilweise in der ausbleibenden Leistungsauswahl im Schulsystem gesehen, die dazu führe, dass eine immer gröBere Anzahl von Schüler_innen das Gymnasium besucht und mit einem Abitur abschließt, aber tatsächlich nicht mit den Anforderungen im Hochschulstudium oder später auf dem Arbeitsmarkt zurechtkommt (DIHT 1992a).

Je mehr wir eine leistungs- und begabungsorientierte Auswahl an das Ende von Bildungswegen verlagern oder sogar auf Abschlußprüfungen verzichten, umso härter wird der Schock beim Übergang in das Arbeitsleben. (DIHT 1992a)

Ziel sei es, wieder eine größere Gruppe von Jugendlichen für eine berufliche Bildung zu gewinnen, da ausgebildete Facharbeiter als „Rückgrat“ der Unternehmen gelten (BDI et al. 1993) und nicht von Hochschulabsolvent_innen ersetzt werden können.

Des Weiteren wird aber auch der generelle gewachsene gesellschaftliche Bedarf an höher Qualifizierten, der sich durch den Strukturwandel in Produktion

108 Zur Veranschaulichung und zum Nachweis der Diskurse werden repräsentative Quellen und Zitate angegeben. Dabei ist $\mathrm{zu}$ beachten, dass diese Quellen nicht die einzigen im Untersuchungszeitraum sein müssen, die das Geschriebene belegen. Vielmehr stellt die Beschreibung der Diskursstruktur eine Modellierung typischer Strukturen und Verständnisse dar, die aus allen im Anhang identifizierten Quellen herausgearbeitet wurden. 
und Beschäftigung, aber auch im technologischen Wandel, dem Wandel in der Betriebsorganisation ergibt, festgestellt (BMBW 1991, 1992; DGB 1992; KMK 1997a). Schließlich sollte die Höherqualifikation auch zur Sicherung des Standortwettbewerbs beitragen. So unterstreichen z.B. die Kammern einen wachsenden Bedarf an Hochschulabsolvent_innen für Positionen in den Führungsetagen der Unternehmen, die nicht mehr angemessen über betriebliche Weiterbildung besetzt werden könnten, und fordern damit einen erleichterten Hochschulzugang für beruflich Qualifizierte (DIHT 1990). Zudem bestehe eine Notwendigkeit zur Höherqualifizierung in der Gesellschaft, da das Wirtschaftswachstum von modernen Gesellschaften eng an das Qualifikationsniveau der Bevölkerung gekoppelt sei (HRK 1992b). Insgesamt seien daher sowohl die Höherqualifizierung über ein Studium als auch der Rückgewinn von Interessenten für eine Facharbeiterausbildung Grundlagen für eine gute Position Deutschlands im globalen und innereuropäischen Wettbewerb (DIHT 1990).

Aber nicht nur gesellschaftlich, sondern auch für das Individuum kann der Zugang zur Hochschule große Vorteile bringen, denn Arbeitslosigkeitsrisiko, Karriere- und Entwicklungschancen seien eng verbunden mit dem individuellen Qualifikationsniveau (HRK 1992b).

\section{Strukturkritisch-universalistischer Diskursstrang (1990-1998): Recht aufChancengleichheit und Gleichstellung von Berufs-und höherer Allgemeinbildung}

Im strukturkritischen Diskurs wurden aber nicht allein Argumente funktionaler Natur als Begründung von Durchlässigkeit angebracht, sondern auch solche, die in dieser Arbeit als universalistisch gelten sollen. In Anlehnung an den egalitären Universalismus der Menschenrechte steht in dieser Argumentationslogik das gleiche Recht des Individuums auf (Weiter-)Bildung im Mittelpunkt.

Um das Recht auf Bildung zu verwirklichen, reicht es nicht aus, die Infrastruktur für das Pflichtschulsystem bereitzustellen. Vorschulische Bildung, berufliche Erstausbildung und hochschulische Ausbildung, berufliche, kulturelle und politische Weiterbildung unterliegen ebenfalls der öffentlichen Verantwortung. (DGB 1998b: 11)

Der Geltungsanspruch ist dabei nicht auf partikulare Bevölkerungsgruppen, die spezifische Vorteile wie ein Hochschulstudium genießen dürfen, begrenzt, sondern zielt stattdessen auf die Inklusion aller, insbesondere auch der bisher Ausgeschlossenen. Es geht dann um die gleichen Rechte und Chancen für alle.

Entsprechend dieser Definition, was unter universalistisch gefasst werden kann, wird im Diskursstrang auf gesellschaftlicher Ebene erstens die Notwendigkeit durchlässiger Bildungsstrukturen über das Ideal der Chancengleichheit begründet, zweitens das Ziel, Bildung als Recht und öffentliches Gut zu institutionalisieren, formuliert und drittens gefordert, dass über Durchlässigkeit auch die Gleichwertigkeit der beruflichen Bildung als andersartiger, aber gleichwertiger Bil- 
dungsweg anerkannt wird. Dieser gleiche Wert der beruflichen Bildung solle daher auch stärker bei Fragen des Hochschulzugangs berücksichtig werden (BMBW 1992).

In Bezug auf Chancengleichheit kann im universalistischen Diskursstrang unterschieden werden zwischen einer progressiven und einer eher konservativen Sichtweise. Von stärker konservativer Seite wird Chancengleichheit in den individuellen Entwicklungsmöglichkeiten für beruflich Qualifizierte (auch Fortgebildete) und Absolvent_innen der Sekundarstufe II der höheren Allgemeinbildung gefordert (BMBW 1992). Aus progressiver Sicht wird dagegen Chancengleichheit für beruflich Qualifizierte und Hochschulabsolvent_innen gefordert (DGB 1995).

Die Attraktivität der beruflichen Bildung muß gestärkt und die Entwicklungschancen ihrer Absolventen verbessert werden, damit sie zu einer echten Alternative zum Hochschulstudium wird [...]. Solange ein Hochschulexamen bessere Beschäftigungschancen, höhere Einkünfte und größeres Prestige ermöglicht, kann von einem Durchbruch des Gleichwertigkeitsprinzips keine Rede sein. (DGB 1995)

Von den Progressiven wird also eine Gleichwertigkeit von Berufs- und Hochschulbildung konstruiert, während bei der konservativen Variante eine Gleichwertigkeit von Berufsbildung und allgemeinbildender Gymnasialstufe besteht und somit indirekt eine Hierarchisierung zwischen Berufs- und Hochschulbildung stattfindet. Für den Diskurszeitraum war diese Position aber noch immer als strukturkritisch einzuschätzen, da auch diese Form der Gleichstellung nicht institutionalisiert war.

Aber auch im Schulsystem wird eine Ursache für die Bildungswahl vieler Schüler_innen gesehen. So wird die zu frühe Selektion in unterschiedliche Bildungswege im deutschen Schulsystem als ein Problem und damit auch als Lösungsmöglichkeit erkannt.

Die Umsetzung von Gleichwertigkeit zwischen beruflicher und allgemeiner Bildung wird davon abhängen, inwieweit es uns gelingt, unter bildungsstrukturellen Aspekten die Optionen für mehr Durchlässigkeit, Flexibilität und Chancengleichheit im gesamten Bildungssystem zu verstärken, um Entscheidungen über den Berufs- und Lebensweg nicht im Wesentlichen schon zum Ende der Grundschulzeit festlegen zu müssen oder jedenfalls zu glauben, das tun zu müssen. (BMBW 1992: 66)

Für die Individualebene wird im universalistischen Argumentationsmuster geschlussfolgert, dass durchlässige Bildungsstrukturen und somit die Ermöglichung der Bildungsteilnahme zu einer Stärkung der individuellen gesellschaftlicher Teilhabe und Persönlichkeitsentfaltung führe (DGB 1998b). Zudem solle sichergestellt werden, dass nicht die reinen Bildungszertifikate, sondern die tatsächlichen Kompetenzen, die auf unterschiedlichsten Wegen, also z.B. per Studium oder beruflicher Bildung, erlangt werden können, die individuellen Entwicklungsmöglichkeiten bestimmen. Hier erfolgt eine Absage an den in der Gesellschaft vorherrschenden Kredentialismus (DGB 1992) und eine deutliche Lernergebnisorientierung. 
Ich sage ebenso deutlich, daß Berufstätige mit einer qualifizierten Berufsausbildung auch mehr Chancen des Aufstiegs in der privaten Wirtschaft und im öffentlichen Dienst erhalten müssen und nicht wegen fehlender formaler Bildungszertifikate Benachteiligungen erfahren. (DGB 1992: 250)

\section{Strukturkonservativ-funktionalistischer Diskursstrang (1990-1998): Durchlässigkeit als Überlastung und Primat des Abiturs}

Im strukturkonservativen Diskurs findet eine Beschäftigung mit der Frage der Durchlässigkeit vor allem deshalb statt, weil entsprechende Forderungen im Rahmen der deutschen Bildungsdebatten deutlich werden und somit an die Träger des Diskurses herangetragen werden. So ist auch die Plenardebatte der HRK 1991 ein Zeichen dafür, da in dieser allein die Relevanz der Frage des Hochschulzugangs für beruflich Qualifizierte ohne schulische Berechtigung diskutiert wurde. Dabei wurde deutlich, dass beruflich Qualifizierte nicht als natürliche Zielgruppe für die Hochschule betrachtet werden.

Dazu stellten sie eher ein Problem dar, denn sie würden die bereits seit den 1970er Jahren bestehende Überlast der Hochschulen noch verstärken. Hochschulen seien nicht entsprechend ausgestattet, um noch eine Studierendengruppe aufzunehmen (HRK 1991). ${ }^{109}$ Beruflich Qualifizierte werden somit nicht als relevante und legitime Zielgruppe anerkannt, sondern als Gefahr für das Funktionieren des Hochschulsystems konstruiert.

Durchlässigkeit ist nicht nur eine Gefahr für die Hochschulen. Zusätzlich sei auch das „,für Berufstätige besonders schwerwiegende Risiko des Scheiterns beim Wechsel von einem ausgeübten Beruf in die Hochschule“ (HRK 1994) zu bedenken. Insofern sind restriktive Durchlässigkeitsregelungen auch als Schutz für diese Individuen selbst zu sehen.

Die mangelnde Attraktivität der beruflichen Bildung, die im strukturkritischen Diskurs als ein Hauptargument für Durchlässigkeitsforderungen formuliert wird, könne aber nicht als Grundlage für die Öffnung der Hochschulen dienen. Dieses Problem müsse von Vertretern der beruflichen Bildung selbst gelöst werden (HRK 1992b). So sei es deren Aufgabe, in Wirtschaft, Verwaltung und öffentlichem Dienst, vergleichbar qualifizierten Absolvent_innen beider Bildungswege gleiche reale Möglichkeiten für die Beschäftigung, Bezahlung, Weiterqualifizierung und Beförderung zu geben." (KMK 1994)

109 Allerdings wurde für eine bestimmte Gruppe der Anspruch auf einen Hochschulzugang als legitim anerkannt, und zwar für diejenigen, die durch das politische System der DDR und die darin stattfindende politisch bestimmte Kontingentierung der Studienplätze benachteiligt wurden (HRK 1991). 
Tabelle 10 Argumentationslogiken der deutschen Diskurse (1990-1998)

\begin{tabular}{|c|c|c|}
\hline \multicolumn{2}{|c|}{ Strukturkritisch } & \multirow{2}{*}{$\begin{array}{l}\text { Strukturkonservativ } \\
\text { Funktionalistisch }\end{array}$} \\
\hline Universalistisch & Funktionalistisch & \\
\hline $\begin{array}{l}\text { Gesellschaft: } \\
\text { Chancengleichheit als gesell- } \\
\text { schaftliche Norm } \\
\text { Bildung als öffentliches Gut } \\
\text { und Recht } \\
\text { Gleichwertigkeit beruflicher } \\
\text { Bildung }\end{array}$ & $\begin{array}{l}\text { Gesellschaft: } \\
\text { Mangel an Facharbeitern durch } \\
\text { mangelnde Attraktivität der be- } \\
\text { ruflichen Bildung („Sackgas- } \\
\text { sensyndrom“) } \\
\text { Bedarf an Höherqualifizierung } \\
\text { durch strukturellen Wandel der } \\
\text { Wirtschaft } \\
\text { Standortwettbewerb - Globali- } \\
\text { sierung }\end{array}$ & $\begin{array}{l}\text { Gesellschaft: } \\
\text { Durchlässigkeit als Bedrohung } \\
\text { der Qualität der Hochschulbil- } \\
\text { dung durch Überlast } \\
\text { Durchlässigkeit kein Weg zur } \\
\text { Steigerung der Attraktivität der } \\
\text { beruflichen Bildung }\end{array}$ \\
\hline $\begin{array}{l}\text { Individuum: } \\
\text { Recht auf (Weiter-)Bildung zur } \\
\text { Erlangung gesellschaftlicher } \\
\text { Teilhabe und Persönlichkeits- } \\
\text { entfaltung } \\
\text { Sicherung gleichwertiger beruf- } \\
\text { licher Entwicklungsmöglichkei- } \\
\text { ten auf Basis tatsächlicher } \\
\text { Kompetenzen, unabhängig von } \\
\text { Zertifikaten }\end{array}$ & $\begin{array}{l}\text { Individuum: } \\
\text { Durchlässigkeit als Möglichkeit } \\
\text { individueller Karrieremöglich- } \\
\text { keiten } \\
\text { Verringerung des individuellen } \\
\text { Arbeitslosigkeitsrisikos }\end{array}$ & $\begin{array}{l}\text { Individuum: } \\
\text { Schutz vor individuellem } \\
\text { Versagen }\end{array}$ \\
\hline
\end{tabular}

Quelle: Darstellung der Verfasserin

Gleichwertigkeit von beruflicher und höherer Allgemeinbildung als ein weiteres Argument des strukturkritischen Diskurses wird im strukturkonservativen Diskurs nicht mit Fragen der Durchlässigkeit verbunden und auch nicht als Norm, die erreicht werden müsse, formuliert.

\section{Strukturmerkmale der deutschen Diskurse (1990-1998)}

Wie und für wen soll nun Durchlässigkeit erreicht werden? Welche Aspekte von Durchlässigkeit stehen im Vordergrund? Im nächsten Abschnitt werden die typischen Deutungsangebote für die Diskursstränge, d.h. die Lösungsmöglichkeiten des Durchlässigkeitsproblems, die Zielgruppe, für die Durchlässigkeit geschafffen werden soll, und auch die konstruierten Subjektpositionen verglichen. Auf diese Weise werden das Verständnis von Durchlässigkeit in den beiden deutschen Diskursen und die Deutungskämpfe zwischen diesen sichtbar.

Nachfolgend werden die typischen Positionen zu den Durchlässigkeitsaspekten, die entsprechend den Argumentationslogiken differieren, als Elemente der jeweiligen Diskursstrukturen verglichen und schließlich jeweils in einer Überblickstabelle zusammengefasst. Diese Diskursstrukturen stellen wiederum einen Querschnitt durch den ersten Untersuchungszeitraum dar. Dies ist zulässig, da sich keine großen Änderungen im Zeitverlauf gezeigt haben. 


\title{
Zugang
}

Wenn Durchlässigkeit thematisiert wurde, diskutierten die Akteure im ersten Zeitraum primär über Fragen des Hochschulzugangs beruflich Qualifizierter. Die Hauptforderung im Rahmen des strukturkritischen Diskurses, sowohl im universalistischen als auch im funktionalistischen Diskursstrang, lautete, das bestehende Monopol der höheren Allgemeinbildung über das Berechtigungswesen für den Hochschulzugang aufzubrechen. Der Hochschulzugang solle auch über berufliche Qualifizierungswege möglich sein. So müsse der gleiche Wert der beruflichen Bildung bei Fragen des Hochschulzugangs stärker berücksichtig werden. Es sei nicht mehr nur die Allgemeinbildung mit ihrem gymnasialen Fächerkanon, die auf die Hochschule vorbereiten könne (DGB 1996; BMBW 1992; DIHT 1990). Hier wird deutlich, dass ein Gegenentwurf zur bis dahin geltenden hegemonialen Deutung, dass insbesondere die gymnasiale Bildung auf die Hochschule vorbereitet (vgl. Wolter 1994), konstruiert wird. Die Selbstverständlichkeit der Hegemonie der Allgemeinbildung für den Hochschulzugang wird hinterfragt.

\begin{abstract}
Unsere Überlegungen zur Umsetzung der Gleichwertigkeit stützen sich auf die These der Gleichwertigkeit der bildenden Qualität sowie des Anforderungs- und des Lernniveaus beruflicher Bildung im Vergleich zur allgemeinen Bildung. Das bedeutet, daß berufspraktische wie fachtheoretische Elemente der dualen Ausbildung ihren Anforderungen entsprechend den klassischen Inhalten des Fächerkanons allgemeinbildender Schulen vergleichbar sind. Vergleichbar heißt aber nicht gleichartig, sondern gleichwertig. (BMBW 1992)
\end{abstract}

Im strukturkonservativen Diskurs dagegen wurde das bestehende Berechtigungswesen als funktionierend und notwendig verteidigt. Der Hochschulzugang solle damit primär über den Weg der höheren Allgemeinbildung erworben werden (KMK 1994).

Deutlich ist in allen Diskursen, dass nur der Weg aus der Berufs- in die Hochschulbildung (aber nicht umgekehrt) thematisiert wird. Dass die berufliche Bildung auch für Individuen mit akademischem Abschluss interessante Weiterentwicklungsmöglichkeiten bieten könne, liegt im Bereich des Unsag- und Undenkbaren. Dieser eindimensionale Blick auf Durchlässigkeit zwischen Berufs- und Hochschulbildung zeigt eindrücklich die bestehenden Hierarchien zwischen den beiden Bildungsbereichen in der Gesellschaft.

Doch wie genau soll gemäß den einzelnen identifizierten Diskurssträngen der Hochschulzugang erfolgen?

\section{Zugang über Berechtigungen in der beruflichen Bildung}

Im universalistischen Strang des strukturkritischen Diskurses wird vor allem die Einführung von Berechtigungen gefordert, die über die berufliche Bildung erworben werden könnte (DGB 1992, 1998c; BMBW 1993b). Nur auf diese Weise sei die Gleichwertigkeit zwischen Berufs- und Hochschulbildung gewährleistet. 
Dieses Monopol der Berechtigungen liegt heute bei den zuständigen Stellen für das Schul- und Hochschulwesen. Hier ist - das war mir sehr einleuchtend - unter dem Begriff der Gleichwertigkeit und der Gleichbehandlung [...] zum Ausdruck gebracht worden, daß dieses Monopol so nicht weiterbestehen kann. Auf der Grundlage von Berufsbildungsgesetz und Handwerksordnung erworbene Qualifikationen müßten hier auch in ihrer Berechtigung gesehen werden. (BMBW 1993b: 80)

Aus einer eher progressiven Perspektive solle die berufliche Erstausbildung mit dem Abitur gleichgestellt werden und zum Zugang zu einer Hochschule berechtigen. So sollen Absolvent_innen einer Berufsausbildung, die drei Jahre Berufserfahrung gesammelt haben, auch ohne weitere Verfahren Zugang zur Hochschule erhalten dürfen (DGB 1998c). Im Gegensatz dazu wird aus einer eher konservativen Sicht zwar kein expliziter Zugang für Absolvent_innen einer Erstausbildung gefordert, wohl aber die Möglichkeit des Erwerbs der fachgebundenen Hochschulreife oder der Fachhochschulreife via Aufstiegsfortbildungen oder erworbenen Zusatzqualifikationen (BMBF 1997b). Während also einerseits ein besserer Zugang nur für die beruflich Qualifizierten, die sich bereits erfolgreich weitergebildet haben, gefordert wird, wird andererseits eine Gleichwertigkeit von beruflicher Erstausbildung und Abitur konstruiert. Dagegen sei eine Gleichstellung der beruflichen Ausbildung mit dem mittleren Schulabschluss nicht weitgehend genug, da viele Auszubildenden diesen Abschuss bereits erworben haben. Vielmehr könne durch diese Gleichstellung die Ungleichwertigkeit zementiert und der Weg zur Hochschule nicht eröffnet, sondern eher versperrt werden (DGB 1992).

Im strukturkonservativen Diskurs wird eine Gleichstellung von Abitur und Berufsabschluss weitgehend abgelehnt. Die Entscheidung über die Aufnahme eines Studiums solle bei den Akteuren der höheren Bildung liegen. Zwar weist die KMK (1997a) darauf hin, dass mit dem gestiegenen Anforderungsniveau, dem Upskilling (vgl. Protsch 2014 zum Konzept des Upskilling), in der beruflichen Bildung eine Gleichstellung im Hinblick auf Berechtigungen denkbar wäre. Diese Ansicht wurde aber nicht von allen Ländern (z.B. nicht von Bayern) geteilt, so dass auch innerhalb der KMK (1997a) Konflikte über die Gleichwertigkeit deutlich wurden, die das Ausstellen von Hochschulzugangsberechtigungen über die berufliche Bildung verhindern. Aber nicht an allen Lernorten könnten die für ein Hochschulstudium notwendigen Kompetenzen gleichermaßen erworben werden, insofern wäre es eine politische Frage festzulegen, wann und wo dies der Fall sei.

Schlüsselqualifikationen, wie sie die Grundlage der neugeordneten Berufe bilden, sind geeignet, Studierfähigkeit zu begünstigen; sie sind für alle Bildungsgänge von entscheidender Bedeutung. [...] Nicht alle Fachgebiete sind dabei gegenseitig substituierbar. Für bestimmte Qualifikationen gilt, daß sie nur an bestimmten Gegenstandsbereichen erworben werden können. Gleichzeitig ist zu beachten, daß zwischen der Entwicklung von Schlüsselqualifikationen und den Organisationsformen des Lernens ein enger Zusammenhang besteht. Die hierfür notwendigen Auswahlentscheidungen sind auf politischer Ebene zu treffen. (KMK 1994) 
Es ginge demnach nicht um die Frage der Gleichwertigkeit der tatsächlich erworbenen Kompetenzen in der beruflichen Bildung im Vergleich der Allgemeinbildung, sondern um politisch ausgetragene ideologische Kämpfe, wobei die Entscheidungskompetenz über Berechtigungen ganz klar bei der KMK läge.

Ein weiteres Problem mit generellen Berechtigungen für Abschlüsse der beruflichen Bildung wird in der fehlenden Differenzierung der beruflichen Bildung gesehen.

In der Struktur des Bildungs- und Ausbildungssystems wird das duale System der Berufsausbildung als Einheit betrachtet. Formal gibt es keine Differenzierung weder bei der Zulassung zur Berufsausbildung noch bei der Zuerkennung von Berechtigungen in Verbindung mit dem Berufsabschluß. Auch die Ausbildungsdauer führt nicht zu Niveaudifferenzierungen. [...] Diese Ausgangslage erschwert generelle Gleichwertigkeitsregelungen im Bereich der dualen Ausbildung. (KMK 1997a: 8)

Eine Möglichkeit, eine Differenzierung vorzunehmen, wäre die Einführung von standardisierten Abschlussprüfungen, um die unterschiedlichen Niveaus in der beruflichen Ausbildung abzubilden. Deutlich wird demnach die Einschätzung, dass die unterschiedlichen Arten der beruflichen Bildung unterschiedliche Niveaus hätten und damit unterschiedlich auf den Zugang zur Hochschule vorbereiten könnten. Da dies aber nicht in formalen Regelungen festgehalten sei, sei die Anerkennung von Gleichwertigkeit und damit auch die Vergabe von Berechtigungen schwierig.

Im funktionalistischen Diskursstrang des strukturkritischen Diskurses werden generelle Berechtigungen für die berufliche Bildung dagegen abgelehnt (BDI et al. 1993; DIHT 1992a). Vielmehr müsse getestet werden, ob die zukünftigen Studierenden auch die „Studierfähigkeit“ besitzen, um ein Studium erfolgreich zu absolvieren.

Wenn ich von Leistung spreche, gilt dies selbstverständlich auch für die Absolventen der beruflichen Bildung. Diese können und sollen davon nicht ausgenommen werden. Sie müssen sich einer Bewertung ihrer Studierfähigkeit an den Hochschulen unterziehen, d.h. konkret belegen, daß sie durch ihren beruflichen Bildungs- und Werdegang nebst ihrer Weiterbildung tatsächlich das erreicht haben, was man Studierfähigkeit nennt. (DIHT 1992a)

\section{Zugang über Eignungsprüfungen}

Ziel aus funktionalistischer Perspektive im strukturkritischen Diskurs ist es, denjenigen mit den größten Erfolgsaussichten den Studienzugang zu gewähren, unabhängig vom Ort ihrer Vorbildung. Auf diese Weise würde auch unnötigen Abbrüchen und damit Ressourcenverschwendung vorgebeugt werden (DIHT 1992a). Die Erfolgsaussichten werden dabei im Rahmen der Strukturkritik im funktionalistischen Diskursstrang von der „Studierfähigkeit“ der Individuen abhängig gemacht.

Studierfähigkeit ist im deutschen Durchlässigkeitsdiskurs ein zentrales Deutungsmuster, das sowohl im strukturkritischen als auch im -konservativen Diskurs 
verwendet wird. Dabei wird Studierfähigkeit als eine Kompetenz oder Fähigkeit konstruiert, die bereits vor dem Beginn des Studiums anhand von Eignungstests oder absolvierten Bildungswegen festgestellt werden könnte. Diese nachzuweisende Fähigkeit ist demnach als etwas konstruiert, das vom Individuum vor dem Studium ausgebildet wird oder eben nicht. Es handelt sich bei dieser Vorstellung somit um ein eher starres Konzept. Eine alternative Sicht wäre ein Konstrukt von Studierfähigkeit, das nicht statisch, sondern dynamisch funktioniert und sich erst beim Studium zeigt. Zulassungsbedingungen wie das Probestudium beruhen auf einem solchen Verständnis (vgl. Wolter 1994). So wird hier die Möglichkeit gesehen, dass die Individuen sich erst während des Studiums beweisen und auch erst durch das Studium die dafür notwendigen Fähigkeiten erlangen. Dieses alternative Verständnis wird im Diskurs nicht expliziert, findet sich aber stärker im universalistischen Diskursstrang wieder, da hier entsprechend dem Ideal des Rechts auf Bildung und der Annahme des gleichen bildenden Werts der Berufs- und Hochschulbildung allen der Beginn eines Studiums zugestanden wird.

Im funktionalistischen strukturkritischen Diskursstrang können dabei zwei Sichtweisen in Bezug auf die Zielgruppe der Eignungstests unterschieden werden. Eine erste, stärker konservative Sichtweise verlangt die Prüfung der Studierfähigkeit allein für beruflich Qualifizierte. Unterschieden wird dabei zwischen den beruflich Qualifizierten mit Aufstiegsfortbildungsabschluss, die eher ein Beratungsgespräch als eine Prüfung ablegen sollten, und denen mit beruflichem Bildungsabschluss ohne Weiterbildung. Letztere sollten ihre Fähigkeit via Prüfung zeigen können (BMBF 1995; KMK 1997a). Konservativ ist diese Zugangslösung insofern, als der Hochschulzugang über die Allgemeinbildung anerkannt wird, der Zugang über den beruflichen Bildungsweg aber zusätzlich abgesichert werden soll. Allein die Studierfähigkeit von beruflich Qualifizierten müsste demnach getestet werden.

Eine zweite Sichtweise innerhalb der funktionalistischen Strukturkritik wird im ersten Zeitraum von den Kammern vertreten. So wird die Einführung eines leistungsbezogenen Auswahltests an den Hochschulen gefordert. Dies aber nicht nur für berufliche Qualifizierte, sondern auch für Absolvent_innen der höheren Allgemeinbildung an den Schulen. Dies sei notwendig, da eine vorherige Leistungsselektion im Schulbereich offenbar nicht mehr ausreichend stattfinde und das Abitur somit nur noch als notwendige, aber nicht mehr hinreichende Voraussetzung für ein Studium angesehen wird. Zeichen der unzureichenden Vorbereitung via Abitur, der gesunkenen „Solidität“, seien die bestehenden „Drop-outs“ im Hochschulsystem, ,die Studienabbrecher, die Dauerwechsler bei Studienfächern, arbeitslose Hochschulabsolventen oder Beschäftigte weit unterhalb ihres Ausbildungsniveaus“ (DIHT 1992a).

Mit der Eingangsprüfung würde aber nicht allein eine fehlende vorherige Leistungsselektion korrigiert, sondern auch Einfluss genommen werden auf die Bildungswahl der Schüler_innen. Denn wenn der Hochschulzugang unabhängig vom Bildungsweg nur über die eine Eignungsprüfung erreicht werden können, 
wäre die Attraktivität der beruflichen Bildung im Vergleich zum Abitur nicht mehr geringer.

Wenn die Verantwortlichen der Politik nicht willens sind, Bildungsströme im allgemeinbildenden Schulwesen zu lenken, zu kanalisieren oder zu bremsen, ist es erst recht ihre Verpflichtung, auf anderen Wegen den Zug der Lemminge ins Studium zu bremsen. Eine Hochschuleingangsprüfung für alle erscheint mir dazu der einzige Weg, der Jugendlichen und Eltern frühzeitig das Risiko ihrer Bildungswahl verdeutlicht. (DIHT 1992a)

Gleichzeitig würde eine generelle Eignungsprüfung auch zu einer größeren Gleichwertigkeit beruflicher Bildung und der allgemeinbildenden Sekundarstufe II herstellen, da beide Bildungswege über eine „leistungsgerechte“ (DIHT 1990) Prüfung formal gleichgestellt wären.

In diesen Aussagen zur Hochschulzugangsprüfung und zur Notwendigkeit der Lenkung des Bildungswahlverhaltens durch frühe Selektion im Schulbereich werden zwei unterliegende Deutungen sichtbar. Zum einen ein Verständnis von Begabung, die als natürlich gegeben und zeitlich stabil und somit nicht veränderbar verstanden wird. Aufgrund dieser Annahme ist eine frühe Selektion im Bildungssystem eine Voraussetzung für die bestmögliche und differenzierte Förderung der Individuen mit unterschiedlichen Begabungsniveaus. Diese Annahme von Begabung spiegelt sich daher wiederum im verwendeten Konstrukt Studierfähigkeit wider, die ebenfalls durch Tests geprüft werden soll und als vorher feststellbar und nicht als dynamisches Konzept konstruiert wird. Zum anderen wird die funktionalistische Argumentation durch die meritokratische Grundannahme der leistungsgerechten Auswahl charakterisiert, die den begrenzten Zugang zu Positionen und Bildungschancen legitimiert.

Während im universalistischen Diskursstrang somit der Weg über Berechtigungen bevorzugt wird, soll im funktionalistischen strukturkritischen Diskursstrang der Zugang über Prüfungen ausgebaut und vereinheitlicht werden, was vor allem dem dritten Bildungsweg entspricht.

Im strukturkonservativen Diskurs wird der dritte Bildungsweg dagegen als gut ausgebaut bzw. als eine Form des Hochschulzugangs beschrieben, bei dem in den letzten Jahren viel passiert sei, so dass hier kein großer Handlungsbedarf bestehe (HRK 1994). Die Hochschulen positionierten sich ganz klar für den Hochschulzugang via Eignungsprüfung (nach dem Vorbild Niedersachsen), die sowohl aus einem allgemeinbildenden als auch einem fachlichen Prüfungsteil bestehen sollte. Die Aufgabe der Prüfung sei, „Anhaltspunkte für die Erfolgsaussichten eines Studiums“ (HRK 1994) zu liefern. Zudem sollte die Prüfung an der aufnehmenden Institution Hochschule stattfinden und von Prüfern aus der höheren Allgemeinbildung (Hochschule, gymnasiale Oberstufe, Erwachsenenbildung) abgenommen werden. Die Beteiligung von Verbänden, also Vertretern der beruflichen Bildung, wurde explizit abgelehnt (HRK 1992b). Zwar wurde von den Hochschulen 1992 anerkannt, dass mitunter auch beruflich Qualifizierte ohne schulische 
Berechtigung über die Voraussetzungen für ein Studium verfügen könnten, aber diese müssten noch durch eine Prüfung abgesichert werden.

Berufliche Ausbildung vermittelt in ihrer Eigenständigkeit Qualifikationen, die ganz anders geartet sind als die der allgemeinbildenden Schulen. Qualifizierte Berufstätigkeit vermittelt in manchen Bereichen jedoch Fähigkeiten und Fertigkeiten, die auch für ein Studium Voraussetzung sein können. (HRK 1992b)

Im strukturkonservativen Diskurs wurde damit wiederum der Zugang zur Hochschule mit dem Konstrukt der Studierfähigkeit verbunden, wobei diese als eine Kompetenz konstruiert ist, die stark auf Allgemeinbildung aufbaut und ausschließlich von Vertreter_innen der Allgemeinbildung geprüft werden könne (HRK 1992b, 1994; KMK 1997a).

\section{Zugang über Allgemeinbildung}

Die Bedeutung der Allgemeinbildung für die Studierfähigkeit wurde im strukturkonservativen Diskurs außerdem dadurch hervorgehoben, dass Studiereignung in Abhängigkeit von Kompetenzen in drei Schlüsselbereichen definiert wurde: sichere Kenntnisse der deutschen Sprache, fremdsprachliche Kompetenzen sowie gute Kenntnisse der Mathematik. So wurde auch von der KMK (1997b) der Erwerb der Fachhochschulreife über berufliche Bildung genau davon abhängig gemacht, ob die an den gymnasialen Curricula angelehnten Bedingungen in der beruflichen Bildung in Form einer Mindeststundenanzahl in diesen Fächern erfüllt seien. Die Fixierung auf Allgemeinbildung blieb demnach bestehen.

Die Kultusministerkonferenz hat festgestellt, daß im Hinblick auf die Studierfähigkeit der Absolventen der allgemeinbildenden Bildungsgänge und der Fachgymnasien/berufsbezogenen Bildungsgänge drei Kompetenzbereiche von herausragender Bedeutung sind: sprachliche Ausdrucksfähigkeit, insbesondere die schriftliche Darlegung eines konzisen Gedankengangs, verständiges Lesen komplexer fremdsprachlicher Sachtexte und sicherer Umgang mit mathematischen Symbolen und Modellen. Zur Sicherung des Erwerbs dieser grundlegenden Kompetenzen komme den Fächern Deutsch, Fremdsprache und Mathematik besondere Bedeutung zu. (KMK 1997a)

Während die Einführung dieser Regelung 1997 grundsätzlich den Erwerb von Berechtigungen für einen Fachhochschulzugang über berufliche Bildung ermöglichte, wurde, wie bereits dargestellt, die Frage des generellen Erwerbs von Berechtigungen zum Hochschulzugang über Berufsbildung kritisch betrachtet.

Ausgehend von dieser geteilten Annahme einer besonderen Bedeutung der Allgemeinbildung für die Studierfähigkeit wird auch der zweite Bildungsweg als ein wichtiges Mittel zur Schaffung von Durchlässigkeit im Bildungssystem gesehen, insbesondere im Hinblick auf den Hochschulzugang. So hätte dieser Bildungsweg für beruflich Qualifizierte auch den Vorteil, dass der Hochschulzugang nicht nur fachgebunden wäre. Zudem hätten Studierende des zweiten Bildungswegs durch ihre bereits bewiesene hohe Motivation erhöhte Aussichten auf Erfolg im Studium. Mit dem zweiten Bildungsweg und insbesondere dem Erwerb 
der allgemeinen Hochschulreife wäre die grundsätzliche Voraussetzung für ein erfolgreiches Studium erfüllt (KMK 1994).

Die allgemeine Hochschulreife beansprucht, inhaltlich und formal ausreichende Voraussetzungen dafür zu gewährleisten, daß jedes Studium erfolgreich betrieben und absolviert werden kann. Die allgemeine Hochschulreife hat sich in diesem Sinne im Wesentlichen bewährt [...]. Zusätzlichen Anforderungen, die sich aus den Erfordernissen eines bestimmten Studienganges her ableiten, muß das Grundstudium gerecht werden. (KMK 1994)

Im Gegensatz zu dieser Position wird der zweite Bildungsweg sowohl im universalistischen als auch im funktionalistischen strukturkritischen Diskurs als Umweg über Allgemeinbildung, als sogenannte „Ochsentour“ (DGB 1992) und damit als langer unbequemer und umständlicher Weg kritisiert (DGB 1992; BMBW 1992; DIHT 1990). Die gegebenen Strukturen des zweiten Bildungswegs reichen damit nicht aus, um im Rahmen des strukturkritischen Diskurses von einem durchlässigen Bildungssystem zu reden.

\section{Zielgruppe}

Entsprechend den hier dargestellten unterschiedlichen Vorstellungen über die Ermöglichung des Zugangs zur Hochschulbildung differieren auch die Ansichten darüber, für wen der Zugang eröffnet bzw. Durchlässigkeit ermöglicht werden soll.

Aus der universalistischen Logik heraus sollen alle zur Hochschule zugelassen werden, die sich weiterbilden wollen und eine Berechtigung besitzen, welche über jegliche Bildungswege erreichbar ist. Insofern gehören zur Zielgruppe, für die die Durchlässigkeitsstrukturen verbessert werden soll, alle beruflich Qualifizierten mit einem Erstausbildungs- oder Fortbildungsabschluss. Im Mittelpunkt steht in diesem Diskursstrang das Individuum als Person, dem über Bildung Chancengleichheit und Teilhabe ermöglicht wird.

Im funktionalistischen strukturkritischen Diskursstrang dagegen wird deutlich, dass vor allem die Gruppe der hoch- und fortgebildeten beruflich Qualifizierten, die sich bereits im Beruf und der beruflichen Bildung bewährt hat, als neue primäre Zielgruppe für ein Studium und Durchlässigkeit gesehen wird. Es sind nicht die beruflich Qualifizierten an sich wie beim universalistischen Diskursstrang, die generell zum Studium berechtigt werden sollten. Insofern wurde auch hier davon ausgegangen, dass es einer besonderen Qualifizierung auf Seiten der Berufsbildung bedarf, um ein Studium tatsächlich zu beginnen und abzuschließen. Wiederum wurde durch diesen Fokus auf besonders Leistungsfähige, also eine beruflichen Elite, eine Ungleichwertigkeit zwischen beruflicher und höherer Allgemeinbildung reproduziert. Des Weiteren wurde allgemein im funktionalistischen Diskurs durch die Befürwortung von Eignungsprüfungen verdeutlicht, dass diese auch die Funktion der Qualitätssicherung für die Hochschulbil- 
dung darstellen müssen. Diese sei notwendig, wenn die Hochschulen ihren Aufgaben - Forschung sowie Ausbildung für die Forschung und den Beruf - weiterhin entsprechen wollten.

Dieser Auftrag und Anspruch ist mit einer unbegrenzten Ausweitung des Hochschulzugangs ohne bestimmte Leistungskriterien nicht vereinbar. Ein Studium für jedermann hätte deutliche Qualitätseinbrüche bei der Hochschulausbildung zur Folge. Die Hochschulen dürfen nicht zu Dienstleistungsbetrieben für den privaten Bildungskonsum umfunktioniert werden. Die Reform des Hochschulzugangs steht damit wesentlich im Zeichen eines auch weiterhin hohen Durchschnittsniveaus in der Hochschulausbildung. (DIHT 1990)

In dem Zitat wird deutlich, dass Hochschulbildung nur für eine Leistungselite geöffnet werden soll, einem eindeutigen Zweck in der Gesellschaft diene und nicht als Recht auf Bildung zu verstehen sei. Dies steht im starken Gegensatz zum universalistischen strukturkritischen Diskurs. Vor allem wird eine starke Hierarchie von Bildungsbereichen konstruiert. Während einerseits auf eine größere Gleichwertigkeit von allgemeinbildender Sekundarstufe II und beruflicher Bildung gezielt wurde, wurde gleichzeitig die hochschulische Bildung als klare Ausbildung der Leistungselite der beruflichen und Allgemeinbildung konstruiert. Im Diskurs wird daher eine deutliche Hierarchisierung zwischen akademischer und beruflicher Bildung vorgenommen. Dies wurde auch deutlich in der Frage der Anerkennung auf dem Arbeitsmarkt. Denn es sollten zwar Karrierechancen in der beruflichen Bildung weiterentwickelt werden, um die Attraktivität für Schüler_innen zu erhöhen, aber für Aufstiege in die Führungsetage von Unternehmen sei ein Hochschulstudium zunehmend Voraussetzung (DIHT 1992a).

Die Zielgruppe der beruflich Qualifizierten, die im strukturkonservativen Diskurs konstruiert wurde, ist eine ganz ähnliche. Auch hier sollen es besonders qualifizierte berufliche Bewerber_innen sein, allerdings sollten diese ihr hohes Allgemeinbildungsniveau via zweiten Bildungsweg oder in einer Extra-Prüfung nachgewiesen haben, bevor sie einen fachbezogenen Zugang zur Hochschule erhalten, da der Nachweis von Allgemeinbildung generell als notwendig für den Hochschulzugang angesehen wird. Diese Anforderungen seien notwendig, da das Risiko des Scheiterns bei beruflich Qualifizierten im Studium besonders hoch sei (HRK 1992b). Wenn diese Bedingungen nicht erfüllt sind, seien beruflich Qualifizierter eine zusätzliche Belastung und Gefahr für die Hochschulbildung (HRK 1991).

Im Hinblick auf das in jedem Studium enthaltene, für Berufstätige besonders schwerwiegende Risiko des Scheiterns hält die HRK eine fachbezogene Hochschuleingangsprüfung für in beruflicher Tätigkeit qualifizierte Studienbewerber ohne Hochschulzugangsberechtigung für geboten. (HRK 1992b)

Tabelle 11bietet einen Überblick der zuvor dargestellten unterschiedlichen Sichtweisen im Kontext von Zugangsfragen. 
Tabelle 11 Vergleich der Diskursstränge zur Frage des Zugangs in Deutschland (1990-1998)

\begin{tabular}{|c|c|c|c|}
\hline & \multicolumn{2}{|c|}{ Strukturkritisch } & \multirow{2}{*}{$\begin{array}{l}\text { Strukturkonservativ } \\
\text { Funktionalistisch }\end{array}$} \\
\hline & Universalistisch & Funktionalistisch & \\
\hline \multirow[t]{4}{*}{ Zugang } & \multicolumn{2}{|c|}{$\begin{array}{l}\text { Infragestellung des Monopols des Berechtigungswe- } \\
\text { sens über höhere Allgemeinbildung }\end{array}$} & $\begin{array}{l}\text { Monopol des Berechti- } \\
\text { gungswesen bei höherer } \\
\text { Allgemeinbildung }\end{array}$ \\
\hline & $\begin{array}{l}\text { Berechtigungen über } \\
\text { Berufsbildung (Erstaus- } \\
\text { oder Fortbildung) }\end{array}$ & $\begin{array}{l}\text { Leistungsauswahl durch } \\
\text { Eignungstests für beruflich } \\
\text { Qualifizierte (und Abitu- } \\
\text { rient_innen) }\end{array}$ & $\begin{array}{l}\text { Hochschulzugang über } \\
\text { Ausweis des Allgemeinbil- } \\
\text { dungsniveaus über zweiten } \\
\text { Bildungsweg oder Eig- } \\
\text { nungsprüfung }\end{array}$ \\
\hline & $\begin{array}{l}\text { Zweiter Bildungsweg als } \\
\text { Zeichen fehlender } \\
\text { Gleichwertigkeit abge- } \\
\text { lehnt }\end{array}$ & $\begin{array}{l}\text { Zweiter Bildungsweg als } \\
\text { Ressourcenverschwendung } \\
\text { abgelehnt }\end{array}$ & $\begin{array}{l}\text { Zweiter Bildungsweg als } \\
\text { bevorzugter Weg }\end{array}$ \\
\hline & $\begin{array}{l}\text { Dritter Bildungsweg nicht } \\
\text { notwendig, wenn Be- } \\
\text { rechtigungen über Be- } \\
\text { rufsbildung } \\
\text { Eingangsprüfungen als } \\
\text { Zeichen für fehlende } \\
\text { Gleichwertigkeit }\end{array}$ & $\begin{array}{l}\text { Kritik an uneinheitlicher } \\
\text { Praxis sowie ungenügen- } \\
\text { dem Ausbau des Dritten } \\
\text { Bildungswegs } \\
\text { Eignungsprüfungen, um } \\
\text { Studierfähigkeit zu testen } \\
\text { (nur von beruflich Qualifi- } \\
\text { zierten vs. von allen Stu- } \\
\text { dierwilligen) }\end{array}$ & $\begin{array}{l}\text { Dritter Bildungsweg ausrei- } \\
\text { chend ausgebaut: } \\
\text { Eignungsprüfungen mit All- } \\
\text { gemeinbildungsanteil unter } \\
\text { Kontrolle der Akteuren der } \\
\text { höheren Allgemeinbildung }\end{array}$ \\
\hline \multirow[t]{2}{*}{ Zielgruppe } & $\begin{array}{l}\text { Allgemein: alle, die sich } \\
\text { bilden wollen und die } \\
\text { Berechtigung erworben } \\
\text { haben }\end{array}$ & $\begin{array}{l}\text { Allgemein: alle, die ihre } \\
\text { Studierfähigkeit bewiesen } \\
\text { haben und damit wahr- } \\
\text { scheinlich erfolgreich sein } \\
\text { werden: Leistungselite }\end{array}$ & $\begin{array}{l}\text { Allgemein: alle mit Ausweis } \\
\text { des notwendigen Niveaus } \\
\text { höherer Allgemeinbildung }\end{array}$ \\
\hline & $\begin{array}{l}\text { Durchlässigkeit: alle be- } \\
\text { ruflich Qualifizierten }\end{array}$ & $\begin{array}{l}\text { Durchlässigkeit: besonders } \\
\text { begabte und hochgebildete } \\
\text { beruflich Qualifizierte (mit } \\
\text { z.B. Fortbildungsabschluss) }\end{array}$ & $\begin{array}{l}\text { Durchlässigkeit: beruflich } \\
\text { Qualifizierte mit Ausweis } \\
\text { des notwendigen Niveaus } \\
\text { an Allgemeinbildung }\end{array}$ \\
\hline $\begin{array}{l}\text { Subjekt- } \\
\text { konstruktion }\end{array}$ & $\begin{array}{l}\text { Individuum als Person, } \\
\text { der über Bildung Chan- } \\
\text { cengleichheit und Teil- } \\
\text { habegerechtigkeit er- } \\
\text { möglicht wird }\end{array}$ & $\begin{array}{l}\text { Individuum als (Humanka- } \\
\text { pital-)Ressource }\end{array}$ & $\begin{array}{l}\text { Individuum als Belastung } \\
\text { bei mangelnder Allgemein- } \\
\text { bildung }\end{array}$ \\
\hline
\end{tabular}

Quelle: Darstellung der Verfasserin

\section{Anrechnung}

Während die Frage des Zugangs kontrovers und umfassend diskutiert wurde, so war Anrechnung von Vorbildung im Zusammenhang mit Durchlässigkeit zwischen Berufs- und Hochschulbildung so gut wie kein Thema in den Diskursen. Einzelne Ausnahmen konnten im Rahmen des funktionalistischen strukturkritischen Diskurses und im strukturkonservativen Diskurs festgestellt werden. So 
wurde Anrechnung beruflicher Bildung auf ein Hochschulstudium im funktionalistisch-strukturkritischen Diskursstrang für die Individuen eingefordert, die bereits den zweiten Bildungsweg absolviert haben. Auf diese Weise würde die Doppelqualifikation anerkannt und eine Benachteiligung beruflich Qualifizierter verhindert werden (DIHT 1990).

Die KMK (1997a) kritisierte dagegen im Rahmen des strukturkonservativen Diskurses die Einseitigkeit der geführten Durchlässigkeitsdebatte, denn es bedürfe auch einer besseren Anerkennung der Allgemeinbildung im Berufsbildungssystem. Hier wurde eine bessere und systematische Anrechnung von erbrachten Leistungen der Allgemeinbildung der Sekundarstufe II z.B. durch eine Verkürzung der Ausbildungszeit gefordert. Die Anrechnung beruflicher Bildung auf ein Hochschulstudium (ohne zusätzlichen Abschluss des zweiten Bildungswegs) wird dagegen nicht explizit diskutiert. Dies zeigt, dass die Vorstellung, berufliche Bildung könne Fähigkeiten ausbilden, die so gestaltet sind, dass Teile des Studiums ersetzt werden können, im ersten Untersuchungszeitraum noch nicht im Bereich des Denkbaren bzw. des Sagbaren lag.

\section{Organisationale Verbindung}

Die Bedeutung von organisationalen Verbindungen für die Schaffung von Durchlässigkeit zwischen Berufs- und Hochschulbildung differiert in den drei Diskurssträngen (vgl. Tabelle 12). Während integrierte Bildungsgänge wie duale Studiengänge z.B. an Berufsakademien im strukturkonservativen Diskurs kein Thema sind, werden sie im funktionalistischen strukturkritischen Diskurs als besonders wertvoll und hilfreich zur Förderung von Durchlässigkeit und vor allem auch der Attraktivität der beruflichen Bildung erachtet.

Gleichwertigkeit von beruflicher und allgemeiner Bildung heißt aber auch mehr Durchlässigkeit der Bildungswege. Wir dürfen uns nicht nur mit den klassischen Übergängen zwischen den einzelnen selbständigen Bildungssektoren befassen. Was Not tut, sind auch neue integrative Modelle, wie sie erfolgreich durch viele Formen belegt werden. Ich verweise auf die Berufsakademien [...].(DIHT 1992a)

Im universalistischen Diskurs werden sie als fördernde Möglichkeit anerkannt, aber ihnen wird weniger Bedeutung beigemessen als im funktionalistischen Diskursstrang.

In Letzterem entwickeln die Kammern zusammen mit Unternehmen ein Rahmenmodell, das die Integration einer beruflichen Ausbildung mit einem beruflichen Weiterbildungsabschluss sowie dem Fachhochschulabschluss vorsieht und insgesamt 5,5 Jahre dauern soll, um wettbewerbsfähig mit normalen Studiengängen zu sein und um auch die Zielgruppe der Abiturient_innen anzusprechen (DIHT 1992b). Integrierte Bildungsformen wie duale Studiengänge oder das Rahmenmodell sollen die Attraktivität der beruflichen Bildung erhöhen und die Entscheidung für entweder berufliche oder akademische Bildung auflösen. Das Rahmenmodell setzt in besonderem Maße auf Flexibilität. So sei es möglich, nach der 
beruflichen Ausbildung oder Weiterbildung aufzuhören, ein Durchstieg bis zum Studium sei nicht notwendig. Auch wenn die Flexibilität zur Absicherung der Bildungsteilnehmer_innen führe, da es weniger wahrscheinlich sei, ohne Abschluss den Bildungsgang zu verlassen (DIHT 1992b), wird im Diskurs deutlich, dass die Verbindung und Integration der Bildungsbereiche vor allem dazu dient, gute Abiturient_innen oder herausragende Abgänger_innen der Sekundarstufe I für einen beruflichen Bildungsweg zu begeistern.

Die Durchlässigkeit, Vernetzung oder sogar Integration der Bildungssektoren Berufsbildung und Hochschulbildung ist das entscheidende Attraktivitätsargument bei den angesprochenen Zielgruppen. Damit muss nicht zwangsläufig in jedem Fall ein Qualifikationsdurchstieg bis zum Hochschulabschluss verbunden sein. (DIHT 1992b)

Insofern soll das Rahmenmodell strategisch auf zweierlei Weise genutzt werden: erstens den Einfluss der Unternehmen in den Hochschulen auszubauen und zweitens das Bildungsverhalten zu lenken. Auch der Fakt, dass wiederum nur eine Auslese derjenigen, die dem integrierten Bildungsgang beginnen, auch das Hochschulstudium beenden soll, spiegelt die Idee der Elitebildung via akademische Bildung wider.

Tabelle 12 Vergleich der Diskursstränge zur Bedeutung organisationaler Verbindungen in Deutschland (1990-1998)

Strukturkritisch

Universalistisch

Förderung doppeltqualifizierender Bildungsgänge (Berufsausbildung mit Abitur)
Duales Studium zur Steigerung der Attraktivität der beruflichen Bildung (Bsp: Verbindung von Aus-, Aufstiegsfortbildung und $\mathrm{FH}$-Studium) Gegen Institutionalisierung von Doppelqualifikationen auf Sek-II-Niveau
Strukturkonservativ

Funktionalistisch

Nicht relevant

Quelle: Darstellung der Verfasserin

Im universalistischen Diskursstrang wurde das Potenzial von integrierten Bildungsgängen wie dualen Studiengängen anerkannt, wenn sichergestellt wird, dass die Abschlüsse breit verwendbar und nicht nur auf die spezifischen betrieblichen Bedürfnisse ausgerichtet sind (DGB 1996). Gefördert werden sollten vor allem doppeltqualifizierende Bildungsgänge wie das Abitur mit Berufsausbildung. So sollten die aus der DDR bestehenden Bildungsgänge in den neuen Bundesländern erhalten werden, da sie eine gute Möglichkeit sein können, Durchlässigkeit voranzubringen. Integrierte Bildungsformen auf Sekundarstufe II, d.h. Doppelqualifikationen wie das Abitur mit Berufsausbildung, werden dagegen im funktionalistischen Diskursstrang abgelehnt, da hier die notwendige Berufspraxis als nicht ausreichend angesehen wird (BDI et al. 1993). 


\section{Umgang mit der Heterogenität von Bedürfnissen}

Ähnlich selten wie Fragen der Anrechnung werden auch Strukturen, die heterogenen Bedürfnissen von Lernenden gerecht werden, im Durchlässigkeitsdiskurs im ersten Untersuchungszeitraum thematisiert. Dabei unterscheidet sich der strukturkritische vom -konservativen Diskurs dahingehend, ob der Umgang mit heterogenen Bedürfnissen im Zusammenhang mit Durchlässigkeit zwischen Berufs- und Hochschulbildung diskutiert wird oder nicht (vgl. auch Tabelle 13). In Letzterem werden zwar allgemein Strukturen, die das Lernen im Hochschulsystem erleichtern sollen, gefordert, aber nicht im Zusammenhang mit Durchlässigkeit (HRK 1994; KMK 1997a). Maßnahmen für beruflich Qualifizierte an den Hochschulen sind kein Thema. Dies ist auch wenig verwunderlich, weil sie nicht als ,natürliche' Zielgruppe der Hochschule anerkannt wurden. Während die HRK die Beratung an den Hochschulen verbessern wollte, sah die KMK (1997a) die Notwendigkeit, eine bessere Beratung in der Schule für die Planung des weiteren Weges in der Berufs- und Hochschulbildung zu institutionalisieren. Auch hier wurde diese Beratung nicht im Kontext von Durchlässigkeit thematisiert, jedoch kann eine gute Beratung über Bildungsmöglichkeiten Einfluss auf eine tatsächlich gelebte Durchlässigkeit haben. Auch die Institutionalisierung der Möglichkeiten des Teilzeitstudiums wurde 1997 von der HRK gefordert (HRK 1997a). Dies sei notwendig, da eine Erhebung des Studierendewerks gezeigt habe, dass viele Studierende neben den Studium noch andere Tätigkeiten, vor allem Erwerbstätigkeiten und Betreuungsaufgaben, nachgingen, die diese an einem Vollzeitstudium hinderten. Als Normalform des Studiums wird weiterhin das Vollzeitstudium angesehen, jedoch sollte auch eine Flexibilisierung von Studiengängen über Modularisierung erreicht werden, so dass die Streckung des Studiums in Teilzeit möglich würde (HRK 1997a). Auch wenn in der Erwerbstätigkeit eine Hauptursache für die Notwendigkeit von Teilzeitstudiengängen gesehen wird, so wird die Institutionalisierung eines Teilzeitstudiums nicht als Möglichkeit zur Erleichterung des Studiums für beruflich Qualifizierte diskutiert.

Im strukturkritischen Diskurs dagegen werden, wenn auch außerordentlich vereinzelt, Strukturen zur Unterstützung beruflich Qualifizierter an den Hochschulen angesprochen. Im universalistischen Diskursstrang sollen die beruflich Qualifizierten durch Mentoringsysteme sowie studienbegleitende Hilfen und Beratung unterstützt werden (DGB 1992). Wie genau das funktionieren könnte, wird nicht ausgeführt. Deutlich ist aber, dass die Unterstützung auch während des Studiums mitgedacht wird. Es geht somit auch darum, die Individuen im Bildungsprozess selbst zu halten und zu unterstützen.

Im funktionalistischen Diskursstrang stand die bessere Gewährung von Informationen und Beratung über Bildungsoptionen und berufliche Entwicklungswege im Vordergrund (BMBW 1993b), so dass die Individuen informierte Bildungsentscheidungen entsprechend ihren Fähigkeiten treffen können, um Umwege zu verhindern. Hier findet die Unterstützung eher vor dem Studium statt. Zudem wird der Ausbau von Fernstudiengängen gefordert (BDI et al. 1993), da 
diese am besten an die Bedürfnisse beruflich Qualifizierter anpassbar seien. Auf diese Weise wäre die Verbindung von Studium und Arbeit möglich.

Tabelle 13 Vergleich der Diskursstränge zur Frage des Umgangs mit Heterogenität in Deutschland (1990-1998)

\begin{tabular}{|c|c|c|}
\hline \multicolumn{2}{|c|}{ Strukturkritisch } & \multirow{2}{*}{$\begin{array}{l}\text { Strukturkonservativ } \\
\text { Funktionalistisch }\end{array}$} \\
\hline Universalistisch & Funktionalistisch & \\
\hline Wenig relevant & Wenig relevant & $\begin{array}{l}\text { Wenig relevant, nicht für beruf- } \\
\text { lich Qualifizierte }\end{array}$ \\
\hline $\begin{array}{l}\text { Mentoringsysteme, studienbe- } \\
\text { gleitende Hilfen, Beratung }\end{array}$ & $\begin{array}{l}\text { Fernstudiengänge zur besse- } \\
\text { ren Vereinbarkeit von Beruf } \\
\text { und Weiterqualifizierung }\end{array}$ & $\begin{array}{l}\text { Vorbereitung auf das Studium } \\
\text { (Erlangen der Studierfähigkeit) } \\
\text { findet außerhalb der Hochschu- } \\
\text { len statt }\end{array}$ \\
\hline
\end{tabular}

Quelle: Darstellung der Verfasserin

\section{Akteurspositionen und Zusammenfassung}

In diesem Abschnitt sollen die untersuchten Akteure in Relation zu den identifizierten Diskurssträngen gebracht werden. Wer ist hauptsächlicher Träger welchen Diskurses? Zudem sollen in der Zusammenschau die wichtigsten Ziele der Diskursstränge und die aus den Diskursstrukturen herauslesbaren Strategien zusammengefasst und verglichen werden.

\section{Akteurspositionen}

Wie in den Vergleichen der Strukturen der unterschiedlichen Diskursstränge anhand der Zitate und Quellenverweise bereits deutlich wurde, sind manche Akteure stärker als Träger der strukturkritischen universalistischen oder funktionalistischen Diskursstränge zu identifizieren und andere eher dem strukturkonservativen Diskurs zuzurechnen. Nichtsdestoweniger soll an dieser Stelle darauf hingewiesen werden, dass in den Aussagen aller Akteure Elemente sowohl den strukturkritischen als auch den strukturkonservativen Diskursen zuzurechnen sind. Insofern ist eine eindeutige Zuordnung nicht möglich. Um die Positionen trotzdem grafisch darzustellen, verwende ich die positional map nach Clarke (2012). Anhand dieser kann man in Abbildung 5 ablesen, wo sich die Akteure am ehesten positionieren lassen. Die Zuordnung der Akteure erfolgt über den gesamten Zeitraum und in Zusammenschau aller Aussagen. Die x-Achse zeigt den Verlauf von eher funktionalistischer zu einer universalistischen Argumentationslogik und die yAchse, ob die Positionen der Akteure mehrheitlich strukturkritisch oder -konservativ einzuordnen sind. Dabei wird auf der $\mathrm{y}$-Achse unterschieden zwischen vollständig (5), sehr deutlich (4), deutlich (3), mehrheitlich (2), vorwiegend (1) strukturkritisch bzw. -konservativ und auf der x-Achse zwischen (fast) vollständig (3), deutlich (2) und vorwiegend (1) funktionalistisch bzw. universalistisch. 
Am Beispiel der KMK kann man sehen, dass die Aussagen der Kultusminister nicht ausschließlich, aber deutlich dem konservativen Diskurs zuzuordnen und dass die Aussagen gleichzeitig zwar deutlich aber nicht ausschließlich funktionalistisch gerahmt sind. Das heißt, dass diese, wenn auch nur zu einem geringen Teil, auch universalistisch waren. Ein Beispiel für eine Aussage, die dem strukturkritischen Diskurs und gleichzeitig dem universalistischen Diskursstrang zuzuordnen ist, ist der Hinweis der KMK auf die Bedeutung von Gleichwertigkeit der Berufsbildung:

Die Gleichwertigkeit von allgemeiner und beruflicher Bildung muß aber in pädagogischer, bildungs- und gesellschaftspolitischer Hinsicht weiterentwickelt werden mit dem Ziel der Angleichung des öffentlichen Stellenwertes beider Bildungsbereiche sowie der Schaffung der hierfür erforderlichen Voraussetzungen. (KMK 1994)

Abbildung 5 Positional map der deutschen Akteure (1990-1998)

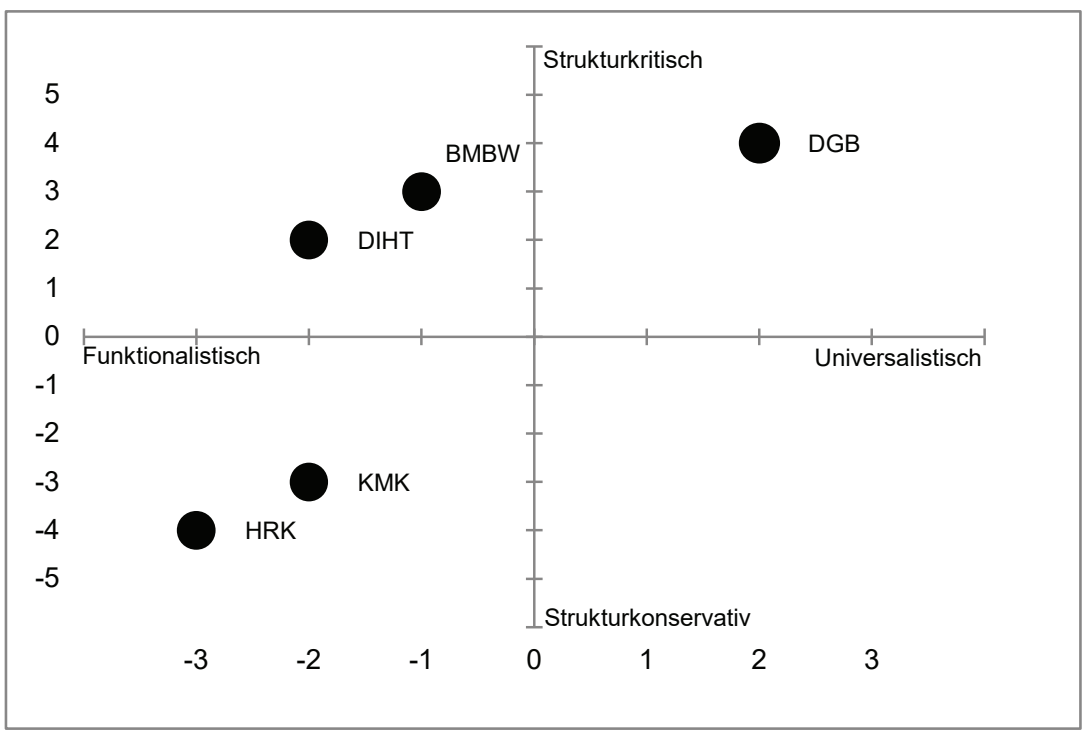

Quelle: Darstellung der Verfasserin

In der positional map wird deutlich, dass kein Akteur rein strukturkritisch oder -konservativ argumentiert, wobei der DGB am stärksten dem strukturkritischen und die HRK am stärksten dem konservativen Diskurs zu zurechnen sind. Insgesamt kann anhand der Aussagen festgestellt werden, dass die Vertreter der höheren Allgemeinbildung, also die KMK und die HRK, noch stärker als Träger des strukturkonservativen Diskurses gelten können, während die Gewerkschaften (DGB), das Ministerium (BMBW/BMBF) und die Kammern (DIHT) als Träger 
des strukturkritischen Diskurses identifiziert werden. Innerhalb des strukturkritischen Diskurses finden sich von allen untersuchten Akteuren Aussagen, die der funktionalistischen Begründung zuzuordnen sind. Als Hauptträger dieses Diskursstrangs können aber vor allem die Kammern und das Bildungsministerium gelten. Die universalistische Argumentationslogik war in den Diskursen am wenigsten vertreten, Aussagen, die diesem Diskursstrang zuzurechnen sind, fanden sich vor allem bei dem DGB, dem BMBW/BMBF sowie teilweise auch der KMK. Als Träger des Diskurses können aber primär der DGB und sekundär das Ministerium gelten.

\section{Zusammenschau der Diskurse und der rekonstruierten Strategien}

Im Folgenden werden die unterschiedlichen Strategien, die sich aus der Struktur und Logik der Diskursstränge ergeben, dargelegt werden. Diese Zusammenschau macht zum einen noch einmal die Deutungskämpfe in den deutschen Durchlässigkeitsdebatten sowie die unterschiedliche Verantwortungszuschreibung deutlich und dient gleichzeitig dazu, die Diskursstränge zusammenzufassen.

Die Träger des strukturkritischen universalistischen Diskurses können als Anwälte der beruflichen Bildung gesehen werden, die für eine gestiegene gesellschaftliche Wertschätzung er beruflichen Bildung arbeiten. Zdiel ist primär die Gleichstellung der beruflichen Bildung in der Gesellschaft und sekundär die Institutionalisierung des Rechts auf Bildung und damit auch Durchlässigkeit im Bildungssystem. Im Rahmen des Diskurses wird der DGB als traditioneller Verfechter der Gleichwertigkeitsforderung dargestellt. Im Gegensatz dazu wurde das BMBW eher als neuerer Verfechter von Gleichwertigkeit und Durchlässigkeit positioniert. Diese Position wird damit begründet, dass durch die erfolgte Modernisierung der Ausbildungsberufe, die mit einem erhöhtem Anforderungsniveau einherging, Gleichwertigkeit tatsächlich eine gerechtfertigte Forderung sei (BMBF 1996a). Das Bundesministerium selbst war ein wichtiger Akteur bei der Modernisierung und trug somit viel dazu bei, die gesellschaftliche Stellung der beruflichen Bildung zu ändern.

Die Hauptverantwortung dafür, Durchlässigkeit zu erreichen, wird in diesem Diskursstrang primär auf Akteure außerhalb der beruflichen Bildung übertragen. So wären es die Länder, die ihre Regelungen zum Hochschulzugang vereinheitlichen und auch Qualifikationen und Berechtigungen der beruflichen Bildung anerkennen sollten. Des Weiteren werden die Länder aufgefordert, ihre Definition von Studierfähigkeit, die als zu eng an dem gymnasialen Fächerkanon gebunden erscheint, zu ändern und stärker berufliche Bildung anzuerkennen (DGB 1998c). Die Hochschulen werden ebenso kritisiert für ihre fehlende Anerkennung der beruflichen Bildung und der damit einhergehenden zurückhaltenden Institutionalisierung von Aufnahmeverfahren für beruflich Qualifizierte. Die Regierung soll daher im Rahmen des Hochschulrahmengesetzes ihren Einfluss in Fragen von Durchlässigkeit insbesondere des Hochschulzugangs wirksam machen (DGB 1990). Die Arbeitgeber_innen sollen sich dagegen verstärkt für die Entwicklungs- 
und Karrierechancen für Absolvent_innen der beruflichen Bildung in Form einer vorausschauenden Personalpolitik einsetzen. Sie sind zusammen mit dem öffentlichen Dienst die Hauptakteure, die Gleichwertigkeit auf dem Arbeitsmarkt umsetzen können. Gleichwertigkeit ist neben Durchlässigkeit eine der Hauptforderungen des universalistischen Diskursstrangs. Berufsbildung müsse als Alternative zur Hochschulbildung gestaltet werden: Arbeitsbedingungen, Weiterbildungs-, Berufs-, Karriere- und Einkommenschancen (auch Besoldungs- und Laufbahnstruktur des öffentlichen Dienstes) seien anzupassen. Berufliche Fortbildungsmodelle sollten so entwickelt werden, dass sie den Einstieg in Führungsetagen ermöglichen. Die Vergabe von Positionen und die Personalpolitik allgemein sollten anhand beruflicher Leistung anstatt auf Basis von schulischen und hochschulischen Berechtigungen geschehen (DGB 1992).

Die Träger des funktionalistischen strukturkritischen Durchlässigkeitsdiskurses stellen sich ebenfalls als Verfechter der Gleichwertigkeit von beruflicher und Allgemeinbildung dar, wobei die Gleichwertigkeit nicht mit der Hochschulbildung, sondern mit der höheren Allgemeinbildung auf Sek-II-Niveau eingefordert wird. Primäres Ziel im Rahmen des funktionalistischen Diskursstrangs sind vor allem die Erhöhung der Attraktivität der Berufsbildung und die Sicherung des Arbeitskräftepotenzials sowie der Wettbewerbsfähigkeit des Landes. Ziel ist dabei nicht generell, den Hochschulzugang auszuweiten, sondern das Bildungswahlverhalten der Schüler_innen in Richtung Berufsbildung zu beeinflussen. Der Hochschulzugang dagegen solle einer Leistungselite vorbehalten bleiben, wobei eine größere Gerechtigkeit und die Gleichwertigkeit von Berufs- und höherer Allgemeinbildung über die Implementierung einer Leistungsauswahl beim Hochschulzugang sichergestellt werden soll. Insofern werden insbesondere die Länder sowie die Regierung dazu aufgefordert, wieder eine stärkere Leistungsauswahl im Bildungssystem zu institutionalisieren und differenzierte Bildungswege zu entwickeln, um die Individuen entsprechend ihren Begabungen zu kanalisieren. Ein weiterer Weg, um die Attraktivität des Berufsbildungsweges zu erhöhen, ist eine Verbesserung der Karrieremöglichkeiten beruflich Qualifizierter. So wird von den öffentlichen Arbeitgebern eine Reform des Laufbahn- und Besoldungsrechts gefordert. Gleichwertige Karrierechancen für Berufs- und Hochschulbildung wie im universalistischen Diskursstrang wird allerdings nicht eingefordert, da Hochschulbildung noch immer als Ausbildungsweg für die gesellschaftlichen Elitepositionen gesehen wird (DIHT 1990).

Für den strukturkonservativen Diskurs kann festgehalten werden, dass die hauptsächlichen Träger des Diskurses, die KMK und die HRK, sich selbst als entscheidende Akteure bei Fragen der Durchlässigkeit zwischen beruflicher Bildung und Hochschulbildung konstruierten und dabei ihre Entscheidungsmacht als legitim und notwendig verteidigten. Auf diese Weise versuchen sie ihre gesellschaftliche Gatekeeperposition für den Zugang zur Hochschulbildung und damit höheren gesellschaftlichen Positionen zu verteidigen. So zielten die Hochschulen 
als aufnehmende Institution auf die Bewahrung der die Qualität der Hochschullausbildung. Dies werde von der KMK als Hüter des Berechtigungswesens durch klare Kriterien für Studierfähigkeit unterstützt. Die Strategie der Träger des strukturkonservativen Diskurses lag darin, dass nur geringer Handlungsbedarf in Bezug auf Durchlässigkeit konstruiert wurde, da bereits viele Möglichkeiten bestünden.Zudem zielte der Diskurs auf die Stärkung der Vorrangstellung der Allgemeinbildung und die Sicherung der Entscheidungsmacht der Akteure der höheren Allgemeinbildung. Während die Entscheidungen über Berechtigungen und $\mathrm{Zu}-$ lassungen nicht aus der Hand gegeben werden sollten, wurden die Akteure der beruflichen Bildung dagegen aufgefordert, mit Reformen in der Berufsbildung selbst mehr für die Attraktivität der beruflichen Bildung zu tun.

Zusammenfassend kann für den ersten Zeitraum festgestellt werden, dass Durchlässigkeit kontrovers diskutiert wurde. Zwei Diskurse und insgesamt drei Argumentationslogiken standen sich gegenüber mit ganz unterschiedlichen Vorstellung davon, was Durchlässigkeit ist, was sie im deutschen Bildungssystem und der Gesellschaft bewirken könnte und sollte, wie diese hergestellt werden soll und wer dies leisten müsste. Vor allem wurde deutlich, dass Durchlässigkeit primär über Fragen des Zugangs ausgehandelt wurde und andere Aspekte kaum thematisiert wurden.

\subsection{Gesellschaftliche Wissensbestände zu Durchlässigkeit in Zeiten von Bologna und Kopenhagen (1999-2012)}

Wie haben sich nun die Vorstellungen von Durchlässigkeit mit dem Beginn und der fortschreitenden Evolution der europäischen Bildungsprozesse in Deutschland entwickelt? Kam es zu einer Veränderung der Diskursstruktur, der Argumentationslogiken, der Akteurspositionen, des Verständnisses von Durchlässigkeit? In diesem Teilkapitel sollen die institutionellen Entwicklungen im Hinblick auf Durchlässigkeit, die sich vor allem in den Veränderungen der gesellschaftlichen Wissensbestände anhand der Diskurse manifestieren, für die Zeit von 1999 bis 2012 dargestellt werden. Dafür wird analog zum ersten Zeitraum zuerst ein Überblick über die unterschiedlichen Debatten, in dem Durchlässigkeit thematisiert wurde, gegeben. In einem zweiten Schritt werden aus den verschiedenen Aussagen der untersuchten Akteure die Diskurse zu Durchlässigkeit im zweiten Untersuchungszeitraum rekonstruiert und entlang ihrer Strukturen dargestellt. Schließlich sollen ebenfalls die Akteurspositionen sowie die aus den Diskursen rekonstruierten Strategien der Diskursträger rekonstruiert und verglichen werden. 


\subsubsection{Schlaglichter der deutschen Debatten zu Durchlässigkeit (1999-2012)}

Im Folgenden sollen die wichtigsten Debatten, in denen Durchlässigkeit behandelt wurde, kurz dargestellt werden. Diese Darstellung dient der besseren Kontextualisierung der Diskurse und gibt einen Überblick über die Entwicklung im zweiten Untersuchungszeitraum.

Im ersten Untersuchungszeitraum wurde Durchlässigkeit zu großen Teilen im Kontext der Debatte um Gleichwertigkeit und die Erhöhung der Attraktivität der beruflichen Bildung behandelt (vgl. Abbildung 4). Im Gegensatz dazu wurde das Thema Durchlässigkeit im Zeitraum von 1999 bis 2012 deutlich häufiger in verschiedenen bildungspolitischen Debatten aufgegriffen. Insgesamt lassen sich sechs größere Debatten identifizieren:

1. die Debatte um Anrechnung,

2. die Debatte um die Reform des Berufsbildungsgesetzes (BBiG),

3. die Debatte zur Qualifizierungsinitiative der Bundesregierung,

4. die Debatte zu den Regelungen des Hochschulzugangs beruflich Qualifizierter,

5. die Debatte zu den Auswirkungen der Bologna-Reformen und

6. die Debatte zum europäischen und deutschen Qualifikationsrahmen.

In Abbildung 6 werden die Debatten chronologisch im zweiten Zeitraum zusammen mit den wichtigsten politischen Entscheidungen auf nationaler und europäischer Ebene dargestellt. Die Darstellung der einzelnen Debatten erfolgt an dieser Stelle zwar getrennt, sie fanden aber teilweise parallel statt und weisen auch Bezüge zueinander auf.

\section{Debatte um Anrechnung}

Während in den ersten zwei Jahren direkt nach der Bologna-Erklärung das Hochschulsystem an sich und dessen antizipierte Veränderungen im Mittelpunkt vieler Bildungsdebatten standen und Mobilität eher europaweit als national zwischen Bildungsbereichen interessierte, war es trotzdem dieser primär auf das Hochschulsystem gerichtete Prozess, der einen wichtigen und vor allem in Deutschland vorher nicht weiter beachteten Aspekt von Durchlässigkeit zwischen beruflicher und Hochschulbildung in die Diskussion einführte: die Frage der Anrechnung außerhochschulischer Leistungen im Hochschulbereich. Denn neben der Einführung von B.A. und M.A. wurde die Einführung eines Leistungspunktesystems vereinbart, mit dessen Hilfe auch außerhochschulisch erworbene Lernergebnisse anzurechnen seien (vgl. Kapitel 5).

In der deutschen Diskussion wurden außerhochschulisch erworbene Kenntnisse dabei vornehmlich auf berufliche Qualifikationen reduziert, und die Frage 
der Anrechnung beruflicher Qualifikationen im Studium wurde daher in mehreren bildungspolitischen Beschlüssen aufgegriffen: Die Anrechnung beruflicher Kompetenzen wurde in der Bund-Länder-Kommission für Bildungsplanung und Forschungsförderung im Juni 2001 als Ziel ausgegeben und auch vom Wissenschaftsrat 2002 empfohlen (DGB et al. 2002). So forderten 2002 eben auch eine Akteurskoalition - bestehend aus dem DGB, dem Kuratorium der Deutschen Wirtschaft für Berufsbildung, in dem der DIHK Mitglied ist, dem Bundesministerium für Wirtschaft und Technologie (BMWT) sowie dem BMBF - die Kultusministerkonferenz auf, sich mit dem Problem der Anrechnung näher zu befassen und am Beispiel der IT-Weiterbildung umzusetzen (DGB et al. 2002). Es gab demnach einen breiten gesellschaftlichen Konsens über die Wichtigkeit von Anrechnung, da diese eine bessere und auch notwendige Verbindung von unterschiedlichen Lernorten und Bildungswegen und letztlich auch eine besser Ausschöpfung des „Bildungspotenzials“ ermögliche.

Die Grundlage für die Umsetzung von Anrechnung an den Hochschulen wurde 2002 von der KMK geschaffen. Sie beschloss, dass bis zu 50 \% eines Studiums durch außerhochschulische Leistungen erbracht werden können, wenn erstens eine Hochschulzugangsberechtigung besteht, zweitens die Gleichwertigkeit des Niveaus und Inhalts geprüft und drittens die Qualität des Anerkennungsverfahren durch Akkreditierung gesichert sei (KMK 2002). Da die Hochschulen letztlich als aufnehmende Institutionen die entscheidenden Akteure bei der Anrechnung beruflicher Qualifikationen sind, richteten sich im Jahr 2003 das BMBF, die KMK und auch die HRK an die einzelnen Hochschulen mit der Forderung, für gleichwertige Studien- und Prüfungsleistungen insbesondere in beruflichen Fortbildungsqualifizierungen Leistungspunkte zu vergeben und auf ein Studium anzurechnen (BMBF et al. 2003).

$\mathrm{Da}$ in Deutschland neben Einstufungsprüfungen kaum Erfahrungen in Sachen Anrechnung von Kompetenzen bestand, Leistungspunktesysteme gerade erst in den Hochschulen eingeführt wurden und auch außerhalb der Hochschule keine weiteren Leistungspunktesysteme existierten, die einfach anerkannt und überführt werden konnten, startete das BMBF mit einigen Partnerorganisationen zusammen die Initiative „Anrechnung beruflicher Kompetenzen auf Hochschulstudiengänge" (ANKOM). Ziel war es, insbesondere für den Bereich der beruflichen Fortbildungsgänge „qualitätsgesicherte Anrechnungsverfahren zu entwickeln und die Implementierung an Hochschulen zu erproben" (Freitag 2009a: 34). ${ }^{110}$ Zwei Jahre nach dem Start der ANKOM-Initiative wurde 2007 auch für die berufliche Bildung eine Initiative gestartet, die die Anrechnung vor allem innerhalb des Berufsbildungssystems erleichtern sollte: DECVET - „Entwicklung eines Leistungspunktesystems in der beruflichen Bildung“". Auch wenn der Fokus primär auf Anrechnungsmöglichkeiten innerhalb der Berufsbildung lag, so bestehen in einem auf Lernergebnisse ausgerichtetem Leistungspunktesystem in der

110 Literatur zu ANKOM und den ersten Evaluationen der Initiativen: Buhr et. al. (2008); Freitag (2009b); Freitag et. al. (2011). 
Berufsbildung auch Anknüpfungspunkte für die Anrechnung in der Hochschulbildung, zumal die Idee der Anrechnung im Hochschulbereich über Leistungspunkte, die in der Berufsbildung erworben wurden, seit Beginn der Debatte bestand (vgl. DGB et al. 2002).

Tatsächlich wurden die Diskussionen um Anrechnung im Vergleich zu den ersten Jahren immer konkreter. Es ging weniger darum, dass generell außerhochschulische Qualifikationen anerkannt werden müssten, sondern konkret um die Wege und Instrumente, über die dies geschehen sollte. In diesem Kontext wurde auch 2008 der Anrechnungsbeschluss der KMK von 2002 bestätigt und erweitert. So werden drei Formen von Anrechnung unterschieden: die Einzelfallprüfung, die pauschale Anrechnung bei bestehenden Kooperationsabkommen zwischen Organisationen der Berufsbildung und einzelnen Hochschulen sowie Einstufungsprüfungen (KMK 2008).

Die Bedeutung des Themenkomplexes Anrechnung wird ebenfalls deutlich, wenn man sich die vielfach neu geschaffenen rechtlichen Regelungen in den Landeshochschulgesetzen anschaut, die bis auf einige Regelungen zur Einstufungsprüfung alle im zweiten Untersuchungszeitraum entstanden sind. Dabei kann zwischen Regelungen zur Einstufungsprüfung, zur Externenprüfung und zu Anrechnungsverfahren allgemein unterschieden werden (vgl. z.B. Freitag 2012).

\section{Debatte um die Reform des BBiG}

Während die Debatte um Anrechnung fast den gesamten Untersuchungszeitraum anhielt, wurde Durchlässigkeit auch immer wieder zu bestimmten Anlässen thematisiert, wie auch im Kontext der Reform des Berufsausbildungsgesetzes. In der Diskussion um die Novellierung des seit 1969 bestehenden Gesetzes forderten vor allem die Akteure der Berufsbildung, insbesondere der DGB (2004a), dass Durchlässigkeit zur Hochschule im Gesetz selbst stärker gefördert werden sollte. Dies wäre möglich, wenn Zugangswege zur Hochschule und Anrechnungsmodalitäten festgeschrieben werden würden.

Damit würden nicht nur formale Zugangsmöglichkeiten verbessert und auf bundesweit einheitliche Strukturen gestellt, sondern für Erwerbstätige mit abgeschlossener Ausbildung und Berufserfahrung auch ein Weg zur Verkürzung von Studienzeiten eröffnet. (DGB 2004a: 10)

Im Gesetz selbst, das 2005 verabschiedet wurde, fanden die Forderungen keinen Eingang. Neben Durchlässigkeit zur Hochschule wurde auch Durchlässigkeit und vor allem Anrechnung innerhalb der beruflichen Bildung gefordert und im Gesetz gefördert (z.B. veränderte Regelungen bei der Externenprüfung). Eine verstärkte Durchlässigkeit in die berufliche Bildung aus der Hochschule wurde aber nicht thematisiert. 


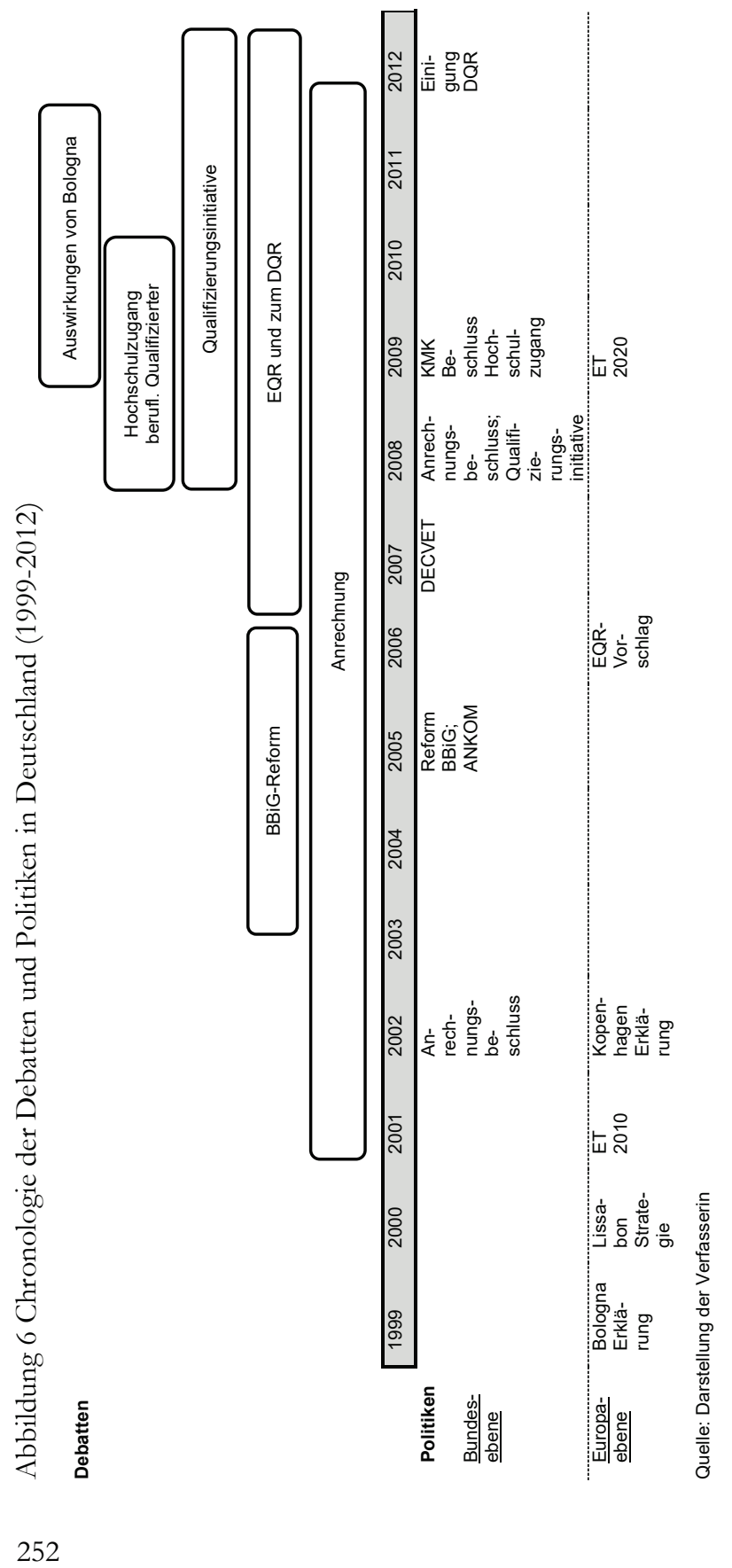




\section{Debatte im Rahmen der Qualifizierungsinitiative}

Auch im Kontext der seit dem Bildungsgipfel 2008 von der Bundesregierung und den Ländern in Dresden gestarteten Qualifizierungsinitiative wurde Durchlässigkeit zur Hochschulbildung immer wieder diskutiert. Die Qualifizierungsinitiative soll der Förderung von Bildung und Weiterbildung im gesamten Lebenslauf dienen und besteht aus verschiedensten Programminitiativen. Viele Bildungsthemen werden behandelt: Ausbau frühkindlicher Bildung, Übergang in die Ausbildung, Weiterbildung, Förderung der Ausbildung in MINT-Fächern, aber auch die Förderung von Durchlässigkeit zwischen Berufs- und Hochschulbildung. Diesbezügliche Programme, die in der Initiative gestartet wurden, sind die Einführung von Aufstiegsstipendien oder auch der Wettbewerb „Aufstieg durch Bildung: Offene Hochschulen“. Mithilfe von Aufstiegsstipendien sollen besonders leistungsstarke beruflich Qualifizierte mit einer mindestens zweijährigen Berufserfahrung und einer erworbenen Hochschulzugangsberechtigung, durch finanzielle Unterstützung zum Studium motiviert und in diesem gefördert werden. Mit dem Wettbewerb „Aufstieg durch Bildung: Offene Hochschulen“ sollen innovative Hochschulkonzepte finanziell gefördert werden, die durch einen stärkeren Fokus auf die Nachfrageseite, insbesondere auch auf berufstätige Personen, dazu beitragen, lebenslanges wissenschaftliches Lernen und Durchlässigkeit zu fördern. Dies kann auf unterschiedlichste Weise erfolgen: durch berufsbegleitende Studiengänge oder Studienmodule, duale Studiengänge oder andere Formen der Praxisintegration (BMBF 2014).

Seit dem Beginn der Qualifizierungsinitiative gab es immer wieder Anlässe, die zu einer Diskussion über Durchlässigkeit zwischen den Akteuren der Berufsund Hochschulbildung geführt haben. Diese Anlässe waren aber weniger Auslöser von Konflikten zwischen den Akteuren, sie wurden vielmehr genutzt, um das jeweilige Verständnis von Durchlässigkeit und der Problemlage zu unterstreichen und zu aktualisieren. Auffällig ist zudem, dass beide Programme und damit auch ein größerer Teil der Debatte in diesem Kontext sich vor allem um den Aspekt des Umgangs mit heterogenen Bedürfnissen der Studierenden und insbesondere beruflich Qualifizierten befassten. Im zweiten Untersuchungszeitraum ist demnach neben Anrechnung auch die Frage des Umgangs mit Heterogenität fast gänzlich neu und auch zentral auf die Agenda gekommen.

\section{Debatte zu den Regelungen des Hochschulzugangs beruflich Qualifizierter}

Bereits im ersten Untersuchungszeitraum wurde die Unübersichtlichkeit der Regelungen zum Hochschulzugang für beruflich Qualifizierte ohne schulische Hochschulzugangsberechtigung wiederholt kritisiert. Zudem wurde eine Verbreiterung des Zugangs immer wieder gefordert (vgl. Kapitel 8.1). Während diese 
Forderungen auch nach 1998 anhielten, waren sie nicht mehr der Hauptaspekt von Durchlässigkeit, über den debattiert worden ist, und die Forderungen gingen meist in den anderen Debatten auf. Eine Ausnahme bildeten allerdings die Jahre 2008 bis 2010. 2008 war das Jahr, in dem diese Forderungen nochmal besonders deutlich wurden. Anlässlich des Bildungsgipfels (vgl. DGB 2008b), aber auch in einer gemeinsamen Erklärung des DIHK und der HRK (2008) wurden die Länder wiederum aufgefordert, transparente und einheitliche Wege in das Studium für beruflich Qualifizierte zu etablieren, da die unterschiedlichen Regelungen Barrieren für interessierte beruflich Qualifizierte darstellten. 2009 reagierte dann die KMK auf diese seit Jahren bestehenden Forderungen mit einem viel beachteten Beschluss zum „Hochschulzugang für beruflich qualifizierte Bewerber ohne schulische Hochschulzugangsberechtigung“" In diesem sollten bundesweit einheitliche Mindeststandards für die Regelungen des Hochschulzugangs beruflich Qualifizierter festgelegt werden, die dann von den Ländern umgesetzt werden sollten. Die Regelungen sehen vor, dass Absolvent_innen einer Aufstiegsfortbildung eine allgemeine Hochschulzugangsberechtigung erhalten. Zudem können Absolvent_innen einer beruflichen Erstausbildung eine fachgebundene Hochschulreife nach einer studienrelevanten Berufspraxis von drei Jahren nach Abschluss der Ausbildung und dem Bestehen einer Eignungsprüfung oder eines Probestudium erhalten (KMK 2009d).

Mit diesem Beschluss wird erstmals ein beruflicher Abschluss, und zwar der der Aufstiegsfortbildung, dem allgemeinbildenden Abitur als Hochschulzugangsberechtigung gleichgestellt, was als Paradigmenwechsel angesehen werden kann. Und auch wenn die Länder bereits in den 1990er Jahren begonnen hatten, in den Hochschulgesetzen den Zugang für beruflich Qualifizierte zu liberalisieren, so kann festgestellt werden, dass der KMK-Beschluss insbesondere die Liberalisierung der Regelungen für die Absolventinnen der Aufstiegsfortbildung in vielen Ländern befördert hat (vgl. Ulbricht 2012a). Letztlich sind KMK-Beschlüsse nicht bindend, aber da sie nach dem Einstimmigkeitsprinzip beschlossen werden, stellen sie einen Minimalkonsens der Länder und damit wirksame Mindeststandards dar. Die Reaktion auf den lange ersehnten Beschluss der Kultusminister war daher auch weitgehend positiv (vgl. BMBF 2011a). Und als Folge wurden die einzelnen Länder angehalten, diese Regelungen auch tatsächlich umzusetzen.

\section{Debatte zu den Auswirkungen der Bologna-Reformen}

2009 war aber nicht nur das Jahr, in dem der KMK-Beschluss zum Hochschulzugang erlassen wurde, auch fanden bundesweit Studierendenproteste als Reaktion auf die Hochschulreformen der vorangegangenen Jahre statt. Auch zehn Jahre nach dem Start des Bologna-Prozesses wurde in der Bildungspolitik in den Jahren 2009 bis 2011 generell viel über die Hochschulreformen und die notwendigen Verbesserungen diskutiert. In der Kritik standen vor allem das neu einge- 
führte gegliederte Studienstruktursystem (B.A, M.A.), welches zu stark vorstrukturiert sowie verschult sei und zu einem zu hohem Leistungsdruck auf die Studierenden führe. ${ }^{111}$ Zudem seien die Ziele der Reformen, eine bessere Betreuung der Studierenden oder mehr Mobilität, nicht erreicht worden.

Natürlich wurden die Bologna-Reformen schon vorher diskutiert, aber gerade in dieser Zeit wurde auch das Thema Durchlässigkeit immer wieder aufgegriffen. Dabei ging es zu großen Teilen um Durchlässigkeit innerhalb des Hochschulsystems. Während die KMK (2009i) allgemein die Einführung des Bachelors und des Masters als einen wichtigen Schritt zu mehr Durchlässigkeit und Flexibilität im Hochschulsystem betrachtete, forderte insbesondere der DGB (2010c), dass der Zugang zum Master nicht beschränkt, sondern für alle zugänglich sein müsste, damit keine neuen Barrieren im Hochschulsystem entstehen würden.

Aber auch die Durchlässigkeit von der Berufs- zur Hochschulbildung wurde in der Debatte um die Bologna-Reformen diskutiert. Zum einen geschah dies im Zusammenhang mit der sogenannten sozialen Dimension, d.h., dass der Zugang für nichttraditionelle Studierende weiter verbessert werden müsste. Damit einher ging die Forderung nach einer besseren Betreuung der Studierenden, wofür die Hochschulen auch die finanzielle Ausstattung benötigten (DGB 2006a, 2008b). Zudem wurden in der Debatte die Hochschulen immer stärker als Lernort im Rahmen des lebenslangen Lernens konstruiert, was wiederum mit einer stärkeren Öffnung der Hochschulen einhergehen müsste (HRK 2007c). Da vor allem die Gruppe der nichttraditionellen Studierenden in Deutschland im internationalen Vergleich relativ klein ausfiel, wurden die neu entstandenen Pilotprogramme, wie der Wettbewerb „Aufstieg durch Bildung: Offene Hochschulen“ oder die ANKOM-Initiative, als besonders wichtig hervorgehoben. Es sollten in den nationalen Bologna-Berichten, die an die Follow-up Group geschickt werden, daher ein größeres Augenmerk auf qualitative Veränderungen im Sinnes dieser Programme anstatt auf die quantitativen Veränderungen gerichtet werden (KMK 2011b).

\section{Debatte über den deutschen Qualifikationsrahmen}

Der letzte große Themenkomplex, in dem Durchlässigkeit wiederholt eine bedeutende Rolle spielte, war die Debatte um den europäischen, aber vor allem den deutschen Qualifikationsrahmen. Als mit dem Maastricht-Kommuniqué 2004 im Kopenhagen-Prozess die Einführung eines europäischen Qualifikationsrahmens beschlossen wurde, dessen ausgearbeitete Version später von der europäischen Kommission 2006 vorgeschlagen wurde, wurde auch in Deutschland entschieden, einen bildungsbereichsübergreifenden deutschen Qualifikationsrahmen (DQR) zu erarbeiten. Ziel war es, einen kompatiblen ebenfalls lernergebnisorientierten

111 Einen Überblick der studentischen Kritik ist hier zu finden auf der Homepage von Bundesweiter Bildungsstreik (2014). 
Rahmen zu entwerfen, der dann zu einer größeren Transparenz und Durchlässigkeit zwischen einzelnen nationalen Bildungsbereichen, aber auch europäischen Bildungssystemen führen sollte. Zu Beginn der Debatte wurde die Frage des Qualifikationsrahmens als eine Möglichkeit gesehen, Reformen im deutschen Bildungssystem anzustoßen und die deutsche Berufsausbildung international besser zu platzieren (DIHK 2006b). Beide Hoffnungen werden bis heute mit dem DQR verbunden. Nachdem die Erarbeitung eines deutschen Qualifikationsrahmens beschlossen war, wurde ein Arbeitskreis mit den wichtigsten Akteuren im deutschen Bildungssystem, inklusive der in dieser Arbeit betrachteten Akteure, berufen, der im Februar 2009 einen ersten Diskussionsvorschlag für den DQR vorlegte. Ziel war es, über die verschiedenen Bildungsbereiche hinweg zu einem gemeinsamen Verständnis des DQR, aber vor allem zu einer gemeinsamen Begrifflichkeit zu kommen (Esser 2012).

Der deutsche Diskussionsvorschlag beinhaltete zwei Kompetenzkategorien, die Fachkompetenz und die personale Kompetenz, die beide in jeweils zwei Unterkategorien aufgehen: in Wissen und Fertigkeiten sowie in Sozial- und Selbstkompetenz. Im Gegensatz dazu unterscheidet der EQR Kenntnisse, Fertigkeiten und Kompetenzen. Der Unterschied zum EQR liegt darin begründet, dass im DQR die personale Kompetenz in Sozial- und Selbstkompetenz unterschieden wurde, was den Bezug zur (beruflichen) Handlungskompetenz, auf die letztlich die deutsche Kompetenzdefinition abzielte, ermöglichte. Der europäische Kompetenzbegriff wurde als nicht adäquat für die Beschreibung der Handlungskompetenz angesehen, die im deutschen Bildungs- und insbesondere Berufsbildungssystem erlangt wird (vgl. DGB 2009c). Die gemeinsame und konsensuale Erarbeitung des Kompetenzbegriffs und der Deskriptoren für die acht unterschiedlichen Niveaustufen fand unter weitgehendem Ausschluss der (Fach-)Öffentlichkeit statt, so dass die Diskussionen erst nach der Veröffentlichung des Vorschlags wieder verstärkt geführt wurden.

In der zweiten Erarbeitungsphase (2009 und 2010) wurde der Entwurf des DQR nun in vier ausgewählten Berufs- und Tätigkeitsfeldern (Gesundheit, Handel, Metall/Elektro und IT-Bereich) durch Expert_innen der Bildungs- und Berufsbereiche erprobt, um die Anwendbarkeit der gestuften Kompetenzmatrix zu prüfen. Dabei wurden die bestehenden Qualifikationen exemplarisch den unterschiedlichen Niveaustufen zugeordnet ${ }^{112}$. Auf Basis dieser Arbeit in den vier Branchen sollte dann eine generelle Zuordnung von deutschen Bildungsqualifikationen in einem dritten Schritt erfolgen. Diese Phase endete vorläufig am 31.01.2012 mit einer Vereinbarung zur Umsetzung des EQR auf einen deutschen Qualifikationsrahmen zwischen Bund, Ländern sowie Spitzenvertretern folgender Organisationen: Zentralverband des Deutschen Handwerks, Bundesvereini-

112 Eine Dokumentation der Arbeit der vier Arbeitsgruppen kann auf der offiziellen Homepage zum deutschen Qualifikationsrahmen nachgelesen werden (vgl. BMBF/KMK 2014). 
gung der Deutschen Arbeitgeberverbände, Deutscher Industrie und Handelskammertag, Deutscher Gewerkschaftsbund, Bundesinstitut für Berufsbildung (BMBF et al. 2012).

Im Gegensatz zu den zuvor dargestellten Debatten waren die zum DQR durch eine starke Polarisierung der Positionen gekennzeichnet. Zwar arbeiteten die unterschiedlichen Akteure der Berufs- und Hochschulbildung erstmals alle gemeinsam an einem bildungsbereichsübergreifenden Instrument, dies ging aber einher mit vielen Meinungsverschiedenheiten. Im Hinblick auf Durchlässigkeit können drei größere Streitpunkte identifiziert werden: a) die Rolle und zukünftige Bedeutung des DQR, b) der verwendete Kompetenzbegriff bzw. die ausgearbeiteten Deskriptoren der Niveaustufen und c) die Zuordnung nationaler Bildungsabschlüsse.

\section{a) Bedeutung des $D Q R$}

Die Hauptunterschiede in den Auffassungen zur zukünftigen Bedeutung des DQR lassen sich in zwei Positionen zusammenfassen. Auf der einen Seite stehen Akteure wie der DGB oder der DIHK, die letztlich im DQR einen Reformmotor für das deutsche Bildungssystem hin zu mehr Durchlässigkeit und Gleichwertigkeit von beruflicher Bildung und Hochschulbildung sehen, wobei der Qualifikationsrahmen tatsächliche Konsequenzen für bestehende und zukünftige Regulierungen im Bildungsbereich haben sollte (vgl. z.B. DIHK 2006a, 2010c; BMWT et al. 2009;DGB 2008a, 2009c). Auf der anderen Seite stehen die Akteure, die vor einer „Überfrachtung“ des Qualifikationsrahmens warnen (KMK 2010a). Dieser sei ein Informationsinstrument und würde als Instrument nur entwickelt, um deutsche Abschlüsse mit dem EQR referenzieren zu können. Diese Position wird vor allem von den Akteuren der höheren Allgemeinbildung vertreten, der KMK (2009g, 2010a) und der HRK (2007c, 2009b).

\section{b) Verwendeter Kompetenzbegriff}

Auch wenn der für den DQR verwendete Kompetenzbegriff und die dazugehörigen Deskriptoren von den im Arbeitskreis sitzenden Akteuren gemeinsam beschlossen wurden, kam es auch hier zu Diskussionen. So sah die HRK (2010a, b) die spezifischen Charakteristika der Kompetenzen aus der Hochschulbildung nicht ausreichend berücksichtigt. „Die spezifische wissenschaftliche Problemlösungskompetenz ist als Qualifikationsanspruch auf den höheren Niveaus zu undeutlich formuliert" (HRK 2010b). Andere Akteure, darunter der DGB, zeigten sich von der Kritik irritiert und sahen darin vielmehr den Versuch der Hochschulen, die „Zuordnungshoheit“ für die höchsten Niveaustufen (6-8) zu behalten (DGB 2010b). Im Hinblick auf Durchlässigkeit war diese Diskussion insofern wichtig, da es darum ging, auch für die obersten Niveaustufen eine Beschreibung $\mathrm{zu}$ finden, die bildungsbereichsübergreifend funktioniert und nicht einen Bereich gegenüber einem anderen begünstigt. So müsste, wenn der DQR auch für Fragen 
der Anrechnung oder des Zugangs relevant werden sollte, auch eine gute Erfassung der Kompetenzen in allen Bildungsbereichen ermöglicht werden. Dies gilt umso mehr, zumal der DQR perspektivisch auch für non-formal und informell erworbene Kompetenzen gelten soll. Sind Deskriptoren dann so gelagert, dass bestimmte Kompetenzen begünstigt werden, die eher in spezifischen z.B. akademischen Bereichen erworben werden, wirkt sich dies auch auf die Möglichkeiten der Anerkennung von Kompetenzen aus.

Die Erarbeitung der Kompetenzbegriffe im Vergleich zu den europäischen Begrifflichkeiten sollte zu einer besseren Passfähigkeit für das deutsche Bildungssystem mit seinem starken Berufsbildungsbereich führen. Während die europäische Kompetenzdefinition von einigen Akteuren als zu stark auf kognitives Wissen fokussierend empfunden wurde, war die Integration von Sozial- und Selbstkompetenz ein wichtiger Schritt. (DGB 2009c). Von anderen Akteuren wie den Hochschulen wird der breitere Kompetenzbegriff eher als Schwierigkeit empfunden: Die Beschreibung der unterschiedlichen Niveaustufen ist schließlich entscheidend für die Zuordnung von Abschlüssen bzw. von Kompetenzen. Im bisherigen Prozess ging es vor allem darum, die bestehenden formalen Bildungsabschlüsse in die Kompetenzmatrix einzuordnen. Hier zeigten sich die größten Auseinandersetzungen zwischen den Akteuren.

\section{c) Zuordnung nationaler Bildungsabschlüsse}

Hauptstreitpunkt in Bezug auf den DQR war die Zuordnung beruflicher Abschlüsse in Relation zu schulrechtlichen Abschlüssen, insbesondere der allgemeinen Hochschulreife (Esser 2012).

Die KMK (2010d) präferierte die Zuordnung der allgemeinen und fachgebundenen Hochschulreife auf Niveau 5 zusammen mit ,anspruchsvollen“ beruflichen Abschlüssen, während die Fachhochschulreife und weitere berufliche Abschlüsse der Stufe 4 zugeordnet werden sollten. Die Akteure der beruflichen Bildung dagegen sahen die Zuordnung von den drei- bis dreieinhalbjährigen Ausbildungsberufen zusammen mit allen Formen der Hochschulreife auf Stufe 4 vor und kritisierten die KMK-Forderungen stark. Eine Einigung konnte nicht erreicht werden, so dass von den beteiligten Akteuren vereinbart wurde, die schulischen Abschlüsse in Deutschland nicht dem Qualifikationsrahmen zuzuordnen, und somit allein für die Abschlüsse der Berufs- und Hochschulbildung eine Zuordnung gefunden wurde (vgl. DQR 2013).

Zudem wurde beschlossen, dass bestehende Zuordnungen nach fünf Jahren erneut beraten werden, und zwar auf Basis kompetenzorientierter Ordnungsmittel, die im Entscheidungszeitraum nicht zur Verfügung standen, sowie vor dem Hintergrund nationaler und internationaler Erfahrungen (vgl. BMBF et al. 2012). In diesen fünf Jahren sollte auch die Frage der Zuordnung der schulischen Abschlüsse gelöst werden.

Betrachtet man alle Debatten im zweiten Untersuchungszeitraum zusammen, fällt auf, dass zwei besonders dominant waren: die Diskussionen zur Anrechnung 
und die Diskussionen über die Entwicklung des DQR. Aber es war vor allem Letztere, die besonders stark polarisierte.

Anhand der Debatten wird bereits deutlich, dass erstens Durchlässigkeit während der Europäisierungsprozesse eine große Rolle in der deutschen Bildungspolitik spielt. Zweitens kann festgestellt werden, dass bereits innerhalb der Debatten kontrovers über Durchlässigkeit diskutiert wurde. Daraus ist zu schließen, dass auch im zweiten Untersuchungszeitraum Deutungskämpfe in den Durchlässigkeitsdiskursen vorzufinden sein werden. Wie genau diese aber nach 1998 strukturiert sind, soll nachfolgend dargestellt werden.

\subsubsection{Deutsche Diskurse 1999-2012: Durchlässigkeit als Norm zwischen Bildungsschisma und neuer Gleichwertigkeit}

Wie bereits im Zeitraum vor 1999 können für den zweiten Untersuchungszeitraum zwei antagonistische Diskurse, ein strukturkritischer und ein strukturkonservativer, identifiziert werden, die eine Weiterentwicklung der bestehenden Diskurse darstellen. Auch können analog zum ersten Zeitraum wiederum die drei Argumentationslogiken - strukturkritisch-universalitisch, strukturkritisch-funktionalistisch und strukturkonservativ-funktionalistisch - unterschieden werden, die die Diskurse strukturieren. Im Folgenden werden daher zuerst die einzelnen Argumentationslogiken, anschließend die Strukturen der einzelnen Diskursstränge anhand des heuristischen Durchlässigkeitskonzepts und schließlich die Akteurspositionen und die rekonstruierten Strategien expliziert.

\section{Argumentationslogiken der Diskurse}

Auch für den zweiten Zeitraum soll dargestellt werden, warum und in welchen Zusammenhängen Durchlässigkeit und damit auch das Verhältnis von Berufs- zu Hochschulbildung thematisiert wird sowie was das Problem im Hinblick auf Durchlässigkeit ist. Dies wird in den Argumentationslogiken sichtbar, welche als Weiterentwicklung der bereits für den ersten Untersuchungszeitraum identifizierten Logiken angesehen werden können. Doch wie sieht die Weiterentwicklung aus? Haben sich die Argumentationen und deren Bedeutung innerhalb der Diskurse geändert? Welche Argumentationslogiken dominieren die Diskurse? Um diese Fragen zu beantworten, werden die einzelnen Argumentationslogiken wiederum einzeln dargestellt und anschließend in Tabelle 14 zusammengefasst.

\section{Strukturkritisch-funktionalistischer Diskursstrang (1999-2012): Durchlässigkeit als funktionales Erfordernis der Arbeitswelt}

Der strukturkritisch-funktionalistische Diskursstrang ist im Zeitraum nach 1998 der die Diskurse dominierende Strang, der von allen Akteuren getragen wird. Das heißt, wenn Durchlässigkeit zwischen Berufs- und Hochschulbildung thematisiert 
wurde, kam dies sehr häufig im Rahmen dieser Argumentationslogik vor. Doch wie wird der Bedarf von Durchlässigkeit begründet und warum ist Durchlässigkeit ein wichtiges zu behandelndes Problem?

Erhöhte Durchlässigkeit zwischen Berufs- und Hochschulbildung sei im funktionalistischen strukturkritischen Diskursstrang notwendig, da sowohl auf gesellschaftlicher Ebene als auch auf Betriebsebene ein Strukturwandel stattgefunden hat bzw. stattfindet. Stichworte sind der gestiegene Wettbewerb durch die Globalisierung, der Wandel hin zur Wissensgesellschaft, der technologische und die demografische Entwicklung. Fazit ist, dass gesellschaftlich eine höhere Qualifizierung der Individuen über den gesamten Lebenslauf notwendig ist und dass durch eine bessere Verzahnung der Bildungsbereiche mehr Humanressourcen genutzt werden können und auf diese Weise dem Fachkräftemangel begegnet werden kann (vgl. z.B. BMBF 2002, 2009c, 2011a; DIHK 2007c, 2010c; HRK 2011a; DGB 2002, 2008b, 2009b).

Aus Sicht des DIHK handelt es sich um ein perspektivisch wichtiges Thema. Denn die neue Arbeitswelt verlangt eine erhöhte Durchlässigkeit der Bildungswege, da die Erwerbsbiografien völlig neue Muster einnehmen. Weiter kann sich Deutschland die Dualität zweier Bildungssäulen [Berufs-und Hochschulbildung, NB] ohne Brückenschläge zwischen ihnen nicht leisten. Und schließlich kommt aufgrund der Alterung der Belegschaften auf die deutsche Erwerbsbevölkerung ein Engpass an Arbeitskräften hinzu, wodurch eine Wiederaufnahme von Qualifikationswegen in der zweiten Hälfte der Erwerbsbiografie häufiger erforderlich wird. (DIHK 2007c)

Ein erhöhter Anpassungsdruck wird zusätzlich anerkannt durch internationale Forderungen nach durchlässigen Strukturen, wie von europäischer Ebene oder der OECD, die diese als Mittel zur Anpassung an den strukturellen Wandel anmahnen (KMK et al. 2003).

Aber auch die funktionale Bedeutung von Durchlässigkeit für die Individuen wird betrachtet. Eine Verbindung der Bildungsbereiche sei notwendig, um Karriere- und Erwerbsverläufe effizient zu gestalten und individuell entwickeln zu können. Zudem ermöglichen Durchlässigkeit und die damit einhergehenden individuellen Bildungsoptionen eine Erhöhung der individuellen „Beschäftigungsfähigkeit" und würden das Arbeitslosigkeitsrisiko minimieren (BMBF 2001b). Darüber hinaus sind es vor allem Hochschulabschlüsse, die neben den Fachkenntnissen auch einen gewissen Status verleihen, der in der Arbeitswelt anerkannt wird (DIHK 2007c).

Ob gesamtgesellschaftlich oder individuell, argumentiert wird in beiden Dimensionen fast ausschließlich vom Bedarf des Arbeitsmarkts aus, an den die Bildungsstrukturen und die individuellen Bildungsbiografien angepasst werden müssen.

Es darf nicht sein, dass nur die Logik von Bildungsinfrastrukturen im Vordergrund steht. Das Endziel darf nicht aus den Augen gelassen werden, dass vor allem der Mittelstand darauf angewiesen ist, Fortbildungsabsolventen auf hohem Niveau weiterzubilden. Denn gerade in mittelständischen Unternehmen arbeiten Personen, die ihre 
Qualifizierung in der beruflichen Aus- und Weiterbildung durchlaufen haben. (DIHK 2007c)

Ein weiterer Grund, die Durchlässigkeit zwischen beruflicher Bildung und Hochschulbildung zu erhöhen, wird zudem darin gesehen, dass das deutsche Berufsbildungssystem sowohl national als auch international eine höhere Wertschätzung erhalten solle (vgl. z.B. DIHK 2006b; BMBF 2004b, 2010a).

Schließlich wird auch von Seiten der Hochschulen die Chance erkannt, beruflich Qualifizierte als Zielgruppe aufzunehmen, weil Teilnahmegebühren für Kurse zur wissenschaftlichen Weiterbildung die Einnahmen der Hochschulen erhöhen (HRK 2011b).

Im Vergleich zum ersten Zeitraum wird deutlich, dass zwar ebenfalls der Strukturwandel der Wirtschaft als Grund angeführt wird, aber insgesamt der Fokus weniger darauf liegt, die Anziehungskraft der Berufsbildung zu erhöhen und mehr Individuen zu einer Facharbeiterausbildung zu motivieren, als tatsächlich mehr beruflich Qualifizierte in die Hochschulbildung zu bekommen, weil die höhere Qualifikation im Arbeitsleben gefordert wird. Neu ist zudem die Bedeutung, die lebenslanges Lernen im Diskurs über Durchlässigkeit in Deutschland einnimmt und dass von Seiten der Hochschulen beruflich Qualifizierte als Einnahmequelle und somit als Ressource betrachtet werden.

\section{Strukturkritisch-universalistischer Diskursstrang (1999-2012): Durchlässigkeit als Recht auf Bildung basierend auf Gleichwertigkeit}

Charakteristisch für den universalistischen strukturkritischen Diskurs ist das Verständnis der Bedeutung von Bildung für die Gesellschaft. Durchlässigkeit wird dann nicht allein aus funktionalistischen auf die Arbeitswelt ausgerichteten Gründen gefordert, sondern aus dem Verständnis heraus, dass Bildung ein universales Menschenrecht darstellt. Barrieren, die den Zugang zu Bildung beeinträchtigen, stellen eine Einschränkung dieses Rechts dar. Insofern kann Durchlässigkeit als eine strukturelle Voraussetzung für die Gewährung des Bildungsrechts gelten.

Die Umsetzung dieses Rechts ermöglicht wirtschaftliche und politische Emanzipation und Teilhabe. Daher dürfen sich Angebote der Weiterbildung nicht nach dem Gesetz von Angebot und Nachfrage richten. (DGB 2006a: 38)

Das Recht auf Bildung begründet sich aus der Bedeutung von Bildung sowohl für das Individuum als auch die Gesellschaft. Individuell dient Bildung neben der Entfaltung des Arbeitskraftpotenzials auch der Entfaltung der Persönlichkeit, der individuellen Emanzipation, und der Schaffung von Teilhabechancen in der Gesellschaft (vgl. DGB 2002, 2008a). Ziel ist daher, gleiche Bildungschancen, aber auch Erwerbschancen für alle Individuen zu ermöglichen.

Gleiche Bildungschancen zu realisieren ist und bleibt daher oberstes Ziel von gewerkschaftlicher Bildungspolitik. Dabei geht es den Gewerkschaften nicht allein um formal gleiche Zugangsrechte, sondern auch um die besondere Förderung derjenigen, die in unserer Gesellschaft benachteiligt sind. (DGB 2002: 24) 
Gesellschaftlich wird Bildung nicht nur als notwendig angesehen, um dem Strukturwandel in Demografie, Technologie und Arbeitswelt durch eine Höherqualifikation weiter Teile der Bevölkerung zu begegnen. Vielmehr ist die Frage der Bildung eng verbunden mit der Frage des gesellschaftlichen Zusammenhalts. Die Ermöglichung von Bildung für alle wird als notwendig angesehen, um Bildungsarmut und soziale Ungleichheiten zu verringern (vgl. DGB 2008b). Durchlässigkeit ist somit ein wichtiger Faktor, um soziale Chancengleichheit zu erreichen (vgl. BMBF 2009b; KMK 2011b).

Voraussetzung dafür, dass tatsächliche Chancengleichheit zwischen Absolvent_innen der unterschiedlichen Bildungsbereiche entstehen kann, ist, dass berufliche Bildung und Hochschulbildung als gleichwertig anerkannt werden. Dies wurde als ein Hauptproblem angesehen, das durch Reformen zu beheben sei. Der bestehenden Gleichwertigkeit der Kompetenzen von Berufs- und Hochschulbildung muss dann auch Rechnung getragen werden, indem die beruflich Qualifizierten die gleichen Chancen im Bildungs- und Erwerbssystem erhalten (DGB 2002, 2009c; BMBF 2003a, 2010a; KMK 2011a, b; DIHK 2008b).

Die Durchlässigkeit am Übergang zur Hochschule ist damit nicht nur eine Maßnahme zur Ausschöpfung des Potenzials an Wissen in einem rohstoffarmen Land, sie fördert ebenso die Chancengleichheit der Qualifizierungswege: Allgemeinbildung und Berufsbildung, die Gleichwertigkeit von allgemeiner und beruflicher Bildung. (KMK 2009h: 2)

Auch in der Bezeichnung von Abschlüssen könne die Gleichwertigkeit sichtbar gemacht werden, wenn Fortbildungsprüfungen zusätzlich auch als Bachelorabschluss betitelt werden (DIHK/HRK 2008).

Gleichwertigkeit im Zusammenhang mit dem Recht auf Bildung ist somit die Grundlage der Durchlässigkeitsforderungen im universalistischen strukturkritischen Diskurs. In diesem Zusammenhang wird insbesondere auf den deutschen Qualifikationsrahmen eine große Hoffnung gelegt.

Ob der Deutsche Qualifikationsrahmen auch im Sinne der Gewerkschaften erfolgreich wird, hängt davon $a b$, ob die für uns entscheidenden Ziele, nämlich Förderung von Durchlässigkeit und Gleichwertigkeit zwischen allgemeiner und beruflicher Bildung, gefördert werden oder nicht. (DGB 2010b)

Die universalistische Argumentationslogik hat sich insgesamt im Vergleich zum ersten Zeitraum nicht stark geändert. Allein die gesellschaftliche Bedeutung durchlässiger Bildungsstrukturen für den sozialen Zusammenhalt und die Verringerung sozialer Ungleichheiten wurden stärker expliziert. Insgesamt kann aber eine Zunahme der universalistischen Argumentation festgestellt werden, wobei die funktionalistische strukturkritische weiterhin die klar dominierende bleibt. 
Tabelle 14 Argumentationslogiken der deutschen Diskurse (1999-2012)

\begin{tabular}{|c|c|c|}
\hline \multicolumn{2}{|c|}{ Strukturkritisch } & \multirow{2}{*}{$\begin{array}{c}\text { Strukturkonservativ } \\
\text { Funktionalistisch }\end{array}$} \\
\hline Universalistisch & Funktionalistisch & \\
\hline $\begin{array}{l}\text { Gesellschaft: } \\
\text { Durchlässigkeit als Weg zu } \\
\text { größerer Chancengleichheit, } \\
\text { zur Verringerung von sozialen } \\
\text { Ungleichheiten und Bildungsar- } \\
\text { mut } \\
\text { Gleichwertigkeit der Bildungs- } \\
\text { wege }\end{array}$ & $\begin{array}{l}\text { Gesellschaft: } \\
\text { Durchlässigkeit als Antwort auf } \\
\text { den Strukturwandel, Fachkräf- } \\
\text { temangel,auf externe Anforde- } \\
\text { rungen (OECD/EU/Bologna } \\
\text { und Kopenhagen) } \\
\text { Einnahmequelle für Hochschu- } \\
\text { len }\end{array}$ & $\begin{array}{l}\text { Gesellschaft: } \\
\text { Unkontrollierte Durchlässigkeit } \\
\text { (für beruflich Qualifizierte) als } \\
\text { Bedrohung der Qualität der } \\
\text { Hochschulbildung, als Ressour- } \\
\text { cenverschwendung, d.h. Durch- } \\
\text { lässigkeit nur unter Kontrolle der } \\
\text { Akteure der höheren Allgemein- } \\
\text { bildung }\end{array}$ \\
\hline $\begin{array}{l}\text { Individuum: } \\
\text { Durchlässigkeit zur Umsetzung } \\
\text { des Rechts auf (Weiter-) } \\
\text { Bildung, } \\
\text { zur Erlangung gesellschaftli- } \\
\text { cher Teilhabe und Persönlich- } \\
\text { keitsentfaltung sowie } \\
\text { zur Sicherung gleichwertiger } \\
\text { beruflicher Entwicklungsmög- } \\
\text { lichkeiten }\end{array}$ & $\begin{array}{l}\text { Individuum: } \\
\text { Durchlässigkeit als Möglichkeit } \\
\text { für individuelle Karrieren, Ver- } \\
\text { ringerung des individuellen Ar- } \\
\text { beitslosigkeitsrisikos bzw. Er- } \\
\text { höhung der Beschäftigungsfä- } \\
\text { higkeit }\end{array}$ & $\begin{array}{l}\text { Individuum: } \\
\text { Kontrolle des Zugangs als } \\
\text { Schutz vor individuellem Versa- } \\
\text { gen }\end{array}$ \\
\hline
\end{tabular}

Quelle: Darstellung der Verfasserin

\section{Strukturkonservativ-funktionalistischer Diskursstrang (1999-2012): Durchlässigkeit nach Maßstab der höheren Allgemeinbildung zur Sicherung des Qualitätsniveaus}

Auch die Argumentationslogik im strukturkonservativen Diskurs hat sich kaum geändert im Vergleich zum ersten Zeitraum. So ist wiederum eine funktionalistische Argumentation leitend. Auf gesellschaftlicher Ebene stellt Durchlässigkeit zwischen Berufs- und Hochschulbildung in dieser Logik eine mögliche Bedrohung der Qualität der Hochschulbildung dar, die es zu sichern gilt. Dies ist am besten gewährleistet, wenn Durchlässigkeit nicht unkontrolliert, sondern nach dem Maßstab der Akteure der höheren Allgemeinbildung erfolgt (vgl. HRK 2007c, 2008b; KMK 2000b, 2011b). Zudem kann unkontrollierte Durchlässigkeit zu einer unnötigen Ressourcenverschwendung führen, da Individuen, die nicht für ein Studium geeignet seien und dieses abbrechen würden, unnötig Personalund Sachressourcen verbrauchten (vgl. HRK 2010a 2010b).Wiederum wird die Frage der Gleichwertigkeit von beruflicher und Hochschulbildung im strukturkonservativen Diskursstrang nicht als Ideal thematisiert. Auf individueller Ebene wird unkontrollierte Durchlässigkeit ebenfalls wieder als Gefahr gesehen, die den 
Individuen falsche Hoffnungen macht und individuelles Versagen mit all den persönlichen emotionalen Konsequenzen nach sich ziehen kann (HRK 2010b). Auch wenn die strukturkonservative funktionalistische Argumentationslogik stark der aus dem ersten Zeitraum ähnelt, kann doch ein großer Unterschied festgestellt werden, der auch die weitere Diskursstruktur deutlich beeinflusst. So wird Durchlässigkeit als relevantes Thema zum einen wahrgenommen und eine kontrollierte Durchlässigkeit nach Maßstab der höheren Allgemeinbildung akzeptiert. Im ersten Zeitraum dagegen wurde generell die Relevanz der Diskussion über Durchlässigkeit infrage gestellt und letztlich kaum behandelt.

\section{Vergleich der Strukturmerkmale der deutschen Diskurse}

Wie wird Durchlässigkeit im zweiten Untersuchungszeitraum konstruiert? Welche Aspekte von Durchlässigkeit stehen nach 1999 im Vordergrund? Insgesamt kann im Gegensatz zum ersten Zeitraum eine Verbreiterung der Diskussion über Durchlässigkeit und damit auch des Verständnisses festgestellt werden. So wird nicht nur über die Fragen des Zugangs, sondern erstmals auch ausführlich über Möglichkeiten der Anrechnung gesprochen. Ebenfalls viel stärker in den Fokus rückt die Beschäftigung mit Strukturen, die sich auf die heterogenen Bedürfnisse der Studierenden und der beruflich Qualifizierten im Besonderen beziehen. Schließlich wurde Durchlässigkeit wie im ersten Zeitraum auch im Zusammenhang mit organisationalen Verbindungen behandelt.

Nachfolgend werden die den unterschiedlichen Argumentationslogiken entsprechenden Diskursstrukturen für die Durchlässigkeitsaspekte einander gegenübergestellt. Dabei erfolgt die Darstellung der Strukturen als Querschnitt durch den gesamten Zeitraum, wobei auf explizite Entwicklungen, wenn es sie denn gab, hingewiesen wird. Am Ende jeden Abschnittes werden die wichtigsten Erkenntnisse tabellarisch festgehalten.

\section{Zugang}

Wie auch im ersten Zeitraum waren Fragen des Hochschulzugangs im gesamten zweiten Zeitraum präsent, wobei ein Höhepunkt der Diskussion mit dem KMKBeschluss von 2009 festzustellen ist. Durch diesen können Inhaber_innen eines beruflichen Fortbildungsabschlusses die allgemeine Hochschulzugangsberechtigung zugesprochen bekommen. Dieser Beschluss zeigt, dass sich die Bedeutung der Diskursstränge im zweiten Zeitraum in Fragen des Zugangs verschoben hat. Berechtigungen über Berufsbildung wurden zwar vereinzelt im ersten Zeitraum gefordert, wurden aber von den Akteuren der höheren Allgemeinbildung zurückgewiesen. Die Einführung von Berechtigungen im beruflichen Bildungswesen stellt damit einen großen Paradigmenwechsel und die Beendigung des Berechtigungsmonopols der höheren Allgemeinbildung dar. Nachfolgend 
werden die unterschiedlichen Aussagen zu den differenten Zugangsmöglichkeiten, der Zielgruppendefinition sowie der Konstruktion des Bildungssubjektes verglichen (vgl. auch Tabelle 15).

\section{Zugang über Berechtigungen in der beruflichen Bildung}

Wie bereits auch im ersten Untersuchungszeitraum wird im universalistischen strukturkritischen Diskursstrang ein Weg zu mehr Durchlässigkeit gesehen, indem der Zugang für beruflich Qualifizierte über Berechtigungen geöffnet wird. Wiederum können zwei Varianten unterschieden werden: Die eine fordert eine Gleichstellung der beruflichen Erstausbildung mit der allgemeinen Hochschulreife (u.a. DGB 2006a, 2009b), die andere sieht die Gleichstellungsmöglichkeiten eher zwischen Fortbildungsabschlüssen und der allgemeinen Hochschulreife (BMBF 2004a, 2009b; KMK 2009d). Ziel ist die Gleichstellung der beruflichen Bildung mit der allgemeinen Hochschulreife, so dass diese einzige Form der Hochschulzugangsberechtigung abgelöst wird. Auf diesen Weg würde tatsächlich eine Gleichwertigkeit von Berufs- und höherer Allgemeinbildung erreicht werden (vgl. DGB 2004b; BMBF 2001b). Dass ein Teil der Forderungen des universalistischen Diskursstrangs, die Berechtigung über berufliche Fortbildungsabschlüsse, als KMK-Beschluss umgesetzt wurde, zeigt den gewonnenen Einfluss des Diskursstrangs. Allerdings bleibt zu berücksichtigen, dass diese Gleichstellung gleichzeitig eine rechtliche Ungleichwertigkeit beruflicher Erstausbildungen im Vergleich zu allgemeinbildenden Abschlüssen der allgemeinbildenden Sekundarstufe II zementiert.

Im funktionalistischen strukturkritischen Diskursstrang werden im Vergleich zum ersten Zeitraum Berechtigungen nicht mehr explizit als schädlich ausgewiesen, aber sie werden auch nicht gefordert. Im strukturkonservativen Diskurs wird darauf hingewiesen, dass weiterhin die schulischen Berechtigungen die Norm für den Hochschulzugang darstellen (HRK 2011b). Zudem werden Reformen beruflicher Ausbildungsgänge durch z.B. die Integration von Zusatzqualifikationen, so dass diese die von der KMK aufgestellten Kriterien von 1997 (vgl. KMK 1997b) zur Erreichung einer (Fach-)Hochschulreife erfüllen, als nicht notwendig erachtet (KMK 2000b). Eine Gleichstellung von beruflichen Erstausbildungsgängen allgemein und der allgemeinen Hochschulreife, ebenso wie Doppelqualifikationen werden abgelehnt (KMK 2000b, 2009g, 2010d). Es wird deutlich, dass die Sonderstellung des Abiturs im Vergleich zu einer beruflichen Ausbildung im Sekundarstufenbereich II erhalten werden soll. Dies wird auch ersichtlich in dem KMKBeschluss, in dem Absolvent_innen mit beruflichen Erstabschluss erst nach dreijähriger Berufserfahrung und zusätzlicher Zulassungsprüfung an den Hochschulen Zugang erhalten sollen (KMK 2009d). Insofern wird auch hier eine klare Hierarchisierung zwischen Berufs- und höherer Allgemeinbildung festgeschrieben. 


\section{Zugang über Eignungsprïfungen}

Der Zugang über Eignungsprüfungen an den Hochschulen spielt in den beiden funktionalistischen Diskurssträngen sowohl im strukturkonservativen als auch im strukturkritischen Diskurs eine wichtige Rolle.

Im strukturkonservativen Diskurs wird deutlich, dass beruflich Qualifizierte, die nicht über schulische Berechtigungen zum Zugang zu den Hochschulen verfügen, unbedingt von Seiten der Hochschulen auf ihre Studiereignung geprüft werden müssen (vgl. z.B. HRK 2008b; DIHK/HRK 2008; KMK 2009h). Die Entscheidung über die Aufnahme liege klar bei der aufnehmenden Institution und solle so auch unter Kontrolle der Akteure der höheren Allgemeinbildung bleiben.

Im Mittelpunkt der Überlegungen zum Hochschulzugang beruflich Qualifizierter steht auch im funktionalistischen strukturkritischen Diskurs die Ausgestaltung des Dritten Bildungswegs. Zugang solle erfolgen anhand einer Prüfung unter Berücksichtigung der beruflichen Qualifikationen (vgl. BMBF 2009c; DIHK 2007c). Dies könne auf unterschiedliche Weise geschehen: durch Quoten für beruflich Qualifizierte, durch Berücksichtigung von Profilnähe und Notendurchschnitt bei der Entscheidung über die Zulassung. Zulassungskriterien dürfen dabei aber nicht „sachfremd“ sein, wie sie zum Teil in den bestehenden Regelungen $\mathrm{zu}$ finden waren, in denen Alter oder Wohnort über den Zugang zu einer bestimmten Hochschule mit entscheidend waren (vgl. DIHK/HRK 2008). Wichtig sei zudem eine Vereinheitlichung der bestehenden Regelungen, um eine größere Transparenz und auch Effizienz zu gewährleisten (vgl. BMBF 2001b, DGB 2004a, 2008b; DIHK/ZDH 2006; DIHK 2007b; HRK 2008b).

Zentral bei der Frage des Zugangs ist die Selektion, die durch die Hochschulen erfolgen soll. Das Bild, welches dabei im Diskurs konstruiert wird, ist das der Hochschule, die - wie ein Arbeitgeber seine Angestellten - ihre Studierenden so auswählt, dass sie zur Hochschule passen und ihren Job gut erledigen, d.h. erfolgreich und möglichst schnell studieren.

Die Zulassung sollte zukünftig ein besonderes Matching zwischen Eignung des Be-

werbers und Profil der Hochschule ermöglichen. (DIHK 2007c)

Dabei erscheint die Beantwortung der folgenden Frage als zentral: Wie lässt sich garantieren, dass die Bewerber auch wirklich einen Studienerfolg meistern (DIHK 2007c)?

In den Zugangsvorstellungen beider funktionalistischen Diskurse wird zudem ersichtlich, dass das Konstrukt einer vor dem eigentlichen Studium existierenden Studierfähigkeit, einer quasi natürliche Begabung, studieren zu können, weiterhin von Bedeutung ist. Die Zulassungsprüfung und die Berücksichtigung weiterer Kriterien sollen genau diese angenommene Fähigkeit beurteilen. Die Vorstellung im funktionalistischen strukturkritischen Diskursstrang von einer Vorselektion bleibt daher erhalten. Aber im Gegensatz zum Diskurs Anfang der 1990er Jahre ist das Ziel, mehr Individuen an den Hochschulen zu qualifizieren und diese nicht auf eine kleine elitäre Minderheit zu begrenzen. Problematisch ist 
die Frage der Selektion im Bildungsbereich, die im funktionalistischen strukturkritischen Diskursstrang gefordert wird, wenn der Studienanwärter nicht passend erscheint und ihm damit der Zugang verwehrt bleibt. Auch bei anderen Kriterien wie den Quoten oder der Profilnähe, um die Passfähigkeit für eine Studienrichtung auszuloten, wird im Diskurs nicht reflektiert, was mit den Individuen geschieht, die nicht im eigenen Berufsprofil verbleiben wollen, oder wenn die Quoten erfüllt sind. Aus diesem Grund wird im universalistischen strukturkritischen Diskursstrang jegliche Form der Selektion und „Kapazitätsbeschränkung“ beim Zugang abgelehnt (DGB 2006a). Dies entspräche auch nicht dem generellen Recht auf Bildung.

\section{Zugang über Allgemeinbildung}

Wie auch im ersten Zeitraum wird im strukturkonservativen Diskurs der Zugang zur Hochschule über den Weg der höheren Allgemeinbildung als Norm konstruiert. So gebe es für beruflich Qualifizierte genügend Wege, sowohl in beruflichen Schulen als auch über den zweiten Bildungsweg eine Hochschulzugangsberechtigung zu erhalten (KMK 2009e, h). Im strukturkritischen Diskurs findet der zweite Bildungsweg im Zusammenhang mit Durchlässigkeit im zweiten Zeitraum keine Erwähnung. Dies zeigt eindrücklich, dass dieser Weg weder im universalistischen noch im funktionalistischen Diskursstrang als durchlässigkeitsfördernd angesehen wird.

\section{Zielgruppe}

Welches Bild wird in den Forderungen von den beruflichen Absolvent_innen, die als relevante Studienbewerber betrachtet werden, in den Diskurssträngen konstruiert? Wer soll Zugang bekommen?

Im strukturkonservativen Diskurs sollen die beruflich Qualifizierten ihre Befähigung am besten über das Erwerben einer allgemeinen Hochschulberechtigung nachweisen, die weiterhin als Norm hochgehalten wird. Unabhängig von dieser Gruppe soll besonders befähigten beruflich Qualifizierten (z.B. Aufstiegsfortgebildeten), die eine bestimmte Neigung (Profilnähe) und ihre Befähigung durch Prüfungen, die auch ein bestimmtes Niveau an Allgemeinbildung nachfragen, ausweisen, der Zugang gewährt werden (HRK 2008b). Eine Studienbefähigung würde sich auch zeigen, wenn sich Individuen im Berufsleben z.B. im Bereich der Forschung herausragend weiterentwickelt haben (HRK 2010a).

Anspruchsvolle Qualifizierungen im Fortbildungsbereich sind in besonderer Weise geeignet, die angestrebte stärkere Verknüpfung zwischen Hochschulen und verschiedenen Qualifizierungswegen sowie Lernorten außerhalb der Hochschulen zu erproben (KMK et al. 2003)

Ebenso ist es natürlich richtig, dass beruflich qualifizierte Individuen, die sich in der Forschung auf ihrem Arbeitsplatz in herausragender Weise weiterentwickelt haben, dann natürlich auch studieren können müssen, wenn sie das wollen. Vielleicht wollen 
sie das ja gar nicht, aber wenn sie wollen, wenn sie einen Mehrwert in einem Studium sehen, dann muss das möglich sein. (HRK 2010a)

Deutlich wird, wie auch im ersten Untersuchungszeitraum, die zugrunde liegende Annahme, dass Studierfähigkeit etwas ist, das vor dem Studium bereits besteht und geprüft werden kann. Zudem wird Studierfähigkeit auch weiterhin als eine Kompetenz konstruiert, die stark auf Allgemeinbildung oder gar Forschung aufbaut und von den Vertretern der Allgemeinbildung geprüft oder bestätigt werden sollte. Ausnahme stellen hier nur die Aufstiegsfortbildungen dar. Insgesamt sind es aber nicht die einfachen Absolvent_innen der Berufsausbildung, sondern es ist eine klare Leistungselite der beruflich Qualifizierten, der Zugang gewährt werden soll. Würde eine Selektion ausbleiben, hätte das negative Folgen für die Studierenden und die Hochschulbildung.

Aus Verantwortung gegenüber dem Einzelnen und im Interesse der Qualität der Lehre sollten die Zugangs- und Auswahlkriterien so definiert werden, dass sie Eignung und Neigung des Lerners oder der Lernerin Rechnung tragen. (HRK 2008b)

So wird insbesondere von der HRK (2010b) ein Bedrohungsszenario konstruiert: Wenn aufgrund politischen Liberalisierungsdrucks keine geeignete Vorselektion stattfände, würde die Qualität der Hochschulbildung leiden.

Der Druck zur Deregulierung des Zugangs zum Hochschulbereich würde so groß, dass realistische Anforderungen an die Vorqualifikation aus dem Blick geraten und Studierende wie Hochschulen überfordert würden. Notwendige Nachqualifikationen wären nicht leistbar und das Qualitätsniveau von Lehre und Studium müsste sinken. (HRK 2010b)

Auch sei ein solches Vorgehen unverantwortlich gegenüber Individuen, die nicht für ein Studium ,geeignet" sind, denen aber die Berechtigung zugesprochen werden soll. Diese wären überfordert, was sich in einer Erhöhung der Abbruchquoten widerspiegeln würde.

In den Hochschulen gibt es eine ganz starke Diskussion darüber, dass Studienabbruch sehr viel mit falschen Vorstellungen vom Studium, von den Leistungsanforderungen, von der Arbeitsweise oder auch vom Berufsfeld zu tun hat. Das spielt natürlich auch hier eine Rolle. Insofern geht es an der Stelle wirklich um die Verantwortung für den Abschluss und nicht nur für den Zugang. (HRK 2010a)

Im funktionalistischen strukturkritischen Diskurs wird die Zielgruppe ebenfalls vorselektiert. Entscheidend für die Selektion ist dabei aber weniger das Niveau der Allgemeinbildung. Zugang sollen leistungsstarke, eigenverantwortliche und erfolgreiche beruflich Qualifizierte erhalten, die ihre „Studierfähigkeit“ und ihre hohe Motivation über eine Prüfung oder durch einen guten Notendurchschnitt und Profilnähe nachgewiesen haben (DIHK/HRK 2008; DIHK 2008b, BMBF 2003b, 2009b, 2009a). Letztlich müssten diese Personen durch ihr Profil eine gute Passung mit dem Studiengang an der spezifischen Hochschule aufweisen. Auf 
diese Weise wird sichergestellt, dass eine möglichste hohe Wahrscheinlichkeit für einen erfolgreichen Abschluss des Studiums besteht.

Wie bereits verdeutlicht, wird im universalistischen Diskursstrang eine Selektion anders als über erworbene Berechtigungen, die auf allen Bildungswegen zu erhalten sein müssten, als Einschränkung des Rechts auf Bildung angesehen. Insofern wird auch kein Bild eines typischen beruflich Qualifizierten konstruiert, für den sich die Hochschulen öffnen sollten.

Stattdessen soll allen Studieninteressierten ein Zugang zur Hochschule nach ihren persönlichen Interessensschwerpunkten ermöglicht werden. Der DGB wendet sich daher gegen jede Form von Kapazitätsbeschränkungen, Eingangstests und andere Auswahlverfahren. (DGB 2006a: 35)

Jeder beruflich Qualifizierte, ob mit Erstausbildung oder mit Fortbildung, soll bei Interesse an die Hochschule dürfen. Die Frage nach bestimmten Voraussetzungen, die im Vorhinein geprüft werden müssten, stellt sich nicht. Dies gilt umso mehr, als mit dem Verständnis eines Rechts auf Bildung (das nicht in der Schule aufhört, sondern für alle Bereiche des lebenslangen Lernens gilt), dem stärker naturalistischen Begabungsglauben, der in den funktionalistischen Diskursen sichtbar wird, eine Vorstellung der universellen Bildungsfähigkeit entgegengesetzt wird (vgl. Lenhardt 2003).

\section{Rolle des Qualifikationsrabmens}

Nicht nur die Debatten um den KMK-Beschluss 2009 beeinflussen den Diskurs über die Zugangsmöglichkeiten beruflich Qualifizierter in die Hochschulbildung, sondern auch die Diskussion um die Einführung des nationalen Qualifikationsrahmens. Insbesondere im Rahmen des universalistischen Diskursstrangs wird verstärkt Hoffnung in den deutschen Qualifikationsrahmen gesetzt.

Der DGB erwartet, dass ein DQR die Durchlässigkeit in und zwischen den Bildungsbereichen, insbesondere die Zugänge zum tertiären Bereich verbessert. (DGB 2008a)

Vorsichtig bis abwehrend ist eine Passage im Einführungstext, wonach die DQRNiveaustufen, das bestehende System der Zugangsberechtigungen nicht ersetzen' sollen. Hier äußern sich die Ängste derer, die auch künftig nicht darauf verzichten wollen, dass Mittlere Reife, Abitur oder Diplom Instrumente der Auslese sind. Der DGB hält es dagegen mit der Entschließung des Hauptausschusses des Bundesinstituts für Berufsbildung (BIBB), in der von der, Verankerung des DQR als Bezugsrahmen im deutschen Bildungssystem ‘ die Rede ist. Genau das ist die Chance der europäischen Entwicklung: Dass Bewegung in die verkrusteten Strukturen und die voneinander abgeschotteten Bereiche des deutschen Bildungssystems kommt und ein neues Bezugssystem der Durchlässigkeit und Gleichwertigkeit entsteht. (DGB 2009a)

Der Qualifikationsrahmen solle dabei perspektivisch auch viel stärker als Instrument gelten, das Einfluss auf Zulassungsentscheidungen und die Ordnungsarbeit, d.h. bei der Entstehung neuer oder Reformierung bestehender Abschlüsse, hat. 
Denn dadurch, dass im Qualifikationsrahmen die Gleichwertigkeit der beruflichen Bildung mit der höheren Allgemeinbildung verankert wird, werden Übergangsmöglichkeiten transparent und können auch stärker mit Berechtigungen verbunden sein (DGB 2009a).

Viel stärker als in den anderen Diskursen ist im universalistischen strukturkritischen Diskurs vor allem der Gewerkschaften die Frage von Durchlässigkeit eng mit der Frage der gleichwertigen Anerkennung der beruflichen und Hochschulbildung nicht nur im Bildungssystem, sondern auch auf dem Arbeitsmarkt verbunden (DGB 2009a). Verstärkt wurde diese Diskussion vor allem in den Debatten um den Qualifikationsrahmen, der explizit einen Bezug zum Beschäftigungssystem haben sollte. So forderte der DGB (2009a), dass auch die Leistung beruflich Qualifizierter anerkannt werden müsse und dass die bestehende Hierarchisierung aufgebrochen werden solle, die auf der Annahme beruhe, die höchsten Positionen in der Arbeitswelt und das höchste Qualifikationsniveau sei nur via Hochschule zu erreichen. Hier wird dem deutschen Qualifikationsrahmen eine wichtige Rolle zugesprochen, weil darin die Gleichwertigkeiten in den Kompetenzen, die durch berufliche und akademische Bildung erworben werden, aufgezeigt werden sollen. Insofern ist für diesen Diskurs auch typisch, dass zwar Durchlässigkeit im Bildungssystem gefordert wird, aber nicht, dass alle beruflich Qualifizierten, die sich höher bilden wollen, auch notwendigerweise an die Hochschulen müssen (DGB 2010e). Wichtig ist aber, dass sie die Möglichkeit dazu haben. Gleichzeitig soll aber auch der Weg der beruflichen Weiterbildung am Arbeitsplatz und in Bildungsgängen als gleichwertige Alternative zum akademischen Weg anerkannt werden

Auch im funktionalistischen strukturkritischen Diskursstrang wird der DQR als nützliches Instrument angesehen, weil darin das tatsächliche Qualifikationsniveau abgebildet werden soll. Dadurch könne die notwendige Selektion auf der Grundlage von Informationen erfolgen (DIHK 2010c; BMBF 2008a, 2010a).

Im strukturkonservativen Diskurs wird dagegen betont, dass der DQR kein Berechtigungsinstrument sei und keine bindenden Wirkungen von ihm ausgehen können, zumal dieser auch gar nicht die notwendigen Informationen, sondern allein Orientierungen für Zulassungsentscheidungen bieten könne.

Insofern birgt auch die Ausweisung der Referenzniveaus in Qualifikationsbescheinigungen die Gefahr, dass ihr Informationswert überschätzt wird: Für die Einschätzung einer Qualifikation sind zahlreiche weitere Informationen wie Inhalte, Profil und Lernort der Ausbildung erforderlich. (HRK 2007c) 
Tabelle 15 Vergleich der Diskursstränge zur Frage des Zugangs in Deutschland (1999-2012)

\begin{tabular}{|c|c|c|c|}
\hline & \multicolumn{2}{|c|}{ Strukturkritisch } & \multirow{2}{*}{$\begin{array}{c}\text { Strukturkonservativ } \\
\text { Funktionalistisch }\end{array}$} \\
\hline & Universalistisch & Funktionalistisch & \\
\hline \multirow[t]{4}{*}{ Zugang } & $\begin{array}{l}\text { Ausweitung des Berechti- } \\
\text { gungswesens auf Berufs- } \\
\text { bildung }\end{array}$ & $\begin{array}{l}\text { Matching von Hochschule } \\
\text { und Absolvent über Eig- } \\
\text { nung (Leistung) und Nei- } \\
\text { gung (Profilnähe) }\end{array}$ & $\begin{array}{l}\text { Zugang unter Kontrolle der } \\
\text { Akteure der höheren Allge- } \\
\text { meinbildung }\end{array}$ \\
\hline & $\begin{array}{l}\text { Hochschulzugang über } \\
\text { Berechtigungen (Erstaus- } \\
\text { bildung oder Fortbildung) }\end{array}$ & $\begin{array}{l}\text { Berechtigungen nicht im } \\
\text { Fokus }\end{array}$ & $\begin{array}{l}\text { Monopol des Berechti- } \\
\text { gungswesens bei höheren } \\
\text { Allgemeinbildung }\end{array}$ \\
\hline & $\begin{array}{l}\text { Zweiter Bildungsweg nicht } \\
\text { thematisiert (nicht durch- } \\
\text { lässigkeitsfördernd) }\end{array}$ & $\begin{array}{l}\text { Zweiter Bildungsweg nicht } \\
\text { thematisiert (nicht durch- } \\
\text { lässigkeitsfördernd) }\end{array}$ & $\begin{array}{l}\text { Zweiter Bildungsweg als } \\
\text { wichtiger Weg in die Hoch- } \\
\text { schulen }\end{array}$ \\
\hline & $\begin{array}{l}\text { Gegen jede Form von Prü- } \\
\text { fungen, Kapazitätsbe- } \\
\text { schränkungen und Aus- } \\
\text { wahlverfahren (Einschrän- } \\
\text { kung des Rechts auf Bil- } \\
\text { dung und Zeichen für feh- } \\
\text { lende Gleichwertigkeit) }\end{array}$ & $\begin{array}{l}\text { Zugang primär über Zulas- } \\
\text { sungsverfahren der Hoch- } \\
\text { schulen, in denen Stu- } \\
\text { dieneignung und Passung } \\
\text { geprüft werden } \\
\text { Vereinheitlichung dieser } \\
\text { Verfahren für stärkere } \\
\text { Transparenz und Effizienz }\end{array}$ & $\begin{array}{l}\text { Zugang über Zulassungs- } \\
\text { prüfung an Hochschulen } \\
\text { anhand Profilnähe und All- } \\
\text { gemeinbildungsniveau }\end{array}$ \\
\hline \multirow[t]{2}{*}{ Zielgruppe } & $\begin{array}{l}\text { Allgemein: alle, die sich } \\
\text { bilden wollen und die } \\
\text { Berechtigung erworben } \\
\text { haben }\end{array}$ & $\begin{array}{l}\text { Allgemein: Alle, die ihre } \\
\text { Studierfähigkeit bewiesen } \\
\text { haben und damit wahr- } \\
\text { scheinlich erfolgreich sein } \\
\text { werden: Leistungselite }\end{array}$ & $\begin{array}{l}\text { Allgemein: alle, die durch } \\
\text { Prüfung der Akteure der } \\
\text { höheren Allgemeinbildung } \\
\text { als geeignet klassifiziert } \\
\text { werden }\end{array}$ \\
\hline & $\begin{array}{l}\text { Durchlässigkeit: alle (be- } \\
\text { ruflich Qualifizierten) ohne } \\
\text { weitere Selektionskriterien }\end{array}$ & $\begin{array}{l}\text { Durchlässigkeit: Begabte/ } \\
\text { Leistungsfähige und ei- } \\
\text { genverantwortlich beruflich } \\
\text { Qualifizierte, die den richti- } \\
\text { gen Match zur Hochschule } \\
\text { darstellen }\end{array}$ & $\begin{array}{l}\text { Durchlässigkeit: beruflich } \\
\text { Qualifizierte mit Ausweis } \\
\text { des notwendigen Niveaus } \\
\text { höherer Allgemeinbildung }\end{array}$ \\
\hline $\begin{array}{l}\text { Subjektkon- } \\
\text { struktion }\end{array}$ & $\begin{array}{l}\text { Individuum als Person, der } \\
\text { über Bildung Chancen- } \\
\text { gleichheit und Teilhabege- } \\
\text { rechtigkeit ermöglicht wird }\end{array}$ & $\begin{array}{l}\text { Individuum als (Humanka- } \\
\text { pital-) Ressource für den } \\
\text { Arbeitsmarkt }\end{array}$ & $\begin{array}{l}\text { Individuum als Risiko für } \\
\text { Qualität des Hochschulstu- } \\
\text { diums bei mangelnder Eig- } \\
\text { nung (mangelnder Allge- } \\
\text { meinbildung) }\end{array}$ \\
\hline
\end{tabular}

Quelle: Darstellung der Verfasserin

Auch die Lernergebnisorientierung als zentrales Prinzip für den DQR wird für Fragen des Zugangs zum Studium als nicht ausreichend angesehen. Stattdessen wird darauf hingewiesen, entsprechend dem alten vorherrschenden Paradigma, dass neben Kompetenzen auch der vermittelte Inhalt (z.B. Allgemeinbildung), das 
Profil des Bildungsgangs und der Lernort weiterhin entscheidend sind (HRK 2007c, 2010a). Für die Träger des strukturkonservativen Diskurses sollen daher europäische Politiken und Standards (wie der DQR) keinen größeren verbindlichen Einfluss auf den Zugang von beruflich Qualifizierten zur Hochschule haben. Entscheidend sei die aufnehmende Institution. Allerdings hat die Debatte um den DQR die Diskurse bereits beeinflusst. Zusammenfassend werden in Tabelle 15 die wichtigsten Charakteristika der Diskurstränge zu den Zugangsfragen dargestellt.

\section{Anrechnung}

Während Anrechnung im ersten Untersuchungszeitraum so gut wie gar nicht thematisiert wurde, war sie der mit am häufigsten diskutierte Aspekt nach 1999. Dies ist von großer Bedeutung, weil Anrechnung ein Weg ist, die Gleichwertigkeit von Erlerntem aus unterschiedlichen Bildungswegen anzuerkennen. Doch was wird tatsächlich als gleichwertig anerkannt?

Aufgekommen ist die Frage von Anrechnung im deutschen Diskurs durch den Bologna-Prozess (vgl. Kapitel 5), in dem gefordert wird, dass auch außerhochschulisch erworbene Kompetenzen anerkannt werden (vgl. KMK 2002, BMBF et al. 2003).

In der gemeinsamen Erklärung der Europäischen Bildungsminister vom 19. Juni 1999 von Bologna, an der auf deutscher Seite das Bundesministerium für Bildung und Forschung und die Kultusminister der Länder beteiligt waren, heißt es, dass Leistungspunkte zur Anrechnung auf Studienanforderungen auch außerhalb der Hochschulen, z.B. durch lebenslanges Lernen erworben werden können. (DGB et al. 2002)

Die Bedeutung der europäischen Prozesse wird in allen Diskurssträngen sichtbar, da in ihnen europäische Standards als Instrumente, die Anrechnung erleichtern können, anerkannt werden. Gemeinsam ist den Diskurssträngen auch, dass, zuerst eher unspezifisch, die Notwendigkeit von Anrechnung anerkannt wurde und erst im Verlauf konkreter über die Fragen, was genau und auf welche Weise angerechnet werden sollte, diskutiert wurde. Doch wie unterscheiden sich die Positionen im Hinblick auf Anrechnung bzw. welche unterschiedlichen Schwerpunkte werden in den Diskursen gesetzt?

\section{Was wird angerechnet und warum?}

Im funktionalistischen strukturkritischen Diskurs werden Fragen zu Anrechnung im Zeitverlauf immer wichtiger, zumal Anrechnung eine Möglichkeit darstellt, Bildungszeiten zu verkürzen und effizient zu gestalten, so dass die Humanressourcen nicht mit dem doppelten Lernen der gleichen Kompetenzen vergeudet werden (DIHK 2007c).

Was soll aber genau angerechnet werden? Anstatt abschlusszentrierter Anerkennung sollen gleichwertige Kompetenzen aus der beruflichen Bildung im Hochschulsystem Berücksichtigung finden. Im Fokus stehen hier insbesondere 
Kompetenzen, die in Aufstiegsfortbildungen erworben worden sind, die dann auch eher in Fachhochschulstudiengängen als in universitären Studiengängen angerechnet werden sollten. Auch das ANKOM Projekt zielte auf die Erprobung der Anrechnung und Erfassung von äquivalenten Kompetenzen von Aufstiegsfortbildungen und Studiengängen (BMBF 2005, 2009c). Der Fokus auf Aufstiegsfortbildungen zeigt, dass vor allem Kompetenzen aus diesen postsekundären Bildungsgängen als gleichwertig anerkannt werden.

Ein wichtiges bildungspolitisches Anliegen ist, dass die in der beruflichen Fortbildung
erworbenen Qualifikationen auch bei einem Hochschulstudium berücksichtigt wer-
den. Das Bundesministerium für Bildung und Forschung wird dazu Vorhaben för-
dern, in denen transparente, praktikable und übertragbare Verfahren zur Feststellung
von in der Beruflichen Bildung erworbenen Qualifikationen und deren Anrechnung
auf Studiengänge entwickelt werden. (BMBF 2005 19)

Die Möglichkeit, informelle und non-formale Kompetenzen anzuerkennen, wurde zwar auch diskutiert, kam aber erst in den letzten Jahren vor allem mit der Debatte um den DQR stärker auf die Agenda. Die Anerkennung hochschulischer Kompetenzen im Berufsbildungssystem wird dagegen allein vom DIHK mit reflektiert. Interessanterweise sind es hier die Studienabbrecher_innen, die als relevante Gruppe ins Auge gefasst werden.

Wir haben bisher nur über die Seite von der beruflichen Bildung hin zur Fachhochschule gesprochen. Es gibt aber auch noch die andere Seite von der Fachhochschule zur beruflichen Bildung. Ich erwähne in diesem Zusammenhang nur das große Problem der Studienabbrecher. Es gibt diesbezüglich auch Modellprojekte. Die berufliche Bildung wird sich auch öffnen müssen, um z.B. Lernleistungen, die man schon im Studium nachgewiesen hat, bei Fachhochschul- oder Hochschulabbrechern dann in einer betrieblichen Lehre zu integrieren bzw. anzurechnen. Wir stehen da noch am Anfang. (DIHK 2006a)

Anscheinend ist die Idee, dass Hochschulabsolvent_innen an ihr Studium eine berufliche Bildung anschließen, nicht vorstellbar. Vielmehr ist die Zielgruppe die der weniger Erfolgreichen im Hochschulsystem. Im Grunde entspricht das genau dem Gegenteil der Zielgruppe für die Hochschulen. In diesem Zusammenhang wird wiederum die bestehende Hierarchie der Bildungsbereiche unterstrichen: die Hochschulbildung gilt weiterhin als der Weg, der zur höchstmöglichen Bildung führt.

Im universalistischen Diskurs wird die Bedeutung von Anrechnung für die gesellschaftliche und vor allem auch hochschulische Anerkennung und Förderung der Gleichwertigkeit der vermittelten Kompetenzen in beiden Bildungsbereichen betont. Angerechnet werden sollen dann Kompetenzen aus der beruflichen Erst- und Fortbildung (DGB 2004a). Ziel sei es, flexible Bildungswege zu schaffen, und zwar entsprechend den individuellen Bedürfnissen der Lernenden (DGB 2002). Dieser Fokus auf die Lernenden stellt ein Charakteristikum des universalistischen Diskurses da. Vor diesem Hintergrund ist auch zu verstehen, dass 
die Bedeutung der Anrechnung nicht von nur formalen, sondern auch von informellen und non-formalen Kompetenzen als sehr hoch eingeschätzt wird (DGB 2004a, 2009a, c).

Im strukturkonservativen Diskurs wird deutlich, dass eine Anrechnung von beruflichen Fähigkeiten in der Hochschule nur stattfinden wird, wenn die die Entscheidungsgewalt darüber bei den Hochschulen verbleibt und eine Hochschulzulassung für das Individuum mit Anrechnungswünschen vorliegt (KMK 2002). Die Vertreter der höheren Allgemeinbildung wollen also ihren Einfluss auf diesen Prozess behalten. Zudem kommt so allen ,geeigneten“Bewerber_innen, die ihre Befähigung bewiesen haben, Anrechnung zugute. Weiterhin kann eine Anrechnung nur bis zu $50 \%$ eines hochschulischen Abschlusses betragen (KMK 2002). Auf diese Weise soll sichergestellt werden, dass ein wesentlicher Teil des Abschlusses tatsächlich am Lernort Hochschule gemacht wurde, nur so könne auch die Qualität des Hochschulabschlusses gewährleistet sein (KMK 2002, 2008). An dieser Stelle wird deutlich, dass für die Träger des strukturkonservativen Diskurses die Hochschule als Lernort und die Kontrolle über den Lernprozess von besonderer Bedeutung sind. Gleichwertige Kompetenzen allein reichten nicht aus, eine akademische Sozialisation müsse demnach über einen Hochschulabschluss stattfinden. Dabei wird bezweifelt, dass die für den akademischen Bereich wichtigen und typischen Kompetenzen tatsächlich außerhalb der Hochschule erworben werden können (HRK 2009b). Diese Bedenken wurden vor allem in den Debatten zum Qualifikationsrahmen deutlich, in welchen speziell die HRK eine starke Abgrenzung zwischen beruflicher und hochschulischer Bildung vornahm (vgl. HRK 2010a). Die KMK dagegen versuchte, die allgemeine Hochschulreife durch ihren Zuordnungsvorschlag auf Stufe 5 im Qualifikationsrahmen von der beruflichen Bildung abzugrenzen. Insgesamt wird demnach im Gegensatz zum universalistischen Diskursstrang eine Anrechnung von Kompetenzen der beruflichen Erstausbildung nicht in Betracht gezogen.

Eine Validierung von Kompetenzen und die Anerkennung ganzer Abschlüsse wie in Frankreich ist im strukturkonservativen Diskurs ebenfalls nicht vorgesehen. Interessanterweise, auch wenn so gut wie nicht thematisiert (außer KMK 2008), gibt es in Deutschland aber zumindest rechtlich in vier Bundesländern (Baden-Württemberg, Sachsen, Sachsen-Anhalt und Thüringen) die Möglichkeit, über Externenprüfung ganze Hochschulabschlüsse zuerkannt zu bekommen (Freitag 2012: 21). Eine hochschulische Prüfung muss jedoch stattfinden.

\section{Wie soll Anrechnung erfolgen?}

$\mathrm{Zu}$ Beginn des Untersuchungszeitraums waren die Vorstellungen in den Diskursen zu einem Anrechnungsvorgehen noch relativ vage. So sollen Leistungspunkte für berufliche Weiterbildung gegeben und dann anerkannt werden (BMBF 2003a, b; BMBF 2009c, BMBF et al. 2003). Mit Initiativen wie ANKOM, an der auch die Kammern beteiligt waren, wurden die Verfahrensvorschläge konkreter. 
Prinzipiell wird in allen Diskursen darauf verwiesen, dass europäische Standards hilfreich bei der Einführung und Umsetzung von Anrechnungsverfahren sein können. So sollten die Modularisierung, die Einführung von Leistungspunktesystemen sowie generell eine stärkere Lernergebnisorientierung die Anrechnungsverfahren unterstützen, da nur so die Kompetenzen, die über andere Lernwege in anderen Kontexten über andere Inhalte erworben wurden, vergleichbar gemacht werden können. Auch der DQR könne hilfreich sein (DGB 2006b, 2008a, 2009a). Insgesamt wird der DQR eher als Orientierung für Anrechnungsentscheidungen gesehen, und die genaue Verbindung zwischen dem Rahmen und den Anerkennungsinstrumenten sollte zukünftig ausgearbeitet werden. Ob der DQR in Fragen der Anrechnung mehr als ein Informationsinstrument sein würde, bleibt in den Diskursen offen (vgl. KMK 2009g).

Nachdem die Ähnlichkeiten der Diskurse im Hinblick auf das konkrete Anrechnungsvorgehen dargestellt wurden, sollen nun die Spezifika folgen.

Im funktionalistischen strukturkritischen Diskurs wird eine pauschale und standardisierte Form der Anrechnung bevorzugt. Dies sei besser für die Transparenz, aber auch die Verlässlichkeit der Anrechnungsregelungen im Gegensatz zu individualisierten Verfahren, die zu aufwendig und wesentlich weniger transparent seien.

Anrechnung sollte - im Dienste der Transparenz und Verlässlichkeit - automatisiert erfolgen, ohne eine individuelle Prüfung vornehmen zu müssen. Also sollten Qualifizierungsinhalte en bloc und standardisiert angerechnet werden. (DIHK 2007c)

Tatsächlich geht es dann aber verstärkt um die Anrechnung formalen Lernens, da nur formale Qualifikationen in vergleichbarer Form vorliegen. So wird z.B. vorgeschlagen, dass es standardisierte Verfahren geben soll für Abschlüsse von Aufstiegsfortbildungen, die bundeseinheitlich geregelt sind (DIHK 2007c, 2012). Gerade bei den standardisierten Verfahren sei dann eine enge Kooperation mit der Wirtschaft Grundvoraussetzung.

Im universalistischen strukturkritischen Diskursstrang sollen Anrechnungsverfahren gesetzlich festgeschrieben werden. So wurde gefordert, konkrete Anrechnungsmodalitäten im zu reformierenden Berufsbildungsgesetz auf Bundesebene festzuschreiben (DGB 2004a). Weiterer Vorschläge sind zudem, dass Anrechnung sowohl in den Landeshochschulgesetzen (BMBF 2009c) als auch bei der Akkreditierung von Studiengängen und in den Prüfungsordnungen selbst festgeschrieben werde (DIHK/HRK 2008). Auch wird eine stärkere Einbindung von Hochschullehrer_innen in die Prüfungen der beruflichen Fortbildung als sinnvoll angesehen. Auf diese Weise könnten Äquivalenzen besser sichtbar gemacht werden und auf hochschulischer Seite ein genauerer Einblick in die Inhalte der Fortbildungen erlangt werden. Zudem sollten die Fortbildungsgänge in das hochschulische Leistungspunktesystem integriert werden.

Im Hinblick auf die Anrechnung von beruflichen Weiterbildungsleistungen in Studiengängen (gemäß Bologna-Vereinbarung und Vereinbarung von BMBF, HRK und 
KMK zur IT-Weiterbildung) sollte die Möglichkeit geschaffen werden, am hochschulischen ECTS-System teilzunehmen und im Hinblick auf gewünschte Äquivalenzen sollte ausdrücklich die Möglichkeit geschaffen werden, in beruflichen Fortbildungsprüfungen auch Hochschullehrer beteiligen zu können. (DGB 2004a)

Da auch die Anrechnung von non-formal und informell erworbenen Kompetenzen im universalistischen Diskursstrang als wichtig eingeschätzt wird, lässt sich schlussfolgern, dass auch die Einführung stärker individualisierter Verfahren, die dies leisten können, gewünscht wird.

Im strukturkonservativen Diskurs werden standardisierte Verfahren durch Kooperationen von Bildungspartner_innen gegenüber aufwendigen individualisierten Verfahren bevorzugt. Da Anrechnung für jede aufnehmende Institution ein sehr sensibles Thema sei, ist die Grundvoraussetzung dafür, dass diese durchgeführt werden könne, die Sicherung der Qualität des Anrechnungsverfahrens, aber auch in den Bildungsbereichen selbst. Nur so könnten die Qualität der Hochschulbildung und der, Verbraucherschutz' für die Studierenden gewährleistet werden.

Maßnahmen zur Förderung dieser [Anrechnungs-, NB] Möglichkeiten müssen natürlich dem Gesichtspunkt der Qualitätssicherung Rechnung tragen. Dabei geht es sowohl darum, die notwendige Qualität der hochschulischen Ausbildung sicherzustellen als auch darum, den Schutz der Studieninteressenten vor unseriösen Bildungsangeboten zu gewährleisten. (KMK 2008: 1)

Dem Thema Anrechnung wurde im Zeitverlauf im strukturkonservativen Diskurs eine immer stärkere Aufmerksamkeit gewidmet. Dies gilt auch für die HRK, wobei Anrechnung meist in Zusammenarbeit mit anderen Akteuren wie der KMK oder dem DIHK thematisiert wurde.

Es kann festgestellt werden, dass Anrechnung ein fester Bestandteil des Verständnisses von Durchlässigkeit auch im strukturkonservativen Diskurs geworden ist. Dies wird aber von folgenden Prämissen abhängig gemacht: Die Entscheidung über die Anrechnung und das Verfahren an sich verbleibt an den Hochschulen (KMK 2002, 2008; 2011b; HRK 2007c). Aus diesem Grund wird auch in der Diskussion über den DQR zwar dessen Orientierungsfunktion anerkannt, aber einen anderen bindenden Charakter dürfe dieser nicht bekommen (HRK 2007c, 2010a). Anrechnung kann nur für einen Teil des Studiums erfolgen. Die Personen, deren Kompetenzen für eine Anrechnung infrage kommen, werden zuvor ebenfalls durch Kriterien der Vertreter der höheren Allgemeinbildung ausgewählt, da der Hochschulzugang Bedingung für Anrechnung ist (KMK 2008). Wenn also die Entscheidungsgewalt bei den Vertretern des strukturkonservativen Diskurses liegt und die Qualität der Verfahren gesichert ist, dann ist Anrechnung durchaus denkbar. Dass einer Beteiligung der anderen Bildungsbereiche für ein besseres Verständnis der Kompetenzen notwendig sein könnte, wird nicht explizit thematisiert, obwohl dies in den Pilotprojekten der ANKOM-Initiative durchaus der Fall war. Ebenfalls war die Anrechnung von informellen und non-formalen Kompetenzen weniger von Bedeutung. 
Tabelle 16Vergleich der Diskursstränge zur Bedeutung von Anrechnung in Deutschland (1999-2012)

\begin{tabular}{|c|c|c|c|}
\hline & \multicolumn{2}{|c|}{ Strukturkritisch } & \multirow{2}{*}{$\begin{array}{c}\text { Strukturkonservativ } \\
\text { Funktionalistisch }\end{array}$} \\
\hline & Universalistisch & Funktionalistisch & \\
\hline Anrechnung & $\begin{array}{l}\text { Im Hochschulsystem als } \\
\text { Zeichen von Gleichwertig- } \\
\text { keit gesetzlich festschrei- } \\
\text { ben }\end{array}$ & $\begin{array}{l}\text { Im Hochschul- und Berufs- } \\
\text { bildungssystem zur Stei- } \\
\text { gerung von Effizienz durch } \\
\text { Reduzierung von Bil- } \\
\text { dungszeiten }\end{array}$ & $\begin{array}{l}\text { Im Hochschulsystem nach } \\
\text { Entscheidung der aufneh- } \\
\text { menden Institution }\end{array}$ \\
\hline $\begin{array}{l}\text { Anrechnung } \\
\text { was }\end{array}$ & $\begin{array}{l}\text { Berufliche Kompetenzen } \\
\text { (aus Erst- und Fortbildung) } \\
\text { Formale, sowie non-for- } \\
\text { male und informelle }\end{array}$ & $\begin{array}{l}\text { Berufliche Kompetenzen } \\
\text { primär aus Fortbildung } \\
\text { Fokus stärker auf formale } \\
\text { Kompetenzen } \\
\text { Akademische Kompeten- } \\
\text { zen von Studienabbre- } \\
\text { cher_innen in der Berufs- } \\
\text { bildung }\end{array}$ & $\begin{array}{l}\text { Berufliche Kompetenzen } \\
\text { primär aus Fortbildung } \\
\text { („nach Inhalt und Niveau } \\
\text { gleichwertig“) } \\
\text { Nicht mehr als } 50 \% \text { eines } \\
\text { Abschlusses (Bedeutung } \\
\text { des Lernortes Hochschule) }\end{array}$ \\
\hline $\begin{array}{l}\text { Anrechnung } \\
\text { wie }\end{array}$ & $\begin{array}{l}\text { Gesetzliche Verankerung } \\
\text { (im BBiG bzw. als Voraus- } \\
\text { setzung für Akkreditierung } \\
\text { von Studiengängen) } \\
\text { Stärkere Anpassung an In- } \\
\text { dividuen, daher kombi- } \\
\text { nierte Verfahren, } \\
\text { Beteiligung von Hoch- } \\
\text { schullehrer_innen in der } \\
\text { beruflichen Fortbildung } \\
\text { (um Gleichwertigkeit sicht- } \\
\text { bar zu machen) } \\
\text { Durch Modularisierung } \\
\text { und ECTs Anrechnung } \\
\text { ermöglichen } \\
\text { Über den DQR Anrech- } \\
\text { nungsoptionen sichtbar } \\
\text { machen }\end{array}$ & $\begin{array}{l}\text { Über klare transparente } \\
\text { Kriterien in Prüfungsord- } \\
\text { nungen } \\
\text { Standardisierte Anrech- } \\
\text { nungsverfahren bevorzugt } \\
\text { Durch gestufte Studien- } \\
\text { struktur, Modularisierung } \\
\text { und ECTs sowie DQR } \\
\text { bessere Möglichkeiten der } \\
\text { Anrechnung }\end{array}$ & $\begin{array}{l}\text { Voraussetzung: Qualitäts- } \\
\text { sicherung der Verfahren } \\
\text { und der Bildungsbereiche } \\
\text { Verfahren: eher pauschal } \\
\text { über Kooperationen zwi- } \\
\text { schen einzelnen Organisa- } \\
\text { tionen als individualisiert } \\
\text { Durch gestufte Studien- } \\
\text { struktur, Modularisierung } \\
\text { und ECTs bessere Mög- } \\
\text { lichkeiten der Anrechnung } \\
\text { DQR: nur Hinweis/ } \\
\text { Orientierung - kein Instru- } \\
\text { ment der Anrechnung }\end{array}$ \\
\hline
\end{tabular}

Quelle: Darstellung der Verfasserin

Insgesamt bleibt festzuhalten, dass im zweiten Untersuchungszeitraum Anrechnung ein wichtiges Thema im Zusammenhang mit der Förderung von Durchlässigkeit zwischen Berufs- und Hochschulbildung ist und damit eine gewisse Gleichwertigkeit von Erlerntem aus beiden Bereichen anerkannt wird. Dabei wird insgesamt eher eine Gleichwertigkeit von postsekundärer Berufs- und Hochschulbildung gesehen und weniger der Erstausbildung, selbst wenn bestimmte Ausbildungen nur von Abiturient_innen gemacht werden und damit eine 
Bildung zusätzlich zu einem allgemeinbildenden Sekundarstufe-II-Abschluss gemacht wird. Diese unterschiedlichen Perspektiven werden in Tabelle 16 zusammengefasst.

\section{Organisationale Verbindung}

Wenn in den deutschen Durchlässigkeitsdiskursen über organisationale Verbindungen zwischen Berufs- und Hochschulbildung gesprochen wird, werden primär Formen der Integration der Bildungsbereiche in einen Bildungsgang thematisiert, wobei nur die strukturkritischen Diskursstränge dies Thema im Zusammenhang von Durchlässigkeit aufnehmen.

Im funktionalistischen strukturkritischen Diskursstrang werden integrierte Studienangebote als Möglichkeit der effizienten Verbindung von Berufs- und Hochschulbildung angesehen (KMK et al. 2002, BMBF 2002). Dabei ist auffällig, dass die Integration von beruflicher und Hochschulbildung als Weg, um Durchlässigkeit in Deutschland voranzubringen, viel weniger präsent ist als noch im ersten Zeitraum. ${ }^{113}$ Es wird aber weiterhin ein Ausbau der als erfolgreiches Modell wahrgenommenen dualen Studiengänge gefordert, denn diese verkürzen nicht nur die Studienzeiten, sondern führen auch zu einer engeren Bindung an die Unternehmen und nähern die beiden Bildungsbereiche einander an (DIHK 2007c). Zusätzlich wird vom Bildungsministerium (2007) vorgeschlagen, neue ein- bis zweijährige Kurzstudiengänge einzuführen, die berufliche Praxis und Hochschulbildung miteinander verbinden. Es fällt auf, dass insgesamt der funktionalistische strukturkritische Diskurs viel stärker darauf abzielt, Bildungsmöglichkeiten für beruflich Qualifizierte im Hochschulbereich, demnach für deren Höherqualifizierung, zu schaffen.

Im universalistischen strukturkritischen Diskursstrang werden organisationale Verbindungen zwischen Berufs- und Hochschulbildung nur vereinzelt im Zusammenhang mit Durchlässigkeit aufgegriffen, wobei drei verschiedenen Verbindungen angeregt wurden. Wie auch im funktionalistischen strukturkritischen Diskurs wird ein Ausbau dualer Studiengänge befürwortet (DGB 2002). Zudem werden mehr Doppelqualifikationen, die das Abitur und eine berufliche Ausbildung verbinden, gefordert (DGB 2006a). Dies war auch schon ein Appell im Rahmen dieses Diskurses vor 1999. Eine weitere Idee der besseren Verbindung der höheren Allgemeinbildung mit der Berufsbildung ist die Integration von allgemeinbildender Schule und des schulischen Anteils der Berufsausbildung des dualen Systems in eine "Schule für alle“, wobei der allgemeinbildende Teil der Berufsschule zusammen mit dem allgemeinen weiterführenden Schulangebot vermittelt werden soll. Ziel ist es, die Trennung zwischen Berufs- und Allgemeinbil-

113 Das bedeutet nicht, dass Debatten zu bzw. duale Studiengänge als solche in Deutschland insgesamt abgenommen hätten. Wegen der guten Karriereaussichten der Absolvent_innen, deren sofortigem Übergang in den Arbeitsmarkt, werden duale Studiengänge immer populärer (Graf et al. 2014). Allein im Zusammenhang mit Durchlässigkeit werden sie weniger thematisiert. 
dung aufzuheben und unterschiedlichste Lernerfahrungen miteinander zu kombinieren. Zudem soll auf diesen Weg in beiden Bereichen die allgemeine Hochschulreife erworben werden (DGB 2006a). Dieser Vorschlag wäre ein klarer Weg, die getrennten Bildungsbereiche miteinander zu verknüpfen. Deutlich wird, dass im Gegensatz zu den anderen Diskursen die Integration nicht im tertiären Bereich stattfinden muss, sondern auch auf Sekundarstufe-II-Niveau.

Tabelle 17 Vergleich der Diskursstränge zur Bedeutung organisationaler Verbindungen in Deutschland (1999-2012)

\begin{tabular}{lcc}
\multicolumn{2}{c}{ Strukturkritisch } & Strukturkonservativ \\
Universalistisch & Funktionalistisch & Funktionalistisch \\
\hline $\begin{array}{l}\text { Förderung doppeltqualifizierender } \\
\text { Bildungsgänge (Berufsausbildung }\end{array}$ & Ausbau dualer Studiengänge & Nicht relevant \\
mit Abitur) & & \\
Integration beruflicher Schulbildung & \\
mit Sek II der Allgemeinbildung & \\
(eine Schule für alle) & \\
\hline
\end{tabular}

Quelle: Darstellung der Verfasserin

\section{Umgang mit der Heterogenität von Bedürfnissen}

Während im ersten Zeitraum die Frage des institutionalisierten Umgangs mit heterogenen Bedürfnissen vor allem auch beruflich Qualifizierter so gut wie gar nicht im Diskurs angesprochen wurde, ändert sich dies deutlich im zweiten Zeitraum. So wird dieser Aspekt (anders als die Frage der organisationalen Verbindungen) in allen Diskurssträngen, wenn auch mit unterschiedlichen Schwerpunkten, behandelt. Dabei ist aber festzustellen, dass dieses Thema nicht von Beginn an, sondern erst ca. ab 2008, dem Beginn der Debatten zur Qualifizierungsinitiative, und vor allem auch im Zusammenhang bzw. Nachgang der Debatten um den Hochschulzugang, die ihren Höhepunkt mit dem KMK-Beschluss 2009 hatten, verstärkt aufgekommen ist. Allein im universalistischen strukturkritischen Diskurs wird die Frage nach dem Umgang mit heterogenen Bedürfnissen über den gesamten Zeitverlauf immer wieder diskutiert. Deren Notwendigkeit wird zudem mit Verweis auf die immer wieder geforderte Stärkung der „sozialen Dimension" im Bologna-Prozess unterstrichen.

Die Bologna-Staaten müssen die soziale Dimension des Europäischen Hochschulraumes stärken. Ein Europa des Wissens braucht künftig nicht weniger, sondern deutlich mehr gut ausgebildete Akademikerinnen und Akademiker. Deutschland hat hier besonderen Nachholbedarf. [...] Der Hochschulzugang für beruflich Qualifizierte ist zu erleichtern. Dafür müssen nicht nur die Länder ihrer Gesetze ändern. Die Hochschulen müssen berufsbegleitende Studiengänge auf- und ausbauen. Im Beruf erworbene Kompetenzen müssen angerechnet werden. Zudem brauchen wir ein Erwachsenen-BAföG, das Menschen, die aus dem Beruf kommen, ein Studium ermöglicht. (DGB 2009d) 
Nachfolgend sollen erstens die unterschiedlichen Motive, warum dieses Thema an Relevanz gewonnen hat, und anschließend die geforderten Strukturen verglichen werden.

Das Motiv, das im universalistischen strukturkritischen Diskursstrang deutlich wird, ist, dass die Einführung von Strukturen, die die Bildungsteilnehmer_innen, insbesondere auch die beruflich Qualifizierten, unterstützen und ein erfolgreiches Lernen ermöglichen, eine Notwendigkeit ist, um das Recht auf Bildung durchzusetzen. Dies sei zudem nur in einem öffentlich finanzierten Bildungswesen möglich.

Der DGB steht für ein sozial integratives Bildungssystem. Dies umfasst die optimale Förderung der Individuen entsprechend ihrer sozialen, kulturellen und ökonomischen Bedürfnisse und die Eröffnung von Bildungswegen unabhängig vom sozialen Hintergrund. Wir wollen ein öffentlich finanziertes und öffentlich organisiertes Bildungssystem, weil nur dieses das Grundrecht auf Bildung garantieren kann. (DGB 2006a: 28 )

Im funktionalistischen strukturkritischen Diskurs dagegen stehen zwei Motive im Mittelpunkt. Zum einen geht es darum, das Hochschulstudium für die neue Zielgruppe der beruflich Qualifizierten attraktiver zu gestalten, so dass sich viele für eine durch den demografischen und Strukturwandel notwendig gewordene Weiterqualifikation entscheiden. Der verstärkte Umgang mit heterogenen Bedürfnissen wird vor allem dann erforderlich, wenn die Zahl der traditionellen Studierenden nicht mehr ausreicht. Die treibende Kraft ist demnach das Marktprinzip.

Die Hochschulen müssen erst mal merken, dass sie irgendwann in der Situation sind, auch die Absolventen der beruflichen Bildung definitiv als Klientel zu benötigen. Das haben sie jetzt noch nicht, jetzt haben sie die doppelten Abiturientenjahrgänge. Aber wenn die demografische Entwicklung so richtig zuschlägt, werden sie sich zur Decke strecken, und dann geht es nicht nur darum, ihnen irgendwie den Zugang zu ermöglichen, sondern auch für sie passgenaue Studiengänge zu entwickeln. (DIHK 2010c:43)

Zum anderen geht es aber auch darum, diese Zielgruppe bestmöglich auszusuchen und auf die Anforderungen im Studium vorzubereiten, so dass diese möglichst effizient und erfolgreich studieren (DIHK/HRK 2008, DIHK 2012).

Im strukturkonservativen Diskurs wird auch die Notwendigkeit anerkannt, dass man, wenn es eine neue Studierendengruppe gibt, sich auf diese vorbereiten müsse. Ziel ist es, die Gefährdung, die durch diese Gruppe für den Hochschulbetrieb und die Qualität ausgeht, zu verringern. So betonen die Hochschulen, dass eine solche Zugangsneuordnung

[.]nur dann sinnvoll ist und ihre Ziele erreichen kann, wenn die Studienbedingungen den besonderen Qualifikationsvoraussetzungen und der besonderen Lebenssituation vieler beruflich Qualifizierter Rechnung tragen. (HRK 2008b) 
Auch wenn jeweils unterschiedliche Motive in den Diskursen mit dem Thema Umgang mit der Heterogenität der Studierenden einhergeht, werden doch vielfach ähnliche Strukturen gefordert. Insgesamt können mehrere Strukturformen unterschieden werden: die Frage der Information und Beratung vor und während der Aufnahme eines Studiums, die Studienorganisation, die pädagogische Ausgestaltung der Studiengänge und die Finanzierung.

\section{Informations- und Beratungsstrukturen}

Bereits im ersten Untersuchungszeitraum wird im universalistischen Diskursstrang darauf verwiesen, dass für beruflich Qualifizierte Beratungs- und Betreuungsstrukturen bereitgestellt werden müssten. Dies wird auch nach 1999 von den anderen Diskurssträngen anerkannt (DGB 2006a, HRK 2011a). Insbesondere für die Frage des Zugangs ist dies auch im funktionalistischen Diskurs wichtig, da hier Informationsangebote wie ,self-assessment“-Verfahren zur Testung der eigenen Studienbefähigung angedacht werden (DIHK/HRK 2008). Diese bieten auch den potenziellen Studierenden die Möglichkeit zu prüfen, welche Angebote zu ihnen passen. Das für den Zugang wichtige geforderte Matchingverfahren zwischen Bewerber/in und Hochschule findet auf diese Weise für beide Seiten statt. Im strukturkonservativen Diskurs sollen die Informations- und Beratungsangebote auch dazu führen, dass die Interessierten prüfen, ob sie den Herausforderungen des Studiums gewachsen sind (DIHK/HRK 2008).

\section{Studienorganisation}

Einig ist man sich auch darüber, dass mit einer neuen Studierendengruppe der beruflich Qualifizierten auch die Studienorganisation flexibilisiert werden sollte. Im strukturkritischen Diskurs wird gefordert, dass viel stärker berufsbegleitende Angebote, also auch Teilzeit- oder Fernstudiengänge, Abendkurse etc. angeboten werden müssten. Auch Strukturen, die das Studieren mit Familie erleichtern, sollen ausgebaut werden. (vgl. BMBF 2001b, 2011a; DIHK/HRK 2008; DIHK 2007a; DGB 2006a; HRK 2007a, 2009a, 2011a). Dies sei notwendig, denn beruflich Qualifizierte unterscheiden sich von traditionellen Studierenden, die direkt nach der Schule an die Hochschulen kommen. Der typische beruflich Qualifizierte, den man an die Hochschule holen will, ist jemand, der oder die bereits erfolgreich im Beruf ist und sich weitergebildet hat. Da diese Individuen dann meist auch älter sind, haben sie oft schon Familienverpflichtungen und können nicht einfach auf Einkommen zugunsten eines Vollzeitstudiums verzichten. Daher sind die Angebote, die ein weiteres Arbeiten im Beruf ermöglichen, als besonders günstig anzusehen.

Niemand, der 28 ist, im Berufsleben steht, schon Meister ist, eine Familie gegründet hat, wird alles liegen und stehen lassen, um dann einen akademischen Abschluss zu erringen. (DIHK/HRK 2008) 
Ein höherer Anteil beruflich qualifizierter Studienanfänger erfordert weitere Investitionen in Studienberatung und Studienplätze, in propädeutische und ergänzende Lehrangebote, in eine flexible Studienorganisation sowie in familienfreundliche Infrastrukturen. (HRK 2008b)

\section{Pädagogik}

Wenn es um die Gestaltung der Studieninhalte bzw. der Pädagogik geht, gehen die Positionen in den Diskursen auseinander. Im funktionalistischen strukturkritischen Diskurs sowie im strukturkonservativen Diskurs werden vor allem Einführungskurse, so genannte Brückenkurse gefordert, um die zukünftigen Studierenden vorzubereiten (HRK 2008b). Auf diesem Weg kann ein erfolgreiches Studium erleichtert (funktionalistisch-strukturkritisch), aber auch die Qualität der Hochschulbildung gesichert werden (strukturkonservativ).

Gleichzeitig empfehlen HRK und DIHK, dass die Hochschulen im Rahmen ihrer Möglichkeiten Vorbereitungs- und Brückenkurse ausbauen, um Defizite beruflich Qualifizierter, vor allem in wissenschaftlichem Arbeiten und fachlich nicht abgedeckten Inhalten auszugleichen. (DIHK/HRK 2008)

Deutlich wird dabei, dass diese Zielgruppe als eine Risikogruppe eingeschätzt wird, die einer besonderen Anpassung bedarf, da sie „Defizite“ aufweise. Die vermeintlich fehlende Passung wird individualisiert und somit als individuelle Defizite, die es zu behandeln gilt, ausgewiesen. Gerade im strukturkonservativen Diskurs wird darauf aufmerksam gemacht, dass häufig ein Fehlen von Allgemeinbildung problematisch sei.

Die Erfahrungen zeigen auch, dass es durchaus Einmündungsschwierigkeiten gibt. Typischerweise, wenn in einem Fach Grundlagen einerseits in der Mathematik und den Naturwissenschaften gefragt sind und andererseits in Englisch. Auf weitere Fremdsprachen brauchen wir gar nicht zu kommen. Das ist Anlass für die Hochschulen, diese Studenten entweder in der Studieneingangsphase besonders zu unterstützen oder Vorkurse anzubieten, sofern sie die Kraft dazu haben. Die großen Hochschulen haben das, die kleinen Hochschulen haben das nicht unbedingt. (HRK 2010a)

Diese Aussagen zeigen, dass die Gruppe der beruflich Qualifizierten homogenisiert wird. Sie werden vor allem im strukturkonservativen Diskurs als Problemfälle beschrieben, die durch eine unzureichende Allgemeinbildung auffallen. Durch diese Typisierung werden beruflich Qualifizierte als weniger kompetent dargestellt und als eine Bürde, um die sich die Hochschulen nun auch noch kümmern müssen. Es kommt dadurch wieder zu einer Trennung von auf der einen Seite traditionellen Studierenden, die über den allgemeinbildenden Weg in die Hochschulen kommen und entsprechend vorbereitet sind, und den beruflich Qualifizierten. Dass aber die Kompetenzniveaus und Bedürfnisse in beiden Gruppen heterogen sind und auch die traditionellen Studierenden Unterstützung zu Beginn des Studiums benötigen könnten, wird nicht reflektiert. Es geht bei diesen Einführungskursen somit darum, dass die beruflich Qualifizierten an die Erfordernisse der Hochschulen, an das gewohnte Kompetenzniveau, angepasst 
werden. Es handelt sich demnach um eine einseitige Anpassung der beruflich Qualifizierten an die Anforderungen der Hochschulen. Eine Anpassung Letzterer im Hinblick auf verwendete Pädagogik oder Studieninhalte ist nicht vorgesehen.

Im universalistischen strukturkritischen Diskurs werden ebenfalls Brückenkurse als hilfreich angesehenen, um den Einstieg beruflich Qualifizierter zu erleichtern (DGB 2006a). Aber es geht nicht nur um eine Anpassung der Studierenden an die neue Lernumwelt, sondern auch eine Anpassung dieser an die unterschiedlichsten Studierenden.

An Hochschulen müssen neue Konzepte der Orientierung und begleitenden Beratung geschaffen werden. Förderangebote sind an den individuellen Lerngeschwindigkeiten und Lernbiographien auszurichten. (DGB 2006a)

Auf diese Weise müsste auch die Lehre für die neue Studierendengruppe geändert werden. Eine solche Anpassung der Pädagogik stellt eine Möglichkeit zusätzlich zu Brückenkursen dar, alle Studierenden entsprechend ihren Kompetenzen zu bilden.

Ich denke aber auch beispielsweise an die Entwicklung flexibler Lehrmethoden, die individuell erlernte Strategien und Besonderheiten der verschiedenen Lern- und Zielgruppen berücksichtigen. (KMK 2011b: 9)

Im Gegensatz zu Brückenkursen explizit für beruflich Qualifizierte stellen neue Lehrmethoden keine spezifischen Maßnahmen nur für beruflich Qualifizierte dar, sondern eine Änderung, die allen Studierenden entsprechend den Idealen inklusiver Bildung gerecht werden soll. So werden diese weniger als Extra-Gruppe mit Extra-Unterrichtsanforderungen stigmatisiert.

\section{Finanzen}

Auch in Fragen der finanziellen Unterstützung sind die Forderungen im universalistischen Diskursstrang am weitreichendsten. Hier sehen vor allem die Gewerkschaften einen doppelten Ansatzpunkt. Einerseits soll das Erwachsenen-Bafög ausgebaut werden und ohne Altersbegrenzung zugänglich sein (DGB 2009e). Hier wird deutlich, dass keine zusätzlichen Strukturen für beruflich Qualifizierte geschaffen werden sollen, sondern die bestehenden einfach an die neuen neue Umstände an den Hochschulen anzupassen seien. Andererseits sollen die Hochschulen an sich finanziell gefördert werden, um die notwendigen Strukturen für beruflich Qualifizierte aufzubauen. So lautet der Vorschlag, dass Studienplätze für beruflich Qualifizierte den Hochschulen Extra-Geld einbringen und damit Anreiz sind, diese auch tatsächlich als relevante Studierendengruppe anzuerkennen und zu behandeln (DGB 2011a). Im Gegensatz zum funktionalistischen strukturkritischen Diskursstrang werden Stipendiensysteme nicht als Lösung gesehen, da diese nur einer hochselektiven Gruppe zugute kommen. Die fördernden Bedingungen sollen stattdessen allen helfen und einen Rechtsanspruch, wie beim Bafög, darstellen. 
Wie gerade angedeutet, wird im funktionalistischen strukturkritischen Diskurs dagegen eine selektive Förderung bevorzugt. Finanzielle Mittel sollten gewährt werden, um besonders begabte beruflich Qualifizierte für ein Studium zu interessieren (BMBF 2009b, 2010b). Die Einführung der Aufstiegsstipendien im Rahmen der Qualifizierungsinitiative steht für diesen Ansatz. Gefördert wird damit nur eine Leistungselite der beruflichen Bildung. Es geht wiederum, wie auch im Aspekt des Zugangs, um eine Begabungsselektion im Gegensatz zu einer generellen finanziellen Berechtigung, wie im universalistischen Diskurs vorgesehen.

Tabelle 18 Vergleich der Diskursstränge zur Frage des Umgangs mit Heterogenität in Deutschland (1999-2012)

\begin{tabular}{|c|c|c|c|}
\hline & \multicolumn{2}{|c|}{ Strukturkritisch } & \multirow{2}{*}{$\begin{array}{c}\text { Strukturkonservativ } \\
\text { Funktionalistisch }\end{array}$} \\
\hline & Universalistisch & Funktionalistisch & \\
\hline Motiv & $\begin{array}{l}\text { Unterstützung beim Zu- } \\
\text { gang und im Studium } \\
\text { für alle zur Sicherung } \\
\text { des Rechts auf Bildung }\end{array}$ & $\begin{array}{l}\text { Attraktivität der Hoch- } \\
\text { schule für neue Klientel } \\
\text { erhöhen sowie Anpas- } \\
\text { sung der Klientel an } \\
\text { Hochschulstrukturen, um } \\
\text { effizientes Arbeiten zu er- } \\
\text { möglichen }\end{array}$ & $\begin{array}{l}\text { Störung des Hoch- } \\
\text { schulbetriebs und } \\
\text { Qualitätsgefährdung } \\
\text { verhindern }\end{array}$ \\
\hline Beratung & $\begin{array}{l}\text { Beratung und Informa- } \\
\text { tion über Studienmög- } \\
\text { lichkeiten }\end{array}$ & $\begin{array}{l}\text { Beratung/Information für } \\
\text { eine bessere Passung - } \\
\text { vor dem Studienbeginn } \\
\text { (Verantwortung beim Indi- } \\
\text { viduum) }\end{array}$ & $\begin{array}{l}\text { Beratung und Informa- } \\
\text { tion - um keine fal- } \\
\text { schen Erwartungen zu } \\
\text { wecken }\end{array}$ \\
\hline Studienorganisation & $\begin{array}{l}\text { Flexibilisierung der Stu- } \\
\text { dienorganisation: } \\
\text { Berufsbegleitende Bil- } \\
\text { dungsgänge, Teilzeit-, } \\
\text { Fernstudium }\end{array}$ & $\begin{array}{l}\text { Flexibilisierung: } \\
\text { Berufsbegleitende Bil- } \\
\text { dungsgänge, Teilzeit-, } \\
\text { Fernstudium } \\
\text { Einführung, familien- } \\
\text { freundliche Infrastruktur }\end{array}$ & $\begin{array}{l}\text { Teilzeit-, Fernstudium } \\
\text { etc. }\end{array}$ \\
\hline Pädagogik & $\begin{array}{l}\text { Anpassung bzw. Um- } \\
\text { stellung von Pädagogik }\end{array}$ & $\begin{array}{l}\text { Brückenkurse, um Erfolg } \\
\text { zu sichern }\end{array}$ & $\begin{array}{l}\text { Brückenkurse, um De- } \\
\text { fizite abzubauen }\end{array}$ \\
\hline Finanzen & $\begin{array}{l}\text { individuelle Unterstüt- } \\
\text { zung durch reformiertes } \\
\text { Bafög ohne Altersbe- } \\
\text { grenzung sowie finanzi- } \\
\text { elle Förderung der } \\
\text { Hochschulen für Stu- } \\
\text { dienplätzen für beruf- } \\
\text { lich Qualifizierte }\end{array}$ & $\begin{array}{l}\text { Finanzielle Unterstützung } \\
\text { für besonders Begabte/ } \\
\text { Leistungselite }\end{array}$ & $\begin{array}{l}\text { Finanzielle Unterstüt- } \\
\text { zung der Hochschulen } \\
\text { für zusätzlicher Arbeit } \\
\text { mit neuer Klientel }\end{array}$ \\
\hline
\end{tabular}

Quelle: Darstellung der Verfasserin

Individuelle finanzielle Förderung explizit für beruflich Qualifizierte wird im strukturkonservativen Diskurs nicht gefordert. Vielmehr wird darauf hingewie- 
sen, dass eine größere finanzielle Unterstützung der Hochschulen eine notwendige Voraussetzung ist, um eine neue Gruppe von Studierenden, die Extra-Strukturen benötigen, in die Hochschulen aufzunehmen (HRK 2011a). So lässt sich festhalten, dass auch im strukturkonservativen Diskurs die Notwendigkeit gesehen wird, bestehende Strukturen an die neue Studierendengruppe beruflich Qualifizierter anzupassen. Dies ist zwar erst eine Entwicklung in den letzten Jahren, aber sie zeigt, dass auch im Verständnis der Träger des strukturkonservativen Diskurses beruflich Qualifizierte in den Hochschulen angekommen sind, wenn auch teilweise nur als Belastung.

Zusammenfassend kann festgehalten werden, dass sich gerade in den letzten Jahren des zweiten Untersuchungszeitraums das Verständnis durchgesetzt hat, dass beruflich Qualifizierte in ihren Bedürfnissen unterstützt werden müssen, um Durchlässigkeit erfolgreich zu gestalten bzw. Durchlässigkeit nicht zum Risiko für die Hochschulqualität werden zu lassen (vgl. auch Tabelle 18). Auch wenn vielfach ähnliche Formen von unterstützenden Strukturen in den Diskursen gefordert wird, kann ein großer Unterschied vor allem in der Frage gesehen werden, ob beruflich Qualifizierte als gewünschte oder belastende Zielgruppe konstruiert wird. Darüber hinaus wird deutlich, dass die Strukturen so angelegt sein können, dass sie beruflich Qualifizierte von den „traditionellen“ Studierenden abheben und eine Differenz zwischen diesen Gruppen konstruieren oder diese stärker inkludieren. Letzteres wird primär im universalistischen Diskursstrang getan.

\section{Akteurspositionen und Zusammenfassung}

Wie auch für den ersten Zeitraum sollen nachfolgend die untersuchten Akteure in Relation zu den identifizierten Diskurssträngen gebracht werden. Wer ist hauptsächlicher Träger welches Diskurses? Des Weiteren werden die Ziele und die aus den Diskursen rekonstruierten Strategien zusammengefasst und verglichen. Dargestellt werden die Akteurspositionen wieder in einer positional map in Abbildung 7, wobei die helleren markierten Positionen, die des ersten Untersuchungszeitraums sind und somit die Veränderungen im Zeitverlauf sichtbar werden. Es wird wiederum auf der y-Achse unterschieden zwischen vollständig (5), sehr deutlich (4), deutlich (3), mehrheitlich (2), vorwiegend (1) strukturkritisch bzw. -konservativ und auf der x-Achse zwischen (fast) vollständig (3), deutlich (2) und vorwiegend (1) funktionalistisch bzw. universalistisch.

\section{Akteurspositionen}

Wie in der Darstellung der Diskursstränge gezeigt werden konnte, sind wiederum nicht einzelne Akteure mit ihren Aussagen nur einem Diskurs oder Diskursstrang zuzuordnen. 


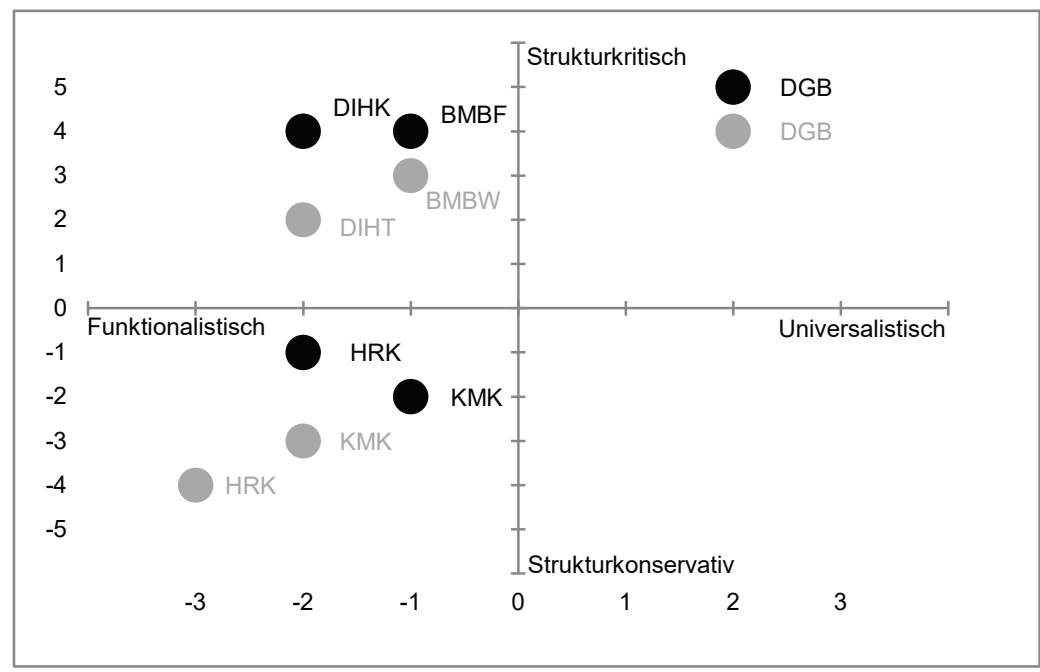

Die helleren eingetragenen Akteurspositionen sind die aus dem Zeitraum 1990 bis 1998.

Quelle: Darstellung der Verfasserin

Deutlich wird auch, dass insgesamt klar funktionalistische Argumentationslogiken (x-Achse) weiterhin vorherrschen, wobei insbesondere die DIHK und die HRK vielfach funktionalistisch argumentieren. Aber auch die Aussagen des BMBF, der HRK und der KMK sind eher den funktionalistischen Mustern zuzuordnen. Allein in den Aussagen des DGB können stärker universalistische Argumentationsmuster gefunden werden. Im Kontinuum zwischen funktional und universalistisch wird jedoch sichtbar, dass auch in den Aussagen von BMBF und KMK deutlich universalistische Muster sichtbar sind. Betrachtet man die Verteilung auf der $\mathrm{y}$-Achse, ergibt sich ein ähnliches Bild wie im ersten Zeitraum. Aber auch Bewegungen der Akteure werden sichtbar. Zum einen ist festzustellen, dass sich die Aussagen der Akteure alle in Richtung strukturkritischen Diskurs verlagert haben. Dennoch können weiterhin die Vertreter der höheren Allgemeinbildung, die KMK und die HRK, als Träger des strukturkonservativen Diskurses identifiziert werden. Aber es wird in den Aussagen ebenfalls deutlich, dass auch von den Trägern des strukturkonservativen Diskurses eine begrenzte Notwendigkeit von Durchlässigkeit, bedingt vor allem durch einen Strukturwandel, anerkannt wird. Auch das Bildungsministerium und die Kammern sind in ihren Aussagen stärker strukturkritisch als im ersten Zeitraum. Die Zuordnung vom DGB zum eher universalistischen strukturkritischen Diskursstrang und des BMBF und des DIHK zum funktionalistischen strukturkritischen Diskursstrang bleibt gleich. 


\title{
Zusammenschau der Diskurse und der rekonstruierten Strategien
}

In diesem Abschnitt sollen die aus den Diskursen rekonstruierten diskursspezifischen Ziele und Strategien und die differenten Verantwortungszuschreibung gegenübergestellt werden. Dieser Abschnitt dient dazu, die drei Diskursstränge, die in Deutschland für den Zeitraum 1999-2012 modelliert wurden, in ihrer Typik zusammenzufassen.

Der universalistische strukturkritische Diskursstrang zielt darauf ab, Durchlässigkeit von der Berufs- zur Hochschulbildung basierend auf der Anerkennung der Gleichwertigkeit der Bildungswege sowie dem Recht auf Bildung durchzusetzen. Bildung dient dabei sowohl der individuellen Persönlichkeitsentfaltung als auch zur Lösung gesellschaftlicher Probleme wie sozialer Ungleichheit. Zudem sollen traditionelle Hierarchien im Bildungssystem zwischen Berufs- und Hochschulbildung aufgebrochen werden.

Erreicht werden soll Durchlässigkeit als eine Form des Rechts auf Bildung primär über die Wege der Verrechtlichung - z.B. einen selektionsfreien Zugang ins Hochschulsystem über Berechtigungen, die in der Berufs- und der Allgemeinbildung erworben werden, die Festschreibung von Anrechnungsmöglichkeiten in Gesetzen, gesetzliche Berechtigungen z.B. finanzielle Unterstützung im Rahmen des Bafög. Wichtig ist, dass die Förderung und Unterstützungsmaßnahmen nicht nur selektiven Gruppen, sondern allen zugute kommen. Auch neue Instrumente wie der deutsche Qualifikationsrahmen sollen perspektivisch mehr sein als ein reines Informationsinstrument sein. Er soll bei Anrechnungs- und Zugangsfragen sowie bei der Festlegung von Gehältern genutzt werden.

\begin{abstract}
So heißt es etwa in dem Text, dass, das Erreichen einer Niveaustufe entkoppelt von tarif- und besoldungsrechtlichen Auswirkungen' sei. Aus DGB-Sicht wird die Entwicklung ganz anders aussehen: Sobald Beschäftigte ihre tatsächlich vorhandenen Kompetenzen mit Brief und Siegel nachweisen können, werden sie auch auf eine entsprechende Bezahlung pochen. Da kommt absehbar Arbeit auf die Tarifvertragsparteien zu. DGB (2009a)
\end{abstract}

Auf diese Weise kommt dem Qualifikationsrahmen im universalistischen Diskursstrang eine zentrale Rolle zur Feststellung und Durchsetzung der Gleichwertigkeit von Berufs- und Hochschulbildung zu. Die Frage von Gleichwertigkeit und auch deren Anerkennung außerhalb des Bildungssystems ist zentral für den universalistischen Diskursstrang.

Aus der großen Bedeutung von regulierenden Maßnahmen für Durchlässigkeit ergibt sich, dass primär die staatlichen Instanzen verantwortlich gezeichnet werden. So sollen entsprechende Gesetze auf Bundes- und Landesebene festgeschrieben werden. Die Hochschulen sind dann in einem zweiten Schritt verantwortlich, die entsprechenden Gesetze auch adäquat umzusetzen und z.B. Studienbedingungen an die unterschiedlichen Gruppen von Studierenden anzupassen oder auch Anrechnungen fair vorzunehmen. 
Im funktionalistischen strukturkritischen Diskurs wird Durchlässigkeit primär als funktionale Notwendigkeit zur Lösung gesellschaftlicher Probleme konstruiert, wobei den Bedarfen des Arbeitsmarkts eine zentrale Rolle zukommt. Im Rahmen dieses Diskurses erfolgt eine Ökonomisierung von Bildung, indem diese nicht als Mittel der Persönlichkeitsentwicklung und Ausbildung von kritischen Bürgern wie im universalistischen Diskurs gesehen wird. Vielmehr steht die Erhöhung des individuellen Humankapitals im Vordergrund. Das Individuum wird zur Ressource. Aber nicht nur der Zweck von Bildung wird ökonomisiert. Auch der Weg zu Durchlässigkeit verlangt im funktionalistischen strukturkritischen Diskurs eine Einführung von Marktprinzipien zur Effizienzsteigerung z.B. bei der Selektion der Studierenden, die als Match zur Hochschule beschrieben werden. Gerade bei Zugangsfragen wird eine Selektion basierend auf Leistung und Begabung als notwendig erachtet, um die Besten an die Hochschulen zu führen und erfolgreiches Studieren zu sichern. Auch eine stärkere Beteiligung von Wirtschaftskreisen wird als Weg gesehen, Durchlässigkeit zu erreichen, vor allem über integrierte Bildungsgänge wie ein duales Studium, aber auch bei Fragen der Anrechnung, bei der standardisierte Verfahren und Kooperationen zwischen Wirtschaftsakteuren, Kammern und Hochschulen einen effizienten und transparenten Weg liefern sollen. Verantwortlich werden weniger staatliche Akteure gezeichnet, und Regulierung wird als weniger wichtig angesehen. Allein bei Fragen der Finanzierung ist öffentliche Unterstützung gefragt. Stattdessen liegt die Verantwortung einerseits bei den Hochschulen, die ihre Strukturen teilweise anpassen müssen und die Selektion der Studierenden vorzunehmen haben, und andererseits bei den Individuen selbst, die sich um die passende Hochschule und auch um eine gute individuelle Vorbereitung an die Anforderungen des Studiums zu kümmern haben. Eine Anpassung der pädagogischen Konzepte an individuelle Bedürfnisse ist dagegen nicht vorgesehen. Aber die Studienstruktur soll flexibilisiert werden, um eine zusätzliche Erwerbstätigkeit, also die gleichzeitige Bereitstellung des Humankapitals auf dem Arbeitsmarkt, besser zu ermöglichen.

Der funktionalistische strukturkonservative Diskurs hat zum Ziel, einerseits Durchlässigkeit zu begrenzen und den traditionell geltenden Maßstab der höheren Allgemeinbildung für die Regelungen des Hochschulsystems zu festigen sowie die traditionell bestehenden gesellschaftlichen Hierarchien, in denen höherer Allgemeinbildung mehr Wert beigemessen wird als Berufsbildung, zu erhalten. Strategie der Träger des Diskurses ist zum einen die Konstruktion eines Gefahrenszenarios, welches bei unkontrollierter Durchlässigkeit von Berufs- zur Hochschulbildung Wirklichkeit werden kann. Die Gefahr besteht zum einen für die Qualität der Hochschulbildung und damit auch für die traditionellen Studierenden durch beruflich Qualifizierte, die wegen des fehlenden Allgemeinbildungsniveaus nicht adäquat auf die Hochschulen vorbereitet sind. Zum anderen besteht sie auch für die Individuen selbst, die durch unkontrollierte Zugänge unnötige Misserfolge verkraften müssen. Daher ist es wichtig, dass die Akteure der höheren 
Allgemeinbildung Verantwortung übernehmen und die Bedingungen von Durchlässigkeit kontrollieren. So bleibt bei Fragen des Hochschulzugangs das Allgemeinbildungsniveau Maßstab der Studierfähigkeit, zudem sind die Zugangsentscheidungen über die aufnehmenden Institutionen oder die durch die KMK bestimmten gesetzlich festgelegten Regelungen zu fällen. Auch die Letztentscheidung über die Anrechnung beruflicher Qualifikationen bleibt bei den aufnehmenden Institutionen, zudem wird die Anrechnungshöhe begrenzt, so dass der hochschulische Lernort und damit die akademischen Akteure bei der Vergabe akademischer Titel entscheidend bleiben. Schließlich sind Strukturen wie Brückenkurse einzufügen, die die bestehenden Defizite beruflich Qualifizierter verringern. Letztere werden als homogene Gruppe konstruiert, die nur an der Hochschule bestehen kann und keine Gefahr mehr ist, wenn sie über die Vermittlung von Allgemeinbildung (zweiter Bildungsweg, Brückenkurse etc.) ihr Niveau anpasst. Während eine Einmischung in Fragen der Durchlässigkeit durch Akteure außerhalb der höheren Allgemeinbildung weitestgehend abgelehnt wird, wird eine breite finanzielle staatliche Unterstützung gefordert, da nur so die Hochschulen diese neue Gruppe Studierende aufnehmen könnten.

Im Gegensatz zum universalistischen strukturkritischen Diskurs beruht im strukturkonservativen Diskurs die Strategie darin, zum einen neue Instrumente wie den deutschen Qualifikationsrahmen in ihrer Macht als neues bildungspolitisches Klassifikationsinstrument zu begrenzen und bestehende Regelungen aufrechtzuerhalten. Zum anderen wird darauf abgezielt, die Position der höheren Allgemeinbildung, d.h. vor allem des Abiturs und der akademischen Abschlüsse, im DQR zu stärken. So wird einerseits versucht, die Position des Abiturs auf einer höheren Stufe als die sekundären beruflichen Abschlüsse einzuordnen und damit eine Ungleichwertigkeit festzuschreiben. Zum anderen wird versucht, die Alleinstellungsmerkmale der akademischen Bildung als konstitutiv für die höchsten Qualifikationsstufen des Qualifikationsrahmens (Stufe 6-8) festzuschreiben, indem die Deskriptoren stark wissens- und forschungsbasiert beschrieben werden und weniger im Hinblick auf Handlungskompetenzen, die außerhalb der Hochschule erworben werden können.

Insgesamt kann festgehalten werden, dass sich einiges seit 1999 getan hat. So sind allgemein die Aussagen der Akteure strukturkritischer geworden - im Sinne einer Besserstellung der beruflichen Bildung bei Fragen der Durchlässigkeit zwischen Berufs- und Hochschulbildung. Allerdings gibt es auch weiterhin klare Deutungskämpfe über Durchlässigkeit und die Hierarchien der Bildungsbereiche. Eine ausführlichere Analyse des Wandels der nationalen Durchlässigkeitsstrukturen erfolgt im nächsten Abschnitt. 


\subsection{Analyse des institutionellen Wandels}

Im folgenden Abschnitt soll einerseits eine Bewertung der diskursiven Veränderungen seit 1990 vorgenommen werden. Auf diese Weise werden Änderungen vor allem auf der kulturell-kognitiven und zum Teil auch auf der normativen institutionellen Dimension zusammengefasst. Gleichzeitig sollen aber auch wichtige regulative Veränderungen, die in diesem Zeitrahmen stattgefunden haben, betrachtet werden, um einen umfassenderen Einblick in die Änderungen der Durchlässigkeitsstrukturen in Deutschland zu erhalten. Der Einbezug der regulativen Dimension ist wichtig, um einzuschätzen, inwiefern die vorgefundenen Veränderungen in den Wissensvorräten sich auch in den rechtlichen Rahmenbedingungen und damit auch viel stärker den Opportunitätsstrukturen der Individuen niederschlagen. Klare kausale Wirkungszusammenhänge zwischen der Veränderung der diskursiven Struktur und den rechtlichen Regelungen können an dieser Stelle nicht aufgezeigt werden. Es wird aber sehr wohl deutlich, dass in Deutschland Veränderungen in den Diskursen regulativen Änderungen oft vorausgehen.

In der Gesamtschau ist es dann auch möglich einzuschätzen, ob es seit 1990 zu einer Fortführung eingeschlagener Entwicklungspfade, zu deren Veränderung oder sogar zu einer gänzlichen Abkehr von historisch gewachsenen Institutionen gekommen ist. Während eine Weiterführung bedeuten würde, dass die gewachsene Hierarchie der Bildungsbereiche zugunsten der höheren Allgemeinbildung in Deutschland und das Schisma zwischen Berufs- und höherer Allgemeinbildung mit der institutionellen Trennung der Bildungsbereiche ohne Übergänge weiterbestehen würden, hieße eine Abkehr von diesen institutionellen Prinzipien faktisch entweder eine Gleichwertigkeit oder sogar Höherbewertung der beruflichen Bildung und/oder eine aufgehobene Trennung. Ohne der Zusammenfassung der Entwicklungen vorzugreifen, ist nach der Beschreibung der diskursiven Strukturen für den deutschen Fall festzustellen, dass beide Szenarien nicht zutreffen. Insofern handelt es sich, wenn man der analytischen Trennung folgt, am ehesten um eine Veränderung institutioneller Entwicklungspfade, die aber nicht zu einer Umkehr oder Abkehr und auch nicht zu einer bloßen Verstärkung der deutschen gewachsenen Durchlässigkeitsinstitutionen geführt haben.

Nachfolgend sollen daher die entscheidenden Veränderungen zusammengefasst werden. Gleichzeitig soll aber verdeutlicht werden, worin eine Weiterführung des institutionellen Pfads und die zum Teil damit zusammenhängenden Barrieren für Durchlässigkeit zwischen Berufs- und Hochschulbildung in Deutschland bestehen. 


\subsubsection{Veränderungen der institutionellen Strukturen in Richtung Durchlässigkeit}

Der Wandel der Durchlässigkeitsstrukturen seit 1990 unter Berücksichtigung der historischen Entwicklungen, die in Kapitel 6 zusammengefasst wurden, steht hier im Mittelpunkt. So soll gezeigt werden, welcher Wandel sich in Bezug auf die in den Diskursen rekonstruierten Wissensvorräte zu Durchlässigkeit feststellen lässt. Zudem werden auch wichtige regulative Strukturen in die Beurteilung des Wandels mit einbezogen. Einen Überblick über die regulativen Entwicklungen gibt Tabelle 39 im Anhang.

Allein beim Vergleich der Debatten in den zwei Zeiträumen werden Veränderungen sichtbar. Durchlässigkeit wurde zwar im ersten Zeitraum diskutiert, entwickelte sich aber im zweiten Zeitraum zu einem wichtigen bildungspolitischen Thema. Dies lässt sich daran feststellen, dass Durchlässigkeit nicht nur in einer Debatte, sondern im Kontext vieler bildungspolitischer Themen diskutiert wurde. Insofern kann eine gestiegene Relevanz von Durchlässigkeit festgehalten werden.

Geändert haben sich auch die Argumentationen für Durchlässigkeit, wobei die dominierende Logik eine funktionalistische geblieben ist. Im ersten Zeitraum waren es vor allem die mangelnde Attraktivität der Berufsbildung und die Angst vor einem daraus resultierenden Facharbeitermangel, die durch eine erhöhte Durchlässigkeit zum Hochschulstudium behoben werden sollten. Ziel war damit nicht primär, mehr Individuen in die Hochschulbildung zu bringen, sondern die berufliche Ausbildung als Bildungswahl wieder attraktiver zu machen. Im zweiten Zeitraum wurde auch ein Fachkräftemangel, der durch einen Strukturwandel der Wirtschaft sowie technologischen und demografischen Wandel hervorgerufen wird, als dominierender Grund für den Ausbau von Durchlässigkeitsstrukturen erkannt. Diesmal steht aber der Bedarf an akademisch Qualifizierten im Vordergrund, und beruflich Qualifizierte werden als ,Begabungsreserve' gesehen. Insofern geht es darum, beruflich Qualifizierte tatsächlich in die Hochschulbildung zu bekommen.

Wie haben sich das Verständnis von und die Wissensvorräte zu Durchlässigkeit geändert? Im ersten Zeitraum wurde, wenn über Durchlässigkeit debattiert wurde, über Fragen des Hochschulzugangs und zum Teil über integrierte Bildungswege nachgedacht. Im zweiten Zeitraum hat sich das Verständnis von Durchlässigkeit und dessen, was dazugehört, deutlich gewandelt: Neben Fragen des Zugangs zur Hochschulbildung und auch Fragen organisationaler Verbindungen in Form von integrierten Bildungsgängen ist die Anrechnung beruflicher Qualifikationen in der Hochschulbildung ein zentrales Thema in den Durchlässigkeitsdiskursen. Neu ist auch, dass seit 2008 verstärkt darüber nachgedacht wird, welche Strukturen etabliert werden müssen, um beruflich Qualifizierte für ein Hochschulstudium zu gewinnen bzw. erfolgreich durch ein solches zu bringen. Im Folgenden sollen kurz die wichtigsten Änderungen im Diskurs sowie entsprechende rechtliche Regelungen dargestellt werden. 


\section{Zugang}

Betrachtet man die aus den Diskursen rekonstruierten Wissensvorräte im Hinblick auf Fragen des Zugangs, lassen sich folgende Veränderungen festhalten: Im ersten Zeitraum wurde teilweise darüber nachgedacht, ob das Thema Hochschulzugang für beruflich Qualifizierte überhaupt diskutierwürdig sei (HRK 1991) und ob beruflich Qualifizierte an die Hochschulen kommen sollten. Im zweiten Zeitraum geht es weniger um die Frage des $\mathrm{Ob}$ als viel stärker um die Fragen des Weges in die Hochschule, welche beruflich Qualifizierten es sein sollen und wer die Entscheidungsgewalt über den Hochschulzugang bekommt. Eine entscheidende Veränderung sowohl in der kulturell-kognitiven als dann auch sichtbar in der regulativen Dimension ist die viel größere Akzeptanz der Berechtigungen zum Hochschulzugang, die über die Berufsbildung erworben werden. Dies stellt einen Paradigmenwechsel dar, da das Berechtigungsmonopol der Allgemeinbildung nicht nur diskursiv infrage gestellt wird, sondern auch Berechtigungen für Berufsbildung regulativ eingeführt werden. Während im ersten Zeitraum eine erste Welle des Ausbaus der Möglichkeiten des Hochschulzugangs primär über Prüfungen anging (vgl. auch im Tabelle 3 Online-Anhang), ${ }^{114}$ wurden im zweiten Zeitraum, insbesondere nach dem KMK-Beschluss 2009, in vielen Bundesländern die Berechtigung für den allgemeinen und nicht nur den eingeschränkten Hochschulzugang für beruflich Fortgebildete (Meister, Techniker und äquivalente Abschlüsse) eingeführt (vgl. Ulbricht 2012a). Wenn auch nicht über Berechtigung zur allgemeinen Hochschulreife, so wurde im zweiten Zeitraum zudem auch, sowohl diskursiv als auch regulativ, der Zugang für Absolvent_innen mit Erstausbildung liberalisiert - die teilweise über eine Prüfung, aber teilweise auch mit einer direkten Anerkennung einer eingeschränkten, also meist fachbezogenen Hochschulzulassung einherging (vgl. Ulbricht: 2012a). Zuvor gab es für beruflich Qualifizierte vor allem den zweiten Bildungsweg in die Hochschulen, den Weg über die Allgemeinbildung, der bereits vor 1990 mit den Abendgymnasien, den Berufsund Fachoberschulen, den Kollegs sowie den Nichtschülerprüfungen bestand.

Es wird somit deutlich, dass sich in Sachen des Zugangs nicht nur die Kulturell-Kognitive und Normative, sondern auch die Regulative zu mehr Durchlässigkeit zwischen Berufs- und Hochschulbildung verändert haben.

114 Waren es 1990 nur sieben Länder, so wiesen 1994 alle Bundesländer mindestens eine Regelung, und zwar dem Zulassungstyp der Prüfung entsprechend, aus, die es beruflich Qualifizierten mit Fortbildung (in allen Ländern) und beruflich Qualifizierten ohne Fortbildung (in allen Ländern außer in BW und BY) ermöglichten, ein Studium aufzunehmen (vgl. Tabelle 3 im OnlineAnhang). Der Zulassungstyp des Probestudiums wurde in drei weiteren Ländern eingeführt, und vier Länder (HH, NW, RP, ST) berechtigten beruflich Fortgebildete (mit Meister oder äquivalentem Abschluss) den eingeschränkten Hochschulzugang ohne vorherige Eignungsprüfung (vgl. Ulbricht 2012a). Der erste Untersuchungszeitraum stellt daher eine Phase der breiten regulativen Institutionalisierung des dritten Bildungswegs in den deutschen Bundesländern dar. 


\section{Anrechnung}

Anrechnung ist ein zentraler Bestandteil des Verständnisses von Durchlässigkeit im zweiten Zeitraum. Dies stellt eine große Veränderung im Vergleich zu der Zeit vor 1999 dar, in dem Anrechnung beruflicher Kompetenzen im Hochschulstudium kein Bestandteil des Diskurses war. ${ }^{115}$ Mit der Frage nach Anrechnung wird die Möglichkeit betrachtet, dass beruflich Qualifizierte nicht nur in der Lage sein könnten zu studieren, sondern dass sie bereits etwas können, das auch im Studium vermittelt wird. Dass gleichwertige Kompetenzen in beiden Bildungsbereichen erworben werden können, wird somit im zweiten Untersuchungszeitraum denkbar. Bereits vor 1999 war im Hochschulrahmengesetz (\$19) die Möglichkeit einer Einstufungsprüfung ${ }^{116}$ festgeschrieben, aber tatsächlich wurde sie nur in wenigen Bundesländern (Bremen, Hessen, Hamburg und Nordrhein-Westfalen) durchgeführt (siehe Freitag 2012). Im zweiten Zeitraum wurde hingegen 2002 von der KMK beschlossen, dass bis zu $50 \%$ eines Studiums durch außerhochschulisch erworbene Kompetenzen angerechnet werden dürfen (KMK 2002). Als eine Reaktion darauf startete 2005 die vom BMBF geförderte ANKOM-Initiative, eine bundesweites Pilotinitiative, in der primär anhand von Modellprojekten geprüft werden sollte, inwieweit formal im beruflichen Weiterbildungssystem erworbene Kompetenzen anrechenbar sind. Aber auch informell erworbene Kompetenzen sollten auf ihre Anrechenbarkeit geprüft werden (vgl. Hartmann/Stamm-Riemer 2006). Deutlich wird, dass eine Äquivalenz vor allem zwischen beruflicher Weiterbildung und Hochschulbildung und nicht beruflicher Erstausbildung gesehen wird. Mit den Novellierungen der Landeshochschulgesetze als Folge des KMKBeschlusses zur Erweiterung des Hochschulzugangs von 2009 wurde Anrechnung auch weitgehend in den Landesgesetzen festgeschrieben (Hanft/Müskens 2013). Zudem wurden im Zuge der Bologna-Reformen Leistungspunktesysteme und Modularisierung eingeführt, die als hilfreich für Anrechnung gelten.

Aber auch wenn sich vieles getan hat, gibt es noch keine bundesweit einheitlichen Verfahren, und die Praxis der Anrechnung ist noch sehr abhängig von den einzelnen Hochschulorganisationen, zumal die Implementierung von Anrechnungsverfahren ein langwieriger Prozess ist, der von vielen Akteuren innerhalb der Hochschule und durch Ressourcenzuwendung unterstützt werden muss (Hanft/Müskens 2013). Trotz der noch nicht selbstverständlichen Praxis der Anrechnung ist aber festzuhalten, dass sich vor allem in der kulturell-kognitiven Dimension ein großer Wandel vollzogen hat, da nun Anrechnung als Bestandteil

115 Eine Ausnahme bildet hier die Forderung der DIHK für beruflich Qualifizierte, die über den zweiten Bildungsweg an die Hochschulen kommen.

116 Die Einstufung erfolgte meist für das zweite Semester (siehe Freitag 2012). Voraussetzungen für die Teilnahme waren zumindest in NRW und Bremen ein Mindestalter (25 Jahre), mehrere Jahre Berufserfahrung und eine abgeschlossene berufliche Erstausbildung und teilweise die Beschränkung auf einen bestimmten Wohnort ( 555 Bremisches Hochschulgesetz 1988, \ 66 Hochschulgesetz für NRW 1993 bei Freitag 2012). 
durchlässiger Bildungsstrukturen gilt und damit prinzipiell die Gleichwertigkeit von Erlerntem aus der Berufs- und höheren Allgemeinbildung anerkannt wird.

\section{Organisationale Verbindung}

Im Hinblick auf organisationale Verbindungen zwischen Berufs- und Hochschulbildung sind in den Diskursen nur geringe Veränderungen festzustellen. So werden die integrativen Formen weniger dafür gedacht, begabte Individuen in die Berufsbildung zu „locken“, wobei diese im vorgestellten Rahmenmodell der DIHT im ersten Zeitraum nicht einmal einen Hochschulabschluss machen müssten, sondern sie werden als wirkliche Verbindung von Berufs- und Hochschulbildung gesehen. Regulativ wurden im zweiten Zeitraum duale Studiengänge weiterhin kontinuierlich ausgebaut und auch im Rahmen der Qualifizierungsinitiative vom Bund gefördert. Zudem wurden die dualen Studiengänge auch an Berufsakademien auf Bachelor und Master umgestellt, so dass nun eine vereinfachte Bildungsmobilität innerhalb des Hochschulwesens rechtlich möglich ist.

\section{Heterogenität}

Wesentlich bedeutender als Zeichen der institutionellen Veränderung der Durchlässigkeitsstrukturen als die Entwicklung in Hinsicht auf organisationale Verbindungen sind die Veränderungen im Hinblick auf den Umgang mit den heterogenen Bedürfnissen der Lernenden. Im ersten Zeitraum wurden beruflich Qualifizierte weitestgehend nicht als Gruppe von Studierenden definiert, denen durch auf ihre Bedürfnisse ausgerichtete Strukturen das Studium erleichtert werden sollte. Im zweiten Zeitraum kann dagegen eine weitestgehende Akzeptanz darüber festgestellt werden, dass beruflich Qualifizierte Teile der Studierendenschaft ausmachen können. Mit dieser Akzeptanz einer neuen Gruppe von Studierenden geht auch die Einsicht einher, dass bestimmte Strukturen, die bisher auf den traditionellen jungen Vollzeitstudierenden ausgerichtet sind, für beruflich Qualifizierte nicht passend sein könnten. Übereinstimmend wird anerkannt, dass vor allem die Studienorganisation flexibilisiert werden soll, damit sie besser mit der Berufstätigkeit und den Familienverpflichtungen der meist älteren, beruflich vorqualifizierten Studierenden vereinbart werden kann. Dies soll vor allem durch zeitliche und örtliche Flexibilisierung geschehen. Auch eine explizite Hilfe bei der Finanzierung eines Studiums wird gefordert. Informations- und Beratungsangebote für beruflich Qualifizierte sowie Einführungskurse sollen ebenfalls ausgebaut werden.

Regelungen, die diesen Forderungen entsprechen, gab es vor dem zweiten Untersuchungszeitraum kaum. Die Ausnahme bildet das Fernstudium. Der Fernunterricht weist eine lange Tradition auf und kann bis ins 18. Jahrhundert zurückverfolgt werden (vgl. Lehmann 2012). Staatlich institutionalisiert wurde das Fernstudium in Deutschland mit der Gründung der Fernuniversität Hagen im Jahr 
1974. Neben der Gründung der Fernuniversität Hagen ist aber auch seit dem ersten HRG in $\ 13$ die Aufforderung verankert, bei der Reform von Studium und Lehre Fernstudienmöglichkeiten einzurichten. Und auch in den einzelnen Landeshochschulgesetzen wurde bereits vor 1990 das Fernstudium reguliert bzw. festgelegt, dass es der Förderung bedarf (Vgl. Tabelle 4 im Online-Anhang).

Im Gegensatz zum Fernstudium ist das Teilzeitstudium als Studienmöglichkeit in den gesetzlichen Regelungen wenig präsent. ${ }^{117}$ Ein Ausbau dieser Möglichkeit wurde zum Teil am Ende des ersten vor allem im zweiten Untersuchungszeitraum in den einzelnen Bundesländern eingeführt, wobei in einigen Landeshochschulgesetzen explizit beruflich Qualifizierte als Zielgruppe gefördert werden sollen (vgl. Tabelle 5 im Online-Anhang). Wie bei der Umsetzung von Anrechnung war auch die Einführung der Leistungspunktesysteme und der Modularisierung hilfreich bei der Umsetzung und Ermöglichung von Teilzeitstudiengängen.

Auch wenn die erste Form der Studienberatung bereits 1904 eingeführt wurde, so erfolgte der große Ausbau der Beratung in den 1970er Jahren, so dass sich auch im Hochschulrahmengesetz seit 1976 ein Paragraf (\$ 14 HRG 1976118) auf die Aufgabe der Studienberatung der Hochschulen bezieht (vgl. Rott/Wickel 1995). Ob gesetzlich geregelt oder nicht, hatten Anfang der 1980er Jahre so gut wie alle Hochschulen (auch viele Fachhochschulen) Beratungseinrichtungen institutionalisiert (Rott/Wickel 1995: 247). Nach der Analyse der relevanten Paragrafen der Landeshochschulgesetze ${ }^{119}$ kann festgestellt werden, dass die Studienberatung generell rechtlich institutionalisiert, aber im gesamten Untersuchungszeitraum nicht auf spezifische Gruppen ausgerichtet ist. Inwiefern in der Praxis auch die spezifischen Bedürfnisse der Studierenden mit beruflichem Hintergrund in Betracht gezogen wurden, kann an dieser Stelle nicht gesagt werden. An einigen Hochschulen, wie in Oldenburg, wurden aber in den letzten Jahren explizit Beratungs- und Begleitungsprogramme für beruflich Qualifizierte entwickelt (vgl. Hanft/Brinkmann 2013). Die Bedeutung der Studienberater_innen wird auch in der Forschung unterstrichen, denn diese können gerade auch für ,nicht traditionelle‘ Studierende die Rolle von Gatekeepern übernehmen, die die Studierenden

117 Die einzige Ausnahme ist Berlin, wo bereits 1986 im Landeshochschulgesetz geschrieben stand, dass Teilzeitstudiengänge eingerichtet werden können, um ein Studium neben dem Beruf zu ermöglichen (LHG Berlin 1986). Inwiefern es aber trotz fehlender Erwähnung in den Gesetzestexten Möglichkeiten des Teilzeitstudiums an einzelnen Hochschulen vor $1999 \mathrm{gab}$, kann mit dieser Analyse nicht gezeigt werden. So schreibt auch die HRK (1997a), dass es bis zu diesen Zeitraum wenige Studien gibt. „Die Hochschulstatistik, Studien- und Prüfungsordnungen sowie die aktuellen hochschulpolitischen Diskussionen zur Studiendauer betrachten weiterhin alle Studierenden als Vollzeit-Studierende, obwohl vermutlich 20-30\% der Studierenden lediglich ein Teilzeitstudium betreiben.“

118 Eine Übersicht über die für die Analyse verwendeten Hochschulgesetze findet sich im OnlineAnhang IV.

119 Siehe Fußnote 118. 
entweder er- oder entmutigen und somit die Gruppe der beruflich Qualifizierten besser oder schlechter integrieren (Alheit 2009).

Schließlich wurde 2008, im zweiten Zeitraum, im Rahmen der Qualifizierungsinitiative der Bundesregierung das Aufstiegsstipendium für begabte beruflich Qualifizierte und damit die erste Form der finanziellen Unterstützungsleistung speziell für beruflich Qualifizierte eingeführt.

Insgesamt wird deutlich, dass vor allem im Diskurs, also in den gesellschaftlichen Wissensvorräten zu Durchlässigkeit zwischen Berufs- und Hochschulbildung, Strukturen, die auf die heterogenen Bedürfnisse von beruflich qualifizierten Studierenden ausgerichtet sind, deutlich an Bedeutung gewonnen haben und Teil des Durchlässigkeitsverständnisses geworden sind. Auch die diesbezüglichen Regelungen wurden vor allem im zweiten Zeitraum, wenn auch noch nicht im Hinblick auf alle in den Diskursen geforderten Strukturen, ausgebaut.

Als Fazit kann damit festgehalten werden, dass sowohl in den Wissensvorräten, die vor allem die kulturell-kognitive und die normative Dimension der deutschen Durchlässigkeitsstrukturen ausmachen, als auch in der Regulative deutliche Entwicklungen in Richtung einer größeren Durchlässigkeit zwischen Berufs- und Hochschulbildung in Deutschland feststellbar sind. Das Verständnis von Durchlässigkeit wurde z.B. stark erweitert, indem neben Fragen des Zugangs und der organisationalen Verbindungen auch Anrechnung und Strukturen, die die Heterogenität der Studierenden beachten, zentral sind. Als besonders wichtiger Schritt in Richtung Durchlässigkeit ist dabei die Abkehr vom alleinigen Weg in die Hochschulbildung über die Allgemeinbildung zu sehen. Dieser Weg wurde bereits in den 1990er Jahren vorbereitet (vgl. Wolter 1994), aber mit der Einführung von Berechtigungen zum Hochschulzugang in der beruflichen Bildung manifestiert. Durch den Beginn der Anrechnungsdiskussionen, die Einführung diesbezüglicher Regelungen, aber auch durch die Einführung des deutschen Qualifikationsrahmens, in dem alle Qualifikationsniveaus auch auf beruflichem Weg erreichbar sein sollen, kommt es zu einer Neujustierung der Wertigkeiten von Bildungswegen zugunsten beruflicher Bildungswege, da diese zumindest teilweise als äquivalent anerkannt werden. Es zeigt sich zudem, dass Veränderungen auf der diskursiven Ebene meist Änderungen auf der rechtlichen vorausgehen.

\subsubsection{Fortführung institutioneller Entwicklungspfade - Batrieren für Durchlässigkeit}

Trotz dieser Entwicklungen hin zu mehr Durchlässigkeit können doch weiterhin in den (fehlenden) regulativen und den kulturell-kognitiven sowie normativen Strukturen, insbesondere der konstruierten Zielgruppe, Entwicklungen gezeigt werden, die dazu beitragen, das Bildungsschisma und die traditionelle Hierarchie 
zwischen Berufs- und Hochschulbildung zu reproduzieren, und die Barrieren für Durchlässigkeit darstellen.

Allgemein kann es bereits als Zeichen für eine weiterhin bestehende Hierarchisierung zugunsten der Hochschulbildung gewertet werden, dass im Diskurs fast ausschließlich eine Richtung von Durchlässigkeit diskutiert wird: von der Berufs- zur Hochschulbildung. Der Weg andersherum erscheint außer für Studienabbrecher_innen als nicht sinnvoll für individuelle Bildungswege. Auch notwendige Veränderungen in der Berufsbildung, die in den bildungspolitischen Diskussionen von 1990 bis 2012 durchaus Thema waren, werden allerdings nicht in Verbindung mit Durchlässigkeit zur Hochschulbildung gebracht. Die Veränderungen zur Schaffung von Durchlässigkeit zwischen Berufs- und Hochschulbildung soll demnach primär von Seiten der Akteure der höheren Allgemeinbildung durchgeführt werden. Veränderungen auf Seiten der Berufsbildung dienen der Modernisierung und Erhöhung der Attraktivität dieses Bildungsbereichs.

Ein weiteres Zeichen für die Fortführung institutioneller Entwicklungen, ohne auf die Details der Diskursstrukturen und Regelungen einzugehen, ist auch das Bestehen des strukturkonservativen Diskurses an sich, der die höhere Allgemeinbildung und ihre Stellung im Bildungssystem als höherwertig verteidigt. Die Existenz dieses Diskurses zeigt, dass in den gesellschaftlichen Wissensvorräten noch immer traditionelle strukturkonservative Deutungsmuster verankert sind. So sind z.B. die Gründe, warum Durchlässigkeit kritisch gesehen wird, im Verlauf des letzten Jahrhunderts weitestgehend gleich geblieben. Eine Öffnung der Hochschulen für nicht traditionelle Studierende wird als Gefahr für die Qualität der akademischen Ausbildung konstruiert (vgl. Hegelheimer 1986; Wolter 1994; Schwabe-Ruck 2010). Zudem wird auch immer noch die Gefahr des Scheiterns für den einzelnen nicht, traditionellen' Studierenden als Argument angeführt. Dass sich bereits in Studien gezeigt hat, dass die wenigen beruflich Qualifizierten an den deutschen Hochschulen nicht weniger erfolgreich sind (vgl. Wolter 2010a), spielt dabei keine Rolle.

Auch die dominierende funktionalistische Rahmung für Durchlässigkeit in beiden Zeiträumen stellt eine Weiterführung traditioneller Argumentationen dar, die auch als Barriere gesehen werden können. Beruflich Qualifizierte sollen in dieser Logik weniger als Person ein Recht auf Weiterbildung an den Hochschulen erhalten, sondern werden als Ressource gesehen, deren Höherqualifizierung an den Hochschulen in der jeweiligen Zeitperiode notwendig ist. So zeigt SchwabeRuck (2010), dass die verschiedenen Wellen, in denen die Hochschulen für beruflich Qualifizierte geöffnet werden sollten, vor allem mit einem erhöhtem Fachkräftebedarf begründet wird, der nicht mehr über die traditionellen Wege gestillt werden kann. Problematisch ist dies insofern, als Durchlässigkeit dann reversibel ist und Forderungen danach sowie institutionalisierte Bildungsstrukturen nur so lange aufrechterhalten werden müssen, wie es aus funktionalen Gründen notwendig erscheint. 
Analog zu der Zusammenfassung der institutionellen Änderungen sollen nun auch die eher als unverändert beschreibbaren Strukturen und damit häufig auch Barrieren zu Durchlässigkeit anhand der einzelnen Durchlässigkeitsaspekte dargestellt werden. Die Darstellung insbesondere der diskursiven Strukturen erfolgt dabei oft, aber nicht ausschließlich mit Bezug zum strukturkonservativen Diskurs.

\section{Zugang}

Auch wenn gerade im Hinblick auf Zugangsfragen eine große Entwicklung festgestellt wurde, so kann doch gezeigt werden, dass im Diskurs und in der Regulative noch keine Gleichstellung der Berufsbildung mit der höheren Allgemeinbildung stattgefunden hat. Berechtigungen in der Berufsbildung gelten für die Fortgebildeten, also die bereits hochqualifizierten beruflichen Absolvent_innen. Beruflich Erstausgebildete können nur fachgebunden studieren und müssen für den Zugang meist noch zusätzlich eine Prüfung ablegen. Die Norm bleibt im Diskurs und auch in den Hochschulzugangsmöglichkeiten der Weg über die Allgemeinbildung. Eine Gleichstellung des Abiturs mit der beruflichen dreijährigen Erstausbildung findet nicht statt und wird auch nur im universalistischen Diskurs gefordert. Als Zeichen, dass die Akteure der höheren Allgemeinbildung auch keine Gleichwertigkeit zwischen der Erstausbildung und dem Abitur anstreben, kann der Zuordnungsvorschlag der Kultusminister gesehen werden, da das Abitur auf Stufe Fünf und nicht auf Vier wie die beruflichen dreijährigen Qualifikationen der Erstausbildung im Qualifikationsrahmen eingeordnet werden soll. Insofern wird weiterhin in den Diskursen eine Ungleichwertigkeit zwischen Berufsausbildung und höherer Allgemeinbildung, vor allem dem Abitur, konstruiert. In der regulativen Dimension wurde diese Ungleichwertigkeit allerdings bisher nicht im neuen Klassifikationssystem, dem Qualifikationsrahmen, übernommen.

Eine weitere Barriere für Durchlässigkeit kann in dem weiterhin fest verankerten Verständnis von Studierfähigkeit gesehen werden, welches in Deutschland bereits eine lange Tradition hat, die Begrenzung des Studienzugangs zu begründen (vgl. Wolter 1989, 1994). Dabei ist das Verständnis von Studierfähigkeit in den Diskursen sichtbar eng gekoppelt an einen statischen naturalisierten Begabungsglauben (Lenhardt 2003). Studierfähigkeit müsse bereits vor dem Studium durch Prüfung oder bereits erworbene Zertifikate feststellbar sein und bestimmt, wer für ein Studium befähigt sei und wer nicht. Hier offenbart sich die Annahme, dass es eine bestimmbare Fähigkeit gebe, die nicht veränderbar sei und daher vor dem Studium festgestellt werden könne und müsse. Dass sich die als notwendig erachteten Fähigkeiten mitunter erst im Studium entwickeln könnten, wird kaum (Ausnahme: Probestudium) für möglich gehalten. Eine solche Vorstellung würde eher der Vorstellung der universellen Bildungsfähigkeit (vgl. Lenhardt 2003) entsprechen. Die Vorstellung von Studierfähigkeit findet sich vor allem im strukturkonservativen, aber auch im funktionalistischen strukturkritischen Diskursstrang. 
Im ersten wird zudem die Studierfähigkeit weiterhin stark von der Existenz eines hohen Allgemeinbildungsniveaus abhängig gemacht.

Insgesamt wird demnach deutlich, dass sowohl in den Diskursen als auch in der regulativen Dimension die Hierarchisierung zwischen Berufs- und höherer Allgemeinbildung nicht aufgelöst wurde. Zudem kommt es auf Basis traditioneller Deutungsmuster zur Begrenzung des Hochschulzugangs und zu einer kontinuierlichen Grenzziehung zwischen Berufs- und höherer Allgemeinbildung, da Studierfähigkeit bei Letzterer angenommen und bei Ersterer geprüft werden müsste.

\section{Anrechnung}

Auch wenn die Veränderung in Richtung Anrechnung als ein wichtiges Zeichen der Aufhebung des Bildungsschismas angesehen werden können, sind auch hier Entwicklungen festzustellen, die einer Gleichstellung von Berufs- und Hochschulbildung und der Aufhebung des Schismas entgegenstehen. Deutlich wird dies vor allem in der Regelung, dass nur bis zu $50 \%$ eines Hochschulstudiums angerechnet werden können. Das heißt, der Lernort Hochschule bleibt der bedeutende und unabhängig davon, wie viele gleichwertige Kompetenzen das Individuum erlangt hat, bleibt der Besuch der Hochschule für ein akademisches Zertifikat obligatorisch. Insofern wird die Gleichwertigkeit von Lernwegen künstlich begrenzt. Zudem wird in den Diskursen deutlich, dass in Deutschland Anrechnung von beruflicher Bildung fast ausschließlich Anrechnung von Kompetenzen einer beruflichen Fortbildung bedeutet. Dass bestimmte berufliche Erstausbildungen, die z.B. sehr anspruchsvoll sind und meist nur von Abiturient_innen absolviert werden, ebenfalls Anrechnungspotenzial besitzen könnten, wird nur im universalistischen strukturkritischen Diskurs anerkannt.

\section{Organisationale Verbindung}

Im Hinblick auf organisationale Verbindungen fällt auf, dass ausschließlich im universalstrukturkritischen Diskursstrang eine organisationale Verbindung im Sekundarbereich angestrebt wird. Eine Möglichkeit der Verbindung wäre die Institutionalisierung einer Berufsausbildung mit Abitur. Solche Bildungswege werden aber traditionell, so hier auch im ersten und im zweiten Untersuchungszeitraum, vor allem von den Akteuren der höheren Allgemeinbildung abgelehnt. Die Sonderrolle des allgemeinbildenden Abiturs soll erhalten bleiben. Die dem Abitur gleichkommende Berechtigung zum allgemeinen Hochschulzugang kann über die Berufsbildung nur postsekundär über Fortbildungsgänge erworben werden, aber nicht im Sekundarbereich.

Die fehlende Institutionalisierung einer solchen Organisationsform bzw. der Abbau anstatt Ausbau des DDR-Modells der Berufsausbildung mit Abitur nach der deutschen Vereinigung kann auch als Barriere für Durchlässigkeit und als 
Festschreibung der Trennung von sekundärer Berufs- und Allgemeinbildung gesehen werden. Dies gilt umso mehr, als sich in anderen Ländern gezeigt hat, dass eine solche Institutionalisierung im Sekundärbereich dazu beiträgt, die Hochschulbildung stärker für beruflich Qualifizierte zu öffnen (vgl. Graf 2013).

\section{Heterogenität}

Ähnlich wie bei Fragen der Anrechnung ist als großer Entwicklungsschritt in Richtung Durchlässigkeit zu werten, dass in den Diskursen und teilweise auch in der regulativen Dimension Strukturen geschaffen wurden, die dazu dienen sollen, beruflich Qualifizierten ein Hochschulstudium zu erleichtern. Auffällig ist jedoch, dass zwar vor allem organisatorische Studienbedingungen zur Flexibilisierung von Lernort oder Lernzeiten angedacht werden, aber eine Anpassung der Lehrmethodik, der pädagogischen Strukturen, an eine Studierendenschaft mit heterogenen Hintergründen allein im universalistischen strukturkritischen Diskurs als notwendig erachtet wird. Stattdessen geht es vor allem im strukturkonservativen Diskurs darum, die beruflich Qualifizierten einseitig an die Anforderungen der Hochschulen im Rahmen von Brückenkursen anzupassen und Defizite abzubauen. Es wird damit deutlich, dass es weitgehend nicht als Aufgabe der Hochschule angesehen wird, die Logik der Hochschullehre zu verändern oder gar die Erfahrungen, die beruflich Qualifizierte an die Hochschulen mitbringen im Studium zu nutzen. Vielmehr sollen Letztere am besten so wie die traditionellen Studierenden funktionieren und nicht weiter auffallen.

Weiterhin wird in den Diskursen kontinuierlich eine Differenz zwischen den ,traditionellen' Studierenden und den beruflich Qualifizierten konstruiert. Die Trennung von Berufs- und höherer Allgemeinbildung wird demnach auch innerhalb der Hochschule nicht aufgehoben. So kommt es zu einer Homogenisierung und Gegenüberstellung der Studierendengruppen. Dies birgt die Gefahr der Stigmatisierung beruflich Qualifizierter, zumal diese vor allem im strukturkonservativen Diskurs als eine Problemgruppe dargestellt werden, die spezifischer Zuwendung bedarf und sich durch Defizite auszeichnet, die in Brückenkursen ausgeglichen werden müssen.

Es konnte gezeigt werden, dass in den gesellschaftlichen Wissensvorräten, rekonstruierbar aus den Diskursen, aber auch in den Regelungen noch immer das Bildungsschisma, die Trennung von Berufs- und höherer Allgemeinbildung, sowie eine Hierarchisierung feststellbar sind. Besonders deutlich wird dies erstens an der einseitigen Richtung, in der Durchlässigkeit gedacht wird. Zweitens ist der weitestgehend ähnlich strukturierte strukturkonservative Diskurs vor und nach 1999 ein Zeichen dafür, dass in Deutschland weiterhin Vorstellungen verbreitet sind, die eine Höherwertigkeit der höheren Allgemeinbildung im Vergleich zur Berufsbildung und eine Notwendigkeit der Begrenzung und Kontrolle des Hochschulzugangs von beruflich Qualifizierten verfechten. 


\subsubsection{Zusammenfassung und Schlussfolgerungen}

Anhand der Zusammenschau der Veränderungen aber auch der gleichgebliebenen, das Bildungsschisma stärkenden diskursiven und regulativen Strukturen, kann eine Veränderung institutioneller Entwicklungspfade festgestellt werden, ohne dass diese aber eine gänzliche Abkehr von traditionellen Entwicklungslinien bedeutet.

Die bestehenden Deutungskämpfe zeigen, dass Durchlässigkeit in Deutschland ein stark umkämpftes Feld ist. Während insbesondere Akteure der höheren Allgemeinbildung ihre Positionen im Bildungswesen sowie auf dem Arbeitsmarkt verteidigen, werden diese insbesondere von den Akteuren der Berufsbildung infrage gestellt. Im zweiten Zeitraum ist dabei ein Wandel nicht nur im Verständnis festzustellen, sondern auch darin, dass bestimmte Arten, über Durchlässigkeit zu denken, z.B. diese vollständig zu negieren, nicht mehr legitim ist: Es hat sich ein diskursiver Wandel vollzogen. Dies zeigt sich darin, dass auch in den Aussagen der Akteure der höheren Allgemeinbildung immer stärker die Notwendigkeit der Durchlässigkeit anerkannt wird und dass beruflich Qualifizierte als Zielgruppe, wenn auch als eine besonders betreuungswürdige, akzeptiert werden. Insofern zeigt die Diskursanalyse deutlich, wie sich die Macht über die Deutungsangebote in Richtung der Vertreter der Gleichwertigkeit von der beruflichen Bildung und der Notwendigkeit von Durchlässigkeit verschoben hat. Ersichtlich wird auch, dass oft ein diskursiver Wandel einem regulativen vorausgeht. Es wird mit dem Verweis auf die historische Entwicklung z.B. in den 1970er Jahren, in denen durch den Bildungsrat sehr fortschrittliche Veränderungen in Richtung Durchlässigkeit und Gleichwertigkeit gefordert wurden, ohne dass die rechtlichen Rahmenbedingungen sich stark verändert hätten, aber auch offenbar, dass einem diskursiven Wandel nicht notwendigerweise auch ein regulativer folgt. Dennoch soll an dieser Stelle deutlich werden, dass es erstens wichtig ist, das Denk- und Sagbare anhand der Diskursstrukturen in einer Gesellschaft zu analysieren, um institutionellen Wandel in der Gesellschaft beschreiben zu können. Zweitens soll unterstrichen werden, dass es, wenn Strukturen verändert werden sollen, wichtig ist, auch die gesellschaftlichen Wissensvorräte zu analysieren und Veränderungen auch auf diskursiver Ebene in die Gesellschaft einzubringen.

Inwiefern diese Veränderungen in Deutschland dazu geführt haben, dass sich Frankreich und Deutschland in ihren Durchlässigkeitsstrukturen annähern, und welchen Einfluss die europäischen Bildungsprozesse auf den Prozess der sichtbaren Macht- und Deutungsverschiebung gehabt haben, wird in Kapitel 10 untersucht. Nachfolgend sollen aber zuerst einmal das Durchlässigkeitsproblem in Frankreich und dessen institutionelle Entwicklungen im Vordergrund stehen. 



\section{Der französische Fall: Bildungssystem, Geschichte und das Problem der Durchlässigkeit}

In dem nun folgenden Kapitel stehen das französische Bildungssystem und die identifizierte Durchlässigkeitsproblematik für den französischen Fall im Fokus. Es dient als Grundlage für die Analyse des institutionellen Wandels der Durchlässigkeitsstrukturen in Frankreich (Kapitel 9).

Ist Durchlässigkeit zwischen Berufs- und Hochschulbildung lediglich ein Problem in Ländern mit stark segmentierten Bildungsbereichen im Sekundar- und Tertiärbereich wie in Deutschland? Nachfolgend soll dargelegt werden, dass auch in einem weniger segmentierten und stratifizierten System, wie in Frankreich, Durchlässigkeit durchaus auch ein Problem darstellen kann. Wie dieses aussieht, wird in einem ersten Schritt (Kapitel 8.1) skizziert. Um das Durchlässigkeitsproblem besser einordnen zu können, werden in einem zweiten Schritt (Kapitel 8.2) die Organisationsfelder Berufs- und Hochschulbildung und deren Steuerung beschrieben. Zudem werden die bestehenden Bildungswege zwischen den Organisationsfeldern erörtert. Schließlich soll ein letzter Abschnitt der historischen Einbettung der Durchlässigkeitsproblematik dienen (Kapitel 8.3), denn nur vor dem historischen Hintergrund der institutionellen Entwicklung der beruflichen und höheren Allgemeinbildung in Frankreich ist das heutige System mit seinen Problemen zu verstehen. Auch dient die Kenntnis der historischen Evolution einer Analyse des institutionellen Wandels, denn nur dergestalt können endogene Strukturen als pfadabhängige Faktoren identifiziert werden und ermöglichen die Einschätzung dahingehend, ob sich das Bildungssystem entlang bestehender Pfade oder durch die Schaffung neuer Strukturen entwickelt.

\subsection{Das Problem der „,segregativen Demokratisierung“ in Frankreich}

Frankreich zeichnet sich durch ein sehr differenziertes Bildungssystem aus, welches über viele Verbindungen zwischen den einzelnen Bildungswegen verfügt ${ }^{120}$. Es besteht dabei keine strikte Trennung zwischen den Organisationsfeldern der Berufs- und Hochschulbildung, wie in Deutschland, welche die Durchlässigkeit zwischen den Bildungsbereichen beeinträchtigen könnte. Wie bereits als Überblick dargestellt (vgl. Kapitel 4.1), werden kurz wichtige Faktoren, welche das

120 Ein Glossar zum französischen Bildungssystem findet sich im Anhang in Tabelle 40 . 
rechtliche Verhältnis zwischen Berufs- und Hochschulbildung in Frankreich charakterisieren, zusammengefasst: In Frankreich gibt es kein breit ausgebautes postsekundäres berufliches Weiterbildungssystem wie in Deutschland ${ }^{121}$. Daher findet berufliche Bildung im postsekundären Bereich vielfach an den französischen Hochschulen (oder im Betrieb ohne Abschluss) statt. Mit der Zeit wurden verschiedene Wege eingeführt, vorgängiges Lernen und damit auch beruflich Gelerntes im Bildungssystem anerkennen zu lassen. Schließlich wurde in Frankreich 1985 mit der Einführung des beruflichen Abiturs, dem baccalauréat professionnel, ein Weg zur Hochschulberechtigung über die Berufsbildung im Sekundarbereich institutionalisiert, so dass auch auf diesem Weg Berufs- und Hochschulbildung rechtlich miteinander verknüpft sind. Kurz, das französische Bildungssystem kann als relativ durchlässig zwischen den Organisationsfeldern Berufs- und Hochschulbildung beschrieben werden. Vor diesem Hintergrund stellt sich die Frage, inwiefern in Frankreich von einem Durchlässigkeitsproblem zwischen beruflicher und hochschulischer Bildung überhaupt ausgegangen werden kann? Ist dies lediglich ein Problem von Ländern, die sich durch eine starke Segmentierung der beiden Bildungsbereiche auszeichnen?

Die Antwort ist, dass auch im französischen Bildungssystem durchaus ein strukturelles Durchlässigkeitsproblem zwischen Berufs- und Hochschulbildung identifiziert werden kann. Ein rechtlicher Anspruch auf Zugang bedeutet nicht, dass ebenfalls alle ein Studium beginnen und dass diejenigen, die eins anfangen, dieses auch erfolgreich abschließen. Das Durchlässigkeitsproblem in Frankreich kristallisiert sich anders als in Deutschland somit nicht in den fehlenden institutionalisierten Zugangs- und Anrechnungswegen, sondern wird sichtbar in den Studienanfänger- und vor allem den Studienabbruchquoten. Erstere sind bei beruflich Qualifizierten im Vergleich zu Studierenden, welche über ein technologisches oder allgemeines Abitur in die Hochschulen kamen, systematisch kleiner und letztere systematisch höher.

Im Folgenden soll kurz das Ausmaß dieses Problems anhand von Statistiken illustriert werden. $\mathrm{Ob}$ und auch wo Abiturient_innen studieren, ist in Frankreich stark abhängig von Typ des erreichten baccalauréat, wobei drei Abiturzweige unterschieden werden: das allgemeine, das technische und das berufliche. Insgesamt sind es im Jahr 2011 ungefähr 75 \% der Abiturient_innen, die ihre Erstausbildung im Hochschulbereich fortsetzen. Fast alle Inhaber_innen des allgemeinen Abiturs, $77 \%$ der Abiturient_innen des technologischen Zuges und ungefähr $28 \%$ der beruflichen Abiturient_innen begannen ein Hochschulstudium (DEPP 2012). $20 \%$ der Abiturient_innen brechen ihr Studium jedoch vorzeitig ab (MESR 2012d). Besonders signifikant ist das Problem des Studienmisserfolges an der Universität im licence-Zyklus, dem französischen Pendant zum dreijährigen Bachelorzyklus, ausgeprägt (vgl. DEPP 2013b; Lemaire 2004). Von den Abiturient_innen, die sich 2008 eingeschrieben haben, haben lediglich $39 \%$ den licence-

121 Ausnahmen bilden die Meisterausbildungen mit dem Abschluss brevet de maitrise, die von den Kammern abgenommen werden. 
Abschluss innerhalb von vier Jahren (DEPP 2013b: 267) erworben. Die Zahl der Absolvent_innen mag sich noch erhöht haben, zeigt allerdings deutlich, dass es besonders schwierig ist, an den Universitäten einen ersten tertiären Abschluss zu erhalten. An den weiteren Hochschulorganisationen des ersten Zyklus ist die Abschlussquote wesentlich höher. An den beruflich orientierten Hochschulorganisationen, dem technischen Hochschulinstitut IUT (institut universitaire technologique) erhalten $77 \%$ (DEPP 2013b: 261) ihren Abschluss in drei Jahren und an den Sektionen für höhere technische Bildung in Oberschulen STS (sections de technicien supérieur) sind es $73 \%$ (DEPP 2013b: 255). ${ }^{122}$

Auch die Abbruchquote ${ }^{123}$ ist signifikant abhängig vom Abiturtyp, mit dem man das Studium aufgenommen hat. Gemäß einer Panelanalyse der Direction de l'Évaluation et de la Prospective, welche 1995 begann und welche die Schüler_innen seit der sixième ${ }^{124}$ (dem ersten Jahr der Sekundarschule in Frankreich) begleitet hat, haben von diesen Schüler_innen ungefähr 9\% der Abiturient_innen des allgemeinbildenden Abiturs, die ihr Studium aufgenommen haben, das französische Hochschulwesen ohne Abschluss verlassen, $29 \%$ der Abiturient_innen mit dem technologischen baccalauréat und $56 \%$ der Berufsabiturient_innen (MESR 2012c: 4). Diese unterschiedlichen Abbruchquoten, in denen die Absolvent_innen eines beruflichen Abiturs systematisch schlechter abschneiden, sind sowohl an den Universitäten als auch an den IUT und den STS vorzufinden (vgl. Tabelle 42 und Tabelle 43 im Anhang).

Betrachtet man die Abiturient_innen des Panels 1995 insgesamt, so lässt sich feststellen, dass $19 \%$ aller Berufsabiturient_innen dieses Jahrgangs einen Hochschulabschluss erhalten haben gegenüber $64 \%$ der Abiturient_innen im technologischen Zug und $89 \%$ im allgemeinbildenden Zug. Im Vergleich zu den Abschlusszahlen der Abiturient_innen des Panels 1989 stellt das zwar jeweils eine Steigerung der Abschlussquote dar, aber die Abbruchquote, insbesondere der beruflich Qualifizierten, ist immer noch immens und systematisch höher als bei den beiden anderen Abiturtypen.

Es sind demnach insbesondere die Abiturient_innen aus dem beruflichen aber auch dem technologischen Zug, welche von dem Problem des Scheiterns im tertiären Bildungssystem betroffen sind. Es zeigt sich folglich eine starke Diskrepanz zwischen formalen Rechten und der Realität, welche die Durchlässigkeit des französischen Bildungswesens infrage stellt. So scheint die Hürde, welche früher

122 Die Zahlen bedeuten nicht, dass die übrigen Studierenden alle ihr Studium abbrechen, partiell ist das der Fall, zum Teil studieren diese aber auch in anderen Studiengängen weiter.

123 In Deutschland besteht sogar eine höhere Studienabbruchquote als in Frankreich (vgl. Heublein et al. 2012). In dieser sind aber nicht systematisch deutlich mehr beruflich Qualifizierte betroffen als in Frankreich.

124 In Frankreich werden die Klassen ab dem collège bis zum Abschluss des Abiturs rückwärts gezählt. Das College beginnt mit der sixième (6. Schuljahr) und endet mit der troisième (9. Schuljahr). Die Oberstufe umfasst dann die Jahre seconde (10. Schuljahr), première (11. Schuljahr) und die terminale (12. Schuljahr). 
darin bestand, ein Abitur und damit eine Hochschulzugangsberechtigung zu erhalten, mit der Reform des Schulsystems und der Einführung des baccalauréat professionnel auf das Hochschulsystem, genauer auf das Erreichen eines ersten tertiären Abschlusses, verschoben worden zu sein. Es wird ebenfalls deutlich, dass Durchlässigkeit nicht nur Zugang bedeutet, sondern auch zu einem erfolgreichen Abschluss führen sollte.

Tabelle 19 Bildungskarrieren aller Abiturient_innen nach Typ des Abiturs des Panels $95^{125}$ (in \%)

\begin{tabular}{|c|c|c|c|c|c|c|c|}
\hline \multirow[t]{2}{*}{$\begin{array}{l}\text { Typ des } \\
\text { Abiturs }\end{array}$} & \multirow[t]{2}{*}{$\begin{array}{c}\text { Kein } \\
\text { Studium }\end{array}$} & \multirow{2}{*}{$\begin{array}{l}\text { Beginn } \\
\text { eines } \\
\text { Studium }\end{array}$} & \multicolumn{3}{|c|}{$\begin{array}{l}\text { Erhalt eines Hochschulab- } \\
\text { schlusses }\end{array}$} & \multirow{2}{*}{$\begin{array}{l}\text { Ohne Hoch- } \\
\text { schuldiplom } \\
\text { bei Studien- } \\
\text { beginn }\end{array}$} & \multirow[t]{2}{*}{$\begin{array}{l}\text { Abbruch } \\
\text { quote }\end{array}$} \\
\hline & & & Total & $\begin{array}{c}\text { davon auf } \\
\text { Niveau } \\
\text { bac }+2\end{array}$ & $\begin{array}{l}\text { davon auf } \\
\text { Niveau } \\
\text { bac }+3 \text { und } \\
\text { höher }\end{array}$ & & \\
\hline $\begin{array}{l}\text { bac } \\
\text { général }\end{array}$ & 2 & 98 & 89 & 11 & 78 & 9 & 9 \\
\hline bac techno & 10 & 90 & 64 & 35 & 29 & 26 & 29 \\
\hline bac pro & 57 & 43 & 19 & 16 & 3 & 24 & 56 \\
\hline Gesamt & 14 & 86 & 69,1 & 19 & 43,5 & 17,1 & 20 \\
\hline
\end{tabular}

Quelle: (MESR 2012d: 4) sowie eigene Berechnung der Abbruchquote

Die Benachteiligung verstärkend kommt hinzu, dass sich insbesondere im beruflichen Abiturzweig aber auch im technologischen, dessen Absolvent_innen die meisten Probleme im tertiären System aufweisen, verstärkt Jugendliche aus Haushalten mit einem geringen sozio-ökonomischen Status (SES) kommen, während die Abiturient_innen des allgemeinbildenden Zweigs, insbesondere in der mathematischen Sektion, welche die besten Chancen haben, einen Hochschulabschluss zu erwerben, meist eine privilegierte soziale Herkunft besitzen. So zeigen die verschiedensten Studien, dass sich mit der Kanalisierung der Schüler_innen in die verschiedenen Abiturzweige gleichzeitig eine soziale Selektion vollzieht und dass diese im Übergang vom baccalauréat zum Hochschulwesen erhalten bleibt (vgl. Beaud/Pialoux 2001; Brauns 1998; Duru-Bellat/Kieffer 2001, 2008; Merle 2000; Pinto 2008). Merle (2000) nennt diese Entwicklung ,segregative Demokratisierung". Dabei wird darauf hingewiesen, dass die Ungleichheiten in Frankreich zwar auch noch immer darin bestehen, wer einen Abschluss bekommt und wer nicht. Aber bei einer Abiturient_innenquote von über $75 \%$ besteht die soziale Ungleichheit weniger darin, ob man ein Abitur erhält, sondern welches Abitur und

125 Das Panel 95 begleitet Lernende, die 1989 von der Grundschule in die Gemeinschaftsschule College wechselten. Meist sind die Schüler_innen zu diesem Zeitpunkt 11 Jahre alt.

126 Die Abbruchquote bezieht sich nur auf die Abiturient_innen, die ein Studium begonnen haben. 
welcher tertiäre Bildungsgang daran angeschlossen werden kann (Duru-Bellat 2005). Es handelt sich demnach nicht allein um ein Problem der Bildungsdurchlässigkeit, sondern auch der sozialen Durchlässigkeit des französischen Bildungssystems.

Doch warum und inwiefern sind Absolvent_innen aus dem beruflichen Abiturzweig im tertiären Bildungssystem in Frankreich besonders benachteiligt? Die Beantwortung dieser Frage soll im Folgenden anhand der Beschreibung der Organisationsfelder Berufs- und Hochschulbildung sowie einer kurzen Zusammenfassung der geschichtlichen Entwicklung deutlicher werden. Auf diese Weise wird das französische Durchlässigkeitsproblem konkretisiert.

\subsection{Beschreibung der Organisationsfelder}

Das französische Bildungssystem ist ein stark differenziertes Bildungssystem, welches diese für Deutschland typische Segmentierung zwischen beruflicher Bildung und allgemeiner Bildung nicht in demselben Maß kennt. Parallel zum allgemeinbildenden System hat sich in Frankreich eine Qualifikationsleiter der beruflich orientierten Bildung entwickelt (vgl. Powell et al. 2009). So gibt es auf allen Qualifikationsniveaus sowohl beruflich orientierte als auch stärker allgemeinbildende Bildungsgänge. Im Folgenden soll im Überblick das französische Bildungswesen anhand seiner Organisationen im Berufsbildungs- und Hochschulbereich beschrieben werden sowie die Gestaltung der Steuerung der Bildungsbereiche. Zuerst jedoch erfolgt ein Überblick über die unterschiedlichen Bildungswege in der Sekundarstufe II allgemein, da diese entscheidend für die weiteren Bildungskarrieren und damit auch das französische Durchlässigkeitsproblem sind.

\subsubsection{Bildungswege nach dem, collège}

Die Sekundarstufe I findet in Frankreich im collège unique statt, der einheitlichen Mittelschule, in der noch alle Schüler_innen zusammen lernen. Das collège unique endet mit der troisième (im deutschen System entspräche das der 9. Klasse). Am Ende der troisième schreiben alle Schüler_innen, welche zumeist 15 Jahre alt sind, ein Examen und erhalten das BEPC (brevet d'études de premier cycle). Im Anschluss an das collège sieht das französische Bildungssystem drei Bildungswege vor (vgl. CEDEFOP 2008; Koch 1998):

1. den Weg in die Berufsbildung mit anschließendem Übergang in den Arbeitsmarkt (voie professionelle) nach Beendigung der Sekundarstufe II: Abschlüsse, die erworben werden können, sind das Berufsbefähigungszeugnis CAP (certificat d'aptitude professionnelle) oder ein berufsorientiertes Abitur 
bac Pro (baccalauréat professionnel). Bis zur Reform 2008 gab es auch noch das Berufsbildungszeugnis BEP (brevet d'études professionnelles).

2. den Weg über die technisch-fachliche Ausbildung, welche nach einem technischen Abitur (baccalauréat technologique) zu einem berufsorientierten technischen Kurzstudium überleiten sollte: Das technische Abitur kann in verschiedenen Schwerpunkten erworben werden: u.a. in der Hotellerie, Medizintechnik, Labortechnik, Industrietechnik, Landwirtschaftstechnik, Wirtschaft und Management.

3. den Weg über das allgemeinbildende Abitur (baccalauréat général) mit einem anschließenden längeren Hochschulstudium ${ }^{127}$. Auch für das allgemeinbildende Abitur gibt es unterschiedliche Schwerpunkte: der naturwissenschaftliche, der literarische und der sozial- und wirtschaftswissenschaftliche Abiturschwerpunkt.

Diejenigen, die nicht den beruflichen Weg gehen, werden in den Oberschulen (lycées générales und technologiques) noch ein Jahr in der seconde gemeinsam unterrichtet, die Spezialisierung in ein allgemeinbildendes oder technisches Abitur erfolgt dann in den anschließenden zwei Schuljahren.

Es ist wichtig zu wissen, dass die Kanalisierung der Schüler_innen in die verschiedenen Bildungswege nach dem collège weniger einer Interessenwahl dieser entspricht. Die Selektion vollzieht sich vielmehr entsprechend der schulischen Leistungen. Die Schüler_innen im beruflichen Zweig sind somit diejenigen, welche bereits in der Sekundarschule die meisten Leistungsprobleme hatten. Insofern ist die berufliche Ausbildung im Sekundarbereich gesellschaftlich nicht hoch angesehen. Aber auch die Schüler_innen im technologischen Zweig haben meist nicht das hohe schulische Niveau der Abiturient_innen des lycée général. Es handelt sich dementsprechend um eine Auswahl „par l'échec“, eine Auswahl über den Misserfolg (Brauns 1998; Groh-Samberg et al. 2012; Kieffer 2008; Koch 1998). Diese Selektion in die nicht allgemeinbildenden Abiturzweige geht dabei einher mit einem Curriculum, welches weniger allgemeinbildend ist und sich auf ein begrenztes berufliches Feld (baccalauréat professionne) oder eine Wirtschaftsrichtung (baccalauréat technologique) spezialisiert (Braun 1998). Der Übergang in die Berufsbildung ist daher eher eine Sanktion für nicht gezeigte Leistung, während der allgemeinbildende Weg der Königsweg bleibt (vgl. CEDEFOP 2008). Übergänge zwischen den Bildungszweigen, sogenannte passerelles, wurden institutionalisiert, so dass es vom Prinzip her möglich ist, auch vom beruflichen Zweig in den technologischen und vom technologischen in den allgemeinbildenden zu wechseln und umgekehrt (vgl. Onisep 2010, 2014).

Die Aufteilung auf die unterschiedlichen Bildungswege sieht in Frankreich 2011 folgendermaßen aus: $57 \%$ wählen den Weg über das technologische und allgemeinbildende Abitur und $37 \%$ den beruflichen Zweig, wobei 13,2\% aller

127 Dabei sind weniger die beruflich orientierten meist zweijährige Kurzstudiengänge gemeint, sondern die stärker allgemeinbildenden Studiengänge an Universitäten und grandes écoles. 
Schüler_innen der SEKII in einen CAP Bildungsgang wechseln, während 23,4 \% einen berufliches Abitur anstreben (DEPP 2013a: 41).

\subsubsection{Berufsbildung der Sekundarstufe II}

Berufsbildung findet in Frankreich sowohl im sekundären als auch im tertiären Bildungsbereich statt. Die Abschlüsse, die dem Tertiärbereich zugerechnet werden können, werden im Rahmen der Beschreibung des Hochschulsystems genauer vorgestellt, so dass im Folgenden das Berufsbildungssystem im Sekundarbereich im Blickpunkt steht. Im beruflichen Sekundarschulbereich können drei Abschlüsse unterschieden werden: das klassische Berufsbildungszertifikat, das CAP (Certificat d'aptitude professionnelle), das BEP (brevet d'études professionnelles) seit 1966 sowie seit 1985 das Berufsabitur, das baccalauréat professionnel (bac pro).

Die Berufsausbildung, welche mit einem CAP-Abschluss endet, dauert zwei Jahre und beginnt gemeinhin nach dem collège. Dieses Berufsbefähigungszeugnis ist das älteste Berufsbildungszertifikat in Frankreich. Ein CAP-Abschluss ist der erste Berufsabschluss, den man in Frankreich erwerben kann und stellt damit auch die Trennlinie zwischen den Berufspositionen für gelernte und ungelernte Arbeitnehmer_innen in Frankreich dar (vgl. Powell et al. 2009).

Die Berufsausbildung, welche zum Berufsbildungszeugnis BEP führte, wurde 1966 kreiert, um einen beruflichen Abschluss mit einer weniger engen Spezialisierung als beim CAP zu schaffen. Das CAP wurde damit zu einem Berufszertifikat, welches auf einen bestimmten klar umgrenzten Beruf vorbereitet, während das BEP eher auf ein Berufsfeld hinführt. Es galt daher auch als anspruchsvoller als das CAP (vgl. Powell et al. 2009). Auch wenn das BEP als Arbeitsmarkteinstiegszertifikat gedacht war, wurde es mehr und mehr zu einem Sprungbrett zum Berufsabitur. Nach der Reform des beruflichen Abiturs 2008/2009 ist das BEP kein eigenständiger Berufsabschluss mehr. Es kann nur noch nach dem erfolgreichen Abschluss der ersten zwei Jahren auf dem Weg zum beruflichen Abitur erworben werden.

Das Berufsabitur wurde 1985 geschaffen. Ursprünglich wurde es nach einem BEP und eher seltener auch nach einem CAP Abschluss durch den Besuch zweier weiterer Jahre am lycée professionelle, dem Berufsgymnasium, erworben. Im Gegensatz zu den anderen Abiturabschlüssen, erhielt man das berufliche Abitur demnach vier Jahre nach dem Ende des college. Nach der Reform der Berufsausbildung von 2008 wurde die Lernzeit nach dem collège auf 3 Jahre verkürzt, u.a. mit dem Ziel, die Zahl der Absolvent_innen des Berufsabiturs zu erhöhen. Die angenommene abschreckende Wirkung zweier weiterer Jahre Lernzeit nach einem ersten beruflichen Abschluss sollte verringert werden. Gleichzeitig wurde auch eine gröBere Symbolisierung von Gleichwertigkeit mit den anderen beiden Abiturzweigen angestrebt, da diese ebenfalls in drei Jahren erreicht werden (vgl. Kapitel 9). 
Insgesamt bleibt festzuhalten, dass es mit der Einführung des bac pro $1985 \mathrm{zu}$ einer weiteren Bildungsexpansion kam, welche auch als die zweite Bildungsexpansion beschrieben wird (vgl. Pinto 2008). Haben 1970 nur rund $20 \%$ und 1985 nur $29 \%$ eines Jahrgangs das Abitur erreicht, so sind es mittlerweile um die $76 \%$ eines Jahrgangs, die eine der drei Abiturarten erreichen (vgl. Abbildung 8).

Abbildung 8 Entwicklung des Abiturient_innenanteils nach Typ des Abiturs (\%)

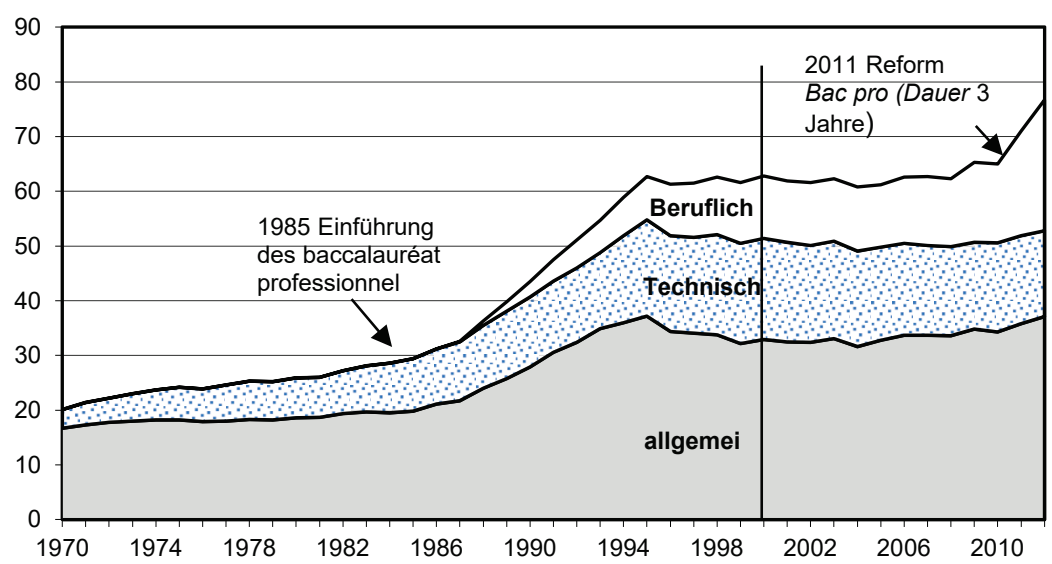

Quelle: (DEPP 2013b: 247), Übersetzung NB

Im Unterschied zu Deutschland findet in Frankreich die Berufsbildung primär vollzeitschulisch an den lycées professionelles, den beruflichen Oberschulen, statt, wobei drei bis zehn Praxiswochen pro Jahr Teil der Ausbildung sind. Typisch für die schulische Ausbildung ist die Vermittlung von einem großen Teil Allgemeinbildung mit spezialisierten technischen Fachkenntnissen, welche insbesondere auf einen bestimmten Beruf ausgerichtet sind (vgl. CEDEFOP 2008). Als Schwäche des französischen schulischen Berufssystems wird jedoch der mangelnde Praxisbezug in der Ausbildung gesehen (vgl. Wirp 2004), der dazu führt, dass die Übergänge in den Arbeitsmarkt sich schwierig gestalten, vor allem auch schwieriger im Vergleich zu stärker dual ausbildenden Ländern (vgl. Brauns et al. 1999a; Brzinsky-Fay 2007; Wolbers 2007).

Aber auch wenn das französische Berufsbildungssystem primär schulisch ausbildet, so können alle beruflichen Abschlüsse im Sekundär- und Tertiärbereich, welche ebenfalls im Register der Berufszertifikate (le répertoire national des certifications) eingetragen sind, auch alternierend, d.h. über ein Lehrausbildung erworben werden. Dies ist seit dem Gesetz loi Séguin von 1987 möglich. Traditionell waren es eher Branchen aus dem Handwerk, welche Lehrausbildungen angeboten haben. Dies hat sich jedoch vor allem durch die vermehrte Einführung von alternance im Hochschulbereich geändert. Jetzt handelt es sich oft um Ingenieurs-, 
Wirtschafts- und Informatikabschlüsse (vgl. CEDEFOP 2008). Vor 1987 war nur das CAP über die Lehre zu erwerben. Im Rahmen einer Lehrlingsausbildung findet die Ausbildung sowohl im Betrieb als auch durch den Besuch von Ausbildungszentren (CFA) statt. In diesen CFA wird der schulische Teil der Ausbildung vermittelt.

In den letzten Jahren gab es vor allem auch durch massive Förderung der Regierung (vgl. Abriac et al. 2009; Arrighi/Brochier 2009) einen Anstieg an sogenannten alternierenden Ausbildungen. Wie in Tabelle 20 ersichtlich, waren es 1995 noch 293.512 Auszubildende. Bis 2011/12 ist die Zahl auf 436.334 angestiegen. In diesem Zusammenhang sind die meisten auf dem französischen Bildungsniveau 5 angesiedelt und in einem CAP-Ausbildungsgang eingeschrieben. Aber auch im Hochschulbereich (Bildungsniveau III-I) ist die Zahl deutlich gestiegen.

Tabelle 20 Entwicklung der Lehrlingszahlen in den centres de formation d'apprentis (CFA)

\begin{tabular}{lccccccc}
\hline $\begin{array}{l}\text { Abschlussjahre/ } \\
\text { Bildungsniveau }\end{array}$ & $\mathbf{1 9 9 5}$ & $\mathbf{2 0 0 0}$ & $\mathbf{2 0 0 4}$ & $\mathbf{2 0 0 8}$ & $\mathbf{2 0 0 9}$ & $\mathbf{2 0 1 0}$ & $\mathbf{2 0 1 1}$ \\
\hline $\begin{array}{l}\text { Bildungsniveau V } \\
\text { (CAP/BEP) }\end{array}$ & 232.135 & 245.333 & 225.274 & 231.659 & 209.767 & 191.857 & 189.560 \\
$\begin{array}{l}\text { Bildungsniveau IV } \\
\text { (bac pro) }\end{array}$ & 41.327 & 69.355 & 80.623 & 98.470 & 111.900 & 123.018 & 123.888 \\
$\begin{array}{l}\text { BildungsNiveau III } \\
\text { (bac +2 Jahre) }\end{array}$ & 15.273 & 35.553 & 39.560 & 58.572 & 59.532 & 62.074 & 67.193 \\
$\begin{array}{l}\text { Bildungsniveau I/II } \\
\text { (ab bac +3 Jahre) }\end{array}$ & 4.777 & 15.633 & 23.531 & 38.949 & 43.543 & 49.331 & 55.693 \\
\hline Gesamt & 293.512 & 365.874 & 368.988 & 427.650 & 424.742 & 426.280 & 436.334 \\
\hline \hline
\end{tabular}

Quelle: (DEPP 2013b: 155), Übersetzung NB

\section{Steuerung}

Nachdem das berufliche Bildungswesen anhand der differenten Organisationsformen dargestellt wurde, soll nachfolgend die Steuerung des Berufsbildungssystems beschrieben werden. Welche Akteure haben Einfluss auf die Durchlässigkeitsstrukturen in Frankreich und sind damit auch wichtige Akteure für die nachfolgende Diskursanalyse? Frankreich dient als Beispiel für ein staatsdominiertes Bildungssystem (Brauns 1998) und auch Greinert (2005) klassifiziert das französische Berufsbildungssystem als ein staatlich reguliertes bürokratisches Modell. Jedoch haben insbesondere Reformen in der Berufsbildung in den letzten Jahrzehnten zu einer zunehmenden Dezentralisierung der Steuerungsfunktionen geführt, so dass nicht nur von einer zentralstaatlichen Steuerung gesprochen werden kann (vgl. Méhaut 2004; Powell et al. 2012c; Tanguy 1988; Van Zanten/Plaisance 
1993). Der Staat ist dessen ungeachtet primär dafür zuständig, die großen Leitlinien festzulegen ${ }^{128}$ und die staatlichen Zeugnisse auszustellen (vgl. Powell et al. 2009). ${ }^{129}$ Auch neue Organisationsformen werden von zentralstaatlicher Seite eingeführt. Den weiterhin signifikanten Einfluss des Bildungsministeriums fasst Minister Melenchon 2002 zusammen:

L'Education Nationale - et la France d'une manière générale - n'est pas une République fédérative. Par conséquent, il y a la légitimité d'un pouvoir politique qui s'exerce complètement. Par conséquent, d'accord ou pas d'accord, le lycée des métiers se fera pour une raison: parce que je l'ai décidé et que je suis légitime à le décider parce que c'est cela, la République. (MDEP 2002) ${ }^{130}$

An Einfluss gewonnen haben jedoch ebenfalls die Regionen. Zum einen sind sie seit 1993 für berufliche Weiterbildung zuständig und seit 2004 auch primär für die Berufsausbildung der Jugendlichen und Arbeitslosen unter 26 Jahren (vgl. CEDEFOP 2008; vgl. Powell et al. 2009). Mittlerweile finanzieren die Regionen den Bau von Gebäuden, Sanierungsarbeiten sowie die materielle Infrastruktur für Berufsschulen. Zudem koordinieren sie in Abstimmung mit den Arbeitgeber_innen- und Arbeitnehmer_innenverbänden sowie der regionalen Landwirtschafts-, Industrie-, Handels- und Handwerkskammern die Ausrichtung der regionalen Berufsbildungspolitik und deren Angebote, um besser den regionalen Bedürfnissen gerecht zu werden (vgl. Agulhon 2005; CEDEFOP 2008). Zusammenfassend kann konstatiert werden, dass die Rolle des Zentralstaats in der beruflichen Bildung auf der regulatorischen Ebene stärker ist als auf der finanziellen Ebene (Bel 2001).

Auch der Einfluss der Sozialpartner wird in Frankreich traditionell unterschätzt. Es besteht zwar keine korporatistische Zusammenarbeit wie in Deutschland, aber insbesondere in der Lehrausbildung werden die Kammern und Sozialpartner bei der Programmentwicklung mit einbezogen. So sitzen sie in den Verwaltungsräten der Lehrlingsausbildungszentren (CFA) und koordinieren diese mit. Die Möglichkeit der Einflussnahme im betrieblichen Teil der Ausbildung kommt noch hinzu. Zudem müssen sich die Unternehmen an der Finanzierung der Berufsbildung, insbesondere der Lehrausbildung in Frankreich beteiligen, da

128 Gerade die Leitlinien und Rahmengesetze sind jedoch bedeutend für Fragen der Durchlässigkeit, so dass trotzdem der Staat als Hauptakteur der Berufsbildung und nicht die Regionen in den Diskursen in dieser Studie untersucht wurden.

129 Auch diese Position steht mittlerweile auf dem Prüfstand. So wird die Einführung von innerbetrieblichen Zertifikaten (certificats de qualification professionnelle, CQP) in der Weiterbildung als ein Zeichen dafür gesehen, dass sich die Vorrangstellung des Staates auflöst (Coutrot/Lautman 2005).

130 „Die Nationale Bildung - und Frankreich im Allgemeinen - ist nicht föderal organisiert. Deshalb ist die ausgeübte politische Macht [der zentralistischen Regierung, NB] vollkommen legitim. Daher, egal ob alle zustimmen oder nicht, wird das Lycée de métiers eingeführt und zwar aus einem Grund: weil ich es so entschieden habe und ich legitimiert bin, diese Entscheidung zu treffen, denn so ist das, so ist die Republik.“ (MDEP 2002, Übersetzung NB) 
es eine gesetzlich eingeführte Ausbildungsabgabe gibt. Während die schulische Ausbildung primär durch öffentliche Mittel finanziert wird, verteilen sich die Kosten der Lehrlingsausbildung auf die Unternehmen, den Staat und die Regionen (CEDEFOP 2008: 62-64). Schließlich beraten Sozialpartner zusammen mit staatlichen Akteuren und den Kammern die Curricula und Methoden (réferentiels) sowie die notwendigen Prüfungsanforderungen für berufliche Zertifikate (vgl. CEDEFOP 2008).

\subsubsection{Hochschulbildung}

Das tertiäre Bildungssystem in Frankreich ist stark differenziert und wird durch eine doppelte Hierarchie strukturiert: zum einen durch das Verhältnis zwischen Eliteausbildung an den grandes écoles und dem Rest des Hochschulsystems und zum anderen durch die Trennung von selektiven und nicht selektiven Bildungssektoren. Für die verschiedenen Bildungsmöglichkeiten im Tertiärsystem (vgl. Tabelle 6 im Online-Anhang) bestehen verschiedene Organisationsformen: die grandes écoles und die dazugehörigen Vorbereitungsklassen (CPGE), die Universitäten, die technischen Hochschulen (IUT) und die Sektionen für höhere technische Bildung (STS) sowie fachspezifische Hochschulen im Gesundheitswesen, für die Lehrer_innenbildung, für die Ausbildung von Ingenieur_innen und Wirtschafts- und Verwaltungshochschulen. Typisch für das französische Hochschulsystem ist eine Differenzierung in viele spezialisierte Hochschulorganisationen, deren Abschlüsse je ihren eigenen Prestigewert in der französische Gesellschaft besitzen (Convert 2003). Verkürzt dargestellt, bedeutet das: Am meisten Prestige besitzen die Abschlüsse der grandes écoles, gefolgt von Studienabschlüssen an den Universitäten auf Masterniveau, dann die beruflichen licence-Abschlüsse gefolgt von den an den IUT und schließlich die an den STS ${ }^{131}$ (Convert 2003).

Folgt man der Trennung zwischen selektiven und offenen Studiengängen, dann umfasst der ,offene' Sektor die Universitäten, zu deren Zugang das Vorliegen einer Hochschulzugangsberechtigung, das französische Abitur, ausreicht. Es gibt nur wenige Ausnahmen wie Numerus Clausus Fächer, z.B. Medizin. Nach der Umstellung 2005 auf das vom Bologna-Prozess induzierte dreizyklische Studiensystem kann man an den Universitäten folgende Abschlüsse erhalten: licence, master, doctorat. Bis auf das Doktorstudium können licence und master sowohl allgemeinbildend als auch berufsorientiert ausgerichtet sein. Ein Studium an der Universität gilt als stärker allgemeinbildend ausgerichtet und bereitet primär auf das Erreichen eines Langzeitstudiengangs, also den Master, vor.

Der Abschluss Licence entspricht dem Bachelor und wird gemeinhin in drei Jahren erworben. Der berufsorientierte Abschluss licence professionnelle dauert ein Jahr und setzt den Abschluss der ersten beiden Jahre eines allgemeinbildenden

131 Eine Übersicht über die unterschiedlichen Studiengänge und deren Zugangsvorasusetzungen findet sich im Online-Anhang in Tabelle 6. 
licence-Studiums oder den Abschluss eines zweijährigen beruflichen Studiengangs voraus. Die licence professionnelle wurde im Rahmen der Bologna-Reformen in Frankreich eingeführt (Maillard/Veneau 2008) Der Zugang zur licence professionelle ist selektiv, d.h., die Hochschulen wählen die Studierenden aufgrund begrenzter Studienplätze aus.

Der Master-Abschluss wird in zwei Jahren erworben. Zugang zum ersten Masterjahr haben diejenigen mit einem ersten hochschulischen Abschluss auf Niveau der licence $(\mathrm{bac}+3)$ in der gleichen Fachrichtung. Andere Bewerber_innen müssen die studienrelevanten Kenntnisse vorher validieren lassen. Zum Teil gibt es auch Numerus clausus Fächer, in welchen der Zugang begrenzt wird. Der Zugang zum zweiten Jahr des Masters ist dagegen stärker selektiv und wird meist über eine Zwischenprüfung geregelt (vgl. Onisep 2013). Analog wie bei der licence ist der Zugang zum beruflichen Master (master professionned) 132 selektiv.

Der selektive Hochschulbereich besteht aus zwei sehr differenten Bildungssträngen. Zum einen wären da die exklusiven grandes écoles. Der Besuch dieser Bildungsorganisationen bereitet auf die Spitzenpositionen in Staat, Wirtschaft und Wissenschaft vor (vgl. Shavit et al. 2007). Die Aufgabe der grandes écoles besteht daher darin, den Bedarf an hochqualifizierten Fachkräften für die französische Gesellschaft zu decken. Zugang zu ihnen wird meist über einen nationalen hochselektiven concours, ein Auswahlverfahren, gewährt, auf den sich die Studierenden in der Regel zwei Jahre in Vorbereitungsklassen, den classes préparatoires (CPGE), vorbereiten. Es sind vor allem die besten Abiturient_innen des allgemeinbildenden Zuges, welche sich in die Vorbereitungsklassen einschreiben (Brauns 1998). Noch immer ist die Verwirklichung von Chancengleichheit und Elitenformation tief im Selbstverständnis der französischen Bildungspolitik verankert (Dobbins 2011). So begründet sich die gesellschaftliche Akzeptanz dieser Elitenauswahl in dem Auswahlverfahren, dem concours, welches das Prinzip der Gleichheit bei der Auslese berücksichtigt (Brauns 1998). Die Studierenden stellen jedoch nicht nur eine leistungsselektive, sondern auch eine sozial selektive Gruppe dar. Noch immer reproduziert sich in den grandes écoles die nationale Elite (Duru-Bellat/Kieffer 2008).

Der andere selektive Strang des Hochschulsystems besteht aus den technischen Kurzstudiengängen einmal an den technischen Hochschulinstituten „Instituts Universitaires de Technologie" (IUT), welche an die Universitäten gekoppelt sind und den Sektionen für höhere technische Bildung in Oberschulen ,Sections de Techniciens Supérieur" (STS). Die Zertifikate, das höhere Technikerzertifikat in universitärer Ausbildung DUT (Diplome universitaire de technology) an den IUT und das höhere Technikerzertifikat in schulischer Ausbildung BTS (Brevet de technicien superieur) in den STS werden in zwei Studienjahren erworben. Für den Zugang zu diesen

132 Vor den Bologna-Reformen entsprach der master professionnel einem DESS Abschluss (Diplôme $d^{\prime}$ Etudes Supérieures Specialisées), der ebenfalls beruflich orientiert ausgerichtet war. 
Studiengängen ist zum einen das Abiturzeugnis wichtig. Oft findet auch eine Prüfung der Bewerber_innen durch die Hochschulen über Eignungsverfahren z.B. dem Schreiben eines dossier, einer thematischen Abhandlung, statt.

2012 waren fast 2,4 Millionen Studierende an den Hochschulen in Frankreich eingeschrieben (DEPP 2013b: 171). Die meisten Studierenden nimmt die Universität auf, gefolgt von den technischen Kurzstudiengängen STS und IUT. Wie in Tabelle 21 ersichtlich, haben sich 2012 ungefähr ein Drittel aller Abiturient_innen in Universitätsstudiengänge eingeschrieben, wobei die meisten von ihnen ein allgemeinbildendes Abitur haben. In die technischen Hochschulinstitute (IUT) gingen 7,3\% aller Abiturient_innen und in die STS 18,6\%.Die Vorbereitungsklassen der grandes ecoles wurden von 6,6\% aller Abiturient_innen besucht, wobei es sich primär um jene des allgemeinbildenden Zweigs handelten. Die Abiturient_innen des technischen Zweigs wechseln zu großen Teilen an die STS und in die Universitäten. Die beruflichen Abiturient_innen, welche sich für ein Studium entschlossen haben, wechseln ebenfalls zu großen Teilen an die STS und immerhin $8 \%$ aller beruflichen Abiturient_innen gingen an die Universität.

Nach Beaud (2008: 51) kam es in den letzten 30 Jahren zu einer Veränderung der Hierarchie der Studiengänge im ersten Studienzyklus. Er nennt es die ,revanches des filières courtes". Unangetastet bleiben die grandes écoles und die dazugehörigen classes préparatoires (CPGE) an der Spitze der Hierarchie, jedoch hat die Bedeutung des ersten Studienzyklusses der Universitäten, die allgemeine licence, in Frankreich an Gewicht verloren, und zwar gegenüber den technischen Kurzstudiengängen, speziell an den IUT. Erklärt wird das mit der Selektivität dieser Studiengänge.

Tabelle 21 Direkte Einschreibungsrate der Abiturient_innen 2012 in die unterschiedlichen Studiengänge (in \%)

\begin{tabular}{lccccc}
\hline Art des Abiturs & $\begin{array}{c}\text { Universi- } \\
\text { täten } \\
\text { ohne IUT }\end{array}$ & IUT & CPGE & STS & andere \\
\hline Alle Abiturarten & 32,1 & 7,3 & 6,6 & 18,6 & 7,4 \\
Bac général & 53,4 & 10,6 & 13,0 & 8,6 & 12,5 \\
Bac techno & 18,7 & 9,6 & 1,7 & 40,8 & 5,6 \\
Bac pro & 8,0 & 0,8 & 0 & 19,3 & 0,6 \\
\hline
\end{tabular}

Quelle: DEPP (2013b: 207), Übersetzung NB

Wie bereits beschrieben, ist die Auslese von Studierenden im französischen Bildungswesen ein zentrales Merkmal und wird als Verfahren der Positionierung der Individuen in der Gesellschaft als legitim anerkannt (Brauns 1998). Aber auch die besseren Studienbedingungen und Erfolgsquoten sowie die Akzeptanz auf dem Arbeitsmarkt trugen dazu bei. Die Folge dieser Entwicklung ist, dass immer mehr Abiturient_innen um die begrenzten Plätze an den selektiven Kurzstudiengängen 
konkurrieren, wobei die Abiturient_innen des allgemeinbildenden Zuges die besten Möglichkeiten haben, aufgenommen zu werden, während die Abiturient_innen der beiden anderen Abiturzweige, wenn Konkurrenz besteht, eher das Nachsehen haben. Dies gilt insbesondere für die technischen Universitäten (IUT), welche immer mehr als Sprungbrett genutzt werden können, um danach entweder auf die grands écoles zu gehen oder an der Universität weiterzustudieren (vgl. Brauns 1998; Duru-Bellat 2007). Aus der Hierarchie der Abiturzweige heraus sind die leistungsstärksten Schüler_innen im allgemeinbildenden Abitur zu finden und weisen demgemäß einen Wettbewerbsvorteil bei der Studienplatzvergabe auf. Als Konsequenz werden diejenigen, welche eigentlich einen beruflich orientierten Kurzstudiengang machen wollten, an die Universitäten gedrängt, obwohl sie aufgrund ihrer schulischen Vergangenheit weniger auf ein Studium an der Universität vorbereitet sind als auf einen beruflich orientierten Kurzstudiengang.

Die Universität hat somit das Problem, dass sie in zweifacher Konkurrenz steht. Zum einen zieht es die besten Abiturient_innen eines Jahrgangs an die grandes écoles, zum anderen verlieren sie auch geeignete Studierende an die Kurzstudiengänge. Das Resultat sind viele Studierende, welche als Notlösung ein Studium an der Universität aufnehmen, dieses aber nicht beenden (Lemaire 2004).

Besonders prekär ist die Lage für die Studierenden, welche mit einem beruflichen oder technologischen Abitur an die Universität gekommen sind. Nur unter $5 \%$ der beruflichen Abiturient_innen und unter $16 \%$ der technischen Abiturient_innen schaffen es, den Abschluss innerhalb von vier Jahren zu erwerben (vgl. Tabelle 22). Auch wenn es heißt, dass die Universität nicht selektiv sei, so wird dies durch die Statistiken stark infrage gestellt. Universitäten sind zwar beim Zugang nicht selektiv, jedoch wird dafür umso stärker im Verlauf des Studiums aussortiert. Die Selektion findet demnach nur später statt.

Tabelle 22 Studienerfolg in der licence innerhalb von vier Jahren je nach Abiturschwerpunkt im Abiturjahrgang 2007

\begin{tabular}{lcc}
\hline \multicolumn{1}{c}{$\begin{array}{c}\text { Art des Abiturs } \\
\text { (+Schwerpunkt) }\end{array}$} & $\begin{array}{c}\text { Anteil an den eingeschrie- } \\
\text { benen Studierenden (\%) }\end{array}$ & $\begin{array}{c}\text { davon Abschluss nach } \\
\text { insgesamt 4 Jahren (\%) }\end{array}$ \\
\hline Bac general: Littéraire & 21,4 & 43,7 \\
Bac general: Economique & 29,3 & 47,9 \\
Bac general: Scientifique & 24,6 & 48,4 \\
Bac techno STG & 10,6 & 12,5 \\
Bac techno, andere Schwer- & & \\
punkte & 5,3 & 15,7 \\
Bac Professionnel & 4,1 & 4,6 \\
Nicht-Abiturient_innen & 4,7 & 30,6 \\
Insgesamt & 100 & 39,0 \\
\hline
\end{tabular}

Quelle: DEPP 2013b:267, Übersetzung NB 
Ein Grund, der für die Probleme der beruflichen und zum Teil auch technischen Abiturient_innen an den Hochschulen gesehen wird, ist, dass ihre eher fachspezifische Ausbildung mit weniger Allgemeinbildungsanteilen als im allgemeinen Abitur nicht auf ein Universitätsstudium vorbereitet, welches sich in seiner Pädagogik stark auf Vorlesungen und eine große Arbeitsautonomie der Studierenden gründet (vgl. Beaud 2008; Vincens 2008). In den selektiven Kurzstudiengängen dagegen wird sich viel stärker um die Studierenden gekümmert. Sie werden während des Studiums begleitet, ihre Arbeit wird kontinuierlich kontrolliert sowie evaluiert und sie haben durch die Präsenz der Lehrenden ebenfalls bei Problemen Ansprechpartner (vgl. Beaud 2008; Vincens 2008).

Geht man von zwei Modellen aus, wie Hochschulorganisationen funktionieren können, einmal nach dem Prinzip des verantwortlichen Studierenden und einmal nach dem Prinzip der verantwortlichen Institution ${ }^{133}$ (Vincens 2008), so entspricht die französische Universität dem Modell des verantwortlichen Studierenden. Die Student_innen dürfen sich gemäß ihren Interessen und Fähigkeiten einschreiben. Der Staat setzt die Anforderungen, welche zum Erreichen eines Diploms erfüllt werden müssen fest und die Universität muss die festgelegte Norm, welche die Qualität des Diploms beschreibt, einhalten. Die Lehre in einer derartigen Institution ist dabei undifferenziert in Bezug auf die schulische Herkunft oder unterschiedliche Fähigkeiten der Studierenden, da davon ausgegangen wird, dass diese ihr Studium verantwortlich entsprechend ihrer Möglichkeiten auswählen und ebenso selbstverantwortlich sind, den Anforderungen gerecht zu werden. Dieses Model setzt demnach ein großes $\mathrm{Ma}$ an Autonomie der Studierenden voraus, ebenso ein gutes Wissen über die Anforderungen und die Kompetenz einzuschätzen, ob man den Anforderungen genügt.

Das Paradox im französischen Hochschulsystem ist, dass die Studiengänge, welche am ehesten den bereits erworbenen Kompetenzen der Abiturient_innen der technologischen und berufsorientierten Zweige entsprechen, durch die Konkurrenz mit den Absolvent_innen des bac général für diese nicht mehr in ausreichendem Maße zugänglich sind. Um dieser Entwicklung entgegen zu wirken, wurden 2013 rechtliche Bestimmungen erlassen (das loi ESR - loi relative à l'enseignement supérieur et à la recherche), die einer durch mehrere Hochschulakteure festzulegenden Minimalquote beruflich Qualifizierter des bac pro und den Absolvent_innen des bac technologique Zugang zu den STS und IUT gewährt.

133 Die verantwortliche Institution dagegen wählt über Auswahlverfahren die Studierenden aus, welche im Rahmen des Selektionsprozesses die Fähigkeiten beweisen, die zum Erreichen des Diploms nötig sind. Der Staat bestimmt auch hier das Curriculum, die Mittel aber auch die Zahl der aufzunehmenden Studierenden. Die Organisation hat dann nach der Selektion die Aufgabe, dafür zu sorgen, dass die zugelassenen Studierenden erfolgreich abschließen. Dieses Modell steht dementsprechend für die selektiven Studiengänge (Vincens 2008). 


\section{Steuerung}

Das französische Bildungssystem wird idealtypisch als ein zentralstaatlich durch die Staatsbürokratie gesteuertes Bildungssystem beschrieben (Clark 1983). Für die berufliche Bildung wurde bereits gezeigt, dass mittlerweile auch weitere Akteure wichtiger geworden sind. Auch in der Hochschulbildung ist nicht mehr nur die Regierung mit dem Hochschul- und Forschungsministerium einziger Akteur, aber die zentralstaatliche Ebene ist weiterhin entscheidend. So fallen folgende zentrale Aufgaben dem Staat zu: die Finanzierung der Hochschulbildung, die Vorgabe von Zielvorstellungen, die Regelungen von Zugangskriterien, die Schaffung neuer und Reform alter Bildungsorganisationen und die Überwachung der Qualität von Diplomen. Eine Folge dieser zentralstaatlichen Macht ist, dass hochschulpolitische Änderungen direkt durch Regierungspolitik beeinflussbar sind (Dobbins 2011). Der große regulative Spielraum des Zentralstaats wird in der französischen Gesellschaft akzeptiert und zudem als Garant dafür gesehen, dass Chancengleichheit über einheitliche Regelungen erreicht wird (Dobbins 2011).

Französische Universitäten verändern sich zwar inkrementell, aber in zentralen Dimensionen deutlich (Musselin/Paradeise 2009) - dies gilt ebenfalls für Fragen der Steuerung. Seit 1984 haben die Hochschulen zunehmend an Autonomie gewonnen. Eine oft vernachlässigte aber sehr bedeutende Reform war die Einführung der Vierjahresverträge zwischen Hochschulen und dem Staat (vgl. Mignot-Gérard/Musselin 2001; Musselin 2001; Musselin/Paradeise 2009). In diesen Verträgen werden Zielvereinbarungen zwischen dem Staat und den einzelnen Universitäten festgehalten. Wie die Ziele erreicht werden, blieb von da an stärker eine Entscheidung der öffentlichen Hochschulen selbst. Der letzte Höhepunkt der Zunahme an Autonomie fand 2007 mit der Verabschiedung des Gesetzes LRU (loi relative aux libertés et responsabilités des universités) statt, da den Hochschulen auch eine stärkere finanzielle Autonomie zugesprochen wurde. Mit diesem Gesetz ging ebenfalls einher, dass die Konferenz der Hochschulpräsidenten CPU (conférence des présidents d'université), die oft beratend bei Hochschulentscheidungen von der Regierung herangezogen werden, auch unabhängiger vom Bildungsministerium wurde. Bis 2007 war immer der oder die Bildungsminister_in Vorsitzende_r der CPU. Trotz der gewachsenen Autonomie der Hochschulen beschreibt Vinokur (2008) diese als noch immer in mehreren Punkten eingeschränkt: Vor allem liegt es daran, dass es der Staat ist, welcher die Abschlüsse definiert und zertifiziert Auch die Definition der Zugangskriterien zur Hochschule sowie die Höhe der Studiengebühren liegt in nationaler Verantwortung.

Neben den beiden Hauptakteuren im Organisationsfeld Hochschulbildung kann auch ein zunehmender Einfluss der Unternehmen festgestellt werden, vor allem in der Ausgestaltung der Curricula der beruflichen Bildungsgänge (an den STS, den IUT sowie in der licence professionelle und dem master professionneD. Dies liegt vor allem an der wachsenden beruflichen Ausrichtung tertiärer Bildungsgänge (vgl. Powell et al. 2009), welche allerdings bereits lange vor dem Beginn des 
Bologna-Prozesses seinen Anfang nahm (vgl. Agulhon 2003; Powell et al. 2009). Am stärksten sind die Einflussmöglichkeiten in alternierend angebotenen Studiengängen, da hier die Unternehmen als Arbeitgeber_innen sowie bei der Festlegung der Curricula ihren Einfluss geltend machen können. Zudem sind sie Mitglieder der Koordinierungsgremien der Ausbildungsorganisationen (CFA), an welchen der hochschulische Teil des Studiums bei Studiengängen en alternance stattfindet.

Die Gewerkschaften haben im Bereich der Steuerung der Hochschulpolitik keine einflussreiche Position. Einzelnen Organisationen sind zwar beratend in Kommissionen zur Hochschulentwicklung tätig, aber Mitbestimmungsrechte liegen nicht vor. Allein als Sozialpartner sind sie in der Koordination der CFA, der Ausbildungszentren für die Student_innen en alternance, vertreten. Trotz ihrer Zersplitterung und der geringen Zahl an Gewerkschaftsmitgliedschaften in Frankreich, können französische Gewerkschaften immer wieder die Betroffenen im Bildungsbereich mobilisieren, was letztlich manchmal auch Einfluss auf die Regierungspolitik haben kann.

Zusammenfassend bleibt für die Steuerung der Hochschulbildung die immer noch zentrale Rolle des Staates hervorzuheben und die gewachsene Autonomie der Hochschulen.

\subsubsection{Bildungswege zwischen Berufs- und Hochschulbildung}

Während bisher speziell die einzelnen Organisationsfelder vorgestellt wurden, soll jetzt deren Verbindung in den Blick genommen werden. Ziel ist zum einen zu zeigen, warum Frankreich regulativ als fortschrittlich im Hinblick auf durchlässige Bildungsstrukturen gelten kann, auch wenn bereits deutlich gemacht wurde, dass Regelungen nicht immer mit tatsächlicher Durchlässigkeit des Bildungssystems einhergehen. Zum anderen wird beschrieben, dass die berufliche Bildung primär im Sekundär- aber auch im Tertiärbereich als Weg für diejenigen gilt, welche es in den anspruchsvolleren (meist allgemeinbildenderen) Bildungsgängen nicht geschafft haben, erfolgreich zu sein.

\section{Wege in die Hochschulbildung}

Ähnlich wie im deutschen System benötigt man für den Zugang zum Hochschulwesen eine Hochschulzugangsberechtigung, welche man durch das französische Abitur erwirbt. Im Gegensatz zu Deutschland ist das baccalauréat seit 1985 mit der Einführung des beruflichen Abiturs über alle sekundären Bildungswege prinzipiell erreichbar. De jure haben alle Träger_innen eines Abiturs die Berechtigung zum Zugang zum tertiären Bildungssystem.

Die Stellung des Abiturs in Frankreich ist eine besondere. Es gilt als „Drehund Angelpunkt" (Koch 1998: 60) der französischen Bildungskarrieren. Es hat 
ein „prestige presque obsessionnel“ (Prost 1983: 133 bei Koch 1998: 60) Traditionell ist das Abitur in Frankreich ein Abschluss, welcher mit sozialem Aufstieg verbunden wird und noch immer einen starken symbolischen Charakter besitzt (Duru-Bellat 2007). Dadurch, dass das Abitur mit dem Recht des Zugangs zum Hochschulwesen verbunden ist und es auch noch immer als erstes Diplom des tertiären Bildungssektors gilt, werden an das Abitur Ansprüche angelegt, die in dem Bildungssystem, wie es jetzt ausgestaltet ist, nicht gehalten werden können. So fragt Duru-Bellat (2007: 4) inwiefern die traditionell an das Abitur geknüpften Aspirationen aufrecht zu erhalten seien, wenn $80 \%$ eines Jahrgangs ${ }^{134}$ diese Bildungsstufe erreichen sollen?

In Bezug auf die zukünftigen Bildungsmöglichkeiten ist das 1985 neu geschaffene Berufsabitur deutlich nicht konkurrenzfähig im Vergleich mit den anderen. Auch wenn es konzipiert wurde als Berufsabschlussqualifikation, so ist es doch ein Abitur, welches rechtlich den anderen Abiturzweigen gleichgestellt ist. In der Praxis ist dies jedoch eine Illusion, welche den beruflichen Abiturient_innen vorspielt, sie könnten erfolgreich studieren. Groh-Samberg et. al. (2012: 203) zeigen, dass diese Illusion und die Benachteiligung speziell für beruflich Qualifizierte von den Betroffenen sehr sensibel wahrgenommen wird. Die gemachten benachteiligenden Erfahrungen führen zu einer „Enttäuschung meritokratischer Erwartungen und republikanischer Versprechen" (Groh-Samberg et al. 2012: 205).

Duru-Bellat (2007: 56) deutet daraufhin, dass eben dieser Mythos des Abiturs mitunter dazu führt, dass eine ungünstige Studienwahl getroffen wird, denn die jungen Leute nehmen an, dass sie mit dem Abitur die Wahl haben, frei zu entscheiden, was sie wollen. Eine realistischere Einschätzung, was möglich ist und was nicht, könnte dagegen mitunter die Negativerfahrung des Studienabbruchs verhindern. Meist handelt es sich, wie bereits dargestellt, durch die Auswahlkriterien der selektiven beruflichen Studiengänge jedoch nicht um eine freie Wahl, die insbesondere die beruflichen und technologischen Abiturient_innen dazu bringt, sich für die für sie vermeintlich ungünstigen Studiengänge an Universitäten zu entscheiden.

Aber nicht nur über die formalen Bildungsinstitutionen ist der Zugang zur Hochschulbildung in Frankreich gewährleistet. Mit dem Diplôme d'accès aux études universitaires (DAEU), die in verschiedenen Versionen seit den 1950er Jahren bestehende Hochschuleingangsprüfung ${ }^{135}$ und 1994 per Dekret reformiert wurde, ist es in Frankreich möglich, auf dem zweiten Bildungsweg die Hochschulzugangsberechtigung zu erwerben (MEN 2000a). Das DAEU gewährt dabei die gleichen Rechte, welche auch mit einem ,normalen' baccalauréat verbunden sind.

134 Seit 1985 wird von der französischen Politik das Ziel, dass $80 \%$ eines Jahrgangs ein Abitur erreichen sollen, immer wieder ausgerufen.

135 Bereits 1954 wurde die Hochschuleingangsprüfung (ESEU - examen spécial d.entrée à l.université) eingeführt, welche 1986 erweitert wurde zum examen d'accès à l.enseignement supérieur (EAES) MEN 2000a). 
Somit können Absolvent_innen sowohl an die Hochschulen als auch an staatlichen concours auf Stellen im öffentlichen Dienst teilnehmen. 2012 wurde das DAEU allerdings von lediglich 5.569 Individuen erworben (DEPP 2013b: 267). ${ }^{136}$

Schließlich verfügt Frankreich im Vergleich zu Deutschland und vielen weiteren europäischen Ländern über einen großen Erfahrungsschatz hinsichtlich der Identifizierung, Bewertung und Anerkennung informell erworbener Kompetenzen (vgl. Bjørnåvold 2001: 127; Roy 2005). So existieren gesetzlich verankerte Verfahren, zur Erfassung beruflich und außerberuflich erworbener Kompetenzen, die sowohl einen Zugang zu Bildungsgängen gewähren als auch auf den Erwerb von ganzen Qualifikationen abzielen. Im Jahr 1985 wurde das Gesetz über die "bilans de compétences" erlassen, welches der Erfassung und Dokumentation informell erworbener beruflicher und persönlicher Kompetenzen dient. Ziel der bilans ist es, eine Hilfestellung für berufliche Entwicklungen, Umorientierungen, Weiterbildungen und Wiedereingliederungen in den Arbeitsmarkt zu bieten. Seit 1991 hat zudem jeder Arbeitnehmer den Anspruch auf einen Bilanzierungsurlaub für die Dauer der Durchführung einer bilan de compétence ${ }^{137}$ (vgl. Bjørnåvold 2001: 129; Frank 2003: 191; Gutschow 2003: 128f.). Mit der bilan selbst ist zwar kein Hochschulzugang möglich, aber es stellt eine gute Voraussetzung dar, um die Möglichkeiten der Weiterbildung zu eruieren und Anrechnungsoptionen zu sehen.

Zudem wurden in Frankreich weitere Verfahren entwickelt, welche beruflich und außerberuflich erworbene Kompetenzen nicht nur bilanzieren, sondern auch validieren und somit ihre Gleichwertigkeit zu den im formalen Bildungssystem erworbenen Kompetenzen anerkennen. Die zwei wichtigsten Verfahren sind die Validierung beruflicher Kompetenzen (VAP - Validation des acquis professionnels) und die Validierung beruflich und außerberuflich erworbener Kompetenzen (,"Validation des acquis de l'expérience - VAE"). Das VAP Verfahren wurde erstmals 1984 erlassen und 1992 per Gesetz reformiert und ermöglicht es Individuen einen Abschluss der beruflichen Bildung (Diplôme) zu erlangen, auf der Grundlage von Kompetenzen, die sie durch Berufserfahrung erworben haben. Durch dieses Verfahren, welches eine fünfjährige Berufserfahrung voraussetzt, kann die Ausbildungszeit zum Erwerb eines Diploms verkürzt werden, indem den Interessenten einzelne relevante Kompetenzen anerkannt und damit Teilprüfungen erlassen werden (Stegemann 2008) ${ }^{138}$.

2002 wurde dieses Gesetz mit der Einführung eines Systems zur Anerkennung auch informellen Lernens (Validation des acquis de l'expérience - VAE) erweitert. Im Gegensatz zur VAP werden bei dem Verfahren der VAE nicht allein

$136 \mathrm{Zu}$ bedenken bleibt bei der geringen Anzahl jedoch auch, dass durch die Bildungsexpansion immer mehr Individuen in Frankreich über ein Abitur verfügen und somit auch die Gruppe derer, die über DE.A.U an die Hochschulen kommen können, geringer wird.

137 Für eine genauere Beschreibung, wie die Bilanzierung der Kompetenzen in Frankreich durchgeführt wird, siehe Stegemann (2008).

138 Das gesamte Diplom kann auf diesem Weg nicht erlangt werden, da mindestens ein Ausbildungsmodul auf formalem Wege im Rahmen einer Prüfung absolviert werden muss. 
berufsbezogene, sondern ebenso persönliche und außerberufliche Erfahrungen beim Erwerb von beruflichen Qualifikationen berücksichtigt und umfassen somit auch Kompetenzen, welche im Rahmen von ehrenamtlichem, politischem oder gewerkschaftlichem Engagement erworben worden sind (vgl. Reitnauer 2004: 133; Triby 2005: 50). Ebenso neu ist die Möglichkeit, dass via VAE die gesamte berufliche Qualifikation und nicht nur Teilprüfungen über die Anrechnung beruflich und außerberuflich erworbener Kompetenzen anerkannt werden können (Reitnauer 2004: 133). ${ }^{139}$. Die Anerkennung ist allerdings lediglich für Diplome möglich, die auch im nationalen Register der Berufszertifikate (,Répertoire national des certifications professionnelles - RNCP") aufgeführt werden. Im Tertiärsystem (ohne die STS) haben ungefähr 4.000 Personen 2011 ihre Erfahrungen validieren lassen, wobei ca. 2400 von ihnen ein ganzes Zertifikat erhalten haben. Die meisten Validierungen im Hochschulbereich fanden statt, um einen master (42\%) und oder eine licence professionnelle (37\%) zu erhalten (DEPP 2013b: 281). Seit der Einführung 2002 hat sich die Zahl der Validierungen im Hochschulbereich deutlich erhöht (vgl. Abbildung $12 \mathrm{im}$ Anhang). Relevanter ist die Validierung allerdings in der Berufsbildung. So haben 2011 fast 18.360 Individuen ihre Erfahrungen validieren lassen, 5.467 von Ihnen allein, um einen BTS-Abschluss ${ }^{140}$ oder Teile davon anerkannt zu bekommen (DEPP 2013b: 278). Auch im Hinblick auf berufliche Bildungsgänge des Sekundarbereichs fand ein starker Anstieg an Validierungen statt (DEPP 2013b: 279).

Zusammenfassend lassen sich drei verschiedene Wege in die Hochschulen feststellen: Erstens der klassische Weg über ein Abitur, zweitens über den Erwerb einer Hochschulzugangsberechtigung auf dem zweiten Bildungsweg und schließlich über die Validierung im Lebensverlauf erworbener Kompetenzen, wobei über letzteres Verfahren Teile von oder ganze Studienabschlüsse erworben werden können.

\section{Wege in die Berufsbildung}

Wie bereits deutlich geworden sein sollte, wird die Berufsbildung, vor allem im Sekundärbereich, als Weg des Misserfolgs betrachtet. Zugang ist nach dem collège allen offen, aber gemeinhin wird der Weg in die Berufsbildung von in der Schule weniger erfolgreichen Jugendlichen beschritten. Im Hochschulbereich sieht die Situation etwas anders aus. Wie beschrieben, sind die beruflich orientierten Kurzstudiengänge selektiv bei ihrer Zulassung. Notwendig ist neben einer Hochschulzugangsberechtigung auch ein guter Notendurchschnitt oder das Bestehen weite-

139 Für eine genauere Beschreibung des Verfahrens siehe auch Stegemann (2008).

140 Ich folge hier der Unterteilung der französischen Statistik, auch wenn in dieser Arbeit die STS wie auch die DUT und licenec pro zum hochschulichen Bereich gezählt werden. 
rer Aufnahmeprüfungen. Auch die grands écoles können im weiteren Sinne als beruflich orientierte Bildung angesehen werden ${ }^{141}$. So ist das Studium dort stärker anwendungsorientiert als in den Universitäten. Der Zugang zu diesen ist hochselektiv und von dem Bestehen eines concours abhängig.

Abgesehen aber von den grands écoles, welche von jeher eine Sonderstellung im französischen Bildungswesen einnehmen, kann auch für den Tertiärbereich festgestellt werden, dass der Weg in die beruflich orientierten Studiengänge, vor allem in die STS - weniger in die IUT und in die licence pro - auch ein Weg für diejenigen darstellt, welche in den Studiengängen an den Universitäten, die tendenziell eher auf ein Langzeitstudium mit Masterabschluss vorbereiten, Studienprobleme aufweisen. Die Umorientierung in berufliche Studiengänge wird dann als Weg gesehen, den Studierenden noch die Möglichkeit auf einen Abschluss zu ermöglichen und zudem stärker auf die Integration in den Arbeitsmarkt vorzubereiten. Insbesondere im letzten Jahrzehnt wurden Übergänge, sog. passerelles zwischen stärker allgemeinbildenden Studiengängen der Universitäten an die beruflich orientierten Kurzstudiengänge ausgebaut, so dass die Studierenden mit Problemen besser überwechseln können, d.h. unter Anerkennung auch bisher erbrachter Leistungen, ohne das Studium von vorn beginnen zu müssen.

Auch wenn die selektiven Kurzstudiengänge durch ihre guten Arbeitsmarktperspektiven (Abriac et al. 2009) und die besseren Chancen auf Studienerfolg (DEPP 2013b) sowie auch die gewachsenen Möglichkeiten auf ein Weiterstudium beliebter geworden sind (Beaud 2002), sind doch die längeren Studien an den Universitäten, welche zu einem Master führen, vom Prestige und der Hierarchie eindeutig höher angesiedelt. Insofern wiederholt sich im Tertiärsystem quasi das typisch französische Schema, wer nicht gut genug ist für stärker allgemeinbildende Studiengänge, wechselt in die kürzeren weniger prestigeträchtigen beruflichen.

\subsection{Verhältnis von Berufs- und Hochschulbildung aus historischer Perspektive}

Um die bestehende Hierarchie in Frankreich, insbesondere zwischen Berufs- und allgemeiner Bildung, welche als eine wichtige Ursache des Durchlässigkeitsproblems in Frankreich angesehen werden kann, zu verstehen, soll nachfolgend ein kurzer Überblick über die Geschichte der Hierarchisierung von Bildungsberei-

141 Meist haben die grands écoles ein spezifisches Berufsfeld, auf das ihre Studierenden hin ausgebildet werden. Beispiele sind die Écoles normales supérieures für die Ausbildung von Wissenschaftler_innen, École polytechnique für die Ausbildung von Ingenieur_innen, Ecole Nationale des Ponts et Chaussées für Ingenieur_innen im Straßen und Brückenbau, École nationale des chartes für Archivare, die École du Louvre für Kunsthistoriker_innen und Archäolog_innen. Zudem kommen auch Teile des Lehrkörpers der grandes écoles aus der Praxis. 
chen in Frankreich erfolgen, wobei ein besonderes Augenmerk auf die Entwicklung der Entwertung und Stigmatisierung der beruflichen Bildung in Frankreich liegt. Historischer Ausgangspunkt ist die bildungspolitische Situation seit der französischen Revolution, da diese sich als entscheidend auch für die Entwicklung des französischen Bildungssystems bis heute darstellt (vgl. Brauns 1998; Greinert 2005; Musselin 2007). Seit der französischen Revolution zeichnet sich das französische Bildungswesen den starken Einfluss des Zentralstaates aus, wobei durch diesen Zentrismus drei große Ziele vereint werden sollen: die Bildung einer Leistungselite, die Gewährung von Chancengleichheit sowie die Sozialisation der französischen Staatsbürger (vgl. Brauns 1998).

\subsubsection{Französisches Bildungswesen bis 1958: Klare Trennung zwischen höherer Allgemein- und beruflicher Bildung}

Das französische Bildungswesen kann bis zum Beginn der fünften Republik seit 1959 als zweigeteilt gelten. Auf der einen Seite gab es das Sekundarschulwesen, das zum allgemeinen baccalauréat und in die Hochschulbildung führte. Auf der anderen Seite existierte ein Volksschulwesen, an das beruflich technische Ausbildungen angeschlossen werden konnten (Brauns 1998). Übergänge zwischen Volks- und Sekundarschule waren nicht üblich (Braun 1998).

\section{Sekundarschulwesen und Hochschulsystem}

Das baccalauréat als zentrales Diplom, welches den Zugang zur Hochschulbildung ermöglichte, wurde als nationales Diplom 1808 durch ein napoleonisches Dekret eingeführt. Um ein baccalauréat zu erhalten, musste man mindestens 16 Jahre alt sein. Es bildete - wie auch heute noch - den Abschluss des sekundären Schulwesens und gleichzeitig den ersten tertiären Abschluss. Dies wurde auch daran deutlich, dass die Jury, die dieses Diplom abnahm, bis ins 20. Jahrhundert hinein lediglich aus Universitätsprofessoren bestand. Auf das Abitur konnte man sich in den lycées, den Gymnasien, vorbereiten, die ebenfalls durch Napoleon 1802 gegründet wurden als Ausbildungsanstalt für eine staatliche Elite (vgl. Troger 2009). Das Sekundarschulwesen mit dem lycée war bis 1930 kostenpflichtig (Brauns 1998; vgl. Troger 2009). Insofern wird deutlich, dass das Sekundarschulwesenden den klaren Auftrag der Eliteausbildung hatte. Nach dem lycée konnten die Absolvent_innen entweder bereits in Positionen des Staatsdienstes, wie z.B. als Lehrkräfte, wechseln, oder ein Studium anschließen.

Das Hochschulsystem nach der französischen Revolution bestand aus zwei Sektoren. In der französischen Revolution wurden alle Universitäten aufgelöst und erst unter Napoleon als unabhängige Fakultäten entlang von Disziplinen (in Verbindung mit dem staatlich kontrollierten Sekundarschulsystem wiedergegründet). Der Name dieser Konstruktion aus Fakultäten und Sekundarschulsystem 
war die imperiale Universität (Koppetsch 1994; Musselin 2007). Im Gegensatz zur deutschen Universität, welche als relativ autonome Organisation beschrieben werden kann, waren die Fakultäten primär eine Verlängerung des Sekundarschulsystems. Forschung fand vor allem außerhalb der Universitäten statt (Koppetsch 1994; Musselin 2007).

Der zweite Sektor bestand aus einer wachsenden Zahl elitärer beruflicher Spezialschulen, den späteren grandes écoles, welche den staatlichen Bedarf an hochausgebildeten Personal stillen sollten. Analog zum steigenden Bedarf an hochqualifizierten Fachkräften wurden jeweils neue Spezialschulen gegründet, so dass das Hochschulsystem hochgradig funktional ausdifferenziert wurde. In diesen Spezialschulen fand und findet bis heute die Elitenbildung statt. Deren anhaltende Bedeutung im französischen System ist jedoch lediglich vor dem Hintergrund der Schwäche der Universitäten zu verstehen (vgl. Koppetsch 1994).

\section{Volksschulwesen und Berufsbildung:}

Auch die Entwicklung der beruflichen Bildung wurde nachhaltig durch die französische Revolution geprägt, denn das bestehende System der Gilden und Zünfte wurde verboten, so dass keine Form von organisierter Ausbildung der Bevölkerung mehr stattfand. Lehrausbildungen waren eine rein private Angelegenheit zwischen Betrieb und Lehrling (Schriewer 1986 bei Greinert 2005). Bis Ende des 19. Jahrhunderts blieb ebenfalls der Staat dem beruflichen Bildungswesen fern und auch danach war der Zentralstaat nur in Teilen in die berufliche Bildung involviert (vgl. Brauns 1998; Greinert 2005; Troger 2004).

Auf das Volksschulwesen aufbauend, welches 1882 mit Einführung der Schulpflicht für die Jahre 6 bis 13 verpflichtend wurde (Brauns 1998), konnten zwei berufliche Wege unterschieden werden: einmal der Weg über die technischen Berufsschulen und einmal der Weg über das Berufsbefähigungszeugnis, welches 1911 beschlossen wurde.

Der erste Zweig war der prestigereichere, welcher später nach sukzessiver Niveauerhöhung (Schriewer 1982) in den technologischen Abiturzweig einmünden wird. Ausgangspunkt bestand einerseits darin, das zunehmende Qualifikationsdefizit der französischen Arbeiterschaft, andererseits aber auch soziale und politische Unruhen zu verhindern (Brauns 1998, Greinert 2005.) Im Rahmen dieser staatlichen Initiativen wurden Lehrlingsschulen „écoles manuelles d'apprentissage" gegründet, welche die notwendigen Fachkenntnisse vermitteln sollten. So entstanden 1880 zahlreiche berufsorientierte Ausbildungseinrichtungen, wie die,Ecoles Primaires Supérieure Professionnelles" und die "Ecoles Nationales Professionnelles" (ENP). Diese rekrutierten ihre Schüler_innen aus den besten Absolvent_innen der Volksschulen über concours-Verfahren und führten in vollzeitschulischer Ausbildung von drei oder vier Jahren zu prestigereichen Abschlüssen (Brauns 1998). Diese Ausbildungen können als Eliteausbildung der Volksschüler_innen in der 
beruflichen Bildung angesehen werden (Greinert 2005). Ausgebildet wurden Techniker_innen, Aufseher_innen und Vorarbeiter_innen.

Da die meisten Franzosen trotz Schulpflicht zu Beginn des 20. Jahrhunderts weder über einen Schul- noch beruflichen Abschluss verfügten, führte der französische Staat eine alternierende Lehrlingsausbildung, die mit einem Berufsbefähigungszeugnis CAP endete, ein, wobei die Lehrlinge ab 1919 mit dem loi Astier auch verpflichtend die Berufsschule besuchen mussten. Damit das CAP nicht zum Nachteil der auszubildenden Betriebe werden konnte, wurde 1925 zudem die Ausbildungsabgabe „taxe d' apprentissage“ eingeführt (Troger 2004). Ungeachtet der staatlichen Bemühungen besuchten jedoch nur wenige Berufsschüler_innen die Berufsschulkurse und erhielten ein Zertifikat (Brauns 1998), da der Staat nicht gewillt war, die rechtlichen Regelungen gegen die Betriebe durchzusetzen (Greinert 2005). Problematisch war zudem, dass das CAP ganz in den Händen der Unternehmen und Kammern lag ohne bestehende einheitlichen Regelungen und Standards (Brucy 2005).

Der mangelnde Durchsetzungswille der Regierung und die fehlende Ausbildungswilligkeit der Unternehmen führte kurz vor Beginn des Zweiten Weltkrieges zur sogenannten Krise der Berufsausbildung, ,crise d'apprentissage“, da nicht genügend qualifizierte Arbeiter_innen für die Aufrüstung zur Verfügung standen. So kam es 1939 letztlich zu einer Trennung der Facharbeiterausbildung vom Arbeitsprozess und einer Einrichtung von Berufsschulen - eine Idee, welche bereits länger innerhalb der Arbeiterbewegung bestand (Brauns 1998: 50). Von der Vichy-Regierung ausgebaut wurden die Berufsschulen, in welchen das CAP innerhalb von drei Jahren vollzeitschulisch erworben wurde, in ,centres $d^{\prime}$ apprentissage" (CA) umbenannt (vgl. Brauns 1998; Greinert 2005). Brucy (2005) beschreibt, dass es mit dem Ausbau der CA auch zu einem großen Anstieg beruflich Ausgebildeter kam. Das CAP war zu dieser Zeit ein angesehener Abschluss, der berufliche Sicherheit und sozialen Aufstieg innerhalb der Arbeiterschaft versprach. Die CAP-Abschlüsse stellten einen Kompromiss zwischen Aufstiegsaspirationen der Arbeiter_innen und ihrem Sicherheitsbedürfnis dar. Dies galt umso mehr, als dass $195086 \%$ der auf dem Arbeitsmarkt aktiven Bevölkerung keinen Abschluss (auBer dem der Grundschule) besaßen (Brucy 2005: 26).

Bis hierhin waren die wenigen Formen der Berufsausbildung zwar hierarchisch klar dem Sekundarschulweg mit Abitur und Studium unterlegen, aber boten über die technischen höheren Schulen, z.B. die Écoles nationales professionnelles (ENP) eine reputierte Form der Ausbildung hin zu höheren Technikerpositionen und mit dem CAP einen geschätzten Berufsabschluss, welcher den internen Aufstieg in den Organisationen des Arbeitsmarkts versprach (vgl. Brauns 1998; Brucy 2005; Tanguy 2000) 


\subsubsection{Entstehung des modernen französischen Bildungssystems: Prozesse der Entwertung beruflicher Bildung im Sekundarbereich}

Als kritische Phase und als der Weg in die Stigmatisierung für die beruflich orientierten Bildungsgänge wird die zunehmende Integration dieser in den allgemeinschulischen Bildungsbereich gesehen (vgl. Brauns 1998; Brucy 2005; Tanguy 2000; Troger 2004). Pardoxerweise wurde diese Entwicklung ausgelöst durch die Reformen der 5. Republik, welche zu einer Demokratisierung, d.h. einer erhöhten Bildungsbeteiligung einerseits und größerer sozialer Mobilität andererseits führen sollte. Brauns (1998: 56, Hervorhebungen im Original) beschreibt die Zielsetzung folgendermaßen:

Das Postulat der ,Demokratisierung' verband sich daher mit dem Ziel struktureller Reformen, die zu einer Abschaffung des traditionellen Dualismus von ,primaire` und ,secondaire' führen sollten. ${ }^{142}$

So waren es die Reformen Berthoine (1959) und Fouchet (1963) und später dann Haby (1975), welche zum einen die Mindestschulzeit von 16 Jahren und das college unique, d.h. eine einheitliche Sekundarschule, eingeführt haben. Dieses sollte von allen Schüler_innen besucht werden, wobei nach zwei Jahren eine Orientierung in die unterschiedlichen Bildungszweige erfolgen sollte (Fouchet Reform): Einmal in den allgemeinbildenden Zweig, welcher zum allgemeinen Abitur führte, dann in die lange technische Bildung, während der das neu eingerichtete (1966) baccalauréat technologique erworben werden konnte und in die kurzen technischen bzw. beruflichen Bildungsgänge hin zum CAP-Abschluss und seit 1966 auch zum BEP, die jeweils nach 2 Jahren erworben werden konnten. Nach dem BEP war es dann möglich für die besten Schüler_innen, in einem Übergangsjahr in ein bac techno zu wechseln. Gleichzeitig wurden mit den Reformen in den 1950ern und 1960ern auch die anerkannten höheren technischen Abschlüsse der ENP und EPCI in das baccalauréat technologique und einige längere Abschlüsse in den postsekundären Abschluss BTS umgewandelt. Die Auswahl in diese Bildungsgänge auf Sekundarniveau erfolgte aber nicht mehr selectiv über einen concours, sondern in Form der Orientierungsphasen im collège unique (Brauns 1998, Brucy 2005). Entsprechend des beruflichen Charakters der Diplome, welche zum baccalauréat technologique umgewandelt wurden, war das bac techno daher ursprünglich als Arbeitsmarkteinstiegszertifikat gedacht. Nur besonders leistungsstarke Absolvent_innen sollte auch der Weg in die technischen Kurzstudiengänge an den STS und seit 1967 auch an den IUT offenstehen (Prost 2000). Aber immer mehr bac techno Absolvent_innen begannen ein Studium.

142 Mit „primaire“ und „secondaire“ ist in diesem Zitat die Trennung zwischen Volksschule und Sekundarschule gemeint. 
Als besonders problematisch für die Wertschätzung beruflicher Bildung in der Gesellschaft wird die „Integration in den allgemein-schulischen Bildungsapparat" (Brauns 1998: 79) gesehen und damit einhergehend eine Anpassung an traditionelle französische Bildungsideale der hohen Wertschätzung abstrakter Wissensinhalte im Gegensatz zur ökonomisch am Arbeitsmarkt orientierten Bildung. Es kam somit zu einer Anpassung an diese Tradition durch eine zunehmende Bildungsdauer, einer Theoretisierung von Inhalten und Distanz zum Arbeitsmarkt, welche sich auch in der Rekrutierung der Lehrer_innenschaft des beruflichen Zweigs widerspiegelte, die stärker an formalen Bildungsqualifikationen als an berufspraktischen Erfahrungen ausgerichtet war. Dies war zur Zeit der Gründung der centres des apprentis (später college d'enseignement technique) noch nicht der Fall.

Damit wurde die französische Berufsausbildung der „Logik“ des Schulwesens und seinen Spielregeln unterstellt sowie der darin gründenden faktischen und wertorientierten Distanz zum Wirtschafts- und Beschäftigungssystem (Schriewer 1982: 253).

Als nachteilig wurde ebenfalls angesehen, dass dem beruflichen Zweig die Autonomie bei der Auswahl ihrer Schüler_innen verloren ging. Die typischen Aufnahmeprüfungen mit Wettbewerbsverfahren wurden ersetzt durch die Phase der orienation am collège, wobei die schlechtesten Schüler_innen in den beruflichen Zweig und die besten in den allgemeinbildenden sortiert werden. Zuvor waren es die besten (an die höheren technischen Schulen) und besseren Schüler_innen der Volkschulen. So änderte sich auch die Klientel der Berufsbildung (Brucy 2005). Dadurch, dass der allgemeinbildende Zweig als einziger Referenzpunkt im Bildungssystem gesehen wurde, und da die technische und berufliche Bildung nicht in ihrer Einzigartigkeit anerkannt wurde, wurden diese entwertet, obwohl sie eigentlich durch die Integration in das allgemeinbildende Bildungswesen aufgewertet werden sollten (Brucy 2005: 2). Somit kam es einerseits zur Stigmatisierung der Berufsbildung als non general und zum anderen durch die auf Leistungsauswahl begründete negative Selektion die Definition des Weges in die berufliche Bildung als „selbstverschuldetes Schicksal“ (Brauns 1998: 69).

Aber auch die Entwicklungen am Arbeitsmarkt verstärkten das negative Image der beruflichen Bildung in Frankreich (Troger 2004). Für Troger wirkten sich zum einen die demografische Entwicklung und zum anderen die neuen technischen Kurzstudiengänge negativ auf die Arbeitsmarktchancen beruflich Qualifizierter aus, da es für sie durch die neu institutionalisierten Abschlüsse (DUT, STS) schwieriger wurde, Zugang zum mittleren Management durch interne Aufstiegsmöglichkeiten im Betrieb zu erhalten. Insofern war die erste Wahl der Schüler_innen und Eltern immer die allgemeine Bildung, denn ,to opt for technical and vocational education meant taking the risk of being locked into lowerranking social status for the remainder of one's life" (Troger 2004: 12).

Die nächste relevante Entwicklung im Bildungssystem war die Entstehung des bac pro 1985, welche einerseits als Reaktion auf die Forderungen der Unter- 
nehmen gesehen wurden (Brucy 2005). Da sich die Anforderungen in den Betrieben erhöht hätten und viele bac techno Absolvent_innen nicht in den Arbeitsmarkt übergingen, sondern weiterstudierten, forderte die Wirtschaft ein neues nationales Diplom auf einem höherem Niveau als BEP und CAP (Brucy/Troger 2000). Die Politik formulierte zur gleichen Zeit das Ziel, dass $80 \%$ eines Jahrgangs Niveau IV, also Abiturniveau, erreichen sollten. Die Einführung eines weiteren beruflichen Diploms war daher die Lösung (Prost 2000). Dieses Diplom wurde, auch wenn es als Arbeitsmarkeinstiegszertifikat kreiert wurde (Prost 2000), baccalauréat professionel genannt und berechtigte damit auch zum Studium. Es sollte nach dem BEP aber auch dem CAP über zwei weitere Ausbildungsjahre erworben werden und dann zu mittleren Arbeiterpositionen hinführen. Durch die Entscheidung, dieses Diplom als baccalauréat zu benennen, sollte auch der berufliche Bildungszweig im Sekundarschulwesen aufgewertet werden (Brauns 1998). Ziel war es jedoch nicht, die Berufsabiturient_innen in ein Studium zu führen. Dafür wurden auch die Curricula nicht konzipiert. Denn im Gegensatz zum bac techno, welches im Sekundarwesen II noch ein Jahr mit dem allgemeinbildenden Zweig zusammen lernt und auch mehr Allgemeinbildung insgesamt im Lehrplan aufweist, ist dies im beruflichen Abitur nicht der Fall. Wie bereits dargelegt, wurde das bac pro gut angenommen, aber entgegen des Ziels einer direkten Arbeitsmarktintegration fingen auch zunehmend bac pro-Absolvent_innen mit einem Studium an, jedoch nur mit vergleichsweise geringem Erfolg.

Was bedeuten die Entwicklungen für die Durchlässigkeit von Berufs- und Hochschulbildung? Bis zu den Reformen in der fünften Republik war Durchlässigkeit zwischen Berufs- und Hochschulbildung durch die eindeutige institutionalisierte Trennung des Volkschulwesens mit anschließender Berufsausbildung und dem allgemeinbildenden Sekundarschulwesen, welches zum Abitur und damit zur Hochschulberechtigung führte, nicht gegeben. Die Hochschuleingangsprüfung, das heutige DAEU, wurde zum Ende der vierten und zu Beginn der fünften Republik in Frankreich eingeführt (MEN 2000a) und war ein erster Weg in die Hochschule ohne Abitur.

Durch die Integration der beruflichen Wege in das Sekundarschulwesen seit Beginn der fünften Republik 1958, wurde diese strikte Trennung mehr und mehr aufgelöst. Primär galt das vor allem für die Bildungswege, welche zu den höheren Technikerpositionen führten (ENP und EPCI) und frühzeitig mit dem neuen Abschluss baccalauréat technologique eine Hochschulzugangsberechtigung erhielten. Allerdings verloren diese Bildungsgänge im Laufe der Zeit und zunehmender Integration in dem allgemeinbildenden Bildungszweig ihre berufsorientierte Bestimmung, so dass das bac techno immer weniger ein Berufseinstiegszertifikat als ein Zertifikat wurde, welches zu höheren Studien führt. Aber auch der berufliche Weg zum Facharbeiter wurde durch die Einführung des baccalauréat professionnel mit dem Hochschulbereich verbunden, auch wenn das Zertifikat als Arbeitsein- 
stiegszertifikat konzipiert wurde. Es kam somit historisch regulativ zu einer immer stärkeren Durchlässigkeit zwischen Berufs- und Hochschulbildung in Frankreich.

Die Integration in das Sekundarschulwesen hatte aber auch negative Konsequenzen für die berufliche Bildung und als Ergebnis auch für die reale Durchlässigkeit zwischen Berufs- und Hochschulbildung. Dadurch, dass der allgemeinbildende Weg der Referenzpunkt im französischen Bildungssystem ist und der Weg in die beruflichen Bildungszweige seit der Integration in das Sekundarschulwesen nicht mehr über ein Wettbewerbsverfahren, sondern über eine Negativauswahl der Schüler_innen mit Leistungsproblemen im allgemeinbildenden Weg führt, kam es immer stärker zu einer Abwertung beruflicher Bildung in der Gesellschaft. Ihr Eigenwert wurde nicht anerkannt. Die negativ selektierte Klientel und das Ziel des beruflichen Bildungswegs des direkten Einstiegs in den Arbeitsmarkt und den dementsprechend ausgerichteten Curricula und Pädagogik führten zu einer systematischen Benachteiligung von Berufsabiturient_innen im Hochschulsystem, die nicht auf dieses vorbereitet wurden. Die Benennung des Abschlusses als baccalauréat war mehr symbolisch als Aufwertung der Berufsbildung gemeint, denn als tatsächliche Reform der Berufsbildung hin zur Hochschulbildung. Verstärkt wurde diese Entwicklung zudem dadurch, dass die beruflich orientierten Kurzstudiengänge, welche noch am ehesten an die Vorbildung der beruflichen Abiturient_innen anschließen durch die Selektion vermeintlich leistungsstärkerer Schüler_innen der anderen Bildungszweige, studierwillige beruflich Qualifizierte zum Teil an die Universitäten drängen, auf deren Anforderungen diese nicht vorbereitet sind. Die Konsequenz ist die systematisch erhöhte Abbruchquote beruflich Qualifizierter im französischen Hochschulsystem, insbesondere an den Universitäten. 


\section{Entwicklung institutioneller Durchlässigkeit in Frankreich}

In diesem Kapitel werden aufbauend auf das dargestellte Durchlässigkeitsproblem und dessen historische Entwicklung (Kapitel 8) die Veränderungen der institutionellen Durchlässigkeitsstrukturen in Frankreich von 1985 bis 2013 analysiert. Im Fokus der Analyse steht die Durchlässigkeit aus dem beruflichen Zweig der Sekundarbildung in das Hochschulsystem. Dabei wird das baccalauréat technologique nicht als Teil der beruflichen Sekundarausbildung behandelt, da der technologische Weg im Sekundarbereich durch eine sukzessive Niveauanhebung mittlerweile stark in den allgemeinbildenden Bereich integriert wurde (vgl. Kapitel 8.3.). Das bac techno, auch wenn es als solches geschaffen wurde, stellt kein typisches Arbeitsmarkteintrittszertifikat mehr dar, was als klares Definitionskriterium beruflicher Bildungswege in Frankreich gelten soll. Vielmehr wird es als ein Abschluss in Richtung Hochschulstudium angesehen ${ }^{143}$. Auch die starke allgemeinbildende curriculare Ausrichtung des bac techno im Vergleich zum bac pro und der fehlende Kontakt mit der Arbeitswelt unterstützen diese Sichtweise.

Für den französischen Fall werden wiederum zwei Zeiträume unterschieden, einmal von 1985 bis 1998 und von 1999 bis 2012. Somit erfolgt die Analyse in diesem Kapitel in drei Schritten. Analog wie für die deutsche Analyse werden zuerst die Entwicklungen in den beiden Zeiträumen dargestellt. Dabei werden sowohl die durchlässigkeitsrelevanten Debatten als auch die Struktur der Diskurse analysiert. Es soll gezeigt werden, in welchen Kontexten über Durchlässigkeit zwischen Berufs- und Hochschulbildung debattiert und wie Durchlässigkeit konstruiert wird, aber auch welche Deutungskämpfe und damit Infragestellungen der Legitimität bestehender Institutionen sichtbar werden. In einem dritten Teil soll die Frage nach dem Wandel der Durchlässigkeitsstrukturen in Frankreich zusammenfassend beantwortet werden.

Im Zentrum der Darstellung institutioneller Entwicklungen in Frankreich stehen solche, welche anhand der Diskursanalyse von Dokumenten der das Berufs- und Hochschulbildungssystem stark mitbestimmenden Akteursgruppen in Frankreich rekonstruiert wurden, und damit primär die kulturell-kognitive und partiell auch normative Dimension. Wie bereits in Kapitel 4 auf Basis der Steuerungsmodi der Berufs- und Hochschulbildung (vgl. auch Kapitel 8) begründet, wurden folgende Akteure in die Analyse mit einbezogen: Für die zentralstaatliche Ebene sind dies insbesondere die Bildungsministerien ${ }^{144}$ (MEN, MESR,

143 Diese Ausrichtung auf das Hochschulstudium wird ebenfalls auf der offiziellen Beschreibung des Bildungsgangs und seiner Ausrichtung von Ministeriumsseite so beschrieben (MEN 2014).

144 Wie in Kapitel 4 bereits dargelegt, habe ich für den ersten Zeitraum auch einige Dokumente, die vom Premierminister und dem Staatspräsidenten kamen, mitanalysiert. Dies war auf Grund der Datenlage notwendig. 
MENRT, MENJS, MENSR, MDEP, MJENR), wobei sich deren Namen und damit auch die Abkürzung oft mit den Regierungswechseln geändert haben. Für die Vertreter der Hochschulebene wurden Dokumente der Konferenz der französischen Hochschulpräsidenten, die Conférence des présidents d'université (CPU) sowie die Association des Directeurs d'IUT (ADIUT) als Vertreter der berufsorientierten technischen Hochschulen analysiert. Als Vertreter der Gewerkschaften wurde die Confédération générale du travail (CGT), welche den zweitgrößten Gewerkschaftsbund in Frankreich darstellt, sowie die größte Gewerkschaftsunion für den Erziehungsbereich, die Fédération Syndicale Unitaire (FSU) und für die Unternehmensseite der Arbeitgeberverband Mouvement des entreprises de France MEDEF (vorher CNPF) als Akteure ausgewählt.

Aber auch regulative Entwicklungen werden in die Analyse eingearbeitet zum einen geschieht dies dadurch, dass ein Überblick über die relevantesten politischen Debatten und Aktionen vermittelt wird, in denen Durchlässigkeit thematisiert wird. Zum anderen werden insbesondere im letzten Abschnitt des Kapitels, in welchem die Frage nach dem Wandel beantwortet werden soll, ebenfalls die regulativen Veränderungen in die Analyse einbezogen. Ziel des Kapitels ist es, die Entwicklungen Frankreichs im Hinblick auf Durchlässigkeit zwischen Berufsund Hochschulbildung zu beurteilen und einzuordnen. Wurden bestehende Durchlässigkeitsstrukturen stabilisiert (path stabilization)? Kam es zu deutlichen Veränderungen, welche aber nichts an den Kernprinzipien für Durchlässigkeit geändert haben (path departure)? Oder kann der französische Fall beschrieben werden als einer, in welchem es zu einer Abkehr historischer Pfade und der Entwicklung neuer Kernprinzipien (path cessation) kam?

\subsection{Gesellschaftliche Wissensbestände zu Durchlässigkeit vor Bologna und Kopenhagen (1990-1998)}

Um die Entwicklung der gesellschaftlichen Wissensbestände in Frankreich aufzuzeigen und damit die kulturell-kognitive sowie ebenfalls normative institutionelle Dimension der Durchlässigkeitsstrukturen zu beleuchten, wird eruiert, inwiefern Durchlässigkeit zwischen beruflicher und akademischer Bildung thematisiert und problematisiert wird. Dabei werden jedoch nicht allein die Diskussionen zum Problem des Studienmisserfolgs bzw. -abbruchs beruflich Qualifizierter untersucht, sondern auch (wie im deutschen Fall) die Dokumente bildungspolitischer Akteure daraufhin analysiert, ob allgemein Durchlässigkeit zwischen beruflicher Sekundarbildung und postsekundärer akademischer Bildung angesprochen wird.

An dieser Stelle soll, wie bereits auch in Kapitel 4, darauf hingewiesen werden, dass die für den ersten Untersuchungszeitraum durchlässigkeitsrelevanten Dokumente allein von Regierungsorganisationen untersucht wurden. Die für die Datenauswahl analysierten Dokumente der anderen bildungspolitischen Akteure 
wiesen keinen Durchlässigkeitsbezug auf. Insgesamt konnten jedoch auch nur wenige bildungspolitische Originaldokumente unabhängig vom Thema Durchlässigkeit für diesen Zeitraum gefunden werden, deren Ursprung nicht öffentliche Stellen waren. Dies bedeutet zweierlei für die Analysen des ersten Untersuchungszeitraums: Die hier vorgestellten Debatten und Diskursstrukturen reflektieren einerseits, wie auch in den Dokumenten der staatlichen Organisationen sichtbar wird, die geringe Relevanz des Themas Durchlässigkeit im ersten Untersuchungszeitraum. Sie können aber schließlich lediglich für einen Teil des koordinativen Diskursraums als repräsentativ gelten, wobei mit der Regierung der bedeutendste Akteur (vgl. Kapitel 8.2.) untersucht und der hegemoniale Diskurs zu dieser Zeit dargestellt wird. Unterschätzt werden könnten allerdings mögliche Gegendiskurse. Anhand der Untersuchung der Diskursstrukturen des zweiten Zeitraums und einer Medienanalyse ist dies nicht sehr wahrscheinlich - aber es ist möglich.

Um die zu rekonstruierenden Diskursstrukturen besser in die bildungspolitischen Diskussionen einzubetten, werden zuerst die Debatten, die Kontexte, in denen Durchlässigkeit thematisiert wird, vorgestellt, um nachfolgend die genauen Charakteristika der Diskursstrukturen zu beleuchten.

\subsubsection{Schlaglichter der französischen Debatten zu Durchlässigkeit (1985-1998)}

In welchen Kontexten wurde über Durchlässigkeit im ersten Untersuchungszeitraum in Frankreich diskutiert? Insgesamt lassen sich drei Debatten identifizieren, welche für die Fragen von Durchlässigkeit zwischen Berufs- und Hochschulbildung in Frankreich von Relevanz sind, wobei nur in einer Debatte Durchlässigkeit von Berufs- zur Hochschulbildung explizit gefordert wird. Folgende Debatten lassen sich voneinander abgrenzen:

1) Debatte zur Modernisierung der Sekundarbildung und Erhöhung der Attraktivität beruflicher Bildung

2) Debatten zur Modernisierung der Hochschulen

3) Debatte zur Weiterbildung und der Ermöglichung lebenslangen Lernens

Die Darstellung der Debatten erfolgt zwar getrennt, sie fanden aber parallel statt und es wurde auch aufeinander Bezug genommen.

\section{Debatte zur Modernisierung der Sekundarbildung und Erhöhung der Attraktivität beruflicher Bildung}

Da der Untersuchungszeitraum für den französischen Fall zur Zeit der Einführung des beruflichen Abiturs einsetzt, ist anzunehmen, dass Fragen der Durchlässigkeit von Berufs- zur Hochschulbildung in den öffentlichen Debatten ausführlich diskutiert wurden. Tatsächlich wurde aber die Etablierung des bac pro nicht 
mit der Ermöglichung des Zugangs zur Hochschule begründet. Vielmehr sollte die Einführung einer höheren Wertschätzung beruflicher Bildung und zu einer Höherqualifizierung beruflich Qualifizierter führen (Brauns 1998). Die Diskussionen zum bac pro sind daher anders als erwartet, nicht durch Aussagen, welche sich mit Durchlässigkeit zwischen beruflicher und hochschulischer Bildung befassen, gekennzeichnet. Vielmehr wurde insbesondere mit der Einführung des beruflichen Abiturs aber auch in den Debatten über die Weiterentwicklung der Berufsbildung als Ziel ausgegeben, die gesellschaftlich bestehenden Hierarchisierungen zwischen beruflichen praktischen und eher allgemeinbildenden Bildungsgängen aufzuheben (Président 1989, MEN 1994, Premier ministre 1995). ${ }^{145}$

Das Ende der 1980er und die 90er Jahre in der Bildungspolitik in Frankreich waren reformreiche Jahre. Neben der Einführung des bac pro waren sie stark geprägt durch die Einführung des Gesetzes loi d'orientation sur l'éducation 1989, dem sogenannten loi Jospin, (Loi Jospin 1989), in welchem u.a. neue Prinzipien für das französische Bildungswesen festgehalten wurden: eine stärkere Individualisierung der Bildung, d.h. eine stärkere personalisierte Ausrichtung der Bildung, aber auch ein größerer Entscheidungs- und Verantwortungsspielraum der Individuen, eine stärkere Dezentralisierung und die Festschreibung der sogenannten „contractualisation", d.h. der Zielvereinbarungen zwischen den Bildungsorganisationen und dem Staat. So wurden zu dieser Zeit auch die vierjährigen Verträge der Hochschulen mit dem Staat (die contrats quadriennaux) erweitert ${ }^{146}$. Das wohl einflussreichste Ziel, welches mit dem loi Jospin festgeschrieben wurde, ist, dass $80 \%$ einer Kohorte ein Abitur und alle mindestens das Niveau des französischen Facharbeiters (CAP/BEP) erreichen sollten.

Dieses Ziel war zwar bereits vorher formuliert worden (MEN 1985) und war ein Grund, warum das Berufsabitur eingeführt wurde (Prost 2000). Mit dem Gesetz von 1989 wurde diese Norm aber als nationales Bildungsziel festgeschrieben.

Diese Debatte wurde aufgenommen, da sie gewissermaßen eine Debatte des Nicht-Thematisierens darstellt. Obwohl beruflich Qualifizierte ein baccalauréat erhalten können, soll dieses lediglich ein Arbeitsmarkteinstiegszertifikat sein und der Übergang zur Hochschule ist kein Thema.

\section{Debatte zur Modernisierung der Hochschulen}

Die meisten Diskussionen im Bildungswesen Ende der 1980er und in den 90er Jahren in Frankreich drehten sich insbesondere um die Frage der Modernisierung

145 Wie auch für die Diskursanalyse im deutschen Fall gilt ebenfalls für den französischen, dass zur Veranschaulichung und zum Nachweis der Diskurse repräsentative Quellen und Zitate angegeben werden. Dabei ist zu beachten, dass diese Quellen nicht die einzigen im Untersuchungszeitraum sein müssen, welche das Geschriebene belegen. Vielmehr stellt die Beschreibung der Diskursstruktur eine Modellierung typischer Strukturen, Verständnisse dar, welche aus allen im Anhang identifizierten Quellen herausgearbeitet wurden.

146 Bereits mit dem Gesetz loi Savary von 1984 existierten erste Zielvereinbarungen, jedoch nur in Bezug auf Forschung und nicht den ganzen Universitätsbetrieb. 
der Universitäten und der Anpassung der Hochschullandschaft an steigende Studierendenzahlen und deren besseren Vorbereitung auf den Arbeitsmarkteinstieg (MENJS 1988 a, b). Mit dem plan université 2000 wurde vor allem der Ausbau der Hochschullandschaft vorangetrieben. Neben der Gründung neuer Universitäten und einer Vielzahl neuer technischer Hochschulinstitute, den IUTs, fand auch die Neugründung von in den Universitäten angesiedelten beruflichen Hochschulinstituten (instituts universitaire professionnels, IUP) in diesem Zeitraum statt. Der Ausbau der IUTs und IUPs sollte zu einer Diversifizierung der Bildungswege und einer stärkeren beruflichen Ausrichtung führen (MEN/MESR 1988; MESR 1993). Insofern wurde innerhalb des Hochschulsystems noch stärker als davor auf eine Mischung zwischen stärker allgemeinbildenden und beruflich orientierten Studiengängen gesetzt.

Ein weiterer Aspekt der Modernisierungsinitiativen, welche vor allem durch die Bildungsminister Jospin (1988-1992) und später von Bayrou (1993-1997) durchgeführt wurden, befasste sich mit dem Problem des échec, dem systematischen Studienmisserfolg einer Vielzahl von Studierenden im ersten Zyklus (den ersten beiden Studienjahren) - speziell an den Universitäten. Im ersten Untersuchungszeitraum wird dieses Thema der Studienprobleme zwar mit dem Anstieg der Studierendenzahlen und damit einer gewachsenen Heterogenität der Student_innenschaft begründet, aber beruflich qualifizierte Abiturient_innen im Gegensatz zu den Absolvent_innen des technologischen Abiturs wurden dennoch in den untersuchten Dokumenten nicht explizit als gefährdete Gruppe angesprochen. Eine Ausnahme bildet ein extra für das Hochschulbildungsministerium verfasster Bericht von Daniel Laurent (1995), in welchem ebenfalls auf die gestiegene Zahl der Berufsabiturient_innen, welche ein Studium an Universitäten aufnehmen anstatt direkt in den Arbeitsmarkt zu wechseln, hingewiesen wird. Insbesondere diese Gruppe - zusammen mit dem Absolvent_innen des technischen Abiturs - werden als mögliche zukünftige Studierdende mit Problemen identifiziert $^{147}$. Insofern ist insgesamt gesehen auch diese Debatte eine des nicht Thematisierens. Beruflich Qualifizierte mit einem bac pro, auch wenn sie auch schon in den 1990er Jahren auf die Hochschulen gewechselt sind, werden nicht als relevant anerkannt.

\section{Debatte zur Weiterbildung und der Ermöglichung lebenslangen Lernens}

Während die beiden vorherigen Debatten das Verhältnis der beruflichen Bildung zur Hochschulbildung mitbestimmen, so ist aber auch festzustellen, dass die Frage der Durchlässigkeit beruflicher Bildung auf Sekundarniveau zur Hochschulbildung nicht thematisiert wurde. Allein die Debatte zur Weiterbildung und

147 Der Bericht, da er nicht von einem der für die Analyse ausgesuchten Akteure verfasst wurde, ging nicht in die Feinanalyse der Diskurse ein. 
der Ermöglichung lebenslangen Lernens bildet eine Ausnahme. So wurde in den Dokumenten sporadisch aber immer mal wieder die Förderung von Bildung über den gesamten Lebensverlauf (Président 1989) und damit auch die Stärkung beruflicher Weiterbildung im Hochschulbereich durch den Ausbau der Validierungsverfahren für berufliche Kenntnisse auch an Hochschulen als ein relevantes Ziel anerkannt und u.a. 1992 mit der Reform des VAP (vgl. Kapitel 8.2.4) vorangebracht. Die Förderung lebenslangen Lernens solle eine Hauptaufgabe auch der Hochschulen werden (MENSR 1996a, 1997), wodurch auch beruflich Qualifizierte, welche bisher nicht studieren konnten oder wollten, eine Möglichkeit bekommen sich weiterzubilden, wobei dadurch gleichzeitig eine Anpassung an die ansteigenden Arbeitsanforderungen auf dem Arbeitsmarkt erfolgen soll.

Abbildung 9 zeigt zusammenfassend die drei Debatten und bildungspolitischen Reformen im Überblick, wobei in Frankreich durch die kontinuierlichen Reformanstrengungen die Debatten quasi keinen richtigen Start und Endpunkt hatten, sondern mit neuen Reforminitiativen immer wieder aufgegriffen wurden. Die gestrichelten Linien um die Debattenbox mit der helleren Schrift sollen anzeigen, dass die Debatte zwar für die Erklärung des französischen Falles von Relevanz war, aber letztlich nicht explizit auf Durchlässigkeit zwischen Berufs- und Hochschulbildung abzielte. Auffällig ist, dass auch wenn einige regulativen Reformen im Hinblick auf Durchlässigkeit zwar stattgefunden hatten - wie die Reform des D.A.E.U., dem zweiten Bildungsweg in die Hochschule -, diese jedoch nicht in den Dokumenten sichtbar wurden.

\subsubsection{Französischer Diskurs 1985-1998: Durchlässigkeit via Weiterbildung für Berufserfahrene}

Anhand der Debatten konnte verdeutlicht werden, dass im ersten Zeitraum Durchlässigkeit zwischen beruflicher Sekundar- und Hochschulbildung kaum diskutiert wurde. Aus den Aussageereignissen, welche primär zu der Debatte zur Ermöglichung des lebenslangen Lernens zählen, aber auch den beiden anderen Debatten konnte trotzdem ein Diskurs identifiziert werden, der Durchlässigkeit zwischen Berufs- zur Hochschulbildung für Berufserfahrene fordert. Wie genau sah nun aber dieser Durchlässigkeitsdiskurs bis 1998 aus?

Die Darstellung der Diskursstruktur erfolgt analog wie im deutschen Fall in mehreren Schritten und stellt den Diskurs als Querschnitt für den ersten Untersuchungszeitraum dar. Dies ist möglich, da keine größeren Veränderungen für diesen Zeitraum festzustellen sind. Zuerst werden die rekonstruierten Argumentationsmuster dargestellt. Es wird gezeigt, warum Durchlässigkeit thematisiert wird. Warum wird fehlende Durchlässigkeit als Problem erkannt? In einem zweiten Schritt geht es insbesondere um das Verständnis von Durchlässigkeit, welches auch in den Lösungsvorschlägen sichtbar wird. Wie soll Durchlässigkeit erreicht werden? Welche Aspekte von Durchlässigkeit spiegeln sich in den Aussagen zu 


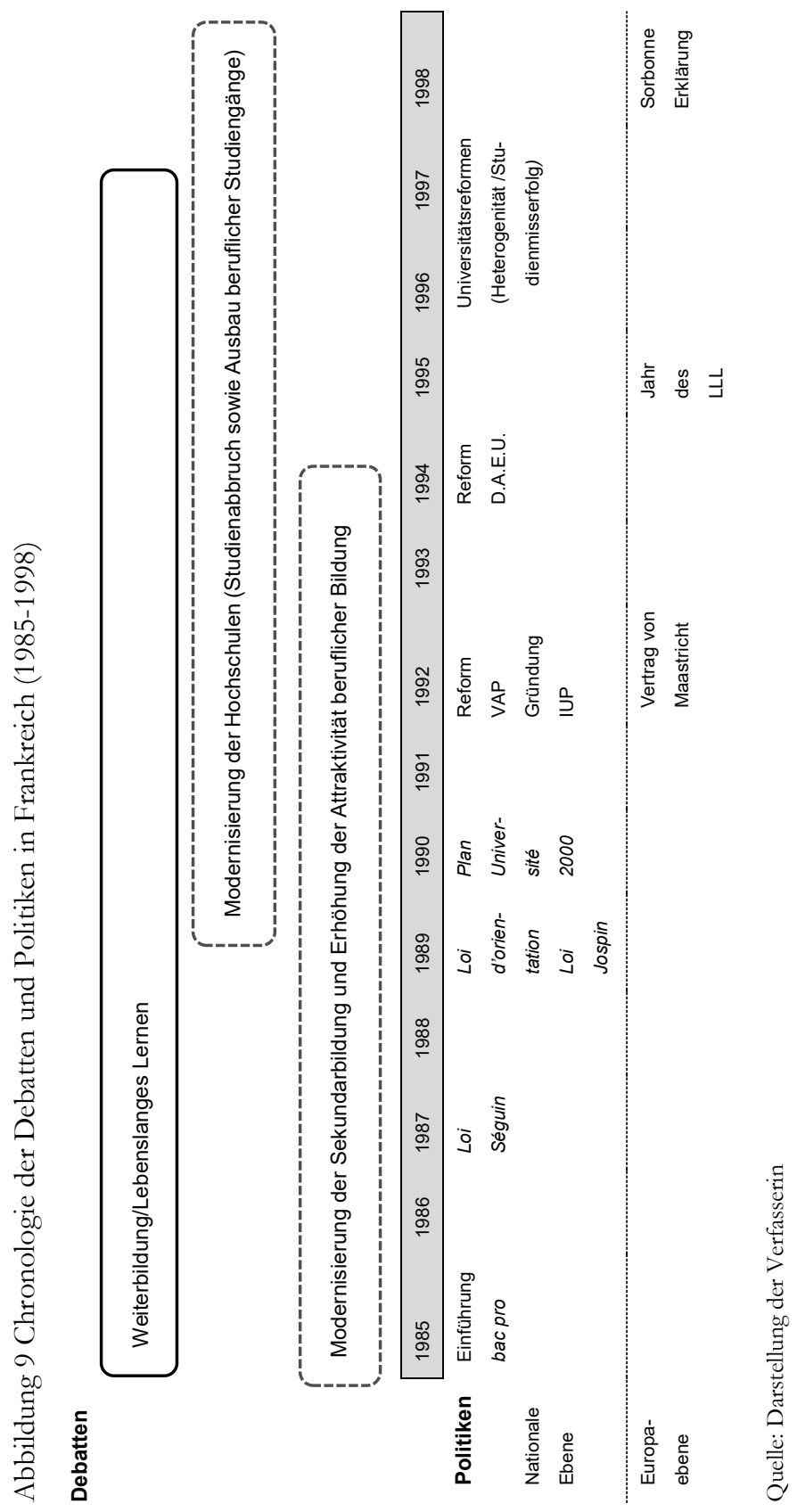


Durchlässigkeit wider? Für wen soll Durchlässigkeit geschaffen werden, d.h., welche Rollenerwartungen (normative Dimension) werden konstruiert? Schließlich sollen auch offenbare Strategien aus dem Diskurs dargelegt werden, wobei dieser Analyseteil im ersten Untersuchungszeitraum nur sehr kurz ausfallen kann.

\section{Argumentationslogiken des französischen Diskurses}

Für den Zeitraum 1985-1998 konnte in Frankreich ein Diskurs identifiziert werden, der als reformorientierter Diskurs benannt werden soll. Im Gegensatz zu Deutschland, wo es eine klare Unterscheidung zwischen einem Diskurs gibt, der bestehende Strukturen kritisiert und einem anderen, der diese konservieren will, ist eine solche Kombination in Frankreich in den untersuchten Aussagen nicht festzustellen. Insofern gilt der Diskurs auch als reformorientiert, da er bestehende Strukturen nicht grundsätzlich infrage stellt aber in Richtung einer größeren Durchlässigkeit reformieren soll. Dieser Diskurs unterscheidet zwei Argumentationslogiken, die dann jeweils auch die Diskursstränge bestimmen. Einmal wird Durchlässigkeit stärker aus funktionalistischer Perspektive und einmal aus universalistischer Perspektive gefordert. Diese beiden Argumentationslogiken begründen, warum in Frankreich über Durchlässigkeit diskutiert wird und warum Durchlässigkeit als Problem in den untersuchten Aussageereignissen wahrgenommen wird.Tabelle 23 fasst die Argumentation abschließend zusammen.

Tabelle 23 Argumentationslogiken des französischen Diskurses (1985-1998)

Reformorientierter Diskurs

universalistisch

funktionalistisch

Gesellschaft:

(Weiter-)bildung als Recht

(Weiter-)bildung als öffentliche Aufgabe

(Weiter-)bildung als zweite Chance, um beste-

hende Ungleichheit zu korrigieren

Individuum:

Recht auf (Weiter-)bildung zur Erlangung des höchsten Bildungsniveaus auch als zweite

Chance auf ein Studium
Gesellschaft:

Bedarf der Höherqualifizierung durch strukturellen Wandel der Wirtschaft

Individuum:

Verringerung des individuellen Arbeitslosigkeitsrisiko

Quelle: Darstellung durch die Verfasserin

Die Besonderheit und das Typische für den ersten Untersuchungszeitraum besteht darin, dass Durchlässigkeit von beruflicher Sekundar- und Hochschulbildung, im Sinne einer Fortführung der Erstausbildung, d.h. eines direkten Anschlussstudiums an die berufliche Ausbildung, nicht explizit thematisiert wird. Durchlässigkeit zwischen Berufs- und Hochschulbildung wird stattdessen erst relevant nach dem Einstieg in das Erwerbsleben bei Fragen der Weiterbildung. Die Ermöglichung der tertiären Weiterbildung wird als eine normale Entwicklung des 
Bildungssystems und komplementäre Aufgabe zur Erstausbildung konstruiert (Président 1987).

La formation continue sera un élément essentiel du rôle de l'enseignement supérieur dans la société française du XXIe siècle. (MENSR 1997) ${ }^{148}$

Im Diskurs werden dabei zwei mögliche Formen der Weiterbildung für die Hochschulbildung diskutiert. Einerseits die Pause nach einem ersten tertiären Abschluss und dann ein Wiedereinstieg, und zweitens der Einstieg in das Hochschulsystem ohne vorheriges Studium aber mit Berufserfahrung. Letzteres ist für die Frage der Durchlässigkeit zwischen beruflicher und Hochschulbildung von Bedeutung.

Diese Form von Weiterbildung im Hochschulsystem wird aus zwei Perspektiven begründet - einerseits funktionalistisch: der Eintritt aus dem Erwerbsleben in die Hochschule dient der Deckung der Bedarfe des Arbeitsmarkts und dem Bedarf der Höherqualifizierung der Arbeitnehmerschaft aufgrund des strukturellen Wandels im Wirtschaftssystem - ausgelöst durch technologischen Wandel und Tertiärisierung (Président 1987, 1989, 1990). Aus dieser Perspektive ist Weiterbildung auch als eine Absicherung gegen die individuelle Gefahr der Arbeitslosigkeit zu sehen (Président 1989).

Andererseits wird Durchlässigkeit in Form von hochschulischer Weiterbildung auch universalistisch begründet als individuelles Recht auf Bildung, d.h. das Recht, sich so hoch wie möglich im Lebensverlauf zu bilden. Aus diesem Grund sei Bildung ein öffentliches Gut, welches vom Staat finanziert werden muss (Président 1989). Zudem stelle die Realisierung von Weiterbildung an den Hochschulen auf Basis beruflicher Erfahrungen auch eine Form von Chancengleichheit dar, da auf diese Weise Individuen, denen zuvor das ein Hochschulstudium nicht möglich war, diese Möglichkeit erhalten (MENSR 1997).

La reconnaissance de ce droit à un temps de formation en cours de vie, pour ceux qui n'auraient pas eu la chance de bénéficier d'une formation initiale universitaire, comme pour ceux qui ressentiraient le besoin d'un complément de formation, selon le principe du chèque-éducation, changera profondément l'image et la réalité de l'université.

(MENSR 1997) ${ }^{149}$

Lebenslange Bildung ist somit zentral für die Ermöglichung von Chancengleichheit und trägt gleichzeitig dazu bei, das Individuum als Person und Bürger und in beruflicher Perspektive weiterzuentwickeln (Président 1989).

148 „Weiterbildung wird ein essentieller Bestandteil der Funktion der Hochschulbildung in der französicshen Gesellschaft des 21. Jahrhunderts sein.“ (MENSR 1997, Übersetzung NB)

149 „Die Anerkennung des Rechtes auf Bildung im gesamten Lebensverlauf nach dem Prinzip eines einzulösenden Bildungsgutscheins für diejenigen, die bis dahin nicht die Chance einer initialen Ausbildung an der Universität hatten sowie für diejenigen, die den Bedarf sehen, ihre Bildung weiter zu vervollständigen, wird das gesellschaftliche Bild und die Realität der Universität grundlegend verändern.“ (MENSR 1997, Übersetzung NB) 


\section{Strukturmerkmale und Strategien des französischen Diskurses}

In diesem Abschnitt soll die Struktur des Diskurses daraufhin untersucht werden, wie und für wen Durchlässigkeit im ersten Zeitraum thematisiert wird. Welche Aspekte werden angesprochen und können zudem Strategien rekonstruiert werden. Eine Übersicht der Struktur findet sich am Ende des Abschnitts in Tabelle 24.

Mit dem Fokus auf Weiterbildung beruflich Qualifizierter wird Durchlässigkeit über Zugang und Anrechnung konzeptualisiert. Zugang sollen dann aus universalistischer Perspektive die Berufserfahrenen erhalten, die sich weiterbilden wollen bzw. aus funktionalistischer Perspektive diejenigen, die sich auch durch strukturellen Wandel des Wirtschaftssystems weiterbilden müssen, um konkurrenzfähig auf dem Arbeitsmarkt zu bleiben. Ein genaueres Bild der Zielgruppe wird nicht spezifiziert. Es sind Personen mit mehrjähriger Berufserfahrung ${ }^{150}$. Es wird deutlich, dass im funktionalistischen Diskursstrang die beruflich qualifizierten Individuen, welche sich weiterbilden, als Ressource für den Arbeitsmarkt betrachtet werden. Im Unterschied dazu steht im universalistischen Diskursstrang das Individuum als Person im Vordergrund, welches eine Chance erhält sich weiterzubilden und potenzielle vorherige Benachteiligungen zu kompensieren (Président 1989, MENSR 1997).

Beruflich Qualifizierte der Sekundarstufe II, die Berufsabiturient_innen, werden im Diskurs nicht als Zielgruppe für die Hochschule thematisiert. Ihre Aufgabe ist die Integration in den Arbeitsmarkt und nicht in die Hochschulen. Auch wenn bereits im ersten Zeitraum Berufsabiturient_innen an die Hochschulen gingen und mit dem Problem der systematischen Studienabbrüche zu kämpfen hatten (vgl. Laurent 1995), wird dies nicht öffentlich diskutiert. Zwar beschreibt Präsident Mitterand 1989, dass allen Abiturient_innen ein Studium ermöglicht werden soll, aber in der weiteren Rede werden auch hier berufliche Abiturient_innen nicht als Zielgruppe für die Hochschule näher erwähnt.

L'Etat doit s'organiser quoiqu'il en coûte pour que tous les bacheliers qui le demandent puissent être admis à poursuivre des études supérieures dans des conditions d'accueil et d'encadrement dignes de notre pays. (Président 1989) ${ }^{151}$

Beruflich Erfahrene dagegen sollen im Diskurs nicht nur den Zugang zur Hochschule erhalten, sondern auch die Möglichkeit bekommen, ihre beruflichen Erfahrungen validieren und damit anzuerkennen und anrechnen zu lassen. Anrechnung soll über das Verfahren VAP erfolgen (MENSR 1996a, 1997).

150 Das Validierungsverfahren VAP, das 1992 eingeführt wurde, kann erst nach 5 Jahren Berufserfahrung durchgeführt werden.

151 „Egal was es kostet, der Staat muss dafür sorgen, dass alle Abiturient_innen, die danach verlangen, die Möglichkeit erhalten, ihre Bildung mit dem Studium fortzusetzen unter Zugangsund Betreuungsbedingungen, die unserem Lande würdig sind.“ (Président 1989, Übersetzung $\mathrm{NB})$ 
Auf diese Weise werden explizit Kenntnisse und Kompetenzen, welche beruflich erworben wurden, als gleichwertig im Vergleich zu Kompetenzen die einerseits mit dem bac general oder techno und andererseits auch mit Kompetenzen, welche innerhalb der Hochschule erworben werden, anerkannt. Gleichwertigkeit zwischen beruflichen und hochschulisch erworbenen Fähigkeiten wird im Diskurs allerdings nur implizit thematisiert, dadurch dass explizit Validierung gefordert wird. Voraussetzung, dass eine derartige Validierung möglich ist, ist zum einen die Institutionalisierung von Validierungsprozeduren an den Hochschulen. Dies soll über die Vier-Jahresverträge sichergestellt werden (MENSR 1997).

Eine weitere Voraussetzung ist zum anderen die kohärente und lesbare Gestaltung von Studiengängen, welche eine Rückkehr in die Bildung über beruflicher Erfahrungen ermöglicht (MENJS 1988b).

Fragen der Heterogenität der Studierenden werden zwar im ersten Zeitraum immer wieder diskutiert, jedoch nicht im Hinblick auf beruflich Qualifizierte. Als besonders zu betreuende Gruppe wird immer wieder die der technischen Abiturient_innen angesprochen (MENJS 1988c, Premier ministre 1995). Es wird konstatiert, dass die bestehenden Strukturen nicht an die differenten Voraussetzungen der Bildungsteilnehmer_innen angepasst sind und dass dies durch eine stärkere Betreuung Studierender, eine größere Flexibilität im Studium und Beratung sowie Lesbarkeit der Bildungsgänge verbessert werden könnte (MENJS 1988b, 1989b; MEN 1994; 1996a; 1997b Premier ministre 1995). An dieser Stelle soll nicht weiter auf den Umgang mit heterogenen Bedürfnissen eingegangen werden, da dieser Diskurs nicht in Bezug auf beruflich Qualifizierte geführt wird. Es soll jedoch deutlich werden, dass auch im ersten Zeitraum ein gewisses Bewusstsein für die Problematik heterogener Bedürfnisse bestand.

Ähnlich verhält es sich bei der Frage organisationaler Verbindungen. Auch dieser Aspekt wird nicht in Verbindung zur Durchlässigkeit mit beruflich Qualifizierten gebracht. Zwar wird insbesondere im ersten Zeitraum ein Ausbau beruflich orientierter Studiengänge, eine für Frankreich typische Verbindung von beruflicher und Hochschulbildung, vorangetrieben. Dieser Ausbau soll zu einer Diversifizierung der Bildungswege führen, welche den Abiturient_innengruppen, vor allem den technischen Abiturient_innen, zugute kommen soll (MESR 1988). Auch die Einführung von alternance, d.h. die Ermöglichung des Lernens in Betrieb und Ausbildungszentrum auf allen Bildungsstufen, wird nicht in Verbindung mit Durchlässigkeit thematisiert. Primäres Ziel dieser Entwicklungen ist eine stärkere Arbeitsmarktorientierung.

Insbesondere durch diese nicht Thematisierung beruflicher Abiturient_innen als Zielgruppe wird jedoch die gesellschaftliche Bewertung des beruflichen Abiturs als Arbeitsmarkteinstiegszertifikat verstärkt. Obwohl diese bereits zu Beginn der 1990er Jahre in die Hochschulen wechselten, werden sie nicht anerkannt und ihre bestehenden Probleme nicht öffentlich wahrgenommen und als relevant betrachtet. 
Eine Übersicht der Charakteristika des französischen Diskurses findet sich in Tabelle 24.

Tabelle 24 Struktur des Durchlässigkeitsdiskurses (1985-1998): Durchlässigkeit über Weiterbildung

\begin{tabular}{|c|c|c|}
\hline & universalistisch & funktionalistisch \\
\hline Zugang & Über Validierung von Berufserfahrung & \\
\hline $\begin{array}{l}\text { Zielgruppe } \\
\text { (konstruierte Rolle) }\end{array}$ & $\begin{array}{l}\text { Individuen mit Berufserfahrung zur in- } \\
\text { dividuellen Weiterentwicklung }\end{array}$ & $\begin{array}{l}\text { Individuum mit Berufserfahrung, das } \\
\text { die eigenen Fähigkeiten an verän- } \\
\text { derten Arbeitsmarktbedingungen an- } \\
\text { passt }\end{array}$ \\
\hline Subjektposition & Individuum als Person & Individuum als Humankapital \\
\hline Anrechnung wo & Im Hochschulsystem & Im Hochschulsystem \\
\hline Anrechnung was & \multicolumn{2}{|c|}{$\begin{array}{l}\text { Berufserfahrung sowohl aus formaler beruflicher Bildung als auch vor allem } \\
\text { aus Berufspraxis }\end{array}$} \\
\hline Anrechnung wie & \multicolumn{2}{|c|}{$\begin{array}{c}\text { Über neu institutionalisiertes Validierungsverfahren (VAP) und Einrichtung ko- } \\
\text { härenter und transparenter Studiengänge }\end{array}$} \\
\hline Heterogenität & \multicolumn{2}{|c|}{$\begin{array}{l}\text { Heterogenität von Studierenden wird anerkannt (aber nicht in Bezug auf be- } \\
\text { ruflich Qualifizierte der SekII) }\end{array}$} \\
\hline $\begin{array}{l}\text { Institutionelle } \\
\text { Verbindung }\end{array}$ & \multicolumn{2}{|c|}{$\begin{array}{l}\text { Ausbau beruflich orientierter Studiengänge (aber nicht in Bezug auf beruflich } \\
\text { Qualifizierte der Sek II, sondern vor allem für Abiturient_innen des techni- } \\
\text { schen Zweigs) }\end{array}$} \\
\hline Strategie & $\begin{array}{l}\text { Institutionalisierung des Rechts auf Bil- } \\
\text { dung zur individuellen Weiterentwick- } \\
\text { lung und Kompensation von möglichen } \\
\text { Benachteiligungen }\end{array}$ & $\begin{array}{l}\text { Weiterbildung um wirtschaftlichen } \\
\text { Entwicklungen zu entsprechen und } \\
\text { individuelle Risiken zu minimieren }\end{array}$ \\
\hline
\end{tabular}

Quelle: Darstellung der Verfasserin

Zusammenfassend lässt sich für den ersten Zeitraum sagen, dass in diesem Durchlässigkeit zwischen Berufs- und Hochschulbildung kaum eine Rolle spielt. Das berufliche Abitur wird nicht in seiner Funktion als Hochschulzugangsberechtigung anerkannt und berufliche Abiturient_innen werden durch die permanente nicht Thematisierung stigmatisiert und als nicht gleichberechtigt zu den Absolvent_innen der beiden anderen Abiturzweige konstruiert. Berufliche Erfahrung nach einer beruflichen Erstausbildung allerdings wird als adäquate Grundlage für ein Hochschulstudium und zum Teil auch einer Anrechnung von Kompetenzen anerkannt. An dieser Stelle wird die Bedeutung, welche Berufserfahrung im Unternehmen in Frankreich spielt (vgl. Maurice et al. 1986) deutlich und deren 
Gleichwertigkeit im Vergleich zu Hochschulzugangsberechtigungen des technischen und allgemein Abiturs sowie Studienleistungen durch die Einführung des Validierungsverfahren institutionalisiert. Im universalistischen Diskursstrang zielt dabei Weiterbildung über Validierung an den Hochschulen auf die Institutionalisierung des Rechts auf Bildung zur individuellen Weiterentwicklung und auf die Kompensation von möglichen vorherigen Benachteiligungen im Bildungsverlauf. Im funktionalistischen Diskursstrang kann die Strategie eher darin gesehen werden, Weiterbildung über Validierung beruflicher Erfahrung als Möglichkeit der Risikominimierung zu konstruieren: einmal gesellschaftlich zur Anpassung an einen Strukturwandel und den Fachkräftebedarf, aber auch individuell als Minimierung des persönlichen Arbeitslosigkeitsrisikos.

\subsection{Gesellschaftliche Wissensbestände zur Durchlässigkeit in Zeiten von Bologna und Kopenhagen (1999-2012)}

Wie hat sich der Durchlässigkeitsdiskurs nach 1998 geändert? Diese Frage soll in diesem Kapitelabschnitt im Zentrum stehen. Kam es zu einer Veränderung der Argumentationslogiken und des Verständnisses von Durchlässigkeit? Im Unterschied zum ersten Zeitraum konnten diesmal Dokumente aller ausgewählten Akteure der Bildungspolitik mit in die Analyse aufgenommen werden, wobei der Diskurs eindeutig durch die Bildungsministerien dominiert wird.

Zuerst werden die durchlässigkeitsrelevanten Debatten vorgestellt, um im Anschluss darauf aufbauend die Diskursstrukturen und schließlich die im Diskurs herauskristallisierten Strategien und Akteurspositionen zu analysieren.

\subsubsection{Schlaglichter der französischen Debatten zu Durchlässigkeit (1999-2012)}

Ähnlich wie die Jahre davor wurde auch nach 1998 im französischen Bildungssystem eine Vielzahl von Reformen durchgeführt, welche u.a. auch ein Großteil der Debatten und damit der Diskurse um Durchlässigkeit mitbestimmten. Wie auch im ersten Zeitraum lassen sich kaum deutliche Anfangs- und Endpunkte von durchlässigkeitsrelevanten Debatten feststellen, da die kontinuierlichen Reformen oder auch Events wie der jährliche Schulabschluss mit den zentralen Abiturprüfungen oder dem Semesterstart zu einem wiederholten Aufgreifen der relevanten Themen führten. Folgende große Debatten lassen sich feststellen: 
1) Debatte zur Aufwertung beruflicher Bildung

2) Debatte zur Modernisierung der Hochschulen, wobei das Problem des studentischen Misserfolgs immer einen großen Teil dieser Debatte ausmachte

3) Debatte zur Umsetzung der Bologna-Reformen und Einführung der licence pro

4) Debatte zum Ausbau der Möglichkeiten für lebenslanges Lernen

Auch wenn manche Debatten den gesamten Zeitraum präsent waren, so bedeutet dies nicht, dass sie immer gleich geführt wurden. Insbesondere durch Regierungswechsel wurden partiell unterschiedliche Schwerpunkten gesetzt. Wenn sich dadurch die Diskurse verändert haben, wird das in der Analyse kenntlich gemacht.

\section{Debatte zur Attraktivität der Berufsbildung}

Die Frage der Erhöhung der Attraktivität der Berufsbildung im französischen Bildungssystem und der Gesellschaft war der erste größere Kontext, in welchem Durchlässigkeit von beruflicher (Sekundar-)Bildung zur Hochschulbildung diskutiert wurde. Besonders stark geführt wurde die Debatte zum Jahrtausendwechsel und in den ersten Jahren danach. Dann war sie weniger virulent und aber erstarkte ab 2007 mit der Reform des Berufsabiturs und deren Konsequenzen erneut (vgl. Abbildung 10). Das Ziel der Erhöhung der Wertschätzung von beruflicher Bildung in Frankreich kann dabei als Konsens der bildungspolitischen Akteure angesehen werden.

Die 1999 vom Bildungsminister Allègre (1997-2000) proklamierte Charte de Lycée zielte in der Reform des französischen Schulwesens auf eine größere Gleichwertigkeit der drei Abiturzweige. Auch wenn das Berufsabitur weiterhin als beruflicher Abschluss, welcher primär auf den Arbeitsmarkt zielt, angesehen wurde, war ein Teil des Reformprogramms auch eine bessere Vorbereitung der beruflichen Abiturient_innen auf ein Studium in den BTS (MENRT 1999a).

Le baccalauréat professionnel a pour objectif principal l'insertion professionnelle directe de ses titulaires dans l'emploi à un niveau de qualification reconnu. [...] Aujourd'hui, un nombre significatif de bacheliers professionnels poursuivent cependant des études celles-ci devant donc pouvoir s'effectuer dans des conditions qui en facilitent la réussite, la préparation au BTS semble pouvoir être privilégiée pour construire une solution pédagogique appropriée. (MENRT 1999a) ${ }^{152}$

152 „Das Berufsabitur zielt primär auf die direkte berufliche Integration seiner Inhaber_innen in den Arbeitsmarkt auf einem rechtlich anerkannten Qualifikationsniveau. [...] Dennoch beginnt eine signifikante Gruppe der Berufsabiturient_innen heutzutage ein Studium. Dieses sollten sie daher auch unter Bedingungen, die einen Studienerfolg erleichtern, absolvieren können. Die Vorbereitung auf den BTS-Abschluss erscheint eine geeignete pädagogische Lösung bieten zu können.“ (MENRT 1999a, Übersetzung NB) 
Dies stellte einen klaren Unterschied zu den Debatten in den Jahren zuvor dar, da beruflich Qualifizierte als Zielgruppe des tertiären Bildungssystems erstmals anerkannt wurden.

Die Bedeutung der beruflichen Bildung wurde zu Zeiten der sozialistischen Regierung von Lionel Jospin auch darin deutlich, dass 2000 ein Minister für berufliche Bildung ernannt wurde. Vor dem Hintergrund einer sehr guten konjunkturellen Lage wurde vor allem auf einen Fachkräftemangel hingewiesen, dem nur entgegengewirkt werden kann, wenn man die Attraktivität der beruflichen Bildung erhöht. Ziel war daher insbesondere, mehr Jugendliche für die Berufsbildung zu begeistern. Der Minister für die berufliche Bildung, Melenchon, sah u.a. folgende Reforminitiativen als essenziell an (vgl. MDEP 2000b, g, 2002): Berufliche Bildung müsse lesbarer werden, d.h., die Diplome und deren Bedeutung sowie deren Zusammenhang mit weiteren beruflichen Abschlüssen sollten besser ersichtlich werden. Zudem dürfe berufliche Bildung keine Sackgasse sein. Durchlässigkeit müsse zu den andern Abiturzweigen, aber auch innerhalb der beruflichen Bildung sowie zu dem nächst höheren Bildungsniveau gewährleistet sein. Nur wenn diese Anschlussfähigkeit gegebensei, sei berufliche Bildung auch attraktiv genug und könne zur tatsächlichen Wahl der Jugendlichen werden, anstatt lediglich als schulische Negativauslese zu fungieren (vgl. MDEP 2000a, b, f).

Die sogenannte „Fluidität“, welche gefordert wird, schließt auch explizit die tertiäre Bildung mit ein; so solle es Wege vom CAP bis zur licence professionelle oder auch dem Ingenieursdiplom geben. Diese Aufstiegsmöglichkeiten im Bildungssystem müssen zudem für alle beruflichen Ausrichtungen möglich sein. Die Einführung des lycée des métiers, einem auf einen größeren Berufsbereich fokussierten Berufsgymnasium, in welchem die zu diesem Berufszweig gehörenden Abschlüsse erworben werden können, sollte ebendiesen Maßstäben entsprechen und die Attraktivität der Berufsbildung erhöhen. Ziel war es in einer Organisation technologische Abiturabschlüsse, berufliche Sekundarabschlüsse vom CAP bis zum Abitur sowie tertiäre berufliche Abschlüsse, BTS und licence pro zu vergeben (vgl. MDEP 2000d, e, g). Auf diese Weise wird zum einen das größere Ganze eines Berufsfeldes auch den Lernenden ersichtlich. Außerdem werden so auch bereits die Absolvent_innen der sekundären Abschlüsse über Möglichkeiten des Weiterlernens hautnah informiert (MDEP 2001b). Der Titel lycée des métiers sollte dabei nur ausgewählten Berufsschulen verliehen werden, welche einen ganzen Kanon an Eignungsvoraussetzungen erfüllen mussten. Lycée des métiers sollte ein Qualitätslabel mit hoher Anziehungskraft sein und auch die konservativen Nachfolgeregierungen sahen in dieser Organisationsform einen Hebel zur Erhöhung der Attraktivität der Berufsbildung (vgl. MJENR 2002b; MEN 2007a, d). Die Hauptkritik der Gewerkschaften an dem lycée des métiers wendete sich vornehmlich gegen die Umbenennung von Berufsschulen mit diesem Label. Es sollten nicht nur einige von den mit dem Label einhergehenden (auch finanziellen) Vergünstigungen profitieren, sondern alle Berufsschulen sollten möglichst auf dasselbe gute 
Qualitätsniveau gebracht werden, um nicht noch innerhalb der Berufsbildung ein Zweiklassensystem einzuführen (CGT 2009).

Nach der Einführung der lycées de métiers war das Gesetz loi d'orientation et de programme pour l'avenir de l'école (loi Fillon) von 2005 (Loi Fillon 2005) ein weiteres wichtiges Ereignis für die Frage der Durchlässigkeit von beruflicher Bildung und Hochschulbildung im Kontext der Debatte zur Attraktivität, auf welches ebenfalls in den nachfolgenden Debatten immer wieder hingewiesen wurde. Das loi Fillon bestätigte dabei erneut das Ziel, dass $80 \%$ eines Jahrgangs einen Abiturabschluss erreichen sollte. Es schrieb auch das von Lionel Jospin zum ersten Mal ausgegebene und mit dem Verweis auf die Lissabon-Stategie immer wieder bestätigte Ziel fest, dass $50 \%$ eines Jahrgangs mit einem Hochschulabschluss das Bildungssystem verlassen sollen. Dies wurde vor allem damit begründet, dass das 21. Jahrhundert das Jahrhundert der Wissensgesellschaften ist und lediglich eine ausreichende Ausbildung eine Voraussetzung ist, um die damit verbundenen Herausforderungen zu meistern (MENSR 2004b).

Neben dieser Zielmarge wurde auch als ein weiteres Ziel des französischen Schulwesens die Stärkung beruflicher Bildung ausgewiesen. Dies sollte dadurch gewährleistet werden, dass für jeden BEP-Abschluss überdies ein passender bac pro-Abschluss bestehen müsse und dass die besonders guten der bac pro-Absolvent_innen, auch einen Zugang zu einem passenden BTS Studiengang erhalten sollten. Diese festgeschriebenen Ziele wurden ebenfalls in den darauffolgenden Jahren immer wieder aufgegriffen und bestätigt.

Pour rendre les filières professionnelles plus attractives, le BEP sera clarifié avec un BEP par branche, l'accès au Bac Pro en 3 ans sera généralisé pour ceux qui le souhaitent, et les titulaires de Bac Pro mention B ou TB auront de plein droit accès à une place en STS, en vue d'un BTS correspondant à leur spécialité. (MENSR 2004b) ${ }^{153}$

Erneute Konjunktur erlangte das Thema der Gleichstellung beruflicher Bildung mit den technischen und allgemeinen Bildungswegen durch die Reform des Berufsabiturs unter dem konservativen Bildungsminister Darcos (2007-2009).

Durch diese Reform wird seit dem Beginn des Schuljahrs 2009 das Berufsabitur in Frankreich genauso wie die anderen beiden Abiturzweige in lediglich drei Jahren erworben, während die Schüler_innen in diesem Zweig zuvor insgesamt vier Jahre benötigten. Das offizielle Ziel der Reform besteht darin, das berufliche Abitur aufzuwerten und auf eine Stufe mit den anderen Abiturzweigen zu stellen, welche ebenfalls nach drei Jahren erworben werden. Das Berufsabitur soll das zentrale Diplom der beruflichen Ausbildung in der Sekundarstufe werden und dazu beitragen, das Ziel zu erreichen, dass $80 \%$ eines Jahrgangs ein Abitur erwerben (MEN 2008d, 2009). Mit dieser Reform wird das berufliche Diplom Brevet

153 „Um die beruflichen Abschlüsse attraktiver zu gestalten, wird das BEP auf einen BEP-Abschluss pro Berufsbranche klarifiziert, wird der Zugang zu einem Berufsabitur in drei Jahren für alle, die dies wünschen, verallgemeinert, und die Inhaber_innen eines Berufsabiturs mit guten oder sehr gutem Abschlusszeugnis werden ein volles Zugangsrecht für einen Platz an den STS in ihrer Fachrichtung erhalten.“ (MENSR 2004b, Übersetzung NB) 
d'etudes professionnelles (BEP), welches zuvor nach zwei Jahren im beruflichen Zyklus erworben werden konnte, quasi abgeschafft und ist nur noch ein intermediäres Zertifikat auf dem Weg zum Abitur (vgl. Bouyx 2009). Dieser Schritt wurde als notwendig angesehen, um diejenigen, welche bisher nach den 2 Jahren ihr BEP erhielten, aber nicht mehr motiviert genug waren, um noch weitere zwei Jahre zur Schule zu gehen, auch auf das Abitur-Niveau zu bringen (vgl. Interview MEDEF). Insofern soll es die Durchlässigkeit zum Abitur erhöhen. Die Frage der Durchlässigkeit zum Hochschulstudium wurde mit der Reform von Seiten der Regierung nicht angesprochen. Auch hier galt das Berufsabitur weiterhin als Abschluss für den Arbeitsmarkteinstieg. Der Durchstieg in den Hochschulbereich wurde weiterhin als Ausnahme angesehen. Die Reform des Berufsabiturs wurde insbesondere von Seiten der Gewerkschaften stark kritisiert. Die Hauptkritikpunkte bestanden dabei darin, dass vor allem das ,gesparte Jahr“" zur Haushaltssanierung jedoch nicht zur Gleichstellung der Bildungswege beitrage und die Demokratisierung im Schulsystem gefährde (CGT 2009).

L'arrière pensée de la contre réforme de la voie professionnelle mise en œuvre à partir de la rentrée 2008, ne réside pas seulement dans la recherche d'économie budgétaire, elle repose aussi sur des présupposés idéologiques et politiques qui accélèrent un processus déjà amorcé, en rupture avec une „ambition scolaire“ démocratique. (CGT 2009) $)^{154}$

Weiterhin kritisieren die Gewerkschaften, dass die Reform den Arbeitsmarkteinstieg erschwert und die zweijährige Erstausbildung entwertet, welche insbesondere von den Schichten mit einem niedrigen sozioökonomischen Status gemacht wird. Durch die mit der Reform einhergehende verringerte Vermittlung von Allgemeinbildung erhalten die Absolvent_innen eine geringere Chance zu Karriere und Weiterbildungsaufstiegen. Auch die Antwort auf die Frage, was die Reform für die Möglichkeit der Abiturient_innen aus diesem Abiturzweig, ein Hochschulstudium anzufangen und erfolgreich abzuschließen, bedeutet, wird von den Gewerkschaften stark negativ bewertet. Zudem verstärkt es die bestehende Perspektive auf die Abiturzweige: Während die anderen auf ein Hochschulstudium vorbereiten, dient das Berufsabitur allein dem Einstieg in den Arbeitsmarkt (CGT 2009, 2011).

La généralisation du bac professionnel 3 ans interpelle les positions respectives du bac professionnel et du bac technologique. Alors que le premier permet l'insertion, le second vise la poursuite d'étude. (CGT 2009) $)^{155}$

154 „Der Hintergedanke dieser rückwärtsgewandten Reform des beruflichen Bildungsweges, die mit dem Schuljahresbeginn 2008 umgesetzt wird, ist nicht nur die Suche nach budgetären Kürzungsmöglichkeiten, er ruht auch in politischen und ideologischen Setzungen, die den sich bereits abzeichnenden [Reform, NB] Prozess beschleunigen, welcher mit der demokratischen ,Schulambition“ bricht.“ (CGT 2009, Übersetzung NB)

155 „Die Verallgemeinerung [der Dauer] des Berufsabiturs in drei Jahren ruft die jeweiligen unterschiedlichen Funktionen des beruflichen und technischen Abiturs hervor. Während das 
Diese Trennung der Ausrichtung der unterschiedlichen Abiturzweige wurde auch in den Reforminitiativen für den allgemeinen und den technischen Abiturzweig deutlich, welche 2008 angestoßen wurden. Ein Hauptziel der Reform war eine bessere ,Orientierung' in das Hochschulwesen, so dass unnötige Studienabbrüche oder -wechsel vermieden werden können. Die Orientierung in den Hochschulbereich war bei den Reformen des bac pro hingegen kein Thema. ,Orientierung' ist anscheinend nicht notwendig. Und wenn die Absolvent_innen tatsächlich weiterstudieren wollen sollten, dann wären die BTS Studiengänge die einzige in den Debatten antizipierte Möglichkeit (MJENR 2002e; MESR 2011a, b; CPU 2011: $55)$.

\section{Debatte zur Modernisierung des Hochschulsystems}

Während im ersten Zeitraum das Problem des Studienmisserfolgs in den Debatten noch nicht auf die Gruppe der beruflich Qualifizierten bezogen wurde, änderte sich dies nach 1999. Insofern ist die zweite bedeutende Debatte, in deren Rahmen Durchlässigkeit von der sekundären Berufsbildung zur Hochschulbildung thematisiert wird, jene der Modernisierung der Hochschulbildung unter Verringerung der Misserfolgsraten Studierender. Studienmisserfolg wurde dabei als Studienabbruch, als stark verlängerte Studiendauer aber auch als unnötiger Studienwechsel gesehen. Während das Problem échec, des Studienmisserfolges, im Regierungsprogramm Plan U3M (Plan für die Universität im 3. Jahrtausend) nur ein Problem unter vielen darstellte und es wiederkehrend jährlich vor allem zu Semesterbeginn auf die Agenda kam, wurde die Lösung dieses Problems ab 2007 mit dem Gesetz Loi relative aux libertés et responsabilités des universités, (Loi LRU 2007) und dem dazugehörigen Plan Réussite en licence (Erfolg in der licence) eines der Hauptziele für den Hochschulbereich, welches von der Regierung angegangen wurde.

Neben der Erhöhung der Autonomie der Hochschulen schrieb das LRUGesetz auch die Verringerung der Misserfolgsquote sowie eine stärkere berufliche Ausrichtung des ersten Studienzyklus fest. Um das Problem des Studienmisserfolges anzugehen wurde daher auch das Aktionsprogramm „Erfolg in der licence“ ins Leben gerufen.

Im Dezember 2007 kündigte die Bildungsministerin Valerie Pécresse dieses Reformprogramm mit folgenden Zielen an (MESR 2007e):

- Das Halbieren der Studienabbruchquote des ersten Jahres der licence innerhalb der nächsten 5 Jahre

- Die licence soll ein nationales Diplom werden, welches sowohl für den Einstieg in den Arbeitsmarkt als auch für ein Anschlussstudium gut vorbereitet

erste den Arbeitsmarkteinstieg ermöglicht, zielt das zweite auf den Beginn eines Studiums ab.“ (CGT 2009, Übersetzung NB) 
- Erreichen des Zieles, dass $50 \%$ eines Jahrgangs das Qualifikationsniveau der licence erreichen

Für das Programm wurden für die Jahre 2008 bis 2012 insgesamt 730 Millionen Euro eingeplant. Dies entspricht einer Budgetsteigerung für die licence-Studiengänge um $43 \%$ (vgl. MESR 2007e).

Mithilfe von drei Strategien sollen diese Ziele erreicht werden (MESR 2007e). Der Inhalt der Curricula der licence an den Universitäten soll überarbeitet werden. Ziel ist es, im ersten Jahr grundlegende Techniken und Kompetenzen, die für ein Studium gebraucht werden, zu vermitteln, um dann anschließend nach Evaluierung des Leistungsstands im zweiten und dritten Jahr eine Studienspezialisierung vorzunehmen. Der zweite Schritt besteht darin, eine bessere Orientierung an die Universität und in der Universität zu gewährleisten. Die letzte Strategie besteht darin, insbesondere die Abiturient_innen der technischen und beruflichen Abiturzweige in die beruflich orientierten Kurzstudiengänge der IUT und STS umzuorientieren, um ihre Erfolgschancen zu vergrößern. So werden die Rektoren der IUT und STS angehalten, insbesondere diese Abiturient_innen aufzunehmen. Um ebendies zu erreichen, wird allen technischen Abiturient_innen, welche mit gut oder sehr gut bestehen, das Anrecht auf einen Platz der IUT zugesprochen. Ebenso ein Anrecht auf Zugang zu den STS haben Abiturient_innen der beruflichen und technischen Zweige, wenn sie ihr Abitur gut oder sehr gut bestanden haben. Im Rahmen dieses Programms wurde zudem die Einführung von Semestern und für die Studiengänge der STS die Einführung des Kreditpunktesystems beschlossen, um auf diesem Weg Übergänge zwischen den einzelnen ersten Studienzyklen an der Universität, den IUT und den STS zu gewährleisten (MESR 2007e).

Die bildungspolitischen Akteure begrüßten im Großen und Ganzen das Reformziel der Regierung, die Studierenden mit Studienproblemen im ersten Zyklus stärker zu unterstützen. Die Wirtschaftsakteure unterstrichen hier insbesondere auch die Bedeutung alternierender tertiärer Bildungsgänge als eine Lösung gegen „Studienmisserfolg“ (MEDEF 2011). Die Gewerkschaften betonten, dass mit den Reformen schließlich alle Absolvent_innen eines Abiturs, egal aus welchem Zweig, ein Anrecht haben, sich im tertiären Bildungssystem weiterzubilden. Dementsprechend sollten die Abiturient_innen erstens bereits im Schulsystem so gut ausgebildet werden, dass dies auch möglich ist und zweitens sollten die Bildungswege im Hochschulsystem so diversifiziert werden, dass für jeden ein passendes Angebot gefunden werden kann. Die Bedeutung der Studien- und Lebensbedingungen der Student_innen für den Studienerfolg werden gleichfalls hervorgehoben (FSU 2011).

Auch die Hochschulen begrüßten die Reforminitiativen des Gesetzes LRU 156 und des Aktionsprogrammes (CPU 2007a). Für die Universitäten, welche ihre

156 Das LRU-Gesetz wurde vielfach kritisiert. Jedoch weniger in Bezug auf die Frage der Durchlässigkeit. Aus diesem Grund sind die weiteren Konfliktlinien hier nicht weiter dargestellt. 
Studierenden nicht auswählen können, war es jedoch wichtig, dass sie nicht das ,Auffangbecken' werden von allen Student_innen, welche von den anderen Organisationen abgelehnt werden (CPU 2007a). So wurde von den Universitäten insbesondere die Einführung der sogenannten aktiven Orientierung mit dem Programm „Admission Post Bac" (APB), (Zulassung nach dem Abitur) begrüßt, da hier der Fokus auf die Studienorientierung bereits in den Schulen erhöht wird (CPU 2007a, ADIUT/UNPIUT 2012). Die technischen Hochschulen (IUT) unterstützen ebenfalls das Programm und sahen ihren personalisierten Umgang mit den Studierenden als ein Erfolgsmodell, welches auch an den Hochschulen eingeführt werden sollte. Negativ standen die IUT allerdings jedweder Form von staatlich geregelter Quotierung für die Aufnahme von Studierenden in ihre Organisationen gegenüber (ADIUT 2012). Die Selektion zusammen mit dem pädagogischen Konzept wird als Erfolgsrezept der IUTs dargestellt, da sich die IUTs mit einer wesentlich geringeren Misserfolgsquote auszeichnen.

Trotz der Abwehr von Quotenregelungen, welche die Unabhängigkeit der Hochschulorganisationen bei der Selektion einschränken, wurde 2013 eine solche nach dem Regierungswechsel von der sozialistischen Regierung mit dem Gesetz Loi relative à l'enseignement supérieur et à la recherche eingeführt. So ist geregelt, dass die besten Absolvent_innen des beruflichen und technischen Abiturs ein Anrecht auf Zugang in die selektiven Studiengänge der STS und IUT besitzen, wobei die zu installierenden Quoten in Abhängigkeit vom Fach und der Region festgelegt werden (vgl. Loi ESR 2013).

\section{Debatte zur Umsetzung der Bologna-Reformen und Einführung der licence professionnelle}

Während der Bologna-Prozess in den Hochschuldebatten in Frankreich seit Beginn des Prozesses eine Rolle spielte, so war er in den Debatten zu Durchlässigkeit vor allem wichtig wegen der durch ihn angestoßenen Einführung der licence professionnelle in Frankreich. Besonders virulent waren die Debatten in den ersten Jahren des neuen Jahrtausends. Mit der Einführung der licence pro war auch die Hoffnung verbunden, die Studierenden, die Studienprobleme im eher allgemeinbildend orientierten licence-Studium hatten, und keinen Master schaffen würden, in einen beruflich orientierten Abschluss umzuleiten und diese auf diese Weise auch mit einem berufsorientierenden Abschluss das Studium erfolgreich beenden zu lassen (Interviews MESR2, CPU3). Durch die licence professionnelle sind berufliche Abschlüsse vom Sekundär- bis Tertiärniveau auf allen Qualifikationsniveaus erreichbar (MDEP 2000a). Zudem wurde insbesondere auch eine stärkere Verbindung der beruflichen Sekundarschulen mit dem Universitätssystem gefordert. Licence pro sollten auch in Kooperation der STS mit den Universitäten vergeben werden. Auf diese Weise wäre ebenfalls die institutionelle Durchlässigkeit im Berufsbildungszweig erhöht (MDEP 2001b, 2002) 


\section{Debatte um lebenslanges Lernen}

Wie auch im ersten Untersuchungszeitraum stellte die Debatte um die Ermöglichung von Weiterbildung, dem sogenannten lebenslangen Lernen, einen weiteren Kontext dar, in dem Durchlässigkeit zwischen Berufs- und Hochschulbildung diskutiert wurde. Einen Höhepunkt nahm die Debatte nach dem europäischen Ratsbeschluss in Lissabon, in welchem die Bedeutung des lebenslangen Lernens für die „Wissensgesellschaften“ Europas herausgehoben wurde. So wurde in Frankreich das französische Modell einer nachhaltigen beruflichen Bildung als Modell für Europa gesehen, in dem im Gegensatz zu einer neoliberalen Version, eine hohes Ausgangsbildungsniveau die Voraussetzung für Weiterbildung auch für die Validierung weiterer beruflicher Kenntnisse bildet (MDEP 2001a).

Eine weiterreichende Umsetzung der europäischen Zielvorgabe der Ermöglichung des Lebenslangen Lernens wurde mit dem Loi de Modernisation (Loi Modern 2002) im Jahr 2002 vorgenommen. Das bestehende Verfahren des VAP wurde reformiert, so dass mit dem VAE anstatt nur Teile von Abschlüssen nun ganze Diplome über die Validierung beruflicher aber auch anderweitiger nicht formal im Bildungssystem also non-formal und informell erworbener Erfahrungen erworben werden können. Das bestehende Monopol des Bildungssystems für die Vermittlung der für Bildungszertifikate notwendigen Kompetenzen wurde auf diese Weise infrage gestellt. Mit dem VAE soll es zu einer stärkeren Anerkennung beruflich erworbener Kompetenzen kommen. Die Durchlässigkeit geht in diesem Fall soweit, dass der berufliche Kompetenzerwerb dem hochschulischen gleichgesetzt wird und mit der Verleihung eines Hochschuldiploms enden kann.

Auch nach der Reform waren die Frage von Weiterbildung und der Ausbau von Validierungsmöglichkeiten beruflicher Erfahrungen insbesondere auch an Hochschulen ein immer wiederkehrendes Thema. Es wurde mehrheitlich von den bildungspolitischen Akteuren begrüßt, wobei zu Beginn die Hochschulen die Möglichkeit der Validierung eher kritisch betrachteten (CPU 1999). Es wurde die Möglichkeit, dass viele Abschlüsse auf diese Weise zertifiziert werden können, infrage gestellt (CPU 2002). Im Verlauf des zweiten Untersuchungszeitraums aber unterstrichen auch die Hochschulen die Bedeutung vom lebenslangen Lernen an den Hochschulen und die Rolle, welche die Validierungsprozeduren dabei spielen, insbesondere für die Gruppe, die im Rahmen der Erstausbildung nicht studieren konnten (CPU 2008, 2009).

Während die Gewerkschaften die Validierung beruflicher Erfahrungen begrüßten (CGT 2005b, 2012), so forderten sie aber auch eine verstärkte Beteiligung von Gewerkschaften bei der Umsetzung der Validierung. Zudem wurde kritisiert, dass, auch wenn ein Recht auf Weiterbildung festgeschrieben ist, nicht alle Angestellten die gleichen Zugangsmöglichkeiten zu den Validierungsprozeduren haben. Dies wurde mit der Komplexität der Validierungsprozeduren begründet, welche bereits ein hohes Vorbildungsniveau erfordern (CGT 2012). 


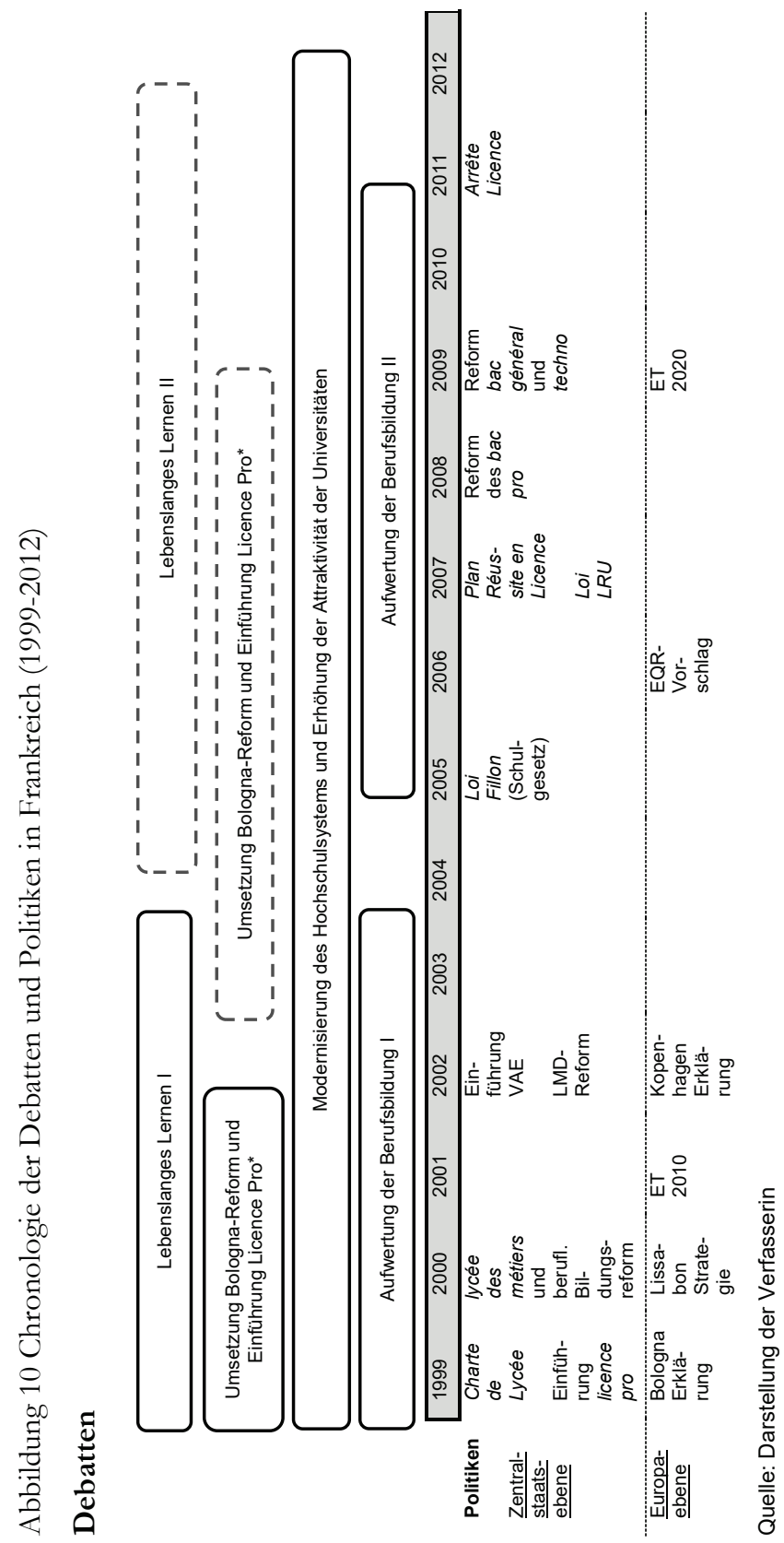


In Abbildung 10 werden die Debatten, Politiken und ferner die gleichzeitigen Reformen auf europäischer Ebene zusammenfassend dargestellt. Es wird deutlich, dass insbesondere die Frage der Modernisierung der Hochschulen und damit auch die des Studierendenmisserfolgs durchgängig thematisierte wurden. Ebenfalls wurde fast den ganzen Zeitraum die Frage der Attraktivität der beruflichen Bildung im Zusammenhang mit Durchlässigkeit debattiert. Die anderen beiden Debatten waren vor allem zu Beginn des neuen Jahrtausends stärker und wurden danach nur noch vereinzelt aufgegriffen, weshalb die Debattenbox in der Darstellung ebenfalls hellgrau gezeichnet wurde. Im Vergleich zum Zeitraum vor 1999 lässt sich insgesamt ein deutlicher Anstieg an Kontexten, in denen Durchlässigkeit zwischen beruflicher (Sekundar-)Bildung und Hochschulbildung debattiert wurde, konstatieren. Anzumerken ist, dass Durchlässigkeit beruflich Qualifizierter in die Hochschulbildung kein die französischen Bildungsdebatten bestimmendes Thema war und im Vergleich zu Deutschland von viel geringerer Relevanz. Anhand der Debatten sollte ebenfalls deutlich geworden sein, dass es vor allem Gesetzesinitiativen sind, die zum Auslöser von Durchlässigkeitsdebatten werden. So wird auch im zweiten Zeitraum die herausstechende Bedeutung der Bildungsministerien als dominierende Akteure unterstrichen.

\subsubsection{Französischer Diskurs 1999-2012: Durchlässigkeit im Spannungsverhältnis universalistischer und funktionalistischer Vorstellungen}

Anhand der Debatten wurde deutlich, dass Durchlässigkeit zwischen beruflicher Sekundar- und Hochschulbildung in der Zeit nach 1999 ein Thema der französischen Bildungspolitik geworden ist. Auch wenn die Notwenigkeit gesehen wird, Strukturen zu schaffen, welche beruflich Qualifizierten den Zugang und Abschluss eines Studiums ermöglichen sollen, so wird doch auch über den gesamten zweiten Zeitraum deutlich, dass Absolvent_innen eines beruflichen Abiturs primär für den direkten Übergang in den Arbeitsmarkt ausgebildet werden. Dies hat sich im Vergleich zum ersten Zeitraum nicht verändert. Geändert hat sich allerdings, dass Berufsabiturient_innen direkt und nicht allein mit Berufserfahrung über den Weg der Weiterbildung an die Hochschule gelangen können sollen. Insgesamt ist der untersuchte Diskurs zur Durchlässigkeit in Frankreich im Gegensatz zu Deutschland jedoch kein die Bildungsdebatten dominierender. Insbesondere die Frage des erfolgreichen Studierens wird primär auf die anderen beiden Abiturzweige bezogen. Dies sollte bei der Betrachtung des französischen Durchlässigkeitsdiskurses immer mitgedacht werden, da sich die Beschreibung des bildungspolitischen Diskurses auf Durchlässigkeit zwischen Berufs- und Hochschulbildung fokussiert ist. 
Wie auch im ersten Zeitraum stehen sich anders als in Deutschland nicht zwei konfligierende Diskurse gegenüber, sondern es kann ein hegemonialer Diskurs rekonstruiert werden, in welchem je nach Argumentationsmuster Reformen von Durchlässigkeit zu divergenten Bedingungen befürwortet werden. Nachfolgend soll der Diskurs und seine Entwicklung genauer vorgestellt werden: Dies erfolgt zuerst anhand der Argumentationslogiken, anschließend steht die Diskursstruktur, in welcher die Vorstellung zu Durchlässigkeit und dessen, was dazugehört, im Mittelpunkt und schließlich werden die rekonstruierten Akteurspositionen aufgezeigt.

\section{Argumentationslogiken des französischen Diskurses 1999-2012}

Auch wenn das Berufsabitur primär als Arbeitsmarkteinstiegszertifikat gesehen wird, wird doch in Frankreich darüber diskutiert, wie beruflich Qualifizierte mit Berufsabitur Zugang zum und einen erfolgreichen Abschluss im Hochschulsystem erhalten sollten. Wie wird diese Position im Diskurs zu Durchlässigkeit begründet. Wieso wird Durchlässigkeit von beruflicher Sekundarbildung zum Hochschulsystem problematisiert? Antworten auf diese Fragen spiegeln sich in den zwei Argumentationslogiken, der universalistischen und der funktionalistischen Logik, welche jeweils einen der beiden Diskursstränge des hegemonialen französischen Durchlässigkeitsdiskurses bestimmen, wider. Nachfolgend werden die Argumentationsmuster nacheinander beschrieben und anschließend in Tabelle 25 aggregiert.

\section{Universalistische Perspektive: Durchlässigkeit als Recht auf Bildung}

Wenn in Frankreich über Durchlässigkeit diskutiert wird, dann zumeist aus universalistischer Perspektive. Doch wie wird dann für Durchlässigkeit argumentiert?

Eine bedeutende Argumentationsfigur ist die sogenannte Demokratisierung der Hochschulbildung (vgl. MDEP 2000f, 2001b; MEN 2008c; MESR 2010c; CPU 2002; FSU 2011). Die Metapher der Demokratisierung impliziert einerseits das Ziel der Erhöhung des Bildungsniveaus allgemein, aber auch eine „chancengerechte Teilhabe“ (Brauns 1998) aller Bevölkerungsgruppen an der (Hochschul-)Bildung. Ein Recht auf Bildung. Dieses Ziel spiegelt sich im Diskurs vor allem in dem Bezug auf zwei Institutionen wieder: dem französischen Abitur, dem baccalauréat, und dem „selektionsfreien“ Zugang zur Universität.

Das baccalauréat, welches gleichzeitig als erster tertiärer Abschluss gilt, dient dabei als Symbol dieses Rechts auf Bildung. Im Diskurs wird immer wieder die Position deutlich, dass jeder und jede, welcher oder welche ein Abitur hat, auch studieren können soll, unabhängig von weiteren Eigenschaften der Person. Mit einem Abitur sind somit für alle die gleichen Rechte verbunden. Das baccalauréat als Berechtigung zur Hochschule vereint damit die republikanischen Ideale Frankreichs „Freiheit, Gleichheit, Brüderlichkeit“ sowie der Meritokratie (MENSR 
2004a). Alle mit Abitur haben die gleichen Rechte, alle mit Abitur sollen die Freiheit haben, sich an den Hochschulen weiterzubilden und haben durch Bildung die Freiheit, sich eine eigene Meinung zu bilden und als Persönlichkeit zu entwickeln, alle mit Abitur wurden in das französische System als Staatsbürger sozialisiert und alle mit Abitur haben sich dieses ebenfalls mit schwerer Arbeit verdient.

Il est à la fois un diplôme sanctionnant la fin des études secondaires et le premier diplôme d'études supérieures. Instrument de l'égalité républicaine, le baccalauréat est aussi l'expression d'une forme d'excellence fondée sur le travail et le mérite. (MENSR $2004 a)^{157}$

Die Argumentationsfigur in Bezug auf das berufliche Abitur ist die Folgende: Wenn es formal ein Abitur ist, dann sollen auch die beruflichen Absolvent_innen alle dem Abitur zugesprochenen Rechte genießen (MENRT 1999a). Eine Beschränkung dieses Rechts würde die Bedeutung des baccalauréat infrage stellen. Die Gewerkschaften und teilweise auch die sozialistischen Regierungen gehen noch einen Schritt weiter und unterstreichen, dass mit dem bac nicht nur allein das Recht, sondern auch die Befähigung für ein Hochschulstudium vermittelt werden soll. Mit dem Konzept der nachhaltigen Verberuflichung betont die sozialistischen Regierung, dass lediglich auf Basis einer breiten Allgemeinbildung eine berufliche Ausbildung aufgebaut werden soll, denn nur so sei ein lebenslanges Lernen zu gewährleisten (MDEP 2000g). Auch Gewerkschaften betonen die Rolle der Allgemeinbildung als Grundlage jeder weiteren Bildung (CGT 2005b). Insofern muss ein Absolvent tatsächlich in die Lage versetzt werden, erfolgreich studieren zu können (CGT 2005b).

Auf der Basis der bestehenden Zugangsvoraussetzung des Abiturs wird zudem aus Gründen der Chancengleichheit und des Ziels der Demokratisierung der selektionsfreie Zugang an den Universitäten als beizubehaltende Regelung unterstrichen (MESR 2007 a, b). Dies solle auch gelten, obwohl die Trägerschaft des Abiturs immer heterogener wird und damit sich auch die Anforderungen an die Universität verändert haben.

L'égalité des chances, elle se joue d'abord à l'université. Alors, j'ai déjà eu l'occasion de le dire, la sélection à l'entrée de l'université n'est pas compatible avec un projet de formation démocratique. (MESR 2007c) ${ }^{158}$

Il est donc utile de rappeler que ce sont les universités qui permettent aujourd'hui à un maximum de jeunes d'accéder à un niveau de connaissance et de compétences et à un statut social que n'avaient pas leurs parents. En accueillant tous les étudiants qui souhaitent s'y former, sans sélection et pour des droits d'inscription modestes, les

157 „Es ist ein Diplom, das gleichzeitig das Ende der Sekundarbildung als auch das erste Diplom der Tertiärbildung repräsentiert. Als Instrument der republikanischen Gleichheit ist das Abitur auch Ausdruck von Exzellenz, die auf Arbeit und Leistung basiert.“ (MENSR 2004a, Übersetzung NB)

158 „Chancengleichheit spielt sich zuallererst an den Universitäten ab. Also ich hatte es ja schon gesagt, die Selektion beim Zugang zur Universität ist nicht kompatibel mit einem demokratischen Bildungsprojekt.“ (MESR 2007c, Übersetzung NB) 
universités françaises relèvent largement le défi de la démocratisation de l'enseignement supérieur depuis 40 ans. (CPU 2009) ${ }^{159}$

Bildung und damit auch Hochschulbildung gilt als ein öffentliches Gut, welches von Staatsseite für jeden Bürger und jede Bürgerin gewährleistet werden muss. Hier wird immer wieder die Bedeutung der Universitäten im Gegensatz zu den stärker beruflich orientierten Hochschulbildungsgängen, welche stärker selektiv sind, unterstrichen (MESR 2007c; CPU 2009).

Andererseits wird aber auch besonders deren Rolle bei der Demokratisierung des tertiären Sektors betont. Gerade die beruflichen Bildungsgänge wurden ausgebaut, um die größer werdende Zahl der Studierendenschaft aufzunehmen (ADIUT 2012; MESR 2008a). Dies sollen die Studiengänge immer noch leisten. Insofern sollen die beruflich Qualifizierten auch insbesondere in diese Studiengänge und nicht in die Universitäten einmünden. An dieser Stelle erfolgt im Diskurs wiederum eine Einschränkung des Universalismus. Die beruflichen Abiturient_innen sollen zwar in den Hochschulbereich kommen, aber dann eher in die beruflichen Bildungsgänge und weniger in die Hochschule, wobei ihnen das Recht auf letzteres durch den selektionsfreien Zugang nicht verwehrt werden kann und soll.

Demokratisierung über Bildung soll nicht allein durch den Zugang zur Hochschulbildung durch eine gewachsene Zahl an Abiturient_innen erfolgen, sondern auch über die Weiterbildung und den Zugang beruflich Qualifizierter mit Berufserfahrung (MEN 2001a). Auch auf Weiterbildung hat jeder französische Bürger ein Anrecht und insbesondere beruflich Qualifizierte mit Berufserfahrung sollen auf dem Weg der Weiterbildung an die Hochschulen kommen bzw. Hochschulabschlüsse erhalten. Dabei wird wie im ersten Zeitraum auch die Realisierung einer zweiten Chance auf Hochschulbildung betont.

Pour tous ceux qui n'ont pu en formation initiale acquérir un diplôme de l'enseignement supérieur, l'Université a fait de la formation tout au long de la vie l'une de ses priorités. Elle est en effet un acteur de plus en important dans la formation continue. (CPU 2009: 5) ${ }^{160}$

Aus Gesellschaftsperspektive wird Durchlässigkeit für beruflich Qualifizierte und damit die Verringerung ihres systematischen Misserfolgs an den Hochschulen als

159 „Es ist nützlich daran zu erinnern, dass es die Universitäten sind, die es heute einer Mehrheit der Jugendlichen ermöglichen, ein Kompetenzniveau und sozialen Status zu erreichen, den ihre Eltern nicht hatten. Indem alle Studierenden, die den Wunsch haben sich zu bilden, aufgenommen werden, ohne Selektion und mit nur geringen Einschreibegebühren, sind es die französischen Universitäten, die zu großen Teilen die Herausforderung der Demokratisierung seit über 40 Jahren annehmen.“ (CPU 2009, Übersetzung NB)

160 „Die Universität macht für alle, die während ihrer Erstausbildung kein Hochschuldiplom erhalten konnten, lebenslanges Lernen zu einer ihrer Prioritäten. Tatsächlich ist sie ein immer wichtiger werdender Akteur für die Ermöglichung lebenslangen Lernens.“ (CPU 2009: 5, Übersetzung NB) 
eine Frage der sozialen Kohäsion der Gesellschaft dargestellt. Gerade die beruflich Qualifizierten, welche oft aus Schichten mit geringen sozioökonomischen Ressourcen kommen, dürfen nicht ausgeschlossen werden. Die Frage von Durchlässigkeit wird damit auch zur Frage der Verringerung sozialer Ungleichheit (MESR 2011c; CPU 2010a).

\begin{abstract}
Parce qu'un système éducatif qui n'ouvre pas de perspectives aux jeunes issus de milieux populaires est un système éducatif qui se sclérose, parce que l'inégalité scolaire menace directement la cohésion sociale, parce qu'enfin tous les talents ne se révèlent pas à 16 ou 18 ans, nous avons le devoir d'offrir à tous les étudiants, non pas la garantie, mais les conditions véritables d'une poursuite d'études et d'une insertion professionnelle réussie. (MESR 2011b) ${ }^{161}$
\end{abstract}

Aber nicht nur für die Gesellschaft als Ganzes, sondern auch für das einzelne Individuum ist Durchlässigkeit relevant. Jeder solle eine so hohe Bildung wie möglich erhalten - dies sei durch das Recht auf Bildung zu gewährleisten. Das gilt sowohl für die Erstausbildung als auch für die Weiterbildung (MDEP 2000g; MESR 2010a; CGT 2012). Durch die von der Politik z.B. im loi Fillon ausgegebenen Benchmarks wird auch an dem Ziel des hohen nationalen Bildungsniveaus, dass mindestens $80 \%$ eines Jahrgangs ein Abitur und $50 \%$ einen Hochschulabschluss erreichen sollen, festgehalten (MDEP 2001b; MEN 2007a). Bildung und insbesondere auch Hochschulbildung wird dabei als wichtig angesehen für die Persönlichkeitsentwicklung, die Möglichkeit der Emanzipation des Individuums, der Entwicklung der Kritikfähigkeit sowie der demokratischen Bürgererziehung.

L'éducation et la formation tout au long de la vie doivent permettre aux jeunes et plus largement à tous les individus de répondre à leurs besoins de formations, leur permettre de devenir des citoyens maitrisant leur propre devenir, des acteurs décisifs des transformations sociales à opérer, capables de peser collectivement sur les évolutions économiques, technologiques, sociales et culturelles, capables de faire valoir des droits nouveaux, d'intervenir sur le contenu et l'organisation du travail, de faire reconnaittre et évoluer leurs qualifications. (FSU 2002) ${ }^{162}$

161 „Da ein Bildungssystem verkalkt, welches nicht auch den Jugendlichen aus unteren sozialen Schichten Bildungsperspektiven bietet, da schulische Ungleichheit konkret den sozialen gesellschaftlichen Zusammenhalt bedroht, da sich nicht alle Talente mit 16 oder 18 Jahren zeigen, haben wir die Aufgabe allen Studierenden nicht nur zu garantieren, dass sie ein Studium aufnehmen können und einen erfolgreichen Einstieg in den Arbeitsmarkt absolvieren, sondern auch die Aufgabe die dafür notwendigen Bedingungen zu schaffen." (MESR 2011b, Übersetzung NB)

162 „Aus- und Weiterbildung muss Jugendlichen und allgemein allen Individuen ermöglichen, ihren Qualifikationsbedarf zu decken, muss ihnen erlauben, Bürger zu werden, die ihre Zukunft selbst in die Hand nehmen, die als entscheidende Akteure soziale Transformationen betreiben, die in der Lage sind, gemeinschaftlich in ökonomische, technologische, soziale und kulturelle Entwicklungen einzugreifen, neue Rechte geltend zu machen, Inhalte und Organisation der Arbeit zu beeinflussen und Anerkennung für ihre Qualifikationen einzufordern und diese weiterzuentwickeln.“ (FSU 2002, Übersetzung NB) 
Schließlich solle durch Durchlässigkeit, im Sinne der Ermöglichung eines erfolgreichen Studienabschlusses für beruflich Qualifizierte, auch individuelle soziale Mobilität gewährleistet werden (MESR 2011b). Die Metapher von Hochschulbildung als ,sozialer Fahrstuhl' wird dabei immer wieder benutzt, wobei vermehrt darauf hingewiesen wird, dass der Fahrstuhl nicht mehr in dem Maße funktioniere wie früher (CPU 2007c; MESR 2007b). Dieses Problem solle behoben werden.

Or l'université est aujourd'hui le principal ascenseur social de ce pays et je n'ai pas l'intention d'en faire descendre les étudiants talentueux à qui l'on n'aurait pas su donner le temps de la réussite ni les moyens pour la conquérir. (MESR 2007b) ${ }^{163}$

Mit der Metapher des, sozialen Fahrstuhls' wird durch die Hochschulen jedoch ebenfalls deutlich gemacht, dass nicht alle Studierenden auf das höchste Niveau kommen können, sondern auch mit den Abschlüssen der kürzeren Studiengänge, vor allem auf licence-Niveau, das französische Hochschulsystem verlassen sollen (CPU 2011).

Ein dritter Argumentationsstrang bezieht sich auf das Problem der geringen Wertschätzung beruflicher Bildung. Durchlässigkeit wird dann als ein Weg angesehen, die Wertschätzung zu erhöhen und eine Gleichwertigkeit der Bildungswege in dem gesellschaftlichen Verständnis des Bildungssystems zu verankern (MENRT 1999a).Berufliche Bildung solle nicht mehr nur der Bildungsweg für die schwachen Schüler_innen sein, der Weg der Reintegration von Problemschüler_innen in das Bildungssystem, sondern auch eine echte Wahlmöglichkeit mit Zukunftsperspektive (MDEP 2001b). Im Diskurs um Gleichwertigkeit wird dabei vor allem auch auf die unterschiedliche Pädagogik hingewiesen, die in Frankreich nicht anerkannt sei (MENRT 1999d). Im Gegensatz zum theorielastigen deduktiven Pädagogikstil der Allgemeinbildung sei berufliche Bildung vor allem praxisorientiert und durch einen eher induktiven Lern- und Lehrstil gekennzeichnet, welcher einigen Menschen einfach besser liegt. In Frankreich werde nun aber vor allem der erste Bildungsstil, das Abstrakte, besonders hoch geschätzt. Dies müsse sich ändern (MDEP 2001c).

Obwohl einerseits auf die Gleichwertigkeit von Berufsbildung und anderen Bildungsgängen hingewiesen wird, welche nur entsteht, wenn berufliche Bildung nicht mehr als ,Auffangbecken' für schwache Schüler_innen gesehen wird (MDEP 2001b), wird andererseits im Diskurs selbst aber auch immer wieder Berufsbildung als der Weg für schwächere Schüler_innen oder Studierende konstruiert. Auch im Hochschulwesen sollen die Studierenden mit Problemen in berufliche Zweige wechseln, um dort einen Abschluss zu erreichen (MEN 2000b; MESR 2011a). Das Bild von Berufsbildung, welches der Bildungsweg der „nicht so Erfolgreichen" sei, wird somit kontinuierlich festgeschrieben. Letztlich bleibt

163 „Nun nimmt hauptsächlich die Universität die Aufgabe des sozialen Fahrstuhls in diesem Land wahr und ich habe nicht die Absicht, talentierte Studierende, denen wir nicht ausreichend Zeit und Mittel für den Erfolg geben konnten, darin wieder absteigen zu lassen.“ (MESR 2007b, Übersetzung NB) 
somit auch eine Trennung zwischen den eher allgemeinbildend akademischen und den stärker beruflichen Bildungsgängen erhalten, auch wenn sämtliche Studiengänge an den Hochschulen stärker auch auf Berufe vorbereiten sollen.

Zusammenfassend ist aus universalistischer Perspektive eine mangelnde Durchlässigkeit problematisch sowohl aus gesellschaftlicher als auch aus individueller Perspektive: Gesellschaftlich bedeutet mangelnde Durchlässigkeit - zum einen fehlende Demokratisierung und damit auch fehlende Chancengleichheit. Und zum anderen schwächt eine mangelnde Durchlässigkeit für berufliche Abiturient_innen die Bedeutung des Abiturs im französischen Bildungssystem. Fehlende Durchlässigkeit gefährdet die soziale Kohäsion und vergrößert soziale Ungleichheit bzw. führt zu gesteigerter Diskriminierung beruflich Qualifizierter im französischen Bildungssystem. Auch wird gesellschaftlich die Wertschätzung von Berufsbildung gefährdet, da berufliche Bildung dann nicht die vollen Bildungsund Entfaltungsmöglichkeiten bietet, wie die technologische und allgemeine Bildung. Auf individueller Ebene bedeutet mangelnde Durchlässigkeit für beruflich Qualifizierte die Einschränkung des Rechts auf Bildung, verringerte Möglichkeiten der Persönlichkeitsentwicklung sowie eingeschränkte Möglichkeiten der sozialen individuellen Mobilität.

\section{Funktionalistische Perspektive: Durchlässigkeit als funktionale Notwendigkeit für eine berufliche Leistungselite}

Im zweiten Diskursstrang wird Durchlässigkeit von Berufs- und Hochschulbildung als funktionales Erfordernis einer modernen europäischen Gesellschaft im 21. Jahrhundert konstruiert. So bestehe eine wirtschaftliche Notwendigkeit der Höherqualifizierung (MENRT 1999d, CGT 2005b). Die festgelegten Zielvorgaben, dass $80 \%$ eines Jahrgangs mindestens ein Abitur und $50 \%$ einen Hochschulabschluss erreichen sollen, werden so vor allem mit dem sich vollziehenden strukturellen Wandel begründet (MEN 2008b). Die gegenwärtige Zeit wird als ein Zeitalter der Wissensökonomien, in dem sich die Technologien und auch der Wissensbestand permanent ändern, beschrieben. Eine gute Ausbildung der Bevölkerung sei demgemäß eine Voraussetzung, um Wirtschaftswachstum zu sichern und so im internationalen Wettbewerb zu bestehen. Dieser Wettbewerb werde durch die Globalisierung noch verstärkt, da auch die Zahl der Wettbewerber gestiegen ist. Bildung und insbesondere Hochschulbildung gelten als der wichtigste Weg der Sicherung des Wohlstands und der Wettbewerbsfähigkeit der Nation. In diesem Zusammenhang wird auch immer wieder auf die 2000 vom europäischen Rat gefasste Lissabon-Erklärung hingewiesen, denn auch dort wurde Bildung als der Schlüssel für die Stärkung der Wirtschaften Europas angesehen (vgl. MDEP 2000c, e; MESR 2007b; ADIUT 2012). Insbesondere im Kontext der Weiterbildung, der Ermöglichung des lebenslangen Lernens, werden diese auf die Lissabon-Strategie bezogenen Argumentationsfiguren herangezogen.

Mais il faut aussi répondre à un besoin de requalification permanente des salariés en activité pour tenir compte de l'évolution accélérée des techniques de la production et 
des services. Bien sûr, il s'agit là de la protection et du développement du niveau de performance de nos économies. (MDEP 2000c) ${ }^{164}$

La France a besoin de diplômés nombreux pour élever son niveau global de qualification et participer à la bataille mondiale de la connaissance. (MESR 2008d) ${ }^{165}$

Durchlässigkeit zur Hochschulbildung wird ebenfalls als eine Möglichkeit betrachtet, Berufsbildung attraktiver zu machen, um so den Fachkräftebedarf der Wirtschaft an beruflich Qualifizierten zu befriedigen (MDEP 2000b). Wenn Berufsbildung nicht mehr als Sackgasse wahrgenommen wird, da sie Aufstiegschancen in das Hochschulsystem bietet, werden sich mehr Individuen für einen beruflichen Bildungsweg interessieren (MDEP 2000a). Im Diskurs sind die Aufstiegsmöglichkeiten vor allem in der Sicherung von Plätzen für berufliche Abiturient_innen in den beruflichen Kurzstudiengängen an den STS vorgesehen (vgl. MENRT 1999a; MDEP 2000a; MEN 2007d; MESR 2010a, 2012a; CPU 2007a). Durchlässigkeit kann hier als Weg der Lenkung des Bildungsverhaltens von Schüler_innen in die berufliche Bildung gesehen werden - durch die Zusicherung von Studienplätzen. Der Argumentationsstrang zur Förderung der Attraktivität von Berufsbildung war vor allem in den Jahren 1999-2003 stark ausgeprägt. Der Hintergrund, welcher ebenfalls in den Diskursen immer wieder deutlich wurde, war die gute konjunkturelle Lage in Frankreich.

Durchlässigkeit wurde jedoch nicht nur als Mittel zur Erhöhung der Attraktivität der beruflichen Bildung, sondern auch der Hochschulbildung insbesondere des universitären ersten Zyklus, der licence, angesehen. An dieser Stelle steht weniger die Sicherung des Zugangs beruflich Qualifizierter im Fokus als Durchlässigkeit im Sinne der Ermöglichung eines Studienerfolges und damit der Verhinderung von Studienabbrüchen und -wechseln. Eine Verringerung des systematisch an den Universitäten stattfindenden Studienmisserfolgs würde zu einem anderen Image der licence führen und damit wieder zu einer größeren Attraktivität (MESR 2011a, c). Andererseits solle eine erhöhte Attraktivität auch zu einer anderen Zusammensetzung der Studierendenschaft führen. Es sollen zwar möglichst alle Studierenden ihr Studium schaffen, aber die licence soll insbesondere attraktiver werden für die Absolvent_innen des allgemeinen Abiturzweigs. Ein Studium im ersten Zyklus an der Universität soll nicht mehr lediglich eine Notlösung für diejenigen darstellen, die in den prestigereicheren selektiven Studiengängen nicht genommen werden (vgl. MJENR 2002e; MEN 2000b; CPU 2007a). Die Stagnation der Studierendenzahlen an den Universitäten gegenüber den technischen Kurzstudiengängen wurde dabei als Zeichen dieser mangelnden Attraktivität interpretiert.

164 „Um auf den beschleunigten technischen Wandel im Produktions- und Dienstleistungsbereich zu reagieren, ist es wichtig, den permanenten Bedarf der Umqualifizierung der betrieblichen Angestellten zu berücksichtigen.“ (MDEP 2000c, Übersetzung NB)

165 „Frankreich braucht eine Vielzahl diplomierter Absolvent_innen, um das gesammte Qualifikationsniveau [der Bevölkerung, NB] anzuheben und im weltweiten Kampf um das Wissen teilzunehmen.“ (MESR 2008d, Übersetzung NB) 
Auch aus internationaler Perspektive sei es notwendig, die französischen Universitäten wieder wettbewerbsfähiger zu machen (MESR 2007b). Auch hier wird eine erhöhte Studienerfolgsquote als relevanter Lösungsansatz anerkannt.

In der Argumentation um die Erhöhung der Attraktivität der Universitätsbildung wird deutlich, dass zwar einerseits der Studienerfolg aller (auch die beruflichen Abiturient_innen werden dabei angesprochen) angestrebt wird. Andererseits soll aber speziell eine bestimmte Gruppe an die Hochschule gezogen werden, die zukünftigen Studierenden mit einem allgemeinen Abitur. Beruflich Qualifizierte sollten sowieso eher in den technischen Kurzstudiengängen unterkommen. Dies würde einfacher sein, wenn die Konkurrenz mit den Absolvent_innen des allgemeinen Abiturzweigs nicht so hoch wäre (vgl. CPU 2011; MENSR 2006a; MEN 2007d; MESR 2011a)

Aber auch die funktionale Bedeutung von Durchlässigkeit aus Individualperspektive wird betrachtet. Ein Studium bzw. die Möglichkeit des Studienabschlusses biete einen größeren Schutz vor Arbeitslosigkeit, sie trage damit dem Erhalt der Beschäftigungsfähigkeit bei und ermögliche einen effizienten Karriere- und Bildungsverlauf (MDEP 2000g).

Tabelle 25 Argumentationslogiken des französischen Diskurses (1999-2012)

\section{Reformorientierter Diskurs}

\section{universalistisch}

funktionalistisch

\section{Gesellschaft:}

Umsetzung republikanischer Ideale: Freiheit,

Gleichheit, Brüderlichkeit

Chancengleichheit, meritokratisches Ideal

(Hochschul-)Bildung als öffentliches Gut

Demokratisierung der Hochschulbildung

Keine Selektion beim Universitätszugang

Soziale Kohäsion der Gesellschaft, Verringerung sozialer Ungleichheit/Diskriminierung

Gleichwertigkeit beruflicher Bildung

Individuum:

Erreichen eines möglichst hohen Bildungsniveaus

Recht auf (Weiter-)Bildung zur Persönlichkeitsentwicklung, demokratischen Bürgererziehung, und Emanzipation

Ermöglichung sozialer Mobilität (Hochschulbildung als sozialer Fahrstuhl)

\section{Gesellschaft:}

Wirtschaftliche Notwendigkeit: Bedarf der Höherqualifizierung durch strukturellen Wandel Wissensökonomien

Wettbewerb durch Globalisierung

Attraktivität der Berufsbildung: Arbeitskräftebedarf in der beruflichen Bildung

Attraktivität der Universitätsbildung (Stagnation der Studierendenzahlen im Vergleich zu technologischen Kurzstudiengängen)

\section{Individuum:}

Schutz vor Arbeitslosigkeit und Ermöglichung von Aufstiegen

Quelle: Darstellung durch die Verfasserin 
Zusammenfassend kann festgestellt werden, dass Durchlässigkeit als funktionales Erfordernis sowohl für die Gesellschaft als auch für das Individuum konstruiert wird. Primär wird diese Notwendigkeit aus Wirtschaftsperspektive, dem Arbeitskräftebedarf begründet, der letztlich zu einer besseren Wettbewerbssituation von Frankreich in der Welt führ. Einerseits gibt es allgemein den Bedarf der Höherqualifizierung von Arbeitskräften - hervorgerufen durch den strukturellen Wandel -, andererseits auch etinen expliziten Bedarf an beruflich qualifizierten Fachkräften, welche über eine Erhöhung der Attraktivität der Berufsbildung auch via Durchlässigkeit gestillt werden soll. Zudem bietet insbesondere Hochschulbildung einen individuell größeren Schutz vor Arbeitslosigkeit, so dass allen der Weg über die Weiterbildung eröffnet werden sollte. Schließlich ist eine mangelnde Durchlässigkeit in Form systematischer Studienabbrüche und -wechsel ein Problem vor allem für den ersten Zyklus der Universität. Diese Ineffizienz gilt es, zu beheben, um dergestalt ebenfalls die Wettbewerbsfähigkeit der französischen Universitäten im Kampf um die besten Köpfe zu erhöhen.

Rekapitulierend werden die Argumentationsmuster beider Diskursstränge in Tabelle 25 gegenübergestellt.

\section{Strukturmerkmale des französischen Diskurses}

Im ersten Zeitraum war die Diskursstruktur in großem Maß auf die Aspekte des Zugangs und der Anrechnung begrenzt. Wie hat sich die Vorstellung von Durchlässigkeit zwischen beruflicher (Sekundar-)Bildung und Hochschulbildung im zweiten Zeitraum entwickelt? Wie und für wen soll Durchlässigkeit erreicht werden? In diesem Abschnitt werden die typischen Deutungsangebote für die Diskursstränge, die Lösungsmöglichkeiten des Durchlässigkeitsproblems, die konstruierte Zielgruppe, für die Durchlässigkeit relevant ist, und damit auch das damit einhergehende Rollenverständnis dargestellt. Auf diese Weise wird auch das Verständnis von Durchlässigkeit in Frankreich deutlich.

Nach 1999 wird Durchlässigkeit in Frankreich entlang von vier Aspekten diskutiert: dem Zugang, der Anrechnung, der organisationalen Verbindung von Berufs- und Hochschulbildung und dem Umgang mit heterogenen Bedürfnissen der Studierenden. Insbesondere die letzten beiden Aspekte strukturieren einen Großteil des Diskurses. Im Folgenden wird die Struktur des Diskurses anhand der Aspekte von Durchlässigkeit jeweils im Vergleich der universalistischen und funktionalistischen Perspektive dargestellt. 


\section{Zugang}

Der Zugang für beruflich Qualifizierte zu einem Hochschulstudium soll in beiden Diskurssträngen vor allem über zwei Wege erfolgen: einerseits über Berechtigungen und andererseits über die Validierung von Berufserfahrungen ${ }^{166}$. Indes sind Unterschiede zwischen den Diskurssträngen, insbesondere mit Blick auf konstruierte Deutungen zum Berechtigungswesen festzustellen.

Im universalistischen Diskursstrang stehen das Abitur und die damit verbundene allgemeine Berechtigung des Zugangs zur Hochschule im Vordergrund. Der Zugang mit dem beruflichen Abitur soll somit wie bei den anderen Abiturzweigen ebenfalls in alle tertiären Bildungswege eröffnet werden (FSU 2011). Tatsächlich wird im Diskurs jedoch nur der Zugang zu den Universitäten und den technischen Kurzstudiengängen an STS und IUT diskutiert. Der Zugang zu den grandes ecoles über die Vorbereitungsklassen (CPGE) ist kein Thema. Insofern wird die bestehende klare Hierarchisierung zwischen allgemeinbildenden Bildungsgängen und beruflichen Bildungsgängen auch im universalistischen Diskursstrang gestützt. Der Bildungsweg über die grandes ecoles, welcher in Frankreich zu den prestigereichsten Arbeitsplätzen führt, bleibt den beruflich Qualifizierten verschlossen und zielt nur auf die Absolvent_innen des allgmeinen Abiturs.

Auch wenn beruflich Qualifizierte in die universitären Studiengänge, wenn sie dies wünschen, Zugang erhalten sollen, wird doch deren Zugang in die technischen Kurzstudiengänge als vorteilhafter bewertet. So sollen alle, welche diesen Weg in die Kurzstudiengänge gehen wollen, auch die Möglichkeit erhalten, und nicht mangels Alternativen an die Universitäten verwiesen werden. Insofern sollen berufliche Abiturient_innen durch spezifische Regelungen bei der Studienplatzvergabe, wie z.B. einer Quote, an den STS bevorteilt werden (MESR 2012a). Im universalistischen Diskursstrang wird zudem deutlich, dass wenn das berufliche Abitur ein gleichwertiges Abitur sein solle, dieses auch mit einem Bildungsniveau abschließen müsse, welches die Absolvent_innen angemessen auf ein Studium vorbereitet. Dieses Mindestniveau an Bildung entspreche dem französischen Verständnis einer nachhaltigen beruflichen Bildung, denn nur unter diesen Umständen sei ein Lernen an der Hochschule in Erst- oder Weiterbildung möglich (vgl. MDEP 2000e).

Wie auch schon im ersten Untersuchungszeitraum ist ein Weg des Zugangs von der Berufs- zur Hochschulbildung über Weiterbildung erreichbar. Im universalistischen Diskursstrang ist dies vor allem ein Weg, um denen, welche vorher nicht studieren konnten, ein Studium zu ermöglichen (MEN 2001a). Der Zugang erfolgt dabei über die Validierung beruflicher Erfahrungen, über das Verfahren VAP und später VAE.

166 Auch wenn das VAE die Validierung nicht nur beruflich erworbener Kompetenzen ermöglicht, wird im Folgenden allein die Validierung von Berufserfahrung thematisiert, weil dies für die Frage der Durchlässigkeit von Berufs- zur Hochschulbildung von zentraler Bedeutung ist. 
Der zweite Bildungsweg, das Nachholen eines Abiturs, ist zwar in Frankreich ein Weg an die Hochschule, wird jedoch im Diskurs so gut wie gar nicht als ein Weg in die Hochschule konstruiert (siehe aber MESR 2008c), wobei natürlich vor allem die Gruppen ohne Abitur die Zielgruppen sind (zu großen Teilen, die Individuen ganz ohne Schulabschluss oder mit einem CAP oder BEP). Stattdessen wird vielmehr auf die Möglichkeit des Wechsels von Schüler_innen aus dem beruflichen Bildungszweig in die technologischen und allgemeinbildenden Zweige als möglicher Weg an die Hochschulen hingewiesen (MDEP 2000d). Insofern ist der Weg über die Allgemeinbildung in die Hochschule nach oder auch vor einem beruflichen Abschluss im Rahmen der Erstausbildung eine diskutierte Möglichkeit. Tatsächlich wäre dieser Wechsel innerhalb der Sekundarbildung dann nicht mehr als eine Stärkung von Durchlässigkeit zwischen sekundärer Berufsbildung zur Hochschulbildung zu sehen.

Im funktionalistischen Diskursstrang sollen berufliche Abiturient_innen aus Effizienzgründen in die beruflich orientierten Kurzstudiengänge, speziell in die STS an den Oberschulen, orientiert werden. Voraussetzung ist zudem eine große Nähe zu ihrem Ausbildungsfach, da hier die größten Erfolgsaussichten bestehen (MENRT 1999a). Aus dem identischen Grund sollen lediglich die besten der beruflichen Abiturient_innen Studienplätze erhalten (vgl. MENSR 2006a; MEN 2007d; MESR 2011a; ADIUT 2009a). Auch wenn berufliche Abiturient_innen per se berechtigt sind, sich generell im Hochschulsystem an den verschiedensten Studiengänge zu bewerben, sollen sie durch eine sogenannte ,aktive Orientierung، in die für sie richtigen Bildungsgänge gelotst werden (CPU 2007c, 2012). Im Rahmen dieser ,aktiven Orientierung' kann den beruflichen Abiturient_innen zwar der Zugang in Universitäten nicht verweigert werden, aber die Individuen sollen in Gesprächen und durch Abbruchstatistiken für den „richtigen“ Weg überzeugt werden (vgl. MESR 2009b; CPU 2007a). ${ }^{167}$ Im funktionalistischen Diskursstrang wird damit gewissermaßen die mit dem Abitur einhergehende Berechtigung im Falle von Berufsabiturient_innen eingeschränkt und zwar in Bezug auf die Studiengänge und Fachnähe, Erfolgswahrscheinlichkeiten sowie auf die berufliche Leistungselite. Auf diese Weise sollen die Erfolgsaussichten erhöht werden. Letztlich wird im funktionalistischen Diskurs eine klare Vorstellung darüber deutlich, welche Gruppen für welche Hochschulorganisationen geeignet sind und Zugang erhalten sollten: Die Berufsabiturient_innen in die STS, die technischen Abiturien_innen in die STS und IUT und die Absolvent_innen des allgemeinen Abiturs primär in die Universität oder die grandes écoles (CPU 2012; MESR 2008d; MJENR 2002e). Die Differenzierung des Hochschulsystems soll somit genutzt werden, um die jeweils passenden Studiengänge auszuwählen.

167 In einer Studie zeigen Beaud und Pialaux (2001) wie Orientierungsgespräche ablaufen können. Auch wenn diese Studie noch vor der Einführung der aktiven Orientierung durchgeführt wurde, gibt sie einen Hinweis, wie an den Universitäten versucht wird, die beruflichen Abiturient_innen von einem Universitätsstudium abzulenken. 
Warum wird der Zugang beruflich Qualifizierter generell thematisiert? Dies liegt zum einen an der gestiegenen Zahl der Jugendlichen, welche sich für ein berufliches Abitur entscheiden. Wenn die vorgegebenen Benchmarks von $50 \%$ eines Jahrgangs, die einen tertiären Abschluss erhalten sollen, erreicht werden sollen, dann muss auch ein Anteil dieser Jugendlichen einen Hochschulabschluss erreichen (MEN 2007a; MESR 2007b).

Nous voulons amener à terme $50 \%$ d'une classe d'âge à un diplôme supérieur alors que ce taux plafonne aujourd'hui à $43 \%$ chez les 25-34 ans. Pour y parvenir, améliorer la réussite des étudiants dans l'enseignement supérieur et notamment dans le cycle licence constitue la première priorité. (MESR 2012a) ${ }^{168}$

Neben einer Berechtigung sehr guter Berufsabiturient_innen für die Kurzstudiengänge wird im funktionalistischen Diskursstrang auch der Zugang zur Hochschulbildung über Weiterbildung im Rahmen der Validierung beruflicher Kompetenzen als eine Möglichkeit konstruiert. Im Gegensatz zu den Berufsabiturient_innen steht bei dieser Gruppe von Studierenden aber die Gefahr des möglichen Studienmisserfolges nicht im Fokus. Diese fehlende Problematisierung zeigt allerdings auch, dass die Gruppe der Weiterbildungsstudierenden als eher qualifiziert angesehen wird, ein Studium zu schaffen als die Berufsabiturient_innen.

Die im Diskurs konstruierte Zielgruppe für die Hochschulbildung sind im universalistischen Diskursstrang alle Individuen, welche ein Studium beginnen wollen und generell alle, die durch ein französisches Abitur, egal welcher Ausrichtung, eine Studienberechtigung erworben haben. Das Individuum wird in diesem Fall als Person wahrgenommen, welche es bei ihrer Weiterentwicklung als Mensch, Bürger und Arbeiternehmer_in zu unterstützen gilt. Insofern werden berufliche Abiturient_innen oder Erfahrene als legitime Studierende anerkannt, wobei darauf hingewiesen wird, dass die Berufsabiturient_innen einer besondere Unterstützung bedürfen und sie, auch wenn sie das Recht besitzen, an einer Universität zu studieren, besser in den Kurzstudiengängen der beruflichen Oberschulen (STS) aufgehoben seien (MESR 2012a).

C'est un écart „,terrible“, inacceptable là encore. Un bachelier professionnel qui veut entreprendre des études supérieures aurait donc 6 fois plus de risque d'échouer qu'un bachelier général. On ne peut pas laisser se satisfaire de cette situation. (MESR 2012a) ${ }^{169}$

168 „Wir wollen, dass $50 \%$ eines Jahrgangs über kurz oder lange ein Hochschuldiplom erwirbt, aber momentan erreichen höchstens $43 \%$ bei den 25-34 Jährigen dieses Niveau. Um unser Ziel zu erreichen, hat die Verbesserung des Studienerfolges im Hochschulbereich und insbesondere im licence-Zyklus erste Priorität.“ (MESR 2012a, Übersetzung NB)

169 „Es ist eine ,fürchterliche“ Diskrepanz und inakzeptabel dazu auch. Ein/e Berufsabiturient_in, der/die studieren möchte, hat eine sechsmal höhere Wahrscheinlichkeit nicht zu bestehen als ein/e Absolvent_in des allgemeinen Abiturs. Wir können uns nicht mit dieser Situation zufrieden geben.“ (MESR 2012a, Übersetzung NB) 
L'élévation du niveau de connaissances, la réussite des étudiants, quel que soit leur bac, quel que soit leur parcours, s'inscrivent dans l'agenda défini par le Président de la République. (MESR 2012a) ${ }^{170}$

Tabelle 26 Struktur des französischen Diskurses 1999-2012: Zugang und Zielgruppe

\begin{tabular}{|c|c|c|}
\hline & universalistisch & funktional \\
\hline Zugang & $\begin{array}{l}\text { Allgemeine Berechtigung über das } \\
\text { berufliche Abitur } \\
\text { Berechtigung auch auf Zugang in se- } \\
\text { lektive Studiengänge der STS (Ein- } \\
\text { schränkung der Selektion) } \\
\text { via Validierung (VAP oder VAE) }\end{array}$ & $\begin{array}{l}\text { Recht auf Zugang in technologische } \\
\text { Kurzstudiengänge nach Leistungsse- } \\
\text { lektion über den Wechsel in technolo- } \\
\text { gischen und allgemeinen Abiturzweig } \\
\text { via Validierung (VAP oder VAE) }\end{array}$ \\
\hline $\begin{array}{l}\text { Zielgruppe } \\
\text { allgemein }\end{array}$ & $\begin{array}{l}\text { alle, die sich bilden wollen und die } \\
\text { Berechtigung, das Abitur egal welcher } \\
\text { Ausrichtung besitzen }\end{array}$ & $\begin{array}{l}\text { alle diejenigen, die sehr wahrschein- } \\
\text { lich erfolgreich abschließen werden: } \\
\text { v.a. Absolvent_innen des allgemeinen } \\
\text { und technologischen Abiturs }\end{array}$ \\
\hline $\begin{array}{l}\text { Zielgruppe } \\
\text { Durchlässigkeit }\end{array}$ & $\begin{array}{l}\text { Berufsabiturient_innen Berufserfahre- } \\
\text { ne über Validierung }\end{array}$ & $\begin{array}{l}\text { allein für die sehr guten Berufsabitu- } \\
\text { rient_innen, } \\
\text { Berufserfahrene über Validierung }\end{array}$ \\
\hline $\begin{array}{l}\text { Subjektposition } \\
\text { der Studierenden }\end{array}$ & $\begin{array}{l}\text { Individuum als Person muss bei sei- } \\
\text { ner Weiterentwicklung als aufgeklär- } \\
\text { ter, kritikfähiger Mensch, als demo- } \\
\text { kratischer Bürger, unterstützt werden }\end{array}$ & $\begin{array}{l}\text { Individuum als (Humankapital-) Res- } \\
\text { source, die benötigt wird, eigenver- } \\
\text { antwortlich handelt bei ausreichender } \\
\text { Information }\end{array}$ \\
\hline
\end{tabular}

Quelle: Darstellung durch die Verfasserin

Im funktionalistischen Diskursstrang steht das Individuum als Ressource im Mittelpunkt. An die Hochschule sollen vor allem die Absolvent_innen des allgemeinen und technologischen Abiturs, da diese auch ausreichend auf ein Studium vorbereitet werden, so dass sie dieses erfolgreich abschließen können. Im funktionalistischen Diskurs wird deutlich, dass die Individuen selbst für die Wahl ihres Studiums verantwortlich sind, wobei diese Wahl durch bereit gestellte Informationen unterstützt wird (MEDEF 2011; CPU 2011).

Le principe d'accès à l'université étant la non sélection des entrants, il est impératif de donner aux futurs étudiants les moyens de construire eux-mêmes leur décision d'orientation et d'étayer d'éléments objectifs la construction de leur projet. (MEDEF 2011) $)^{171}$

170 „Die Erhöhung des Wissensstandes, der Erfolg aller Studierenden, egal aus welchem Abiturzweig, egal was ihr Bildungsweg ist, ist in die Agenda des Präsidenten der Republik eingeschrieben.“ (MESR 2012a, Übersetzung NB)

171 „Wenn das Zugangsprinzip an der Universität das der Nicht-Selektion der Studierenden ist, dann besteht ein Imperativ darin, die zukünftigen Studierenden mit den Fähigkeiten auszustatten, dass 
Wird trotz der gegebenen Informationen die, falsche' Wahl getroffen, sind es auch die Individuen, welche die Konsequenzen zu tragen haben. Beruflich Qualifizierte sind vor diesem Hintergrund lediglich dann Teil der Zielgruppe für Hochschulbildung, wenn sie besonders gute Noten beim Berufsabschluss vorweisen können und mit ihren Erfahrungen bzw. ihrem Bildungsstand besonders gut zu dem Bildungsgang passen. Allein bei beruflich Qualifizierten wird im Diskurs thematisiert, dass nur eine Auswahl studieren solle. Diese muss neben dem Wunsch ebenfalls die entsprechenden Fähigkeiten ausweisen. Bei den anderen Abiturzweigen werden der Wunsch und die Fähigkeit für ein Studium nicht infrage gestellt, sondern als selbstverständlich vorausgesetzt. Eigentlich gehören Berufsabiturient_innen nach dem Abschluss des Abiturs direkt in den Arbeitsmarkt, wobei den besten unter ihnen der Zugang zur Hochschule nicht verwehrt werden sollte. Der Zugang dieser Gruppe nach ein paar Jahren Berufserfahrung dagegen wird nicht als problematisch angesehen, sondern wird begrüßt, da durch den strukturellen Wandel die Anpassung des vorhandenen Bildungsniveaus kontinuierlich notwendig sei.

\section{Anrechnung}

Auch die Frage von Anrechnung beruflicher Qualifikationen wurde im Diskurs thematisiert. Allerdings stand sie anders als im ersten Zeitraum nicht im Zentrum der Debatten, sondern wurde immer wieder erwähnt, vor allem im Kontext der Debatte um die Ermöglichung lebenslangen Lernens. Eine weitere Perspektive war die Vereinfachung von Studiengangswechsel innerhalb des Hochschulsystems und damit der Anrechnung von formalem Lernen.

Bis auf die Begründung, warum Anrechnung ermöglicht werden soll, sind keine Unterschiede zwischen den Diskurssträngen festzustellen. Im universalistischen Diskurs wird die Bedeutung der Weiterbildung zur Erlangung eines Studienabschlusses betont und damit eine Weiterführung der Demokratisierung des französischen Bildungswesens verbunden (MDEP 2000e, 2001a). Im funktionalistischen Diskursstrang wird die Ermöglichung von Anrechnung eher im Kontext der Weiterentwicklung der französischen Gesellschaft hin zu einer Wissensökonomie behandelt, in welcher lebenslanges Lernen der Individuen eine Voraussetzung sei. Dabei wird auch immer wieder Frankreichs Vorreiterrolle in Europa betont (vgl. MDEP 2000g; CPU 2008).

Ort der Anrechnung ist das Hochschulsystem, dem für die Zukunft eine große Bedeutung in Sachen Ermöglichung von Weiterbildung zugesprochen wird. Als Verfahren zur Anrechnung und Ermöglichung eines Studienabschlusses beruflich Erfahrener wird insbesondere auf die 2002 institutionalisierte neue Form der Validierung (VAE) hingewiesen. Um einen Wechsel innerhalb des

sie selbst ihre Hochschulwahl treffen und sich auf objektive Fakten stützend ihr eigenes [Bildungs-, NB] Vorhaben konstruieren.“ (MEDEF 2011, Übersetzung NB) 
Hochschulsystems zu erleichtern, werden die durch die Europäisierung induzierten Standards, die eingeführte Semestereinteilung im gesamten Hochschulsystem, die Einführung von Modularisierung, Ergebnisorientierung und Leistungspunktesystemen als vorteilhaft anerkannt (ADIUT/UNPIUT 2012; MEDEF 2011). Auf diese Weise ist es möglich, auch ohne Zeitverlust umorientiert weiter zu studieren (MEN 2001b). Diese Standards unterstützen vor allem auch die Hochschulorganisationsform übergreifenden Wechsel. Insbesondere die Studiengänge der STS an den Oberschulen waren zuvor in ihren Strukturen noch nicht kompatibel mit denen der Universitäten.

Tabelle 27 Struktur des französischen Diskurses 1999-2012: Anrechnung

\begin{tabular}{lll} 
& \multicolumn{1}{c}{ universalistisch } & \multicolumn{1}{c}{ funktional } \\
\hline Anrechnung & $\begin{array}{l}\text { Im Hochschulsystem } \\
\text { Anrechnung als Möglichkeit einer zwei- } \\
\text { ten Chance auf einen Studienab- } \\
\text { schluss, Zeichen der Demokratisierung } \\
\text { des Bildungssystems }\end{array}$ & $\begin{array}{l}\text { Anrechnung zur Erhöhung der Effizi- } \\
\text { enz (Weiterstudium ohne Zeitverlust, } \\
\text { keine unnötigen Doppelqualifikationen) } \\
\text { und als Ermöglichung der Weiterent- } \\
\text { wicklung der Wissensökonomie }\end{array}$ \\
$\begin{array}{l}\text { Anrechnung } \\
\text { was }\end{array}$ & $\begin{array}{l}\text { Berufliche Kompetenzen, sowohl formale beim Wechsel von Studiengängen als } \\
\text { auch non-formale und informelle bei Berufserfahrenen }\end{array}$ \\
$\begin{array}{l}\text { Anrechnung } \\
\text { wie }\end{array}$ & $\begin{array}{l}\text { Über Validierungsverfahren VAE } \\
\text { Anrechnung zwischen Studiengängen durch Einführung von Semestern, Modu- } \\
\text { larisierung, Kompetenzbasierung und Kreditpunktesystemen }\end{array}$ \\
\hline
\end{tabular}

Quelle: Darstellung der Verfasserin

Wie auch im ersten Zeitraum wird die im Konzept der Anrechnung inhärente Frage der Gleichwertigkeit von unterschiedlichen Feldern erlangten Kompetenzen (vgl. Kapitel 2), in diesem Fall zwischen Berufs- und Hochschulbildung, nicht explizit diskutiert. Dies ist insofern erstaunlich, da dies ein Weg gewesen wäre, die Debatte um die Attraktivität der beruflichen Bildung und deren Anschlussfähigkeit im französischen Bildungssystem zu stärken. Dessen ungeachtet wird die Möglichkeit anerkannt, dass auch durch den Erwerb beruflicher Erfahrungen gleichwertige Fähigkeiten zu einem Studium vermittelt werden. Während also einerseits vielen Absolvent_innen der beruflichen Sekundarbildung die Befähigung durch die Berufsbildung zum Studium abgesprochen wird (ADIUT 2009a), ist dies weitaus weniger der Fall, wenn die Kompetenzen über mehrere Jahre im Beruf erworben worden sind. In Tabelle 27 findet sich die Zusammenfassung für den Diskurs zum Thema Anrechnung.

\section{Organisationale Verbindungen}

Die Frage der organisationalen Verbindungen zwischen Berufs- und höherer Allgemeinbildung werden in Frankreich in Bezug zu Durchlässigkeit in zwei Formen 
diskutiert. Die erste Form ist die der integrierten Bildungsgänge, welche berufliche und stärker allgemeinbildende Bildung und Hochschulbildung in einem Bildungsgang integrieren. Hier werden die beruflichen Kurzstudiengänge STS, die lycées des métiers oder die licences professionnelles im Kontext von Durchlässigkeit thematisiert. Die andere Form ist die der institutionalisierten Übergänge zwischen beruflichen und stärker allgemeinbildenden Bildungsgängen (vgl. Kapitel 2). In Frankreich werden solche institutionalisierten Formen passerelles genannt und wurden in den letzten Jahren im Hochschulsystem ausgebaut. Nachfolgend soll zuerst der französische Diskurs zu den integrierten Bildungsgängen und anschließend zu den institutionalisierten Übergängen dargestellt werden. Eine Zusammenfassung der Diskursstrukturen zu organisationalen Verbindungen erfolgt in Tabelle 28.

Die erste Form des integrierten Bildungsgangs in Frankreich kann anhand der Differenzierung des Hochschulsystems in stärker allgemeinbildende und stärker beruflich orientierte Kurzstudiengänge erklärt werden. Wie in Kapitel 8 beschrieben, sind diese Kurzstudiengänge eng mit den meist regionalen Bedürfnissen der Wirtschaft verbunden, vermitteln theoriebasierte berufliche Bildung, können en alternance, d.h. in Wechsel der Lernorte, durchgeführt werden und enden mit einem beruflich orientierten Abschluss, welcher auf klare Arbeitsmarktpositionen verweist, da diese Abschlüsse im Referentiell festgehalten sind. Insofern können die STS und IUT entsprechend dem heuristischen Konzept aus Kapitel 2 als integrierte Organisationsformen in Frankreich gelten. In der Existenz dieser Studiengänge wird ebenfalls das größte Potenzial für eine Durchlässigkeit von der Berufs- zur Hochschulbildung gesehen. Wie in der Beschreibung des französischen Diskurses im Hinblick auf Zugangsfragen deutlich wurde, sind es vor allem die technischen Kurzstudiengänge in den Oberschulen (STS), welche als primäre Einmündungsmöglichkeit beruflich Qualifizierter im Hochschulbereich gesehen werden (vgl. MENRT 1999a; MESR 2011b, 2012a; CPU 2007a). Dies gilt für den universalistischen, in dem aber das Recht der Abiturient_innen auch an andere Hochschulorganisationen zu studieren unterstrichen wird, aber auch für den funktionalistischen Diskursstrang. Begründet wird dies durch die besseren Betreuungsmöglichkeiten, die Struktur des Studiums, die stärker an schulische Strukturen angelehnt ist, sowie die gleich von Beginn an praktischere Ausrichtung des Studiums, welche dem Kenntnisstand und den Wünschen beruflicher Abiturien_innen am ehesten entsprechen.

Il fallait faire bouger les lignes sans rien bouleverser de ce qui a fait le succès des BTS, cet équilibre complexe entre exigence académique et compétences professionnelles, organisation des enseignements encore proche de celle du lycée (essentielle à la réussite de nombreux élèves) et préparation à la poursuite d'études universitaires. (MESR $2011 b)^{172}$

172 „Wir müssen Bewegung in veraltete Strukturen bringen, ohne das, was den Erfolg der BTS ausmacht, umzustürzen, dieses komplexe Gleichgewicht zwischen akademischen 
Da diese Studiengänge jedoch selektiv sind und damit berufliche Abiturient_innen in Konkurrenz zu anderen Abiturgruppen stehen, wird vor allem im universalistischen Diskursstrang eine gesetzliche Regelung gefordert, welche die Autonomie der Hochschulorganisationen zugunsten der beruflichen Abiturient_innen einschränkt (MESR 2012a).

Eine weitere Form der Integration stärker allgemeinbildender Hochschulstudiengänge mit beruflichen Studiengängen wurde durch die Einführung der licence professionnelle vorangetrieben. Im Hinblick auf Durchlässigkeit wird die licence pro in zweierlei Hinsicht thematisiert. So soll sie als Einmündungsmöglichkeit für diejenigen an den Hochschulen gelten, die perspektivisch keinen guten Abschluss im licence-Bereich mit anschließendem Weiterstudium machen werden, also auch für die Gruppe, welche mit einem Studienmisserfolg umgehen müsste.

Cette mesure [introduction de la licence pro, NB] devrait permettre une amélioration de la qualité des sorties post-deug à l'université et concourir à limiter l'échec. (MENRT 1999c) ${ }^{173}$

Beruflich Qualifizierte werden dabei nur indirekt angesprochen, wenn sie zu der Gruppe gehören, die nicht bereits vorher schon aus der allgemeinbildenden licence ausgeschieden sind und einen beruflich orientierten Abschluss erhalten möchten. Im funktionalistischen Diskursstrang wird die Einführung der licence pro stärker als bessere Vorbereitung auf den Arbeitsmarkt für die Gruppen gesehen, welche aufgrund ihrer Leistungen wohl nicht erfolgreich weiterstudieren würden. Die Annäherung zwischen Hochschulbildung und stärker beruflicher Ausbildung dient somit primär der Integration in den Arbeitsmarkt.

Praktisch hat sich gezeigt, dass dieser Plan der Verbindung der allgemeinbildenden licence mit der beruflich orientierten nicht wie gewünscht funktioniert hat (CPU 2011), da die meisten der begrenzten Plätze durch Absolvent_innen der STS und der IUT belegt werden.

Zum anderen werden licence pro-Studiengänge im Diskurs als eine Möglichkeit gesehen, die bisher eher getrennten Welten der Universitätsfakultäten, an welchen die licence pro Studiengänge primär institutionalisiert sind ${ }^{174}$, und der beruflichen Oberschulen, an welchen der berufliche Hochschulabschluss BTS erworben werden kann, zu verbinden. Die Einführung der licence pro wird somit vor allem auch im universalistischen Diskursstrang als symbolische Verbindung von beruflicher Sekundarbildung mit Hochschulbildung gesehen. Sie soll, vorausgesetzt STS und Universitäten arbeiten im Rahmen der licence pro zusammen, ein Zeichen sein, dass

Anforderungen und beruflichen Fähigkeiten, eine Organisation der Lehre eng an die der Oberschulen angelehnt (essentiell für den Studienerfolg einer großen Zahl Studierender) und die Vorbereitung auf ein daran anschließendes Studium.“" (MESR 2011b, Übersetzung NB)

173 „Diese Maßnahme [Einführung der licence pro, NB] erlaubt eine Verbesserung der Abgänge nach dem DEUG-Abschluss an der Universität und leistet einen Beitrag, den Studienmisserfolg zu begrenzen.“ (MENRT 1999c, Übersetzung NB)

174 Auch an den IUT wurden licence pro-Studiengänge eingeführt. 
auch berufliche Bildungswege bis zum Bildungsniveau bac +3 führen und keine Sackgassen darstellen (MDEP 2001b, 2002).

Et c'est très important pour moi de pouvoir afficher qu'il va y avoir et qu'il y a déjà 70 licences professionnelles installées dans des lycées professionnels, parce que c'est du post-bac, c'est de l'enseignement supérieur, et parce que cela va permettre d'en finir avec cette image d'une filière courte qui, au bout du compte, s'apparente désormais à un préjugé. On ne peut vaincre ce préjugé que par des mesures d'ordre concret, qui permettent aux familles de projeter l'avenir de leurs enfants et qui permettent aussi aux enseignants de se dire que, quand ils recommandent cette voie à un jeune, ils lui proposent une voie qui va lui permettre d'aller le plus loin possible. (MDEP 2002) ${ }^{175}$

[...] il ne doit pas y avoir des BEP sans BAC pro pas de BAC pro sans perspective d'accès au BTS, pas de BTS ou de DUT sans ouverture vers la licence professionnelle, pas de licence professionnelle conduisant à une impasse ou un cul de sac. (MDEP 2000a) ${ }^{176}$

Eine dritte Form der organisationalen Verbindung wurde zu Beginn des neuen Jahrtausends eingeführt, das lycée des métiers. Ziel dieser Organisationsform ist die Verbindung von beruflichen und technischen Bildungsgängen in einem breiteren Berufsfeld, so dass in dieser Oberschule vom CAP und BEP zum bac techno, zum bac pro, zum BTS und schließlich zur licence pro alles erworben werden kann (MDEP 2001c; MEN 2007d). Insofern verbindet das lycée des métiers technische und berufliche Sekundarbildung mit Hochschulbildung bis zum licence-Bereich, wobei für eine licence noch eine zusätzlich Kooperation mit den Universitäten institutionalisiert wird. Zudem sollen im lýcee de métiers die Abschlüsse in der Erstausbildung, der Weiterbildung oder über das Validierungsverfahren VAE erhalten werden. Dieses lycée des métiers wird vornehmlich von der sozialistischen Regierung zu Beginn des zweiten Untersuchungszeitraums als wichtiger Weg zur Erhöhung der Attraktivität beruflicher Bildungszweige angesehen, da in dieser Schule die Entwicklungsmöglichkeiten bzw. die Aufstiegsoptionen mit einem beruflichen Abschluss deutlich werden und auch die Schüler_innen der beruflichen Bildung

175 „Es ist sehr wichtig für mich zu zeigen, dass wir dahin gelangen werden und dass es bereits 70 licences professionnelles, die an beruflichen Oberschulen angesiedelt sind, gibt. Denn das ist der Bereich nach dem Abitur, das gehört zur Hochschulbildung und dies erlaubt es, mit den Vorstellungen eines kurzen [beruflichen, NB] Bildungsweges aufzuräumen, was letzten Endes einem Vorurteil gleichkommt. Man kann diese Vorurteile nur mithilfe konkreter Maßnahmen besiegen, die es dann den Familien ermöglichen, eine Zukunft ihrer Kinder zu planen und den Lehrern und Lehrerinnen erlauben, wenn sie diesen Bildungsweg [an den STS] ihren Jugendlichen vorschlagen, einen Bildungsweg zu empfehlen, der es allen ermöglicht, sich so hoch wie möglich weiterzubilden." (MDEP 2002, Übersetzung NB)

176, ,...] es darf kein BEP geben ohne ein [passendes, NB] bac pro, kein bac pro ohne Perspektive auf Zugang zu einem BTS, kein BTS oder DUT ohne mögliche Öffnung in Richtung licence professionnelle, keine licence professionnelle, die in eine Sackgasse führt.“ (MDEP 2000a, Übersetzung NB) 
selbst diese Entwicklungsmöglichkeiten in ihrer Schule kennenlernen. Durchlässigkeit zwischen beruflicher Sekundar- und Hochschulbildung wird erleichtert (MDEP 2002; MEN 2007d).

Im universalistischen Diskursstrang wird das lycée des métiers vor allem als Möglichkeit zur Erhöhung der Chancengleichheit auf einen Hochschulabschluss für beruflich Qualifizierte, ihre Höherqualifizierung und Motivierung zum Weiterlernen konstruiert. Aus diesem Grund wird auch von den Gewerkschaften gefordert, dass nicht nur ausgewählte Oberschulen, sondern alle beruflichen Oberschulen in lycée des métiers umgewandelt werden sollten (CGT 2005b). Im Rahmen des funktionalistischen Diskursstranges werden hingegen speziell die Selektionsmöglichkeiten, die sich durch eine Kooperation von Sekundar- und Tertiärbildung, wie im lycée des métiers ergeben, als positiv bewertet, da durch derartige Kooperationsformen frühzeitig die für ein Studium fähigen Absolvent_innen ausgesucht werden können (ADIUT 2009a). Nicht nur innerhalb der lycées des métiers wird insofern im funktionalistischen Diskursstrang eine Kooperation als sinnvoll angesehen. Auch zwischen ,normalen' beruflichen Oberschulen und den technischen Studiengängen sollten solche Kooperationsformen für ein besseres Matching von Studienplatz und beruflich Qualifizierten genutzt werden, um auf diese Weise die Erfolgschancen zur erhöhen (ADIUT 2009a).

Die Institutionalisierung von alternance, und somit die Integration beruflicher Praxis in ein Hochschulstudium, wird schließlich auch als vorteilhaft für einen erfolgreichen Studienabschluss angesehen. Vor allem wird dies im funktionalistischen Diskurs thematisiert. Hier wird argumentiert, dass besonders Berufsabiturient_innen durch ihre Vorbildung weniger eine rein hochschulische Ausbildung erfolgreich meistern, sondern die Verbindung mit der Praxis benötigen, um Lernen zu können (ADIUT 2009a, 2012).

Die zweite Form der organisationalen Verbindung, die der institutionalisierten Übergänge, ist besonders im Kontext der Frage des Studienmisserfolgs für Durchlässigkeit von Belang. Im französischen Diskurs wird immer wieder auf die Notwendigkeit des Ausbaus und der besseren Abstimmung institutionalisierter Übergänge zwischen den einzelnen unterschiedlichen Studiengängen der differenten Organisationsformen hingewiesen. Inwiefern ist das für Durchlässigkeit zwischen beruflicher Sekundarbildung und Hochschulbildung wichtig? Passerelles werden im französischen Diskurs vor allem als Möglichkeit gesehen, eine frühzeitige Korrektur oder ,Umorientierung' während des Studiums vorzunehmen und damit einen erfolgreichen Studienabschluss zu begünstigen (vgl. CPU 2010b; MESR 2009b, 2010a). Dies gilt für alle Studierenden, aber auch besonders für beruflich Qualifizierte, welche viel stärker von Studienabbrüchen betroffen sind. Die institutionalisierten Übergänge, welche es den Studierenden ermögli $\neg$ chen, ohne großen Zeitverlust und unter Anerkennung der bisherigen Leistun $\neg$ gen Stu $\neg$ diengänge auch zwischen Organisationsformen zu wechseln, werden für beruflich Qualifizierte primär als Weg von der Universität an die STS gesehen. Faktisch sind solche passerelles aber auch in die andere Richtung vorgesehen 
(MESR 2010b). Für Berufsabiturient_innen, welche merken, dass der ausge $\neg$ wählte Studiengang doch nicht ihren Vorstellungen und Wünschen entspricht, aber auch für solche, welche in einem ersten Schritt nicht in den STS genom $\neg$ men wurden, stellen pas $\neg$ serelles somit auch eine zweite Chance auf einen Studien $\neg$ erfolg dar.

Tabelle 28 Vergleich der Diskursstränge zur Bedeutung organisationaler Verbindungen in Frankreich (1999-2012)

\begin{tabular}{|c|c|c|}
\hline & universalistisch & funktionalistisch \\
\hline \multirow[t]{4}{*}{$\begin{array}{l}\text { Integrierte Bil- } \\
\text { dungsformen }\end{array}$} & $\begin{array}{l}\text { STS als Verbindung beruflicher Orien- } \\
\text { tierung und tertiärer Bildung im Hoch- } \\
\text { schulbereich als primärer Ort für ein } \\
\text { Studium der bac pro-Absolvent_innen, } \\
\text { wobei Zugang über klare Berechtigun- } \\
\text { gen/Quoten geregelt werden soll }\end{array}$ & $\begin{array}{l}\text { STS als Verbindung beruflicher } \\
\text { Orientierung und tertiärer Bildung im } \\
\text { Hochschulbereich als primärer Ort } \\
\text { für ein Studium für ausgewählte } \\
\text { sehr guten bac pro-Absolvent_innen }\end{array}$ \\
\hline & $\begin{array}{l}\text { licence pro als institutionalisierte Wei- } \\
\text { terbildungsmöglichkeit beruflich Qualifi- } \\
\text { zierter }\end{array}$ & $\begin{array}{l}\text { licence pro als Weg aus Allgemein- } \\
\text { bildung in die Berufsbildung für die } \\
\text { weniger Leistungsstarken, die kein } \\
\text { längeres Studium schaffen }\end{array}$ \\
\hline & $\begin{array}{l}\text { Lycée des métiers/Verbindung Berufs- } \\
\text { schulen mit Hochschulbereich: um Bil- } \\
\text { dungsoptionen aufzuzeigen und zum } \\
\text { Weiterlernen zu motivieren sowie } \\
\text { Durchlässigkeit zu vereinfachen }\end{array}$ & $\begin{array}{l}\text { Lycée des métiers/Verbindung von } \\
\text { Berufsschulen mit Hochschulbereich } \\
\text { nutzen zur frühen Auswahl der ,fähi- } \\
\text { gen' bac pro-Absolvent_innen und } \\
\text { die Attraktivität der beruflichen Bil- } \\
\text { dungswege zu erhöhen }\end{array}$ \\
\hline & & $\begin{array}{l}\text { Alternierende Bildungsgänge im } \\
\text { Hochschulsystem durch Verbindung } \\
\text { von Praxis und Theorie stärker an } \\
\text { Lernfähigkeiten Berufsabitu- } \\
\text { rient_innen orientiert und erhöhen } \\
\text { Erfolgswahrscheinlichkeit und } \\
\text { gleichzeitig Bedürfnisse des Arbeits- } \\
\text { markts integriert }\end{array}$ \\
\hline $\begin{array}{l}\text { Institutionalisierte } \\
\text { Verbindungen }\end{array}$ & $\begin{array}{l}\text { passerelles im Hochschulbereich zwi- } \\
\text { schen Bildungsgängen auf einem Ni- } \\
\text { veau } \\
\text { Ziel Ermöglichung der Umorientierung, } \\
\text { der Wahl des besten Studienangebots } \\
\text { entsprechend den Bedürfnissen (die } \\
\text { sich auch ändern können), als Recht } \\
\text { auf Bildung } \\
\text { Recht auf Weiterbildung ausgehend } \\
\text { von jedem Bildungsgang }\end{array}$ & $\begin{array}{l}\text { passerelles im Hochschulbereich } \\
\text { zwischen Bildungsgängen auf einem } \\
\text { Niveau } \\
\text { Ziel: Korrektur individueller Studien- } \\
\text { wahl, Ressourcen- und Effizienzsi- } \\
\text { cherung }\end{array}$ \\
\hline
\end{tabular}

Quelle: Darstellung der Verfasserin 
Durch die Einrichtung der institutionalisierten Übergänge werden die Hochschulorganisationen angehalten, sich verstärkt auf Studienwechsler einzustellen, bestimmte Kurse zur Einführung anzubieten, um den Wechsel so einfach wie möglich zu gestalten (vgl. MESR 2010b, MESR 2011b). Auch die Semestereinführung sowie die Einführung der Modularisierung und Leistungspunktesysteme, welche generell für eine Anrechnung als wichtig erachtet werden, werden als Erleichterung der Strukturierung von Übergängen angesehen. Sowohl im universalistischen als auch im funktionalistischen Diskursstrang werden passerelles als relevantes Instrument konstruiert, um das Problem des Studienmisserfolges anzugehen. Im funktionalistischen Diskursstrang sollen sie vor allem dazu dienen, individuelle Fehler bei der Studienwahl zu korrigieren und die Investitionen, welche bereits getätigt wurden, zu retten (MEN 2000b). Zudem erhöhen sie durch bestehende institutionalisierte Regelungen, die für den Wechsel vorgesehen sind, die Effizienz bei Studiengangwechsel, denn ohne passerelles begannen die Studierenden zuvor, wenn der vorherige Studiengang nicht sehr fachnah war, das Studium von vorn. Im universalistischen Diskurs werden passerelles als wichtig erachtet, da sie es den Individuen ermöglichen, ohne Bestrafung einen für sie besser passenden Studiengang zu wählen und ihr Recht auf Bildung und persönliche Entwicklung wahrzunehmen (MESR 2009b).

Zusammenfassend (vgl. auch Tabelle 28) werden in Frankreich zwei Formen von organisationalen Verbindungen im Diskurs zur Durchlässigkeit thematisiert, die integrierten Bildungsgänge und die institutionalisierten Übergänge. Insbesondere die STS als stärker an die beruflichen Vorerfahrungen der Berufsabiturient_innen anknüpfende Studiengänge werden als primärer Weg zur Durchlässigkeit im Sinne eines möglichst erfolgreichen Studiums konstruiert. So sollen auch die passerelles beruflich Qualifizierten, die Probleme an der Hochschule haben, einen Wechsel in die STS ermöglichen.

\section{Umgang mit heterogenen Bedürfnissen}

Von Beginn an war die Frage des Umgangs mit heterogenen Bedürfnissen einer immer heterogener werdenden Studierendenschaft ein typisches Strukturmerkmal für den französischen Diskurs. Auch im ersten Zeitraum war diese Frage präsent, allerdings nicht in Bezug auf beruflich Qualifizierte. Dies änderte sich im zweiten Zeitraum, wobei einige Reforminitiativen sich weiterhin explizit primär an die Absolvent_innen des bac général und technologique wenden. Dies gilt z.B. für die verstärkte Einführung der Orientierungsphasen im lycée géneral und technologique, welche die Studien- und Berufswahl vorbereiten sollen (Loi lycée 2010).

Insgesamt unterscheide ich mehrere strukturelle Elemente, welche im französischen Diskurs in Bezug auf den Umgang mit heterogenen Bedürfnissen thematisiert werden. Erstens werden Beratungs- und Informationsstrukturen thematisiert, wobei diese als besonders wichtig im Diskurs gelten. Zweitens wird die Frage der Studienorganisation und inwiefern diese auf heterogene Bedürfnisse angepasst werden sollte, thematisiert. Drittens und das ist wiederum ein wichtige 
Punkt im französischen Diskurs ist die Anpassung pädagogischer Strukturen und Konzepte an die heterogene Studierendenschaft und schließlich wird auch die Frage der Studienfinanzierung als Thema aufgenommen. In Tabelle 29 am Ende dieses Abschnittes werden die wichtigsten Strukturmerkmale der Diskursstränge zusammengefasst. Bevor die Bedeutung der strukturellen Elemente nachfolgend dargestellt werden, sollen zuerst die unterschiedlichen Motive, welche sich aus der typischen Argumentationsstruktur der funktionalistischen und universalistischen Diskursstränge ergeben, rekonstruiert werden.

Im universalistischen Diskursstrang wird die bessere Anpassung an die Heterogenität der Studierenden als eine Möglichkeit gesehen, alle Studierende sowie Interessierte und damit explizit auch beruflich Qualifizierte bei der Studienwahl und im Studium selbst so zu unterstützen, so dass alle ihr Studium mit einem erfolgreichen Abschluss beenden und sich so hoch wie möglich bilden können (MDEP 2000b). Diese Form der Unterstützung wird als notwendig erachtet, um das Recht auf Bildung durchzusetzen und ungleiche Voraussetzungen auszugleichen.

Sans proposer l'allongement des études comme un impératif pour chaque élève, il est néanmoins indispensable de garantir que, le cas échéant, chacun pourra aller aussi loin que le lui permettront ses mérites. (MDEP 2000f) ${ }^{177}$

Im funktionalistischen Diskursstrang geht es insbesondere darum, die Absolvent_innen der Sekundarstufe II erstens über selektive Fördermöglichkeiten in die richtigen Bildungswege oder den Arbeitsmarkt zu lenken, um somit vor allem den vielversprechenden Talenten eine spezifische für sie passende Bildung, d.h. voraussichtlich erfolgreiche Ausbildung im Hochschulwesen, zu ermöglichen. Und zweitens sollen die Individuen so vorbereitet werden, dass ein Studienerfolg wahrscheinlich wird. Ziel ist somit primär die Erhöhung der Effizienz im Hochschulwesen und gleichzeitig durch eine verringerte Quote an Misserfolgen insbesondere an den Universitäten deren Attraktivität und Wettbewerbsfähigkeit zu erhöhen.

Selbst wenn unterschiedliche Motive in den Diskursen zum Thema des Umgangs mit der Heterogenität der Studierenden rekonstruiert wurden, werden doch vielfach ähnliche Strukturen gefordert. Diese sollen nun mit dem jeweiligen Fokus der Diskursstränge dargestellt werden.

\section{Beratung und Information}

Strukturen zur Beratung und besseren Information über Studien- und Berufswege sowie Anschlussmöglichkeiten sollen vor und während des Studiums ausgebaut werden. Dies gilt für beide Diskursstränge und über den gesamten zweiten Zeitraum hinweg.

177 „Ohne vorzuschlagen, dass weiterführende Bildung für alle ein Imperativ sei, muss trotzdem jedem garantiert sein, wenn Bedarf besteht, so weit [im Bildungssystem, NB] gehen zu können wie es die persönliche Leistungsfähigkeit erlaubt.“ (MDEP 2000f, Übersetzung NB) 
Besonders wichtig im Vergleich zu den anderen Strukturelementen wird ein Ausbau dieser Strukturen allerdings im funktionalistischen Diskurs angesehen. So sollen die Schüler_innen der Sekundarstufe II durch bessere Information in die Lage versetzt werden, eigenverantwortlich ihre berufliche Zukunft und die dazugehörigen Bildungswege zu planen (MJENR 2002e; MEDEF 2011; MEN 2009). Insbesondere im Zusammenhang mit dem Plan réussite en licence wird ein besonders starker Fokus auf die sogenannte Orientierungsphase gelegt. Dabei soll eine ,aktive Orientierung' stattfinden, in welcher die Individuen auf die Erfolgswahrscheinlichkeiten in einem Studium vor dem Hintergrund des eigenen Bildungsprofils direkt in die für sie wahrscheinlich erfolgreicheren Studienfächer gelenkt werden (MESR 2009b).

C'est aussi à ces jeunes bacheliers que nous devons des réponses: s'ils n'ont pas d'informations sur les parcours qui s'offrent à eux, s'ils n'ont pas de données sur leurs chances de réussite dans les différentes filières, ils ne pourront ni mûrir leur choix ni même faire un choix éclairé. (MESR 2009b) ${ }^{178}$

Für beruflich Qualifizierte bedeutet dieses die Lenkung in die STS aber auch primär in den Arbeitsmarkt (MJENR 2002e). Deutlich wird, dass durch neue Portale mit genaueren Informationen über Studiengänge und deren Berufsmöglichkeiten sowie durch eine verstärkte Orientierung innerhalb der Schulen durch extra institutionalisierte Beratungsgespräche dem Individuum geholfen werden soll, die richtige Studienwahl zu treffen (MEN 2009). Interessanterweise wurde an den beruflichen Gymnasien die Orientierung nicht ausgebaut, insofern wohl angenommen wird, dass die Orientierung durch die Berufsausbildung bereits statt gefunden hat. Die Beratung soll auch an den Hochschulorganisationen selbst stattfinden, so dass Studienbewerber_innen bereits vorher ihr Profil an die Hochschulen schicken können oder zu Informationsgesprächen eingeladen werden, in welchen geprüft wird, ob ein Studium unter ihrenVoraussetzungen an der Universität anzuraten wäre (MEN 2008b). Auch wenn die Hochschulen keine Selektion zu Beginn durchführen dürfen, ist dies ein Weg, mögliche, Problemfälle‘ bereits vor Studienbeginn , auszusortieren'.

Mit dieser Politik gehen folgende Konsequenzen einher. Studienmisserfolg wird erstens stärker individualisiert, da genügend Informationen zu einer richtigen Wahl hätten führen müssen (CPU 2011; MEDEF 2011; MENSR 2006b). Zweitens wird durch eine gezielte (Ab-)Lenkung von Studierenden in die als jeweils passend erscheinenden Studiengänge oder den Arbeitsmarkt nicht die Möglichkeit eingeräumt, dass sich die tatsächliche Passung zwischen Studium und Individuum erst im Studium zeigt.

178 „Es sind vor allem die jungen Abiturient_innen, denen wir Antworten schulden: wenn sie keine Informationen über die Bildungswege angeboten bekommen, wenn sie keine Statistiken über ihre Erfolgswahrscheinlichkeiten in den unterschiedlichen Fächern zur Verfügung haben, können sie ihre [Bildungs-, NB]Wahl weder entwickeln noch eine aufgeklärte Entscheidung treffen.“ (MESR 2009b, Übersetzung NB) 
Im universalistischen Diskursstrang wird die Rolle von Beratung und Information ebenso unterstrichen. Allerdings zielen hier diese Strukturen eher auf den Abbau von Ungleichheiten in der Gesellschaft, also von Informationsasymmetrien. So sind es häufig die Absolvent_innen der beruflichen Bildungsgänge, welche nicht genug über die Möglichkeiten der Weiterbildung entweder direkt oder auch später informiert sind. Insofern soll eine bessere Information dazu führen, dass auch eine Selbstzensur der Absolvent_innen, welche sich ein Studium nicht zutrauen, abgebaut wird. Am Beispiel des lycée des métiers wird dieses verdeutlicht: In dieser Organisation können die beruflich Qualifizierten durch die Vielzahl der Abschlussmöglichkeiten sich besser über Bildungsoptionen informieren und somit auch die Vorstellungskraft über die eigenen Bildungsmöglichkeiten erweitern (MDEP 2002; ADIUT 2009a).

Eine Voraussetzung dafür, dass Bildungsmöglichkeiten überhaupt erkannt werden, stellt die ,Lesbarkeit ${ }^{`}$ der Bildungsgänge dar. Hier wird betont, dass Reformen notwendig sind, um diese in einem so differenzierten Bildungssystem für alle Individuen zu erhöhen (MDEP 2000a, d). Auch eine Transparenz der Bildungswege ist eine Möglichkeit zu weiterer Bildung zu motivieren und eine Selbstzensur zu verhindern (MESR 2009a).

Rendre lisible les parcours, c'est combattre une des discriminations sociales sur laquelle butent nos publics. La culture de projet, la capacité à prévoir, à s'auto-orienter n'est pas, quoi qu'on en pense une vertu si bien partagée. L'opacité, l'anonymie parfois, sont d'authentiques facteurs de discrimination sociale. (MDEP 2000a) ${ }^{179}$

Dabei sei es besonders wichtig, diese Informationen über die Anschlussfähigkeit beruflicher Bildungsgänge bereits im collège zu vermitteln, um die Negativselektion in die beruflichen Bildungsgänge nach dem collège vorzubeugen (MDEP 2002).

Schließlich wird im universalistischen Diskurs ebenfalls gefordert, dass insbesondere über Bildungsmöglichkeiten, wie das D.A.E.U., zum Nachholen des Abiturs oder über die Verfahren der Validierung, das VAE, besser informiert und beraten werden muss, um bestehende Ungleichheiten zu verhindern (CGT 2012; MESR 2008c).

\section{Studienorganisation}

Sowohl im universalistischen als auch im funktionalistischen Diskursstrang wird die Bedeutung einer stärkeren Flexibilisierung der Studienorganisation über Fernoder Teilzeitstudium, E-Learning unterstrichen. Im universalistischen Diskursstrang wird betont, dass auf diese Weise ebenfalls den unterschiedlichen Lernrhytmen der Individuen besser entsprochen werden kann (CPU 2011). Auch eine

179 „Bildungswege lesbar zu machen, bedeutet auch soziale Diskriminierung, die sich in der Gesellschaft festgesetzt hat, zu bekämpfen. Eine Kultur zu planen, die Fähigkeit vorauszuschauen, sich selbst zu orientieren, ist nicht, wie man annehmen würde, eine weitverbreitete Tugend. Die Undurchsichtigkeit und zum Teil die Anonymität sind wesentliche Gründe für soziale Diskriminierung.“ (MDEP 2000a, Übersetzung NB) 
Vereinbarkeit von beruflicher Weiterbildung und Arbeitstätigkeit ist durch eine stärkere Entstandardisierung der Bildungsorganisation gewährleistet. Auf diese Weise werden bestimmte Gruppen auch bei Weiterbildungsentscheidungen inkludiert und nicht durch starre Regelungen benachteiligt.

Il faut une université-pôle de ressources et de formation pour toute la société, accueil-
lant aussi bien ceux qui peuvent étudier à plein temps que ceux qui - notamment
parce qu'ils travaillent - doivent gérer au plus près leur temps disponible. Une univer-
sité doit être organisée pour qu'on puisse facilement revenir s'y former dès que l'on
en a besoin. (MENJR 2002e) (180 $^{\text {(MEN }}$

Im funktionalistischen Diskursstrang dient die Flexibilisierung der Erhöhung des Fachkräftepotenzials und ist daher unterstützenswert. Insgesamt wird betont, dass die Einführung der Modularisierung und der Leistungspunktesysteme Faktoren sind, welche einer weiteren Flexibilisierung helfen (MJENR 2002e). Im Rahmen des funktionalistischen Diskurses wird zudem darauf hingewiesen, dass die Studienorganisation des technischen Kurzstudium an den STS am ehesten für die studierwilligen beruflich Qualifizierten sei, da diese noch stark an die schulischen Strukturen angelehnt ist und keine große Autonomie von den Studierenden im Vergleich zu einem Universitätsstudium verlangt wird (MENRT 1999a).

\section{Pädagogik}

Nicht nur die Studienstruktur, sondern ebenfall die pädagogische Ausrichtung bzw. pädagogische Konzepte werden im Durchlässigkeitsdiskurs als wichtig für die Ermöglichung eines erfolgreichen Studiums beruflich Qualifizierter angesehen. Im Rahmen des funktionalistischen Diskursstranges wird ähnlich wie bei der Studienorganisation darauf hingewiesen, dass die Sektionen der technischen Hochschulbildung in den Oberschulen (STS) das am meisten an die Bedürfnisse der beruflich Qualifizierten angepasste pädagogische Konzept aufweisen. Dieses ist gekennzeichnet durch relativ kleine Gruppen, eine enge Betreuung über das ganze Studium hinweg. Zudem ist das Studium an STS weniger theorie- dafür stärker praxisorientiert, was auch den Fähigkeiten von beruflich Qualifizierten besser entspräche (ADIUT 2009a, MESR 2011b). Durch die Diversifizierung der Hochschulbildung in Frankreich wird somit im funktionalistischen Diskurs eine klare Zuteilung von Absolvent_innen der Sekundarstufe II zu den jeweiligen Bildungsorganisationen vorgenommen und als funktional angesehen. Die damit aber auch einhergehende Einschränkung von Weiterbildungsmöglichkeiten und Karrierewegen wird nicht thematisiert.

180 „Es bedarf einer Universität, als ein Ressourcen- und Bildungspool für die gesamte Gesellschaft, die sowohl diejenigen, die Vollzeit studieren können, als auch diejenigen, die - insbesondere, weil sie arbeiten - ihre verfügbare Zeit stärker einteilen müssen, aufnimmt. Eine Universität muss so organisiert sein, dass man sich ohne Probleme dort bilden kann, sobald es notwendig ist." (MENJR 2002e ; Übersetzung NB) 
Zusätzlich sollen Brückenkurse beruflich Qualifizierte auf ein Studium vorbereiten und bestehende Defizite abbauen, denn eigentlich werden Absolvent_innen des Berufsabiturs auf den direkten Eintritt in den Arbeitsmarkt vorbereitet und nicht für den Weg in die Hochschulen (ADIUT 2009a). Insofern sollen bestehende individuelle Defizite durch Einführungskurse gemindert werden, um die Erfolgsaussichten dieser Studierendengruppe zu verbessern. Problematisch ist diese Konstruktion insbesondere dann, wenn die Individuen durch die Einführungskurse als different zu den anderen Studienanfänger_innen durch z.B. extra Klassen stigmatisiert werden (ADIUT 2009a). Zudem wird damit deutlich vor allem auch in der Charakterisierung der Berufsabiturient_innen, welche in einem Pilotprojekt an IUTs studiert haben, dass das berufliche Abitur - auch wenn es diesen Namen trägt - kein hochschulvorbereitendes Zertifikat darstellt (ADIUT 2009a).

[...] de plus, l'équipe pédagogique [...] a été confrontée à des problèmes de comportement fréquents (incapacité à rester en place, irrespect, impossibilité de demander du travail personnel, ...). (ADIUT 2009a) ${ }^{181}$

Im funktionalistischen Diskursstrang wird zudem auch eine Veränderung der Pädagogik an den Universitäten gefordert, um die Attraktivität der licence-Studiengänge zu erhöhen, jedoch zielen diese Änderungen weniger auf beruflich Qualifizierte, als auf die Studierenden aus dem allgemeinen und technischen Abiturzweig. Deutlich wird jedoch auch, dass durch Veränderungen, wie die Einführung kontinuierlicher Leistungskontrollen, schneller auf Problemstudierende reagiert und diese dann dementsprechend beraten oder in passendere Hochschulorganisationen umorientiert werden sollen (MESR 2010a).

Im universalistischen Diskursstrang steht die Umorientierung bei Fragen der Pädagogik weniger im Vordergrund, vielmehr sollen die Potenziale der Studierenden geweckt sowie entdeckt und sie befähigt werden, sich im Rahmen des Studiums weiterzuentwickeln (MESR 2011b). Vor allem die Fähigkeiten beruflich Qualifizierter werden in der Erarbeitung der Studiengänge nicht entsprechend berücksichtigt. Dies müsse sich ändern (FSU 2011)

Les bacheliers d'aujourd'hui ont des acquis différents qui sont insuffisamment pris en compte dans l'élaboration des formations. Cela exige une réflexion sur la diversification des filières et sur les contenus, des moyens pour mettre en œuvre des innovations pédagogiques permettant la réussite, un effort particulier en direction des baccalauréats technologiques et professionnels, mais aussi un accès élargi à la formation continue des salariés et demandeurs d'emploi, ainsi qu'à la VAE. (FSU 2011) ${ }^{182}$

181, „...] zudem sind die Lehrkräfte [...] häufig mit Verhaltensproblemen konfrontiert (Unfähigkeit still zu sitzen, fehlender Respekt, Unmöglichkeit selbstständig Aufgaben zu lösen, ...).“ (ADIUT 2009a, Übersetzung NB)

182 „Abiturient_innen verfügen heutzutage über ganz unterschiedliche Fähigkeiten, die nur unzureichend bei der Erarbeitung von Bildungsgängen berücksichtigt werden. Dies erfordert eine Reflexion über die Diversifizierung der Fächer und ihrer Inhalte, Mittel um pädagogische 
Die veränderte Pädagogik, welche gefordert wird, zielt insofern vor allem auf eine stärkere Begleitung der Studierenden, eine individuelle Betreuung, die Abkehr von einem Studium, welches in großem $\mathrm{Ma}$ auf Vorlesungen beruht, hin zu stärkerer Arbeit in Tutorien und Kleingruppen. Voraussetzung, dass eine derartige Reform auch erfolgreich ist, ist generell eine höherer Anerkennung und Wertschätzung der Lehre an den Universitäten, welche sich auch finanziell zeigen kann (ADIUT 2011). Auf diese Weise sind ebenfalls die Lehrenden motiviert, alle Studierenden mitzunehmen.

Im universalistischen Diskurs wird zudem betont, dass auch die STS sich mit ihrer Pädagogik auf eine größere Zahl beruflich qualifizierter Studierender einstellen müssen (MESR 2010a). Dessen ungeachtet sollen STS nicht zu Hochschulorganisationen werden sollen, die nur Berufsabiturient_innen aufnehmen, sondern auch die Absolvent_innen der anderen Abiturzweige (CGT 2005b).

Chacun connaît le succès de cette filière de formation qui est synonyme d'insertion, mais aussi de première marche vers la poursuite d'études. Elle doit aujourd'hui s'adapter pour conduire davantage de jeunes vers la réussite, en particulier en répondant mieux à la demande d'un nombre croissant de bacheliers technologiques et professionnels de poursuivre des études supérieures. (MESR 2010a) ${ }^{183}$

Nicht nur an den STS, sondern auch an den Universitäten ist eine Umstellung notwendig. So soll allgemein eine stärker propädeutische Ausbildung im ersten Studienjahr dazu führen, dass alle Studierenden durch die Sicherung eines entsprechenden Ausgangsniveaus ebenfalls eine Chance auf ein erfolgreiches Studium haben.

\section{Finanzierung}

Fragen der Finanzierung des Studiums spielen in Frankreich in Bezug auf Durchlässigkeit keine signifikante Rolle. Eine Unterstützung bei der Finanzierung wird für alle Studierenden als notwendig erachtet und gilt als Voraussetzung, sich voll und ganz auf das Studium zu konzentrieren (FSU 2006, 2011). Wie berufliche Weiterbildung in Form eines Studiums finanziell unterstützt wird, war nicht Teil des Diskurses. Zudem gelten für berufliche Abiturient_innen die gleichen Unterstützungsmöglichkeiten wie für die des allgemeinen und technischen Abiturs.

Insgesamt nimmt im Verlauf des zweiten Untersuchungszeitraums die Frage des Umgangs mit der Heterogenität der Studierenden zu, wobei sie von Beginn

Innovationen, die einen Studienerfolg begünstigen, umzusetzen, einen besonderen Einsatz für die technischen und beruflichen Abiturient_innen aber auch einen erweiterten Zugang zur Weiterbildung sowie zum VAE für Angestellte und Arbeitssuchende.“ (FSU 2011, Übersetzung NB)

183 „Jeder kennt den Erfolg dieses Bildungsgangs [STS, NB], der als Synonym gilt für einen Arbeitsmarkteinstieg aber ebenfalls die erste Stufe zu einem weiteren Studium darstellt. Um die Jugendlichen zum Studienerfolg zu bringen, muss er sich anpassen, insbesondere um besser auf die Anforderungen der steigenden Zahl technischer und beruflicher Abiturient_innen, die studieren wollen, gerecht zu werden.“ (MESR 2010a, Übersetzung NB) 
an präsent war. Im Zeitverlauf werden zudem auch politische Programme wie „réussite en licence" aufgesetzt, um die Reformen ebenfalls in den Hochschulen durchzusetzen. Deutlich ist, dass im funktionalistischen Diskursstrang ein Hauptaugenmerk auf der Beratung und Information der Studierenden liegt, damit diese an die für sie passenden Hochschulorganisationen gelangen und dort mit einer höheren Wahrscheinlichkeit erfolgreich studieren. An dieser Stelle soll entsprechend der Differenzierung des Hochschulsystems ebenfalls eine differenzielle Pädagogik - zugeschnitten auf die unterschiedlichen Zielgruppen weiterentwickelt werden. Durch einen derartigen Fokus wird zwar auf die Heterogenität der Studierenden eingegangen, diese soll aber wenn möglich gleichzeitig durch die $\mathrm{Zu}$ teilung zu passenden Organisationen aufgehoben werden, so dass letztlich die Reformen zu einer verringerten Heterogenität in den einzelnen Hochschulorganisationen führen sollte. Gleichzeitig wird in diesem Diskursstrang den unterschiedlichen Abiturient_innen auf Grundlage ihrer Vorbildung eine Befähigung zum Studium zugeschrieben, welche als stabil gilt.

Im universalistischen Diskursstrang steht dagegen die Befähigung aller Studierenden in der jeweiligen Hochschulorganisation selbst im Fokus. Zudem sollen Information und Beratung dazu beitragen, dass Bildungsmöglichkeiten überhaupt erst wahrgenommen werden, welche möglicherweise durch ein begrenztes Wissen über Bildungsmöglichkeiten im individuellen Umfeld nicht bekannt waren. Auf diese Weise soll Selbstzensur aufgrund der gesellschaftlichen Zuschreibungen oder Unwissenheit verhindert werden. Zusammengefasst werden die Argumente in Tabelle 29 dargestellt.

\section{Akteurspositionen und Zusammenfassung}

Nachdem die Struktur der Diskursstränge verglichen wurde, sollen in dem folgenden Abschnitt die untersuchten Akteure in Relation zu den identifizierten Diskurssträngen gebracht werden. Wer ist hauptsächlicher Träger welchen Dis-kursstranges? Zudem sollen in der Zusammenschau die wichtigsten Ziele, die in den Diskurssträngen rekonstruiert werden konnten, und die aus den Strukturen herausgearbeiteten Strategien verglichen und damit auch zusammengefasst werden.

\section{Akteurspositionen}

Bevor die Akteurspositionen für den zweiten Untersuchungszeitraum in Frankreich dargestellt werden, soll zuvor auf zwei Punkte aufmerksam gemacht werden. Erstens ergibt sich wie auch im deutschen Fall aus dem in dieser Arbeit dargestellten Diskursverständnis, dass einzelne Akteure nicht direkt ebenfalls Träger eines Diskursstranges sind, sondern ihre Aussagen zumeist auch Fragmente anderer Diskursstränge beinhalten. Insofern werden die untersuchten Akteure auf einem Kontinuum zwischen den identifizierten Diskurssträngen verortet. Dabei ist im Vergleich zu Deutschland auffällig, dass es viel schwieriger ist, Akteure zu 
verorten, da die Verbindung beider Perspektiven im Diskurs sehr stark ist, wobei manche Aspekte eher stärker aus universalistischer Perspektive (Zugang) und andere stärker aus funktionalistischer Perspektive (Umgang mit Heterogenität) argumentiert werden. Um die Positionen trotzdem graphisch darzustellen, verwende ich wiederum die positional map nach Clarke (2012). In dieser kann abgelesen werden, wo sich die Akteure am ehesten positionieren lassen. Die Zuordnung der Akteure erfolgt über den gesamten Zeitraum und in Zusammenschau aller Aussagen. Die einzige Ausnahme bilden die Bildungsministerien, bei denen unterschieden wird, ob die Aussagen zu Zeit einer konservativen oder sozialistischen Regierung stattfanden, da sich hier Unterschiede gezeigt haben. Die x-Achse zeigt den Verlauf von eher funktionalistischer zu einer universalistischen Argumentationslogik und die y-Achse, ob die Positionen der Akteure mehrheitlich reformorientiert pro Durchlässigkeit oder gegen Durchlässigkeit sind.

Zweitens und das macht den französischen Fall insbesondere im Vergleich zum deutschen so besonders, ist die Tatsache, dass kein klarer Gegendiskurs zum reformorientierten Diskurs identifiziert werden konnte. Nun könnte behauptet werden, dass wenn immer wieder das Berufsabitur als Arbeitsmarkteinstiegszertifikat konstruiert wird, dies ein Teil eines solchen Diskurses sei. In der vorliegenden Arbeit wird dies nicht so gewertet, da in den untersuchten Aussagen Hinweise auf einen klaren Gegendiskurs darüber hinaus fehlten. Dieser müsste sich - außer in dem Hinweis, dass Berufsabiturient_innen durch ihre berufliche Ausrichtung primär in den Arbeitsmarkt integriert werden sollten - auch darin äußern, dass beruflich Qualifizierten der Zugang zur Hochschule verwehrt bleiben soll, dass Durchlässigkeit zwischen Berufs- und Hochschulbildung sich negativ auf die Entwicklung im Bildungssystem in Frankreich auswirken würde, oder dass Anrechnung nicht möglich wäre. Die Frage von Integration von Berufs-, Hochschulbildung wäre ebenfalls kein Thema oder würde negativ bewertet werden. Dies war in den Dokumenten der untersuchten Akteure jedoch nicht der Fall. Es scheint, dass derartige Positionen zumindest im bildungspolitischen Diskurs nicht legitim sind. Daher wurde der identifizierte reformorientierte Diskurs als hegemonial beschrieben.

Trotzdem wurde auf den Achsen der positional map (vgl. Abbildung 11), in welcher die Akteurspositionen dargestellt werden, zwischen stärker reformorientierten und Durchlässigkeit ablehnenden Positionen unterschieden, wobei die untersuchten Akteure alle im Feld der reformorientierten Positionen verortet werden können. Akteure, die stärker in Richtung Durchlässigkeit ablehnende Positionen verortet wurden, zeichneten sich durch eine stärkere Betonung des Arguments, dass beruflich Qualifizierte in den Arbeitsmarkt einmünden sollten oder einer nicht Thematisierung des Durchlässigkeitsproblems, aus. Dabei wird an der y-Achse unterschieden zwischen vollständig (5), sehr deutlich (4), deutlich (3), mehrheitlich (2), vorwiegend (1) reformorientiert bzw. durchlässigkeitskritisch und an der x-Achse zwischen (fast) vollständig (3) deutlich (2) und vorwiegend (1) funktionalistisch bzw. universalistisch unterschieden. 
Tabelle 29 Vergleich der Diskursstränge zur Frage des Umgangs mit Heterogenität in Frankreich (1999-2012)

\begin{tabular}{|c|c|c|}
\hline & universalistisch & funktionalistisch \\
\hline Motiv & $\begin{array}{l}\text { Unterstützung ins und im Studium für } \\
\text { alle zur Sicherung des Rechts auf Bil- } \\
\text { dung }\end{array}$ & $\begin{array}{l}\text { Selektion der Studierenden in die pas- } \\
\text { senden Organisationen, (An-)passung } \\
\text { der Klientel an Hochschulstrukturen, } \\
\text { um effizientes Arbeiten zu ermögli- } \\
\text { chen, Attraktivität von Universität } \\
\text { erhöhen }\end{array}$ \\
\hline $\begin{array}{l}\text { Beratung/ } \\
\text { Information }\end{array}$ & $\begin{array}{l}\text { Information über Aufstiegschancen zur } \\
\text { Vermeidung einer starken Selbstselek- } \\
\text { tion/Selbstzensur - in z.B. lycée des } \\
\text { métiers, } \\
\text { Voraussetzung: Lesbarkeit der Bil- } \\
\text { dungsgänge und ihrer Aufstiegsmög- } \\
\text { lichkeiten (Selbstzensur verhindern) } \\
\text { Auch Unterstützung für Prozeduren } \\
\text { wie DAEU bzw. VAE, um Benachteili- } \\
\text { gungen abzubauen }\end{array}$ & $\begin{array}{l}\text { Aktive Orientierung (Beratungs- und } \\
\text { Informationssysteme) für eine Len- } \\
\text { kung beruflicher Abiturient_innen in } \\
\text { die STS } \\
\text { spezifische Studienberatung im bac } \\
\text { géneral und techno } \\
\text { Verantwortung liegt beim Individuum }\end{array}$ \\
\hline $\begin{array}{l}\text { Studien- } \\
\text { organisation }\end{array}$ & $\begin{array}{l}\text { Studienorganisation: } \\
\text { Flexibilisierung der Studienorganisa- } \\
\text { tion um unterschiedlichen Lernrhyt- } \\
\text { men gerecht zu werden } \\
\text { Entstandardisierung von Bildungswe- } \\
\text { gen: durch Teilzeit-, Fernstudium för- } \\
\text { dern }\end{array}$ & $\begin{array}{l}\text { Flexibilisierung: } \\
\text { berufsbegleitende Bildungsgänge, } \\
\text { Teilzeit, Fernstudium } \\
\text { Organisationsstrukturen vor allem in } \\
\text { STS an beruflich Qualifizierte ange- } \\
\text { passt }\end{array}$ \\
\hline Pädagogik & $\begin{array}{l}\text { Anpassung bzw. Umstellung von Pä- } \\
\text { dagogik an die unterschiedlichen Be- } \\
\text { dürfnisse nicht nur an STS auch an } \\
\text { Universitäten } \\
\text { Stärker propädeutische Ausrichtung } \\
\text { im ersten Studienjahr an Universitäten } \\
\text { Pädagogisches Konzept: innovative } \\
\text { personalisierte Lösungen und Hilfe, } \\
\text { Tutorien, Kleingruppen, Begleitung, } \\
\text { (höhere Wertschätzung von Lehre) }\end{array}$ & $\begin{array}{l}\text { Einführungskurse/Brückenkurse um } \\
\text { Erfolg zu sichern } \\
\text { Durch Diversifizierung des Systems } \\
\text { passende Pädagogik für beruflich } \\
\text { Qualifizierte an STS } \\
\text { Und um Attraktivität des licence-Studi- } \\
\text { engangs zu erhöhen: bessere Päda- } \\
\text { gogik wie kontinuierliche Leistungs- } \\
\text { kontrollen, Tutorien }\end{array}$ \\
\hline Finanzen & $\begin{array}{l}\text { Nicht explizit für beruflich Qualifizierte, } \\
\text { sondern generell für alle notwendig, } \\
\text { um gutes Studieren zu ermöglichen }\end{array}$ & \\
\hline
\end{tabular}

Quelle: Darstellung der Verfasserin

Wie bereits angedeutet, ist es für den französischen Durchlässigkeitsdiskurs und auch für die Aussagen der einzelnen Akteure typisch, dass in großem Maß eine universalistische mit einer funktionalistischen Perspektive verbunden wird. Allein in den wenigen Aussagen der Wirtschaftsakteure wird deutlich stärker funktionalistisch zu Durchlässigkeit argumentiert. 
Ebenfalls noch stärker funktionalistisch als universalistisch, wenn auch nicht deutlich, kann die Position der Hochschulakteure eingeschätzt werden. Es sind die Vertreter_innen der Universitäten (CPU) und der technischen Hochschulen (ADIUT), welche - auch wenn sie allgemein ein Recht auf das Studium für alle Abiturient_innen anerkennen - doch immer wieder deutlich machen, das Berufsabiturient_innen nicht zu ihren Organisationen passen und ein größeres Misserfolgsrisiko darstellen. Daher sollten beruflich Qualifizierte durch bessere Information oder auch Vorbereitung vor allem an die technischen Sektionen der Oberschulen (STS) studieren können, jedoch nicht bei ihnen. Sie vertreten das Verständnis, dass es durch die diversifizierte Hochschullandschaft in Frankreich eine klare funktionale Zuteilung von Abiturient_innen gibt, wobei vor allem die Absolvent_innen des allgemeinbildenden Zweigs als Klientel bevorzugt werden. Diese versprechen eine höhere Erfolgswahrscheinlichkeit und erhöhen die Attraktivität der eigenen Organisationsform. Beruflich Qualifizierte interessieren lediglich als Berufserfahrene über Verfahren der Validierung ihrer praktischen Kompetenzen. Dessen ungeachtet sind es insbesondere die Universitäten, die deutlich machen, dass sie durch ihre republikanische Tradition des selektionsfreien Zugangs versuchen, allen Studierenden ein erfolgreiches Studium und eine persönliche Weiterentwicklung zu gewährleisten. Daher sind es vor allem die Universitäten, die zu einer deutlichen Demokratisierung des Bildungswesens in Frankreich beigetragen haben und dies weiterhin tun. Allerdings sollten die Universitäten nicht verpflichtet sein, die Studierenden aufzunehmen, die eigentlich gar nicht an die Universität wollen. Für diese Gruppen sollten die selektiven Studiengänge an STS und IUT stärker geöffnet werden. Aus Sicht der Universitäten wird damit auch klar deutlich, dass sie ihr Image, dass der licence Studiengang nur die zweite Wahl ist, auch durch eine bessere Pädagogik verändern wollen, welche die Diversität der Lernenden, welche aber möglichst nur aus den technischen und allgemeinbildenden Abiturzweigen kommen, stärker berücksichtigt. Die IUT dagegen versuchen sich im Diskurs vornehmlich auch von den ebenfalls beruflich ausgerichteten STS abzugrenzen. Eine Einschränkung ihrer Autonomie bei der Selektion ihrer Studierenden wird nicht als sinnvoll erachtet, da die bestehende Leistungsselektion zu Beginn des Studiums zu den bisherigen Erfolgen der IUT führt. Durch den erhöhten Aufwand, welcher bei der Betreuung der Berufsabiturient_innen gesehen wird, werden diese stärker als Ballast gesehen, welche die Abschlussquoten verringern. Insofern wird der Diskurs auch strategisch von den Hochschulakteuren genutzt ihre Position in der Hierarchie der Studienabschlüsse in Frankreich zu behaupten oder zu verbessern, wobei die Aufnahme von Berufsabiturient_innen als nachteilig und prestigemindernd konstruiert wird.

Die Aussagen der untersuchten Gewerkschaften (CGT/FSU) können im Gegensatz dazu stärker zu dem universalistischen Diskursstrang zugeordnet werden. In ihre Aussagen nehmen insbesondere das Recht auf Bildung und Weiterbildung einen großen Platz ein. Zudem soll die Gleichwertigkeit des beruflichen Zweigs 
gestärkt werden. Berufliche Bildungswege sollen nicht als Wege für die leistungsschwächeren Schüler_innen konstruiert werden und insgesamt viel stärkere Möglichkeiten der Weiterbildung im Studium bieten. Dafür müsste auch ein Berufsabitur zu derartigen Fähigkeiten führen, dass ein Studium erfolgreich abgeschlossen werden kann. Insofern wurden von Gewerkschaftsseiten insbesondere die Reform der Dauer des baccalauréat professionnel von vier auf drei Jahre in hohem $\mathrm{Maß}$ kritisiert als Sparmaßnahme der Regierung, welche den Individuen eher schadet als nützt. Zudem wird von Gewerkschaftsseite eine zu starke Ausrichtung auf den Arbeitsmarkt als ungünstig für die individuellen Entwicklungsmöglichkeiten dargestellt. Für diese ist ein hohes Niveau an Allgemeinbildung notwendig, welches ebenfalls durch die Verkürzung des bac pro verringert wird. Aber auch die spezifischen Fähigkeiten, welche im Rahmen einer technischen und beruflichen Ausbildung erworben werden sollten in der Gesellschaft stärkere Wertschätzung erfahren und zudem in den Studiengängen als Leistungsreservoir anerkannt werden.

Auch in den Aussagen der Bildungsministerien, namentlich unter sozialistischer Regentschaft, wird die Bedeutung der Aufwertung beruflicher Bildungszweige als wichtig anerkannt. Durchlässigkeit und damit Aufstiegschancen im Bildungssystem innerhalb dieses Weges werden dementsprechend als besonders wichtig erachtet. Wie in der positional map ersichtlich, können die Aussagen der Bildungsministerien insgesamt stärker dem universalistischen Diskursstrang zugerechnet werden, während aber in den Aussagen zu Zeiten konservativer Regierungen (2002-2012) einerseits die primäre Ausrichtung des Berufsabiturs auf den Arbeitsmarkt stärker unterstrichen wird, wird zu Zeiten der sozialistischen Regierung (1999-2002 und 2012) stärker auf die Notwendigkeit der Demokratisierung und der Ermöglichung eines Studium für alle Abiturient_innen betont. Diese Argumente lassen sich zudem in den Aussagen der konservativen Regierung finden, jedoch liegt der Fokus in der Bildungspolitik hier zum einen stärker auf einer funktionalen Lenkung der Lernenden entsprechend ihrer Fähigkeiten, welche ihnen anhand ihrer Sekundarabschlüsse zugeschrieben werden. Zudem wird durch die Verkürzung des Abiturs, das einerseits einer größeren Gleichwertigkeit zwischen den Zweigen der Oberstufe führen soll, gleichzeitig die Vorbereitung beruflicher Abiturient_innen auf die Hochschule verkürzt, während die Reformen, die eine verstärkte Orientierung in die Hochschulen in der Sekundarstufe II einführen nur für das technische und allgemeine lycée gelten. Im Gegensatz zum ersten Zeitraum werden jedoch auch Berufsabiturient_innen als Zielgruppe der Hochschulbildung anerkannt, wobei dies für die besten unter ihnen gilt. Diesen soll insbesondere durch den Weg in die STS und der Möglichkeit der Umorientierung aus den Universtäten, bei einem erfolgreichen Abschluss geholfen werden. Ähnliche Argumente finden sich in den Aussagen der sozialistischen Regierung, aber diese betonen noch stärker den Bedarf, auch berufliche Abiturient_innen zu einem Studium zu motivieren, über Bildungsmöglichkeiten zu in- 
formieren und bestehende Mechanismen der Selbstselektion oder sozialen Benachteiligung zu verringern. Die Verbindung zwischen sozialer Benachteiligung und den verringerten Bildungschancen beruflich Qualifizierter in Frankreich wird stärker als Problem thematisiert, während unter konservativer Führung das Problem der Studienabbrüche behoben werden soll. Da aber mehr beruflich Qualifizierte als früher in die Hochschule wechseln und von dem Problem des Studienabbruchs besonders betroffen sind, sind sie ebenfalls eine Zielgruppe, um die sich die Politik kümmern muss. Dies gilt umso mehr, wenn politische Bildungsziele, dass $50 \%$ eines Jahrgangs einen tertiären Abschluss erhalten sollen, zu erfüllen sind.

Abbildung 11 Positional map der französischen Akteure (1999-2012)

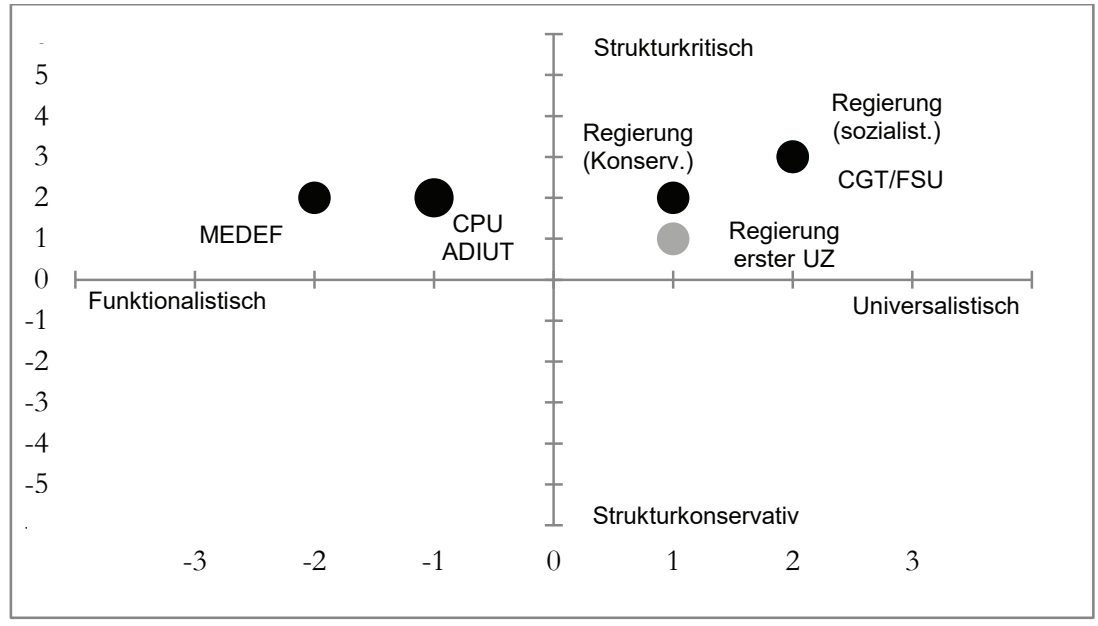

Quelle:Darstellung der Verfasserin

Im Vergleich zum Diskurs vor 1999, welcher allein die Position der Bildungsministerien beinhaltet, ist jedoch eindeutig sowohl von sozialistischer als auch von konservativer Regierungsseite ein Wandel hin zu einer stärkeren Wahrnehmung der Gruppe der Berufsabiturient_innen festzustellen. Dass mit dem beruflichen Abitur ebenso ein Recht auf ein Studium verbunden ist und dass es die Aufgabe der Regierung ist, dieses zu gewährleisten, wird erst seit dem zweiten Zeitraum im Diskurs deutlich. Im ersten Zeitraum wurde aber kein Diskurs, welcher explizit gegen Durchlässigkeit argumentierte, gefunden. Insofern kann die Position der Regierung vor 1999 als noch reformorientiert gelten, da die Notwendigkeit angesehen wird, beruflich Qualifizierten, wenn auch auf dem Weg der Validierung Zugang zu Hochschulabschlüssen zu gewähren.

Insgesamt wird deutlich, dass im französischen Diskurs Durchlässigkeit stark an ein universalistisches Bildungsverständnis geknüpft ist, jedoch im Fall der be- 
ruflich Qualifizierten oft im Zusammenhang mit funktionalistischen Vorstellungen einer zertifikatsbasierten Selektion in bestimmte Bildungsgänge einhergeht. Auf diese Weise wird die bestehende Hierarchie zwischen den Bildungszweigen in Frankreich festgeschrieben wird.

\section{Zusammenschau der Diskurse und der rekonstruierten Strategien}

In diesem Abschnitt sollen die aus den Diskurssträngen rekonstruierten diskursspezifischen Ziele und Strategien sowie die differenten Verantwortungszuschreibungen gegenübergestellt werden. Diese Zusammenschau dient dazu, die beiden Diskursstränge, welche in Frankreich für den Zeitraum 1999-2012 modelliert wurden, in ihrer Typik zusammenzufassen. Da sich jedoch in Frankreich keine zwei unterschiedlichen Diskurse, sondern nur Diskursstränge innerhalb des reformorientierten Diskurses gegenüberstehen, werden weniger Konfliktlinien als im deutschen Fall deutlich, die sich im Anschluss in Strategien niederschlagen könnten.

Der universalistische Diskursstrang hat seine Wurzeln in den republikanischen Idealen Frankreichs, welche immer wieder betont werden. Insbesondere die Ermöglichung von Chancengleichheit durch das institutionalisierte Recht auf Bildung und damit auch das Ziel der Demokratisierung des gesamten Bildungssystems stechen hervor. Bildung dient dabei sowohl der individuellen Persönlichkeitsentfaltung, der demokratischen Bürgererziehung und der Emanzipation der Individuen als auch zur Lösung gesellschaftlicher Probleme wie sozialer Ungleichheit und Diskriminierung. Ziel ist es, über Durchlässigkeit zu einer stärkeren gesellschaftlich anerkannten Gleichwertigkeit zwischen Berufsbildung und insbesondere der höheren Allgemeinbildung zu gelangen und damit traditionelle Hierarchien zu verringern sowie jedem Individuum zu ermöglichen, sich so hoch wie möglich zu bilden.

Erreicht werden soll Durchlässigkeit als eine Form des Rechts auf Bildung primär über Berechtigungen: Beispiele sind das Recht auf Hochschulzugang über das Abitur, das Recht auf Weiterbildung durch klar institutionalisierte Anrechnungsverfahren, wie das VAE-Verfahren, das Recht auf Aufnahme an den Universitäten durch den institutionalisierten selektionsfreien Zugang sowie die rechtliche Einschränkung der Bewerberauswahl eigentlich selektiver Hochschulorganisationen wie den STS und IUT. Durchlässigkeit soll gefördert werden durch eine stärkere Information der beruflichen Lernenden über Bildungsoptionen, um Formen der negativen Selbstselektion oder fehlende Information durch das individuelle Umfeld zu kompensieren. Diese können z.B. durch die Verbindung von Oberschulen und Hochschulen, wie beim lycée des métiers, besonders hilfreich sein, da die direkten Bildungswege innerhalb des eigenen Lernumfeldes erfahren werden. Zudem sind im gesamten Bildungssystem Verbindungen zwischen den Bildungsgängen zu installieren, welche eine ,Umorientierung im Laufe des Lernprozesses unkompliziert ermöglichen. Schließlich sollen Unterstützungsstrukturen 
aufgebaut werden, welche den spezifischen Bedürfnissen der Bildungsteilnehmer_innen gerecht werden. Neben Informationen gehören auch eine flexibilisierte Lernorganisation und eine auf die Heterogenität der Individuen angepasste Pädagogik dazu, welche ein individuelles Lernen ermöglichen. Insbesondere bei letzteren sollen die spezifischen Kompetenzen, die beruflich Qualifizierte in den Lernprozess mitbringen, wesentlich besser für das weitere Lernen genutzt werden. Wichtig ist, dass Durchlässigkeit nicht nur für eine bestimmte Gruppe institutionalisiert werden soll, sondern, dass jeder das Recht hat, sich so weit wie möglich weiterzubilden. Dabei sollen alle Individuen im Verlauf der Erst- aber auch der Weiterbildung unterstützt und motiviert werden.

Im funktionalistischen Diskursstrang wird Durchlässigkeit primär als funktionale Notwendigkeit konstruiert, zur Lösung gesellschaftlicher Probleme, wobei den Bedarfen des Arbeitsmarkts eine zentrale Rolle zukommt. Das Individuum wird zur Ressource und Bildung wird ein Weg zur Absicherung individueller Risiken wie Arbeitslosigkeit und gesellschaftlicher Ziele wie Wirtschaftswachstum. Durchlässigkeit soll dabei aber nicht für jeden ermöglicht werden, sondern für diejenigen, welche wahrscheinlich auch den nächsten Bildungsschritt erfolgreich absolvieren. Bei Zugangsfragen wird insofern eine Selektion basierend auf Leistung als notwendig erachtet. Neben dieser Selektion ist eine weitere Voraussetzung, dass entsprechend der unterschiedlichen angenommenen Leistungsdifferenzen auch ein differenziertes Hochschulsystem besteht, das den Individuen gemäß ihrer Vorbildung die passende Weiterbildung ermöglicht. Die Heterogenität der Studierenden innerhalb einzelner Hochschulorganisationsformen soll demnach deutlich geringer gehalten werden, als wenn alle frei ihren Studiengang wählen könnten. Dies soll die Effizienz der Bildungsgänge erhöhen. Dabei werden für beruflich Qualifizierte insbesondere die beruflich orientierten Studiengänge an den Oberschulen als am angemessensten angesehen. Durchlässigkeit für beruflich Qualifizierte bedeutet im funktionalistischen Diskursstrang, Zugang zu den STS zu erhalten. Ermöglicht werden soll dies durch eine gezielte Information und ,aktive Orientierung' der Berufsabiturient_innen, eine Zusammenarbeit der STS mit den Berufsklassen der Sekundarstufe, um geeignete Potenziale früh zu erkennen, sowie eine Umlenkung derjenigen, die in anderen Bildungsgängen zu scheitern drohen in ebendiese stärker beruflich orientierten Bildungsgänge. Um eine Passung zwischen Hochschule und Lernenden zu vergrößern, sollen Strukturen eingerichtet werden, welche die Lernenden auf das notwenige Niveau des Studiengangs bringen. Da durch die Berechtigungen, welche das Abitur mit sich bringt und den Zugangsregelungen an den französischen Hochschulen, keine klare Zuteilung möglich ist wie nach dem collège, liegt die Verantwortung der richtigen Passung zwischen Studierenden und Hochschule bei beiden Akteuren. Primär sind es die Individuen, welche sich mithilfe von ihnen erreichbaren Informationen über ihre zukünftigen Bildungswege entscheiden und dann auch selbstverantwortlich für die Konsequenzen ihrer Entscheidung sind. Da aber gleichzeitig 
auch die beruflich orientierten Hochschulen, die für sie passenden Bewerber wählen, kommt ihnen ebenfalls die Verantwortung zu, die richtigen auszulesen und ihre Lehre auf diese Gruppe auszurichten.

Betrachtet man den Diskurs insgesamt, ist festzustellen, dass die Frage, warum über Durchlässigkeit zwischen Berufs- und Hochschulbildung gesprochen wurde, zumeist mit universalistischen Vorstellungen beantwortet wurde. Betrachtet man in diesem Zusammenhang allerdings, welche Formen von Durchlässigkeit in den Diskursen sichtbar werden bzw. wie Durchlässigkeit umgesetzt werden soll, sind eher die funktionalistischen Vorstellungen, die eine stärkere Selektion und damit einhergehend eine Segregation der Studierenden entsprechend ihrer Vorbildung in die unterschiedlichen Bildungszweige des differenzierten Hochschulsystems fordern.

\subsection{Analyse des institutionellen Wandels}

In diesem Abschnitt folgt analog zum deutschen Fall eine Bewertung der Veränderungen der nationalen Durchlässigkeitsstrukturen in Frankreich von 1985 bis 2012/2013. Betrachtet werden dabei nicht nur die Veränderungen auf der kulturell-kognitiven Dimension sowie auch zu einem geringeren Anteil auf der normativen Dimension, welche im Rahmen der Diskursanalyse offengelegt wurden, sondern auch regulative Veränderungen in diesem Zeitraum, um ein umfassenderen Einblick in die Änderungen der Durchlässigkeitsstrukturen zu erhalten. Ein Überblick über die rechtlichen Entwicklungen im Vergleich der Zeiträume findet sich in Tabelle $44 \mathrm{im}$ Anhang. Der Einbezug der regulativen Dimension ist wichtig, um einzuschätzen, inwiefern die vorgefundenen Veränderungen in den Wissensvorräten sich auch in den rechtlichen Rahmenbedingungen und damit ebenfalls viel stärker den Opportunitätsstrukturen der Individuen widerspiegeln bzw. inwiefern auch die regulativen Möglichkeiten auf der kulturell-kognitiven Ebene wahrgenommen werden. Lionel Jospin fasst es so zusammen:

Il faut se garder de l'illusion bien française qui voudrait qu'un texte législatif suffise à régler les problèmes. Cette loi serait lettre morte, si elle n'était pas portée par la volonté, par l'accord de tous ceux qui, au sein de l'Ecole, ou avec elle, devront quotidiennement la mettre en œuvre. Une évolution ne se décrète pas, elle se construit. (MENJS 1989b : P10) ${ }^{184}$

184 „Man muss sich vor der typisch französischen Illusion hüten, die annimmt, dass ein Gesetzestext ausreicht, um Probleme zu lösen. Dieses Gesetz wird aber wertlos sein, wenn es nicht auch durch den Willen und der Zustimmung derjenigen getragen wird, die in der Schule oder zusammen mit dieser das Gesetz tagtäglich umsetzen müssen. Wandel lässt sich nicht per Dekret veranlassen, ein Wandel muss erarbeitet werden.“ (MENJS 1989b: P10, Übersetzung NB) 
Auf Grundlage der ganzheitlichen Betrachtung der Veränderungen soll analysiert werden, inwiefern diese zu einer Fortführung eingeschlagener Entwicklungspfade, ihrer Veränderung oder sogar einer gänzlichen Abkehr historisch gewachsener Institutionen geführt haben.

Eine Weiterführung würde für den französischen Fall bedeuten, dass die klare Hierarchisierung zwischen den Bildungszweigen erhalten bleibt und dass Hochschulbildung primär über den Weg der Allgemeinbildung erreicht wird. Gleichzeitig bedeutet jedoch eine Weiterführung auch die weitere sukzessive Niveauerhöhung der beruflichen Bildung (vgl. Schriewer 1982) und deren weitere Integration in das nach den Prinzipien des allgemeinbildenden Zweigs funktionierenden Bildungswesen. Eine Abkehr von den historisch gewachsenen Prinzipien würde eine Gleichwertigkeit oder gar Höherbewertung beruflicher Bildung im Bildungswesen bedeuten und eine problemlose Durchlässigkeit für alle beruflich Qualifizierten in das Hochschulsystem. Eine Veränderung institutioneller Entwicklungspfade dagegen bedeutet eine weitgehende Beibehaltung zentraler Prinzipien im Bildungswesen aber mit eindeutigen Veränderungen.

Anders als im deutschen Fall ist eine Bewertung der französischen Entwicklung weniger eindeutig. Denn es konnte gezeigt werden, dass sich insbesondere die kulturell-kognitiven Strukturen hin zu einer stärkeren Durchlässigkeit im Sinne einer Anerkennung beruflich Qualifizierter der Sekundarbildung als Zielgruppe der Hochschullandschaft entwickelt haben. Jedoch blieben typische Kernprinzipien, wie die klare Hierarchie zwischen beruflichen Bildungswegen im Sekundarbereich und der Allgemeinbildung, weiterbestehen. Zudem knüpfen ebenfalls Veränderungen, wie die Verkürzung des Berufsabiturs auf drei Jahre, die eigentlich zu mehr Gleichwertigkeit und damit einer Enthierarchisierung führen sollen, gleichzeitig an die paradoxe Entwicklung des französischen Bildungssystems an, in welcher letztlich die zunehmende Integration beruflicher Bildungswege in die Strukturen des allgemeinbildenden Systems zu deren Entwertung und einer Verringerung von Durchlässigkeit führen.

Nachfolgend sollen demgemäß die Veränderungen der institutionellen Strukturen zu mehr Durchlässigkeit als auch die Fortführung institutioneller Entwicklungspfade dargestellt werden. Dabei werden auch mögliche Barrieren für Durchlässigkeit, welche mit der Weiterführung oder auch Veränderung von Strukturen einhergehen, herausgearbeitet. Im Gegensatz zum deutschen Fall werden die Veränderungen und Weiterentwicklungen zusammen dargestellt, da zum Teil Veränderungen in Richtung Durchlässigkeit die Weiterentwicklung bestimmter Pfade bedeuten können. Zudem können, da es in Frankreich das klassische Schisma zwischen Berufs- und Hochschulbildung nicht gibt, Barrieren viel stärker sowohl in den Veränderungen als auch in der Kontinuität institutioneller Strukturen verortet werden. 


\subsubsection{Veränderungen institutioneller Durchlässigkeitsstrukturen und Fortfuihrung institutioneller Entwicklungspfade}

Wie kann man nun die institutionellen Entwicklungen in Frankreich im Hinblick auf Durchlässigkeit zusammenfassen? Betrachtet man die Relevanz des Themas Durchlässigkeit zwischen beruflicher Bildung und Hochschulbildung ist im Vergleich der Untersuchungszeiträume eine eindeutige Bedeutungszunahme des Themas festzustellen. Drehten sich die sehr wenigen Aussagen zu Durchlässigkeit vor 1998 vornehmlich um den Zugang beruflich Erfahrener zur Hochschule über Validierungsprozesse, so änderte sich dies deutlich im zweiten Zeitraum. Durchlässigkeit zur Hochschule nicht nur von Berufserfahrenen, sondern auch von Absolvent_innen des beruflichen Bildungszweigs des Sekundarschulwesens wurde zu einem Thema, mit dem sich die französischen bildungspolitischen Akteure in differenten Kontexten (vgl. Kapitel 9.2.1) beschäftigen.

In beiden Zeiträumen konnte ein hegemonialer Diskurs, in welchem Reformen für größere Durchlässigkeit befürwortet werden, rekonstruiert werden. Der Diskurs besteht jeweils aus einem universalistischen und funktionalistischen Diskursstrang. Die rekonstruierten Argumentationslogiken ähneln sich im Vergleich der Zeiträume, wobei durch den Fokus auf Berufsabiturient_innen und deren Probleme im Hochschulbereich weitere Argumentationsmuster hinzugekommen sind. Typisch für den universalistischen Diskursstrang ist in beiden Zeiträumen die Betonung von Chancengleichheit und dem Recht auf Bildung, welches durchgesetzt werden müsse, um den Idealen der französischen Republik gerecht zu werden. Bildung dient dabei vor allem der Persönlichkeitsentwicklung und demokratischen Bürgererziehung. Im funktionalistischen Diskursstrang stehen in beiden Zeiträumen die Bedarfe des Arbeitsmarkts im Vordergrund. Bildung dient der Erhöhung des Humankapitals, welche notwendig ist, um den Veränderungen durch Strukturwandel, internationalen Wettbewerb auf gesellschaftlicher und individueller Ebene zu begegnen. Neu ist vor allem im funktionalistischen Diskurs die Argumentation, dass durch Durchlässigkeit die berufliche Bildung aufgewertet wird und dass Durchlässigkeit, wenn sie zu einem erfolgreichen Abschluss führt, auch die Attraktivität der Universitäten erhöht. Insgesamt überwiegen allerdings universalistische Begründungen.

Wie veränderte sich das Verständnis, die Wissensvorräte zu Durchlässigkeit? Im ersten Zeitraum wurde Durchlässigkeit von beruflich Erfahrenen lediglich im Zusammenhang mit dem Zugang zur Hochschule und der Anrechnung beruflicher Erfahrungen thematisiert. Im zweiten Zeitraum gab es deutliche Änderungen. Wenn über Durchlässigkeit zwischen Berufs- und Hochschulbildung gesprochen wird, dann im Zusammenhang mit Fragen des Zugangs, der Anrechnung, aber auch der organisationalen Verbindungen und im besonderen Maße über den Umgang mit heterogenen Bedürfnissen. Die wichtigsten Änderungen sowie Kontinuitäten im Diskurs sowie den entsprechenden rechtlichen Regelungen werden für die Aspekte nachfolgend zusammengefasst. 


\section{Zugang}

Für den ersten Zeitraum konnte insbesondere in Bezug auf Fragen des Zugangs eine starke Diskrepanz zwischen der regulativen und der im Diskurs rekonstruierten normativen und kulturell-kognitiven institutionellen Dimension festgestellt werden. Während durch die Institutionalisierung des Berufsabiturs 1985 auch ein Weg in die Hochschulen über berufliche Sekundarbildung eingeführt wurde, werden im Diskurs bis 1998 dessen Absolvent_innen im Zusammenhang zu Durchlässigkeit nicht erwähnt. Das Berufsabitur gilt als Arbeitsmarkteinstiegszertifikat und allein beruflich Erfahrene gelten als legitime Studierende.

Diese Diskrepanz verringert sich im zweiten Zeitraum, da auch Berufsabiturient_innen das Recht auf ein Studium aufgrund des Abiturs zugestanden wird und diese sogar partiell motiviert werden sollen, Weiterbildungsmöglichkeiten zu erkennen und wahrzunehmen. Andererseits wird im funktionalistischen Diskursstrang auch weiterhin die berufliche Ausbildung primär als Einstieg in den Arbeitsmarkt betrachtet. Berufsabiturient_innen stellen somit noch immer nicht die klassische Zielgruppe hochschulischer Bildung dar. Dies liegt insbesondere auch daran, dass der berufliche Bildungszweig im Sekundarschulwesen weiterhin als der Bildungsbereich gilt, in welchem die Schüler_innen mit Leistungsproblemen einmünden. Durch dieses angenommene (fehlende) Leistungsniveau werden im funktionalistischen Diskursstrang insofern nur die besten Absolvent_innen des Berufsabiturs als studierfähig eingeschätzt. Zudem werden Berufsabiturient_innen im funktionalistischen Diskursstrang als Gruppe konstruiert, welche lediglich in beruflich orientierten Hochschulorganisationen, den STS, ein Studium absolvieren sollen, weshalb diese Organisationen auch verstärkt diese Gruppe aufnehmen solle. Es lässt sich somit zeigen, dass sich die Diskrepanz zwischen der regulativen und kulturell-kognitiven sowie normativen Dimension zwar verringert hat, aber noch immer besteht, da im funktionalistischen im Gegensatz zum universalistischen Diskursstrang nicht die gleichen Bildungsmöglichkeiten mit dem beruflichen Abitur wie mit dem technischen und allgemeinbildenden verbunden werden.

Im Gegensatz zum funktionalistischen Diskursstrang wird allerdings im universalistischen Diskursstrang auf die Frage, inwiefern das Berufsabitur auch für ein Hochschulstudium befähigt, die Lösung konstruiert, dass, wenn es ein Abitur sein soll, dieses auch mit der Vermittlung eines notwendig hohen Allgemeinbildungsniveaus verbunden sein muss. Zudem muss ein Ziel darin bestehen, die gesellschaftliche Wertschätzung beruflicher Bildungswege zu erhöhen, um somit auch die Negativselektion in diese zu verhindern. Die Verkürzung des Berufsabiturs von vier auf drei Jahre ist dabei eine Reform, welche zwar die Gleichwertigkeit zwischen den Abiturzweigen durch eine gleiche Dauer symbolisieren soll, aber sich gleichzeitig negativ auf die Erfolgschancen beruflich Qualifizierter im Studium auswirken könnte. Mit dieser Verkürzung wird den Berufsabitu- 
rient_innen, welche bereits eine in Bezug auf ihre bisherigen Lernergebnisse negativ selektierte Gruppe sind, die nötige Zeit zur Vermittlung von Allgemeinbildung genommen, die für eine Vorbereitung auf ein Studium in Frankreich als besonders wichtig gilt. Diese Reform kann im Sinne institutioneller Kontinuität als ein weiterer Schritt identifiziert werden, die berufliche Bildung in die Strukturen analog zu denen des allgemeinbildenden Systems zu konstruieren, was gemeinhin in Frankreich mit dem Ziel verbunden war, die Gleichwertigkeit der Bildungswege und die Chancengleichheit der Bildungsteilnehmer_innen zu erhöhen. Gleichzeitig kann auch diese Integration als eine negative Entwicklung für Durchlässigkeit angesehen werden, da notwendige Lernzeiten gekürzt werden.

In den rechtlichen Regelungen gab es kaum Veränderungen, allerdings wurde zum Schluss des zweiten Untersuchungszeitraums die Selektionsautonomie der beruflichen Hochschulorganisationen zugunsten der Absolvent_innen des bac pro und bac techno an STS und IUT eingeschränkt, so dass durch festzulegende Quoten auch beruflich Qualifizierte aufgenommen werden müssen, während bisher stärker die Absolvent_innen der anderen Abiturzweige bevorteilt wurden (Loi ESR 2013).

Insgesamt können somit größere Veränderungen, die ebenfalls mit einer Verringerung von Barrieren für Durchlässigkeit einhergehen, aber auch Kontinuitäten festgestellt werden. Als wichtiger Schritt in Richtung Durchlässigkeit kann die größere Selbstverständlichkeit, dass auch beruflich Qualifizierte studieren, gewertet werden. Andererseits bleibt die traditionelle Hierarchisierung zwischen beruflichen und technischen und allgemeinen Bildungswegen bestehen, welche bereits durch die Selektion in die berufliche Bildung der schwächeren Schüler_innen in der Sekundarstufe beginnt. Dies wird als größte Barriere für Durchlässigkeit anerkannt. Königsweg in Frankreich bleibt eindeutig der allgemeinbildende. Um diese Absolvent_innen wird daher auch im Hochschulbereich gebuhlt. Die Ungleichwertigkeit der Bildungswege reproduziert sich dann in der anvisierten Eingrenzung der Bildungsmöglichkeiten für beruflich Qualifizierte im Hochschulsystem im funktionalistischen Diskurs.

Die Gefahr einer so eindeutigen Zuteilung besteht darin, dass wenn letztlich die Studierenden an den STS sich primär aus den beruflichen Abiturient_innen rekrutieren, dieses zu einem „Reststudium“ wird, analog zu dem beruflichen Bildungszweig im Sekundarbereich. Eine Mischung der Studierenden wäre anzustreben, um einer Stigmatisierung und Devaluierung des BTS-Abschlusses zu verhindern. Ansonsten wäre zwar Durchlässigkeit in den Tertiärbereich gewährleistet, diese wäre jedoch nicht notwendigerweise verbunden mit sozialer Durchlässigkeit, wenn man davon ausgeht, dass die Absolvent_innen der anderen Abiturzweige in besser bewertete Studiengänge wechseln. 


\section{Anrechnung}

Die Evolution im Hinblick auf Anrechnung kann in Frankreich als eindeutig durchlässigkeitsfördernd festgestellt werden. Insbesondere die gesetzlichen Entwicklungen von dem Validierungsverfahren VAP zum Verfahren des VAE, welches nicht nur berufliche, sondern auch informelle Erfahrungen anerkennt (vgl. Loi Modern 2002) machen dies deutlich. Anrechnung soll beruflich Erfahrene zu einem Hochschulabschluss führen und wird in beiden Zeiträumen als relevant anerkannt, wobei Fragen der Anrechnung im zweiten Zeitraum im Vergleich zu den anderen Aspekten weniger thematisiert wurden. Deutlich wird aber in beiden Zeiträumen die Anerkennung beruflicher Kompetenzen als gleichwertig zu den im formalen Bildungssystem erworbenen.

Neben den Validierungsverfahren wird auch die Einführung von Semestern, Leistungspunktesystemen als vorteilhaft für Verfahren der Anerkennung angesehen. Insofern ist auch diese Entwicklung als durchlässigkeitsfördernd einzuschätzen.

\section{Organisationale Verbindung}

Im französischen Durchlässigkeitsdiskurs werden organisationale Verbindungen zwischen Berufs- und Hochschulbildung erst im zweiten Zeitraum im Hinblick auf Durchlässigkeitsfragen diskutiert. Auch hier wird ähnlich wie bei Fragen des Zugangs zum Teil eine Diskrepanz zwischen der bestehenden regulativen Dimension und der kulturell-kognitiven sichtbar. Der Ausbau einiger Formen organisationaler Verbindungen, die im Diskurs thematisiert werden, hatte bereits vorher angefangen und wurde vor allem im ersten Untersuchungszeitraum verstärkt. So besteht seit 1987 (Loi Seguin) in allen beruflich orientierten Bildungsgängen, auch im Tertiärsystem, die Möglichkeiten einen Abschluss en alternance zu erwerben. Diese Form der Integration wird im zweiten Zeitraum als vorteilhaft anerkannt, da er den Fähigkeiten und Lernweisen bereits beruflich Qualifizierter besser entspricht.

Weiterhin wurden die beruflich orientierten Bildungsgänge STS bereits in den 1960er Jahren gegründet und auch in den 1990er Jahren zusammen mit den IUT ausgebaut. In den 1990er Jahren wurde der Ausbau aber speziell damit begründet, dass die Bildungsgänge stärker für die Absolvent_innen des technologischen Abiturzweigs, die auch immer stärker in die Hochschulen kamen, ausgebaut werden sollten. Berufliche Abiturient_innen wurden nicht erwähnt. Dies änderte sich deutlich im Diskurs im zweiten Zeitraum, in welchem vor allem im funktionalistischen Diskurs Berufsabiturient_innen, wenn sie ein Studium aufnehmen, vor allem an den STS studieren sollen, da die berufliche Ausrichtung im Studium am besten ihren Fähigkeiten entspräche.

Wie bereits bei der Erörterung der Vorstellungen zum Zugang deutlich wurde, bringt eine derartige Zuordnung von Studiengängen zu Abschlussgruppen 
der Sekundarbildung nicht nur Vorteile, wenn sie zu einer Entwertung der Studiengänge durch eine zu große Homogenisierung führt. Es wird zudem deutlich, dass die beruflichen Wege vor allem für die als nicht traditionell gesehenen Studierendengruppen betrachtet werden. Im ersten Zeitraum und davor waren es die technischen Abiturient_innen und im zweiten die beruflichen. Andererseits, wenn die Studiengänge, die berufliche und akademische Elemente verbinden, weiterhin als attraktiv gelten und weitere Bildungsoptionen im Anschluss ermöglichen, kann dies trotzdem ein wertvoller Weg für Durchlässigkeit und eine Aufwertung beruflicher Bildung sein.

Andere Formen der Integration wurden erst im zweiten Zeitraum eingeführt, so dass auch regulativ eine Veränderung hin zu einer größeren Durchlässigkeit festzustellen ist. Insbesondere die lycées des métiers werden als ein wichtiger Schritt für Durchlässigkeit von beruflicher Sekundar- zur Hochschulbildung anerkannt, da mögliche Bildungsoptionen innerhalb einer Organisation für die Lernenden sichtbar werden und damit auch zur Weiterbildung motivieren können.

Neu sowohl in der regulativen als auch der kulturell kognitiven Dimension im Hinblick auf Durchlässigkeit sind die verbesserten Verbindungen zwischen den Studiengängen (die sogenannten passerelles), welche im Diskurs vor allem als Möglichkeit einer frühzeitigen Korrektur oder, Umorientierung` während des Studiums gesehen werden, um einen erfolgreichen Studienabschluss zu begünstigen. Dies gilt für alle Studierenden, aber auch besonders für beruflich Qualifizierte, die viel stärker von Studienabbrüchen betroffen sind. Die Einführung von Studienzyklen, Leistungspunkten und Semestern helfen dabei, einen leichteren Übergang zu ermöglichen, wobei dieser durch extra Einführungskurse an den aufnehmenden Organisationen sowie einer Anerkennung von bereits Gelerntem einhergeht. Institutionalisierte Übergänge können somit als Möglichkeiten gelten, ein erfolgreiches Studium durch Korrekturen der Studienfachwahl für alle Studierenden und somit auch die beruflich Qualifizierten zu ermöglichen und damit auch als wichtige Entwicklung für Durchlässigkeit gelten.

Während regulativ allerdings passerelles in jede Richtung institutionalisiert wurden, wird im Diskurs vor allem die ,Umorientierung' ${ }^{6}$ von den allgemeinen in die beruflichen Studiengänge vorgesehen. Problematisch wird dies dadurch, dass es wiederum zu einer Entwertung beruflicher Bildungsgänge kommt, da diese diejenigen aufnehmen sollen, die ein stärker theoretisch ausgerichtetes Studium nicht schaffen. Eine Folge könnte dann eine Fortschreibung der Hierarchisierung zwischen allgemeinbildenden und beruflichen Bildungsgängen im Tertiärbereich sein.

\section{Umgang mit heterogenen Bedürfnissen}

Eine deutliche Veränderung zwischen dem ersten und dem zweiten Zeitraum kann im Hinblick auf die Thematisierung des Umgangs heterogener Bedürfnisse als Voraussetzung für Durchlässigkeit konstatiert werden. Während im ersten 
Zeitraum solche Überlegungen nicht präsent im Diskurs zu Durchlässigkeit waren, zeigten sie sich im zweiten Zeitraum als wichtiger Bestandteil. Dabei ist allerdings anzumerken, dass die zunehmende Heterogenität Studierender bereits im ersten Zeitraum diskutiert wurde, allerdings nicht in Bezug auf beruflich Qualifizierte. Partiell werden daher im zweiten Zeitraum nur bestehende Diskurse auf die neue Gruppe von (Problem-)Studierenden ausgeweitet.

So waren Fragen der Anpassung der Pädagogik an eine neue Klientel, die nicht mehr nur von den klassischen Gymnasien kommt, bereits Thema der Reform Bayrou von 1997 (Arrêté Bayrou 1997). Mit dieser wurden Tutorien und z.B. Kurse zur Einführung ins wissenschaftliche Arbeiten eingeführt. Als Weiterführung dieser Reform können dann die mit dem Plan ,réussite en licence“ eingeführten Maßnahmen gesehen werden: verstärkte kontinuierliche Kontrollen, bessere Betreuung, Kleingruppenunterricht. Ziel ist es und das wird auch im Diskurs betont, die Studierenden so zu unterstützen, dass sie einen erfolgreichen Abschluss schaffen.

Ein besonderer Fokus im zweiten Zeitraum liegt bei Fragen der Heterogenität vor allem auf einer besseren Beratung und Information, so dass die Phase der sogenannten Orientierung zu einer individuell richtigen Studienwahl führt. Im Diskurs war vor allem die funktionalistische Version die bestimmende, in welcher Beratung und Information genutzt werden sollen, beruflich Qualifizierte entweder weg vom Hochschulsystem oder in die technischen Studiengänge an den Oberschulen (STS) zu lenken, da dort die Erfolgswahrscheinlichkeiten am größten sind. Die Wahl wird zwar durch Beratung unterstützt, aber letztlich ist das Individuum verantwortlich und zwar auch für negative Konsequenzen. Durch die Lenkung beruflich Qualifizierter nur in die STS wird deutlich, dass das bac pro nicht als gleichwertig zu den anderen Abiturzweigen angesehen wird, die sich durch eine breitere Studienwahl auszeichnen. Während demnach Beratung und Information zwar genutzt werden sollen, um den individuellen Bedürfnissen und Wünschen gerecht zu werden, wird gleichzeitig offenbar, dass tatsächlich weniger die individuellen Wünsche als Erfolgschancen und die Form des Abiturs entscheidend sind, welche Studienangebote als passend empfohlen werden.

Auch regulativ wurden die verstärkte Beratung und Informationsstrukturen institutionalisiert, wobei sie an den allgemeinen und technischen Gymnasien jedoch nicht an den beruflichen Extra-Beratungsstunden für die Studienorientierung eingeführt wurden. Zweierlei wird daraus ersichtlich: zum einen zeigt sich wiederum, dass beruflich Qualifizierte im Vergleich zu den Absolvent_innen der anderen Abiturzweige primär in den Arbeitsmarkt einmünden sollen. Zweitens werden Informationsasymmetrien vergrößert, da für die Berufsabiturient_innen, die meist durch ihre benachteiligende soziale Herkunft generell weniger über ihre Bildungsoptionen informiert sind, keine zusätzlich Informationenstrukturen institutionalisiert wurden.

Insgesamt lässt sich zusammenfassen, dass im französischen Diskurs generell als wichtig anerkannt wird, dass es nicht ausreicht, nur den Zugang zum Studium 
zu gewähren, sondern dass weitere unterstützende Strukturen für den Studienerfolg notwendig sind und auch nur dann tatsächlich zu Durchlässigkeit führen. Ein solches Verständnis ist als durchlässigkeitsfördernd zu bewerten. Allerdings wird durch eine stärker funktionalistische Sichtweise auf den Umgang mit heterogenen Bedürfnissen im Diskurs, diese Frage für beruflich Qualifizierte primär auf deren Lenkung in den Arbeitsmarkt oder in die STS begrenzt. Dabei soll das differenzierte System der Hochschulbildung letztlich dazu führen, dass die unterschiedlichen Bildungsgruppen jeweils in die ,passenden' unterschiedlichen Studiengänge einmünden. Die Gefahr dieser Vorstellungen besteht dann darin, dass Studiengänge wie die STS abgewertet werden könnten, da diese als Auffangbecken für die beruflichen Abiturient_innen werden, in welche die anderen Absolvent_innen nicht mehr einmünden wollen. Bestehende Hierarchien aus der Sekundarstufe II würden damit reproduziert.

Insofern kann auch im Hinblick auf diesen Aspekt ein Wandel in Richtung größerer Durchlässigkeit festgestellt werden, wobei gleichzeitig die Durchlässigkeit vor allem auch im dominierenden funktionalistischen Diskurs wiederum eingeschränkt wird und bestehende Hierarchien reproduziert werden.

\subsubsection{Zusammenfassung und Schlussfolgerungen}

Es konnte im Rahmen dieses Kapitels gezeigt werden, dass in Frankreich eine deutliche Veränderung institutioneller Durchlässigkeitsstrukturen zugunsten einer größeren Durchlässigkeit von beruflich Qualifizierten der Sekundarstufe II zum Hochschulsystem stattgefunden hat. Dabei fanden weniger Veränderungen auf der regulativen Dimension als auf der kulturell-kognitiven und normativen Dimension statt, da bereits wichtige Voraussetzungen für Durchlässigkeit regulativ institutionalisiert waren. Die wichtigste Veränderung ist, dass berufliche Abiturient_innen nicht mehr nur ein Recht auf den Hochschulzugang haben, sondern dass dieses auch im Selbstverständnis der bildungspolitischen Akteure angekommen ist. Die Diskrepanz, die vor allem im ersten Zeitraum noch zwischen den einzelnen institutionellen Dimensionen lag, wurde verringert, wenn auch nicht aufgehoben. Im Diskurs wird kontinuierlich deutlich, dass selbst wenn rechtlich das bac pro den anderen Abiturarten gleichgestellt ist, sich dies aber auf den kulturell-kognitiven und normativen Dimensionen nicht widergespiegelt. Typisch für die Vorstellungen zu Durchlässigkeit in Frankreich ist das im Diskurs immer wieder herauskristallisierte Spannungsverhältnis zwischen universalistischen und funktionalistischen Diskursträngen. So sind primär die Begründungen, warum auch beruflich Qualifizierte Zugang zur Hochschulbildung bekommen sollten, stärker durch universalistische Argumentationen geprägt, während die konkreten Vorstellungen, wie Durchlässigkeit für beruflich Qualifizierte aussehen könnte, nicht gänzlich aber doch stärker funktionalistisch angelegt sind. Die Zielgruppe wird auf eine Leistungselite der beruflich Qualifizierten, die wenn überhaupt nur 
in die STS einmünden sollen, begrenzt und homogenisiert dargestellt. Insofern wird die Hierarchisierung zwischen den Bildungszweigen auch im Durchlässigkeitsdiskurs weiterhin reproduziert. Aus diesem Grund sollen in der vorliegenden Arbeit auch die Entwicklungen in Frankreich stärker als eine Weiterführung bestehender Pfade eingeordnet werden, auch wenn sich das französische System stärker in Richtung Durchlässigkeit bewegt hat. Diese Bewegung ist jedoch Teil des französischen Entwicklungspfads. Es kommt zwar zu einer Höherqualifizierung im französischen Bildungssystem, allerdings entspricht diese Entwicklung, ohne Hierarchien wirklich aufzubrechen, einer segregativen Demokratisierung (Merle 2000).

Auch wenn es sich um einen hegemonialen Diskurs in Frankreich handelt, werden dennoch Konflikte bzw. Strategien zur Durchsetzung von Deutungen sichtbar. Dies gilt vor allem für die Akteure der Hochschulbildung, die zwar entsprechend dem französischen Paradox das Recht auf Bildung auch für beruflich Qualifizierte bejahen, aber gleichzeitig diese Zielgruppe nicht bei sich aufnehmen möchte, da ihre Bildungsgänge (an den Universitäten und IUT) zu anspruchsvoll für diese Gruppe von Studierenden seien. Die Gruppe der beruflich Qualifizierten wird dabei homogenisiert und als anders im Vergleich zur ,normalen' Klientel beschrieben, was zu einer weiteren Stigmatisierung beruflich Qualifizierter führt.

Die Gewerkschaften auf der anderen Seite weisen ebenfalls auf die Besonderheit dieser Studierendengruppe hin, allerdings sollen beruflich Qualifizierte bereits durch eine bessere Ausbildung in der Schule und Aufwertung beruflicher Bildung unterstützt werden. Zum anderen werden auch die Hochschulorganisationen und der Staat stärker in die Pflicht genommen, auch diesen Individuen ihr Recht auf Bildung durch zusätzliche Strukturen und eine stärkere Anerkennung beruflichen Leistungsvermögens im Studium zu ermöglichen.

Aufgrund ihrer herausragenden Steuerungsposition im französischen Bildungswesen sind vor allem die Bildungsministerien entscheidende Akteure. Hier konnte festgestellt werden, dass vornehmlich unter sozialistischen Regierungen die Stellung der beruflichen Bildung verbessert und Durchlässigkeit erhöht werden sollte. Konservative Regierungen dagegen, auch wenn sie die universalistischen Grundannahmen in ihren Aussagen betonten, setzen sich stärker für dem funktionalistischen Diskursstrang zuzählende Durchlässigkeitsvarianten ein, welche eher zu einer Reproduktion bestehender Hierarchien der Bildungszweige führen. Insofern wird erstens deutlich, dass insbesondere Politikwechsel in Frankreich einen deutlichen Einfluss auf Durchlässigkeitsstrukturen haben können, da viele Regelungen zentralstaatlich verabschiedet werden. Zweitens können somit auch in Frankreich trotz eines hegemonialen Diskurses Deutungskämpfe über Durchlässigkeit festgestellt werden.

Sichtbar wurde im Hinblick auf die Entwicklungen auch, dass regulative Änderungen nicht gleichzeitig einhergehen müssen mit Änderungen der kulturellkognitiven Dimension. Insofern wird auch im französischen Fall deutlich, wie wichtig eine Analyse der unterschiedlichen institutionellen Dimensionen für die 
Beurteilung nationaler Durchlässigkeitsstrukturen ist. Inwiefern die für Frankreich festgestellten Veränderungen auch durch die Europäisierungsprozesse beeinflusst wurden und ob sich die Strukturen zwischen Deutschland und Frankreich angenähert haben, soll im nun folgenden Kapitel 10 gezeigt werden. 



\section{Durchlässigkeit in Deutschland und Frankreich und der Einfluss europäischer Bildungsprozesse: Die Frage nach der Konvergenz}

In den Länderkapiteln konnte gezeigt werden, welche Aspekte sich jeweils in Deutschland und Frankreich in den letzten Jahren im Hinblick auf die institutionellen Dimensionen zu Durchlässigkeit zwischen beruflicher und Hochschulbildung gewandelt haben. Darauf aufbauend soll in diesem Vergleichskapitel erstens die Frage beantwortet werden, inwiefern sich die institutionelle Ausgestaltung der Bildungssysteme in Frankreich und Deutschland mit dem Wandel angenähert haben. Im Zentrum der Analyse steht hier demgemäß die Sigma-Konvergenz, d.h. eine Verringerung der Varianz der Durchlässigkeitsstrukturen zwischen den Staaten. Diese Frage stellt sich insbesondere, da beide Länder nicht nur Mitgliedsstaaten, sondern auch treibende Kräfte der in dieser Arbeit untersuchten europäischen Bildungsprozesse, dem Bologna- und Kopenhagen-Prozess, sind. Zudem weisen sie sehr unterschiedliche Bildungssysteme mit einem divergenten Verhältnis zwischen beruflicher und Hochschulbildung auf, auf welche die europäischen Prozesse Einfluss nehmen könnten. Es bleibt demzufolge zu untersuchen, inwiefern sich das Verständnis von einem durchlässigen Bildungssystem und dessen diskursive Behandlung wie auch die gesetzlichen Regelungen zu Durchlässigkeit angleichen. Durch diesen Fokus stehen insbesondere die kulturell-kognitive Dimension sowie die regulative Dimension im Vordergrund, wobei in den Diskursen ebenso Normen und Standards gesetzt und damit sichtbar werden. Die vergleichende Analyse von institutionalisierten Durchlässigkeitsstrukturen ist besonders relevant, da auf diese Weise nationale Selbstverständlichkeiten und Regelungen als kontingente Konstrukte aufgedeckt werden können. Kulturelle Unterschiede werden deutlich und nationale Entwicklungen in eine neue Perspektive gerückt.

In einem zweiten Teil dieses Kapitels wird danach gefragt werden, wie diese Entwicklungen zu erklären sind. Welchen Einfluss haben die europäischen Bildungsprozesse Bologna und Kopenhagen? Um die Frage nach dem Einfluss zu beantworten, wird zuerst die Delta-Konvergenz (Holzinger et al. 2007: 17-23) untersucht, d.h. die Annäherung der nationalen Strukturen an europäische Vorgaben. Eine Annäherung kann als erster Hinweis für einen Einfluss Europas gelten. Um diesen Hinweis abzusichern, werden mithilfe der Analyse der Diskurse und durch Expert_inneninterviews die Einflussannahmen hinterfragt und plausibilisiert. Können die Europäisierungsprozesse als Gelingensbedingung für größere Durchlässigkeit angesehen werden? Haben sich durch europäische Prozesse diskursive Kräfteverhältnisse verändert? Welche Rolle spielen dagegen nationale Entwicklungslogiken. Dieser Abschnitt soll daher auch Aufschluss über die potenzielle Wirkmächtigkeit von europäischen Prozessen und deren Begrenzung durch nationale Entwicklungspfade geben. 


\subsection{Konvergenz zwischen den nationalen Bildungssystemen?}

In diesem Teilabschnitt steht die Frage im Zentrum, inwiefern sich die jeweiligen nationalen Entwicklungen in Deutschland und Frankreich zu einer stärkeren Annäherung oder auch Konvergenz der durchlässigkeitsrelevanten Institutionen im Vergleich geführt haben. Wie bereits in der Fallauswahl dargestellt, handelt es sich bei den beiden Ländern Deutschland und Frankreich um deutlich verschiedene Bildungssysteme, deren Differenzen sich auf den unterschiedlichen institutionellen Dimensionen manifestieren und vor allem durch ihr jeweiliges eigenes Verhältnis zwischen beruflicher Bildung und Hochschulbildung gekennzeichnet sind. Trotz der Unterschiede konnte in dieser Arbeit ebenfalls gezeigt werden, dass in beiden Ländern Barrieren zu Durchlässigkeit zwischen beruflicher Bildung (auf Sekundarschulniveau) und Hochschulbildung bestehen. Diese sind zwar unterschiedlich, gehen aber jeweils mit gesellschaftlichen Hierarchisierungen zwischen beruflicher Bildung und höherer Allgemeinbildung einher. Die identifizierten Probleme werden nachfolgend noch einmal kurz zusammengefasst.

In Deutschland besteht das Durchlässigkeitsproblem durch die traditionell gewachsene Segmentierung und ebenfalls gegenseitige Abschottung der Organisationsfelder Berufs- und Hochschulbildung. Diese gewachsenen Strukturen verhindern Bildungszu- und -übergänge von einem Bildungsbereich in den anderen ebenso wie die Anerkennung des Gelernten. Aus diesem Grund werden auch die Absolvent_innen aus dem jeweils anderen Bildungsbereich nicht als legitime oder selbstverständliche Bildungsteilnehmer_innen in der Hochschule oder der Berufsbildung angesehen. Bildungsstrukturen zielen allein auf die ,traditionellen Teilnehmer_innen. Diese strikte Trennung der Bildungsbereiche spiegelt sich ferner in einer weitgehenden Trennung der Arbeitsmarktpositionen wider. Wie in einem „qualificational space“ (Maurice et al. 1986) üblich, sind bestimmte Berufe lediglich über klar definierte Bildungswege zu erlernen. In Deutschland sind dabei oft die hierarchisch höheren Karrrierepositionen über die Hochschulbildung erreichbar. Eine gesellschaftliche Hierarchisierung der Bildungsbereichen wird sichtbar.

In Frankreich besteht das Problem darin, dass berufliche Abiturient_innen viel stärker von Studienabbrüchen und Umorientierungen betroffen sind im Vergleich zu den Abiturient_innen der anderen Bildungszweige. Dies liegt zum einen daran, dass beruflich Qualifizierte geringe Chancen auf den Zugang zu den selektiven beruflichen Hochschulgängen haben, auf die sie potenziell besser vorbereitet wären als auf selektionsfreie Studiengänge an den Universitäten. Zum anderen ist das Berufsabitur nicht als Weg in die Hochschule, sondern auf den Arbeitsmarkt konzipiert, so dass beruflich Qualifizierte nicht adäquat auf ein Hochschulstudium vorbereitet werden und auch nicht als legitime/-r Bildungsteilnehmer_in den Organisationen der Hochschulbildung angesehen werden, was sich auch in 
den bestehenden Bildungsstrukturen der Organisationen, die auf die ,Normalstudierenden' zielen, widerspiegelt. Im französischen Bildungssystem wird damit deutlich, dass Durchlässigkeit nicht nur bedeutet, Wege in die Bildung zu konstruieren, sondern zudem Strukturen zu stellen, die Bildungserfolge ermöglichen.

Die Frage nach der Annäherung der Bildungssysteme im Hinblick auf ihre Durchlässigkeitsinstitutionen wird dabei in zwei Schritten beantwortet. Zuerst wird die ,Ausgangsposition` in Form von Ähnlichkeiten und Unterschieden zwischen den beiden Ländern vor 1999, also vor den untersuchten europäischen Bildungsprozessen dargestellt. In einem zweiten Schritt erfolgt der Vergleich der sich seit 1999 bis 2013 gewandelten Durchlässigkeitsstrukturen in beiden Ländern. Die Vergleiche werden auf Grundlage der ausführlichen Länderanalysen durchgeführt und basieren daher vor allem auf den diskursanalytischen Auswertungen sowie auf den durchgeführten Dokumentenanalysen ${ }^{185}$. Entlang der Durchlässigkeitsaspekte (vgl. Kapitel 2) werden jeweils die wichtigsten regulativen Strukturen sowie die jeweilige diskursive Behandlung des Durchlässigkeitsproblems verglichen, welche die nationalen Selbstverständlichkeiten, Deutungen, Problematisierungen, Argumentationslogiken, aber auch Normen und damit die kulturell-kognitive und zum Teil auch normative Dimension offenlegen. Deutlich werden soll dabei ebenfalls, inwiefern die Entwicklungen dazu beigetragen haben, Barrieren zu Durchlässigkeit abzubauen bzw. Barrieren zu verstetigen.

\subsubsection{Nationale Durchlässigkeitsstrukturen im Vergleich (1985/90-1998)}

Die Zeit vor Beginn der Bologna- und Kopenhagenprozesse ist gekennzeichnet durch eine signifikante Unterschiedlichkeit zwischen Deutschland und Frankreich sowohl in der regulativen als auch in der diskursiven Durchlässigkeitsstruktur. Gerade bei letzterer ist wichtig zu bedenken, dass in Deutschland die Frage nach Durchlässigkeit wesentlich prominenter diskutiert wurde als in Frankreich, wo Durchlässigkeit von beruflicher Bildung in die Hochschulbildung nur ganz am Rande Thema war.

\section{Argumentationslogiken im Vergleich}

Als erster Schritt werden die Argumentationslogiken der untersuchten Diskurse vor 1999 in Deutschland und Frankreich verglichen. In Deutschland war vor 1999 die Diskussion zu Durchlässigkeit stark konfliktbehaftet, was sich auch in den

185 Eine ausführliche Darstellung der Entwicklungen mit den konkreten Verweisen auf die untersuchten Dokumente findet sich in den Länderkapiteln 8 und 10. Für den Vergleich werden die Entwicklungen weitestgehend ohne die konkreten Verweise zu den Dokumenten zusammengefasst. 
zwei Diskursen, dem strukturkritischen und strukturkonservativen Diskurs widerspiegelt. Auch auf Diskursebene wird somit das deutsche Bildungsschisma (Baethge 2006) abgebildet, da in den beiden Diskursen auch ein unterschiedliches Verständnis von Durchlässigkeit und der Bedeutung von beruflicher Bildung im Vergleich zur Hochschulbildung offenbart wird. Dies unterstreicht umso stärker die kulturell-kognitive Wirkmächtigkeit des Bildungsschismas. In Frankreich zeigte sich allein ein Diskurs, welcher eine bestimmte Form der Durchlässigkeit und zwar im Rahmen der beruflichen Weiterbildung befürwortet.

Wenn man die Rahmungen der Diskurse bzw. der Diskurstränge betrachtet, so wird deutlich, dass in Frankreich sowohl universalistisch als auch funktionalistisch für eine größere Durchlässigkeit argumentiert wurde. In Deutschland war der universalistische Diskursstrang, welcher einen Teil des strukturkritischen Diskurses ausmachte, viel weniger stark ausgeprägt. Die Argumente in Frankreich und Deutschland ähnelten sich jedoch. Durchlässigkeit wurde diskutiert als eine Frage der Teilhabegerechtigkeit und Chancengleichheit in der Gesellschaft und eine Voraussetzung, dass das Recht auf Bildung wahrgenommen werden könne.

Aber auch die funktionalistischen Rahmungen für eine größere Durchlässigkeit waren partiell ähnlich. In beiden Ländern wurde konstatiert, dass der Strukturwandel der Wirtschaft dazu führe, dass die Bevölkerung höher qualifiziert werden müsse, um die neuen anspruchsvolleren Arbeiten auszuführen. Gleichzeitig wurde in Deutschland überdies funktional argumentiert, dass durch Durchlässigkeit die berufliche Bildung attraktiver werde, die Wertschätzung in der Gesellschaft ansteige und somit ein Fachkräftemangel in der Berufsbildung umgangen werden könne. Auch wenn in Frankreich bereits vor 1999 eine größere gesellschaftliche Anerkennung beruflicher Bildung gewünscht wurde, so wurde dies nicht mit dem Ziel der Durchlässigkeit verbunden.

Die funktionalistische Argumentation gegen Durchlässigkeit, die betonte, dass beruflich Qualifizierte eine Gefahr für das Funktionieren der Hochschulbildung darstellten, zumal die Hochschulen ausreichend mit dem ,Ansturm' der traditionellen Studierenden durch die Bildungsexpansion beschäftigt seien, fand sich im deutschen strukturkonservativen Diskurs. Und auch das eher funktionalistische Verständnis, das von einer notwendigen Arbeitsteilung nach entsprechender Begabung in der Gesellschaft ausgeht und besagt, dass lediglich eine Leistungselite an die Hochschulen gehöre, war im Zusammenhang von Durchlässigkeit zwischen beruflicher und Hochschulbildung im ersten Zeitraum nur im deutschen Diskurs anzutreffen. Dies lässt sich aber auch durch den sehr begrenzten Fokus des französischen Diskurses auf die Weiterbildung von Berufserfahrenen im ersten Zeitraum erklären.

\section{Vergleich anhand der Durchlässigkeitsaspekte}

Neben den Rahmungen der Diskurse ist jedoch ebenfalls wichtig zu vergleichen, wie Durchlässigkeit geregelt wurde und anhand welcher Aspekte sie diskutiert 
wurde. Womit befassten sich die bildungspolitischen Akteure, wenn sie über Durchlässigkeit gesprochen haben? Ohne auf die genauen Inhalte einzugehen, wird bereits anhand der behandelten Aspekte ein deutlicher Unterschied zwischen Frankreich und Deutschland offenbar. Während in Deutschland die Fragen von Zugang, institutionellen Verbindungen und in geringem Maß der Umgang mit Heterogenität diskutiert wurde, wurde in Frankreich die Frage des Zugangs vornehmlich mit der Frage der Anrechnung verbunden. Nachfolgend sollen nun überdies die genaueren Regelungen und Diskursstrukturen verglichen werden.

\section{Zugang und Anrechnung}

Die Untersuchung der bestehenden rechtlichen Regelungen vor 1999 zeigt besonders große Unterschiede zwischen Deutschland und Frankreich in Bezug auf den Zugang zum Hochschulstudium als auch auf Anrechnungsmöglichkeiten auf. Während in den 1990er Jahren in Deutschland zuerst ein langsamer Ausbau des dritten Bildungswegs in den Bundesländern, d.h. von Zugangsmöglichkeiten zur Hochschule vor allem via Eignungsprüfungen und partiell über ein Probestudium, stattgefunden hatte, existierte in Frankreich durch das Berufsabitur bereits die Möglichkeit, über die Berufsbildung eine Berechtigung für das Hochschulsystem zu erhalten. Den Weg über das Nachholen eines Abiturs gab es in beiden Ländern. Zudem konnten beruflich Qualifizierte in Frankreich bereits seit 1984 über den Weg der Anrechnung beruflicher Erfahrungen ein Studium aufzunehmen. Dieser Weg wurde 1992 erweitert, so dass sogar große Teile eines Studiums angerechnet werden konnten. In Deutschland gab es bis auf die wenig praktizierte im Hochschulrahmengesetz festgehaltene Möglichkeit der Einstufungsprüfung (Wolter/Reibstein 1991: 46), welche als funktionales Äquivalent zur VAP Praktik von 1984 gesehen werden kann, keine Möglichkeiten berufliche Erfahrungen und berufliche Bildungsabschlüsse in einem Hochschulstudium anzuerkennen. In Frankreich war somit eine regulative Durchlässigkeit vom Berufsbildungssystem (auf Sekundarniveau) in Fragen des Zugangs und Anrechnung weitestgehend gewährleistet, während in Deutschland ein rechtlicher Ausbau des Zugangs erst begann.

Bedeutende Unterschiede werden auch in den diskursiven Strukturen zu diesen beiden Aspekten sichtbar. In Deutschland drehte sich der Diskurs insbesondere darum, welche Rolle die höhere Allgemeinbildung für den Zugang zum Studium haben sollte und wie festgestellt werden könne, ob und wann beruflich Qualifizierte die Voraussetzungen mitbringen, studieren zu dürfen. Im Diskurs wurden unterschiedliche Möglichkeiten des Zugangs beruflich Qualifizierter zur Hochschulbildung diskutiert. Einmal sollten Zugangsberechtigungen nach der Erst- oder Aufstiegsfortbildung eingeführt werden, was mit einer Infragestellung des Berechtigungsmonopols der Allgemeinbildung einherging (strukturkritischer Diskurs). Weitere Möglichkeiten wurden in Eignungsprüfungen und schließlich in dem Erwerb von höherer Allgemeinbildung gesehen. In Frankreich drehte sich 
der Diskurs um den Zugang von Erwerbstätigen, welche im Rahmen einer beruflichen Weiterbildung an die Hochschule kommen sollten, und zwar über die Möglichkeit der Anrechnung ihrer beruflichen Erfahrung.

Die konstruierte Zielgruppe und damit auch konstruierte Norm der beruflich Qualifizierten an den Hochschulen waren in Frankreich damit nicht die Berufsabiturient_innen, sondern beruflich Erfahrene in Weiterbildung. Berufsabiturient_innen wurden nicht als legitime Gruppe für die Hochschulen anerkannt. Ihr Weg nach dem Abitur sollte in den Arbeitsmarkt gehen. Hier besteht eine Inkongruenz zwischen den regulativ ermöglichten Zielgruppen und der in den Diskursen konstruierten Norm. In Frankreich wurden die beruflich Qualifizierten, welche sich weiterbilden können sollen, nicht näher charakterisiert. Eine Beschränkung der Gruppe fand nicht statt. Vielmehr wurde deutlich, dass über die Anrechnungsmöglichkeit speziell auch solche Individuen an die Hochschulen gelangen sollten, die bisher keine Chance dazu hatten. In Deutschland variierte die konstruierte Zielgruppe je nach Diskursstrang: zwischen allen beruflich Qualifizierten und einer Elite der Berufsausbildung. Insgesamt kann man aber zusammenfassen, dass die Zielgruppe eher aus besonders gut ausgebildeten beruflich Qualifizierten bestehen sollte, welche ihre Fähigkeiten durch eine abgeschlossene Weiterbildung, den Nachweis eines hohen Allgemeinbildungsniveaus oder einem notwendigen Fachwissenstest nachweisen. In Deutschland ähnelte die so diskursiv konstruierte Zielgruppe noch stark der in den Gesetzen festgehaltenen (vgl. Tabelle 30).

Tabelle 30 Vergleich der durchlässigkeitsrelevanten Zielgruppe für die Hochschulbildung vor 1999

\section{Regulative Strukturen}

\begin{tabular}{lll}
\hline Zielgruppe & FR: alle beruflich Qualifizierten & $\begin{array}{l}\text { FR: keine genauere Beschreibung der } \\
\text { Gruppe, die sich weiterbilden möchte }\end{array}$ \\
& $\begin{array}{l}\text { DE: beruflich Qualifizierte, mit Abitur } \\
\text { (2. Bildungsweg) oder mit Fortbildungs- } \\
\text { abschluss und Leistungsnachweis via } \\
\text { Eignungsprüfung (3. Bildungsweg) }\end{array}$ & $\begin{array}{l}\text { DE: besonders Begabte beruflich Quali- } \\
\text { fizierte, mit nachgewiesenen Leistungs- }\end{array}$ \\
=> deutlicher Unterschied & niveau deutlicher Unterschied \\
\hline
\end{tabular}

Quelle: Darstellung der Verfasserin

Es wird deutlich, dass in Deutschland im Gegensatz zu Frankreich sowohl regulativ als auch diskursiv eine berufliche Qualifizierung auf Sekundarniveau für die Aufnahme eines Studiums nicht ausreichte. Eine Konstruktion von Gleichwertigkeit von beruflicher Bildung und höherer Allgemeinbildung auf Sekundarniveau fand somit nicht statt. Beruflich Qualifizierte, auch wenn sie genauso lange im Bildungssystem verbracht haben konnten wie Abiturient_innen in Deutschland, wurden von den bildungspolitischen Akteuren mehrheitlich nicht als äquivalent fähig für ein Hochschulstudium angesehen. Nur Aussagen der deutschen 
Gewerkschaften bildeten hier eine Ausnahme. Das Nicht-Einbeziehen von Berufsabiturient_innen, sondern allein der Fokus auf Erwerbstätige mit Weiterbildungsambitionen deutet eine ähnliche Ungleichwertigkeit für Frankreich an. Expliziert wurde sie jedoch durch die fehlende Charakterisierung dieser Gruppe nicht. Es scheint, dass Erfahrungen im Berufsleben eine Voraussetzung für einen gleichwertigen Zugang zum Hochschulwesen waren - im Gegensatz zu alleiniger beruflicher Bildung auf Sekundarniveau. Letztlich wurde jedoch berufliche Erfahrung in Frankreich sowohl in den Diskursen als auch in den rechtlichen Regelungen damit auch höher geschätzt als in Deutschland, wo Anrechnung gar keine Rolle spielte.

\section{Institutionelle Verbindung und Umgang mit Heterogenität}

Deutlich ähnlicher als beim Zugang und der Anrechnung sahen die rechtlichen Entwicklungen zu Durchlässigkeit im Hinblick auf institutionelle Verbindungen in Deutschland und Frankreich vor 1999 aus. In beiden Ländern begannen in den letzten Jahren des 20. Jahrhunderts der Ausbau und eine stärkere rechtliche Anerkennung integrierter Bildungsformen. So darf in Frankreich seit 1987 auch im Hochschulwesen en alternance gelernt werden. In Deutschland wuchs gleichzeitig die Zahl der dualen Studiengänge - Abschlüsse an Berufsakademien wurden als tertiäre Abschlüsse anerkannt. Im Diskurs dagegen waren institutionalisierte Verbindungen zwischen Berufs- und Hochschulbildung in Frankreich kein Thema. In Deutschland dagegen wurde in dem Ausbau von dualen Studiengängen ein Lösungsansatz für das deutsche Durchlässigkeitsproblem gesehen.

Die Frage, wie möglichen unterschiedlichen Bedürfnissen von Studierenden, welche eine berufliche Ausbildung oder Berufserfahrung vorweisen, besser begegnet werden könne, wurde in Frankreich nicht und in Deutschland kaum diskutiert. Allein im strukturkritischen deutschen Diskurs wurde dieses Thema gestreift. Gemeinsam ist auch in beiden Ländern, dass bereits vor 1999 Möglichkeiten des Fernstudiums rechtlich institutionalisiert waren. In Frankreich wurde zwar auch in den 1990ern über eine bessere Anpassung der Hochschulen an die gewachsene Zahl der Studierenden und der damit gewachsenen Heterogenität diskutiert, aber nicht explizit im Zusammenhang mit beruflich qualifizierten Studierendenr. Gleiches gilt für die gesetzlichen Regelungen. So wurden Beratungs- und Betreuungsangebote stärker an den Hochschulen gefordert, aber wiederum nicht im Kontext von Durchlässigkeit zwischen Berufs- und Hochschulbildung. In Deutschland dagegen war die Diskussion noch stark auf die Frage fokussiert, ob beruflich Qualifizierte überhaupt an die Hochschulen kommen sollten, so dass es in den Diskursen kaum relevant war, wie ein Studium entsprechend der Bedürfnisse beruflich Qualifizierter gestaltet werden müsste. Diesbezügliche gesetzliche Änderungen fanden auch nicht statt. 


\section{Der Vergleich im Überblick}

Zusammenfassend ist zu konstatieren, dass vor dem Beginn der Bologna- und Kopenhagen-Prozesse die Durchlässigkeitsstrukturen in Deutschland und Frankreich als deutlich unterschiedlich charakterisiert werden können (vgl. Tabelle 31). Der größte Unterschied bestand in der geringen Relevanz des Themas in Frankreich im Vergleich zu Deutschland und dass in Deutschland die Diskurse stark konfliktbehaftet waren. Auch wenn vor allem die universalistische Rahmung der Diskurse Ähnlichkeiten aufzeigten und überdies die Notwendigkeit der Höherqualifizierung der Bevölkerung in beiden Ländern funktional begründet wurde, finden sich keine Übereinstimmungen für weitere Argumentationen, welche besonders in Deutschland wichtig waren, wie die Erhöhung der Attraktivität beruflicher Bildung.

Zudem lassen sich für Durchlässigkeit signifikante Unterschiede in den Aspekten des Zugangs und der Anrechnung finden. Deutlich wird dies in der rechtlichen Ausgestaltung bzw. dem Fehlen eines Berechtigungswesen für den Hochschulzugang über den Weg der Berufsbildung sowie von Anrechnungsmöglichkeiten in Deutschland. Auch wenn in beiden Ländern die Verbindung von Hochschulbildung mit dem Lernen am Arbeitsplatz verstärkt wurde, so wurde dies lediglich in Deutschland als durchlässigkeitsfördernd angesehen. Ähnlichkeiten bestanden sonst nur in den jeweils fehlenden Regelungen und Aussagen zur Anpassung von bestehenden Bildungsstrukturen an die Bedürfnisse beruflich Qualifizierter.

Auch die Barrieren für Durchlässigkeit zwischen Berufs- und Hochschulbildung waren in Frankreich und Deutschland vor 1999 vollkommen unterschiedlich zu verorten. In Frankreich befanden sich die Barrieren weniger in der regulativen institutionellen Dimension. Viel stärker dagegen kann die fehlende Thematisierung der Berufsabiturient_innen als Zielgruppe als normative und kulturellkognitive Barriere angesehen werden. Dies gilt umso mehr, da bereits in den 1990er Jahren Berufsabiturient_innen in Frankreich an die Hochschulen gingen und auch schon zu diesem Zeitpunkt mit dem Problem des Studienmisserfolgs kämpften. Sie wurden aber nicht als legitime Studierende wahrgenommen, denen es zu helfen gilt, da sie in den Arbeitsmarkt einmünden sollten.

In Deutschland bestanden dagegen die Barrieren für Durchlässigkeit in der regulativen, normativen und kulturell-kognitiven Dimension. In der regulativen Dimension wird dieses an den vielfältigen Zugangsbeschränkungen, den fehlenden rechtlich institutionalisierten Anrechnungsmöglichkeiten sowie den fehlenden Maßnahmen, die beruflich Qualifizierte als legitime Studierendengruppe im Studium unterstützt, deutlich. Der/die Normalstudierende war immer noch ein Individuum, welches über die höhere Allgemeinbildung in das Hochschulwesen gekommen ist und nur besonders leistungsfähige, begabte beruflich Qualifizierte wurden als potenzielle Zielgruppe anerkannt. 
Tabelle 31 Unterschiede und Ähnlichkeiten in den nationalen Durchlässigkeitsstrukturen vor 1999

\begin{tabular}{|c|c|c|}
\hline Aspekte & Regulative Strukturen & Diskursive Strukturen \\
\hline \multirow[t]{3}{*}{ Zugang } & $\begin{array}{l}\text { FR: Zugang via Berechtigung (bac } \\
\text { pro) und Anrechnung }\end{array}$ & $\begin{array}{l}\text { FR: Zugang aus dem Berufsleben für } \\
\text { Weiterbildung }\end{array}$ \\
\hline & $\begin{array}{l}\text { DE: fehlendes Berechtigungssystem } \\
\text { und Zugang über uneinheitliche Pra- } \\
\text { xis von Eignungsprüfungen } \\
\text { Aber FR und DE: Zugang über das } \\
\text { Nachholen des Abiturs }\end{array}$ & $\begin{array}{l}\text { DE: Infragestellung des bestehenden } \\
\text { Berechtigungswesens, Zugang über } \\
\text { Leistungstests, aber auch Zugang in } \\
\text { beruflichen Weiterbildungsphasen }\end{array}$ \\
\hline & $\Rightarrow$ deutlicher Unterschied & $\Rightarrow$ deutlicher Unterschied \\
\hline \multirow[t]{3}{*}{ Anrechnung } & $\begin{array}{l}\text { FR: Anrechnungsmöglichkeiten von } \\
\text { breiten Studiumsteilen (VAP) }\end{array}$ & $\begin{array}{l}\text { FR: Anrechnung zur Ermöglichung } \\
\text { von Durchlässigkeit }\end{array}$ \\
\hline & $\begin{array}{l}\text { DE: fehlende institutionalisierte An- } \\
\text { rechnungsstrukturen von Berufsbil- } \\
\text { dung in Hochschulbildung (Aus- } \\
\text { nahme Einstufungsprüfung) }\end{array}$ & DE: Anrechnung nicht relevant \\
\hline & $\Rightarrow$ deutlicher Unterschied & $=>$ deutlicher Unterschied \\
\hline \multirow[t]{4}{*}{$\begin{array}{l}\text { Organisationale } \\
\text { Verbindung }\end{array}$} & $\begin{array}{l}\text { in beiden Ländern Beginn einer stär- } \\
\text { keren Institutionalisierung integrierter } \\
\text { Studiengänge: }\end{array}$ & \\
\hline & FR: Alternance mit dem Loi Sequin & FR: nicht relevant für Durchlässigkeit \\
\hline & DE: Duales Studium & DE: wichtiger Lösungsansatz \\
\hline & $=>\underline{\text { Ähnlich }}$ & $\Rightarrow$ deutlicher Unterschied \\
\hline \multirow[t]{4}{*}{$\begin{array}{l}\text { Anpassung an } \\
\text { Heterogenität }\end{array}$} & $\begin{array}{l}\text { In beiden Ländern wenig institutiona- } \\
\text { lisiert (bis auf Fernstudium) bezogen } \\
\text { auf beruflich Qualifizierte }\end{array}$ & $\begin{array}{l}\text { In beiden Ländern Aspekt im Hinblick } \\
\text { auf beruflich Qualifizierte B kaum } \\
\text { (DE) bzw. nicht (FR) im Diskurs }\end{array}$ \\
\hline & $\begin{array}{l}\text { FR: Institutionalisierung von Bera- } \\
\text { tungsangeboten, Tutorien aber nicht } \\
\text { explizit für beruflich Qualifizierte }\end{array}$ & $\begin{array}{l}\text { FR: Heterogenität der Studierenden } \\
\text { wichtig aber nicht für beruflich Qualifi- } \\
\text { zierte als Zielgruppe }\end{array}$ \\
\hline & DE: Ausbau Studienberatung & $\mathrm{DE}:$ generell weniger wichtig \\
\hline & $=>$ Unterschiede trotz Ähnlichkeiten & => Unterschiede trotz Ähnlichkeiten \\
\hline
\end{tabular}

Quelle: Darstellung der Verfasserin

Weitere Barrieren in der kulturell-kognitiven Dimension konnten in den diskursiven Strukturen herausgearbeitet werden. Zum einen wurden sie bereits durch die zwei Diskurse deutlich, wobei der konservative Diskurs generell eine Barriere 
für Durchlässigkeit darstellte, da die beruflich Qualifizierten als Gefahr konstruiert wurden, die nur dann nicht bestünde, wenn sie als angehende Studierenden ein angemessenes $\mathrm{Ma} ß$ an höherer Allgemeinbildung nachweisen könnten. Aber auch in der Zusammenschau beider deutschen Diskurse wurde deutlich, dass das im Diskurs verwendete Verständnis einer im Vorhinein prüfbaren Studierfähigkeit als eine doppelte Barriere für Durchlässigkeit gelten konnte: Zum einen ist dies der Fall, weil das Konstrukt Studierfähigkeit, die im Vorhinein nachgewiesen werden muss, prinzipiell eine Exklusion von Individuen, welche als nicht studierfähig charakterisiert werden, beinhaltet. Zum anderen gilt dies auch, weil die Studierfähigkeit mit dem Abitur als bewiesen gilt, jedoch nicht mit einer erfolgreich abgeschlossenen Erstausbildung. Eine weitere Barriere zeigte sich in dem fehlenden Diskurs zur Anrechnung, da mit der Nicht-Thematisierung der beruflichen Bildung eine gleichwertige Kompetenzvermittlung abgesprochen wird. Damit einher geht auch die im Diskurs sichtbare Barriere zu Durchlässigkeit durch die kontinuierliche Grenzziehung zwischen beruflicher und Hochschulbildung, welche die Differenzen der Bildungswege unterstreicht, anstatt mögliche Anschlusspunkte hervorzuheben. Auch aus der kurzen Zusammenschau der bestehenden Barrieren werden insofern die bestehenden Unterschiede zwischen dem französischen und deutschen System deutlich.

\subsubsection{Nationale Durchlässigkeitsstrukturen im Vergleich (1999-2012)}

Wie in den Länderkapiteln aufgezeigt werden konnte, fanden in dem Zeitraum zwischen 1999 und 2013 sowohl in Frankreich als auch in Deutschland Veränderungen der Durchlässigkeitsstrukturen statt. Inwiefern diese Veränderungen zu einer Konvergenz oder stärkeren Ähnlichkeit dieser Strukturen der beiden Länder geführt haben, soll im Folgenden dargestellt werden. Wie im Abschnitt zuvor wird dies anhand des Vergleichs der vorherrschenden Argumentationslogiken in den Diskursen, den regulativen und diskursiven Durchlässigkeitsstrukturen entlang der Aspekte und der sich daraus abzeichnenden Barrieren für die Jahre 1999-2012 erfolgen.

\section{Argumentationslogiken im Vergleich}

In beiden Ländern wurde nach 1999 viel stärker als zuvor über Durchlässigkeit zwischen beruflicher und Hochschulbildung diskutiert. Ähnlich wie im ersten Zeitraum ist festzustellen, dass wiederum Überschneidungen in den Argumentationen innerhalb der Diskurse existent sind. In Deutschland und Frankreich finden sich sowohl universalistische als auch funktionalistische Argumentationslogiken für (und auch gegen) Durchlässigkeit. 
In Frankreich ist das Verhältnis zwischen der funktionalistischen Argumentationslogik und der universalistischen viel ausgeglichener ist als in Deutschland, wo eindeutig die funktionalistische Logik dominiert. Hier wird ein großer und wichtiger Unterschied auf der kulturell-kognitiven Dimension zwischen den beiden Ländern deutlich. Das Recht auf Bildung für jeden Bürger, das Ziel der Chancengleichheit und dem damit verbundenen Ziel der Demokratisierung aller Bildungsgänge, also auch der Hochschulbildung, ist wirksam im französischen Selbstverständnis verankert. Aus diesem Grund ist ebenfalls der selektionsfreie Zugang zu den Universitäten eine feste Säule der universalistischen Rahmung in Frankreich. Auch in Deutschland zielt der universalistische Diskursstrang auf die Gewährleistung des Rechts auf Bildung, auf Chancengleichheit und die Verringerung von sozialer Ungleichheit. Daher ist auch das in den universalistischen Diskurssträngen konstruierte Bildungssubjekt ein ähnliches: Im Mittelpunkt steht das Individuum als Mensch, welcher seine Persönlichkeit über Bildung weiterentwickelt und so zu einem kritikfähigen aufgeklärten Bürger wird, wobei in Frankreich der demokratische Erziehungsauftrag von Bildung wesentlich stärker betont wird als in Deutschland.

Auch das Bildungssubjekt, welches in den funktionalistischen Diskurssträngen konstruiert wurde, ist in beiden Ländern ähnlich. Hier wird das Individuum vor allem als Humankapitalressource betrachtet, welches entsprechend seiner/ihrer Fähigkeiten für bestimmte Arbeitsmarktpositionen ausgebildet wird. Der Bildungsprozess und die Auswahl des Bildungswegs erfolgen auf Basis ausreichender Informationen eigenverantwortlich ebenso wie der Umgang mit den daraus entstehenden Konsequenzen. Das funktionalistische Hauptargument in beiden Ländern ist der durch den Strukturwandel und viel stärker auch der in diesem Zeitraum durch den globalen Wettbewerb hervorgerufene Bedarf der akademischen Höherqualifizierung der Bevölkerung für den Arbeitsmarkt. Damit einher geht auch das Argument, dass eine Höherqualifizierung zu einer Erhöhung der jeweils individuellen Beschäftigungsfähigkeit führe. In Frankreich im Gegensatz zu Deutschland spielen dabei festgelegte klare Zielmarken der Höherqualifizierung eine große Rolle. Mindestens 80 \% eines Jahrgangs sollen das Abiturniveau erreichen und $50 \%$ einen tertiären Abschluss.

In Deutschland hat sich im Vergleich zum vorherigen Zeitraum die Argumentation dahingehend geändert, dass stärker einem akademischen Fachkräftemangel entgegengewirkt werden soll als einem Mangel an beruflich qualifizierten Facharbeiter_innen. Das Ziel der Erhöhung der Attraktivität der Berufsbildung bleibt allerdings weiterhin erhalten. In Frankreich wird zu Beginn des neuen Jahrtausends viel stärker als im Zeitraum davor eine Erhöhung der Attraktivität der beruflichen Bildung als notwendig erachtet, um einen Facharbeitermangel in bestimmten Branchen zu verhindern. Durchlässigkeit ist demgemäß das Argument dafür, dass die berufliche Bildung nicht mehr als, Sackgasse' angesehen werde. In Frankreich wird Durchlässigkeit nicht nur als attraktivitätssteigernd für die Be- 
rufsbildung angesehen, sondern soll auch durch eine höhere Erfolgswahrscheinlichkeit der beruflichen Studierenden bzw. deren Lenkung in berufliche Studiengänge zu einer höheren Attraktivität der Universitäten für die Absolvent_innen des allgemeinen Abiturs im Vergleich zu den anderen Hochschulorganisationen führen.

In Deutschland zeichnet sich der strukturkonservative Diskurs primär durch eine funktionale Argumentationslogik aus. Ziel ist der Erhalt der Vormachtstellung der höheren Allgemeinbildung. In Frankreich gibt es keinen deutlich strukturkonservativen Diskurs, jedoch werden in den funktionalistischen Argumentationen des vorherrschenden Diskurses ebenso Einschränkungen für Durchlässigkeit deutlich, welche den Argumenten des strukturkonservativen deutschen Diskurses ähneln. Durchlässigkeit zwischen Berufs- und Hochschulbildung sei nur solange positiv, wie sie das Funktionieren der Hochschulorganisationen und deren Qualität nicht stört. Eine derartige Störung sei dann gegeben, wenn die beruflich Qualifizierten nicht die Fähigkeit für ein Hochschulstudium besitzen und es daher zu einer Ressourcenverschwendung an ,nicht passende“ Studierende kommen würde, die entweder in anderen Organisationen oder außerhalb der Hochschulbildung besser aufgehoben wären. So gibt es zwar den deutlichen Unterschied, dass sich in Deutschland der strukturkritische und -konservative Diskurs gegenüberstehen und in Frankreich nur ein Diskurs vorherrscht, aber innerhalb dieses Diskurses sind Überschneidungen mit den funktionalistischen Rahmungen des deutschen konservativen Diskurses festzustellen.

Es bleibt festzuhalten, dass in den Argumentationslogiken der Diskurse in Deutschland und Frankreich zwar der deutliche Unterschied in dem Gewicht funktionalistischer und universalistischer Argumentationen besteht, wobei in Frankreich die universalistischen deutlich wichtiger sind im Diskurs. Viele der herausgearbeiteten Argumentationsmuster ähneln sich allerdings.

\section{Vergleich anhand der Durchlässigkeitsaspekte}

Selbst wenn das Thema der Durchlässigkeit beruflich Qualifizierter zum Hochschulwesen in Frankreich wesentlich prominenter diskutiert wird als vor 1999, ist wie auch im ersten Zeitraum diese Problemstellung noch immer deutlich stärker in den deutschen Bildungsdebatten präsent. Im Gegensatz zum Zeitraum zuvor aber werden in den Diskussionen zur Durchlässigkeit in beiden Ländern jeweils alle vier Aspekte, die Frage des Zugangs, der Anrechnung, der organisationalen Verbindungen und auch der Umgang mit heterogenen Bedürfnissen angesprochen. Das Verständnis von Durchlässigkeit, welches in den Diskursen in Deutschland und Frankreich sichtbar wird, beinhaltete demnach in beiden Ländern eine mehr oder weniger ähnliche Behandlung aller vier Durchlässigkeitsaspekte. Eben diese Unterschiede und Ähnlichkeiten sollen im Folgenden zusammen mit dem Vergleich der rechtlichen Regelungen dargestellt werden, um die 
Frage nach einer Annäherung der Durchlässigkeitsstrukturen weiter zu beantworten.

Tabelle 32 Diskursiv behandelte Aspekte von Durchlässigkeit im Vergleich vor und nach 1999

\begin{tabular}{lcccc} 
& \multicolumn{2}{c}{ Vor 1999} & \multicolumn{2}{c}{ Ab 1999} \\
Aspekte & Deutschland & Frankreich & Deutschland & Frankreich \\
\hline Zugang & $\checkmark$ & $\checkmark$ & $\checkmark$ & $\checkmark$ \\
Anrechnung & & $\checkmark$ & $\checkmark$ & $\checkmark$ \\
Institutionelle Verbindung & $\checkmark^{*}$ & $\checkmark^{*}$ & $\checkmark$ \\
Umgang mit Heterogenität & $\checkmark^{* *}$ & $\checkmark$ & $\checkmark$ \\
\hline Anerkennung auf dem & $\checkmark^{*}$ & $\checkmark^{*}$ & $\checkmark$ \\
Arbeitsmarkt & & & \\
\hline * lediglich im strukturkritischen Diskurs & $* *$ lediglich sehr begrenzt im Fokus & \\
Quelle: Darstellung der Verfasserin & & &
\end{tabular}

\section{Zugang}

Waren vor 1999 die Unterschiede im Hinblick auf die rechtlichen Regelungen zum Zugang und der Anrechnung besonders groß, so ist insbesondere durch die vorgenommenen Reformen in Deutschland hier eine Annäherung der Strukturen festzustellen. Es kann jedoch nicht von einer Konvergenz gesprochen werden, da Unterschiede bestehen bleiben.

In beiden Ländern ist der Zugang zum Hochschulsystem über Berechtigungen, die im beruflichen Bildungswesen erworben werden können, möglich. Der Unterschied besteht vor allem darin, dass in Frankreich die Berechtigung mit dem Berufsabitur nach einer Erstausbildung erworben werden kann und in Deutschland der Abschluss einer beruflichen Weiterbildung nach dem Erstabschluss notwendig ist. Insofern werden in Deutschland höhere Anforderungen allein in Bildungsjahren für den Hochschulzugang gestellt. Aber auch ohne Berechtigung und mit Erstausbildung ist es mittlerweile in Deutschland möglich, Zugang zu einem fachnahen Studium an Fachhochschulen zu erhalten, wenn eine Prüfung oder ein Studium auf Probe stattgefunden hat. Diese Prüfungen zielen wiederum auf die Feststellung der Studierfähigkeit. In Frankreich ist zusätzlich noch der Zugang über die Validierung von Berufserfahrung im ganzen Land möglich, während in Deutschland dies durch den Bildungsföderalismus nur in einigen Ländern mit der Einstiegsprüfung der Fall ist.

Die Diskurse zum Zugang verlaufen in beiden Ländern noch sehr unterschiedlich, wobei sowohl in Frankreich als auch in Deutschland alle beruflich Qualifizierten von der Erstausbildung bis zur Weiterbildung im Fokus stehen. In 
Frankreich, wo sich das Durchlässigkeitsproblem vor allem in den Studienmisserfolgen zeigt, wird zwar die generelle Berechtigung zum Hochschulzugang durch das Berufsabitur nicht infrage gestellt, aber eine Kanalisierung beruflich Qualifizierter weg von den Universitäten in die beruflich orientierten Hochschulgänge der BTS angestrebt. Zudem wird ein notwendiges Ausbildungsniveau mit einem ausreichenden $\mathrm{Ma} \beta$ an Allgemeinbildung als Voraussetzung für ein Studium gesehen. In Frankreich wird weiterhin, wie auch vor 1999, der Zugang beruflich Erfahrener über Anrechnungsverfahren als wünschenswert betrachtet.

Der deutsche Diskurs ist wiederum stärker konfliktbehaftet, was die Definition der Gruppe betrifft, die Zugang erhalten sollte und auf welche Weise dies geschehen könne. Einerseits sollen im universalistischen Diskursstrang alle die Möglichkeit zum Studium erhalten, andererseits werden im funktionalistischen strukturkritischen Diskursstrang das Leistungsniveau sowie eine Passung von Hochschule sowie Studierenden und im strukturkonservativen Diskurs ein hohes Niveau an Allgemeinbildung als Voraussetzungen für den Zugang zum Studium definiert. Wie auch im ersten Zeitraum wird somit der Zugang von einer konstruierten ,Studierfähigkeit“ abhängig gemacht.

Die Frage eines ausreichenden Allgemeinbildungsniveaus stellt sich in Frankreich wie auch in Deutschland. In Deutschland wird aber allein im strukturkonservativen Diskurs die ausreichende Vermittlung dieses Niveaus in der Berufsbildung infrage gestellt. Zudem werden berufliche Bildung und Allgemeinbildung im strukturkonservativen Diskurs als Gegensatz konstruiert. In Frankreich wird ein hohes Allgemeinbildungsniveau allgemein als Voraussetzung für ein Hochschulstudium wahrgenommen, welches allerdings im Rahmen des Berufsabiturs vermittelt werden müsse. An dieser Stelle spiegelt sich auch die französische Tradition der viel stärker schulisch und theoretisch ausgerichteten Berufsbildung wider (Greinert 2005). Ungeachtet dieser Ausrichtung wird auch in Frankreich eine starke Differenz zwischen den stärker allgemeinbildenden Schulzweigen des bac général und bac technologique und dem bac professionnel konstruiert, wobei die Gruppe der Berufsabiturient_innen eher eingeschränkt als Zielgruppe für die Hochschulbildung v dargestellt wird.

Sowohl in Frankreich als auch in Deutschland gehören beruflich Qualifizierte im Diskurs zu der Gruppe der nicht traditionellen Studierenden. Die gesetzlich genormte Zielgruppe für Durchlässigkeit hat sich aber in beiden Ländern angeglichen. In den Diskursen in Frankreich und Deutschland werden je nach Diskursstrang unterschiedliche Zielgruppen beruflich Qualifizierter für die Hochschulbildung konstruiert (vgl. auch Tabelle 33), wobei sich die Konstruktion dieser Gruppe jeweils in einem Kontinuum zwischen allen beruflich Qualifizierten und einer Leistungselite widerfindet. An dieser Stelle haben sich die diskursiven Strukturen und die Deutungskonflikte in den beiden Ländern angenähert. Wie auch in der Regulative besteht insbesondere der Unterschied darin, dass in Deutschland viel stärker als in Frankreich eine Erstausbildung als nicht ausreichend für ein Studium erscheint. In Frankreich wird jedoch auch im Diskurs die 
generelle Berechtigung des Abiturs auf die guten Absolvent_innen des Berufsabiturs eingeschränkt, da das bac pro weiterhin vorrangig als Arbeitsmarkteinstiegszertifikat gesehen wird.

Tabelle 33 Vergleich der durchlässigkeitsrelevanten Zielgruppe für die Hochschulbildung nach 1999

Regulative Strukturen

\begin{tabular}{|c|c|}
\hline \multirow[t]{3}{*}{ Zielgruppe } & alle beruflich Qualifizierten \\
\hline & $\begin{array}{l}\text { FR: ab sekundärer Berufserstausbildung } \\
\text { (bac pro) } \\
\text { DE: alle, die mehr als Berufserstausbil- } \\
\text { dung nachweisen können (Erfahrung, } \\
\text { Weiterbildung, Abitur) }\end{array}$ \\
\hline & => Ähnlicher aber mit Unterschieden \\
\hline
\end{tabular}

\section{Diskursive Strukturen}

Beruflich Qualifizierte als nicht traditionelle Gruppe, Kontinuum von allen beruflich Qualifizierten bis zu einer Leistungselite

FR: primär (leistungsstarke) Berufsabiturient_innen, beruflich Qualifizierte mit Berufserfahrung DE: beruflich Qualifizierte nicht direkt nach Erstausbildung $=>$ Ähnlicher aber mit Unterschieden

Quelle: Darstellung der Verfasserin

An dieser Stelle wird daher auch eine deutliche Inkongruenz zwischen den institutionellen Dimensionen in Frankreich sichtbar. In der regulativen Dimension sind es alle beruflich Qualifizierten, die über Berechtigung oder Validierung Zugang zu den Hochschulen erhalten. In der normativen Dimension gehören stärker die sehr guten Absolvent_innen des Berufsabiturs und Berufserfahrene mit Weiterbildungswunsch zur Zielgruppe. Letztere Gruppe hat auch kaum eine andere Wahl, da es im Untersuchungszeitraum keine ausgebaute national anerkannte berufliche Weiterbildung außerhalb des Hochschulsystems in Frankreich gab. In der kulturell-kognitiven Dimension wird diese Inkongruenz aufgenommen, da einerseits ein französisches baccalauréat als Abschluss selbstverständlich auch den $\mathrm{Zu}-$ gang zur Hochschulbildung ermöglicht und in den Universitäten weiterhin nicht beim Zugang selektiert werden soll. Andererseits werden Absolvent_innen der sekundären beruflichen Bildung in Frankreich als eine Auswahl leistungsschwacher Problemschüler_innen stigmatisiert, welche mit der praktischen Berufsausbildung für den Arbeitsmarkt und nicht für ein Studium vorbereitet werden.

In Deutschland besteht trotz der konflikthaften Ausgangssituation um Durchlässigkeit eine viel geringere Inkongruenz zwischen den institutionellen Dimensionen. Dies liegt u.a. daran, dass der universalistische Diskursstrang viel schwächer ausgeprägt ist und sowohl der funktionalistisch strukturkritische als auch der strukturkonservative Diskurs(-strang) eine Selektion für den Zugang zum Hochschulsystem fordern. Diese Selektion findet sich überdies in den rechtlichen Regelungen wieder. 


\section{Anrechnung}

Bei dem Vergleich der rechtlichen und diskursiven Strukturen im Hinblick auf Anrechnung kann ebenfalls eine Annäherung der deutschen Strukturen an die französischen festgestellt werden. Anrechnung ist mittlerweile in Deutschland Thema geworden, wenn über Durchlässigkeit zwischen Berufs- und Hochschulbildung nachgedacht wird. In beiden Ländern wird somit anerkannt, dass auch in der beruflichen Sphäre äquivalente Fähigkeiten wie in der Hochschulbildung erlernt werden können. Anrechnung soll in beiden Ländern zudem an den Hochschulen stattfinden.

In der regulativen Dimension sind trotz dieser Annäherungen klare Unterschiede festzustellen. Während in Deutschland beschlossen wurde, dass bis zu $50 \%$ eines Studiums durch außeruniversitär erworbene Fähigkeiten angerechnet werden können, gibt es kein einheitlich geregeltes Verfahren, wie dies zu geschehen habe. Es wurden zwar Pilotprogramme eingesetzt, um Anrechnungsmöglichkeiten aufzuzeigen, aber eine bundesweite Umsetzung von Anrechnungsverfahren findet nicht statt. In Frankreich dagegen gibt es die national geregelten Verfahren des VAE und VAP, wobei beim VAE $100 \%$, dementsprechend das ganze Diplom, über Anrechnung erworben werden kann. In den diskursiven Strukturen wird ersichtlich, dass in beiden Ländern Anrechnung außerhochschulischer Leistungen ein wichtiges Thema nach 1999 ist. Wie aber Anrechnung diskutiert wird, ist dagegen deutlich unterschiedlich.

In Frankreich wird die Möglichkeit der Anrechnung bereits viel selbstverständlicher thematisiert, zumal es auch geregelte Verfahren dafür gibt. In Deutschland stellte Anrechnung dagegen im Diskurs vor allem zu Beginn des neuen Jahrtausends noch immer etwas Besonderes dar. Zudem wird viel stärker darauf hingewiesen, dass Anrechnung ein Zeichen von Gleichwertigkeit von Berufsbildung und höherer Allgemeinbildung darstelle. Auch der Lernort spielt in beiden Ländern bei Fragen der Anrechnung eine vollkommen unterschiedliche Rolle. In Deutschland bleibt der Lernort der Hochschule weiterhin wichtig, denn nur bis zu $50 \%$ eines Diploms können durch nicht akademische Bildung erworben werden. Im Diskurs wird diese Einschränkung nicht aufgehoben. In Frankreich dagegen wird der Lernort Hochschule mit der Einführung des VAE 2002 weniger wichtig, da ein Hochschuldiplom erworben werden kann, ohne auch an der Hochschule gewesen zu sein.

Auch was genau angerechnet werden soll, unterscheidet sich in beiden Ländern: In Deutschland ist der Fokus viel stärker auf ganze Bildungsgänge gerichtet und eine pauschale Anrechnung steht im Zentrum der Diskussionen. Im strukturkritischen Diskurs wird davon ausgegangen, dass auch bereits Kompetenzen aus der Erstausbildung anrechnungsfähig sind, während im konservativen Diskurs Anrechnung, wenn überhaupt, dann am ehesten von Kompetenzen, welche in der beruflichen Weiterbildung erworben wurden, stattfinden solle. Die Anrech- 
nung von non-formalen und informellen Kompetenzen, welche dann eher individualisiert stattfinden würde, wird vor allem im universalistischen strukturkritischen Diskursstrang gefordert.

In Frankreich wird die Anrechnung beruflicher Kompetenzen aus einem sekundären Berufsabschluss nicht für relevant erachtet. Im Fokus stehen vielmehr berufliche und seit 2002 ebenfalls außerberufliche Erfahrungen. Insofern wird in Frankreich auch stärker von einer individualisierten Anrechnung ausgegangen. Dieser Fokus auf Berufserfahrung im Gegensatz zur beruflichen Bildung zeigt aber auch die Geringschätzung der beruflichen Ausbildungsgänge auf Sekundarniveau in Frankreich. Anrechnungsfähige Kompetenzen werden erst nach dem Abschluss in der tatsächlichen Praxis erworben. Der Erwerb äquivalenter Kompetenzen in der beruflichen Erstausbildung ist unvorstellbar oder zumindest in Diskurs unsagbar. Hier wird aber auch ein Unterschied zum deutschen Diskurs deutlich, in welchem die berufliche Erstausbildung eine höhere Wertschätzung erfährt.

Auch durch diesen unterschiedlichen Fokus auf informelle, non-formale und formale Kompetenzen bei der Anrechnung kann erklärt werden, dass vor allem in Deutschland auch viel stärker im Diskurs auf die Voraussetzungen für die Anrechnung beruflicher Bildung hingewiesen wird. Neben einer stärkeren Ergebnisorientierung sowohl beruflicher als auch hochschulischer Bildungsgänge wird vor allem eine Qualitätssicherung der beruflichen Bildung gefordert, damit pauschales Anrechnen möglich sei. Schließlich ist in beiden Ländern Anrechnung auch relevant, wenn über Wechsel innerhalb des Hochschulsystems nachgedacht wurde. Dies ist jedoch für die in der vorliegenden Arbeit betrachtete Durchlässigkeit nur bedingt relevant $-z . B$. wenn es um die Ermöglichung institutioneller Übergänge während des Studiums geht, um Abbrüche zu vermeiden.

\section{Organisationale Verbindungen}

Insgesamt kann für den Aspekt der Verbindung von Berufs- und Hochschulbildung auf Organisationsebene, wie auch vor 1999, eine ähnliche Entwicklung in Frankreich und Deutschland festgestellt werden. Dies zeigt sich beim Ausbau dualer Studienformen in Deutschland und dem Zuwachsvon Hochschulstudiengängen en alternance in Frankreich. Zudem wurde die Organisationsform lycée des métiers in Frankreich aufgebaut, welche berufsbereichsübergreifend alle beruflichen Bildungsgänge vom CAP, bac professionnel und technologique bis zur licence professionnelle anbietet. Zudem wurde mit dem Bologna-Prozess die licence professionnelle eingeführt, wodurch vermehrt berufliche Bildung an den Universitäten, die traditionell als stärker theoretisch akademisch und forschungsorientiert gelten, stattfindet.

Auch werden in beiden Ländern Wechsel zwischen tertiären Organisationsformen und damit institutionelle Übergänge, verstärkt ermöglicht. In Deutschland wurde mit der Einführung des B.A. und M.A. speziell der Wechsel nach Ab- 
schlüssen erleichtert, so dass ein Wechsel von der stärker an Berufspraxis orientierten Fachhochschule an die Universität möglich wurde. In Frankreich wurde partiell ferner der Wechsel vor dem Abschluss eines Diploms mit der Übernahme bereits erbrachter Leistungen ermöglicht. Durchlässigkeitsrelevant sind diese Wechselmöglichkeiten vor allem dann, wenn ein Studienmisserfolg durch den Studiengangwechsel in eine andere Hochschulorganisation verhindert werden soll.

Im Gegenteil zum Zeitraum davor werden integrierte Bildungsformen in beiden Ländern als durchlässigkeitsfördernd angesehen. In Deutschland liegt der Fokus im Diskurs vor allem auf den dualen Studiengängen. Auch in Frankreich werden Studiengänge en alternance als ein möglicher Weg gesehen, um beruflich Qualifizierten im Hochschulwesen Erfolg zu ermöglichen, da durch die Praxisphasen die Stärken beruflich Qualifizierter besser zur Geltung kommen sollen. Insgesamt ist aber in Frankreich der Ausbau des lycée des metiérs stärker im Fokus des Diskurses. Durch die Integration der sonst getrennten beruflichen Bildungsgänge aus dem Sekundär- und Tertiärbereich sowie dem technologischen Abitur wird das lycée des metiérs auch als Möglichkeit gesehen, zum einen bereits die besten beruflich Qualifizierten auszuwählen und so deren Studienerfolg zu erleichtern, zum andern aber auch als ein Weg, beruflich Qualifizierte erst einmal Entwicklungsmöglichkeiten aufzuzeigen und mögliche bestehende Ängste abzubauen.

Unterschiedlich zwischen Deutschland und Frankreich ist auch die Rolle institutionalisierter Übergänge innerhalb des Hochschulbereichs im Diskurs. In Deutschland werden diese Verbindungen nicht im Hinblick auf Durchlässigkeit zwischen beruflicher und Hochschulbildung betrachtet ${ }^{186}$. In Frankreich ist sie dagegen schon relevant, da in einem Wechsel der Studiengänge auch eine Möglichkeit gesehen wird, die Studierenden mit Problemen und damit auch beruflich Qualifizierte zu einem Studienerfolg in dem für sie passenderen Fach zu bringen. Es wird deutlich, dass die Aspekte je nachdem wie das nationale ,Durchlässigkeitsproblem' gelagert ist, anders debattiert werden.

\section{Umgang mit Heterogenität}

Für beide Länder ist festzustellen, dass die Frage, wie man mit den Bedürfnissen der bereits beruflich qualifizierten Studierenden umgeht, wesentlich wichtiger ist als vor 1999. So wurden neue rechtliche Regelungen getroffen und auch in den Aussagen zu Durchlässigkeit spielt der Umgang mit Heterogenität eine immer größere Rolle. Unterschiedlich zwischen Frankreich und Deutschland sind allerdings die genauen rechtlichen Veränderungen bzw. die diskursiven Schwerpunkte. Insgesamt lässt sich feststellen, dass in Frankreich die Frage, wie man beruflich Qualifizierte erfolgreich zum Studienabschluss bringt, eng mit der Frage, wie man

186 Die Nichtthematisierung im Kontext von Durchlässigkeit zwischen beruflicher Bildung und Hochschulbildung bedeutet allerdings nicht, dass die Frage institutionalisierter Übergänge im Hochschulsystem in den deutschen Bildungsdebatten nicht wichtig gewesen ist, zumal sie auch mit dem viel diskutierten Problem der Selektivität der Masterstudiengänge zusammenhängt. 
mit den heterogenen Bedürfnissen beruflich Qualifizierter umgeht, verbunden wird und wesentlich prominenter gewesen ist als im deutschen Diskurs.

Betrachtet man die rechtlichen Regelungen findet in beiden Ländern eine stärkere Flexibilisierung des Studiums, inklusive des Ausbaus der Teilzeitstudiumsmöglichkeiten sowie E-Learning, statt. Die Umsetzung derartiger Maßnahmen obliegt in beiden Ländern den Hochschulen. In Frankreich wird vor allem seit dem Plan Réussite en Licence auf einen Ausbau der Studienberatung vor und während des Studiums, eine stärkere Studienbetreuung sowie eine Veränderung der Pädagogik weg von großen Vorlesungen hin zu individualisierten Lehrmethoden wie Tutorien gedrängt. Diese Veränderungen sollen insbesondere an den Universitäten stattfinden. Aber auch in den Schulen wurde per Gesetz mehr Zeit für eine Berufsorientierung festgelegt. In Deutschland dagegen wurde zusätzlich zu den Flexibilisierungsmöglichkeiten, das Aufstiegsstipendium, eine finanzielle Unterstützung leistungsselektierter beruflich Qualifizierter beim Studium auf den Weg gebracht. Insofern sind differente Schwerpunkte in den Regelungen zwischen Frankreich und Deutschland festzustellen.

Diese unterschiedlichen Schwerpunkte lassen sich ebenfalls in den Diskursen wiederfinden. Aber es gibt auch Ähnlichkeiten. Sowohl in Frankreich als auch in Deutschland wird davon ausgegangen, dass beruflich Qualifizierte stark dazu beitragen, die Heterogenität der Studierenden zu erhöhen, auf welche nun vermehrt reagiert werden müsse. So werden z.B. in beiden Ländern in den jeweiligen funktionalen Diskurssträngen, in denen die Passung der Studierenden mit dem gewählten Bildungsweg im Vordergrund stehen, Informationsangebote und Beratungen vor dem Studium als besonders wichtig erachtet. Als hilfreich wird ebenfalls eine Vorbereitung auf das Studium durch Propädeutika angesehen, um mögliche Defizite der beruflich Qualifizierten zu verringern.

Im französischen Diskurs ist insbesondere die Beratung vor dem Studium, die Studienorientierung, relevant, die zu einer gezielten Lenkung der Berufsabiturient_innen in den Arbeitsmarkt oder die beruflichen Studiengänge führen soll. Zwar wird im universalistischen Diskursstrang auch die Möglichkeit der Hilfe für die leistungsschwächeren Studierenden über Begleitungsarrangements, eine abgestimmte Pädagogik, einen veränderter Lehrplan sowie Beratung im Studium behandelt, aber die Bedeutung der (Um-) ${ }^{\circ}$ Orientierung' beruflich Qualifizierter aus den Universitäten war wesentlich präsenter. Trotz der starken universalistischen Argumentationen sind die häufigsten Lösungsvorschläge eher dem funktionalistischen Diskursstrang zuzuordnen. Beruflich Qualifizierte haben zwar ein Recht auf ein Studium, aber durch den verstärkten Fokus auf die, Orientierung ${ }^{6}$ vor Studienbeginn inklusive der Informationen über die (geringen) Erfolgswahrscheinlichkeiten beruflich Qualifizierter in den Studienfächern stand die funktionalistische Selektion und weniger die Unterstützung der Studierenden im Bildungsweg im Vordergrund.

In Deutschland kann je nach Diskurs(-strang) ebenfalls ein Kontinuum zwischen einem stärkeren Fokus auf die Studieninformation vor dem Studium im 
funktionalistischen, Vorbereitungskursen zum Abbau von Defiziten im konservativen Diskurs sowie einer stärkeren Unterstützung und entsprechenden Anpassung der Lehrmethoden im universalistischen Diskursstrang wiedergefunden werden. Gemeinsam wird in den Diskursen die Bedeutung der Flexibilisierung der Studienorganisation betont, um z.B. ein Arbeiten neben dem Studium zu ermöglichen. Auch wird der Bedarf von Finanzierungsmöglichkeiten für eine Leistungselite im funktionalistischen und für alle beruflich Ausgebildeten im universalistischen Diskursstrang erkannt.

\section{Der Vergleich im Überblick}

In Deutschland und Frankreich fanden viele Entwicklungen statt, welche im Vergleich zu der Periode vor 1999 zu einer deutlichen Annäherung der Durchlässigkeitsstrukturen in den beiden Ländern geführt haben. Aber auch deutliche Unterschiede bleiben bestehen (vgl. Tabelle 34).

Mit Blick auf die Barrieren zu Durchlässigkeit kann festgestellt werden, dass in beiden Ländern eine Verringerung dieser durch bessere Zugangsmöglichkeiten und Anrechnungsbedingungen erfolgte.

Eine große Barriere für Durchlässigkeit in Frankreich besteht weiterhin in der Inkongruenz zwischen den institutionellen Dimensionen. So besteht diese zum einen in den stärker universalistischen Rahmungen, welche eine Förderung aller beruflich Qualifizierten vorsieht und den stärker funktionalistischen Reformvor schlägen, welche auf eine stärkere Selektion, eine Lenkung der Berufsabiturient_innen in den Arbeitsmarkt abzielen, anstatt auf eine größere Unterstützung. Auch bei der Rolle des Abiturs wird die Inkongruenz deutlich. In der regulativen

Tabelle 34 Unterschiede und Ähnlichkeiten in den Durchlässigkeitsstrukturen nach 1999

\begin{tabular}{|c|c|c|}
\hline Aspekte & Regulative Strukturen & Diskursive Strukturen \\
\hline $\begin{array}{l}\text { Zugang } \\
\text { Ähnlichkeiten }\end{array}$ & $\begin{array}{l}\text { Berechtigungen zum Hochschulsystem } \\
\text { über Berufsausbildung }\end{array}$ & $\begin{array}{l}\text { Zugang nach Erstausbildung und Wei- } \\
\text { terbildung }\end{array}$ \\
\hline \multirow[t]{2}{*}{ Unterschiede } & $\begin{array}{l}\text { FR: Berechtigung mit Berufsabitur, Zu- } \\
\text { gang leistungsstarker Berufsabitu- } \\
\text { rient_innen in BTS bei Fachnähe, Mög- } \\
\text { lichkeit der Validierung von Berufser- } \\
\text { fahrung }\end{array}$ & $\begin{array}{l}\text { FR: keine Selektion an Universitäten, } \\
\text { baccalauréat als generelle Berechti- } \\
\text { gung einerseits, Kanalisierung einer } \\
\text { Leistungselite der Berufsabitu- } \\
\text { rient_innen in berufliche Studiengänge } \\
\text { anderseits, keine spezifischen Anforde- } \\
\text { rungen an Berufserfahrene }\end{array}$ \\
\hline & $\begin{array}{l}\text { DE: uneingeschränkte Berechtigung al- } \\
\text { lein nach Weiterbildungsabschluss, mit } \\
\text { Erstausbildung in Fachnähe mit Berufs- } \\
\text { erfahrung und über Eignungsprüfung }\end{array}$ & $\begin{array}{l}\text { DE: Selektion einer Gruppe von beruf- } \\
\text { lich Qualifizierten nach ihrer Studierfä- } \\
\text { higkeit (Allgemeinbildung oder Leis- } \\
\text { tungsniveau und Passung mit Hoch- } \\
\text { schule), kaum Forderung des selekti- } \\
\text { onsfreien Zugangs für alle }\end{array}$ \\
\hline
\end{tabular}




\begin{tabular}{|c|c|c|}
\hline Aspekte & Regulative Strukturen & Diskursive Strukturen \\
\hline $\begin{array}{l}\text { Anrechnung } \\
\text { Ähnlichkeiten }\end{array}$ & $\begin{array}{l}\text { Anrechnung als Aufgabe der Hoch- } \\
\text { schulen }\end{array}$ & $\begin{array}{l}\text { Anrechnung beruflich erworbener Kom- } \\
\text { petenzen wichtig }\end{array}$ \\
\hline \multirow[t]{2}{*}{ Unterschiede } & $\begin{array}{l}\text { FR: national einheitliche Verfahren VAP } \\
\text { und VAE, Anrechnung bis zu } 100 \%\end{array}$ & $\begin{array}{l}\text { FR: Lernort Hochschule weniger be- } \\
\text { deutend, Fokus Validierung beruflicher } \\
\text { Erfahrung, Einbeziehung aller Lernfor- } \\
\text { men (formal, informell und non formal), } \\
\text { individualisiert }\end{array}$ \\
\hline & $\begin{array}{l}\text { DE: Einführung von Anrechnungsmög- } \\
\text { lichkeiten in Deutschland bis zu } 50 \% \text {, } \\
\text { jedoch ohne Vorgabe eines national } \\
\text { einheitlichen Verfahrens, Pilotpro- } \\
\text { gramme }\end{array}$ & $\begin{array}{l}\text { DE: Anrechnung begrenzt, Lernort } \\
\text { Hochschule wichtig, Fokus auf Anrech- } \\
\text { nung formaler beruflicher Bildungsab- } \\
\text { schlüsse, pauschale Verfahren, An- } \\
\text { rechnung als Zeichen von Gleichwertig- } \\
\text { keit }\end{array}$ \\
\hline $\begin{array}{l}\text { Institutionelle } \\
\text { Verbindung } \\
\text { Ähnlichkeiten }\end{array}$ & $\begin{array}{l}\text { Ausbau integrierter Bildungsformen, } \\
\text { v.a. dualer Formen, erleichterte Verbin- } \\
\text { dungen zwischen Studiengängen unter- } \\
\text { schiedlicher Organisationsformen }\end{array}$ & $\begin{array}{l}\text { Integrierte Bildungsformen in beiden } \\
\text { Ländern als ein Weg zu größeren } \\
\text { Durchlässigkeit }\end{array}$ \\
\hline \multirow[t]{2}{*}{ Unterschiede } & $\begin{array}{l}\text { FR: weitere Form der Integration: lycée } \\
\text { de metiers und licence pro }\end{array}$ & $\begin{array}{l}\text { FR: neben Alternance, v.a. Lycée de } \\
\text { metiers, Bedeutung von Passerelles } \\
\text { (inst. Übergängen) zwischen berufli- } \\
\text { chen und stärker rein akademischen } \\
\text { Hochschulstudiengängen }\end{array}$ \\
\hline & DE: Duales Studium & $\begin{array}{l}\text { DE: duale Studiengänge weniger wich- } \\
\text { tig im Diskurs trotz Ausbau, } \\
\text { institutionelle Übergänge nicht im Fo- } \\
\text { kus für Durchlässigkeit }\end{array}$ \\
\hline $\begin{array}{l}\text { Umgang mit } \\
\text { Heterogenität } \\
\text { Ähnlichkeiten }\end{array}$ & $\begin{array}{l}\text { Ausbau von Maßnahmen in beiden Län- } \\
\text { dern mit unterschiedlichen Schwer- } \\
\text { punkten, aber gemeinsam Ermögli- } \\
\text { chung des Teilzeitstudiums }\end{array}$ & $\begin{array}{l}\text { Umgang mit Heterogenität zunehmend } \\
\text { wichtiger, in funktionalen Diskursträn- } \\
\text { gen Berufsberatung sowie Propädeuti- } \\
\text { ka wichtig, Ermöglichung von Teilzeit- } \\
\text { studium }\end{array}$ \\
\hline \multirow[t]{2}{*}{ Unterschiede } & $\begin{array}{l}\text { FR: Programm vor allem für Universitä- } \\
\text { ten (plan Réussite en Licence), Ausbau } \\
\text { Studienberatungssysteme, Studienbe- } \\
\text { treuung im Studium, Veränderung der } \\
\text { Pädagogik }\end{array}$ & $\begin{array}{l}\text { FR: insgesamt sehr wichtig, starker Fo- } \\
\text { kus auf Studierendenorientierung und } \\
\text { Information (gezielte Lenkung der be- } \\
\text { rufl. Qualifizierten), weniger prominent } \\
\text { Unterstützung für Studierende mit } \\
\text { Problemen durch Beratung im Studium, } \\
\text { Vorbereitung, Pädagogik }\end{array}$ \\
\hline & $\begin{array}{l}\text { DE: Ausbau Studienfinanzierung für be- } \\
\text { ruflich Qualifizierte }\end{array}$ & $\begin{array}{l}\text { DE: je nach Diskurs eher Fokus auf Stu- } \\
\text { dienorganisation und Pädagogik } \\
\text { oder Beratung und Information, diskurs- } \\
\text { übergreifend: Finanzierung und Teil- } \\
\text { zeitstudium }\end{array}$ \\
\hline
\end{tabular}

Quelle: Darstellung der Verfasserin

Dimension berechtigt jedes baccalauréat zu einem Zugang zum Hochschulwesen. Diese Verbindung des Abiturs mit dem Zugang ist zudem Teil des französischen 
Selbstverständnisses. Auf das Berufsabitur bezogen kann allerdings eine Widersprüchlichkeit festgestellt werden. Denn im Diskurs wird zwar der Zugang zur Hochschule nicht versagt, aber das Berufsabitur oft nur als Arbeitsmarkteinstiegszertifikat gesehen. Mehrheitlich wird eine Leistungselite beruflich Gebildeter und Berufserfahrener als Zielgruppe konstruiert. Die bestehende Norm, dass jede/-r oder fast jede/-r (80\%) ein Abitur erreichen sollen, aber nur $50 \%$ einen tertiären Abschluss, bestätigt, dass nicht jede/-r Abiturient_in auch als Zielperson für die Hochschule angesehen wird. Während einerseits die Einführung des Berufsabiturs in Frankreich und die Verkürzung der Abiturdauer eine Steigerung der Wertschätzung beruflicher Bildung nach sich ziehen und Gleichwertigkeit demonstrieren sollen, bleibt berufliche Bildung auf Sekundarniveau weiterhin im französischen Selbstverständnis der Bildungsbereich, in welchen die Schüler_innen gelangen, die am collège Probleme hatten. Die Stigmatisierung der sekundären Berufsbildung in Frankreich als Auffangbecken von leistungsschwächeren Individuen untergräbt somit ebenfalls die Anerkennung des bac pro als ,richtiges' Abitur, welches für ein Studium befähigt. Eine Barriere besteht somit in der gesellschaftlich verankerten Hierarchisierung der Bildungswege, die in den Diskursen reproduziert wird.

Auch wenn sie weniger geworden sind, bestehen auch in Deutschland noch Barrieren zur Durchlässigkeit. In der regulativen Dimension sind diese u.a. zu verorten in der fehlenden Gleichstellung beruflicher Bildung und der höheren Allgemeinbildung auf Sekundarniveau im Berechtigungswesen und in der fehlenden Institutionalisierung eindeutiger Anrechnungsverfahren. Wie auch in Frankreich ist das Abitur eine zentrale Institution für den Zugang zum Hochschulwesen in Deutschland. Im Gegensatz zu Frankreich ist es aber nur eingeschränkt möglich, mit dem Berufsabschluss auch eine Hochschulzugangsberechtigung zu erhalten. Die Ausnahmen, die bestehen, gelten lediglich, wenn ein ausreichendes $\mathrm{Maß}$ an Allgemeinbildung vermittelt wurde. Hier wird wiederum die Ungleichwertigkeit von Berufs- und höherer Allgemeinbildung deutlich.

Wie auch im ersten Zeitraum ist das Bestehen eines strukturkonservativen Diskurses, welcher auf das Beibehalten der Hierarchien und Trennung zwischen höherer Allgemeinbildung und Berufsbildung zielt, als deutliche Barriere zu erkennen. Innerhalb der deutschen Bildungspolitik existiert noch immer die Annahme, dass vornehmlich die höhere Allgemeinbildung für ein Hochschulstudium befähigt und dass Individuen mit einem anderen Bildungshintergrund ihre Eignung unter Beweis stellen müssen. Auch das Verständnis der Studierfähigkeit, welche vor einem Studium feststellbar sei, ist eine weiterhin bestehende Barriere. So müsse bereits vor dem Studium durch Prüfung oder bereits erworbene Zertifikate feststellbar sein, wer für ein Studium befähigt sei und wer nicht. Hier offenbart sich der für das deutsche Bildungssystem bestimmende Glaube an eine biologisch festgelegte bestimmbare Begabung, die statisch und nicht veränderbar ist und insofern vor dem Studium geprüft werden kann. Dass sich die als notwendig erachteten Fähigkeiten mitunter erst im Studium entwickeln könnten, wird 
kaum (Ausnahme Probestudium) als möglich erachtet. Dies gilt signifikant für den konservativen Diskurs, jedoch auch für den funktionalistischen strukturkritischen Diskursstrang. Das Konzept der Studierfähigkeit spiegelt sich daher auch in den rechtlichen Zugangsregelungen wider.

Ähnlich wie in Frankreich werden beruflich Qualifizierte in Deutschland stigmatisiert, da sie als Gefahr für die Qualität der Hochschulbildung konstruiert werden. Aber auch die Konstruktion der beruflich Qualifizierten als nicht traditionelle Studierende, die besondere Zuwendung benötigen, damit das Studium funktioniert, kann als Stigmatisierung gesehen werden und abschreckend wirken. Die Barriere besteht dann, wenn beruflich Qualifizierte als extra Gruppe im Kontrast zu den vermeintlich homogenen traditionellen Studierenden dargestellt werden und damit die Differenz auch an den Hochschulen zwischen den Gruppen vergrößert wird. Schließlich kann auch die dominierende funktionalistische Rahmung für Durchlässigkeit als Barriere gesehen werden. Beruflich Qualifizierte sollen in dieser Logik weniger als Person ein Recht auf Weiterbildung an den Hochschulen erhalten, sondern werden als Ressource gesehen, deren Höherqualifizierung an den Hochschulen momentan notwendig ist. Problematisch ist dies insofern, als dass Durchlässigkeit dann reversibel ist und nur solange aufrechterhalten werden muss, wie es aus funktionalen Gründen notwendig erscheint.

Was bedeuten aber die aufgezeigten Barrieren für die Frage nach der Annäherung der Durchlässigkeitsstrukturen in beiden Ländern? Einerseits konnte gezeigt werden, dass partiell ähnliche Barrieren verringert wurden und auch ähnliche Barrieren bestehen. Die gesellschaftlich verankerte Hierarchisierung höherer Allgemeinbildung im Vergleich zur Berufsbildung (auf Sekundarniveau), die Stigmatisierungen beruflich Gebildeter als Gefahr für die Qualität der Hochschulbildung oder als besondere Gruppe in Differenz zu den traditionellen Studierenden sind in diesem Kontext deutliche Beispiele. Andererseits wurden entsprechend der unterschiedlichen Problemlagen auch ganz unterschiedliche Barrieren festgestellt. Insgesamt konnte somit gezeigt werden, dass es zwar zu einer Annäherung der Durchlässigkeitsstrukturen gekommen ist, aber nicht zu einer Konvergenz, da viele nationaltypische Strukturen weiterhin bestehen.

\subsection{Durchlässigkeit durch Europäisierung? Nationale Entwicklungen und europäische Vorgaben}

Bisher konnte in dieser Arbeit gezeigt werden, dass erstens die europäischen Bildungsprozesse Bologna und Kopenhagen eine verbesserte Durchlässigkeit zwischen Berufs- und Hochschulbildung in den Mitgliedsländern anstreben (vgl. Kapitel 5). Zudem wurde zweitens ebenfalls die Entwicklung der institutionellen Durchlässigkeitsstrukturen für Deutschland (Kapitel 7) und Frankreich (Kapitel 9) analysiert und fallspezifisch dargestellt. Drittens konnte im Anschluss gezeigt 
werden, dass sich die Durchlässigkeitsstrukturen zwischen Frankreich und Deutschland zwar annähern, wobei nationale Eigenheiten und Unterschiede aber weiterhin deutlich bleiben.

Dieser Kapitelabschnitt soll nun einen Beitrag zur Erklärung des Wandels leisten und Aufschluss darüber geben, inwiefern die Europäisierungsprozesse einen Anteil an der jeweiligen Entwicklung in den Ländern und an der festgestellten Annäherung der institutionellen Strukturen in beiden Ländern hatten. Dies erfolgt in drei Schritten. In einem ersten Schritt soll dabei für die beiden Länder gezeigt werden, inwiefern diese bereits vor 1999 die in den europäischen Dokumenten sichtbaren Durchlässigkeitsanforderungen erfüllen. Ausgehend von den in Kapitel 5 herausgearbeiteten durchlässigkeitsrelevanten Standards, welche in den europäischen Dokumenten eingefordert wurden, wird zunächst verglichen, inwiefern das deutsche und französische System bereits vor den Prozessen den diffundierenden Durchlässigkeitsnormen entsprachen. Auf diese Weise wird die ,goodness of fit ${ }^{* 187}$ (Börzel/Risse 2003) der Bildungssysteme herausgearbeitet, so dass auch die Stärke des normativen Anpassungsdrucks auf die Länder sichtbar wird. Der, goodness of fit"-Ansatz geht davon aus, dass der Handlungsdruck umso größer wird in den Mitgliedsstaaten, je stärker die Inkompatibilität zwischen den europäischen Vorgaben und den nationalen Regelungen ist. Hier bleibt anzumerken, dass aber auch die Wahrnehmung und das diskursiven Framing entscheiden, ob Reformen als notwendig erachtet werden oder nicht, unabhängig von der Passung der nationalen rechtlichen Regelungen zu den europäischen Standards (Radaelli/Schmidt 2005).

Insbesondere da Frankreich und Deutschland zu den Vorreiterstaaten der beiden europäischen Bildungsprozesse gehörten und die Prozesse mit der Methode der offenen Koordinierung arbeiten, also damit auch stark an bestehenden Praktiken und Standards in den Mitgliedsstaaten angelehnt sind, macht es Sinn zu eruieren, inwiefern in den gemeinsamen verfassten Dokumenten nicht bereits in den Ländern bestehende Standards eingeschrieben wurden. Dann wäre der Handlungsdruck wesentlich geringer. Zudem ist vornehmlich der Austausch zwischen den Ländern ein Mechanismus, wie die europäischen Bildungsprozesse funktionieren. Die Deklarationen entstanden nicht im luftleeren Raum oder wurden von einer europäischen Regierung festgelegt, sondern wurden unter der Beteiligung der Mitgliedsstaaten erstellt. Es ist daher wichtig, diesen zirkulären Prozess der Entstehung mitzudenken, auch wenn in der vorliegenden Arbeit nicht ausgeführt

187 Der ,goodness of fit"-Ansatz wird eher in Bereichen der positiven Integration, d.h. in Politikbereichen angewendet, in denen die EU klare Regelungskompetenzen hat und ein Politikmodell vorgibt. (vgl. Auel 2006) Aber auch wenn die europäischen Bildungsprozesse Bologna und Kopenhagen nicht Teil der supranationalen Politiksetzung der EU sind, kann von ihnen doch normativer Druck ausgehen. Zudem wird durch die Darstellung der Passung zwischen Vorgaben und der institutionellen Strukturen der Status quo für die Untersuchungsfrage dargestellt, von dem aus die nationalen Entwicklungen in Relation zu den Vorgaben analysiert werden. 
werden kann, warum welche Standards in den europäischen Dokumenten gefordert werden. Zudem ist es genau diese Art der Prozesse der Aushandlung, der Vergleiche untereinander, welche die Diffusion von Ideen und Argumentationslogiken, die Übernahme von Standards anregen und somit zu nationalen Lernund Sozialisationsprozessen führen können (vgl. Radaelli 2000: 19).

Um mögliche Diffusionsprozesse zu identifzieren, wird insofern in einem logischen zweiten Schritt analysiert, inwiefern die Veränderung der nationalen Durchlässigkeitsstrukturen zu einer Annäherung an die europäischen Standards geführt hat. Im Zentrum der Analyse steht die Delta-Konvergenz. In diesem Schritt werden zusätzlich nicht nur die Standards, sondern auch die Argumentationslogiken mit den in den europäischen Dokumenten vorherrschenden verglichen, um auch hier einen möglichen Einfluss Europas auszumachen. Gleichen sich die Argumentationslogiken und ähneln sich die Konstruktionen der Subjekte, für die Durchlässigkeit geschaffen werden soll?

In einem dritten Schritt werden die Annahmen hinsichtlich des Einflusses der europäischen Prozesse, welche aus der vorhergehenden Analyse entstanden sind, anhand der Bedeutungszuschreibung der europäischen Prozesse in den Diskursen und in den geführten Expert_inneninterviews ${ }^{188}$ geprüft. Die Kombination der Untersuchung von Diskursen und Expert_inneninterviews erscheint sinnvoll, da politische Akteure nicht immer zugeben, dass Reformen durch exogene Kräfte beeinflusst wurden. „SSJilent borrowing“ (Waldow 2009: 478) ist keine Seltenheit. Wurde in den Diskursen oder Interviews direkt auf europäische Prozesse oder Standards verwiesen? Welche Rolle messen die Expert_innen den europäischen Prozessen zu? Die Zielsetzung besteht darin, aufzuzeigen, welche Rolle die europäischen Bildungsprozesse für den institutionellen Wandel in Deutschland und Frankreich spielten. Haben sie zu einer Veränderung der diskursiven Kräfteverhältnisse geführt? Und inwiefern bestätigen sie auch den angenommenen isomorphen Veränderungsdruck und erklären die Annäherung der Durchlässigkeitsstrukturen der beiden Länder.

\subsubsection{Passung zwischen nationalen Strukturen und europäischen Vorgaben vor 1999}

Wie bereits zuvor dargelegt, wird sowohl im Bologna- als auch im KopenhagenProzess die Etablierung von Standards eingefordert, welche zu einer Erhöhung der Durchlässigkeit zwischen beruflicher und Hochschulbildung führen könne. Sie wurden mehr (im Kopenhagen-Prozess) oder weniger (im Bologna-Prozess) direkt mit dem Ziel der Förderung von Durchlässigkeit verbunden. Dabei ist festzuhalten, dass viele der Standards nicht nur die Förderung eines spezifischen Durchlässigkeitsaspekts bewirken, sondern auf mehrere Einfluss haben können.

188 Eine Auflistung der Expert_inneninterviews befindet sich im Anhang (vgl. Tabellen 45-47) zusammen mit einem Hinweis der Zitierabkürzungen. 
Generell beziehen sie sich vor allem auf Strukturen innerhalb von Bildungsorganisationen und können auf die Organisation des Bildungsgangs an sich und auf das Bildungssubjekt ausgerichtet sein. Es wurden aber auch Standards gefordert, die sowohl zur Verbindung der Organisationsfelder Hochschul- und Berufsbildung beitragen als auch direkt auf der Ebene des gesamten Bildungssystems angesiedelt sind. Für ersteres wurde in den europäischen Dokumenten eine stärkere Integration beruflicher Bildung an den Hochschulen gefordert - für letzteres kann der Qualifikationsrahmen gelten, da dieser alle Bildungsbereiche und Lernformen in einen Rahmen integrieren soll. Um die Frage nach dem potenziellen von den Bildungsprozessen ausgehenden Handlungsdruck auf nationaler Ebene zu klären, wurden die europäischen Forderungen mit den rechtlichen und diskursiven Durchlässigkeitsstrukturen in Frankreich und Deutschland vor 1999 verglichen.

Insgesamt ist auffällig, dass insbesondere Deutschland eine signifikante Inkompatibilität der bestehenden Regelungen mit den europäischen Standards aufwies. Viele der in den europäischen Prozessen geforderten Strukturen waren gar nicht oder kaum in den rechtlichen Strukturen verankert (vgl. Tabelle 35) und wurden ebenfalls nicht thematisiert. Allein die Integration beruflicher Bildung im Hochschulsystem konnte durch das Anwachsen der dualen Studiengänge und der Anerkennung von Abschlüssen der Berufsakademien als tertiäre Abschlüsse bereits vor 1999 vorgefunden werden.

Strukturen, die auf Ebene der Bildungsorganisationen angesiedelt sind, waren vor 1999 nicht institutionalisiert. Die Bildungsgänge wurden basierend auf den Prinzipien der Inputorientierung und nicht ergebnisorientiert strukturiert, so dass bedeutend war, was, wie lange und wo und wie gelernt wurde. Dieses erschwerte die Vergleichbarkeit zwischen verschiedenen Bildungswegen, die möglicherweise ähnliche Kompetenzen vermitteln. Weder in der Hochschul- noch in der Berufsbildung existierten ein bestehendes Leistungspunktesystem oder modularisierte Bildungsgänge, wobei seit 1998 die Möglichkeit bestand, Leistungspunktesysteme an den Hochschulen einzuführen. Auch die Studienstruktur war noch einstufig, so dass Hochschulabschlüsse regulär 4 bis 5 Jahre Regelstudienzeit veranschlagten. Bis auf kaum institutionalisierte Ausnahmen, wie Einstufungsprüfungen an den Hochschulen, fand auch keine Anrechnung zwischen Berufs- und Hochschulbildung statt. Die durchlässigkeitsfördernden institutionellen Strukturen, welche das Bildungssubjekt im Fokus haben, waren in Deutschland ebenfalls kaum vorhanden. So waren zwar Fernstudienmöglichkeiten, aber kaum Teilzeitstudiengänge existent und Beratungs- sowie Unterstützungsstrukturen waren nicht für beruflich Qualifizierte an den Hochschulen ausgelegt. Schließlich gab es in Deutschland auch keinen Qualifikationsrahmen, welcher eine stärkere Transparenz zwischen den Bildungswegen hätte schaffen können.

In Frankreich vermittelte sich ein deutlich anderes Bild. So fanden sich mehr durchlässigkeitsfördernde Strukturen im Hinblick auf die Organisation der Bil- 
dungsgänge. Es gab sowohl Anrechnungsstrukturen als auch bereits ein mehrstufiges Studiensystem. Berufliche Bildungsabschlüsse, welche im französischen Referentiell gelistet waren, wurden zudem bereits kompetenzorientiert beschrieben. Auch die berufliche Bildung hatte gesetzlich geregelt durch das Loi Sequin (vgl. Kapitel 9) einen Platz an den Hochschulen. Und schließlich gab es ebenfalls schon seit 1969 einen französischen Qualifikationsrahmen, welcher zwar sechs und nicht acht Stufen beinhaltete, aber auch bildungsbereichsübergreifend wirksam war (Bouder/Kirsch 2007). Deutlich geringer fiel in Frankreich die Übereinstimmung mit den geforderten Strukturen aus, welche auf das Bildungssubjekt, also die Lernenden, zielten. Zwar fand ein Ausbau von Beratungsangeboten für Studierende statt - jedoch nicht in Bezug auf beruflich Qualifizierte. Ähnliches gilt für die geforderten Unterstützungsstrukturen, welche zum Teil durch die Einführung des Tutoriums begannen. Zudem gab es zwar die Möglichkeit des Fernstudiums, aber Teilzeitstudienmöglichkeiten waren kaum existent.

Zusammenfassend wird deutlich, dass insbesondere in Deutschland ausgehend von der vor 1999 bestehenden Gesetzeslage ein großer Handlungsbedarf bestand, während in Frankreich einige Standards bereits regulativ festgehalten waren. Angenommen werden kann, dass daher insbesondere auch in Deutschland die europäischen Prozesse viele Einflussmöglichkeiten hatten, insofern auch der normative Handlungsdruck durch die inkompatiblen Strukturen als besonders hoch eingeschätzt werden kann. Für Frankreich ist anzunehmen, dass der Handlungsdruck wesentlich geringer war und damit auch der wahrscheinliche Einfluss der europäischen Prozesse auf die Durchlässigkeitsstrukturen.

\subsubsection{Annäherung an europäische Durchlässigkeitsvorgaben seit 1999?}

Wie genau haben sich nun aber die institutionellen Strukturen in beiden Ländern nach 1999 in Relation zu den europäischen Standards geändert? In diesem Fall handelt es sich um die Frage nach der sogenannten Delta-Konvergenz. Diese soll im nächsten Schritt ermittelt werden, um einen Hinweis auf die mögliche Bedeutung der europäischen Bildungsprozesse zu erhalten.

Es werden für die Analyse nicht allein die rechtlichen Entwicklungen einbezogen, sondern auch die Informationen aus der Diskursanalyse. Werden die geforderten Standards in den Diskursen im Zusammenhang mit Durchlässigkeit thematisiert? Zudem sollen auch die Argumentationslogiken verglichen werden ebenso wie die diskutierte Zielgruppe für Durchlässigkeit. Die Einbeziehung der diskursiven Ebene ist notwendig, da erstens gesetzliche Regelungsprozesse zeitaufwendig sind und daher ein Einfluss europäischer Politik auch ohne rechtliche Implementierung möglich. Zudem verlaufen Diffusionsprozesse auf allen institutionellen Dimensionen, also auch in der kulturell-kognitiven und normativen Dimension (DiMaggio/Powell 1983; Radaelli 2000 sowie Kapitel 3 in dieser Arbeit). 
Untersucht man die rechtliche Implementierung und diskursive Behandlung der europäischen Standards von 1999 bis 2012 im Vergleich zu dem Zeitraum vor 1999 ist ein starker institutioneller Wandel für Deutschland festzustellen, aber auch in Frankreich hat eine Annäherung der Durchlässigkeitsstrukturen an die geforderten Standards stattgefunden. In Tabelle 35 ist dies nachzuvollziehen. Sowohl in Frankreich als auch in Deutschland wurden alle Standards mindestens in den Diskursen thematisiert und vielfach auch bereits rechtlich institutionalisiert. In Frankreich und Deutschland wurde jeweils begonnen, die Studiengänge lernergebnisorientierter umzustellen. Vor allem in Deutschland ist die Einführung von einer stärkeren Lernergebnisorientierung bei der Bildungsgangentwicklung als Paradigmenwechsel zu sehen (Graf 2013), da diese bis dato eher inputorientiert aufgebaut waren. Mittlerweile müssen tertiäre Bildungsgänge, welche akkreditiert werden wollen, stärker lernergebnisorientiert beschrieben werden. Eine ähnliche Entwicklung für die Hochschulbildung fand in Frankreich statt (Interview MESR2). Auch wurden die stärker beruflich orientierten Studiengänge bereits kompetenzorientiert entwickelt. In beiden Ländern wurde ebenfalls das Diploma supplement eingeführt. Auch wurde in beiden Ländern die Studienzyklenstruktur auf B.A. und M.A. sowie Doktorphase umgestellt, wobei in Frankreich weiterhin Abschlüsse außerhalb dieser Struktur, wie die beruflichen Abschlüsse BTS und DUT, erworben werden können.

Auffällig ist, dass viele der Standards, welche sowohl im Bologna- als auch im Kopenhagen-Prozess gefordert wurden, insbesondere in Bezug auf die Organisation der Bildungsgänge bereits stärker im Hochschulsystem als im Berufsbildungssystem institutionalisiert wurden. Dies gilt insbesondere für die Einführung der Leistungspunktesysteme und die Modularisierung der Bildungsgänge. In Deutschland wurde jedoch durch Pilotprogramme, wie das DECVET-Programm, der Frage nachgegangen, wie Leistungspunktesysteme in die berufliche Bildung integriert werden können (vgl. Bergzog et al. 2010; Loebe 2012). Auch in den Diskursen werden Leistungspunkte in der Berufsbildung als durchlässigkeitsfördernd angesehen. Die Umsetzung von Modularisierung im Sinne von Bildungsbausteinen fand in Deutschland ebenfalls an den Hochschulen und im begrenzteren Maße, z.B. in Berufsvorbereitungsmaßnahmen oder beruflichen Weiterbildungsgängen, in der beruflichen Bildung statt. In Frankreich spielte die Einführung von ECVET für Durchlässigkeitsfragen keine Rolle, wohl aber die Einführung von ECTS in beruflich orientierten Studiengängen, wie den BTS.

In Bezug auf Verfahren zur Anerkennung und Anrechnung von vorgängigem Lernen haben sich ebenfalls beide Länder weiterentwickelt. Wie aber in diesem Kapitel deutlich wurde, kann Frankreich diesbezüglich als Vorreiter gelten, während in Deutschland erst langsam Anrechnung eine Rolle in den Bildungsdiskursen und in den rechtlichen Reglungen spielte. Mit der Einführung von VAE wurde in Frankreich zudem ein Verfahren implementiert, welches den Erwerb ganzer Diplome auf Basis von vorgängigem Lernen, egal ob formal, non-formal 
und informell, ermöglicht. In Deutschland dagegen wurde für die Hochschulbildung zwar festgelegt, dass $50 \%$ eines Abschlusses durch Anrechnung erworben werden können, jedoch gibt es keine flächendeckend standardisierten institutionalisierten Anrechnungsverfahren. Allein Pilotprogramme wurden aufgesetzt, um zu ermitteln, wie und ob Anrechnung beruflicher Bildung in Hochschulbildung überhaupt funktionieren könne. Hierbei ging es jedoch vor allem um formal Erlerntes (vgl. Freitag et al. 2011). Für non-formal und informell erlernte Kompetenzen wurden in Deutschland noch keine Verfahren entwickelt. Bis 2013 wurden zu diesem Thema aber Arbeitsgruppen mit differentesten Bildungsakteuren eingerichtet, um einen Weg der Validierung informeller und non-formaler Kenntnisse für die Hochschulbildung in Deutschland zu erarbeiten. Für die berufliche Bildung gibt es bereits die Externenprüfung, welche die Möglichkeit bietet, via Prüfung einen beruflichen Abschluss zu erreichen, wenn mindestens das 1,5-fache der Ausbildungszeit in dem entsprechenden Beruf als Erfahrung gesammelt wurde (vgl. BBiG 2004). Da es sich um konkrete Berufserfahrung handelt, ist unklar, inwiefern auch akademische Kenntnisse angerechnet werden.

Betrachtet man die Strukturen, welche stärker auf den Lernenden zielen, ist in beiden Ländern ebenfalls eine Entwicklung in Richtung europäischer Vorgaben festzustellen. In Frankreich wurden insbesondere die Beratungsstrukturen vor und während des Studiums ausgebaut. Zudem wurde auch dem Prinzip der Orientierung auf den Lernenden, durch verstärkte Einführung von Tutorien und Verfahren der individuellen Studierendenbetreuung nachgekommen. Sämtliche dieser Maßnahmen, die den Umgang mit den heterogenen Bedürfnissen von Lernenden befördern, wurden in Frankreich durch den Plan Réussite en Licence verstärkt (vgl. Annoot 2012).

In Deutschland wurde im Diskurs ebenfalls Beratung vor und während des Studiums sowie eine individuelle Betreuung vor allem im strukturkritischen Diskurs als wichtig erachtet, aber gesetzliche Initiativen, die diese Strukturen für beruflich Qualifizierte einführen, gab es nicht. Stattdessen wurde jedoch mit dem Aufstiegsstipendium eine Möglichkeit der Studienfinanzierung für beruflich Qualifizierte eingeführt. In beiden Ländern wurde ebenfalls ein verstärkter Fokus auf den Ausbau von Teilzeitstudiengängen gelegt.

Der europäischen Forderung einer stärkeren Integration beruflicher Bildung mit Hochschulbildung kamen beide Länder nach, wobei diese auch schon vor 1999 bestand. In Deutschland wurden duale Studiengänge verstärkt ausgebaut und ein stärkerer Fokus auf berufspraktische Phasen während des Studiums gelegt. In Frankreich wurde neben den bestehenden Möglichkeiten der beruflich orientierten Studiengänge an der Universität die licence pro als beruflicher Abschluss eingeführt.

Der letzte Standard, welcher an dieser Stelle eingehender betrachtet wird, ist der Qualifikationsrahmen. In Frankreich bestand ein derartiger bereits seit 1969 und man hatte sich entschlossen diesen Rahmen von 6 auf 8 Stufen zu erweitern, um so eine größere Kompatibilität und Vergleichbarkeit mit dem europäischen 
Rahmen zu erreichen. Der französische Rahmen ist auf den Arbeitsmarkt ausgerichtet und umfasst sowohl berufliche als auch hochschulische Qualifikationen, nicht aber das technologische und allgemeine Abitur. Im Diskurs zu Durchlässigkeit spielte der Qualifikationsrahmen in Frankreich keine Rolle (vgl. Kapitel 9).

Ganz anders sah dies in Deutschland aus (vgl. Kapitel 7). Hier spielten der Qualifikationsrahmen und seine Entwicklung eine enorme Rolle, auch wenn seine mögliche Wirkung umstritten blieb. So wurde einerseits im strukturkritischen Diskurs seine Bedeutung für die Kenntlichmachung der Gleichwertigkeit von beruflicher und höherer Allgemeinbildung inklusive Hochschulbildung, für Anrechnungsmöglichkeiten und Bildungswegentscheidungen betont. Im konservativen Diskurs wurde er stattdessen auf die Rolle des Transparenzinstruments reduziert, welches möglichst ohne weiteren Einfluss bleiben sollte. In den Debatten, insbesondere über die Zuordnung von Abschlüssen in den Qualifikationsrahmen wurde das weiterhin bestehende Bildungsschisma erneut deutlich. Zum einen wurde z.B. bei der Diskussion um die Deskriptoren der Stufen viel stärker auf die Eigenheiten der Berufs- und Hochschulbildung abgezielt, anstatt auf die Gemeinsamkeiten, welche der Qualifikationsrahmen unterstreichen sollte. Zum anderen sollte mit der Einordnung des allgemeinbildenden Abiturs auf Stufe 5, einer Stufe über der beruflichen Bildung und der Fachhochschulreife, die historisch bestehende Sonderstellung und damit auch eine gesellschaftliche Hierarchisierung gesichert werden. Die letztliche Nicht-Zuordnung des Abiturs zeigte daher die weiterhin bestehende Uneinigkeit über die Wertigkeit beruflicher und höherer Allgemeinbildung auf Niveau der zweiten Sekundarstufe, aber auch dass die höhere Allgemeinbildung sich nicht mehr ohne weiteres durchsetzen kann. Nach langen Jahren der Aushandlung hat Deutschland schließlich im Januar 2012 den Qualifikationsrahmen beschlossen, im Mai 2013 eingeführt mit der Zuordnung der formalen Qualifikationen in Deutschland ${ }^{189}$ und somit die europäischen Vorgaben im Hinblick auf formale Qualifikationen erfüllt.

Insgesamt lässt sich somit eine starke Annäherung an die europäischen Vorgaben in beiden Ländern feststellen, wobei sich in Deutschland deutlich mehr getan hat, aber der Handlungsdruck auch größer war angesichts der Ausgangssituation vor 1999. Es ist jedoch schwierig von einer Delta-Konvergenz zwischen den nationalen Strukturen und europäischen Vorgaben zu sprechen und zwar aus zweierlei Gründen. Erstens sind teilweise noch nicht alle Vorgaben in Deutschland und Frankreich umgesetzt, was am Beispiel der ECVET gesehen werden kann. Zum anderen sind aber auch die europäischen Vorgaben teilweise allgemein und unspezifisch gehalten (vgl. Kapitel 5), so dass zwar vielleicht eine Entwicklung in Richtung der Vorgaben festgestellt werden kann, aber nicht klar ist, ab wann diese als erfüllt gelten können.

189 Die Tabelle der Zuordnung der Abschlüsse zum DQR findet sich im Online-Anhang in Tabelle 2. 


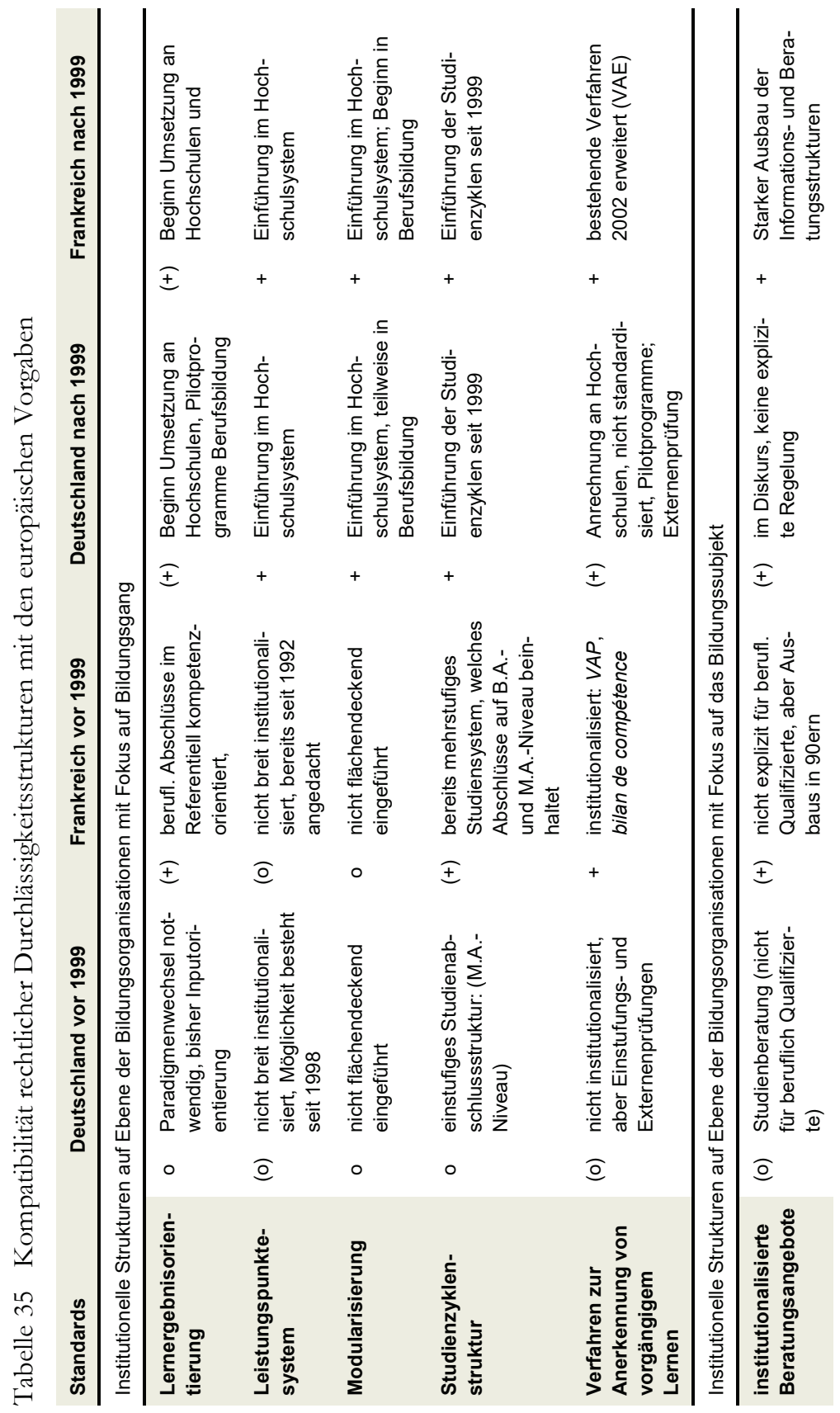




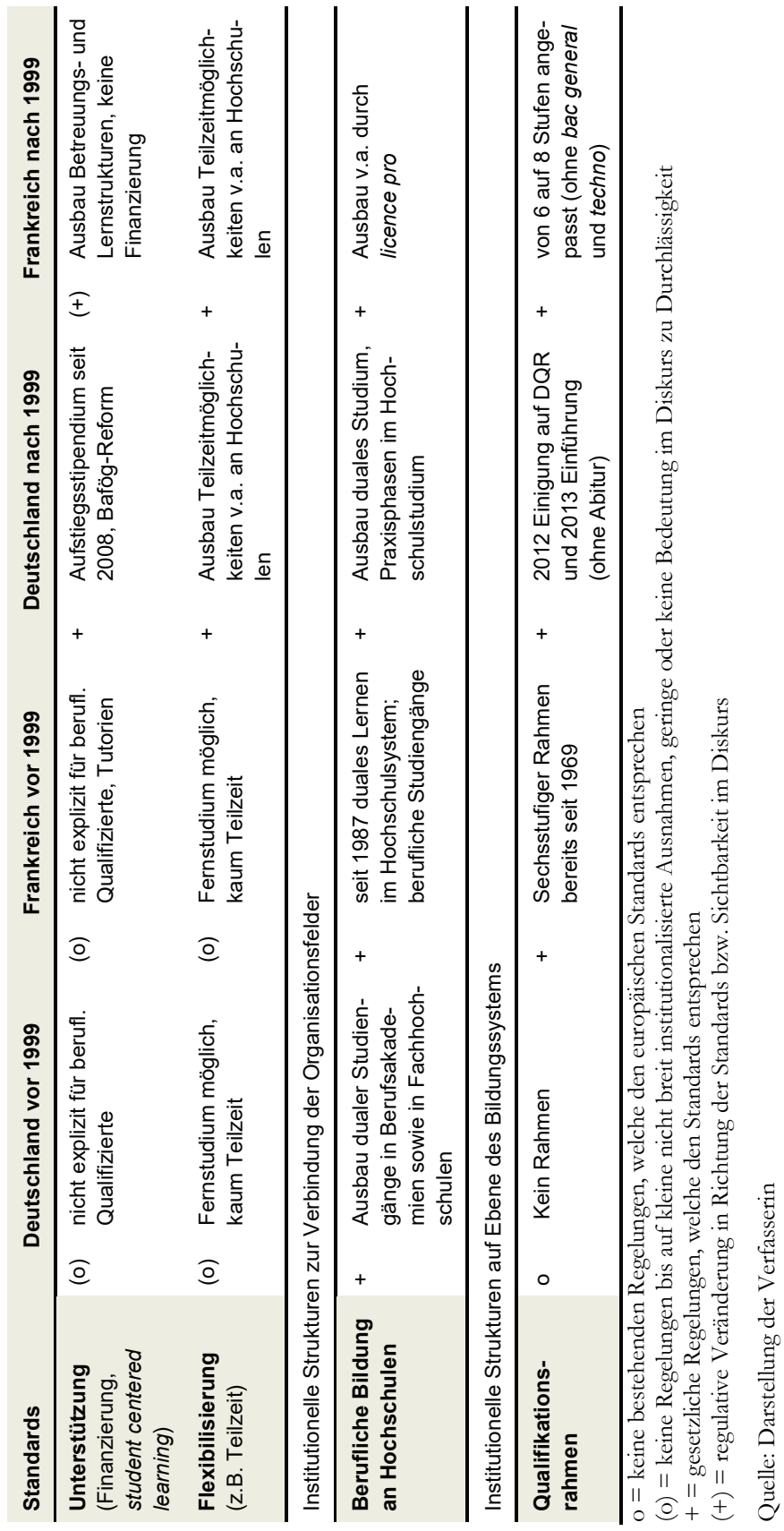


Wenn man in einem nächsten Schritt analysiert, wie Durchlässigkeit in den Deklarationen im Vergleich zu den nationalen Diskursen behandelt wird, können ebenfalls Ähnlichkeiten festgestellt werden. In beiden Ländern wie auch auf europäischer Ebene wird Durchlässigkeit zwischen Berufs- und Hochschulbildung mehr und weniger explizit über alle vier Aspekte - den Zugang, die Anrechnung, die organisationalen Verbindungen und den Umgang mit heterogenen Bedürfnissen - thematisiert, wobei auf europäischer Ebene insbesondere der Aspekt des Zugangs und der Anrechnung kontinuierlich im Fokus stand. Wie bereits gezeigt wurde, wurde vor 1999 Durchlässigkeit auf nationaler Ebene nicht anhand aller vier Aspekte thematisiert, insofern kann auch hier eine Entwicklung in Richtung europäischer Praxis festgestellt werden.

Tabelle 36 Thematisierte Aspekte von Durchlässigkeit in Deutschland, Frankreich und den europäischen Bildungsprozessen

\begin{tabular}{lccccc} 
& Europa & \multicolumn{2}{c}{ Deutschland } & \multicolumn{2}{c}{ Frankreich } \\
\hline Aspekte & Ab 1999 & vor 1999 & ab 1999 & Vor 1999 & ab 1999 \\
Zugang & $\checkmark$ & $\checkmark$ & $\checkmark$ & $\checkmark$ & $\checkmark$ \\
Anrechnung & $\checkmark$ & $\checkmark$ & $\checkmark$ & $\checkmark$ \\
$\begin{array}{l}\text { Organisationale } \\
\text { Verbindung }\end{array}$ & $\checkmark$ & $\checkmark^{*}$ & $\checkmark^{*}$ & $\checkmark$ \\
$\begin{array}{l}\text { Umgang mit } \\
\text { Heterogenität }\end{array}$ & $\checkmark$ & $\sqrt{ }^{* *}$ & $\checkmark$ & & $\checkmark$ \\
\hline
\end{tabular}

* lediglich im strukturkritischen Diskurs $\quad$ ** nur sehr begrenzt im Fokus

Quelle: Darstellung der Verfasserin

Interessant ist, dass die bereits verglichenen Standards keine Vorgaben machen, wie der Zugang geregelt werden sollte, ob z.B. über Berechtigungen oder Prüfungen. Sie stellen dagegen eher Möglichkeiten dar, wie der Zugang erleichtert werden könnte. So kann der Qualifikationsrahmen Transparenz schaffen über das Bildungsniveau von Abschlüssen und mit den lernergebnisorientierten Beschreibungen von Bildungsgängen, Hinweise geben, ob Voraussetzungen für den $\mathrm{Zu}$ gang zu einem Bildungsgang gegeben sind. Aber auch ohne explizite Vorgaben, wie Zugang geregelt werden sollte, wird dieser zur Hochschulbildung für beruflich Qualifizierte immer wieder gefordert, wobei dies im Kopenhagen- viel deutlicher als im Bologna-Prozess der Fall war. Im letzteren sollte die Gruppe der Studierenden die Diversität der Gesellschaft widerspiegeln, so dass auch diejenigen einbezogen werden, welche bisher nicht traditionell an die Hochschulen gelangten, wie beruflich Qualifizierte.

Die Zielgruppe für Durchlässigkeit in Richtung Hochschulbildung bestand in den europäischen Dokumenten aus beruflich Qualifizierten bzw. nicht traditionellen Studierenden, die eine gewisse Leistungsfähigkeit mitbringen. Wie die 
Leistung gemessen wird, blieb aber offen. Offenbar ist aber, dass zwischen einer Gruppe der Fähigen und nicht-Fähigen unterschieden wird. Wie in den funktionalistischen Diskurssträngen ist somit auch in Europa Zugang an Leistung (insofern an das meritokratische Prinzip) geknüpft. Insgesamt wird in den europäischen Dokumenten ein Bild des Lernenden, des Bildungssubjekts, konstruiert, welches ebenfalls dem der funktionalistischen Diskursstränge in den Ländern ähnelt. Der Lernende gilt für seine Bildungsentscheidungen und Bildungs(miss)erfolge weitestgehend als selbstverantwortlich, wobei die Entscheidungen durch gezielte Informationsangebote vorbereitet werden (vgl. Powell et al. 2012a).

Eine weitere Ähnlichkeit zwischen den nationalen Diskursen und den europäischen Dokumenten ist, dass zwischen einer Gruppe der traditionell Studierenden und den nicht traditionellen unterschieden wird, wobei für letztere ein besonderer Zuwendungsbedarf festgehalten wird. Diese nicht-traditionellen Studierenden können beruflich Qualifizierte oder eine andere bisher nicht repräsentierte Bevölkerungsgruppe sein. Dadurch, dass ihnen jedoch besondere Zuwendung zukommen soll, wird die Differenz zwischen den Traditionellen und den Neuen reproduziert. In den europäischen Dokumenten, aber auch insbesondere im französischen Diskurs zur Durchlässigkeit werden diese Unterstützungsleistungen begleitet mit einem allgemeinen Wechsel zu einer stärkeren Lernendenzentrierung in den Bildungsgängen, welche sich nicht nur auf beruflich Qualifizierte, sondern allgemein auf alle Bildungsteilnehmer_innen bezieht. In Deutschland spielte diese allgemeine Lernendenzentrierung eine weniger starke Rolle im Durchlässigkeitsdiskurs, d.h., hier wurde der Zuwendungsbedarf für nicht traditionelle Studierende weniger eingebettet in einen allgemeinen Wandel hin zu einer stärkeren Lernendenzentrierung in der Bildung ${ }^{190}$, so dass beide Gruppen, die Traditionellen und nicht Traditionellen, eher als homogene Gruppen betrachtet werden, die entweder Hilfe benötigen oder nicht. Die stärkere Betonung einer allgemeinen Lernendenzentrierung wie in Frankreich dagegen schwächt die Homogenisierungstendenzen $\mathrm{ab}$, insofern alle Lernenden als Individuum für unterstützende Maßnahmen beim Lernen im Blickpunkt stehen sollen.

Als letzter Schritt sollen im Folgenden auch die Argumentationslogiken der nationalen Diskurse mit den Legitimationen, welche in den europäischen Dokumenten verwandt wurden, verglichen werden ${ }^{191}$. Durchlässigkeit und die dazu führenden Maßnahmen und Standards werden auch auf europäischer Ebene sowohl funktionalistisch als auch universalistisch begründet (vgl. Kapitel 5). Wie in

190 Lernendenzentrierung war zwar durchaus in Deutschland im Zusammenhang mit dem Bolognaund Kopenhagen-Prozess ein Thema, auch wenn kritisiert wurde, dass diese bisher nicht genug im Mittelpunkt der deutschen Reformanstrengungen stand (vgl. Himpele et al. 2010: 155).

191 Der Vergleich beruht auf den bereits in Kapitel 5 erarbeiteten Legitimationsmustern aus den europäischen Dokumenten sowie den Rahmungen und Argumentationen in den Fallstudien (Kapitel 7 und 9). 
Deutschland stehen die funktionalistischen Begründungen jedoch stark im Vordergrund. Auch die Inhalte der Argumentationen ähneln denen in Frankreich und Deutschland. Im Fokus der Legitimationen standen primär die Bedarfe des Arbeitsmarkts. Bedingt durch einen Strukturwandel der Wirtschaft und technologischen Wandel müssen die Bildungssysteme in Europa durchlässig werden, um auch global wettbewerbsfähig zu sein. Durchlässigkeit wird demnach für eine Höherqualifizierung der Bevölkerung benötigt. Diese solle über den gesamten Lebensverlauf ermöglicht werden. Lebenslanges Lernen und die Verbindung mit der Lissabon-Strategie waren in den Dokumenten zentral. Verschärft werde der bereits bestehende Druck durch die demografischen Entwicklungen. Diese Argumentation ist allerdings vor allem in Deutschland und auf der europäischen Ebene präsent, während sie in Frankreich keine große Rolle spielt. Zudem sollte die Attraktivität der beruflichen Bildung durch eine größere Durchlässigkeit erhöht werden. Diese Argumentationen fanden sich ebenfalls in Frankreich und Deutschland.

Die weniger stark verwendeten universalistischen Argumentationen in den europäischen Dokumenten fanden sich auch in den deutschen und französischen Diskursen wieder. Sie betonen, dass Durchlässigkeit eine Frage der Chancengleichheit ist, insbesondere für bisher unterrepräsentierte Gruppen. Zudem könne Durchlässigkeit zu einer größeren Gleichwertigkeit zwischen beruflicher Bildung und Hochschulbildung führen. Im Gegensatz zu den Diskursen in Deutschland und Frankreich wurde Durchlässigkeit jedoch nicht mit dem Recht auf Bildung begründet. Dies ist ein großer Unterschied, da fehlende Durchlässigkeit auf diese Weise nicht als Beeinträchtigung von Grundrechten dargestellt wird. Ebenfalls nicht im Vordergrund stand die Bedeutung von Durchlässigkeit für das Individuum in den europäischen Dokumenten. Im Gegensatz zu Frankreich, wo auf die Bedeutung von Bildung und die Entwicklung eines aufgeklärten, kritikfähigen demokratisch erzogenen Bürgers hingewiesen wurde.

Gab es ebenfalls Argumente, die für die nationalen Diskurse neu waren bzw. wichtiger wurden, welche in den europäischen Dokumenten vorkamen? Deutlich wurde dies vor allem anhand der kontinuierlichen Referenz auf lebenslanges Lernen und den stattfindenden Globalisierungsdruck, wobei lebenslanges Lernen in Frankreich bereits vorher im Zusammenhang mit Durchlässigkeit thematisiert wurde und vor allem in Deutschland an Bedeutung zunahm. Während in Deutschland und Frankreich im ersten Zeitraum stärker der Wettbewerb zwischen den europäischen Ländern als Argument betrachtet wurde, war dies im Zeitraum der europäischen Prozesse vor allem der Wettbewerb mit Ländern auBerhalb Europas. In Frankreich wurde zudem die Attraktivität der beruflichen Bildung im zweiten Zeitraum im Durchlässigkeitsdiskurs relevanter. Da die universalistischen Argumentationen in den europäischen Dokumenten nicht sehr stark ausgeprägt waren, sind hier auch keine möglichen Entwicklungen für die nationalen Diskurse nachzuvollziehen. Chancengleichheit und Gleichwertigkeit waren auch bereits vorher wichtig in den nationalen Diskursen. 
Was ergibt sich aus allen diesen Vergleichen? Welche Rolle für die nationalen Entwicklungen kann für die europäischen Prozesse angenommen werden? Erstens konnte gezeigt werden, dass die nationalen Durchlässigkeitsstrukturen stark an die europäischen Vorgaben, den Standards, angenähert haben. Die Vorgaben auf der normativen institutionellen Dimension auf europäischer Ebene fanden sich demnach sowohl in der regulativen und normativen als auch in der kulturellkognitiven institutionellen Dimension von Durchlässigkeit auf nationaler Ebene wieder.

Zweitens wurde deutlich, dass wenn über Durchlässigkeit gesprochen wird, dann meist in Bezug auf alle vier Durchlässigkeitsaspekte. Sowohl in Frankreich als auch in Deutschland konnte eine Veränderung in der kulturell-kognitiven Dimension in Richtung dieser Praxis seit 1999 festgestellt werden. In beiden Ländern als auch in den europäischen Dokumenten werden durchlässige Bildungssysteme somit ähnlich behandelt.

Drittens konnte eine größere Übereinstimmung der konstruierten Zielgruppen und der Bildungssubjekte sowie vor allem der Argumentationen mit den nationalen funktionalistischen Diskurssträngen festgehalten werden. Dies gibt Hinweise auf Übereinstimmungen in der normativen und kulturell-kognitiven Dimension. Was die Argumentationslogiken angeht, kann eine größere Übereinstimmung zwischen Deutschland und europäischer Ebene festgestellt werden, da diese in beiden Ländern dominiert. Wenn Argumente aus Europa übernommen wurden, dann sind es die funktionalistischen, wobei auch hier bereits viele vor 1999 auf nationaler Ebene zu finden waren. An dieser Stelle sei erneut darauf hingewiesen, dass Europäisierung nicht nur eindimensional wirkt, sondern dass es auch die Nationalstaaten sind, welche diese Prozesse bestimmen. Insofern ist es nicht verwunderlich, dass auch auf europäischer Ebene auf bekannte nationale Argumentationen verwiesen wird.

Zusammenfassend existieren demnach deutliche Hinweise, dass die europäischen Prozesse einen Einfluss auf die nationalen Durchlässigkeitsstrukturen genommen haben könnten. Können diese bestätigt werden? Diese Frage soll im folgenden Abschnitt behandelt werden.

\subsubsection{Einfluss der Bologna- und Kopenhagen-Prozesse auf die nationalen Durchlässigkeitsstrukturen}

Wie zuvor dargestellt werden konnte, legt der Vergleich der Durchlässigkeitsentwicklungen mit denen auf europäischer Ebene die Schlussfolgerung nahe, dass die europäischen Bildungsprozesse in beiden Ländern Einfluss auf die Durchlässigkeitsstrukturen hatten. Inwiefern sich diese Annahme auch in den direkten Bezügen in den Diskursen auf die europäischen Prozesse und durch die Aussagen der interviewten Bildungsexpert_innen in beiden Ländern bestätigt, soll im Fol- 
genden gezeigt werden. Diese Kontrolle ist insofern wichtig, als dass die Annäherung an die Strukturen sich ebenso aufgrund endogener Entwicklungen vollzogen haben könnten.

\section{Wirkung der Europäisierungsprozesse in Deutschland}

Sowohl in den Diskursen als auch in den Interviews wurde ein signifikanter Einfluss des Bologna- sowie des Kopenhagen-Prozesses auf die deutsche Entwicklung von Durchlässigkeitsstrukturen bekräftigt, wobei die unterschiedlichen Akteure den Einfluss partiell unterschiedlich gewichteten.

\section{Bologna-Prozess}

Was den Bologna-Prozess betrifft, wird von allen untersuchten Akteuren im Diskurs dessen große Bedeutung für die Beschäftigung mit Fragen von Anrechnung außeruniversitärer Kompetenzen - und zwar sowohl formaler als auch non-formaler und informeller erlernter Kompetenzen - unterstrichen. Dies erfolgte diskursübergreifend. Der Bologna-Prozess wird dabei als Auslöser für die deutsche Entwicklung anerkannt. So sei der Prozess nicht nur wichtig dafür, dass das Thema aufs Tableau kam, sondern auch dafür, dass durch die konkreten Vorgaben, wie die Einführung von Lernergebnisorientierung, Leistungspunktesystemen und Modularisierung ebenfalls Instrumente für eine Anrechnungspraxis mitgeliefert wurden.

Neben der Förderung von Mobilität wurde Durchlässigkeit als ein zentrales Ziel des Bologna-Prozesses anerkannt (z.B. BMBF 2007, 2011a; DGB 2009d). So sollte die im Verlauf des Prozesses zu stärkende sogenannte soziale Dimension, d.h. die Ermöglichung einer stärkeren Teilhabe bisher unterrepräsentierter Gruppen und damit auch beruflich Qualifizierter ebenfalls in Deutschland ernstgenommen und umgesetzt werden (BMBF 2009a; DGB 2009d). Auch wurde mit dem Bologna-Prozess das Ziel einer stärkeren zielgruppenorientierten Flexibilisierung in den Diskursen verbunden (KMK 2011b). Also auch im Hinblick auf den Umgang mit heterogenen Bedürfnissen wurde im deutschen Diskurs auf Bologna verwiesen, jedoch erst gegen Ende des zweiten Untersuchungszeitraums, wesentlich seltener und auch nicht von allen Akteuren. Zudem wurde mit dem BolognaProzess auch die Förderung der Ermöglichung lebenslangen Lernens verbunden, welche wiederum als Voraussetzung durchlässige Struktur bedarf.

Insgesamt kann konstatiert werden, dass der Bologna-Prozess im Diskurs stark zur Legitimierung der in Deutschland stattfindenden Reformen, wie der Ermöglichung von Anrechnung durch den Anrechnungsbeschluss der KMK 2002 (vgl. BMBF 2009c; DGB et al. 2002; KMK 2008) und der Einführung der Standards, wie Leistungspunkte, Modularisierung und Beginn der Lernergebnisorientierung aber auch der Studienzyklenstruktur, benutzt wurde (vgl. DGB 2004a). Zudem wurde der Prozess diskursübergreifend als förderlich für Durchlässigkeit zwischen Berufs- und Hochschulbildung angesehen. 
Auch in den Interviews wurde dem Bologna-Prozess eine relevante Rolle für die Entwicklung der Durchlässigkeitsstrukturen in Deutschland seit 1999 bescheinigt. Allein die HRK betonte, dass Durchlässigkeit zwischen beruflicher Bildung und Hochschulbildung nicht als ein primäres Ziel des Bologna-Prozesses angesehen werde (Interview HRK).

Aber in Richtung Durchlässigkeit war jetzt sozusagen Bologna jetzt gar nicht so, also hat zumindest das Thema nicht auf die Agenda gebracht bei den Hochschulen. (Interview HRK)

Insgesamt wurde dem Bologna-Prozess für deutsche Durchlässigkeitsreformen eine Initiator- und Katalysatorrolle zugesprochen (Interviews BIBB1, BMBF), dabei sei das Thema Durchlässigkeit nicht mit dem Bologna-Prozess aufs Tableau gekommen, aber durch diesen unterstützt worden. So haben die bildungspolitischen Akteure Europäisierungsprozesse genutzt, um überfällige Reformen umzusetzen (Interviews BIBB1, BIBB3).

Wir haben einen neuen Drive bekommen - durch Europa. Ganz klar. Nationale Bildungsdauerbaustellen, die wir in Sachen Durchlässigkeit hatten, z.B. auch OutcomeOrientierung - sind durch Europa befördert worden. Dem konnte sich Deutschland dann nicht mehr entziehen in seinen jeweiligen Partikularinteressen, die sich hier abbilden in Sozialparteien: Arbeitgeber, Arbeitnehmer, die Schulseite, und, und und ist ja bekannt. Haben wir einen Drive bekommen. Ich habe den Eindruck, dass Bologna etwas losgetreten hat, wo man heute sagen kann - frei nach Goethe: Den Geist, den sie gerufen haben, werden sie nicht mehr los. (Interview BIBB1)

Wichtig waren europäische Prozesse, wie Bologna, zudem, da sie durch die geforderten Standards zeigten, wie man nationale Durchlässigkeitsreformen umsetzen kann (Interview BMBF). Wenn über Bologna gesprochen wurde, wurde in den Interviews auf dessen Einfluss insbesondere auf Strukturen, die den Zugang zur Hochschule und die Anrechnung erleichtern, hingewiesen. So wurde unter Einfluss von Bologna mit der Einführung der Lernergebnisorientierung begonnen, durch welche eine bessere Vergleichbarkeit zwischen Bildungsgängen ermöglicht wird. Auch die Einführung von Leistungspunktesystemen wurde genannt. Zudem wurde dem Bologna-Prozess zugeschrieben, dass er die Anrechnung berufsbezogener Kompetenzen in Deutschland voranbrachte (Interviews BIBB1, BMBF). Vor diesem Hintergrund wurden auch Pilotprojekte wie ANKOM auf dem Weg gebracht.

Der Bologna-Prozess wurde zwar mehrheitlich aber nicht nur positiv für die deutsche Entwicklung von Durchlässigkeit gesehen. So wurde einerseits angemerkt, dass im Gegensatz zum Diplom der Bachelor weniger interessant sein könnte für beruflich Qualifizierte mit Fortbildungsabschluss und so ein geringeres Interesse bestünde, an die Hochschulen zu gehen (Interview BIBB4). Im Hinblick auf die organisationale Verbindung von Berufs- und Hochschulbildung wurden auch negative Konsequenzen festgestellt. Mit den neuen Akkreditierungsregelungen, die mit der Umsetzung des Bologna-Prozess eingeführt wurden, wurden zum 
Teil innovative duale Studiengänge nicht mehr im neuen Studiensystem akkreditiert und anerkannt, da sie bestimmte Mindeststandards wie ein Mindestmaß an Stunden in der Hochschule im Vergleich zu den praxisorientierten Phasen nicht erfüllten (Interview BMBF).

Einen Einfluss des Bologna-Prozesses im Hinblick auf Durchlässigkeitsstrukturen, welche auf die Unterstützung der Lernenden zielen, wurde in den Interviews nicht angezeigt - auch wenn diese in den Diskursen als relevant für Durchlässigkeit eingestuft wurden. Dies bedeutet jedoch nicht, dass die Studierendenzentrierung nicht auch im Rahmen der Umsetzung des Bologna-Prozesses in Deutschland allgemein relevant wurde, nur weniger in Verbindung mit Durchlässigkeit. Zu erklären ist dies vielleicht damit, dass in Deutschland zeitlich recht spät erst in den letzten Jahren die Frage des Umgangs mit Heterogenität überhaupt auf die Durchlässigkeitsagenda kam.

\section{Kopenhagen-Prozess}

Der Einfluss des Kopenhagen-Prozesses auf Durchlässigkeitsstrukturen in Deutschland wurde im Diskurs und auch in den Interviews zu großen Teilen in der Entwicklung und Einführung des deutschen Qualifikationsrahmens gesehen. Mit dem DQR zusammenhängend wird die Einführung der Debatten um Lernergebnisorientierung in der Berufsbildung auch als Folge des Kopenhagen-Prozesses beschrieben.

Wie bereits im deutschen Fall dargestellt, wurde dabei der DQR nicht von allen Akteuren als wichtiges Instrument zur Schaffung von Durchlässigkeit im deutschen Bildungssystem angesehen. Das war vor allem der Fall im strukturkonservativen Diskurs. So betonten sowohl die HRK als auch die KMK, dass die Hauptrolle des DQR Hilfe bei der Schaffung eines europäischen Bildungsraums sei und weniger die interner Bildungsreformen (z.B. HRK 2009b, 2010a, KMK 2010a). Zudem sollte der DQR nicht mit Hoffnungen überfrachtet werden, da es letztlich lediglich als Transparenzinstrument dienen sollte und könne, woraus noch lange keine Durchlässigkeit entstünde (KMK 2009g). Andere Akteure hingegen sahen die Möglichkeit, dass durch den Kopenhagen-Prozess und die daraus folgende Entwicklung des DQR, Durchlässigkeit, Gleichwertigkeit und eine Höherbewertung der beruflichen Bildung im Vergleich zur Hochschulbildung in Deutschland und in Europa beitragen könnte (u.a. BMBF 2010a, DGB 2008a, 2009a, 2010e, DIHK 2010b, c).

Der DGB erwartet, dass ein DQR die Durchlässigkeit in und zwischen den Bildungsbereichen, insbesondere die Zugänge zum tertiären Bereich verbessert. Insgesamt geht es um mehr Chancengleichheit und die Herstellung der Gleichwertigkeit von allgemeiner und beruflicher Bildung im Bildungssystem. Die weitgehende Abschottung beruflicher von hochschulischen Bildungsgängen, der nur in Ausnahmefällen mögliche Übergang von der Berufs- zur Hochschulbildung und die kaum bestehenden Möglichkeiten der Anrechnung beruflich erworbenen Kompetenzen auf Studiengänge bedürfen einer Veränderung. (DGB 2009a: 16) 
Deutlich wurde, dass der einzuführende Qualifikationsrahmen per se nicht zu Durchlässigkeit führt, im Gegensatz z.B. zu Anrechnungsverfahren an den Hochschulen, aber als ein mehr oder weniger wichtiges Instrument beschrieben wurde, das Durchlässigkeit befördert, je nachdem wie es in Verbindung mit anderen Instrumenten und Regelungen verwendet wird. Insofern wurde die mögliche Unterstützung von Durchlässigkeit via DQR auf verschiedene Aspekte bezogen: Vor allem handelt es sich um Zugangs- aber auch um Anrechnungsfragen, da er bestehende Kompetenzen transparent machen und somit die Entscheidung für den Zugang und die Anrechnung erleichtern soll. Gleichzeitig könnte er aber auch benutzt werden, um Curricula zu entwickeln und institutionelle Verbindungen zu fördern (vgl. Kapitel 7).

In den Interviews spiegelten sich weitestgehend die Einschätzungen, die auch in den Diskursen sichtbar wurden, wider. Der Kopenhagen-Prozess wurde primär mit dem deutschen Qualifikationsrahmen verbunden, wobei der Qualifikationsrahmen als eine sehr wichtige Entwicklung für Durchlässigkeit in Deutschland (Interviews BIBB1, BIBB2, BIBB5, BMBF) und sogar als Katalysator sowie ,historischer Durchbruch“ (Interview BIBB1) angesehen wurde. Die Bedeutung des DQR wird zudem bestätigt, da anhand der vier Testbereiche gezeigt wurde, dass auch im deutschen segmentierten System ein bildungsbereichsübergreifender Rahmen funktionieren kann (Interview BIBB1, BIBB5).

Auch wenn die Rolle des DQR für Durchlässigkeit weitestgehend als sehr wichtig eingestuft wurde, wurde dies wie auch im untersuchten Diskurs von der HRK (Interview HRK) infrage gestellt. Der Qualifikationsrahmen sei ein Transparenzinstrument und könnte nachteilig für Durchlässigkeit wirken, wenn allein über den Rahmen die Befähigung zu Bildungsgängen abgeleitet würde. Dies könnte zu systematischen Enttäuschungssituationen führen. Viel stärker solle der DQR eine Orientierungs- und Ermutigungsfunktion für die Individuen im Lebensverlauf ausfüllen.

Wir finden, er hat eine ganz wichtige Funktion da, wo es um Ermutigung geht, weiterzulernen und sich andere Qualifikationsformen und Qualifikationsstufen zu erschließen. Und auch sich mit seiner Bildungsbiographie besser verorten zu können. (Interview HRK)

Zudem wurde auf die Gefahr hingewiesen, dass die pauschale Zuordnung von Abschlüssen auf ein alleiniges Niveau sich auch negativ für Durchlässigkeit auswirken könnte, da sie Anrechnungsprozesse behindern könnte. Dies ist der Fall, wenn man davon ausgeht, dass man mit einem Abschluss Fertigkeiten, Kenntnisse und Kompetenzen auf unterschiedlichen Niveaustufen erwirbt. Durch die klare Zuordnung können vielmehr wieder Grenzen gezogen werden, welche bei einer lernergebnisorientierten Beschreibung von Qualifikationen so nicht bestünden. Letztlich sei dann der DQR als Matrix zur lernergebnisorientierten Beschreibung von Niveaustufen ausreichend (Interview BMBF).

Mit dem Kopenhagen-Prozess wurde in den Interviews jedoch nicht nur der DQR verbunden, sondern auch die Leistungspunkte, die ECVET, eine stärkere 
Kompetenz- also Lernergebnisorientierung sowie Modularisierung in der Berufsbildung, die Durchlässigkeit befördern können (Interviews BIBB1, BIBB3, BMBF).

In den Interviews wie auch in den Diskursen wurde somit ersichtlich, dass die europäischen Bildungsprozesse einen signifikanten Einfluss auf die deutschen Reformen hatten. Aber es wurde ebenso betont, dass die Problematik der Durchlässigkeit und der Verbindung von Berufs- sowie Hochschulbildung bereits seit längerem bestand und auch im Bewusstsein der Akteure war. Insofern können die Durchlässigkeitsentwicklungen als Zusammenspiel europäischer Impulse, nationaler wirtschaftlicher und gesellschaftlicher Entwicklungen begründet werden (Interviews BIBB5, HRK). Europäisierungsprozesse wurden dann auch konkret genutzt, um Reformen voranzubringen, zumal sie partiell auch klare Instrumente für die nationale Umsetzung mitlieferten (Interviews BMBF, BIBB1, BIBB2, BIBB5). Es handele sich aber nicht nur um einen top-down-Prozess, da zum einen deutschen Akteure auch auf europäischer Ebene aktiv an den Prozessen mitwirken (Interviews BIBB1, BIBB 2, BMBF), aber auch weil es nicht zu einer automatischen Umsetzung kommt, sondern diese an die nationalen Reformbedarfe angepasst werde (Interview BIBB 3).

\section{Wirkung der Europäisierungsprozesse in Frankreich}

Auch in Frankreich haben sich die Durchlässigkeitsstrukturen in Richtung europäischer Vorgaben geändert, wobei ein geringerer Handlungsdruck bestand als in Deutschland. Inwiefern werden diese Änderungen in den Diskursen und den Interviews auf europäische Einflüsse zurückgeführt? Es wurde deutlich, dass dem Bologna-Prozess im Gegensatz zu Deutschland eine deutlich wichtigere Rolle zugeschrieben wurde, während der Kopenhagen-Prozess insgesamt als wenig einflussreich beschrieben werden kann. So fand er in den Diskursen zur Durchlässigkeit keine Erwähnung und war sogar auch teilweise bei den befragten Expert_innen unbekannt.

\section{Bologna-Prozess}

Im französischen Diskurs wird ein Einfluss des Bologna-Prozesses auf verschiedene Durchlässigkeitsaspekte sichtbar. Zum einen wurde der Bologna-Prozess als förderlich für den Ausbau einer stärkeren Flexibilisierung, Individualisierung und damit auch Studierendenorientierung sowie verbesserten Teilzeitmöglichkeiten betrachtet. Dies sei insbesondere durch die Einführung von Modularisierung und der Leistungspunkte an den Hochschulen möglich (MEN 2001b, CPU 2012). Von Gewerkschaftsseite wurde allerdings kritisiert, dass die durch Bologna induzierte Semestereinführung an den Hochschulen nur dann zur Flexibilisierung führen kann, wenn auch die Lehrveranstaltungen in den Semestern angepasst und nicht nur alle zwei Semester angeboten werden (FSU 2011). 
ECTS und Modularisierung wurden zudem auch als wertvolle Instrumente angesehen, welche eine Anrechnung von Kompetenzen allgemein erleichtern können (MEN 2001b, MESR 2011b MEDEF 2011; CPU 2008; ADIUT 2009b). Die erweiterten Anrechnungsmöglichkeiten wurden dabei als ein Weg gesehen, um lebenslanges Lernen an den Hochschulen zu vereinfachen. Die Idee von Anrechnung und der Ermöglichung von lebenslangem Lernen an sich wird jedoch nicht dem Bologna-Prozess zugeschrieben. Zum einen wurde die Lissabon-Strategie (2001) für den Bedeutungszuwachs des Ziels der Ermöglichung von lebenslangem Lernen als wesentlich wichtiger erachtet (MDEP 2000e, MEN 2008b). Zum anderen wurde aber auch Frankreich als Vorreiter für die Schaffung von Strukturen, die lebenslanges Lernen ermöglichen, dargestellt (MDEP 2000e, 2001b; CPU 2008). So sollte z.B. ein Verfahren, wie das VAE, zusammen mit einem Register alle Berufsabschlüsse, die darin kompetenzorientiert beschrieben sind, in ganz Europa eingeführt werden (CPU 2008).

Schließlich wurde der Bologna-Prozess in den Diskursen auch noch als förderlich für die organisationalen Verbindungen, zum einen über die Integration beruflicher Bildung und Hochschulbildung über die licence pro aber auch über eine bessere Verbindung zwischen beruflich orientierten und allgemeinbildenden Studiengängen, den sogenannten passerelles, angesehen. Die verbesserte Verbindungsmöglichkeit bestehe durch das Leistungspunktesystem und die Modularisierung, was auch zu einer Erleichterung von Umorientierungen im Studienverlauf führe (MEN 2001b; CPU 2008). Die Einführung der licence pro wurde ebenfalls als eine Reaktion auf den Bologna-Prozess beschrieben und sollte ganz im Sinne der im Bologna-Prozess geforderten „employabilty“ zu einer stärkeren Ausrichtung auf den Arbeitsmarkt auch nach dem Beginn eines eher allgemeinbildenden Studiums führen (MDEP 2001b, 2002; CGT 2005). Der Bologna-Prozess wurde dabei allerdings nicht nur als positive Entwicklung für das Bildungssystem beschrieben. Vor allem die Gewerkschaften kritisierten eine zunehmende Vermarktlichung der Bildung, da diese nur auf ihre Verwertbarkeit reduziert wird (FSU 2001a).

In den Interviews wurde der Bologna-Prozess ebenfalls als wichtig für die Entwicklung des französischen Bildungssystems im Hinblick auf Durchlässigkeit angesehen. Zum einen werde durch ECTS, Kompetenzorientierung und Modularisierung Anrechnung erleichtert (Interviews MESR2, CNCP, CPU1, CPU3). Diese Bologna induzierten Entwicklungen führen dazu, dass sich in den Hochschulen berufliche und allgemeinbildende Studiengänge annähern (Interview CPU3). Sie erleichtern Übergänge und somit auch Umorientierungen im Hochschulsystem. Mit den passerelles ist es nun möglich, nicht erst nach Abschlüssen zu wechseln, sondern auch während des Studiums, und gleichzeitig gelernte Kompetenzen anerkannt zu bekommen (Interview MESR2). So sind Instrumente, wie ECTS, als wichtig eingeschätzt worden, um zudem eine größere Flexibilität der Bildungswege und damit eine Anpassung an die Bedürfnisse der Studierenden zu erleichtern. Damit unterstützen sie die Reversibilität von Bildungsentscheidungen (Interview MEDEF). Es wurde angemerkt, dass mit dem Bologna-Prozess zwar 
eine bessere Durchlässigkeit, im Sinne einer höheren Erfolgsquote, ermöglicht wurde. Allerdings führen das neu eingeführte System der kontinuierliche Prüfungen ${ }^{192}$ und Kompensationsmöglichkeiten dazu, dass zum einen die Kohärenz der Studiengänge sowie die Qualität geringer geworden sei. Dies wirke sich letztlich darin aus, dass zwar mehr ein Licence Niveau erreichen, aber die große Auslese auf den Master verschoben wurde (Interview Sauvons).

Schließlich sei durch Bologna die Struktur licence, master, doctorat (LMD) die neue Norm (Interviews ParisV, CMH1, MESR1, CEREQ4, SNUEP), wodurch auch viele Absolvent_innen der beruflichen Studiengänge ihr Studium nach dem zweijährigen Abschluss verlängern (Interview CGPME). Dies wird zum Teil negativ bewertet, da dies zu einer Abwertung ebenjener zweijährigen beruflichen Abschlüsse führe (Interview SNUEP). Die Einführung der dreistufigen Zyklenstruktur wird damit klar auf Bologna zurückgeführt. Ein deutlicher Einfluss wurde dem Bologna-Prozess auch bei der Einführung der licence pro zugeschrieben, da durch Bologna die Frage der stärkeren beruflichen Ausrichtung in den Fokus trat (Interviews MEDEF, CEREQ1, Sauvons, SNUEP). Für Frankreich typisch sei die Einführung eines neuen Studiengangs, so dass die bestehenden stärker allgemeinbildend ausgerichteten licence-Studiengänge wenig geändert wurden (Interview CPU 1). Diese Entwicklung wird jedoch auch positiv gesehen, da so berufliche Abschlüsse auf allen Niveaustufen erreicht werden können (Interview CEREQ4). Die licence pro ist demnach eine Reaktion auf die neue Zyklenstruktur, so dass es auch auf diesem neuen Standardniveau der licence einen beruflichen Bildungsgang gibt sowie eine Antwort auf die geforderte beruflicher Ausrichtung. Ursprünglich war die licence pro angedacht als Weg, aus dem allgemeinbildenden Hochschulzweig in einen beruflich orientierten zu wechseln, also Übergänge von Allgemeinbildung zur beruflichen Hochschulbildung zu erleichtern und vor allem auch denen zu helfen, welche Probleme in der licence générale haben. Diese Hoffnung hat sich nicht bestätigt, da es vor allem Absolvent_innen der IUT und BTS sind, die eine licence pro machen (Interviews CPU 3, MESR2).

Zusammenfassend bleibt festzuhalten, dass der Bologna-Prozess sowohl im Diskurs als auch von den Expert_innen als einflussreich für die französischen Durchlässigkeitsstrukturen gesehen wurde. Dies gilt für Strukturen der Anrechnung, der organisationalen Verbindungen (durch passerelles und licence pro) sowie für den Umgang mit heterogenen Bedürfnissen. Deutlich wurde in den Interviews jedoch auch, dass diese Reformen durch Europa vereinfacht wurden, aber durch Frankreich auch viel auf die europäische Agenda kam (Interviews MESR2, CPU1). Im Zusammenhang mit Europa nicht erwähnt wurde der Ausbau von

192 Zuvor waren Abschlussprüfungen am Ende des Studienjahres üblich, durch die die Selektion der Studierenden erfolgte. 
Informations- und Beratungsangeboten. Hier kann eine stärkere national induzierte Entwicklung angenommen werden ${ }^{193}$, zumal in den Diskursen vor 1999 ebenfalls diese Strukturen gestärkt werden sollten.

\section{Kopenhagen-Prozess}

Wie bereits konstatiert, war der Kopenhagen-Prozess im untersuchten Durchlässigkeitsdiskurs in Frankreich nicht relevant und auch in den Interviews spielte er kaum eine Rolle.

In den Interviews gab es drei Reaktionen auf die Frage nach dem Kopenhagen-Prozess. Erstens war dieser den Interviewten gar nicht bekannt. Zweitens wurde dem Prozess kein großer Einfluss auf französische Bildungsstrukturen zugesprochen. Dies galt für die Mehrheit der Expert_innen und wurde u.a. damit erklärt, dass in Frankreich bereits ein Qualifikationsrahmen, welcher nur angepasst werden müsse, bestehe (Interviews CPU1, CNCP), Prinzipien der Anrechnungs- und Validierungsverfahren bereits institutionalisiert seien (Interviews MESR2, CNCP) und ECVET nicht eingeführt sei (Interview MEDEF). In der dritten Position wurde zwar ein Einfluss wahrgenommen, jedoch wurde dieser kaum in Verbindung mit Durchlässigkeitsstrukturen dargestellt (Interview CGPME). Primär wurde die Möglichkeit der Validierung und Zertifizierung von beruflichen Fähigkeiten unabhängig von Abschlüssen via Modularisierung ECVET als positive Entwicklung eingeschätzt. Der einzige positive Effekt auf Durchlässigkeit wurde durch die Einführung von ECVET und ECTS und die verstärkte Kompetenzorientierung in der beruflichen Bildung gesehen, da dies zu einem größeren Dialog zwischen den Bildungsbereichen führen könne (Interview MESR2).

Insgesamt kann für Frankreich konstatiert werden, dass die Entwicklung der Durchlässigkeitsstrukturen in Richtung der europäischen Vorgaben auch durch den europäischen Einfluss zustande kam. Allerdings ist der Einfluss begrenzt gewesen. So kann z.B. die auch in Europa geforderte Weiterentwicklung von Validierungsverfahren in Frankreich mit dem VAE eher als französische als eine von europäischen Prozessen induzierte Weiterentwicklung angesehen werden. Im Gegensatz zu Deutschland sind es aber nicht beide Prozesse, die ihre Wirkung entfalten konnten, sondern lediglich der Bologna-Prozess.

\section{Nationale Entwicklungen der Durchlässigkeitsstrukturen und Einflussfaktoren im Vergleich}

Bisher konnte im Rahmen dieses Kapitels gezeigt werden, dass sich erstens die Durchlässigkeitsstrukturen zwischen Deutschland und Frankreich angenähert haben, aber sich weiterhin signifikant unterscheiden. Zweitens wurde ermittelt, dass

193 Annoot (2012) beschreibt allerdings, dass insbesondere Unterstützungsangebote in den Hochschulen auch im Zusammenhang mit dem Bologna-Prozess stehen. 
sich in beiden Ländern die Strukturen in Richtung europäischer Vorgaben zu Durchlässigkeit entwickelt haben, wobei in Deutschland der Einfluss europäischer Prozesse deutlich stärker eingeschätzt werden kann als in Frankreich. In den Länderkapiteln wurde drittens gezeigt, inwiefern sich insgesamt ein Wandel der nationalen Durchlässigkeitsstrukturen vollzogen hat. Dabei wurden für Frankreich zwar Veränderungen der Durchlässigkeitsstrukturen festgestellt, welche allerdings als Weiterentwicklung des bestehenden französischen institutionellen Pfads eingeschätzt werden können. Für Deutschland wird ebenfalls keine Abkehr von den Kernprinzipien des institutionellen Entwicklungspfads, aber doch eine deutliche Änderung festgestellt. Nachfolgend sollen nun die Ergebnisse der nationalen Kapitel mit denen in diesem Kapitel zusammen interpretiert werden, um die nationalen Entwicklungen auch unter Einbezug des analysierten Europäisierungseinflusses zu erklären.

\section{Europäisierungsprozesse als window of opportunity in Deutschland}

Durchlässigkeit zwischen Berufs- und Hochschulbildung war auch bereits vor 1999 in Deutschland ein Thema, mit dem sich bildungspolitische Akteure beschäftigt haben. Allerdings bleibt festzustellen, dass das Durchlässigkeitsverständnis relativ begrenzt war auf Fragen des Zugangs sowie organisationaler Verbindungen und auch nur einzelne rechtliche Regelungen in Sachen Zugang angestoBen wurden. Der stark durch funktionalistische Argumente geprägte strukturkritische Diskurs sah in Fragen der Durchlässigkeit vor allem die Möglichkeit, das Fachkräfteproblem der beruflichen Bildung zu lösen und weniger das Ziel, tatsächlich beruflich Qualifizierte - und wenn dann nur für eine Elite - ein Studium an den deutschen Hochschulen zu ermöglichen.

Der Zeitraum nach 1999 dagegen ist durch eine starke Dynamik sowohl in den Diskursen als auch in den rechtlichen Änderungen gekennzeichnet (vgl. Kapitel 7). In den Diskursen sowie in den Expert_inneninterviews wird ersichtlich, dass diese Dynamik stark mit dem Einsetzen der europäischen Bildungsprozesse zusammenhing. So waren zwar unter Expert_innen einige der später auch von den politischen Akteuren geäußerten Forderungen und Ideen zur Durchlässigkeit bereits verbreitet, allerdings boten erst die europäischen Prozesse den richtigen Nährboden, diese Ideen und Forderungen auch bildungspolitisch zu fordern und zu fördern. Die Diskrepanz zwischen den europäischen Vorgaben und dem deutschen System war nicht nur in der regulativen Dimension kennzeichnend, sondern wurde auch im Diskurs als gravierende Diskrepanz konstruiert, so dass mit Verweis auf die europäische Ebene eine Entwicklungsnotwendigkeit aufgezeigt wurde. Gerade im Hinblick auf die Frage von Anrechnung wird dies sowohl in den Regelungen als auch im Diskurs deutlich. Aber auch die Debatten um den Qualifikationsrahmen erhöhten die Dynamik im Durchlässigkeitsdiskurs, da durch dieses Klassifikationsinstrument die Akteure der Berufs- und Hochschulbildung gezwungen waren, sich miteinander über Wertigkeiten von Bildungsab- 
schlüssen und Definitionen von Schlüsselbegriffen auseinanderzusetzen. Als hilfreich kann ebenfalls die ähnliche vorherrschende funktionalistische Argumentationslogik in der deutschen Bildungspolitik sowie den europäischen Prozessen angesehen werden. Insofern kann Europäisierung auch als eine Gelingensbedingung ${ }^{194}$ für die Veränderung der Durchlässigkeitsstrukturen in Deutschland gelten.

Wie jedoch bereits in Kapitel 5 gezeigt wurde, sind die europäischen Vorgaben nicht sehr konkret, sondern lassen einen großen Interpretationsspielraum für die nationale Umsetzung zu. Zudem treffen europäische Ideen und Standards auf Bildungssysteme, welche durch national gewachsene Institutionen und damit in Verbindung stehende Machtverhältnisse gekennzeichnet sind (vgl. Kapitel 6). So konnte in der vorliegenden Arbeit dargelegt werden, dass europäische Prozesse zwar einen sichtbaren Einfluss auf die Diskurse um Durchlässigkeit hatten, aber dass diese Einflüsse nicht ohne Konflikte einfach in die deutschen Durchlässigkeitsstrukturen integriert wurden. Vielmehr war dieser Prozess verbunden mit deutlichen Deutungskämpfen über die Entwicklung der Strukturen, was sich in der Gegenüberstellung des strukturkritischen und -konservativen Diskurses zeigt. Und auch wenn in Rückschau der Veränderungen geschlussfolgert werden kann, dass es durch Europäisierung auch zu einer Verschiebung der Macht über die Deutungsangebote in Richtung der Vertreter des strukturkritischen Diskurses kam, zeigen sich gleichzeitig deutlich Verharrungsstrukturen des Bildungsschismas und der damit zusammenhängenden Hierarchisierung beruflicher und höherer Allgemeinbildung in den institutionellen Entwicklungen (vgl. Kapitel 7.3.2).

\section{Entwicklungen in Frankreich getrieben durch nationalen Problemdruck}

Im Gegensatz zu Deutschland kann der Einfluss der Europäisierung in Frankreich, auch wenn gezeigt wurde, dass es hier ebenfalls Veränderungen in Richtung europäischer Vorgaben gab und europäische Standards als durchlässigkeitsfördernd betrachtet werden, als nicht entscheidend für die institutionellen Veränderungen angesehen werden.

Auch in Frankreich konnte ein Wandel der Durchlässigkeitsstrukturen hin zu einer größeren Durchlässigkeit von beruflicher Sekundar- zur Hochschulbildung festgestellt werden. Allerdings waren die Veränderungen weniger auf der regulativen als auf der normativen und kulturell-kognitiven institutionellen Dimension festzustellen und gingen mit einer Verringerung der Diskrepanz zwischen den institutionellen Strukturen einher. Während im ersten Zeitraum berufliche Abiturient_innen bereits berechtigt waren, ein Hochschulstudium aufzunehmen, wurden sie als Zielgruppe und Problem erst im zweiten Zeitraum tatsächlich wahrgenommen. Dies stellte die größte Veränderung dar. So wurde nach der Einführung des baccalauréat professionnel, dessen Ausbau, dem Anwachsen der Absolvent_innen

194 Das Konzept der institutionellen Gelingensbedingung kann bei Solga et al. (2013) nachgelesen werden. 
und damit auch dem Anstieg an Studierenden aus dem beruflichen Zweig, die mit großen Problemen in den Hochschulen zu kämpfen hatten, der Problemdruck für die französische Politik immer größer. Auch wenn das Berufsabitur als Arbeitsmarkteinstiegszertifikat eingeführt wurde, wurde dieses gleichzeitig symbolisch aufgeladen und aufgewertet mit der Benennung Abitur, welches in Frankreich traditionell als Inbegriff der Möglichkeiten sozialer Mobilität und des Hochschulzugangs gilt (Brauns 1998; Duru-Bellat 2007). Zudem ist in Frankreich durch das traditionell universalistische institutionalisierte Grundverständnis der Rolle von Bildung eine Ausgrenzung von Gruppen von den Möglichkeiten der Weiterbildung nicht möglich. Jeder soll die Chance haben, sich so hoch wie möglich zu qualifizieren und das gilt umso mehr, wenn der Abschluss ein Abitur ist. ${ }^{195}$ Dies kann auch als Hauptgrund angesehen werden, warum es keinen offenen Gegendiskurs zum hegemonialen Durchlässigkeitsdiskurs gibt.

Dennoch kann festgestellt werden, dass Strukturen durch den Einfluss europäischer Prozesse und deren diffundierende Standards, wie ECTS, Modularisierung, Semestereinführung, verändert wurden, aber neue Aspekte und entscheidende Strukturen durch die Europäisierung nicht auf die französische Agenda kamen. Anrechnung war schon im ersten Zeitraum ein relevantes Thema, wurde weiterentwickelt und kann durch eingeführte europäische Standards leichter umgesetzt werden. Auch die Frage der institutionalisierten Übergänge, durch die bessere Verbindungen zwischen den Studiengängen und eine leichtere Umorientierung der Studierenden zu einer höheren Erfolgsquote führen soll, wurde durch europäische Standards der Semester, Leistungspunkte etc. erleichtert aber nicht grundsätzlich neu eingeführt. Die Frage der veränderten Pädagogik durch eine stärkere Lernendenzentrierung wird zwar auch in den europäischen Argumenten gefordert, ist jedoch bereits im ersten Zeitraum vor den Prozessen im französischen Diskurs präsent, wenn auch nicht in Bezug auf beruflich Qualifizierte. Insofern werden zwar europäische Forderungen aufgegriffen, aber sie stellen vielfach auch Weiterentwicklungen nationaler Institutionen dar.

So wird auch die gesamte Entwicklung in Frankreich als Weiterentwicklung nationaler Pfade klassifiziert, denn die Öffnung der Hochschule für Berufsabiturient_innen ist eine logische Konsequenz des französischen Selbstverständnisses des Rechts auf Bildung und des Symbols des französischen Abiturs. Ähnliches hatte sich bereits für die Absolvent_innen des technischen Abiturs, die ursprünglich auch direkt in den Arbeitsmarkt einmünden sollten, vollzogen. Gleichzeitig wird aber die bestehende Hierarchisierung der Bildungszweige nicht aufgebrochen, sondern auf der nächsthöheren Bildungsebene reproduziert. Insbesondere

195 Die einzige Eingrenzung wird durch eine meritokratische Leistungsauswahl begründet, wobei auch diese im Fall der Zugangsberechtigungen für berufliche Kurzstudiengänge außer Kraft gesetzt wurde. 
die Lenkung beruflich Qualifizierter in die STS birgt die Gefahr einer verstärkten Segregation von Bildungswegen und der Entwertung dieses Studiengangs.

\section{Fazit}

Was kann aus der Analyse des Wandels in den beiden untersuchten Fällen gelernt werden? Es wird deutlich, dass Europäisierungsprozesse zwar Einfluss ausüben, aber wie stark dieser tatsächlich institutionellen Wandel beeinflusst, ist von mehreren Faktoren abhängig. Zum einen bietet der festgestellte misfit einen Anhaltspunkt, wie groß der Handlungsdruck für die Nationalstaaten ist. Für Deutschland wurde ein wesentlich stärkerer misfit festgestellt und in Deutschland wurde ebenfalls ein größerer Einfluss der Bologna- und Kopenhagen-Prozesse erkannt.

Allerdings ist mitentscheidend, ob bereits ein Klima des Wandels existiert und damit auch Akteursgruppen, welche einen Wandel unterstützen und dann auch die europäischen Vorgaben diskursiv nutzen. In Deutschland sind es vor allem die Akteure der Berufsbildung, welche die europäischen Prozesse nutzen, um ihren Forderungen nach mehr Gleichwertigkeit und Durchlässigkeit Nachdruck zu verleihen und Positionen, die Durchlässigkeit ablehnen, zu delegitimieren. Bestehende Forschung zeigt zum Einfluss von Europäisierungsprozessen, dass völlig divergente Interessen zu Diskurskoalitionen führen können (Busemeyer 2009; Trampusch 2008). Eine solche Analyse wurde in dieser Arbeit nicht durchgeführt. Vielmehr konnte gezeigt werden, wie um die Deutungshoheit von Durchlässigkeit gerungen wird und wie sich Deutungen zugunsten eines breiteren Verständnisses von Durchlässigkeit verschieben und eine Anpassung der legitimen Zielgruppe für den Hochschulzugang, zu der im zweiten Zeitraum auch beruflich Qualifizierte gehören, stattfand.

In Frankreich dagegen, auch wenn sich die Strukturen ebenfalls in Richtung einer größeren Übereinstimmung mit europäischen Vorgaben geändert haben, demnach in Richtung einer Delta-Konvergenz entwickeln, waren die europäischen Prozesse weit weniger einflussreich für die spezifische Entwicklung der diskursiven und regulativen Durchlässigkeitsstrukturen. Die europäischen Prozesse waren unterstützend, aber wichtige Ideen und Standards, die auch von europäischen Prozessen gefordert wurden, waren bereits vor dem Beginn der europäischen Prozesse in den französischen Diskursstrukturen existent und wurden zum Teil nur später mit der Anerkennung von Berufsabiturient_innen auch auf diese Gruppe ausgeweitet. Entwicklungen in Richtung Delta-Konvergenz bedeuten daher nicht, dass es auch die europäischen Modelle sind, die zu einer Änderung führen. Es wird somit deutlich, dass in der Forschung bei der Analyse der DeltaKonvergenz keine einfachen Rückschlüsse auf den tatsächlichen Einfluss internationaler Modelle und Prozesse gezogen werden können, wenn nicht auch nationale Entwicklungen vor und nach dem Aufkommen der Modelle analysiert werden.

Insbesondere am Beispiel Frankreich, welches auch ein zentraler Akteur der Entwicklung der Bologna- und Kopenhagen-Prozesse war, muss noch einmal auf 
die Zirkularität zwischen europäischen und nationalen Entwicklungen hingewiesen werden. Eine Untersuchung dieser zirkulären Wirkungsverhältnisse war nicht Teil der Analyse, so dass nicht gesagt werden kann, wie die europäischen Standards festgeschrieben worden sind, dass aber nationale Modelle einflussreich bei der Festlegung von Standards und Normen gewesen sind, ist anzunehmen (vgl. Powell et al. 2012a). Insofern ist es durchaus möglich, dass französische Bildungsvorstellungen ebenfalls Teil des europäischen Modells sind und Europäisierung dann auch als Bestätigung nationaler Bildungsvorstellungen angesehen wird. Insbesondere bei Fragen der Anrechnung ist dies auch im französischen Diskurs zu erkennen. Ein klarer Rückschluss ausgehend von einer Zunahme der Delta-Konvergenz auf den tatsächlichen Einfluss der Europäisierungsprozesse ist nicht möglich.

Während in beiden Ländern eine Entwicklung in Richtung europäischer Vorgaben deutlich war, kam es aber nicht im gleichen $\mathrm{Maß}$ zu einer Entwicklung in Richtung Sigma-Konvergenz, also einer Annäherung der Strukturen zwischen den Ländern. Insofern kann nicht von einem Automatismus zwischen Delta- und Sigma-Konvergenz ausgegangen werden. Zwar gibt es deutliche Ähnlichkeiten in Teilen der Durchlässigkeitsstrukturen, welche auch durch die Umsetzung der europäischen Standards, wie Semester, Leistungspunkte und Modularisierung, zu erklären sind, aber gleichzeitig sind ebenso eindeutige national unterschiedliche Entwicklungen zu erkennen. Dies liegt zum einen daran, dass das europäische Durchlässigkeitsmodell nicht sehr spezifisch, sondern eher allgemein gehalten ist und damit genügend Interpretationsspielraum bei der Umsetzung ermöglicht. Zum anderen sind es die ganz unterschiedlichen institutionellen Ausgangslagen, welche die spezifische nationale Weiterentwicklung stark beeinflussen.

Im Sinne neoinstitutioneller Studien der Weltkultur, die eine starke Konvergenz zwischen den Ländern in Bezug auf bestimmte allgemeine Prinzipien annehmen, kann zwar gezeigt werden, dass Durchlässigkeit im Sinne einer Ermöglichung des Weiterlernens in anderen Bildungsbereichen als Prinzip anerkannt und auch in den Strukturen sichtbar wird. So ist sowohl in den deutschen und französischen als auch in den europäischen Dokumenten ein Verständnis von Durchlässigkeit zu rekonstruieren, welches die Aspekte Zugang, Anrechnung, organisationale Verbindungen und Umgang mit Heterogenität beinhaltet. Aber die konkrete Ausgestaltung in den Ländern wird durch die spezifischen kulturellen Bedeutungswelten (Schriewer 2007), die historisch gewachsenen Institutionen beeinflusst. 



\section{Resümee und Ausblick}

Die vorliegende Studie zielt darauf ab, den Wandel institutioneller Durchlässigkeitsstrukturen zwischen Berufs- und Hochschulbildung in Deutschland und Frankreich vor dem Hintergrund des Einflusses europäischer Bildungsprozesse $\mathrm{zu}$ analysieren. Im Folgenden werden die zentralen Erkenntnisse der Studie auf Basis einer Zusammenfassung der wichtigsten Analyseschritte dargestellt. Dabei stehen die Ergebnisse der drei herausgearbeiteten Forschungsfragen im Vordergrund. Welche Entwicklungen fanden in Deutschland und Frankreich statt? Haben sich die Bildungsstrukturen der Länder einander angenähert und inwiefern sind die jeweiligen Veränderungen durch den Einfluss der Bologna- und Kopenhagen-Prozesse zu erklären? Im zweiten Teil dieses abschließenden Kapitels werden die Grenzen der empirischen Untersuchung, die darauf abzuleitenden Forschungslücken sowie von dieser Arbeit abzuleitende gesellschaftliche Implikationen erörtert.

\subsection{Anlage der Untersuchung und Ergebnisse}

Ausgangspunkt der Studie war die Feststellung, dass sich moderne Bildungssysteme in Europa unterschiedlichsten Herausforderungen stellen müssen. Für diese Herausforderungen wird ein generell, insbesondere aber zwischen Berufs- und Hochschulbildung durchlässiges Bildungssystem, in dem es möglich ist, ein Leben lang an die Abschlüsse und erworbenen Kompetenzen anzuknüpfen, als eine wichtige Voraussetzung gesehen. Durchlässigkeit des Bildungssystems kann neben der Funktion der Ermöglichung von Bildung als Bürgerrecht (Dahrendorf 1965) auch als eine Voraussetzung für die Verringerung sozialer Ungleichheiten in Gesellschaften gesehen werden. Zudem werden durchlässige Bildungssysteme als relevant angesehen, um die Qualifikationsanforderungen der Gesellschaft zu befriedigen, welche sich durch den Wandel hin zu einer Wissensgesellschaft, den technologischen Wandel und die demografischen Entwicklungen ergeben (vgl. z.B. Baethge et al. 2007). Schließlich wird auch von internationaler und insbesondere europäischer Seite eine Ausrichtung der Bildungssysteme auf die Ermöglichung lebenslangen Lernens gefordert. Als besonders wichtig können dabei die europäischen Prozesse Bologna und Kopenhagen identifiziert werden, welche eine neue Dynamik in die europäischen Bildungsprozesse gebracht haben (vgl. Kapitel 5). Auch diese fordern die Einrichtung durchlässiger Bildungsstrukturen, um europäische Gesellschaften zu wettbewerbsfähigen Wissensökonomien zu transformieren.

Wie reagieren nun europäische Nationalstaaten auf diese Herausforderungen und inwiefern wandeln sich die institutionellen Strukturen ihrer Bildungssysteme 
in Richtung einer größeren Durchlässigkeit? Um diese Frage zu untersuchen, wurden mit Deutschland und Frankreich zwei Länder vergleichend in den Blick genommen. Ein Vergleich der institutionellen Entwicklung dieser Länder bietet sich an, da sie wichtige und einflussreiche Mitgliedsstaaten der Europäischen Union sind sowie Mitbegründer und Initiatoren der Bologna- und Kopenhagen-Prozesse. Zudem zeichnen sich beide Länder durch traditionell einflussreiche Bildungsmodelle aus (Greinert 2005, Goldschmidt 1991), welche die transnationalen europäischen Bildungsprozesse mit beeinflussen können (vgl. Powell et al. 2012b). Die Fallauswahl (vgl. Kapitel 4.1) begründet sich aber auch aus einer Logik der Unterschiedlichkeit. Die beiden Länder besitzen differente Bildungstraditionen, Ideale und institutionelle Ausgestaltungen der Berufs- und Hochschulsysteme. Damit einhergehend unterscheidet sich auch das Verhältnis zwischen Berufs- und Hochschulbildung in Deutschland und Frankreich.

In Deutschland besteht ein System, in welchem berufliche Bildung und höhere Allgemeinbildung traditionell getrennt organisiert sind (vgl. Kapitel 6). Durch diese strikte Trennung sind sowohl $\mathrm{Zu}$ - als auch Übergänge von einem Bereich in den anderen schwierig und es werden im Bildungssystem bislang die Qualifikationen, welche in einem Bereich erworben worden sind, wenig bis gar nicht in einem anderen anerkannt bzw. angerechnet. Die Forderung nach Durchlässigkeit stellt Deutschland somit vor einen hohen Handlungsbedarf.

In Frankreich (vgl. Kapitel 8) ist eine derartig strikte Trennung zwischen Berufs- und Hochschulbildung nicht vorhanden. Beruflich orientierte Bildung findet erstens zu weiten Teilen auch im Hochschulsystem statt. Zweitens ist über die berufliche Bildung im Sekundarbereich auch der Zugang zum Hochschulsystem gewährleistet. Drittens bestehen im Bildungssystem Möglichkeiten, vorgängiges Lernen und damit auch berufliche Erfahrungen anrechnen zu lassen. Allerdings ist festzustellen, dass auch in Frankreich, wo keine Trennung zwischen Berufsund Hochschulbildung im Sinne eines Bildungsschismas besteht, durchaus Probleme der Durchlässigkeit zwischen diesen Bildungsbereichen existieren. So zeigt sich das Problem in Frankreich darin, dass im Hochschulsystem die beruflichen Abiturient_innen systematisch stärker von Studienmisserfolgen und -abbrüchen betroffen sind als die Absolvent_innen der anderen beiden Abiturzweige. Die Hierarchisierung zwischen den allgemeinen und den beruflichen Bildungswegen im Sekundarbereich zugunsten der Allgemeinbildung und damit eine leistungsbasierte Negativselektion der Schüler_innen in die beruflichen Bildungszweige bildet eine Grundlage dieser Entwicklung. Gleichzeitig erfolgt mit dieser Kanalisierung der Schüler_innen in die verschiedenen Abiturzweige eine soziale Selektion, welche im Übergang vom Abitur zum Hochschulwesen erhalten bleibt (vgl. Brauns 1998, Duru-Bellat/Kieffer 2008).

Ungeachtet der differenten institutionellen Ausgestaltung der Bildungssysteme stellen sich somit folgende Fragen, die in der vorliegenden Studie beantwortet werden: 
1. Inwiefern haben sich die institutionellen Durchlässigkeitsstrukturen in den letzten Jahrzehnten geändert und wie können diese Entwicklungen beschrieben werden: Wurden bestehende Durchlässigkeitsstrukturen stabilisiert? Gab es institutionellen Entwicklungen im Verhältnis von Berufs- und Hochschulbildung, welche dennoch nichts an den gewachsenen Kerncharakteristika der nationaltypischen Durchlässigkeitsstrukturen geändert haben? Oder konnten in dieser Arbeit eine Abkehr von historischen Pfaden und eine Entwicklung neuer Kernprinzipien in Deutschland und Frankreich festgestellt werden?

2. In welchem Ausmaß geht mit den festzustellenden nationalen Veränderungen auch eine Annäherung der institutionellen Durchlässigkeitsstrukturen zwischen den Ländern einher?

3. Welchen Einfluss hatten die europäischen Bildungsprozesse auf die festgestellten Entwicklungen bzw. inwiefern waren diese eher endogen bedingt?

Um diese Fragen zu beantworten, wurde in der vorliegenden Arbeit zunächst der analytische, theoretische und methodische Rahmen für die weitere Analyse abgesteckt. In Kapitel 2 wurde zunächst ein dieser Arbeit zugrunde zu legendes Konzept von Durchlässigkeit auf Basis vorhandener Sekundärliteratur erarbeitet, denn Durchlässigkeit stellt in der Soziologie wie auch in der Erziehungswissenschaft keinen klar definierten Begriff dar. Es gibt daher verschiedene Möglichkeiten, wie über institutionelle Durchlässigkeit nachgedacht wird und wie Durchlässigkeit gefördert werden kann. Die vorangehende Analyse des Durchlässigkeitskonzepts ist notwendig, um eine zu stark eingeschränkte institutionelle Betrachtung zu vermeiden, die nur einen Aspekt untersucht und damit potenzielle weitere strukturelle Durchlässigkeitsprobleme übersieht. In dieser Arbeit wird zwischen vier verschiedenen Aspekten unterschieden:

1. Zugang

2. Anrechnung von Gelerntem aus dem jeweils anderen Bildungsbereich

3. Organisationale Verbindungen zwischen den Bildungsbereichen in Form von a) institutionalisierten Übergängen oder b) integrierten Bildungsgängen

4. Umgang mit heterogenen Bedürfnissen der Lernenden

Es ist zu betonen, dass diese analytisch getrennten Aspekte sich nicht gegenseitig ausschließen, sondern z.T. aufeinander aufbauen und die Durchlässigkeitswirkung des jeweils anderen Aspekts unterstützen können. Die Darstellung der einzelnen Aspekte machte deutlich, dass Durchlässigkeit als mehrdimensionales Konzept verschiedenste Institutionalisierungsmöglichkeiten durchlässigkeitsfördernder Bildungsstrukturen aufweist. Dabei kann unterschieden werden zwischen Strukturen, welche stärker innerhalb der Bildungsorganisationen implementiert werden können (Aspekte 2 und 4), während andere mehr die Verbindungen der 
unterschiedlichen Bildungsbereiche betreffen (Aspekte 1 und 3a). Zudem unterscheiden sich die Aspekte mit ihren verschiedenen Varianten in Bezug darauf, ob sie eher individuen- oder gruppenzentrierte Strukturen eröffnen. Insgesamt wird die starke Ambiguität des Durchlässigkeitskonzepts deutlich.

Neben der Untersuchung der Frage, wie institutionelle Durchlässigkeit konzeptualisiert werden kann, wurde in Kapitel 2 gleichfalls verdeutlicht, dass institutionelle Durchlässigkeit als eine Voraussetzung für ein sozial durchlässiges Bildungssystem gelten kann. Die Frage der Durchlässigkeit kann dabei aus einer stärker strukturfunktionalistischen oder aber einer stärker institutionen- und konflikttheoretischen Perspektive betrachtet werden. Aus letzterer Perspektive, die auch die leitende dieser Arbeit ist, wird deutlich, dass die Aufgabe von Bildungsinstitutionen die legitimierte Verteilung von Individuen auf Positionen qua Bildungsabschluss ist. Fehlende institutionelle Durchlässigkeit ist daher mit der Problematik sozialer Schließung verbunden.

In Kapitel 3 wurde anschließend der theoretische Rahmen entwickelt, der die Ansätze des soziologischen Neoinstitutionalismus und des historischen Institutionalismus mit einer wissenssoziologischen Diskursanalyse verbindet. Ziel war es, eine institutionen- und konflikttheoretische Perspektive auf die Entwicklungsprozesse der Bildungssysteme einzunehmen. Aus dieser wird betont, dass institutioneller Wandel von Durchlässigkeitsstrukturen keine bloße funktionale Anpassung an gesellschaftliche Notwendigkeiten wie z.B. an die demografische Entwicklung darstellt, sondern auch Ausdruck veränderter Machtverhältnisse in der Gesellschaft einerseits und veränderter Legitimität bestehender Institutionen andererseits sein kann.

Der Begriff der Institution wird in der vorliegenden Arbeit entsprechend dem soziologischen Neoinstitutionalismus als ein differenziertes Konstrukt gesehen, in welchem drei Dimensionen von Institutionen, die kulturelle-kognitive, die normative und die regulative (Scott 2008) unterschieden werden, wobei institutioneller Wandel auf allen drei Dimensionen stattfinden kann, allerdings in unterschiedlichem Maße. Auch wenn der Fokus in dieser Arbeit durch die Verbindung neoinstitutioneller Analysen mit der Diskursanalyse vornehmlich auf den Veränderungen des Wissens zu Durchlässigkeit, also auf der kulturell-kognitiven Dimension liegt, wurden gleichsam normative und regulative Änderungen mitberücksichtigt, um mögliche Inkonsistenzen aufzuzeigen. Zudem bietet der soziologische und historische Neoinstitutionalismus eine Varianz an theoretischen Erklärungen und Konzepten an, wie Wandel zustande kommt und beschrieben werden kann. So stellt sich die Frage nach dem Einfluss der europäischen Bildungsprozesse aufgrund der im soziologischen Neoinstitutionalismus verankerten theoretischen Annahme, dass eine europäische Zusammenarbeit zu einer verstärkten Diffusion von Ideen, Normen und Standards zwischen den Mitgliedsstaaten beiträgt, was bestehende Strukturen in den Ländern unter Veränderungsdruck setzen kann. 
Durch Einbeziehung des historischen Institutionalismus werden parrallel die jeweils historisch gewachsenen Strukturen und Abhängigkeiten bei der Analyse des Wandels mitberücksichtigt.

Die diskursanalytische Perspektive rückt den prozesshaften Charakter sowie die Konflikthaftigkeit, die mit der Transformation oder auch dem Erhalt von institutionellen Ordnungen verbunden ist, in den Vordergrund. Institutionen werden durch Diskurse erzeugt und legitimiert, sie setzen Diskurse voraus und sie werden durch diese aufrechterhalten oder gewandelt. Zum anderen sind es aber auch Institutionen, die gleichsam Diskurse beschränken. Von diesem Verhältnis zwischen Diskurs und Institutionen ausgehend wird deutlich, dass mithilfe der Diskursanalyse der Prozess der Institutionalisierung und die damit zusammenhängenden möglichen Deutungskämpfe analysiert werden können. An dieser Stelle wird die Rolle der diskursiven Herstellung der Realität offenbar: Denn nur, wenn exogene Faktoren auch diskursiv genutzt werden und neue Ideen und Leitbilder hervorbringen, können sie ihre Wirkung entfalten (vgl. Schmidt/Radaelli 2004).

Methodisch wurde eine empirische Untersuchung auf Basis eines qualitativ explorativen, vergleichenden Fallstudiendesigns durchgeführt, in welcher verschiedene Forschungsmethoden miteinander verbunden werden (vgl. Kapitel 4). Die Arbeit basiert primär auf der diskursanalytischen Auswertung von mehr als 250 Dokumenten der bildungspolitischen Akteure in Frankreich und Deutschland. Mithilfe dieser Analyse konnten das Verständnis und die Vorstellungen von Durchlässigkeit sowie deren Änderungen rekonstruiert werden, was gleichzeitig die Analyse der kulturell-kognitiven und teilweise der normativen institutionellen Dimension beinhaltete. Auf diese Weise wurden auch die verschiedenen Deutungskonflikte zu Durchlässigkeit sichtbar. Die Diskursanalyse wurde ergänzt um eine Dokumentenanalyse rechtlicher Texte, um auch die regulative Entwicklung in den untersuchten Ländern nachzuvollziehen. Expert_inneninterviews wurden durchgeführt, um ein besseres Verständnis des Bildungssystems in Frankreich zu erhalten und um die Forschungsfrage auf Durchlässigkeit einzugrenzen. Zudem wurden diese zusammen mit der diskursanalytischen Auswertung benutzt, um im Rahmen des process tracing den Einfluss der europäischen Prozesse zu verstehen. Die theoriegeleitete qualitative Inhaltsanalyse der Deklarationen und Kommuniqués der Bologna- und Kopenhagen-Prozesse stellt die Grundlage für die Analyse des Einflusses europäischer Prozesse dar, indem sie die inhaltliche Rekonstruktion der Durchlässigkeitsforderungen, -vorstellungen, -standards und -argumentationen ermöglichte, welche sodann mit den nationalen Entwicklungen verglichen wurden. Der vergleichende Ansatz der Arbeit diente weiterhin dazu, nationale Selbstverständlichkeiten und Regelungen als kontingente Konstrukte aufzudecken, kulturelle Unterschiede zu verdeutlichen und nationale Entwicklungen in eine neue Perspektive zu rücken. So konnte z.B. gezeigt werden, dass fehlende Durchlässigkeit zwischen Berufs- und Hochschulbildung nicht nur 
ein Problem in Ländern ist, die wie Deutschland stark segmentierte Bildungssysteme aufweisen, sondern auch in Ländern, in denen die Bildungsbereiche weniger stark stratifiziert sind. Gleichzeitig konnte durch den Vergleich festgestellt werden, inwiefern exogene Einflüsse wie die europäischen Bildungsprozesse unterschiedlich oder auch ähnlich auf unterschiedliche Länder wirken und inwiefern sich im Laufe der Zeit auch die Bildungsstrukturen der Länder selbst angleichen.

Im Folgenden werden die wichtigsten Ergebnisse der Länder- und Vergleichsanalysen in Bezug auf die Forschungsfragen zusammengefasst.

\subsubsection{Entwicklung der nationalen Durchlässigkeitsstrukturen in Deutschland und Frankreich}

Welche institutionellen Entwicklungen konnten in Deutschland und Frankreich festgestellt werden und wie können diese beschrieben werden? Zur Beantwortung dieser Frage wurde für beide Fälle zunächst die historische Gewachsenheit der jeweiligen Durchlässigkeitsproblematik betrachtet (vgl. Kapitel 6 und 8). Anschließend wurden in Kapitel 7 und 9 die nationalen Durchlässigkeitsstrukturen jeweils in den beiden getrennten Untersuchungszeiträumen vor und nach dem Beginn der europäischen Bildungsprozesse Bologna und Kopenhagen analysiert und schließlich miteinander verglichen. Die festgestellten Veränderungen wurden dann daraufhin bewertet, inwiefern sie zu einer Weiterführung der historischen institutionellen Entwicklungspfade, zu einer Änderung oder zu einer Abkehr von diesen beigetragen haben. Nachfolgend werden die zentralen Ergebnisse dargestellt.

\section{Deutschland}

Seit 1990 haben sich die institutionellen Strukturen in Deutschland, sowohl die in den Diskursen rekonstruierte kulturell-kognitive und normative als auch die regulative Dimension, deutlich in Richtung einer größeren Durchlässigkeit zwischen Berufs- und Hochschulbildung entwickelt, wobei die größten Veränderungen im Zeitraum nach 1999 festgestellt wurden (vgl. Kapitel 7).

Dennoch wurde in Deutschland die Trennung und Hierarchisierung der Berufs- und Hochschulbildung nicht aufgelöst. Vielmehr herrschten sowohl im ersten als auch im zweiten Zeitraum deutliche Deutungskämpfe darüber, ob, unter welchen Bedingungen und warum Durchlässigkeit zwischen Berufs- und Hochschulbildung notwendig sei. So konnten ein strukturkritischer Diskurs, in welchem eine größere Durchlässigkeit befürwortet wird, sowie ein strukturkonservativer, der einer stärkeren Durchlässigkeit und Gleichwertigkeit zwischen Berufsund Hochschulbildung kritisch gegenübersteht, herausgearbeitet werden. Unterschieden wurde im strukturkritischen Diskurs zudem zwischen einem funktiona- 
listischen und einem universalitischen Diskursstrang. Bei ersterem wird Durchlässigkeit zwischen Berufs- und Hochschulbildung primär als notwendige Voraussetzung dafür angesehen, dem Arbeitsmarkt genügend qualifizierte Fachkräfte zur Verfügung zu stellen. Im universalistischen Diskursstrang wird Durchlässigkeit vor allem als Grundlage zur Umsetzung des Rechts auf Bildung, der gesellschaftlichen Konstruktion von Gleichwertigkeit zwischen den Bildungsbereichen sowie einer Verringerung sozialer Ungleichheiten identifiziert. An dieser Stelle wird deutlich, dass sich die in Kapitel 2 bereits dargestellten Perspektiven auf Bildung und Ungleichheit auch in den Diskurssträngen widerspiegeln, wobei der funktionalistische Diskursstrang eindeutig der dominierende war. Die Argumentationsmuster im strukturkonservativen Diskurs waren ebenfalls funktionalistisch, allerdings wird hier die Durchlässigkeit von der Berufs- zur Hochschulbildung als ein Risiko für die Qualität der Hochschulbildung konstruiert.

Die wichtigsten institutionellen Veränderungen in Richtung einer größeren Durchlässigkeit, aber auch die bestehenden institutionellen Strukturen, welche das Bildungsschisma aufrechterhalten, können für Deutschland wie folgt zusammengefasst werden:

Erstens haben sich die Argumentationen hinsichtlich Durchlässigkeit geändert, wobei die dominierende Logik eine funktionalistische geblieben ist. Im ersten Zeitraum waren es insbesondere ein festgestellter Mangel an Attraktivität der Berufsbildung und die Angst vor einem daraus resultierenden Facharbeiter_innenmangel, welcher durch eine erhöhte Durchlässigkeit zum Hochschulstudium behoben werden sollte. Die Zielsetzung war damit nicht primär, mehr Individuen in die Hochschulbildung zu bringen, sondern allein durch die Eröffnung der Möglichkeit die berufliche Ausbildung wieder attraktiver zu gestalten. Im zweiten Zeitraum wurde ebenfalls ein Fachkräftemangel als dominierender Grund für den Ausbau von Durchlässigkeitsstrukturen erkannt, hervorgerufen durch einen Strukturwandel der Wirtschaft sowie technologischen und demografischen Wandel. Diesmal stand jedoch der Bedarf an akademisch Qualifizierten im Vordergrund, während beruflich Qualifizierte als Begabungsreserve gesehen wurden. Insofern ging es hier tatsächlich darum, beruflich Qualifizierte in die Hochschulbildung zu führen.

Auch das Verständnis von Durchlässigkeit wurde im zweiten Zeitraum gegenüber dem ersten deutlich erweitert: Vor 1999 wurde Durchlässigkeit im Zusammenhang mit Fragen des Zugangs und z.T. integrierter Bildungswege thematisiert. Im zweiten Zeitraum sind neben Fragen des Zugangs und der organisationalen Verbindungen auch die Aspekte Anrechnung und Strukturen, welche die Heterogenität der Studierenden beachten, in den Diskursen und den Regelungen zentral.

Als besonders wichtiger Schritt in Richtung Durchlässigkeit ist dabei die Abkehr vom alleinigen Weg in die Hochschulbildung über die Allgemeinbildung zu sehen. Berechtigungen zum Hochschulzugang können nun auch über die Berufs- 
bildung erworben werden. Dies stellt einen Paradigmenwechsel dar, da das Berechtigungsmonopol der Allgemeinbildung nun nicht mehr nur diskursiv infrage gestellt wurde, wie bereits auch schon im ersten Zeitraum. Vielmehr wurden die geforderten Änderungen auch regulativ umgesetzt.

Die Thematisierung von Anrechnung sowie die Einführung der Regel, dass bis zu $50 \%$ der Leistungen eines Studiengangs durch außerhochschulisch erworbene Kompetenzen angerechnet werden können, stellt einen weiteren wichtigen Schritt in Richtung der Stärkung von Durchlässigkeit und einer De-Hierarchisierung von Berufs- und Hochschulbildung in Deutschland dar. Die Möglichkeit, in Berufs- und Hochschulbildung gleichwertige Kompetenzen zu erwerben, wird somit im zweiten Untersuchungszeitraum nicht nur denk- und sagbar, sondern auch regulativ festgehalten. Durch die Anrechnungsdiskussionen, die Einführung diesbezüglicher Regelungen, aber auch des deutschen Qualifikationsrahmens, in welchem alle Qualifikationsniveaus ebenfalls auf beruflichem Weg erreichbar sein sollen, kommt es zu einer Neujustierung der Wertigkeiten von Bildungswegen zugunsten beruflicher Bildung, da diese partiell als äquivalent anerkannt wird.

Organisationale Verbindungen zwischen den Bildungsbereichen werden im zweiten Zeitraum in Form von dualen Studiengängen verstärkt ausgebaut. Allerdings werden diese weniger prominent als im ersten Zeitraum als eine Möglichkeit zur Schaffung von Durchlässigkeit anerkannt.

Schließlich kann im zweiten Zeitraum eine weitestgehende Akzeptanz beruflich Qualifizierter als Teil der Studierendenschaft ausgemacht werden. Mit dieser Akzeptanz einer neuen Gruppe von Studierenden geht auch die Einsicht einher, dass bestimmte Strukturen, welche bisher auf die traditionellen jungen Vollzeitstudierenden ausgerichtet sind, für beruflich Qualifizierte einer Anpassung bedürfen. So werden eine stärkere Flexibilisierung, bessere Informationsstrukturen und auch ein Stipendiensystem für beruflich Qualifizierte gefordert und z.T. eingeführt.

Trotz dieser durchlässigkeitsfördernden Veränderungen können sowohl in den Regelungen als auch in den Diskursen Elemente festgestellt werden, welche die Durchlässigkeit zwischen Berufs- und Hochschulbildung beschränken und die bestehende Hierarchisierung reproduzieren:

Zu nennen ist hier zum einen die Einseitigkeit des Diskurses, in dem Durchlässigkeit primär in Richtung von der Berufs- in die Hochschulbildung diskutiert wird, wodurch Letztere als das anzustrebende Bildungsziel konstruiert wird. Zum anderen ist der strukturkonservative Diskurs selbst bereits ein Zeichen dafür, dass das Bildungsschisma weiterhin besteht. Die Existenz dieses Diskurses zeigt deutlich, dass in den gesellschaftlichen Wissensvorräten noch immer traditionelle strukturkonservative Deutungsmuster verankert sind. So sind die Gründe, aus denen Durchlässigkeit kritisch gesehen wird, im Verlauf des letzten Jahrhunderts weitestgehend die gleichen geblieben. So wird z.B. eine Öffnung der Hochschulen für nicht traditionelle Studierende als Gefahr für die Qualität der akademischen 
Ausbildung und mögliche Resourcenverschwendung konstruiert. In den rekonstruierten Vorstellungen und den Regelungen zum Zugang zur Hochschulbildung wird zudem deutlich, dass eine Gleichstellung der sekundaren Berufsbildung mit der höheren Allgemeinbildung der Sekundarstufe II, dem Abitur, nicht stattfindet, da die direkte Berechtigung zum Hochschulzugang nur über einen beruflichen Weiterbildungsabschluss erfolgt.

Auch in den Diskussionen um den deutschen Qualifikationsrahmen wird von den Akteuren der höheren Allgemeinbildung diese Ungleichwertigkeit reproduziert. Hier wird angestrebt, das Abitur höher einzustufen als die beruflichen Abschlüsse des Sekundarbereichs II. Gleichzeitig kann auch das hier manifestierte Verständnis von Studierfähigkeit, welche bereits vor dem Studium festgestellt werden soll, als Barriere für Durchlässigkeit gesehen werden: Während die Studierfähigkeit von Absolvent_innen der höheren Allgemeinbildung als nachgewiesen gilt, wird dieselbe bei beruflich Qualifizierten als vorab zu prüfen eingeschätzt. Im Hinblick auf Anrechnung wird in den Diskursen und Regelungen deutlich, dass der Lernort der Hochschule als wichtiger betrachtet wird als die Kompetenzen, welche ein Individuum bereits vorweisen kann, da die Anrechnung von auBerhochschulisch erworbenen Kompetenzen auf 50 \% eines Studiums begrenzt wurde. Auch die Berücksichtigung beruflich Qualifizierter als neue Studiengruppe, auf deren Bedürfnisse stärker als zuvor geachtet werden müsse, wird eingeschränkt, da es vor allem im strukturkonservativen Diskurs einseitig darum geht, die beruflich Qualifizierten im Rahmen von Brückenkursen an die Anforderungen der Hochschulen anzupassen und damit deren konstruierten Defizite abzubauen. Eine Anpassung der Pädagogik und der Curricula, die berufliche Erfahrungen im Studium wertschätzen und nutzen, wird kaum gefordert. Vielmehr sollen beruflich Qualifizierte am besten genau so wie die ,traditionellen“ Studierenden ,funktionieren' und keine Mehrarbeit mit sich bringen. Schließlich wird kontinuierlich eine Differenz zwischen den, traditionellen' und den beruflich qualifizierten Studierenden konstruiert, wobei Letztere als besonders hilfsbedürftig gelten. Die diskursive Trennung von Berufs- und höherer Allgemeinbildung wird demnach auch innerhalb der Hochschule nicht aufgehoben.

Es bleibt daher zu konstatieren, dass Durchlässigkeit in Deutschland weiterhin ein umkämpftes Feld ist. Während einerseits insbesondere Akteure der höheren Allgemeinbildung ihre Positionen im Bildungswesen sowie auf dem Arbeitsmarkt verteidigen, werden diese insbesondere von den Akteuren der Berufsbildung infrage gestellt. In der Diskursanalyse wurde deutlich, dass sich die Macht über die Deutungsangebote in Richtung der Vertreter von Gleichwertigkeit zwischen Berufs- und Hochschulbildung und der Notwendigkeit von Durchlässigkeit verschoben hat. So ist im zweiten Zeitraum ein Wandel nicht nur im Verständnis von Durchlässigkeit festzustellen, sondern auch darin, dass bestimmte Denkansätze über Durchlässigkeit, so z.B., diese vollständig zu negieren, nicht mehr legitim sind. Trotz dieser Änderungen kann jedoch aus einer analyti- 
schen Perspektive keine Abkehr oder Umkehr der institutionellen Entwicklungspfade festgestellt werden. Die institutionellen Änderungen haben aber auch nicht zu einer Verfestigung des so genannten Bildungsschismas beigetragen. Vielmehr können die Entwicklungen als eine teilweise Aufweichung der historisch gewachsenen Trennung der Berufs- und Hochschulbildung angesehen werden, wobei gleichzeitig zentrale institutionelle Strukturen, die die Trennung der beiden Bildungsbereiche stützen, weiterbestehen bzw. reproduziert werden.

\section{Frankreich}

Auch für den französischen Fall konnten in Kapitel 9 Veränderungen der institutionellen Durchlässigkeitsstrukturen zugunsten von beruflich Qualifizierten festgestellt werden.

Betrachtet man die Relevanz des Themas Durchlässigkeit zwischen beruflicher Bildung und Hochschulbildung, so ist im Vergleich der Untersuchungszeiträume eine klare Bedeutungszunahme festzustellen. Drehten sich die sehr wenigen Aussagen zur Durchlässigkeit vor 1998 vor allem um den Zugang beruflich Erfahrener zur Hochschule über Validierungsprozesse, so wurde im zweiten Zeitraum Durchlässigkeit öfter im Zusammenhang mit Absolvent_innen des beruflichen Abiturzweigs diskutiert. Absolvent_innen des Berufsabiturs hatten zwar auch zuvor formal die Berechtigung für einen Hochschulzugang, wurden allerdings nicht als Zielgruppe anerkannt. Somit wurde sichtbar, dass in Frankreich im ersten Zeitraum eine deutliche Diskrepanz zwischen der regulativen Dimension einerseits und der kulturell-kognitiven sowie normativen Dimension andererseits bestand. Diese Diskrepanz verringerte sich im zweiten Zeitraum, wurde jedoch nicht aufgehoben. Die wichtigste Veränderung ist, dass berufliche Abiturient_innen nicht mehr nur ein Recht auf den Hochschulzugang hatten, sondern dass diese Tatsache auch im Selbstverständnis der bildungspolitischen Akteure angekommen ist. Auch die große Gefahr des Studienabbruchs dieser spezifischen Gruppe wurde problematisiert. Andererseits wurde und wird das Berufsabitur weiterhin primär als Arbeitsmarkteinstiegszertifikat konstruiert.

Im Gegensatz zu Deutschland konnte in beiden Zeiträumen ein hegemonialer Diskurs rekonstruiert werden, in welchem Reformen für größere Durchlässigkeit befürwortet werden. Die Diskurse bestanden jeweils aus einem universalistischen und einem funktionalistischen Diskursstrang. Im universalistischen Diskursstrang werden in beiden Zeiträumen die Ziele der Chancengleichheit und des Rechts auf Bildung als integrale Bestandteile der Ideale der Französischen Republik betont. Im funktionalistischen Diskursstrang stehen in beiden Zeiträumen die Bedarfe des Arbeitsmarkts im Vordergrund. Bildung dient der Erhöhung des Humankapitals, welches notwendig ist, um den Veränderungen durch wirtschaftlichen Wandel sowie internationalen Wettbewerb auf gesellschaftlicher und individueller Ebene zu begegnen. Insgesamt überwiegen in Frankreich universalistische Begründungen. 
Im ersten Zeitraum wurde Durchlässigkeit zwischen Berufs- und Hochschulbildung primär im Zusammenhang mit Fragen des Zugangs und der Anrechnung thematisiert. Dieses eher begrenzte Verständnis wurde im zweiten Zeitraum deutlich erweitert, indem nun zusätzlich auch organisationale Verbindungen und der Umgang mit den spezifischen Bedürfnissen beruflich Qualifizierter als durchlässigkeitsfördernd thematisiert wurden.

Betrachtet man die Frage der organisationalen Verbindung, so kann insbesondere die Institutionalisierung der lycées des métiers als ein wichtiger Schritt hin zu Durchlässigkeit von beruflicher Sekundar- zur Hochschulbildung gesehen werden. In diesen Organisationen werden mögliche Bildungsoptionen für die Lernenden sichtbar und können damit auch zur Weiterbildung motivieren. Als Entwicklung im zweiten Zeitraum sowohl in der regulativen als auch in der kulturell kognitiven Dimension sind zudem die verbesserten Verbindungen zwischen den Studiengängen der differenten Hochschulformen (die sogenannten passerelles) anzusehen. Diese wurden im Diskurs vor allem als Möglichkeiten einer frühzeitigen Korrektur oder Umorientierung während des Studiums thematisiert werden, da sie einen erfolgreichen Studienabschluss begünstigen sollen. Im französischen Diskurs wird im zweiten Zeitraum demnach als wichtig anerkannt, dass es nicht ausreicht, nur den Zugang zum Studium zu gewähren, sondern weitere unterstützende Strukturen für den Studienerfolg notwendig sind, um tatsächlich zu Durchlässigkeit zu führen.

Allerdings wird im Diskurs durch eine stärker funktionalistische Sichtweise in Bezug auf den Aspekt Umgang mit heterogenen Lernenden diese Frage für beruflich Qualifizierte primär auf deren Lenkung in den Arbeitsmarkt oder in die technischen Kurzstudiengänge (STS) begrenzt. Dabei soll das differenzierte System der Hochschulbildung letztlich dazu führen, dass die unterschiedlichen Abiturgruppen jeweils in die für sie als passend konstruierten verschiedenen Studiengänge einmünden. Die traditionelle Hierarchisierung zwischen beruflichen, technischen und allgemeinen Bildungswegen, die bereits durch die Selektion der schwächeren Schüler_innen in die berufliche Sekundarbildung beginnt, bleibt bestehen. Dies wird in der vorliegenden Arbeit als größte Barriere für Durchlässigkeit erkannt. Königsweg in Frankreich bleibt eindeutig der allgemeinbildende Zweig, dessen Absolvent_innen alle Bildungswege offen stehen.

Es bestehen in Frankreich zwar keine eindeutigen Deutungskonflikte zwischen strukturkritischen und -konservativen Diskursen wie in Deutschland, allerdings wird im Diskurs das Spannungsverhältnis zwischen universalistischen und funktionalistischen Vorstellungen deutlich. So sind die Begründungen, warum auch beruflich Qualifizierte Zugang zur Hochschulbildung erhalten und diese erfolgreich abschließen sollten, stärker durch universalistische Argumentationen geprägt, während die konkreten Vorstellungen, wie Durchlässigkeit für beruflich Qualifizierte aussehen könnte, nicht gänzlich, aber doch stärker funktionalistisch angelegt sind: Die Zielgruppe soll hier auf eine Leistungselite der beruflich Qualifizierten begrenzt und primär an die Kurzstudiengänge (STS) gelenkt werden, 
während die Abiturient_innen des technologischen und allgemeinbildenden Zweigs für ein Studium an den Universitäten, den technischen Hochschulen (IUT) oder den grandes ecoles interessiert werden sollen. Insofern wird die Hierarchisierung zwischen den Bildungszweigen auch im Durchlässigkeitsdiskurs weiterhin reproduziert. Aus diesem Grund klassifiziert die vorliegende Arbeit auch die Entwicklungen in Frankreich im Wesentlichen als eine Weiterführung bestehender institutioneller Pfade, obwohl sich das französische System stärker als zuvor in Richtung Durchlässigkeit für beruflich Qualifizierte bewegt hat. Diese Bewegung wird jedoch als integraler Bestandteil des französischen Entwicklungspfads gedeutet (vgl. Kapitel 8 und 9). Die Öffnung der Hochschule für Berufsabiturient_innen ist eine logische Konsequenz des französischen Selbstverständnisses des Rechts auf Bildung und des Symbols des französischen Abiturs, welches zur Aufnahme einer tertiären Bildung berechtigt und im französischen Selbstverständnis traditionell auch als ein Symbol für soziale Aufstiegsmöglichkeiten gilt. Es kommt damit im französischen Bildungssystem zwar zu der Möglichkeit einer Höherqualifizierung beruflich Qualifizierter, allerdings ist dies eine Entwicklung, die die bestehenden Hierarchien nicht aufzubrechen vermag.

Deutlich wurde anhand des französischen Falls ebenso, dass regulative Änderungen nicht automatisch einhergehen müssen mit Änderungen der kulturellkognitiven Dimension. Dies ist ein Beleg für die Bedeutung einer differenzierten Analyse der institutionellen Dimensionen für die Beurteilung nationaler Durchlässigkeitsstrukturen.

\subsubsection{Vergleich institutioneller Durchlässigkeit in Deutschland und Frankreich}

Sowohl in Deutschland als auch in Frankreich konnte ein Wandel in Richtung größerer Durchlässigkeit zwischen Berufs- und Hochschulbildung festgestellt werden. Doch was bedeutet dies im Hinblick auf das Verhältnis der institutionellen Strukturen zwischen den Ländern? Kam es zu einer Annäherung, zu einer Entwicklung in Richtung Sigma-Konvergenz? Um diese Frage zu beantworten, wurde in Kapitel 11 zuerst die Ausgangssituation beider Länder im ersten Untersuchungszeitraum verglichen, um dann eine Aussage über die Entwicklung im zweiten Zeitraum treffen zu können. Betrachtet wurden sowohl die zentralen regulativen als auch die dominanten diskursiven Strukturen, wobei die Argumentationslogiken ebenso wie die spezifischen Vorstellungen von Durchlässigkeit verglichen wurden.

Für den ersten Zeitraum konnten sowohl in der regulativen Dimension als auch in den diskursiven Strukturen starke Diskrepanzen zwischen Deutschland und Frankreich festgestellt werden. Insgesamt wurde in Frankreich Durchlässigkeit formal besser umgesetzt als in Deutschland. Aber auch im Verständnis von Durchlässigkeit konnten signifikante Unterschiede gefunden werden. Die größten 
Unterschiede bestanden in der geringeren Relevanz des Themas in Frankreich im Vergleich zu Deutschland und darin, dass in Deutschland die Durchlässigkeitsdiskurse konfliktbehaftet waren. Ähnlichkeiten bestanden allerdings in den in beiden Ländern fehlenden Regelungen und Aussagen zur Anpassung bestehender Bildungsstrukturen an die Bedürfnisse beruflich Qualifizierter.

Im zweiten Zeitraum kann eine deutliche Annäherung der Durchlässigkeitsstrukturen in den beiden Ländern festgestellt werden. Es besteht zwar weiterhin der Unterschied des in Deutschland im Vergleich zu Frankreich stärker umkämpften Diskursfeldes, gleichzeitig sind jedoch die universalistischen und funktionalistischen Argumentationslogiken mit den darin konstruierten Bildungssubjekten in den Diskursen in Deutschland und Frankreich ganz ähnlich. In den funktionalistischen Diskurssträngen dominiert der Blick auf das Individuum als Humankapitalressource für den Arbeitsmarkt, während in den universalistischen stärker das Individuum als Person im Vordergrund steht, welches mithilfe von Bildung seine Persönlichkeit weiterentwickelt und so zu einem aufgeklärten, kritikfähigen Bürger wird. Ein deutlicher Unterschied besteht allerdings in der Verteilung funktionalistischer und universalistischer Argumentationen, wobei in Frankreich die universalistischen deutlich wichtiger waren als in Deutschland.

Die eindeutige Annäherung der Durchlässigkeitsstrukturen wird auch sichtbar im Vergleich der im Diskurs oder auch Regularien gefundenen Aspekte von Durchlässigkeit. Sie umfassen in beiden Ländern Fragen des Zugangs, der Anrechnung und organisationaler Verbindungen sowie die Frage nach dem Umgang mit heterogenen Bedürfnissen beruflich Qualifizierter. Wenn man die genauen Inhalte und Regelungen zu den Aspekten analysiert, werden allerdings neben den wachsenden Ähnlichkeiten auch deutliche Unterschiede sichtbar.

In beiden Ländern ist der Zugang zum Hochschulsystem über Berechtigungen, die im beruflichen Bildungswesen erworben werden können, möglich. Der Unterschied besteht vor allem darin, dass in Frankreich die Berechtigung mit dem Berufsabitur nach einer Erstausbildung erworben werden kann, in Deutschland hingegen der Abschluss einer beruflichen Weiterbildung nach dem Erstabschluss notwendig ist. Sowohl in Frankreich als auch in Deutschland gehören außerdem beruflich Qualifizierte im Diskurs zu der Gruppe der nicht traditionellen Studierenden.

Bei dem Vergleich der rechtlichen und diskursiven Strukturen im Hinblick auf Anrechnung kann ebenfalls eine Annäherung der deutschen Strukturen an die französischen festgestellt werden. Anrechnung ist mittlerweile in Deutschland zum Thema geworden, wenn über Durchlässigkeit zwischen Berufs- und Hochschulbildung nachgedacht wird. In beiden Ländern wird somit anerkannt, dass in der beruflichen Sphäre äquivalente Fähigkeiten zu denen der Hochschulbildung erlangt werden können. Anrechnung soll in beiden Ländern an den Hochschulen stattfinden. Die Anrechnungsmöglichkeiten sind in Frankreich jedoch viel stärker ausgebaut und auch viel selbstverständlicher als in Deutschland, wo keine geregelten Verfahren wie das französische Validierungsverfahren (VAE) existieren. 
Zudem werden in Deutschland klare Begrenzungen der Anrechnungsmöglichkeiten festgelegt, während in Frankreich ganze Hochschuldiplome auf diesem Weg erworben werden können. In Frankreich wird außerdem stärker von einem individuumszentrierten Anrechnungsverfahren ausgegangen als in Deutschland.

Im Gegensatz zum Zeitraum zuvor werden integrierte Bildungsformen in beiden Ländern nun als durchlässigkeitsfördernd angesehen. In Deutschland ruht der Fokus im Diskurs insbesondere auf dualen Studiengängen. Auch in Frankreich werden Studiengänge en alternance als ein möglicher Weg gesehen, um beruflich Qualifizierten im Hochschulwesen zum Erfolg zu verhelfen. Ebenfalls als wirksam werden die lycées des métier konstruiert. Ein Unterschied wird allerdings deutlich in der Thematisierung institutionalisierter Übergänge zwischen allgemeinbildenden und beruflichen Studiengängen, welche in Frankreich als ein wichtiger Weg konstruiert werden, um auch beruflich qualifizierte Studierende durch ,Umorientierung' in stärker berufliche Studiengänge zum Erfolg zu bringen. In Deutschland spielt für die Frage von Durchlässigkeit diese Art von organisationaler Verbindung keine Rolle.

Schließlich ist für beide Länder zu konstatieren, dass das Thema Umgang mit den Bedürfnissen der bereits beruflich qualifizierten Studierenden wesentlich wichtiger geworden ist als vor 1999, wobei diese Fragen in Deutschland vergleichsweise spät aufkamen und in Frankreich einen größeren Stellenwert im Diskurs einnahmen. In beiden Ländern wurden neue rechtliche Regelungen getroffen, und auch in den Aussagen zu Durchlässigkeit spielt der Umgang mit Heterogenität eine immer größere Rolle. Sowohl in Frankreich als auch in Deutschland wird davon ausgegangen, dass beruflich Qualifizierte die Heterogenität der Studierendenschaft signifikant erhöhen. Darauf müsse vermehrt reagiert werden, da diese Gruppe nicht zu den traditionellen Studierenden gehöre. Betrachtet man die rechtlichen Regelungen, findet in beiden Ländern eine Stärkung der Flexibilisierungsmöglichkeiten des Studiums statt. Jedoch bestehen unterschiedliche Schwerpunkte in den Vorstellungen dazu, welche Strukturen verändert werden müssten. In Frankreich ruht der Fokus sowohl diskursiv als auch regulativ stärker auf der Bereitstellung von Informations- und Beratungsangeboten sowie einer veränderten Pädagogik - weg von großen Vorlesungen hin zu stärker interaktiven und individualisierten Lehrmethoden wie Tutorien. In Deutschland wurde regulativ mit dem Aufstiegsstipendium eine finanzielle Unterstützung leistungsselektierter beruflich Qualifizierter beim Studium auf den Weg gebracht. Eine Anpassung der Pädagogik wurde hingegen kaum gefordert, wohl aber Strukturen, welche die Passung zwischen beruflich Qualifizierten und Hochschulen erhöhen, wie Informations- und Beratungsangebote sowie Vorbereitungskurse. Die Anpassung soll dabei primär von den Lernenden erbracht werden.

Insgesamt konnte somit gezeigt werden, dass es zwar zu einer Annäherung der Durchlässigkeitsstrukturen gekommen ist, aber nicht zu einer vollständigen Sigma-Konvergenz, da viele nationaltypische Strukturen weiterhin bestehen. Die 
nationalen Entwicklungen werden durch die jeweils spezifischen Durchlässigkeitsproblematiken und nationalen Bildungsstrukturen mitbestimmt.

\subsubsection{Einfluss europäischer Bildungsprozesse}

Die dritte große Forschungsfrage, welche im Rahmen dieser Arbeit beantwortet wurde, behandelt den Einfluss der europäischen Bildungsprozesse Bologna und Kopenhagen auf die nationalen Entwicklungen in Deutschland und Frankreich. Um diese Frage zu beantworten, waren mehrere Analyseschritte notwendig.

Erstens wurden in dieser Arbeit die Deklarationen und Kommuniqués der Bologna- und Kopenhagen-Prozesse, in denen die Nationalstaaten sich auf gemeinsame Ziele und Standards geeinigt haben, daraufhin analysiert, wie genau Durchlässigkeit thematisiert wird. Dieser Schritt war notwendig, um den Prozessen zugeschriebene Charakteristika nicht ungeprüft zu übernehmen, sondern tatsächlich zu zeigen, welche konkreten Vorstellungen sowie Vorgaben zu Durchlässigkeit in den Prozessen eine Rolle spielen. Dabei wurde gleichzeitig untersucht, inwiefern sich die Durchlässigkeitsvorgaben in beiden Prozessen ähneln bzw. widersprechen.

Es konnte in Kapitel 5 gezeigt werden, dass sich trotz einiger deutlicher Unterschiede in den europäischen Bildungsprozessen bezüglich der Weise und des Umfangs, in dem Durchlässigkeit thematisiert wird (stärker im Kopenhagen als im Bologna Prozess), eine weitgehende Übereinstimmung im Verständnis von Durchlässigkeit feststellen lässt. Aus diesem Grund wird in der vorliegenden Arbeit von einem europäischen Kernkonzept von Durchlässigkeit gesprochen. Wesentliche Charakteristika dieses Konzepts werden nachfolgend zusammengefasst: Das europäische Durchlässigkeitsmodell zeichnet sich durch eine dominierende funktionalistische Begründungslogik für Durchlässigkeit aus. Dabei ist die Förderung von lebenslangem Lernen in beiden Prozessen der Hauptkontext, in dem die Einführung durchlässiger Bildungsstrukturen als notwendig angesehen wurde. Nur mit einer Bevölkerung, die sich kontinuierlich weiterbildet, könne den Herausforderungen durch technologische, wirtschaftliche und demografische Entwicklungen begegnet und damit in der globalen Wettbewerbssituation erfolgreich agiert werden. Im Kopenhagen-Prozess sollen außerdem durchlässige Bildungsstrukturen dafür sorgen, die Attraktivität beruflicher Bildung zu erhöhen. Zudem solle der Zugang zur Hochschule für bisher unterrepräsentierte Bevölkerungsgruppen geöffnet werden, um Chancengleichheit und soziale Kohäsion zu fördern. Diese stärker universalistische Argumentation war jedoch im Vergleich zur funktionalistischen deutlich unterrepräsentiert.

Durchlässigkeit wurde in beiden Bildungsprozessen sowohl im Zusammenhang mit Fragen des Zugangs, der Anrechnung und organisationaler Verbindungen als auch mit dem Umgang mit heterogenen Lernenden thematisiert. Das re- 
konstruierte europäische Modell von Durchlässigkeit ist allerdings kein klar definiertes, sondern lässt bei der Interpretation und Umsetzung einigen Spielraum. Die Aspekte werden breit thematisiert und die Umsetzungsvorschläge wenig spezifisch gefasst. So wird nicht beschrieben, wie die Zugangsregelungen zur Hochschule ausgestaltet sein sollten: ob über Berechtigungen oder hochschulische Regelungen, ob individuumszentriert oder gruppenspezifisch. Auch wie Anrechnung stattfinden soll, d.h., inwiefern sie stärker individuell ausgerichtet oder pauschalisiert institutionalisiert sein sollte, wird in den untersuchten Dokumenten offengelassen. Allerdings werden klare Standards wie Leistungspunktesysteme, Lernergebnisorientierung, Qualifikationsrahmen und Modularisierung eingefordert, welche bei der Umsatzung von Anrechnungs- und Validierungsverfahren helfen sollen. Nicht näher spezifiziert werden die zu institutionalisierenden Formen der organisationalen Verbindung zwischen Berufs- und Hochschulbildung. Etwas spezifischer gefasst sind dagegen die Standards, welche den Umgang mit Heterogenität der Lernenden erleichtern und zu einem Bildungserfolg führen sollen; hier werden Maßnahmen zur Flexibilisierung der Lernorganisation, zur Beratung und Unterstützung z.B. durch Lernendenzentrierung und Finanzierungsmöglichkeiten vorgeschlagen. Ob die Finanzierung aber z.B. stärker universalistisch ausgestaltet für alle Lernenden oder in Form leistungsbasierter Stipendien institutionalisiert werden soll, bleibt wiederum offen.

Trotz der Offenheit der Durchlässigkeitsvorgaben auf europäischer Seite wird jedoch deutlich, dass Durchlässigkeit ein Thema ist, mit dem sich die Mitgliedsstaaten beschäftigen müssen. Bildungssysteme, welche keine Mobilität zwischen Bildungsbereichen ermöglichen, büßen durch die europäischen Bildungsprozesse an Legitimität ein und gelten als Bremser der wirtschaftlichen und sozialen Entwicklung in Europa.

Der zweite Schritt zur Analyse des Einflusses der europäischen Bildungsprozesse beruht auf dem Vergleich der herausgefilterten nationalen Entwicklungen mit den europäischen Durchlässigkeitsvorgaben (vgl. Kapitel 10). Dabei wurde zuerst der institutionelle misfit und damit die Frage analysiert, inwiefern bereits vor dem Beginn der europäischen Prozesse eine Passung zwischen den nationalen Strukturen und den rekonstruierten Vorgaben bestand. Anschließend wurde die Delta-Konvergenz untersucht, d.h. die Frage, inwiefern die nationalen Strukturen sich den europäischen Vorgaben im Verlauf des zweiten Untersuchungszeitraums angeglichen haben. Die Erkenntnisse hieraus wurden als erster Hinweis auf den Einfluss der europäischen Prozesse gewertet.

Im Vergleich der regulativen und diskursiven Strukturen vor dem Beginn der Europäisierungsprozesse mit den europäischen Vorgaben wurde deutlich, dass in Deutschland ein wesentlich größerer misfit bestand als in Frankreich. Dort waren bereits einige zentrale Forderungen institutionalisiert. Der anschließende Vergleich mit den nationalen Entwicklungen zeigte, dass sich die nationalen Durchlässigkeitsstrukturen zum Teil in Frankreich aber besonders signifikant in Deutschland an die europäischen Vorgaben angenähert haben. Dies gilt sowohl 
für die regulative und die normative als auch für die kulturell-kognitive Dimension. Wenn über Durchlässigkeit gesprochen wird, dann meist in Bezug auf alle vier Durchlässigkeitsaspekte. Zudem wurden Zugangsmöglichkeiten und organisationale Verbindungen ausgebaut sowie Anrechnungsmöglichkeiten und Strukturen, welche den Umgang mit heterogenen Bedürfnissen erleichtern, eingeführt bzw. erweitert. Darüber hinaus konnte eine Übereinstimmung der europäischen Argumentationen mit den nationalen funktionalistischen Diskurssträngen festgestellt werden. Dabei ähneln funktionalistisch gerahmten Begründungen in den europäischen Dokumenten besonders stark den funktionalistischen Argumentationen in Deutschland. Anhand dieser Beobachtungen lässt sich schlussfolgern, dass die nationalen Entwicklungen in Richtung eines europäischen Durchlässigkeitsmodells speziell in Deutschland, aber auch in Frankreich, als ein Hinweis auf den Einfluss der europäischen Prozesse angesehen werden kann.

In einem dritten Schritt wurde diese Annahme geprüft und zwar auf Basis der nationalen Diskurse und der geführten Expert_inneninterviews. Diese wurden daraufhin analysiert, inwiefern die jeweiligen nationalen Entwicklungen in Frankreich bzw. in Deutschland von den Akteur_innen tatsächlich durch europäische Einflüsse erklärt werden. Für Deutschland konnte gezeigt werden, dass die europäischen Bildungsprozesse eine starke Dynamik der Entwicklung der nationalen Durchlässigkeitsstrukturen ausgelöst haben. Auch wenn bereits vor 1999 Durchlässigkeit zwischen Berufs- und Hochschulbildung ein wichtiges Thema der Bildungspolitik darstellte, boten erst die europäischen Prozesse den richtigen Nährboden, um diese Ideen und politischen Lösungsansätze auch zu fordern und zu fördern. Die Diskrepanz zwischen den europäischen Vorgaben und dem deutschen System war nicht nur in der regulativen Dimension signifikant, sondern wurde auch im strukturkritischen Diskurs als solche konstruiert, indem mit Verweis auf die europäische Ebene eine starke Entwicklungsnotwendigkeit aufgezeigt wurde. Speziell im Hinblick auf die Frage von Anrechnung wurde dies wird dies deutlich, wobei hier vor allem dem Bologna Prozess eine entscheidende Rolle zukommt. Der Einfluss des Kopenhagen- Prozesses wurde v.a. in der Entwicklung und Einführung des deutschen Qualifikationsrahmens gesehen. Ihm wurde sowohl im Diskurs als auch in den Interviews eine Katalysatorrolle zuerkannt. $\mathrm{Zu}$ begründen ist die Katalysatorfunktion damit, dass der DQR als Klassifikationssystem gelten kann. Und da Klassifikationssysteme der Legitimation und Aufrechterhaltung sozialer Kategorisierungen und damit auch Ungleichheiten dienen (Vgl. Tilly 1998) sind sie stark umkämpft. So wurde von den unterschiedlichen Akteuren versucht durch Kategorisierungen und Grenzziehungen bestehende Hierarchien zwischen Berufs- und Hochschulbildung zu verändern bzw. aufrechtzuerhalten. Gleichzeitig führte die Auseinandersetzung mit dem Qualifikationsrahmen auch dazu, dass die Akteure der Berufs- und Hochschulbildung gezwungen waren, sich miteinander über Wertigkeiten von Bildungsabschlüssen und die Definition von Schlüsselbegriffen auseinanderzusetzen. 
Insgesamt wurde allerdings auch deutlich, dass europäische Vorgaben nicht einfach übernommen wurden, sondern der Prozess der Veränderung verbunden war mit Deutungskämpfen über die Entwicklung der Strukturen, was sich in der Gegenüberstellung von strukturkritischem und strukturkonservativem Diskurs zeigt. Und auch wenn für Deutschland in Rückschau der Veränderungen geschlussfolgert werden kann, dass die Europäisierung zu einer Verschiebung der Macht über die Deutungsangebote in Richtung der Vertreter des strukturkritischen Diskurses geführt hat, zeigen sich gleichzeitig Verharrungsstrukturen des Bildungsschismas und der damit zusammenhängenden Hierarchisierung beruflicher und höherer Allgemeinbildung in den institutionellen Entwicklungen. Trotzdem können abschließend die europäischen Bildungsprozesse Bologna und Kopenhagen für Deutschland jedoch als eine Gelingensbedingung (vgl. Solga et al. 2013) für die Veränderung der institutiopnellen Strukturen hin zu mehr Durchlässigkeit angesehen werden.

Im Vergleich zu Deutschland kann der Einfluss des Bologna- und vor allem des Kopenhagen-Prozesses in Frankreich als deutlich weniger entscheidend für die wichtigen institutionellen Veränderungen für Durchlässigkeitt angesehen werden, obwohl auch hier gezeigt werden konnte, dass sich die Durchlässigkeitsstrukturen in Richtung europäischer Vorgaben entwickelt haben. So wurden ebenfalls europäische Standards wie Leistungspunktesysteme, Modularisierung und Semestereinteilung aufgrund der europäischen Prozesse institutionalisiert, was als unterstützend für die Schaffung von Durchlässigkeit angesehen werden kann. Allerdings kamen durch die Europäisierung keine gänzlich neuen Aspekte auf die französische Agenda. So etwa war Anrechnung dort schon im ersten Zeitraum ein wichtiges Thema, wurde 2002 weiterentwickelt und kann durch inzwischen eingeführte europäische Standards allenfalls leichter umgesetzt werden. Gleiches gilt für die institutionalisierten Übergänge. Die Veränderung der Pädagogik in Richtung einer Lernendenzentrierung wird zwar auch in den europäischen Argumenten gefordert, war aber im französischen Diskurs bereits im ersten Zeitraum präsent, wenn auch nicht in Bezug auf beruflich Qualifizierte. Insofern werden zwar europäische Forderungen aufgegriffen, diese stellen aber vielfach lediglich Weiterentwicklungen nationaler institutioneller Entwicklungen dar.

Schließlich ist die bedeutendste Entwicklung hin zu mehr Durchlässigkeit zwischen Berufs- und Hochschulbildung in Frankreich in der Verringerung der bestehenden Diskrepanz zwischen den regulativ institutionalisierten Rechten beruflich Qualifizierter und der normativen und kulturell-kognitiven Dimension zu sehen. Die Anerkennung von Berufsabiturient_innen als legitime Studierende wird in der vorliegenden Arbeit als ein von den europäischen Einflüssen unabhängiger Prozess bewertet. So wurde nach der Einführung des baccalauréat professionnel und dessen Ausbau, dem einhergehenden Anstieg beruflicher Abiturient_innen und damit auch einem Anstieg an beruflich qualifizierten Studierenden an den Hochschulen, die mit großen Problemen des Studienabbruchs zu kämpfen hatten, der Problemdruck für die französische Politik immer größer. Vor 
dem Hintergrund der symbolischen Bedeutung des Abiturs in Frankreich sowie des tief verankerten französischen Selbstverständnisses des Rechts auf Bildung sind die Öffnung des Hochschulbereichs für Berufsabiturient_innen und deren Anerkennung, wenn auch größtenteils als Problemstudierende, logische Konsequenzen. Allerdings, und das entspricht ebenfalls der Weiterentwicklung nationaler institutioneller Pfade, wird die bestehende Hierarchisierung der Bildungszweige nicht aufgebrochen, sondern auf der nächsthöheren Bildungsebene reproduziert. Während die beruflich Qualifizierten in die technischen Kurzstudiengänge an die STS gelenkt werden sollen, werden die Abiturient_innen des technologischen und vor allem allgemeinbildenden Abiturzweiges eher als Studierende der grandes ecoles, der Universitäten und der IUT gesehen. Insgesamt konnte somit durch den Bologna-Prozess und die damit eingeführten Standards eine Erleichterung der Durchsetzung von Durchlässigkeit erreicht werden, ohne aber den eingeschlagenen institutionellen Entwicklungspfad entscheidend zu verändern.

Die vergleichende Analyse des Einflusses europäischer Prozesse in Frankreich und Deutschland führte schließlich nicht nur zu einer fallspezifischen Einschätzung, sondern auch zu verallgemeinerbaren Erkenntnissen:

1. Die Stärke des Einflusses europäischer Prozesse ist durch verschiedenste Faktoren zu erklären. So vermittelt der festgestellte misfit einen Eindruck des vorhandenen Handlungsdrucks. Allerdings ist mitentscheidend, ob bereits ein Klima des Wandels vorherrscht und damit auch Akteursgruppen existieren, welche einen Wandel unterstützen und dabei auch die europäischen Vorgaben diskursiv nutzen. Insofern spielt die jeweilige nationale institutionelle Ausgangslage eine signifikante Rolle.

2. Am französischen Beispiel wurde deutlich, dass Entwicklungen in Richtung Delta-Konvergenz, also eines europäischen Modells, nicht automatisch bedeuten, dass es die europäischen Prozesse selbst sind, die zu einer Änderung führen. Über die Analyse der Delta-Konvergenz allein können demnach keine Rückschlüsse auf den tatsächlichen Einfluss internationaler Modelle und Prozesse gezogen werden, ohne gleichzeitig auch die nationalen Entwicklungen vor und nach dem Aufkommen der Modelle zu analysieren.

3. Insbesondere bei intergouvernementalen Prozessen ist die Interdependenz der verschiedenen Analyseebenen, also die Zirkularität zwischen nationalen und europäischen Entwicklungen nicht zu vernachlässigen. Modelle entstehen nicht im luftleeren Raum, sondern werden durch die institutionellen Erfahrungen der beteiligten Akteure maßgeblich beeinflusst (vgl. Powell et al. 2012a).

4. Es existiert kein Automatismus zwischen Delta- und Sigma-Konvergenz. Ein Zusammenhang besteht am ehesten dann, wenn spezifische 
Standards vorgegeben werden - wie etwa bei der Einführung von Semestern oder Leistungspunkten. Da aber das europäische Durchlässigkeitsmodell der Bologna und Kopenhagen Prozesse eher unspezifisch gehalten wurde, bleibt genügend Interpretationsspielraum bei der nationalen Umsetzung. Zudem sind es wiederum die ganz unterschiedlichen nationalen institutionellen Ausgangslagen, welche die spezifische nationale Weiterentwicklung stark beeinflussen.

Insofern zeigt sich schließlich in der vorliegenden Studie, dass allgemeine Prinzipien und Zielvorstellungen wie die Förderung von Durchlässigkeit entsprechend den Annahmen des soziologischen Neoinstitutionalismus durch europäische Prozesse diffundieren. Das Verständnis dieser Prinzipien, die diskursive Wahrnehmung wie auch deren konkrete Umsetzung sind allerdings durch national gewachsene Institutionen und bestehende Herrschaftsverhältnisse mitbestimmt.

\subsection{Limitationen, weiterführende Forschung und Implikationen}

Nachdem oben zusammengefasst wurde, wie die in diesem Buch gestellten Forschungsfragen beantwortet werden konnten, sollen auch die Grenzen dieser Studie sowie weitere offene Forschungsfragen identifiziert werden. Abschließend werden dann gesellschaftliche Implikationen der Ergebnisse der vorliegenden Arbeit erörtert.

\subsubsection{Grenzen der Studie und mögliche Erweiterungen}

Die vorliegende Studie bietet Einblick in die nationalen Entwicklungen und erklärt, welchen Einfluss die europäischen Prozesse Bologna und Kopenhagen auf diese Entwicklungen hatten. Die Studie kann allerdings keinen Beitrag dazu leisten, das Zustandekommen der europäischen Prozesse und der Modelle zu erklären.

Weiterhin muss unterstrichen werden, dass in dieser Arbeit zur Analyse der institutionellen Durchlässigkeitsentwicklungen ein bestimmter Ausschnitt der gesellschaftlichen Wirklichkeit fokussiert wurde. Der Fokus ruhte dabei vor allem auf der Entwicklung der nationalen Diskurse der bildungspolitischen Elite, wobei auch regulative Entwicklungen in die Analyse inkludiert wurden. Dieses Vorgehen stellte insbesondere die nationale politische Entscheidungsebene ins Zentrum, die auch für die regulativen und z.T. für die normativen Veränderungen im Bildungssystem zuständig ist. Allerdings musste in Deutschland die föderale 
Ebene, auf der mitunter ebenfalls starke Unterschiede in den Durchlässigkeitsstrukturen bestehen können (vgl. Ulbricht 2012a), unterbelichtet bleiben. Insofern wären eine weitergehende Untersuchung und ein Vergleich allein der föderalen Unterschiede in Deutschland spannend.

Nicht untersucht wurden zudem die weiteren existierenden Diskursarenen. Auch wenn diese Arenen nicht unabhängig voneinander bestehen und sich gegenseitig beeinflussen, ist zu fragen, wie z.B. der öffentliche Mediendiskurs zu Durchlässigkeit ausfällt. Verläuft dieser ähnlich und ist ähnlich strukturiert wie der Elitendiskurs oder gibt es deutliche Unterschiede? Insbesondere wäre es untersuchungswert, den Mediendiskurs zu analysieren, welcher ein breiteres Publikum erreicht, da dieser die Vorstellungen der Bürger_innen zur Durchlässigkeit zwischen Berufs- und Hochschulbildung in einem stärkeren Maße mitbestimmen kann als ein Elitendiskurs, der möglicherweise überhaupt nicht wahrgenommen wird.

Ebenfalls nicht näher in den Blick genommen wurden die direkt von Durchlässigkeit betroffenen Professionen. Auch für diese kann angenommen werden, dass sie bestimmte Vorstellungen und Interessenlagen zur Durchlässigkeit aufweisen. Zu untersuchen wäre hier z.B. der Diskurs der Professionen der Schul-, Hochschul- und Berufsschullehrer_innen. Eine solche Untersuchung wäre von größter Bedeutung, denn die Professionellen sind gatekeeper. Sie sitzen an der Schnittstelle, welche es den Lernenden erleichtern oder erschweren kann, Zugang $\mathrm{zu}$ und Erfolg in einem anderen Bildungsbereich zu haben (vgl. Pfahl 2011).Gleichsam kann durch eine Analyse der Praxis der Professionen auch untersucht werden wie mögliche Differenz zwischen den unterschiedlichen Bildungsteilnehmer_innen mitkonstruiert wird (vgl. Budde 2012).

Durch den Blick auf Diskurse und Regelungen war der Analysefokus auf die gesamtgesellschaftliche Ebene und die der Organisationsfelder (vgl. Kapitel 4) gerichtet - nicht aber auf die einzelnen Organisationen, in denen besonders die normative institutionelle Dimension, also die der vorherrschenden Standards untersucht werden können. Eine wichtige Fragestellung für eine weitere Untersuchung wäre hier etwa, auf welche Weise Durchlässigkeit in den Bildungsorganisationen gefördert und umgesetzt wird. Es gibt verschiedenste Studien gerade für den deutschen Fall, die sich mit einzelnen Aspekten von Durchlässigkeit beschäftigen (vgl. u.a. Banscherus/Pickert 2013; Buhr et al. 2008; Hanft/Brinkmann 2013). Interessant wäre allerdings eine Rückkopplung solcher Analysen mit den Ergebnissen der vorliegenden Arbeit. Welche Aspekte spielen in den Bildungsorganisationen tatsächlich eine Rolle? Wie werden diese umgesetzt: individuums- oder gruppenzentriert? Entsprechen die Praktiken eher strukturkritischen oder strukturkonservativen Vorstellungen? Sind sie stärker universalistisch oder funktionalistisch orientiert? All diese Fragen könnten aufbauend auf diese Studie weiter erforscht werden. Hier wäre es wichtig zu untersuchen, inwiefern zwischen den Regelungen, den in den Diskursen der bildungspolitischen Akteure rekonstruierten Vorstel- 
lungen und den vorherrschenden Standards sowie Praktiken in den Organisationen eine Übereinstimmung festzustellen ist. Oder sind die Standards und Praktiken in den Organisationen, auch wenn in der Bildungspolitik Durchlässigkeit zwischen Berufs- und Hochschulbildung wichtiger wird, von den bildungspolitischen Vorstellungen entkoppelt? Die Beantwortung dieser Fragen ist zentral, um die tatsächlichen Bildungsmöglichkeiten der Lernenden im Hinblick auf Durchlässigkeit bewerten zu können.

Ebenso wie die Organisationsebene wird auch die Individualebene in dieser Arbeit außer in Beteiligungsraten kaum betrachtet. So wäre es zielführend, zum einen zu untersuchen, wie die Institutionen sich ändern, und zum anderen, inwiefern sich diese Veränderungen in den Handlungsmustern der Individuen widerspiegeln. Zu eruieren wäre ferner, welche durchlässigkeitsfördernden Praktiken in den Organisationen besonders positiv auf die Beteiligung beruflich Qualifizierter im Hochschulbereich wirken. Weiterführend wäre es wertvoll zu untersuchen, welche Subjektivierungswirkungen von den Bildungsdiskursen zu Durchlässigkeit ausgehen (vgl. z.B. Pfahl 2011 zur Frage der Subjektivierung von Behinderung). Wie beschreiben beruflich Qualifizierte ihre Rolle als Studierende? Sehen sie sich als selbstverständliche und legitime Studierendengruppe? Wie nehmen sie ihr akademisches Umfeld wahr? Welche Strategien und Praktiken entwickeln sie, um im Studium erfolgreich zu sein? Unterscheiden sie sich darin von anderen Studierendengruppen? Und in welchem Verhältnis steht all dies zu dem in den Diskursen rekonstruierten Verständnis von Durchlässigkeit und der dort beschriebenen Rolle beruflich Qualifizierter in der Hochschule?

Während sich die oben genannten Forschungsvorschläge primär auf weiterführende Studien zur Analyse der nationalen Entwicklungen beziehen, sind aus vergleichender Perspektive ebenfalls Erweiterungen denkbar. So könnten weitere europäische Staaten und deren Entwicklung im Hinblick auf Durchlässigkeit untersucht und mit den Ergebnissen dieser Studie verglichen werden. Es wäre interessant zu analysieren, wie Durchlässigkeit in anderen Ländern verstanden wird, ob überall dieselben Aspekte eine Rolle spielen oder ob neue Aspekte identifiziert werden können. Sinnvoll wäre hier zunächst der Vergleich mit Ländern, die ebenso wie Deutschland eine starke Trennung zwischen Berufs- und Hochschulbildung aufweisen. Dabei könnte untersucht werden, inwieweit die jeweiligen Entwicklungen unterschiedlich oder gleich verlaufen sind und warum dies jeweils der Fall war (vgl. Graf 2013). Spannend wäre jedoch auch die Betrachtung osteuropäischer Länder, die ähnlich wie die DDR durch das sowjetische Bildungssystem und ein spezifisches Verhältnis zwischen Berufs- und Hochschulbildung geprägt waren. Wie haben sich Verhältnisse dort nach dem Zusammenbruch der Sowjetunion und unter dem Einfluss der europäischen Bildungsprozesse entwickelt?

All diese Ansätze machen deutlich, dass noch viele weitere Fragen und Perspektiven zur Durchlässigkeit zwischen Berufs- und Hochschulbildung zu untersuchen sind. Die vorliegende Arbeit kann hier nur ein Schritt sein, um bestehende 
Forschungslücken zu schließen, auf welchen aber für die weitere Forschung durchaus aufgebaut werden kann.

\subsubsection{Gesellschaftspolitische Implikationen}

Bereits in der Einleitung und in Kapitel 2 wurde der Zusammenhang zwischen einem institutionell durchlässigen Bildungssystem und Bildungsmobilität und damit den gesellschaftlichen Strukturen sozialer Ungleichheit unterstrichen. So kann institutionalisierte Durchlässigkeit im Bildungssystem als eine wichtige Voraussetzung dafür gelten, das Dahrendorfsche Ideal des Rechts auf Bildung umzusetzen. Veränderungen in Richtung eines durchlässigen Bildungssystems können zudem einhergehen mit einer Erhöhung der Lebenschancen von Individuen und helfen, den kumulativen Prozess von Bildungsungleichheiten über den Lebenslauf zu begrenzen. Denn ohne die Möglichkeit, an bestehende Abschlüsse und Kompetenzen im Lebensverlauf anzuknüpfen, und ohne institutionalisierte Übergangsmöglichkeiten zwischen einzelnen Bildungsbereichen würden weiterhin systematisch unterschiedliche Lebenschancen für die Lernenden bestehen und jede (Fremd- und Selbst-)Selektion im Bildungssystem wäre eine finale. Durchlässigkeit im Bildungssystem zielt hingegen auf den Abbau individueller Bildungssackgassen, so dass eine barrierefreie Bildungsbiografie sowie soziale Aufstiege ermöglicht werden. Die vorliegende Analyse ist in vielfältiger Hinsicht wertvoll für Überlegungen, wie Bildungssysteme durchlässiger gestaltet und soziale Ungleichheiten abgebaut werden können.

Zum einen wurde deutlich, dass nationale Durchlässigkeitsstrukturen differenziert und mehrdimensional sind und punktuelle Veränderungen nicht automatisch bessere Strukturen nach sich ziehen. Beide Länderstudien unterstreichen, dass nicht nur formale Regeln wichtig sind, sondern auch das gesellschaftlich verankerte Verständnis, die kulturell-kognitive und die normative institutionelle Dimension.

So zeigt sich in Frankreich, dass es nicht ausreicht, Regeln zu institutionalisieren, wenn diese in Diskrepanz zum gesellschaftlichen Selbstverständnis stehen. Denn dann sind deren Auswirkungen allenfalls begrenzt und können nicht die vermeintlich intendierten Ergebnisse nach sich ziehen. Die formalen Zugangsregelungen helfen den beruflich Qualifizierten nur dann, wenn diese auch als legitime Gruppe von Studierenden anerkannt und entsprechend vorbereitet bzw. unterstützt werden. Ist dies nicht der Fall, sind formale Durchlässigkeitsregelungen nur eine Farce, die den Individuen Möglichkeiten vorspiegelt, die ihnen in Wirklichkeit nicht offenstehen.

In Deutschland zeigt sich vor allem in der kulturell-kognitiven Dimension einerseits die starke Verankerung des Bildungsschismas, welches die Veränderungen in Richtung Durchlässigkeit zwischen Berufs- und Hochschulbildung be- 
grenzt. Andererseits wurde jedoch auch deutlich, dass sich gesellschaftliche Vorstellungen ändern und bestimmte vorher als selbstverständlich erachtete vermeintliche Wahrheiten delegitimiert werden können. Es ist also wichtig - in der Gesellschaft generell wie auch auf Seiten der Forschung - allgemeine Selbstverständlichkeiten zu hinterfragen, vor allem dann, wenn diese nachweislich individuelle Lebenschancen negativ beeinflussen oder zu anderen gesellschaftlichen Problemen führen können. Auf diese Weise können grundlegende institutionelle Entwicklungen in Gang gesetzt werden. Im deutschen Fall wird deutlich, dass vielen regulativen Änderungen zur Durchlässigkeit auch solche in den Diskursen vorausgingen. Es tritt allerdings auch klar hervor, dass Veränderungen mitunter sehr langsam vor sich gehen und dabei nicht von einem Automatismus ausgegangen werden kann. So wurden Ideen, welche bereits in den 1970er Jahren präsent waren, wie die Eingrenzung des Berechtigungsmonopols der Allgemeinbildung, damals kaum und erst viele Jahre später in Teilen umgesetzt.

Gezeigt wurde in dieser Arbeit ebenfalls, dass verschiedene Begründungen dafür, Durchlässigkeit umzusetzen, auch mit bestimmten Implikationen verbunden sind. So steht in der rekonstruierten funktionalistischen Perspektive nicht das Recht auf Bildung der Individuen im Vordergrund, sondern die Bedarfe des Arbeitsmarkts. Sowohl in Deutschland als auch in Frankreich steht die funktionalistische Argumentationslogik allerdings nicht allein, sondern wird von einer universalistischen ergänzt. Dies ist insofern wichtig, als Durchlässigkeit aus rein funktionalistischer Perspektive reversibel ist, und zwar dann, wenn der gesellschaftliche Bedarf nicht mehr gesehen wird. Aus universalistischer Perspektive jedoch ist das Recht auf Bildung nicht verhandelbar und damit irreversibel. Daraus kann abgeleitet werden, dass die Regelungen, wenn sie auch dazu beitragen sollen, das Recht auf Bildung zu institutionalisieren, nicht nur einer funktionalistischen Logik folgen sollten.

Weiterhin wurde in dieser Studie herausgearbeitet, dass ein durchlässiges Bildungssystem sich durch mehr auszeichnet als nur die Möglichkeit des Zugangs zu anderen Bildungsbereichen. Möglichkeiten der Anrechnung sowie organisationale Verbindungen können Durchlässigkeit ebenfalls fördern. Wichtig ist zudem eine Akzeptanz der Gruppe neuer Lernender und eine entsprechende Strukturierung der Lernmöglichkeiten bzw. Vorbereitung der Lernenden. Nur wenn durch spezifische Strukturen der Beratung, der Information, des Lehrens und Lernens auf die Diversität der Lernenden Rücksicht genommen wird, kann Durchlässigkeit im Sinne eines erfolgreichen Lernens ermöglicht werden. Unerlässlich bei der Etablierung derartiger Strukturen ist es darauf zu achten, dass sie nicht zu einer weiteren Segregation oder Stigmatisierung einer bestimmten Gruppe von Lernenden beitragen. So sollten Beratungs-, Unterstützungs- und Vorbereitungsangebote für alle Lernenden der Organisation gelten, denn die ,traditionellen“ Bildungsteilnehmer_innen können unterstützende Strukturen genauso benötigen wie neue Gruppen von Lernenden, die sich ebenfalls durch eine Heterogenität ihrer Bedürfnisse auszeichnen. 
Es existiert für die Ermöglichung durchlässiger Bildungsstrukturen kein Patentrezept. Vielmehr gibt es, wie in Kapitel 2 gezeigt, verschiedenste Möglichkeiten, Durchlässigkeit zu fördern, wobei eine Mischung aus stärker individuumszentrierten und gruppenspezifischen Strukturen als erfolgsversprechend angesehen werden kann. Die jeweiligen Ausrichtungen gehen mit differenten Vor- und Nachteilen einher. Gruppenspezifische Strukturen können dabei oft als barriereärmer gelten. Die Umsetzung einer Regelung für viele ist mit weniger Aufwand sowohl für die umsetzende Organisation als auch für das Individuum verbunden, zumal solche Regelungen oft mit Berechtigungen für das Individuum einhergehen. Implizit sind aber derartigen gruppenspezifischen Strukturen Homogenitätsannahmen über die Gruppen inhärent, welche dann nachteilig sein können, wenn es keine Strukturen gibt, die das ausgleichen und somit dazu dienen, die Heterogenität der Individuen innerhalb der Gruppen anzuerkennen. Individuumszentrierte Strukturen sind geeignet, genau dies zu leisten.

Abschließend sei darauf hingewiesen, dass die Rolle des Bildungssystems bei der Verringerung sozialer Ungleichheiten nicht überbewertet werden sollte (vgl. auch Solga 2012). Für die Frage nach dem Verhältnis von Berufs- und Hochschulbildung ist neben der Schaffung von Durchlässigkeit im Bildungssystem auch deren jeweilige Stellung in der Gesellschaft signifikant. Ungleichheiten können maßgeblich auch dann verringert werden, wenn Gleichwertigkeit von Berufs- und Hochschulbildung auch außerhalb des Bildungssystems anerkannt wird. Dies gilt im besonderen Maße auf dem Arbeitsmarkt. Wenn unabhängig vom Bildungsweg Absolvent_innen beider Bereiche bei gleichen Kompetenzen auch die gleichen Chancen auf Arbeitsmarktpositionen und Gehälter haben, ist ebenfalls ein großer Schritt in Richtung der Gleichwertigkeit von Berufs- und Hochschulbildung getan, der mit einer Verringerung bildungsbedingter sozialer Ungleichheiten einhergeht. 



\section{Literaturverzeichnis}

Abriac, Dominique, Roland Rathelot und Ruby Sanchez (2009). L'apprentissage, entre formation et insertion professionnelles. Formations et emploi, Edition 2009, Insee références, Paris: INSEE, 57-74.

ADIUT [Assemblée des Directeurs d'Instituts Universitaires de Technologie] (2009a). Bacheliers Professionnels en IUT. Note au 13 septembre 2009. http://www.iut.fr/files/fck/File/documents/publications/assises\%20ESR/ 9-ADIUT-BPRO-20091013.pdf, [abgerufen am 05.03.2014].

ADIUT (2009b). Note sur la question BTS - DUT, Organismes préparant au BTS IUT. http://www.iut.fr/files/fck/File/documents/publications/assises\%20ESR/18-ADIUT-Note_BTS-20091214.pdf, [abgerufen am 05.03.2014].

ADIUT (2011). Contribution ADIUT à la définition de la stratégie Licence Jean-Francois Mazoin et Christian Cuesta. http://cache.media.enseignementsuprecherche.gouv.fr/file/Plan_reussir_en_licence/71/1/adiut_181711.pdf, [abgerufen am 07.03.2014].

ADIUT (2012). Quelle place pour les bacheliers technologiques en IUT? http://www.iut.fr/files/fck/File/documents/publications/assises\%20ESR/ 5-ADIUT-BTN_MLN-Note-20120906.pdf, [abgerufen am 05.03.2014].

ADIUT und UNPIUT (2012). Contribution de l'ADIUT et de l'UNPIUT aux Assises de l'Enseignement Supérieur et de la Recherche. Août à Novembre 2012. http://www.iut.fr/files/fck/ADIUT_UNPIUT-Contribution_Assises_ESR2012-VF.pdf, [abgerufen am 07.03.2014].

Agulhon, Catherine (2003). L'enseignement professionnel à la croisée de l'école et de l'entreprise. Habilitation à diriger des recherches sous la directions d'Eric Plaisance (Paris 5) et de José Rose (CEREQ).

Agulhon, Catherine (2005). Les politiques régionales de formation professionnelle. Du référentiel commun à la politique locale. Education et sociétés. 16 (2): 279-292.

Agulhon, Catherine (2007). La professionnalisation à l'université, une réponse à la demande sociale? Recherche et formation. 54: 11-27.

Agulhon, Catherine (2011). Les licences professionnelles, une construction locale et éclectique. Reflet des contradictions des réformes universitaires. Cabiers de la recherche sur l'éducation et les savoirs. 10: 183-203.

Aktionsprogramm (1976). Entschließung des Rates und der im Rat vereinigten Minister für Bildungswesen vom 9. Februar 1976 mit einem Aktionsprogramm im Bildungsbereich. Amtsblatt Nr. C 038 vom 19/02/1976 S. 0001-0005. http://eur-lex.europa.eu/LexUriServ/LexUriServ.do?uri=CELEX:41976X0 219:DE:HTML, [abgerufen am 12.02.2014].

Alesi, Bettina, Sandra Bürger, Barbara Kehm und Ulrich Teichler (2005). Stand der Einfübrung von Bachelor- und Masterstudiengängen im Bologna-Prozess sowie in 
ausgewäblten Ländern Europas im Vergleich zu Deutschland. Berlin: Bundesministerium für Bildung und Forschung (BMBF).

Alheit, Peter (2009). Exklusionsmechanismen des universitären Habitus. Unsichtbare Barrieren für Studierende auf dem ,zweiten Bildungsweg'. Hessische Blätter für Volksbildung. 59 (3): 215-226.

Allmendinger, Jutta (1989). Educational Systems and Labor Market Outcomes. European Sociological Review. 5 (3): 231-250.

Angermüller, Johannes (2010). Rezension: Andrea D. Bührmann, Werner Schneider. Vom Diskurs zum Dispositiv: Eine Einführung in die Dispositivanalyse. http://www.litkult.de/Dispositivanalyse.html, [abgerufen am 13.03.2014].

Angermüller, Johannes und Ronny Scholz (2013). Semantische und kommunikative Dimensionen diskursiven Wandels. Ein integrativer Ansatz zur Analyse der Makro- und Mikrostrukturen am Beispiel des BolognaDiskurses. In: Dietrich Busse und Wolfgang Teubert (Hrsg.): Linguistische Diskursanalyse: neue Perspektiven. Wiesbaden: Springer, 287-318.

Annoot, Emmanuelle (2012). La réussite à l'université : du tutorat au plan licence. Bruxelles: De Boeck.

Archer, Margaret S. (1979). Social Origins of Educational Systems. London: Sage.

Archer, Margaret S. (1986). The Sociology of Education. In: Ulf Himmelstrand

(Hrsg.): The Social Reproduction of Organisation and Culture. London, Beverly Hills: Sage, 59-87.

Arrighi, Jean-Jacques und Damien Brochier (2009). L'apprentissage an sein de $l^{\prime}$ Education nationale : une filière sortie de la clandestinité. NEF $\mathrm{n}^{\circ}$ 40, avril 2009 : Céreq.

Auel, Katrin (2006). Europäisierung nationaler Politik. In: Hans-Jürgen Bieling (Hrsg.): Theorien der europäischen Integration. Wiesbaden: VS Verlag für Sozialwissenschaften, 293-318.

Autorengruppe Bildungsberichterstattung (2008). Bildung in Deutschland 2008. Bielefeld: Bertelsmann.

Autorengruppe Bildungsberichterstattung (2010). Bildung in Deutschland 2010. Bielefeld: Bertelsmann.

Autorengruppe Bildungsberichterstattung (2012). Bildung in Deutschland 2012. Bielefeld: Bertelsmann.

Autorengruppe Bildungsberichterstattung (2014). Bildung in Deutschland 2014. Bielefeld: Bertelsmann.

Baethge, Martin (2006). Das deutsche Bildungs-Schisma: Welche Probleme ein vorindustrielles Bildungssystem in einer nachindustriellen Gesellschaft hat. In: SOFI-Mitteilungen No. 34. Soziologisches Forschungsinstitut an der Universität Göttingen, 13-27.

Baethge, Martin, Heike Solga und Markus Wieck (2007). Berufsbildung im Umbruch: Signale eines überfälligen Aufbruchs. Berlin: Friedrich-Ebert-Stiftung. 
Baker, David P. (2011). Forward and Backward, Horizontal and Vertical: Transformation of Occupational Credentialing in the Schooled Society. Research in social stratification and mobility. 29 (1): 5-29.

Baker, David P. und Gerald K. LeTendre (2005). National Differences, Global Similarities: World Culture and the Future of Schooling. Stanford: Stanford University Press.

Balzer, Carolin und Alessandra Rusconi (2007). From the European Commission to the Member States and Back - A Comparison of the Bologna and the Copenhagen Process. In: Kerstin Martens, Alessandra Rusconi und Kathrin Leuze (Hrsg.): New Arenas of Education Governance - The Impact of International Organizations and Markets on Educational Policy Making. Basingstoke: Palgrave, 57-75.

Banscherus, Ulf (2007). Hochschulzugang für nicht-traditionelle Studierende Ein Luxusproblem? In: BdWi/fzs/GEW (Hrsg.): Bildung - Beruf - Praxis. Bildungsreform zwischen Elfenbeinturm und Verwertungslogik. Marburg: BdWiStudienheft 4, 45-47.

Banscherus, Ulf, Caroline Kamm und Alexander Otto (2015). Information, Beratung und Unterstützung von nicht-traditionellen Studierenden. Angebote der Hochschulen und deren Bewertung durch die Zielgruppe. . In Anke Hanft, Olaf Zawacki-Richter und Willi. B. Gierke (Hrsg.), Herausforderung Heterogenität beim Übergang in die Hochschule. Münster u.a.: Waxmann: 81 96.

Banscherus, Ulf und Anne Pickert (2013). Unterstützungsangebote für nichttraditionelle Studieninteressierte: eine Bestandsaufnahme. In: Helmut Vogt (Hrsg.): Wächst zusammen, was zusammen gebört? Wissenschaftliche Weiterbildung berufsbegleitendes Studium - lebenslanges Lernen. Beiträge zur wissenschaftlichen Weiterbildung Nr. 53. Hamburg, 128-134.

Baron, Sonja C. (2007). Das duale System der Berufsausbildung unter dem Einfluss der europäischen Berufsbildungspolitik. Entwicklungsprozesse und Herausforderungen. Dissertation am Fachbereich Bildungswissenschaften der Universität Duisburg-Essen.

Baumert, Jürgen, Kai Maaz, Cornelia Gresch, Nele McElvany, Yvonne Anders, Kathrin Jonkmann, Marko Neumann und Rainer Watermann (2010). Der Übergang von der Grundschule in die weiterführende Schule. Leistungsgerechtigkeit und regionale, soziale und ethnisch-kulturelle Disparitäten. Zusammenfassung der zentralen Befunde. In: Kai Maaz, Jürgen Baumert, Cornelia Gresch und Nele McElvany (Hrsg.): Der Übergang von der Grundschule in die weiterfübrende Schule. Leistungsgerechtigkeit und regionale, soziale und ethnisch-kulturelle Disparitäten. Bonn u.a.: Bundesministerium für Bildung und Forschung, Referat Bildungsforschung, 5-22. 
BDI, BDA, DIHT, HDE, ZdH, BGA, BFB und DBV [Bundesverband der Deutschen Industrie, Bundesvereinigung der Deutschen Arbeitgeberverbände, Deutscher Industrie- und Handelstag, Hauptverband des Deutschen Einzelhandels, Zentralverband des Deutschen Handwerks, Bundesverband des Deutschen Groß- und Außenhandels, Bundesverband der Freien Berufe und Deutscher Bauernverband] (1993). Differenzierung Durchlässigkeit Leistung. Bonn: DCM.

Beaud, Stéphane (2002). 80 \% an Bac et après ? Paris: La Découverte.

Beaud, Stéphane (2008). Enseignement supérieur : la « démocratisation scolaire » en panne. Formation emploi. 101: 149-165.

Beaud, Stéphane und Michel Pialoux (2001). Les « bacs pro » à l'université. Récit d'une impasse. Revue Française de Pédagogie. 136: 87-95.

Bechmann, Sebastian (2007). Gesundheitssemantiken der Moderne: Eine Diskursanalyse der Debatten über die Reform der Krankenversicherung. Berlin: edition sigma.

Becker-Ritterspach, Florian (2006). Wissenstransfer und -integration im Transnationalen Konzern. In: Ursula Mense-Petermann und Gabriele Wagner (Hrsg.): Transnationale Konzerne: Ein neuer Organisationstyp? Wiesbaden: VS Verlag für Sozialwissenschaften, 153-187.

Becker, Howard S. (1992). Cases, Causes, Conjunctures, Stories and Imagery. In: Charles Ragin und Howard S. Becker (Hrsg.): What is a Case? Exploring the Foundations of Social Inquiry. Cambridge: Cambridge University Press, 223-233.

Becker, Peter und Radostina Primova (2009). Die Europäische Union und die Bildungspolitik. Diskussionspapier der FG 1, 2009/07. Berlin: Forschungsgruppe EU-Integration, Stiftung Wissenschaft und Politik, Deutsches Institut für Internationale Politik und Sicherheit.

Becker, Rolf und Andreas Hadjar (2009). Meritokratie - Zur gesellschaftlichen Legitimation ungleicher Bildungs-, Erwerbs- und Einkommenschancen in modernen Gesellschaften. In: Rolf Becker (Hrsg.): Lebrbuch der Bildungssoziologie. Wiesbaden: Springer, 35-59.

Becker, Rolf, Sigrid Haunberger und Frank Schubert (2010). Studienfachwahl als Spezialfall der Ausbildungsentscheidung und Berufswahl. Zeitschrift für Arbeitsmark.tForschung. 42 (4): 292-310.

Beicht, Ursula und Verena Eberhard (2013). Bedeutung der Teilnahme an teilqualifizierenden Bildungsgängen und Maßnahmen am Übergang von der Schule zur Berufsausbildung. In: BIBB (Hrsg.): Datenreport zum Berufsbildungsbericht 2013. Informationen und Analysen zur Entwicklung der beruflichen Bildung. Bonn: BIBB, 100-109.

Bel, Maïten (2001). Cadres institutionnels et rôle des organisations dans la construction de la formation professionnelle des jeunes. Education et sociétés. 7 (1): 37-50.

Bell, Daniel (1975). Die nachindustrielle Gesellschaft. Frankfurt am Main: Campus. 
Bell, Daniel (1994). The Coming of Post-Industrial Society. In: David B. Grusky (Hrsg.): Social Stratification: Class, Race, and Gender in Sociological Perspective. Boulder, CO: Westview Press, 686-697.

Bellenberg, Gabriele (2012). Schulformwechsel in Deutschland - Durcblässigkeit und Selektion in den 16 Schulsystemen der Bundesländer innerbalb der Sekundarstufe I. Gütersloh: Bertelsmann Stiftung.

Below, Susanne von (2002). Bildungssysteme und soziale Ungleichheit. Das Beispiel der neuen Bundesländer. Opladen: Leske + Budrich.

Benavot, Aron, Yun-Kyung Cha, David Kamens, John W. Meyer and Suk-Ying Wong (1991). Knowledge for the masses: World models and national curricula, 1920-1986. American Sociological Review. 56 (1): 85-100.

Ben-David, Joseph (1992). Centers of Learning: Britain, France, Germany, United States. New Brunswick, NJ: Transaction Publishers.

Bennett, Colin J. (1991). What is Policy Convergence and What Causes it? British Journal of Political Science. 21 (2): 215-233.

Bergen Communiqué (2005). The European Higher Education Area - Achieving the Goals Communique of the Conference of European Ministers Responsible for Higher Education, Bergen, 19-20 May 2005. http://www.ehea.info/Uploads/ Declarations/Bergen_Communique1.pdf, [abgerufen am 10.02.2014].

Berger, Peter L. und Thomas Luckmann (1986). Die gesellschaftliche Konstruktion der Wirklichkeit, 6. Auflage. Frankfurt am Main; Fischer Taschenbuch.

Berger, Peter L. und Thomas Luckmann (2007). Die gesellschaftliche Konstruktion der Wirklichkeit: Eine Theorie der Wissenssoziologie. Frankfurt am Main: Fischer Taschenbuch.

Berggreen-Merkel, Ingeborg (2006). Nationale Identitäten und ein gemeinschaftlicher Bildungsanspruch der EU - ein unauflöslicher Widerspruch? Tertium Comparationis. 12 (1): 24-47.

Bergzog, Thomas, Andreas Diettrich, Christiane Köhlmann-Eckel und Egon Meerten (Hrsg.) (2010). Die Pilotinitiative DECVET. Kompetenzen anrechnen Durchlässigkeit verbessern. Bonn: BMBF.

Berlin Communiqué (2003). Realising the European Higher Education Area. Communique of the Conference of Ministers responsible for Higher Education in Berlin on 19 September 2003. http://www.ehea.info/Uploads/Declarations/Berlin_ Communique1.pdf, [abgerufen am 10.02.2014].

Bernhard, Nadine, Lukas Graf und Justin J.W. Powell (2013). Auswirkungen des neuen europäischen Bildungsmodells auf die Verknüpfung zwischen Berufsund Hochschulbildung in Deutschland, Österreich und Frankreich. In: Karin Amos, Josef Schmid, Josef Schrader und Ansgar Thiel (Hrsg.): Europäischer Bildungsraum. Europäisierungsprozesse in Bildungspolitik und Bildungspraxis. BadenBaden: Nomos, 175-192. 
Bernhard, Nadine, Lukas Graf und Justin J.W. Powell (2010). Wenn sich Bologna und Kopenhagen treffen - Erhöhte Durchlässigkeit zwischen Berufs- und Hochschulbildung? WZB Mitteilungen. 130: 26-29.

BIBB [Bundesinstitut für Berufsbildung] (2010). Datenreport zum Berufsbildungsbericht 2010. Bonn. http://datenreport.bibb.de/media2010/a12 voe_datenreport_bbb_2010.pdf, [abgerufen am 11.03.2014].

BIBB (2011). Datenreport zum Berufsbildungsbericht 2011. Bonn. http://datenreport.bibb.de/media2011/Datenreport_2011.pdf, [abgerufen am 10.02.2014].

BIBB (2013). Datenreport zum Berufsbildungsbericht 2013. Bonn. http://datenreport.bibb.de/media2013/BIBB_Datenreport_2013.pdf, [abgerufen am 15.03.2014].

Bieber, Tonia (2010a). Berufsbildungs- und Hochschulpolitik in der Schweiz, Österreich und Deutschland. Swiss political science review. Sonderheft 2010. 4: 597830.

Bieber, Tonia (2010b). Europe à la Carte? Swiss Convergence towards European Policy Models in Higher Education and Vocational Education and Training. Swiss Political Science Review. 16 (4): 773-800.

Bieber, Tonia (2011). Convergence through Communication and Competition? The Internationalization of Secondary and Higher Education Policies in Switzerland. TranState Working Papers No. 147: Sfb 597 „Staatlichkeit im Wandel“. Bremen: Universität Bremen.

Bischoff-Wanner, Claudia (2002). Vor- und Nachteile einer Akademisierung der Berufsausbildung in den personenbezogenen Dienstleistungsberufen. BIBB Dokumentation Vierter Fachkongress 2002. http://www.forschungsnetzwerk.at/downloadpub/berufsbildung_21jhdt_08_2_01.pdf, [abgerufen am 3.8.2016].

Bjørnåvold, Jens (Hrsg.) (2001). Lernen sichtbar machen. Ermittlung, Bewertung und Anerkennung nicht formal erworbener Kompetenzen in Europa. Luxemburg: Amt für Amtliche Veröffentlichungen der Europäischen Gemeinschaften.

Blanck, Jonna M., Benjamin Edelstein, und Justin J. W. Powell (2013). Persistent School Segregation or Change Towards Inclusive Education? The Impact of the Convention on the Rights of People with Disabilities on Reform Processes in Germany's Bundesländer. Swiss Journal of Sociology, 39(2), 267-292. Blankertz, Herwig (1963). Berufsbildung und Utilitarismus. Düsseldorf: Schwann.

Blings, Jessica (2012). EQR-Implementierung und Lernergebnisorientierung - ein Weg in Richtung mehr Durchlässigkeit? In: Jessica Blings und Klaus Ruth (Hrsg.): Transparenz. und Durchlässigkeit durch den EQR? Perspektiven zur Implementierung. Bielefeld: Bertelsmann, 9-19.

Blings, Jessica und Klaus Ruth (Hrsg.) (2012). Transparen₹ und Durchlässigkeit durch den EQR? Perspektiven zur Implementierung. Bielefeld: Bertelsmann. 
Bluhm, Katharina (2005). Institution, Organisation und Strategie. Konzepte institutioneller Einbettung von Unternehmenshandeln. In: Michael Faust, Maria Funder und Manfred Moldaschl (Hrsg.): Die ,Organisation' der Arbeit. München: Rainer Hampp Verlag, 259-281.

BMBF [Bundesministerium für Bildung, Wissenschaft, Forschung und Technologie] (1995). Berufsbildungsbericht 1995. In: Deutscher Bundestag: Drucksache 13/1300. Bonn.

BMBF (1996a). Bericht der Bundesregierung „Stärkung und Modernisierung der beruflichen Bildung". In: Deutscher Bundestag: Drucksache 13/4213. Bonn.

BMBF (1997b). Lebensbegleitendes Lernen: Situation und Perspektiven der beruflichen Weiterbildung. In: Deutscher Bundestag: Drucksache 13/8527. Bonn.

BMBF (2001b). Situation und Perspektiven der beruflichen Ausbildung und des Systems der Weiterbildung als Stufen eines lebenslangen Lernens. In: Deutscher Bundestag: Drucksache 14/5060. Bonn.

BMBF (2002). Berufsbildungsbericht 2002. In: Deutscher Bundestag: Drucksache 14/8950. Berlin.

BMBF (2003a). Berufsbildungsbericht 2003. In: Deutscher Bundestag: Drucksache 15/1000. Berlin.

BMBF (2003b). Bildung, Forschung, Innovation - der Zukunft Gestalt geben. Bildungs- und forschungspolitische Schwerpunkte des BMBF. http://www.bmbf.de/pub/gesamtpapier_bildung_forschung_innovation.pdf [abgerufen am 16.10.2012].

BMBF (2004a). Berufsbildungsbericht 2004. In: Deutscher Bundestag: Drucksache 15/3299. Berlin.

BMBF (2004b). Catenhusen: „Wichtiger Schritt zur Verbesserung der Mobilität bei der beruflichen Bildung in Europa". Pressemitteilung vom 15.12.2004.: BMBF. Berlin.

BMBF (2005). Berufsbildungsbericht 2005. In: Deutscher Bundestag: Drucksache 15/5285. Berlin.

BMBF (2007). „Berufliche Bildung ist Zukunftssicherung“. Rede von Andreas Storm. 5. BIBB Fachkongress am 12.09. 2007. Düsseldorf: BMBF http://www. bmbf.de/pub/psts_20070912.pdf [abgerufen am 10.03.2014].

BMBF (2008a). Deutscher Qualifikationsrabmen/ Europäischer Qualifikationsrahmen. In: Deutscher Bundestag: A-Drs. 16(18)378. Berlin.

BMBF (2009a). Andreas Storm: „Modernisierungsschub für Hochschulen nutzen". Pressemitteilung vom 30.01.2009. http://www.bmbf.de/press/2457.php [abgerufen am 16.10.2012].

BMBF (2009b). Aufstieg durch Bildung. Die Qualifirierungsinitiative für Deutschland. Berlin. http://www.bmbf.de/pub/qualifizierungsinitiative_breg.pdf [abgerufen am 10.03.2014].

BMBF (2009c). Berufsbildungsbericht 2009. In: Deutscher Bundestag: Drucksache 16/12640. Berlin.

BMBF (2010a). Der Deutsche Qualifikationsrabmen für lebenslanges Lernen. Rede der Bundesministerin für Bildung und Forschung anlässlich der Eröffnung der 2. Fachtagung 
zum Deutschen Qualifikationsrabmen am 19.10.2010. Berlin. http://www.bmbf. de/pub/reden/mr_2010_10_19.pdf [abgerufen am 11.03.2014].

BMBF (2010b). Von der Krankenschwester zur Gesundheitsmanagerin. Pressemitteilung vom 30.11.2010. http://www.bmbf.de/press/3003.php [abgerufen am 16.10.2012].

BMBF (2011a). Aufstieg durch Bildung: offene Hochschulen. Einleitendes Grußwort von Cornelia Quennet-Tbielen. http://www.wettbewerb-offene-hochschulen-bmbf. de/dateien/Tagungsdokumentation_offene_Hochschulen_barrierefrei.pdf [abgerufen am 11.03.2014].

BMBF (2014). Bund-Länder-Wettbewerb „Aufstieg durch Bildung: offene Hochschulen“, http://www.wettbewerb-offene-hochschulen-bmbf.de, [abgerufen am 07.01.2014].

BMBF, BMWT, KMK, WMK, DGB, BDA, ZDH, DIHK und BIBB [Bundesministerium für Bildung und Forschung; Bundesministerium für Wirtschaft und Technologie; Kultusministerkonferenz; Wirtschaftsministerkonferenz; Deutscher Gewerkschaftsbund; Bund Deutscher Arbeitnehmerverbände; Zentralverband des Deutschen Handwerks; Deutscher Industrieund Handelskammertag; Bundesinstitut für Berufsbildung] (2012). Vereinbarung. Berlin.

BMBF und KMK (2014). Expertenvotum zur zweiten Erarbeitungsphase des DQR, http://www.deutscherqualifikationsrahmen.de/de/expertenvoten/experten votum-zur-zweiten-erarbeitungsphase-des-dq_gmelt2ej.html, [abgerufen am 10.02.2014].

BMBW [Bundesministerium für Bildung und Wissenschaft] (1991). Berufsbildungsbericht 1991. In: Deutscher Bundestag: Drucksache 12/348. Bonn.

BMBW (1992). Gleichwertigkeit beruflicher und allgemeiner Bildung. Rede von Fritz. Schaumann. 1. BMBW-Fachtagung am 8.07.1992. Bonn: Bundesministerium für Bildung und Wissenschaft.

BMBW (1993b). Gleichwertigkeit beruflicher und allgemeiner Bildung. Rede von Alfred Hardenacke. 2. BMBW-Fachtagung am 29.09.1993. Dortmund.

BMWT, DIHK und ZVDH [Bundesministerium für Wirtschaft und Technologie; Deutscher Industrie- und Handelskammertag; Zentralverband des Deutschen Handwerks] (2009). Auf Augenböhe - Die Qualitätssicherungssysteme der beruflichen Bildung stehen denen der Hochschulen in nichts nach. Gemeinsame Pressemitteilung vom 16.12.2009. www.bmwi.de/DE/Presse/presse mitteilungen, did=324338.html view $=$ renderPrint [abgerufen am 24.10.2012].

Bogner, Alexander, Beate Littig und Wolfgang Menz (Hrsg.) (2014). Interviews mit Experten: eine praxisorientierte Einführung. Wiesbaden: Springer-Verlag. Bogner, Alexander und Wolfgang Menz (2009). Das theoriegenerierende Experteninterview: Erkenntnisinteresse, Wissensformen, Interaktion. In: Alexander Bogner, Beate Littig und Wolfgang Menz (Hrsg.): Das 
Experteninterview: Theorie, Methode, Anwendung. Wiesbaden: Verlag für Sozialwissenschaften, 61-98.

Bohlinger, Sandra (2006). Lernergebnisorientierung als Ziel beruflicher Qualifizierung? Absehbare und nicht absehbare Folgen der Einführung des Europäischen Qualifikationsrahmens. bwp@Berufs- und Wirtschaftspädagogik - online.11, http://www. bwpat.de/ausgabe11/bohlinger_bwpat11.pdf, [abgerufen am 20.01.2016].

Böhlke, Effi, Pascale Laborier, Andreas Knie und Dagmar Simon (Hrsg.) (2009). Exzellenz durch Steuerung? Neue Herausforderungen für das deutsche und das französische Wissenschaftssystem. Berlin, (Discussion Papers/Wissenschaftszentrum Berlin für Sozialforschung, Forschungsschwerpunkt Gesellschaft und wirtschaftliche Dynamik, Forschungsgruppe Wissenschaftspolitik 2009-602). http://nbn-resolving.de/urn:nbn:de:0168-ssoar-238176 [abgerufen am 02.08.2016]

Boli, John und George M. Thomas (1997). World Culture in the World Polity: A Century of International Non-Governmental Organization. American Sociological Review. 62: 171-190.

Bologna Declaration (1999). Bologna Declaration, 19 June 1999. Bologna: Joint Declaration by Ministers Responsible for Higher Education. http://www.ehea.info/Uploads/Declarations/BOLOGNA_DECLARATI ON1.pdf, [abgerufen am 10.02.2014].

Bordeaux Communiqué (2008). The Bordeaux Communiqué on enhanced European cooperation in vocational education and training. Communique of the European Ministers for vocational education and training, the European social partners and the European Commission, meeting in Bordeaux on 26 November 2008 to review the priorities and strategies of the Copenhagen process. http://ec.europa.eu/education/policy/ vocational-policy/doc/bordeaux_en.pdf, [abgerufen am 10.02.2014].

Börzel, Tanja und Thomas Risse (2003). Conceptualising the Domestic Impact of Europe. In: Kevin Featherstone und Claudio M. Radaelli (Hrsg.): The Politics of Europeanisation. Oxford: Oxford University Press, 57-82

Bouder, Annie (2006). Der Europäische Qualifikationsrahmen. Ein kritischer Blick mit französischen Augen. Berufsbildung in Wissenschaft und Praxis. 5: 8-12.

Bouder, Annie und Jean-Louis Kirsch (2007). The French Vocational Education and Training System: like an unrecognised prototype? European Journal of Education. 42 (4): 503-521.

Bourdieu, Pierre (1987). Die feinen Unterschiede - Kritik der gesellschaftlichen Vernunft. Frankfurt am Main: Suhrkamp Taschenbuchverlag.

Bourdieu, Pierre (2009). Ökonomisches Kapital, kulturelles Kapital, soziales Kapital. In: Heike Solga, Justin J.W. Powell und Peter A. Berger (Hrsg.): Soziale Ungleichbeit - Klassische Texte zur Sozialstrukturanalyse. Frankfurt am Main: Campus, 111-125.

Bourdieu, Pierre und Passeron, Jean-Claude (1971). Die Illusion der Chancengleichheit. Suttgart: Klett. 
Bouyx, Benoit (2009). Notice 14 - La formation professionnelle initiale: le parent pauvre? In Bernard Toulemonde (Hrsg.): Le système éducatif en France. Paris: La documentation Francaise.

bpb Lexikon (2014). Subsidiaritätsprinzip, http://www.bpb.de/nachschlagen/ lexika/recht-a-z/22960/subsidiaritaetsprinzip, [abgerufen am 10.02.2014].

Brändle, Tobias (2010). 10 Jahre Bologna-Prozess: Chancen, Herausforderungen und Problematiken. Wiesbaden: VS Verlag für Sozialwissenschaften.

Brauns, Hildegard (1998). Bildung in Frankreich. Eine Studie zum Wandel berkunftsund geschlechtssperifischen Bildungsverbaltens. Opladen: Leske + Budrich.

Brauns, Hildegard, Markus Gangl und Stefani Scherer (1999a). Education and Unemployment: Patterns of Labour Market Entry in France, the United Kingdom and Germany. Mannheim: Arbeitspapiere - Mannheimer Zentrum für Europäische Sozialforschung.

Brauns, Hildegard, Susanne Steinmann, Annick Kieffer und Catherine Marry (1999b). Does Education Matter? France and Germany in Comparative Perspective. European Sociological Review. 15 (1): 61-89.

Brint, Steven und Jerome Karabel (1989). The Diverted Dream. Community Colleges and the Promise of Educational Opportunity in America, 1900-1985. New York, Oxford: Oxford University Press.

Brint, Steven und Jerome Karabel (1991). Institutional Origins and Transformations: The Case of American Community Colleges. In: Walter W. Powell und Paul J. DiMaggio (Hrsg.): The New Institutionalism in Organizational Analysis. Chicago: University of Chicago Press, 337-360.

Brucy, Guy (2005). L'enseignement technique et professionnel français. Cahiers de la recherche sur l'éducation et les savoirs. 2005 (4): 13-34.

Brucy, Guy und Vincent Troger (2000). Un siècle de formation professionnelle en France : la parenthèse scolaire ? Revue française de pédagogie. 131 (1): 9-21.

Bruges Communiqué (2010). The Bruges Communiqué on enbanced European Cooperation in Vocational Education and Training for the period 2011-2020. Communique of the European Ministers for Vocational Education and Training, the European Social Partners and the European Commission, meeting in Bruges on 7 December 2010 to review the strategic approach and priorities of the Copenhagen process for 2011-2020. http://ec.europa.eu/education/policy/vocational-policy/ doc/brugescom_en.pdf, [abgerufen am 10.02.2014].

Brzinsky-Fay, Christian (2007). Lost in transition? Labour market entry sequences of school leavers in Europe. European Sociological Review. 23 (4): 409-422.

Bublitz, Hannelore (2001). Archäologie und Genealogie. In: Markus S. Kleiner (Hrsg.): Michel Foucault. Eine Einfübrung in sein Denken. Frankfurt am Main: Campus, 27-39.

Bublitz, Hannelore (2006). Differenz und Integration. Zur diskursanalytischen Rekonstruktion der Regelstrukturen sozialer Wirklichkeit. In: Reiner Keller, Andreas Hirseland, Werner Schneider und Willy Viehöver (Hrsg.): Handbuch 
Sozialwissenschaftliche Diskursanalyse. Band 1: Theorien und Methoden. Opladen: Leske + Budrich, 227-261.

Bucharest Communiqué (2012). Bucharest Communiqué: Making the Most of Our Potential: Consolidating the European Higher Education Area, EHEA Ministerial Conference. $\quad$ http://www.ehea.info/Uploads/\%281\%29/Bucharest $\% 20$ Communique\%202012\%281\%29.pdf, [abgerufen am 10.02.2014].

Budapest-Vienna Declaration (2010). Budapest-Vienna Declaration on the European Higher Education Area, March 12, 2010. http://www.ehea.info/Uploads/ Declarations/Budapest-Vienna_Declaration.pdf, [abgerufen am 10.02.2014]. Budde, Jürgen (2012). Problematisierende Perspektiven auf Heterogenität als ambivalentes Thema der Schul- und Unterrichtsforschung. Zeitschrift für Pädagogik. 58 (4): 522-540

Buhr, Regina, Walburga K. Freitag, Ernst A. Hartmann, Claudia Loroff, Karl Heinz Minks, Kerstin Mucke und Ida Stamm-Riemer (Hrsg.) (2008). Durchlässigkeit gestalten! Wege zwischen beruflicher und hochschulischer Bildung. Münster: Waxmann.

Bührmann, Andrea D. und Werner Schneider (2007). Mehr als nur diskursive Praxis? - Konzeptionelle Grundlagen und methodische Aspekte der Dispositivanalyse. Forum Qualitative Sozialforschung. 8 (2): Art. 28.

Bührmann, Andrea D. und Werner Schneider (2008). Vom Diskurs zum Dispositiv: eine Einführung in die Dispositivanalyse. Bielefeld: transcript.

Bundesagentur für Arbeit (2014). Wege zum Zweiten Bildungsweg, http://www.arbeitsagentur.de/web/content/DE/Veroeffentlichungen/The menheftedurchstarten/WeiterdurchBildung/Bildungswege/NachholenvonS chulabschluessen/WegezumZweitenBildungsweg/index.htm, [abgerufen am 20.03.2014].

Bundesweiter Bildungsstreik (2014). Forderungen der Studierenden zum Bildungsstreik 2009, http://www.bildungsstreik.net/aufruf-zum-bildungsstreikfur-solidaritat-und-freie-bildung/aufruf/forderungen-der-studierenden/, [abgerufen am 25.03.2014].

Busemeyer, Marius R. (2009). Die Europä̈isierung der deutschen Berufsbildungspolitik Sachzwang oder Interessempolitik? Internationale Politikanalyse. Berlin: FriedrichEbert-Stiftung.

Busemeyer, Marius R. und Kathleen Thelen (2008). From Collectivism towards Segmentalism: Institutional Change in German Vocational Training. MPIfG Discussion Paper 08/13.

Campbell, John L. (2004). Institutional Change and Globalization. Princeton, Oxford: Princeton University Press.

Campinos-Dubernet, Myriam (1995). Qualifizierende Organisation und Mobilität. Die Betriebstechniker in der chemischen Industrie. Berufsbildung (CEDEFOP). (5): 19-29. 
CEDEFOP (2008). Berufsbildung in Frankreich: Kurzbeschreibung. Luxemburg: Amt für amtliche Veröffentlichungen der Europäischen Gemeinschaften. http://www.cedefop.europa.eu/EN/Files/5190_de.pdf, [abgerufen am 10.02.2014].

CGT [Confédération générale du travail] (2005b). Projet des repères revendicatifs de la CGT Educ'action concernant les voies professionnelles et technologiques. http://ddata.over-blog.com/xxxyyy/0/03/60/91/cgt/projet-de-repe-revoie-pro-et-techno.doc, [abgerufen am 06.03.2014].

CGT (2009). Lycée: les dangers de la réforme. Dossier spécial de la CGT-Educ'action. http://www.cgteduccreteil.org/actualite/article/lycee-les-dangers-de-lareforme, [abgerufen am 06.03.2014].

CGT (2011). Bac Pro 3 ans, réforme STI2D. CGT Educ'action. Non à la disparition d'une formation et professionnelle de qualité http://educaction7627.fr/IMG/pdf/4_p-_enseignement_pro-__rentree_ 2011.pdf, [abgerufen am 06.04.2014].

CGT (2012). Fiche $n^{\circ} 13$ : Droit à l'éducation. http://www.cgt.fr/spip.php? page $=$ article_dossier1\&id_article $=29 \# 13$.

Chabbott, Colette (2003). Constructing education for development. International organizations and education for all. New York: Routledge.

Charmaz, Kathy (2006). Constructing grounded theory: A practical guide through qualitative analysis. London: Pine Forge Press.

Checkel, Jeffrey T. (2005). It's the Process Stupid! Process Tracing in the Study of European and International Politics. Working Paper 26.

Clark, Burton R. (1983). The Higher Education System. Academic Organization in CrossNational Perspective. Berkeley: University of California Press.

Clarke, Adele E. (2012). Situationsanalyse. Wiesbaden: Springer.

Clement, Ute (2006). Der Europäische Qualifikationsrabmen - mögliche Konsequenzen für Deutschland. http://www.fes.de/aspol/docs/20060309_clement_text.pdf, [abgerufen am 15.03.2014].

Collier, David (2011). Understanding Process Tracing. PS: Political Science and Politics. 44 (4): 823-830.

Collins, Randall (1979). The Credential society : an bistorical sociology of education and stratification. New York: Academic Press.

Convert, Bernard (2003). Des hiérarchies maintenues : Espace des disciplines, morphologie de l'offre scolaire et choix d'orientation en France, 1987-2001. Actes de la recherche en sciences sociales. 149 (4): 61-73.

Copenhagen Declaration (2002). Declaration of the European Ministers of Vocational Education and Training, and the European Commission, convened in Copenhagen on 29 and 30 November 2002, on enhanced European cooperation in vocational education and training: „The Copenhagen Declaration“. http://ec.europa.eu/education/policy/ vocational-policy/doc/copenhagen-declaration_en.pdf, [abgerufen am 10.02.2014]. 
Cordes, Alexander und Birgit Gehrke (2012): Strukturwandel und Qualifikationsnachfrage. Aktuelle Entwicklungen forschungs- und wissensintensiver Wirtschaftszweige in Deutschland und im internationalen Vergleich. Studien zum deutschen Innovationssystem Nr. 10-2012. Hannover: Niedersächsisches Institut für Wirtschaftsforschung e.V.

Cort, Pia (2009). The EC Discourse on Vocational Training: How a 'Common Vocational Training Policy' Turned into a Lifelong Learning Strategy. Vocations and Learning. 2 (2): 87-107.

Council of the European Union (2009). Council Conclusions on a strategic framework for European cooperation in education and training (,ET 2020").

Coutrot, Laurence und Jacques Lautman (2005). Doctrines et pratiques patronales. L'adéquation : des pratiques de formations aux niveaux. In: JeanFrançois Giret, Alberto Lopez und José Rose (Hrsg.): Des formations pour quels emplois ? Paris: La Découverte, 47-60.

CPU [Conférence des présidents d'université] (1999). L'accueil et l'insertion des étudiants à l'université. Rennes, 1.er décembre 1999. Conférence des présidents d'université. http://www.cpu.fr/wp-content/uploads/2013/09/Actes_ AccueilInsertion_Rennes99.pdf, [abgerufen am 07.03.2014].

CPU (2002). L'Étudiant dans l'Université du XXIe siècle. Les actes du Colloque annuel de la CPU. http://www.cpu.fr/wp-content/uploads/2013/09/Actes_ ColloqueCPU2002.pdf, [abgerufen am 07.03.2014].

CPU (2007a). Avis de la CPU sur l'orientation active et l'admission post-bac. Prises de position CPU. http://www.cpu.fr/wp-content/uploads/2013/08/Position CPU-Orientation_active_et_admission_Postbac_22-11-2007_.pdf, [abgerufen am 07.03.2014].

CPU (2007c). Propositions du Colloque de Metr. Prises de position CPU. http://www.cpu.fr/wp-content/uploads/2013/08/Propositions_Colloque_ Metz_2007_01.pdf, [abgerufen am 07.03.2014].

CPU (2008). Les Universités Européennes : nouvelles frontières, nouvelles perspectives. Bruxelles 2008. Conclusions. Conférence des présidents d'université. http://www.cpu.fr/wp-content/uploads/2013/03/page1-188.pdf.

CPU (2009). L'université française, moteur du progrès social. Dossier CPU. Conférence des présidents d'université. http://www.cpu.fr/wp-content/uploads/2013/ 09/fiche_argu_ascenseur_social_def_01.pdf.

CPU (2010a). L'Université, lieu de tous les savoirs, lieu de tous les métiers d'anjourd'bui et de demain. Dossier CPU. Conférence des présidents d'université. http:// www.cpu.fr/wp-content/uploads/2013/09/dossier_pro_2010-BAT.pdf, [abgerufen am 07.03.2014].

CPU (2010b). La diversité au coeur des universités : la réussite et l'excellence pour tous. http://www.cpu.fr/wp-content/uploads/2013/09/CP_CPU_Diversite.pdf. 
CPU (2011). Une ambition : la licence. Colloque annuel de la Conférence des présidents d'université, Toulouse, les 11, 12 et 13 mai 2011. http://www.cpu.fr/wp-con tent/uploads/2013/09/actes-web_toulouse.pdf, [abgerufen am 07.03.2014].

CPU (2012). Contribution de la CPU aux assises de l'enseignement supérieur et de la recherche. http://www.cpu.fr/wp-content/uploads/2014/02/121114Contribution-CPU.pdf, [abgerufen am 07.03.2014].

Crespy, Amandine und Vivian A. Schmidt (2014). The clash of Titans: France, Germany and the discursive double game of EMU reform. Journal of European Public Policy, 21(8), 1085-1101.

Csigó, Monika (2006). Institutioneller Wandel durch Lernprozesse. Eine neoinstitutionalistische Perspektive. Wiesbaden: VS Verlag für Sozialwissenschaften.

Culpepper, Pepper D. und Kathleen Thelen (2008). Institutions and Collective Actors in the Provision of Training: Historical and Cross-National Comparisons. In: Karl Ulrich Mayer und Heike Solga (Hrsg.): Skill Formation: Interdisciplinary and Cross-National Perspectives. New York: Cambridge University Press. 21-49

Curaj, Adrian, Peter Scott, Lazăr Vlasceanu und Lesley Wilson (2012). European bigher education at the crossroads: between the Bologna process and national reforms. Dordrecht: Springer.

Dahrendorf, Ralf (1965). Bildung als Bürgerrecht. Plädoyer für eine aktive Bildungspolitik. Osnabrück: Nannery-Verlag GmbH.

Davis, Kingsley und Wilbert E. Moore (1945). Some principles of stratification. American sociological review. 10 (2): 242-249.

Deißinger, Thomas (1998). Beruflichkeit als, organisierendes Prinzip' der deutschen Berufsausbildung. Markt Schwaben: Eusl-Verlagsgesellschaft.

Deißinger, Thomas (Hrsg.) (2001a). Berufliche Bildung zwischen nationaler Tradition und globaler Entwicklung. Beiträge zur vergleichenden Berufsbildungsforschung. BadenBaden: Nomos.

Deißinger, Thomas (2001b). Zur Frage der Bedeutung des Berufsprinzips als ,organisierendes Prinzip' der deutschen Berufsausbildung im europäischen Kontext: Eine deutsch-französische Vergleichsskizze. Tertium Comparationis. 7 (1): 1-18.

Denzin, Norman K. (1978). Triangulation: A case for methodological evaluation and combination. In Norman K. Denzin (Hrsg.): Sociological methods. A Sourcebook. New York: McGraw-Hill, 339-357.

DEPP [Direction de l'évaluation, de la prospective et de la performance] (2012). Repères et références statistiques sur les enseignements, la formation et la recherche - édition 2012.

DEPP (2013a). L'état de l'École 2013.

DEPP (2013b). Repères et références statistiques sur les enseignements, la formation et la recherche - édition 2013. 
DGB [Deutscher Gewerkschaftsbund] (1990). DGB zum Berufsbildungsbericht 1990: Berufsbildungsbericht 1990 gibt keine Antworten auf Probleme der Gegenwart und Zukunft. Gewerkschaftliche Bildungspolitik. (3): 56-64.

DGB (1991). DGB zum Berufsbildungsbericht 1991: Qualifizierte Berufsbildung für alle in einem vereinten Deutschland als Auftrag. Gewerkschaftliche Bildungspolitik. (3): 56-61.

DGB (1992). Qualifizierte berufliche Bildung für alle! Artikel von Ulf Fink. Gewerkschaftliche Bildungspolitik. (11): 248-253.

DGB (1995). DGB zum Berufsbildungsbericht 1995: Mit Ausbildung für Arbeit von morgen die Zukunft gestalten. Gewerkschaftliche Bildungspolitik. (4): 80-83.

DGB (1996). Vorschläge und Forderungen des DGB zur Sicherung und Modernisierung der beruflichen Bildung. Gewerkschaftliche Bildungspolitik. (4): 35.

DGB (1998b). Gewerkschaften im Dialog: „Bewegt Bildung ein Appell des DGB zum Handeln“. Gewerkschaftliche Bildungspolitik. (7/8): 10-14.

DGB (1998c). KMK: Hochschulreife durch Zusatzqualifikationen zur Berufsausbildung. Artikel von Ursula Herdt und Bernd Schwiedrzik. Gewerkschaftliche Bildungspolitik. (11/12): 29-30.

DGB (2002). Zukunftsprojekt Bildung: Eine Weiterbildungsoffensive, damit Menschen die Zukunft gestalten können. Gewerkschaftliche Bildungspolitik. (5/6): 19-27.

DGB (2004a). DGB Stellungnahme zum Referentenentwurf zur Reform der beruflichen Bildung vom 19. Mai 2004. Abteilung Bildung und Qualifizierung. Berlin. http://www.dgb.de/themen/++co++mediapool-9870760fbc4559648da0d 395ba45af85 [abgerufen am 11.03.2014].

DGB (2004b). Reform beruflicher Bildung. Gewerkschaftliche Bildungspolitik. (1): 844.

DGB (2005). Berufsbildungsgesetz: Licht und Schatten. Pressemitteilung vom 18.02.2005. http://www.dgb.de/presse/++co++a4b94c90-155b-11df-4ca900093d10fae2 [abgerufen am 23.10.2012].

DGB (2006a). Beschlïsse zur Bildungspolitik. Bereich Bildung, Qualifizierung, Forschung. Berlin.

DGB (2006b). Europa entwickeln - berufliche Bildung gestalten Entwicklung eines Europäischen und Nationalen Qualifikationsrahmens (EQR/NQR). Redebeiträge von Hans-Detlev Küller:: In: Deutscher Bundestag: Protokoll 16/22 des Ausschusses für Bildung, Forschung und Technikfolgenabschätzung. Berlin

DGB (2008a). Deutscher Qualifikationsrabmen. Vorschlag des Deutschen Gewerkschaftsbundes. Berlin.

DGB (2008b). „Mit guter Bildung in die Zukunft"-Gewerkschaftliche Anforderungen an den Bildungsgipfel. Beschluss des DGB-Bundesvorstandes vom 07.10.2008. Berlin. 
DGB (2009a). Der Deutsche Qualifikationsrabmen (DQR). Chancen und Risiken aus gewerkschaftlicher Sicht. DGB-Bundesvorstand. Berlin.

DGB (2009b). Heute für morgen: Fachkräfte qualifizieren. Pressemitteilung vom 30.03.2009. http://www.dgb.de/presse/++co++b748ad42-1560-11df-4ca900093d10fae2 [abgerufen am 23.10.2012].

DGB (2009c). Öffentliches Fachgespräch zum Thema „Deutscher Qualifikationsrabmen“. Redebeiträge von Hermann Nehls:: In: Deutscher Bundestag: Protokoll 16/85 des Ausschusses für Bildung, Forschung und Technikfolgenabschätzung. Berlin.

DGB (2009d). Sebrbrock fordert Korrektur der Studienreform in Deutschland. Pressemitteilung vom 28.04.2009. http://www.dgb.de/themen/++co++ f165a3d2-24cd-11df-4f82-001ec9b03e44 [abgerufen am 23.10.2012].

DGB (2009e). Statement von Ingrid Sehrbrock auf der Pressekonferenz, zu „Ein Jahr nach dem Bildungsgipfel: W as ist uns gute Bildung wert?". Pressekonferenz am 22.10.2009. Dresden.

DGB (2010b). Debatte um Deutschen Qualifikationsrabmen (DQR). Redebeiträge von Hermann Nebls: http://www.wir-gestalten-berufsbildung.de/wir-ueberuns/newsletter/ausgaben-2010/arbeiten-am-deutschen-qualifikations rahmen-ausgesetzt/ [abgerufen am 23.10.2012].

DGB (2010c). Neuer Kurs für Bologna. DGB-V orschläge für einen nationalen Aktionsplan. Beschluss des 19. DGB-Kongresses. http://www.dgb.de/themen/++co $++58432 f 9$ e-64b0-11df-74dc-00188b4dc422 [abgerufen am 23.10.2012].

DGB (2010e). Stellungnahme zu „Europä̈scher Qualifikationsrabmen/ Deutscher Qualifikationsrahmen“. In: Deutscher Bundestag: A-Drs. 17(18)83g. Berlin.

DGB (2011a). Deutschland ist weit von einer Bildungsrepublike entfernt. Pressemitteilung vom 13.09.2011. http://www.dgb.de/presse/++co++c7681cb0-ddea-11e07685-00188b4dc422 [abgerufen am 23.10.2012].

DGB, KWB, BMWT und BMBF [Deutscher Gewerkschaftsbund, Kuratorium der Deutschen Wirtschaft für Berufsbildung, Bundesministerium für Wirtschaft und Technologie, Bundesministerium für Bildung und Forschung] (2002). Umsetzung von Leistungspunktesystemen in der beruflichen Weiterbildung am Beispiel der IT-Fortbildungsverordnung. Gewerkschaftliche Bildungspolitik. (3/4): 23.

DHQR [Deutscher Hochschulqualifikationsrahmen] (2005). Qualifikationsrahmen für Deutsche Hochschulabschlïsse (Im Zusammenwirken von Hochschulrektorenkonferen₹, Kultusministerkonferen₹, und Bundesministerium für Bildung und Forscbung erarbeitet und von der Kultusministerkonferenz. am 21.04.2005 beschlossen). http://www.kmk.org/fileadmin/veroeffentlichungen_beschlues se/2005/2005_04_21-Qualifikationsrahmen-HS-Abschluesse.pdf, [abgerufen am 10.02.2014].

Diaz-Bone, Rainer (2002). Kulturvelt, Diskurs und Lebensstil. Eine diskurstheoretische Erweiterung der Bourdieuschen Distinktionstheorie. Opladen: Leske + Budrich. 
Diaz-Bone, Rainer (2003). Entwicklungen im Feld der foucaultschen Diskursanalyse. Forum Qualitative Sozialforscbung. 4 (3): Art. 1. http://www.qualitative-research.net/index.php/fqs/article/view/683/1476, [abgerufen am 03.08.2016].

DIHK [Deutscher Industrie- und Handelskammertag] (2006a). Europa entwickeln - berufliche Bildung gestalten Entwicklung eines Europäischen und Nationalen Qualifikationsrabmens. Redebeiträge von Sybille von Obernitz und Barbara Fabian.: In: Deutscher Bundestag: Protokoll 16/22 des Ausschusses für Bildung, Forschung und Technikfolgenabschätzung. Berlin.

DIHK (2006b). Reformdruck für das deutsche Bildungssystem: Der Europäische Qualifikationsrahmen. Pressemitteilung vom 12.10.2006. Berlin.

DIHK (2007c). Wege der Beförderung von Anrechnung beruflicher Kompetenzen auf Hochschulstudiengänge. Statement von Dr. Knut Diekmann zur Abschlußtagung der ANKOM-Initiative. Bonn.

DIHK (2008b). Spitzenverbände der deutschen Wirtschaft begrüßen Qualifizierungsinitiative der Bundesregierung. Pressemitteilung vom 09.01.2008. Berlin.

DIHK (2010b). Öffentliche Anbörung „Europä̈scher Qualifikationsrabmen/Deutscher Qualifikationsrabmen (EQR/DQR)". Stellungsnabme des DIHK. In: Deutscher Bundestag: A-Drs. 17(18)83d. Berlin.

DIHK (2010c). Öffentliche Anbörung zum Thema „Europä̈scher Qualifikationsrabmen/Deutscher Qualifikationsrahmen". Redebeiträge von Sybille von Obernitz: In: Deutscher Bundestag: Protokoll 17/19 des Ausschusses für Bildung, Forschung und Technikfolgenabschätzung. Berlin.

DIHK (2012). Mit Berufserfahrung an die Hochschule - Durchlässigkeit erböben. Pressemitteilung vom 02.08.2012. Berlin.

DIHK und HRK [Deutscher Industrie- und Handelskammertag, Hochschulrektorenkonferenz] (2008). Für mehr Durchlässigkeit zwischen beruflicher Bildung und Hochschulbildung! Gemeinsame Erklärung des DIHK und der HRK. http://www.dihk.de/ressourcen/downloads/dihk_hrk_durchlaessig keit.pdf, [abgerufen am 27.03.2014].

DIHK und ZDH [Deutscher Industrie- und Handelskammertag, Zentralverband des Deutschen Handwerks] (2006). DIHK und ZDH: Europäischen Qualifikationsrabmen schnell verabschieden. Pressemitteilung vom 14.11.2006. Berlin.

DIHT [Deutscher Industrie- und Handelstag] (1990). 11 Thesen für eine größere Durchlässigkeit der Bildungsebenen. DIHT.

DIHT (1992a). Gleichwertigkeit beruflicher und allgemeiner Bildung. Rede von Franz. Schoser. Dokumentation der 1. BMBW Fachtagung am 8.07.1992. Bonn.

DIHT (1992b). Modellkonzept „Duale Berufsbildung im Verbund“. DIHT.

DiMaggio, Paul J. (1988). Interest and Agency in Institutional Theory. In: Lynne G. Zucker (Hrsg.): Institutional Patterns and Organizations: Culture and Environment. Cambridge: Ballinger, 3-21. 
DiMaggio, Paul J. (1991). Constructing an Organizational Field as a Professional Project: U.S. Art Museums, 1920-1940. In: Walter W. Powell und Paul J. DiMaggio (Hrsg.): The New Institutionalism in Organizational Analysis. Chicago: University of Chicago Press, 267-292.

DiMaggio, Paul J. und Walter W. Powell (1983). The Iron Cage Revisited: Institutional Isomorphism and Collective Rationality in Organizational Fields. American Sociological Review. 48 (2): 147-160.

Dobbin, Frank (1994). Cultural Models of Organization: The Social Construction of Rational Organizing Principles. In: Diana Crane (Hrsg.): The Sociology of Culture. Boston: Basil Blackwell, 117-143.

Dobbin, Frank, Beth Simmons und Geoffrey Garrett (2007). The Global Diffusion of Public Policies: Social Construction, Coercion, Competition, or Learning? Annual Review of Sociology. 33: 449-472.

Dobbins, Michael (2009). Comparing Higher Education Policies in Central and Eastern Europe : To converge or not to converge? Dissertation eingereicht an der Universität Konstanz im Fachbereich für Politik- und Verwaltungswissenschaft.

Dobbins, Michael (2011). Frañösische Hochschulpolitik: Wandel durch Internationalisierung? TranState Working Papers No. 152: Sfb 597 „Staatlichkeit im Wandel“. Bremen: Universität Bremen.

Dobbins, Michael und Christoph Knill (2009). Higher Education Policies in Central and Eastern Europe: Convergence towards a Common Model? Governance: An International Journal of Policy, Administration, and Institutions. 22 (3): 397-430.

Dobischat, Rolf, Marcel Fischell und Anna Rosendahl (2008). Auswirkungen der Studienreform durch die Einfübrung des Bachelorabschlusses auf das Berufsbildungssystem - Eine Problemskizze. Düsseldorf: Hans-Böckler-Stiftung.

Dostal, Werner (2001). Demografie und Arbeitsmarkt 2010 - Perspektiven einer dynamischen Erwerbsgesellschaft. In: Hans Jörg Bullinger (Hrsg.): Zukunft der Arbeit in einer alternden Gesellschaft. Stuttgart.

DQR (2013). Deutscher Qualifikationsrahmen für lebenslanges Lernen. Liste der zugeordneten Qualifikationen. Aktualisierter Stand: 1. August 2013, S. 2, http://www.dqr.de/media/content/Liste_der_zugeordneten_Qualifikatione n_01_08_2013.pdf [abgerufen am 03.08.2016].

Duden (1976). DUDEN - Das große Wörterbuch der deutschen Sprache in sechs Bänden. Herausgegeben und bearbeitet vom Wissenschaftlichen Rat und den Mitarbeitern der Dudenredaktion unter Leitung von Günther Drosdowski. Band 2: Cl-F. Mannheim, Wien, Zürich: Bibliographisches Institut.

Dunkel, Torsten, Isabelle Le Mouillour und Ulrich Teichler (2009). Through the Looking-Glass. Diversification and Differentiation in Vocational Education and Training and Higher Education. In: CEDEFOP (Hrsg.): Modernising Vocational Education and Training. Fourth Report on Vocational Training Research in 
Europe: Background Report. Volume 2. Luxembourg: Office for Official Publications of the European Communities, 239-68.

Duru-Bellat, Marie (2005). Democratisation of Education and reduction in inequalities of opportunities : an obvious link? Paper presented at the European Conference on Educational Research, University College Dublin, 7-10 September 2005, http://www.leeds.ac.uk/educol/documents/143161. htm, [abgerufen am 25.02.2014].

Duru-Bellat, Marie (2007). L'orientation dans le système éducatif francais, au collège et an lycée. Rapport pour le HCE, http://www.hce.education.fr/gallery_files/site/ 21/52.pdf [abgerufen am 02.08.2016]

Duru-Bellat, Marie und Annick Kieffer (2001). The Democratization of Education in France: Controversy over a Topical Issue. Population: An English Selection. 13 (2): 189-218.

Duru-Bellat, Marie und Annick Kieffer (2008). Du baccalauréat à l'enseignement supérieur en France : déplacement et recomposition des inégalités. Population 2008/1 (63): 123-157.

Ebbinghaus, Bernhard (2005). Can Path Dependence Explain Institutional Change? Two Approaches Applied to Welfare State Reform. MPIfG-DP 05. Köln: Max-Planck-Institut für Gesellschaftsforschung.

Ebner, Christian, Lukas Graf und Rita Nikolai (2013). New Institutional Linkages between Dual Vocational Training and Higher Education. A Comparative Analysis of Germany, Austria, and Switzerland. In: Michael Windzio (Hrsg.): Integration and Inequality in Educational Institutions. Dordrecht: Springer, 281-298.

Ecker-Ehrhardt, Matthias (2006). Argumente, Koalitionen, Kognitionen. Konstruktivistische Diskursanalyse am Beispiel der deutschen Debatte über die Osterweiterung der Europäischen Union. Dissertation. Berlin: Freie Universität Berlin.

Eckert, Manfred und Arnulf Zöller (Hrsg.) (2006). Der europäische Berufsbildungsraum - Beiträge der Berufsbildungsforschung: 6. Forum der Arbeitsgemeinschaft Berufsbildungsforschungsnetz (AG BFN) 19.-20. September 2005, Universität Erfurt. Bielefeld: Bertelsmann.

Ehmke, Timo, Fanny Hohensee, Heike Heidemeier und Manfred Prenzel (2004). Familiäre Lebensverhältnisse, Bildungsbeteiligung und Kompetenzerwerb. In: Manfred Prenzel, Jürgen Baumert, Werner Blum, Rainer Lehmann, Detlev Leutner, Michael Neubrand, Reinhard Pekrun, Jürgen Rost und Ulrich Schiefele (Hrsg.): PIS A 2003. Der Bildungsstand der Jugendlichen in DeutschlandErgebnisse des zweiten internationalen Vergleichs. Münster: Waxmann, 225-253.

Erikson, Robert und John H Goldthorpe (1992). The constant flux: A study of class mobility in industrial societies. New York: Oxford University Press.

Ertl, Hubert (2003). The European Union and Education and Training: An Overview of Policies and Initiatives. In: David Phillips und Hubert Ertl 
(Hrsg.): Implementing European Union Education and Training Policy. A Comparative Study of Issues in Four Member States. Dordrecht: Kluwer, 13-39.

Esser, Friedrich Hubert (2012). Die Umsetzung des Deutschen Qualifikationsrahmens. Hintergrund, Sachstand und anstehende Aufgaben. Berufsbildung in Wissenschaft und Praxis. 41 (3): 47-51.

Europäische Kommission (2005). Auf dem Weg zu einem Europäischen Qualifikationsrabmen für Lebenslanges Lernen, 08.07.2005.

Europäischer Rat (2000). Schluffolgerungen des Vorsitzes - Lissabon, 23. und 24. März 2000 (Lissabon-Strategie). http://www.bologna-berlin2003.de/pdf/ BeschluesseDe.pdf, [abgerufen am 10.02.2014].

Europäischer Rat (2002). Detailliertes Arbeitsprogramm zur Umsetzung der Ziele der Systeme der allgemeinen und beruflichen Bildung in Europa. http://www.eubildungspolitik.de/uploads/dokumente_bildungspolitik/2002_06_eu-arbeits programm2010_de.pdf, [abgerufen am 10.02.2014].

Europäischer Rat (2009). Schlussfolgerungen des Rates vom 12. Mai 2009 zu einem strategischen Rahmen für die europäische Zusammenarbeit auf dem Gebiet der allgemeinen und beruflichen Bildung (,ET 2010")(2009/C 119/02). Brüssel.

European Commission (2008). Der Europäische Qualifikationsrabmen für lebenslanges Lernen (EQR). Brussels: European Commission.

European Council (2001). Presidency Conclusions, Stockholm European Council, 23 and 24 March 2001. http://www.consilium.europa.eu/uedocs/cms_data/docs/ pressdata/en/ec/00100-r1.\%20ann-r1.en1.html, [abgerufen am 10.02.2014].

European Ministers Responsible for Education (1974). Resolution of the ministers of education, meeting within the Council, of 6 June 1974 on cooperation in the field of education.

EWG-Vertrag (1957). The Treaty of Rome. http://ec.europa.eu/economy_ finance/emu_history/documents/treaties/rometreaty2.pdf, [abgerufen am 12.02.2014].

Fahle, Klaus und Peter Thiele (2003). Der Brügge-Kopenhagen-Prozess - Beginn der Umsetzung der Ziele von Lissabon in der beruflichen Bildung. Berufsbildung in Wissenschaft und Praxis. 4/2003: 9-12.

Fend, Helmut (1980). Theorie der Schule. München: Urban \& Schwarzenberg.

Fend, Helmut (2006). Nene Theorie der Schule : Einführung in das Verstehen von Bildungssystemen. Wiesbaden: VS Verlag für Sozialwissenschaften.

Finger, Claudia (2011). The social selectivity of international mobility among German university students: A multi-level analysis of the impact of the Bologna process. Discussion Papers, Wissenschaftszentrum Berlin für Sozialforschung, Forschungsschwerpunkt Bildung, Arbeit und Lebenschancen, Abteilung Ausbildung und Arbeitsmarkt, No. SP I 2011-503.

Fink-Eitel, Hinrich (1990). Michel Foucault ఇur Einführung. Hamburg: Junius.

Flick, Uwe (1998). Qualitative Forscbung. Reinbek b. Hamburg: Rowohlt Taschenbuch Verlag. 
Flick, Uwe (2004). Triangulation: Eine Einführung. Wiesbaden: VS Verlag für Sozialwissenschaften.

Flick, Uwe (2014). Gütekriterien qualitativer Sozialforschung. In Nina Baur und Jörg Blasius (Hrsg): Handbuch Methoden der empirischen Sozialforschung: Wiesbaden: VS Verlag für Sozialwissenschaften, 411-423.

Follow up Copenhagen (2006). Follow-up of the Copenhagen Process. Towards the Helsinki Communiqué. http://www.minedu.fi/export/sites/default/OPM/ Tapahtumakalenteri/2006/10/eu_16_1710/Communique-Background_ document.pdf, [abgerufen am 10.02.2010].

Foucault, Michel (1976). Mikrophysik der Macht. Über Strafjustiz, Psychiatrie und Medizin. Berlin: Merve.

Foucault, Michel (1978). Dispositive der Macht. Über Sexualität, Wissen und Wahrheit. Berlin: Merve.

Foucault, Michel (1981). Archäologie des Wissens. Frankfurt am Main: Suhrkamp.

Foucault, Michel (2003). Sexualität und Wabrheit. Erster Band: Der Wille zum Wissen (14. durchgesehene und korrigierte Auflage). Frankfurt am Main: Suhrkamp.

Foucault, Michel (2007). Die Ordnung des Diskurses. Mit einem Essay von Ralf Konersmann (10. Auflage). Frankfurt am Main: Fischer.

Frank, Irmgard (2003). Erfassung und Anerkennung informell erworbener Kompetenzen - Entwicklung und Perspektiven in Deutschland und in ausgewählten europäischen Ländern. In: Wolfgang Wittwer und Steffen Kirchhof (Hrsg.): Informelles Lernen und Weiterbildung. Neue Wege zur Kompetenzentwicklung. München/Unterschleißheim: Hermann Luchterhand Verlag, 168-209.

Frankenberg, Peter (2008). Profil und Passung - Hochschulpolitische Thesen zur Studierendenauswahl. In: Christoph Heine, Hans-Jörg Didi, Klaudia Haase und Heidrun Schneider (Hrsg.): Profil und Passung. Studierendenauswabl in einem differenzierten Hochschulsystem. HIS Forum Hochschule 14/2008, 9-15.

Freikamp, Ulrike (2008). Bewertungskriterien für eine qualitative und kritischemanzipatorische Sozialforschung. In: Ulrike Freikamp, Matthias Leanza, Janne Mende, Stefan Müller, Peter Ullrich und Heinz-Jürgen Voß (Hrsg.): Kritik. mit Methode? Forschungsmethoden und Gesellschaftskritik. Bonn: Dietz, 215232.

Freitag, Walburga K. (2008). Soziale und strukturelle Durchlässigkeit als bildungspolitische Herausforderung. In: Hilde von Balluseck (Hrsg.): Professionalisierung der Frühpädagogik: Perspektiven, Entwicklungen, Herausforderungen. Opladen: Barbara Budrich, 111-122.

Freitag, Walburga K. (2009a). Europäische Bildungspolitik und Anrechnung auf Hochschulstudiengänge. Eine Skizze der Rahmenbedingungen und gegenwärtiger Effekte. In: Walburga K. Freitag (Hrsg.): Neue Bildungswege in die Hochschule. Anrechnung beruflich ervorbener Kompetenzen für Erziehungs-, Gesundheitsund Sozialberufe. Bielefeld: Bertelsmann, 12-38. 
Freitag, Walburga K. (2009b). Anrechnung als Schlüssel für die Durchlässigkeit zur Hochschule und zur Realisierung lebenslangen Lernens? In: Margret Bülow-Schramm (Hrsg.): Hochschulzugang und Übergänge in der Hochschule: Selektionsprozesse und Ungleichbeiten. Frankfurt am Main: Lang, 97-113.

Freitag, Walburga K. (2009c). Hochschulzugang öffnen - Mehr Chancen für Studierende obne schulische Hochschulzugangsberechtigung. Schriftliche Stellungnahme zur Anhörung im Landtag NRW am 3. September 2009 in Düsseldorf. Hannover: HIS GmbH.

Freitag, Walburga K. (2012). Zweiter und Dritter Bildungsweg in die Hochschule. Forschungsstand und Forschungsbedarfe. Düsseldorf: Hans-Böckler-Stiftung. http://www.boeckler.de/pdf_fof/S-2010-374-5-1.pdf, [abgerufen am 16.05.2012].

Freitag, Walburga K., Ernst A. Hartmann, Claudia Loroff, Ida Stamm-Riemer, Daniel Völk und Regina Buhr (Hrsg.) (2011). Gestaltungsfeld Anrechnung. Hochschulische und berufliche Bildung im Wandel. Münster: Waxmann.

Freitag, Walburga K.; Buhr Regina; Danzeglocke, Eva-Maria; Schröder, Stefanie; Völk, Daniel (Hrsg.) (2015). Übergange gestalten. Durchlässigkeit zwischen beruflicher und hochscbulischer Bildung erböhen. Münster: Waxmann.

Friedberg, Erhard und Christine Musselin (1993). L'État face aux universités en France et en Allemagne. Anthropos.

Frommberger, Dietmar (2009). „Durchlässigkeit“ in Bildung und Berufsbildung: Begriff, Begründungen, Modelle und Kritik. bwp@ Berufs- und Wirtschaftspädagogik - online. Profil 2: 1-18. http://www.bwpat.de/profil2/ frommberger_profil2.pdf, [abgerufen am 27.06.2012].

Frommberger, Dietmar und Holger Reinisch (2013). Zur Weiterentwicklung der Durchlässigkeit in der beruflichen Bildung in Deutschland zwischen den Impulsen einer Europäischen Berufsbildungspolitik und nationalen Traditionen. Überlegungen und Befunde am Beispiel der DECVETPilotinitiative. bwp@ Berufs- und Wirtschaftspädagogik - online. 25: 1-25. http://www.bwpat.de/ausgabe25/frommberger_reinisch_bwpat25.pdf, [abgerufen am 20.01.2016]

FSU [Fédération syndicale unitaire] (2001a). Déclaration de M. Gérard Aschieri, secrétaire général de la FSU, sur l'engagement de la FSU en faveur d'un droit à l'éducation et à la formation tout au long de la vie, Paris le 1er décembre 2001. http://discours.vie-publique.fr/notices/023002123.html, [abgerufen am 06.03.2014].

FSU (2002). Déclaration de M. Gérard Aschieri, secrétaire général de la FSU, sur les politiques européennes de l'éducation et de la formation, Florence le 6 novembre 2002. http://discours.vie-publique.fr/notices/033000788.html, [abgerufen am 06.03.2014].

FSU (2006). Déclaration de M. Gérard Aschieri, secrétaire général de la FSU, sur la réussite des étudiants à l'université, l'insertion professionnelle et la précarité, Paris le 24 mai 2006. 
http://discours.vie-publique.fr/notices/063002414.html, [abgerufen am 06.03.2014].

FSU (2011). Les propositions du SNESUP pour le cycle licence. Texte adopté par le BN du 24 mars par 16 voix pour et 2 abstention. http://cache.media.enseignementsup-recherche.gouv.fr/file/Plan_reussir_ en_licence/59/9/propositions_SNESUP_cycle_licence_mars2011_173599. pdf, [abgerufen am 06.03.2014].

Galonska, Christian (2004). Zur Inhaltsanalyse von Tageszeitungen am Beispiel der gesellschaftlichen Verantwortung von Wirtschaftseliten. Workshop des Forschungsverbundes „Desintegrationsprozesse“. Bremen: Universität Bremen.

Géhin, Jean-Paul und Philippe Méhaut (1993). Apprentissage on formation continue? Stratégies éducatives des entreprises en Allemagne et en France. Paris: Editions L'Harmattan.

Gei, Julia, Andreas Krewerth und Joachim Gerd Ulrich (2011). Reformvorschläge zum Übergang Schule-Berufsausbildung nur bedingt konsensfähig. Ergebnisse einer Expertenbefragung. Berufsbildung in Wissenschaft und Praxis. 40 (2): 9-13.

George, Alexander L. und Andrew Bennett (2005). Case Studies and Theory Development in the Social Sciences. Cambridge, MA: MIT Press.

Gerhards, Jürgen (1992). Dimensionen und Strategien öffentlicher Diskurse. Journal für Sozialforschung. 32 (3/4): 307-318.

Gerring, John (2004). What is a Case Study and What is it Good for? American Political Science Review. 98 (2): 341-353.

Glaser, Barney G. (1992). Emergence vs. Forcing: Basics of Grounded Theory Analysis. Mill Valey: Sociology Press.

Gläser, Jochen und Grit Laudel (1999). Theoriegeleitete Textanalyse? Das Potential einer variablenorientierten qualitativen Inhaltsanalyse. WZB Discussion Paper P 99-401. Berlin: Wissenschaftszentrum Berlin für Sozialforschung.

Gläser, Jochen und Grit Laudel (2006). Experteninterviews und qualitative Inhaltsanalyse: als Instrumente rekonstruierender Untersuchungen. Wiesbaden: VS Verlag für Sozialwissenschaften.

Goldschmidt, Dietrich (1991). Idealtypische Charakterisierung sieben westlicher Hochschulsyteme. Zeitschrift für Sozialforschung und Errziebungssoziologie. 11 (1): 317.

Goldthorpe, John H. (1996). Problems of „Meritocracy“. In: Robert Erikson und Jan O. Jonsson (Hrsg.): Can Education be Equalized? Boulder, CO: Westview Press, 255-287.

Gornitzka, Åse (2005). Coordinating policies for a Europe of knowledge. ARENA Working Paper 16/2005.

Graf, Lukas (2009). Applying the Varieties of Capitalism Approach to Higher Education: Comparing the Internationalization of German and British Universities. European Journal of Education. 44 (4): 569-585. 
Graf, Lukas (2013). The Hybridization of Vocational Training and Higher Education in Austria, Germany and Switzerland. Opladen: Budrich UniPress.

Graf, Lukas, Justin J.W, Powell, Johann Fortwengel und Nadine Bernhard (2014). Duale Studiengänge im globalen Kontext: Internationalisierung in Deutschland und Transfer nach Brasilien, Frankereich, Katar, Mexiko und in die USA. Berlin: DAAD.

Green Cowles, Maria, James A. Caporaso und Thomas Risse (Hrsg.) (2001). Transforming Europe : Europeanization and Domestic Change. Ithaca, N.Y.: Cornell University Press.

Greenwood, Royston und C.R. Hinings (1996). Understanding radical organizational change: Bringing together the old and new institutionalism. Academy of Management Review. 21: 1022-1954.

Greinert, Wolf-Dietrich (2005). Mass Vocational Education and Training in Europe. CEDEFOP Panorama, 118. Luxembourg: European Communities.

Greinert, Wolf-Dietrich (2006). Geschichte der Berufsausbildung in Deutschland. In: Rolf Arnold und Antonius Lipsmeier (Hrsg.): Handbuch der Berufsbildung. Wiesbaden: VS Verlag für Sozialwissenschaften, 499-508.

Groh-Samberg, Olaf, Ariane Jossin, Carsten Keller und Ingrid Tucci (2012). Biografische Drift und zweite Chance. Bildungs- und Berufsverläufe von Migranten in Deutschland und Frankreich. Kölner Zeitschrift für Soziologie und Sozialpsychologie. Sonderheft 31. 52: 186-210.

Grollmann, Philipp (2012). Duale Ausbildung - Nischenexistenz oder auf dem Vormarsch? In: Eva Kuda, Jürgen Strauß, Georg Spöttl und Bernd Kaßebaum (Hrsg.): Akademisierung der Arbeitswelt? Zur Zukunft der beruflichen Bildung. Hamburg: VSA-Verlag, 300-312.

Grollmann, Philipp, Georg Spöttl und Felix Rauner (2006). Europä̈sierung Beruflicher Bildung - eine Gestaltungsaufgabe. Münster: LIT.

Gutschow, Katrin (2003). Erfassen, Beurteilen und Zertifizieren non-formell und informell erworbener beruflicher Kompetenzen in Frankreich: Die Rolle des bilan de compétences. In: Gerald A. Straka (Hrsg.): Zertifizierung non-formell und informell erworbener beruflicher Kompetenzen. Ergebnisse der Facbtagung „Erfassen, Beurteilen und Zertifizieren non-formell und informell erworbener beruflicher Kompetenzen". Münster: Waxmann, 127-139.

Hackl, Elsa (2001). Towards a European Area of Higher Education: Change and Convergence in European Higher Education. EUI Working Papers. RSC No. 2001/09. San Domenico: Robert Schuman Centre for Advanced Studies, European University Institute.

Hadjar, Andreas und Rolf Becker (Hrsg.) (2006). Die Bildungsexpansion. Erwartete und unerwartete Folgen. Wiesbaden: VS Verlag für Sozialwissenschaften.

Hall, Peter A. (1993). Policy Paradigms, Social Learning, and the State. The Case of Economic Policymaking in Britain. Comparative Politics. 25 (3): 275-296. 
Hall, Peter A. und David Soskice (Hrsg.) (2001). Varieties of Capitalism - The Institutional Foundation of Comparative Advantage. New York: Oxford University Press.

Hanf, Georg (2011). Der Europäische Qualifikationsrahmen im Rahmen des Kopenhagen-Prozesses und seine Umsetzung in Deutschland. In: Kerstin Odendahl (Hrsg.): Europäische (Bildungs-)Union? Berlin: Berliner WissenschaftsVerlag , 449-467.

Hanf, Georg und Klaus Fahle (2005). Der Europäische Qualifikationsrabmen Konsultationsprozess läuft. BIBB. http://www.bibb.de/de/21696.htm, [abgerufen am 15.03.2014].

Hanft, Anke und Katrin Brinkmann (Hrsg.) (2013). Offene Hochschulen. Die Neuausricbtung der Hochschulen auf Lebenslanges Lernen. Münster: Waxmann.

Hanft, Anke und Wolfgang Müskens (2013). Anrechnung beruflicher Kompetenzen auf Hochschulstudiengänge: Ein Überblick. In: Anke Hanft und Katrin Brinkmann (Hrsg.): Offene Hochschulen. Die Neuausricbtung der Hochschulen auf Lebenslanges Lernen. Münster: Waxmann, 223-234.

Hanft, Anke, Annika Maschwitz und Monika Hartmann-Bischoff (2013). Beratung und Betreuung von berufstätigen Studieninteressierten und Studierenden zur Verbesserung des Studienerfolgs. In: Anke Hanft und Katrin Brinkmann (Hrsg.): Offene Hochschulen. Die Neuausricbtung der Hochschulen auf Lebenslanges Lernen. Münster: Waxmann, 13-29.

Hanft, Anke; Pellert, Ada; Cendon, Eva; Wolter, Andrä (Hrsg.) (2015a). Weiterbildung undLebenslanges Lernen an Hochschulen. Ergebnisse der wissenschaftlichen Begleitung zur ersten Förderphase der ersten Wettbewerbsrunde des Bund-LänderWettbewerbs „Aufstieg durch Bildung offene Hochschulen“. Oldenburg.

Hanft, Anke; Zawacki-Richter, Olaf; Gierke, Willi B. (Hrsg.) (2015b). Herausforderung Heterogenität beim Übergang in die Hochscbule, Münster: Waxmann. Hannan, Michael T. und Glenn R. Carroll (1995). An Introduction to Organizational Ecology. In: Glenn R. Carroll und Michael T. Hannan (Hrsg.): Organizations in Industry: Strategy, Structure and Selection. New York: Oxford University Press, 17-31.

Hartmann-Bischoff, Monika und Stefanie Brunner (2013). Studieren mit beruflicher Qualifikation - Beratung, Vorbereitung und Begleitung. In: Anke Hanft und Katrin Brinkmann (Hrsg.): Offene Hochschulen - Die Neuausricbtung der Hochscbulen auf Lebenslanges Lernen. Münster: Waxmann, 120-126.

Hartmann, Ernst A., Michaela Knust, Claudia Loroff und Ida Stamm-Riemer (2009). Towards Permeability between Vocational and Academic Education. Experiences and Analyses from Current Initiatives in Germany. European Journal of Education. 44 (3).

Hartmann, Ernst A. und Ida Stamm-Riemer (2006). Die BMBF-Initiative „Anrechnung beruflicher Kompetenzen auf Hochschulstudiengänge“ - ein 
Beitrag zur Durchlässigkeit des deutschen Bildungssystems und zum Lebenslangen Lernen. Hochschule und Weiterbildung. 2006 (1): 52-60.

Hasse, Raimund und Georg Krücken (2005). Neo-Institutionalismus (2., vollständig überarbeitete Auflage). Bielefeld: transcript.

Haugg, Kornelia (2008). Im Dialog Durchlässigkeit der Bildungssysteme und Anrechnung von Kompetenzen fördern. In: Regina Buhr, Walburga K. Freitag, Ernst A. Hartmann, Claudia Loroff, Karl-Heinz Minks, Kerstin Mucke und Ida Stamm-Riemer (Hrsg.): Durchlässigkeit gestalten! Wege zwischen beruflicher und hochschulischer Bildung. Münster: Waxmann, 38-41.

Hay, Colin (2006). Constructivist institutionalism. In: R.A.W. Rhodes, Sarah Binder und Bert Rockmann (Hrsg.): The Oxford Handbook of Political Institutions. Oxford: Oxford University Press, 56-74.

Hecker, Ursula (1994). Berufsausbildung nachbolen. Wege zum nachträglichen Berufsabschluss für ungelernte (junge) Erwachsene. Ergebnisse, Veröffentlichungen und Materialien aus dem BIBB, Berlin.

Hegelheimer, Armin (1986). Gleichwertigkeit von allgemeiner und beruflicher Bildung. Bad Honeff: Bock Verlag.

Heine, Christoph (2012). Übergang vom Bachelor- zum Masterstudium. Studien zum deutschen Innovationssystem. 02-2012. Berlin: HIS Forum Hochschule 7/12. http://www.e-fi.de/fileadmin/Innovationsstudien_2012/StuDIS_02_ HIS.pdf, [abgerufen am 26.03.2014].

Hellmann, Kai-Uwe (2006). Organisationslegitimität im Neo-Institutionalismus. In: Konstanze Senge und Kai-Uwe Hellmann (Hrsg.): Einfübrung in den NeoInstitutionalismus. Wiesbaden: VS Verlag für Sozialwissenschaften, 75-89.

Helmrich, Robert (2008). Fachkräfteengpässe. Anforderungen an das Bildungssystem. In: Regina Buhr, Walburga K. Freitag, Ernst A. Hartmann, Claudia Loroff, Karl-Heinz Minks, Kerstin Mucke und Ida Stamm-Riemer (Hrsg.): Durchlässigkeit gestalten! Wege zwischen beruflicher und hochschulischer Bildung. Münster: Waxmann, 28-37.

Helsinki Communiqué (2006). The Helsinki Communiqué on Enhanced European Cooperation in Vocational Education and Training. Communique of the European Ministers of Vocational Education and Training, the European Social partners and the European Commission, convened in Helsinki on 5 December 2006 to review the priorities and strategies of the Copenhagen Process. http://ec. europa.eu/education/policy/vocational-policy/doc/helsinki_en.pdf, [abgerufen am 10.02.2014].

Héritier, Adrienne (2001). Differential Europe: New Opportunities and Restrictions for Policy-Making in Member states. In: Adrienne Héritier, Dieter Kerwer, Christoph Knill, Dirk Lehmkuhl, Michael Teutsch und Anne-Cécile Douillet (Hrsg.): Differential Europe: European Union Impact on National PolicyMaking. Boulder, CO: Rowman \& Littlefield, 1-22. 
Heublein, Ulrich, Johanna Richter, Robert Schmelzer und Dieter Sommer (2012). Die Entwicklung, der Schwund und Studienabbruchquoten an den deutschen Hochschulen. Statistische Berechnungen auf der Basis des Absolventenjabrgangs 2010. HIS: Forum Hochschule. http://www.his.de/pdf/pub_fh/fh-201203.pdf, [abgerufen am 15.03.2014].

Hillmert, Steffen (2008). When traditions change and virtues become obstacles: Skill formation in Britain and Germany. In: Karl Ulrich Mayer und Heike Solga (Hrsg.): Skill Formation: Interdisciplinary and Cross-National Perspectives. New York: Cambridge University Press, 50-81.

Hillmert, Steffen (2011). Bildungszugang, Bildungskonsequenzen und soziale Ungleichheit im Lebenslauf. GWP - Gesellschaft. Wirtschaft. Politik. 60 (2).

Hillmert, Steffen und Marita Jacob (2003). Social inequality in higher education. Is vocational training a pathway leading to or away from university? European Sociological Review. 19 (3): 319-334.

Hillmert, Steffen und Marita Jacob (2005). Zweite Chance im Schulsystem? Zur sozialen Selektivität bei ,späteren' Bildungsentscheidungen. In: Peter A. Berger und Heike Kahler (Hrsg.): Institutionalisierte Ungleichbeiten. Wie das Bildungswesen Chancen blockiert. Weinheim/München: Juventa Verlag, 155-176.

Himpele, Klemens, Andreas Keller und Sonja Staack (Hrsg.) (2010). Endstation Bologna? Zehn Jahre europäischer Hochschulraum. Bielefeld: Bertelsmann.

Holzinger, Katharina, Helge Jörgens und Christoph Knill (Hrsg.) (2007). Transfer, Diffusion und Konvergenz von Politiken. Wiesbaden: VS Verlag für Sozialwissenschaften.

Holzinger, Katharina und Christoph Knill (2005). Explaining Cross-national Policy Convergence: Concepts, Causes, and Conditions. Journal of European Public Policy. 12: 755-796.

Hörner, Wolfgang (2006). Zur unterschiedlichen Logik der Berufsbildungssysteme in Frankreich und Deutschland. In: DeutschFranzösisches Institut (Hrsg.): Frankreich Jahrbuch 2005: Bildungspolitike im Wandel. Wiesbaden: VS Verlag für Sozialwissenschaften, 139-154.

HRK [Hochschulrektorenkonferenz] (1991). Protokoll der 165. Plenarversammlung der Hochschulrektorenkonferenz am 4.11.1991. Bonn: HRK.

HRK (1992b). Öffnung der Hochschulen für Berufstätige obne formale Hochschulzugangsberechtigung. Entschließung des 167. Plenums am 6.07.1992.

HRK (1994). Die Studienberatung in den Hocbscbulen in der Bundesrepublik Deutscbland. Entschließung des 173. Plenums vom 4. Juli 1994. Bonn. http:// www.hrk.de/positionen/gesamtliste-beschluesse/position/convention/diestudienberatung-in-den-hochschulen-in-der-bundesrepublik-deutschland/ [abgerufen am 13.03.2014].

HRK (1997a). Position der HRK zum Teilzeitstudium. Entschließung des 183. Plenums vom 10. November 1997. Bonn. http://www. 
hrk.de/positionen/gesamtliste-beschluesse/position/convention/positionder-hrk-zum-teilzeitstudium/ [abgerufen am 13.03.2014].

HRK (2007c). Stellungnabme des HRK Senats vom 13.02.2007 zum Europäischen Qualifikationsrabmen und zur bevorstehenden Erarbeitung eines nationalen Qualifikationsrabmens http://www.hrk.de/uploads/tx_szconvention/Stellung nahme_Qualifikationsrahmen.pdf [abgerufen am 16.10.2012].

HRK (2008b). Neuordnung des Hochschulzugangs für beruflich Qualifizierte. Entschließung der Mitgliederversammlung am 18.11.2008. HRK. Bonn. http://www.hrk.de/positionen/gesamtliste-beschluesse/position/ convention/neuordnung-des-hochschulzugangs-fuer-beruflich-qualifizierte/ [abgerufen am 13.03.2014].

HRK (2009b). Öffentliches Fachgespräch zum Thema „Deutscher Qualifikationsrahmen“. Redebeiträge von Achim Hopbach. In: Deutscher Bundestag: Protokoll 16/85 des Ausschusses für Bildung, Forschung und Technikfolgenabschätzung. Berlin.

HRK (2010a). Öffentliche Anbörung zum Thema „Europäischer Qualifikationsrahmen/Deutscher Qualifikationsrahmen". Redebeiträge von Jan Rathjen. In: Deutscher Bundestag: Protokoll 17/19 des Ausschusses für Bildung, Forschung und Technikfolgenabschätzung. Berlin.

HRK (2010b). Stellungnahme „Europäischer Qualifikationsrahmen“. Deutscher Bundestag: A-Drs. 17(18)83c. Berlin

HRK (2011a). Anrecbnung beruflicher Kompetenzen auf ein Studium wird wichtiger. Pressemitteilung vom 13.07.2011. Bonn. http://www.hrk.de/presse/presse mitteilungen/pressemitteilung/meldung/anrechnung-beruflicher-kompeten zen-auf-ein-studium-wird-wichtiger-38/ [abgerufen am 13.03.2014].

HRK (2011b). Grußwort der Hochschulrektorenkonferen₹: Rede von Prof. Dr. Margret Wintermantel. Auftaktveranstaltung „Wettbewerb Aufstieg durch Bildung: offene Hocbscbulen" am 9.12.2011.

HRK (2013). Hochschulen in Zablen. http://www.hrk.de/uploads/media/2013-0430_Faltblatt-final_2013_fuer_Internet.pdf, [abgerufen am 25.02.2014].

Husemann, Rudolf, Johannes Münch und Claudia Pütz (1995). Mit Berufsausbildung zur Hochschule: Argumente zur Gleichwertigkeit allgemeiner und beruflicher Bildung. Frankfurt am Main: Gesellschaft zur Förderung arbeitsorientierter Forschung und Bildung.

Jäger, Siegfried (1999). Kritische Diskursanalyse - Eine Einführung. 2., überarbeitete und erweiterte Auflage. Münster: Unrast Verlag.

Jahn, Detlef (2005). Fälle, Fallstricke und die komparative Methode in der vergleichenden Politikwissenschaft. Manuskriptversion.

Jakobi, Anja P. (2009). International Organizations and Lifelong Learning. From Global Agendas to Policy Diffusion. Basingstoke: Palgrave Macmillan.

Jakobi, Anja P. und Kerstin Martens (2007). Diffusion durch internationale Organisationen: Die Bildungspolitik der OECD. In: Katharina Holzinger, 
Helge Jörgens und Christoph Knill (Hrsg.): Transfer, Diffusion und Konvergenz von Politiken. Wiesbaden: VS Verlag für Sozialwissenschaften, 247-270.

Jakobi, Anja P. und Alessandra Rusconi (2008). Crossing Frontiers to and within Higher Education: A Lifelong Learning Perspective on the Bologna Process. WZB Discussion Paper, No. SP I, 2008-502.

Jakobi, Anja P. und Alessandra Rusconi (2009). Lifelong Learning in the Bologna process: European developments in higher education. Compare: A Journal of Comparative and International Education. 39 (1): 51-65.

Jakobi, Anja und Janna Teltemann(2011). Convergence in Education Policy? A Quantitative Analysis of Policy Change and Stability in OECD Countries. Compare 41(5): 579-595.

Jansen, Dorothea (2006). Organisationssoziologische Theorien: Traditionen und aktuelle Diskussionen. Hagen: Fernuniversität Hagen.

Kamens, David H., John W. Meyer and Aaron Benavot (1996). Worldwide Patterns in Academic Secondary Education Curricula. Comparative Education Review, 40(2), 116-138.

Keller, Andreas (2003). Von Bologna nach Berlin. Perspektiven eines Europäischen Hochschulraums im Rabmen des Bologna-Prozesses am Vorabend des europäischen Hochschulgipfels 2003 in Berlin. http://ids.hof.uni-halle.de/documents/ t592.htm, [abgerufen am 15.02.2014].

Keller, Reiner (2005). Wissenssoziologische Diskursanalyse als interpretative Analytik. In: Reiner Keller, Andreas Hirseland, Werner Schneider und Willy Viehöver (Hrsg.): Die diskursive Konstruktion von Wirklichkeit. Zum Verbältnis von Wissenssoziologie und Diskursforschung. Konstanz: UVK, 49-76.

Keller, Reiner (2006). Wissenssoziologische Diskursanalyse. In: Reiner Keller, Andreas Hirseland, Werner Schneider und Willy Viehöver (Hrsg.): Handbuch Sozialwissenschaftliche Diskursanalyse. Band 1: Theorien und Methoden. Opladen: Leske + Budrich, 115-146.

Keller, Reiner (2007a). Diskurse und Dispositive analysieren. Die Wissenssoziologische Diskursanalyse als Beitrag zu einer wissensanalytischen Profilierung der Diskursforschung. Forum Qualitative Sozialforschung. 8 (2).

Keller, Reiner (2007b). Diskursforschung. Eine Einführung für SozialwissenschaftlerInnen. 3. aktualisierte Auflage. Opladen: Leske + Budrich.

Keller, Reiner (2008). Wissenssoziologische Diskursanalyse. Grundlegung eines Forschungsprogramms. Wiesbaden: VS Verlag für Sozialwissenschaften.

Keller, Reiner (2009). Müll: Die gesellschaftliche Konstruktion des Wertvollen. Die öffentliche Diskussion über Abfall in Deutschland und Frankreich. Wiesbaden: VS Verlag für Sozialwissenschaften.

Keller, Reiner, Andreas Hirseland, Werner Schneider und Willy Viehöver (2001). Zur Aktualität sozialwissenschaftlicher Diskursanalyse. Eine Einführung. In: Reiner Keller, Andreas Hirseland, Werner Schneider und Willy Viehöver 
(Hrsg.): Handbuch Sozialwissenschaftliche Diskursanalyse. Band 1: Theorien und Methoden. Opladen: Leske + Budrich, 7-28.

Kerckhoff, Alan C. (2001). Education and Social Stratification Processes in Comparative Perspective. Sociology of Education. 74 (Extra Issue): 3-18.

Kieffer, Annick (2008). Applying the ISCED-97 to France: Some Issues and

Propositions. In: Silke L. Schneider (Hrsg.): The International Standard Classification of Education. Mannheim: Mannheimer Zentrum für Europäische Sozialforschung (MZES), 103-121.

Klatetzki, Thomas (2006). Der Stellenwert des Begriffs „Kognition“ im NeoInstitutionalismus. In: Konstanze Senge und Kai-Uwe Hellmann (Hrsg.): Einfübrung in den Neo-Institutionalismus. Wiesbaden: VS Verlag für Sozialwissenschaften, 48-61.

Klemm, Jana und Georg Glasze (2004). Methodische Probleme Foucaultinspirierter Diskursanalysen in den Sozialwissenschaften. Tagungsbericht: „Praxis-Workshop Diskursanalyse“. Forum Qualitative Sozialforschung. 6 (2), Art. 24.

KMK [Kultusministerkonferenz] (1991). Rahmenvereinbarung über die Berufsscbule. Beschluss der Kultusministerkonferenz vom 15.03.1999. Bonn.

KMK (1994). Zu Fragen der Gleichwertigkeit von allgemeiner und beruflicher Bildung. Beschluß der Kultusministerkonferenz vom 02.12.1994. Bonn. http://www.kmk.org/fileadmin/veroeffentlichungen_beschluesse/1994/19 94_02_12-ErGIWk.pdf [abgerufen am 13.03.2014].

KMK (1997a). Gleichwertigkeit von allgemeiner und beruflicher Bildung. http://www.kmk.org/presse-und-aktuelles/meldung/gleichwertigkeit-vonberuflicher-und-allgemeiner-bildung.html [abgerufen am 13.03.2014].

KMK (1997b). Vereinbarung über den Erwerb der Fachbochschulreife in beruflichen Bildungsgängen. Beschluss der Kultusministerkonferenz vom 05.06.1998 i.d.F. vom 09.03.2001. Bonn/Berlin. http://www.kmk.org/fileadmin/ veroeffentlichungen_beschluesse/1997/1997_06_05-Fachoberschulreifeberufliche-Bildung.pdf [abgerufen am 13.03.2014].

KMK (2000b). Strukturelle Weiterentwicklung der dualen Berufsausbildung - Gemeinsame Grundlagen und Orientierungen. Länderpositionen ₹u Folgeaktivitäten aus dem Beschluss der Arbeitsgruppe „Aus und Weiterbildung" im Bündnis für Arbeit, Ausbildung und Wettbewerbsfähigkeit. Bonn.

KMK (2002). Anrechnung von außerhalb des Hochschulwesens erworbenen Kenntnissen und Fäbigkeiten auf ein Hochschulstudium. Beschluss der Kultusministerkonferenz vom 28.06.2002. http://www.kmk.org/fileadmin/veroeffentlichungen_ beschluesse/2002/2002_06_28-Anrechnung-Faehigkeiten-Studium-1.pdf [abgerufen am 23.10.2012].

KMK (2008). Anrechnung von außerhalb des Hochschulwesens erworbenen Kenntnissen und Fäbigkeiten auf ein Hocbschulstudium. Beschluss der Kultusministerkonferenz vom 18.09.2008. http://www.kmk.org/fileadmin/veroeffentlichungen_ 
beschluesse/2008/2008_09_18-Anrechnung-Faehigkeiten-Studium-2.pdf [abgerufen am 23.10.2012].

KMK (2009d). Hochschulingang für beruflich qualifizierte Bewerber obne schulische Hochschulzugangsberechtigung. Beschluss der Kultusministerkonferenz vom 06.03.2009. http://www.kmk.org/fileadmin/veroeffentlichungen_beschlues se/2009/2009_03_06-Hochschulzugang-erful-qualifizierte-Bewerber.pdf [abgerufen am 23.10.2012].

KMK (2009e). Kultusministerkonferenz. verbessert Durchlässigkeit und Anschlussfäbigkeit im Bildungssystem. Pressemitteilung vom 10.09.2009. http://www.kmk. $\mathrm{org} /$ presse-und-aktuelles/meldung/kultusministerkonferenz-verbessertdurchlaessigkeit-und-anschlussfaehigkeit-im-bildungssystem.html [abgerufen am 23.10.2012].

KMK (2009g). Öffentliches Fachgespräch zum Thema "Deutscher Qualifikationsrahmen“. Redebeiträge von Lothar Herstix und Karl-Heinz Held. In: Deutscher Bundestag: Protokoll 16/85 des Ausschusses für Bildung, Forschung und Technikfolgenabschätzung. Berlin.

KMK (2009h). Studium über berufliche Bildung. Wege und Berechtigungen. http://www.kmk.org/fileadmin/pdf/PresseUndAktuelles/2009/09-09_ Hochschulzugang_Berufliche_Bildung.pdf [abgerufen am 23.10.2012].

KMK (2009i). Weiterentwicklung des Bologna-Prozesses. Beschluss der 327. Kultusministerkonferenz am 15.10.2009. http://www.kmk.org/presse-undaktuelles/meldung/weiterentwicklung-des-bologna-prozesses.html [abgerufen am 23.10.2012].

KMK (2012b). Grundstruktur des Bildungswesens in der Bundesrepublik Deutschland. Bonn: KMK.

KMK (2010a). Ausblick und Abschluss. Rede von Lothar Herstix auf der 2. Fachtagung zum Deutschen Qualifikationsrahmen 19.10.2010. Tagungsdokumentation.

KMK (2010d). Öffentliche Anbörung zum Thema „Europä̈scher Qualifikationsrabmen/Deutscher Qualifikationsrahmen". Redebeiträge von Lothar Herstix. In: Deutscher Bundestag: Protokoll 17/19 des Ausschusses für Bildung, Forschung und Technikfolgenabschätzung. Berlin.

KMK (2011a). Ergebnisse der 335. Plenarsitzung der Kultusministerkonferenz am 20./21. Oktober 2011 in Berlin. http://www.kmk.org/presse-und-aktuelles/ meldung/ergebnisse-der-335-plenarsitzung-der-kultusministerkonferenz-am2021-oktober-2011-in-berlin.html [abgerufen am 23.10.2012].

KMK (2011b). Grußwort der Kultusministerkonferen₹. Rede von Prof. Dr. Birgitta Wolff. Auftaktveranstaltung „Wettbewerb Aufstieg durch Bildung: offene Hochschulen" am 9.12.2011.

KMK (2013). Das Bildungswesen in der Bundesrepublik Deutscbland 2011/2012. Darstellung der Kompetenzen, Strukturen und bildungspolitischen Entwicklungen für den Informationsaustausch in Europa. Bonn: KMK. 
KMK (2014a). Berufsoberscbule, http://www.kmk.org/bildung-schule/beruflichebildung/berufsoberschule.html, [abgerufen am 20.03.2014].

KMK (2014b). Fachoberschule, http://www.kmk.org/bildung-schule/beruflichebildung/fachoberschule.html, [abgerufen am 20.03.2014].

KMK, BDI, BDA, DIHK und ZDH [Kultusministerkonferenz; Bundesverband der Deutschen Industrie, Bundesvereinigung der Deutschen Arbeitgeberverbände; Deutscher Industrie- und Handelstag; Zentralverband des Deutschen Handwerks] (2002). Leistungsfähigkeit des Bildungssystems verbessern. Pressemitteilung vom 28.11.2002. Berlin. http://www.kmk.org/ index.php?id $=900 \&$ type $=123$ [abgerufen am 13.03.2014].

KMK, BMBF und HRK [Kultusministerkonferenz, Bundesministeriums für Bildung und Forschung, Hochschulrektorenkonferenz] (2003). Empfehlung des $B M B F$, der KMK und der HRK an die Hochschulen zur Vergabe von Leistungspunkten in der beruflichen Fortbildung und Anrecbnung auf ein Hochschulstudium. Empfehlung des 200. Plenums vom http://www.hrk.de/positionen/gesamtliste-beschluesse/position/conven tion/empfehlung-des-bmbf-der-kmk-und-der-hrk-an-die-hochschulen-zurvergabe-von-leistungspunkten-in-der/ [abgerufen am 16.10.2012].

Knill, Christoph, Eva Maria Vögtle und Michael Dobbins (2013). Hochschulpolitische Reformen im Zuge des Bologna-Prozesses: Eine vergleichende Analyse von Konvergenzdynamiken im OECD-Raum. Springer.

Koch, Richard (1998). Duale und schulische Berufsausbildung zwischen Bildungsnachfrage und Qualifikationsbedarf. Ein deutsch-französischer Vergleich. Berichte zur beruflichen Bildung, Heft 217. Bielefeld: Bundesinstitut für Berufsbildung. Der Generalsekretär.

Kohl, Matthias, Sylvia Kestner und Regina Buhr (2013). Übergänge und Durchlässigkeit in der Aufstiegsfortbildung - Steuerungspotenzial der Zulassungs- und Anrechnungsvorschriften in bundeseinheitlich geregelten Fortbildungsordnungen.bwp@Berufs- und Wirtschaftspädagogik - online. 25: 1-22, [abgerufen am 20.01.2016].

Konersmann, Ralf (2007). Der Philosoph mit der Maske. Michel Foucaults L'ordre du discours. In: Michel Foucault (Hrsg.): Die Ordnung des Diskurses. Frankfurt am Main: Fischer, 51-94.

Konsortium Bildungsberichterstattung (2006). Bildung in Deutschland. Ein indikatorengestütəter Bericht mit einer Analyse zu Bildung und Migration. Bielefeld: W. Bertelsmann Verlag.

Koppetsch, Cornelia (1994). Bildungstraditionen in Deutschland und Frankreich 1800-1870. Beiträge zur Hochschulforschung. 2: 187-213.

Kozma, Tamas (2014). The Bologna Process in Central and Eastern Europe. A comparative view. In: Tamas Kozma, Magdolna Rébay, Andrea Óhidy und Éva Szolár (Hrsg.): The Bologna process in Central and Eastern Europe. Wiesbaden: Springer VS, 13-32. 
Kraus, Katrin (2001). Lebenslanges Lernen - Karriere einer Leitidee. Bielefeld: Bertelsmann.

Kraus, Katrin (2007). Die „Berufliche Ordnung“ im Spannungsfeld nationaler Tradition und europäischer Integration. Zeitschrift für Pädagogik. 53 (3): 382398.

Krücken, Georg (2003). Learning the „New, New Thing“: On the Role of Path Dependency in University Structures. Higher Education. 46 (3): 315-339.

Krücken, Georg (2007). Organizational Fields and Competitive Groups in Higher Education: Some Lessons from the Bachelor/Master Reform in Germany. Management Revue. 18 (2): 187-203.

Krüger, Heinz-Hermann und Werner Helsper, W. (2014). Elite und Exzellenz im Bildungssystem - Nationale und internationale Perspektiven. Einleitung. Zeitschrift für Erziehungswissenschaft, 17(3), 1-10.

Laurent, Daniel (1995). L'évolution de l'enseignement supérieur et l'adaptation de l'université aux exigences de son environnement économique et social. Rapport inédit au Ministre de l'Education Nationale de la France.

Lawrence, Thomas B., Bernhard Leca, und Tammar B. Zilber (2013). Institutional Work: Current Research, New Directions and Overlooked Issues. Organization Studies, 34(8), 1023-1033.

Lawrence, Thomas B. und Roy Suddaby (2006). Institutions and Institutional Work. In: Stewart R. Clegg, Cynthia Hardy, Thomas B. Lawrence und Walter R. Nord (Hrsg.): Handbook of Organization Studies. London: Sage, 215-254.

Lawrence, Thomas B., Roy Suddaby und Bernhard Leca (2009). Institutional Work: Actors and Agency in Institutional Studies of Organizations. Cambridge: Cambridge University Press.

Lederle, Sabine (2008). Die Ökonomisierung des Anderen: Eine neoinstitutionalistisch inspirierte Analyse des Diversity Management-Diskurses. Wiesbaden: VS Verlag für Sozialwissenschaften.

Lehmann, Burkhard (2012). Aus der Ferne Lehren und Lernen. Zu den Grundzügen eines außergewöhnlichen Bildungsformats. In: Angela Fogolin (Hrsg.): Bildungsberatung im Fernlernen. Beiträge aus Wissenschaft und Praxis. Bielefeld: Bertelsmann, 19-41.

Leibfried, Stephan (2010). Die Exzellenzinitiative Zwischenbilanz, und Perspektiven. Frankfurt am Main, New York: Campus.

Leipold, Helmut (1996). Zur Pfadabhängigkeit der institutionellen Entwicklung Erklärungsansätze des Wandels von Ordnung. In: Dieter Cassel (Hrsg.): Entstehung und Wettbewerb von Systemen. Berlin: Duncker und Humblot, S: 93115.

Lemaire, Sylvie (2004). Que deviennent les bacheliers après leur baccalauréat? Note d'information, Ministère de l'Éducation nationale, de l'Enseignement supérieur et de la Recherche. 
Lemke, Thomas (2008). Gouvernementalität und Biopolitik. Wiesbaden: VS Verlag für Sozialwissenschaften.

Lenhardt, Gero (2002). Die verspätete Entwicklung der deutschen Schule. Pädagogische Korresponden₹: (29): 5-22.

Lenhardt, Gero (2003). Begabung - Qualifikation - Schulpraxis. Die verspätete Entwicklung der deutschen Schule. In: Grete Anzengruber und Editha Reiterer (Hrsg.): Begabungsglaube. Analyse, Kritik, Schulrealität. Wien: Verein der Förderer der Schulhefte. 36-61.

Leuven Communiqué (2009). Leuven/Louvain-la-Neuve Communiqué: The Bologna Process 2020 - The European Higher Education Area in the new decade Communiqué of the Conference of European Ministers Responsible for Higher Education, Leuven and Louvain-la-Neuve, 28-29 April 2009.

Liebeskind, Uta (2011). Universitäre Lehre. Deutungsmuster von ProfessorInnen im deutsch-französischen Vergleich. Konstanz: UVK.

Loebe, Herbert (Hrsg.) (2012). Kompetenzorientierung und Leistungspunkte in der Berufsbildung. Bielefeld: wbv.

London Communiqué (2007). London Communiqué - Towards the European Higher Education Area: responding to challenges in a globalised world, 18 May 2007. http://www.ehea.info/Uploads/Declarations/London_Communique18May 2007.pdf, [abgerufen am 10.02.2014].

Lönz, Michael (2008). Der Zweite Bildungsweg als Teil eines reversiblen Systems des lebenslangen Lernens? In: Rudolf Egger, Regina Mikula, Sol Haring, Andrea Felbinger und Angela Pilch-Ortega (Hrsg.): Orte des Lernens. Wiesbaden: VS Verlag für Sozialwissenschaften. 127-135.

Loroff, Claudia, Ida Stamm-Riemer und Ernst A. Hartmann (2011). Anrechnung: Modellentwicklung, Generalisierung und Kontextbedingungen. In: Walburga K. Freitag, Ernst A. Hartmann, Claudia Loroff, Ida Stamm-Riemer, Daniel Völk und Regina Buhr (Hrsg.): Gestaltungsfeld Anrechnung - Hochschulische und berufliche Bildung im Wandel. Münster: Waxmann, 77-117.

Lörz, Markus, Heiko Quast und Jan Roloff (2015). Konsequenzen der BolognaReform : warum bestehen auch am Übergang vom Bachelor- ins Masterstudium soziale Ungleichheiten? Zeitschrift für Soziologie, 44 (2): 137-155.

Lutz, Burkart (1976). Bildungssystem und Beschäftigungsstruktur in Deutschland und Frankreich. In: Hans'Gerhard Mendius, Werner Sengenberger, Burkart Lutz, Norbert Altmann, Fritz Böhle, Inge Asendorf·Krings, Ingrid Drexel und Christoph Nuber: Betrieb, Arbeitsmarkt, Qualifikation I. Frankfurt am Main: aspekte, 83-153.

Maastricht Communiqué (2004). Maastricht Communique on the Future Priorities of Enhanced European Cooperation in Vocational Education and Training (VET). Review of the Copenhagen Declaration of 30 November 2002.

Maeße, Jens (2009). Konsensstrategien in der Hochschulpolitik. Wie der BolognaProzess Gefolgschaft rekrutiert. Die Hochschule. 18 (2), 18-32. 
Maeße, Jens (2010). Die vielen Stimmen des Bologna-Prozesses. Zur diskursiven Logik eines bildungspolitischen Programms. Bielefeld: transcript.

Maggetti, Martino und Fabrizio Gilardi (2014). How Policies Spread: A Meta-Analysis of Diffusion Mechanisms. Working Paper. University of Zurich.

Mahoney, James (2000). Path Dependence in Historical Sociology. Theory and Society. 29: 507-548.

Mahoney, James (2010). After KKV: The New Methodology of Qualitative Research. World Politics. 62 (01): 120-147.

Maillard, Dominique und Patrick Veneau (2008). Les licences professionnelles. Quelle acception de la professionalisation à l'université ? In: Cereq Relief (Hrsg.): Les chemins de la formation vers l'emploi 1ère biennale Formation-EmploiTravail, 63-66.

Maillard, Dominique, Patrick Veneau und Colette Grandgérard (2004). Les licences professionnelles : quelle acception de la "professionalisation » à l'université ? Relief. Cereq. http://www.cereq.fr/index.php/publications/Relief/Les-licencesprofessionnelles-quelle-acception-de-la-professionnalisation-a-l-universite, [abgerufen am 15.03.2014].

Mannheim, Karl (1969). Ideologie und Utopie. Frankfurt am Main: Schulte-Bulmke

March, James G. und Johan P. Olsen (1989). Rediscovering Institutions: the Organizational Basis of Politics. New York, London: The Free Press.

Martens, Kerstin und Klaus Dieter Wolf (2006). Paradoxien der Neuen Staatsräson. Die Internationalisierung der Bildungspolitik in der EU und der OECD. Zeitschrift für Internationale Beziehungen. 13 (2): 145-176.

Martin, Simon (2005). Der MEDEF. München: GRIN Verlag GmbH.

Maurice, Marc, François Sellier und Jean-Jaques Silvestre (1986). The Social Foundations of Industrial Power. A Comparison of France and Germany. Cambridge, Massachusetts, London: The MIT Press.

Mayer, Karl Ulrich (1990). Lebensverläufe und sozialer Wandel - Anmerkungen zu einem Forschungsprogramm. Kölner Zeitschrift für Soziologie und Sozialpsychologie. Sonderbeft 31. 7-21.

Mayer, Karl Ulrich (2004). Whose Lives? How History, Societies and Institutions Define and Shape Life Courses. Research in Human Development. 1 (3): 161-187.

Mayer, Karl Ulrich, Walter Müller und Reinhard Pollak (2007). Germany: Institutional Change and Inequalities of Access in Higher Education. In: Yossi Shavit, Richard Arum und Adam Gamoran (Hrsg.): Stratification in Higher Education. A Comparative Study. Stanford: Stanford University Press, 240-265.

Mayer, Karl Ulrich und Heike Solga (1994). Mobilität und Legitimität. Zum Vergleich der Chancenstrukturen in der alten DDR und der alten BRD oder: Haben Mobilitätschancen zu Stabilität und Zusammenbruch der DDR beigetragen? Kölner Zeitschrift für Soziologie und Sozialpsychologie. 46 (2): 193-208.

Mayer, Karl Ulrich und Heike Solga (Hrsg.) (2008). Skill Formation: Interdisciplinary and Cross-National Perspectives. New York: Cambridge University Press. 
Mayring, Philipp (2002). Einfübrung in die qualitative Sozialforschung. Eine Anleitung zи qualitativem Denken. Weinheim: Beltz.

Mayring, Philipp (2003). Qualitative Inhaltsanalyse: Grundlagen und Techniken. Weinheim und Basel: Beltz.

MDEP [Ministère délégué à l'enseignement professionnel] (2000a). Déclaration de M. Jean-Luc Mélenchon, ministre délégué à l'enseignement professionnel, sur l'approfondissement de la professionnalisation dans l'enseignement supérieur, notamment par la mise en oeuvre de la licence professionnelle, Paris le 15 juin 2000. http://discours.vie-publique.fr/notices/003002213.html, [abgerufen am 05.03.2014].

MDEP (2000b). Déclaration de M. Jean-Luc Mélénchon, ministre délégué à l'enseignement professionnel, sur l'enseignement professionnel, notamment les métiers, les formations professionnelles, les diplômes, le statut des éléves et des enseignants, Paris le 19 septembre 2000. http://discours.vie-publique.fr/notices/003002951.html, [abgerufen am 05.03.2014].

MDEP (2000c). Déclaration de M. Jean-Luc Mélenchon, ministre délégué à l'enseignement professionnel, sur la formation professionnelle initiale et continue et l'harmonisation des diplômes au sein de l'Union européenne, Biarritz le 4 décembre 2000. http://discours.vie-publique.fr/notices/013000145.html, [abgerufen am 05.03.2014].

MDEP (2000d). Déclaration de M. Jean-Luc Mélenchon, ministre délégué à l'enseignement professionnel, sur la rentrée de l'enseignement professionnel, notamment les moyens dégagés pour la mise en place des équipements et matériels informatiques et la situation des professeurs de l'enseignement professionnel, Paris le 4 septembre 2000. http://discours.vie-publique.fr/notices/003002316.html, [abgerufen am 05.03.2014].

MDEP (2000e). Déclaration de M. Jean-Luc Mélenchon, ministre délégué à l'enseignement professionnel, sur la validation des acquis professionnels, Nice le 30 novembre 2000. http://discours.vie-publique.fr/notices/013000130.html, [abgerufen am 05.03.2014].

MDEP (2000f). Déclaration de M. Jean-Luc Mélenchon, ministre délégué à l'enseignement professionnel, sur le bilan de son action, notamment les formations et la pédagogie, l'enseignement secondaire et le supérieur technologique et professionnel, Paris août 2000. http://discours.vie-publique.fr/notices/003002592.html, [abgerufen am 05.03.2014].

MDEP (2000g). Déclaration de M. Jean-Luc Mélenchon, ministre délégué à l'enseignement professionnel, sur les avancées de l'enseignement professionnel, le système éducatif et les acteurs de l'enseignement professionnel, Paris décembre 2000. http://discours.viepublique.fr/notices/013000183.html, [abgerufen am 05.03.2014].

MDEP (2001a). Déclaration de M. Jean-Luc Mélenchon, ministre délégué à l'enseignement professionnel, sur l'éducation et la formation tout au long de la vie et la validation des acquis 
professionnels, Paris le 30 novembre 2001;. http://discours.viepublique.fr/notices/023000276.html, [abgerufen am 05.03.2014].

MDEP (2001b). Déclaration de M. Jean-Luc Mélenchon, ministre délégué à l'enseignement professionnel, sur la réforme de l'enseignement professionnel, Aix-en-Provence les 2, 3 et 4 juin 2001. http://discours.vie-publique.fr/notices/013002401.html, [abgerufen am 05.03.2014].

MDEP (2001c). Lettre de M. Jean-Luc Mélenchon, ministre délégué à l'enseignement professionnel, sur l'évolution du collège notamment l'orientation scolaire, la formation professionnelle et l'accès à l'enseignement supérieur, Paris le 16 mars 2001. http://discours.vie-publique.fr/notices/013001171.html, [abgerufen am 05.03.2014].

MDEP (2002). Déclaration de M. Jean-Luc Mélenchon, ministre délégué à l'enseignement professionnel, sur la rénovation du CAP, le lycée des métiers et le diplôme européen, Paris el 12 février 2002. http://discours.vie-publique.fr/notices/023001905.html, [abgerufen am 05.03.2014].

MEDEF [Mouvement des entreprises de France] (2011). Avis du MEDEF sur le plan Nouvelle Licence. Avril 2011. http://cache.media.enseignementsuprecherche.gouv.fr/file/Plan_reussir_en_licence/67/0/MEDEF_176670.pdf, [abgerufen am 06.03.2014].

Méhaut, Philippe (2004). Decentralising the vocational training system: more than just a shift in scale.bwp@Berufs- und Wirtschaftspädagogik - online. 7. http://www.bwpat.de/7eu/mehaut_fr_bwpat7.shtml, [abgerufen am 20.01.2016].

MEN [Ministère de l'éducation nationale] (1985). Discours de M. Jean-Pierre Chevènement, ministre de l'éducation nationale, sur la promotion et la revalorisation de l'enseignement technique et professionnel, à l'Assemblée nationale le 8 octobre 1985. http://discours.vie-publique.fr/notices/853171300.html, [abgerufen am 05.03.2014].

MEN (1994). Déclaration de M. François Bayrou, ministre de l'éducation nationale, sur les propositions de réforme du système scolaire dans l'enseignement primaire et secondaire afin de lutter contre l'échec scolaire, Paris le 9 mai 1994. http://discours.viepublique.fr/notices/943163300.html, [abgerufen am 05.03.2014].

MEN (2000a). Diplome d'accès aux Ėtudes universitaires. Note d'information 00-17. ftp://trf.education.gouv.fr/pub/edutel/dpd/ni0017.pdf, [abgerufen am 15.03.2014].

MEN (2000b). Déclaration de M. Jack Lang, ministre de l'éducation nationale, sur les orientations pour l'enseignement supérieur, notamment le budget 2001, l'amélioration des conditions de vie des étudiants, la modernisation et le rayonnement du système d'enseignement supérieur, la recherche et la mobilité européenne, Paris le 11 octobre 2000. http://discours.vie-publique.fr/notices/003002984.html, [abgerufen am 05.03.2014]. 
MEN (2001a). Déclaration de M. Jack Lang, ministre de l'éducation nationale, sur l'amélioration des conditions matérielles de la rentrée universitaire, le renouveau des formations proposées par l'enseignement et l'ouverture des universités, Paris le 4 octobre 2001. http://discours.vie-publique.fr/notices/013003624.html, [abgerufen am 05.03.2014].

MEN (2001b). Déclaration de M. Jack Lang, ministre de l'éducation nationale, sur les orientations pédagogiques pour l'enseignement supérieur, notamment la mobilité étudiante en Europe et dans le monde, et l'adoption de "points capitalisables" dans l'organisation des études, Paris le 24 avril 2001. http://discours.viepublique.fr/notices/013001347.html, [abgerufen am 05.03.2014].

MEN (2007a). Conférence de presse de M. Xavier Darcos, ministre de l'éducation nationale, sur la rentrée scolaire et les grandes orientations de la politique de l'enseignement, à Paris le 29 août 2007. http://discours.vie-publique.fr/notices/073002528.html, [abgerufen am 05.03.2014].

MEN (2007d). Déclaration de M. Xavier Darcos, ministre de l'éducation nationale, sur les priorités mises en oeuvre pour valoriser l'enseignement professionnel, Châlons-enChampagne le 6 septembre 2007. http://discours.vie-publique.fr/notices/ 073002603.html, [abgerufen am 05.03.2014].

MEN (2008b). Déclaration de M. Xavier Darcos, ministre de l'éducation nationale, sur l'orientation scolaire et professionnelle dans l'espace européen, Lyon le 17 septembre 2008. http://discours.vie-publique.fr/notices/083002855.html, [abgerufen am 05.03.2014].

MEN (2008c). Déclaration de M. Xavier Darcos, ministre de l'éducation nationale, sur la réforme du lycée, les enseignements de la classe de seconde et l'orientation scolaire, Paris le 21 octobre 2008. http://discours.vie-publique.fr/notices/083003307.html, [abgerufen am 05.03.2014].

MEN (2008d). Déclaration de M. Xavier Darcos, ministre de l'éducation nationale, sur la rénovation de la voie générale et technologique et la réforme du lycée général technologique, Paris le 12 novembre 2008. http://discours.vie-publique.fr/notices/083004 019.html, [abgerufen am 05.03.2014].

MEN (2009). Conférence de presse de M. Xavier Darcos, ministre de l'éducation nationale, sur le calendrier du baccalauréat 2009 et l'enseignement professionnel, Paris le 10 juin 2009. http://discours.vie-publique.fr/notices/093001757.html, [abgerufen am 06.03.2014].

MEN (2014). Le baccalauréat technologique, http:/ / www.education.gouv.fr/cid147/ le-baccalaureat-technologique.html, [abgerufen am 20.02.2014].

MEN und MESR [Ministère de l'éducation nationale, Ministère chargé de la recherche et de l'enseignement supérieur] (1988). Conseil des ministres du 09 Mars 1988 Perspectives pour l'éducation nationale et l'enseignement supérieur. http://discours.vie-publique.fr/notices/886004788.html, [abgerufen am 05.03.2014]. 
MENJS [Ministère de l'éducation nationale, de la jeunesse et des sports] (1988a). Conseil des ministres du 09 Mars 1988 Perspectives pour l'éducation nationale et l'enseignement supérieur. http://discours.vie-publique.fr/ notices/883342500.html, [abgerufen am 05.03.2014].

MENJS (1988b). Déclaration de M. Lionel Jospin, ministre de l'éducation nationale de la jeunesse et des sports, sur les grandes lignes de la politique universitaire concernant l'évaluation, la rénovation des cycles supérieurs, le fonctionnement des universités et l'amélioration de la condition des étudiants et des personnels universitaires, Paris le 30 juin 1988. http://discours.vie-publique.fr/notices/883342500.html, [abgeru-fen am 05.03.2014].

MENJS (1988c). Déclarations de MM. Lionel Jospin, ministre de l'éducation nationale de la jeunesse et des sports, et Robert Chapuis, secrétaire d'Etat chargé de l'enseignement technique, sur l'enseignement technique supérieur et la politique contractuelle, Paris le 29 septembre 1988. http://discours.vie-publique.fr/notices/883371000.html, [abgerufen am 05.03.2014].

MENJS (1989b). Déclarations de M. Lionel Jospin, ministre de l'éducation nationale de la jeunesse et des sports, sur le projet de loi d'orientation sur l'éducation, à l'Assemblée nationale le 7 juin et au Sénat le 27 juin 1989. http://discours.viepublique.fr/notices/893212700.html, [abgerufen am 03.05.2014].

MENRT [Ministère de l'éducation nationale, de la recherche et de la technologie] (1999a). Conférence de presse de M. Claude Allègre, ministre de l'éducation nationale de la recherche et de la technologie, sur l'enseignement professionnel intégré, la réforme des diplômes et le statut des éléves et des enseignants, Paris le 24 juin 1999 (en annexe les formations professionnelles et les mesures sur l'enseignement professionnel). http://discours.viepublique.fr/notices/993001973.html, [abgerufen am 05.03.2014].

MENRT (1999c). Déclaration de M. Claude Allègre, ministre de l'éducation nationale, de la recherche et de la technologie, sur l'enseignement supérieur, notamment la collaboration entre établissements d'enseignement supérieur et organismes de recherche, les filiéres, les diplômes, la reconnaissance du concept de technologie et l'harmonisation européenne, Paris le 25 février 1999. http://discours.vie-publique.fr/notices/993002607.html, [abgerufen am 05.03.2014].

MENRT (1999d). Déclaration de M. Claude Allègre, ministre de l'éducation nationale, de la recherche et de la technologie, sur les réformes de l'enseignement professionnel pour le XXIème siècle, Lille le 29 septembre 1999. http://discours.vie-publique.fr/ notices/993002832.html, [abgerufen am 05.03.2014].

MENSR [Ministère de l'éducation nationale, de l'enseignement supérieur et de la recherche] (1996a). Déclaration de M. François Bayrou, ministre de l'éducation nationale de l'enseignement supérieur et de la recherche, sur les propositions gouvernementales pour la réforme de l'université, à la Sorbonne, Paris le 18 juin 1996. http://discours.vie-publique.fr/notices/963171600.html, [abgerufen am 05.03.2014]. 
MENSR (1997). Présentation à la presse du Rapport d'Étape de la réforme de l'Université, 4 février 1997, Janvier 1997. http://guilde.jeunes-chercheurs. org/Reflexions/Documents/Rapport-EG-01-97.html [abgerufen am 04.03.2014]

MENRT [Ministère de l'éducation nationale, de la recherche et de la technologie] (1999a). Conférence de presse de M. Claude Allègre, ministre de l'éducation nationale de la recherche et de la technologie, sur l'enseignement professionnel intégré, la réforme des diplômes et le statut des éléves et des enseignants, Paris le 24 juin 1999 (en annexe les formations professionnelles et les mesures sur l'enseignement professionnel). http://discours.vie-publique.fr/notices/9930

01973.html, [abgerufen am 05.03.2014].

MENRT (1999c). Déclaration de M. Claude Allègre, ministre de l'éducation nationale, de la recherche et de la technologie, sur l'enseignement supérieur, notamment la collaboration entre établissements d'enseignement supérieur et organismes de recherche, les filiéres, les diplômes, la reconnaissance du concept de technologie et l'barmonisation européenne, Paris le 25 février 1999. http://discours.vie-publique.fr/notices/993002607.html, [abgerufen am 05.03.2014].

MENRT (1999d). Déclaration de M. Claude Allègre, ministre de l'éducation nationale, de la recherche et de la technologie, sur les réformes de l'enseignement professionnel pour le XXIème siècle, Lille le 29 septembre 1999. http://discours.vie-publique.fr/ notices/993002832.html, [abgerufen am 05.03.2014].

MENSR [Ministère de l'éducation nationale, de l'enseignement supérieur et de la recherche] (2004a). Conférence de presse de M. Francois Fillon, ministre de l'éducation nationale, de l'enseignement supérieur et de la recherche, sur l'organisation du baccalauréat 2004, Paris le 3 juin 2004. http://discours.vie-publique.fr/notices/04300 1707.html, [abgerufen am 05.03.2014].

MENSR (2004b). Projet de loi d'orientation sur l'avenir de l'École. Dossier de presse 03/12/2004. http://www.education.gouv.fr/cid600/projet-de-loi-d-orienta tion-sur-l-avenir-de-l-ecole.html, [abgerufen am 2.8.2016]

MENSR (2006a). Conférence de presse de M. Gilles de Robien, ministre de l'éducation nationale, de l'enseignement supérieur et de la recherche, sur l'organisation des épreuves du baccalauréat 2006, Paris le 6 juin 2006. http://discours.vie-publique.fr/ notices/063002012.html, [abgerufen am 05.03.2014].

MENSR (2006b). Déclaration de M. Gilles de Robien, ministre de l'éducation nationale, de l'enseignement supérieur et de la recherche, sur „le portail étudiant" comme outil de l'action gouvernementale en faveur de l'insertion professionnelle des jeunes, Paris le 17 mai 2005. http://discours.vie-publique.fr/notices/063001772.html, [abgerufen am 05.03.2014].

Merle, Pierre (2000). Le concept de démocratisation de l'institution scolaire : une typologie et sa mise à l'épreuve. Population 55 (1): 15-50.

MESR [Ministère chargé de la recherche et de l'enseignement supérieur] (1988). Conférence de presse de $M$. Jacques Valade, ministre chargé de la recherche et de 
l'enseignement supérieur, présentant le rapport „Demain l'Universite" "sur les orientations fondamentales de l'enseignement supérieur, à Paris le 7 janvier 1988. http://dis cours.vie-publique.fr/notices/883059600.html, [abgerufen am 05.03.2014].

MESR (1993). Conseil des ministres du 16 Juin 1993 Les Orientations de la politique de la recherche et de l'enseignement supérieur. http://discours.vie-publique.fr/ notices/936003587.html, [abgerufen am 05.03.2014].

MESR (2007a). Déclaration de Mme Valérie Pécresse, ministre de l'enseignement supérieur et de la recherche, publiée sur le site internet du ministère le 16 novembre 2007 et intitulée „Aux étudiants, je veux dire..." http://discours.vie-publique.fr/notices/0730 03558.html, [abgerufen am 06.03.2014].

MESR (2007b). Déclaration de Mme Valérie Pécresse, ministre de l'enseignement supérieur et de la recherche, sur le projet de loi sur l'autonomie des universités, Paris le 11 juillet 2007. http://discours.vie-publique.fr/notices/073002231.html, [abgerufen am 06.03.2014].

MESR (2007c). Déclaration de Mme Valérie Pécresse, ministre de l'enseignement supérieur et de la recherche, sur les enjeux de la réforme de l'enseignement supérieur, à Paris le 31 mai 2007. http://discours.vie-publique.fr/notices/073001845.html, [abgerufen am 06.03.2014].

MESR (2007e). Plan pour la réussite en licence : 730 millions d'euros d'ici 2012. http://www.enseignementsup-recherche.gouv.fr/cid20651/plan-pour-lareussite-en-licence-730-millions-d-euros-d-ici-2012.html, [abgerufen am 05.03.2014].

MESR (2008a). 3000 bacheliers technologiques supplémentaires accueillis dans les IUT. Intervention de Valérie Pécresse. Ministre de l'Enseignement supérieur et de la Recherche. Assemblée générale des Directeurs d'IUT. http://www.enseigne mentsup-recherche.gouv.fr/cid22269/3000-bacheliers-technologiquessupplementaires-accueillis-dans-les-iut.html, [abgerufen am 06.03.2014].

MESR (2008c). Doubler le nombre d'inscrits au Diplôme d'Accès aux Etudes Universitaires. Discours de Valérie Pécresse. 31.01.2008. http://www.enseigne mentsup-recherche.gouv.fr/cid20885/doubler-le-nombre-d-inscrits-au-diplo me-d-acces-aux-etudes-universitaires.html, [abgerufen am 06.03.2014].

MESR (2008d). Rentrée 2008: l'Université change. Valérie Pécresse, ministre de l'Enseignement supérieur et de la Recherche a présenté les nouveautés de la rentrée universitaire à l'occasion de la conférence de presse tenue au ministère. 18.09.2008. http://www.enseignementsup-recherche.gouv.fr/cid22452/rentree-2008-1universite-change.html, [abgerufen am 06.03.2014].

MESR (2009a). Conférence de presse de Mme Valérie Pécresse, ministre de l'enseignement supérieur et de la recherche, sur la réussite des étudiants à l'université et la plateforme Admission Post Bac, Paris le 17 septembre 2009. http://discours.viepublique.fr/notices/093002566.html, [abgerufen am 06.03.2014].

MESR (2009b). Déclaration de Mme V alérie Pécresse, ministre de l'enseignement supérieur et de la recherche, sur l'autonomie et la recherche universitaire, l'orientation des étudiants et 
les formations universitaires, Paris le 15 juillet 2009. http://discours.viepublique.fr/notices/093002100.html, [abgerufen am 06.03.2014].

MESR (2010a). Déclaration de Mme Valérie Pécresse, ministre de l'enseignement supérieur et de la recherche, sur la rentrée universitaire, les projets pour l'enseignement supérieur et la politique d'aide aux étudiants, Paris le 13 septembre 2010. http://discours.viepublique.fr/notices/103001995.html.

MESR (2010b). La nouvelle Licence, un diplôme pour l'emploi. Discours de Valérie Pécresse, ministre de l'Enseignement supérieur et de la Recherche. 17.12.2010. http:// www.enseignementsup-recherche.gouv.fr/cid54280/la-nouvelle-licence-undiplome-pour-l-emploi.html, [abgerufen am 06.03.2014].

MESR (2010c). Les IUT, composantes essentielles des universités. Discours de Valérie Pécresse. 19.05.2010. http://www.enseignementsup-recherche.gouv.fr/cid517 03/les-iut-composantes-essentielles-des-universites.html, [abgerufen am 06.03.2014].

MESR (2011a). Déclaration de Mme Valérie Pécresse, ministre de l'enseignement supérieur et de la recherche, sur l'orientation active des lycéens, les contours de la nouvelle licence et l'autonomie des universités, Paris le 13 mai 2011. http://discours.viepublique.fr/notices/113001186.html, [abgerufen am 06.03.2014].

MESR (2011b). Déclaration de Mme Valérie Pécresse, ministre de l'enseignement supérieur et de la recherche, sur la rénovation des sections de techniciens supérieurs (STS), Paris le 1er mars 2011. http://discours.vie-publique.fr/notices/113000537.html, [abgerufen am 06.03.2014].

MESR (2011c). Déclaration de Mme Valérie Pécresse, ministre de l'enseignement supérieur et de la recherche, sur les objectifs de la réforme du diplôme de la licence pour la poursuite d'études dans l'enseignement supérieur, Paris le 8 février 2011. http:/ / discours.viepublique.fr/notices/113000356.html, [abgerufen am 06.03.2014].

MESR (2012a). Déclaration de Mme Geneviève Fioraso, ministre de l'enseignement supérieur et de la recherche, sur l'échec en licence universitaire, le décrochage des étudiants, l'orientation des étudiants et la revalorisation des bourse d'études, Paris le 225 septembre 2012. http://discours.vie-publique.fr/notices/123001739.html, [abgerufen am 06.03.2014].

MESR (2012c). Les bacheliers professionnels dans l'enseignement supérieur. Note d'information $n^{\circ} 12.04$, juin 2012.

MESR (2012d). Parcours dans l'enseignement supérieur : devenir après le baccalauréat des élèves entrés en Gème en 1995. Note d'information n¹2.05, juin 2012.

Meuser, Michael und Ulrike Nagel (2009). Experteninterview und der Wandel der Wissensproduktion. In: Alexander Bogner, Beate Littig und Wolfgang Menz (Hrsg.): Experteninterviews - Theorien, Methoden, Anwendungsfelder (3. grundlegend überarbeitete Auflage). Wiesbaden: VS Verlag für Sozialwissenschaften, 35-60.

Meyer, Alan D., Geoffrey R. Brooks und James B. Goes (1990). Environmental jolts and industry revolutions: Organizational responses to discontinuous change. Strategic Management Journal. 11 (5): 93-110. 
Meyer, Heinz-Dieter und Brian Rowan (Hrsg.) (2006). The New Institutionalism in Education. Albany: State University of New York.

Meyer, John W. (1977). The Effects of Education as an Institution. American Journal of Sociology. 83 (1): 55-77.

Meyer, John W. (1992). From Constructionism to Neo-Institutionalism: Reflections on Berger and Luckmann. Perspectives. The Theory Section Newsletter of the American Sociological Association. 15 (2): 11-12.

Meyer, John W. (2004). Word Society, the Welfare State and the Life Course: An Institutionalist Perspective. Social World Working Paper. Bielefeld: University of Bielefeld.

Meyer, John W.und Ronald L.Jepperson (2000). The 'Actors' of Modern Society: The Cultural Construction of Social Agency. Sociological Theory, 18 (1): 100-120.

Meyer, John W., David H. Kamens und Aaron Benavot (1992). School Knowledge for the Masses. Washington: Falmer Press.

Meyer, John W., Francisco O. Ramirez, Richard Rubinson und John Boli-Bennett (1977). The World Educational Revolution, 1950-1970, Sociology of Education. 50 (October 1977): 242-58.

Meyer, John W. und Francisco O. Ramirez (2007). The World Institutionalization of Education. In: Jürgen Schriewer (Hrsg.): Weltkulturen und kulturelle Bedeutungswelten. Frankfurt: Campus, 279-297.

Meyer, John W. und Brian Rowan (1977). Institutionalized Organizations: Formal Structure as Myth and Ceremony. The American Journal of Sociology. 83 (2): 340363.

Meyer, John W. und Brian Rowan (1992). The Structure of Educational Institutions. In: John W. Meyer und W. Richard Scott (Hrsg.): Organizational Environments. Ritual and Rationality. Newbury Park, CA: Sage, 71-97.

Mignot-Gérard, S. und Christine Musselin (2001). L'offre de formation universitaire : à la recherche de nouvelles régulations. Education et sociétés. 8: 11-25.

Mill, John Stuart (1872). A System of Logic, Ratiocinative and Inductive. 8. Auflage. London: Longmans, Green, Reader, and Dyer.

MJENR [Ministère de la jeunesse, de l'éducation nationale et de la recherche] (2002b). Conférence de presse de MM. Luc Ferry, ministre de la jeunesse, de l'éducation nationale et de la recherche et Xavier Darcos, ministre délégué à l'enseignement scolaire, sur la politique de l'enseignement, notamment les chantiers prioritaires de la rentrée scolaire, la lutte contre l'échec scolaire et l'acquisition des savoirs fondamentaux, Paris le 2 septembre 2002. http://discours.vie-publique.fr/notices/023002796.html, [abgerufen am 05.03.2014].

MJENR (2002e). Déclaration de M. Luc Ferry, ministre de la jeunesse, de l'éducation nationale et de la recherche, sur la réussite de l'entrée dans l'enseignement supérieur, son adaptation à l'Europe, l'ouverture de perspectives pour la recherche universitaire et le développement de l'autonomie des universités, Paris le 7 octobre 2002. 
http://discours.vie-publique.fr/notices/023003693.html, [abgerufen am 05.03.2014].

Moebus, Martine und Eric Verdier (1998). Les diplômes professionnels en Allemagne et en France : conception et jeux d'acteurs. Paris: Editions L'Harmattan.

Moodie, Gavin F. (2008). From Vocational to Higher Education: An International Perspective. Ballmor: Open University Press.

Mucke, Kerstin und Franziska Kupfer (2011). Durchlässigkeit umsetzen für lebensbegleitendes Lernen - Schlussfolgerungen aus der Sicht der beruflichen Bildung. In: Walburga K. Freitag, Ernst A. Hartmann, Claudia Loroff, Ida Stamm-Riemer, Daniel Völk und Regina Buhr (Hrsg.): Gestaltungsfeld Anrechnung. Hochschulische und berufliche Bildung im Wandel. Münster: Waxmann, 221-238.

Müller, Walter, Reinhard Pollak, David Reimer und Steffen Schindler (2009). Hochschulbildung und soziale Ungleichheit. In: Rolf Becker und Andreas Hadjar (Hrsg.): Lebrbuch der Bildungssoziologie. Wiesbaden: VS Verlag für Sozialwissenschaften, 281-320.

Musselin, Christine (2001). La longue marche des universités françaises. Paris: P.U.F.

Musselin, Christine (2005). Le marché des universitaires : France, Allemagne, États-Unis. Les Presses de Sciences Po.

Musselin, Christine (2007). France. In: James J.F. Forest und Philip G. Altbach (Hrsg.): International Handbook of Higher Education. Dordrecht: Springer, 711728.

Musselin, Christine (2008). Ten Years After the Sorbonne Declaration - What has Changed in European Study Structures? In: Barbara M. Kehm (Hrsg.): Hochschule im Wandel. Die Universität als Forschungsgegenstand. Festschrift für Ulrich Teicbler. Frankfurt am Main: Campus, 309-318.

Musselin, Christine und Catherine Paradeise (2009). France: From incremental transitions to institutional change. In: Catherine Paradeise, Emanuela Reale, Ivar Bleiklie und Ewan Ferlie (Hrsg.): University Governance. Dordrecht: Springer, 21-49.

Nee, Victor (2001). Sources of the New Institutionalism. In: Mary C. Brinton und Victor Nee (Hrsg.): The New Institutionalism in Sociology. Stanford: Stanford University Press, 1-16.

Nickel, Sigrun und Sindy Duong (2012). Studieren obne Abitur: Monitoring der Entwicklungen in Bund, Ländern und Hochschulen. CHE-Arbeitspapier Nr. 157. Gütersloh.

Nickel, Sigrun und Britta Leusing (2009). Studieren obne Abitur: Entwicklungspotenziale in Bund und Ländern. Eine empirische Analyse. CHEArbeitspapier Nr. 123. Gütersloh: CHE.

North, Douglass C. (1990). Institutions, Institutional Change and Economic Performance. Cambridge: Cambridge University Press. 
North, Douglass C. (1994). Institutional Change: A Framework of Analysis. Economics Working Paper. Economic History Series, Nr 9412001. Washington.

North, Douglass C. (1995). Five Propositions about Institutional Change. In: Jack Knight und Itai Sened (Hrsg.): Explaining Social Institutions. Ann Arbor: University of Michigan Press, 15-26.

Onisep (2010). En 1re générale et technologique: une orientation réversible, http://www.onisep.fr/Espace-pedagogique/LEGT/Orientation/Le-kit-duprofesseur-principal-de-LEGT/En-1re-generale-et-technologique-une-orien tation-reversible, [abgerufen am 25.02.2014].

Onisep (2013). Master : le point sur l'inscription, l'organisation et la validation, http://www.onisep.fr/Choisir-mes-etudes/Apres-le-bac/Filieres-d-etudes/ Reussir-en-master/Master-le-point-sur-l-inscription-l-organisation-et-la-vali dation, [abgerufen am 25.02.2014].

Onisep (2014). Changer de voie, http://www.onisep.fr/voie-pro/Lyceenetudiant-apprenti/Changer-de-voie, [abgerufen am 25.02.2014].

Parreira do Amaral, Marcelo (2011). Emergenz eines Internationalen Bildungsregimes? International Educational Governance und Regimetheorie. Münster: Waxmann.

Parsons, Talcott (1959). Abdruck aus: Sozialstruktur und Persönlichkeit. Die Schulklasse als soziales System: Einige ihrer Funktionen in der amerikanischen Gesellschaft. Harvard Educational Review. 29 (4): 8.297-318.

Petrini, Francesco (2004). Die gemeinsame Politik der Berufsausbildung in der EWG von 1961 bis 1972. Europäische Zeitschrift für Berufsbildung. 32: 50-60.

Pfahl, Lisa (2011). Techniken der Behinderung. Der deutsche Lernbehinderungsdiskurs, die Sonderschule und ihre Auswirkungen auf Bildungsbiografien. Bielefeld: transcript.

Pfahl, Lisa und Justin J.W. Powell (2010). Draußen vor der Tür: Die Arbeitsmarktsituation von Menschen mit Behinderung. Aus Politik und Zeitgescbichte. 32 (23).

Pfahl, Lisa und Justin J.W. Powell (2011). Legitimating school segregation. The special education profession and the discourse of learning disability in Germany. Disability \& society 26 (4): 449-462.

Philips, Nelson, Thomas B. Lawrence und Cynthia Hardy (2004). Discourse and institutions. Academy of Management Review. 29 (4): 635-652.

Phillips, David und Hubert Ertl (Hrsg.) (2003). Implementing European Union and Training Policy. A Comparative Study of Issues in Four Member States. Dordrecht: Kluwer.

Pinto, Vanessa (2008). «Démocratisation» et «professionnalisation» de l'enseignement supérieur. mowvements. 3 (55/56): 12-23.

Pollak, Reinhard (2010). Kaum Bewegung, viel Ungleichheit. Heinrich Böll Stiftung: Eine Studie zu sozialem Auf- und Abstieg in Deutschland Berlin: agit-druck.

Powell, Justin J.W. (2011). Barriers to Inclusion: Special Education in the United States and Germany. Boulder, CO: Paradigm Publishers. 
Powell, Justin J.W. und Heike Solga (2010). Analyzing the Nexus of Higher Education and Vocational Training in Europe: A Comparative-Institutional Framework. Studies in Higher Education. 35 (6): 705-721.

Powell, Justin J.W., Nadine Bernhard und Lukas Graf (2012a). Amerikanisierung oder Europäisierung der (Aus-)Bildung? Die Bologna- und KopenhagenProzesse und das neue europäische Modell der Hochschul- und Berufsbildung. In: Rolf Becker und Heike Solga (Hrsg.): Soziologische Bildungsforschung. Kölner Zeitschrift für Soziologie und Sozialpsychologie. Sonderheft 52. Wiesbaden: Springer, 437-458.

Powell, Justin J.W., Nadine Bernhard und Lukas Graf (2012b). The Emerging European Model in Skill Formation: Comparing Higher Education and Vocational Training in the Bologna and Copenhagen Processes. Sociology of Education. 85 (3): 240-258.

Powell, Justin J.W., Lukas Graf, Johann Fortwengel und Nadine Bernhard (2014).

Duale Studiengänge im globalen Kontext: Internationalisierung in Deutschland und Transfer nach Brasilien, Frankreich, Katar, Mexiko und in die USA. Berlin: DAAD. https://www.daad.de/medien/der-daad/medien-publikationen/ publikationen-pdfs/dokmat-band-77.pdf [abgerufen am 02.08.2016]

Powell, Justin J.W. (2004). Barriers to Inclusion. The Institutionalization of Special Education in Germany and the United States. Dissertation eingereicht am Fachbereich Politik- und Sozialwissenschaften. Berlin: Freie Universität Berlin.

Powell, Justin J.W. und Christine Trampusch (2012). Europeanization and the Varying Responses in Collective Skill Systems. In: Marius R. Busemeyer und Christine Trampusch (Hrsg.): The Comparative Political Economy of Collective Skill Systems. Oxford: Oxford University Press, 284-316.

Powell, Justin J.W., Laurence Coutrot, Lukas Graf, Nadine Bernhard, Annick Kieffer und Heike Solga (2009). Comparing the Relationship between Vocational and Higher Education in Germany and France. WZB Discussion Paper SP I 2009-506. Berlin: Wissenschaftszentrum Berlin für Sozialforschung.

Powell, Justin J.W. und Claudia Finger (2013). The Bologna Process's Model of Mobility in Europe: The Relationship of its Spatial and Social Dimensions. European Educational Research Journal. 12 (2): 270-285.

Powell, Justin J.W., Lukas Graf, Nadine Bernhard, Laurence Coutrot und Annick Kieffer (2012c). The Shifting Relationship between Vocational and Higher Education in France and Germany: Towards Convergence? European Journal of Education. 47 (3): 405-423.

Powell, Justin J.W. und Heike Solga (2008). Internationalization of Vocational and Higher Education Systems - A Comparative-Institutional Approach. WZB Discussion Paper SP I 2008-501. Berlin: Wissenschaftszentrum Berlin für Sozialforschung. 
Powell, Justin J.W. und Heike Solga (2011). Why are Participation Rates in Higher Education in Germany so Low? Institutional Barriers to Higher Education Expansion. Journal of Education and Work. 24 (1-2): 49-68.

Powell, Walter W. (1991). Expanding the Scope of Institutional Analysis. In: Walter W. Powell und Paul J. DiMaggio (Hrsg.): The New Institutionalism in Organizational Analysis. Chicago: University of Chicago Press, 183-203.

Powell, Walter W. und Paul J. DiMaggio (Hrsg.) (1991). The New Institutionalism in Organizational Analysis. Chicago: University of Chicago Press.

Prague Communiqué (2001). Towards the European Higher Education Area. Communique of the meeting of European Ministers in charge of Higher Education in Prague on May 19th 2001. http://www.ehea.info/Uploads/Declarations/ PRAGUE_COMMUNIQUE.pdf, [abgerufen am 10.02.2014].

Premier ministre [Premier ministre] (1995). Déclaration de M. Alain Juppé, Premier ministre, sur les pistes de réflexion de la commission Fauroux sur l'éducation, Paris le 11 septembre 1995. http://discours.vie-publique.fr/notices/953237600.html, [abgerufen am 05.03.2014].

Président (1987). Allocution de M. François Mitterrand, Président de la République, sur la crise économique et l'importance de la formation professionnelle, à la mairie de Lisieux, mardi 23 juin 1987. http://discours.vie-publique.fr/notices/ 877014700.html, [abgerufen am 05.03.2014].

Président (1989). Allocution de M. François Mitterrand, Président de la République, sur la priorité que constitue la formation professionnelle dans la politique gouvernementale et son importance pour l'avenir de la France, Paris, Cité des Sciences de la Villette, le 12 septembre 1989. http://discours.vie-publique.fr/notices/897019200. html, [abgerufen am 05.03.2014].

Président (1990). Allocution de M. François Mitterrand, Président de la République, notamment sur la nécessité d'accroitre le nombre des étudiants diplômés de qualité pour rester dans la compétition internationale, sur la construction de locaux et la mission des universitaires, La Sorbonne, le 29 juin 1990. http://discours.viepublique.fr/notices/907016000.html, [abgerufen am 05.03.2014].

Prost, Antoine (1983). Les lycees et leurs études au senil du XXle siecle. Rapport au Ministère de l'Education nationale. Paris.

Prost, Antoine (2000). The creation of the Baccalaureat Professionnel: a case study of decision making in French education. Journal of Education Policy. 15 (1): 19-31.

Protsch, Paula (2011). Zugang ₹u Ausbildung: Eine historisch vergleichende Perspektive auf den segmentierten Ausbildungsmarkt in (West-)Deutschland. WZB Discussion Paper SP I 2011-502. Berlin: Wissenschaftszentrum Berlin für Sozialforschung. Protsch, Paula (2014). Segmentierte Ausbildungsmärkte. Opladen: Budrich UniPress. Przeworski, Adam und Henry Teune (1970). The Logic of Comparative Social Inquiry. New York: Wiley. 
Quenzel, Gudrun (2005). Konstruktionen von Europa. Die europäische Identität und die Kulturpolitik der Europäischen Union. Bielefeld: transcript.

Radaelli, Claudio M. (2000). Policy Transfer in the European Union: Institutional Isomorphism as a Source of Legitimacy. Governance: An International Journal of Policy and Administration. 13 (1): 25-43.

Radaelli, Claudio M. (2003). The Europeanisation of Public Policy. In: Kevin Featherstone und Claudio M. Radaelli (Hrsg.): The Politics of Europeanisation. Oxford: Oxford University Press, 27-56.

Radaelli, Claudio M. und Vivien A. Schmidt (Hrsg.) (2005). Policy Change and Discourse in Europe. London: Routledge.

Raffe, David (2003). Bringing Academic Education and Vocational Training Closer Together. In: Jürgen Oelkers (Hrsg.): Futures of Education II: Essays from an Interdisciplinary Symposium. Frankfurt: Peter Lang, 49-65.

Raffe, David, Cathy Howieson, Ken Spours und Michael Young (1998). The Unification of Post-Compulsory Education: Towards a Conceptual Framework. British Journal of Educational Studies. 46 (2): 169-187.

Rakic, Vojin (2001). To converge or not converge: the European Union and higher education policies in the Netherlands, Belgium/Flanders and Germany. Higher Education Policy 14: 225-240.

Ramirez, Francisco O. und John Boli-Bennett (1982). Global patterns of educational institutionalization. In: Altbach, Philip G., Robert F. Arnove und Gail P. Kelly (Hrsg) Comparative Education. New York: Macmill, 15-38.

Rauhvargers, Andrejs, Cynthia Deane und Wilfried Pauwels (2009). Bologna Process Stocktaking Report 2009. Bologna Follow-up Group (28-29 April 2009). Leuven/Louvain-la-Neuve, Ministerial Conference: Education and Culture DG.

Rauner, Felix (2012). Akademisierung beruflicher und Verberuflichung akademischer Bildung - widersprüchliche Trends im Wandel nationaler Bildungssystemebwp@Berufs- und Wirtschaftspädagogik - online. 23: 1-19. http://www. bwpat.de/ausgabe23/rauner_bwpat23.pdf, [abgerufen am 20.01.2016].

Rauner, Felix, Philipp Grollmann und Georg Spöttl (2006). Den Kopenhagen-Prozess vom Kopf auf die Füßse stellen: Das Kopenbagen-Lissabon-Dilemma. Bremen: Institut Technik und Bildung.

Ravinet, Pauline (2008). From Voluntary Participation to Monitored Coordination: Why European countries feel increasingly bound by their commitment to the Bologna Process. European Journal of Education. 43 (3): 353-367.

Ravinet, Pauline (2009). Comment le processus de Bologne a-t-il commencé ? La formulation de la vision de l'espace Euopéen d'Enseignement Supérieur en 1998. Éducation et Sociétés. 24 (2): 29-44.

Rehberg, Karl-Siegbert (1995). Die „Öffentlichkeit“ der Institutionen. Grundbegriffliche Überlegungen im Rahmen der Theorie und Analyse 
institutioneller Mechanismen. In: Gerhard Göhler (Hrsg.): Macht der Öffentlicbkeit - Öffentlicbkeit der Macht. Baden Baden: Nomos, 181-211.

Reimer, David und Reinhard Pollak (2010). Educational Expansion and Its Consequences for Vertical and Horizontal Inequalities in Access to Higher Education in West Germany. European Sociological Review. 26 (4): 415-430.

Reinalda, Bob und Ewa Kulesza (2006). The Bologna Process - Harmonizing Europe's Higher Education (2. revised edition). Opladen: Barbara Budrich.

Reitnauer, Jochen (2004). Erfahrungslernen als gleichwertige Komponente im Bildungssystem. Das französische System der qualifizierenden Anerkennung beruflich und außerberuflich erworbener Kompetenzen. In: Georg Rothe (Hrsg.): Alternanz - die EU-Konzeption für die Berufsausbildung: Erfabrungslernen Hand in Hand mit Abschnitten systematischer Ausbildung, dargestellt unter Einberiehung von Ergebnissen aus Ländervergleichen. Karlsruhe: Universitätsverlag Karlsruhe, 132-136.

Rixen, Thomas und Lora A. Viola (2014). Putting path dependence in its place: toward a Taxonomy of institutional change. Journal of Theoretical Politics. 26(3): 1-23.

Rogers, Everett M. (1995). Diffusion of Innovation. New York: The Free Press.

Rothe, Daniela (2011). Lebenslanges Lernen als Programm. Frankfurt am Main: Campus.

Rothe, Daniela (2013). Die Bildung Erwachsener oder „Lebenslanges Lernen“. Rekonstruktion der Debatten und Politiken. Vierteljahrsschrift für wissenschaftliche Pädagogik. 89 (1): 7-19.

Rott, Gerhart und Wolfram Wickel (1995). Student counselling in Germany: An overview. International Journal for the Advancement of Counselling. 18 (4): 245-262.

Roy, Simon (2005). France. In: Manuel Souto Otero, Andrew McCoshan and Kerstin Junge (Hrsg.): A European inventory on validation of non-formal and informal learning. A Final Report to DG Education \& Culture of the European Commission. Birmingham: ECOTEC.

Ruß, Uwe (2012). Bildung, Meritokratie und Ungleichbeit: Gibt es einen Zusammenhang zwischen Bildungsungleichbeiten, Meritokratieglauben und der Verteilung der Einkommen in fortgeschrittenen Gesellschaften? WZB Discussion Paper SP I 2012-501. Berlin: Wissenschaftszentrum Berlin für Sozialforschung.

Sabel, C.F. und J. Zeitlin (2007). Learning from Difference: The New Architecture of Experimentalist Governance in the EU. European Governance Papers.

Scharpf, Fritz W. (2000). Notes Toward a Theory of Multilevel Governing in Europe. MPIfG Discussion Paper (Vol. 00/5), Köln: Max Planck Institut für Gesellschaftsforschung.

Scherrer, Christoph (2007). GATS - Commodifying Education via Trade Treaties. In: Kathrin Leuze, Kerstin Martens und Alessandra Rusconi (Hrsg.): New Arenas of Education Governance - The Impacts of International Organizations and Markets on Education Policy Making. Basingstoke: Palgrave, 117-135. 
Scherrer, Christoph (2001). Jenseits von Pfadabhängigkeit und "natürlicher Auslese": Institutionentransfer aus diskursanalytischer Perspektive. WZB Discussion Paper FS II 01-205. Berlin: Wissenschaftszentrum Berlin für Sozialforschung.

Schindler, Steffen (2012). Aufstiegsangst? Eine Studie zur sozialen Ungleichbeit beim Hochschulzugang im historischen Zeitverlauf. Mit einem Kommentar von Walter Müller. Düsseldorf: Vodafone Stiftung Deutschland gGmbH.

Schmidt, Vivien A. (2002). The Futures of European Capitalism. Oxford: Oxford University Press.

Schmidt, Vivien A. (2008). Discursive Institutionalism: The Explanatory Power of Ideas and Discourse. Annual Review of Political Science. 11: 303-326.

Schmidt, Vivien A. (2010). Taking Ideas and Discourse Seriously: Explaining Change Through Discursive Institutionalism as the 'Fourth Institutionalism'. European Political Science Review. 2 (1): 1-25.

Schmidt, Vivian A. (2014). Speaking to the Markets or to the People? A Discursive Institutionalist Analysis of the EU's Sovereign Debt Crisis. The British Journal of Politics \& International Relations, 16(1), 188-209.

Schmidt, Vivien A. und Claudio M. Radaelli (2004). Policy change and discourse in Europe: Conceptual and methodological issues. West European Politics. 27 (2): 183-210.

Schreiber, Daniel (2010). Ist die Externenprüfung eine Form der Anerkennung informellen Lernens? Berufsbildung. 64 (125): 18-19.

Schreiber, Daniel, Katrin Gutschow, Robin Weber-Höller und Julia Gei (2012). Anerkennung beruflicher Kompetenzen am Beispiel der Zulassung zur Abschlussprüfung im Rabmen der Externenregelung. Abschlussbericht 2012. Bonn: BIBB.

Schreier, Margrit (2014). Varianten qualitativer Inhaltsanalyse: Ein Wegweiser im Dickicht der Begrifflichkeiten. Forum Qualitative Sozialforschung 15 (1), http://nbn-resolving.de/urn:nbn:de:0114-fqs1401185 [abgerufen am 02.08.2016]

Schriewer, Jürgen (1982). Alternativen in Europa: Frankreich. Lehrlingsausbildung unter dem Anspruch von Theorie und Systematik. In: Herwig Blankertz, Josef Derbolav, Adolf Kell und Günter Kutscha (Hrsg.): Enayklopädie Erriebungswissenschaft, Band 9, Teil 1: Jugendbildung zwischen Schule und Beruf. Stuttgart: Klett, 250-285.

Schriewer, Jürgen (1986). Intermediäre Instanzen, Selbstverwaltung und berufliche Ausbildungsstrukturen im historischen Vergleich. Zeitscbrift für Pädagogik. 32 (1): 69-90.

Schriewer, Jürgen (1987). Vergleich als Methode und Externalisierung auf Welt: VomUmgang mit Alterität in Reflexionsdisziplinen. In: Baecker, Dirk, Jürgen Markowitz, Rudolf Stichweh, Hartmann Tyrell und Helmut Willke(Hrsg.): Theorie als Passion. Frankfurt am Main: Suhrkamp, 629-668.

Schriewer, Jürgen (Hrsg.) (2007). Weltkultur und kulturelle Bedeutungswelten: Zur Globalisierung von Bildungsdiskursen. Frankfurt am Main, New York: Campus. 
Schwabe-Ruck, Elisabeth (2010). „Zweite Chance“ des Hochschulzugangs. Düsseldorf: Hans-Böckler-Stiftung.

Schwap-Trapp, Michael (2001). Diskurs als soziologisches Konzept. Bausteine für eine soziologisch orientierte Diskursanalyse. In: Reiner Keller, Andreas Hirseland, Werner Schneider und Willy Viehöver (Hrsg.): Handbuch Sozialwissenschaftliche Diskursanalyse. Band 1: Theorien und Methoden. Opladen: Leske + Budrich, 263-286.

Scott, Richard W. (1981). Organizations: Rational, Natural, and Open Systems. Upper Saddle River, NJ: Prentice Hall.

Scott, Richard W. (2001). Institutions and Organizations. Thousand Oaks: Sage Publications.

Scott, Richard W. (2008). Institutions and Organizations. (3. Auflage). Thousand Oaks: Sage Publications.

Searle, John (1995). The Construction of Social Reality. New York: Free Press.

Senge, Konstanze und Kai-Uwe Hellmann (Hrsg.) (2006). Einfübrung in den Neoinstitutionalismus. Wiesbaden: VS Verlag für Sozialwissenschaften.

Serrano-Velarde, Kathia (2009). Mythos Bologna? 10 Jahre Forschung zum Bolognaprozess. Soziologie. 38 (2): 193-203.

Severing, Eckart (2005). Europäisierung der Berufsbildung: Wirkungen auf die duale Ausbildung. In: Manfred Eckert und Manfred Zöller (Hrsg.): Der europäische Berufsbildungsraum: Beiträge der Berufsbildungsforschung. 6. Forum der Arbeitsgemeinschaft Berufsbildungsforschungsnetz (AG BFN) vom 19.-20. September 2005 an der Universität Erfurt, 79-97.

Shavit, Yossi, Richard Arum und Adam Gamoran (2007). More Inclusion than Diversion: Expansion, Differentiation, and Market Structure in Higher Education. In: Yossi Shavit, Richard Arum und Adam Gamoran (Hrsg.): Stratification in Higher Education: A Comparative Study. Stanford: Stanford University Press, 1-35.

Shavit, Yossi und Walter Müller (1998). From School to Work. A Comparative Study of Educational Qualifications and Occupational Destinations. Oxford: Oxford University Press.

Shavit, Yossi und Walter Müller (2000a). Vocational Secondary Education Where Diversion and where Safety Net? European Societies. 2 (1): 29-50.

Shavit, Yossi und Walter Müller (2000b). Vocational Secondary Education, Tracking, and Social Stratification. In: Maureen T. Hallinan (Hrsg.): The Handbook of the Sociology of Education. New York: Kluwer, 437-452.

Simon, Dagmar (2010). L'impact de l'Exzellenzinitiative en Allemagne. Revue internationale d'éducation Sèvres. 54: 127-136.

Snow, David A. und Danny Trom (2002). The Case Study and the Study of Social Movements. In: Bert Klandermans und Suzanne Staggenborg (Hrsg.): Methods of Social Movement Research. Minneapolis/London: University of Minnesota Press, 146-172. 
Solga, Heike (1995). Auf dem Weg in eine kelassenlose Gesellschaft? Klassenlagen und Mobilität zwischen Generationen in der DDR. Berlin: Akademie Verlag.

Solga, Heike (2002). „Ausbildungslosigkeit" als soziales Stigma in Bildungsgesellschaften. Ein soziologischer Erklärungsbeitrag für die wachsenden Arbeitsmarktprobleme von gering qualifizierten Personen. Kölner Zeitschrift für Soziologie und Sozialpsychologie. 54 (3): 476-505.

Solga, Heike (2005a). Meritokratie - die moderne Legitimation ungleicher Bildungschancen. In: Peter A. Berger und Heike Kahler (Hrsg.): Institutionalisierte Ungleichbeiten. Wie das Bildungswesen Chancen blockiert. Weinheim/München: Juventa Verlag, 19-38.

Solga, Heike (2005b). Ohne Abschluss in die Bildungsgesellschaft: Die Erwerbschancen gering qualifizierter Personen aus soziologischer und ökonomischer Perspektive. Opladen: Barbara Budrich.

Solga, Heike (2009). Der Blick nach vorn: Herausforderungen an das deutsche Ausbildungssystem. Thesenpapier zum Symposium des WZB am 19.11.2009. WZB Discussion Paper SP I 2008-507. Berlin: Wissenschaftszentrum Berlin für Sozialforschung.

Solga, Heike (2012). Bildung und materielle Ungleichheiten. Der investive Sozialstaat auf dem Prüfstand. In: Rolf Becker und Heike Solga (Hrsg.): Soziologische Bildungsforschung. Kölner Zeitschrift für Soziologie und Sozialpsychologie, Sonderbeft 52. Wiesbaden: Springer, 459-487.

Solga, Heike und Rolf Becker (2012). Soziologische Bildungsforschung - Eine kritische Bestandsaufnahme. In: Rolf Becker und Heike Solga (Hrsg.): Soziologische Bildungsforschung. Kölner Zeitschrift für Soziologie und Sozialpsychologie, Sonderheft 52. Wiesbaden: Springer, 7-43.

Solga, Heike, Peter A. Berger und Justin Powell (2009). Soziale Ungleichheit Kein Schnee von gestern! Eine Einführung. In: Heike Solga, Justin Powell und Peter A. Berger (Hrsg.): Soziale Ungleichbeit - Klassische Texte zur Sozialstrukturanalyse. Frankfurt: Campus, 11-45.

Solga, Heike, Christian Brzinsky-Fay, Lukas Graf, Cornelia Gresch und Paula Protsch (Hrsg.) (2013). Vergleiche innerhalb von Gruppen und institutionelle Gelingensbedingungen. Vielversprechende Perspektiven für die Ungleichbeitsforschung: WZB Discussion Paper SP I 2013-501. Berlin: Wissenschaftszentrum Berlin für Sozialforschung.

Solga, Heike und Rosine Dombrowski (2009). Soziale Ungleichheiten in schulischer und außerschulischer Bildung: Stand der Forschung und Forschungsbedarf. Arbeitspapier der Hans-Böckler-Stiftung Nr. 171. Düsseldorf.

Solga, Heike und Sandra Wagner (2001). Paradoxie der Bildungsexpansion. Die doppelte Benachteiligung von Hauptschülern. Zeitschrift für Erziehungswissenschaft. 4 (1): 107-127.

Sorbonne Declaration (1998). Sorbonne Joint Declaration: Joint declaration on harmonisation of the architecture of the European higher education system by the four 
Ministers in charge for France, Germany, Italy and the United Kingdom. Paris, the Sorbonne, May 25 1998. http://www.ehea.info/Uploads/Declarations/ SORBONNE_DECLARATION1.pdf, [abgerufen am 10.02.2013].

Sozialdemokratische Partei Deutschlands und Bündnis 90/Die GRÜNEN (1998). Aufbruch und Erneuerung - Deutschlands Weg ins 21. Jahrbundert. Koalitionsvereinbarung zwischen der Sozialdemokratischen Partei Deutschlands und Bündnis 90/Die GRÜNEN, Bonn, 20. Oktober 1998. http://www.trend. infopartisan.net/trd1098/vertrag.html, [abgerufen am 26.03.2014].

Spermann, Alexander (2013). Die Demografiestrategie der Bundesregierung ein Weg zu mehr Wohlstand? Wirtschaftsdienst, 93(3), 165-169.

Spilker, Niels (2012). Lebenslanges Lernen als Dispositiv-Bildung, Macht und Staat in der neoliberalen Gesellschaft. Münster: Westfälisches Dampfboot.

Stamm-Riemer, Ida und Ernst A. Hartmann (2011). Entwicklungen und Trends im ANKOM-Kontext zu Anrechnung und Durchlässigkeit zwischen beruflicher und hochschulischer Bildung in Deutschland und Europa. In: Walburga K. Freitag, Ernst A. Hartmann, Claudia Loroff, Ida Stamm-Riemer, Daniel Völk und Regina Buhr (Hrsg.): Gestaltungsfeld Anrechnung. Hochschulische und berufliche Bildung im Wandel. Münster: Waxmann, 57-76.

Statistisches Bundesamt (2012). Bildung und Kultur. Berufliche Schulen. Fachserie 11 Reibe 2.

Statistisches Bundesamt (2013a). Bildung und Kultur. Allgemeinbildende Schulen. Fachserie 11 Reibe 1.

Statistisches Bundesamt (2013b). Bildung und Kultur. Berufliche Bildung. Fachserie 11 Reibe 3.

Statistisches Bundesamt (2013c). Bildung und Kultur. Studierende an Hochschulen. Fachserie 11 Reibe 4.1.

Stegemann, Anna (2008). Informelles Lernen. Identifizierung, Bewertung und Anerkennung informell erworbener Kompetenzen. Hamburg: Diplomica Verlag $\mathrm{GmbH}$.

Steiner-Khamsi, Gita (2012). Understanding Policy Borrowing and Lending. Building Comparative Policy Studies. In Gita Steiner-Khamsi und Florian Waldow (Hrsg.): Policy borrowing and lending in education. London: Routledge, 317.

Steinke, Ines (1999). Kriterien qualitativer Forschung. Ansätze zur Bewvertung qualitativempirischer Sozialforschung. Weinheim/München: Juventa Verlag.

Stinchcombe, Arthur L. (1997). On the Virtues of the Old Institutionalism. Annual Review of Sociology. 23: 1-18.

Strauss, Anselm und Juliet Corbin (1996). Grounded Theory: Grundlagen Qualitativer Sozialforschung [Original: Basics of Qualitative Research: Grounded Theory Procedures and Technique (1990)]. Weinheim: Beltz, Psychologie Verlags Union. 
Streeck, Wolfgang (2012). Skills and Politics: General and Specific. In: Marius R. Busemeyer und Christine Trampusch (Hrsg.): The Political Economy of Collective Skill Formation. Oxford: Oxford University Press, 317-352.

Streeck, Wolfgang und Kathleen Thelen (Hrsg.) (2005). Beyond Continuity. Institutional Change in Advanced Political Economies. Oxford: Oxford University Press.

Strübing, Jörg (2004). Grounded Theory. Zur sozialtheoretischen und epistemologischen Fundierung des Verfahrens der empirisch begründeten Theoriebildung. Wiesbaden: VS Verlag für Sozialwissenschaften.

Suchman, Mark C. (1995). Managing Legitimacy: Strategic and Institutional Approaches. The Academy of Management Review. 20 (3): 571-610.

Tanguy, Lucie (1988). L'enseignement technique et professionnel, repères dans l'histoire. Formation-Emploi. (27-28): 5-15.

Tanguy, Lucie (2000). Note de synthèse: Histoire et sociologie de l'enseignement technique et professionnel en France: un siècle en perspective. Revue française de pédagogie. 131 (1): 97-127.

Tanguy, Lucie (2005). De l'éducation à la formation: quelles réformes ? Education et sociétés. (2): 99-122.

Teichler, Ulrich (1990a). Europä̈sche Hochschulsysteme: Die Beharrlichkeit vielfältiger Modelle. Frankfurt am Main, New York: Campus.

Teichler, Ulrich (1990b). Hochschulzugang für Berufserfahrene - Erfahrungen und Zukunftsperspektiven. In: Norbert Kluge, Wolf-Dieter Scholz und Andrä Wolter (Hrsg.): Vom Lehrling zum Akademiker. Neue Wege des Hochschulzugangs für berufserfahrene Erwachsene. Oldenburg: BIS, Bibliotheks- und Informationssystem der Universität Oldenburg, 23-48.

Teichler, Ulrich (2002). Hochschulbildung. In: Rudolf Tippelt (Hrsg.): Handbuch Bildungsforschung. Opladen: Leske + Budrich, 349-370.

Teichler, Ulrich (2005). Hochschulstrukturen im Umbruch - eine Bilanz der Reformdynamik seit vier Jahrzebnten. Frankfurt am Main, New York: Campus.

Teichler, Ulrich und Andrä Wolter (2004). Zugangswege und Studienangebote für nicht-traditionelle Studierende. die hochschule. 2/2004: 64-80.

Theisens, Henno C. und Jürgen Enders (2007). State Models, Policy Networks, and Higher Education Policy. Policy Change and Stability in Dutch and English Higher Education (87). In: Georg Krücken, Anna Kosmützky und Marc Torka (Hrsg.): Towards a Multiversity? Universities between Global Trends and National Traditions. Bielefeld: transcript, 87-107.

Thelen, Kathleen (2004). How Institutions Evolve: The Political Economy of Skills in Germany, Britain, the United States, and Japan. Cambridge: Cambridge University Press.

Thielen, Michael (2008). Eine Agenda für Gleichwertigkeit und Durchlässigkeit. In: Regina Buhr, Walburga K. Freitag, Ernst A. Hartmann, Claudia Loroff, Karl Heinz Minks, Kerstin Mucke und Ida Stamm-Riemer (Hrsg.): 
Durchlässigkeit gestalten! Wege zwischen beruflicher und hochschulischer Bildung. Münster: Waxmann, 9-11.

Thomas, George M., John W. Meyer, Francisco Ramirez und John Boli (1987). Institutional Structure. Constituting State, Society and the Individual. California: Sage. Tippets, Jared N. (2011). Changing the Way We do Things Around Here: Strategies Presidents Use for Creating Organizational Change in Higher Education. Dissertation, http://uknowledge.uky.edu/cgi/viewcontent.cgi? article $=1163 \&$ context $=$ gradschool_diss, [abgerufen am 15.03.2014].

Tilly, Charles (1998). Durable inequality. Berkeley, CA: University of California Press

Toens, Katrin (2007). Die Sorbonne-Deklaration - Hintergründe und Bedeutung für den Bologna-Prozess. Die Hochschule. 2: 37-53.

Trampusch, Christine (2008). Jenseits von Anpassungsdruck und Lernen - Die EU-Berufsbildungspolitik und die Europäisierung der deutschen Berufsbildungspolitik. Zeitschrift für Staats- und Europawissenschaften. 6 (4): 577-605.

Trampusch, Christine (2009). Europeanization and Institutional Change in Vocational Education and Training in Germany and Austria. Governance: An International Journal of Policy. 22 (3): 371-397.

Traue, Boris, Lisa Pfahl und Lena Schürmann (2014). Diskursanalyse. In Nina Baur und Jörg Blasius (Hrsg.): Handbuch Methoden der empirischen Sozialforschung. Wiesbaden: VS Verlag, 493-508.

Trenz, Hans-Jörg (2008). Elements of a sociology of European integration. Arena Working Paper. Oslo: ARENA Centre for European Studies.

Triby, Emmanuel (2005). Validierung von Erfahrungen - Umwandlungen und Implikationen. Europäische Zeitschrift für Berufsbildung. 35: 49-62.

Troger, Vincent (2004). Vocational training in French schools: the fragile stateemployer alliance. European Centre for the Development of Vocational Training: Towards a history of vocational education and training (VET) in Europe in a comparative perspective: proceedings of the first international conference October 2002, Florence. Volume 1: the rise of national VET systems in a comparative perspective. Luxembourg: Office for Official Publications of the European Communities. Troger, Vincent (2009). Du latin à la terminale S, l'héritage élitiste des lycées. La Documentation Française. Regards sur l'actualité. 353: 8-14.

Truschkat, Inga (2008). Kompeten₹diskurs und Bewerbungsgespräche: Eine Dispositivanalyse (neuer) Rationalitäten sozialer Differenzierung. Wiesbaden: VS Verlag für Sozialwissenschaften.

Truschkat, Inga; Kaiser, Manuela \& Reinartz, Vera (2005). Forschen nach Rezept? Anregungen zum praktischen Umgang mit der Grounded Theory in Qualifikationsarbeiten [48 Absätze]. Forum Qualitative Sozialforschung / Forum: Qualitative Social Research, 6(2), Art. 22, http://nbn-resolving.de/ urn:nbn:de:0114-fqs0502221 [abgerufen am 20.1.2014]. 
Ulbricht, Lena (2012a). Öffnen die Länder ihre Hochschulen? Annahmen über den Dritten Bildungsweg auf dem Prüfstand. Die Hochschule. 21 (1): 154-168.

Ulbricht, Lena (2012b). Stille Explosion der Studienberechtigungszahlen - die neuen Regelungen für das Studium ohne Abitur. Berufsbildung in Wissenschaft und Praxis. 1/2012: 39-42.

Ulbricht, Lena (2014). Der Hochschulzugang für beruflich Qualifizierte obne Abitur. Politische Entscheidungsprozesse in den deutschen Ländern. Dissertation eingereicht an der Kultur-, Sozial- und Bildungswissenschaftlichen Fakultät der Humboldt-Universität zu Berlin am 08. Juli 2014.

Van Zanten, Agnès-Henriot und Éric Plaisance (1993). Les transformations du système éducatif. Acteurs et politiques. Paris: Editions L'Harmattan.

Vincens, Jean (2008). Réforme de l'Université ou réforme de l'enseignement supérieur. In: Jean Vincens (Hrsg.): Évolution de l'enseignement supérieur: persistance des paradoxes. Toulouse: LIRHE, 189-229.

Vinokur, Annie (2008). Vous avez dit « autonomie» ? Monvements. 3 (55-56): $72-$ 81.

Vögtle, Eva M. (2013). Transnationale Kommunikation als Bedingungsfaktor für hochschulpolitische Konvergenz. In: Karin Amos, Josef Schmid, Josef Schrader und Ansgar Thiel (Hrsg.): Europäischer Bildungsraum. Europäisierungsprozesse in Bildungspolitik und Bildungspraxis. Baden-Baden: Nomos, 93-122.

Vögtle, Eva M., Christoph Knill und Michael Dobbins (2011). To what extent does transnational communication drive cross-national policy convergence? The impact of the bologna-process on domestic higher education policies. Higher Education. 61 (1): 77-94.

Völk, Daniel (2011). Anrechnung aus der Perspektive von Hochschullehrenden. In: Walburga K. Freitag, Ernst A. Hartmann, Claudia Loroff, Ida StammRiemer, Daniel Völk und Regina Buhr (Hrsg.): Gestaltungsfeld Anrechnung. Hochschulische und berufliche Bildung im Wandel. Münster: Waxmann, 177-190.

Waldow, Florian (2007). Ökonomische Strukturyyklen und internationale Diskurskonjunkturen. Zur Entwicklung der schwedischen Bildungsprogrammatik. 19302000. Frankfurt am Main: Lang.

Waldow, Florian (2009). Undeclared imports: silent borrowing in educational policy-making and research in Sweden. Comparative Education. 45 (4): 477-494. Waldow, Florian (2014a). Conceptions of Justice in the Examination Systems of England, Germany, and Sweden: A Look at Safeguards of Fair Procedure and Possibilities of Appeal. Comparative Education Review, 58(2), 322-343.

Waldow, Florian (2014b). Von der Meritokratie zur Parentokratie? Zeitschrift für Erziehungswissenschaft, 17(3), 43-58.

Walgenbach, Peter und Renate E. Meyer (2008). Neoinstitutionalistische Organisationstheorie. Stuttgart: Kohlhammer.

Walkenhorst, Heiko (2008). Explaining Change in EU Education Policy. Journal of European Public Policy. 15 (4): 567-587. 
Walter, Thomas (2006). Der Bologna-Prozess. Ein Wendepunkt europäischer Hocbschulpolitik. Wiesbaden: VS Verlag für Sozialwissenschaften.

Wanken, Simone, Alrun Schlieff und Maren Kreutz (2010). Durchlässigkeit von beruflicher und hochschulischer (Weiter-)Bildung - Die Paradoxie von Anspruch und Wirklichkeit aus steuerungstheoretischer Perspektive. bwp@ Berufs- und Wirtschaftspädagogik - online. 19: 1-18. http://www.bwpat.de/ ausgabe19/wanken_etal_bwpat19.pdf, [abgerufen am 20.01.2016].Weber, Max (1922 [1980]). Wirtschaft und Gesellschaft: Grundriß der verstehenden Soziologie. Tübingen: Mohr.

Weber, Max (1972). Wirtschaft und Gesellschaft. Tübingen: Mohr Siebeck.

Wendt, Alexander (1987). The agent-structure problem in international relations theory. International Organizations. 41 (3): 335-370.

Wirp, Fabian (2004). Das Berufsbildungssystem in Frankreich. Deutscher Industrieund Handelskammertag. http://www.rhein-neckar.ihk24.de/linkableblob/ maihk24/aus_und_weiterbildung/downloads/454162/.3./data/Berufsbildu ngssystem-data.pdf, [abgerufen am 25.02.2014].

Wissenschaftsrat (2004). Empfehlungen zur Reform des Hochschulzugangs. Drs. 5920/04. Berlin.

Witte, Johanna (2006). Change of Degrees and Degrees of Change - Comparing Adaptations of European Higher Education Systems in the Context of the Bologna Process. Enschede: Center for Higher Education Policy Studies (CHEPS), University of Twente.

Witte, Johanna, Marijk Van der Wende und Jereon Huisman (2008). Blurring boundaries: how the Bologna-process changes the relationship between university and non-university higher education in Germany, the Netherlands and France. Studies in Higher Education. 33 (3): 217-231.

Wolbers, Maarten H.J. (2007). Patterns of Labour Market Entry: A Comparative Perspective on School-to-Work Transitions in 11 European Countries. Acta Sociologica. 50 (3): 189-210.

Wolter, Andrä (1989). Von der Elitenbildung zur Bildungsexpansion. Zweibundert Jahre Abitur (1788-1988). Oldenburg.

Wolter, Andrä (1994). Hochschulzugang im Umbruch. Oldenburger Universitätsreden. Oldenburg: Universität Oldenburg.

Wolter, Andrä (2010a). Durchlässigkeit zwischen beruflicher Bildung und Hochschule - Vom Besonderheitenmythos zur beruflichen Kompetenz. In: Klaus W. Birkelbach, Axel Bolder, Karl Düsseldorff und Rolf Dobischat (Hrsg.): Berufliche Bildung in Zeiten des Wandels: Festschrift für Rolf Dobischat zum 60. Geburtstag. Baltmannsweiler: Schneider Verlag Hohengehren, 199-219.

Wolter, Andrä (2010b). Studium und Berufstätigkeit. Historische Entwicklungen, aktuelle Bedarfe und zukünftige Potentiale. HIS-Konferenz „Berufsbegleitend studieren in Deutschland“. Berlin: Max-Planck-Gesellschaft. http://bas. 
dzhw.eu/index_html/fileadmin/pdf/Studium_und_Berufstaetigkeit_Wolter. pdf, [abgerufen am 18.08.2015].

Wolter, Andrä (2011). Hochschulzugang und soziale Ungleichheit in Deutschland. In: Heinrich Böll Stiftung: Öffnung der Hochschule. Chancengerechtigkeit, Diversität, Integration. Berlin: Heinrich Böll Stiftung, 9-15.

Wolter, Andrä (2012). From Individual Talent to Institutional Permeability: Changing Policies for Non-Traditional Access Routes in German Higher Education. In: Maria Slowey und Hans G. Schuetze (Hrsg.): Global Perspectives on Higher Education and Lifelong Learners. London/New York: Routledge, 43-59.

Wolter, Andrä, Ulf Banscherus; Caroline Kamm, Alexander Otto und Anna Spexard (2014): Durchlässigkeit zwischen beruflicher und akademischer Bildung als mehrstufiges Konzept: Bilanz und Perspektiven, Beiträge zur Hochschulforschung, 36 (4), S. 8-39.

Wolter, Andrä und Erika Reibstein (1991). Studierfähig durch Beruf und Weiterbildung? Eine empirische Fallstudie anhand der Bildungs- und Berufsbiographien von Erwachsenen. In: Andrä Wolter (Hrsg.): Die Öffnung des Hochschulzugangs für Berufstätige. Oldenburg: Bibliotheks- und Informationssystem der Universität Oldenburg, 35-97.

Wolter, Andrä und Wolf-Dieter Scholz (1986). Kann Berufstätigkeit zur Studierfähigkeit führen? Ein empirischer Vergleich zur Äquivalenz von höherer Schulbildung und Berufsqualifikation im Hochschulstudium. Zeitschrift für Berufs-und Wirtschaftspädagogik. 82: 323-340.

Wrana, Daniel (2006). Das Subjekt schreiben. Reflexive Praktiken und Subjektivierung in der Weiterbildung. Eine Diskursanalyse. Baltmannsweiler: Schneider Verlag Hohengehren.

Young, Michael und David Raffe (1998). The Four Strategies for Promoting Parity of Esteem. In: Johanna Lasonen und Michael Young (Hrsg.): Strategies for Achieving Parity of Esteem in European Upper Secondary Education. Jyväskylä: Institute for Educational Research, 35-46.

Young, Michael, Ken Spours, Cathy Howieson und David Raffe (1997). Unifying academic and vocational learning and the idea of a learning society. Journal of Education Policy. 12 (6): 527-537.

Zgaga, Pavel (2004). Report to the Minister of Education of the signatory countries commissioned by the Follow-up Group of the Bologna Process. In: $\mathrm{BMBF}$ und KMK (Hrsg.): Realising the European Higher Education Area: Conference of European Ministers Responsible for Higher Education, 18-19 September 2003, Documentation. Bielefeld: Bertelsmann, 90-128.

Zgaga, Pavel (2003). Bologna Process between Prague and Berlin. Berlin: Bologna Process Follow-up Working Group.

Zucker, Lynne G. (1983). Organizations and Institutions. Research in the sociology of organizations. 2: 1-47. 
Zymek, Bernd (2014). Ausleseverfahren und Institutionen der nationalen Elitebildung und ihre internationalen Herausforderungen. Zeitscbrift für Eriziehungswissenschaft, 17(3), 59-79. 


\section{Anhang}

\section{Tabellen und Abbildungen}

Tabelle 37 Institutionelle Charakteristika der Berufs- und Hochschulbildung in Deutschland und Frankreich

\begin{tabular}{|c|c|c|c|c|}
\hline \multirow{2}{*}{$\begin{array}{l}\text { Institutionelle } \\
\text { Merkmale }\end{array}$} & \multicolumn{2}{|c|}{ Frankreich } & \multicolumn{2}{|c|}{ Deutschland } \\
\hline & Berufsbildung & Hochschulbildung & Berufsbildung & Hochschulbildung \\
\hline \multicolumn{5}{|l|}{ Kulturell-kognitiv } \\
\hline $\begin{array}{l}\text { Dominante } \\
\text { Zielperspektive, } \\
\text { Ideale }\end{array}$ & $\begin{array}{l}\text { Theoretische tech- } \\
\text { nische und breite } \\
\text { berufsfeldorientierte } \\
\text { Kompetenz }\end{array}$ & $\begin{array}{l}\text { Klassische Elitebil- } \\
\text { dung, gebildete Per- } \\
\text { sönlichkeit }\end{array}$ & $\begin{array}{l}\text { Berufliche Hand- } \\
\text { lungsfähigkeit, Be- } \\
\text { rufsprinzip }\end{array}$ & $\begin{array}{l}\text { Klassisch Elitebil- } \\
\text { dung, gebildete Per- } \\
\text { sönlichkeit, Verbin- } \\
\text { dung Lehre und For- } \\
\text { schung }\end{array}$ \\
\hline \multicolumn{5}{|l|}{ Normativ } \\
\hline Lernorganisation & $\begin{array}{l}\text { Theorie- und } \\
\text { technikorientiert, } \\
\text { weniger Praxis }\end{array}$ & $\begin{array}{l}\text { Theorieorientiert an } \\
\text { Hochschulen }\end{array}$ & $\begin{array}{l}\text { Praxisintegriert } \\
\text { (Verbindung von } \\
\text { Arbeit und Lernen) }\end{array}$ & $\begin{array}{l}\text { Theorieorientiert an } \\
\text { Hochschulen }\end{array}$ \\
\hline $\begin{array}{l}\text { Repräsentative } \\
\text { Organisations- } \\
\text { form }\end{array}$ & $\begin{array}{l}\text { Berufsschulen } \\
\text { (auch alternance) }\end{array}$ & $\begin{array}{l}\text { Grandes ecoles, } \\
\text { differenziertes } \\
\text { Hochschulwesen }\end{array}$ & $\begin{array}{l}\text { Dualität von Betrieb } \\
\text { und Schule }\end{array}$ & $\begin{array}{l}\text { Forschungs- } \\
\text { universitäten }\end{array}$ \\
\hline Zielgruppe & $\begin{array}{l}\text { Schüler/innen mit } \\
\text { Problemen }\end{array}$ & Klassisch: Elite & Alle & Klassisch: Elite \\
\hline \multicolumn{5}{|l|}{ Regulativ } \\
\hline Governance & $\begin{array}{l}\text { Zentralstaatlich (mit } \\
\text { korporatistischen } \\
\text { Anteilen) }\end{array}$ & Zentralstaatlich & Korporatistisch & $\begin{array}{l}\text { Vor allem föderal } \\
\text { (auf Länderebene) }\end{array}$ \\
\hline Finanzierung & $\begin{array}{l}\text { Primär öffentlich, } \\
\text { anteilig durch } \\
\text { Betriebe }\end{array}$ & Öffentlich & $\begin{array}{l}\text { Primär durch Be- } \\
\text { triebe (aber Berufs- } \\
\text { schulen öffentlich) }\end{array}$ & Öffentlich \\
\hline $\begin{array}{l}\text { Status der } \\
\text { Lernenden }\end{array}$ & Schüler_in & Student_in & $\begin{array}{l}\text { Angestellte Auszu- } \\
\text { bildende }\end{array}$ & Student_in \\
\hline $\begin{array}{l}\text { Durchlässigkeits- } \\
\text { regularien }\end{array}$ & \multicolumn{2}{|c|}{$\begin{array}{l}\text { Berechtigung zur Hochschulbildung durch } \\
\text { Berufsabitur (Sekundarniveau), } \\
\text { institutionalisierte Anrechnungsverfahren }\end{array}$} & \multicolumn{2}{|c|}{$\begin{array}{l}\text { Keine Berechtigung zur Hochschulbildung } \\
\text { via Berufsbildung (auf Sekundarniveau) } \\
\text { Anrechnung neuerdings möglich, aber } \\
\text { keine institutionalisierten Anrechnungs- } \\
\text { verfahren* }\end{array}$} \\
\hline
\end{tabular}

Sowohl die Einführung von Berechtigungen über berufliche (Weiter-)Bildung (2009) und von Anrechnungsmöglichkeiten (2002) stellen die neuesten Entwicklungen für den deutschen Fall dar, so dass die Differenz zwischen Frankreich und Deutschland diesbezüglich als noch größer anzusehen ist.

Quelle: Baethge (2006: appendix); Powell et al. (2009: 10, 31) angepasst und entlang den institutionellen Dimensionen organisiert von NB sowie Team INVEST 
Tabelle 38 Darstellung des europäischen Kernkonzeptes von Durchlässigkeit

\begin{tabular}{|c|c|c|}
\hline & Bologna-Prozess & Kopenhagen-Prozess \\
\hline \multicolumn{3}{|c|}{ Kulturell-Kognitive Dimension } \\
\hline \multirow{2}{*}{$\begin{array}{l}\text { Behandelte Aspekte } \\
\text { von Durchlässigkeit }\end{array}$} & \multicolumn{2}{|c|}{$\begin{array}{c}\text { Zugang (zur Hochschulbildung) } \\
\text { Anrechnung von vorgängigem Lernen } \\
\text { Umgang mit heterogenen Bedürfnissen der Lernenden }\end{array}$} \\
\hline & & $\begin{array}{l}\text { Organisationale } \\
\text { Verbindungen }\end{array}$ \\
\hline \multirow[b]{2}{*}{$\begin{array}{l}\text { Kontext bzw. Ziel } \\
\text { (wofür Durchlässig- } \\
\text { keit) }\end{array}$} & \multicolumn{2}{|c|}{$\begin{array}{l}\text { Lebenslanges Lernen ermöglichen } \\
\text { Schaffung sozialer Inklusion }\end{array}$} \\
\hline & & $\begin{array}{l}\text { Erhöhung der Attraktivität und } \\
\text { Wertschätzung der Berufsbil- } \\
\text { dung, (Gleichwertigkeit zwi- } \\
\text { schen BB und HB) }\end{array}$ \\
\hline \multirow{3}{*}{$\begin{array}{l}\text { Legitimation } \\
\text { (warum Durchlässig- } \\
\text { keit notwendig) }\end{array}$} & \multicolumn{2}{|c|}{$\begin{array}{c}\text { Demographischer Wandel } \\
\text { Soziale Ungleichheit }\end{array}$} \\
\hline & Fehlende Mobilität (in HB) & $\begin{array}{l}\text { Wandel der Arbeitsmarktstruk- } \\
\text { tur (Fachkräftebedarf) }\end{array}$ \\
\hline & Mangelnde Chancengleichheit & \\
\hline \multicolumn{3}{|l|}{ Normative Dimension } \\
\hline \multirow[t]{2}{*}{ Standards } & \multicolumn{2}{|c|}{$\begin{array}{c}\text { Lernergebnisorientierung } \\
\text { Leistungspunktesystem } \\
\text { Qualifikationsrahmen } \\
\text { Verfahren zur Anerkennung von vorgängigem Lernen } \\
\text { Qualitätssicherungssysteme } \\
\text { Institutionalisierte Beratungsangebote } \\
\text { Modularisierung }\end{array}$} \\
\hline & $\begin{array}{l}\text { Studienzyklenstruktur } \\
\text { Unterstützungssysteme } \\
\text { Flexibilisierungsmöglichkeiten }\end{array}$ & $\begin{array}{l}\text { Vermittlung von Schlüsselkom- } \\
\text { petenzen in BB } \\
\text { beruflicher Bildung an den } \\
\text { Hochschulen }\end{array}$ \\
\hline \multirow[b]{2}{*}{ Zielgruppe } & \multicolumn{2}{|c|}{ Beruflich Qualifizierte } \\
\hline & $\begin{array}{l}\text { bisher unterrepräsentierte Grup- } \\
\text { pen } \\
\text { leistungsfähige Individuen }\end{array}$ & $\begin{array}{l}\text { + Studienabbrecher } \\
\text { (ins BB-System) } \\
\text { Kein Leistungskriterium } \\
\text { genannt }\end{array}$ \\
\hline \multicolumn{3}{|l|}{ Regulative Dimension } \\
\hline \multirow[b]{2}{*}{ Finanzen } & \multicolumn{2}{|c|}{ Öffentliche Gelder } \\
\hline & & $\begin{array}{l}\text { + private und europäische } \\
\text { Gelder }\end{array}$ \\
\hline \multirow{2}{*}{ Akteure } & Ministerien/Verwa & gen, Sozialpartner \\
\hline & + Hochschulorganisationen & + Kammern \\
\hline
\end{tabular}

Quelle: Darstellung der Verfasserin 


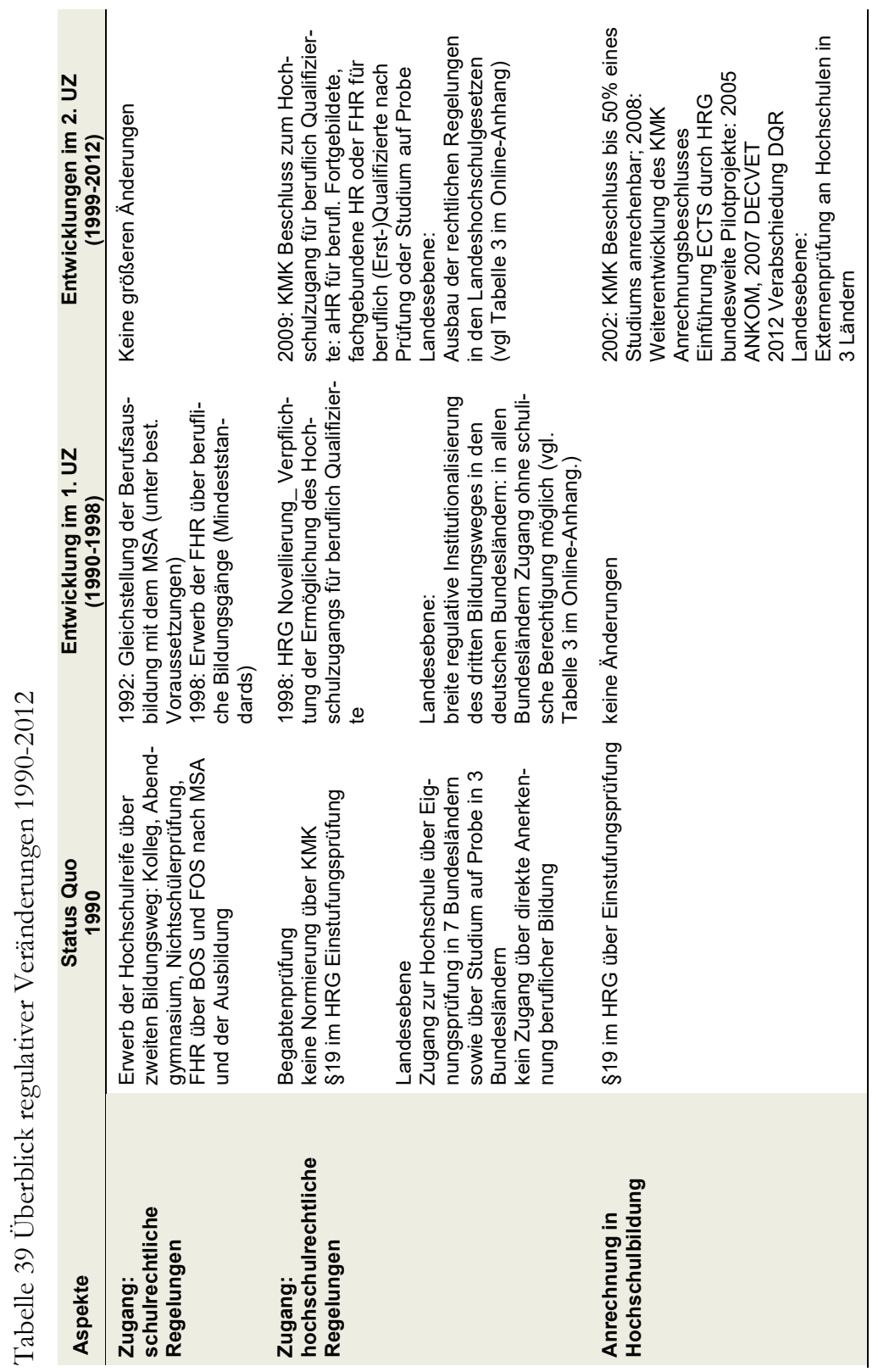




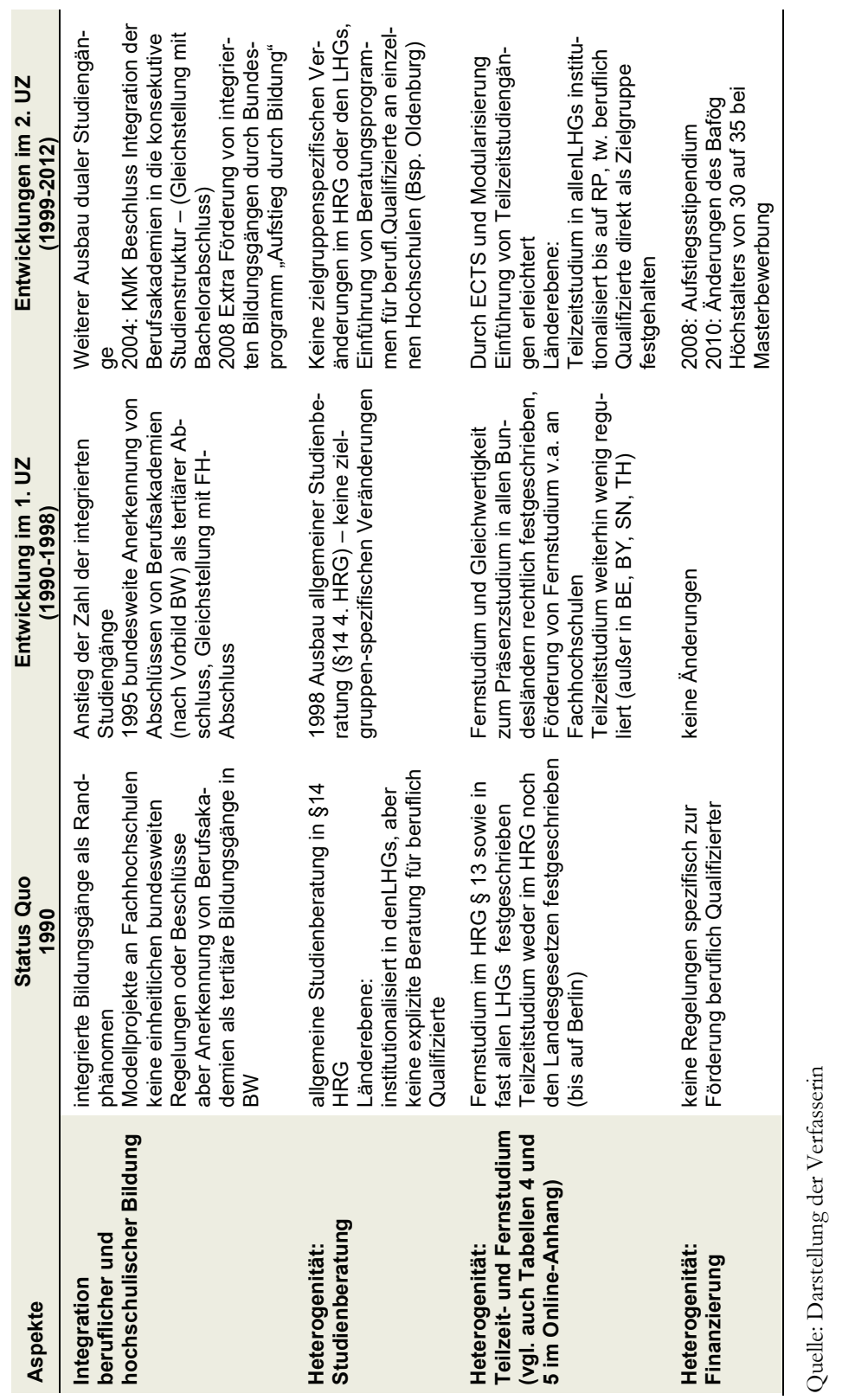


Tabelle 40 Glossar zum französischen Bildungssystem

\begin{tabular}{|c|c|c|}
\hline Französisch & Abkürzung & Deutsch \\
\hline Admission Post Bac & APB & Zulassung nach dem Abitur \\
\hline Alternance & & $\begin{array}{l}\text { alternierende (Aus)bildung zwischen } \\
\text { Schule und Lehrbetrieb }\end{array}$ \\
\hline Baccalauréat général & bac général & Allgemeinbildende Abitur \\
\hline Baccalauréat professionnel & bac pro & Berufsabitur \\
\hline Baccalauréat technologique & bac techno & Technisches Abitur \\
\hline Bilan de compétences & & $\begin{array}{l}\text { Dokumentation informell erworbener beruf- } \\
\text { licher und persönlicher Kompetenzen }\end{array}$ \\
\hline Brevet de maitrise & & Meisterabschluss \\
\hline Brevet de technicien supérieur & BTS & $\begin{array}{l}\text { höheres Technikerzertifikat in schulischer } \\
\text { Ausbildung in den STS }\end{array}$ \\
\hline $\begin{array}{l}\text { Brevet d'études du premier } \\
\text { cycle (du second degré) }\end{array}$ & BEPC & Zertifikat zum Ende der Sekundarstufe I \\
\hline $\begin{array}{l}\text { Brevet d'études professionnel- } \\
\text { les }\end{array}$ & BEP & $\begin{array}{l}\text { 2-jähriger Berufsbildungsabschluss (weni- } \\
\text { ger spezialisiert als der CAP Abschluss) }\end{array}$ \\
\hline Centre d' apprentissage & $\mathrm{CA}$ & Ausbildungszentrum \\
\hline Centre de formation d'apprentis & CFA & Ausbildungszentrum für duale Ausbildung \\
\hline $\begin{array}{l}\text { Certificat d'aptitude profession- } \\
\text { nelle }\end{array}$ & CAP & $\begin{array}{l}\text { Berufsbildungsabschluss im Sekundärbe- } \\
\text { reich, Berufsbefähigungszeugnis }\end{array}$ \\
\hline $\begin{array}{l}\text { Certificat de qualification pro- } \\
\text { fessionnelle }\end{array}$ & CQP & innerbetriebliches Zertifikat \\
\hline $\begin{array}{l}\text { Classes préparatoires aux } \\
\text { grandes écoles }\end{array}$ & CPGE & $\begin{array}{l}\text { Vorbereitungsklassen für die grandes } \\
\text { écoles }\end{array}$ \\
\hline Collège unique & & $\begin{array}{l}\text { Erster Teil der Sekundarschule, den alle } \\
\text { Schüler/innen noch gemeinsam besuchen }\end{array}$ \\
\hline $\begin{array}{l}\text { Commissions professionnelles } \\
\text { consultatives }\end{array}$ & $\mathrm{CPC}$ & $\begin{array}{l}\text { Paritätisch besetzte Ausschüsse, die die } \\
\text { Curricular der beruflichen Bildung mitbe- } \\
\text { stimmen }\end{array}$ \\
\hline Concours & & $\begin{array}{l}\text { leistungsbasiertes Wettbewerbsverfahren } \\
\text { für den Zugang zu den grandes écoles } \\
\text { oder anderen Hochschulen und bestimm- } \\
\text { ten Arbeitsmarktpositionen (meist im öf- } \\
\text { fentlichen Dienst) }\end{array}$ \\
\hline $\begin{array}{l}\text { Diplôme d'Accès aux Etudes } \\
\text { Universitaires }\end{array}$ & DAEU & $\begin{array}{l}\text { Zertifikat, das den Zugang zum Hoch- } \\
\text { schulstudium gewährleistet (zweiter Bil- } \\
\text { dungsweg) }\end{array}$ \\
\hline $\begin{array}{l}\text { Diplôme d'Etudes Supérieures } \\
\text { Specialisées }\end{array}$ & DESS & $\begin{array}{l}\text { beruflich orientiertes Hochschuldiplom, } \\
\text { das im } 5 \text {. Studienjahr erworben wird (jetzt } \\
\text { master professionnel) }\end{array}$ \\
\hline
\end{tabular}




\begin{tabular}{|c|c|c|}
\hline Französisch & Abkürzung & Deutsch \\
\hline $\begin{array}{l}\text { Diplôme universitaire de tech- } \\
\text { nologie }\end{array}$ & DUT & $\begin{array}{l}\text { höhere Technikerzertifikat in universitärer } \\
\text { Ausbildung, technisches Hochschuldiplom }\end{array}$ \\
\hline Doctorat & & Doktorgrad \\
\hline Échec & & Misserfolg \\
\hline $\begin{array}{l}\text { Ecole Nationale des Ponts et } \\
\text { Chaussées }\end{array}$ & & $\begin{array}{l}\text { Nationale Hochschule für den Straßen- } \\
\text { und Brückenbau (grandes école) }\end{array}$ \\
\hline $\begin{array}{l}\text { Ecole Nationale Profes- } \\
\text { sionnelle }\end{array}$ & ENP & $\begin{array}{l}\text { elitäre berufliche Schule des 19. und } 20 . \\
\text { Jahrhunderts zur Ausbildung von Techni- } \\
\text { kern, Vorarbeitern, Aufsehern }\end{array}$ \\
\hline $\begin{array}{l}\text { Ecole Nationale Supérieure } \\
\text { d'Ingénieur }\end{array}$ & ENSI & Hochschule für Ingenieure (grande école) \\
\hline École Polytechnique & & Polytechnische Hochschule (grande école) \\
\hline $\begin{array}{l}\text { Ecole Primaire Supérieure } \\
\text { Professionnelle }\end{array}$ & & $\begin{array}{l}\text { Elitäre berufliche Schule des 19. und } 20 . \\
\text { Jahrhunderts zur Ausbildung von Techni- } \\
\text { kern, Vorarbeitern, Aufsehern }\end{array}$ \\
\hline $\begin{array}{l}\text { Ecoles Manuelle d'Apprentissa- } \\
\text { ge }\end{array}$ & & historische Lehrlingsschule \\
\hline $\begin{array}{l}\text { Ecole Pratique de Commerce } \\
\text { et d'Industrie }\end{array}$ & $\mathrm{EPCl}$ & $\begin{array}{l}\text { Staatlich bezahlte Hochschule für Handel } \\
\text { und Industrie }\end{array}$ \\
\hline $\begin{array}{l}\text { Examen d'accès à l.enseigne- } \\
\text { ment supérieur }\end{array}$ & EAES & Hochschuleingangsprüfung \\
\hline $\begin{array}{l}\text { Examen spécial d.entrée à } \\
\text { l.université }\end{array}$ & ESEU & Hochschuleingangsprüfung \\
\hline Grandes écoles & & Elitehochschulen in Frankreich \\
\hline $\begin{array}{l}\text { Institut universitaire de techno- } \\
\text { logie }\end{array}$ & IUT & $\begin{array}{l}\text { Universitätsinstitut für technologische } \\
\text { Bildung }\end{array}$ \\
\hline $\begin{array}{l}\text { Instituts universitaires de } \\
\text { formation des maitres }\end{array}$ & IUFM & Universitätsinstitut für Lehrerbildung \\
\hline $\begin{array}{l}\text { Instituts universitaires profes- } \\
\text { sionalisés }\end{array}$ & IUP & Universitätsinstitut für berufliche Bildung \\
\hline Licence & & $\begin{array}{l}\text { Bachelor-Abschluss (sowohl allgemeinbil- } \\
\text { dend als auch beruflich orientiert) }\end{array}$ \\
\hline Licence professionnelle & L pro & $\begin{array}{l}\text { Beruflich ausgerichteter Bachelorstudien- } \\
\text { gang }\end{array}$ \\
\hline Lycée & & $\begin{array}{l}\text { Gymnasium/Oberschule (Schulen der } \\
\text { Sekundarstufe II) }\end{array}$ \\
\hline Lycée de métiers & & $\begin{array}{l}\text { berufliche auf ein Berufsfeld konzentrierte } \\
\text { Oberschule, in der verschiedene sekun- } \\
\text { däre und tertiäre Abschlüsse erworben } \\
\text { werden können }\end{array}$ \\
\hline $\begin{array}{l}\text { Lycées d'enseignement profes- } \\
\text { sionnel/ lycée professionnelle }\end{array}$ & & Berufsgymnasium/Berufsschule \\
\hline
\end{tabular}




\begin{tabular}{|c|c|c|}
\hline Französisch & Abkürzung & Deutsch \\
\hline Maîtrise & MSG & Hochschulabschluss nach vier Jahren \\
\hline Master professionnel & & beruflicher Masterabschluss \\
\hline Passerelles & & $\begin{array}{l}\text { institutionalisierte Übergänge zwischen } \\
\text { einzelnen Bildungsgängen }\end{array}$ \\
\hline Première & & $\begin{array}{l}\text { 11. Klasse (vorletzte Klassenstufe der } \\
\text { SEK II) }\end{array}$ \\
\hline Réferentiels & & Inhalt und Methodik eines Bildungsganges \\
\hline $\begin{array}{l}\text { Répertoire national des certifi- } \\
\text { cations professionnelles }\end{array}$ & RNCP & Register der Berufszertifikate \\
\hline Seconde & & 10. Klasse (erste Klassestufe der SEK II) \\
\hline $\begin{array}{l}\text { Sections de techniciens supéri- } \\
\text { eurs }\end{array}$ & STS & $\begin{array}{l}\text { Sektionen für höhere technische Bildung in } \\
\text { Oberschulen }\end{array}$ \\
\hline Sixième & & $\begin{array}{l}\text { Erstes Jahr der Sekundarschule in Frank- } \\
\text { reich }\end{array}$ \\
\hline Terminale & & 12. Klasse (letzte Klassenstufe der SEK II) \\
\hline Troisième & & 9. Klasse (letzte Klassenstufe der SEK I) \\
\hline $\begin{array}{l}\text { Validation des acquis de l'expé- } \\
\text { rience }\end{array}$ & VAE & $\begin{array}{l}\text { Zertifizierung beruflich und außerberuflich } \\
\text { erworbener Kompetenzen }\end{array}$ \\
\hline $\begin{array}{l}\text { Validation des acquis profes- } \\
\text { sionnels }\end{array}$ & VAP & $\begin{array}{l}\text { Verfahren zur Validierung beruflicher Kom- } \\
\text { petenzen }\end{array}$ \\
\hline Voie professionelle & & Beruflicher Bildungsweg \\
\hline
\end{tabular}

Quelle: Aufstellung der Autorin

Tabelle 41 Studienerfolg in der licence innerhalb von vier Jahren je nach Abiturschwerpunkt im Abiturjahrgang 2007

\begin{tabular}{lcc}
\hline Art des Abiturs (+Schwerpunkt) & $\begin{array}{c}\text { Anteil an den eingeschrie- } \\
\text { benen Studierenden (\%) }\end{array}$ & $\begin{array}{c}\text { davon Abschluss nach } \\
\text { insgesamt 4 Jahren (\%) }\end{array}$ \\
\hline Bac general: Littéraire & 21,4 & 43,7 \\
Bac general: Economique & 29,3 & 47,9 \\
Bac general: Scientifique & 24,6 & 48,4 \\
Bac techno STG & 10,6 & 12,5 \\
Bac techno, andere Schwerpunkte & 5,3 & 15,7 \\
Bac Professionnel & 4,1 & 4,6 \\
Nicht-Abiturienten & 4,7 & 30,6 \\
\hline Insgesamt & 100 & 39,0 \\
\hline \hline
\end{tabular}

Quelle: DEPP 2013b:267, Übersetzung NB 
Tabelle 42 Studienerfolg am IUT nach Typ des Abiturs innerhalb von drei Jahren in $\%$ (Kohorte 2008)

\begin{tabular}{lcccc} 
& IUT Produktionssektor & \multicolumn{2}{c}{ IUT Dienstleistungssektor } \\
Abiturarten & Gesamtzahl & $\begin{array}{c}\text { Davon } \\
\text { schluss in } \\
\text { 3 Jahren (\%) }\end{array}$ & Gesamtzahl & $\begin{array}{c}\text { Davon Ab- } \\
\text { schluss in } \\
\text { 3 Jahren (\%) }\end{array}$ \\
\hline Baccalauréat général & 16.030 & 81,1 & 23.228 & 82,1 \\
Baccalauréat technologique & 6.195 & 66,2 & 9.052 & 67,1 \\
Baccalauréat professionnel & 283 & 45,7 & 690 & 49,5 \\
Abiturienten insgesamt & 22.508 & 76,6 & 32.970 & 77,3 \\
Nicht-Abiturienten & 1.223 & 58,5 & 1.069 & 54,5 \\
\hline Total & 23.731 & 75,6 & 34.039 & 76,6 \\
\hline \hline
\end{tabular}

Quelle: DEPP (2013b: 261, Übersetzung NB)

Tabelle 43 Studienerfolg in den STS nach Typ des Abiturs im Jahr 2012

\begin{tabular}{lccc} 
& $\begin{array}{c}\text { Angetreten zur } \\
\text { Prüfung }\end{array}$ & $\begin{array}{c}\text { Zugelassen zur } \\
\text { Prüfung }\end{array}$ & Erfolgsquote (\%) \\
\hline Baccalauréat général & 46.688 & 38.953 & 83,4 \\
Baccalauréat technologique & 71.384 & 54.026 & 75,7 \\
Baccalauréat professionnel & 35.282 & 20.404 & 57,8 \\
Autres diplômes (BT, BMA, & 8.795 & 5.246 & 59,6 \\
étrangers...) & 162.149 & 118.629 & 73,2 \\
\hline Total BTS & &
\end{tabular}

Quelle: DEPP (2013b: 255; Übersetzung NB)

Abbildung 12 Entwicklung der Zahl der Validierungen mit dem VAE Verfahren seit 2002 in Frankreich

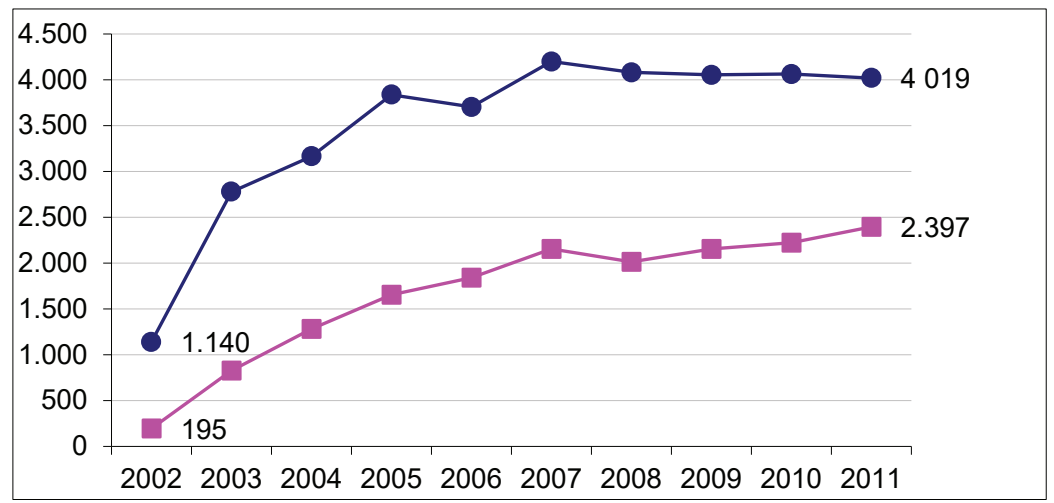

Legende: Die Trendlinie mit den Quadraten sind die Validierungen, die zu vollständigen Diplomen geführt haben. Die Trendlinie mit den Kreisen sind alle Validierungsverfahren.

Quelle: DEPP (2013b: 281; Übersetzung NB) 


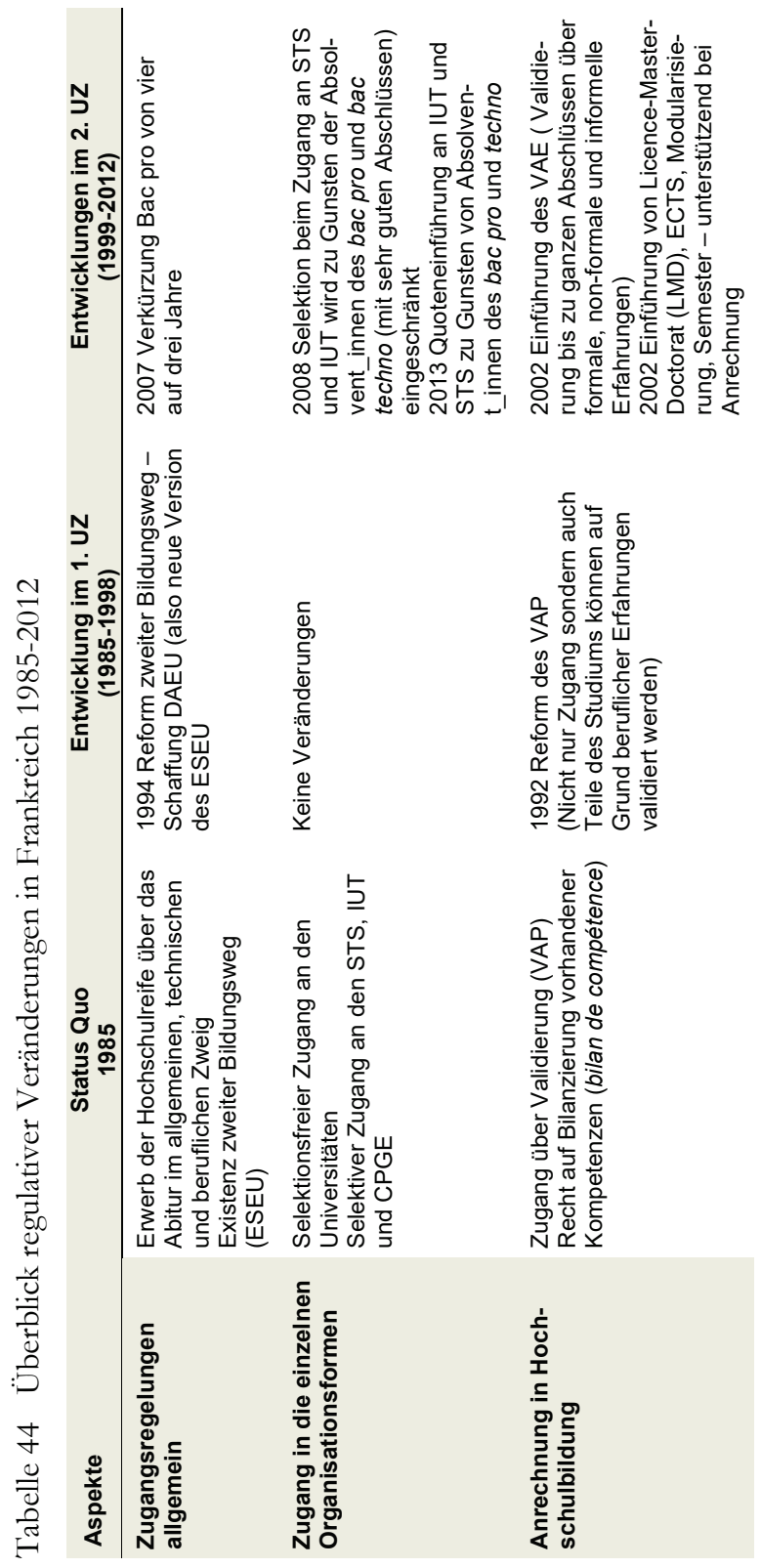




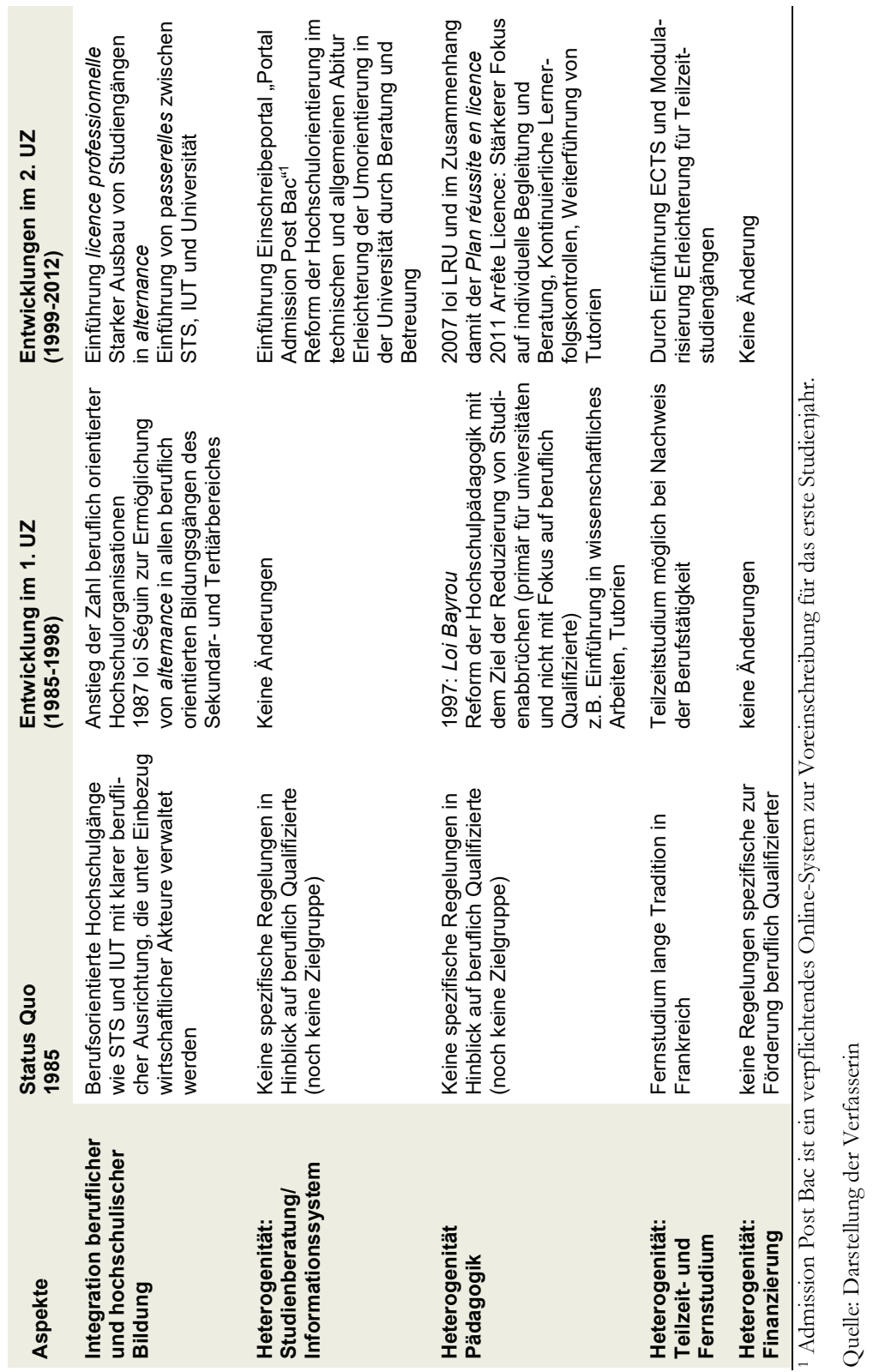




\section{Übersicht über die geführten Expert_inneninterviews}

Tabelle 45 Expert_inneninterviews in Deutschland

\begin{tabular}{|c|c|c|c|}
\hline Organisation & Abkürzung & Datum & Ort \\
\hline $\begin{array}{l}\text { Bundesinstitut für Berufsbildung, Bereich } \\
\text { Internationales* }\end{array}$ & BIBB1 & 16.03.2011 & Bonn \\
\hline $\begin{array}{l}\text { Bundesinstitut für Berufsbildung, Bereich } \\
\text { Internationales }\end{array}$ & BIBB2 & 18.03 .2011 & Bonn \\
\hline $\begin{array}{l}\text { Bundesinstitut für Berufsbildung, Bereich Qualität, } \\
\text { Nachhaltigkeit, Durchlässigkeit* }\end{array}$ & BIBB3 & 16.03 .2011 & Bonn \\
\hline $\begin{array}{l}\text { Bundesinstitut für Berufsbildung, Bereich Qualität, } \\
\text { Nachhaltigkeit, Durchlässigkeit }\end{array}$ & BIBB4 & 17.03 .2011 & Bonn \\
\hline $\begin{array}{l}\text { Bundesinstitut für Berufsbildung, Bereich Ordnung } \\
\text { der Berufsbildung }\end{array}$ & BIBB5 & 15.03 .2011 & Bonn \\
\hline Bundesinstitut für Berufsbildung*** & BIBB6 & 16.03 .2011 & Bonn \\
\hline Hochschulrektorenkonferenz & HRK & 18.03 .2011 & Bonn \\
\hline Bundesministerium für Bildung und Forschung & BMBF & 06.06 .2011 & Berlin \\
\hline
\end{tabular}

* Interview mit 2 Expert_innen

** Gespräche, die nicht aufgenommen und transkribiert werden konnten, Interviewnotizen als Grundlage

*** Die interviewte Person war zum Zeitpunkt des Gespräches bereits in Rente. Das Gespräch bezog sich aber auf die Erfahrungen als Repräsentant_in der Organisation

Die Interviews BIBB1 bis BIBB4 sowie BIBB6 wurden zusammen mit Lukas Graf und Justin Powell und die Interviews HRK und BMBF zusammen mit Lukas Graf durchgeführt. 
Tabelle 46 Expert_inneninterviews mit bildungspolitischen Akteuren in Frankreich

\begin{tabular}{|c|c|c|c|}
\hline Organisation & Abkürzung & Datum & Ort \\
\hline $\begin{array}{l}\text { Confédération générale du patronat des petites et } \\
\text { moyennes entreprises }\end{array}$ & CGPME & 29.3.2010 & Paris \\
\hline Confédération générale du travail** & CGT & $\begin{array}{l}19.3 .2010 \\
24.3 .2010\end{array}$ & Paris \\
\hline $\begin{array}{l}\text { La Commission nationale de la certification profes- } \\
\text { sionnelle }\end{array}$ & $\mathrm{CNCP}$ & 15.3.2010 & Paris \\
\hline La Conférence des présidents d'université* & CPU1 & 19.3.2010 & Paris \\
\hline L'Association des Directeurs d'IUT** & ADIUT & 19.3.2010 & Paris \\
\hline Le Mouvement des entreprises de France* & MEDEF & 22.3.2010 & Paris \\
\hline Ministère de l'Education Nationale *** & MEN & 10.3.2010 & Paris \\
\hline $\begin{array}{l}\text { Ministère de l'Enseignement supérieur et de la } \\
\text { Recherche }{ }^{\star * \star}\end{array}$ & MESR1 & 17.3.2010 & Paris \\
\hline $\begin{array}{l}\text { Syndicat National Unitaire de l'Enseignement } \\
\text { Professionnel* }\end{array}$ & SNUEP & 25.3 .2010 & Paris \\
\hline La Conférence des présidents d'université & CPU2 & 23.05.2011 & Paris \\
\hline La Conférence des présidents d'université & CPU3 & 23.05.2011 & Paris \\
\hline $\begin{array}{l}\text { Ministère de l'Enseignement supérieur et de la } \\
\text { Recherche }\end{array}$ & MESR2 & 20.5 .2011 & Paris \\
\hline Sauvons l'université* & Sauvons & 25.5 .2011 & Paris \\
\hline
\end{tabular}

* Interview mit 2 Expert_innen

** Gespräche, die nicht aufgenommen und transkribiert werden konnten, Interviewnotizen als Grundlage

*** Die interviewte Person war zum Zeitpunkt des Gespräches bereits in Rente. Das Gespräch bezog sich aber auf die Erfahrungen als Repräsentant_in der Organisation 
Tabelle 47 Expert_inneninterviews mit (Bildungs-)Forscher_innen in Frankreich

\begin{tabular}{|c|c|c|c|}
\hline Organisation & Abkürzung & Datum & Ort \\
\hline Centre de sociologie des organisations & $\mathrm{CSO}$ & 26.3 .2010 & Paris \\
\hline Centre Maurice Halbwachs & $\mathrm{CMH} 1$ & 22.3.2010 & Paris \\
\hline Centre Maurice Halbwachs & $\mathrm{CMH} 2$ & 23.3.2010 & Paris \\
\hline Université de Paris V René Descartes & Paris V & 11.3.2010 & Paris \\
\hline Université Paris Ouest Nanterre** & CNRS & 23.3.2010 & Paris \\
\hline $\begin{array}{l}\text { Centre d'études et de recherches sur les qualifica- } \\
\text { tions: Schwerpunkt Licence professionnelle }\end{array}$ & CEREQ1 & 18.5 .2011 & Marseille \\
\hline $\begin{array}{l}\text { Centre d'études et de recherches sur les qualifica- } \\
\text { tions: Schwerpunkt bac pro }\end{array}$ & CEREQ2 & 18.5.2011 & Marseille \\
\hline $\begin{array}{l}\text { Centre d'études et de recherches sur les qualifica- } \\
\text { tions: Schwerpunkt bac pro }\end{array}$ & CEREQ3 & 19.5.2011 & Marseille \\
\hline $\begin{array}{l}\text { Centre d'études et de recherches sur les qualifica- } \\
\text { tions: Schwerpunkt Verberuflichung der } \\
\text { Hochschulbildung }\end{array}$ & CEREQ4 & 19.5.2011 & Marseille \\
\hline $\begin{array}{l}\text { Centre d'études et de recherches sur les qualifica- } \\
\text { tions: Schwerpunkt Weiterbildung }\end{array}$ & CEREQ5 & 19.5.2011 & Marseille \\
\hline $\begin{array}{l}\text { Centre d'études et de recherches sur les qualifica- } \\
\text { tions: Schwerpunkt Internationales }\end{array}$ & CEREQ6 & 19.5.2011 & Marseille \\
\hline
\end{tabular}

